\title{
Synthesis of Functional 1,2,3-Triazoles and Its Applications in Transition Metal Catalysis
}

Wuming Yan

West Virginia University

Follow this and additional works at: https://researchrepository.wvu.edu/etd

\section{Recommended Citation}

Yan, Wuming, "Synthesis of Functional 1,2,3-Triazoles and Its Applications in Transition Metal Catalysis" (2012). Graduate Theses, Dissertations, and Problem Reports. 4941.

https://researchrepository.wvu.edu/etd/4941

This Dissertation is protected by copyright and/or related rights. It has been brought to you by the The Research Repository @ WVU with permission from the rights-holder(s). You are free to use this Dissertation in any way that is permitted by the copyright and related rights legislation that applies to your use. For other uses you must obtain permission from the rights-holder(s) directly, unless additional rights are indicated by a Creative Commons license in the record and/ or on the work itself. This Dissertation has been accepted for inclusion in WVU Graduate Theses, Dissertations, and Problem Reports collection by an authorized administrator of The Research Repository @ WVU.

For more information, please contact researchrepository@mail.wvu.edu. 


\title{
Synthesis of Functional 1,2,3-Triazoles and Its Applications in \\ Transition Metal Catalysis
}

\author{
Wuming Yan \\ Dissertation submitted to the \\ Eberly College of Arts and Sciences \\ at West Virginia University \\ in partial fulfillment of the requirements \\ for the degree of \\ Doctor of Philosophy \\ in \\ Organic Chemistry \\ Xiaodong Shi, Ph.D., Chair \\ Kung K. Wang, Ph.D. \\ Björn Söderberg, Ph.D. \\ Jeffrey L. Petersen, Ph.D. \\ Peter Gannett, Ph.D.
C. Eugene Bennett Department of Chemistry
Morgantown, West Virginia \\ 2012
}

Keywords: Functional 1,2,3-Triazoles, Transition Metal Catalysis, Allene, Conjugated Enyne, Fluorophore, Planar-Intramolecular-Charge-Transfer, Iron Chemistry, Photoredox Catalysis 


\title{
ABSTRACT \\ Synthesis of Functional 1,2,3-Triazoles and Its Applications in Transition Metal Catalysis
}

\author{
Wuming Yan
}

Several new methodologies for synthesizing functional 1,2,3-triazoles which include arylation, vinylation, propargylation and allenation, have been successfully developed. These resulting new methods are of great benefit to the organic chemists who are working on the research fields related to 1,2,3-triazoles. Particularly, the iron catalyzed propargylation and allenation have initiated the iron chemistry with 1,2,3-triazoles as the ligand. For example, our efforts led to the discovery of a practical approach for the synthesis of conjugated enynes that is challenging through conventional methods. In the synthesis of conjugated enyne, 1,2,3-triazoles were revealed as the only ligand effectively promoting this important and challenging transformation (gave up to $95 \%$ yields). Other tested N-heterocycles, including the 1,2,4-triazole, tetrazole, and pyridine, all gave poor results $(<10 \%)$ even with similar binding pattern, which demonstrating the unique role of 1,2,3-triazole in iron catalysis.

In addition, the complexes $\left[\operatorname{Ir}\left(\mathrm{L}^{\wedge} \mathrm{X}\right)(\operatorname{ArTriaz})_{2}\right]$ and $\left[\operatorname{Ir}(\right.$ bbpy $\left.)(\operatorname{ArTriaz})_{2}\right] \mathrm{Cl}$ have been successfully prepared through N-2-aryl-1,2,3-triazoles as the efficient binding ligands in coordination with iridium salt in excellent yields. These air and moisture stable Ir(III) complexes showed effective catalytic reactivity in photoredox catalysis.

The N-2-aryl-1,2,3-triazole derivatives (NATs) were developed as a new class of UV/blue-light-emitting fluorophores. Though both $\mathrm{N}$-1-aryl-1,2,3-triazoles and N-2-aryl1,2,3-triazoles gave strong photo absorption under excitation at $330 \mathrm{~nm}$, only the $\mathrm{N}-2$ analogous showed strong fluorescence emission in the UV/blue range with high efficiency in various solvents. The computational studies along with X-ray crystal structures indicated the significance of the effective conjugation between triazole ring and aryl groups on the N-2 position. The planar intramolecular charge transfer (PICT) mechanism was proposed, which was supported by solvent effect studies. 


\section{DEDICATED TO}

My wife, Yalan XING, my daughter, Emma Jiayun YAN My parents, Qingshen YAN, Baixiang YAN 


\section{ACKNOWLEDGEMENTS}

I would like to express my deepest gratitude to my supervisor, Dr. Xiaodong Michael Shi, I have been so fortunate to have an advisor who gave me the freedom to explore on my own in the science of organic chemistry and at the same time the guidance to recover when I failed. Michael taught me how to become a real and better organic chemist. His patience and support helped me overcome many crisis situations. Without his help, this dissertation would truly not have been possible.

I would like to give my sincere appreciation to Dr. Kung K. Wang and Dr. Björn Söderberg in my academic development. Their invaluable advice and suggestion throughout my years here in WVU are appreciated and acknowledged. I would also like to thank Dr. Jeffrey L. Petersen for his countless number of X-ray crystallographic analyses and guidance during my graduate studies. Moreover, I am deeply grateful to the above mentioned and Dr. Peter Gannett for their roles on my Doctoral Research Committee.

I would like to acknowledge my colleagues who I've had the pleasure of working with, Dr. Haifeng Duan, Dr. Yuxiu Liu, Dr. Yunfeng Chen, Dr. Chen Zhong, Dr. Sujata Sengupta, Dr. Dawei Wang, Wenyan Liao, Tao Liao, Lekn Nath Gautam, Qiaoyi Wang, Xiaohan Ye, Rong Cai, Yanwei Zhang, Sraven Kumar, Siddhita Aparaj, Feroz Mohammad, Sathya Girigani, Naveen Diddi for all the assistance they have given me in the last four years. I would also like to thank Dr. Novruz Akhmedov for his kind and friendly help during NMR experiments.

Though only my name appears on the cover of this dissertation, a great many people have contributed to its production. I would like to express my heart-felt gratitude 
to my family for their unconditional love, support and guidance throughout my life. My parents, my wife and my daughter have been a constant source of love, concern, support and strength all these years. I have to give a special mention for the support and love given by my parents-in-law. Without their understanding, encouragement and support, I could not have finished this thesis.

Financial supports from the C. Eugene Bennett department of chemistry at West Virginia University (particularly for a John R. Conard Fellowship) and the National Science Foundation are also gratefully acknowledged. 


\section{TABLE OF CONTENTS}

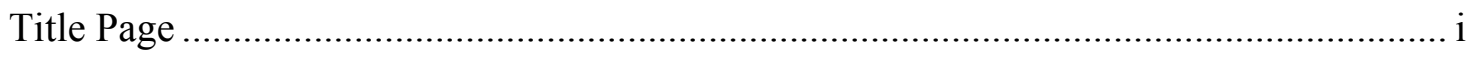

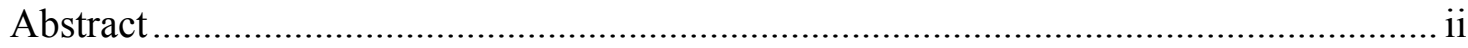

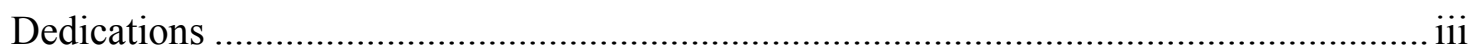

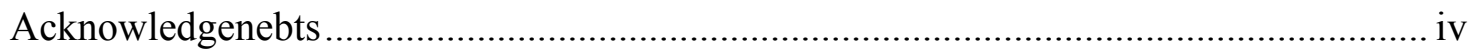

Table of Contents ................................................................................................. vi

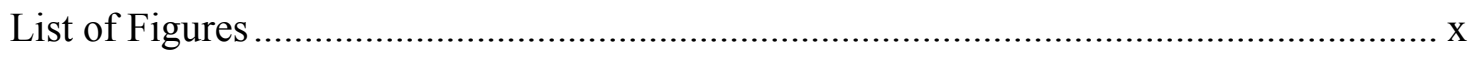

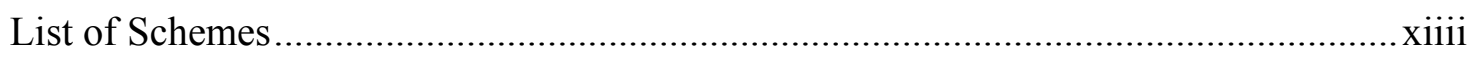

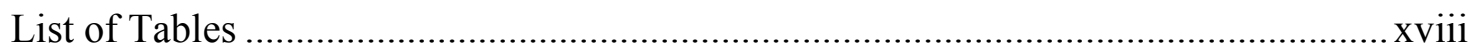

Chapter One: Synthesis of functional 1,2,3-triazoles ........................................... 1

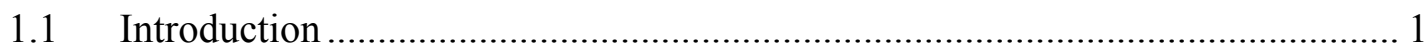

1.1.1 Synthesis of precursor: $\mathrm{NH}-1,2,3$-triazole .............................................. 1

1.1.2 Our strategy: How to reach the N-2 substitution of $\mathrm{NH}-1,2,3$-triazoles? ..... 6

1.2 Efficient synthesis of N-2-aryl-1,2,3-triazole fluorophores via post-triazole

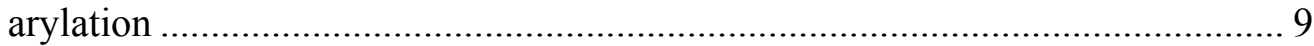

1.2.1 Reported method for synthesizing N-2-aryl-1,2,3-triazole........................ 9

1.2.2 Research Objective and Results........................................................... 10

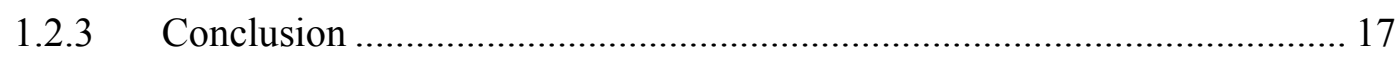

1.3 Highly efficient synthesis of vinyl substituted triazoles by $\mathrm{Au}(\mathrm{I})$ catalyzed

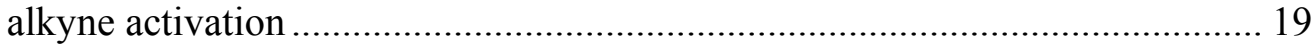

1.3.1 Reported methods for synthesis vinyl substituted triazoles ...................... 19

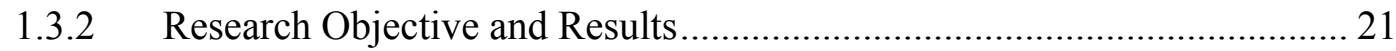

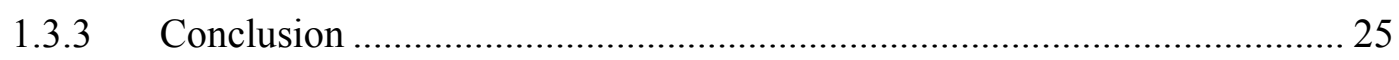

1.4 Iron catalyzed C-O bond activation for the synthesis of propargyl-1,2,3-triazoles

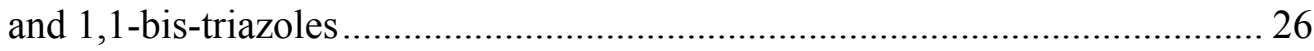

1.4.1 Examples of traditional propargylation ................................................. 26 
1.4.2 Research Objective and Results.......................................................... 27

1.4.3 Application in synthesis of 1,1-bis-triazoles via 'click' chemistry............. 33

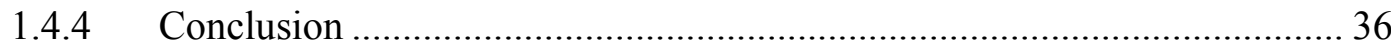

1.5 Synthesis of allene triazole through iron catalyzed regioselective addition to

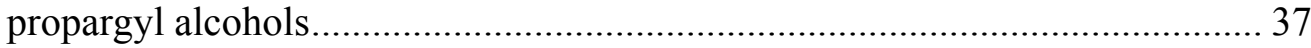

1.5.1 Previous approaches for synthesis allene compounds ............................. 37

1.5.2 Examples of allenes ligands and catalysts. .......................................... 40

1.5.3 Research Objective and Results........................................................... 42

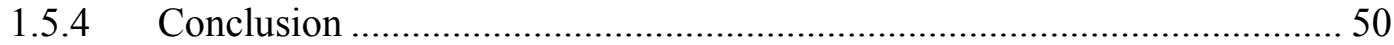

1.6 Mitsunobu reaction of 1,2,3-NH-triazoles: regio- and stereo-selective synthesis

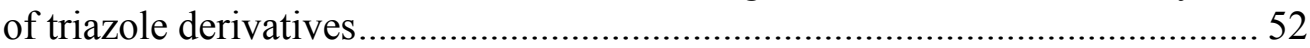

1.6.1 Two practical problems from previous 1,2,3-triazole functionalizations ... 52

1.6.2 Introduction of Mitsunobu reaction ........................................................ 54

1.6.3 An example: Improvement of regioselective $\mathrm{N}-2$ isomers from ligand

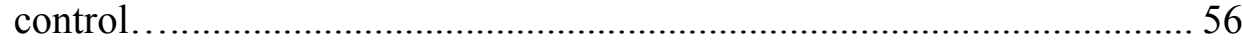

1.6.4 Research Objective and Results....................................................... 57

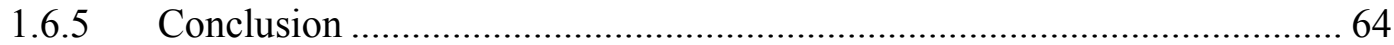

Chapter Two: 1,2,3-trizazoles applied as ligands in transition metal catalysis ........ 66

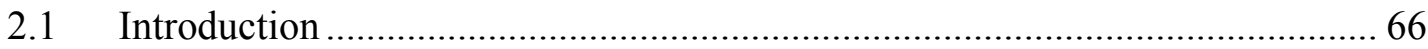

2.1.1 Substituted 1,2,3-triazoles as neutral ligands.......................................... 66

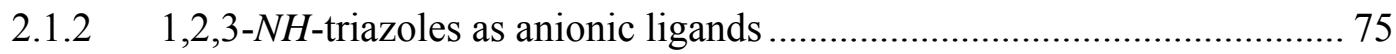

2.1.3 Achievements of our group by using 1,2,3-triazoles as the ligands ........... 77

2.2 Synthesis of $\left[\operatorname{Ir}\left(\mathrm{L}^{\wedge} \mathrm{X}\right)(\operatorname{ArTriaz})_{2}\right]$ and $\left[\operatorname{Ir}(\right.$ bbpy $\left.)(\operatorname{ArTriaz})_{2}\right] \mathrm{Cl}$ complexes and their application in photoredox catalysis ..................................................... 80

2.2.1 Reported examples of triazole-Ir(III) complexes..................................... 80

2.2.2 Synthesis of $\left[\operatorname{Ir}\left(\mathrm{L}^{\wedge} \mathrm{X}\right)(\operatorname{ArTriaz})_{2}\right]$ and $\left[\operatorname{Ir}(\right.$ bbpy $\left.)(\text { ArTriaz })_{2}\right] \mathrm{Cl}$ complexes.. 83

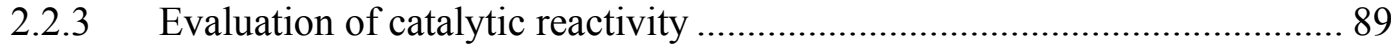

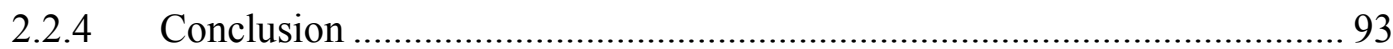


2.3 1,2,3-Triazole as Unique Ligand in Promoting Iron Catalyzed Propargyl Alcohol Dehydration for the Synthesis of Conjugated Enynes

2.3.1 General introduction of Iron chemistry .................................................. 94

2.3.2 Research Objective and Results.............................................................. 98

2.3.3 Tuning the reactivity of conjugated enynes in 1,4-bromolactonization. .. 106

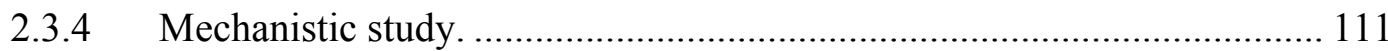

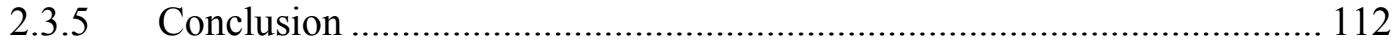

Chapter Three: N-2-aryl-1,2,3-triazoles: a new class of UV-Blue emitting fluorophores through PICT .................................................... 114

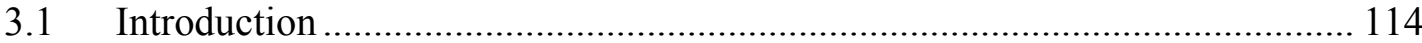

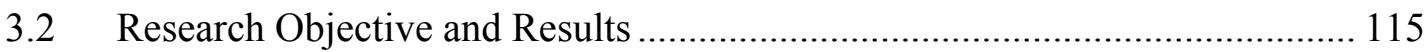

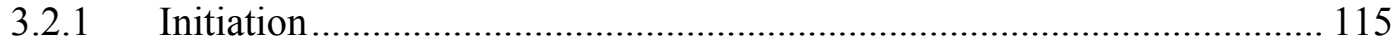

3.2.2 Influence of substituted groups on the C-4 and C-5 positions................. 116

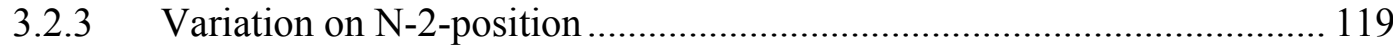

3.2.4 Substituent group electronic effect ….................................................. 122

3.2.5 NATs: Tunable UV/blue emitting small molecule dyes......................... 125

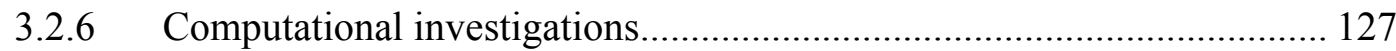

3.2.7 Extended NAT fluorescence probes ….............................................. 129

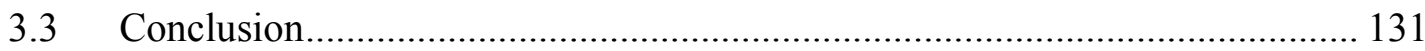

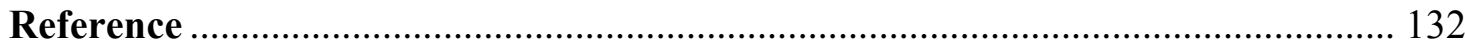

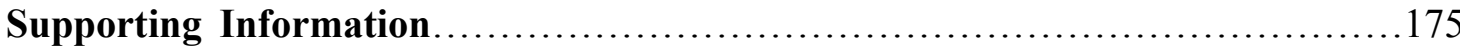

Chapter One: Synthesis of functional 1,2,3-triazoles.............................175

1.2 Efficient synthesis of N-2-aryl-1,2,3-triazole fluorophores via post-triazole arylation........................................................

1.3 Highly efficient synthesis of vinyl substituted triazoles by $\mathrm{Au}(\mathrm{I})$ catalyzed

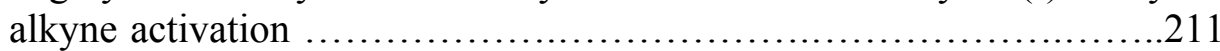

1.4 Iron catalyzed C-O bond activation for the synthesis of propargyl-1,2,3triazoles and 1,1-bis-triazoles..................................229

1.5 Synthesis of allene triazole through iron catalyzed regioselective addition to propargyl alcohols............................................254 
1.6 Mitsunobu reaction of 1,2,3-NH-triazoles: regio- and stereo-selective synthesis of triazole derivatives.......................................296

Chapter Two: 1,2,3-trizazoles applied as ligands in transition metal catalysis 330

2.2 Synthesis of $\left[\operatorname{Ir}\left(\mathrm{L}^{\wedge} \mathrm{X}\right)(\right.$ ArTriaz $\left.) 2\right]$ and $[\operatorname{Ir}($ bbpy $)($ ArTriaz $) 2] \mathrm{Cl}$ complexes and their application in photoredox catalysis..........................330

2.3 1,2,3-Triazole as Unique Ligand in Promoting Iron Catalyzed Propargyl Alcohol Dehydration for the Synthesis of Conjugated Enynes............342

Chapter Three: N-2-aryl-1,2,3-triazoles: a new class of UV-Blue emitting fluorophores through Planar-Intramolecular-Charge-Transfer (PICT)..............398

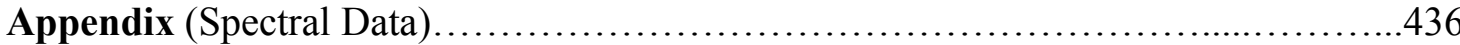




\section{LIST OF FIGURES}

Figure 1: Proposed reaction mechanism of "Click chemistry" ........................................ 3

Figure 2: Mechanism for synthesis of 4,5-disubstituted $\mathrm{NH}$-1,2,3-triazoles. ................... 6

Figure 3: Strategy for selective N-2 substitution (two bulky groups on C-4 and C-5)..... 7

Figure 4: The C-4 aryl group was nearly coplanar with the triazole ring........................ 8

Figure 5: Conformational control for selective N-2 substitution..................................... 8

Figure 6: Products from trans-metallation coupling with arylboronic acids. .................. 15

Figure 7: Comparison of photonic luminescence of selected N-1 and N-2-aryl triazoles (1.2.5b and 1.2.5c)...

Figure 8: Photonic luminescence of N-2-aryl triazoles with different substituted groups on the $\mathrm{C}-5$ position.

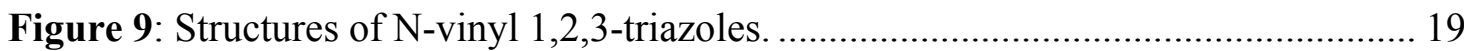

Figure 10: Synthesis of N-vinyl 1,2,3-triazole via elimination of alkyl triazole. ............ 20

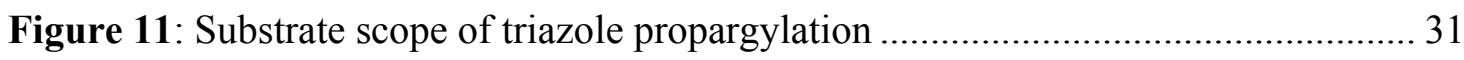

Figure 12: Potential formation of Nazarov cyclization product ................................... 32

Figure 13: Most general method for synthesis allenes via nucleophilic $\mathrm{S}_{\mathrm{N}} 2^{\prime}$ substitution.

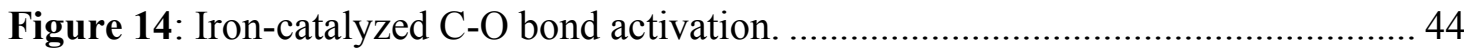

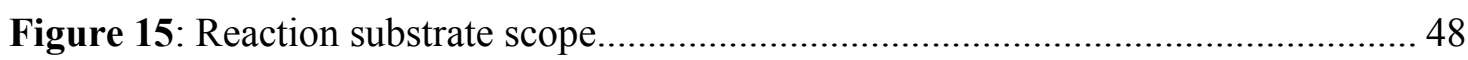

Figure 16: Extending the substrate scope with propargyl acetate................................ 49

Figure 17: Five different functionalizations of 1,2,3-triaozles. .................................... 52

Figure 18: N-1 \& N-2 selectivities of 1,2,3-NH-triazoles.......................................... 53

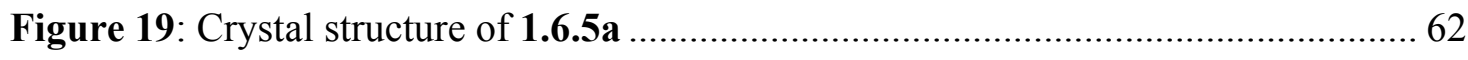

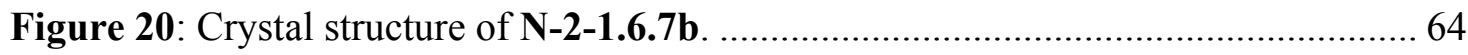

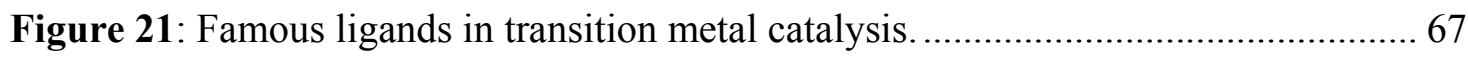

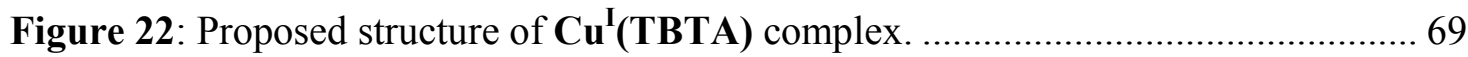

Figure 23: Proposed dinuclear structure of $\mathrm{Rh}(\mathrm{I})$-triazole complexes............................ 76 
Figure 24: Chelating triazoles derived from 2-ethynyl pyridine (A), 2-picolylethyne (B), and 2-azidomethylpyridine (C).

Figure 25: Different kind of biscyclometalated iridium complexes. 81

Figure 26: Enhanced luminescent Ir(III) complexes bearing aryltriazole cyclometallated ligands reported by Zysman-Colman.

Figure 27: Control of the Mutual Arrangement of Cyclometalated Ligands in Cationic Ir(III) Complexes. 83

Figure 28: Four different kinds of biscyclometalated $\operatorname{Ir}(\mathrm{III})$ complexes. 84

Figure 29: Three different potential binding sites for forming $\operatorname{Ir}(\mathrm{III})$ complexes in the 1,2,3-triazole ligands 85

Figure 30: Six different $\left[\operatorname{Ir}\left(\mathbf{L}^{\wedge} \mathbf{X}\right)(\operatorname{ArTriaz})_{2}\right]$ complexes. 86

Figure 31: X-ray crystal structure for triazole bound $\operatorname{Ir}(\mathrm{III})$ complex $\mathbf{2 . 2 . 3 b}$................. 86

Figure 32: X-ray crystal structure for triazole bound $\operatorname{Ir}(\mathrm{III})$ complex $\mathbf{2 . 2 . 3 c}$................... 86

Figure 33: X-ray crystal structure for triazole bound $\operatorname{Ir}(\mathrm{III})$ complex 2.2.3d................. 87

Figure 34: Conformational stability analysis of triazole-Ir(III) complexes...................... 87

Figure 35: UV absorption spectra of triazole-Ir(III) complexes................................... 88

Figure 36: Fluorescence spectra of triazole-Ir(III) complexes at $\lambda_{\text {ex }}=286 \mathrm{~nm} \ldots \ldots \ldots \ldots \ldots . . .88$

Figure 37: Fluorescence spectra of triazole-Ir(III) complexes at $\lambda_{\mathrm{ex}}=369 \mathrm{~nm} \ldots \ldots \ldots \ldots \ldots . . . .89$

Figure 38: Proposed mechanism for oxidative aza-Henry reaction by Stephenson. ....... 90

Figure 39: Mechanistic insight of Aza-Henry reaction with nitroalkene.........................92

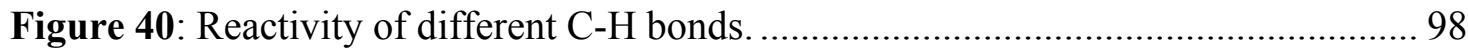

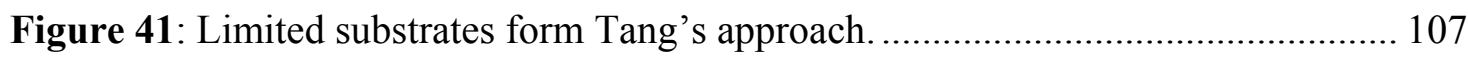

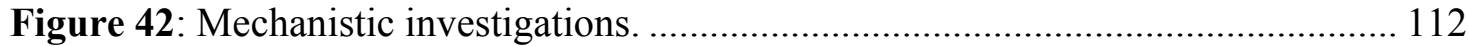

Figure 43: Photoluminescent spectra of N-2-aryl-1,2,3-triazole ................................. 116

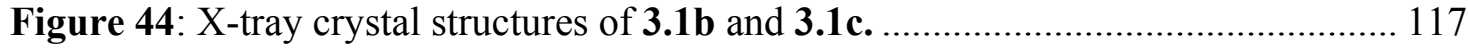

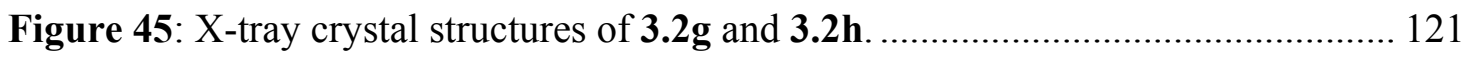

Figure 46: Photoluminescent spectra of N-2-aryl-1,2,3-triazole with various $\mathrm{R}_{\mathrm{N}-2-p-\mathrm{Ph}}$ or

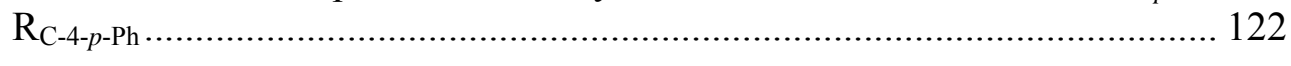

Figure 47: Photoluminescent spectra of N-2-aryl-1,2,3-triazole ................................ 125 
Figure 48: Range of Stock shifts for N-2-aryl-1,2,3-triazole. 126

Figure 49: HOMO and LUMO orbitals of compounds 3.2d and 3.3g..... 127

Figure 50: The most stable conformation of N-1-1e' from computational studies: twisted $\mathrm{N}$-1-aryl ring. 128 


\section{LIST OF SCHEMES}

Scheme 1: Thermal 1,3-dipolar cycloaddition of acetylenes and azides. ......................... 1

Scheme 2: The synthesis of 1,4-disubstituted 1,2,3-triaozles by Click chemistry............ 2

Scheme 3: Example of CuAAC reported by Shapless. .................................................. 2

Scheme 4: Synthesis of $\mathrm{NH}-1,2,3$-triazoles through CuAAC ........................................ 4

Scheme 5: The synthesis of $N H-1,2,3$-triazoles by Zefirov's method. ............................. 4

Scheme 6: Thermal condensation of $\alpha$-nitroalkene and sodium azide (Zard's method). .. 5

Scheme 7: Lewis base-catalyzed cascade nitroalkene-aldehyde-azide condensation........ 5

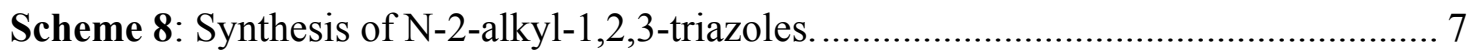

Scheme 9: The alkylation of benzyl ketone triazole. .................................................. 8

Scheme 10: The hydrazine/ $\alpha$-hydoxyketone condensation............................................. 9

Scheme 11: Copper catalyzed transmetalation between triazoles and boronic acids....... 15

Scheme 12: Vinyl azide: challenging substrate for dipolar cycloaddition due to the existence of equilibrium.............................................................................. 20

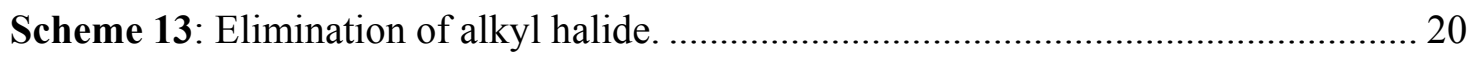

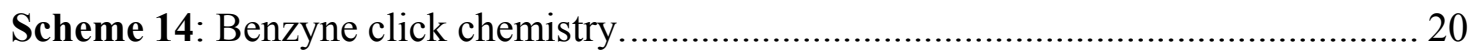

Scheme 15: Substituted scope of different $\mathrm{NH}-1,2,3$-triazoles. ......................................... 24

Scheme 16: Meyer-Schuster rearrangement and its mechanism.................................... 26

Scheme 17: Metal promoted propargylation of arenes................................................... 27

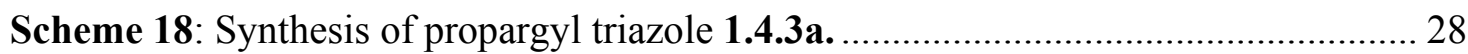

Scheme 19: Challenges in the triazole propargylation................................................. 28

Scheme 20: Unexpected decomposition of terminal propargyl triazole. .......................... 33

Scheme 21: Three Different Types of Bis-triazole Compounds...................................... 34

Scheme 22: Synthesis of unsymmetrical N-C type bis-triazoles................................... 35

Scheme 23: $\mathrm{Cp}_{2} \mathrm{Zr}(\mathrm{H}) \mathrm{Cl}$ catalyzed preparation of chiral allenes. ................................. 38

Scheme 24: Appel-type reactions for synthesis haloellenes........................................ 39 
Scheme 25: Synthesis of haloellenes from propargyl diethanolamine boronates. 39

Scheme 26: Formation of bromoallenes by using $\mathrm{NbBr}_{5}$ 39

Scheme 27: Application of organometallic reagents in preparation of allenes. 40

Scheme 28: Enantioselective addition of (i-Pr) ${ }_{2} \mathrm{Zn}$ to 2-(alkynyl)peprinidine-5carbaldehyde in the presence of chiral allenes. 40

Scheme 29: Asymmetric ring opening of meso-epoxides with $\mathrm{SiCl}_{4}$ 41

Scheme 30: Enantioselective addition of boronic acids to $\alpha$-keto esters catalyzed by $\mathrm{Rh}^{\mathrm{I}}($ AllenePhos) complexes. 41

Scheme 31: Proposed approaches from deprotonation of propargyl triazoles. 42

Scheme 32: Proposed $\mathrm{S}_{\mathrm{N}} 2^{\prime}$ strategy of propargyl acetate with triazole anion. 43

Scheme 33: Lewis acids activation strategy for synthesizing allenes. 43

Scheme 34: First reaction for investigation triazole allene product formation. 44

Scheme 35: TMS-deprotection and X-ray crystal structures 50

Scheme 36: Propargylation of benzotriazole with enantioenriched propargyl alcohol.... 53

Scheme 37: Mitsunobu reaction. 55

Scheme 38: Postulated mechanism for the Mitsunobu reaction. 55

Scheme 39: N-2 arylation was reported by Shi and Buchwald. 56

Scheme 40: Mitsunobu reaction and alkylation of benzotriazole. .................................57

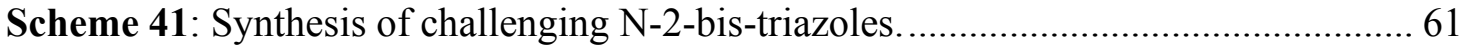

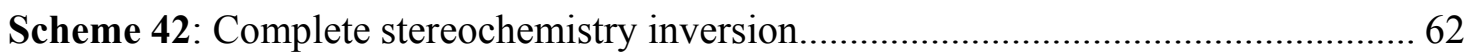

Scheme 43: Asymmetric synthesis of chiral triazole derivatives.................................... 63

Scheme 44: Application of C3-symmetric polytriazoles in click chemistry................... 68

Scheme 45: Synthesis route of "ClickPhine" P,N-ligand and their Pd-complexes.......... 70

Scheme 46: 1,2,3-triazoles as monodentate Lewis-basic ligands for cationic Pd- and Ptpincer complexes .............................................................................. 71

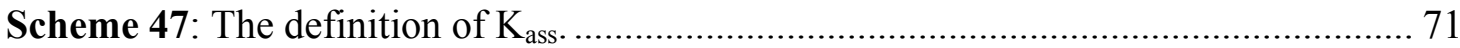

Scheme 48: Suzuki couplings with aryl chlorides catalyzed by the Pd-1,2,3-triazolylidene complex $\mathrm{PdCl}_{2}$ (TMes) $)_{2}$ .73

Scheme 49: CuAAC reactions catalyzed by copper(I)-1,2,3-triazolylidene complexes .. 74 
Scheme 50: Carbene transfer reactions from diazo compounds catalyzed by the gold(I)-

Scheme 51: Ring opening and ring closing metathesis using Grubbs' 1,3,4-triaryl-1,2,3triazol-5-ylidene containing ruthenium catalysts.

Scheme 52: Synthesis of $[\mathrm{Rh}(\mathrm{btz})(\mathrm{COD})]_{2}$ complexes reported by Oro......................... 75

Scheme 53: Synthesis of $[\mathrm{Rh}(\mu-\mathrm{DcTz})(\mathrm{COD})]_{2}$ complex.......................................... 77

Scheme 54: Synthesis of triazole-Rh(I) complexes and its application in Pauson-Khand reactions

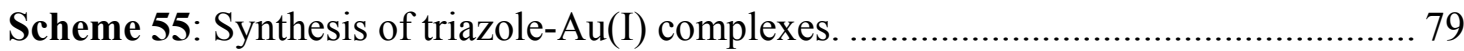

Scheme 56: Asymmetric synthesis of substituted allenes............................................ 79

Scheme 57: General method of synthesis for triazole-Ir(III) complexes. ........................ 85

Scheme 58: An aza-Henry reaction between tertiary $\mathrm{N}$-arylamines and nitroalkanes..... 90

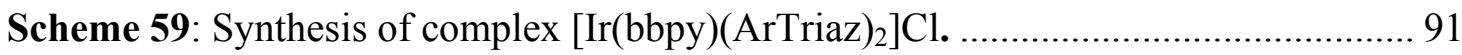

Scheme 60: Aza-Henry reaction of 1,2,3,4-tetrahydroisoquinoline catalyzed by $\left[\operatorname{Ir}(\right.$ bbpy $\left.)(\text { ArTriaz })_{2}\right] \mathrm{Cl}$ complex.

Scheme 61: Aza-Henry reaction with nitroalkene activated by Lewis base. .................. 92

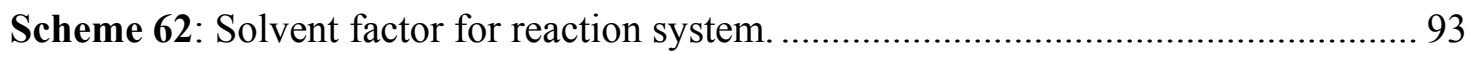

Scheme 63: Iron-catalyzed cross coupling reaction between organic halide/pseudohalide and Grignard reagents. .................................................................... 95

Scheme 64: Different "low-valent” iron complexes. ....................................................... 95

Scheme 65: One "low-valent" iron complex as the catalyst in servral transformations. . 96

Scheme 66: Iron-catalyzed oxidation of unactivated $\mathrm{sp}^{3} \mathrm{C}-\mathrm{H}$ Bonds. ............................... 97

Scheme 67: Classical approaches for synthesizing conjugated enynes. ......................... 99

Scheme 68: The dehydration propargyl alcohol for specific substrates in synthesis of

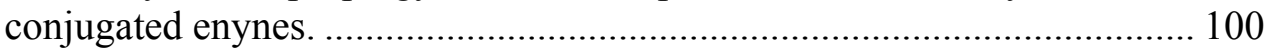

Scheme 69: DABCO-catalyzed 1,4-Bromolactonization of conjugated enynes reported

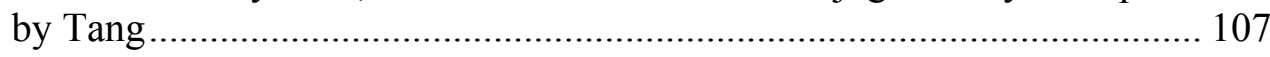

Scheme 70: The 6-endo-trig cyclization of conjugated enynyl acids........................... 108

Scheme 71: The 7-endo-trig cyclization of conjugated enynyl acids........................... 108

Scheme 72: The reactions with enediynyl acids as the reactants.............................. 110 
Scheme 73: Synthesis of enynes 2.3.2s, 2.3.2t and 2.3.2u....................................... 110

Scheme 74: Substituted group dependent enyne activation. ...................................... 111

Scheme 75: Different types of UV/blue emitting small molecules............................... 115

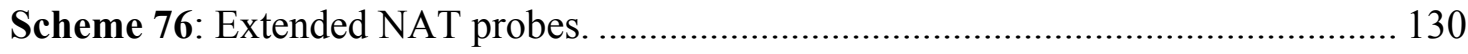




\section{LIST OF TABLES}

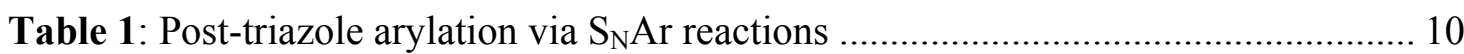

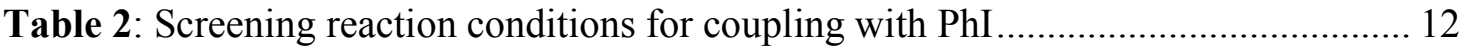

Table 3: Substrate scope for $\mathrm{Cu}$ catalyzed triazole amidation ....................................... 14

Table 4: Screening of catalyst for triazole-alkyne addition ......................................... 22

Table 5: Substate scope of alkynes in the $\mathrm{Au}(\mathrm{I})$ catalyzed triazole addition. ................... 23

Table 6: Catalyst screening in propargylation. .......................................................... 29

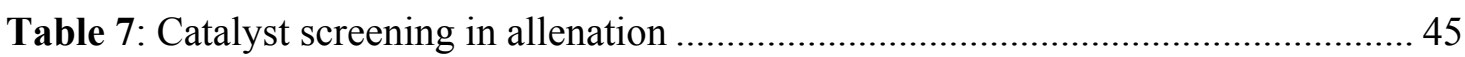

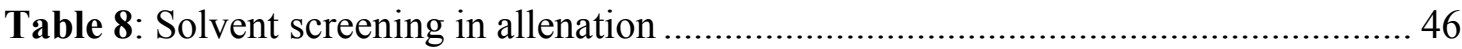

Table 9: Reaction substrate scope with benzotriazole (BTA) ...................................... 58

Table 10: Reaction substrate scope with various triazoles............................................. 59

Table 11: Screening of various triazoles as stabilizing ligands for copper(I) catalyst..... 68

Table 12: $\boldsymbol{K}_{\text {ass }}$ values for Lewis base coordination to Pt-pincer complex compared to $\mathbf{L} 1$

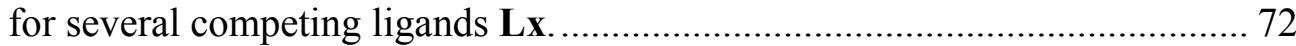

Table 13: Direct propargyl alcohol dehydration: a challenging transformation ............. 101

Table 14: A small detail from allenation of triazole. ................................................... 102

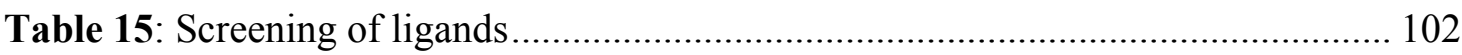

Table 16: Screening of different heteroaromatic ligands ........................................... 103

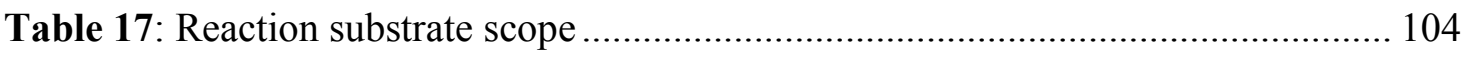

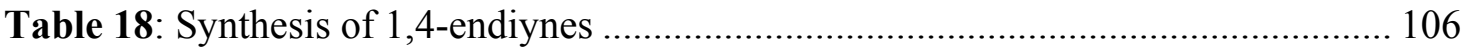

Table 19: Fluorescence emission of compounds with different C-5 substitutions ........ 118

Table 20: Substituted group effect on the N-2 position. ............................................ 120

Table 21: Excitation and emission behavior of substituted NATs............................... 123

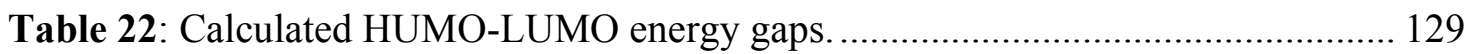




\section{Chapter One: Synthesis of functional 1,2,3-triazoles}

\subsection{Introduction}

\subsubsection{Synthesis of precursor: $\mathrm{NH}-1,2,3$-triazole}

The 1,3-dipolar cycloaddition reaction between two unsaturated reactants gave an access to various five-membered heterocycles, which was developed by Huisgen in the early 1960 's. ${ }^{1}$ The cycloaddition of acetylenes and azides to synthesize 1,2,3-triazoles is by far the most useful member of this family. However, the 1,3-dipolar cycloaddition of terminal alkynes and organic azides afforded the 1,2,3-triazole as the mixture of 1,4adduct and 1,5-adduct under thermal conditions (Scheme 1), ${ }^{2}$ since the activation energies for the formation of both adducts were very close in this concerted process based on the mechanism study. In addition, the 1,4-adduct can be obtained only if the acetylene component is attached to an electron-withdrawing group (EWG), such as a carbonyl or cyano group $(\mathrm{CN}){ }^{3}$ However, these reactions usually required long reaction time and high temperature as alkynes are poor 1,3-dipole receptors. ${ }^{4}$

Scheme 1: Thermal 1,3-dipolar cycloaddition of acetylenes and azides.

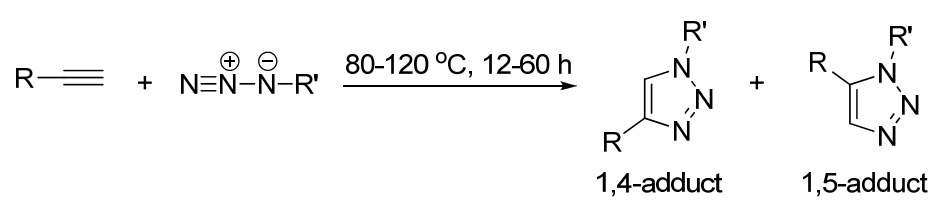

To avoid these disadvantages of Huisgen azide-alkyne cycloaddition, Sharpless and co-workers have successfully discovered that the cycloaddition reaction is dramatically accelerated in the presence of copper catalyst, to give the 1,4-disubstituted 1,2,3-triazoles with excellent regioselectivity and high efficiency (yields are $>95 \%$ ). ${ }^{5}$ Furthermore, this 
copper catalyzed azide-alkyne cycloaddition (CuAAC), which is referred to as "click chemistry", was well tolerant to various other functional groups and can be performed in various organic solvents, and water with a variety of (partially) miscible organic solvents, including DMSO, DMF, $t \mathrm{BuOH}$ and acetone (Scheme 2).

Scheme 2: The synthesis of 1,4-disubstituted 1,2,3-triaozles by Click chemistry.

$$
\mathrm{R}=+\mathrm{N} \equiv \stackrel{\oplus}{\mathrm{N}}-\stackrel{\ominus}{\mathrm{N}}-\mathrm{R}^{\prime} \quad \frac{\mathrm{Cu}(\mathrm{I}), \text { solvent }}{\mathrm{rt}, 12-24 \mathrm{~h}}
$$

Although several $\mathrm{Cu}(\mathrm{I})$ sources can be used directly in this process, such as copper(I) bromide $(\mathrm{CuBr})$ or copper(I) iodide $(\mathrm{CuI})$, the catalyst was better prepared in situ by the reduction of $\mathrm{Cu}(\mathrm{II})$ salts (e.g. $\mathrm{CuSO}_{4}$ ) with reductants such as sodium ascorbate or ascorbic acid (Scheme 3). This transformation exhibited a broad substrate scope and produced the 1,4-disubstituted 1,2,3-triaozles in good to excellent yields with near perfect regioselectivity.

Scheme 3: Example of CuAAC reported by Shapless.

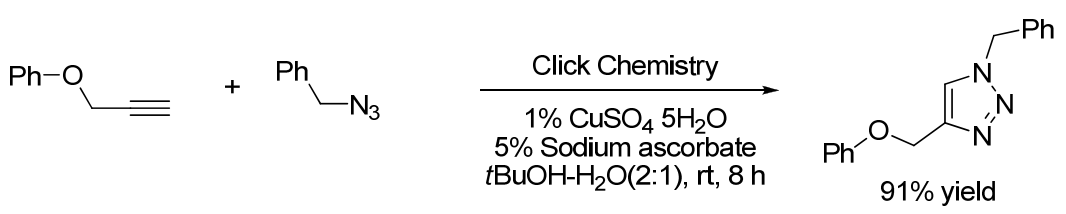

The reaction mechanism has been investigated using density functional theory calculations (DFT). ${ }^{6}$ The copper(I) species generated in situ formed a $\pi$-complex with the triple bond of a terminal alkyne. In the presence of a base, the terminal hydrogen, being the most acidic was deprotonated first to give a $\mathrm{Cu}(\mathrm{I})$ acetylide intermediate (therefore 
indicated why internal alkynes failed to react by this process). Studies have shown that the transition state involved two copper atoms. One copper atom was bonded to the acetylide while the other $\mathrm{Cu}$ atom served to activate the azide. The metal center coordinated with the electrons on the nitrogen atom. The azide and the acetylide were not coordinated to the same $\mathrm{Cu}$ atom in this case. The ligands employed were labile and weakly coordinating. The azide displaced one ligand to generate a copper-azide-acetylide complex (CuAAC). At this point cyclization took place and followed by protonation; the source of proton being the hydrogen which was pulled off from the terminal acetylene by the base. The product was formed by dissociation of copper-azide-acetylide complex and the catalyst ligand complex was regenerated for further reaction cycles (Figure 1).

Figure 1: Proposed reaction mechanism of "Click chemistry"

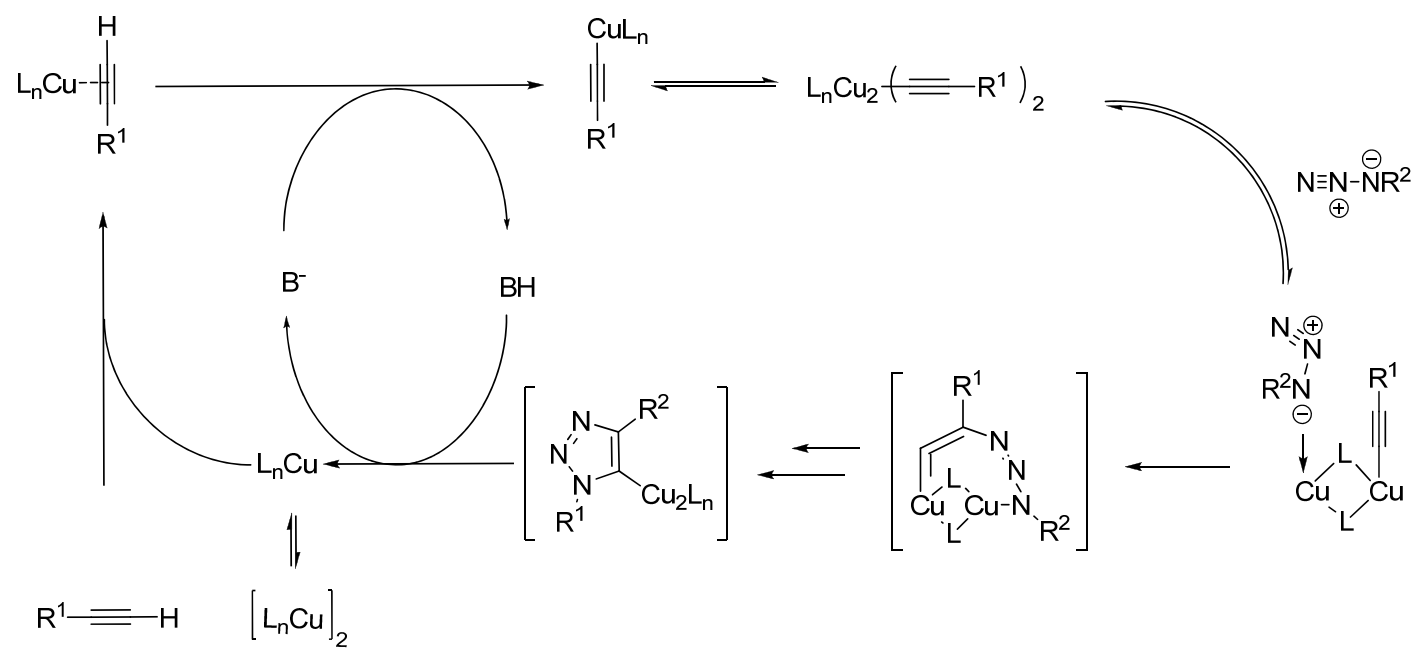

Since the discovery of the CuAAC reaction in 2001, the 1,2,3-triazoles have been the subject of considerable research mainly due to their usefulness as the valuable synthetic intermediates in organic chemistry ${ }^{7}$ and also because of the important biological properties, which have found broad use in biology ${ }^{8}$ and medicinal chemistry. ${ }^{9}$ However, 
despite being a very efficient method for the synthesis of $\mathrm{N}$-substituted triazoles, one major limitation of the $\mathrm{CuAAC}$ was the lack of reactivity toward unsubstituted azides, such as $\mathrm{NaN}_{3}$ and $\mathrm{HN}_{3}$. Therefore, to prepare $\mathrm{NH}-1,2,3$-triazoles by CuAAC, the organic azides were usually applied with removable $N$-protecting groups followed by the deprotection (Scheme 4). ${ }^{10}$

Scheme 4: Synthesis of $N H$-1,2,3-triazoles through CuAAC.

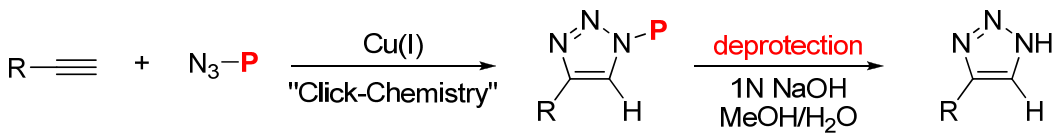

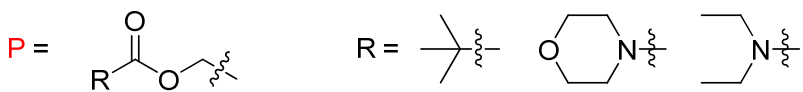

Besides the CuAAC approach, the 1,3-dipolar cycloaddition between $\alpha$-nitroalkene and sodium azide was considering another popular methodology for the synthesis of $\mathrm{NH}$ 1,2,3-triazoles and was first reported by Zefirov in $1971 .{ }^{11}$ With DMSO as the solvent, the authors reported the formation of corresponding triazole at room temperature in roughly $60 \%$ yield along with "considerable quantities" of the corresponding byproduct 1,3,5-triarylbenzene (Scheme 5).

Scheme 5: The synthesis of $N H-1,2,3$-triazoles by Zefirov's method.

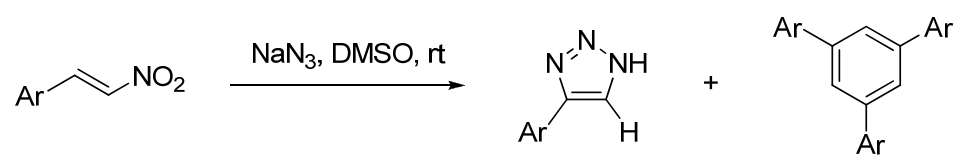

However, Zard and co-worker re-examined this reaction in 2005 and discovered the above result was not reproducible. ${ }^{12}$ As a result, they figured out the optimal condition 
which involved a slow addition of $\alpha$-nitroalkene to a hot solution of the excess amount of sodium azide (2-4 equiv.) at $80-90{ }^{\circ} \mathrm{C}$ (Scheme 6).

Scheme 6: Thermal condensation of $\alpha$-nitroalkene and sodium azide (Zard's method).

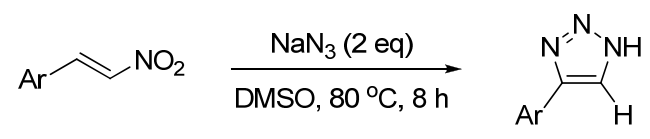

Under the optimized condition, the $\mathrm{NH}$-1,2,3-triazoles could be obtained in good to excellent yields ( $96 \%$ in one single case) with a very small amount of the byproduct 1,3,5-triarylbenzene. However, the challenging access of the olefin precursor $\alpha$ nitroalkene significantly limited the application of this reaction.

Our group reported a Lewis base-catalyzed cascade nitroalkene-aldehyde-azide condensation for the synthesis of 4,5-disubstituted $\mathrm{NH}$-1,2,3-triazoles in 2008 (Scheme 7). ${ }^{13}$ More than 25 new $N H-1,2,3$-triazoles were prepared in good to excellent yields under mild conditions. The C-5 vinyl group of triazole can be readily converted into other functional groups.

Scheme 7: Shi's Approach: Lewis base-catalyzed cascade nitroalkene-aldehyde-azide condensation.
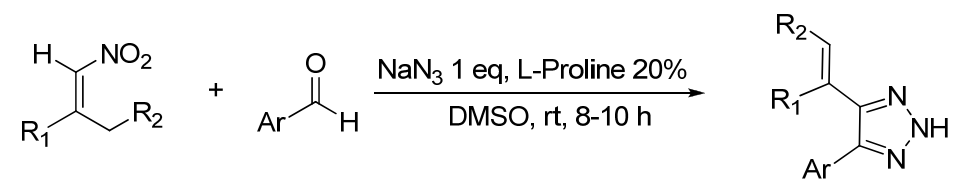

up to $89 \%$ yield

The reaction mechanism was also investigated (Figure 2). The 1-aryl diene intermediate was produced by the Herny reaction of nitroalkene and aldehyde, and then 
quenched by azide anion $\left(\mathrm{N}_{3}{ }^{-}\right)$to give cycloaddition product triazole with elimination of the nitro group $\left(\mathrm{NO}_{2}^{-}\right)$.

Figure 2: Mechanism for synthesis of 4,5-disubstituted $\mathrm{NH}$-1,2,3-triazoles.

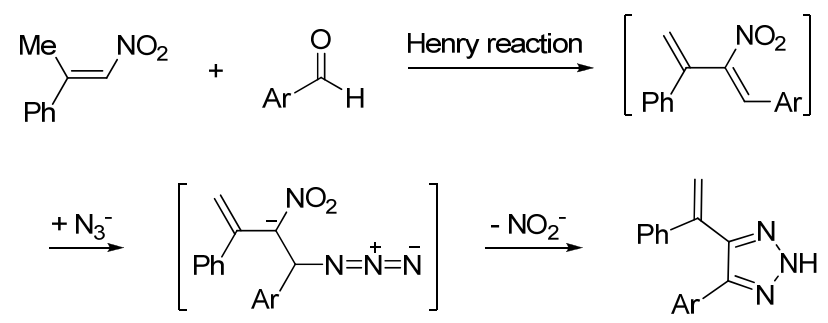

\subsubsection{Our strategy: How to reach the N-2 substitution of $\mathrm{NH}-1,2,3$-triazoles?}

To the best of our knowledge, the copper catalyzed azide-alkyne cycloaddition (CuAAC) gave N-1 substituted triazoles only (see section 1.1.1), due to the nature of azides. Alternatively, N-2 substituted triazoles may be accessed by the conversion of nonsubstituted $\mathrm{NH}$-triazoles with appropriate electrophiles. However, as we know, before 2008, no effective strategy for regioselective N-2 substituted of 1,2,3-triazoles had been reported in the literature.

With a strong dipole moment and high electron density on the nitrogens, the $\mathrm{NH}$ 1,2,3-triazole could be a good nucleophile, which will react with electrophiles under suitable conditions. However, among the reported examples, ${ }^{14}$ the $\mathrm{N}-1$ substituted triazoles were the dominant products. This was caused by the higher electron density associated with two terminal nitrogens $(\mathrm{N}-1$ and $\mathrm{N}-3)$ than the internal nitrogen $(\mathrm{N}-2)$. Therefore, regioselective N-2 substitution was a big challenge in triazole derivatization.

To develop an efficient synthesis of N-2 substituted triazoles, our group has put numerous efforts and concluded successfully a strategy which was that the C-4 and C-5 
substituent groups could deliver the necessary steric hindrance to provide $\mathrm{N}-2$ as the preferred nucleophilic site (Figure 3).

Figure 3: Strategy for selective N-2 substitution (two bulky groups on C-4 and C-5).

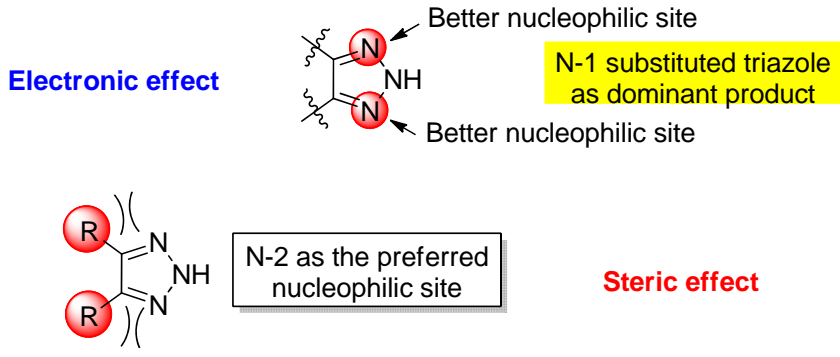

To evaluate the effectiveness of this strategy, our group has developed successfully the N-2 alkylation of $\mathrm{NH}-1,2,3$-triaozles (Scheme 8). ${ }^{15}$

Scheme 8: Synthesis of N-2-alkyl-1,2,3-triazoles.

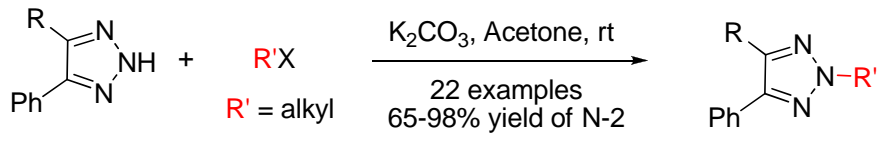

As reported in the paper, the $\mathrm{N}-2$ alkylation reactions usually gave very less amount of N-3 substituted triazoles. From the crystal structures, ${ }^{*}$ the aryl group on the C-4 position was nearly coplanar with the triazole ring. The N-3 position was then blocked for electrophilic addition (Figure 4). Surprisingly, the benzyl ketone triazole gave excellent N-2 selectivity and even formed only the N-2 product in some cases (Scheme 9). Based on the conformational control analysis, the benzoyl group on C-5 position would like to be coplanar with the triazole ring to minimize the electronic repulsion

\footnotetext{
* Check the compounds 2a, N-1-3a, N-1-5a and $\mathbf{N - 2 - 4 a}$ in the supporting information of N-2 alkylation published paper (see ref. 15).
} 
between the keto oxygen and the N-1 nitrogen (Figure 5). Therefore, the phenyl group effectively blocked the N-1 position.

Figure 4: The C-4 aryl group was nearly coplanar with the triazole ring.

Aryl group adopts co-planar conformation to block the N-3 position

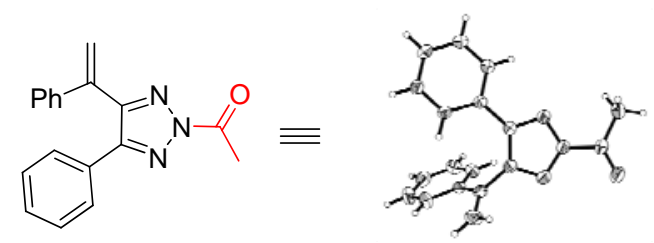

Scheme 9: The alkylation of benzyl ketone triazole.

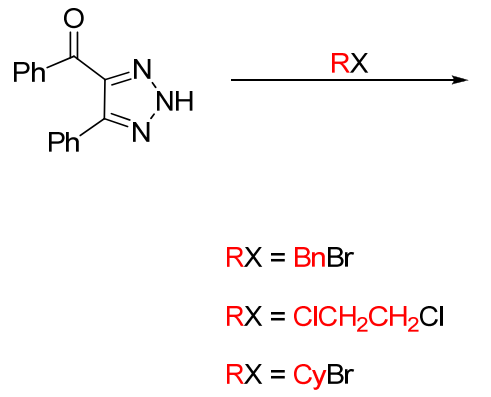<smiles></smiles>

7.6 only product only product<smiles></smiles>

$\mathrm{N}-1$

1

0

0<smiles>[R]n1nnc(C(=O)c2ccccc2)c1-c1ccccc1</smiles>

N-3

0.4

0

0

Figure 5: Conformational control for selective N-2 substitution.<smiles></smiles>

enol isomer<smiles>O=C(c1ccccc1)C1NNC1c1ccccc1</smiles>

keto isomer

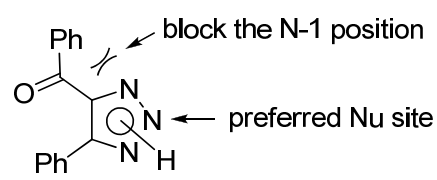

preferred conformation for mimium electronic replusion

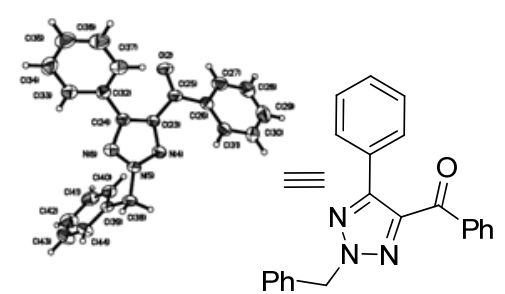




\subsection{Efficient synthesis of N-2-aryl-1,2,3-triazole fluorophores via post- triazole arylation $\uparrow$}

\subsubsection{Reported method for synthesizing N-2-aryl-1,2,3-triazole}

One important class of triazole derivatives is the N-2-aryl triazoles, where the two aromatic rings adopt a coplanar conformation. With the characteristic electron density distribution in the HOMO and LUMO, these bis-aromatic compounds give unique photonic properties. One example is the commercially available o-hydroxyphenyl benzotriazoles (such as Tinuvin P), which has been applied as an efficient light absorber to prevent polymer degradation from light radiation. ${ }^{16}$

Currently, the general approach for N-2-aryl triazole involves the hydrazine/ $\alpha$ hydoxyketone condensation, ${ }^{17}$ as shown in Scheme 10. The need of various aryl hydrazines and $\alpha$-hydoxyketones as starting materials significantly limited the application of this methods and only specific N-2 aryl triazole can be prepared with good yields. Therefore, an effective regioselective N-2 arylation approach will be of great importance for the triazole related research. ${ }^{18}$

Scheme 10: The hydrazine/ $\alpha$-hydoxyketone condensation.

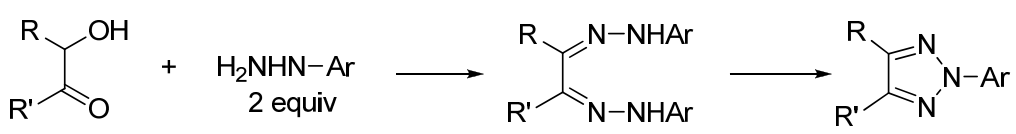

\footnotetext{
$\dagger$ Reproduced in parts with permission from Org. Lett. 2008, 10, 5389-5392. Copyright 2008, with permission from the American Chemical Society.
} 


\subsubsection{Research Objective and Results}

Our group has recently reported the synthesis of 4,5-disubstituted-NH-traizoles through Lewis base catalyzed process. ${ }^{13}$ With these compounds in hand, we recently reported a successful post-triazole $\mathrm{N}-2$ alkylation through substrate conformation control. ${ }^{15}$ Encouraged by these results, we wondered if this strategy could be further extended to the regioselective $\mathrm{N}$-2-arylation.

Table 1: Post-triazole arylation via $\mathrm{S}_{\mathrm{N}} \mathrm{Ar}$ reactions ${ }^{a}$

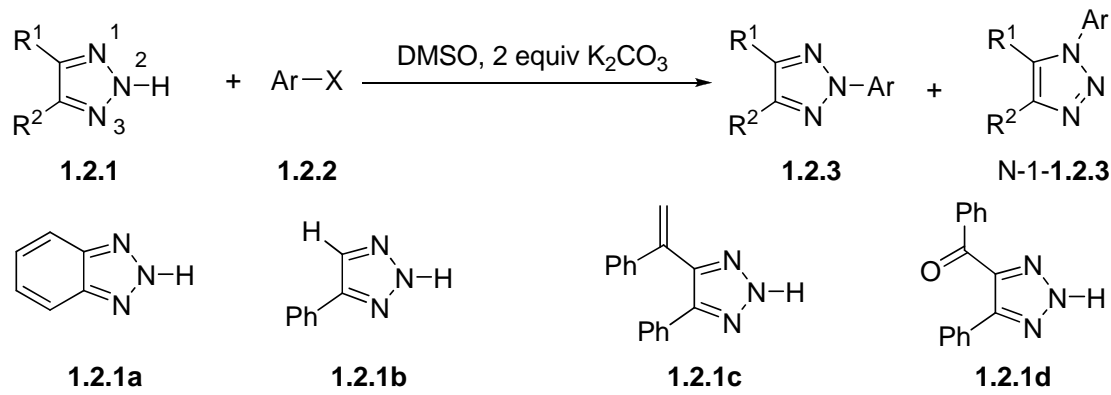

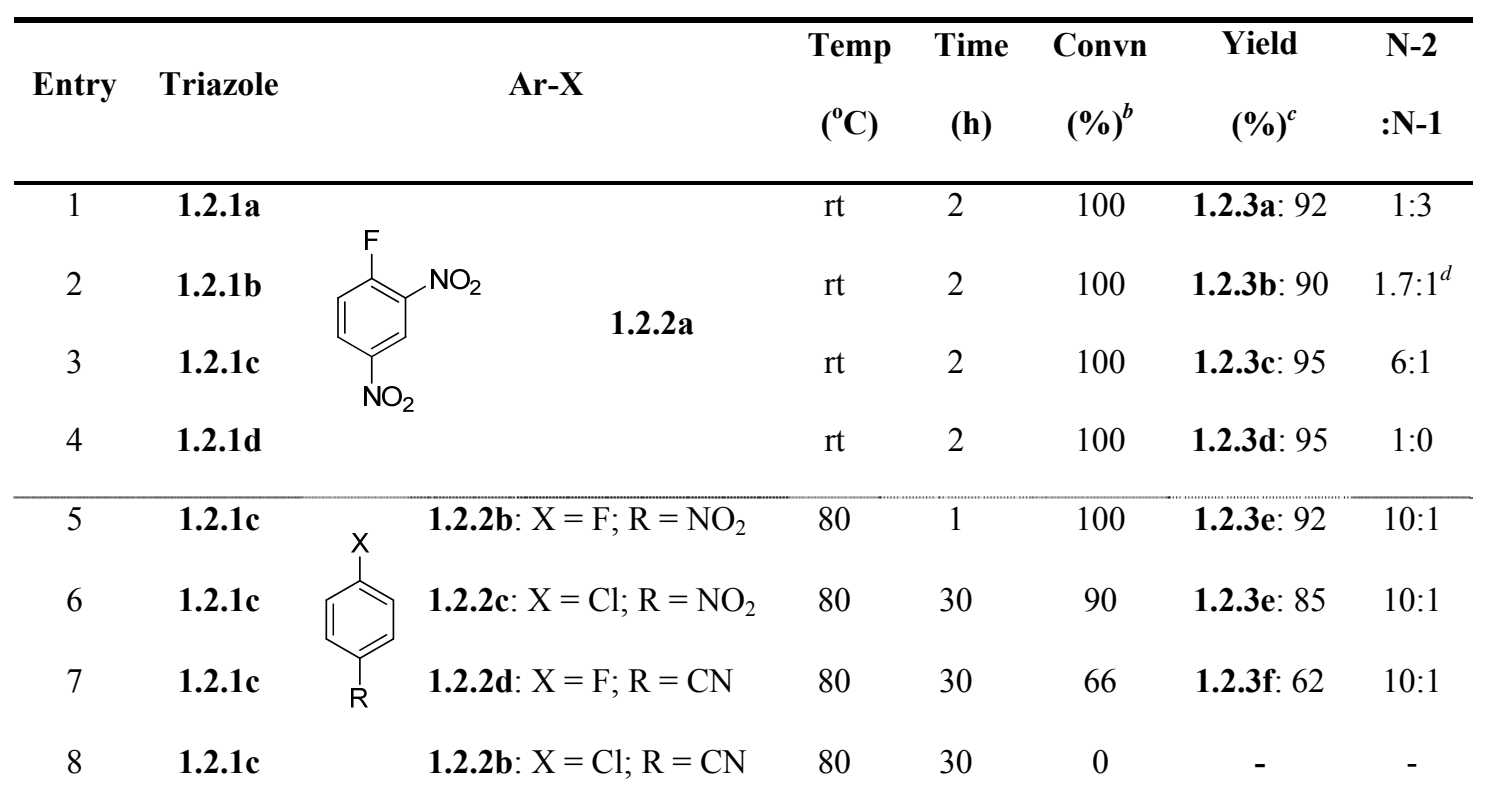

1.2.1:1.2.2 $=1: 1.5, \mathrm{c}=0.2 \mathrm{M} ;{ }^{b}$ based on the consumption of 1.2.1 by NMR; ${ }^{c}$ isolated yields of all isomers; ${ }^{d}$ structure of $\mathbf{N - 1 - 1 . 2 . 3 b}$ was determined by X-ray crystallography. 
To evaluate the regioselectivity, the $\mathrm{S}_{\mathrm{N}} \mathrm{Ar}$ substitution was first studied with a variety of 1,2,3-triazoles. The results are summarized in Table 1. Due to the absence of C-N bond exchange under the reaction condition in the arylation products 1.2.3, the $\mathrm{S}_{\mathrm{N}} \mathrm{Ar}$ reaction provided direct measurement of $\mathrm{C}-4$ and $\mathrm{C}-5$ groups influence on the regioselectivity. As indicated in Table 1, the benzotriazole 1.2.1a gave modest selectivity with $\mathrm{N}-1$ substituted triazole as the major product. On the other hand, the 4-phenyl triazole 1.2.1b improved the $\mathrm{N}-2$ selectivity, giving $\mathrm{N}-2$ arylation as the major product, though with poor selectivity. To our pleasure, the application of 4,5-disubstituted triazole 1.2.1c and 1.2.1d gave $\mathrm{N}-2$ arylation as the dominant products with excellent regioselectivity. As mentioned previously, the selectivity was driven by the conformation control of the C-4 and C-5 substitute groups. However, at higher temperature, the selectivity should be only driven by the steric effect. To our pleasure, increasing the reaction temperature $\left(80^{\circ} \mathrm{C}\right)$ gave improved $\mathrm{N}-2$ selectivity (entry 5,6 and 7 ), which suggested that the regioselectivity of post-triazole arylation could be achieved at higher temperature. Moreover, the less electron-deficient substrates were also suitable for this $\mathrm{S}_{\mathrm{N}} \mathrm{Ar}$ transformation to form the corresponding $\mathrm{N}-2$ arylation products as dominate products (entries 5-7). Although the $\mathrm{S}_{\mathrm{N}} \mathrm{Ar}$ reaction of triazoles gave excellent $\mathrm{N}-2$ regioselectivity, it only worked with electron-deficient aromatic halide substrates.

To extend the generality of N-2 arylation of 1,2,3-triazoles, we then explored the possible coupling strategy for aromatic systems with higher electron density. Numerous copper catalyzed $\mathrm{N}$-aryl halide reactions have been reported in the last several years, but the reaction mechanism was not clear. ${ }^{19}$ The most likely mechanism involves a $\mathrm{Cu}(\mathrm{I})$ oxidative addition to $\mathrm{Cu}(\mathrm{III})$ intermediate followed by reductive elimination, which was 
proposed by Buchwald, Hartwig and Stahl. ${ }^{20}$ Hartwig also suggested that oxidative addition of the carbon halogen bond likely occurs in the catalytic cycles with strong ligand effects. ${ }^{20 \mathrm{~b}}$ Based on these remarkable results, we rationalized that $\mathrm{NH}-1,2,3-$ triazole might be another good nitrogen source for copper mediated aryl halide amidation. With the focus on the ligand effect for optimal performance, reactions between NHtriazole 1.2.1c and phenyl iodide 1.2.5a were then carried out (Table 2).

Table 2: Screening reaction conditions for coupling with $\mathrm{PhI}^{a}$

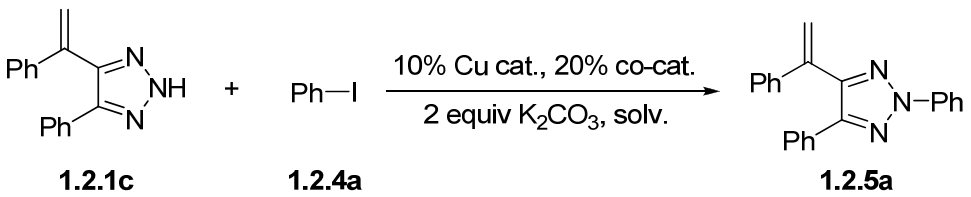

\begin{tabular}{|c|c|c|c|c|c|c|c|}
\hline Entry & Cu cat & Ligand $^{c}$ & Solvent & Temp $\left({ }^{\circ} \mathrm{C}\right)$ & Time (h) & Convn $(\%)$ & ${ }^{d}$ Yield $(\%)^{d}$ \\
\hline 1 & $\mathrm{CuI}$ & - & DMSO & 110 & 24 & low & 0 \\
\hline 2 & $\mathrm{CuI}$ & proline & DMSO & 110 & 24 & 100 & 71 \\
\hline 3 & $\mathrm{CuCl}$ & proline & DMSO & 110 & 24 & 100 & 88 \\
\hline 4 & other $^{b} \mathrm{Cu}$ cat. & proline & DMSO & 110 & 24 & $<92$ & $<79$ \\
\hline 5 & $\mathrm{CuCl}$ & proline & other solvents $^{b}$ & 110 & 24 & $<85$ & $<74$ \\
\hline 6 & $\mathrm{CuCl}$ & proline & DMSO & w 160 & 0.5 & 98 & 92 \\
\hline 7 & $\mathrm{CuCl}$ & glycine & DMSO & w 160 & 0.5 & trace & trace \\
\hline 8 & $\mathrm{CuCl}$ & Me-Gly & DMSO & w 160 & 0.5 & 60 & 30 \\
\hline 9 & $\mathrm{CuCl}$ & DMG & DMSO & w 160 & 0.5 & 98 & 86 \\
\hline 10 & $\mathrm{CuCl}$ & EDA & DMSO & w 160 & 0.5 & 53 & 29 \\
\hline 11 & $\mathrm{CuCl}$ & DMEDA & DMSO & w 160 & 0.5 & 70 & 47 \\
\hline 12 & $\mathrm{CuCl}$ & TMEDA & DMSO & w 160 & 0.5 & trace & trace \\
\hline 13 & $\mathrm{CuCl}$ & DACH & DMSO & w 160 & 0.5 & 53 & 44 \\
\hline
\end{tabular}

1.2.1c:1.2.4a $=1: 1.5, \mathrm{c}=0.2 \mathrm{M} ;{ }^{b}$ see supporting information; ${ }^{c} \mathrm{Me}-\mathrm{Gly}: \mathrm{N}$-methyl glycine; DMG: $\mathrm{N}, \mathrm{N}-$ dimethyl glycine; EDA: ethyleneediamine; DMEDA: N, $\mathrm{N}^{\prime}$-dimethylethylenediamine; TMEDA: N,N, $\mathrm{N}^{\prime}, \mathrm{N}^{\prime}$ tetramethylethylenediamine; DACH: trans-diaminohexane; ${ }^{d}$ Based on the ${ }^{1} \mathrm{H}$ NMR with $1,3,5-$ trimethoxybenzene as internal standard. 
Among all the tested reactions, the coupling product 1.2.5a was not formed, if no ligands were applied. Different nitrogen containing ligands have been investigated in this $\mathrm{Cu}$ mediated amidation of $\mathrm{NH}$-triazole, and we found proline was the best ligand for this transformation. To our satisfaction, excellent regioselectivity was received with N-2 arylation as the dominant product. Both $\mathrm{Cu}(\mathrm{I})$ and $\mathrm{Cu}(\mathrm{II})$ could effectively catalyze this reaction (entries 2-4). Screening of the solvents gave DMSO as the solvent choice. Considering of its low cost, good reaction performance and easy handling, $\mathrm{CuCl}$ was selected as the catalyst for this reaction. The optimal reaction condition is shown in entry 3. The use of microwave irradiation significantly shortened the reaction time form 24 hours to 30 minutes.

With the optimal reaction conditions, different $\mathrm{NH}$-triazoles and aryl iodides were applied to evaluate the reaction substrate scope (Table 3). As shown in Table 3, benzotriazole 1.2.1a gave the $\mathrm{N}-1$ arylation as the dominant product with only $8 \% \mathrm{~N}-2$ product isolated. The N-2 selectivity was increased while 4-phenyl substituted 1.2.1b was applied, giving $60 \% \mathrm{~N}-2$ isomer with another $15 \% \mathrm{~N}-1$ isomer and only trace amount of N-3 isomer. Significant difference was observed when 4,5-disubstituted triazoles were used, where, as expected, in all cases only $\mathrm{N}-2$ isomers were isolated with excellent yields. The substrate scope of this transformation covered different substituted groups on C-5 position of triazoles and various aryl iodides with EDG, EWG and pyridine. Due to the diminished reactivity of aryl bromides in the oxidative addition, application of aryl bromides resulted in significant decrease in the reaction conversion and yield. Moreover, this copper mediated aryl halide amidation of 1,2,3- $\mathrm{NH}$-triazoles did not work well with a heterocyclic halide, because of the chelating interaction between heterocyclic structure 
and copper catalyst. Notably, the substrate $\mathbf{1 . 2 . 1} \mathbf{j}$ gave $45 \%$ of desired N-2 arylation product 1.2.5j and 40\% of N-3-arylation product N-3-1.2.5j (structures were determined by X-ray diffraction), which suggested the $\mathrm{Cu}$-chelation between the phenol oxygen and triazole-N-3 position. The production of the steric disfavored N-3 arylation product strongly supports the $\mathrm{Cu}(\mathrm{III})$ reductive elimination mechanism.

Table 3: Substrate scope for $\mathrm{Cu}$ catalyzed triazole amidation ${ }^{a}$
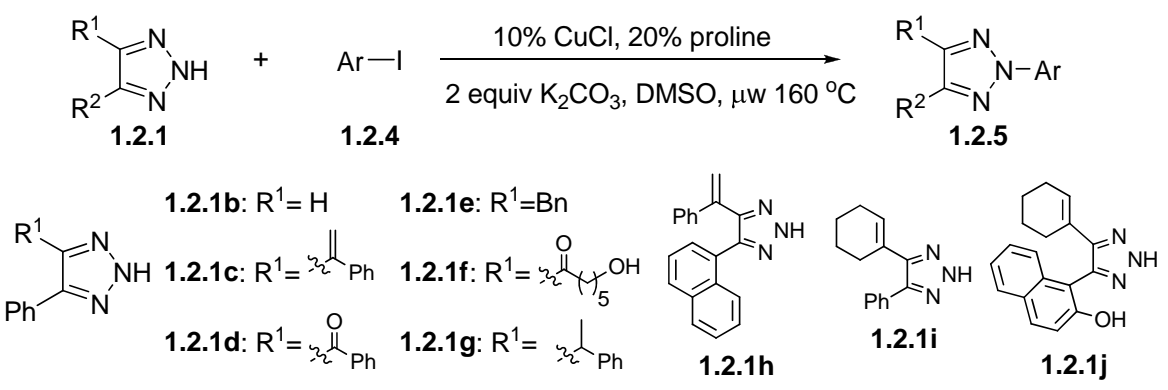

\begin{tabular}{|c|c|c|c|c|c|c|}
\hline & $\overline{\text { ArI }}$ & yield $(\%)^{b}$ & & ArI & & yield $(\%)^{b}$ \\
\hline $1.2 .1 \mathrm{a}$ & $1.2 .4 \mathrm{a}$ & 1.2.5b: $8^{c}$ & 1.2.1f & $1.2 .4 a$ & & 1.2.5f: 60 \\
\hline $1.2 .1 \mathrm{~b}$ & $1.2 .4 \mathrm{a}$ & 1.2.5c: $68^{d}$ & $1.2 .1 \mathrm{~g}$ & $1.2 .4 a$ & & 1.2.5g: 85 \\
\hline $1.2 .1 \mathrm{c}$ & $1.2 .4 \mathrm{a}$ & 1.2.5a: 87 & $1.2 .1 \mathrm{~h}$ & $1.2 .4 \mathrm{a}$ & & 1.2.5h: 87 \\
\hline $1.2 .1 \mathrm{~d}$ & $1.2 .4 \mathrm{a}$ & $\mathbf{1 . 2 . 5 d}: 85^{e}$ & $1.2 .1 \mathrm{i}$ & $1.2 .4 a$ & & 1.2.5i: 80 \\
\hline $1.2 .1 \mathrm{e}$ & $1.2 .4 \mathrm{a}$ & 1.2.5e: 85 & $1.2 .1 \mathrm{j}$ & $1.2 .4 a$ & & 1.2.5j: $45^{e, f}$ \\
\hline $1.2 .1 \mathrm{c}$ & 1.2.4b: $\mathrm{R}=p-\mathrm{Me}$ & 1.2.5k: 86 & & & & \\
\hline $1.2 .1 \mathrm{~d}$ & 1.2.4c: $\mathrm{R}=p-\mathrm{NO}_{2}$ & 1.2.5l: 88 & 1.2 .10 & & $1.2 .4 n$ & 1.2.5r. $/ 0$ \\
\hline $1.2 .1 \mathrm{c}$ & 1.2.4d: $\mathrm{R}=p$-OMe & 1.2.5m: 74 & & & & \\
\hline $1.2 .1 \mathrm{i}$ & 1.2.4e: $\mathrm{R}=o-\mathrm{Me}$ & 1.2.5n: 70 & $\begin{array}{l}1.2 .1 \mathrm{c} \\
1.2 .1 \mathrm{~d}\end{array}$ & & $1.2 .4 \mathrm{i}$ & $\begin{array}{l}1.2 .5 \mathrm{~s}: 70 \\
1.2 .5 \mathrm{t}: 60\end{array}$ \\
\hline $1.2 .1 \mathrm{c}$ & 1.2.4f: $\mathrm{R}=p-\mathrm{I}$ & 1.2.50: $67^{e, g}$ & & & & \\
\hline $\begin{array}{l}1.2 .1 \mathrm{c} \\
1.2 .1 \mathrm{~d}\end{array}$ & $1.2 .4 \mathrm{~g}$ & $\begin{array}{l}1.2 .5 \mathbf{p}: 88 \\
1.2 .5 \mathbf{q}: 83^{e}\end{array}$ & 1.2.1c & & $1.2 .4 \mathrm{j}$ & 1.2.5u: $70^{e}$ \\
\hline
\end{tabular}


The strong dependence on ligands of this transformation* initiated our interest in evaluating trans-metallation as an alternative pathway to further extend the substrate scope, especially for heterocyclic substrates. Therefore, the trans-metallation reactions between triazoles 1.2.1c and phenyl boronic acid 1.2.6a were investigated. Excitingly, transmetallation indeed produced the desired N-2 arylation product. Further screening of reaction conditions revealed THF as the best solvent (see other conditions in supporting information) (Scheme 11). To achieve the efficient turnover of the copper catalyst, oxygen gas was required.

Scheme 11: Copper catalyzed transmetalation between triazoles and boronic acids.

\begin{tabular}{|c|c|c|c|c|c|}
\hline & & & & & \\
\hline & & & & conditions & yields (\%) \\
\hline $2.1 c+$ & $\mathrm{Ph}-\mathrm{B}(\mathrm{OH})_{2}$ & $\stackrel{\text { conditions }}{\longrightarrow}$ & $1.2 .5 \mathrm{a}$ & $\mathrm{Cu}(\mathrm{OAc})_{2}$ (1.5 equiv), DCM, rt, $12 \mathrm{~h}$ & 88 \\
\hline & 1.2.6a & & & $\mathrm{Cu}(\mathrm{OAc})_{2}(0.2$ equiv), THF, rt, $12 \mathrm{~h}$ & 45 \\
\hline & & & & 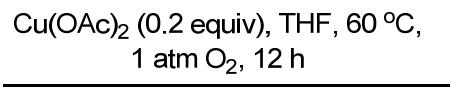 & 89 \\
\hline
\end{tabular}

Figure 6: Products from trans-metallation coupling with arylboronic acids.

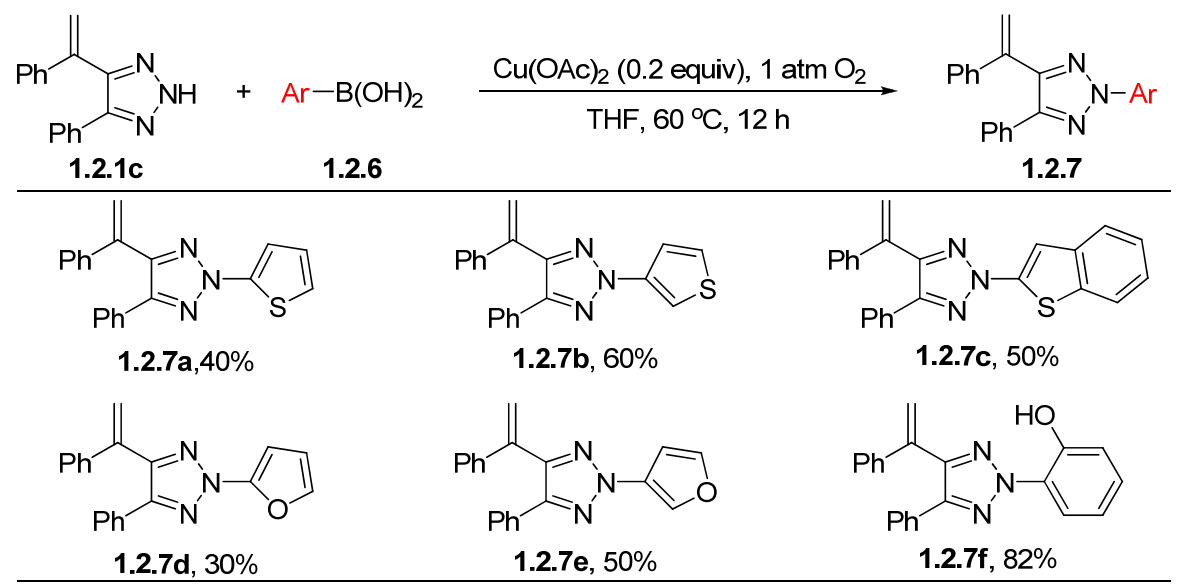

Reaction conditions: 1.2.1c:1.2.6 $=1: 1.5, \mathrm{c}=0.1 \mathrm{M}$, THF, 0.2 equiv. $\mathrm{Cu}(\mathrm{OAc})_{2}, 2$ equiv, pyridine, $\mathrm{O}_{2} 1 \mathrm{~atm}, 50{ }^{\circ} \mathrm{C}, 24 \mathrm{~h}$.

* Check the detail in Table 2 (entry 6-13). 
With triazole 1.2.1c, the N-2 isomer was the only product isolated, while 1.2.1a and 1.2.1b gave less than $40 \% \mathrm{~N}-2$ isomers. We then focused on applying this strategy for the synthesis of N-2 aryl substrates that were difficult to be prepared from aryliodide amidation. The results are summarized in Figure 6.

Finally, with a series of new N-2-aryl-1,2,3-triazoles in hand, the photonic properties which included UV absorption and fluorescence emission have evaluated. Besides the strong UV absorptions, the new triazole derivatives N-2-aryl-1,2,3-triazoles produced strong photonic luminescence and their emission pattern really depended on the substituted groups.

Figure 7: Comparison of photonic luminescence of selected N-1 and N-2-aryl triazoles (1.2.5b and 1.2.5c): the sample was measured in $\mathrm{CH}_{3} \mathrm{OH}, c=10 \mu \mathrm{M} ; \lambda_{\mathrm{ex}}=254 \mathrm{~nm}$.

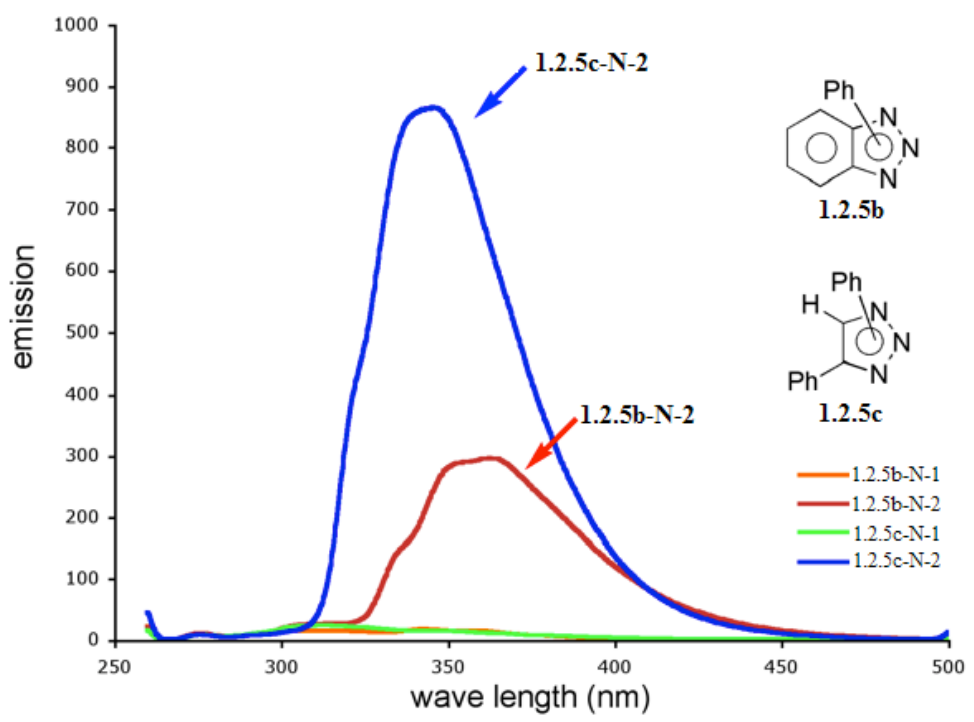

The importance of fluorescence active molecules has been well documented in various research fields. ${ }^{21}$ As shown in the Figure 7, both N-2 phenyl 1,2,3-triazoles (1.2.5b and 1.2.5c) gave strong fluorescence emissions, while $\mathrm{N}-1$ aryl analogues gave 
weak emissions. These results suggested the N-2-aryl group is crucial for photonic emission. More importantly, the 4,5-disubstituted triazoles showed much stronger emission comparing to benzyl triazole derivatives (Figure 8). The N-2-aryl-1,2,3triazoles as a new class of UV-Blue emitting fluorophores have been systematic investigated (see Chapter Three). ${ }^{22}$

Figure 8: Photonic luminescence of N-2-aryl triazoles with different substituted groups on the C-5 position: the sample was measured in $\mathrm{CH}_{3} \mathrm{OH}, c=10 \mu \mathrm{M} ; \lambda_{\text {ex }}=254 \mathrm{~nm}$.

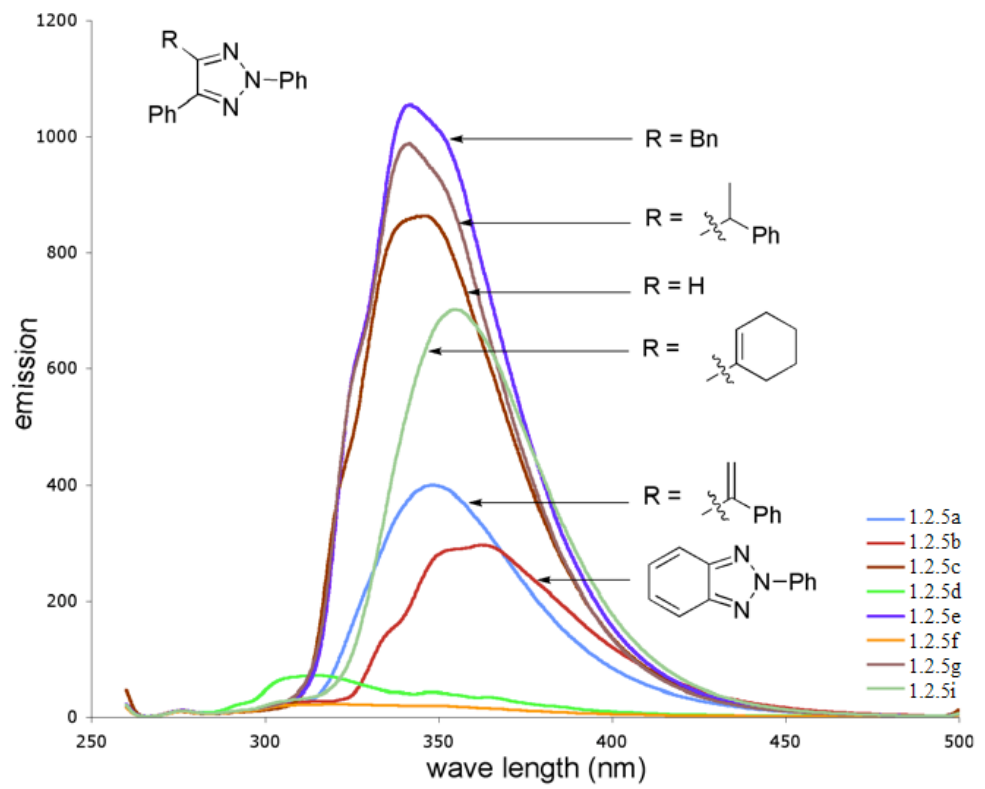

\subsubsection{Conclusion}

In conclusion, the first regioselective post triazole-arylation of $\mathrm{NH}-1,2,3$-triazoles was developed through substrate controlled $\mathrm{N}$-arylation. The importance of ligand effect in the copper mediated coupling with aryl iodide was observed and optimal condition allowed the formation of $\mathrm{N}-2$ aryl products in excellent yields. Alternative approaches, including $\mathrm{S}_{\mathrm{N}} \mathrm{Ar}$ and boronic acid transmetallations, have also been proved with excellent 
regioselectivity. Moreover, the newly prepared N-2 aryl products gave interesting photonic luminescent emission, which re-emphasized the significance of the N-2-aryltriazoles and reported methodologies. 


\subsection{Highly efficient synthesis of vinyl substituted triazoles by Au(I) catalyzed alkyne activation $\uparrow$}

\subsubsection{Reported methods for synthesis vinyl substituted triazoles}

$\mathrm{N}$-vinyl 1,2,3-triazole is a member of vinyl monomer family (Figure 9), ${ }^{23}$ which provides the attractive features of thermal and chemical stability and functional handles from reactive groups in the synthesis of advanced materials. As we can see, in the $\mathrm{N}$-vinyl triazole, it has a very unique structure, reminiscent of both styrene (aromaticity) and vinyl pyridine (lone pairs) ${ }^{24}$ which displays a range of novel properties in the polymerization profiles similar to styrenic/acrylate systems.

Figure 9: Structures of N-vinyl 1,2,3-triazoles.

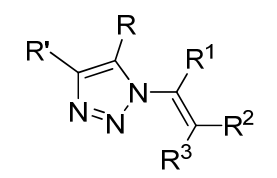

$\mathrm{N}-1$ vinyl-1,2,3-triazole

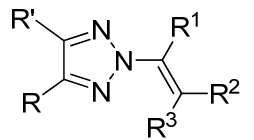

$\mathrm{N}-2$ vinyl-1,2,3-triazole

From the retrosynthetic analysis, the N-vinyl 1,2,3-triazole could be synthesized by the cycloaddition of alkynes with vinyl-substituted azides through either thermo conditions $^{25}$ or metal catalyzed 1,3-dipolar cycloadditions. However, specifically, the metal catalyzed cycloaddition between alkynes and vinyl-substituted azides has never been reported in literature, possibly due to the poor stability of vinyl-substituted azides (high-energy material) and existence of different resonance structures in vinyl-substituted azides (Scheme 12). As a result, the preparation of vinyl-triazole was very challenging.

\footnotetext{
$\dagger$ Reproduced in parts with permission from Bioorg. Med. Chem. Lett. 2009, 19, 3899-
} 3902. Copyright 2009, with permission from Elsevier Ltd. 
Scheme 12: Vinyl azide: challenging substrate for dipolar cycloaddition due to the existence of equilibrium.

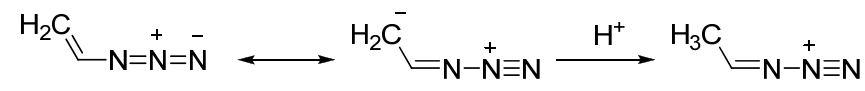

Figure 10: Synthesis of N-vinyl 1,2,3-triazole via elimination of alkyl triazole.

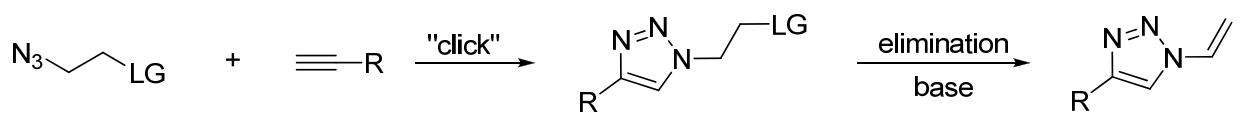

Besides the elimination of alkyl substituted triazole approach (Figure 10), most two general methods have been reported in literature in the preparation of $\mathrm{N}$-vinyl 1,2,3triazoles. One was the elimination of alkyl halide (Scheme 13). ${ }^{26}$ In this elimination approach, significant amounts of bis-triazole byproducts were usually formed and the substrate scope was very limited. Another one was the cyclization of vinyl azides with a highly reactive benzyne intermediate (Scheme 14). ${ }^{27}$ The benzyne click chemistry provided an alternative strategy in preparing triazoles, however, again, it suffered poor yields and limited substrate scope.

Scheme 13: Elimination of alkyl halide.

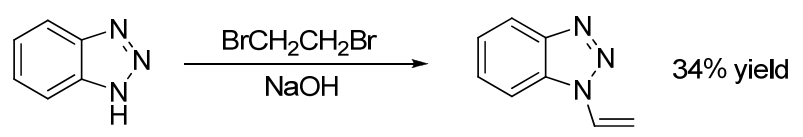

Scheme 14: Benzyne click chemistry.

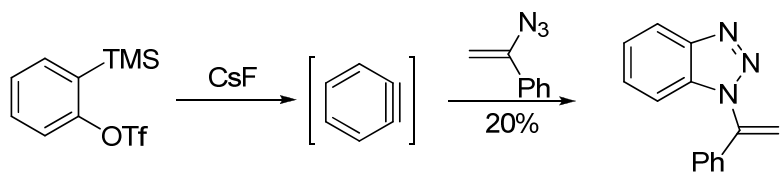


Therefore, efficient synthetic routes to access vinyl-triazoles are highly desirable. Considering the strong nucleophilicity of $\mathrm{NH}-1,2,3$-triazole, we wondered whether the NH-triazole alkyne addition could be achieved as a new strategy for the synthesis of vinyl triazoles with high efficiency and a broad substrate scope.

\subsubsection{Research Objective and Results}

To optimize the reaction condition, the reactions between benzotriazole 1.2.1a and phenylacetylene 1.3.2a were performed in the presence of various catalysts (Table 4). Reactions at room temperature gave either very low yields or no reaction at all for all catalysts tested (included $\operatorname{In}(\mathrm{OTf})_{3}, \mathrm{AuCl}, \mathrm{AuCl}_{3}$ and $\mathrm{PPh}_{3} \mathrm{AuCl}$ ). By increasing the reaction temperature, the $\mathrm{Au}(\mathrm{I})$ catalysts were identified as the best catalysts in activating the triple bond other than other metals catalysts as listed in the Table 4. With 5\% loading of $\mathrm{PPh}_{3} \mathrm{AuOTf}$ at $80{ }^{\circ} \mathrm{C}$, the desired vinyl triazole 1.3.3a was obtained in quantitative yield (entry 5). The reaction gave excellent regio-selectivity, where by only the N-1 substituted Markovnikov's product was observed. As indicated in entry 7, the product was obtained in excellent yield (92\%) even with $0.2 \%$ loading of $\mathrm{PPh}_{3} \mathrm{AuOTf}$ catalyst, though a longer reaction time $(12 \mathrm{~h})$ required. Some other catalysts, such as $\operatorname{In}(\mathrm{OTf})_{3}$, indicated modest reactivity (68\% yield), but the superior efficiency of the $\mathrm{PPh}_{3} \mathrm{AuOTf}$ catalyst made itself an ideal catalyst for this transformation. Different alkynes were then applied to the reaction to investigate the substrate scope. The results are summarized in Table 5 . 
Table 4: Screening of catalyst for triazole-alkyne addition. ${ }^{a}$

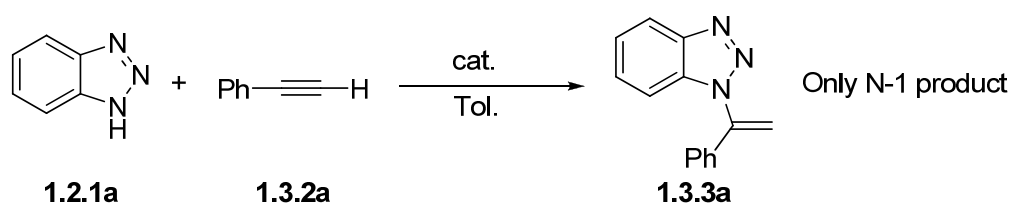

\begin{tabular}{|c|c|c|c|c|c|}
\hline Entry & Catalyst & Loading (\%) & Temp $\left({ }^{\circ} \mathrm{C}\right)$ & Time (hour) & Yields $^{b}(\%)$ \\
\hline 1 & $\mathrm{AuCl}$ & 5 & 80 & 6 & 82 \\
\hline 2 & $\mathrm{AuCl}_{3}$ & 5 & 80 & 6 & 88 \\
\hline 3 & $\mathrm{PPh}_{3} \mathrm{AuOTf}$ & 5 & $\mathrm{rt}$ & 12 & 15 \\
\hline 4 & $\mathrm{PPh}_{3} \mathrm{AuOTf}$ & 5 & 50 & 20 & 60 \\
\hline 5 & $\mathrm{PPh}_{3} \mathrm{AuOTf}$ & 5 & 80 & 5 & $>99 \%$ \\
\hline 6 & $\mathrm{PPh}_{3} \mathrm{AuOTf}$ & 1 & 80 & 6 & 96 \\
\hline 7 & $\mathrm{PPh}_{3} \mathrm{AuOTf}$ & 0.2 & 80 & 12 & 92 \\
\hline 8 & $\mathrm{CuI}$ & 5 & 80 & 12 & N.R. \\
\hline 9 & $\mathrm{Cu}(\mathrm{OAc})_{2}$ & 5 & 80 & 12 & N.R. \\
\hline 10 & $\mathrm{Sc}(\mathrm{OTf})_{2}$ & 5 & 80 & 12 & $<5 \%$ \\
\hline 11 & HOTf & 5 & 80 & 12 & 10 \\
\hline 12 & $\operatorname{In}(\mathrm{OTf})_{3}$ & 5 & 80 & 12 & $68 \%$ \\
\hline 13 & $\mathrm{La}(\mathrm{OTf})_{3}$ & 5 & 80 & 12 & N.R. \\
\hline 14 & AgOTf & 5 & 80 & 12 & 20 \\
\hline 16 & $\mathrm{Pd}(\mathrm{OAc})_{2}$ & 5 & 80 & 12 & N.R. \\
\hline
\end{tabular}

${ }^{a}$ Reactions were carried out by dissolving 1.2.1a (1.0 equiv), 1.3.2a (1.5 equiv) and catalyst in toluene $(0.2$ M); ${ }^{b}$ Determined by crude NMR.

From Table 5, the transformation was suitable for a variety of substituted alkynes. The terminal alkynes gave higher than $90 \%$ yields of only the Markovnikov's products even with $0.2 \%$ catalyst loading (1.3.3a-d). Impressively, the reaction also worked very well with symmetric internal alkynes, which are usually challenging substrates to be activated by metal catalysts. With $1 \%$ catalyst loading, the desired addition products were 
prepared in good yields (1.3.3e-g). Notably, the reaction reached excellent double-bond selectivity and only the trans-addition products were formed in all cases, though the regio-selectivity of the asymmetric alkynes was poor $(\mathbf{1} .3 .3 \mathbf{h}, \mathbf{1 . 3 . 3 i})$. The double-bond stereochemistry was confirmed by NOE experiments. Meanwhile, both alkyl and aryl substituted alkynes were suitable for this reaction.

Table 5: Substate scope of alkynes in the $\mathrm{Au}(\mathrm{I})$ catalyzed triazole addition. ${ }^{a}$

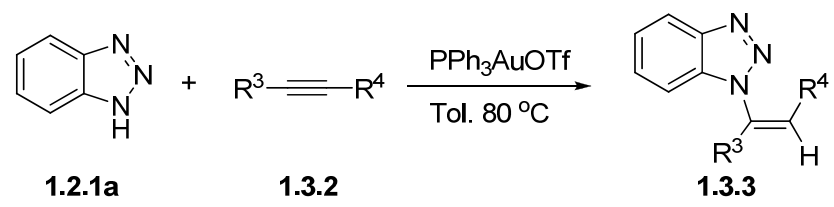

\begin{tabular}{|c|c|c|c|c|c|}
\hline Alkyne & Product & & Cat. (\%) & Time (h) & Yield $^{\mathrm{b}}(\%)$ \\
\hline$p-\mathrm{Me}-\mathrm{Ph}=\mathrm{H}$ & & 1.3.3b: $\mathrm{R}^{3}=p-\mathrm{Me}-\mathrm{Ph}$ & 0.2 & 12 & 94 \\
\hline$p-\mathrm{F}-\mathrm{Ph}=\mathrm{H}$ & & 1.3.3c: $\mathrm{R}^{3}=p-\mathrm{F}-\mathrm{Ph}$ & 0.2 & 10 & 96 \\
\hline$n-\mathrm{Bu}=\mathrm{H}$ & & 1.3.3d: $R^{3}=n-B u$ & 0.2 & 12 & 90 \\
\hline $\mathrm{Ph}=\mathrm{Ph}$ & & 1.3.3e: $\mathrm{R}^{3}=\mathrm{R}^{4}=\mathrm{Ph}$ & 1.0 & 30 & 70 \\
\hline$n-\operatorname{Pr}=n-\operatorname{Pr}$ & & 1.3.3f: $R^{3}=R^{4}=n-P r$ & 1.0 & 20 & 83 \\
\hline $\mathrm{TMS}=\mathrm{Me}$ & & 1.3.3g: $\mathrm{R}^{3}=\mathrm{CH}_{3}, \mathrm{R}^{4}=\mathrm{H}$ & 1.0 & 12 & 82 \\
\hline $\mathrm{Ph}=\mathrm{Me}$ & & & 1.0 & 20 & 84 \\
\hline$n-\mathrm{Pr}=\mathrm{Me}$ & & & 1.0 & 20 & 81 \\
\hline & 1.3.3i: $1: 1$ & ne two regio isomer & & & \\
\hline
\end{tabular}


Scheme 15: Substituted scope of different $\mathrm{NH}$-1,2,3-triazoles.

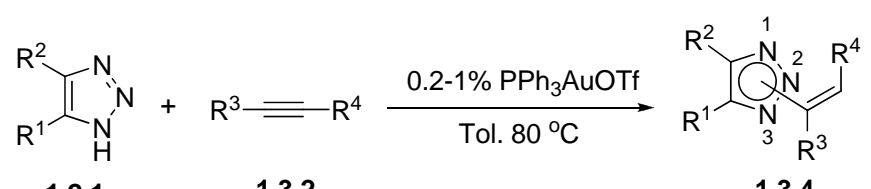

1.2 .1

1.3 .2

1.3.4

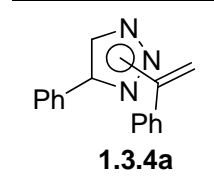

$0.2 \%$ cat., $91 \%$ yield

N1:N2:N3 = 22:78:0

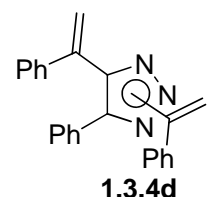

$0.2 \%$ cat., $90 \%$ yield N1:N2:N3 = 24:71:5

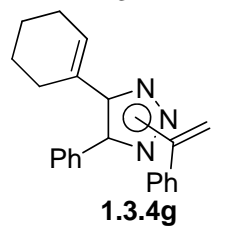

$0.2 \%$ cat., $87 \%$ yield $\mathrm{N} 1: \mathrm{N} 2: \mathrm{N3}=29: 71: 0$

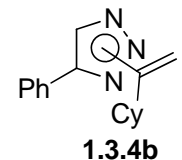

$0.2 \%$ cat., $87 \%$ yield N1:N2:N3 = 20:80:0

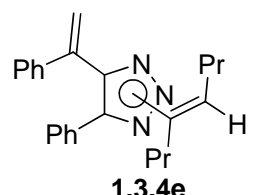

$1.0 \%$ cat., $85 \%$ yield N1:N2:N3 = 22:78:0

$$
\text { Cy }
$$$$
\text { 1.3.4h }
$$

$0.2 \%$ cat., $93 \%$ yield N1:N2:N3 = 18:82:0

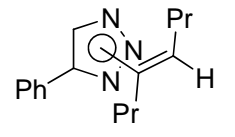

1.3.4c

$1.0 \%$ cat., $81 \%$ yield N1:N2:N3 = 18:82:0<smiles>C=C1N=NC(C(C)c2ccccc2)=C1c1ccccc1</smiles>

$0.2 \%$ cat., $88 \%$ yield N1:N2:N3 = 20:80:0<smiles>C=NC(=C)NC(=O)c1nnc(-c2ccccc2)[nH]1</smiles>

$0.2 \%$ cat., $84 \%$ yield $\mathrm{N} 1: \mathrm{N2}: \mathrm{N3}=0: 100: 0$

${ }^{a}$ Reactions were carried out by dissolving 1.2.1 (1.0 equiv), 1.3.2 (1.5 equiv) and $\mathrm{PPh}_{3} \mathrm{AuOTf}$ in toluene $(0.2 \mathrm{M})$ and heating at $80{ }^{\circ} \mathrm{C}$. ${ }^{\mathrm{b}}$ Ratio of the two products were determined by NMR.

With this highly efficient catalytic triazole addition strategy, we then investigated the N-selectivity with different $\mathrm{NH}$-1,2,3-triazoles (Scheme 15). All NH-triazoles were suitable for this transformation, giving the alkyne addition products in excellent yields. Only trans additions were observed, giving exclusive single double-bond isomers. The substituents on the triazoles showed a strong influence on the N-regio-selectivity, where most C-4-phenyl substituted triazoles gave N-2 isomers as the major products. Increasing the steric hindrance on the $\mathrm{C}-4$ and $\mathrm{C}-5$ positions did not show a dramatic difference towards the regio-selectivity. However, application of carbonyl-substituted NH-triazole 
led to the formation of a single N-2 isomer (1.3.4i), similar to the results from $\mathrm{N}-2$ alkylation ${ }^{15}$ and N-2 arylation (see Section 1.2) of NH-triazoles.

\subsubsection{Conclusion}

In conclusion, the first successful method in achieving vinyl substituted 1,2,3-triazoles through the $\mathrm{Au}(\mathrm{I})$ catalyzed alkyne additions was developed. Various vinyl-triazoles were prepared in good to excellent yields (up to $98 \%$ ) with as low as $0.2 \%$ catalyst loading. Furthermore, excellent stereoselectivity of double bonds was achieved. Considering the great efficiency, excellent stereoselectivity of double bonds, broad substrate scope and controllable triazole nitrogen regioselectivity, it is our belief that the reported method provides a good strategy for the preparation of various vinyl-triazoles and thereby benefiting researches involving 1,2,3-triazoles as building blocks in material science and medicinal chemistry. 


\subsection{Iron catalyzed $\mathrm{C}-\mathrm{O}$ bond activation for the synthesis of propargyl- 1,2,3-triazoles and 1,1-bis-triazoles $\dagger$}

\subsubsection{Examples of traditional propargylation}

The propargylic moiety is a widely distributed structure in medicinal and organic chemistry due to the high synthetic value of the alkyne functionality. ${ }^{28}$ The electron-rich triple bond, in combination with the fairly acidic character of the terminal acetylenic hydrogen atom, makes it a versatile entity for further chemical transformations. ${ }^{29}$ In addition, various natural products, fine chemicals and pharmaceuticals containing propargylic subunits as components of their structures have been reported. ${ }^{30}$

In general, the propargylation could be challenging since the nucleophiles would potentially attack the triple bond other than propargyl position, to give corresponding allene intermediates that further convert into other products, such as Meyer-Schuster rearrangement (Scheme 16). ${ }^{31}$

Scheme 16: Meyer-Schuster rearrangement and its mechanism.

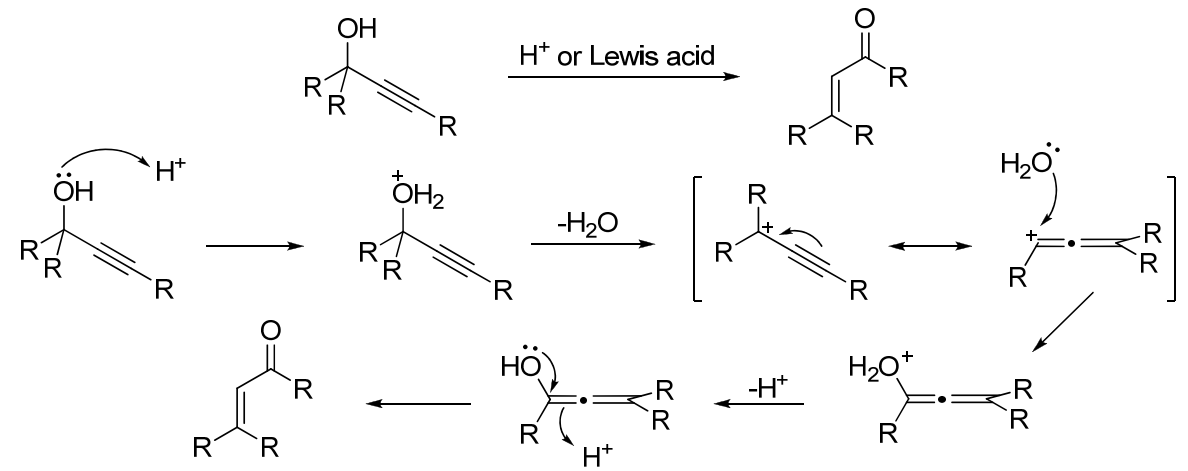

\footnotetext{
$\dagger$ Reproduced in parts with permission from Org. Lett. 2010, 12, 3308-3311. Copyright 2010, with permission from the American Chemical Society.
} 
In last several years, many methods regarding propargylation have been reported. One attractive method was the Brønsted acid catalyzed propargylation. ${ }^{32}$ Among these reactions, two of most common nucleophiles were 1,3-diester ${ }^{33}$ and allylsilane. ${ }^{34}$ Another efficient way for making this propargylic moiety was the direct propargylation of arenes or heteroarenes with propargyl alcohols via transition metal catalyzed Friedel-Crafts ${ }^{35}$ and ene-type ${ }^{36}$ alkylation reactions (Scheme 17).

Scheme 17: Metal promoted propargylation of arenes.<smiles>[R]C(O)C#CP</smiles>

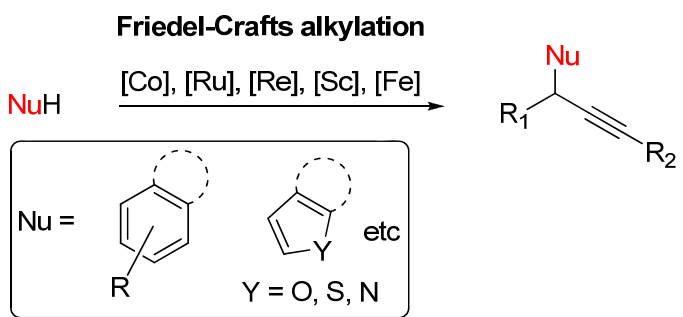<smiles>[R]C#CC([R])O</smiles>

Ene-type alkylation<smiles>[CH-]=C([Mg])[AlH2]</smiles><smiles>[R]C#CC([R])C=[W]</smiles>

With the fast-growing research applications of 1,2,3-triazoles, the effective methods in preparing diverse 1,2,3-triazole derivatives are strongly needed. Therefore, we are committed to the development of new strategies to introduce functional groups on the nitrogen of the triazole ring. At this time, we are focused on the propargylation of $\mathrm{NH}$ 1,2,3-triazoles with propargyl alcohols to synthesize propargyl-1,2,3-triazoles.

\subsubsection{Research Objective and Results}

To investigate the propargylation of $\mathrm{NH}$-1,2,3-triazole, we first studied the $\mathrm{S}_{\mathrm{N}} 2$ reaction between benzotriazole 1.2.1a and propargyl bromide 1.4.2a and obtained propargyl- 
1,2,3-triaozle 1.4.3a in excellent yield (98\%) as shown in Scheme 18. We then wondered whether the propargyl triazoles could be produced via simple $\mathrm{S}_{\mathrm{N}} 2$ reactions of propargyl acetates. The propargyl acetate 1.4.2b was reacted with benzotriazole 1.2.1a to give no reaction at all with a base $\mathrm{Cs}_{2} \mathrm{CO}_{3}$ (Scheme 19). Treating the acetate under harsher conditions (DMSO and strong base) led to the formation of complex reaction mixtures with no desired propargyl triazole 1.4.3b observed. Moreover, attempts in converting propargyl alcohol 1.4.4a into propargyl tolsylate or halide failed, which was likely caused by the undesired side reactions (such as Nazarov cyclization ${ }^{37}$ ) associated with propargyl carbon cations (better leaving groups). We therefore deduced that one practical synthesis of propargyl triazoles could likely occur from the effective C-O bond activation of readily available propargyl alcohol.

Scheme 18: Synthesis of propargyl triazole 1.4.3a.

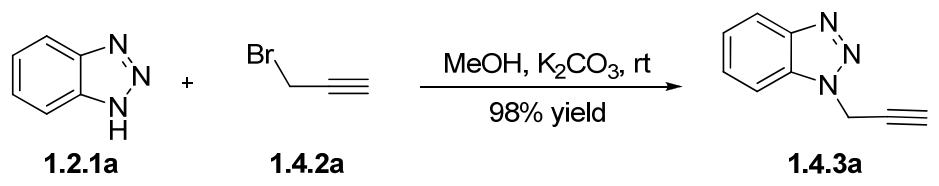

Scheme 19: Challenges in the triazole propargylation.

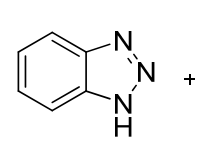

1.2.1a

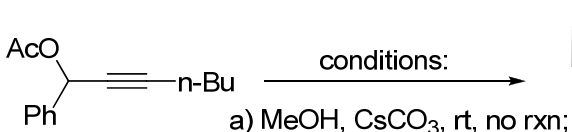

b) DMSO, NaOt-Bu, rt, no rxn

1.4.2b

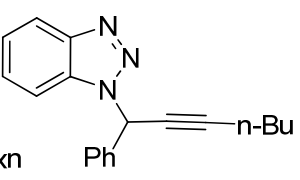

1.4.3b

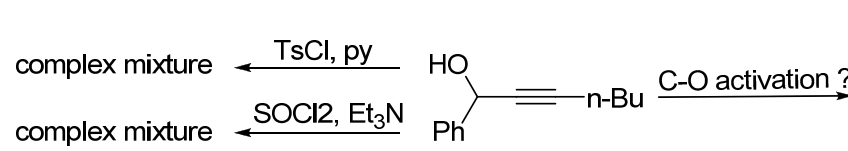

1.4.4a

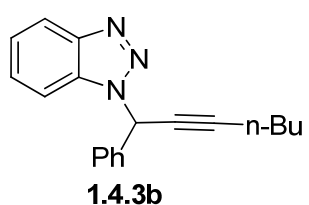

1.4.3b 
A series of different commonly used Lewis acid catalysts were investigated in promoting the propargylation of triazole 1.2.1a and propargyl alcohol 1.4.4a. The results are summarized in Table 6.

Table 6: Catalyst screening. ${ }^{a}$
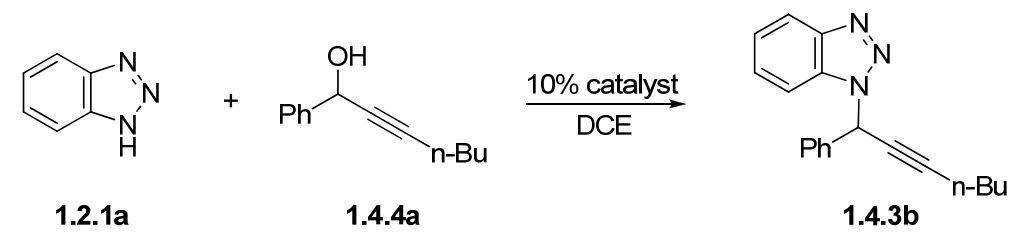

\begin{tabular}{|c|c|c|c|c|c|c|c|c|}
\hline & Catalyst & Loading (\%) & Solv. & Temp $\left({ }^{\circ} \mathrm{C}\right)$ & $\begin{array}{l}\text { Time } \\
\text { (h) }\end{array}$ & Conv. $(\%)^{b}$ & $\begin{array}{l}\text { Yield } \\
(\%)^{c}\end{array}$ & N1:N2 \\
\hline 1 & $\mathrm{FeCl}_{3}$ & 20 & DCE & 60 & 17 & 100 & 90 & $8: 1$ \\
\hline 2 & $\mathrm{Cu}(\mathrm{OAc})_{2}$ & 20 & DCE & 60 & 17 & 57 & $<5$ & \\
\hline 3 & $\mathrm{CuI}$ & 20 & DCE & 60 & 17 & 22 & $<5$ & \\
\hline 4 & $\mathrm{PdCl}_{2}$ & 20 & DCE & 60 & 17 & 13 & $<5$ & \\
\hline 5 & $\mathrm{RuCl}_{3}$ & 20 & DCE & 60 & 17 & 69 & 49 & 1.1:1 \\
\hline 6 & $\mathrm{IrCl}_{3}$ & 20 & DCE & 60 & 17 & 96 & 65 & $2: 1$ \\
\hline 7 & $\mathrm{Co}(\mathrm{OAc})_{2}$ & 20 & DCE & 60 & 17 & 28 & $<5$ & \\
\hline 8 & $\mathrm{LaCl}_{3}$ & 20 & DCE & 60 & 17 & 21 & $<5$ & \\
\hline 9 & $\mathrm{CeCl}_{3}$ & 20 & DCE & 60 & 17 & 30 & $<5$ & \\
\hline 10 & $\mathrm{Ti}(\mathrm{O}-\mathrm{iPr})_{4}$ & 20 & DCE & 60 & 17 & 52 & $<5$ & \\
\hline 11 & $\mathrm{AlCl}_{3}$ & 20 & DCE & 60 & 17 & 26 & $<5$ & \\
\hline 12 & $\mathrm{TfOH}$ & 20 & DCE & 60 & 17 & 100 & 35 & $1.5: 1$ \\
\hline 13 & $\mathrm{H}_{3} \mathrm{PO}_{4}$ & 20 & DCE & 60 & 17 & 73 & 9 & $1: 1$ \\
\hline 14 & $\mathrm{FeCl}_{3}$ & 20 & THF & 60 & 7 & 90 & 70 & $6: 1$ \\
\hline 15 & $\mathrm{FeCl}_{3}$ & 20 & Toluene & 60 & 7 & 100 & 40 & $2: 1$ \\
\hline 16 & $\mathrm{FeCl}_{3}$ & 20 & $\mathrm{MeOH}$ & 60 & 7 & 60 & 44 & $7: 1$ \\
\hline 17 & $\mathrm{FeCl}_{3}$ & 20 & $\mathrm{MeNO}_{2}$ & 60 & 7 & 90 & $<5$ & \\
\hline 18 & $\mathrm{FeCl}_{3}$ & 20 & DMF & 60 & 7 & $<5$ & $<5$ & \\
\hline
\end{tabular}




$\begin{array}{ccccccccc}19 & \mathrm{FeCl}_{3} & 20 & \mathrm{DMSO} & 60 & 7 & <5 & <5 & \\ 20 & \mathrm{FeCl}_{3} & 20 & \mathrm{MeCN} & 60 & 7 & 91 & 85 & 10: 1 \\ 21 & \mathrm{FeCl}_{3} & 20 & \mathrm{MeCN} & \mathrm{rt} & 12 & 62 & 50 & 9: 1 \\ 22 & \mathrm{FeCl}_{3} & 20 & \mathrm{MeCN} & 90 & 5 & 100 & 93 & 11: 1 \\ 23 & \mathrm{FeCl}_{3} & 10 & \mathrm{MeCN} & 90 & 10 & 100 & 90 & 10: 1 \\ 24 & \mathrm{FeCl}_{3} & 5 & \mathrm{MeCN} & 90 & 12 & 90 & 82 & 9: 1\end{array}$

${ }^{a}$ Standard reaction condition: 1 eqiuv propargyl alcohol 1.1.4a, 1.2 equiv triazole 1.2.1a, 0.2 equiv catalyst and solvent; ${ }^{b}$ conversions were determined based on the consumption of 1.4.4a; ${ }^{c}$ yields determined by NMR with 1,3,5-trimethoxybenzene as internal standard, and ratio determined by NMR of crude reaction mixtures.

As shown in Table 6, the significant decomposition of 1.4.4a occurred with most of the Lewis acid catalysts. Some catalysts, such as $\mathrm{RuCl}_{3}$ and $\mathrm{IrCl}_{3}$, promoted this reaction in modest yields, but gave poor regioselectivities of $1: 1$ to $2: 1$ ratio of $\mathrm{N} 1 / \mathrm{N} 2$ isomers (entry 5, 6, 12 and 13). Based on the observed performance of the various catalysts, $\mathrm{FeCl}_{3}$ was revealed as the effective catalyst in promoting this transformation and gave the desired product $\mathbf{1 . 4 . 3 b}$ in good yield with high regioselectivity, with an 8:1 ratio of N1/N2 isomers (entry 1). Further solvent screening revealed that this reaction was totally shut down in polar solvents, like DMSO, DMF. Surprisingly, the reaction gave almost no product formation with the $90 \%$ conversion of propargyl alcohol when $\mathrm{MeNO}_{2}$ applied as the reaction solvent, because of the formation of $\alpha, \beta$-unsaturated ketone product from Meyer-Schuster rearrangement (entry 17). Finally, we found the $\mathrm{MeCN}$ as the optimal solvent (entries 15-20) to give effective conversion even with decreased catalyst loading (entries 23-24). With the optimal condition in hand, different triazoles and propargyl alcohols were applied to investigate the reaction substrate scope (Figure 11). 
Figure 11: Substrate scope of triazole propargylation. ${ }^{a}$
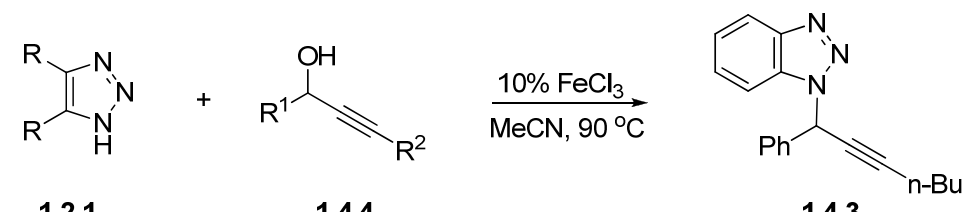

1.2.1

1.4 .4

1.4.3
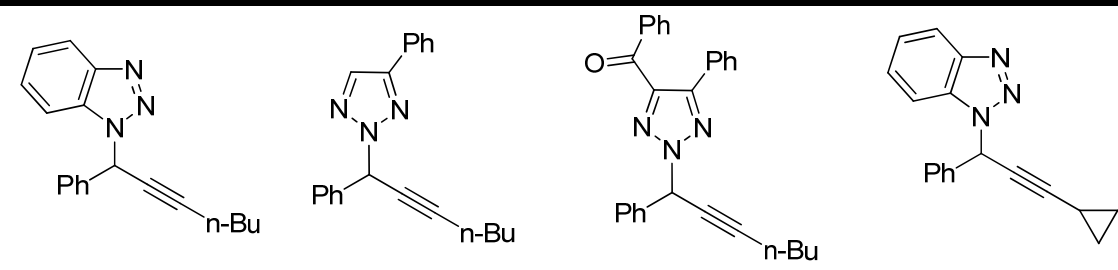

$\triangle N_{N}^{N}$

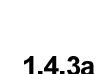

N1: $85 \%$

$$
\begin{gathered}
1.4 .3 b \\
N 2: N 1=85 \%: 10 \%
\end{gathered}
$$

$1.4 .3 \mathrm{c}$

1.4.3d

N1: $89 \%$<smiles>C(#CC(c1ccccc1)c1ccccc1)c1ccccc1</smiles>

N2: $90 \%$

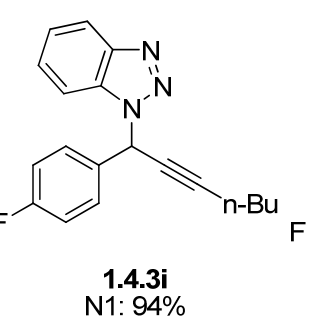

1.4.3e<smiles>O=C(c1ccccc1)c1nn(C(C#Cc2ccccc2)c2ccccc2)nc1-c1ccccc1</smiles><smiles>CCCC#CC(c1cccc2ccccc12)n1nnc2ccccc21</smiles>

1.4.3h

N1: $88 \%$

N1: $94 \%$

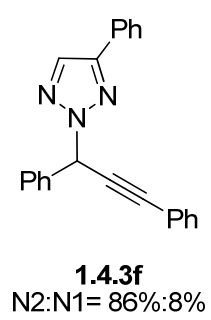

$1.4 .3 \mathrm{~g}$
$\mathrm{~N} 2:-92 \%$<smiles>Cc1ccc(C(C#Cc2ccccc2)n2ncc(-c3ccccc3)n2)cc1</smiles><smiles>c1ccc2[nH]nnc2c1</smiles><smiles>Cc1ccc(C(C#Cc2ccccc2)C#Cc2ccccc2)cc1</smiles><smiles>CC#CC(c1ccccc1)n1ncc(-c2ccccc2)n1</smiles><smiles>CC(C)(C)C#CC(c1ccccc1)n1nnc2ccccc21</smiles>

$\mathrm{N} 2: \mathrm{N} 1=76 \%: 13 \%$

N2:N1 $=84 \%: 8 \%$

1.4 .31
$N 1: 85 \%$<smiles>CCCCC#CC1(n2nnc3ccccc32)C=CCCC1</smiles><smiles>CC#CC1(n2nnc3ccccc32)C=CCCC1</smiles>
N2:N1 $=64 \%: 26 \%$

$1.4 .3 n$
N1: $96 \%$
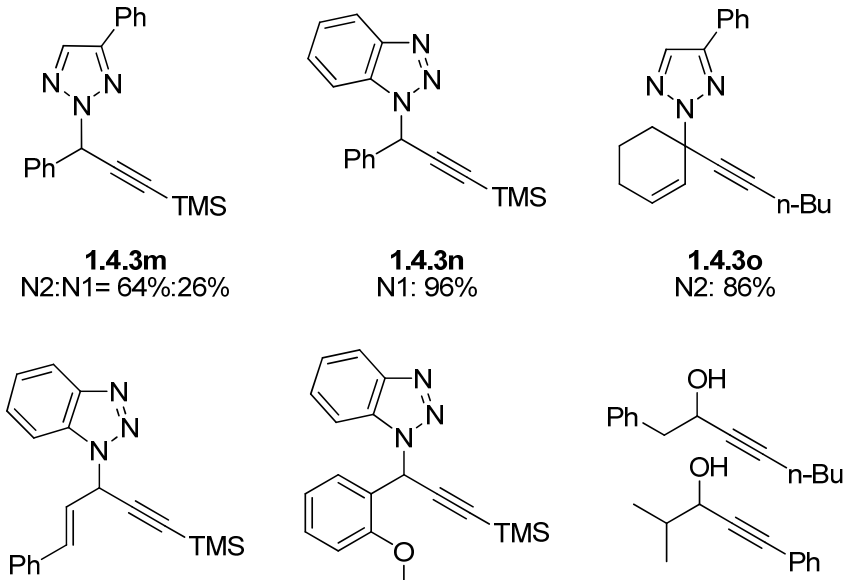<smiles>COc1ccccc1C(C#CC(C)(C)C)n1nnc2ccccc21</smiles><smiles>CCCCC#CC(O)Cc1ccccc1</smiles>

1.4.3p
N1:N2= $1.4 .3 q$
N1: $93 \%$

$1.4 .3 s$
$N 1: 82 \%$

no reaction

a Yields were determined by NMR with 1,3,5-trimethoxybenzene as internal standard, and ratios determined by NMR of crude reaction mixtures. The N-2 1.4.3f structure was confirmed by X-ray crystallography.

As shown in Figure 11, this $\mathrm{Fe}(\mathrm{III})$-catalyzed propargylation was suitable for various different propargyl alcohols, which further enriched the available methods for effective post-triazole functionalization. Similar to our previously reported strategy (see 
Section 1.1.2), the regioselectivity of 1,2,3-triazole strongly depends on the nature of triazole substrates. For example, the benzotriazole gave primarily $\mathrm{N}-1$ substituted products, while keto-modified 4,5-disubstituted triazoles produced the dominant $\mathrm{N}-2$ isomers (i.e., 1.4.3c, 1.4.3g). Meanwhile, the $\mathrm{C}-4$ phenyl triazole gave a mixture of both $\mathrm{N}-1$ and N-2 isomers with roughly 4:1 ratio, favoring the N-2 isomer.

The substituted groups on the propargyl position were critical for this transformation. The reaction generally worked well with aromatic substituted propargyl alcohols, except substrates with strong electron withdrawing groups (the p-nitrobenzene gave no reaction). No reactions were observed with aliphatic substituted propargyl alcohols due to the insufficient reactivity of $\mathrm{C}-\mathrm{O}$ bond. Impressively, the reaction proceeded smoothly with vinyl-substituted substrate (such as 1.4.3r), giving the corresponding triazole substituted enynes in excellent yields. Notably, Nazarov cyclization products were not observed in these reactions (Figure 12), which highlighted the rather mild conditions of the reported method.

Figure 12: Potential formation of Nazarov cyclization product with vinyl-substituted propargyl alcohols.

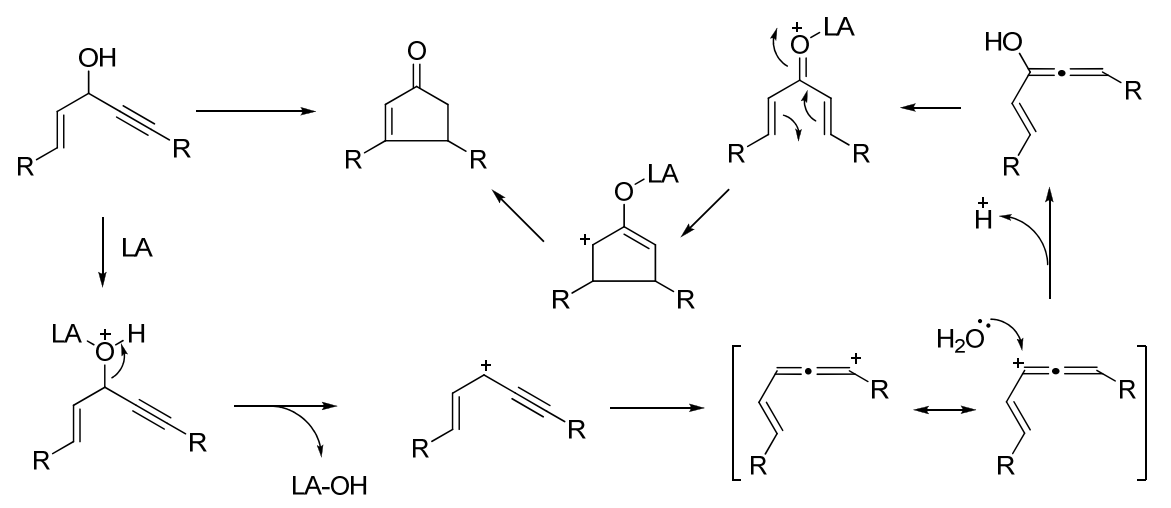


Excellent yields were generally received for internal alkynes with different substituted groups, including alkyl, aryl, TMS, and cyclopropyl. The reaction was also suitable for terminal alkynes and gave the corresponding propargyl triazoles in good yields based on NMR. Surprisingly, these substrates were not stable upon condensation for further purification purpose and gave complex mixtures (Scheme 20). We then thought the terminal propargyl triazoles would undergo further transformations in the presence of $\mathrm{FeCl}_{3}$. To remove the $\mathrm{FeCl}_{3}$ factor, the deprotection of TMS protected propargyl triazole 1.4.3n was carried out. Unfortunately, we obtained exactly the same result, which was the decomposition of terminal propargyl triazole upon condensation. One potential pathway could be triazole-alkyne 5-endo-dig cyclization which has been reported by Chen in our group. ${ }^{38}$

Scheme 20: Unexpected decomposition of terminal propargyl triazole.

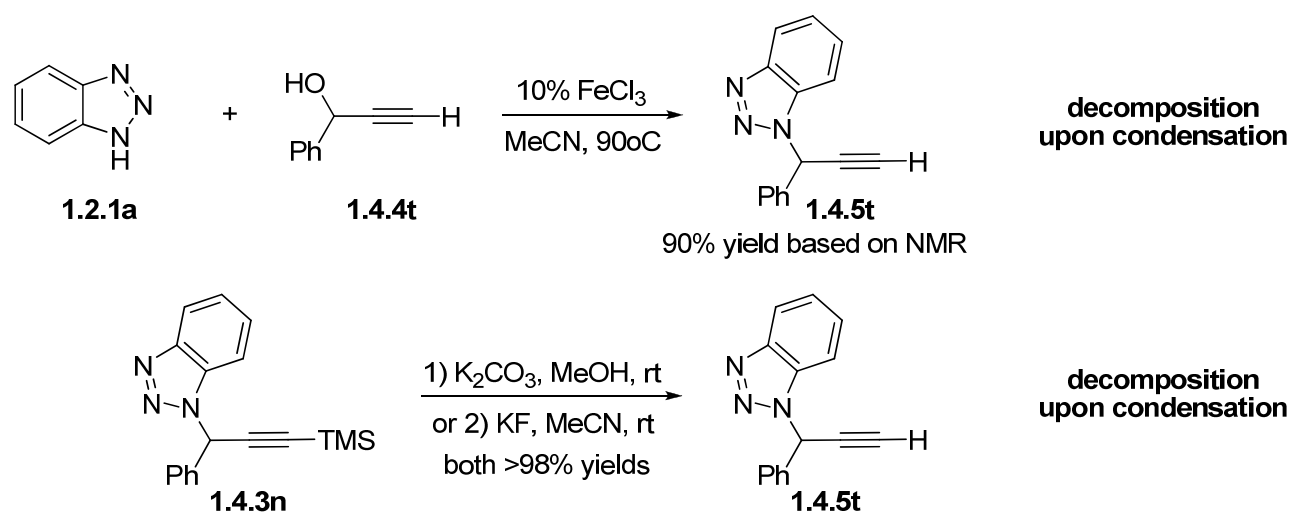

\subsubsection{Application in synthesis of 1,1-bis-triazoles via 'click' chemistry}

During the last four years, our group has been working on the development of 1,2,3triazole derivatives as ligands in transition metal catalysis. ${ }^{39}$ These triazole complexes have shown significant influence on the metal catalyst reactivity by serving as effective 
nitrogen $\sigma$-donor. Our further studies of triazole-metal coordination initiated our interest in the synthesis the bis-triazole compounds as potential interesting bidentate ligands to coordinate with transition metal cations.

Scheme 21: Three Different Types of Bis-triazole Compounds.

a) N-N type bistriazole

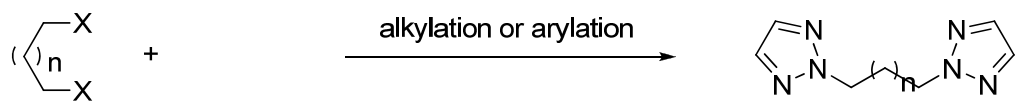

b) C-C type bistriazole

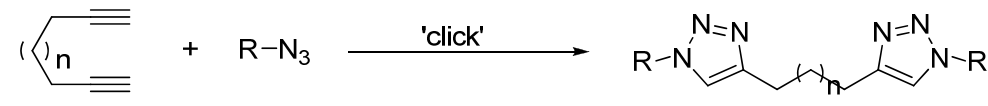

c) N-C type bistriazole

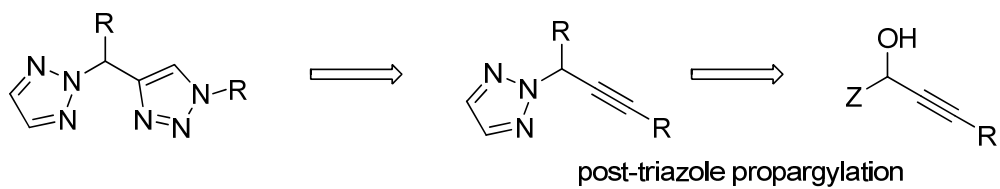

Considering the different kind of substitution pattern, the bis-triazole compounds could be summarized in three different classes: N-N type, ${ }^{40} \mathrm{C}-\mathrm{C}$ type,${ }^{41}$ and $\mathrm{N}-\mathrm{C}$ type (Scheme 21). While the N-N and C-C type bis-triazoles have been successfully synthesized from either post-triazole alkylation (Scheme 21a) ${ }^{15}$ or double click chemistry (Scheme 21b), the N-C type bis-triazoles have not been reported. These unsymmetrical $\mathrm{N}-\mathrm{C}$ type bis-triazoles, especially the 1,1-bis-triazoles, are particularly interesting because of the presence of the stereocenter. A brief synthetic design of this type of compounds is shown in Scheme 21c, where the two triazole moieties are introduced by post-triazole propargylation and sequentially click chemistry. Although the retrosynthesis design looked straightforward, the actual preparation was much more challenging than we expected, especially the click chemistry of propargyl triazoles. 
The click chemistry worked well with terminal alkynes rather than internal alkynes. However, the terminal propargyl triazoles undergo decomposition upon condensation, as we mentioned before. Therefore, we focused on searching a proper displacement of terminal propargyl triazole which can be applied in click chemistry, such as readily prepared TMS protected propargyl triazole.

Scheme 22: Synthesis of unsymmetrical N-C type bis-triazoles. ${ }^{a}$
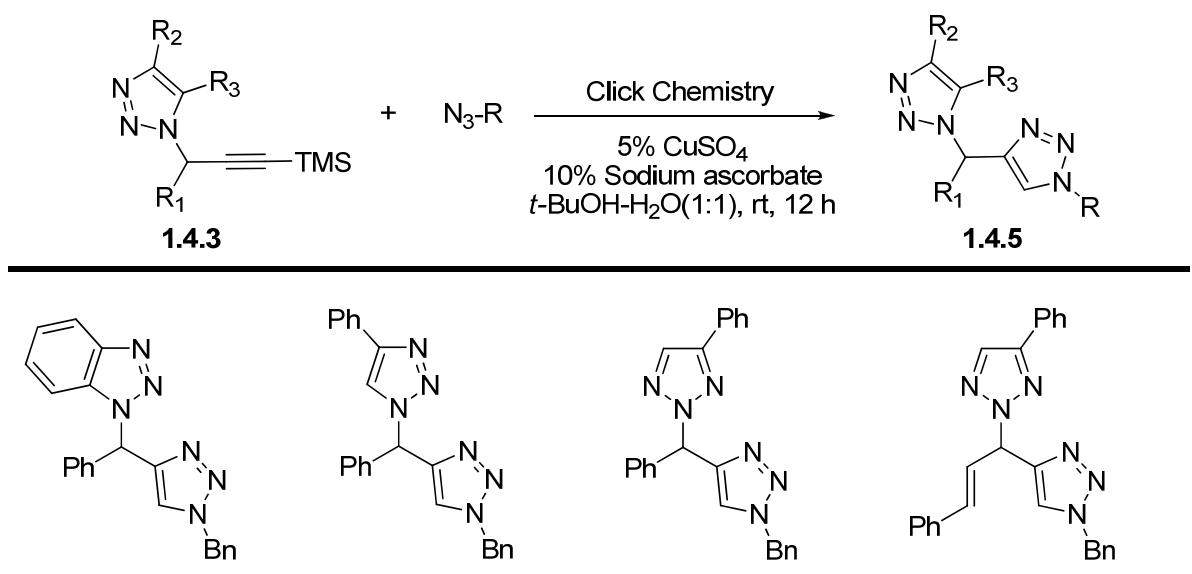

1.4.5a $95 \%$

1.4.5b $94 \%$

1.4.5c $93 \%$

1.4.5d $90 \%$<smiles>C1=CC(c2cn(Cc3ccccc3)nn2)(n2nnc3ccccc32)CCC1</smiles><smiles>COc1ccccc1C(c1cn(Cc2ccccc2)nn1)n1nnc2ccccc21</smiles><smiles>COc1ccccc1C(c1cn(COc2ccccc2)nn1)n1nnc2ccccc21</smiles>

1.4.5g $96 \%$

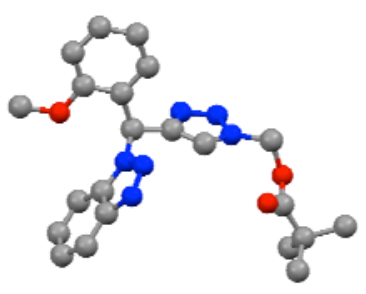

X-ray of $1.4 .5 \mathrm{~g}$

${ }^{a}$ Isolated yields for all cases.

With the click chemistry of TMS protected alkynes, the universal procedures were TMS deprotection under basic condition first and subsequently 'click' chemistry in two individual steps. However, we have combined the TMS deprotection and click chemistry in one single step. Fortunately, the reactions between TMS protected propargyl triazoles 
and corresponding azides gave the desired bis-triazoles (1.4.5a-h) in excellent yields under standard "click chemistry" condition (Scheme 22).

\subsubsection{Conclusion}

In conclusion, a highly efficient $\mathrm{FeCl}_{3}$ catalyzed post-triazole propargylation method was developed, giving the desired propargyl triazole in excellent yields. Application of this strategy led to the synthesis of unsymmetrical 1,1-bis-triazoles. This study not only provided another efficient post-triazole functionalization strategy for the preparation of diverse triazole propargyl analogues, but provided entry to a new class of 1,1-bis-triazole compounds, which would likely be of interest for medicinal chemistry research, transition metal catalysis and material science. 


\subsection{Synthesis of allene triazole through iron catalyzed regioselective addition to propargyl alcohols $\uparrow$}

\subsubsection{Previous approaches for synthesis allene compounds}

During last few decades, allene chemistry has received ample attention from organic and medicinal chemists, due to their synthetic potential in regio- and stereoselective $\mathrm{C}-\mathrm{C}$ and C-X bond forming reactions ${ }^{42}$ and unique axis-to-center chirality transfer properties. ${ }^{43}$ Moreover, allene subunits have been widely found in many natural products and pharmacologically active compounds. ${ }^{44}$ Therefore, the allene chemistry has created the great demand of new methods for synthesis of any kind of allenes as precursors.

All the classical reaction types (addition, ${ }^{45}$ elimination, ${ }^{46}$ substitution ${ }^{47}$ and rearrangement ${ }^{48}$ ) have been applied to the synthesis of allenes and transition metal catalyzed allene synthesis have also been developed. ${ }^{49}$ Among these methods, one of most convenient and general strategy for the synthesis of allenes was the nucleophilic $\mathrm{S}_{\mathrm{N}} 2^{\prime}$ substitution of propargylic compounds with a leaving group. A variety of labile groups, including propargylic acetates, mesylates, carbonates, and halides, etc. have been used as leaving groups (Figure 13).

Figure 13: Most general method for synthesis allenes via nucleophilic $\mathrm{S}_{\mathrm{N}} 2^{\prime}$ substitution.

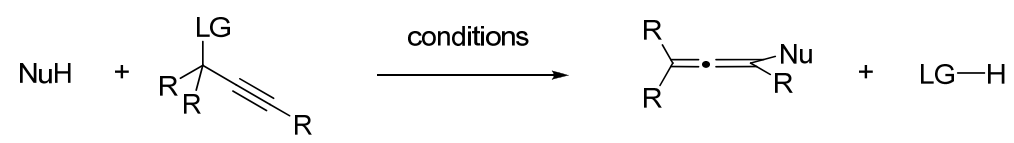

\footnotetext{
${ }^{\dagger}$ Reproduced in parts with permission from Chem. Commun. 2012, 48, 3521-3523. Copyright 2012, with permission from The Royal Society of Chemistry.
} 
In the nucleophilic $\mathrm{S}_{\mathrm{N}} 2^{\prime}$ substitutions, various types of nucleophiles, such as hydrides, halides, and organometallic reagents, have been employed. With hydride nucleophiles, most common hydride sources were aluminum hydride reagents, such as lithium aluminum hydride $\left(\mathrm{LiAlH}_{4}\right)$, and diisobutylaluminum hydride (DIBAL-H). ${ }^{50}$ Recently, $\mathrm{Cp}_{2} \mathrm{Zr}(\mathrm{H}) \mathrm{Cl}$ has been applied to the anti-S $\mathrm{S}_{\mathrm{N}} 2^{\prime}$-type reductive substitution of the in situ generated zinc or magnesium alkoxides of propargylic alcohols furnishing allenes in good yields and high ee's (Scheme 23). ${ }^{51}$ This approach provided stereospecific access to allenes directly from propargylic alcohols and then greatly improved the efficiency.

Scheme 23: $\mathrm{Cp}_{2} \mathrm{Zr}(\mathrm{H}) \mathrm{Cl}$ catalyzed preparation of chiral allenes.

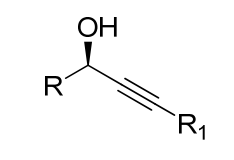

$\mathrm{R}, \mathrm{R} 1=$ alkyl, aryl

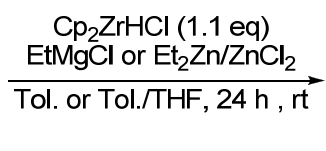

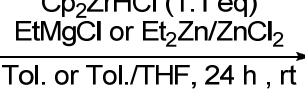

(1)

Haloallenes were synthesized by the $\mathrm{S}_{\mathrm{N}} 2^{\prime}$ substitutions of propargyl alcohols with halide nucleophiles which are useful substrates in substitution reactions ${ }^{52}$ and transitionmetal catalyzed cross-coupling reactions. ${ }^{53}$ The typical reaction condition for the preparation of haloallenes from paropargyl alcohols was Appel-type reaction condition containing halide source (NXS or $\mathrm{CBr}_{4}$ ) and appropriate $\mathrm{OH}$ activating reagent, such as phosphane-like $\mathrm{PPh}_{3},(n-\mathrm{Bu})_{3} \mathrm{P}$ and trisubstituted amines (i-PrNEt) (Scheme 24) ${ }^{54}$ Other conditions also have been reported. For example, treatment of propargyl diethanolamine boronates with $\mathrm{N}$-halosuccinimides gave the corresponding allenyl halides in high yields (Scheme 25). ${ }^{55}$ Moreover, the reaction of secondary or tertiary propargylic alcohols with $\mathrm{NbBr}_{5}$ afforded allenylic bromides in moderate to good yields (Scheme 26). ${ }^{56}$ 
Scheme 24: Appel-type reactions for synthesis haloellenes.

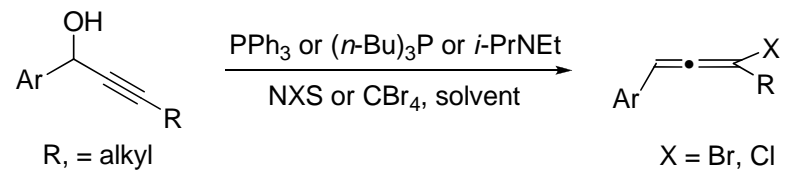

Scheme 25: Synthesis of haloellenes from propargyl diethanolamine boronates.

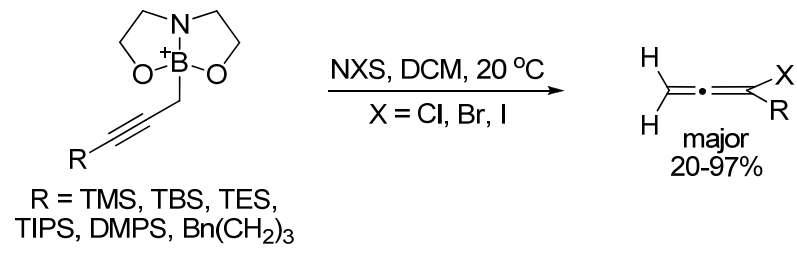

Scheme 26: Formation of bromoallenes by using $\mathrm{NbBr}_{5}$ as the catalyst and bromide source.

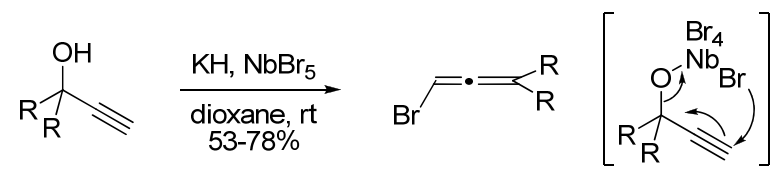

Allenes can be synthesized via the direct $\mathrm{S}_{\mathrm{N}} 2^{\prime}$ addition of organometallic reagents, such as copper, indium, ${ }^{57}$ zinc $^{58}$ etc. to propargylic alcohols. Especially, diorganocopper reagents (Gilman's cuprates) have been widely used to prepare allenes from propargyl moieties with excellent chemoselectivity of $\mathrm{S}_{\mathrm{N}} 2^{\prime}$ substitutions rather than direct $\mathrm{S}_{\mathrm{N}} 2$ reactions. ${ }^{59}$ There are also many different organocopper catalysts involving a catalytic amount of a copper(I) salt (like $\mathrm{CuBr}$ ) with an excess amount of organometallic reagents, which included organolithium, ${ }^{60}$ Grignard reagents, ${ }^{61}$ organozinc, ${ }^{62}$ and organozirconium, have been reported in the literature (Scheme 27). ${ }^{63}$ 
Scheme 27: Application of organometallic reagents in preparation of allenes.

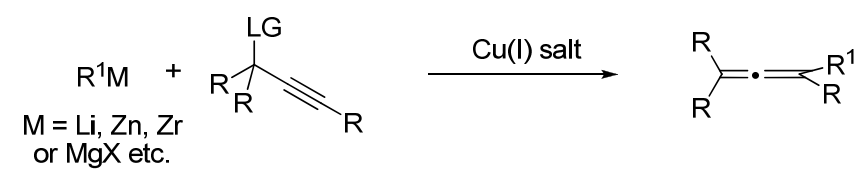

\subsubsection{Examples for allene as either ligand or catalyst.}

It is well known that the allene compound has played an important role in the organic reactions as synthetic intermediate. However, there are only a few examples of allenes being used as catalysts or ligands have been reported in the literature. The first example was reported by Soai and co-workers in 2002. They have developed the asymmetric synthesis of pyrimidin-5-yl alkanol in the addition of $(i-P r)_{2} \mathrm{Zn}$ to pyrimidine-5carbaldehyde with excellent ee's by using chiral 1,3-disubstituted hydrocarbon allenes as catalysts (Scheme 28). ${ }^{64}$ Later, they also pointed out the actual effectiveness of the allene as a chiral modulator was difficult to gauge because other experiments with the same system indicated that a strong nonlinear effect coupled with autocatalysis conspire to amplify even unmeasurable enantiomeric excesses. ${ }^{65}$

Scheme 28: Enantioselective addition of $(i-\operatorname{Pr})_{2} \mathrm{Zn}$ to 2-(alkynyl)peprinidine-5carbaldehyde in the presence of chiral allenes.

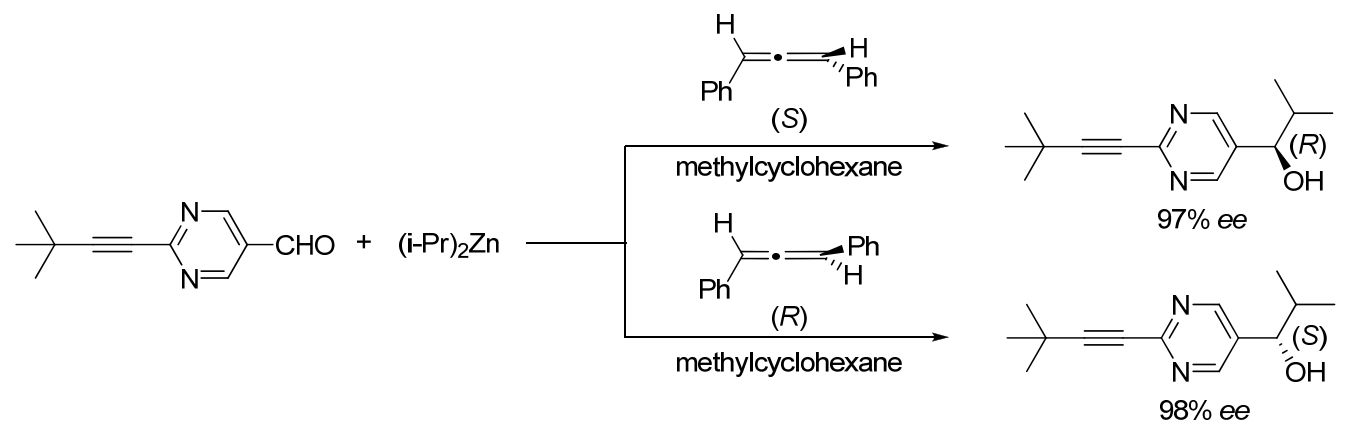


In 2009, Ready and co-workers demonstrated that chirality of chiral allenes can be transferred to products in asymmetric catalysis. In the paper, they prepared a series of allene-containing biphosphine oxide catalysts, which were utilized in the asymmetric ring opening of meso-epoxides reactions with good enantioslectivities (Scheme 29). ${ }^{66}$ The allene-containing biphosphine oxide catalysts revealed the very high efficiency in the reaction $(0.1 \%$ loading only). Moreover, they successfully used chiral allene-containing biphosphine oxides as ligands in transition metal catalysis in 2011 (Scheme 30). ${ }^{67}$

Scheme 29: Asymmetric ring opening of meso-epoxides with $\mathrm{SiCl}_{4}$.

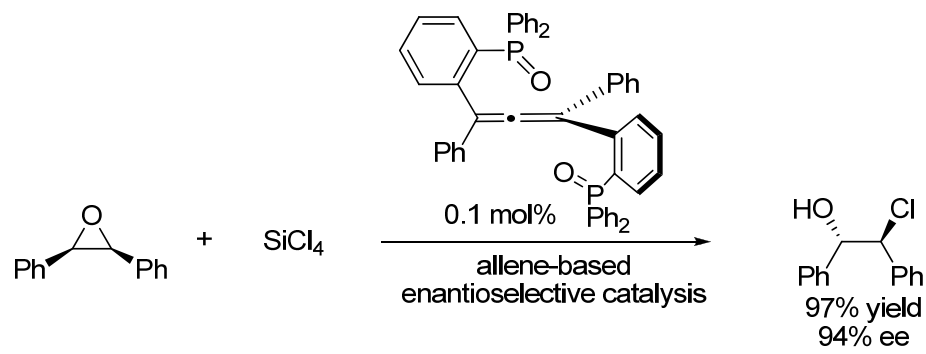

Scheme 30: Enantioselective addition of boronic acids to $\alpha$-keto esters catalyzed by $\mathrm{Rh}^{\mathrm{I}}($ AllenePhos) complexes.
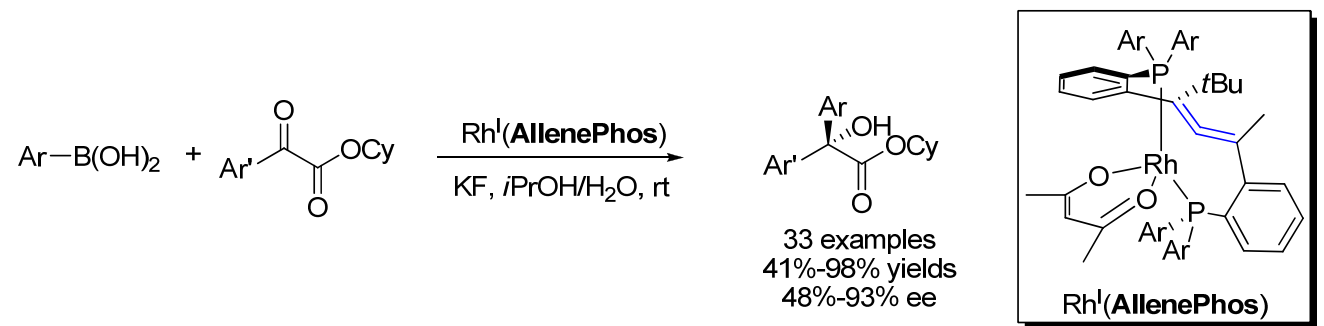

These studies opened the potential door of allenes compounds in the asymmetric catalysis. Considering the triazole binding pattern, we wondered whether the allenyl triazoles have the unique catalytic properties in transition metal catalysis. However, more 
importantly, there is no effective method for synthesis of allenyl triazoles. We subsequently reported for the first time, the successful synthesis of allene triazoles through iron catalyzed regioselective triazole addition to the propargyl alcohol.

\subsubsection{Research Objective and Results}

Based on the literature reported strategies, two common approaches were proposed for the allene-triazole synthesis. One proposed pathway for synthesis of allene-triazole invovles the deprotonation of propargyl triazoles at the propargyl position, followed by the treatment with $\mathrm{H}^{+}$or other electrophiles (Scheme 31).

Scheme 31: Proposed approaches from deprotonation of propargyl triazoles.

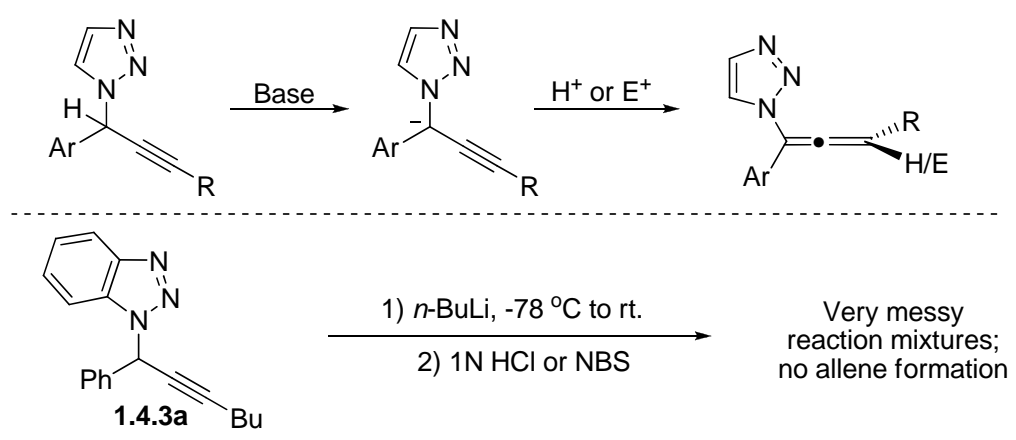

Unfortunately, the treatment of propargyl triazole 1.4.3a with different base like $n \mathrm{BuLi}$ or $\mathrm{KOtBu}$ followed by the quenching with either water or NBS gave a complex reaction mixture with no allene product formation. We then focused on alternative plausible $\mathrm{S}_{\mathrm{N}} 2^{\prime}$ strategy, the triazole addition to propargyl alcohol derivatives. However, the reaction between propargyl acetate 1.5.2a and benzotriazole 1.2.1a gave no allene product even after the reaction mixture was refluxed for 10 hours with triazole anion 
(Scheme 32). These results suggested that the synthesis of an allene-triazole was indeed a great challenge using conventional methods.

Scheme 32: Proposed $\mathrm{S}_{\mathrm{N}} 2^{\prime}$ strategy of propargyl acetate with triazole anion.

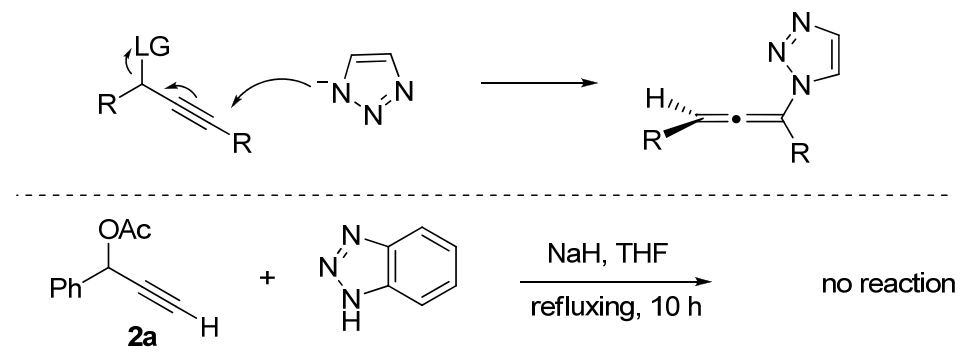

Considering our previous success in the synthesis of propargyl triazoles through iron-catalyzed $\mathrm{C}-\mathrm{O}$ bond activation, we decided to apply this process for the preparation of allene triazoles. According to literature, few examples have been reported for synthesis of allenes from propargyl alcohols by directly using Lewis acids as the catalysts (Scheme 33). ${ }^{68}$

Scheme 33: Lewis acids activation strategy for synthesizing allenes.

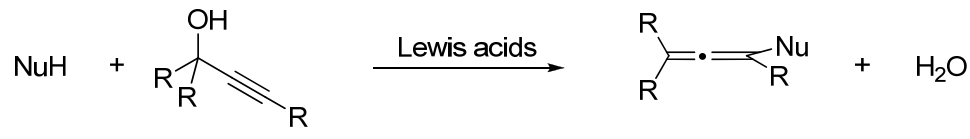

As shown in Figure 14, the propargylation product can be formed if triazole substituted on the propargylic position $\left(\mathrm{S}_{\mathrm{N}} 2\right)$, while the allenation product will be produced if the triazole attacked the triple bond $\left(\mathrm{S}_{\mathrm{N}} 2^{\prime}\right)$. Therefore, the di-substituted propargyl alcohols will be used with the hope to increase the steric hindrance at the propargyl position to direct the triazole to the alkyne based on our understanding of C-O bond activation. Although the di-substitution limited the reaction substrate scope, the 
success of this strategy led to the first synthesis of the allene triazole and open the door for further development.

Figure 14: Iron-catalyzed C-O bond activation.

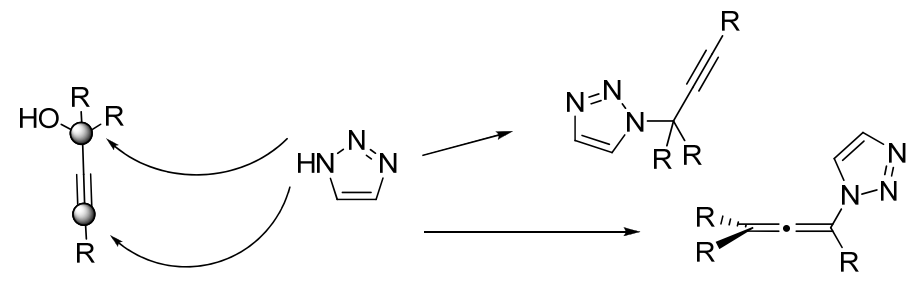

To test our hypothesis, diphenyl substituted propargyl alcohol 1.5.3a was reacted with benzotriazole 1.2.1a in the presence of $\mathrm{FeCl}_{3}$ and $\mathrm{MeCN}$ as reaction solvent, both products from propargylation (1.5.5a) and allenation (1.5.4a) in a 1:3 ratio were obtained with 57\% NMR yield (Scheme 34). The allene product 1.5.4a has been characterized by X-ray crystallography.

Scheme 34: First reaction for investigation triazole allene product formation.<smiles>CC(C)(C#CC(O)(c1ccccc1)c1ccccc1)c1ccccc1</smiles>

1.5.3a<smiles>c1ccc2[nH]nnc2c1</smiles>

1.2.1a

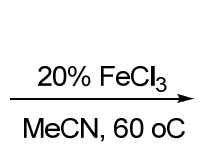

TMS

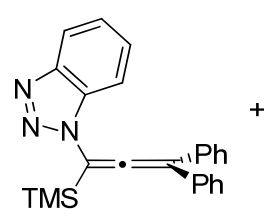

1.5.4a

conv.: $86 \%$, yield: $57 \%, 3: 1$

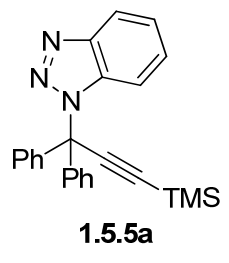

Encouraged by this result, the reactions between propargyl alcohol 1.5.3a and benzotriazole 1.2.1a have been investigated under various catalysts (Table 7). As shown in Table 7, the decomposition of propargyl alcohol 1.5.3a occurred with most Lewis acids catalysts. However, we found $\mathrm{FeCl}_{3}$ was the best choice of catalyst to give modest yield $57 \%$ and fair regioselectivity (3:1 ratio) for this transformation. Compared to other 
transition metals, iron catalysts had many advantages, such as inexpensive, nontoxic and easy to handle.

Table 7: Catalyst screening ${ }^{a}$

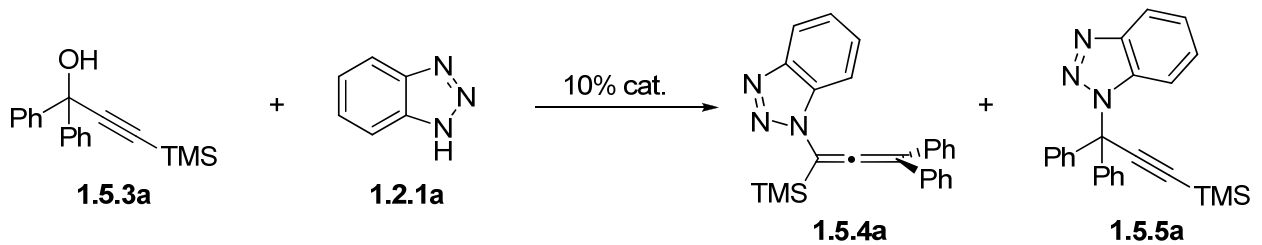

\begin{tabular}{|c|c|c|c|c|c|c|c|c|}
\hline & Catalyst & $\begin{array}{c}\text { Loading } \\
(\%)\end{array}$ & Sol. ${ }^{b}$ & $\begin{array}{c}\text { Temp } \\
\left({ }^{\circ} \mathrm{C}\right)\end{array}$ & $\begin{array}{l}\text { Time } \\
\text { (h) }\end{array}$ & $\begin{array}{l}\text { Tonv. } \\
(\%)^{c}\end{array}$ & $\begin{array}{l}\begin{array}{l}\text { Yield } \\
(\%)^{d}\end{array} \\
(\%)\end{array}$ & $1.5 .4 \mathbf{a}: 1.5 .5 \mathbf{a}^{d}$ \\
\hline 1 & $\mathrm{FeCl}_{3}$ & 10 & $\mathrm{MeCN}$ & 60 & 5 & 86 & 57 & $3: 1$ \\
\hline 2 & $\mathrm{Cu}(\mathrm{OAc})_{2}$ & 10 & $\mathrm{MeCN}$ & 60 & 5 & $<5$ & $<5$ & - \\
\hline 3 & $\mathrm{CuI}$ & 10 & $\mathrm{MeCN}$ & 60 & 5 & $<5$ & $<5$ & - \\
\hline 4 & $\mathrm{PdCl}_{2}$ & 10 & $\mathrm{MeCN}$ & 60 & 5 & 30 & $<5$ & - \\
\hline 5 & $\mathrm{RuCl}_{3}$ & 10 & $\mathrm{MeCN}$ & 60 & 5 & 82 & 36 & $1: 1$ \\
\hline 6 & $\mathrm{IrCl}_{3}$ & 10 & $\mathrm{MeCN}$ & 60 & 5 & 85 & 25 & $1: 2$ \\
\hline 7 & $\mathrm{Fe}(\mathrm{acac})_{3}$ & 10 & $\mathrm{MeCN}$ & 60 & 5 & $<5$ & $<5$ & - \\
\hline 8 & $\mathrm{LaCl}_{3}$ & 10 & $\mathrm{MeCN}$ & 60 & 5 & 19 & $<5$ & - \\
\hline 9 & $\mathrm{CeCl}_{3}$ & 10 & $\mathrm{MeCN}$ & 60 & 5 & 25 & $<5$ & - \\
\hline 10 & $\mathrm{Bi}(\mathrm{OTf})_{3}$ & 10 & $\mathrm{MeCN}$ & 60 & 5 & 71 & 42 & $2: 1$ \\
\hline 11 & $\mathrm{AlCl}_{3}$ & 10 & $\mathrm{MeCN}$ & 60 & 5 & 63 & 7 & $1: 5$ \\
\hline 12 & $\mathrm{TfOH}$ & 10 & $\mathrm{MeCN}$ & 60 & 5 & 75 & 10 & $2: 1$ \\
\hline 13 & $\mathrm{SnCl}_{2}$ & 10 & $\mathrm{MeCN}$ & 60 & 5 & 69 & 29 & $1: 1$ \\
\hline 14 & $\mathrm{FeCl}_{2}$ & 10 & $\mathrm{MeCN}$ & 60 & 5 & 50 & 20 & $1: 1$ \\
\hline 15 & $\mathrm{LiCl}$ & 10 & $\mathrm{MeCN}$ & 60 & 5 & $<5$ & $<5$ & - \\
\hline
\end{tabular}


${ }^{a}$ Standard reaction condition: 1 eqiuv propargyl alcohol $(0.25 \mathrm{mmol}), 1.3$ equiv triazole $(0.325 \mathrm{mmol}), 0.1$ equiv catalyst $(0.025 \mathrm{mmol})$ and $2 \mathrm{~mL}$ solvent; ${ }^{b}$ DCE: 1,2-dichlorethane, EtOAc: ethyl acetate; ${ }^{c}$ conversions were determined based on the consumption of propargyl alcohol; ${ }^{d}$ NMR yields of 4 a with 1,3,5-trimethoxybenzene as internal standard.

Table 8: Solvent screening ${ }^{a}$

\begin{tabular}{|c|c|c|c|c|c|c|c|c|}
\hline & Catalyst & $\begin{array}{c}\text { Loading } \\
(\%)\end{array}$ & Sol. ${ }^{b}$ & $\begin{array}{c}\text { Temp } \\
\left({ }^{\circ} \mathrm{C}\right)\end{array}$ & $\begin{array}{c}\text { Time } \\
\text { (h) }\end{array}$ & 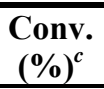 & $\begin{array}{l}\begin{array}{l}\text { Yield } \\
(\%)^{d}\end{array} \\
\end{array}$ & $1.5 .4 \mathbf{a}: 1.5 .5 \mathbf{a}^{d}$ \\
\hline 16 & $\mathrm{FeCl}_{3}$ & 10 & Acetone & 60 & 5 & 83 & 32 & $2: 1$ \\
\hline 17 & $\mathrm{FeCl}_{3}$ & 10 & THF & 60 & 5 & 73 & 42 & $2: 1$ \\
\hline 18 & $\mathrm{FeCl}_{3}$ & 10 & Toluene & 60 & 5 & 30 & $<5$ & - \\
\hline 19 & $\mathrm{FeCl}_{3}$ & 10 & $\mathrm{MeOH}$ & 60 & 5 & 71 & $<5$ & - \\
\hline 20 & $\mathrm{FeCl}_{3}$ & 10 & $\mathrm{MeNO}_{2}$ & 60 & 5 & 78 & 60 & $4: 1$ \\
\hline 21 & $\mathrm{FeCl}_{3}$ & 10 & DMSO & 60 & 5 & $<5$ & $<5$ & - \\
\hline 22 & $\mathrm{FeCl}_{3}$ & 10 & DMF & 60 & 5 & $<5$ & $<5$ & - \\
\hline 23 & $\mathrm{FeCl}_{3}$ & 10 & $\mathrm{CHCl}_{3}$ & 60 & 5 & 80 & 40 & $3: 1$ \\
\hline 24 & $\mathrm{FeCl}_{3}$ & 10 & EtOAc & 60 & 5 & 78 & 56 & $4: 1$ \\
\hline 25 & $\mathrm{FeCl}_{3}$ & 10 & DCE & 60 & 5 & 100 & $90^{e}$ & $>20: 1$ \\
\hline 26 & $\mathrm{FeCl}_{3}$ & 10 & DCE & $\mathrm{rt}$ & 5 & 50 & 40 & $8: 1$ \\
\hline
\end{tabular}

${ }^{a}$ Standard reaction condition: 1 eqiuv propargyl alcohol $(0.25 \mathrm{mmol}), 1.3$ equiv triazole $(0.325 \mathrm{mmol}), 0.1$ equiv catalyst $(0.025 \mathrm{mmol})$ and $2 \mathrm{~mL}$ solvent; ${ }^{b}$ DCE: 1,2-dichlorethane, EtOAc: ethyl acetate; ${ }^{c}$ conversions were determined based on the consumption of propargyl alcohol; ${ }^{d}$ NMR yields of 4a with $1,3,5$-trimethoxybenzene as internal standard; ${ }^{e}$ Isolated yield.

To further improve the regioselectivity of this reaction, different solvents was screened (Table 8). A polar solvent, such as DMSO, DMF, totally quenched the reaction. 
To our surprise, the regioselectivity of the reaction, as indicatd by the ratio of $\mathbf{1 . 5 . 4 a}$ and 1.5.5a increased dramatically ( $>20: 1$ ratio) when 1,2-dichloroethane (DCE) was used as the reaction solvent. Interestingly, the ratio between 1.5.4a and 1.5.5a was dependent on the reaction temperature, where regioselectivity was dropped to $8: 1$ ratio at room temperature (entry 26).

With the optimal condition in hand, a series of different propargyl alcohols were investigated to explore the reaction substrate scope (Figure 15). As shown in Figure 15, the alkyne terminal $\mathrm{R}^{3}$ position could be tolerated TMS, $n-\mathrm{Bu}, t-\mathrm{Bu}$, vinyl and cyclopropanyl groups. Although a longer reaction time $(12 \mathrm{~h})$ was required, the bulky $t$ $\mathrm{Bu}$ group gave 1.5.4e in $91 \%$ yield. No enyne decomposition (1.5.4f, 1.5.4r) or cyclopropane ring opening $(\mathbf{1 . 5 . 4 d}, \mathbf{1 . 5 . 4 s})$ products were observed. The phenyl substituted alkyne gave the corresponding hydration product instead of the formation of desired triazole allene. The terminal alkynes $\left(\mathrm{R}^{3}=\mathrm{H}\right)$ gave a complex reaction mixture under the optimal conditions. This limitation could be easily overcome by TMS deprotection.

Various substitutions on the propargyl position have also been investigated. The dialkyl substituted propargyl alcohols were unreactive enough even at higher temperature for longer time $\left(60{ }^{\circ} \mathrm{C}, 24 \mathrm{~h}\right)$. The fact that aryl-alkyl substituted groups gave no allene triazole product was likely caused by the elimination of propargyl alcohols. For the electron-rich aryl substitution, higher yields were observed at room temperature (1.5.4g-1.5.4k). Heterocycles, such as thiophene (1.5.4l), were suitable for this reaction. Surprisingly, this transformation can tolerate a variety of functional group, such as the phenol substituted propargyl alcohols afforded the corresponding products $(\mathbf{1 . 5 . 4 j}, \mathbf{1 . 5 . 4 k})$ in good yields. 
Figure 15: Reaction substrate scope ${ }^{a}$

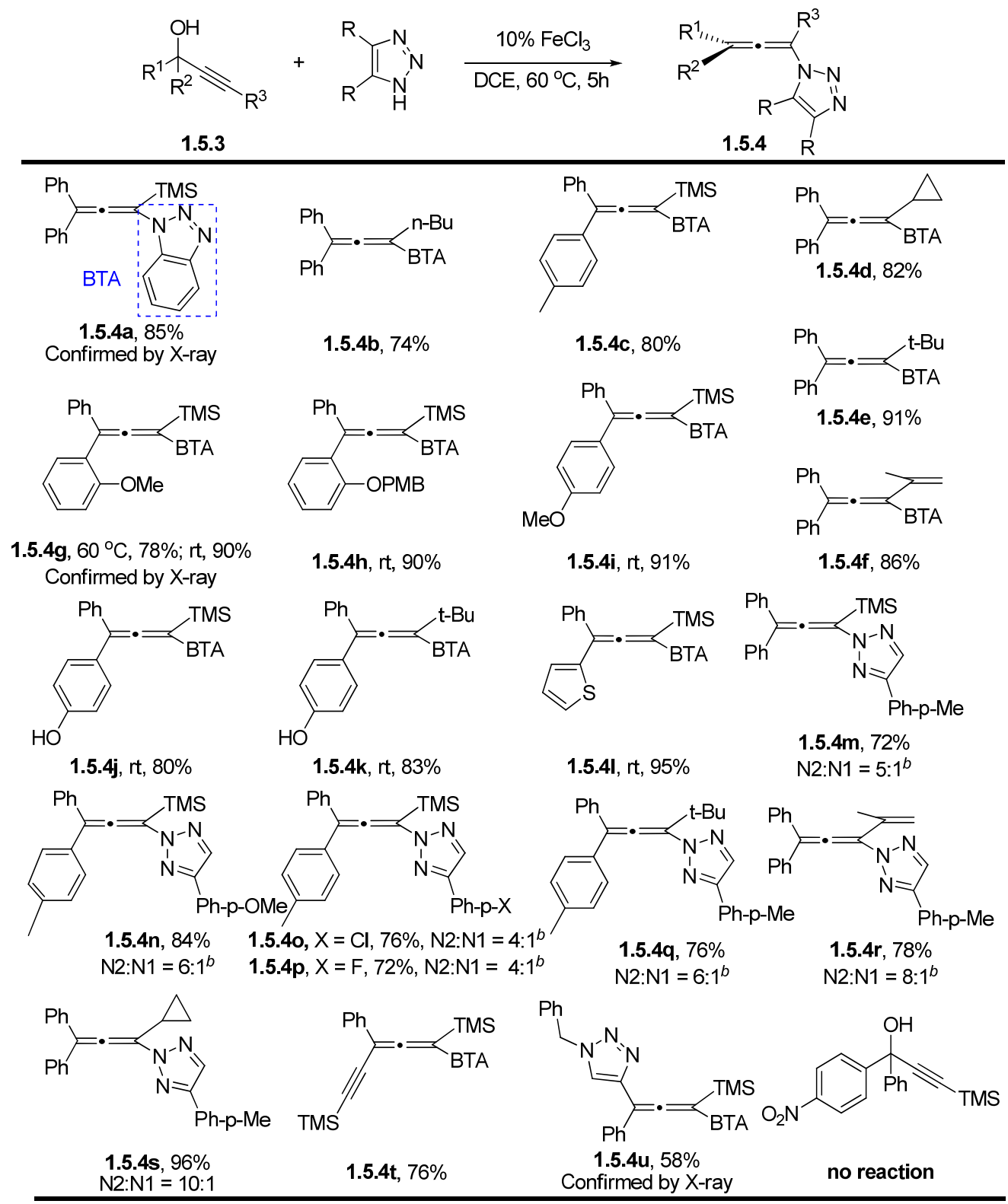

${ }^{a}$ Isolated yield; ${ }^{b}$ Room temperature; ${ }^{c}$ Ratio(N2:N1) was determined by crude NMR of reaction mixture.

Substituents on the triazole indicated little influence on the reaction $(\mathbf{1 . 5 . 4 m - 1 . 5 . 4 s )}$, likely due to the excellent overall nucleophilicity of the NH-triazole. Impressively, the alkyne-substituted propargyl alcohol was also suitable for this reaction, giving the conjugated alkyne-allene triazole in good yield. The sturcture of $\mathbf{1 . 5 . 4 t}$ was confirmed by 
X-ray crystallography. The electron-deficient aryl substituted propargyl alcohols did not work because the strong electron-withdrawing group on the benzene ring decreases the reactivity of $\mathrm{C}-\mathrm{O}$ bond. Interestingly, although the triazole ring is a highly electrondeficient aromatic ring, the allene bis-triazole 1.5.4u was obtained in modest yield under standard conditions, suggesting the potential triazole-Fe coordination in the activation of propargyl C-O bond.

Figure 16: Extending the substrate scope with propargyl acetate. ${ }^{a, b}$

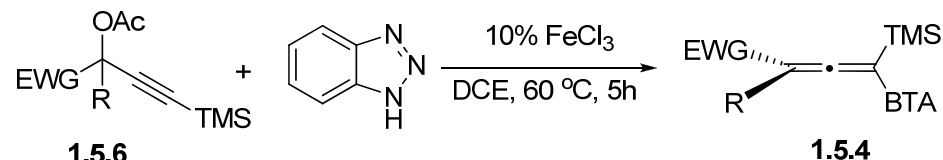

$\mathrm{R}=\mathrm{Ph} ; \mathrm{EWG}=\mathrm{Ph}-\mathrm{p}-\mathrm{NO}_{2} ; \mathbf{1 . 5 . 4 v}, \mathbf{7 1} \% \mathrm{R}=\mathrm{Ph} ; \mathrm{EWG}=\mathrm{Ph}-\mathrm{p}-\mathrm{Cl} ; \mathbf{1 . 5 . 4 x}, \mathbf{7 2} \%$

$\mathrm{R}=\mathrm{Ph} ; \mathrm{EWG}=\mathrm{Ph}-\mathrm{p}-\mathrm{F} ; \mathbf{1 . 5 . 4 w}, 88 \% \quad \mathrm{R}=\mathrm{Ph}-\mathrm{p}-\mathrm{F} ; \mathrm{EWG}=\mathrm{Ph}-\mathrm{p}-\mathrm{F} ; \mathbf{1 . 5 . 4 y}, 84 \%$

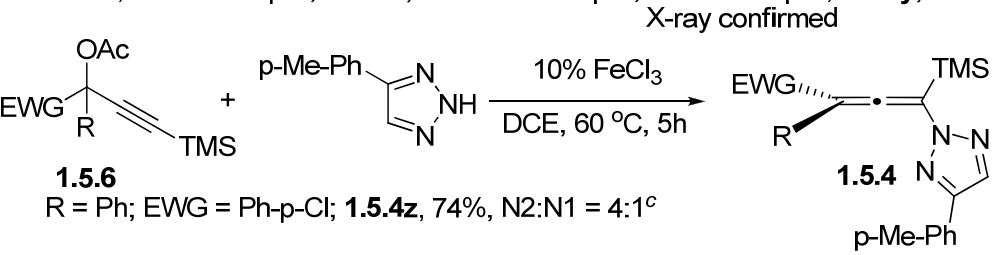

${ }^{a}$ Reaction condition: 1 eqiuv propargyl acetate $(0.25 \mathrm{mmol}), 1.3$ equiv triazole $(0.325 \mathrm{mmol}), 0.1$ equiv catalyst $(0.025 \mathrm{mmol})$ and $2 \mathrm{~mL}$ solvent; ${ }^{b}$ Isolated yield; ${ }^{c}$ Ratio(N2:N1) was determined by crude NMR of reaction mixture.

As we metioned before, the electron-deficient aryl substituted propargyl alcohols were not suitable for this transformation, due to the lower reactivity of the $\mathrm{C}-\mathrm{O}$ bond. To further extend the substrate group of electron-deficient ring, we have tuned the reactivity of propargyl alcohol derivatives. Therefore propargyl acetates 1.5 .6 were prepared to react with triazoles. Excitingly, the desired allene triazoles $(1.5 .4 \mathrm{v}-1.5 .4 z)$ with electronwithdrawing group substituents were obtained in good to excellent yields, as shown in Figure 16. 
Scheme 35: TMS-deprotection and X-ray crystal structures

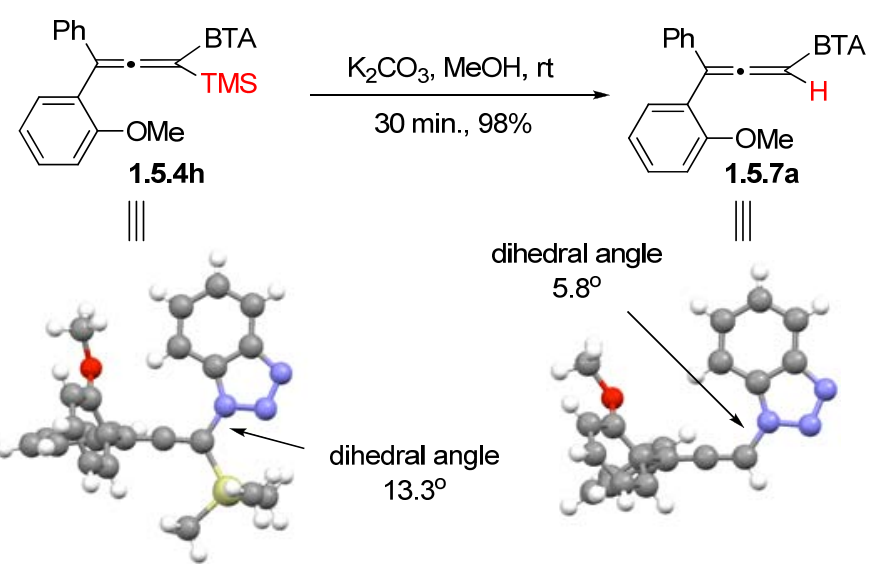

Surprisingly, the allene-triazole shows significantly higher stability than the regular allene. For example, when the allene 1.5.4h was treated with 2 equivalents of $\mathrm{TfOH}$ in wet DCM at room temperature, no allene decomposition (even the TMS deprotection) was observed after 12 hours based on the recovered yield of $\mathbf{1 . 5 . 4 h}(97 \%)$. The TMS group could be readily removed by treatment with base as shown in Scheme 35 .

The X-ray crystal structures not only confirmed the formation of the allene-triazoles, but also revealed the detailed configuration. Among all five allene-triazole crystal structures (1.5.4a, 1.5.4g, 1.5.4u, 1.5.4y and 1.5.7a), excellent conjugations between triazole ring and the allene $\pi$-bond were observed, even with bulky TMS group on the same carbon center. This extended conjugation likely accounted for the improved allene stability.

\subsubsection{Conclusion}

Herein, we reported the first synthesis of allene-substituted 1,2,3-triazole derivatives to fill the "missing piece" of triazole functionalizations. With the improved allene stability, the allene-triazoles are expected to exhibit potential new reactivity that may lead to 
interesting chemical and physical properties, such as ligand development and material construction. 


\subsection{Mitsunobu reaction of 1,2,3- $\mathrm{NH}$-triazoles: regio- and stereo- selective synthesis of triazole derivatives $\uparrow$}

\subsubsection{Two practical problems from previous 1,2,3-triazole functionalizations}

Since 2008, our group have successfully reported five different functionalizations of 1,2,3-triaozles which included alkylation, arylation, vinylation, propargylation and allenation (Figure 17).

Figure 17: Five different functionalizations of 1,2,3-triaozles.

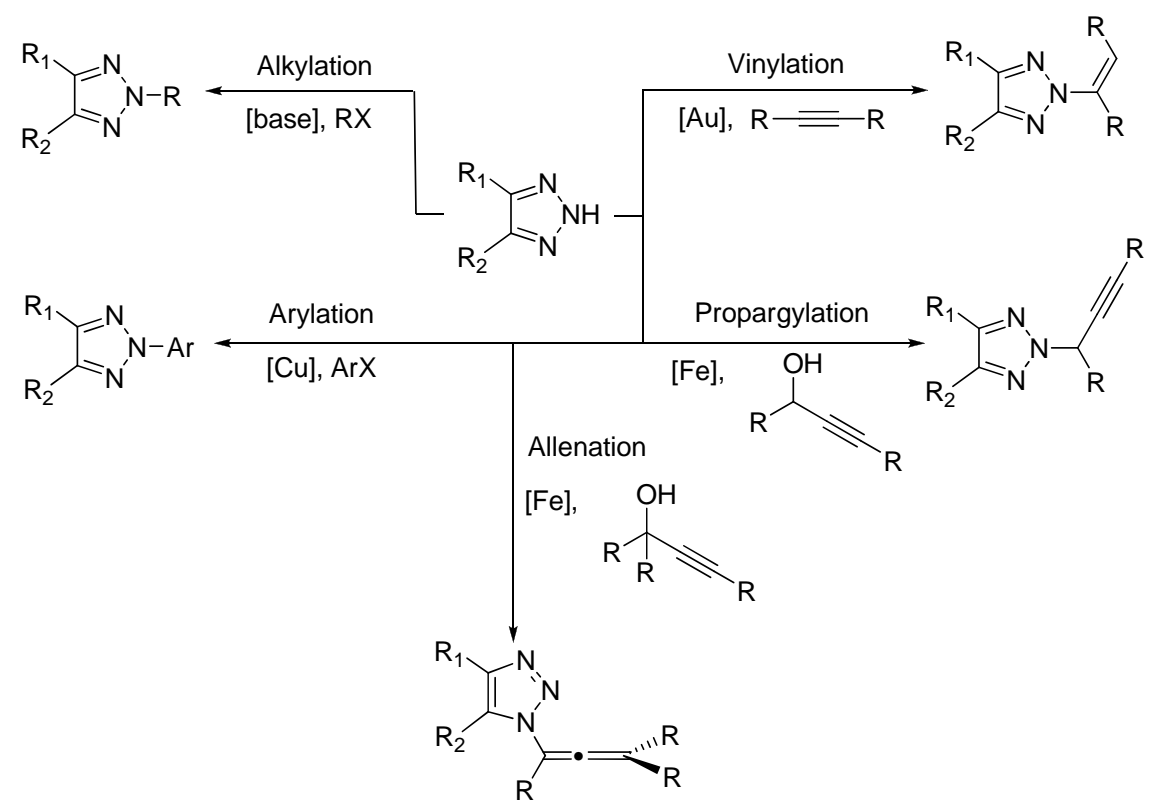

From these studies, we learned that the N-1 and N-2 selectivities of 1,2,3-triazoles heavily depended on the placement of substituents on the 1,2,3-triaozles, such as 4,5disubstituted 1,2,3-NH-triazoles gave absolutely dominant $\mathrm{N}-2$ substituted products and 4-substituted 1,2,3-NH-triazoles produced slightly dominant N-2 regioisomers, while

$\dagger$ Reproduced in parts with permission from Chem. Asian. J. 2011, 6, 2720-2724. Copyright 2011, with permission from Willey-VCH Verlag $\mathrm{GmbH} \&$ Co. KGaA, Weinheim. 
benzotriazole gave N-1 substituted products as significantly major products in all functionalizations (Figure 18). Therefore, the first practical problem was carried out regarding the regioselective synthesis of $\mathrm{N}-2$ triazoles. How can we improve the $\mathrm{N}-2$ regioselectivity of 1,2,3-triazoles, especially for $\mathrm{N}-1$ preferred triazoles, such as $1 \mathrm{H}$ 1,2,3-triazole and benzotriazole. Although some strategies which was focused on the development of the appropriate substituents on the C-4 and C-5 positions to reach good $\mathrm{N}-2$ selectivity have been reported in literature, ${ }^{69}$ new strategies that can encourage N-2 selectivity from "N-1-substitution favored" 1,2.3- $\mathrm{NH}$-triazole were highly desired.

Figure 18: N-1 \& N-2 selectivities of 1,2,3- $\mathrm{NH}$-triazoles.

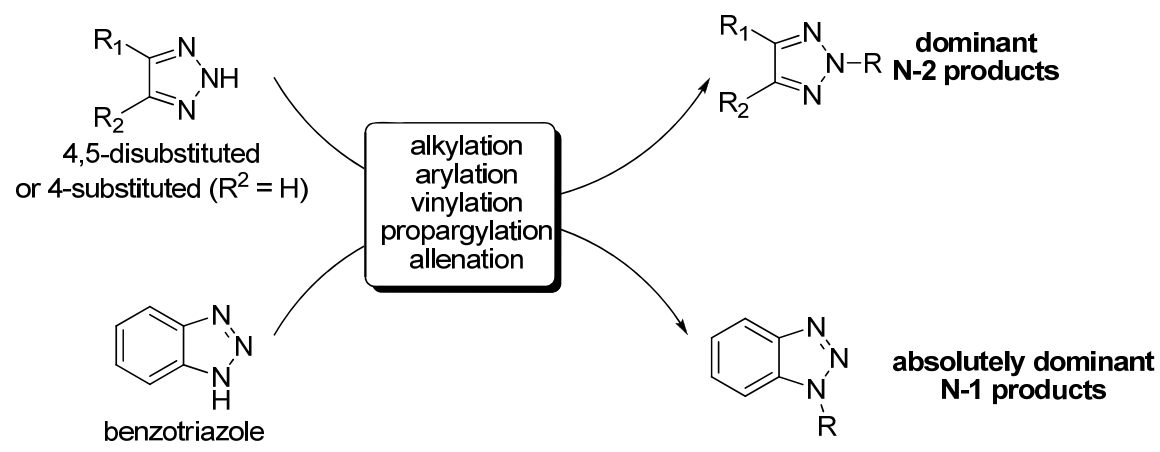

Scheme 36: The propargylation of benzotriazole with enantioenriched propargyl alcohol.

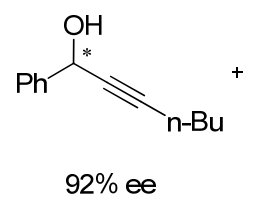<smiles>CC(C#N)Oc1ccc2[nH]nnc2c1</smiles>

$92 \%$ ee

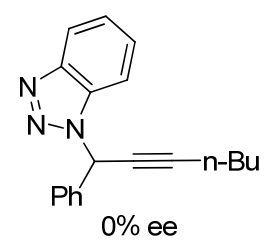

Due to the presence of a stereogenic center, the propargylation of triazoles gave the potential opportunity to synthesize chiral propargyl triazole derivatives. We then investigated the reaction between benzotriazole and an enantioenriched propargyl alcohol 
(92\% ee) (Scheme 36). Unfortunately, we obtained a racemic propargyl triazole product. This result suggested the chiral propargyl triazoles could not be synthesized via propargylation. Then the second practical challenge was the selective synthesis of enantioenriched triazole derivatives.

The N-1 nitrogen of 1,2,3-triazole is usually more basic (higher electron density) than the N-2 nitrogen, however, because the center N-2 nitrogen is much less steric hindered, N-2 substitution is kinetically favored. As a result, the highly stereochemistry sensitive Mitsunobu reactions could potentially favorer the kinetic product and increase the N-2 selectivity. Meanwhile, the rather high acidicity (pKa around $8-10$ ) and the excellent nucleophilicity of $\mathrm{NH}$-1,2,3-triazoles make them suitable coupling partners with alcohols under Mitsunobu conditions. Moreover, the excellent stereoselectivity associated with the Mitsunobu reaction could be achieved, where complete inversion of alcohol stereogenic center occurred through $\mathrm{S}_{\mathrm{N}} 2$ addition. Combining these two ideas, we postulated that the Mitsunobu reaction could be used to install chirality on the triazoles compounds.

\subsubsection{Introduction of Mitsunobu reaction}

The Mitsunobu reaction is an organic reaction which provides the substitution of primary or secondary alcohols with nucleophiles mediated by the combination of a trialkyl or triarylphosphine and a dialkyl azodicarboxylate (DEAD or DIAD). ${ }^{70}$ Because of its scope, stereospecificity, and mild reaction conditions, the Mitsunobu reaction has played a critical role in organic synthesis and medicinal chemistry. ${ }^{71} \mathrm{~A}$ wide range of compounds can be synthesized using a Mitsunobu protocol, such as esters, ${ }^{72}$ amines, ${ }^{73}$ azides,${ }^{74}$ ethers, ${ }^{75}$ cyanides, ${ }^{76}$ thiocyanides,${ }^{77}$ thioesters, ${ }^{78}$ and thioethers ${ }^{79}$. (Scheme 37). 
Scheme 37: Mitsunobu reaction.

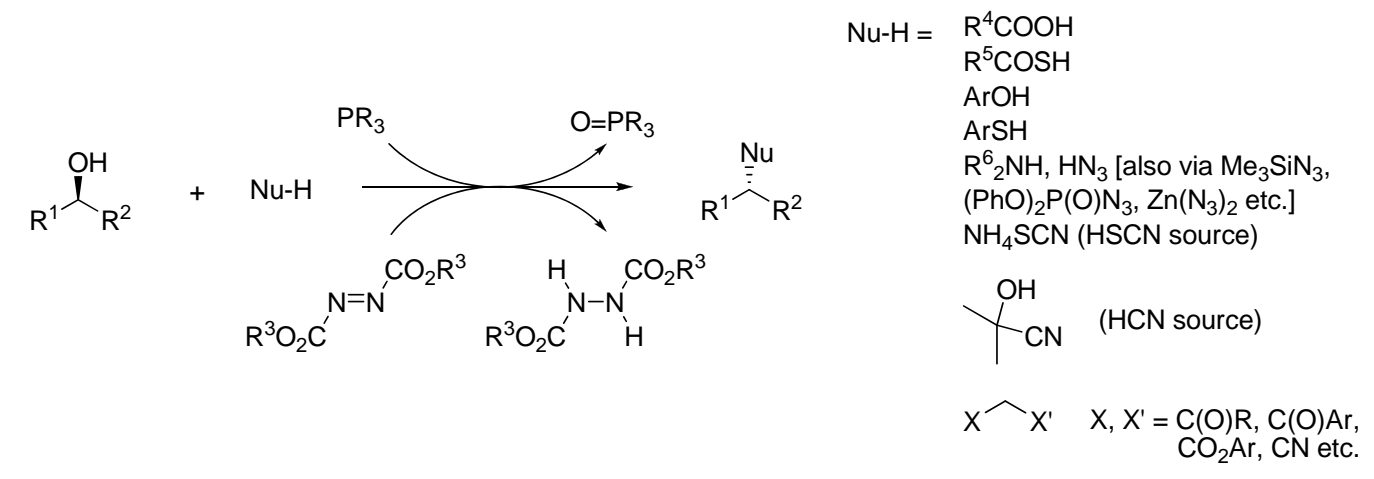

Although the Mitsunobu reaction is widely used in synthetic organic chemistry, the reaction mechanism of Mitsunobu reaction was fairly complex. The reaction intermediates have been the subject of debate. ${ }^{80} \mathrm{~A}$ possible mechanism was shown in

\section{Scheme 38 .}

Scheme 38: Postulated mechanism for the Mitsunobu reaction.

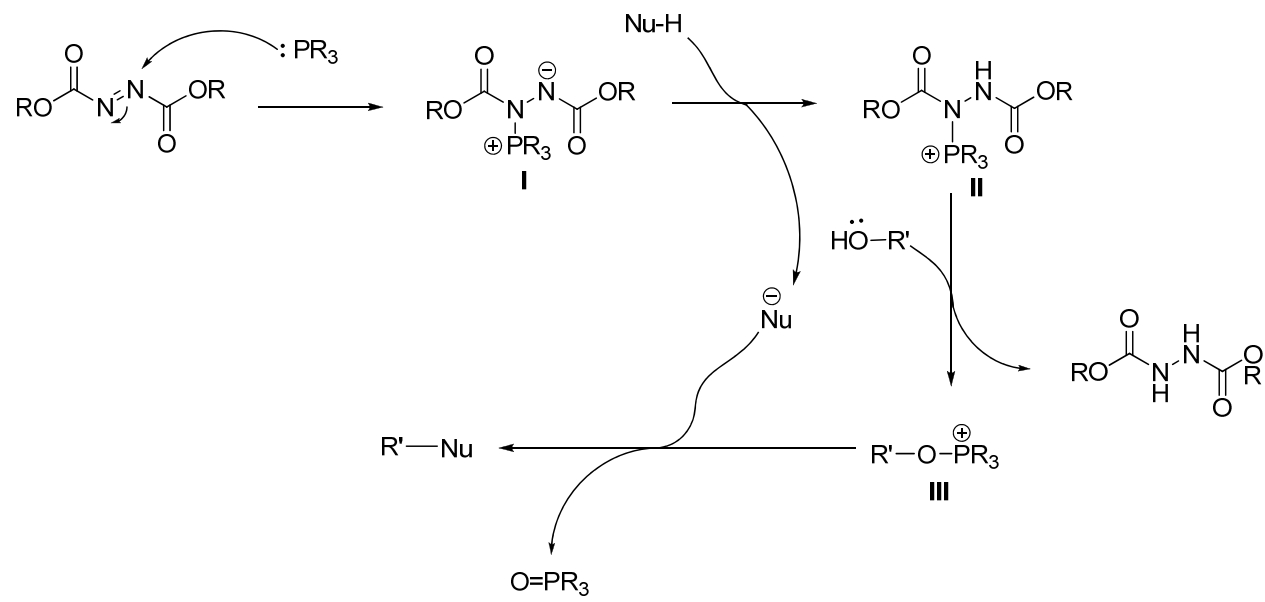

The first step is the irreversible formation of the intermediate betaine $\mathbf{I}$ from the nucleophilic attack of phosphine $\left(\mathrm{PR}_{3}\right)$ upon dialkyl azodicarboxylate, which has been confirmed by $\mathrm{NMR}^{81}$ and ESI-MS. ${ }^{82}$ In the second step, the betaine $\mathbf{I}$ deprotonates the 
hydrogen of the nucleophile to give the species II. The intermediate II then reacts with the alcohol to form the alkoxyphosphonium III. Finally, the desired product is produced by the attack of nucleophilic anion upon intermediate III. The byproducts of Mitsunobu reaction are phosphine oxide and dialkyl hydrazine-1,2-dicarboxylate.

\subsubsection{An example: Improvement of regioselective N-2 isomers from ligand control.}

Despite our successes on the application of conformational control strategy for the synthesis of regioselective N-2 substituted 1,2,3-triazoles (see Section 1.1.2), we realized this strategy is not effective for specific substrates, which were discussed in Section 1.6.1.

Scheme 39: N-2 arylation was reported by Shi and Buchwald.

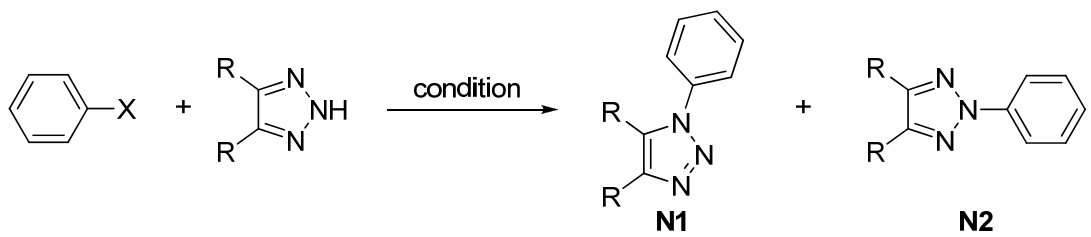

Shi's condition: $\mathrm{CuCl}$, Proline, $\mathrm{K}_{2} \mathrm{CO}_{3}$, DMSO, $85^{\circ} \mathrm{C}, 4 \mathrm{~h}$.

Buchwald's condition: $\mathrm{Pd}_{2}(\mathrm{dba})_{3}, \mathrm{~L}, \mathrm{~K}_{3} \mathrm{PO}_{4}$, toluene, $120^{\circ} \mathrm{C}, 5 \mathrm{~h}$.

\begin{tabular}{lcc} 
& \multicolumn{2}{c}{ ratio (N2/N1) } \\
\cline { 2 - 3 } & Shi's approach & Buchwald's method \\
\hline
\end{tabular}

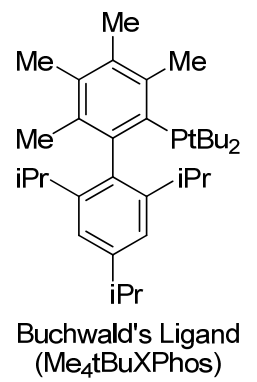

In 2008 , we reported the copper(I) mediated N-2 arylation of 1,2,3-NH-triazoles (Section 1.2). We obtained poor to moderate N-2 regioselectivity for both benzotriazole and 4-substituted 1,2,3-NH-triazoles. Recently, Buchwald ${ }^{83}$ and coworkers described a 
palladium-catalyzed $\mathrm{N}$-arylation of 1,2,3-triazoles with exceptional levels of $\mathrm{N}-2$ regioselectivity by using the very bulky biaryl phosphine ligand (Scheme 39). The ratios of N-2 and N-1 triazole regio-isomers were 1:7.5 and 6:1 for benzotriazole and 4phenyltriazole individually by utilization of our approach, while they were $1: 1$ and $32: 1$ roughly from Buchwald's method. We therefore have known the N-2 selectivity of 1,2,3triazoles could be improved significantly by using a bulky ligand.

\subsubsection{Research Objective and Results}

To verify our hypothesis as mentioned in the section 1.6.1, the reactions between benzyl alcohol 1.6.2a and benzotriazole 1.2.1a were performed under standard Mitsunobu reaction and alkylation conditions separately (Scheme 40).

Scheme 40: Mitsunobu reaction and alkylation of benzotriazole.

(A)

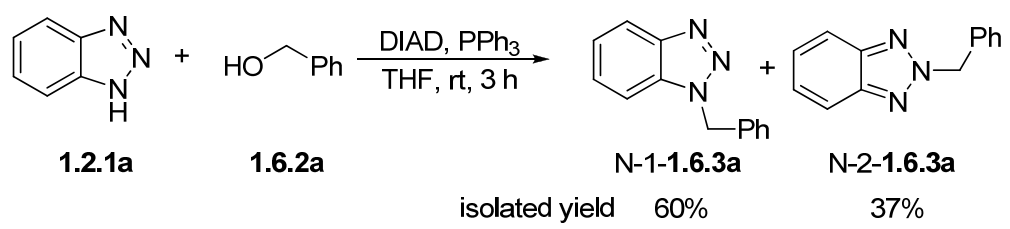

(B)

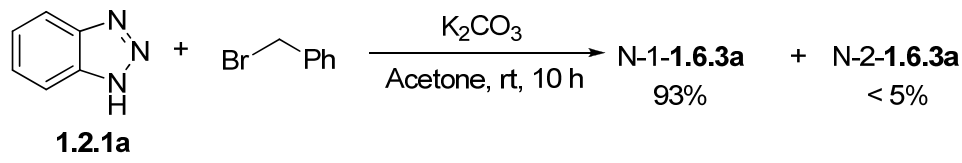

As shown in Scheme 40A, the dehydration product 1.6.3a was obtained in excellent isolated yields (97\%, combined N-1 and N-2 isomers) under Mitsunobu conditions. Compared to the alkylation reaction of benzotriazole, where only trace amount $(<5 \%)$ of N-2-1.6.3a was isolated (Scheme 40B), the Mitsunobu reaction provided significantly 
better overall yields of the N-2 isomer. To verify how different alcohols influenced the reactivity and regioselectivity, alcohols 1.6.2 reacted with 1.2.1a. The results were summarized in Table 9.

Table 9: Reaction substrate scope with benzotriazole (BTA) ${ }^{a}$

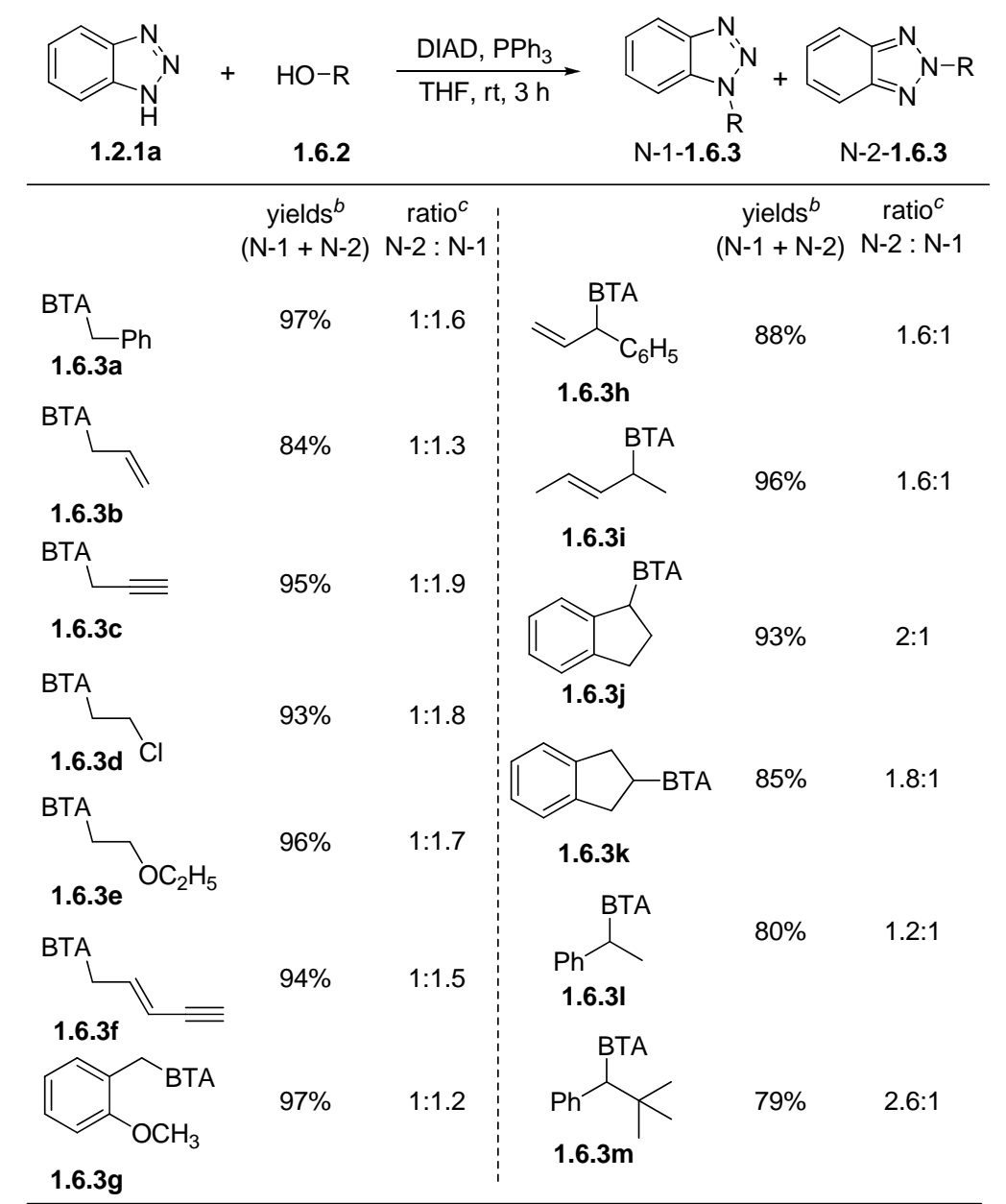

${ }^{a}$ Standard reaction condition: 1 equiv of alcohols 1.6.2, 1.2 equiv of triazoles 1.2.1a, 1.2 equiv of triphenylphosphine $\left(\mathrm{PPh}_{3}\right)$ and 1.2 equiv of diisopropyl azodicarboxylate (DIAD) were mixed in the distilled tetrahydrofuran (THF). ${ }^{b}$ Isolated yields. ${ }^{c}$ Ratios determined by NMR of crude reaction mixtures.

As shown in Table 9, the Mitsunobu reaction was suitable for a number of different alcohols 1.6.2, giving the coupling products in excellent yields ( $>85 \%)$. Significantly improved N-2 products (compared with the alkylation of 1,2,3-triazoles) were obtained in 
all cases. The Mitsunobu reactions produced N-1 isomers as the dominant products in primary alcohols. However, compared to alkylation, they improved the regioselectivities significantly. Although secondary alcohols required longer reaction times (8 to $12 \mathrm{~h}$ ), they gave the N-2 isomers as the major products, which supported our hypothesis that stereochemistry sensitive Mitsunobu reaction help the formation of kinetic N-2 addition even with highly N-1 preferred benzotriazole.

Table 10: Reaction substrate scope with various triazoles. ${ }^{a, b}$

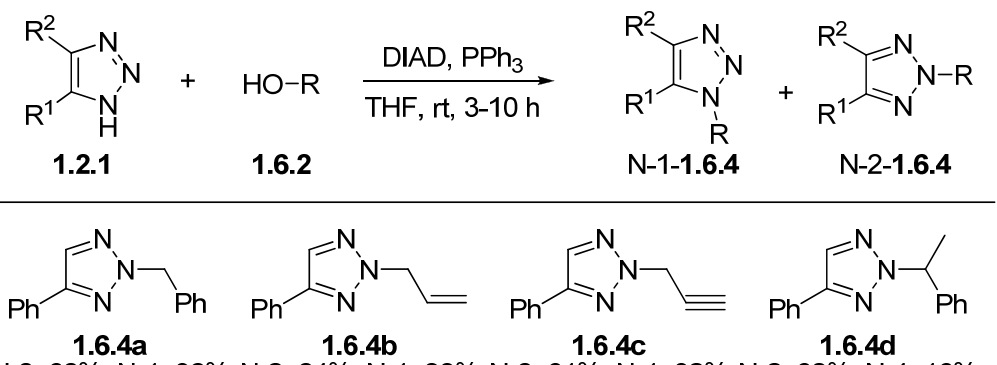
N-2: 62\%; N-1: $36 \%$ N-2: 64\%; N-1: 30\% N-2: 61\%; N-1: $32 \%$ N-2: 68\%; N-1: 16\%<smiles>CC(C)(C)C(c1ccccc1)n1ncc(-c2ccccc2)n1</smiles>

$\mathrm{N}-2: 64 \% ; \mathrm{N}-1: 16 \%$<smiles>C=CCn1cc(C(=C)c2ccccc2)c(-c2ccccc2)n1</smiles>

$\mathrm{N}-2: 62 \% ; \mathrm{N}-1: 21 \%$

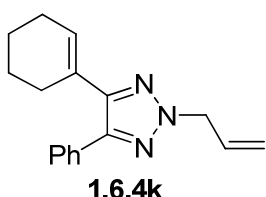

$\mathrm{N}-2: 77 \% ; \mathrm{N}-1: 9 \%$

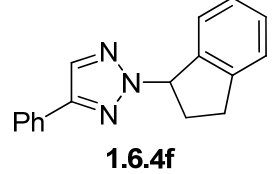

$\mathrm{N}-2: 72 \% ; \mathrm{N}-1: 18 \%$<smiles>C=C(c1ccccc1)c1nn(CCCl)nc1-c1ccccc1</smiles>

$\mathrm{N}-2: 72 \% ; \mathrm{N}-1: 20 \%$

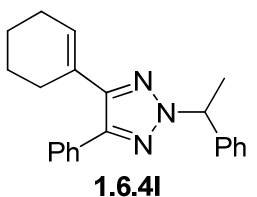

$\mathrm{N}-2: 72 \% ; \mathrm{N}-1:<5 \%$<smiles>C=C(c1ccccc1)c1nn(Cc2ccccc2)nc1-c1ccccc1</smiles>

$\mathrm{N}-2: 67 \% ; \mathrm{N}-1: 16 \%$

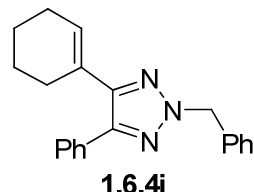

$\mathrm{N}-2: 80 \% ; \mathrm{N}-1: 10 \%$

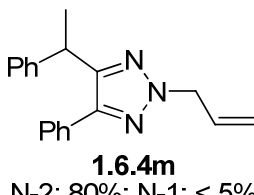

${ }^{a}$ Standard reaction conditions: 1 equiv of alcohols, 1.2 equiv of triazoles, 1.2 equiv of triphenylphosphine $\left(\mathrm{PPh}_{3}\right)$ and 1.2 equiv of diisopropyl azodicarboxylate (DIAD) were mixed in the distilled tetrahydrofuran (THF). ${ }^{b}$ Yields determined by NMR with 1,3,5-trimethoxybenzene as internal standard, and ratios determined by NMR of crude reaction mixtures. 
Different $\mathrm{NH}-1,2,3$-triazoles were subsequently used to investigate their influence on regioselectivity and also further extend the reaction substrate scope (Table 10). Among all the tested $\mathrm{NH}-1,2,3$-triazoles, significantly improved N-2 selectivity was observed. For example, as we have reported in previous alkylation of triazoles, the reaction between the 4-phenyl- $\mathrm{NH}$-1,2,3-triazole (1.2.1b) with benzyl bromide $\left(\mathrm{PhCH}_{2} \mathrm{Br}\right)$ gave $\mathrm{N}-1$ substituted benzyl 1.6.4a as the major product $(\mathrm{N}-2: \mathrm{N}-1=1: 5$ roughly).

Under the Mitsunobu conditions, the desired N-2 isomer became the major product $(\mathrm{N}-2: \mathrm{N}-1=1.7: 1)$. Good to excellent isolated yields of the $\mathrm{N}-2$ isomers were obtained. The Mitsunobu reactions of $\mathrm{NH}$-1,2,3-triazoles not only provided an alternative approach for $\mathrm{NH}$-1,2,3-triazole functionalization, but also, more importantly allowed the N-2 functionalization through altering different alcohols instead of adjusting the substitution groups on 1,2,3-triazoles.

The significance of Mitsunobu reaction in altering the $\mathrm{N}-1 / \mathrm{N}-2$ selectivity was further highlighted in the synthesis of bis-N-2-triazole derivatives as shown in Scheme 41. Generally speaking, the N-1/N-1-bis-triazoles could be readily prepared through the double click chemistry from di-alkynes. On the other hand, the N-2/N-2-bistriazoles, were extremely challenging to prepare due to the unfavored statistic from mathematics calculation. For example, assuming the mono substitution gave $\mathrm{N}-1: \mathrm{N}-2=5: 1$, the ratio of the double substitution of the same reaction would be $\mathrm{N}-1 / \mathrm{N}-1: \mathrm{N}-1 / \mathrm{N}-2: \mathrm{N}-2 / \mathrm{N}-2=$ $25: 10: 1((\mathrm{~N}-1: \mathrm{N}-2) *(\mathrm{~N}-1: \mathrm{N}-2)=\mathrm{N}-1 / \mathrm{N}-1: \mathrm{N}-1 / \mathrm{N}-2: \mathrm{N}-2 / \mathrm{N}-1: \mathrm{N}-2 / \mathrm{N}-2=25: 5: 5: 1$, where $\mathrm{N}-1 / \mathrm{N}-2=\mathrm{N}-2 / \mathrm{N}-1$ ). The theoretical yields for $\mathrm{N}-2 / \mathrm{N}-2$ product would be only $2.7 \%$. The Mitsunobu conditions, by altering the N-1 and N-2 selectivity, gave the great 
opportunity to synthesize the $\mathrm{N}-2 / \mathrm{N}-2$ isomer for the first time through the simple post triazole derivatization.

Scheme 41: Synthesis of challenging N-2-bis-triazoles.

(A)

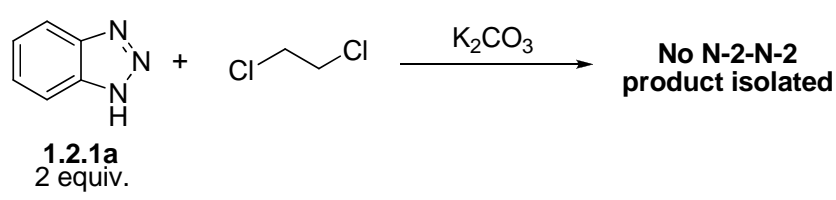

(B)

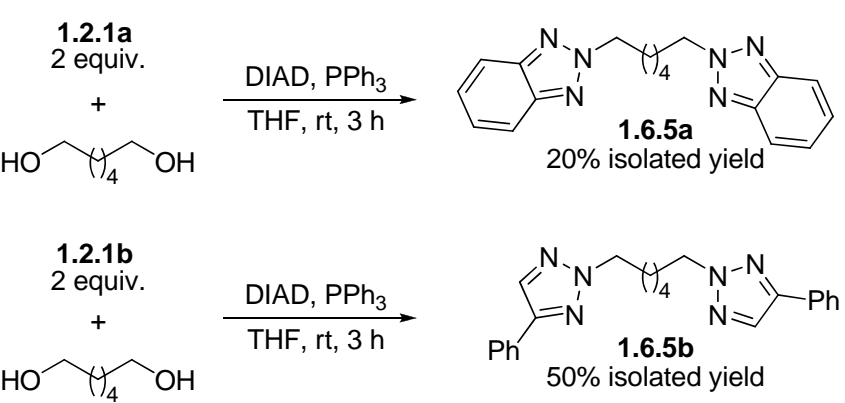

(C)

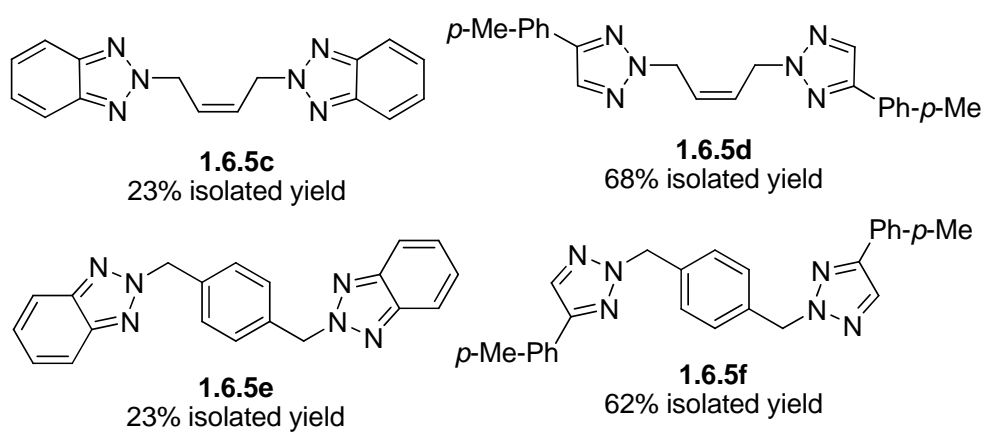

As indicated in Scheme 41, bis-triazole 1.6.5a which has been confirmed by X-ray crystallography was successfully prepared even with benzotriazole, which gives predominantly $\mathrm{N}-1$ products (Figure 19). The yields of the $\mathrm{N}-2 / \mathrm{N}-2$ isomers were significantly improved with the 4-phenyl-triazole (1.6.5b) and derivatives (1.6.5d and 1.6.5f). Notably, only trace amount of the $\mathrm{N}-2 / \mathrm{N}-2$ bis-triazoles were observed while treating the 4-phenyl-triazole with the di-chloro/bromo alkanes due to the unfavored statistic discussed above. 
Figure 19: Crystal structure of 1.6.5a

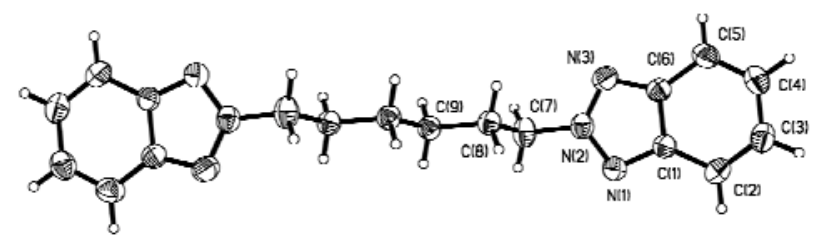

From above studies, the $N H-1,2,3$-triazoles have been confirmed as the good coupling partner of Mitsunobu reaction with good reactivities. We then investigated the stereoselectivity of this transformation. The Mitsunobu reaction can be transfer the complete inversion of alcohol stereogenic center to product via $\mathrm{S}_{\mathrm{N}} 2$ addition. The trans2-methylcyclohexanol 1.6.2b and trans-2-methylcyclopentanol 1.6.2c were reacted with benzotriazole (BTA) 1.2.1a and phenyl-triazole (PTA) 1.2.1b. As expected, excellent stereoselectivity were achieved with only the corresponding cis-products (1.6.6a - 1.6.6d) observed via complete stereochemistry inversion (Scheme 42). ${ }^{*}$

Scheme 42: Complete stereochemistry inversion.
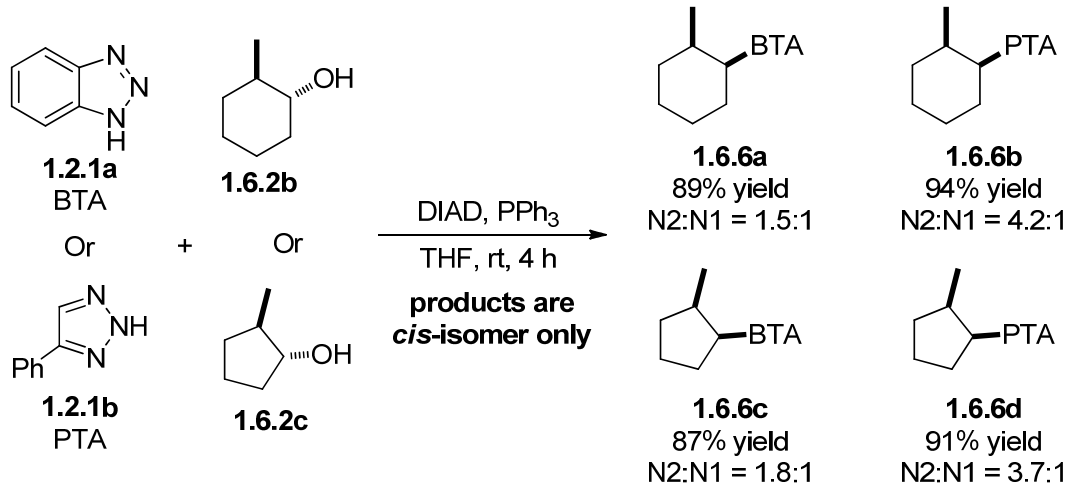

* The relative stereochemistries of 1.6.6a - 1.6.6d were determined by the NMR. 
Encouraged by these exciting results, we synthesized enantiomeric pure 1,2,3triazole derivatives through the coupling of triazoles and chiral alcohols under Mitsunobu conditions. As shown in Scheme 43, the chiral triazoles 1.6.7a and 1.6.7b were prepared from enantiomeric pure alcohol 1.6.2d and quinine 1.6.2e with excellent stereochemistry control. Under the Mitsunobu conditions, the enantiomeric pure alcohol 1.6.2d gave near complete chirality transfer, forming the chiral triazole $\mathbf{1 . 6 . 7 a}$ in $96 \%$ ee (determine by HPLC). ${ }^{*}$

Scheme 43: Asymmetric synthesis of chiral triazole derivatives.
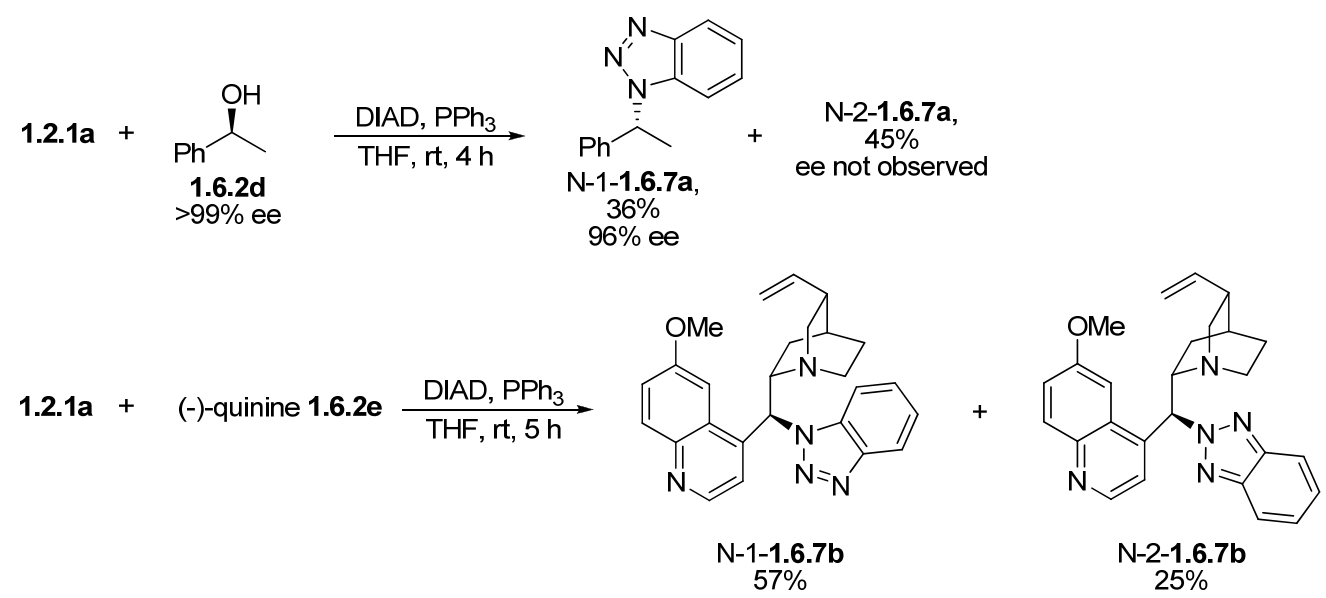

Due to the large difference of polarity between N-1 and N-2 isomers, they were easily separated by column chromatography, which made this method very attractive strategy for the preparation of the enantiomeric pure triazoles. Similar to previous cases, the secondary alcohols improved the yields of the N-2 isomers even with benzotriazole.

\footnotetext{
* The ee of N-2-1.6.7a was not determined due to the difficulty in separation of the two enantiomers in HPLC. However, large optical rotation of N-2-1.6.7a was observed with $[\alpha]_{D}^{25}=-55.40\left(\mathrm{CH}_{2} \mathrm{Cl}_{2}, \quad c \quad 1.0\right)$, which confirmed the likely similar excellent stereochemistry inversion was associated with the product.
} 
The synthesis of $\mathbf{1 . 6 . 7 b}$ (structure confirmed by X-ray crystallography) highlighted the strength of Mitsunobu reaction of $\mathrm{NH}-1,2,3$-traizoles in the preparation of highly functional triazole analogous (Figure 20). To the best of our knowledge, these are the first examples of the synthesis of enantiomeric pure triazoles. We are expected that these compounds can certainly be applied as potential novel ligand and catalysis in asymmetric catalysis.

Figure 20: Crystal structure of $\mathbf{N}-2-1.6 .7 b$.

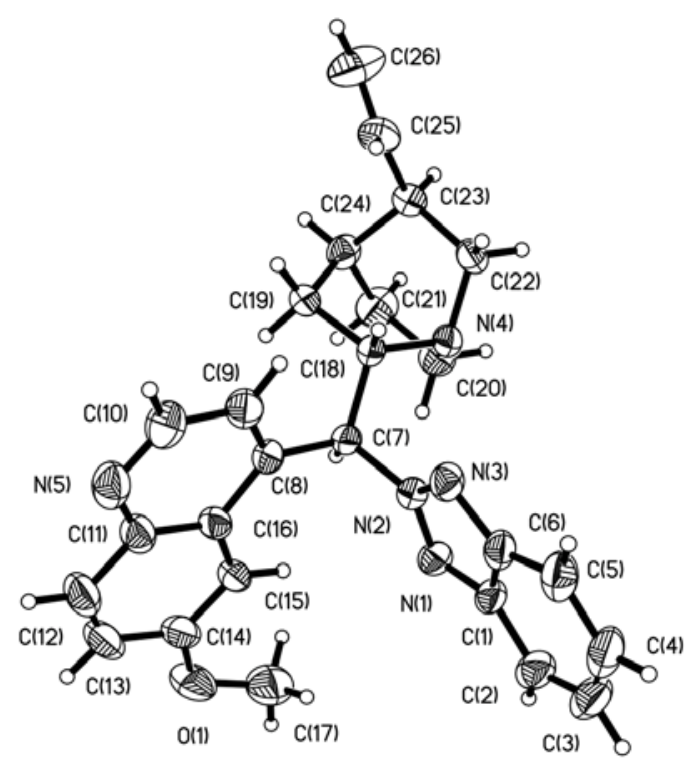

\subsubsection{Conclusion}

In conclusion, the Mitsunobu reactions between NH-triazoles and alcohols is a practical strategy for 1,2,3-triazole functionalization with excellent yields under mild conditions. Unlike previous strategies reported in literature, where different substituents of triazoles were required to achieve good yields of N-2 isomers, the Mitsunobu conditions favored the formation of the kinetic product $\mathrm{N}-2$ isomers, even with highly $\mathrm{N}-1$ preferred $1,2,3-$ 
triazoles (such as benzotriazole). Therefore, this method provided an alternative approach to achieve triazole N-2 substitution without changing the substituents on the triazoles. Moreover, with the excellent stereochemistry control, the reported method allowed asymmetric synthesis of enantiomeric pure triazole derivatives, which could certainly help the further development of 1,2,3-triazole as potential new ligands in transition metal catalysis. 


\section{Chapter Two: 1,2,3-trizazoles applied as ligands in transition metal catalysis}

\subsection{Introduction}

\subsubsection{Substituted 1,2,3-triazoles as neutral ligands}

The transition metal catalysis has revolutionized the traditional way synthetic chemists construct organic molecules in last several decades. ${ }^{84}$ Compared to classical syntheses, transition metal catalysis provided new efficient and sustainable routes for organic synthesis and the production of fine chemicals. One key correlation of successful transition metal mediated catalysis was the fundamental understanding of ligand-metal coordination (referred as "ligand field theory"), which represented an application of molecular orbital theory to transition metal complexes. ${ }^{85}$ In addition to the unique chemical properties of the individual metal elements, the binding ligand plays a crucial role in transition metal catalysis. ${ }^{86}$ Besides providing the spatial arrangement (asymmetric synthesis) at the metal center, ligand influences the electronic environment, thus adjusting metal reactivity. ${ }^{87}$ Ligand variation provide a powerful tool in transition metal catalysis, because key features of transition metal catalysts such as reactivity, selectivity, and stability may be tuned by the steric and electronic properties of the coordinated ligands. ${ }^{88}$ Considerable effort has focused on the development of new novel metal binding ligands, ${ }^{89}$ such as BINAP, ${ }^{90}$ BOX $^{91}{ }^{91}$ DuPhos, ${ }^{92}$ TADDOL $^{93}$ and salen. ${ }^{94}$ All of these ligands which have been extensively and successful applied in organic synthetic chemistry (Figure 21). 
Figure 21: Famous ligands in transition metal catalysis.

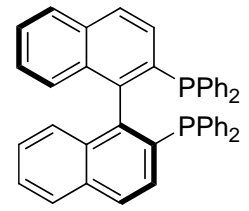

(S)-BINAP

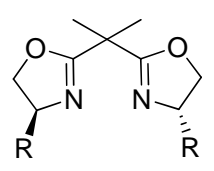

BOX

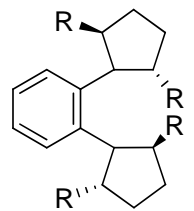

DuPhos

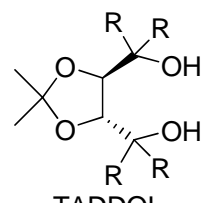

TADDOL

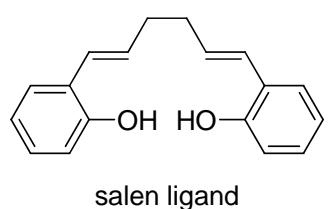

With the strong $\sigma$-donor and weak $\pi$-acceptor properties of the triazole ring, 1,2,3triazoles offer attractive ligands for coordination with transition metals. ${ }^{95}$ However, due to the limited access of functional triazole derivatives, only a few studies that utilize triazoles as ligands were reported in the literature before $2001 .{ }^{96}$ Since the discovery of "click chemistry", the application of 1,2,3-triazoles in several fields have been widely studied. However, these studies regarding the application of 1,2,3-triazoles as ligand in transition metal catalysis still remain undeveloped.

One of the earliest studies which revealed the excellent metal binding ability of 1,2,3-traizoles was reported by Sharpless in 2004. They synthesized the $\mathrm{C}_{3}$-synmmetric polytriazolylamines as ligands for copper(I) catalyst. As they claimed, the loading of copper(I) catalyst can be decreased as low as $0.25-1 \mathrm{~mol} \%$ (normally $5 \mathrm{~mol} \%$ in standard condition of "click chemistry"), because these powerful ligands stabilize the copper(I) catalyst, by protecting it from oxidation and disproportionation, while enhancing its catalytic activity in the click chemistry (Scheme 44). ${ }^{97}$ This new class of ligands is 
capable of binding to the copper(I) species by forming a five member chelate between the N-3 nitrogen of triazole ring and the amine.

Scheme 44: Application of $\mathrm{C}_{3}$-symmetric polytriazoles as the replacement of sodium ascorbate salt in click chemistry.

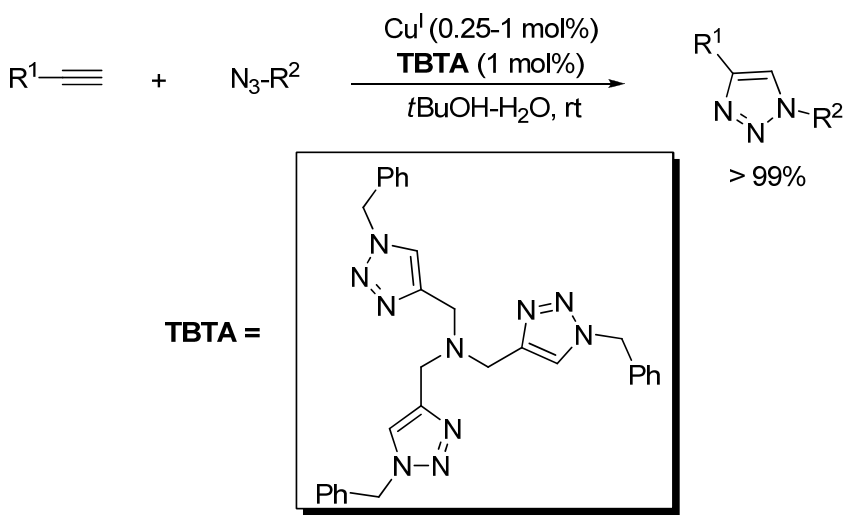

Table 11: Screening of various triazoles as stabilizing ligands for copper(I) catalyst.

$$
\mathrm{Ph}=+\mathrm{N}_{3}-\mathrm{Bn} \frac{\left[\mathrm{Cu}\left(\mathrm{CH}_{3} \mathrm{CN}\right)_{4}\right] \mathrm{PF}_{6}(1 \mathrm{~mol} \%)}{\text { ligand }(1 \mathrm{~mol} \%)} \underset{t \mathrm{BuOH}-\mathrm{H}_{2} \mathrm{O}(2: 1), \mathrm{rt}, 24 \mathrm{~h}}{\mathrm{Ph}}
$$

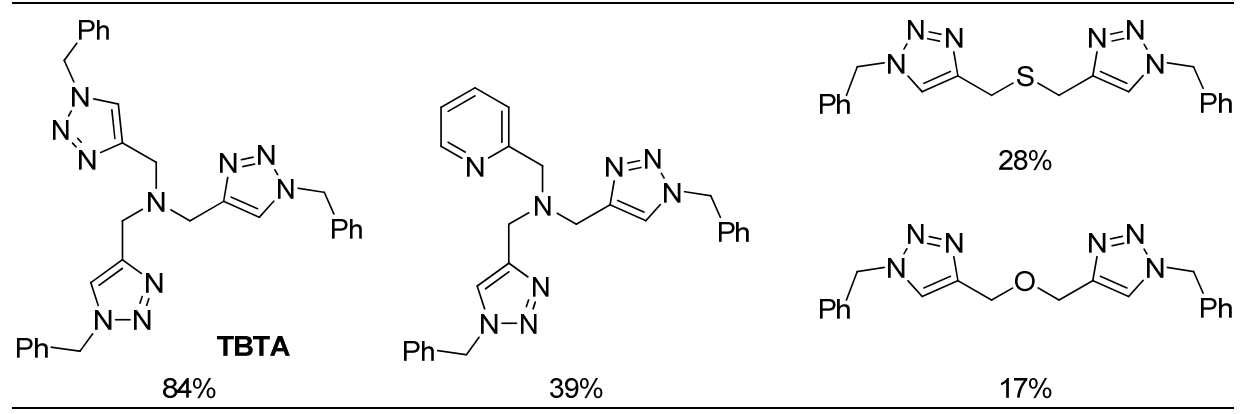

Based on the screening of various ligands (Table 11), the best ligand was tris(benzyltriazolylmethyl)amine (TBTA). Its tetradentate binding ability is believed to completely envelope the copper(I) center, leaving no free binding sites available for potential destabilizing interactions (Figure 22). ${ }^{98}$ The tertiary amine and the 1,2,3- 
triazole functionalities likely work in concert to make TBTA so efficient: the more basic and sterically encumbered nitrogen, accelerates the catalysis by providing additional electron density on the metal center, while the latter, being more labile, comes off the copper center only temporarily to allow the formation of the copper(I)-acetylide/ligand complex, which is then carried through the catalytic cycle.

Figure 22: Proposed structure of $\mathbf{C u}^{\mathrm{I}}$ (TBTA) complex.

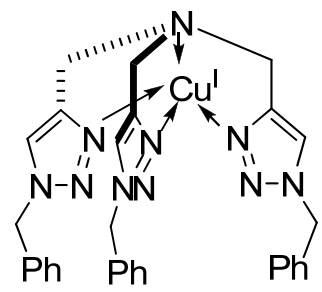

In 2005, Zhang reported a new type of monophosphine ligand (ClickPhos) bearing a triazole heterocycle in the backbone, which was showing high reactivity in the Pdcatalyzed Suzuki-Miyaura coupling and amination reactions of aryl chlorides. ${ }^{99}$ Later, van Marseveen and co-workers introduced a 1,2,3-triazoles as part of bidentate $\mathrm{P}, \mathrm{N}$-type ligand (ClickPhine), ${ }^{100}$ which is an important class of ligands that have been applied in various catalytic transformations. ${ }^{101}$ In the ClickPhine ligand, the 1,2,3-triazole played the role of nitrogen donor. A series of ligands were synthesized from readily available starting materials to give several homogenous and heterogeneous dendritic and polymeric catalysts (Scheme 45).

According to the NMR studies of the precursor palladium chloride IV (when R = $\mathrm{Ph}$ ), the ${ }^{1} \mathrm{H}$ NMR spectra gave broad signals for the allylic protons (except for the central proton) indicated isomerization via $\pi$ rotation or $\pi, \sigma$-rearrangement. The ${ }^{31} \mathrm{P}$ NMR spectrum showed a broad signal at $20.6 \mathrm{ppm}$ and revealed a monodentate binding of the 
ClickPhine ligand with the chloride still coordinated to the palladium. After mixing compound IV with $\mathrm{AgBF}_{4}$, the formation of cationic palladium-allyl triazole complex in which the ligand shows bidentate $\mathrm{P}, \mathrm{N}$ coordination, as confirmed by NMR data. The ${ }^{1} \mathrm{H}$ NMR signal of the triazole proton shifted $0.71 \mathrm{ppm}$ to lower field (from 8.15 to $8.86 \mathrm{ppm}$ ) after ion exchange, and the $\mathrm{CH}_{2}$ group gave rise to an $\mathrm{AB}$ pattern showing that these protons became inequivalent. In addition, the signal in the ${ }^{31} \mathrm{P}$ NMR spectrum shifted from 20.6 to $40.5 \mathrm{ppm}$ and became sharper. This was the first example that provided direct evidence of triazole binding to a transition metal. Furthermore, preliminary experiments found the efficiency of these ClickPhine ligands in the Pd-catalyzed allylic alkylation reaction.

Scheme 45: Synthesis route of "ClickPhine" P,N-ligand and their Pd-complexes.

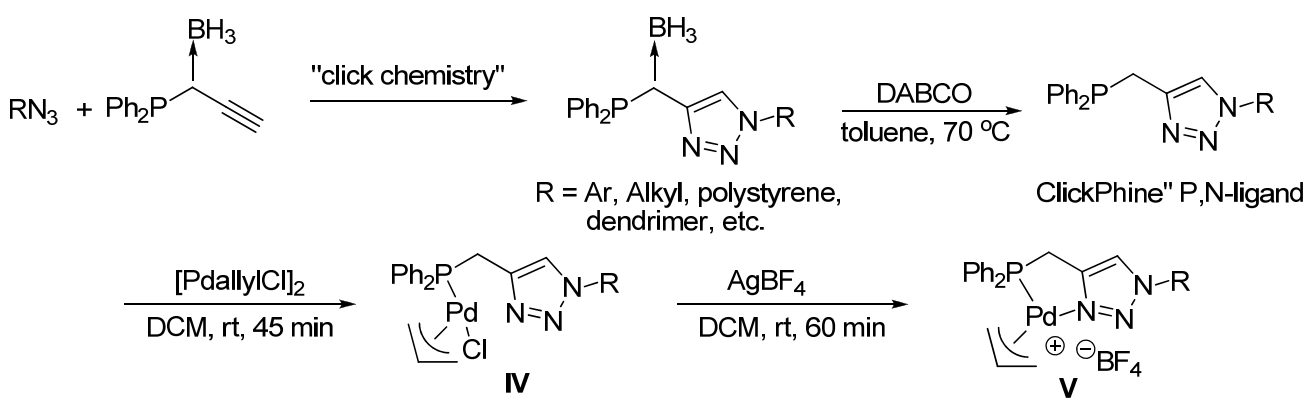

Additional examples of $\mathrm{N}$-coordination of a 1,2,3-triazole to a metal was reported in 2007. Koten, Gebbink and co-workers reported the first examples of 1,4-disubstituted 1,2,3-triazoles as monodentate Lewis-basic ligands for cationic Pd- and Pt- pincer complexes with reported crystal structure (Scheme 46). ${ }^{102}$ The 1,2,3-triazole coordinated metal complexes were accessed by the addition of the triazole to the pincer $\mathrm{Pd}$ and $\mathrm{Pt}$ cations, which is generated in situ upon reaction with $\mathrm{AgBF}_{4}$. The single $\mathrm{X}$-ray 
crystallographic structure of the complex revealed an $\mathrm{M}(\mathrm{II})$ ion which is four coordinate with a distorted square-planar ligand environment by virtue of the donor atoms of the tridentate pincer ligand and the N-3 nitrogen of the triazole.

Scheme 46: 1,2,3-triazoles as monodentate Lewis-basic ligands for cationic Pd- and Ptpincer complexes
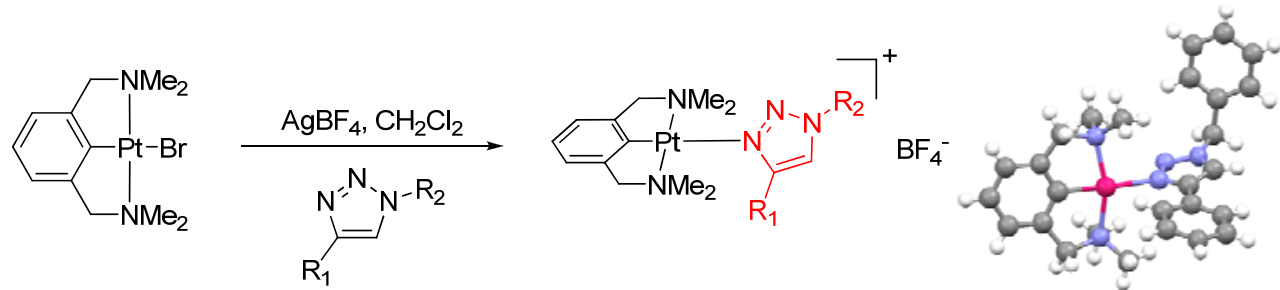

A comparison study of relative coordination strength of 1,2,3-trizaoles with respect to more traditional Lewis bases $\mathrm{PPh}_{3}, 1$-methyl-imidazole, $\mathrm{Et}_{2} \mathrm{~S}$, DMSO, anline, $\mathrm{MeCN}$ and pyridine was performed by means of ${ }^{1} \mathrm{H}$ NMR spectroscopy which represented as the relative association constant, $K_{\text {ass }}$ (Scheme 47).

Scheme 47: The definition of $\mathrm{K}_{\text {ass. }}$.

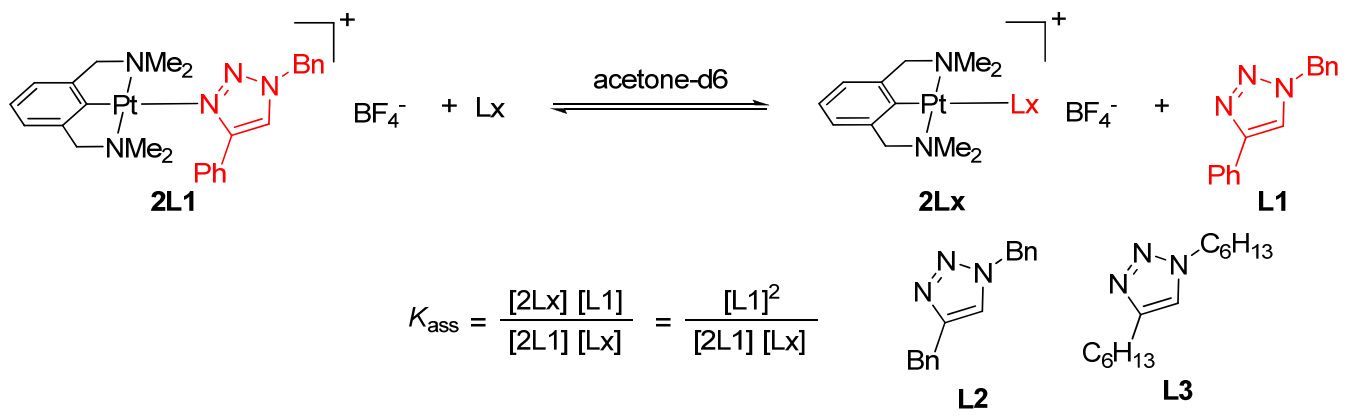

The experiments showed that 1,2,3-triazole $\mathbf{L} 1$ is a stronger ligand than $\mathrm{Et}_{2} \mathrm{~S}$, DMSO, $\mathrm{H}_{2} \mathrm{O}$ and $\mathrm{MeCN}$ (Table 12), but is weaker than $\mathrm{PPh}_{3}$, pyridine and $N$-methyl imidazole in strength. Entries 9-11 indicated that the coordination strengths of triazoles 
ligands toward the pincer Pt cation can be tuned by the appropriate functionalization of 1,4-disubstituted triazoles.

Table 12: $\boldsymbol{K}_{\text {ass }}$ values for Lewis base coordination to Pt-pincer complex compared to $\mathbf{L} \mathbf{1}$ for several competing ligands $\mathbf{L x}$.

\begin{tabular}{ccc|ccc}
\hline Entry & $\mathbf{L x}$ & $\boldsymbol{K}_{\text {ass }}$ & Entry & $\mathbf{L x}$ & $\boldsymbol{K}_{\text {ass }}$ \\
\hline 1 & $\mathrm{H}_{2} \mathrm{O}$ & - & 6 & Pyridine & 19.5 \\
2 & $\mathrm{DMSO}$ & 0.002 & 7 & $\mathrm{PPh}_{3}$ & $>^{a}$ \\
3 & $\mathrm{MeCN}$ & 0.02 & 8 & $N$-methyl imidazole & $>^{a}$ \\
4 & $\mathrm{Et}_{2} \mathrm{~S}$ & 0.03 & 9 & $1 H-1,2,3$-triazole & 4.16 \\
5 & Anline & 0.3 & 10 & $\mathbf{L 2}$ & 6.47 \\
& & & 11 & $\mathbf{L 3}$ & 18.5 \\
\hline
\end{tabular}

${ }^{a}$ Full conversion to $\mathbf{2} \mathbf{L x}$ occurred

Recently, 1,2,3-triazol-5-ylidenes were found to be the good alternative NHC ligands for forming complexes with transition metals, ${ }^{103}$ such as gold, ${ }^{104}$ silver, ${ }^{105}$ rhodium, ${ }^{106}$ ruthenium, ${ }^{107}$ palladium ${ }^{108}$ and copper $^{109}$ which have been applied in the some classical organic synthesis. For example, Fukuzawa and co-workers demonstrated the utility of their new $\mathrm{PdCl}_{2}(\mathrm{TMes})_{2}$ complex in Suzuki reactions (Scheme 48), where the TMes (triazole-based NHC bearing mesityl groups). ${ }^{108 b}$ Indeed, complex $\mathrm{PdCl}_{2}$ (TMes $)_{2}$ was successful in catalyzing the Suzuki reaction between aryl chlorides and various aryl boronic acids in good to excellent yields. Following their previous results with $\mathrm{PdCl}_{2}$ (TMes) $)_{2}$ complex, they extended the chemistry of 1,2,3-triazolylidene ligands by synthesizing new copper complexes, such as $\mathrm{CuCl}(\mathrm{aNHC})$ and $\mathrm{CuCl}(\mathrm{TPh}) .{ }^{109}$ These complexes were evaluated as catalysts for the CuAAC azide-alkyne cycloaddition 
reaction to give 1,4-substituted 1,2,3-triazoles in excellent yields at room temperature with short reaction times (Scheme 49). They also claimed complex $\mathrm{CuCl}(\mathrm{TPh})$ was particularly effective for the reaction between sterically hindered azides and alkynes. Moreover, Crowley ${ }^{104}$ and co-workers disclosed the first gold 'click' 1,2,3-triazolylidene complex and its utility in gold(I)-catalysis (Scheme 50). The air and moisture stable gold(I) complex proved to be a good catalyst for carbene transfer reactions from diazo compounds into $\mathrm{O}-\mathrm{H}, \mathrm{N}-\mathrm{H}$ and $\mathrm{C}-\mathrm{H}$ bonds, hydroalkoxylation of allenes and enyne cyclizations. In 2011, Grubbs ${ }^{107 b}$ applied the stabilized 1,3,4-triaryl-1,2,3-triazol-5ylidene carbenes to displace the phosphine ligand of Hoveyda-Grubbs catalyst, generating a family of 1,3,4-triaryl-1,2,3-triazol-5-ylidene containing ruthenium metathesis catalysts (Scheme 51). These complexes were found to be active roomtemperature catalysts for the ring-opening metathesis polymerization of cyclic olefins and for ring-closing olefin metathesis reactions. The catalytic properties of the Ru-1,2,3triazolylidene complexes are strongly influenced by the nature of the MICs' substituents.

Scheme 48: Suzuki couplings with aryl chlorides catalyzed by the Pd-1,2,3-triazolylidene complex $\mathrm{PdCl}_{2}(\mathrm{TMes})_{2}$
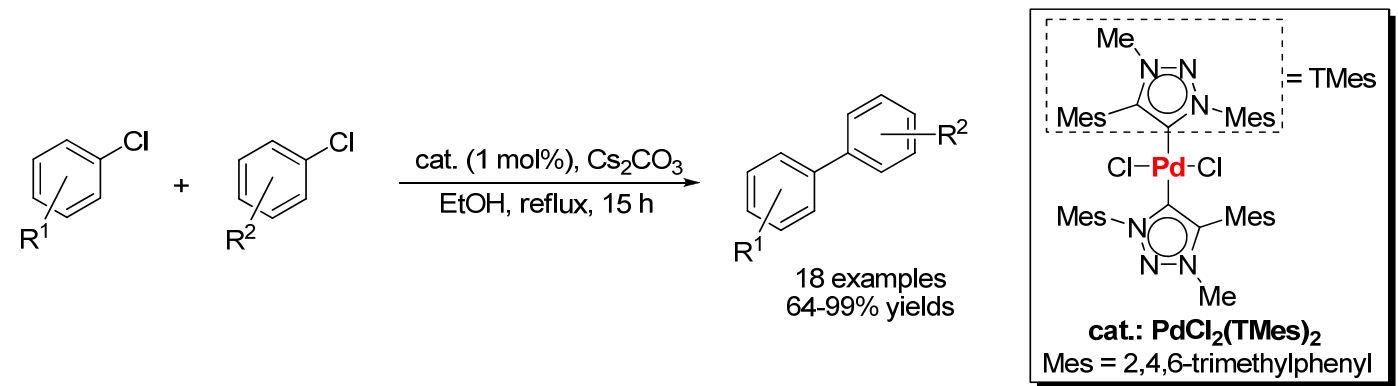
Scheme 49: CuAAC reactions catalyzed by copper(I)-1,2,3-triazolylidene complexes

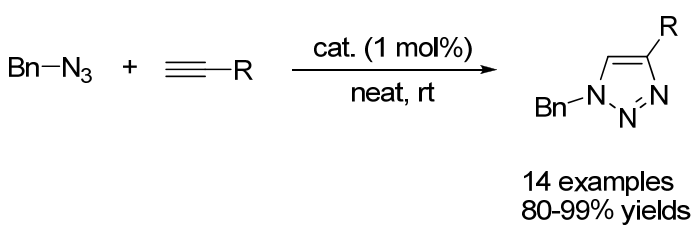

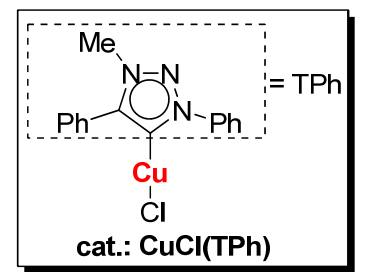

Scheme 50: Carbene transfer reactions from diazo compounds catalyzed by the gold(I)1,2,3-triazolylidene complex.
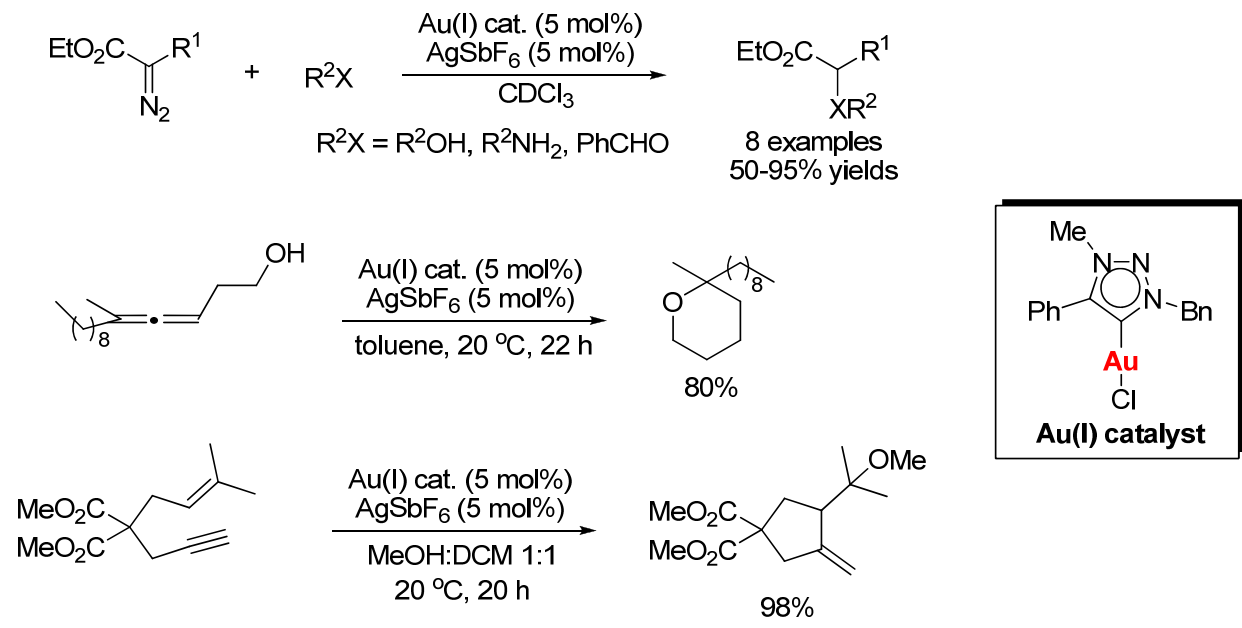

Scheme 51: Ring opening and ring closing metathesis using Grubbs' 1,3,4-triaryl-1,2,3triazol-5-ylidene containing ruthenium catalysts.
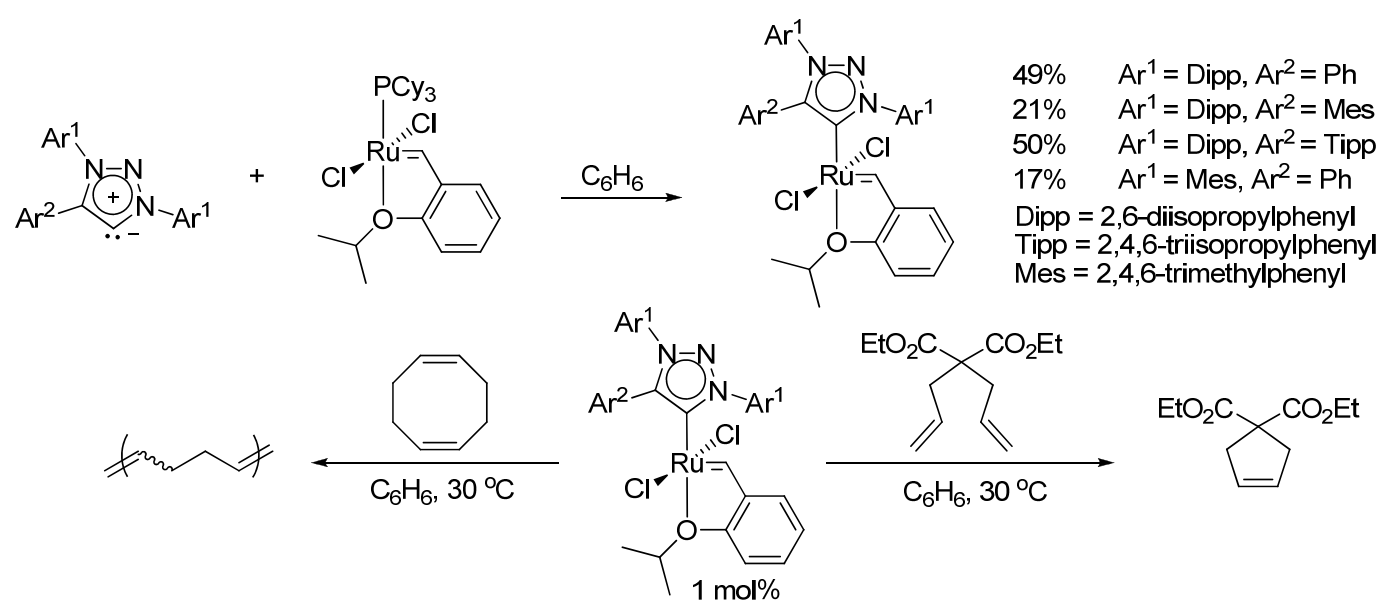<smiles>CCOCC1(COCC)CC=CC1</smiles> 


\subsubsection{1,2,3-NH-triazoles as anionic ligands}

Compared to $\mathrm{N}$-substituted triazoles, the $\mathrm{NH}$-triazoles provide acidic $\mathrm{N}-\mathrm{H}$ protons $(\mathrm{pKa}<$ 10), ${ }^{110}$ therefore, they can be applied as anionic ligands with multiple binding sites. On the basis of reported literatures so far, all efforts regarding triazole as anionic binding ligand have focused on benzotriazoles, ${ }^{111}$ which decreased the overall interests in this class of complexes, due to limited functionality availability. In one of the earliest example of benzotriazole as a ligand, Oro and co-workers discovered the application of benzotriazole and benzotriazolate anion in $\mathrm{Rh}(\mathrm{I})$ complexes. ${ }^{112}$ To investigate the structure and binding patterns of Rh(I)-triazole complexes, they successfully synthesized mono- and di nuclear rhodium complexes of the general formula $\mathrm{RhClL}_{2}(\mathrm{btzH}),[\mathrm{Rh}(\mu-$ btz) $\left.\mathrm{L}_{2}\right]_{2}, \mathrm{Rh}_{2}\left(\mu\right.$-btz) $\left(\mu-\mathrm{N}_{3}\right)\left(\mathrm{L}_{2}\right)_{2}$ and $\mathrm{Rh}_{2}(\mu$-btz $)(\mu-\mathrm{SCN})(\mathrm{COD})_{2}\left[\mathrm{~L}_{2}=\right.$ diolefin, $(\mathrm{CO})_{2}$ or $\left.(\mathrm{CO})\left(\mathrm{PPh}_{3}\right)\right]$ (Scheme 52). For example, the addition of benzotriazole to $[\mathrm{Rh}(\mu-$ $\mathrm{Cl})(\mathrm{COD})]_{2}$ suspended in acetone resulted in the formation of $[\mathrm{RhCl}(\mathrm{COD})(\mathrm{btzH})]$ complex as stable yellow solid in excellent yield (88\%).

Scheme 52: Synthesis of $[\mathrm{Rh}(\mathrm{btz})(\mathrm{COD})]_{2}$ complexes reported by Oro.

$$
\begin{aligned}
& {[\mathrm{Rh}(\mu-\mathrm{Cl})(\mathrm{COD})]_{2}+\text { btzH } \stackrel{\text { acetone }}{\longrightarrow} \underset{88 \% \text { yield }}{[\mathrm{RhCl}(\mathrm{COD})(\mathrm{btzH})]} \stackrel{\mathrm{Et}_{3} \mathrm{~N}}{\longrightarrow} \underset{\text { yellow solid }}{[\mathrm{Rh}(\mu-\mathrm{btz})(\mathrm{COD})]_{2}}}
\end{aligned}
$$

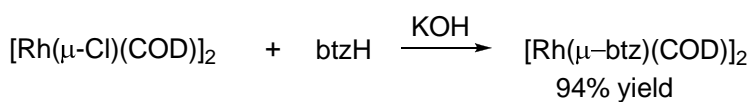

Although the molecular structure of $\mathrm{Rh}(\mathrm{I})$-triazole complex remains to be determined, a square planar arrangement was proposed with the benzotriazole coordinated through a pyridyl nitrogen atom. Moreover, the addition of triethylamine to a 
solution of $[\mathrm{RhCl}(\mathrm{COD})(\mathrm{btzH})]$ in methanol, led to elimination of the acidic proton of the benzotriazole ligand with the formation of $[\mathrm{Rh}(\mu-\mathrm{btz})(\mathrm{COD})]_{2}$. A direct route to prepare $[\mathrm{Rh}(\mu-\mathrm{btz})(\mathrm{COD})]_{2}$, complex, in high yield involved the reaction of $[\mathrm{Rh}(\mu-\mathrm{Cl})(\mathrm{COD})]_{2}$ with benzotriazole and $\mathrm{KOH}$. The molecular weight measurements on $[\mathrm{Rh}(\mu-$ btz)(COD) $]_{2}$ complex indicated a dinuclear complex (Rh-Rh $\left.3.27 \AA ̊\right)$, therefore suggesting that the nitrogen atoms of the benzotriazolate ligand involved in coordination are adjacent to each other (Figure 23). This proposed dinuclear structure for these complexes was confirmed by the X-ray study of $[\mathrm{Rh}(\mu-\mathrm{btz})(\mathrm{COD})]_{2}$.

Figure 23: Proposed dinuclear structure of Rh(I)-triazole complexes.

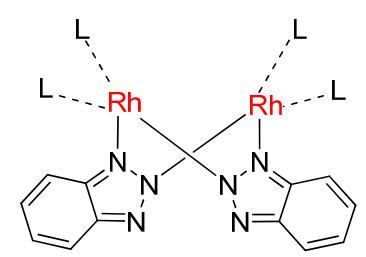

In addition, Oro and co-workers studied the only known example of a 4,5disubstituted-(NH)-1,2,3-triazole coordination with $\mathrm{Rh}(\mathrm{I}) .{ }^{113}$ Neutral bi- and mononuclear dicyanotriazolate $(\mathrm{DcTz})$ complexes of formula $\left[\mathrm{Rh}_{2}(\mu-\mathrm{DcTz})_{2} \mathrm{~L}_{2} \mathrm{~L}_{2}^{\prime}\right]\left(\mathrm{L}_{2}=\mathrm{L}_{2}^{\prime}=\right.$ diolefin; $\mathrm{L}=\mathrm{CO}, \mathrm{L}^{\prime}=\mathrm{PPh}_{3}$ or $\left.\mathrm{P}(\mathrm{OPh})_{3} ; \mathrm{L}=\mathrm{L}^{\prime}=\mathrm{P}(\mathrm{OPh})_{3} ; \mathrm{L}_{2}=\mathrm{COD}, \mathrm{L}^{\prime}=\mathrm{P}(\mathrm{OPh})_{3}\right)$ and $\left[\mathrm{Rh}(\mathrm{DcTz}) \mathrm{L}_{2} \mathrm{~L}^{\prime}\right]\left(\mathrm{L}_{2}=\right.$ diolefin, $\left.\mathrm{L}^{\prime}=\mathrm{PPh}_{3} ; \mathrm{L}=\mathrm{PPh}_{3}, \mathrm{~L}^{\prime}=\mathrm{CO}\right)$ and the ionic derivative $\left[\mathrm{Rh}(\mathrm{dppe})_{2}\right][\mathrm{DcTz}]$ have been synthesized. Based on the study by Rasmussen ${ }^{114}$ showing that the coordinating ability of the ligands can be influenced by the presence of cyan substituents on the heterocyclic ring, the authors investigated the coordinating ability of 4,5-dicyano-1,2,3-triazole (HDcTz). Treatment of [Rh(acac)(COD)] with 4,5-dicyano- 
$1,2,3$ triazolate led to the formation of the $\mathrm{Rh}(\mathrm{I})$ dicyanotriazole complex in high yields (Scheme 53).

Scheme 53: Synthesis of $[\mathrm{Rh}(\mu-\mathrm{DcTz})(\mathrm{COD})]_{2}$ complex.

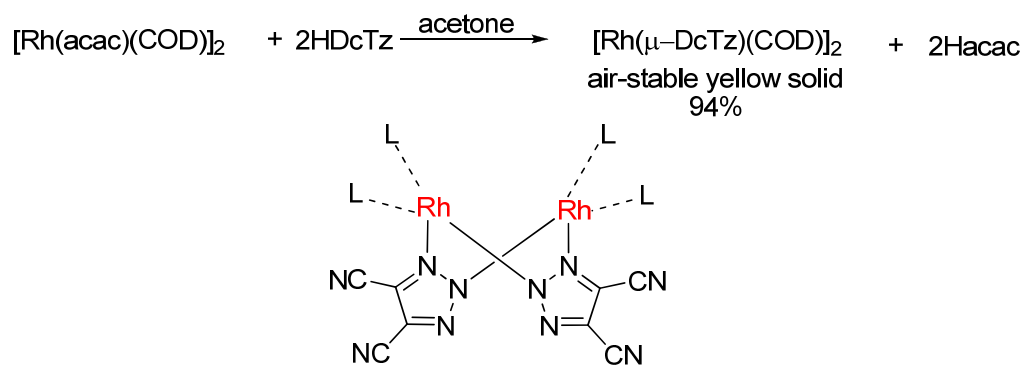

\subsubsection{Achievements of our group by using 1,2,3-triazoles as the ligands}

During the past several years, our group has been working on the development of new catalysts with 1,2,3-triazoles as ligands in transition metal catalysis. These efforts led to the discoveries of triazole- $\mathrm{Rh}$ and triazole-Au catalysts with interesting new reactivities.

On the basis of our success in the synthesis of 4,5-disubstituted-( $\mathrm{NH})-1,2,3$-triazoles through Lewis base-catalyzed cascade nitroalkene-aldehyde-azide condensation. ${ }^{13}$ Duan in our group examined the binding ability of a triazole anion with $\mathrm{Rh}(\mathrm{I})$ by treating the 4,5-disubstituted-(NH)-1,2,3-triazoles with $[\mathrm{Rh}(\mathrm{COD}) \mathrm{Cl}]_{2}$ under basic conditions (Scheme 54). ${ }^{115}$ As expected, the triazole anion effectively substituted the chloride $\left(\mathrm{Cl}^{-}\right)$ and triazole- $\mathrm{Rh}(\mathrm{I})$ complexes $\left(\left[\mathrm{Rh}(\mathrm{COD})(\mathrm{Triaz}]_{2}\right)\right.$ were obtained in near quantitative yields. These complexes showed excellent stability towards both air and moisture, and can be purified by column chromatography. As a new class of transition metal complexes, these compounds display effective catalytic reactivity in the Pauson-Khand reaction. 
Encouraged by the successful synthesis of triazole-Rh(I) complexes, we investigated triazoles as ligands for the synthesis of $\mathrm{Au}(\mathrm{I})$ complexes. In comparison with the more expensive NHC ligands, ${ }^{116}$ the simple triazoles are also known to possess a nitrogen $\sigma$-donor and a lower LUMO aromatic antibonding orbital for metal backbonding. The triazole-Au(I) complexes were prepared successfully in quantitative yields using a preparation procedure similar to triazole-Rh(I) complexes (Scheme 55). ${ }^{117}$ Further studies revealed that the triazole- $\mathrm{Au}(\mathrm{I})$ catalyst gave excellent reactivity, selectivity and improved thermal stabilities in several organic transformations which included hydroamination and synthesis of Hashmi phenol, ${ }^{118} E$ - $\alpha$-haloenones, ${ }^{119}$ enones ${ }^{120}$ and allenes. ${ }^{121}$ Based on the synthesis of allenes form rearrangement of propargyl alcohols, we deducted one of unique properties of triazole-Au complexes was no further activation of allene intermediates with activation of triple bond only, while other regular gold catalysts can activate both (Scheme 56). ${ }^{\text {121a }}$

Scheme 54: Synthesis of triazole-Rh(I) complexes and its application in Pauson-Khand reactions.
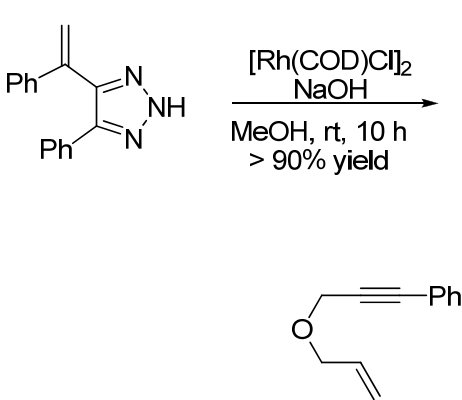

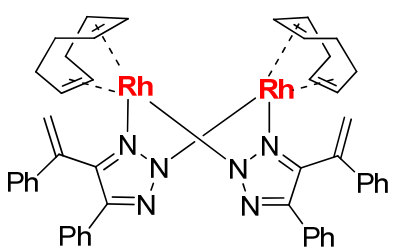

5\% Rh complex, 6\% dppp, xylene air and moisture stralbe Rh(I) complexes characterized by X-ray

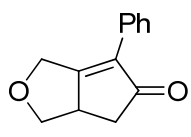

$80 \%$ 
Scheme 55: Synthesis of triazole-Au(I) complexes.

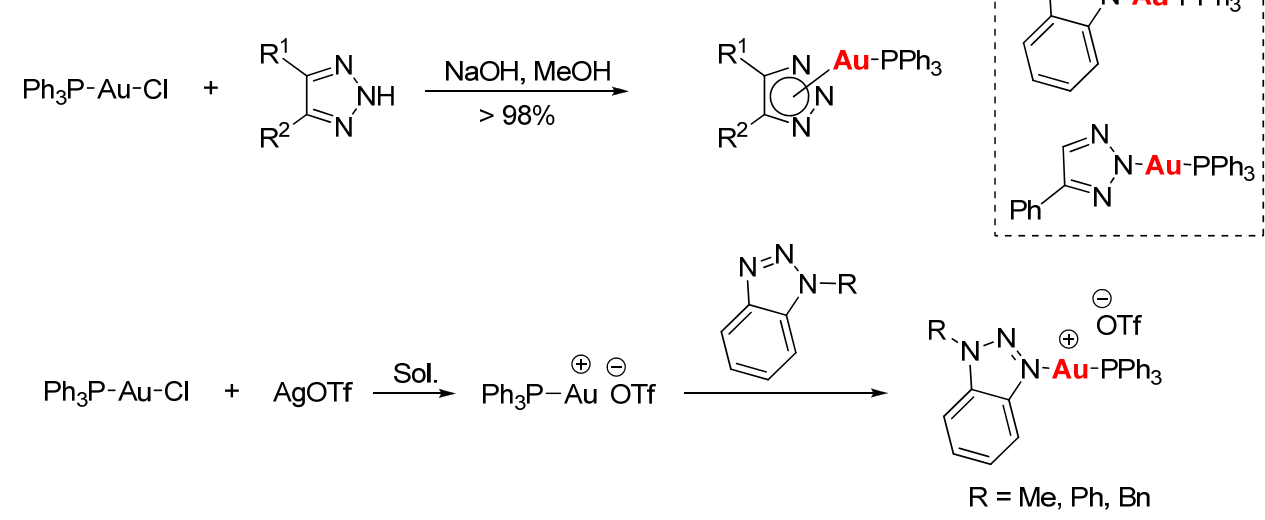

Scheme 56: Asymmetric synthesis of substituted allenes.

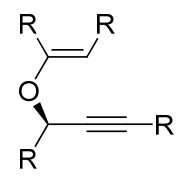

$\stackrel{\text { TA-Au (1.0\%), } \mathrm{CH}_{2} \mathrm{Cl}_{2} \text {, rt }}{\longrightarrow}$

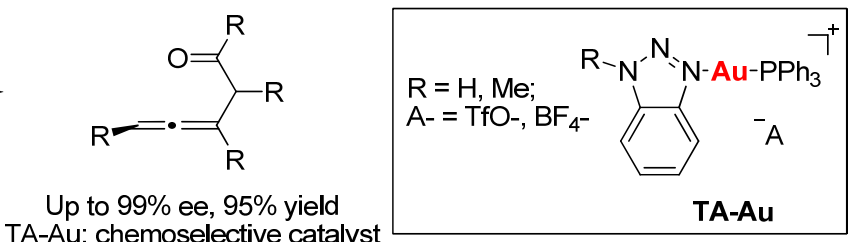




\subsection{Synthesis of $\left[\operatorname{Ir}\left(\mathrm{L}^{\wedge} \mathrm{X}\right)(\text { ArTriaz })_{2}\right]$ and $\left[\operatorname{Ir}(\mathrm{bbpy})(\operatorname{ArTriaz})_{2}\right] \mathrm{Cl}$ complexes and their application in photoredox catalysis}

\subsubsection{Reported examples of triazole-Ir(III) complexes}

1,2,3-triazoles have been extensively studied in the past decade due to the discovery of "click chemistry". The synthesis of 1,2,3-triazole related chelate ligands was first described using the "click chemistry" of amino acid derived alkynes or azides in $2006 .{ }^{122}$ The resultant chelate ligands are bound through a primary amine and either the N-3 nitrogen or, in some cases, the N-2 nitrogen of the triazoles (Figure 24). Since then, several additional examples of this "click-to-chelate" approach have been reported, and this field has been reviewed. ${ }^{123}$ The majority of examples use either 2-ethynyl pyridine, ${ }^{124}$ which tends to give five-membered chelation (Figure 24A), or 2-picolylethyne, ${ }^{125}$ which gives six-membered chelation (Figure 24B). Both of these ligands coordinate through N3 nitrogen as expected, since DFT calculations have shown that N-3 nitrogen is a better donor than N-2 nitrogen. ${ }^{126}$ However, coordination through N-2 nitrogen is also possible using ligands of type $\mathrm{C}^{127}$ but the complexes are less stable than those of type $\mathrm{A}$, as has been confirmed experimentally and computationally for a series of palladium complexes. ${ }^{127 \mathrm{c}}$

Figure 24: Chelating triazoles derived from 2-ethynyl pyridine (A), 2-picolylethyne (B), and 2-azidomethylpyridine $(\mathbf{C})$.

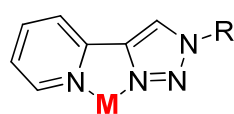

A

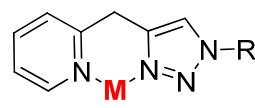

B

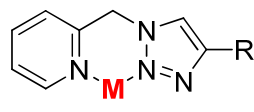

C 
The luminescence of pyridine triazoles is sensitive to the presence of metal ions, ${ }^{128}$ and the ease of tuning the electronic properties of these ligands has been used to control the luminescence of biscyclometalated iridium(III) complexes (Figure 25). ${ }^{129}$ Luminescent $\operatorname{Ir}(\mathrm{III})$ complexes have been used in various applications such as biological labeling reagents, ${ }^{130}$ photosensitizers for light-driven catalytic water reduction, ${ }^{131}$ single oxygen sensitizers, ${ }^{132}$ and sensors. ${ }^{133}$ Moreover, the most important application of the $\operatorname{Ir}(\mathrm{III})$ complexes is as emitters in electroluminescent devices including organic lightemitting devices (OLEDs) ${ }^{134}$ and light-emitting electrochemical cells (LEECs). ${ }^{135}$

Figure 25: Different kind of biscyclometalated iridium complexes.
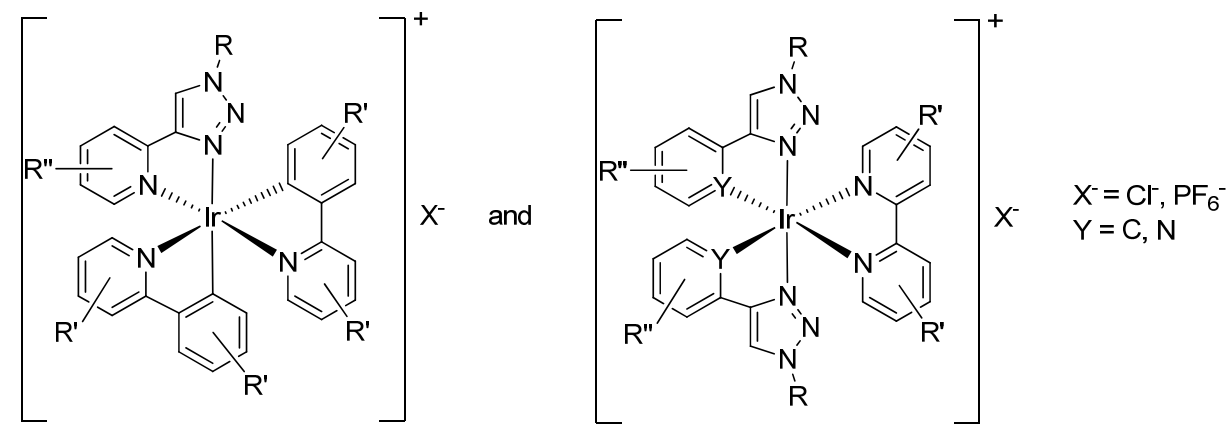

The luminescent properties of biscyclometalated iridium(III) complexes significantly changed when 1,2,3-triazoles was the subunits of iridium(III) complexes structures. For example, Zysman-Colman reported the incorporation of 4-aryl-1-benzyl$1 H$-1,2,3-triazoles (atl) as cyclometallating ligands into new heteroleptic iridium(III) complexes containing diimine $\left(\mathrm{N}^{\wedge} \mathrm{N}\right)$ ancillary ligands $2,2^{\prime}$-bipyridine (bpy) and 4,4'ditert-butyl-2,20-bipyridine (dtBubpy) in 2011. ${ }^{136}$ These complexes emit from the yellow to sky blue in acetonitrile $(\mathrm{MeCN})$ solution at room temperature. Their photoluminescent quantum efficiencies are markedly higher (between 25 and 80\%) than analogous 
$\left(\mathrm{C}^{\wedge} \mathrm{N}\right)_{2} \operatorname{Ir}\left(\mathrm{N}^{\wedge} \mathrm{N}\right)^{+}$type complexes, where $\mathrm{C}^{\wedge} \mathrm{N}$ is a 2-phenylpyridine ligand (Figure 26). The results of a computational investigation suggested that for these complexes, the highest occupied molecular orbital (HOMO) is located on both the aryl fragment of the atl ligands and the iridium metal while the lowest unoccupied molecular orbital (LUMO) is located essentially exclusively on the ancillary ligand.

Figure 26: Enhanced luminescent Ir(III) complexes bearing aryltriazole cyclometallated ligands reported by Zysman-Colman.
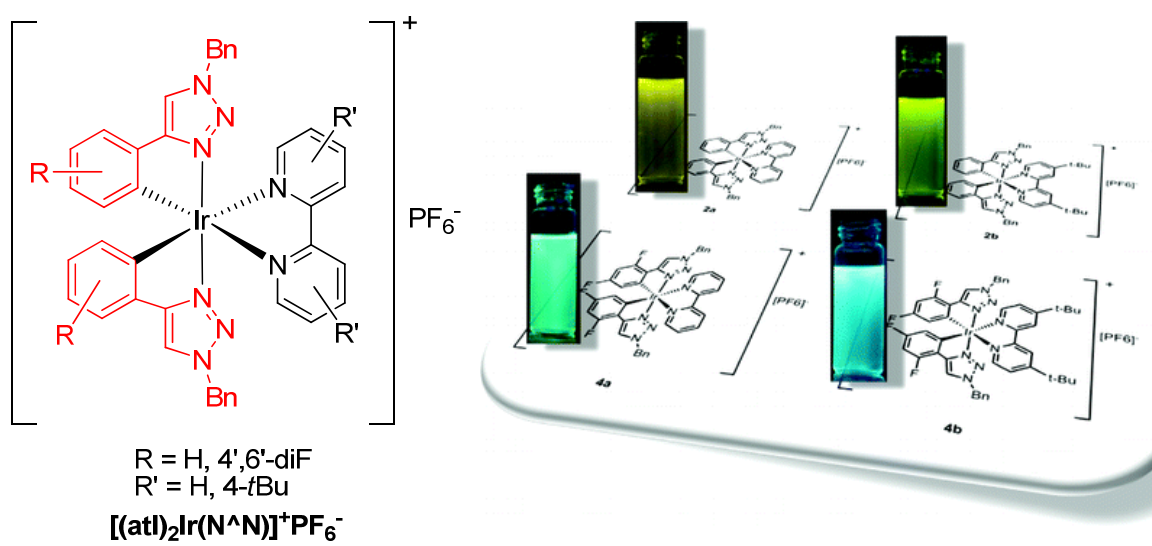

$\left.(\text { atl })_{2} \operatorname{Ir}\left(\mathbf{N}^{\wedge} \mathbf{N}\right)\right]^{+} \mathrm{PF}_{6}^{-}$

Furthermore, De Cola and Fernández-Hernández synthesized and characterized two biscyclometalated $\operatorname{Ir}(\mathrm{III})$ complexes isomers depending on the reaction temperatures which are N,N-trans-[Ir- $\left.(\mathrm{dfptrBz})_{2}(\mathrm{dmbpy})\right]^{+} \mathrm{PF}_{6}^{-}$, with cis-C,C and trans-N,N arrangement of the $\mathrm{C}^{\wedge} \mathrm{N}$ ligands at $140{ }^{\circ} \mathrm{C}$, and $\mathrm{N}, \mathrm{N}$-cis-[Ir- $\left.(\mathrm{dfptrBz})_{2}(\mathrm{dmbpy})\right]^{+} \mathrm{PF}_{6}^{-}$, cis$\mathrm{C}, \mathrm{C}$ and cis-N,N $\mathrm{C}^{\wedge} \mathrm{N}$ ligand orientation at $80{ }^{\circ} \mathrm{C}\left(\mathrm{dmbpy}=4,4^{\prime}\right.$-dimethyl-2,2'-bipyridine and dfptrBz $=1$-benzyl-4-(2,4-difluorophenyl)-1H-1,2,3-triazole). ${ }^{137}$ More interestingly, both isomers showed significantly different photophysical and electroluminescent properties, depending on the mutual arrangement of the $\mathrm{C}^{\wedge} \mathrm{N}$ ligands (Figure 27). 
Figure 27: Control of the Mutual Arrangement of Cyclometalated Ligands in Cationic Ir(III) Complexes.

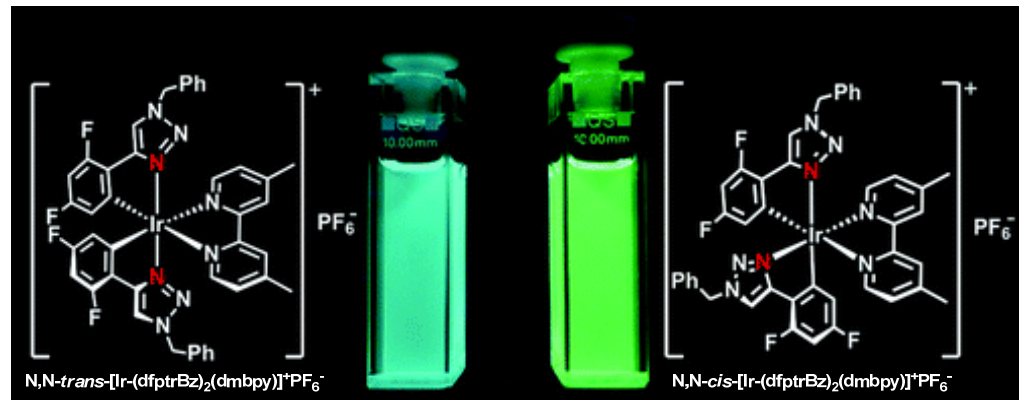

Although these triazole-Ir(III) complexes revealed the unique and important luminescent properties, the triazole-Ir(III) complexes have not yet been explored for use in homogeneous transition metal catalysis. Therefore, we develop new transformations using triazole-Ir(III) complexes as the catalysts to take advantage of their unique luminescent properties to develop systems that are capable of photoredox catalysis.

\subsubsection{Synthesis of $\left[\operatorname{Ir}\left(\mathrm{L}^{\wedge} \mathrm{X}\right)(\operatorname{ArTriaz})_{2}\right]$ and $\left[\operatorname{Ir}(\mathrm{bbpy})(\mathrm{ArTriaz})_{2}\right] \mathrm{Cl}$ complexes}

These biscyclometalated iridium(III) complexes are generally synthesized through a twostep process, the first step being a reaction between the $\mathrm{HC}^{\wedge} \mathrm{N}$ ligand and $\mathrm{IrCl}_{3} \cdot \mathrm{nH}_{2} \mathrm{O}$ to form a dichloro-bridged dinuclear $\operatorname{Ir}(\mathrm{III})$ dimer, $\left[\operatorname{Ir}\left(\mathrm{C}^{\wedge} \mathrm{N}\right)_{2}(\mu-\mathrm{Cl})\right]_{2} \cdot{ }^{138}$ Further reaction of the dichloro-bridged dimer with a third cyclometalating ligand yields triscyclometalated complexes that can be homoleptic, $\left[\operatorname{Ir}\left(\mathrm{C}^{\wedge} \mathrm{N}\right)_{3}\right],{ }^{139}$ if the $\mathrm{C}^{\wedge} \mathrm{N}$ are identical or heteroleptic, $\left[\operatorname{Ir}\left(\mathrm{C}^{\wedge} \mathrm{N}\right)_{2}\left(\mathrm{C}^{\prime \wedge} \mathrm{N}^{\prime}\right)\right]$, if a different $\mathrm{C}^{\prime \wedge} \mathrm{N}^{\prime}$ is used. ${ }^{140}$ The chloride dimer can also react with chelating ligands, leading to a wide range of neutral bis-cyclometalated complexes $\left[\operatorname{Ir}\left(\mathrm{C}^{\wedge} \mathrm{N}\right)_{2}\left(\mathrm{~L}^{\wedge} \mathrm{X}\right)\right]\left(\mathrm{L}^{\wedge} \mathrm{X}=\right.$ anionic ligands as $\beta$-diketonates, picolinates, pyridineazolates, 
etc.) or cationic complexes $\left[\operatorname{Ir}\left(\mathrm{C}^{\wedge} \mathrm{N}\right)_{2}\left(\mathrm{~L}^{\wedge} \mathrm{L}^{\prime}\right)\right]^{+}$(e.g. $\mathrm{L}^{\wedge} \mathrm{L}^{\prime}=$ neutral ligands such as diimines, phosphines, etc.) (Figure 28). ${ }^{141}$

Figure 28: Four different kinds of biscyclometalated Ir(III) complexes.

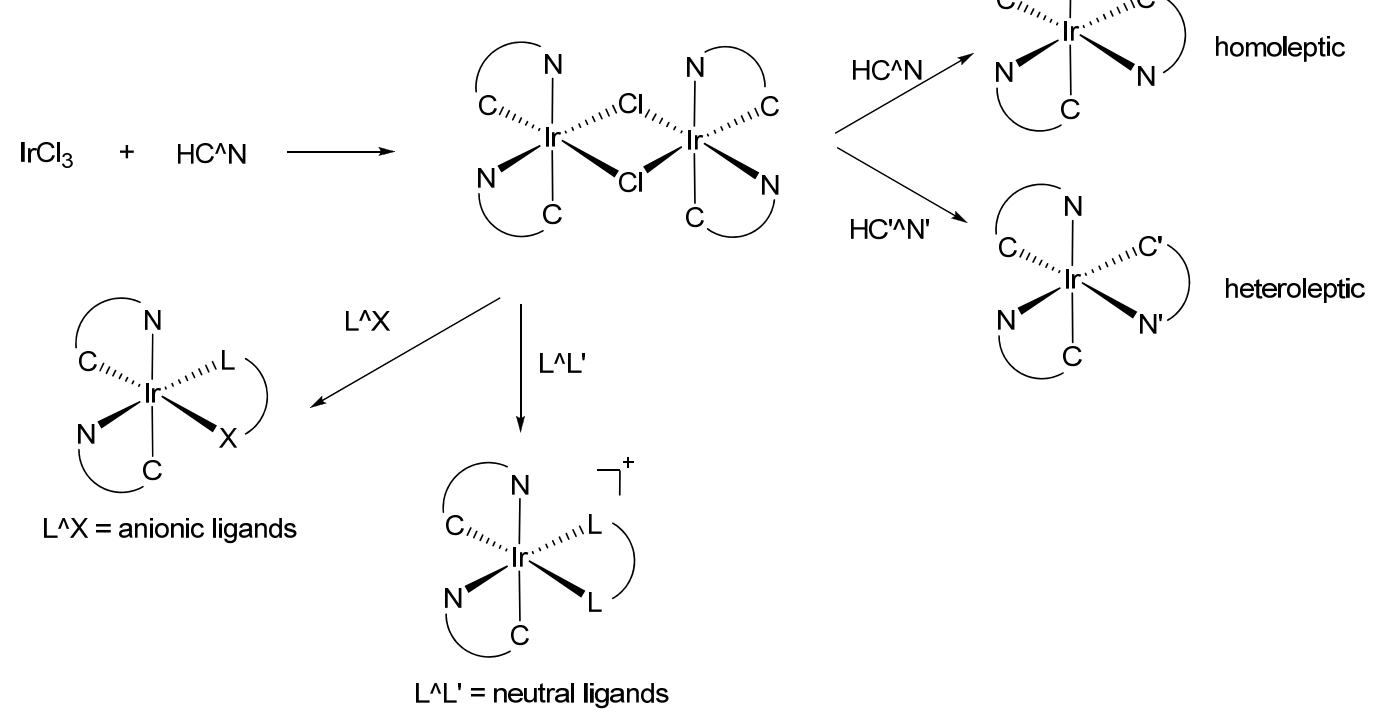

With 4-aryl-1-alkyl-1H-1,2,3-triazoles as cyclometallating ligands $\left(\mathrm{HC}^{\wedge} \mathrm{N}\right)$ into Ir(III) complexes, the triazoles can act as the directing group to facilitate $\mathrm{C}-\mathrm{H}$ activation on the phenyl ring to form cyclometalated N,C chelates. ${ }^{142}$ Based on our previous study for the efficient synthesis of N-2-aryl-1,2,3-triazoles as mentioned in Section 1.2, our efforts turned to the application of these N-2-aryl-1,2,3-triazoles as new cyclometallating ligands in the preparation of iridium(III) complexes. As shown in Figure 29, these are three different potential binding sites $\mathrm{N}(1)^{\wedge} \mathrm{C}\left(6^{\prime \prime}\right), \mathrm{N}(3)^{\wedge} \mathrm{C}\left(2^{\prime \prime}\right)$ and $\mathrm{N}(3)^{\wedge} \mathrm{C}\left(2^{\prime}\right)$ for the formation of iridium(III) complexes. Considering steric effects, the binding site $\mathrm{N}(1)^{\wedge} \mathrm{C}\left(6^{\prime \prime}\right)$ is less steric hindered than the other two sites $\mathrm{N}(3)^{\wedge} \mathrm{C}\left(2^{\prime \prime}\right)$ and $\mathrm{N}(3)^{\wedge} \mathrm{C}\left(2^{\prime}\right)$, we then expect the binding site $\mathrm{N}(1)^{\wedge} \mathrm{C}\left(6^{\prime \prime}\right)$ is more favored to bind with iridium(III) ion. To 
evaluate this hypothesis, the reactions between $\mathrm{IrCl}_{3}$ and 2,4-diphenyl-2H-1,2,3-triazole are carried out (Scheme 57). As we expected, each reaction gave only one single product which was the desired triazole-Ir(III) complexes binding with $\mathrm{N}(1)^{\wedge} \mathrm{C}\left(6^{\prime \prime}\right)$ site in the triazole ligand in excellent yield. These new Ir(III) complexes showed excellent stability towards both air and moisture and could be easily purified by column chromatography.

Figure 29: Three different potential binding sites for forming $\operatorname{Ir}(\mathrm{III})$ complexes in the 1,2,3-triazole ligands.

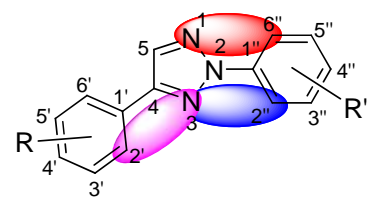

Scheme 57: General method of synthesis for triazole-Ir(III) complexes.

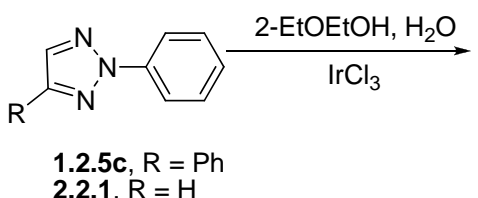

$2.2 .1, R=H$

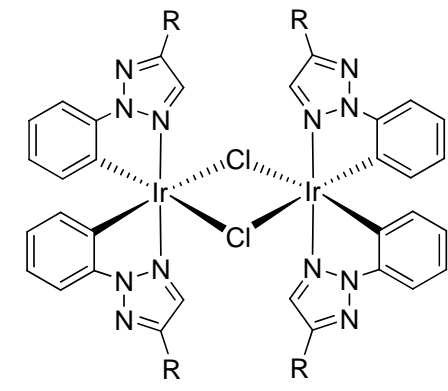

2.2 .2

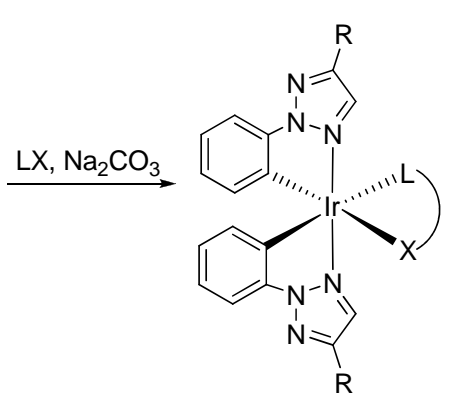

[ $\left.\operatorname{Ir}\left(\mathrm{L}^{\wedge} \mathrm{X}\right)(\text { ArTriaz })_{2}\right]$ 2.2.3 $>91 \%$ yield in 2 steps<smiles>[X][I-]1[I-]N2C=CC=CC2=C1O</smiles>

a<smiles>O=C1NI=C2C=CC=CN12</smiles>

\section{.}


crystallographic analysis, were successfully grown from dichloromethane (DCM) and are shown in Figure 31, Figure 32 and Figure 33 respectively.

Figure 30: Six different $\left[\operatorname{Ir}\left(\mathbf{L}^{\wedge} \mathbf{X}\right)(\text { ArTriaz })_{2}\right]$ complexes.<smiles></smiles>

2.2.3a: $X=O$ 2.2.3b: $\mathrm{X}=\mathrm{NH}$<smiles></smiles>

2.2.3c: $X=O$
2.2.3d: $X=N H$<smiles></smiles>

2.2.3e: $X=O$ 2.2.3f: $\mathrm{X}=\mathrm{NH}$

Figure 31: X-ray crystal structure for triazole bound $\operatorname{Ir}(\mathrm{III})$ complex 2.2.3b.
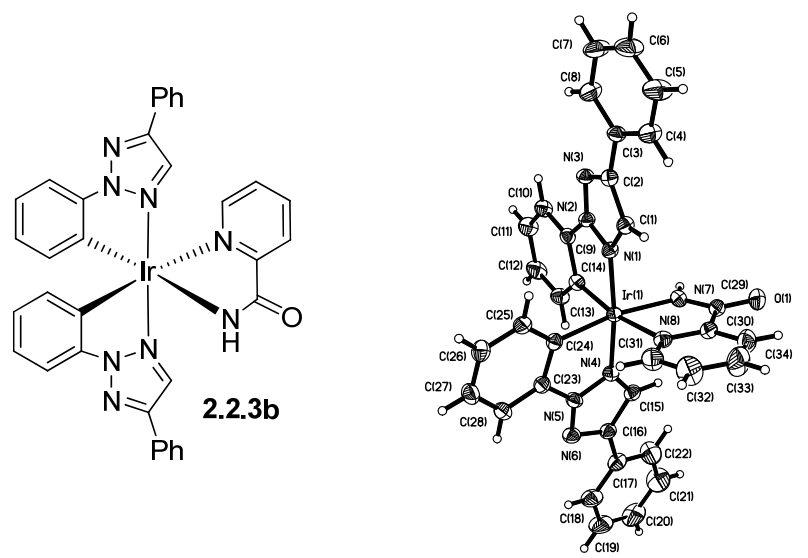

Figure 32: X-ray crystal structure for triazole bound Ir(III) complex 2.2.3c.

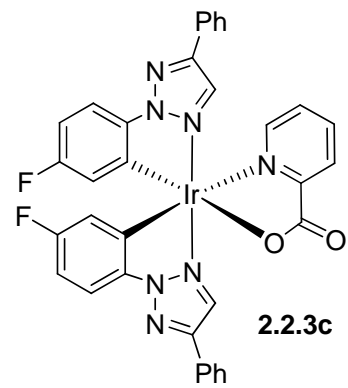

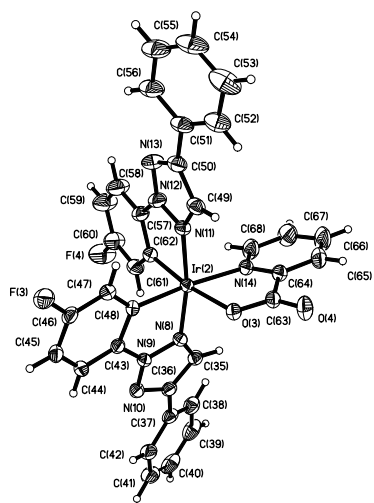


Figure 33: X-ray crystal structure for triazole bound Ir(III) complex 2.2.3d.
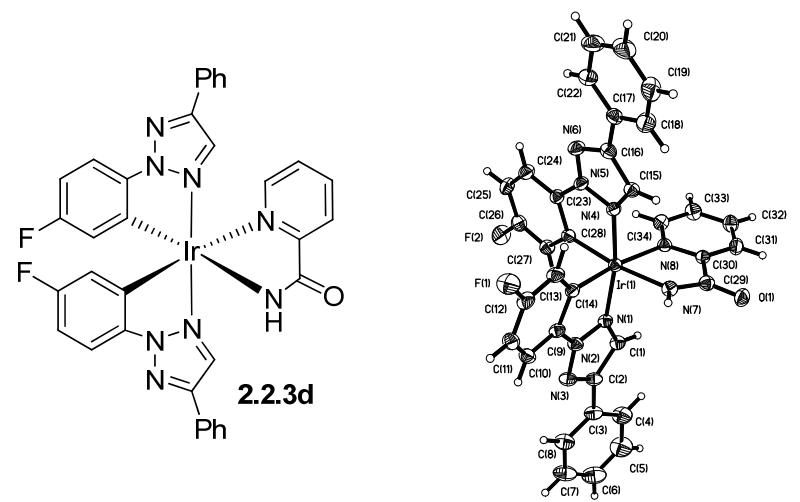

As revealed by the crystal structures, in all three complexes, the phenyl substituent on the C-4 position of the triazole ring are direct away from each other, therefore avoiding the possibility of a $\pi-\pi$ stacking interaction between them (Figure 34).

Figure 34: Conformational stability analysis of triazole-Ir(III) complexes.
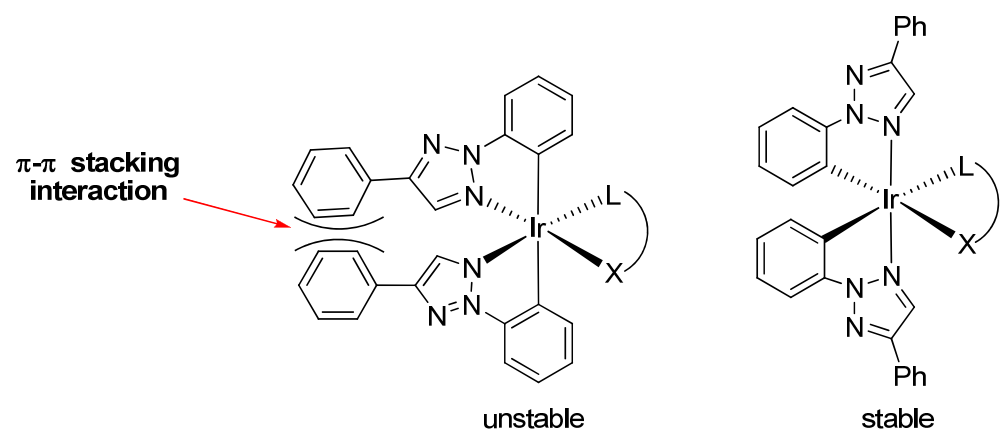

With these triazole-Ir(III) complexes in hand, the UV absorption (Figure 35) and photoluminescence (Figure 36 and Figure 37) have been investigated. From Figure 37, it is clearly noted that the emission wavelength varies from $415 \mathrm{~nm}$ to $527 \mathrm{~nm}$ at excitation $\lambda=369 \mathrm{~nm}$, depending on the ancillary ligand structure, even although their 
UV absorption spectra were almost the same. This phenomenon is consistent to the reported literatures. ${ }^{141 \mathrm{c}}$

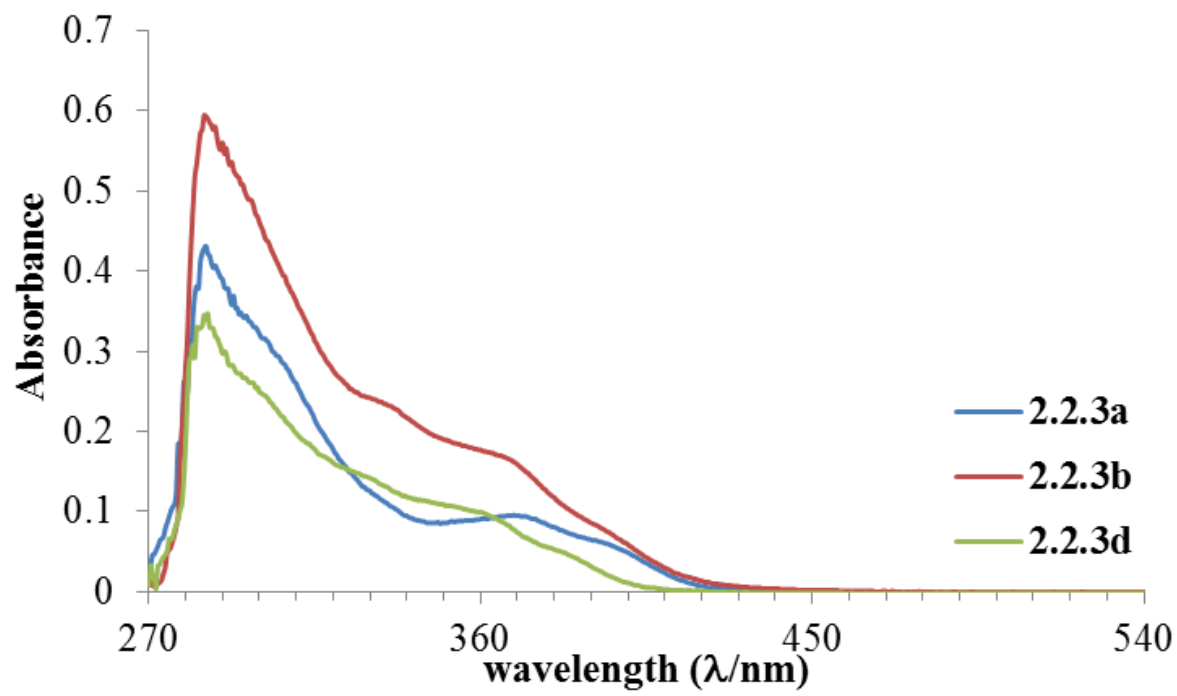

Figure 35: UV absorption spectra of triazole-Ir(III) complexes. Sample preparation: $1.0 \mathrm{x}$ $10^{-5} \mathrm{~mol} / \mathrm{L}$ in $\mathrm{MeOH}$ and measured in $1 \mathrm{~cm}$-length quartz cell).

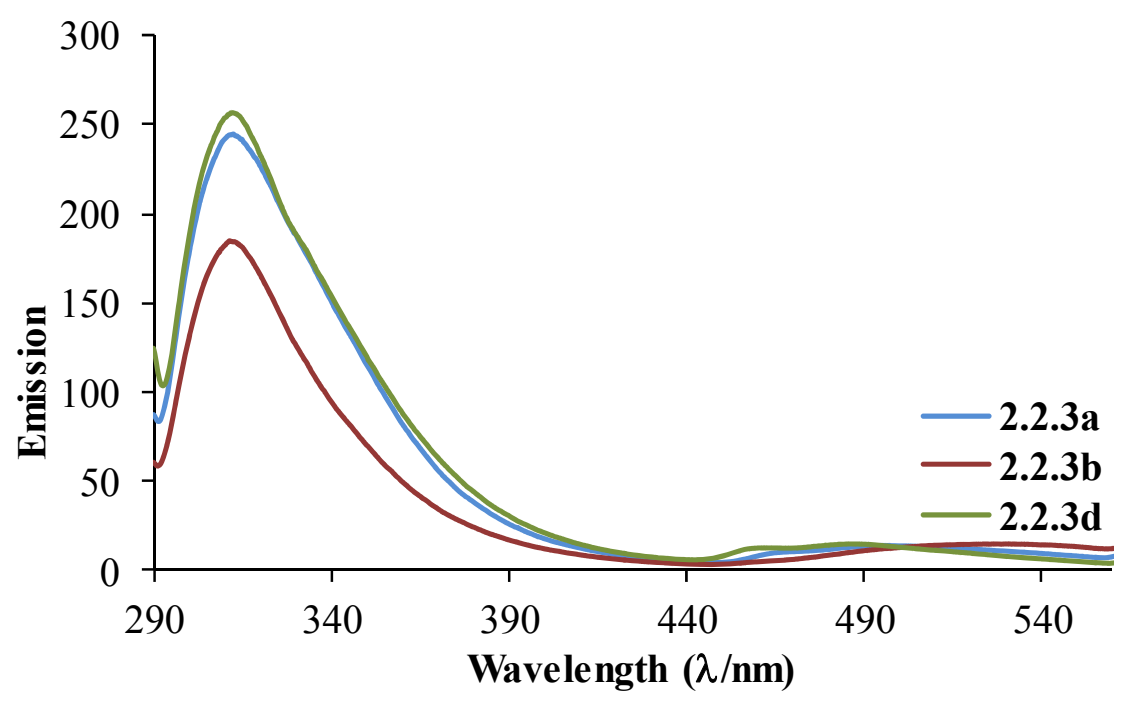

Figure 36: Fluorescence spectra of triazole-Ir(III) complexes. Sample preparation: $1.0 \mathrm{x}$ $10^{-5} \mathrm{~mol} / \mathrm{L}$ in $\mathrm{MeOH}$, excitation at $\lambda=286 \mathrm{~nm}$ with $2.5 \mathrm{~nm}$ slit. 


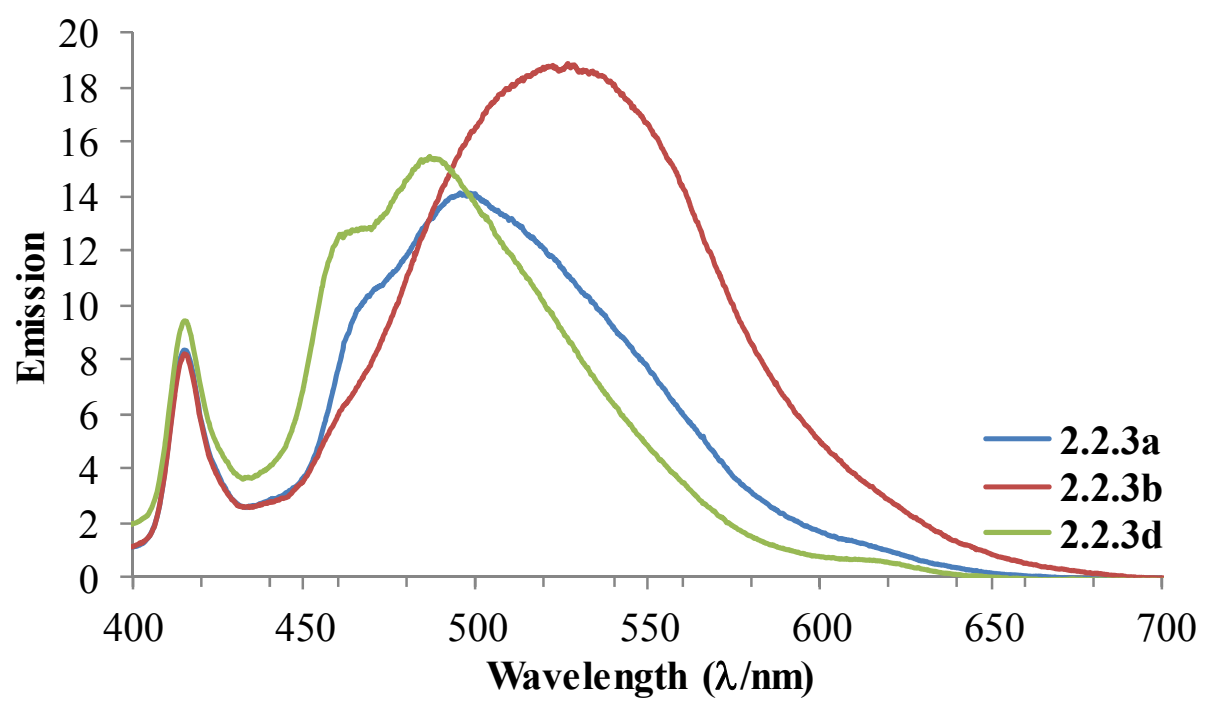

Figure 37: Fluorescence spectra of triazole-Ir(III) complexes. Sample preparation: $1.0 \mathrm{x}$ $10^{-5} \mathrm{~mol} / \mathrm{L}$ in $\mathrm{MeOH}$, excitation at $\lambda=369 \mathrm{~nm}$ with $2.5 \mathrm{~nm}$ slit.

\subsubsection{Evaluation of catalytic reactivity}

During the last decade, visible-light photoredox catalysis has shown great promise as a green and sustainable method. As a result of its high natural abundance, benign environmental impact, cleanliness, and sustainability, ${ }^{143}$ photocatalysis using visible light is a reliable and powerful energy source. ${ }^{144}$ In this context, there are several examples of its usefulness, including asymmetric alkylation of aldehydes, ${ }^{145}[3+2]$ cycloaddition of aryl cyclopropyl ketones, ${ }^{146}[2+2]$ cycloaddition of enones, ${ }^{147}$ reductive dehalogenation, ${ }^{148}$ Oxidation, ${ }^{149}$ radical addition to unsaturated bonds, ${ }^{150}$ and coupling reactions. ${ }^{151}$ Since 2010, Stephenson and co-workers reported the application of visiblelight photoredox catalysis for the formation of $\mathrm{C}-\mathrm{C}$ bonds between tertiary $\mathrm{N}$-arylamines and nitroalkanes via an oxidative aza-Henry reaction. In the presence of $1 \mathrm{~mol} \%$ $\operatorname{Ir}(\text { ppy })_{2}(\mathrm{dtbbpy}) \mathrm{PF}_{6}$, efficient coupling of nitroalkanes with in situ-generated iminium 
ions provides the desired products in up to $96 \%$ yield (Scheme 58). ${ }^{152}$ Mechanistic studies suggest that reductive quenching of the $\mathrm{Ir}^{3+}$ excited state by the tertiary amine leads to the ammonium radical cation, with subsequent catalyst turnover $\left(\operatorname{Ir}^{2+} \rightarrow \mathrm{Ir}^{3+}\right)$ likely affected by atmospheric oxygen (Figure 38).

Scheme 58: An oxidative aza-Henry reaction between tertiary $\mathrm{N}$-arylamines and nitroalkanes.

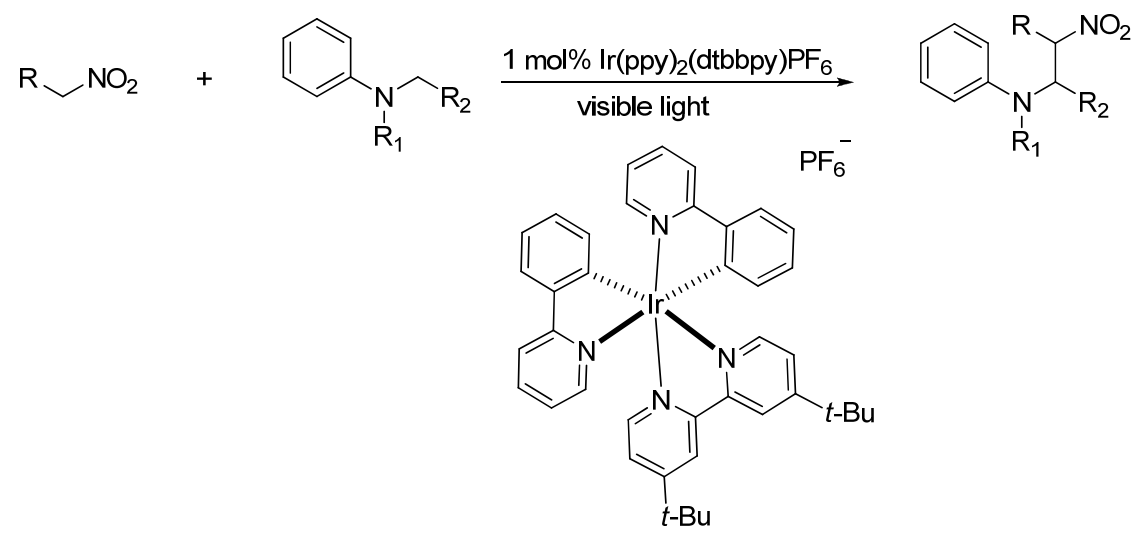

Figure 38: Proposed mechanism for oxidative aza-Henry reaction by Stephenson.

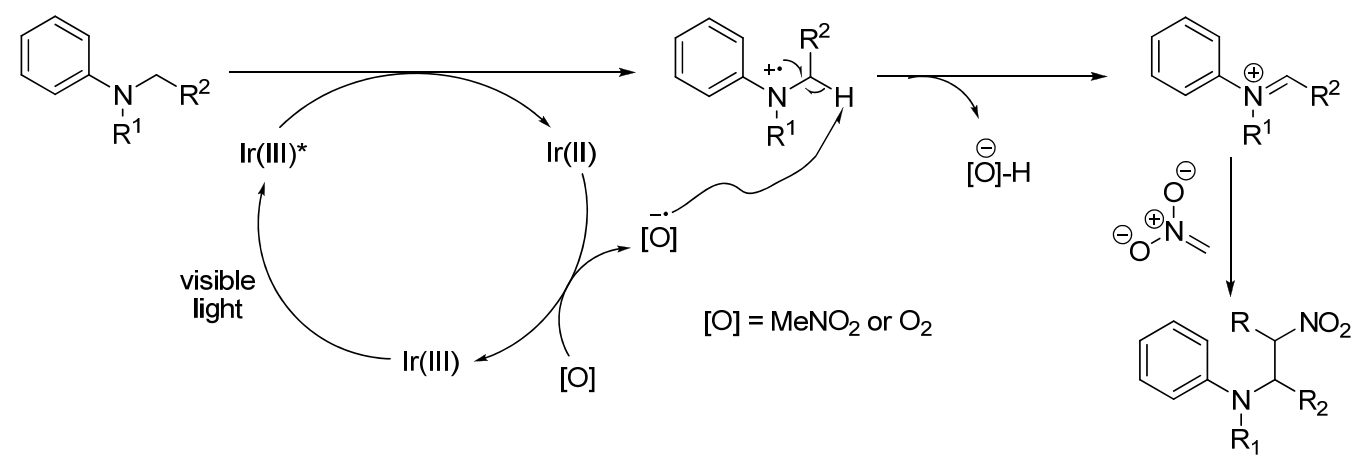

To evaluate the catalytic reactivity of this new class of $\operatorname{Ir}(\mathrm{III})$ complexes, we have synthesized the iridium complex $\left[\operatorname{Ir}(\right.$ bbpy $\left.)(\text { ArTriaz })_{2}\right] \mathrm{Cl}$ (Scheme 59). We then tested its reactivity in aza-Henry reaction of 2-phenyl-1,2,3,4-tetrahydroisoquinoline. 
Scheme 59: Synthesis of complex $\left[\operatorname{Ir}(\right.$ bbpy $\left.)(\text { ArTriaz })_{2}\right] \mathrm{Cl}$.
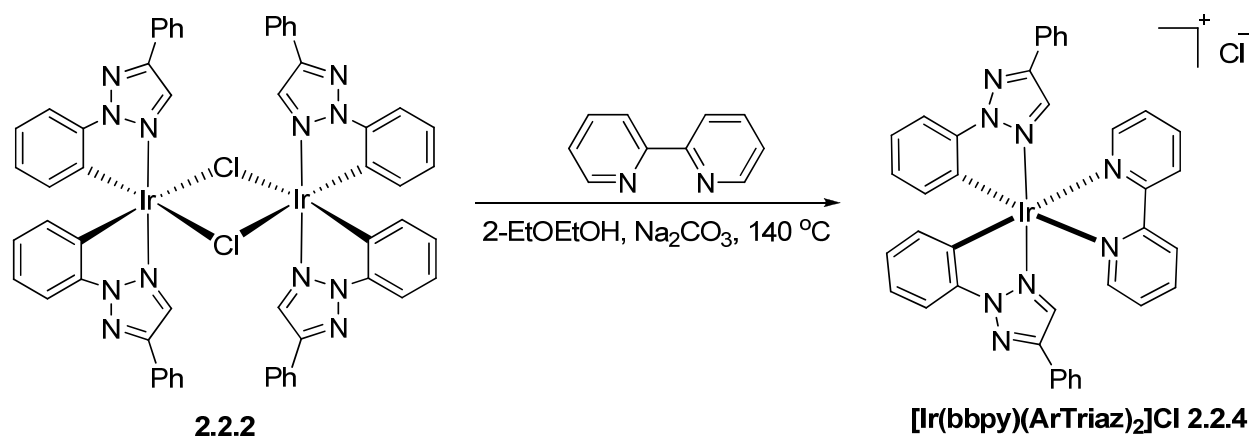

Scheme 60: Aza-Henry reaction of 1,2,3,4-tetrahydroisoquinoline catalyzed by $\left[\operatorname{Ir}(\right.$ bbpy $\left.)(\text { ArTriaz })_{2}\right] \mathrm{Cl}$ complex.

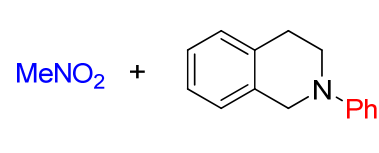

2.2.5a

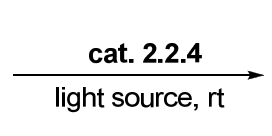

light source, $\mathrm{rt}$<smiles>O=[N+]([O-])CC1c2ccccc2CCN1c1ccccc1</smiles>

2.2.6a
Light source

lamp: $15 \%$

blue LED: $75 \%$

Surprisingly, we found this reaction was wavelength sensitive. As shown in Scheme 60, different light sources gave different efficiency of this transformation. At the same time, we have known $\left[\operatorname{Ir}(\right.$ bbpy $\left.)(\text { ArTriaz })_{2}\right] \mathrm{Cl}$ complexes were the effective catalysts for photoredox catalysis. We then wondered whether the new methodology which combined crossed-conjugate addition and photoredox catalysis could be achieved for the synthesis of new novel nitroalkene derivative. The Lewis base activated crossedconjugate addition of nitroalkene chemistry is well-known in our group. ${ }^{153}$

Our initial attempts were to treat 2-phenyl-1,2,3,4-tetrahydroisoquinoline 2.2.5 and nitroalkenes 2.2.7 in the presence of the Lewis base L-proline. Unfortunately, no products were obtained (Scheme 61). Based on a proposed mechanism as shown in Figure 38, $\mathrm{O}_{2}$ could be the oxidant to turn over the catalytic cycle of Ir catalyst. In our reaction system, 
it failed to generate the iminium ion intermediate which suggests the nitromethane is very critical for the turnover catalytic cycle of iridium catalyst (Figure 39). We then tested the parallel reactions with different reaction solvents (Scheme 62).

Scheme 61: Aza-Henry reaction with nitroalkene activated by Lewis base.

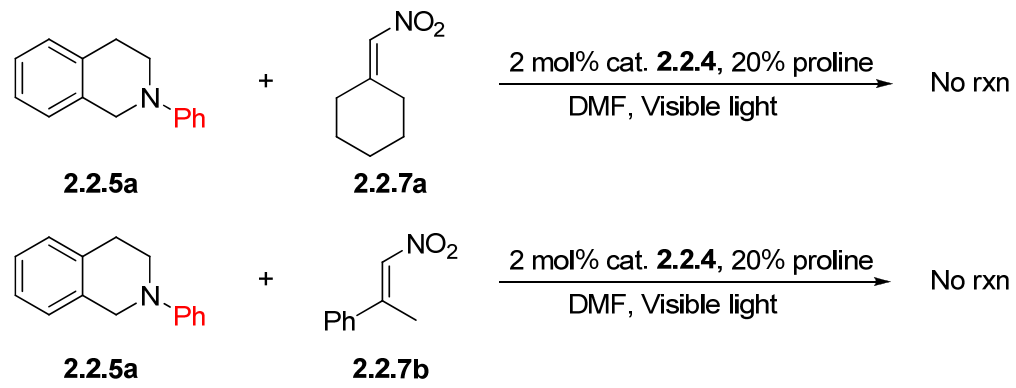

Figure 39: Mechanistic insight of Aza-Henry reaction with nitroalkene.

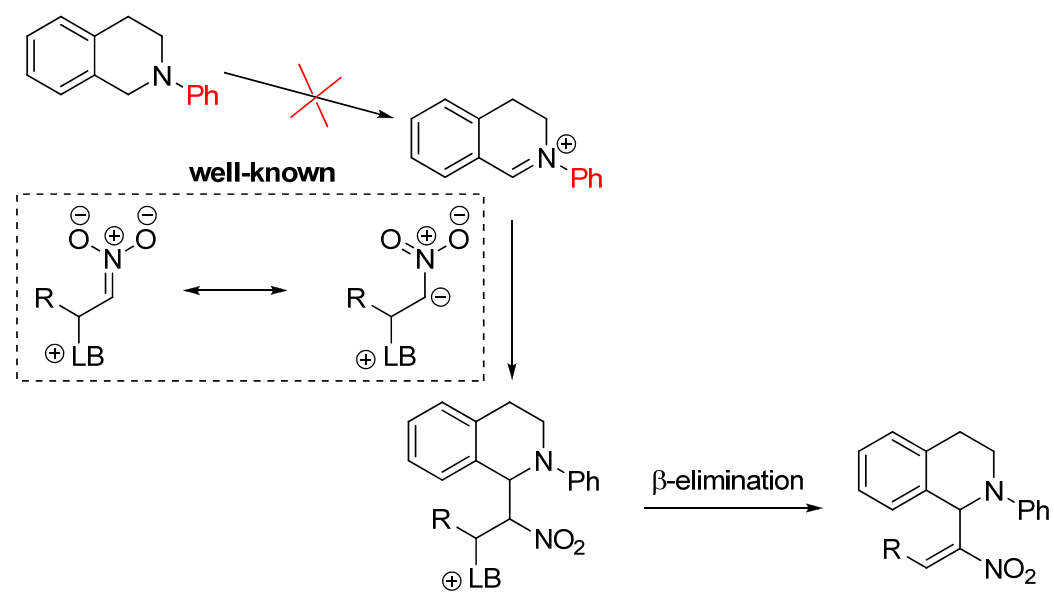


Scheme 62: Solvent factor for reaction system.

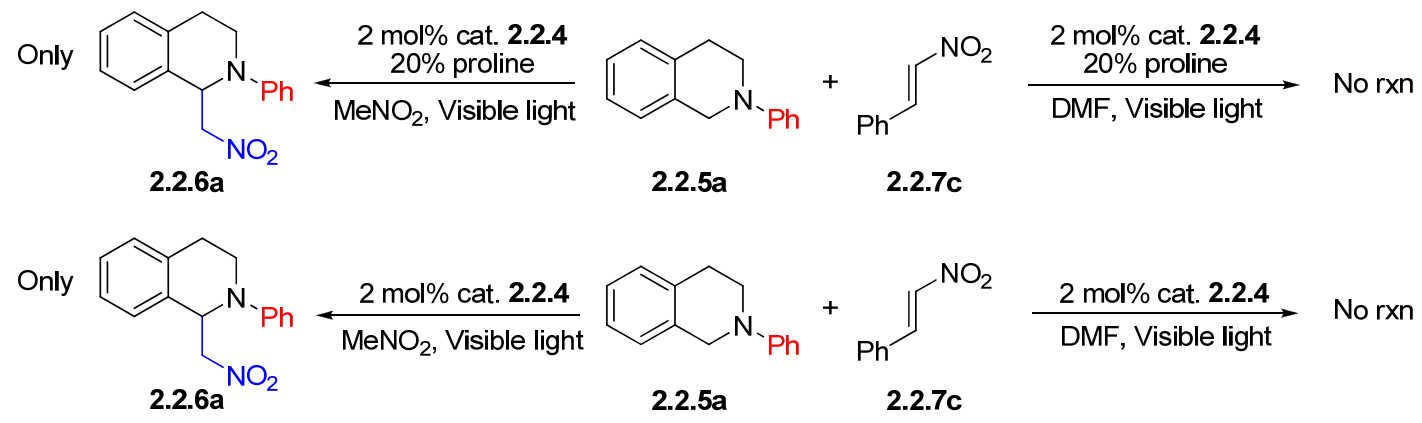

As shown in Scheme 62, the product formed only from nitromethane as the reactant, while the activated nitroalkene was not involved in the reaction. These above results indicated the proper oxidant to make the effective turnover of iridium catalyst is vital for the Aza-Henry reaction with activated nitroalkene. Therefore, we need to put further efforts to investigate the optimal conditions for this new transformation.

\subsubsection{Conclusion}

In conclusion, we have successfully developed the synthesis and structural characterization of N-2-aryl-1,2,3-triazoles as cyclometallating ligands in coordination with $\operatorname{Ir}(\mathrm{III})$ salt to give $\left[\operatorname{Ir}\left(\mathbf{L}^{\wedge} \mathbf{X}\right)(\mathbf{A r T r i a z})_{2}\right]$ and $\left[\operatorname{Ir}(\mathbf{b b p y})(\operatorname{Triaz})_{2}\right] \mathbf{C l}$ complexes in excellent yields. One of the first examples of X-ray structure of N-2-aryl-1,2,3-triazole as the ligands bound with iridium salt is presented. Although the new methodology for azaHenry reaction with activated nitroalkene has yet to be demonstrated, these complexes showed effective catalytic activity in the aza-Henry reaction, indicating the potential of this $\operatorname{Ir}(\mathrm{III})$ triazole complexes as a new class of photoredox catalysts. 


\subsection{1,2,3-Triazole as Unique Ligand in Promoting Iron Catalyzed Propargyl Alcohol Dehydration for the Synthesis of Conjugated Enynes}

\subsubsection{General introduction of Iron chemistry}

The development of efficient, fast, selective, and clean synthetic methods is highly desirable in organic chemistry. With this point, numerous metal-mediated catalysts have been developed and played vital roles in the organic syntheses. Many of them are derived from heavy or rare metals and their toxicity and prohibitive prices constitute severe drawbacks for large-scale applications. As we know, iron is one of the most abundant metals on earth which is closely related to the life of the human being, and consequently one of the most inexpensive and environmentally friendly ones. ${ }^{154}$ Most impotantly, many iron salts and complexes are commercially available, ${ }^{155}$ or are reported in the literature. ${ }^{156}$ Due to its advantages and significance, the development of iron-based chemistry in organic synthesis has led to various iron-catalyzed organic transformations, including nucleophilic additions, ${ }^{157}$ substitutions, ${ }^{158}$ reductions, ${ }^{159}$ oxidations, ${ }^{160}$ hydrogenations, ${ }^{161}$ cycloadditions, ${ }^{162}$ isomerizations, ${ }^{163}$ rearrangements, ${ }^{164}$ and polymerizations. ${ }^{165}$

In the last decade, Fürstner's group explored several organoiron catalyzed cross coupling reactions with Grignard reagents (Scheme 63). ${ }^{166}$ They showed that iron salts allow various types of electrophiles, such as aryl chlorides, aryl tosylates, alkyl halides, alkynyl epoxides, enol triflates and phosphates, acid chlorides and thiolesters, to be activated under mild conditions. Such iron-catalyzed processes occur very rapidly even at low temperature and therefore are distinguished by broad functional group compatibility. Furthermore, recent advances in carbon-heteroatom bond formation and studies relevant 
to the general reactivity of in situ generated and structurally defined "low-valent" iron complexes which have been confirmed by X-ray crystallography are presented (Scheme 64). ${ }^{167}$ These complexes revealed the excellent catalytic reactivity for a variety of Alderene, [4+2], [5+2], and [2+2+2] cycloadditon and cycloisomerization reactions of polyunsaturated substrates which tolerate esters, ketones, sulfonamides, acetals, ethers, cyclopropanes, remote alkenes, amines and aryl halides (Scheme 65).

Scheme 63: Iron-catalyzed cross coupling reaction between organic halide/pseudohalide and Grignard reagents.

$$
\begin{aligned}
& \mathrm{R}-\mathrm{X}+\mathrm{R}+\mathrm{MgBr} \stackrel{[\mathrm{Fe}] \text { cat. }}{\longrightarrow} \\
& \\
& {[\mathrm{Fe}] \text { cat. }=\mathrm{FeCl}_{3}, \mathrm{Fe}(\mathrm{acac})_{3} \text { etc. } } \\
& \mathrm{R}=\text { acyl-, alkenyl-, aryl-, alkyl } \\
& \mathrm{X}=\text { halide, tosylate, epoxide, triflate, phosphate } \\
& \mathrm{R}^{\prime}=\text { aryl, alkyl }
\end{aligned}
$$

Scheme 64: Different "low-valent" iron complexes.

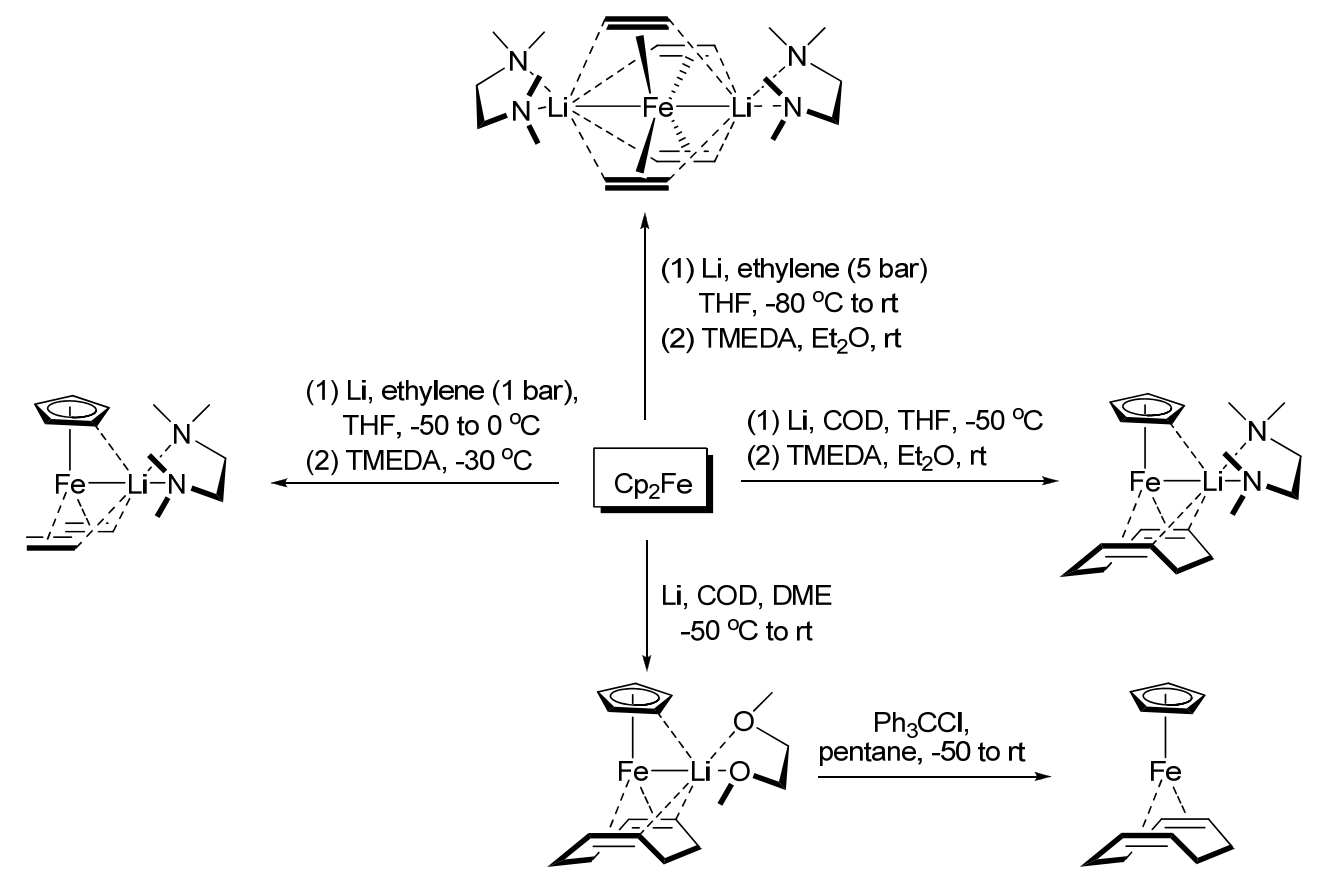


Scheme 65: One "low-valent" iron complex as the catalyst in a variety of transformations.

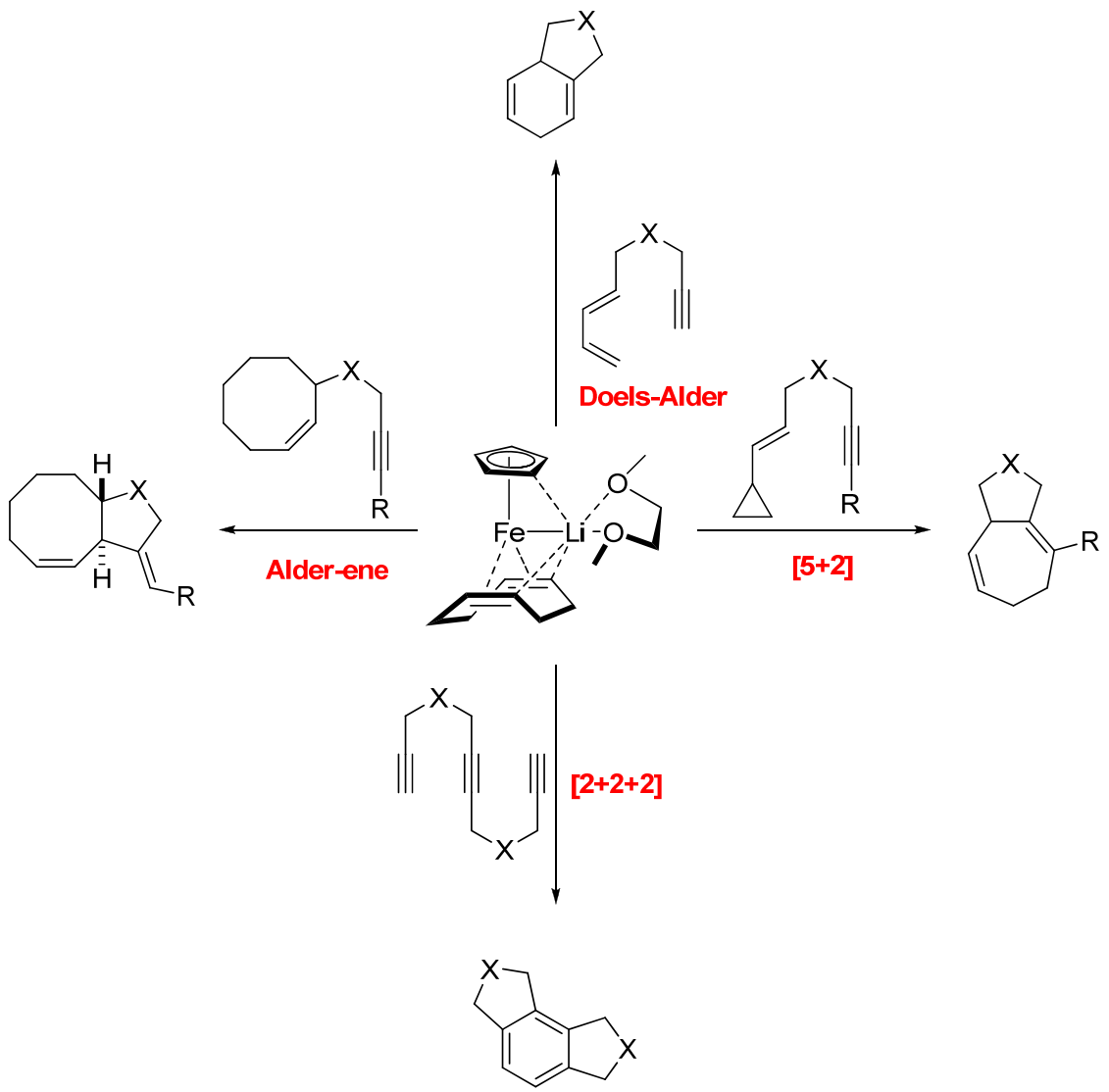

The $\mathrm{C}-\mathrm{H}$ activation has gained many attentions from organic chemists, because it meets not only the efficient and sustainable requirement of organic transformations but also the understanding of the intrinsic features of the broadly existing $\mathrm{C}-\mathrm{H}$ bonds in organic molecules. ${ }^{168}$ Iron-containing enzyme-catalyzed C-H activation has been reported and suggested a potential application of iron catalysis in $\mathrm{C}-\mathrm{H}$ activation. ${ }^{169}$ Combining the advantages of both iron chemistry and $\mathrm{C}-\mathrm{H}$ activation, the significance of ironcatalyzed C-H activation has stimulated rapid development in the past several years. ${ }^{170}$

The $\mathrm{C}-\mathrm{H}$ bond oxidation is one of the most common processes in $\mathrm{C}-\mathrm{H}$ activation. In the synthetic field, iron-catalyzed systems for $\mathrm{C}-\mathrm{H}$ oxidation can be traced back to one 
century ago, and this was summarized as Gif chemistry ${ }^{171}$ and Fenton chemistry, ${ }^{172}$ as well as other non-heme mimic systems. ${ }^{173}$ Recently, iron-catalyzed oxidation was mostly focused on sulfide oxidations, ${ }^{174}$ epoxidation, ${ }^{175}$ and olefin dihydroxylation. ${ }^{176}$ In contrast, iron catalyzed $\mathrm{C}-\mathrm{H}$ bond oxidation has had few significant breakthroughs for a rather long time, until recent years.

In 2007, White and co-worker made a significant improvement in the oxidation of general $\mathrm{sp}^{3} \mathrm{C}-\mathrm{H}$ bonds. ${ }^{177}$ They reported the iron catalyzed aliphatic $\mathrm{C}-\mathrm{H}$ oxidation by using $\left[\mathrm{Fe}(\mathrm{PDP})\left(\mathrm{SbF}_{6}\right)_{2}\right]$ complex. In this report, a highly selective oxidation of nonactivated sp3 C-H bonds via iron catalysis was well investigated (Scheme 66). Iron (PDP) complex [PDP = 2-(\{(S)-2-[(S)-1-(pyridin-2-ylmethyl)pyrrolidin-2-yl]pyrrolidin1-yl \}methyl)pyridine] was essential for this transformation and the higher rigid PDP ligand led to the increase of selectivity. This process was rather clean, since the oxidant was hydrogen peroxide, which was cheap, easily available, and frequently used in traditional iron-catalyzed Gif ${ }^{171}$ and Fenton ${ }^{172}$ chemistry. In this transformation, the electronic feature rather than the steric effect of $\mathrm{C}-\mathrm{H}$ bonds plays an important role, since electron rich tertiary $\mathrm{C}-\mathrm{H}$ bonds showed a higher reactivity than either secondary or primary $\mathrm{C}-\mathrm{H}$ bonds. An electron-withdrawing group (EWG) on the $\alpha$ or $\beta$ carbon of the C-H bond quenched the reactivity (Figure 40).

Scheme 66: Iron-catalyzed oxidation of unactivated $\mathrm{sp}^{3} \mathrm{C}-\mathrm{H}$ Bonds.
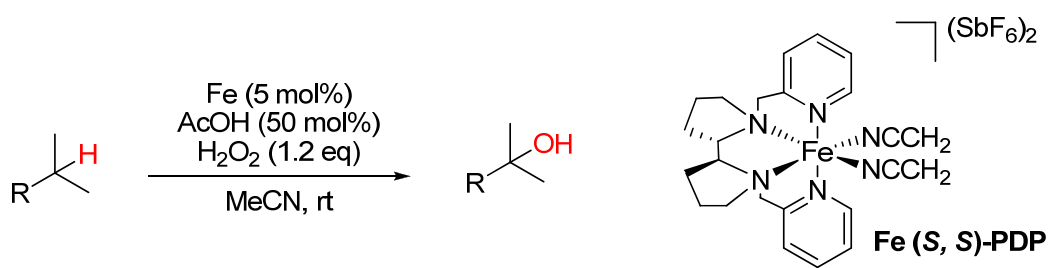
Figure 40: Reactivity of different $\mathrm{C}-\mathrm{H}$ bonds.

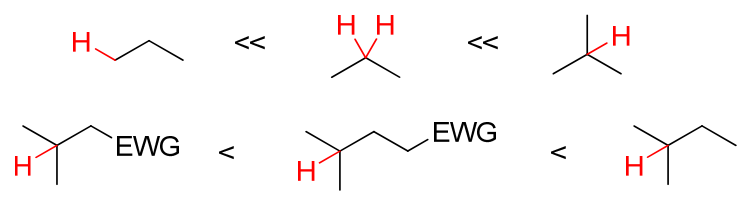

Considering the mechanistic insight among iron catalyzed transformations, the iron species generally could either serve as Lewis acid or as redox center through single electron transfer (SET) process. For example, various iron-containing domains of the enzymes showed their high catalytic abilities in $\mathrm{C}-\mathrm{H}$ oxidations, in which the SET process was considered as a possible pathway to facilitate the $\mathrm{C}-\mathrm{H}$ oxidation. ${ }^{178}$

\subsubsection{Research Objective and Results}

Conjugated enynes are fundamental building blocks in biology, ${ }^{179}$ material science ${ }^{180}$ and fine chemical synthesis. ${ }^{181}$ As a result, the synthesis of enynes has received considerable attention during recent decades, and new methods for their preparation have been developed. Among these various methods, the metal-catalyzed dimerization of terminal alkynes was a straightforward approach (Scheme 67A, R=R", R'=H). ${ }^{182}$ Unfortunately, the complicated regio- and stereoselectivity of the dimerization process significantly limited the wide application of this method. To minimize this drawback, the solution was the coupling of alkynes with structurally defined organometallic alkenes (Scheme 67B) ${ }^{183}$ However, organometallic alkenes are usually difficult to handle and are very toxic in general, especially those that are volatile. 
Scheme 67: Classical approaches for synthesizing conjugated enynes.

A)

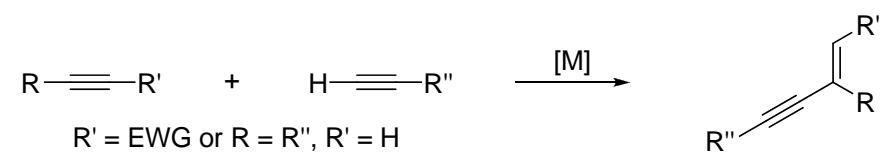

B)

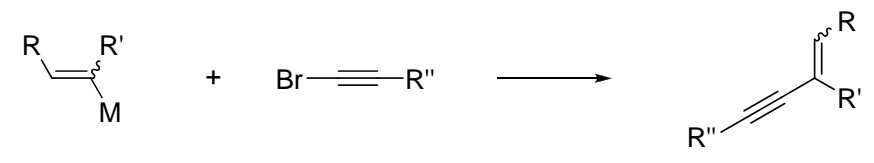

C)

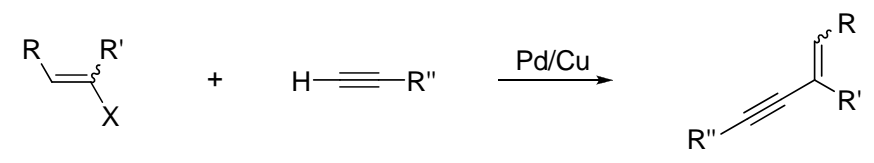

The most employed protocol for the synthesis of conjugated enynes has been the cross-coupling reactions between vinyl halides and terminal acetylenes catalyzed by palladium complexes and copper(I) iodide, namely, the Sonogashira reaction (Scheme 67C). ${ }^{184}$ This elegant process does not require the stoichiometric amount of an organometallic reagent. However, the major limitation of this method was the preparation of the vinyl halide. In view of the limitations and shortcomings of these established methods, we believed that the simple and efficient methods that can install this unsaturated hydrocarbon moiety are highly desirable. ${ }^{185}$

The catalytic propargyl alcohol dehydration, without any doubt, is one of most atom-economic and practical approaches for conjugated enyne synthesis. Although some methods related to propargyl alcohol dehydration strategy have been reported (Scheme 68), such as the phosphoric esters syn-elimination ${ }^{186}$ and cyclopropane ring opening, ${ }^{187}$ these methods only work for limited specific substrates. Therefore, this simple transformation is challenging and problematic. 
Scheme 68: The dehydration propargyl alcohol for specific substrates in synthesis of conjugated enynes.

A) Syclopropane ring opening strategy

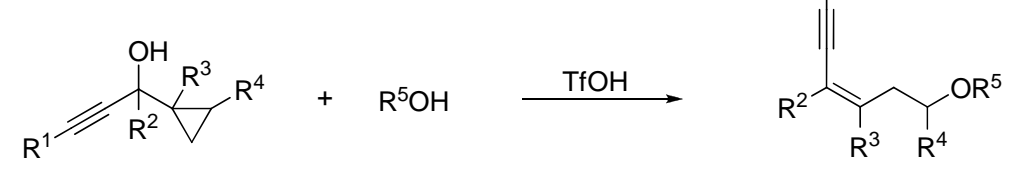

B) Phosphoric esters syn-elimination

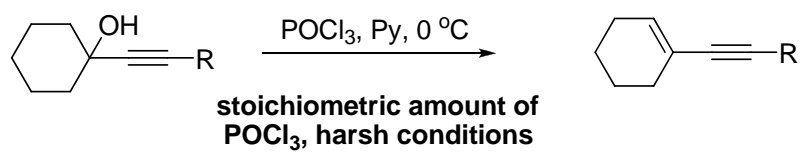

C) A general approach

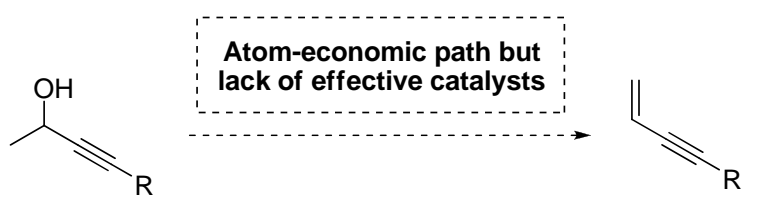

To achieve alcohol elimination, a Lewis acid was generally required (for $\mathrm{OH}$ activation). However, the carbocation intermediate and conjugated enynes products are highly reactive under these conditions. This often leads to undesired decomposition and polymerization. As illustrated in Table 13, the commonly used Lewis acids all gave poor yields of enyne 2.3.2a, even though most of the propargyl alcohol 2.3.1a was consumed. Therefore, to fill this "missing methodology", new catalysts need to be developed either by providing proper acidicity (only activating the C-O bond without formation of "pure" carbocation) or by going through alternative reaction path (such as radical) to avoid the undesired decomposition. The recent studies on triazole-Au complex revealed excellent chemoselectivity of this triazole catalyst and the triazole-borane complexes suggested the ability of triazole to stabilize a single electron reaction center. ${ }^{188}$ Additionally, in the 
synthesis of triazole-allenes (Section 1.5), the higher yield and improved regioselectivity was obtained when we pre-mixed the $\mathrm{FeCl}_{3}$ and triazole before the addition of propargyl alcohol (Table 14). On the basis of these results, we postulated that triazole ligands might provide the needed reactivity for this simple but important transformation.

Table 13: Direct propargyl alcohol dehydration: a challenging transformation. ${ }^{a}$

\begin{tabular}{|c|c|c|c|c|}
\hline & 2.3.1a & & & \\
\hline Entry & Catalyst & $\operatorname{Temp}\left({ }^{\circ} \mathrm{C}\right)$ & Convn $(\%)^{b}$ & Yield $(\%)^{c}$ \\
\hline 1 & $\mathrm{TfOH}$ & 60 & 90 & 30 \\
\hline 2 & $\mathrm{AlCl}_{3}$ & 60 & 10 & $<5$ \\
\hline 3 & $\mathrm{Bi}(\mathrm{OTf})_{3}$ & 60 & 90 & 21 \\
\hline 4 & $\mathrm{NiCl}_{3}$ & 60 & 22 & $<5$ \\
\hline 5 & $\operatorname{In}(\mathrm{OTf})_{3}$ & 60 & 80 & 15 \\
\hline 6 & $\mathrm{CeCl}_{3}$ & 60 & 23 & $<5$ \\
\hline 7 & $\mathrm{LaCl}_{3}$ & 60 & 26 & $<5$ \\
\hline 8 & $\mathrm{RuCl}_{3}$ & 60 & 78 & 22 \\
\hline 9 & $\mathrm{IrCl}_{3}$ & 60 & 75 & 20 \\
\hline 10 & $\mathrm{PdCl}_{2}$ & 60 & 30 & $<5$ \\
\hline 11 & $\mathrm{Cu}(\mathrm{OAc})_{2}$ & 60 & 52 & $<5$ \\
\hline 12 & $\mathrm{Co}(\mathrm{OAc})_{3}$ & 60 & 26 & $<5$ \\
\hline 13 & $\mathrm{Ti}(\mathrm{Oi}-\mathrm{Pr})_{4}$ & 60 & 71 & 9 \\
\hline 14 & $\mathrm{FeCl}_{2}$ & 60 & 30 & $<5$ \\
\hline 15 & $\mathrm{FeCl}_{3}$ & 60 & 95 & 29 \\
\hline 16 & $\mathrm{Fe}(\mathrm{acac})_{3}$ & 60 & 59 & 12 \\
\hline 17 & $\mathrm{FeCl}_{3}$ & $\mathrm{rt}$ & 32 & 11 \\
\hline
\end{tabular}


${ }^{a}$ General reaction conditions: 2.3.1a $(0.25 \mathrm{mmol}, 1.0$ equiv.) and Lewis acid catalyst $(10 \mathrm{~mol} \%)$ in $\mathrm{MeCN}$ $(5 \mathrm{~mL}) ;{ }^{b}$ Conversions were determined based on the consumption of propargyl alcohol; ${ }^{c}$ NMR yields of 2.3.2a with $1,3,5$-trimethoxybenzene as internal standard.

Table 14: A small detail from allenation of triazole.

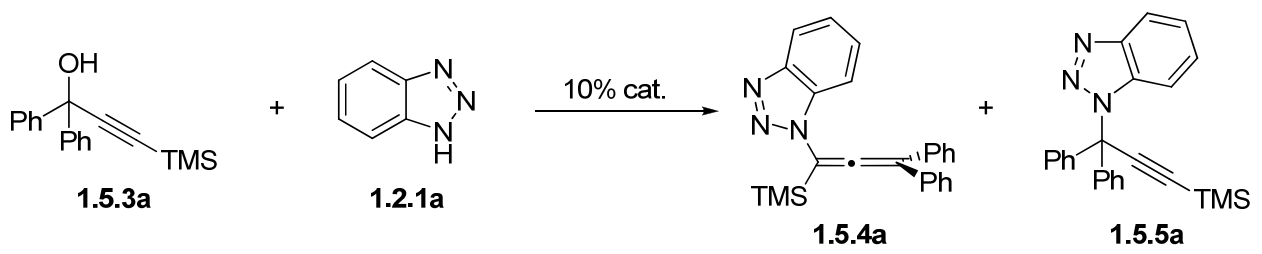

\begin{tabular}{|c|c|c|c|c|c|c|c|}
\hline Catalyst & $\begin{array}{c}\text { Loading } \\
(\%)\end{array}$ & Sol. $^{b}$ & $\begin{array}{c}\text { Temp } \\
\left({ }^{\circ} \mathrm{C}\right)\end{array}$ & $\begin{array}{c}\text { Time } \\
\text { (h) }\end{array}$ & $\begin{array}{c}\text { Conv. } \\
(\%)^{c}\end{array}$ & $\begin{array}{l}\text { Yield } \\
(\%)^{d} \\
\end{array}$ & 1.5.4a:1.5.5 $\mathbf{a}^{d}$ \\
\hline $\begin{array}{c}\mathrm{FeCl}_{3} \\
\text { (w/o pre-coord.) }\end{array}$ & 10 & $\mathrm{MeCN}$ & 60 & 5 & 86 & 57 & $3: 1$ \\
\hline $\mathrm{LiCl}$ & 10 & $\mathrm{MeCN}$ & 60 & 5 & 90 & 70 & $6: 1$ \\
\hline
\end{tabular}

Table 15: Screening of ligands ${ }^{a, b, c}$

$$
\underset{\mathrm{Ph}}{\stackrel{\mathrm{HO}}{=} \mathrm{Bu}} \frac{10 \mathrm{~mol} \% \mathrm{FeCl}_{3}, \mathrm{MeCN}}{20 \mathrm{~mol} \% \mathrm{~L}, 60 \mathrm{oC}, 7 \mathrm{~h}} \underset{\mathrm{Ph}}{\mathrm{2.3.2a}}
$$

L:<smiles>Cn1nnc2ccccc21</smiles>

L1

conv. $63 \%$ yield $43 \%$<smiles>NC(=O)Cn1cc(C(c2ccccc2)n2nnc3ccccc32)nn1</smiles>

L5

conv. $80 \%$

yield $61 \%$<smiles>Oc1ccccc1-c1cn(COP)nn1</smiles>

L8

conv. $73 \%$ yield $41 \%$<smiles>c1ccc(-c2cnn(-c3ccccn3)n2)cc1</smiles>

L2 conv. $49 \%$ yield $10 \%$<smiles>c1ccc(-c2cnn(-c3cccc(-n4ncc(-c5ccccc5)n4)c3)n2)cc1</smiles>

L6

conv. $79 \%$ yield $29 \%$<smiles>CC(C)(O)c1cn(CO[Na])nn1</smiles>

L9

conv. $75 \%$ yield $31 \%$
$\mathrm{Ph}$<smiles>Cc1cn(-c2ccccn2)nn1</smiles>

L3 conv. $78 \%$ yield 33\%<smiles>c1ccc(-c2ccccn2)nc1</smiles>

L4 conv. $31 \%$ yield $<5 \%$

${ }^{a}$ General reaction conditions: $\mathbf{2 . 3 . 1 a}(0.25 \mathrm{mmol}, 1.0$ equiv. $)$, ligands (10 $\left.\mathrm{mol} \%\right)$ and Lewis acid catalyst $(10 \mathrm{~mol} \%)$ in $\mathrm{MeCN}(5 \mathrm{~mL}){ }^{b}$ Conversions were determined 
based on the consumption of propargyl alcohol; ${ }^{c}$ NMR yields of 2.3.2a with 1,3,5trimethoxybenzene as internal standard.

To test our hypothesis, various triazole- $\mathrm{FeCl}_{3}$ mixtures were employed as the catalysts to react with $2.3 .1 a$. To ensure the consistency, all reactions were conducted under identical conditions and the reaction mixtures were measured after 7 hours (Table 15). To our surprise, the simple addition of $\mathrm{N}$-methyl benzotriazole $\mathbf{L 1}$ to $\mathrm{FeCl}_{3}$ significantly decreased undesired decomposition of 2.3.1a, giving the desired enyne 2.3.2a in good yields. Screening the triazole ligands revealed even better reactivity of chelating ligands, giving enyne in excellent yields. On the basis of synthetic simplicity of triazole ligands, we have selected $2-(1 H$-benzo[d][1,2,3]triazol-1-yl)ethanol L10 as the optimal ligand for this transformation.

Table 16: Screening of different heteroaromatic ligands ${ }^{a, b, c}$

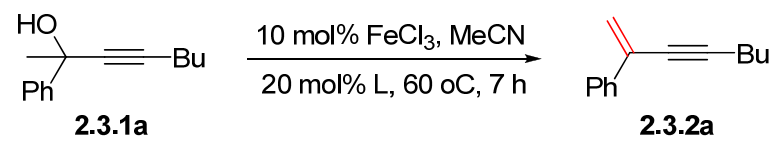

(1)
yield $<5 \%$


Furthermore, we have investigated other heteroaromatic ligands, including pyridine, imidazole, tetrazole and 1,2,4-triazoles. Interestingly, they did not catalyze this transformation at all, even with very similar binding patterns, which demonstrated the unique properties of 1,2,3-triazoles as ligands in adjusting iron cation reactivity (Table 16).

Table 17: Reaction substrate scope. ${ }^{a, b}$

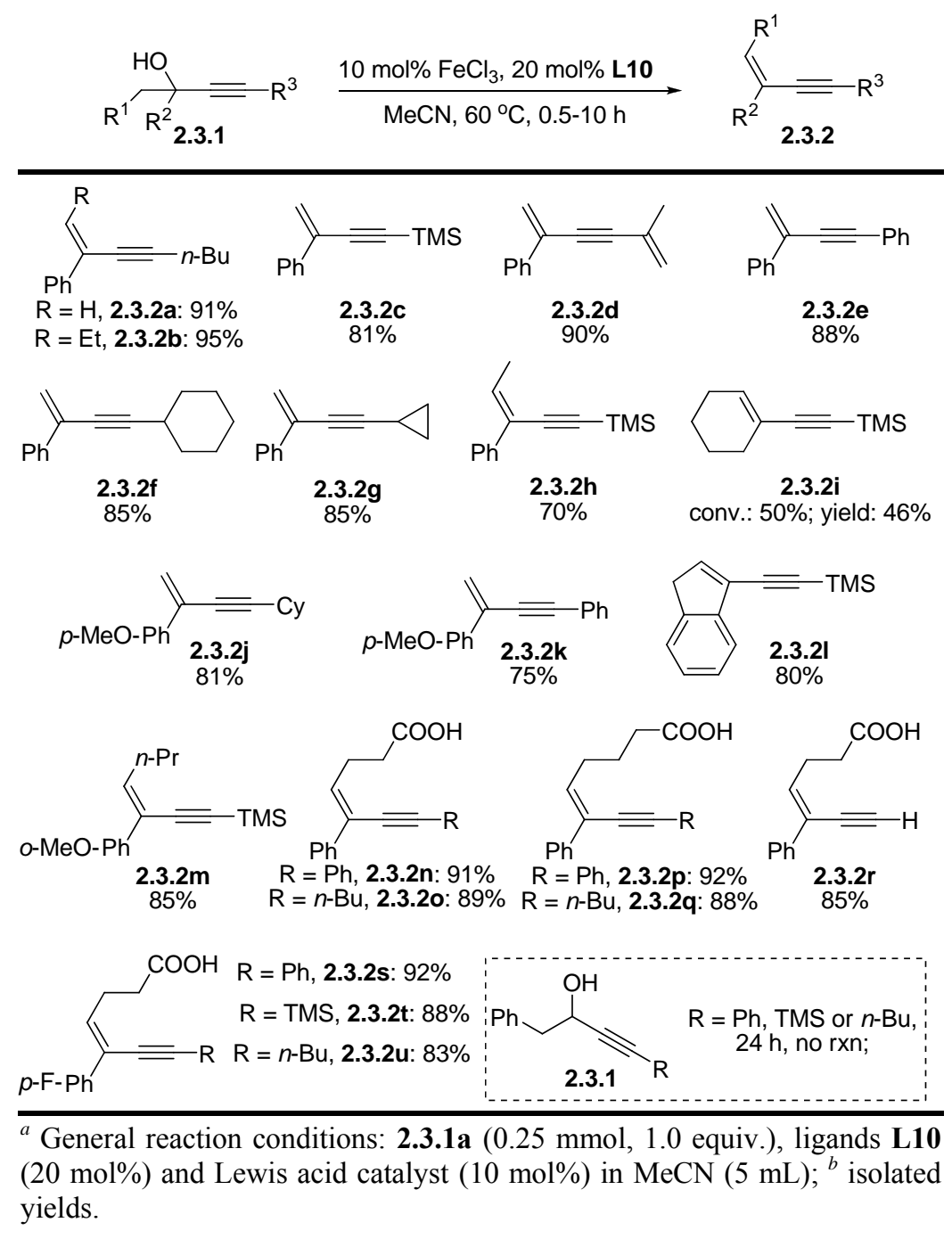

The bi-dentate triazole-based ligands displayed better reactivity. Increasing the ligand loading revealed 2:1 (ligand/Fe) ratio as the optimal conditions, which was 
consistent with the $\mathrm{Fe}^{3+}$ octahedral coordination (six coordination sites, requiring open coordination sites for $\mathrm{OH}$ binding in the transition state). Solvent screening revealed acetonitrile as the optimal solvent. Attempts in obtaining the iron-triazole complex crystal structure have been unsuccessful at this moment. Nevertheless, addition of readily available 1,2,3-triazole to $\mathrm{FeCl}_{3}$ provided a practical and highly efficient catalyst system, which gave the critical chemoselectivity for the C-O bond activation. To the best of our knowledge, this is the first catalytic system reported that can effectively promote this important transformation. ${ }^{189}$ Various functional propargyl alcohols were prepared to investigate the reaction substrate scope. The results are summarized in Table 17.

As indicated in Table 17, both terminal alkyne (2.3.2r) and internal alkyne were suitable for this reaction, giving the desired enynes in excellent isolated yields. The alkyne terminal can tolerate various substituted groups, including alkyl (2.3.2a, 2.3.2b), aryl (2.3.2e), TMS (2.3.2c), cyclopropanyl (2.3.2g) and even vinyl groups (2.3.2d). The propargyl position tolerates an aromatic ring with either electron donating $(\mathbf{2 . 3 . 2 j}, \mathbf{2 . 3 . 2 k})$ or electron withdrawing (2.3.2s to $2.3 .2 \mathrm{u})$ groups. Elimination to the alkenes showed excellent stereoselectivity, with only one double bond isomer observed, which were unambiguously characterized by X-ray crystal structure (2.3.2n). Substrates $2.3 .2 n$ 2.3.2u illustrated the good functional group tolerability of this method. The formation cyclohexenyl enyne 2.3.2i indicated slower reaction rate caused by the lower reactivity of the C-O bond. However, the starting material propargyl alcohol could be fully recovered, indicating the excellent chemoselectivity of the 1,2,3-triazole/Fe catalysts. Enynes with 1,2-disubsitututed alkenes could not be formed because of the low reactivity of C-O bond under the reaction conditions. Raising the reaction temperature caused decomposition of 
the propargyl alcohols. However, the highly reactive 1,4-enediynes could be easily synthesized with this method (Table 18), which highlighted the benefits of the milder reaction condition and improved chemoselectivity of this catalyst.

Table 18: Synthesis of 1,4-endiynes ${ }^{a, b}$

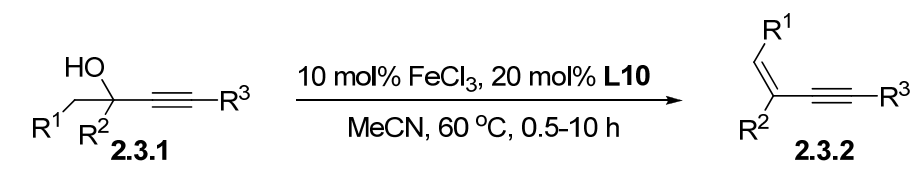

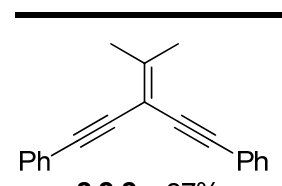

2.3.2v: $87 \%$

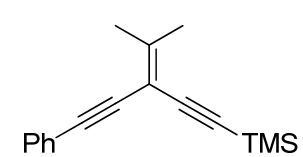

2.3.2w: $90 \%$
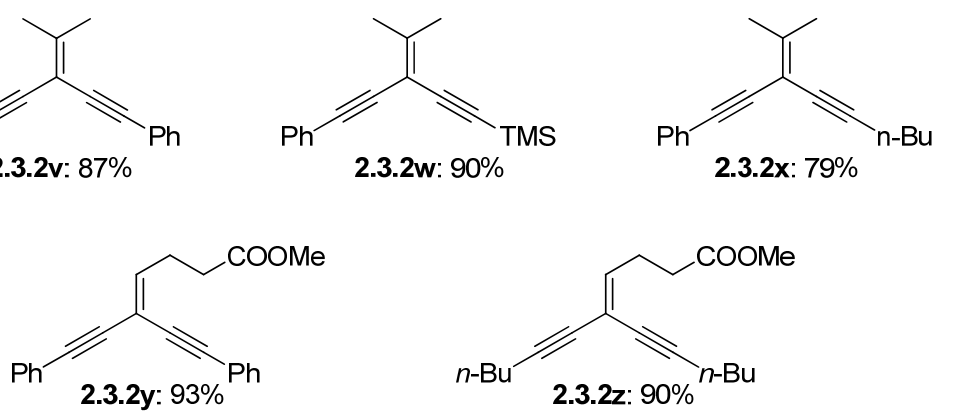

${ }^{a}$ General reaction conditions: identical as above; ${ }^{b}$ Isolated yields.

\subsubsection{Tuning the reactivity of conjugated enynes in 1,4-bromolactonization.}

Halogen-promoted addition of nucleophiles to alkenes and alkynes is one of the most fundamental reactions in organic synthesis. ${ }^{190}$ Arguably, the halolactonization is very useful since the resulting lactone can easily be elaborated. ${ }^{191}$ To date, several $1,4-$ bromolactonizations of conjugated enynes to give the products synthetically valuable bromoallenes have been reported in natural product synthesis. ${ }^{192}$ Moreover, bromoallenes could be the important intermediates in organic transformations. ${ }^{193}$ In 2009 , Tang and coworkers reported a DABCO-catalyzed, highly regio- and diastereoselective 1,4bromolactonization of conjugated enynes (Scheme 69). ${ }^{194}$ In contrast to 1,2-addition to double bond in the conjugated enynes, where two adjacent stereogenic centers are created, 
1,4-addition across conjugated enynes can produce a stereogenic center and an axially chiral allene simultaneously. However, in Tang's paper, the substrates were relatively limited. As shown in Figure 41, the substituted group on $\mathrm{R}_{2}$ position was methyl or hydrogen only and also no substrate with an aromatic substituent on the $\mathrm{R}_{3}$ position has been reported, likely caused by the difficult enyne synthesis.

Scheme 69: DABCO-catalyzed 1,4-Bromolactonization of conjugated enynes reported by Tang

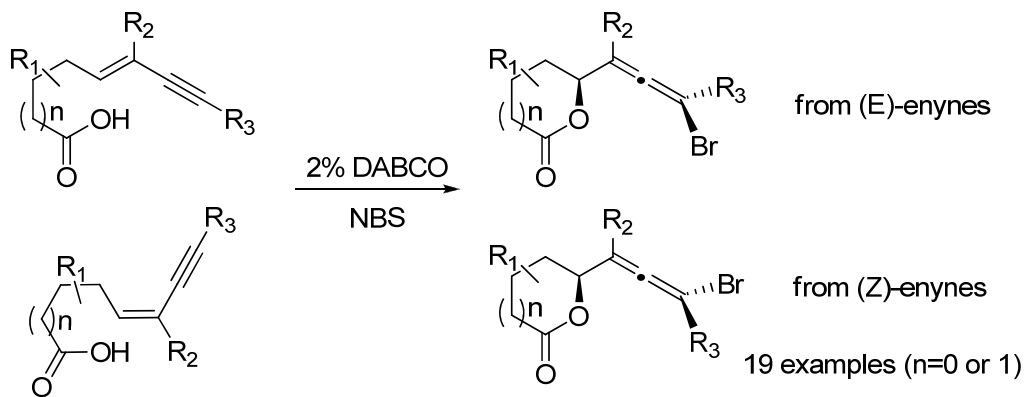

Figure 41: Limited substrates form Tang's approach.<smiles>O=C1CCC(=C=C(Br)Br)O1</smiles>

83\%

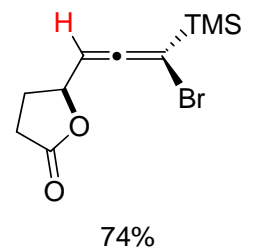

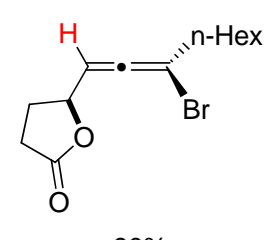

$90 \%$

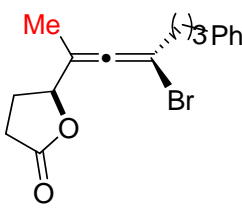

$21 \%$

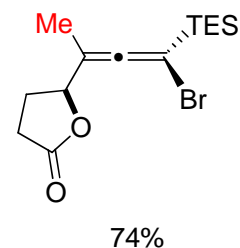

$74 \%$

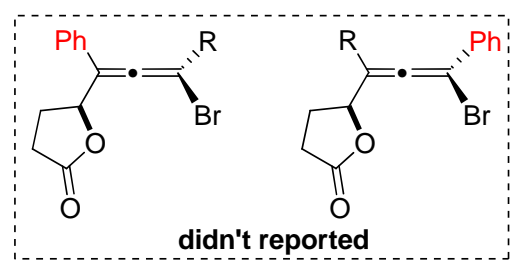

With our reported method here, these $\mathrm{CO}_{2} \mathrm{H}$ or ester $\left(\mathrm{CO}_{2} \mathrm{Me}\right)$ substrates could be readily prepared, we then tried to extend the substrate scope of Tang's approach which is 
DABCO-catalyzed 1,4-Bromolactonization of conjugated enynes. Treating 2.3.2n, 2.3.20 with NBS in the presence of $2 \mathrm{~mol} \%$ DABCO resulted in 6-endo-trig cyclization products, giving the lactones 2.3.3a and 2.3.3b in good yields (Scheme 70). With the terminal alkyne substrate (2.3.2r), it gave complex reaction mixture.

Scheme 70: The 6-endo-trig cyclization of conjugated enynyl acids.
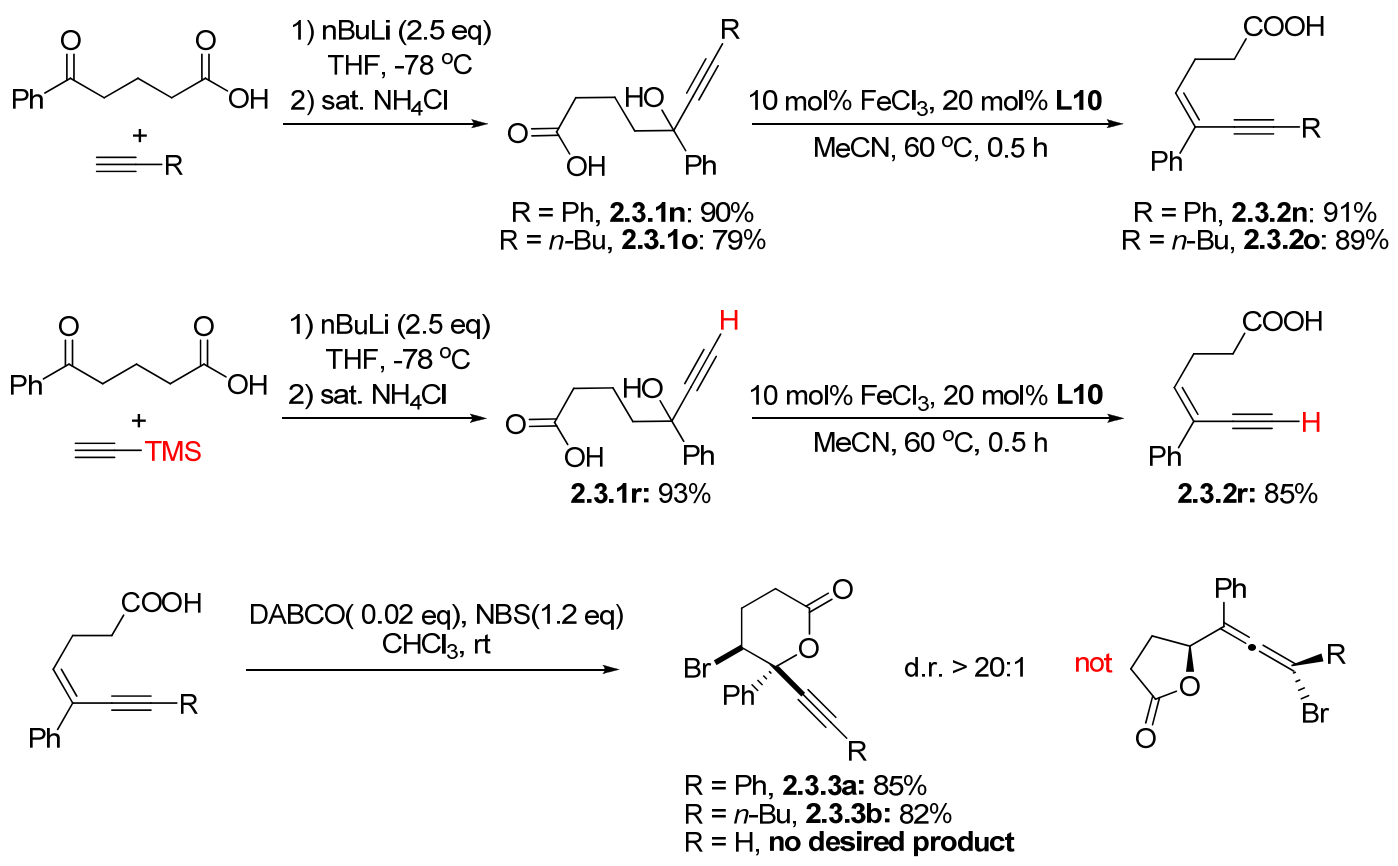

Based on above results, we thought two factors, which are the energetic favorite of cyclization (based on ring strain, six-membered ring is most stable) and relative reactivities of double bond and triple bond in the enynes, may be affected the success of 1,4-bromolatctonization of conjugated enynes. Both extended carbon chain substrates 2.3.2p and 2.3.2q have been subjected to the standard condition of 1,4bromolactonization, unfortunately, no six-membered ring containing bromoallenes were 
formed (Scheme 71). We then focused on tuning the reactivities of double bond and triple bond in conjugated enynes.

Scheme 71: The 7-endo-trig cyclization of conjugated enynyl acids.
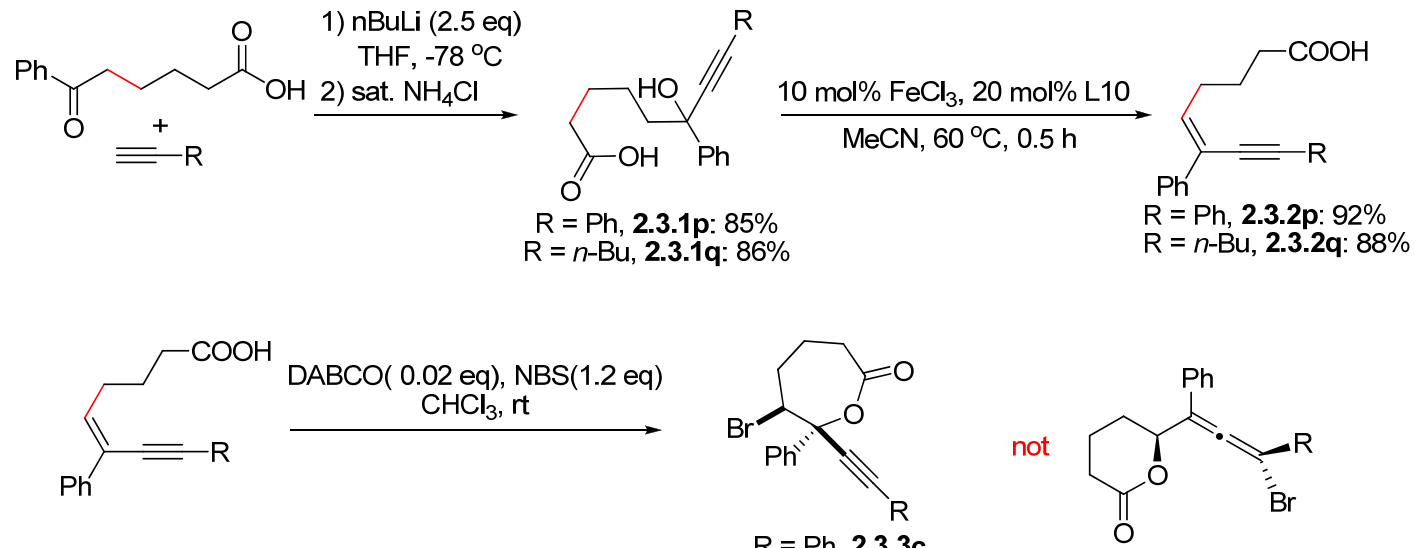

$\mathrm{R}=\mathrm{Ph}, 2.3 .3 \mathrm{c}$
$\mathrm{R}=n-\mathrm{Bu}, 2.3 .3 \mathrm{~d}$

identified by crude NMR

The enediynyl acids $\mathbf{2 . 3 . 2 \mathbf { y } ^ { \prime }}$ and $\mathbf{2 . 3 . 2 z ^ { \prime }}$, which can be prepared in 3 steps, was reacted with NBS and gave no desired products. We thought that the formation of tertiary bromide generated the carbocation intermediate in situ, and then decomposed (Scheme 72). Despite these negative results, we learned that the reactivities of double bond and triple bond were very critical for this transformation at least. Finally, to tune the electronic effect of substituted pattern on the both double bond and triple bond, these enynes 2.3.2s, 2.3.2t and 2.3.2u have been prepared successfully (Scheme 73). With our delight, the treatment 2.3.2s, 2.3.2t, 2.3.2u with NBS resulted in either 6-endo-trig or 5exo-trig cyclization, giving the lactone $\mathbf{2 . 3 . 3 e}$ or bromoallenes $(\mathbf{2 . 3 . 3 f}, \mathbf{2 . 3 . 3 \mathrm { g }}$ ) in excellent yields and diastereoselectivity (>20:1) (Scheme 74). Surprisingly, simply changing the substituted groups at the alkyne terminal, the 6-6-endo-trig or 5-exo-trig could be controlled with excellent stereoselectivity. 
Scheme 72: The reactions with enediynyl acids as the reactants.

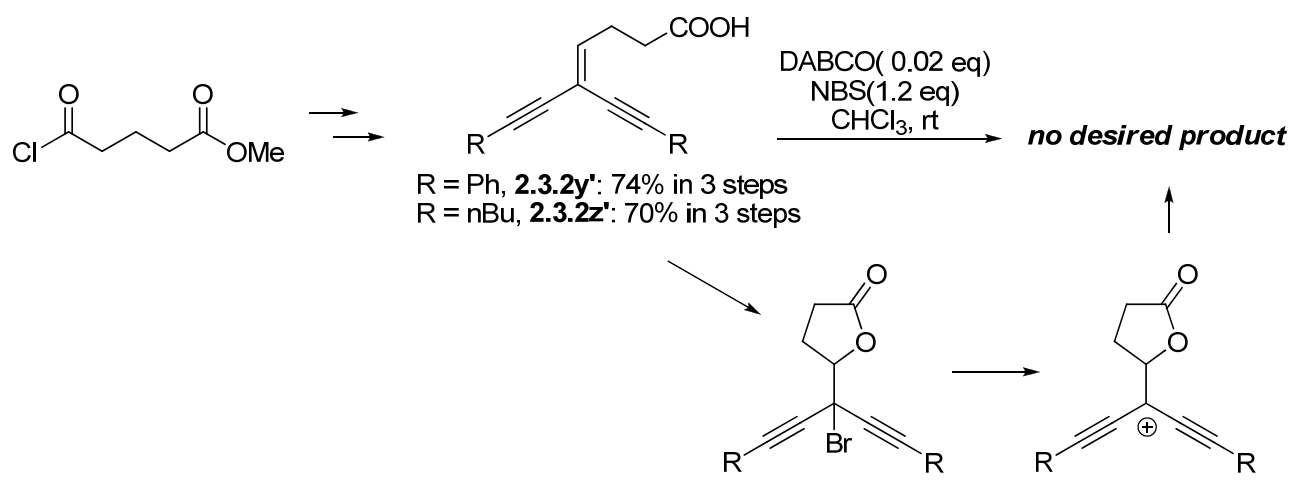

Scheme 73: Synthesis of enynes $2.3 .2 s, 2.3 .2 t$ and 2.3.2u.
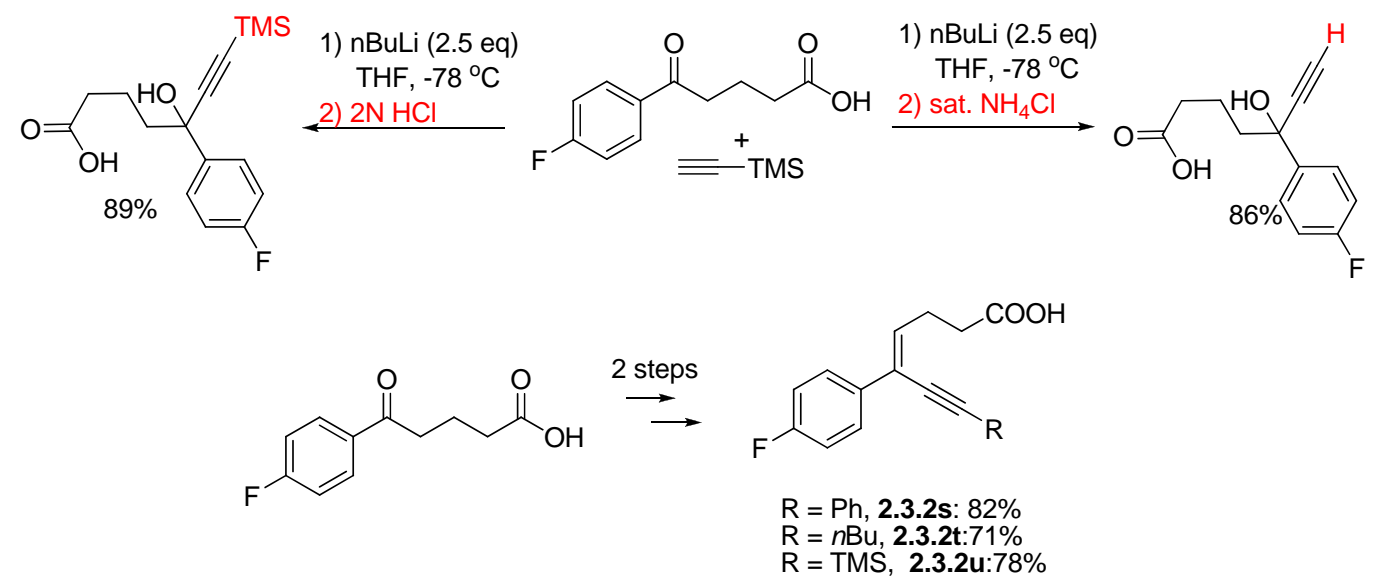
Scheme 74: Substituted group dependent enyne activation. ${ }^{*}$
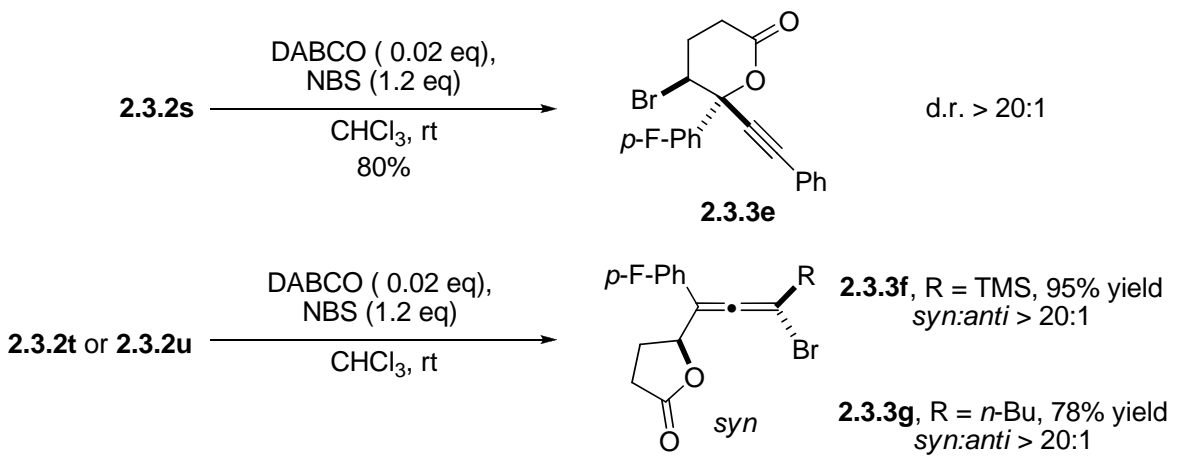

\subsubsection{Mechanistic study.}

According to the literature, iron cation catalyzed reactions should undergo either Lewis acid electrophilic activation or single electron redox process. ${ }^{195}$ To investigate the reaction mechanism of this triazole-ligand coordinated $\mathrm{Fe}^{3+}$ cation catalyst, the cyclopropane substituted propargyl alcohols $(2.3 .4 a, 2.3 .4 b)$ were prepared and treated with $\mathrm{FeCl}_{3}$ under various conditions. As indicated in Figure 42, both Lewis acid catalyzed cyclopropane opening ${ }^{196}$ and radical initiated ring opening ${ }^{197}$ have been reported in literature. The NH-triazole nucleophile and radical trapper 1,4cyclohexyldiene (CHD) were then used to investigate the mechanism of the triazole-Fe catalysts.

\footnotetext{
* Relative stereochemistry of compounds $2.3 .3 \mathrm{e}, \mathbf{2 . 3 . 3 f}$ and $2.3 .3 \mathrm{~g}$ have been fully characterized through comprehensive 1D and 2D NMR analysis. Detailed spectra are provided in supporting information.
} 
Figure 42: Mechanistic investigations.

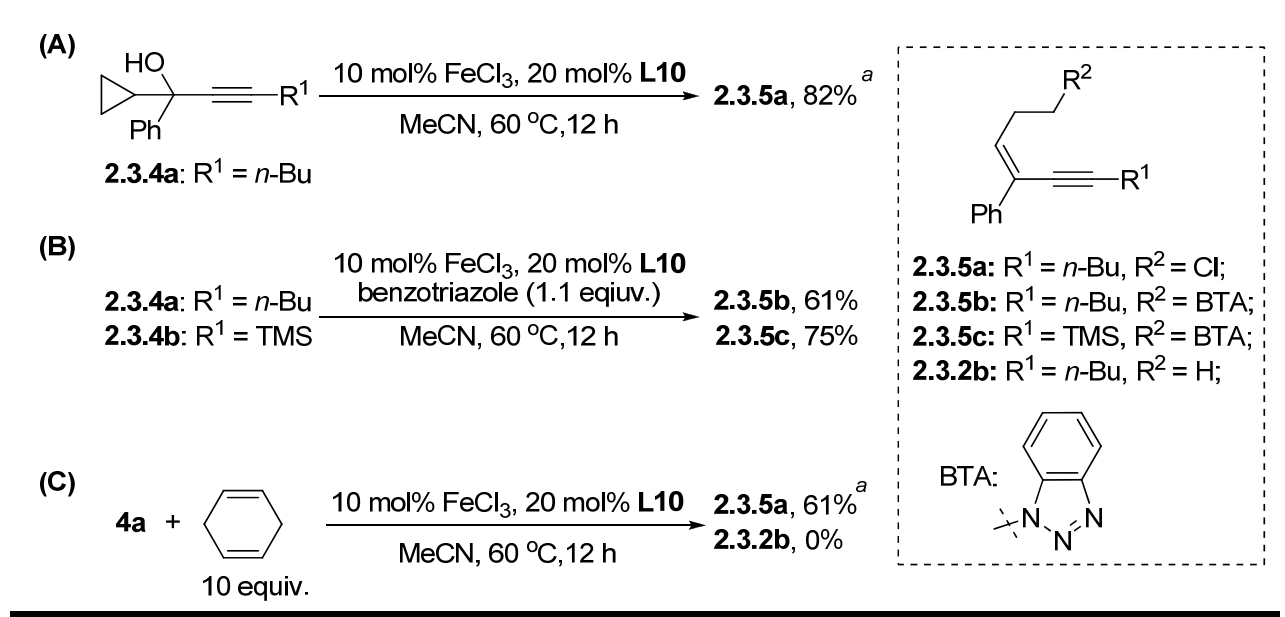

${ }^{a}$ Yields were determined based on the completion of $\mathrm{Cl}^{-} \cdot \mathrm{FeCl}_{3}$.

The reaction of 2.3.4a under the optimal conditions gave the chloro-enyne 2.3.5a as the only enyne product isolated ( $82 \%$ yield, Figure $42 \mathrm{~A})$. With the addition of NHtriazole nucleophile (Figure 42B), triazole enynes 2.3.5b and 2.3.5c were isolated in $61 \%$ and $75 \%$ yield individually as the only double bond isomers $(\mathbf{2 . 3 . 5 c}$ was confirmed by $\mathrm{X}$ ray). Addition of the radical trapper CHD did not lead to the formation of H-quenched enyne 2.3.2b (Figure 42C). Instead, the chloro-enyne 2.3.5a was the only enyne product isolated. These results suggested the reaction proceeds via carbocation ring opening instead of radical mechanism, which is consistent with our hypothesis that 1,2,3-triazole ligands provided the required electronic effect in adjusting Lewis acidity of $\mathrm{Fe}^{3+}$ to achieve the required chemoselectivity for this transformation.

\subsubsection{Conclusion}

In conclusion, 1,2,3-triazoles were identified as unique ligands for iron mediated catalysis. The challenging catalytic dehydration of propargyl alcohols to conjugated enynes was 
successfully achieved with this new iron-based catalytic system. The fact that 1,2,3triazoles were the only heterocycles "working" among the tested ligands illustrate their ability in adjusting iron cation reactivity, thereby providing the foundation for further development of other important iron catalyzed transformations. Moreover, we have tuned the reactivity of conjugated enyne in the 1,4-bromolactonization reaction. 


\section{Chapter Three: N-2-aryl-1,2,3-triazoles: a new class of UV- Blue emitting fluorophores through Planar- Intramolecular-Charge-Transfer (PICT) $\dagger$}

\subsection{Introduction}

Photoactive molecules are important in chemistry, biology and material research. ${ }^{198}$ The last two decades evidenced the great advance in this research area, where fluorescent active dyes have been applied to many different research fields, such as biological target imaging, ${ }^{199}$ effective photosensors ${ }^{200}$ and novel photoactive materials. ${ }^{201}$

Two key features of fluorescence emitting molecules are quantum efficiency and the wavelengths of excitation and emission. Although several fluorophores have been developed over the years, effective UV/blue light emitting small molecules are still $\operatorname{rare}^{202}$ due to the relative high-energy gap required between the interactive orbitals, which may cause either poor photostability or low quantum efficiency. ${ }^{203}$ However, the fast growing research, such as OLED display ${ }^{204}$ and Förster resonance energy transfer (FRET) ${ }^{205}$ studies, in photoactive compounds generated increasing needs for effective UV/blue emitting molecules.

Two of main challenges in developing UV/blue emitting small molecules are the optical activity and photostability dilemma. As shown in Scheme $\mathbf{7 5 A}$, although condensed polyaromatic structures have an effective aromatic conjugation, usually these compounds possess low energy gap between excited and ground states, thus giving emissions at a longer wavelength. Alternatively, bis-aromatic structures (Scheme 75B)

\footnotetext{
${ }^{\dagger}$ Reproduced in parts with permission from Chem. Eur. J. 2011, 17, 5011-5018. Copyright 2011, with permission from Willey-VCH Verlag GmbH \& Co. KGaA, Weinheim.
} 
could provide the needed larger energy gap for potential UV/blue emitting. ${ }^{206}$ However, the steric repulsions between the ortho-substituted groups cause poor conjugations between the two aromatic rings and therefore significantly decrease the photoactivity. To overcome this problem, extended aromatic systems have been developed to enhance the conjugations (Scheme 75C) ${ }^{207}$ Blue light emission has been observed with this type of molecules, such as the commercial available blue dye disodium 4,4'-bis(2sulfostyryl)biphenyl (DSBP, STD-1). ${ }^{208}$ However, the photolabile $\mathrm{C}=\mathrm{C}$ double bonds considerably limit the potential applications of these compounds. ${ }^{209}$

Scheme 75: Different types of UV/blue emitting small molecules.

(A)

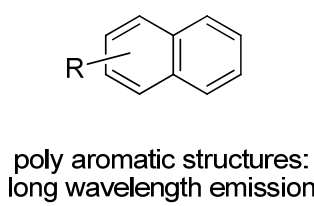

(C)

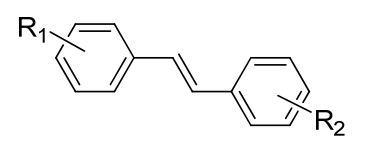

extended conjugation structures: photo-labile double bond
(B)

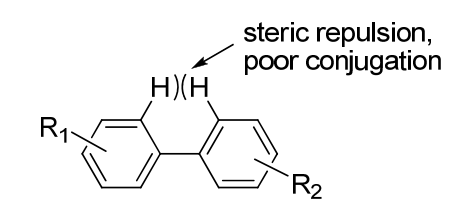

bis-aromatic structures: poor conjugation

(D)

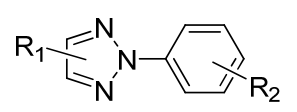

N-2-aryl-1,2,3-triazoles: good conjugation and good photo-stability

\subsection{Research Objective and Results}

\subsubsection{Initiation}

Our interest in developing novel blue-emitting small molecules was initiated by our recent discovery (Figure 43). As shown in Figure 43, the N-2-phenyl triazole 3.1a showed good emission at $362 \mathrm{~nm}$ when it was excited at $254 \mathrm{~nm}$, while the N-1-aryl 
isomer 3.1a' gave almost no emission. This interesting finding suggested suggested that the N-2-aryl-triazoles (NATs) might be applied as new building blocks to reach effective $\mathrm{UV} /$ blue emission by overcoming the challenges associated with other small molecule systems. Therefore, a series of NATs were prepared by using the method which has been described in Section 1.2 and the optical properties of these NATs were investigated as the new UV/blue dyes.

Figure 43: Photoluminescent spectra of N-2-aryl-1,2,3-triazole: sample preparation: $1.0 \mathrm{x}$ $10^{-5} \mathrm{~mol} / \mathrm{L}$ in $\mathrm{MeOH}$, excitation at $254 \mathrm{~nm}$ with $5 \mathrm{~nm}$ slit, quantum yield of 3.1a $\Phi=0.154$, quantum yield of 3.1 a' $\Phi=0.009$

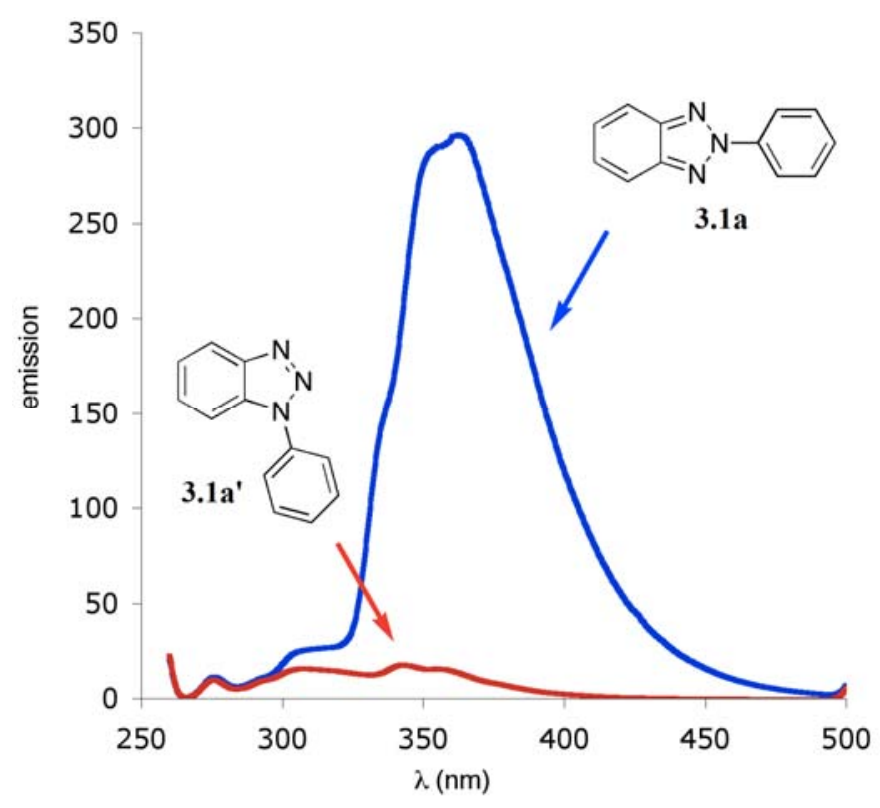

\subsubsection{Influence of substituted groups on the $\mathrm{C}-4$ and $\mathrm{C}-5$ positions}

To evaluate these new fluorophores, one key concern is whether the optical properties (efficiency, excitation and emission wavelengths) of the NATs could be adjusted while maintaining effective emission in the UV/blue range. The structures of NATs allowed 
substitutions on three different positions: C-4, C-5 and N-2. Although the C-4 and C-5 substitutions are away from the N-2 aryl groups, they may strongly influence conjugation at the N-2 position through electronic effect. This concern was confirmed by the X-ray crystal structures of compounds 3.1b and 3.1c shown in Figure 44.

Figure 44: $X$-tray crystal structures of 3.1b and 3.1c.

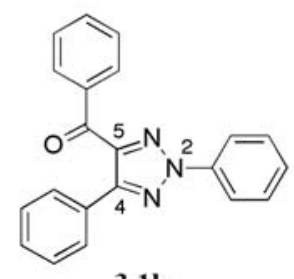

$3.1 \mathrm{~b}$

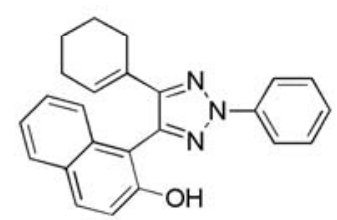

3.1c
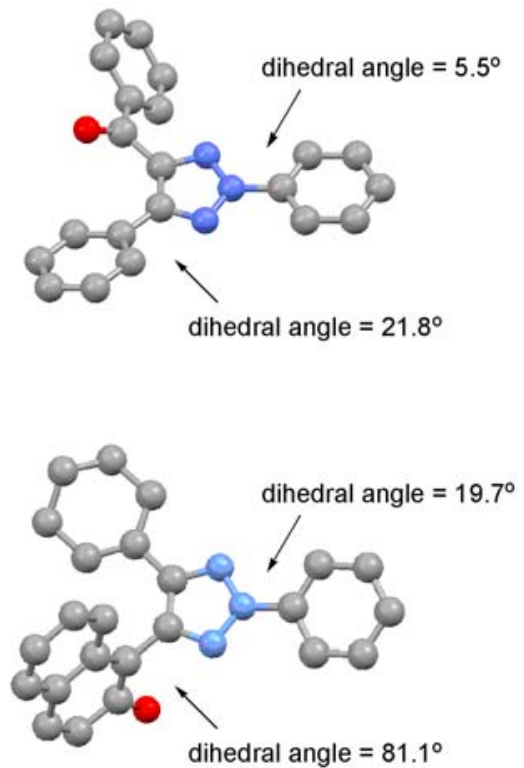

As shown in Figure 44, the N-2-phenyl group in compound 3.1b formed good conjugation with triazole ring with a dihedral angle $5.5^{\circ}$, which supported our proposed strategy that the N-2 aryl triazoles could provide much improved conjugations by avoiding the ortho-steric repulsion associated with typical bis-aromatic systems. The C4-phenyl, on the other hand, showed poorer conjugation (dihedral angle $21.8^{\circ}$ ) in the solid state. This steric influence was further emphasized by the structure of compound 3.1c, where a nearly perpendicular conformation was observed (dihedral angle $81.1^{\circ}$ ) at the C-4 position when the bulky cyclohexenyl group was placed at the C-5 position, even 
with the possible formation of intramolecular hydrogen bonds (between phenol hydroxy and triazole N-2 nitrogen). Moreover, the conformation of the C-4-aryl group also showed the influence on the conjugation at the N-2 position, at least in the solid state: a much bigger dihedral angle was observed for compound 3.1c $\left(19.7^{\circ}\right)$ than 3.1b $\left(5.5^{\circ}\right)$. These crystal structures provided a strong foundation in rationalizing the photoactivity of the NATs. However, it was expected that free $\sigma$-bond rotations at N-2 and C-4 positions could occur while the compounds were dissolved in solution.

Table 19: Fluorescence emission of compounds with different C-5 substitutions. ${ }^{a}$<smiles>C=C(c1ccccc1)c1nn(-c2ccccc2)nc1-c1ccccc1</smiles>

3.1d<smiles>c1ccccc1</smiles>

$3.1 e$

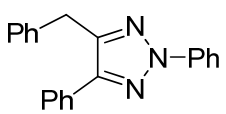

$3.1 f$

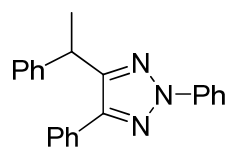

3.1g

\begin{tabular}{|c|c|c|c|c|}
\hline & Absorption (nm) & Emission $\left(\lambda_{\max }\right)$ & $\Phi^{b}$ & $\begin{array}{c}\text { Fluorescence } \\
\text { Intensity }\left(\begin{array}{cc}\mathbf{x} & \left.10^{3}\right)^{c}\end{array}\right.\end{array}$ \\
\hline $3.1 \mathrm{a}$ & $222(0.127), 305(0.222)$ & 362 & 0.15 & 19.6 \\
\hline $3.1 \mathrm{~b}$ & $206(0.128), 263(0.250)$ & 316 & 0.02 & 4.7 \\
\hline $3.1 \mathrm{c}$ & 227 (0.257), $292(0.268)$ & 380,400 & 0.06 & 10.8 \\
\hline $3.1 \mathrm{~d}$ & $220(0.146), 296(0.276)$ & 348 & 0.09 & 23.0 \\
\hline $3.1 \mathrm{e}$ & $220(0.118), 294(0.263)$ & 347 & 0.35 & 46.3 \\
\hline 3.1f & $220(0.111), 290(0.253)$ & 342 & 0.45 & 56.6 \\
\hline $3.1 \mathrm{~g}$ & $221(0.128), 285(0.265)$ & 342 & 0.30 & 52.4 \\
\hline STD-1 & $207(0.452), 234(0.228), 349(0.710)$ & 402,422 & 0.23 & 63.1 \\
\hline STD-2 & $234(0.423), 334(0.068)$ & 365 & 0.56 & 13.9 \\
\hline
\end{tabular}

${ }^{a}$ Sample information: $1.0 \times 10^{-5} \mathrm{~mol} / \mathrm{L}$ in $\mathrm{MeOH}$, excitation at $254 \mathrm{~nm}$ with $5.0 \mathrm{~nm}$ slit; ${ }^{b}$ Quantum yields (Ф) were determined based on $1.0 \times 10^{-5} \mathrm{~mol} / \mathrm{L}$ Quinine sulfate dihydrate (STD-2) in $\mathrm{H}_{2} \mathrm{O}(\Phi=0.56)$; ${ }^{c}$ calculated photo emission integration from the original spectra. All fluorescence measured under identical conditions (see supporting information). STD-1 = disodium 4,4'-bis(2-sulfostyryl)biphenyl (DSBP). 
To evaluate how the various substituted groups on the C-4 and C-5 positions influenced the overall excitation and emission, compounds 3.1d to 3.1g were designed and prepared with different substituted groups on the $\mathrm{C}-5$ positions. The absorptions and emissions of these compounds are summarized in Table 19.

Compared with N-2-phenyl benzotriazole 3.1a, the C-4-N-2-diphenyl triazoles generally gave stronger emissions (3.1e-3.1g). All of these substrates emitted in the UVblue range with $\lambda_{\max }$ around $350 \mathrm{~nm}$. The photoquenching by strong electron withdrawing carbonyl group were observed (3.1b). Notably, the highly steric hindered compound $\mathbf{3 . 1 g}$ gave similar strong emission for the much less hindered compound 3.1f, which suggested the little influence of the bulkiness on the $\mathrm{C}-5$ position for the light emission.

\subsubsection{Variation on $\mathbf{N}-2$-position}

With the N-2-aryl moiety as the fluorophore core, it is expected that the N-2 substituted groups will surely influence the overall optical performance. We wondered if this could be used to adjust the fluorescence emissions and make NAT tunable dyes. Compounds with different substituted groups on the N-2-phenyl ring were prepared to investigate the substituent group effect on their optical properties.

As shown in Table 20, most of the N-2-substituted aromatic compounds $\mathbf{3 . 2}$ gave good UV/blue light emissions. The overall quantum efficiency of NATs is around $30 \%$, which are comparable with commercial blue dyes (for example, DSBP $\Phi=0.23$, measured under the identical conditions). These results suggested the promising future of these compounds as new UV/blue emitting fluorophores. Similar to other literature reports, ${ }^{210}$ photoquenching by $\mathrm{NO}_{2}$ (3.2e) and by a carbonyl group (3.2f) were observed. 
Interestingly, a significant decrease of emission intensity was obtained with ortho-

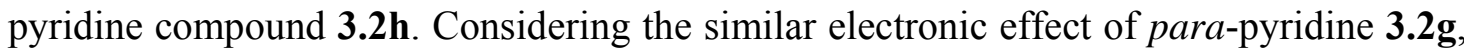
this result was rather surprising. The success in obtaining the X-ray crystal structures for both 3.2g and 3.2h helped to reveal the mechanistic reason behind this result (Figure 44).

Table 20: Substituted group effect on the N-2 position. ${ }^{a}$

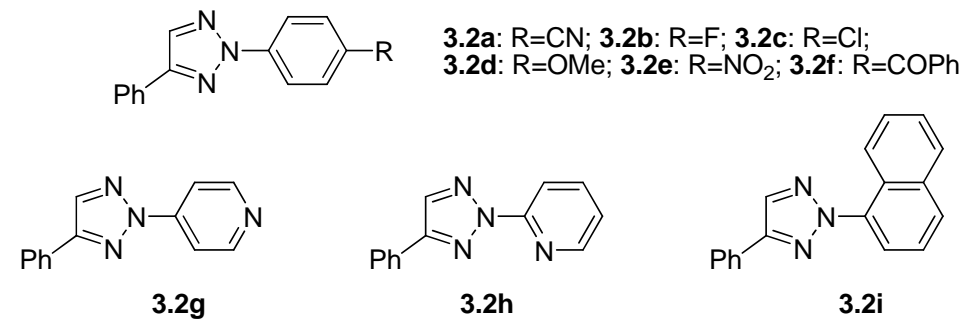

\begin{tabular}{ccccc}
\hline Absorption (nm) & $\begin{array}{c}\text { Emission } \\
\left(\lambda_{\max }\right)\end{array}$ & $\Phi^{b}$ & $\begin{array}{c}\text { Fluorescence } \\
\text { Intensity }(\mathbf{x ~ 1 0})^{\mathbf{c}}\end{array}$ \\
\hline $1 \mathrm{e}$ & $220(0.118), 294(0.263)$ & 347 & 0.35 & 46.3 \\
$2 \mathrm{a}$ & $224(0.154), 309(0.397)$ & 350 & 0.31 & 138 \\
$2 \mathrm{~b}$ & $240(0.056), 294(0.212)$ & 343 & 0.34 & 35.0 \\
$2 \mathrm{c}$ & $298(0.274)$ & 344 & 0.35 & 72.1 \\
$2 \mathrm{~d}$ & $245(0.041), 298(0.226)$ & 374 & 0.31 & 61.6 \\
$2 \mathrm{e}$ & $225(0.181), 336(0.205)$ & 342 & $<0.005$ & $<0.5$ \\
$2 \mathrm{f}$ & $228(0.135), 250(0.14), 317(0.365)$ & 343 & $<0.005$ & $<0.5$ \\
$2 \mathrm{~g}$ & $220.5(0.133), 300(0.242)$ & 341 & 0.28 & 52.2 \\
$2 \mathrm{~h}$ & $238(0.156), 276(0.093)$ & 341 & 0.15 & 1.7 \\
$2 \mathrm{i}$ & $222(0.482), 260(0.115), 296(0.127)$ & 375 & 0.27 & 29.6 \\
\hline
\end{tabular}

Sample information: $1.0 \times 10^{-5} \mathrm{~mol} / \mathrm{L}$ in $\mathrm{MeOH}$, excitation at $310 \mathrm{~nm}$ with $2.5 \mathrm{~nm}$ slit; ${ }^{b}$ Quantum yields $(\Phi)$ were determined based on $1.0 \times 10^{-5} \mathrm{~mol} / \mathrm{L}$ Quinine sulfate dihydrate in $\mathrm{H}_{2} \mathrm{O}(\Phi=0.564) ;{ }^{c}$ calculated photo emission integration from the original spectra. All fluorescence measured under identical conditions (see supporting information). 
Figure 45: X-tray crystal structures of 3.2g and 3.2h.

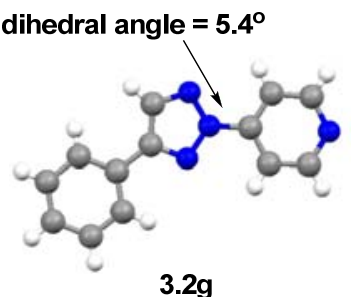

good planar conjugation strong emission $\Phi=0.28$ dihedral angle $=17.9^{\circ}$

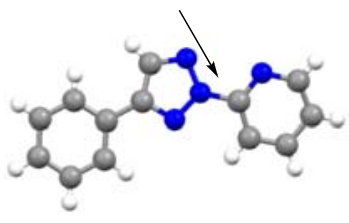

$3.2 \mathrm{~h}$

poor conjugation weak emission $\Phi=0.15$

As shown in Figure 45, the crystal structure of 3.2h exhibited a larger dihedral angle between the pyridine ring and triazole ring, due the electron repulsion between the long pair electrons of the nitrogen atoms on triazole and pyridine rings. This result supports our hypothesis that effective conjugation between triazole ring and N-2 aryl groups is crucial for good fluorescence emission.

The quantum yield $(\Phi)$, which indicates the percentage photon emission, is based on the photons absorbed. Therefore, although quantum yield is a very important parameter used to evaluate fluorescent active molecules, it may not completely represent the performance of the fluorophores since molecules may absorb photons with different efficiency (different absorption $\varepsilon$ ). The fluorescence intensities of NATs were calculated (under the identical conditions) and shown in Table 20.

The emission intensities of NATs are compatible or higher than both commercial the blue dyes quinine sulfate dihydrate and DSBP. Impressively, compounds 3.2a gave very intense emission, which was more than two times stronger than quinine sulfate dihydrate dye; extending conjugation in known to enhance photoabsorption. However, this strategy usually does not work well for blue emitting dyes because conjugation gives a red shift, resulting in the emission at a longer wavelength. To our surprise, this 
undesired red shift did not occur in the NAT system. For compound 3.2i, only slight red shift (13 nm) was observed compared with compound 3.1e. The compound 3.2a, on the other hand, gave almost no red shift even with largely increased emission intensity. These unusual photoactivities highlighted the unique properties of these novel NAT dyes (also see computational studies in later section for the HOMO-LUMO distributions).

\subsubsection{Substituent group electronic effect}

To investigate substituent electronic effects, two series of compounds were compared with substituted groups either on the para-position of the N-2 phenyl or on the paraposition of the C-4 phenyl. The photoluminance spectra are depicted in Figure 46.

Figure 46: Photoluminescent spectra of N-2-aryl-1,2,3-triazole. Sample information: 1.0 x $10^{-5} \mathrm{~mol} / \mathrm{L}$ in $\mathrm{MeOH}$, excitation at $310 \mathrm{~nm}$ with $2.5 \mathrm{~nm}$ slit.

(A)

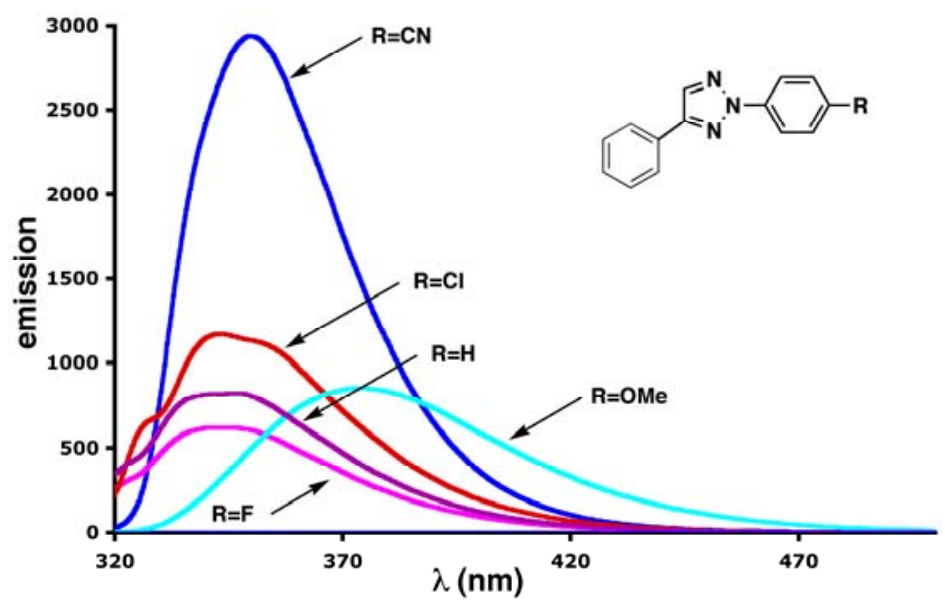

* See the detailed excitation/emission data in supporting information. 
(B)

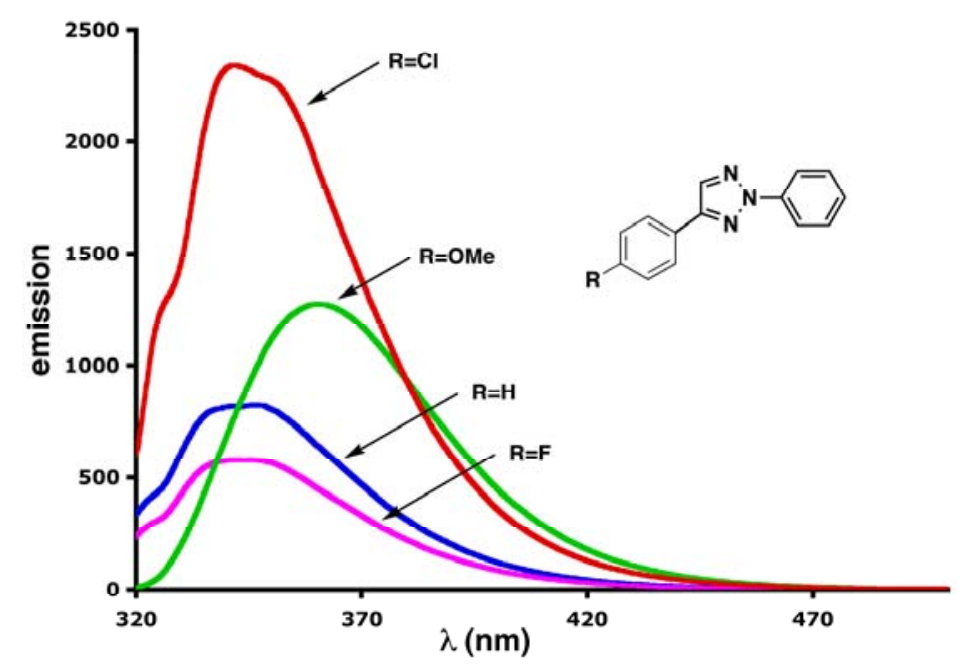

The F-, Cl-, CN- and OMe-substituted NATs all gave strong emission. Interestingly, a very similar trend was observed with substituted groups on either the N-2 phenyl ring (Figure 46A) or on the C-4 phenyl ring (Figure 46B). The NATs with electron deficient substituted groups $(\mathrm{F}, \mathrm{Cl}, \mathrm{CN})$ showed very little wavelength shifts. In contrast, the strong electron donating methoxy group, gave noticeable red shifts when placed on either N-2 (3.2d) or C-4 (3.3f) positions. The CN-substituted compound 3.2a gave the highest emission intensity due to the more efficient light absorption through conjugation. Similar trends were also observed between F- and Cl-substituted NATs. Whereas similar quantum yields were observed, the Cl-substituted NATs gave higher fluorescence emission than F substituted NATs, which was due to the better resonance conjugation between lone pair electrons of $\mathrm{Cl}$ and the phenyl ring. The summarized absorption and emission data are shown in Table 21. 
Table 21: Excitation and emission behavior of substituted NATs. ${ }^{a}$

\begin{tabular}{|c|c|c|c|c|c|c|}
\hline \multicolumn{2}{|c|}{$\begin{array}{l}\text { 3.1e: } R^{1}=H, R^{2}=H \\
\text { 3.2a: } R^{1}=H, R^{2}=C N \\
\text { 3.2b: } R^{1}=H, R^{2}=F \\
\text { 3.2c: } R^{1}=H, R^{2}=C l \\
\text { 3.2d: } R^{1}=H, R^{2}=O M e\end{array}$} & $\begin{array}{l}\text { 3.3a: } R^{1}=F, R^{2}=H \\
\text { 3.3b: } R^{1}=F, R^{2}=C N \\
\text { 3.3c: } R^{1}=F, R^{2}=F \\
\text { 3.3d: } R^{1}=F, R^{2}=C l \\
\text { 3.3e: } R^{1}=F, R^{2}=O M e\end{array}$ & \multicolumn{2}{|c|}{$\begin{array}{l}\text { 3.3f: } R^{1}=O M e, R^{2}=H \\
\text { 3.3g: } R^{1}=O M e, R^{2}=C N \\
\text { 3.3h: } R^{1}=O M e, R^{2}=F \\
\text { 3.3i: } R^{1}=O M e, R^{2}=C l \\
\text { 3.3j: } R^{1}=O M e, R^{2}=O M e\end{array}$} & \multicolumn{2}{|c|}{$\begin{array}{l}\text { 3.3k: } R^{1}=C l, R^{2}=H \\
\text { 3.3l: } R^{1}=C l, R^{2}=C N \\
\text { 3.3m: } R^{1}=C l, R^{2}=F \\
\text { 3.3n: } R^{1}=C l, R^{2}=C l \\
\text { 3.3o: } R^{1}=C l, R^{2}=O M e\end{array}$} \\
\hline & Excitation $\left(\lambda_{\max }\right)$ & Emission $\left(\lambda_{\max }\right)$ & $\Phi^{b}$ & $\begin{array}{l}\text { Fluores } \\
\text { Intensity }\end{array}$ & $\begin{array}{l}\text { cence } \\
\left(\begin{array}{ll}\mathbf{x} & 10^{3}\end{array}\right)^{c}\end{array}$ & $\begin{array}{c}\text { Stoke shift } \\
(\mathbf{n m})\end{array}$ \\
\hline $3.1 \mathrm{e}$ & 298 & 347 & 0.34 & 46. & & 49 \\
\hline $3.2 \mathrm{a}$ & 312 & 350 & 0.31 & 13 & & 38 \\
\hline $3.2 \mathrm{~b}$ & 298 & 343 & 0.34 & 35. & & 45 \\
\hline $3.2 \mathrm{c}$ & 292 & 344 & 0.35 & 72 . & & 52 \\
\hline $3.2 \mathrm{~d}$ & 304 & 374 & 0.31 & 61. & & 70 \\
\hline $3.3 \mathrm{a}$ & 278 & 343 & 0.39 & 35 . & & 65 \\
\hline $3.3 \mathrm{~b}$ & 305 & 351 & 0.51 & 99. & & 46 \\
\hline $3.3 \mathrm{c}$ & 277 & 343 & 0.40 & 45. & & 66 \\
\hline $3.3 \mathrm{~d}$ & 279 & 343 & 0.25 & 79. & & 64 \\
\hline $3.3 \mathrm{e}$ & 280 & 373 & 0.33 & 96. & & 93 \\
\hline 3.3f & 284 & 360 & 0.39 & 82. & & 76 \\
\hline $3.3 \mathrm{~g}$ & 318 & 401 & 0.32 & 11 & & 83 \\
\hline $3.3 \mathrm{~h}$ & 283 & 360 & 0.34 & 78. & & 77 \\
\hline $3.3 \mathrm{i}$ & 287 & 364 & 0.34 & 89. & & 77 \\
\hline 3.3j & 284 & 369 & 0.34 & 11 & & 85 \\
\hline 3.3k & 280 & 341 & 0.35 & 13 & & 61 \\
\hline 3.31 & 306 & 352 & 0.45 & 17 & & 46 \\
\hline $3.3 \mathrm{~m}$ & 281 & 343 & 0.41 & 77. & & 62 \\
\hline $3.3 n$ & 283 & 345 & 0.36 & 99. & & 62 \\
\hline 30 & 285 & 376 & 0.35 & 10 & & 91 \\
\hline
\end{tabular}


${ }^{a}$ Sample information: $1.0 \times 10^{-5} \mathrm{~mol} / \mathrm{L}$ in $\mathrm{MeOH}$, excitation at $310 \mathrm{~nm}$ with $2.5 \mathrm{~nm} \mathrm{slit;}{ }^{b}$ Quantum yields $(\Phi)$ were determined based on $1.0 \times 10^{-5} \mathrm{~mol} / \mathrm{L}$ Quinine sulfate dihydrate (STD-2) in $\mathrm{H}_{2} \mathrm{O}(\Phi=0.56) ;{ }^{c}$ calculated photo emission integration from the original spectra. All fluorescence measured under identical conditions (see supporting information).

\subsubsection{NATs: Tunable UV/blue emitting small molecule dyes}

The large number of NATs spectra allowed us to explore the key factors that control the optical properties of novel tunable NATs UV/blue dyes. First, the emission enhancement by $\mathrm{CN}$ and $\mathrm{Cl}$ groups were confirmed, where strong emissions were observed in all cases with these two groups. When both $\mathrm{CN}$ and $\mathrm{Cl}$ are present (triazole 3.3I), the highest emitting intensity was achieved with $\lambda_{\max }$ at $352 \mathrm{~nm}$. Notably, though the fluorescence enhancement was believed to be a consequence of the extended conjugation, no significant red shift was observed. This UV emission was more than three times stronger than the commercial standard blue dye DSBP, which highlighted the superior photoactivity of these novel UV fluorophores.

Figure 47: Photoluminescent spectra of N-2-aryl-1,2,3-triazole. Sample information: 1.0 x $10^{-5} \mathrm{~mol} / \mathrm{L}$ in $\mathrm{MeOH}$, excitation at $310 \mathrm{~nm}$ with $2.5 \mathrm{~nm}$ slit.

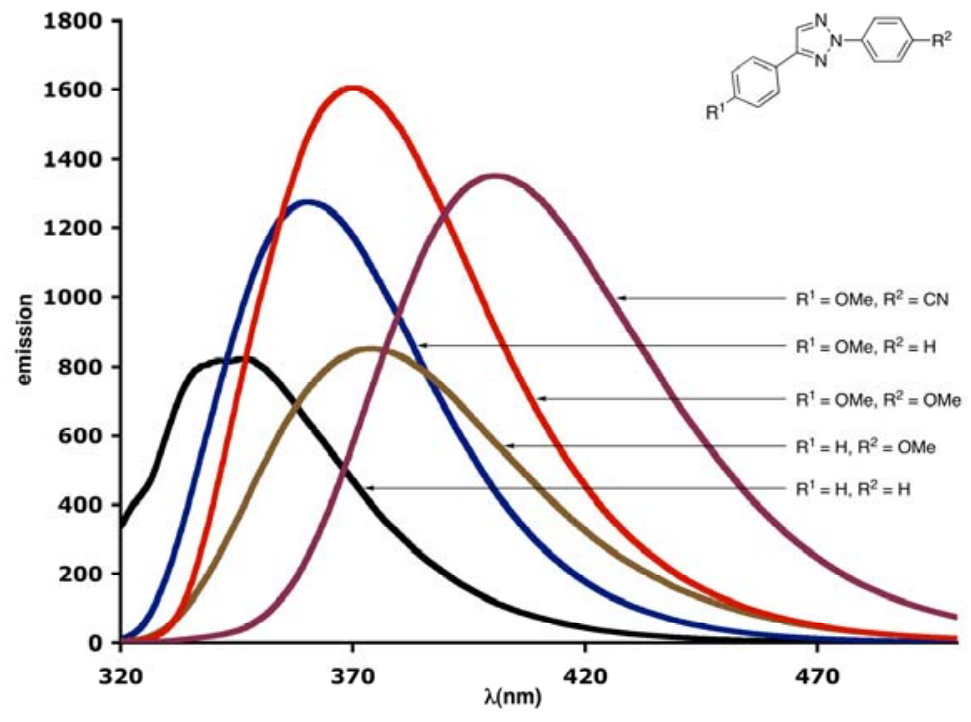


The electron donating OMe is an as the effective shift-tuning group for the NAT dyes. The red shifts were observed with OMe on either N-2 (3.2d) or C-4 (3.3f) positions. No further red shift occurred with OMe on both positions (3.3j), suggesting that cancellation of the inductive effects in $\mathbf{3 . 3 j}$ resulted in no further red shift. This hypothesis was further supported by the emission of compound 3.3g, where a red shift was achieved (emission $\lambda_{\max }$ at $401 \mathrm{~nm}$ ) when combination of $\mathrm{OMe}$ and electron withdrawing $\mathrm{CN}$ were applied on the opposite sites of NATs (Figure 47).

Figure 48: Range of Stock shifts for N-2-aryl-1,2,3-triazole. Sample information: $1.0 \mathrm{x}$ $10^{-5} \mathrm{~mol} / \mathrm{L}$ in $\mathrm{MeOH}$, excitation at $310 \mathrm{~nm}$ with $2.5 \mathrm{~nm}$ slit.

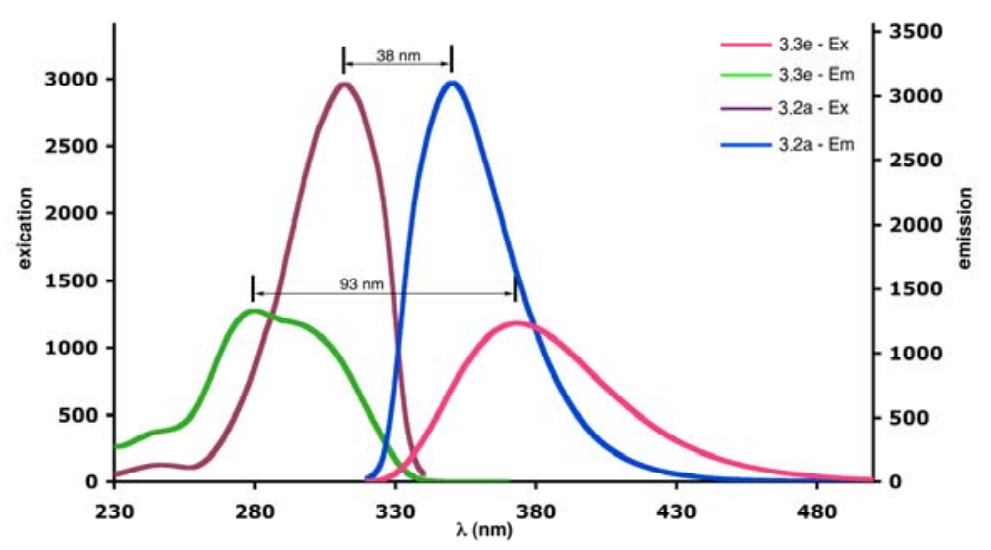

Besides the significant influence on the fluorescence intensity and emission wavelength, the various substituents could also affect the Stock shift. As shown in Table 21, the Stock shifts for these tested compounds ranges from $38 \mathrm{~nm}$ to $93 \mathrm{~nm}$ (Figure 48). The CN modified NATs gave narrow emission band with smaller Stock shifts. Whereas the OMe substituted NATs gave broader emission bands with larger Stock shifts. The ability to modify the Stock shifts is consistent with good photoactivity of the NATs, 
which allowed easy adjustment for the emission wavelength and the gap between excitation and emission.

\subsubsection{Computational investigations}

Time-dependent density function theory (TD-DFT) theoretical calculations were also carried out with the Gaussian $03^{211}$ packages by using the B3LYP/6-31G(d) model. ${ }^{212}$ Unlike the orbitals in other simple bis-aromatic compounds, the HOMOs and LUMOs of the NATs were very similar for all N-2 substituted triazoles with LUMO locate between triazole and N-2 aryl rings. Examples of compounds 3.2d and 3.3g are given in Figure 49.

The N-2-substituted NATs are much less polar compared with the N-1 analogous due to the homogenization of electron density through extended $\pi$-conjugation. The photoexcitation introduces a high extension of planarity to induce the homogeneous distribution of electron density throughout the triazole and N-2 aryl rings. This phenomenon, which has been well documented in the literature, is referred to as planar intramolecular charge transfer (PICT). ${ }^{213}$

Figure 49: HOMO and LUMO orbitals of compounds 3.2d and 3.3g.

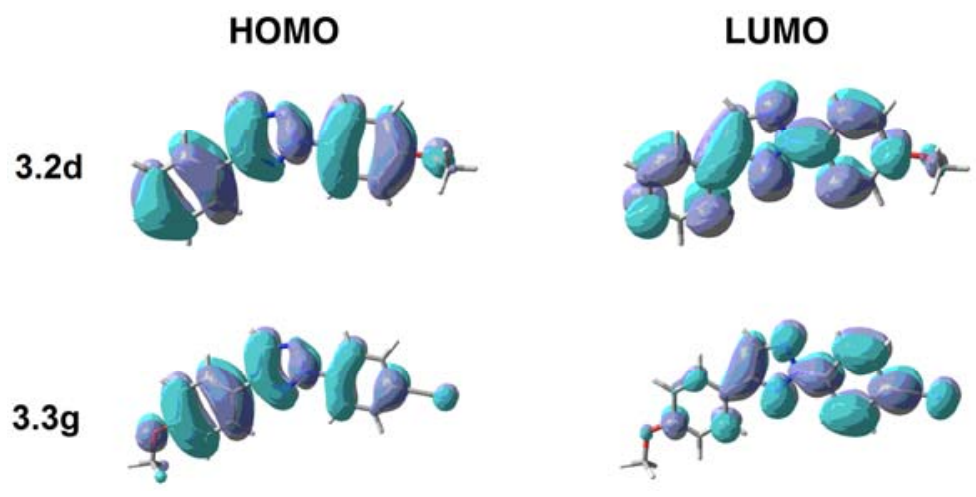


What makes the 1,2,3-triazole special comparing to other aromatic structures is the highly effective conjugation between triazole and N-2 aryl rings, even with the presence of theoretically free-rotatable N-C $\sigma$ bonds. These co-planar structures were confirmed not only by the X-ray crystal structures, as shown in earlier discussions, but also indicated by the computational studies as the most stable conformations for these molecules. Solvent screenings of these dyes has been performed and indicated little change of the optical properties of these dyes in EtOAc, $\mathrm{MeOH}, \mathrm{CH}_{2} \mathrm{Cl}_{2}$, THF or $\mathrm{CH}_{3} \mathrm{CN},{ }^{214}$ which supported the proposed intramolecular charge transfer mechanism. Moreover, the computational studies also indicated that the twisted non-planar conformation was the most energetic favored structures for N-1 isomers (Figure 50), which is consistent with the experimental observation that poor fluorescence emission was observed with all N-1 isomers.

Figure 50: The most stable conformation of $\mathbf{N - 1 - 1 e ' ~ f r o m ~ c o m p u t a t i o n a l ~ s t u d i e s : ~ t w i s t e d ~}$ N-1-aryl ring.

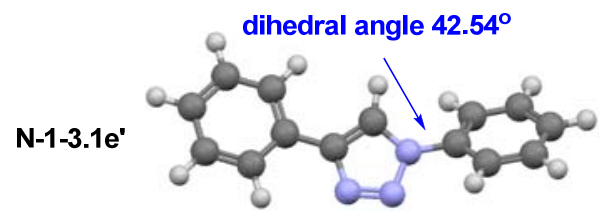

These computational studies revealed the unique features of the NATs $(\mathrm{N}-2$ isomers), which allow them to be effective fluorophores for UV/blue light emitting. The calculated HOMO-LUMO gaps for 3.1e-3.3o (Table 22) are around 3.60-4.78 eV, which 
are in good agreement with the experimental values of $3.90-4.48 \mathrm{eV}$ from optical absorption spectra. $^{*}$

Table 22: Calculated HUMO-LUMO energy gaps. ${ }^{a}$

\begin{tabular}{|c|c|c|c|c|c|}
\hline & Eg $(e V)$ & LUMO-HOMO (eV) & & $\mathbf{E g}(\mathrm{eV})$ & LUMO-HOMO (eV) \\
\hline $3.1 \mathrm{e}$ & 4.16 & 3.95 & 3.3f & 4.37 & 4.34 \\
\hline $3.2 \mathrm{a}$ & 3.97 & 3.60 & $3.3 \mathrm{~g}$ & 3.90 & 3.73 \\
\hline $3.2 \mathrm{~b}$ & 4.16 & 4.06 & $3.3 \mathrm{~h}$ & 4.38 & 4.51 \\
\hline $3.2 \mathrm{c}$ & 4.25 & 4.06 & $3.3 \mathrm{i}$ & 4.32 & 4.51 \\
\hline 3.2d & 4.08 & 3.94 & 3.3j & 4.37 & 3.72 \\
\hline $3.3 \mathrm{a}$ & 4.46 & 4.29 & $3.3 \mathrm{k}$ & 4.43 & 4.27 \\
\hline $3.3 \mathrm{~b}$ & 4.07 & 3.94 & 3.31 & 4.05 & 3.90 \\
\hline $3.3 \mathrm{c}$ & 4.48 & 4.78 & $3.3 \mathrm{~m}$ & 4.41 & 4.04 \\
\hline $3.3 \mathrm{~d}$ & 4.44 & 4.77 & $3.3 \mathrm{n}$ & 4.38 & 4.03 \\
\hline $3.3 \mathrm{e}$ & 4.43 & 4.21 & 3.30 & 4.35 & 4.16 \\
\hline
\end{tabular}

\subsubsection{Extended NAT fluorescence probes}

Encouraged by the good optical activity of these NATs and the mechanistic insight for tuning optical emission, we prepared compounds 3.4a and 3.4b as two new molecular probes (Scheme 76). Considering the UV/blue emission was achieved through the extended conjugations between triazole and N-2 aryl groups, we postulated that the bistriazoles should behave as good fluorophores with even stronger emission efficiency.

* See detailed computational results of all compounds 3.1e to 3.3o in the supporting information 
Compound 3.4a was prepared and tested. As expected, the two triazole rings provide good conjugation with the central phenyl groups (dihedral angle measured from X-ray crystal structure), giving significantly improved UV emission with quantum yield at 0.76 . Notably, the emission $\lambda_{\max }$ is $390 \mathrm{~nm}$, still in the UV/blue range even with the further extended conjugation. The fluorescence intensity of 3.4a was more than 3 times stronger that the commercial blue dye DSBP, while measured at identical condition (excitations at both $254 \mathrm{~nm}$ and $310 \mathrm{~nm}$ ). The styrene groups in 3.4a gave the extra synthetic handle for any further modification, such as polymerization and metathesis coupling, making 3.4a a promising UV probe for further investigation.

Scheme 76: Extended NAT probes.

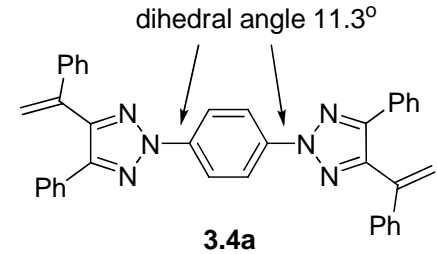

emission $\lambda_{\max }=390 \mathrm{~nm}, \Phi=0.76$; 3.5 times stronger emissition than DSBP: dihedral angle determined by X-ray

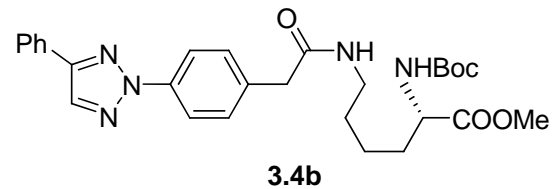

emission $\lambda_{\max }=370 \mathrm{~nm}, \Phi=0.35$; no peptide fluorescence quenching

Effective UV/blue dye for biological systems is rare since the protein substrates may quench the emission. To verify the biocompatibility of the NAT fluorophore, compound 3.4b was synthesized through coupling NAT with protected lysine. The optical properties of this amino acid modified NAT were tested. We were pleased to find that no photoquenching was observed. Notably, compound 3.4b possessed selected protected acid and amine groups, which made it easier to be coupled with biological targets through either the $\mathrm{C}$-terminal or the $\mathrm{N}$-terminal. Considering the unique UV/blue 
emission of this NAT, It is expected that the amino acid modified NAT could be good new UV fluorescence probe for various chemical and biological studies.

\subsection{Conclusion}

We herein report the N-2-aryl-triazole (NAT) as a novel class of effective UV/blue emitting dyes. Through a comprehensive comparison of the substituted groups on C-4, C5 and N-2 positions, a general trend was revealed regarding how to effectively adjust the photoactivity of these compounds. Both emission wavelengths (from $350 \mathrm{~nm}$ to $400 \mathrm{~nm}$ ) and Stocks shift (from $38 \mathrm{~nm}$ to $98 \mathrm{~nm}$ ) could be adjusted with various substituted functional groups. The computational studies and X-ray crystal structures confirmed the effective co-planar structures as the most stable conformation, which was unique to the N-2 isomers. This information could initiate the new strategies for future dye designs. Finally, further derivatizations gave highly efficient UV-emitting bis-triazoles and biocompatible amino acid NAT probes, thus supporting the potential use of these novel dyes in related chemical, material and biological applications. 


\section{Reference}

1 (a) Huisgen, R. Proc. Chem. Soc. 1961, 357-396. (b) Huisgen, R. in 1,3-Dipolar Cycloaddition Chemistry (Ed.: Padwa, A.), Wiley, New York, 1984, pp. 1-176. (c) Padwa, A. in Comprehensive Organic Synthesis, Vol. 4 (Ed.: Trost, B. M.), Pergamon, Oxford, 1991, pp. 1069-1109. (d) for a review of asymmetric 1,3-dipolar cycloaddition reactions, see Gothelf, K. V.; Jorgensen, K. A. Chem. Rev. 1998, 98, 863-909. (e) for a review of synthetic applications, see Mulzer, J. Org. Synth. Highlights 1991, 77-95.

2 (a) Bastide, J.; Henri-Rousseau, V. Bull. Chim. Soc. Fr. 1973, 2294-2296. (b) stepanova, N. P.; Orlova, N. A.; Galishev, V. A.; Turbanova, E. S.; Petrov, A. A. Zh. Org. Khim. 1985, 21, 979-983. (c) Clarke, D.; Mares, D. W.; McNab, H. J. Chem. Soc. Perkin Trans. 1 1997, 1799-1804.

3 (a) Journet, M.; Cai, D.; Kowal, J. J.; Larsen, R. D. Tetrahedron Lett. 2001, 42, 9117-9118. (b) Jarowski, P. D.; Wu, Y. L.; Schweizer, W. B.; Diederich, F. Org. Lett. 2008, 10, 3347-3350. (c) Michinobu, T.; May, J. C.; Lim, J. H.; Boudon, C.; Gis- selbrecht, J.; Seiler, P.; Gross, M.; Biaggio, I.; Diederich, F. Chem. Commun. 2005, 737-739. (d) Cheng, Z. Y.; Li, W. J.; He, F.; Zhou, J. M.; Zhu, X. F. Bioorg. Med. Chem. 2007, 15, 1533-1538. (e) Tsai, C. W.; Yang, S. C.; Liu, Y. M.; Wu, M. J. Tetrahedron 2009, 65, 8367-8372.

4 Bock, V. D.; Hiemstra, H.; van Maarseveen J. H. Eur. J. Org. Chem. 2006, 51-68.

5 (a) Kolb, H. C.; Finn, M. G.; Sharpless, K. B. Angew. Chem. Int. Ed. 2001, 40, 20042021. (b) Tornøe, C. W.; Christensen, C.; Meldal, M. J. Org. Chem. 2002, 67, $3057-$ 
3064. (c) Rostovtsev, V. V.; Green, L. G.; Fokin, V. V.; Sharpless, K. B. Angew. Chem. Int. Ed. 2002, 41, 2596-2599.

6 Himo, F.; Lovell, T.; Hilgraf, R.; Rostovtsev, V. V.; Noodleman, L.; Sharpless, K. B.; Fokin, V. V. J. Am. Chem. Soc. 2005, 127: 210-216.

7 (a) Nakamura, T.; Terashima, T.; Ogata, K.; Fukuzawa, S. Org. Lett. 2011, 13, 620623. (b) Spiteri, C.; Moses, J. E. Angew. Chem. Int. Ed. 2010, 49, 31-33. (c) Kappe, C. O.; Van der Eycken, E. Chem. Soc. Rev. 2010, 39, 1280-1290. (d) Am-blard, F.; Cho, J. H.; Schinazi, R. F. Chem. Rev. 2009, 109, 4207-4220. (e) Shi, F.; Waldo, J. P.; Chen, Y.; Larock, R. C. Org. Lett. 2008, 10, 2409-2412. (f) Moses, J. E.; Moorhouse, A. D. Chem. Soc. Rev. 2007, 36, 1249-1262.

8 (a) Moorhouse, A. D.; Santos, A. M.; Gunaratnam, M.; Moore, M.; Neidle, S.; Moses, J. E. J. Am. Chem. Soc. 2006, 128, 15972-15973 (b) Kumar, R.; El-Sagheer, A.; Tumpane, J.; Lincoln, P.; Wilhelmsson, L. M.; Brown, T. J. Am. Chem. Soc. 2007, 129, 6859-6864. (c) Mamidyala, S. K.; Finn, M. G. Chem. Soc. Rev. 2010, 39, 1252-1261. (d) Waengler, C.; Maschauer, S.; Prante, O.; Schaefer, M.; Schirrmacher, R.; Bartenstein, P.; Eisenhut, M.; Waengler, B. Chembiochem 2010, 11, 2168-2181. (e) Sharma, A.; Neibert, K.; Sharma, R.; Hourani, R.; Maysinger, D.; Kakkar, A. Macromolecules 2011, 44, 521-529.

9 (a) Whiting, M.; Muldoon, J.; Lin, Y. C.; Silverman, S. M.; Lindstrom, W.; Olson, A. J.; Kolb, H. C.; Finn, M. G.; Sharpless, K. B.; Elder, J. H.; Fokin, V. V. Angew. Chem. Int. Ed. 2006, 45, 1435-1439. (b) Moorhouse, A. D.; Moses, J. E. Chemmedchem 2008, 3, 715-723. (c) Tron, G. C.; Pirali, T.; Billington, R. A.; Canonico, P. L.; Sorba, G.; Genazzani, A. A. Med. Res. Rev. 2008, 28, 278-308. (d) 
Gracia, S. R.; Gaus, K.; Sewald, N. Future Med. Chem. 2009, 1, 1289-1310. (e) Willand, N.; Desroses, M.; Toto, P.; Dirie, B.; Lens, Z.; Villeret, V.; Rucktooa, P.; Locht, C.; Baulard, A.; Deprez, B. ACS Chem. Biol. 2010, 5, 1007-1013.

10 Loren, J. C.; krasunski, A.; Fokin, V. V.; Sharpless, K. B. Synlett 2005, 2847-2850.

11 Zefirov, N. S.; Chapovskaya, N. K.; Kolesnikov, V. V. J. Chem. Soc., Dalton Trans. 1971, 1001-1002.

12 Quiclet-Sire, B.; Zard, S. Z. Synthesis 2005, 19, 3319-3326.

13 Sengupta, S.; Duan, H. F.; Lu, W. B.; Petersen, J. L.; Shi, X. D. Org. Lett. 2008, 10, 1493-1496.

14 (a) Katritzky, A. R.; Pastor, A. J. Org. Chem, 2000, 65, 3679-3682. (b) Wang, J.; Li, H.; Zu, L. S.; Wang, W. Org. Lett. 2006, 8, 1391-1394.

15 Chen, Y.; Liu, Y.; Petersen, J. L.; Shi, X. Chem. Commun. 2008, 3254-3256.

16 (a) Maliakal, A.; Lem, G.; Turro, N. J.; Ravichandran, R.; Suhadolnik, J. C.; DeBellis, A. D.; Wood, M. G.; Lau, J. J. Phys. Chem. A. 2002, 106, 7680. (b) Heller, H. J.; Blatttmann, H. R. Pure Appl. Chem. 1973, 36, 141.

17 (a) Tang, W.; Hu, Y. Synth. Commun. 2006, 36, 2461-2468. (b) El Ashry, E. S. H.; Atta, K. F.; Aboul-Ela, S.; Beldi, R. J. Carbohyd. Chem. 2007, 26, 429-437. (c) Trofimova, B. A.; Myachinaa, G. F.; Ermakovaa, T. G.; Kuznetsovaa, N. P.; Volkovaa, L. I.; Sultangareeva, R. G.; Larinaa, L. I.; Klybaa, L. V.; Sukhanovb, G. T.; Sakovichb, G. V. Russ. J. Org. Chem. 2009, 45, 1690-1691.

18 Reported examples for the post-triazole derivation toward N-2-aryl-1,2,3-triazoles: (a) Lacerda, P. S. S.; Silva, A. M. G.; Tome, A. C.; Neves, M.; Silva, A. M. S.; 
Cavaleiro, J. A. S.; Llamas-Saiz, A. L. Angew. Chem., Int. Ed. 2006, 45, 5487-5491.

(b) Kim, D. K.; Kim, J.; Park, H. J. Bio Med. Chem. Lett. 2004, 14, 2401-2405.

19 Selected reviews: (a) Evindar, G.; Batey, R. A. J. Org. Chem. 2006, 71, 1802-1808.

(b) Beletskaya, I. P.; Cheprakov, A. V. Coord. Chem. Rev. 2004, 248, 2337-2364.

(c) Cristau, H. J.; Cellier, P. P.; Spindler, J. F.; Taillefer, M. Chem. Eur. J. 2004, 10, 5607-5622. (d) Ley, S. V.; Thomas, A. W. Angew. Chem. Int. Ed. 2003, 42, 54005449.

20 (a) Altman, R. A.; Koval, E. D.; Buchwald, S. L. J. Org. Chem. 2007, 72, 61906199. (b) Tye, J. W.; Weng, Z.; Johns, A. M.; Incarvito, C. D.; Hartwig, J. F. J. Am. Chem. Soc. 2008, 130, 9971-9983. (c) Huffman, L. M.; Stahl, S. S. J. Am. Chem. Soc. 2008, 130, 9196-9197.

21 Selected examples: (a) Dunn, B.; Zink, J. I. J. Mater. Chem. 1991, 1, 903-913. (b) Calzaferri, G.; Pauchard, M.; Maas, H.; Huber, S.; Khatyr, A.; Schaafsma, T. J. Mater. Chem. 2002, 12, 1-13. (c) de Silva, A. P.; Fox, D. B.; Moody, T. S.; Weir, S. M. Pure Appl. Chem. 2001, 73, 503-511.

22 Yan, W.; Wang, Q.; Lin, Q.; Li, M.; Petersen, J. L.; Shi, X. Chem. Eur. J. 2011, 17, $5011-5018$

23 (a) Thibault, R. J.; Takizawa, K.; Lowenheilm, P.; Helms, B.; Mynar, J. L.; Fréchet, J. M. J.; Hawker, C. J. J. Am. Chem. Soc. 2006, 128, 12084-12085. (b) Takizawa, K.; Nulwala, H.; Thibault, R. J.; Lowenhielm, P.; Yoshinaga, K.; Wooley, K. L.; Hawker, C. J. J. Polym. Sci., Part A: Chem. 2008, 46, 5506-5517. (c) Nulwala, H.; Takizawa, K.; Odukale, A.; Khan, A.; Thibault, R. J.; Taft, B. R.; Lipshutz, B. H.; Hawker, C. J. Macromolecules 2009, 42, 6068-6074. 
24 Nulwala, H.; Burke, D. J.; Khan, A.; Serrano, A.; Hawker, C. J. Macromolecules 2010, 43, 5474-5477.

25 For metal free dipolar addition of vinyl azides example see: Alfred, H.; Benjamin, A. B.; Mark, H.; Paul, M. Tetrahedron Lett. 1981, 22, 863.

26 (a) Wender, P. A.; Cooper, C. B.; Tetrahedron 1986, 42, 2985-2291. (b) Barker, S. J.; Storr, R. C. J. Chem. Soc, Perkin Trans. 1 1990, 3, 485-488. (c) Torres, J.; Lavandera, J. L.; Cabildo, P.; Claramunt, R. M.; Elguero, J. J. Heterocyclic Chem. 1988, 25, 771-782. (d) Katritzky, A. R.; Li, J. Q.; Malhotra, N. C. Liebigs Ann Chem. 1992, 8, 843-853.

27 Shi, F.; Waldo, J. P.; Chen, Y.; Larock, R. C. Org. Lett. 2008, 10, 2409-2412.

28 (a) Matsuzawa, H.; Miyake, Y.; Nishibayashi, Y. Angew. Chem. Int. Ed. 2007, 46, 6488-6491. (b) Patman, R. L.; Williams, V. M.; Bower, J. F.; Krische, M. J. Angew. Chem. Int. Ed. 2008, 47, 5220-5223. (c) Justicia, J.; Sancho-Sanz, I.; ÁlvarezManzaneda, E.; Oltra, J. E.; Cuerva, J. M. Adv. Synth. Catal. 2009, 351, 2295-2300. (d) Shi, S. L.; Xu, L. W.; Oisaki, K.; Kanai, M.; Shibasaki, M. J. Am. Chem. Soc. 2010, 132, 6638-6639. (e) Ding, C. H.; Hou, X. L. Chem. Rev. 2011, 111, 1914-1937. 29 (a) Sonogashira, K. J. Organomet. Chem. 2002, 653, 46-49. (b) Colobert, F.; Castanet, A. S.; Abillard, O. Eur. J. Org. Chem. 2005, 3334-3341. (c) Coutelier, O.; Mortreux, A. Adv. Synth. Catal. 2006, 348, 2038-2042. (d) Shao, Z.; Peng, F. Angew. Chem. Int. Ed. 2010, 49, 9566-9958. (e) Yu, D. Y.; Zhang, Y. G. Adv. Synth. Catal. 2011, 353, 163-169. (f) Beveridge, R. E.; Batey, R. A. Org. Lett. 2012, 14, 540-543.

30 (a) Fusetani, N.; Sugano, M.; Matsunaga S.; Hashimoto, K. Tetrahedron Lett. 1987, 28, 4311-4312. (b) Thompson, A. S.; Corley, E. G.; Huntington, M. F.; Grabowski, 
E. J. J. Tetrahedron Lett. 1995, 36, 8937-8940. (c) Wright, J. L.; Gregory, T. F.; Kesten, S. P.; Boxer, P. A.; Serpa, K. A.; Meltzer, L. T.; Wise, L. D.; Espitia, S. A.; Konkoy, C. S.; Whittemore, E. R.; Woodward, R. M. J. Med. Chem. 2000, 43, 34083419. (c) Ravindar, K.; Reddy, M. S.; Deslongchamps, P. Org. Lett. 2011, 13, 31783181.

31 (a) Swaminathan, S.; Narayan, K. V. Chem. Rev. 1971, 71, 429-438. (b) Georgy, M.; Boucard, V.; Campagne, J. M. J. Am. Chem. Soc. 2005, 127, 14180-14181. (c) Engel, D. A.; Dudley, G. B. Org. Lett. 2006, 8, 4027-4029. (d) Sun, C. X.; Lin, X. C.; Weinreb, S. M. J. Org. Chem. 2006, 71, 3159-3166. (e) Trost, B. M.; Chung, C. K. J. Am. Chem. Soc. 2006, 128, 10358-10359. (f) Sugawara, Y.; Yamada, W.; Yoshida, S.; Ikeno, T.; Yamada, T. J. Am. Chem. Soc. 2007, 129, 12902-12903. (g) Dai, L. Z.; Shi, M. Chem. Eur. J. 2008, 14, 7011-7018. (h) Bandini, M.; Eichholzer, A. Angew. Chem. Int. Ed. 2009, 48, 9533-9537. (i) Wang, D.; Ye, X.; Shi, X. Org. Lett. 2010, 12, 2088-2091.

32 (a) Nishibayashi, Y.; Shinoda, A.; Miyake, Y.; Matsuzawa, H.; Sato, M. Angew. Chem. Int. Ed. 2006, 45, 4835-4839. (b) Sanz, R.; Miguel, D.; Martinez, A.; Alvarez-Gutierrez, J. M.; Rodriguez, F. Org. Lett. 2007, 9, 727-730. (c) Srihari, P.; Reddy, J. S. S.; Satyanarayana K.; Yadav, J. S. Synthesis, 2008, 1853-1860. (d) Zhan, Z. P.; Yu, J. L.; Liu, H. J.; Cui, Y. Y.; Yang, R. F.; Yang, W. Z.; Li, J. P. J. Org. Chem. 2006, 71, 8298-8301. (e) Nishimoto, Y.; Kajioka, M.; Saito, T.; Yasuda, M.; Baba, A. Chem. Commun. 2008, 6396-6398.

33 (a) Cadierno, V.; Gimeno, J.; Nebra, N. Adv. Synth. Catal. 2007, 349, 382-394. (b) Huang, W.; Wang, J.; Shen, Q.; Zhou, X. Tetrahedron 2007, 63, 11636-11643. (c) 
Kuninobu, Y.; Ueda, H.; Takai, K. Chem. Lett. 2008, 37, 878-879. (d) Yadav, J. S.;

Subba Reddy, B. V.; Raghavendra Rao, K. V.; Narender, R. Tetrahedron Lett. 2009, $50,3963-3965$.

34 (a) Zhan, Z. P.; Yang, W. Z.; Yang, R. F.; Yu, J. L.; Li, J. P.; Liu, H. J. Chem. Commun. 2006, 31, 3352-3354. (b) Kabalka, G. W.; Yao, M. L.; Borella, S.; Goins, L. K. Organometallics 2007, 26, 4112-4114. (c) Yadav, J. S.; Subba Reddy, B. V.; Srinivasa Rao, T.; Raghavendra Rao, K. V. Tetrahedron Lett. 2008, 49, 614-618. (d) Boucard, G. M.; Debleds, V.; Dal Zotto, C.; Campagne, J. M. Tetrahedron 2009, 65, $1758-1766$.

35 (a) Nishibayashi, Y.; Yoshikawa, M.; Inada, Y.; Hidai, M.; Uemura, S. J. Am. Chem. Soc. 2002, 124, 11846-11847. (b) Nishibayashi, Y.; Inada, Y.; Yoshikawa, M.; Hidai M.; Uemura, S. Angew. Chem. Int. Ed. 2003, 42, 1495-1498. (c) Murakami, M.; Hori, S. J. Am. Chem. Soc. 2003, 125, 4720-4721. (d) Kennedy-Smith, J. J.; Young, L. A.; Toste, F. D. Org. Lett. 2004, 6, 1325-1327. (e) Bustelo, E.; Dixneuf, P. H. Adv. Synth. Catal. 2005, 347, 393-397. (f) Bustelo, E.; Dixneuf, P. H. Adv. Synth. Catal. 2007, 349, 933-942. (g) Yoshimatsu, M.; Otani, T.; Matsuda, S.; Yamamoto, T.; Sawa, A. Org. Lett. 2008, 10, 4251-4254.

36 Peng, S. Y.; Wang, L.; Wang, J. Org. Biomol. Chem. 2012, 10, 225-228.

37 (a) Nazarov, I. N.; Zaretskaya, I. I. Izv. Akad. Nauk. SSSR, Ser. Khim. 1941, 211 224. (b) Denmark, S. E.; Habermas, K. L.; Jones, T. K. Org. React. 1994, 45, 1-158. (c) Harrington, P. E.; Tius, M. Org. Lett. 1999, 1, 649-652. (d) He, W.; Sun, X.; Frontier, A. J. J. Am. Chem. Soc. 2003, 125, 14278-14279. (e) Frontier, A. J.; Collison, C. Tetrahedron 2005, 61: 7577-7606. (f) Grant, T. N.; West, F. G. J. Am. 
Chem. Soc. 2006, 128, 9348-9349. (g) Malona, J. A.; Cariou, K.; Frontier, A. J. J. Am. Chem. Soc. 2009, 131, 7560-7561.

38 Chen, Y.; Wang, D.; Petersen, J. L.; Akhmedov, N.; Shi, X. Chem. Commun. 2010, 46, 6147-6149 and some unpublished results.

39 (a) Duan, H.; Sengupta, S.; Petersen, J. L.; Shi, X. Organomatellic 2009, 2352-2355.

(b) Duan, H.; Sengupta, S.; Petersen, J. L.; Akhmedov, N.; Shi, X. J. Am. Chem. Soc. 2009, 131, 12100-12102. (c) Chen, Y.; Yan, W.; Akhmedov, N.; Shi, X. Org. Lett. 2010, 12, 344-347. (d) Wang, D.; Ye, X.; Shi, X. Org. Lett. 2010, 12, 2088-2091. (e) Chen, Y.; Wang, D.; Petersen, J. L.; Akhmedov, N.; Shi, X. Chem. Commun. 2010, 46, 6147-6149. (f) Wang, D.; Gautam, L. N. S.; Bollinger, C.; Harris, A.; Li, M.; Shi, X. Org. Lett. 2011, 13, 2618-2621. (g) Wang, D.; Zhang, Y.; Cai, R.; Shi, X. Beilstein J. Org. Chem. 2011, 7, 1014-1020. (h) Wang, D.; Zhang, Y.; Harris, A.; Gautam, L. N. S.; Shi, X. Adv. Syn. Cat. 2011, 353, 2584-2588.

40 (a) Karthikeyan, T.; Sankararaman S. Tetrahedron-Asymmetr. 2008, 19, 2741-2745.

(b) Hu, M. C.; Wang, Y.; Zhai, Q. G.; Li, S. N.; Jiang, Y. C.; Zhang, Y. Inorg. Chem. 2009, 48, 1449-1468. (c) Kamal, A.; Shankaraiah, N.; Reddy, C. R.; Prabhakar, S.; Markandeya, N.; Srivastava, H. K.; Sastry, G. N. Tetrahedron 2010, 66, 5498-5506.

41 (a) Aucagne, V.; Leigh, D. A. Org. Lett. 2006, 8, 4505-4507. (b) Fletcher, J. T.; Bumgarner, B.; Engels, N. D.; Skoglund, D. A. Organometallics 2008, 27, 54305433. (c) Aizpurua, J. M.; Azcune, I.; Fratila, R. M.; Balentova, E.; SagartzazuAizpurua, M.; Miranda, J. I. Org. Lett. 2010, 12, 1584-1587. (d) Doak, B. C.; Scanlon, M. J.; Simpson, J. S. Org. Lett. 2011, 13, 537-539. 
42 (a) Funami, H.; Kusama, H.; Iwasawa, N. Angew. Chem. Int. Ed. 2007, 46, 909-911.

(b) Lee, J. H.; Toste, F. D. Angew. Chem. Int. Ed. 2007, 46, 912-914. (c) Brummond, K. M.; DeForrest, J. E. Synthesis 2007, 6, 795-818. (d) Bongers, N.; Krause, N. Angew. Chem. Int. Ed. 2008, 47, 2178-2181. (e) Ma, S. Acc. Chem. Res. 2009, 42, 1679-1688. (f) Alcaide, B.; Almendros, P.; Aragoncillo, C. Chem. Soc. Rev. 2010, 39, 783-816. (g) Aubert, C.; Fensterbank, L.; Garcia, P.; Malacria, M.; Simonneau, A. Chem. Rev. 2011, 111, 1954-1993. (h) Corma, A.; Leyva-Pérez, A.; Sabater, M. J. Chem. Rev. 2011, 111, 1657-1712. (i) Yu, S.; Ma, S. Chem. Commun. 2011, 47, 5384-5418. (j) Han, X.; Wang, Y.; Zhong, F.; Lu, Y. J. Am. Chem. Soc. 2011, 133, 1726-1729. (k) Li, X. X.; Zhu, L. L.; Zhou, W.; Chen, Z. L. Org. Lett. 2012, 14, 436-439.

43 (a) Marshall, J. A. Chem. Rev. 2000, 100, 3163-3186. (b) Trost, B. M.; Fandrick, D. R.; Dinh, D. C. J. Am. Chem. Soc., 2005, 127, 14186-14187. (c) Imada, Y.; Nishida, M.; Kutsuwa, K.; Murahashi, S. I.; Naota, T. Org. Lett. 2005, 7, 5837-5839. (d) Ogasawara, M.; Okada, A.; Subbarayan, V.; Sörgel, S.; Tamotsu, T. Org. Lett. 2010, 12, 5736-5739. (e) Adachi, Y.; Kamei, N.; Yokoshima, S; Fukuyama, T. Org. Lett. 2011, 13, 4446-4449.

44 (a) Wan, Z.; Nelson, S. G. J. Am. Chem. Soc. 2000, 122, 10470-10471. (b) Krause, N.; Hoffmann-Röder, A. In Modern Allene Chemistry; Krause, N., Hashimi, A. S. K., Eds.; Wiley-VCH: Weinheim, Germany, 2004; pp 997-1039. (c) HoffmannRöder, A.; Krause, N. Angew. Chem. Int. Ed. 2004, 43, 1196-1216. (d) Ondeyka, J. G.; Zink, D. L.; Young, K.; Painter, R.; Kodali, S.; Galgoci, A.; Collado, J.; Tormo, J. R.; Basilio, A.; Vicente, F.; Wang, J.; Singh, S. B. J. Nat. 
Prod. 2006, 69, 377-380. (e) Wang, B.; Pagenkopf, B. L. Org. Lett. 2007, 9, 3703-3706. (f) Sabot, C.; Berard D.; Canesi, S. Org. Lett. 2008, 10, 4629-4632. (e) Pu, X.; Qi, X.; Ready, J. M. J. Am. Chem. Soc. 2009, 131, 10364-10365. (f) Jian, Y. J.; Wu, Y. Org. Biomol. Chem. 2010, 8, 811-821.

45 (a) Luken, C.; Moberg, C. Org. Lett. 2008, 10, 2505-2508. (b) Nagaki, A.; Takizawa, E.; Yoshida, J. J. Am. Chem. Soc. 2009, 131, 1654-1655. (c) Terao, J.; Bando, F.; Kambe, N. Chem. Commun. 2009, 7336-7338. (d) Stephan, D. W.; Erker, G. Angew. Chem. Int. Ed. 2010, 49, 46-76. (e) Xiao, Y.; Zhang, J. Chem. Commun. 2010, 46, 752-754. (f) Chen, G.; Ma, S. Angew. Chem. Int. Ed. 2010, 49, 8306-8308.

46 (a) Yamazaki, T.; Yamamoto, T.; Ichihara, R. J. Org. Chem. 2006, 71, 6251-6253.

(b) Lavallo, V.; Dyker, C. A.; Donnadieu, B.; Bertrand, G. Angew. Chem. Int. Ed. 2008, 47, 5411-5414. (c) Yokota, M.; Fuchibe, K.; Ueda, M.; Mayumi, Y.; Ichikawa, J. Org. Lett. 2009, 11, 3994-3997. (d) Melaimi, M.; Parameswaran, P.; Donnadieu, B.; Frenking, G.; Bertrand, G. Angew. Chem. Int. Ed. 2009, 48, 4792-4795. (e) Zhang, Y.; Hao, H. D.; Wu, Y. Synlett, 2010, 905-908.

47 (a) Stiles, D. T.; Trost, B. M. Org. Lett. 2005, 7, 2117-2120. (b) Shen, L.; Hsung, R. P.; Zhang, Y.; Antoline, J. E.; Zhang, X. Org. Lett. 2005, 7, 3081-3084. (c) Zhao, J.; Liu, Y.; Ma, S. Org. Lett. 2008, 10, 1521-1523. (d) Persson, A. K. A.; Johnston, E. V.; Backvall, J. E. Org. Lett. 2009, 11, 3814-3817. (e) Jian, Y. J.; Wu, Y. Org. Biomol. Chem. 2010, 8, 1905-1909.

48 (a) Ma, M.; Peng, L.; Li, C.; Zhang, X.; Wang, J. J. Am. Chem. Soc. 2005, 127, 15016-15017. (b) Guo, H.; Zheng, Z.; Yu, F.; Ma, S.; Holuigue, A.; Tromp, D. S.; Elsevier, C. J.; Yu, Y. Angew. Chem. Int. Ed. 2006, 45, 4997-5000. (c) Schwier, T.; 
Sromek, A. W.; Yap, D. M. L.; Chernyak, D.; Gevorgyan, V. J. Am. Chem. Soc. 2007, 129, 9868-9878. (d) Armstrong, A.; Daniel, P. G. E. Org. Lett. 2009, 11, 1547-1550. (e) Madelaine, C.; Valerio, V.; Maulide, N. Angew. Chem. Int. Ed. 2010, 49, 1583-1586. (f) Danowitz, A. M.; Taylor, C. E.; Shrikian, T. M.; Mapp, A. K. Org. Lett. 2010, 12, 2574-2577.

49 (a) Evans, P. A.; Lawler, M. J. Angew. Chem. Int. Ed. 2006, 45, 4970-4972. (b) Shu, X. Z.; Liang, Y. M. Angew. Chem. Int. Ed. 2007, 46, 7068-7071. (c) Lo, V. K. Y.; Wong, M. K.; Che, C. M. Org. Lett. 2008, 10, 517-519. (d) Ohmiya, H.; Ito, H.; Sawamura, M. Org. Lett. 2009, 11, 5618-5620. (e) Coeffard, V.; Aylward, M.; Guiry, P. J. Angew. Chem. Int. Ed. 2009, 48, 9152-9155. (f) Kim, H.; Lee, K.; Kim, S.; Lee, P. H. Chem. Commun. 2010, 46, 6341-6343. (g) Bi, H. P.; Liu, X. Y.; Gou, F. R.; Guo, L. N.; Duan, X. H.; Bolte, B.; Odabachian, Y.; Gagosz, F. J. Am. Chem. Soc. 2010, 132, 7294-7296. (h) Kuang, J.; Ma, S. J. Am. Chem. Soc. 2010, 132, 17861787.

50 (a) Saito, S.; Yamamoto, H. Ed.; Thieme, Stuttgart, 2004, p. 15. (b) Wang, S. Y.; Mao, W. W.; She, Z. G.; Li, C. R.; Yang, D. Q.; Lin, Y. C.; Fu, L. W. Bioorg. Med. Chem. Lett. 2007, 17, 2785-2788.

$51 \mathrm{Pu}$, X.; Ready, J. M. J. Am. Chem. Soc. 2008, 130, 10874-10875.

52 (a) Jacobs, T. L.; Fenton, D. M. J. Org. Chem. 1965, 30, 1808-1812. (b) Landor, S. R.; Landor, P. D.; Whiter, P. F. J. Chem. Soc., Perkin Trans. 1 1977, 1710-1714. (c) Franzén, J.; Löfstedt, J.; Falk, J.; Bäckvall, J. E. J. Am. Chem. Soc. 2003, 125, 14140-14148. (d) Alcaide, B.; Almendros, P.; Aragoncillo, C.; Redondo, M. C. J. Org. Chem. 2007, 72, 1604-1608. 
53 (a) Kalli, M.; Landor, P. D.; Landor, S. R. J. Chem. Soc., Perkin Trans. 1, 1973, 1347-1349. (b) Jeffery-Luong, T.; Linstrumelle, G. Tetrahedron Lett.1980, 21, 50195020. (c) Caporusso, A. M.; Filippi, S.; Barontini, F.; Salvadori, P. Tetrahedron Lett. 2000, 41, 1227-1230. (d) Vaz, B.; Alvarez, R.; Brückner, R.; de Lera, A. R. Org. Lett. 2005, 7, 545-548. (e) Woerly, E. M.; Cherney, A. H.; Davis, E. K.; Burke, M. D. J. Am. Chem. Soc. 2010, 132, 6941-6943.

54 (a) Karunakar, G. V.; Periasamy, M. J. Org. Chem. 2006, 71, 7463-7466. (b) Lyakhova, E. G.; Kalinovsky, A. I.; Dmitrenok, A. S.; Kolesnikova, S. A.; Fedorov, S. N.; Vaskovsky, V. E.; Stonik, V. A. Tetrahedron Lett. 2006, 47, 6549-6552. (c) Watanabe, Y.; Yamazaki, T. Synlett, 2009, 3352-3354.

55 Fandrick, D. R.; Reeves, J. T.; Tan, Z.; Lee, H.; Song, J. J.; Yee, N. K.; Senanayake, C. H. Org. Lett. 2009, 11, 5458-5461.

56 (a) Ravikumar, P. C.; Yao, L.; Fleming, F. F. J. Org. Chem. 2009, 74, 7294-7299.

(b) Fleming, F. F.; Ravikumar, P. C.; Yao, L. Synlett, 2009, 1077-1080.

57 (a) Lee, K.; Lee, P. H. Org. Lett. 2008, 10, 2441-2444. (b) Li, Z.; Yang, C.; Zheng, H.; Qiu, H.; Lai, G. J. Organomet. Chem. 2008, 693, 3771-3779.

58 Kobayashi, K.; Naka, H.; Wheatley, A. E. H.; Kondo, Y. Org. Lett. 2008, 10, 33753377.

59 (a) Moreau, J. L.; Gaudemar, M. J. Organomet. Chem. 1976, 108, 159-164. (b) Marek, I.; Mangeney, P.; Alexakis, A.; Normant, J. F. Tetrahedron Lett. 1986, 27, 5499-5502. (c) Alexakis, A.; Marek, I.; Mangeney, P.; Normant, J. F. J. Am. Chem. Soc. 1990, 112, 8042-8047.

60 Ortiz deMontellano, P. R. J. Chem. Soc. Chem. Commun. 1973, 709-710. 
61 (a) Cahiez, C.; Alexakis, A.; Normant, J. F. Synthesis 1978, 528-530. (b) Tadema, G.; Vermeer, P.; Meijer, J.; Brandsma, L. Recl. Trav. Chim. Pays-Bas. 1976, 95, 66-67.

(c) Alexakis, A.; Commerçon, A.; Villiéras, J.; Normant, J. F. Tetrahedron Lett. 1976, 17, 2313-2316.

62 Bertozzi, F.; Crotti, P.; Macchia, F.; Pineschi, M.; Arnold, A.; Feringa, B. L. Tetrahedron Lett. 1999, 40, 4893-4896.

63 (a) Kotora, M.; Noguchi, Y.; Takahashi, T. Collect. Czech. Chem. Commun. 1999, 64, 1119-1124. (b) Duan, Z.; Nishimoto, T.; Ogasawara, M.; Takahashi, T. Synthesis, 2005, 2055-2060.

64 Sato, I.; Matsueda, Y.; Kadowaki, K.; Yonekubo, S.; Shibata, T.; Soai, K. Helv. Chim. Acta 2002, 85, 3383-3387.

65 Kawasaki, T.; Sato, M.; Ishiguro, S.; Takahiro, S.; Yosuke, M.; Sato, I.; Nishino, H.; Inoue, Y.; Soai, K. J. Am. Chem. Soc. 2005, 127, 3274-3275.

66 Pu, X.; Qi, X.; Ready, J. M. J. Am. Chem. Soc. 2009, 131, 10364-10365.

67 Cai, F.; Pu, X.; Qi, X.; Lynch, V.; Radha, A.; Ready, J. M. J. Am. Chem. Soc. 2011, 133, 18066-18069.

68 (a) Gou, F. R.; Bi, H. P.; Guo, L. N.; Guan, Z. H.; Liu, X. Y.; Liang, Y. M. J. Org. Chem. 2008, 73, 3837-3841. (b) Huang, W.; Shen, Q.; Wang, J.; Zhou, X. J. Org. Chem. 2008, 73, 1586-1589. (c) Huang, W.; Hong, L. C.; Zheng, P. Z.; Liu, R. L.; Zhou, X. G. Tetrahedron Lett. 2009, 65, 3603-3610.

69 (a) Kwok, S. W.; Hein, J. E.; Fokin, V. V.; Sharpless, K. B. Heterocycles 2008, 76, 1141-1154. (b) Wang, X. J.; Sidhu, K.; Zhang, L.; Camp-bell, S.; Haddad, N.; Reeves, D. C.; Krishnamurthy, D.; Senanayake, C. H. Org. Lett. 2009, 11, 5490- 
5493. (c) Wang, X. J.; Zhang, L.; Krishna-murthy, D.; Senanayake, C. H.; Wipf, P. Org. Lett. 2010, 12, 4632-4635. (d) Wang, X. J.; Zhang, L.; Lee, H.; Haddad, N.; Krishnamurthy, D.; Senanayake, C. H. Org. Lett. 2009, 11, 5026-5028.

70 (a) Mitsunobu, O.;Yamada, M. Bull. Chem. Soc. Jpn. 1967, 40, 2380-2382. (b) Mitsunobu, O.; Yamada, M.; Mukaiyama, T. Bull. Chem. Soc. Jpn. 1967, 40, 935939. (c) Mitsunobu, O.; Kato, K.; Tomari, M. Tetrahedron 1970, 26, 5731-5736. (d) Mitsunobu, O.; Kimura, J.; Fujisawa, Y. Bull. Chem. Soc. Jpn. 1972, 45, 245-247. (e) Mitsunobu, O.; Wada, M.; Sano, T. J. Am. Chem. Soc. 1972, 94, 679-680. (f) Kurihara, T.; Nakajima, Y.; Mitsunobu, O. Tetrahedron Lett. 1976, 17, 2455-2458. (g) Mitsunobu, O.; Kimura, J.; Iiizumi, K.; Yanagida, N. Bull. Chem. Soc. Jpn. 1976, 49, 510-513.

71 (a) Mitsunobu, O. Synthesis 1981, 1, 1-28. (b) Hughes, D. L. Org. React. 1992, 42, 335-656. (c) Lawrence, S. PharmaChem 2002, 1, 12-14. (d) Valentine, D. H. Jr.; Hillhouse, J. H. Synthesis 2003, 317-334. (e) Dembinski, R. Eur. J. Org. Chem. 2004, 2763-2772. (f) Dandapani, S.; Curran, D. P. Chem. Eur. J. 2004, 10, 3130-3138. (g) Parenty, A.; Moreau, X.; Campagne, J. M. Chem. Rev. 2006, 106, 911-939. (h) But, T. Y. S.; Toy, P. H. Chem. Asian J. 2007, 2, 1340-1355. (i) Kumara Swamy, K. C.; Bhuvan Kumar, N. N.; Balaraman, E.; Pavan Kumar, K. V. P. Chem. Rev. 2009, 109, $2551-2651$.

72 (a) Wu, Y.; Esser, L.; De Brabander, J. K. Angew. Chem. Int. Ed. 2000, 39, 43084310. (b) Pungente, M. D.; Weiler, L. Org. Lett. 2001, 3, 643-646. (c) Wang, Y.; Janjic, J.; Kozmin, S. A. J. Am. Chem. Soc. 2002, 124, 13670-13671. (d) Grab, T.; Braese, S. Adv. Synth. Catal. 2005, 347, 1765-1768. (e) Shirokawa, S. I.; 
Shinoyama, M.; Ooi, I.; Hosokawa, S.; Nakazaki, A.; Kobayashi, S. Org. Lett. 2007, 9, 849-852. (f) Huang, J.; Wu, C.; Wulff, W. D. J. Am. Chem. Soc. 2007, 129, 13366-13367. (g) Deng, L.; Ma, Z.; Zhao, G. Synlett 2008, 728-732. (h) Sze But, T. Y.; Lu, J. N.; Toy, P. H. Synlett 2010, 7, 1115-1117.

73 (a) Koide, K.; Finkelstein, J. M.; Ball, Z.; Verdine, G. L. J. Am. Chem. Soc. 2001, 123, 398-408. (b) Kurosawa, W.; Kan, T.; Fukuyama, T. J. Am. Chem. Soc. 2003, 125, 8112-8113. (c) Yoon, U. C.; Kwon, H. C.; Hyung, T. G.; Choi, K. H.; Oh, S. W.; Yang, S.; Zhao, Z.; Mariano, P. S. J. Am. Chem. Soc. 2004, 126, 1110-1124. (d) Morita, N.; Krause, N. Eur. J. Org. Chem. 2006, 4634-4641. (e) Wang, Y. G.; Takeyama, R.; Kobayashi, Y. Angew. Chem. Int. Ed. 2006, 45, 3320-3323. (f) Sakamoto, I.; Izumi, N.; Yamada, T.; Tsunoda, T. Org. Lett. 2006, 8, 71-74. (g) Miyazaki, T.; Yokoshima, S.; Simizu, S.; Osada, H.; Tokuyama, H.; Fukuyama, T. Org. Lett. 2007, 9, 4737-4740. (h) Chu, Q.; Henry, C.; Curran, D. P. Org. Lett. 2008, $10,2453-2456$.

74 (a) Dong, L.; Roosenberg, J. M.; Miller, M. J. J. Am. Chem. Soc. 2002, 124, 1500115005. (b) Chen, J.; Forsyth, C. J. J. Am. Chem. Soc. 2003, 125, 8734-8735. (c) Mergott, D. J.; Frank, S. A.; Roush, W. R. Proc. Natl. Acad. Sci. U.S.A. 2004, 101, 11955-11959. (d) Gagnon, D.; Lauzon, S.; Godbout, C.; Spino, C. Org. Lett. 2005, 7, 4769-4771. (e) Cohen, F.; Overman, L. E. J. Am. Chem. Soc. 2006, 128, 2594-2603. (f) Klepper, F.; Jahn, E. M.; Hickmann, V.; Carell, T. Angew. Chem. Int. Ed. 2007, 46, 2325-2327. (g) Bauder, C. Org. Biomol. Chem. 2008, 6, 2952-2960.

75 (a) Kemmler, M.; Bach, T. Angew. Chem. Int. Ed. 2003, 42, 4824-4826. (b) Krishnan, S.; Schreiber, S. L. Org. Lett. 2004, 6, 4021-4024. (c) Hodgetts, K. J. 
Tetrahedron 2005, 61, 6860-6870. (d) Goundry, W. R. F.; Lee, V.; Baldwin, J. E. Synlett 2006, 2407-2410. (e) Jackson, T.; Woo, L. W. L.; Trusselle, M. N.; Chander, S. K.; Purohit, A.; Reed, M. J.; Potter, B. V. L. Org. Biomol. Chem. 2007, 5, 29402952. (f) Nygaard, S.; Leung, K. C. F.; Aprahamian, I.; Ikeda, T.; Saha, S.; Laursen, B. W.; Kim, S. Y.; Hansen, S. W.; Stein, P. C.; Flood, A. H.; Stoddart, J. F.; Jeppesen, J. O. J. Am. Chem. Soc. 2007,129, 960-970.

76 (a) Aesa, M. C.; Baán, G.; Novák, L.; Szántay, C. Synth. Commun.1996, 26, 909914. (b) Tsunoda, T.; Uemoto, K.; Nagino, C.; Kawamura, M.; Kaku, H.; Ito, S. Tetrahedron Lett. 1999, 40, 7355-7358. (c) Bussiere, A.; Barragan-Montero, V.; Clavel, C.; Toupet, L.; Montero, J. L. Lett. Org. Chem. 2006, 3, 654-657. (d) Uchida, K.; Yokoshima, S.; Kan, T.; Fukuyama, T. Org. Lett. 2006, 8, 5311-5313. (e) Ghosh, A. K.; Moon, D. K. Org. Lett. 2007, 9, 2425-2427.

77 (a) Iranpoor, N.; Firouzabadi, H.; Akhlaghinia, B.; Azadi, R. Synthesis 2004, 92-96.

(b) Iranpoor, N.; Firouzabadi, H.; Azadi, R.; Akhlaghinia, B. J. Sulfur Chem. 2005, 26, 133-137. (c) Abe, H.; Aoyagi, S.; Kibayashi, C. J. Am. Chem. Soc. 2005, 127, $1473-1480$.

78 (a) Smith, A. B., III; Safonov, I. G.; Corbett, R. M. J. Am. Chem. Soc. 2001, 123, 12426-12427. (b) Trost, B. M.; Crawley, M. L. Chem. Eur. J. 2004, 10, 2237-2252. (c) Curran, D. P.; Zhang, Q.; Richard, C.; Lu, H.; Gudipati, V.; Wilcox, C. S. J. Am. Chem. Soc. 2006, 128, 9561-9573. (d) Wojczykowski, K.; Jutzi, P. Synlett 2006, 3940. (e) Miyauchi, H.; Chiba, S.; Fukamizu, K.; Ando, K.; Narasaka, K. Tetrahedron 2007, 63, 5940-5953. (f) Wuest, F.; Carlson, K. E.; Katzenellenbogen, J. A. Steroids 2008, 73, 69-76. 
79 (a) Chaturvedi, D.; Ray, S. Tetrahedron Lett. 2006, 47, 1307-1309. (b) Chaturvedi, D.; Mishra, N.; Mishra, V. Monatsh. Chem. 2007, 138, 57-60. (c) Chaturvedi, D.; Mishra, N.; Mishra, V. Synthesis 2008, 355-357.

80 (a) McNulty, J.; Capretta, A.; Laritchev, V.; Dyck, J.; Robertson, A. J. Angew. Chem. Int. Ed. 2003, 42, 4051-4054. (b) Schenk, S.; Weston, J.; Anders, E. J. Am. Chem. Soc. 2005, 127, 12566-12576. (c) Kumara Swamy, K. C.; Praveen Kumar, K.; Bhuvan Kumar, N. N. J. Org. Chem. 2006, 71, 1002-1008. (d) Fitzjarrald, V. P.; Pongdee, R. Tetrahedron Lett. 2007, 48, 3553-3557.

81 Von Itzstein, M.; Jenkins, I. D. Aust. J. Chem. 1983, 36, 557-563.

82 Wilson, S. R.; Perez, J.; Pasternak, A. J. Am. Chem. Soc. 1993, 115, 1994-1997.

83 Ueda, S.; Su, M. J.; Buchwald, S. L. Angew. Chem. Int. Ed. 2011, 50, 8944-8947.

84 (a) Ojima, I.; Tzamarioudaki, M.; Li, Z.; Donovan, R. J. Chem. Rev. 1996, 96, 635662. (b) Tamaru, Y.; Kimura, M. Synlett 1997, 749-757. (c) D’Souza, D. M.; Müller, T. J. J. Chem. Soc. Rev. 2007, 36, 1095-1108. (d) Ouchi, M.; Terashima, T.; Sawamoto, M. Acc. Chem. Res. 2008, 41, 1120-1132. (e) Díez-González, S.; Marion, N.; Nolan, S. P. Chem. Rev. 2009, 109, 3612-3676. (f) Zhong, C.; Shi, X. Eur. J. Org. Chem. 2010, 2999-3025. (g) Dobereiner, G. E.; Crabtree, R. H. Chem. Rev. 2010, 110, 681-703.

85 (a) Schläfer, H. L.; Gliemann, G. Basic Principles of Ligand Field Theory, Wiley Interscience: New York, 1969. (b) Pearson, A. J. Metallo-organic Chemistry, Wiley, 1985. (c) Crabtree, R. H. The Organometallic Chemistry of the Transition Metals, 2nd ed.; Wiley, New York, 1994. (d) Spessard, G. O.; Miessler, G. L. Organometallic Chemistry, Prentice Hall, NJ, 1997. 
86 Fu, G. C. Acc. Chem. Res. 2006, 39, 853-860.

87 (a) Gorin, D. J.; Sherry, B. D.; Toste, F. D. Chem. Rev. 2008, 108, 3351-3378. (b) Würtz, S.; Glorius, F. Acc. Chem. Res. 2008, 41, 1523-1533. (c) Daugulis, O.; Do, H. Q.; Shabashov, D. Acc. Chem. Res. 2009, 42, 1074-1086. (d) Shao, Z. H.; Zhang, H. B. Chem. Soc. Rev. 2009, 38, 2745-2755. (e) Teichert, J. F.; Feringa, B. L. Angew. Chem. Int. Ed. 2010, 49, 2486-2528. (f) van Leeuwen, W. N. M.; Kamer, P. C. J.; Claver, C.; Pamies, O.; Dieguez, M. Chem. Rev., 2011, 111, 2077-2118.

88 (a) Gomez Arrayas, R.; Adrio, J.; Carretero, J. C. Angew. Chem. Int. Ed. 2006, 45, 7674-7715. (b) Leuthäusser, S.; Schwarz, D.; Plenio, H. Chem. Eur. J. 2007, 13, 7195-7203. (c) Mellah, M.; Voituriez, A.; Schulz, E. Chem. Rev, 2007, 107, 5133 5209. (d) Hargaden, G. C.; Patrick, J. G. Chem. Rev, 2009, 109, 2505-2550. (e) Rasika Dias, H. V.; Lovely, C. J. Chem. Rev. 2008, 108, 3223-3238. (f) Nolan, S. P. Acc. Chem. Res. 2011, 44, 91-100.

89 (a) Pfaltz, A.; Drury, W. J. Proc. Natl. Acad. Sci. U.S.A. 2004, 101, 5723-5726. (b) Ohkuma, T.; Kitamura, M.; Noyori, R. New Front. Asymmetric Catal. 2007, 1-32. (c) Lundgren, R. J.; Hesp, K. D.; Stradiotto, M. Synlett 2011, 17, 2443-2458. (d) Weller, A. Nat. Chem. 2011, 3, 577-578. (d) Jin, S. S.; Wang, H.; Xu, M. H. Chem. Commun. 2011, 47, 7230-7232.

90 (a) Noyori, R.; Takaya, H. Acc. Chem. Res. 1990, 23, 345-350. (b) Kocovsky, P.; Vyskocil, S.; Smrcina, M. Chem. Rev. 2003, 103, 3213-3245. (c) Berthod, M.; Mignani, G.; Woodward, G.; Lemaire, M. Chem. Rev. 2005, 105, 1801-1836. (d) Tanaka, K. Synlett 2007, 13, 1977-1993. (e) Shimizu, H.; Nagasaki, I.; Matsumura, 
K.; Sayo, N.; Saito, T. Acc. Chem. Res. 2007, 40, 1385-1393. (f) Arshad, N.; Kappe, C. O. Adv. Heterocycl. Chem. 2010, 99, 33-59.

91 (a) Basak, R. Synlett 2003, 8, 1223-1224. (b) Sun, Z. K.; Yu, S. Y.; Ding, Z. D. Ma, D. W. J. Am. Chem. Soc. 2007, 129, 9300-9301. (c) Füerstner, A.; Schlecker, A. Chem. Eur. J. 2008, 14, 9181-9191. (d) Shu, W.; Ma, SS. M. Chem. Commun. 2009, 41, 6198-6200. (e) Poisson, T.; Yamashita, Y.; Kobayashi, S. J. Am. Chem. Soc. 2010, 132, 7890-7892. (f) Nakanishi, M.; Minard, C.; Retailleau, P.; Cariou, K.; Dodd, R. H. Org. Lett. 2011, 13, 5792-5795.

92 (a) Aikawa, K.; Akutagawa, S.; Mikami, K. J. Am. Chem. Soc. 2006, 128, 1264812649. (b) Scriban, C.; Glueck, D. S. J. Am. Chem. Soc. 2006, 128, 2788-2789. (c) Cote, A.; Lindsay, V. N. G.; Charette, A. B. Org. lett. 2007, 9, 85-87. (d) Shaikh, N. S.; Enthaler, S.; Junge, K.; Beller, M. Angew. Chem. Int. Ed. 2008, 47, 2497-2501. (e) Kanai, M.; Wada, R.; Shibuguchi, T.; Shibasaki, M. Pure Appl. Chem. 2008, 80, 1055-1062. (f) Campian, M. V.; Perutz, R. N.; Procacci, B.; Thatcher, R. J.; Torres, O.; Whitwood, A. C. J. Am. Chem. Soc. 2012, 134, 3480-3497.

93 (a) Moteki, S. A.; Wu, D.; Chandra, K. L.; Reddy, D. S.; Takacs, J. M. Org. Lett. 2006, 8, 3097-3100. (b) Robert, T.; Velder, J.; Schmalz, H. G. Angew. Chem. Int. Ed. 2008, 47, 7718-7721. (c) Anderson, C. D.; Dudding, T.; Gordillo, R.; Houk, K. N. Org. Lett. 2008, 10, 2749-2752. (d) Teller, H.; Fluegge, S.; Goddard, R.; Füerstner, A. Angew. Chem. Int. Ed. 2010, 49, 1949-1953. (e) Lam, H. W. Synthesis 2011, 13, 2011-2043. (f) Naeemi, Q.; Dindaroglu, M.; Kranz, D. P.; Velder, J.; Schmalz, H. G. Eur. J. Org. Chem. 2012, 1179-1185. 
94 (a) Jacobsen, E. N. Acc. Chem. Res. 2000, 33, 421-431. (b) Clever, G. H.; Soeltl, Y.; Burks, H.; Spahl, W.; Carell, T. Chem. Eur. J. 2006, 12, 8708-8718. (c) Kemper, S.; Hrobarik, P.; Kaupp, M.; Schlorer, N. E. J. Am. Chem. Soc. 2009, 131, 4172-4173. (d) Brown, M. K.; Blewett, M. M.; Colombe, J. R.; Corey, E. J. J. Am. Chem. Soc. 2010, 132, 11165-11170. (e) Clegg, W.; Harrington, R. W.; North, M.; Pasquale, R. Chem. Eur. J. 2010, 16, 6828-6843. (f) Kurahashi, T.; Fujii, H. J. Am. Chem. Soc. 2011, 133, 8307-8316.

95 (a) Devi, R. N.; Rabu, P.; Golub, V. O.; O’Connor, C. J.; Zubieta, J. Solid State Sci. 2002, 4, 1095-1102. (b) Armstrong, D. R.; Davies, R. P.; Haigh, R.; Hendy, H. M.; Raithby, P. R.; Snaith, R.; Wheatley, A. E. H. Eur. J. Inorg. Chem. 2003, 3363-3366. (c) Busetto, L.; Marchetti, F.; Zucchini, S.; Zanotti, V. Inorg. Chim. Acta 2005, 358, 1469-1484. (d) Bronisz, R. Inorg. Chem. 2005, 44, 4463-4465. (e) Zou, W. Q.; Li, Y.; Zheng, F. K.; Guo, G. C.; Huang, J. S. Acta Cryst. 2006, E62, o3591-o3593.

96 (a) Olson, J. R.; Yamauchi, M.; Bulter, W. M. Inorg. Chim. Acta 1985, 99, 121-125. (b) Purnell, L. G.; Shepard, J. C.; Hodgson, D. J. J. Am. Chem. Soc. 1975, 97, 23762377.

97 Chan, T. R.; Hilgraf, R.; Sharpless, K. B.; Fokin, V. V. Org. Lett. 2004, 6, 2853 2855.

98 Similar mode of binding has been previously suggested for trisoxazoline based ligands. See: Kohmura, Y.; KAtsuki, T. Tetrahedron Lett. 2000, 41, 3941-3945.

99 Liu, D.; Gao, W.; Dai, Q.; Zhang, X. Org. Lett. 2005, 7, 4907-4910.

100 Detz, R. J.; Arévalo Heras, S.; de Gelder, R.; van Leeuwen, P. W. N. M.; Hiemstra, H.; Reek, J. N. H.; van Maarseveen, J. H. Org. Lett. 2006, 8, 3227-3230. 
101 (a) Bell, S.; Wüstenberg, B.; Kaiser, S.; Menges, F.; Netscher, T.; Pfaltz, A. Science 2006, 311, 642-644. (b) Guiry, P. J.; Saunders: C. P. Adv. Synth. Catal. 2004, 346, 497-537.

102 Suijkerbuijk, B. M. J. M.; Aerts, B. N. H.; Dijkstra, H. P.; Lutz, M.; Spek, A. L.; van Koten, G.; Klein Gebbink, R. J. M. Dalton Trans. 2007, 1273-1276.

103 Crowley, J. D.; Lee, A. L.; Kilpin, K. J. Aust. J. Chem. 2011, 64, 1118-1132.

104 Kilpin, K. J.; Paul, U. S. D.; Lee, A. L.; Crowley, J. D. Chem. Commun. 2011, 47, 328-330.

105 (a) Keske, E. C.; Zenkina, O. V.; Wang, R.; Crudden, C. M. Organometallics 2012, 31, 456-461. (b) Mathew, P.; Neels, A.; Albrecht, M. J. Am. Chem. Soc. 2008, 130, $13534-13535$.

106 (a) Poulain, A.; Canseco-Gonzalez, D.; Hynes-Roche, R.; Müller-Bunz, H.; Schuster, O.; Stoeckli-Evans, H.; Neels, A.; Albrecht, A. Organometallics 2011, 30, 1021-1029. (b) Guisado-Barrios, G.; Bouffard, J.; Donnadieu, B.; Bertrand, G. Organometallics 2011, 30, 6017-6021.

107 (a) Prades, A.; Peris, E.; Albrecht, M. Organometallics 2011, 30, 1162-1167. (b) Bouffard, J.; Keitz, B. K.; Tonner, R.; Guisado-Barrios, G.; Frenking, G.; Grubbs, R. H.; Bertrand, G. Organometallics 2011, 30, 2617-2627.

108 (a) Karthikeyan, T.; Sankararaman, S. Tetrahedron Lett. 2009, 50, 5834-5837. (b) Nakamura, T.; Ogata, K.; Fukuzawa, S. I. Chem. Lett. 2010, 39, 920-922. (c) Saravanakumar, R.; Ramkumar, V.; Sankararaman, S. Organometallics 2011, 30, 1689-1694. (d) Inomata, S.; Hiroki, H.; Terashima, T.; Ogata, K.; Fukuzawa, S. Tetrahedron 2011, 67, 7263-7267. 
109 Nakamura, T.; Terashima, T.; Ogata, K.; Fukuzawa, S. I. Org. Lett. 2011, 13, 620623.

110 Poznanski, J.; Najda, A.; Bretner, M.; Shugar, D. J. Phys. Chem. A 2007, 111, 65016509. According to the paper, the pKa of benzotriazole is 8.3 .

111 (a) Katritzky, A. R.; Lan, X.; Yang, J. Z.; Denisko, O. V. Chem. Rev. 1998, 98, 409548. (b) Verma, A. K.; Singh, J.; Sankar, V. K.; Chaudhary, R.; Chandra, R. Tetrahedron Lett. 2007, 48, 4207-4210.

112 Oro, L. A.; Pinillos, M. T.; Tejel, C.; J. Organimet. Chem. 1985, 280, 261-267.

113 Pinillos, M. T.; Elduque, A.; Oro, L. A. J. Organimet. Chem. 1988, 338, 411-419.

114 (a) Rasmussen, P. G.; Hough, R. L.; Anderson, J. E.; Bailey, O. H.; Bayon, J. C. J. Am. Chem. Soc. 1982, 104, 6155-6156. (b) Rasmussen, P. G.; Bailey, O. H.; Bayon, J. C. Inorg Chem. 1984, 23, 338-343.

115 Duan, H.; Sengupta, S.; Petersen, J. L.; Shi, X. Organometallics 2009, 28, 23522355.

116 For literature examples of NHC-Au complexes, see: (a) Kieft, R. L.; Peterson, W. M.; Blundell, G. L.; Horton, S.; Henry, R. A.; Jonassen, H. B. Inorg. Chem. 1976, 15, 1721-1722. (b) Nomiya, K.; Noguchi, R.; Oda, M. Inorg. Chim. Acta 2000, 298, 24-32. (c) Partyka, D. V.; Updegraff, J. B.; Zeller, M.; Hunter, A. D.; Gray, T. G. Organometallics 2007, 26, 183-186. (d) Partyka, D. V.; Robilotto, T. J.; Zeller, M.; Hunter, A. D.; Gray, T. G. Proc. Natl. Acad. Sci. U.S.A 2008, 105 , 14293-14297.

117 Duan, H.; Sengupta, S.; Petersen, J. L.; Akhmedov, N.; Shi, X. J. Am. Chem. Soc. 2009, 131, 12100-12102.

118 Chen, Y.; Yan, W.; Akhmedov, N.; Shi, X. Org. Lett. 2010, 12, 344-347. 
119 Wang, D.; Ye, X.; Shi, X. Org. Lett. 2010, 12, 2088-2091.

120 Wang, D.; Zhang, Y.; Harris, A.; Gautam, L. N. S.; Shi, X. Adv. Syn. Cat. 2011, 353, $2584-2588$.

121 (a) Wang, D.; Gautam, L. N. S.; Bollinger, C.; Harris, A.; Li, M.; Shi, X. Org. Lett. 2011, 13, 2618-2621. (b) Wang, D.; Zhang, Y.; Cai, R.; Shi, X. Beilstein J. Org. Chem. 2011, 7, 1014-1020.

122 Mindt, T. L.; Struthers, H.; Brans, L.; Anguelov, T.; Schweinsberg, C.; Maes, V.; Tourwe, D.; Schibli, R. J. Am. Chem. Soc. 2006, 128, 15096-15097.

123 Struthers, H.; Mindt, T. L.; Schibli, R. Dalton Trans. 2010, 39, 675-696.

124 (a) Meudtner, R. M.; Ostermeier, M.; Goddard, R.; Limberg, C.; Hecht, S. Chem. Eur. J. 2007, 13, 9834-9840.(b) Obata, M.; Kitamura, A.; Mori, A.; Kameyama, C.; Czaplewska, J. A.; Tanaka, R.; Kinoshita, I.; Kusumoto, T.; Hashimoto, H.; Harada, M.; Mikata, Y.; Funabiki, T.; Yano, S. Dalton Trans. 2008, 3292-3300. (c) Fletcher, J. T.; Bumgarner, B. J.; Engels, N. D.; Skoglund, D. A. Organometallics 2008, 27, 5430-5433. (d) Orselli, E.; Albuquerque, R. Q.; Fransen, P. M.; Frohlich, R.; Janssen, H. M.; De Cola, L. J. Mater. Chem. 2008, 18, 4579-4590. (e) Schweinfurth, D.; Pattacini, R.; Strobel, S.; Sarkar, B. Dalton Trans. 2009, 9291-9297.

125 Bratsos, I.; Urankar, D.; Zangrando, E.; Genova-Kalou, P.; Kosmrlj, J.; Alessio, E.; Turel, I. Dalton Trans. 2011, 40, 5188-5199.

126 (a) Bastero, A.; Font, D.; Pericàs, M. A. J. Org. Chem. 2007, 72, 2460-2468. (b) Maisonial, A.; Serafin, P.; Traïkia, M.; Debiton, E.; Théry, V.; Aitken, D. J.; Lemoine, P.; Viossat, B.; Gautier, A. Eur. J. Inorg. Chem. 2008, 2008, 298-305. 
127 (a) Urankar, D.; Pinter, B.; Pevec, A.; De Proft, F.; Turel, I.; Košmrlj, J. Inorg. Chem. 2010, 49, 4820-4829. (b) Crowley, J. D.; Bandeen, P. H.; Hanton, L. R. Polyhedron 2010, 29, 70-83. (c) Kilpin, K. J.; Gavey, E. L.; McAdam, C. J.; Anderson, C. B.; Lind, S. J.; Keep, C. C.; Gordon, K. C.; Crowley, J. D. Inorg. Chem. 2011, 50, 6334-6346.

128 Schweinfurth, D.; Hardcastle, K. I.; Bunz, U. H. F. Chem. Commun. 2008, 22032205.

129 (a) Felici, M.; Contreras-Carballada, P.; Vida, Y.; Smits, J. M. M.; Nolte, R. J. M.; De Cola, L.; Williams, R. M.; Feiters, M. C. Chem. Eur. J. 2009, 15, 13124-13134. (b) Mydlak, M.; Bizzarri, C.; Hartmann, D.; Sarfert, W.; Schmid, G.; De Cola, L. Adv. Funct. Mater. 2010, 20, 1812-1820. (c) Felici, M.; Contreras-Carballada, P.; Smits, J. M. M.; Nolte, R. J. M.; Williams, R. M.; De Cola, L.; Feiters, M. C. Molecules 2010, 15, 2039-2059. (d) de Barros e Silva Botelho, M.; FernandezHernandez, J. M.; de Queiroz, T. B.; Eckert, H.; De Cola, L.; de Camargo, A. S. S. J. Mater. Chem. 2011, 21, 8829-8834. (e) Zanarini, S.; Felici, M.; Valenti, G.; Marcaccio, M.; Prodi, L.; Bonacchi, S.; Contreras-Carballada, P.; Williams, R. M.; Feiters, M. C.; Nolte, R. J. M.; De Cola, L.; Paolucci, F. Chem. Eur. J. 2011, 17, 4640-4647. (f) Juricek, M.; Felici, M.; Contreras-Carballada, P.; Lauko, J.; Bou, S. R.; Kouwer, P. H. J.; Brouwer, A. M.; Rowan, A. E. J. Mater. Chem. 2011, 21, 2104-2111. (g) Liu, S.; Müller, P.; Takase, M. K.; Swager, T. M. Inorg. Chem. 2011, $50,7598-7609$.

130 (a) Lo, K. K. W.; Hui, W. K.; Chung, C. K.; Tsang, K. H. K.; Lee, T. K. M.; Li, C. K.; Lau, J. S. Y.; Ng, D. C. M. Coord. Chem. Rev. 2006, 250, 1724-1736. (b) Yu, M. 
X.; Zhao, Q.; Shi, L. X.; Li, F. Y.; Zhou, Z. G.; Yang, H.; Yia, T.; Huang, C. H. Chem. Commun. 2008, 2115-2117. (c) Zhao, Q.; Yu, M. X.; Shi, L. X.; Liu, S. J.; Li, C. Y.; Shi, M.; Zhou, Z. G.; Huang, C. H.; Li, F. Y. Organometallics 2010, 29, 1085-1091.

131 (a) Metz, S.; Bernhard, S. Chem. Commun. 2010, 46, 7551-7553. (b) Curtin, P. N.; Tinker, L. L.; Burgess, C. M.; Cline, E. D.; Bernhard, S. Inorg. Chem. 2009, 48, 10498-10506. (c) Cline, E. D.; Adamson, S. E.; Bernhard, S. Inorg. Chem. 2008, 47, 10378-10388. (d) Goldsmith, J. I.; Hudson, W. R.; Lowry, M. S.; Anderson, T. H.; Bernhard, S. J. Am. Chem. Soc. 2005, 127, 7502-7510. (e) King, K. A.; Spellane, P. J.; Watts, R. J. J. Am. Chem. Soc. 1985, 107, 1431-1432.

132 (a) DeRosa, M. C.; Hodgson, D. J.; Enright, G. D.; Dawson, B.; Evans, C. E. B.; Crutchley, R. J. J. Am. Chem. Soc. 2004, 126, 7619-7626. (b) Gao, R. M.; Ho, D. G.; Hernandez, B.; Selke, M.; Murphy, D.; Djurovich, P. I.; Thompson, M. E. J. Am. Chem. Soc. 2002, 124, 14828-14829.

133 (a) Zhao, Q.; Li, F. Y.; Liu, S. J.; Yu, M. X.; Liu, Z. Q.; Yi, T.; Huang, C. H. Inorg. Chem. 2008, 47, 9256-9264. (b) Zhao, Q.; Li, F.; Huang, C. H. Chem. Soc. Rev. 2010, 39, 3007-3030.

134 (a) Baldo, M. A.; O’Brien, D. F.; You, Y.; Shoustikov, A.; Sibley, S.; Thompson, M. E.; Forrest, S. R. Nature 1998, 395, 151-154. (b) Orselli, E.; Kottas, G. S.; Konradsson, A. E.; Coppo, P.; Frohlich, R.; Frtshlich, R.; De Cola, L.; van Dijken, A.; Buchel, M.; Borner, H. Inorg. Chem. 2007, 46, 11082-11093. (c) Thompson, M. E.; Djurovich, P. E.; Barlow, S.; Marder, S. In Comprehensive Organometallic Chemistry III; Elsevier: Oxford, 2007; Vol. 12, pp 101-194. 
135 (a) Nazeeruddin, M. K.; Wegh, R. T.; Zhou, Z.; Klein, C.; Wang, Q.; De Angelis, F.; Fantacci, S.; Gratzel, M. Inorg. Chem. 2006, 45, 9245-9250. (b) Lowry, M. S.; Bernhard, S. Chem. Eur. J. 2006, 12, 7970-7977. (c) Di Censo, D.; Fantacci, S.; De Angelis, F.; Klein, C.; Evans, N.; Kalyanasundaram, K.; Bolink, H. J.; Gratzel, M.; Nazeeruddin, M. K. Inorg. Chem. 2008, 47, 980-989. (d) Yang, C. H.; Beltran, J.; Lemaur, V.; Cornil, J.; Hartmann, D.; Sarfert, W.; Frohlich, R.; Bizzarri, C.; De Cola, L. Inorg. Chem. 2010, 49, 9891-9901.

136 Ladouceur, S.; Fortin, D.; Zysman-Colman, E. Inorg. Chem. 2011, 50, 11514-11526. 137 Fernández-Hernández, J. M.; Yang, C. H.; Beltrán, J. I.; Lemaur, V.; Polo, F.; Fröhlich, R.; Cornil, J.; De Cola, L. J. Am. Chem. Soc. 2011, 133, 10543-10558.

138 Nonoyama, M. Bull. Chem. Soc. Jpn. 1974, 47, 767-768.

139 (a) Tamayo, A. B.; Alleyne, B. D.; Djurovich, P. I.; Lamansky, S.; Tsyba, I.; Ho, N. N.; Bau, R.; Thompson, M. E. J. Am. Chem. Soc. 2003, 125, 7377-7387. (b) Holmes, R. J.; Forrest, S. R.; Sajoto, T.; Tamayo, A.; Djurovich, P. I.; Thompson, M. E.; Brooks, J.; Tung, Y. J.; D’Andrade, B. W.; Weaver, M. S.; Kwong, R. C.; Brown, J. J. Appl. Phys. Lett. 2005, 87, 243507/1-243507/3. (c) Ren, X. F.; Kondakova, M. E.; Giesen, D. J.; Rajeswaran, M.; Madaras, M.; Lenhart, W. C. Inorg. Chem. 2010, 49, 1301-1303.

140 (a) McDonald, A. R.; Lutz, M.; von Chrzanowski, L. S.; van Klink, G. P. M.; Spek, A. L.; van Koten, G. Inorg. Chem. 2008, 47, 6681-6691. (b) Deaton, J. C.; Young, R. H.; Lenhard, J. R.; Rajeswaran, M.; Huo, S. Q. Inorg. Chem. 2010, 49, 91519161. (c) Dedeian, K.; Shi, J. M.; Shepherd, N.; Forsythe, E.; Morton, D. C. Inorg. Chem. 2005, 44, 4445-4447. 
141 (a) Lamansky, S.; Djurovich, P.; Murphy, D.; Abdel-Razzaq, F.; Lee, H. E.; Adachi, C.; Burrows, P. E.; Forrest, S. R.; Thompson, M. E. J. Am. Chem. Soc. 2001, 123, 4304-4312. (b) Li, J.; Djurovich, P. I.; Alleyne, B. D.; Yousufuddin, M.; Ho, N. N.; Thomas, J. C.; Peters, J. C.; Bau, R.; Thompson, M. E. Inorg. Chem. 2005, 44, 17131727. (c) You, Y. M.; Park, S. Y. J. Am. Chem. Soc. 2005, 127, 12438-12439. (d) Chou, P. T.; Chi, Y. Chem. Eur. J. 2007, 13, 380-395. (e) Chi, Y.; Chou, P. T. Chem. Soc. Rev. 2010, 39, 638-655.

142 (a) Beyer, B.; Ulbricht, C.; Escudero, D.; Friebe, C.; Winter, A.; Gonzalez, L.; Schubert, U. S. Organometallics 2009, 28, 5478-5488. (b) Boutadla, Y.; Davies, D. L.; Jones, R. C.; Singh, K. Chem. Eur. J. 2011, 17, 3438-3448. (c) Ulbricht, C.; Beyer, B.; Friebe, C.; Winter, A.; Schubert, U. S. Adv. Mater. 2009, 21, 4418-4441. 143 Ciamician, G. Science 1912, 36, 385-394.

144 For selected reviews, see: (a) Gust, D.; Moore, T. A. Acc. Chem. Res. 1993, 26, 198205. (b) Ravelli, D.; Dondi, D.; Fagnoni, M.; Albini, A. Chem. Soc. Rev. 2009, 38, 1999-2011. (c) Zeitler, K. Angew. Chem. Int. Ed. 2009, 48, 9785-9789. (d) Yoon, T. P.; Ischay, M. A.; Du, J. Nat. Chem. 2010, 2, 527-532. (e) Narayanam, J. M. R.; Stephenson, C. R. J. Chem. Soc. Rev. 2011, 40, 102-113.

145 (a) Nicewicz, D. A.; MacMillan, D. W. C. Science 2008, 322, 77-80. (b) Nagib, D. A.; Scott, M. E.; MacMillan, D. W. C. J. Am. Chem. Soc. 2009, 131, 10875-10877. (c) Shih, H. W.; Vander Wal, M. N.; Grange, R. L.; MacMillan, D. W. C. J. Am. Chem. Soc. 2010, 132, 13600-13603. (d) Neumann, M.; Fuldner, S.; Konig, B.; Zeitler, K. Angew. Chem. Int. Ed. 2011, 50, 951-954.

146 Lu, Z.; Shen, M.; Yoon, T. P. J. Am. Chem. Soc. 2011, 133, 1162-1164. 
147 (a) Ischay, M. A.; Anzovino, M. E.; Du, J.; Yoon, T. P. J. Am. Chem. Soc. 2008, 130, 12886-12887. (b) Du, J.; Yoon, T. P. J. Am. Chem. Soc. 2009, 131, 1460414605. (c) Ischay, M. A.; Lu, Z.; Yoon, T. P. J. Am. Chem. Soc. 2010, 132, $8572-$ 8574.

148 (a) Narayanam, J. M. R.; Tucker, J. W.; Stephenson, C. R. J. J. Am. Chem. Soc. 2009, 131, 8756-8757. (b) Furst, L.; Narayanam, J. M. R.; Stephenson, C. R. J. Angew. Chem. Int. Ed. 2011, 50, 9655-9659.

149 (a) Zen, J. M.; Liou, S. L.; Kumar, A. S.; Hsia, M. S. Angew. Chem. Int. Ed. 2003, 42, 577-579. (b) Zhang, M.; Chen, C. C.; Ma, W. H.; Zhao, J. C. Angew. Chem. Int. Ed. 2008, 47, 9730-9733. (c) Su, F.; Mathew, S. C.; Lipner, G.; Fu, X.; Antonietti, M.; Blechert, S.; Wang, X. J. Am. Chem. Soc. 2010, 132, 16299-16301. (d) Su, F.; Mathew, S. C.; Möhlmann, L.; Antonietti, M.; Wang,; Blechert, S. Angew. Chem. Int. Ed. 2011, 50, 657-660. (e) Su, Y.; Zhang, L.; Jiao, N. Org. Lett. 2011, 13, $2168-$ 2171. (f) Zou, Y. Q.; Chen, J. R.; Liu, X. P.; Lu, L. Q.; Davis, R. L.; Jørgensen, K. A.; Xiao, W. J. Angew. Chem. Int. Ed. 2012, 51, 784-788.

150 (a) Tucker, J. W.; Narayanam, J. M. R.; Krabbe, S. W.; Stephenson, C. R. J. Org. Lett. 2010, 12, 368-371. (b) Tucker, J. W.; Nguyen, J. D.; Narayanam, J. M. R.; Krabbe, S. W.; Stephenson, C. R. J. Chem. Commun. 2010, 46, 4985-4987. (c) Furst, L.; Matsuura, B. S.; Narayanam, J. M. R.; Tucker, J. W.; Stephenson, C. R. J. Org. Lett. 2010, 12, 3104-3107. (d) Andrews, R. S.; Becker, J. J.; Gagné, M. R. Angew. Chem. Int. Ed. 2010, 49, 7274-7276. (e) Nguyen, J. D.; Tucker, J. W.; Konieczynska, M. D.; Stephenson, C. R. J. J. Am. Chem. Soc. 2011, 133, 4160-4163. 
151 (a) Rueping, M.; Vila, C.; Koenigs, R. M.; Poscharny, K.; Fabry, D. C. Chem. Commun. 2011, 47, 2360-2362. (b) Rueping, M.; Zhu, S.; Koenigs, R. M. Chem. Commun. 2011, 47, 8679-8681. (c) Hari, D. P.; König, B. Org. Lett. 2011, 13, 38523855. (d) Pham, P. V.; Nagib, D. A.; MacMillan, D. W. C. Angew. Chem. Int. Ed. 2011, 50, 6119-6122. (e) Tucker, J. W.; Narayanam, J. M. R.; Shah, P. S.; Stephenson, C. R. J. Chem. Commun. 2011, 47, 5040-5042. (f) Dai, C.; Narayanam, J. M. R.; Stephenson, C. R. J. Nat. Chem. 2011, 3, 140-145.

152 Condie, A. G.; González-Gómez, J. C.; Stephenson, C. R. J. J. Am. Chem. Soc. 2010, $132,1464-1465$.

153 (a) Sun, X.; Sengupta, S.; Petersen, J. L.; Wang, H.; Lewis, J. P.; Shi, X. Org. Lett. 2007, 9, 4495-4498. (b) Duan, H.; Sun, X.; Liao, W.; Petersen, J. L.; Shi, X. Org. Lett. 2008, 10, 4113-4116. (c) Zhong, C.; Chen, Y.; Petersen, J. L.; Akhmedov, N.; Shi, X. Angew. Chem. Int. Ed. 2009, 48, 1279-1282. (d) Chen, Y.; Zhong, C.; Petersen, J. L.; Akhmedov, N.; Shi, X. Org. Lett. 2009, 11, 2333-2336. (e) Chen, Y.; Zhong, C.; Sun, X.; Akhmedov, N.; Petersen, J. L.; Shi, X. Chem. Commun. 2009, 5150-5152. (f) Zhong, C.; Liao, T.; Tuguldur, O.; Shi, X. Org. Lett. 2010, 12, 20642067. (g) Zhong, C.; Gautam, L. N. S.; Wang, D.; Akhmedov, N.; Petersen, J. L.; Shi, X. Tetrahedron 2011, 67, 4402-4411.

154Cotton, F. A.; Wilkinson, G. Advanced Inorganic Chemistry, 4th ed.; Verlag Chemie: Weinheim, 1982; p 767.

155 (a) Zettler, M. W. In Encyclopedia of Reagents for Organic Synthesis; Paquette, L., Ed.; Wiley: New York, 1995; Vol. 4, p 2871. (b) White, A. D. In Encyclopedia of 
Reagents for Organic Synthesis; Paquette, L., Ed.; Wiley: New York, 1995; Vol. 4, p 2873.

156 Encyclopedia of Inorganic Chemistry; King, B. R., Ed.; Wiley: New York, 1994; Vol. 4.

157 (a) Trost, B. M.; Lee, C. B. J. Am. Chem. Soc. 2001, 123, 3671-3686. (b) Dell'Anna, M. M.; Gallo, V.; Mastrorilli, P.; Nobile, C. F.; Romanazzi, G.; Suranna, G. P. Chem. Commun. 2002, 434-435. (c) He, W.; Sun, X.; Frontier, A. J. J. Am. Chem. Soc. 2003, 125, 14278-14279. (d) Christoffers, J.; Baro, A. Angew. Chem. Int. Ed. 2003, 42, 1688-1690. (e) Watahiki, T.; Akabane, Y.; Mori, S.; Oriyama, T. Org. Lett. 2003, 5, 3045-3048. (f) Ji, S. J.; Zhou, M. F.; Gu, D. G.; Jiang, Z. Q.; Loh, T. P. Eur. J. Org. Chem. 2004, 1584-1587.

158 (a) Barrett, I. C.; Langille, J. D.; Kerr, M. A. J. Org. Chem. 2000, 65, 6268-6269. (b) Dohle, W.; Kopp, F.; Cahiez, G.; Knochel, P. Synlett 2001, 1901-1904. (c) Trost, B. M.; Crawley, M. L. Chem. Rev. 2003, 103, 2921-2943. (d) Hölzer, B.; Hoffmann, R. W. Chem. Commun. 2003, 732-733. (e) Nakamura, M.; Matsuo, K.; Ito, S.; Nakamura, E. J. Am. Chem. Soc. 2004, 126, 3686-3687. (f) Nagano, T.; Hayashi, T. Org. Lett. 2004, 6, 1297-1299. (g) Shinokubo, H.; Oshima, K. Eur. J. Org. Chem. 2004, 2081-2091.

159 (a) Cann, K.; Cole, T.; Slegeir, W.; Pettit, R. J. Am. Chem. Soc. 1978, 100, 39693971. (b) Ragaini, F.; Song, J. S.; Ramage, D. L.; Geoffroy, G. L. Organometallics 1995, 14, 387-400. (c) Boothroyd, S. R.; Kerr, M. A. Tetrahedron Lett. 1995, 36, 2411-2414. (d) Ragaini, F. Organometallics 1996, 15, 3572-3578. (e) Fakhfakh, M. 
A.; Franck, X.; Hocquemiller, R.; Figadère, B. J. Organomet. Chem. 2001, 624, 131135.

160 (a) Murahashi, S. I.; Oda, Y.; Naota, T. Tetrahedron Lett. 1992, 33, 7557-7560. (b) Kim, S. S.; Nehru, K.; Kim, S. S.; Kim, D. W.; Jung, H. C. Synthesis 2002, 24842486. (c) Mekmouche, Y.; Hummel, H.; Ho, R. Y. N.; Que, L., Jr.; Schünemann, V.; Thomas, F.; Trautwein, A. X.; Lebrun, C.; Gorgy, K.; Leprétre, J. C.; Collomb, M. N.; Deronzier, A.; Fontecave, M.; Ménage, S. Chem. Eur. J. 2002, 8, 1196-1204. (d) Baciocchi, E.; Gerini, M. F.; Lapi, A. J. Org. Chem. 2004, 69, 3586-3589. (e) Legros, J.; Bolm, C. Angew. Chem. Int. Ed. 2004, 43, 4225-4228.

161 (a) Inoue, H.; Sato, M. J. Chem. Soc., Chem. Commun. 1983, 983-984. (b) Miller, M. E.; Grant, E. R. J. Am. Chem. Soc. 1984, 106, 4635-4636. (c) Nagorski, H.; Mirbach, M. J. J. Organomet. Chem. 1985, 291, 199-204. (d) Subba Rao, G. S. R.; Sundar, N. S. J. Chem. Res. (S) 1979, 282-283. (e) Hoshino, Y.; Tanaka, H.; Takeno, N. Bull. Chem. Soc. Jpn. 1998, 71, 2923-2928.

162 (a) Kündig, E. P.; Saudan, C. M.; Viton, F. Adv. Synth. Catal. 2001, 343, 51-56. (b) Chavan, S. P.; Sharma, A. K. Synlett 2001, 667-669. (c) Ohara, H.; Itoh, T.; Nakamura, M.; Nakamura, E. Chem. Lett. 2001, 624-625. (d) Viton, F.; Bernardinelli, G.; Kündig, E. P. J. Am. Chem. Soc. 2002, 124, 4968-4969. (e) Edulji, S. K.; Nguyen, S. T. Organometallics 2003, 22, 3374-3381. (f) Heuss, B. D.; Mayer, M. F.; Dennis, S.; Hossain, M. M. Inorg. Chim. Acta 2003, 342, 301-304.

163 (a) Schroeder, M. A.; Wrighton, M. S. J. Am. Chem. Soc. 1976, 98, 551-558. (b) Iranpoor, N.; Mottaghinejad, E. J. Organomet. Chem. 1992, 423, 399-404. (c) Whetten, R. L.; Fu, K. J.; Grant, E. R. J. Am. Chem. Soc. 1982, 104, 4270-4272. (d) 
Long, G. T.; Weitz, E. J. Am. Chem. Soc. 2000, 122, 1431-1442. (e) Cherkaoui, H.;

Soufiaoui, M.; Grée, R. Tetrahedron 2001, 57, 2379-2383.

164 (a) Picione, J.; Mahmood, S. J.; Gill, A.; Hilliard, M.; Hossain, M. M. Tetrahedron Lett. 1998, 39, 2681-2684. (b) Ohara, H.; Kudo, K.; Itoh, T.; Nakamura, M.; Nakamura, E. Heterocycles 2000, 52, 505-510. (c) Taber, D. F.; Kanai, K.; Jiang, Q.; Bui, G. J. Am. Chem. Soc. 2000, 122, 6807-6808.

165 (a) Deng, L.; Margl, P.; Ziegler, T. J. Am. Chem. Soc. 1999, 121, 6479-6487. (b) Chen, X. P.; Qiu, K. Y. Chem. Commun. 2000, 1403-1404. (c) Khoroshun, D. V.; Musaev, D. G.; Vreven, T.; Morokuma, K. Organometallics 2001, 20, 2007-2026. (d) Matyjaszewski, K.; Xia, J. Chem. Rev. 2001, 101, 2921-2990. (e) Kamigaito, M.; Ando, T.; Sawamoto, M. Chem. Rev. 2001, 101, 3689-3745. (f) Kim, S. S.; Nehru, K.; Kim, S. S.; Kim, D. W.; Jung, H. C. Synthesis 2002, 2484-2486. (g) O’Reilly, R. K.; Gibson, V. C.; White, A. J. P.; Williams, D. J. J. Am. Chem. Soc. 2003, 125, $8450-8451$.

166 (a) Füerstner, A.; Leitner, A. Angew. Chem. Int. Ed. 2002, 41, 609-612. (b) Füerstner, A.; Mendez, M. Angew. Chem. Int. Ed. 2003, 42, 5355-5357. (c) Seidel, G.; Laurich, D.; Füerstner, A. J. Org. Chem. 2004, 69, 3950-3952. (d) Martin, R.; Füerstner, A. Angew. Chem. Int. Ed. 2004, 43, 3955-3957. (e) Füerstner, A.; Martin, R. Chem. Lett. 2005, 34, 624-629. (f) Sherry, B. D.; Füerstner, A. Acc. Chem. Res. 2008, 41, 1500 1511.

167 (a) Fürstner, A.; Martin, R.; Majima, K. J. Am. Chem. Soc. 2005, 127, 12236-12237. (b) Fürstner, A.; Majima, K.; Martin, R.; Krause, H.; Kattnig, E.; Goddard, R.; Lehmann, C. W. J. Am. Chem. Soc. 2008, 130, 1992-2004. 
168 For a review on the $\mathrm{C}-\mathrm{H}$ activation, see: (a) Dyker, G. Handbook of $\mathrm{C}-\mathrm{H}$ Transformations. Applications in Organic Synthesis; Wiley-VCH: Weinheim, 2005. (b) Jones, W.; Fehe, F. Acc. Chem. Res. 1989, 22, 91-100. (c) Labinger, J. A.; Bercaw, J. E. Nature 2002, 417, 507-514. (d) Godula, K.; Sames, D. Science 2006, 312, 67-72. (e) Bergman, R. G. Nature 2007, 446, 391-393.

169 (a) Claussen, C. A.; Long, E. C. Chem. Rev. 1999, 99, 2797-2816. (b) Cohen, S. M. Curr. Opin. Chem. Biol. 2007, 11, 151-158.

170 Sun, C. L.; Li, B. J.; Shi, Z. J. Chem. Rev. 2011, 111, 1293-1314.

171 For a review on Gif chemistry, please see: (a) Knight, C.; Perkins, M. J. Chem. Commun. 1991, 925-927. (b) Barton, D. H. R.; Doller, D. Acc. Chem. Res. 1992, 25, 504-512. (c) Barton, D. H. R. Chem. Soc. Rev. 1996, 25, 237-239. (d) Perkins, M. J. Chem. Soc. Rev. 1996, 25, 229-236. (e) Barton, D. H. R. Tetrahedron 1998, 54, 5805-5817. (f) Stavropoulos, P.; Çelenligil-Çetin, R.; Tapper, A. E. Acc. Chem. Res. 2001, 34, 745-752.

172 For a review on Fenton chemistry, please see: (a) Sawyer, D. T.; Sobkowiak, A.; Matsushita, T. Acc. Chem. Res. 1996, 29, 409-416. (b) Walling, C. Acc. Chem. Res. 1998, 31, 155-157. (c) MacFaul, P. A.; Wayner, D. D. M.; Ingold, K. U. Acc. Chem. Res. 1998, 31, 159-162. (d) Goldstein, S.; Meyerstein, D. Acc. Chem. Res. 1999, 32, $547-550$.

173 (a) Fontecave, M.; Ménage, S.; Duboc-Toia, C. Coord. Chem. Rev. 1998, 178-180, 1555-1572. (b) Costas, M.; Chen, K.; Que, L., Jr. Coord. Chem. Rev. 2000, 200-202, 517-544. (c) Costas, M.; Mehn, M. P.; Jensen, M. P.; Que, L., Jr. Chem. Rev. 2004, 104, 939-986. (d) Tshuva, E. Y.; Lippard, S. J. Chem. Rev. 2004, 104, 987-1012. 
174 (a) Korte, A.; Legros, J.; Bolm, C. Synlett 2004, 2397-2399. (b) Bryliakov, K. P.; Talsi, E. P. Angew. Chem. Int. Ed. 2004, 43, 5228-5230. (c) Legros, J.; Bolm, C. Chem. Eur. J. 2005, 11, 1086. (d) Egami, H.; Katsuki, T. J. Am. Chem. Soc. 2007, $129,8940-8941$.

175 (a) Rose, E.; Ren, Q. Z.; Andrioletti, B. Chem. Eur. J. 2004, 10, 224-230. (b) Gelalcha, F. G.; Bitterlich, B.; Anilkumar, G.; Tse, M. K.; Beller, M. Angew. Chem. Int. Ed. 2007, 46, 7293-7296. (c) Anilkumar, G.; Bitterlich, B.; Gelalcha, F. G.; Tse, M. K.; Beller, M. Chem. Commun. 2007, 289-291.

176 (a) Oldenburg, P. D.; Shteinman, A. A.; Que, L., Jr. J. Am. Chem. Soc. 2005, 127, 15672-15673. (b) Bukowski, M. R.; Comba, P.; Lienke, A.; Limberg, C.; de Laorden, C. L.; Mas-Ballesté, R.; Merz, M.; Que, L., Jr. Angew. Chem. Int. Ed. 2006, 45, 3446-3449. (c) Oldenburg, P. D.; Ke, C. Y.; Tipton, A. A.; Shteinman, A. A.; Que, L., Jr. Angew. Chem. Int. Ed. 2006, 45, 7975-7978. (d) Company, A.; G omez, L.; Fontrodona, X.; Ribas, X.; Costas, M. Chem. Eur. J. 2008, 14, 57275731.

177 (a) Chen, M. S.; White, M. C. Science 2007, 318, 783-787. (b) Vermeulen, N. A.; Chen, M. S.; White, M. C. Tetrahedron 2009, 65, 3078-3084. (c) Chen, M. S.; White, M. C. Science 2010, 327, 566-571.

178 (a) Fridovich, I. Science 1978, 201, 875-880. (b) Han, S.; Eltis, L. D.; Timmis, K. N.; Muchmore, S. W.; Bolin, J. T. Science 1995, 270, 976-980. (c) Roach, P. L.; Clifton, I. J.; Hensgens, C. M. H.; Shibata, N.; Schofield, C. J.; Hajdu, J.; Baldwin, J. E. Nature 1997, 387, 827-830. (d) Ford, P. C.; Fernandez, B. O.; Lim, M. D. Chem. Rev. 2005, 105, 2439-2455. 
179 (a) Trost, B. M. Science 1991, 254, 1471-1477. (b) Nicolaou, K. C.; Dai, W. M.; Tsay, S. C.; Estevez, V. A.; Wrasidlo, W. Science 1992, 256, 1172-1178. (c) Layton, M. E.; Morales, C. A.; Shair, M. D. J. Am. Chem. Soc. 2002, 124, 773-775. (d) Frstner, A.; Turet, L. Angew. Chem. Int. Ed. 2005, 44, 3462-3466. (e) Cho, E. J.; Lee, D. Org. Lett. 2008, 10, 257-259. (f) Werness, J. B.; Tang, W. Org. Lett. 2011, $13,3664-3666$.

180 (a) Hwang, G. T.; Son, H. S.; Ku, J. K.; Kim, B. H. J. Am. Chem. Soc. 2003, 125, 11241-11248. (b) Liu, Y.; Nishiura, M.; Hou, Z. M. J. Am. Chem. Soc. 2006, 128, 5592-5593. (c) Pilzak, G. S.; Van Gruijthuijsen, K.; Van Doorn, R. H.; Van Lagen, B.; Sudhölter, E. J. R.; Zuilhof, H. Chem. Eur. J. 2009, 15, 9085-9096. (d) Pasquini, C.; Bassetti, M. Adv. Synth. Catal. 2010, 352, 2405-2410. (e) Cao, Z.; Ren, T. Organometallics 2011, 30, 245-250.

181 (a) Saito, S.; Yamamoto, Y. Chem. Rev. 2000, 100, 2901-2916. (b) Kang, B.; Kim, D. H.; Do, Y.; Chang, S. Org. Lett. 2003, 5, 3041-3043. (c) Zhang, L. M.; Wang, S. Z. J. Am. Chem. Soc. 2006, 128, 1442-1443. (d) Geny, A.; Gaudrel, S.; Slowinsky, F.; Amatore, M.; Choraqui, G.; Malacria, M.; Aubert, C.; Gandon, V. Adv. Synth. Catal. 2009, 351, 271-275. (e) Yu, X. Z.; Du, B.; Wang, K.; Zhang, J. L. Org. Lett. 2010, 12, 1876-1879. (f) Chen, Z. W.; Huang, G.; Jiang, H. F.; Huang, H. W.; Pan, X. Y. J. Org, Chem. 2011, 76, 1134-1139. (g) Tomida, Y.; Nagaki, A.; Yoshida, J. J. Am. Chem. Soc. 2011, 133, 3744-3747.

182 (a) Rubina, M.; Gevorgyan, V. J. Am. Chem. Soc. 2001, 123, 11107-11108. (b) Yang, C.; Nolan, S. P. J. Org. Chem. 2002, 67, 591-593. (c) Nishiura, M.; Hou, Z.; Makatsuki, Y.; Yamaki, T.; Miyamoto, T. J. Am. Chem. Soc. 2003, 125, 1184-1185. 
(d) Ogoshi, S.; Ueta, M.; Oka, M.; Kurosawa, H. Chem. Commun. 2004, 2732-2733.

(e) Katayama, H.; Nakayama, M.; Nakano, T.; Wada, C.; Akamatsu, K.; Ozawa, F. Macromolecules 2004, 37, 13-17. (f) Komeyama, K.; Kawabata, T.; Takehira, K.; Takaki, K. J. Org. Chem. 2005, 70, 7260-7266. (g) Chen, X.; Xue, P.; Sung, H. H. Y.; Williams, I. D.; Peruzzini, M.; Bianchini, C.; Jia, G. Organometallics 2005, 24, 4330-4332. (h) Lee, C. C.; Lin, Y. C.; Liu, Y. H.; Wang, Y. Organometallics 2005, 24, 136-143. (i) Katayama, H.; Yari, H.; Tanaka, M.; Ozawa, F. Chem. Commun. 2005, 4336-4338. (j) Bassetti, M.; Pasquini, C.; Raneri, A.; Rosato, D. J. Org. Chem. 2007, 72, 4558-4561.

183 (a) Stang, P. J.; Kitamura, T. J. Am. Chem. Soc. 1987, 109, 7561-7563. (b) Kang, S. K.; Kim, W. Y.; Jiao, X. Synthesis 1998, 1252-1254. (c) Silveira, C. C.; Braga, A. L.; Vieira, A. S.; Zeni, G. J. Org. Chem. 2003, 68, 662-665. (d) Hoshi, M.; Nakayabu, H.; Shirakawa, K. Synthesis 2005, 1991-2007.

184 (a) Sonogashira, K.; Tohda, Y.; Hagihara, N. Tetrahedron Lett. 1975, 4467-4470. (b) Doucet, H.; Hierso, J. C. Angew. Chem. Int. Ed. 2007, 46, 834-871. (c) Chinchilla, R.; Najera, C. Chem. Rev. 2007, 107, 874-922. (d) Silva, S.; Sylla, B.; Suzenet, F.; Tatibouet, A.; Pauter, A. P.; Rollin, P. Org. Lett. 2008, 10, 853-856. (e) Torborg, C.; Beller, M. Adv. Synth. Catal. 2009, 351, 3027-3043. (f) Chinchilla, R.; Nájera, C. Chem. Soc. Rev. 2011, 40, 5084-5121.

185 For other methods of conjugated enyne synthesis, see: a) Dash, A. K.; Eisen, M. S. Org. Lett. 2000, 2, 737-740. (b) Shi, J. C.; Zeng, X.; Negishi, E. I. Org. Lett. 2003, 5, 1825-1828. (c) Kang, B.; Kim, D. H.; Do, Y.; Chang, S. Org. Lett. 2003, 5, 3041 3043. (d) Saitoh, H.; Ishida, N.; Satoh, T. Tetrahedron Lett. 2010, 51, 633-637. (e) 
Sakai, N.; Komatsu, R.; Uchida, N.; Ikeda, R.; Konakahara, T. Org. Lett. 2010, 12, $1300-1303$.

186 (a) Dieter, R. K.; Chen, N. Y.; Yu, H. Y.; Nice, L. E.; Gore, V. K. J. Org. Chem. 2005, 70, 2109-2119. (b) Yoshida, M.; Hayashi, M.; Shishido, K. Org. Lett. 2007, 9, 1643-1646. (c) Huestis, M. P.; Chan, L.; Stuart, D. R.; Fagnou, K. Angew. Chem. Int. Ed. 2011, 50, 1338-1341.

187 (a) Yamauchi, Y.; Onodera, G.; Sakata, K.; Yuki, M.; Miyake, Y.; Uemura, S.; Nishibayashi, Y. J. Am. Chem. Soc. 2007, 129, 5175-5179. (b) Xiao, H. Q.; Shu, X. Z.; Ji, K. G.; Qi, C. Z.; Liang, Y. M. New J. Chem. 2007, 31, 2041-2043. (c) Mothe, S. R.; Chan, P. W. H. J. Org. Chem. 2009, 74, 5887-5893.

188 Liao, W.; Chen, Y.; Duan, H.; Liu, Y.; Petersen, J. L.; Shi, X. Chem. Commun. 2009, 6436-6438.

189 The Ru(II) based catalyst has been used for propargyl alcohol dehydration, but only limited to terminal alkyne: Cadierno, V.; García-Garrido, S. E.; Gimeno, J. Adv. Syn. Cat. 2006, 348, 101-110.

190 (a) Harding, K. E.; Tiner, T. H. In Comprehensive Organic Synthesis; Trost, B. M., Fleming, I., Eds.; Pergamon Press: Oxford, U.K., 1991; Vol. 4, p 363. (b) Rodriguez, J.; Dulcere, J. P. Synthesis 1993, 1177-1205.

191 For reviews on halolactonization, see: (a) Dowle, M. D.; Davies, D. I. Chem. Soc. Rev. 1979, 8, 171-197. (b) Ranganathan, S.; Muraleedharan, K. M.; Vaish, N. K.; Jayaraman, N. Tetrahedron 2004, 60, 5273-5308.

192 (a) Evans, P. A.; Murthy, V. S.; Roseman, J. D.; Rheingold, A. L. Angew. Chem. Int. Ed. 1999, 38, 3175-3177. (b) Crimmins, M. T.; Tabet, E. A. J. Am. Chem. Soc. 2000, 
122, 5473-5476. (c) Boukouvalas, J.; Pouliot, M.; Robichaud, J.; MacNeil, S.;

Snieckus, V. Org. Lett. 2006, 8, 3597-3599. (d) Sabot, C.; Berard, D.; Canesi, S. Org. Lett. 2008, 10, 4629-4632. (e) Braddock, D. C.; Bhuva, R.; Perez-Fuertes, Y.; Pouwer, R.; Roberts, C. A.; Ruggiero, A.; Stokes, E. S. E.; White, A. J. P. Chem. Commun. 2008, 1419-1421.

193 For recent synthetic applications of bromoallenes, see: (a) Ohno, H.; Hamaguchi, H.; Ohata, M.; Kosaka, S.; Tanaka, T. J. Am. Chem. Soc. 2004, 126, 8744-8754. (b) Xu, B.; Hammond, G. B. Angew. Chem. Int. Ed. 2005, 44, 7404-7407. (c) Hamaguchi, H.; Kosaka, S.; Ohno, H.; Tanaka, T. Angew. Chem. Int. Ed. 2005, 44, 1513-1517. (d) Shen, L. C.; Hsung, R. P.; Zhang, Y. S.; Antoline, J. E.; Zhang, X. J. Org. Lett. 2005, 7, 3081-3084. (e) Vaz, B.; Dominguez, M.; Alvarez, R.; de Lera, A. R. Chem. Eur. J. 2007, 13, 1273-1290. (f) Xia, Y. Z.; Dudnik, A. S.; Gevorgyan, V.; Li, Y. H. J. Am. Chem. Soc. 2008, 130, 6940-6941.

194 (a) Zhang, W.; Werness, J. B.; Tang, W. Org. Lett. 2008, 10, 2023-2026. (b) Zhang, W.; Xu, H. D.; Xu, H.; Tang, W. J. Am. Chem. Soc. 2009, 131, 3832-3833. (c) Zhang, W.; Zheng, S.; Liu, N.; Werness, J. B.; Guzei, I. A.; Tang, W. J. Am. Chem. Soc. 2010, 132, 3664-3665.

195 (a) Li, Z.; Cao, L.; Li, C. J. Angew. Chem. Int. Ed. 2007, 46, 6505-6507. (b) Wang, X. F.; Dong, Y. X.; Wittlin, S.; Creek, D.; Chollet, J.; Charman, S. A.; Tomas, J. S.; Scheurer, C.; Snyder, C.; Vennerstrom, J. L. J. Med. Chem. 2007, 50, 5840-5847. (c) DeMartino, M. P.; Chen, K.; Baran, P. S. J. Am. Chem. Soc. 2008, 130, 11546-11560. (d) Rao, W.; Zhang, X.; Sze, E. M. L.; Chan, P. W. H. J. Org. Chem. 2009, 74, 1740-1743. 
196 (a) Reissig, H.; Zimmer, R. Chem. Rev. 2003, 103, 1151-1196. (b) Xu, G. C.; Liu, L. P.; Lu, J. M.; Shi, M. J. Am. Chem. Soc. 2005, 127, 14552-14553. (c) Zheng, X. M.; Kerr, M. A. Org. Lett. 2006, 8, 3777-3779. (d) Grant, T. N.; West, F. G. J. Am. Chem. Soc. 2006, 128, 9348-9349. (e) Perreault, C.; Goudreau, S. R.; Zimmer, L. E.; Charette, A. B. Org. Lett. 2008, 10, 689-692. (f) Hu, B.; Xing, S. Y.; Wang, Z. W. Org. Lett. 2008, 10, 5481-5484.

197 (a) Kulinkovich, O. G. Chem. Rev. 2003, 103, 2597-2632. (b) Booker-Milburn, K. I.; Jones, J. L.; Sibley, G. E.; Cox, M. R.; Meadows, J. Org. Lett. 2003, 5, 1107-1109. (c) Keaton, K. A.; Phillips, A. J. Org. Lett. 2007, 9, 2717-2719. (d) Noda, D.; Sunada, Y.; Hatakeyama, T.; Nakamura, M.; Nagashima, H. J. Am. Chem. Soc. 2009, 131, 6078-6079. (e) Fürstner, A.; Martin, R.; Krause, H.; Seidel, G.; Goddard, R.; Lehmann, C. W. J. Am. Chem. Soc. 2008, 130, 8773-8787. (f) Van Humbeck, J. F.; Simonovich, S. P.; Knowles, R. R.; MacMillan, D. W. C. J. Am. Chem. Soc. 2010, 132, 10012-10014.

198 For selected recent reviews: (a) Saha, S.; Stoddart, J. F. Chem. Soc. Rev. 2007, 36, 77-92. (b) Sakai, N.; Sisson, A. L.; Burgi, T.; Matile, S. J. Am. Chem. Soc. 2007, 129, 15758-15759. (c) Diaz, D. D.; Cid, J. J.; Vazquez, P.; Torres, T. Chem. Eur. J. 2008, 14, 9261-9273. (d) Armstrong, N. R.; Veneman, P. A.; Ratcliff, E.; Placencia, D.; Brumbach, M. Acc. Chem. Res. 2009, 42, 1748-1757. (e) Singhal, K.; Kalkan, A. K. J. Am. Chem. Soc. 2010, 132, 429-431. (f) Nguyen, B. T.; Anslyn, E. V. Coord. Chem. Rev. 2006, 250, 3118-3127.

199 For selected recent examples: (a) Medintz, I. L.; Uyeda, H. T.; Goldman, E. R.; Mattoussi, H. Nat. Mater. 2005, 4, 435-446. (b) Johnsson, N.; Johnsson, K. ACS 
Chem. Biol. 2007, 2, 31-38. (c) Low, P. S.; Henne, W. A.; Doorneweerd, D. D. Acc.

Chem. Res. 2008, 41, 120-129. (d) Takaoka, Y.; Sakamoto, T.; Tsukiji, S.; Narazaki, M.; Matsuda, T.; Tochio, H.; Shirakawa, M.; Hamachi, I. Nat. Chem. 2009, 1, $557-$ 561. (e) Jayagopal, A.; Halfpenny, K. C.; Perez, J. W.; Wright, D. W. J. Am. Chem. Soc. 2010, 132, 9789-9796. (f) Yang, Y.; Seidlits, S. K.; Adams, M. M.; Lynch, V. M.; Schmidt, C. E.; Anslyn, E. V.; Shear, J. B. J. Am. Chem. Soc. 2010, 132, 1311413116.

200 For selected recent examples: (a) Sohn, Y. S.; Goodey, A.; Anslyn, E. V.; McDevitt, J. T.; Shear, J. B.; Neikirk, D. P. Biosens. Bioelectron. 2005, 21, 303-312. (b) Khong, S. H.; Sivaramakrishnan, S.; Png, R. Q.; Wong, L. Y.; Chia, P. J.; Chua, L. L.; Ho, P. K. H. Adv. Funct. Mater. 2007, 17, 2490-2499. (c) Tian, E. T.; Wang, J. X.; Zheng, Y. M.; Song, Y. L.; Jiang, L.; Zhu, D. B. J. Mater. Chem. 2008, 18, 1116-1122. (d) Ensafi, A. A.; Far, A. K.; Meghdadi, S. J. Hazard. Mater. 2009, 172, 1069-1075. (e) Zhao, Y. S.; Zhan, P.; Kim, J.; Sun, C.; Huang, J. X. Acs Nano 2010, 4, 1630-1636. (f) Sparano, B. A.; Koide, K. J. Am. Chem. Soc. 2007, 129, 47854794.

201 (a) Yan, D. C.; Mohsseni-Ala, J.; Auner, N.; Bolte, M.; Bats, J. W. Chem. Eur. J. 2007, 13, 7204-7214. (b) Zhang, X. Y.; Matsuo, Y.; Nakamura, E. Org. Lett. 2008, 10, 4145-4147. (c) Dan-Hardi, M.; Serre, C.; Frot, T.; Rozes, L.; Maurin, G.; Sanchez, C.; Ferey, G. J. Am. Chem. Soc. 2009, 131, 10857-10859. (d) Wahab, M. A.; Hussain, H.; He, C. Langmuir 2009, 25, 4743-4750. (e) Mercs, L.; Albrecht, M. Chem. Soc. Rev. 2010, 39, 1903-1912.

202 (a) Muller, C. D.; Falcou, A.; Reckefuss, N.; Rojahn, M.; Wiederhirn, V.; Rudati, P.; 
Frohne, H.; Nuyken, O.; Becker, H.; Meerholz, K. Nature 2003, 421, 829-833. (b)

Kuo, J. S.; Kuyper, C. L.; Allen, P. B.; Fiorini, G. S.; Chiu, D. T. Electrophoresis 2004, 25, 3796-3804. (c) Liu, X. M.; He, C. B.; Huang, J. C.; Xu, J. M. Chem. Mater. 2005, 17, 434-441. (d) Tsai, M. H.; Hong, Y. H.; Chang, C. H.; Su, H. C.; Wu, C. C.; Matoliukstyte, A.; Simokaitiene, J.; Grigalevicius, S.; Grazulevicius, J. V.; Hsu, C. P. Adv. Mater. 2007, 19, 862-866.

203 (a) Gaal, M.; List, E. J. W.; Scherf, U. Macromolecules 2003, 36, 4236-4237. (b) Zhang, H. C.; Guo, E. Q.; Fang, Y. J.; Ren, P. H.; Yang, W. J. J. Polym. Sci., Part A: Polym. Chem. 2009, 47, 5488-5497.

204 (a) Fukuda, Y.; Watanabe, T.; Wakimoto, T.; Miyaguchi, S.; Tsuchida, M. Synthetic Met. 2000, 111-112, 1-6. (b) Sugimoto, A.; Ochi, H.; Fujimura, S.; Yoshida, A.; Miyadera, T.; Tsuchida, M. Ieee J. Sel. Top. Quant. 2004, 10, 107-114. (c) Zhou, L.; Park, S.; Bai, B.; Sun, J.; Wu, S. C.; Jackson, T. N.; Nelson, S.; Freeman, D.; Hong, Y. Ieee Electr. Device L. 2005, 26, 640-642. (d) Burn, P. L.; Lo, S. C.; Samuel, I. D. W. Adv. Mater. 2007, 19, 1675-1688. (e) Chang, C. H.; Cheng, H. C.; Lu, Y. J.; Tien, K. C.; Lin, H. W.; Lin, C. L.; Yang, C. J.; Wu, C. C. Org. Electron. 2010, 11, $247-$ 254.

205 (a) Caruso, F.; Donath, E.; Moehwald, H. J. Phys. Chem. B 1998, 102, 2011-2016. (b) Cotlet, M.; Gronheid, R.; Habuchi, S.; Stefan, A.; Barbafina, A.; Müllen, K.; Hofkens, J.; De Schryver, F. C. J. Am. Chem. Soc. 2003, 125, 13609-13617. (c) Cotlet, M.; Vosch, T.; Habuchi, S.; Weil, T.; Mullen, K.; Hofkens, J.; De Schryver, F. J. Am. Chem. Soc. 2005, 127, 9760-9768. (d) Kuzmenkina, E. V.; Heyes, C. D.; Nienhaus, G. U. Proc. Natl. Acad. Sci. U.S.A. 2005, 102, 15471-15476. (e) Clapp, A. 
R.; Medintz, I. L.; Mattoussi, H. ChemPhysChem 2006, 7, 47-57. (f) Mor, G. K.; Basham, J.; Paulose, M.; Kim, S.; Varghese, O. K.; Vaish, A.; Yoriya, S.; Grimes, C. A. Nano Lett. 2010, 10, 2387-2394.

206 (a) Tonzola, C. J.; Kulkarni, A. P.; Gifford, A. P.; Kaminsky, W.; Jenekhe, S. A. Adv. Funct. Mater. 2007, 17, 863-874. (b) Tong, Q. X.; Lai, S. L.; Chan, M. Y.; Zhou, Y. C.; Kwong, H. L.; Lee, C. S.; Lee, S. T. Chem. Mater. 2008, 20, 63106312. (c) Park, Y.; Lee, J. H.; Jung, D. H.; Liu, S. H.; Lin, Y. H.; Chen, L. Y.; Wu, C. C.; Park, J. J. Mater. Chem. 2010, 20, 5930-5936.

207 (a) Gomez, R.; Veldman, D.; Langeveld, B. M. W.; Segura, J. L.; Janssen, R. A. J. J. Mater. Chem. 2007, 17, 4274-4288. (b) Gomez, R.; Segura, J. L. Tetrahedron 2009, $65,540-546$.

208 Iamazaki, E. T.; Atvars, T. D. Z. Langmuir 2007, 23, 12886-12892.

209 (a) Yeh, A. T.; Shank, C. V.; McCusker, J. K. Science 2000, 289, 935-938. (b) Gong, X. O.; Iyer, P. K.; Moses, D.; Bazan, G. C.; Heeger, A. J.; Xiao, S. S. Adv. Funct. Mater. 2003, 13, 325-330.

210 (a) Goodpaster, J. V.; Harrison, J. F.; McGuffin, V. L. J. Phys. Chem. A 2002, 106, 10645-10654. (b) Focsaneanu, K. S.; Scaiano, J. C. Photoch. Photobio. Sci. 2005, 4, $817-821$

211 Gaussian 03 (Revision A.1), Frisch, M. J.; Trucks, G. W.; Schlegel, H. B.; Scuseria, G. E.; Robb, M. A.; Cheeseman, J. R.; Montgomery, Jr., J. A.; Vreven, T.; Kudin, K. N.; Burant, J. C.; Millam, J. M.; Iyengar, S. S.; Tomasi, J.; Barone, V.; Mennucci, B.; Cossi, M.; Scalmani, G.; Rega, N.; Petersson, G. A.; Nakatsuji, H.; Hada, M.; Ehara, M.; Toyota, K.; Fukuda, R.; Hasegawa, J.; Ishida, M.; Nakajima, T.; Honda, 
Y.; Kitao, O.; Nakai, H.; Klene, M.; Li, X.; Knox, J. E.; Hratchian, H. P.; Cross, J.

B.; Adamo, C.; Jaramillo, J.; Gomperts, R.; Stratmann, R. E.; Yazyev, O.; Austin, A. J.; Cammi, R.; Pomelli, C.; Ochterski, J. W.; Ayala, P. Y.; Morokuma, K.; Voth, G. A.; Salvador, P.; Dannenberg, J. J.; Zakrzewski, V. G.; Dapprich, S.; Daniels, A. D. ; Strain, M. C.; Farkas, O.; Malick, D. K.; Rabuck, A. D.; Raghavachari, K.; Foresman, J. B.; Ortiz, J. V.; Cui, Q.; Baboul, A. G.; Clifford, S.; Cioslowski, J.; Stefanov, B. B.; Liu, G.; Liashenko, A.; Piskorz, P.; Komaromi, I.; Martin, R. L.; Fox, D. J.; Keith, T.; Al-Laham, M. A.; Peng, C. Y.; Nanayakkara, A.; Challacombe, M.; Gill, P. M. W.; Johnson, B.; Chen, W.; Wong, M. W.; Gonzalez, C.; Pople, J. A. Gaussian, Inc., Pittsburgh, PA, 2003.

212 Silva-Junior, M. R.; Schreiber, M.; Sauer, S. P.; Thiel, W. J. Chem. Phys. 2008, 129, 104103/1-104103/14.

213 (a) Druzhinin, S. I.; Bursulaya, B. D.; Uzhinov, B. M. J. Photoch. Photobio. A 1995, 90, 53-56. (b) Zachariasse, K. A.; Grobys, M.; von der Haar, T.; Hebecker, A.; Il'ichev, Y. V.; Jiang, Y. B.; Morawski, O.; Khnle, W. J. Photoch. Photobio. A 1996, 102, 59-70.

214 Solvatochromism studies of compound 3.1 e, 3.2 a, 3.2 d, 3.2 e have been carried out in various solvents. Detailed experimental results are shown in the Supporting Information, where little changes were observed in different solvent systems, strongly supported the proposed PICT mechanism. For example, for compound 3.2 a, the quantum yield in different solvents are observed as following: in $\mathrm{MeOH} \Phi$ $=0.31$; in dichloromethane $\Phi=0.37$; in acetonitrile $\Phi=0.34$; in ethyl acetate $\Phi=$ 0.37 ; in THF $\Phi=0.34$. 


\section{Supporting information}

\section{Chapter One: Synthesis of functional 1,2,3-triazoles}

\subsection{Efficient synthesis of $\mathbf{N}$-2-aryl-1,2,3-triazole fluorophores via post-triazole arylation}

\section{General Information}

Unless otherwise noted all commercial materials and solvents were obtained from commercial provider and used without further purification. Air and/or moisture-sensitive reactions were carried out under an atmosphere of nitrogen using oven/flamed-dried glassware and standard syringe/septa techniques. ${ }^{1} \mathrm{H}$ and ${ }^{13} \mathrm{C}$ NMR spectra were recorded with $600 \mathrm{MHz} \mathrm{NMR}$ spectrometer. The Chemical shifts were reported relative to internal tetramethylsilane $(\delta 0.00$ ppm) or $\mathrm{CDCl} 3(\delta 7.26 \mathrm{ppm})$ or $\mathrm{DMSO}(\delta 2.49 \mathrm{ppm})$ for ${ }^{1} \mathrm{H}$ and $\mathrm{CDCl}_{3}(\delta 77.0 \mathrm{ppm})$ or DMSO

( $\delta 39.5 \mathrm{ppm}$ ) for ${ }^{13} \mathrm{C}$. Melting points were measured on a Mel-Temp 1001D apparatus and uncorrected. HRMS were recorded on LTQ-FTUHRA spectrometer. UV-visible spectra were obtained on a Shimadzu UV-2500. Fluorescence spectra for emission and excitation were obtained on a Hitachi 7000 .

$1 H$-benzo[ $[d][1,2,3]$ triazole (1.2.1a) is commercial available. 4-Phenyl- $2 H$-1,2,3-triazole (1.2.1b) was prepared according to Zard's procedure. ${ }^{1}$ Other N-H triazoles (1.2.1c, 1.2.1d, 1.2.1f-1.2.1j) were prepared according to our procedures. ${ }^{2,3}$ 


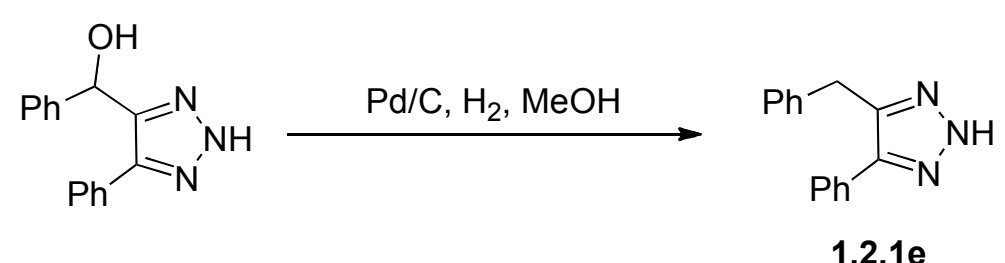

To a $100 \mathrm{~mL}$ round-bottom bottle was added phenyl(5-phenyl-2H-1,2,3-triazol-4-yl)methanol ${ }^{2}$ $(1.0 \mathrm{~g}), 10 \% \mathrm{Pd} / \mathrm{C}(0.4 \mathrm{~g})$ and methanol $(20 \mathrm{~mL})$. The bottle was capped and filled with hydrogen and the reaction mixture was stirred at room temperature for $2 \mathrm{~d}$. After $\mathrm{Pd} / \mathrm{C}$ was filtrated, the filtrate was concentrated. The residue was added water $(15 \mathrm{~mL})$ then extracted with ethyl acetate $(3 \times 15 \mathrm{~mL})$. The combined organic phases were washed with brine and dried over anhydrous $\mathrm{Na}_{2} \mathrm{SO}_{4}$. The solvent was removed under reduced pressure and flash silica gel chromatography (hexane-EtOAc, v/v = 4/1) gave the product. Yield 70\%, ${ }^{1} \mathrm{H}$ NMR $(600 \mathrm{MHz}$, $\left.\mathrm{CDCl}_{3}\right): \delta$ 7.62-7.59 (m, 2H), 7.41-7.33 (m, 3H), $7.27(\mathrm{t}, J=7.8 \mathrm{~Hz}, 2 \mathrm{H}), 7.22-7.17(\mathrm{~m}, 3 \mathrm{H})$, $4.24(\mathrm{~s}, 2 \mathrm{H}) ;{ }^{13} \mathrm{C} \mathrm{NMR}\left(125 \mathrm{MHz}, \mathrm{CDCl}_{3}\right): \delta 144.0,141.3,138.1,130.2,128.7,128.7,128.5$, $128.3,127.6,126.6,31.1$. 


\section{$\underline{\text { General procedure for } S_{N}} \underline{A \text { r reaction of triazole with halobenzene }}$}

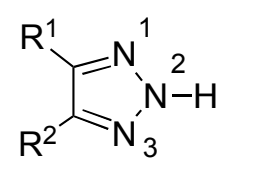

1.2.1

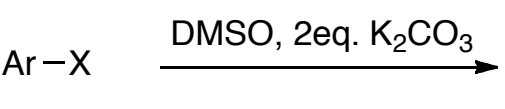

1.2.2

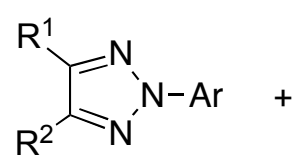

1.2.3

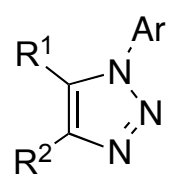

$\mathrm{N}-1-1.2 .3$

To a $50 \mathrm{~mL}$ round-bottom bottle was added triazole $(1 \mathrm{mmol})$, fluoro or chloro-substituted benzene $(1.5 \mathrm{mmol})$, potassium carbonate $(2 \mathrm{mmol})$ and DMSO $(2 \mathrm{~mL})$. The reaction mixture was stirred at room temperature (or heated up to $80{ }^{\circ} \mathrm{C}$ ) and monitored by TLC. After the reaction was completed, water $(20 \mathrm{~mL})$ was added. The mixture was extracted with ethyl acetate (3 $\times 30 \mathrm{~mL})$. The combined organic phases were washed with brine and dried with anhydrous $\mathrm{Na}_{2} \mathrm{SO}_{4}$. The solvent was removed under reduced pressure to get a residue, the residue was purified by recrystallization or flash silica gel chromatography gave the product. 
General procedure for microwave assisted Cu-catalyzed coupling of triazoles with $\underline{\text { aryliodide }}$

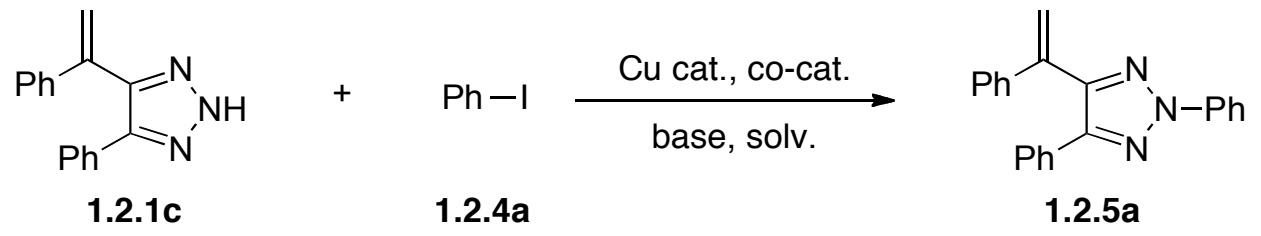

To $10 \mathrm{~mL}$ microwave tube was successively added 4-phenyl-5-(1-phenylvinyl)-2H-1,2,3-triazole (1a) $(98.8 \mathrm{mg}, 0.4 \mathrm{mmol})$, base $(0.8 \mathrm{mmol})$, copper catalyst $(0.04 \mathrm{mmol})$, ligand $(0.08 \mathrm{mmol})$, and iodobenzene $(125 \mathrm{mg}, 0.6 \mathrm{mmol})$ under $\mathrm{N}_{2}$, and then anhydrous solvent $(2 \mathrm{~mL})$ was added by syringes. The tube was put into microwave reactor and heated to certain temperature for 30 min. After cool, to the reaction mixture was added 1,3,5-trimethoxybenzene (15-20 mg, accurate to $0.1 \mathrm{mg})$, water $(15 \mathrm{~mL}), 10 \% \mathrm{HCl}$ solution $(1 \mathrm{~mL})$ and extracted with ethyl acetate $(2 \times 15$ $\mathrm{mL})$. The combined organic phases were washed with brine $(10 \mathrm{~mL})$, dried over anhydrous $\mathrm{Na}_{2} \mathrm{SO}_{4}$ and concentrated in vacuo. The residue was used to NMR analysis directly. The data were listed in Table S1 and Table S2. 
$\underline{\text { Screening reaction conditions }}$

Table S1.2-1: Screening of the reaction condition ${ }^{a}$

\begin{tabular}{|c|c|c|c|c|c|c|c|c|}
\hline Ent & $\operatorname{Cat}(10 \%)$ & $\begin{array}{l}\text { Co-cat } \\
(20 \%)\end{array}$ & $\begin{array}{c}\text { Base } \\
(2.5 \mathrm{eq})\end{array}$ & Sol & $\mathrm{T}\left({ }^{\circ} \mathrm{C}\right)$ & $\mathrm{t}(\mathrm{h})$ & Conv. ${ }^{b}$ & Yield $^{b}$ \\
\hline 1 & $\mathrm{CuI}$ & - & $\mathrm{K}_{2} \mathrm{CO}_{3}$ & DMSO & 110 & 24 & 0 & 0 \\
\hline 2 & $\mathrm{CuI}$ & L-proline & $\mathrm{K}_{2} \mathrm{CO}_{3}$ & DMSO & 110 & 24 & 100 & 71 \\
\hline 3 & $\mathrm{CuCl}$ & L-proline & $\mathrm{K}_{2} \mathrm{CO}_{3}$ & DMSO & 110 & 24 & 100 & 88 \\
\hline 4 & $\mathrm{CuCl}_{2}$ & L-proline & $\mathrm{K}_{2} \mathrm{CO}_{3}$ & DMSO & 110 & 24 & 64 & 42 \\
\hline 5 & $\mathrm{CuSO}_{4}$ & L-proline & $\mathrm{K}_{2} \mathrm{CO}_{3}$ & DMSO & 110 & 24 & 37 & 11 \\
\hline 6 & $\mathrm{Cu}(\mathrm{OAc})_{2}$ & L-proline & $\mathrm{K}_{2} \mathrm{CO}_{3}$ & DMSO & 110 & 24 & 92 & 79 \\
\hline 7 & $\mathrm{CuCl}$ & L-proline & $\mathrm{K}_{2} \mathrm{CO}_{3}$ & $\mathrm{DMF}$ & 110 & 24 & 85 & 74 \\
\hline 8 & $\mathrm{CuCl}$ & L-proline & $\mathrm{K}_{2} \mathrm{CO}_{3}$ & $\mathrm{CH}_{3} \mathrm{CN}$ & 110 & 24 & 73 & 60 \\
\hline 9 & $\mathrm{CuI}$ & L-proline & $\mathrm{K}_{2} \mathrm{CO}_{3}$ & DMSO & mw110 & 0.5 & 62 & 32 \\
\hline 10 & $\mathrm{CuCl}$ & L-proline & $\mathrm{K}_{2} \mathrm{CO}_{3}$ & DMSO & mw110 & 0.5 & 82 & 68 \\
\hline 11 & $\mathrm{CuCl}_{2}$ & L-proline & $\mathrm{K}_{2} \mathrm{CO}_{3}$ & DMSO & mw110 & 0.5 & 80 & 49 \\
\hline 12 & $\mathrm{CuSO}_{4}$ & L-proline & $\mathrm{K}_{2} \mathrm{CO}_{3}$ & DMSO & mw110 & 0.5 & low & trace \\
\hline 13 & $\mathrm{Cu}(\mathrm{OAc})_{2}$ & L-proline & $\mathrm{K}_{2} \mathrm{CO}_{3}$ & DMSO & mw110 & 0.5 & 81 & 51 \\
\hline 14 & $\mathrm{CuCl}$ & L-proline & $\mathrm{K}_{2} \mathrm{CO}_{3}$ & DMSO & mw140 & 0.5 & 96 & 87 \\
\hline 15 & $\mathrm{CuCl}$ & L-proline & $\mathrm{K}_{2} \mathrm{CO}_{3}$ & DMSO & mw160 & 0.5 & 98 & 92 \\
\hline 16 & $\mathrm{CuCl}$ & L-proline & $\mathrm{Cs}_{2} \mathrm{CO}_{3}$ & DMSO & mw160 & 0.5 & 84 & 55 \\
\hline 17 & $\mathrm{CuCl}$ & L-proline & $\mathrm{KOH}$ & DMSO & mw160 & 0.5 & 75 & 45 \\
\hline 18 & $\mathrm{CuCl}$ & L-proline & $\mathrm{K}_{2} \mathrm{CO}_{3}$ & $\mathrm{DMF}$ & mw160 & 0.5 & 87 & 64 \\
\hline 19 & $\mathrm{CuCl}$ & L-proline & $\mathrm{Cs}_{2} \mathrm{CO}_{3}$ & DMF & mw160 & 0.5 & 90 & 71 \\
\hline 20 & $\mathrm{CuCl}^{d}$ & L-proline $e^{e}$ & $\mathrm{~K}_{2} \mathrm{CO}_{3}$ & DMSO & mw160 & 0.5 & 82 & 73 \\
\hline $21^{\mathrm{c}}$ & $\mathrm{CuCl}$ & L-proline & $\mathrm{K}_{2} \mathrm{CO}_{3}$ & DMSO & mw160 & 0.5 & 40 & 10 \\
\hline $22^{c}$ & $\mathrm{CuCl}$ & L-proline & $\mathrm{Cs}_{2} \mathrm{CO}_{3}$ & DMSO & mw160 & 0.5 & 68 & 36 \\
\hline $23^{c}$ & $\mathrm{CuI}$ & L-proline & $\mathrm{Cs}_{2} \mathrm{CO}_{3}$ & DMSO & mw160 & 0.5 & 85 & 53 \\
\hline
\end{tabular}

${ }^{a}$ 1.2.1c:1.2.4a $=1: 1.5, \mathrm{C}=0.2 \mathrm{M} ;{ }^{b}$ Based on the consumption of 1.2.1c and formation of 1.2.5a using 1,3,5-trimethoxybenzene as internal standard; ${ }^{c}$ use $\mathrm{PhBr}$ instead of $\mathrm{PhI} ;{ }^{d}$ loading was decreased to $5 \%{ }^{e}$ loading was decreased to $10 \%$. 
Table S1.2-2: Ligand Screening ${ }^{a}$

\begin{tabular}{|c|c|c|c|c|c|c|c|c|}
\hline Ent & Cat(10\%) & $\begin{array}{l}\text { Co-cat } \\
(20 \%)\end{array}$ & $\begin{array}{c}\text { Base } \\
(2.5 \mathrm{eq})\end{array}$ & Sol & $\mathrm{T}\left({ }^{\circ} \mathrm{C}\right)$ & $\mathrm{t}(\mathrm{h})$ & Conv. ${ }^{b}$ & Yield $^{b}$ \\
\hline 12 & $\mathrm{CuCl}$ & L-proline & $\mathrm{K}_{2} \mathrm{CO}_{3}$ & DMSO & mw160 & 0.5 & 98 & 92 \\
\hline 24 & $\mathrm{CuCl}$ & pyrrolidine & $\mathrm{K}_{2} \mathrm{CO}_{3}$ & DMSO & mw160 & 0.5 & low & trace \\
\hline 25 & $\mathrm{CuCl}$ & glycine & $\mathrm{K}_{2} \mathrm{CO}_{3}$ & DMSO & mw160 & 0.5 & low & trace \\
\hline 26 & $\mathrm{CuCl}$ & Me-GLY & $\mathrm{K}_{2} \mathrm{CO}_{3}$ & DMSO & mw160 & 0.5 & 60 & 30 \\
\hline 27 & $\mathrm{CuCl}$ & DMG & $\mathrm{K}_{2} \mathrm{CO}_{3}$ & DMSO & mw160 & 0.5 & 98 & 86 \\
\hline 28 & $\mathrm{CuCl}$ & EDA & $\mathrm{K}_{2} \mathrm{CO}_{3}$ & DMSO & mw160 & 0.5 & 53 & 29 \\
\hline 29 & $\mathrm{CuCl}$ & DMEDA & $\mathrm{K}_{2} \mathrm{CO}_{3}$ & DMSO & mw160 & 0.5 & 70 & 47 \\
\hline 30 & $\mathrm{CuCl}$ & TMEDA & $\mathrm{K}_{2} \mathrm{CO}_{3}$ & DMSO & mw160 & 0.5 & low & trace \\
\hline 31 & $\mathrm{CuCl}$ & $\mathrm{DACH}$ & $\mathrm{K}_{2} \mathrm{CO}_{3}$ & DMSO & mw160 & 0.5 & 53 & 44 \\
\hline 32 & $\mathrm{CuCl}$ & bipy & $\mathrm{K}_{2} \mathrm{CO}_{3}$ & DMSO & mw160 & 0.5 & 47 & trace \\
\hline 33 & $\mathrm{CuCl}$ & $\mathrm{ABC}$ & $\mathrm{K}_{2} \mathrm{CO}_{3}$ & DMSO & mw160 & 0.5 & 40 & trace \\
\hline 34 & $\mathrm{CuCl}$ & DMABC & $\mathrm{K}_{2} \mathrm{CO}_{3}$ & DMSO & mw160 & 0.5 & 48 & 7 \\
\hline 35 & $\mathrm{CuCl}$ & $\mathrm{BDC}$ & $\mathrm{K}_{2} \mathrm{CO}_{3}$ & DMSO & mw160 & 0.5 & 50 & trace \\
\hline 36 & $\mathrm{CuCl}$ & PDC & $\mathrm{K}_{2} \mathrm{CO}_{3}$ & DMSO & mw160 & 0.5 & 32 & trace \\
\hline
\end{tabular}

${ }^{a}$ 1.2.1c:1.2.4a $=1: 1.5, \mathrm{C}=0.2 \mathrm{M} ;{ }^{b}$ Me-Gly: N-methyl glycine; DMG: N,N-dimethyl glycine; EDA: ethylenediamine; DMEDA: N,N'-dimethylethylenediamine; TMEDA: N,N,N',N'tetramethylethylenediamine; DACH: 1,2-trans-diaminocyclohexane; bipy: 2,2'-bipyiridine; ABC: 2-aminobenzoic acid; DMABC: 2-(dimethylamino)benzoic acid; DBC: 1,2-benzenedicarboxic acid; PDC: pyridine-2,6-dicarboxylic acid. 


\section{General procedure for microwave assisted Cu-catalyzed coupling of triazoles with}

\section{$\underline{\text { aryliodide }}$}

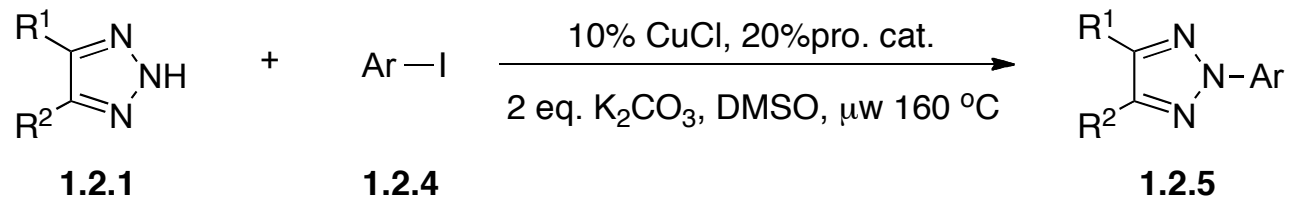

To $10 \mathrm{~mL}$ microwave tube was successively added $\mathrm{N}-\mathrm{H}$ triazole $(0.8 \mathrm{mmol})$, potassium carbonate (220 mg, $1.6 \mathrm{mmol})$, L-proline (18 mg, $0.16 \mathrm{mmol})$, copper (I) chloride (8 mg, 0.08 mmol) and iodobenzene $(250 \mathrm{mg}, 1.2 \mathrm{mmol})$ under $\mathrm{N}_{2}$, and then anhydrous solvent $(2 \mathrm{~mL})$ was added by syringes. The tube was put into microwave reactor and heated to certain temperature for $30 \mathrm{~min}$. After cool, to the reaction mixture was added water $(15 \mathrm{~mL})$ and extracted with ethyl acetate $(3 \times 20 \mathrm{~mL})$. The combined organic phases were washed with brine and dried over anhydrous $\mathrm{Na}_{2} \mathrm{SO}_{4}$. The solvent was removed under reduced pressure and flash silica gel chromatography gave the product. 


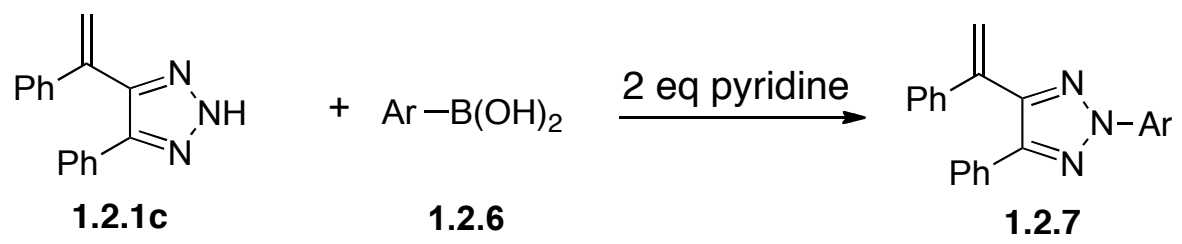

To $25 \mathrm{~mL}$ round-bottom bottle was successively added N-H triazole (1c) $(0.4 \mathrm{mmol})$, arylboronic acid (0.8 mmol), THF $10 \mathrm{~mL}$, pyridine (125 mg, $0.8 \mathrm{mmol})$ and copper (II) acetate (14 mg, 0.08 mmol). The bottle was equipped with a condenser with an oxygen balloon on top of it. The reaction mixture was refluxed and monitored by TLC. After cool, the reaction mixture was concentrated and added water $(15 \mathrm{~mL})$ then extracted with ethyl acetate $(3 \times 20 \mathrm{~mL})$. The combined organic phases were washed with brine and dried over anhydrous $\mathrm{Na}_{2} \mathrm{SO}_{4}$. The solvent was removed under reduced pressure and flash silica gel chromatography gave the product. 


\section{General procedure for UV/VIS and Fluorescence measurements}

UV/VIS absorption, fluorescence emission and excitation spectra were recorded in methanol

solvents in $1 \mathrm{~cm}$-length quartz cell. The solution concentrations were $3 \times 10^{-6} \mathrm{M}$ for absorption spectroscopy and fluorescence spectroscopy. Emission spectra were recorded with an excitation wavelength at $254 \mathrm{~nm}$.

\section{$\underline{\text { Reference }}$}

1. Quiclet-Sire, B.; Zard, S. Z.; Synthesis 2005, 19, 3319.

2. Sengupta, S.; Duan, H.; Lu, W.; Petersen, J. L.; Shi, X. Org. Lett. 2008, 10, 1493.

3. Chen, Y; Liu ,Y; Petersen, J. L.; Shi, X. Chem. Commun. 2008, $28,3254$. 


\section{Compounds Characterization}

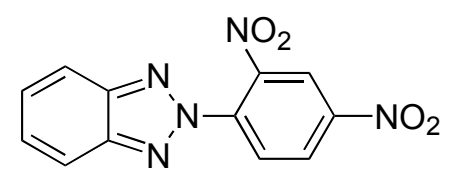

1.2.3a

2-(2,4-dinitrophenyl)-2H-benzo[d] $[1,2,3]$ triazole $1.2 .3 a$ : yield $24 \%$; m.p. $166-167{ }^{\circ} \mathrm{C} ;{ }^{1} \mathrm{H}$ NMR $\left(600 \mathrm{MHz}, \mathrm{CDCl}_{3}\right): \delta 8.70(\mathrm{~d}, J=2.4 \mathrm{~Hz}, 1 \mathrm{H}), 8.60(\mathrm{dd}, J=9.0 \mathrm{~Hz}, 2.4 \mathrm{~Hz}, 1 \mathrm{H}), 8.47(\mathrm{~d}, J=$ $9.0 \mathrm{~Hz}, 1 \mathrm{H}), 7.87-7.85(\mathrm{~m}, 2 \mathrm{H}), 7.46-7.44(\mathrm{~m}, 2 \mathrm{H}) ;{ }^{13} \mathrm{C} \mathrm{NMR}\left(125 \mathrm{MHz}, \mathrm{CDCl}_{3}\right): \delta$ 146.8, 146.1, $143.4,136.3,129.0,127.1,126.7,120.7,118.7$; UV $(\mathrm{MeOH}) \lambda_{\max } 221(\varepsilon=36000), 321(\varepsilon=$ 20000); HRMS Calculated for $\left[\mathrm{C}_{12} \mathrm{H}_{7} \mathrm{~N}_{5} \mathrm{O}_{4}+\mathrm{H}\right]^{+}:$286.05708, Found: 286.05725 .

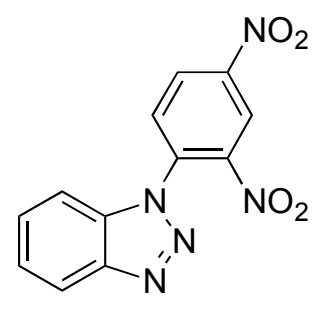

N-1-1.2.3a

1-(2,4-dinitrophenyl)-1H-benzo $[\boldsymbol{d}][\mathbf{1 , 2 , 3}]$ triazole $\mathbf{N}-1-1.2 .3 \mathrm{a}$ : yield $74 \%$; m.p. $185-187{ }^{\circ} \mathrm{C} ;{ }^{1} \mathrm{H}$ NMR (600 MHz, $\left.\mathrm{CDCl}_{3}\right): \delta 9.00(\mathrm{~d}, J=2.4 \mathrm{~Hz}, 1 \mathrm{H}), 8.70(\mathrm{dd}, J=9.0 \mathrm{~Hz}, 2.4 \mathrm{~Hz}, 1 \mathrm{H}), 8.21(\mathrm{~d}, J$ $=8.4 \mathrm{~Hz}, 1 \mathrm{H}), 8.02(\mathrm{~d}, J=9.0 \mathrm{~Hz}, 1 \mathrm{H}), 7.63(\mathrm{t}, J=8.4 \mathrm{~Hz}, 1 \mathrm{H}), 7.52(\mathrm{t}, J=8.4 \mathrm{~Hz}, 1 \mathrm{H}), 7.45(\mathrm{~d}$, $J=8.4 \mathrm{~Hz}, 1 \mathrm{H}) ;{ }^{13} \mathrm{C} \mathrm{NMR}\left(125 \mathrm{MHz}, \mathrm{CDCl}_{3}\right): \delta 147.4,146.3,144.2,134.3,132.5,129.8,128.5$, 
128.4, 125.4, 121.8, 121.2, 108.8; UV (MeOH) $\lambda_{\max } 221(\varepsilon=48000)$; HRMS Calculated for $\left[\mathrm{C}_{12} \mathrm{H}_{7} \mathrm{~N}_{5} \mathrm{O}_{4}+\mathrm{H}\right]^{+}:$286.05708, Found: 286.05709 .

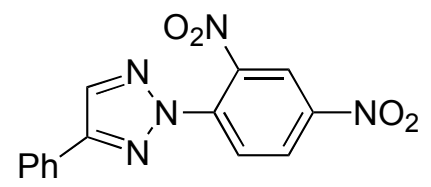

1.2.3b

2-(2,4-dinitrophenyl)-4-phenyl-2H-1,2,3-triazole 1.2.3b: yield $60 \%$; m.p. $187-189{ }^{\circ} \mathrm{C} ;{ }^{1} \mathrm{H}$ NMR (600 MHz, d6-DMSO): $\delta 8.99(\mathrm{~d}, J=2.4 \mathrm{~Hz}, 1 \mathrm{H}), 8.86(\mathrm{~s}, 1 \mathrm{H}), 8.68(\mathrm{dd}, J=9.0 \mathrm{~Hz}, 2.4$ $\mathrm{Hz}, 1 \mathrm{H}), 8.41(\mathrm{~d}, J=9.0 \mathrm{~Hz}, 1 \mathrm{H}), 7.96-7.94(\mathrm{~m}, 2 \mathrm{H}), 7.78-7.77(\mathrm{~m}, 2 \mathrm{H}), 7.76-7.74(\mathrm{~m}, 1 \mathrm{H}) ;{ }^{13} \mathrm{C}$ NMR (125 MHz, d6-DMSO): $\delta$ 151.4, 146.7, 142.2, 137.2, 134.7, 130.7, 130.0, 128.83, 128.81, 127.0, 125.5, 121.8; HRMS Calculated for $\left[\mathrm{C}_{14} \mathrm{H}_{9} \mathrm{~N}_{5} \mathrm{O}_{4}+\mathrm{H}\right]^{+}:$312.07273, Found: 312.07280.

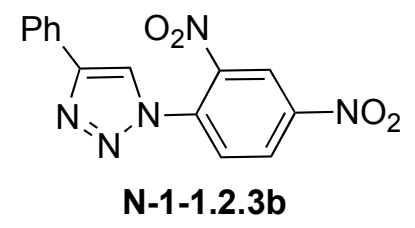

1-(2,4-dinitrophenyl)-4-phenyl-1H-1,2,3-triazole N-1-1.2.3b: yield 38\%; m.p. 202-204 ${ }^{\circ} \mathrm{C} ;{ }^{1} \mathrm{H}$ NMR (600 MHz, d6-DMSO): $\delta 9.30(\mathrm{~s}, 1 \mathrm{H}), 8.98(\mathrm{~d}, J=2.4 \mathrm{~Hz}, 1 \mathrm{H}), 8.76(\mathrm{dd}, J=9.0 \mathrm{~Hz}, 2.4$ $\mathrm{Hz}, 1 \mathrm{H}), 8.25(\mathrm{~d}, J=9.0 \mathrm{~Hz}, 1 \mathrm{H}), 7.91(\mathrm{dd}, J=8.4 \mathrm{~Hz}, 1.2 \mathrm{~Hz}, 2 \mathrm{H}), 7.54-7.46(\mathrm{~m}, 2 \mathrm{H}), 7.40$ (t, $J$ $=8.4 \mathrm{~Hz}, 1 \mathrm{H}) ;{ }^{13} \mathrm{C}$ NMR (125 MHz, d6-DMSO): $\delta$ 148.2, 148.1, 144.1, 133.5, 130.1, 129.8, 129.5, 129.4, 128.6, 126.2, 123.2, 122.1; HRMS Calculated for $\left[\mathrm{C}_{14} \mathrm{H}_{9} \mathrm{~N}_{5} \mathrm{O}_{4}+\mathrm{H}\right]^{+}: 312.07273$, Found: 312.07277. 


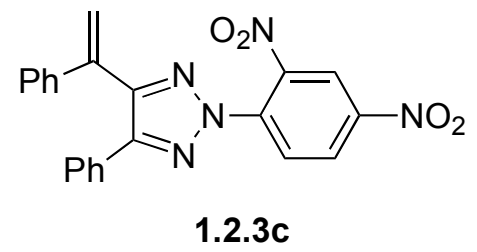

2-(2,4-dinitrophenyl)-4-phenyl-5-(1-phenylvinyl)-2H-1,2,3-triazole 1.2.3c: yield 84\%; m.p. 156-158 ${ }^{\circ} \mathrm{C} ;{ }^{1} \mathrm{H}$ NMR $\left(600 \mathrm{MHz}, \mathrm{CDCl}_{3}\right): \delta 8.62(\mathrm{~d}, J=2.4 \mathrm{~Hz}, 1 \mathrm{H}), 8.52(\mathrm{dd}, J=9.0 \mathrm{~Hz}, 2.4$ $\mathrm{Hz}, 1 \mathrm{H}), 8.37$ (d, $J=9.0 \mathrm{~Hz}, 1 \mathrm{H}), 7.64-7.61(\mathrm{~m}, 2 \mathrm{H}), 7.37-7.34(\mathrm{~m}, 2 \mathrm{H}), 7.32-7.25(\mathrm{~m}, 6 \mathrm{H}), 5.98$ (s, 1H), $5.66(\mathrm{~s}, 1 \mathrm{H}) ;{ }^{13} \mathrm{C} \mathrm{NMR}\left(125 \mathrm{MHz}, \mathrm{CDCl}_{3}\right): \delta 149.6,149.0,145.6,141.9,138.1,137.6$, $135.2,129.4,128.55,128.52,128.51,127.9,126.9,126.7,124.3,120.8,120.2 ; \mathrm{UV}(\mathrm{MeOH}) \lambda_{\max }$ $221(\varepsilon=116000), 266(\varepsilon=83600), 336(\varepsilon=33600) ;$ HRMS Calculated for $\left[\mathrm{C}_{22} \mathrm{H}_{15} \mathrm{~N}_{5} \mathrm{O}_{4}+\mathrm{H}\right]^{+}$: 414.11968, Found: 414.11997.<smiles>O=C(c1ccccc1)c1nn(-c2ccc([N+](=O)[O-])cc2[N+](=O)[O-])cc1-c1ccccc1</smiles>

1.2.3d

(2-(2,4-dinitrophenyl)-5-phenyl-2H-1,2,3-triazol-4-yl)(phenyl)methanone 1.2.3d: yield 92\%; m.p. $164-166{ }^{\circ} \mathrm{C} ;{ }^{1} \mathrm{H}$ NMR (600 MHz, d6-DMSO): $\delta 9.03(\mathrm{~d}, J=2.4 \mathrm{~Hz}, 1 \mathrm{H}), 8.73(\mathrm{dd}, J=9.0$ Hz, $2.4 \mathrm{~Hz}, 1 \mathrm{H}), 8.49(\mathrm{~d}, J=9.0 \mathrm{~Hz}, 1 \mathrm{H}), 8.06(\mathrm{~d}, J=7.3 \mathrm{~Hz}, 2 \mathrm{H}), 7.81-7.67$ (m, 3H), 7.60 (t, $J$ $=7.8 \mathrm{~Hz}, 2 \mathrm{H}), 7.47-7.45$ (m, 3H); ${ }^{13} \mathrm{C}$ NMR (125 MHz, d6-DMSO): $\delta 186.7,151.0,146.9,144.4$, $142.1,135.9,134.4,133.7,130.2,130.1,128.71,128.69,128.5,128.3,127.8,126.2,121.2$; UV 
$(\mathrm{MeOH}) \lambda_{\max } 221(\varepsilon=97600), 266(\varepsilon=76900)$; HRMS Calculated for $\left[\mathrm{C}_{21} \mathrm{H}_{13} \mathrm{~N}_{5} \mathrm{O}_{5}+\mathrm{H}\right]^{+}$: 416.09916, Found: 416.09895.

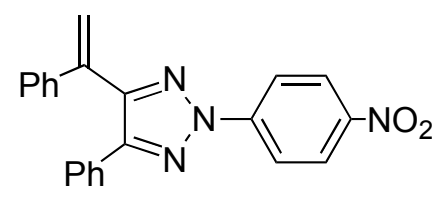

$1.2 .3 e$

2-(4-nitrophenyl)-4-phenyl-5-(1-phenylvinyl)-2H-1,2,3-triazole 1.2.3e: yield 98\%; m.p. 147$149{ }^{\circ} \mathrm{C} ;{ }^{1} \mathrm{H}$ NMR $\left(600 \mathrm{MHz}, \mathrm{CDCl}_{3}\right): \delta 8.35(\mathrm{~d}, J=9.6 \mathrm{~Hz}, 2 \mathrm{H}), 8.32(\mathrm{~d}, J=9.6 \mathrm{~Hz}, 2 \mathrm{H}), 7.65-$ $7.63(\mathrm{~m}, 2 \mathrm{H}), 7.40-7.38(\mathrm{~m}, 2 \mathrm{H}), 7.26-7.24(\mathrm{~m}, 6 \mathrm{H}), 5.97(\mathrm{~s}, 1 \mathrm{H}), 5.67(\mathrm{~s}, 1 \mathrm{H}) ;{ }^{13} \mathrm{C}$ NMR $(125$ $\left.\mathrm{MHz}, \mathrm{CDCl}_{3}\right): \delta 148.4,147.7,146.2,143.5,138.7,128.0,129.3,128.9,128.47,128.42,128.37$, 127.8, 126.8, 125.1, 119.6, 118.8; UV (MeOH) $\lambda_{\max } 336(\varepsilon=28600)$; HRMS Calculated for $\left[\mathrm{C}_{22} \mathrm{H}_{16} \mathrm{~N}_{4} \mathrm{O}_{2}+\mathrm{H}\right]^{+}: 369.13460$, Found: 369.13481 .

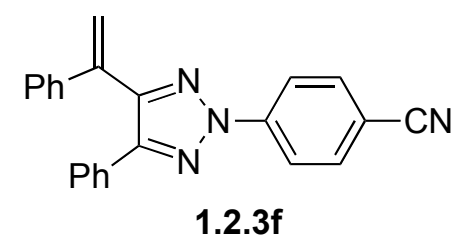

4-(4-phenyl-5-(1-phenylvinyl)-2H-1,2,3-triazol-2-yl)benzonitrile 1.2.3f: yield 62\%; m.p. 134$135^{\circ} \mathrm{C} ;{ }^{1} \mathrm{H}$ NMR (600 MHz, $\left.d 6-\mathrm{DMSO}\right): \delta 8.26(\mathrm{~d}, J=8.4 \mathrm{~Hz}, 2 \mathrm{H}), 8.06(\mathrm{~d}, J=8.4 \mathrm{~Hz}, 2 \mathrm{H})$, 7.65-7.62 (m, 2H), 7.42-7.26 (m, 8H), $6.12(\mathrm{~s}, 1 \mathrm{H}), 5.66(\mathrm{~s}, 1 \mathrm{H}) ;{ }^{13} \mathrm{C}$ NMR $(125 \mathrm{MHz}, d 6-$ DMSO): $\delta 147.1,146.9,141.5,137.9,137.5,134.1,129.0,128.9,128.6,128.4,127.3,126.4$, 
$120.0,118.8,118.2,109.9 ; \mathrm{UV}(\mathrm{MeOH}) \lambda_{\max } 210(\varepsilon=39100), 314(\varepsilon=28600)$; HRMS Calculated for $\left[\mathrm{C}_{23} \mathrm{H}_{16} \mathrm{~N}_{4}+\mathrm{H}\right]^{+}: 349.14477$, Found: 349.14486 .

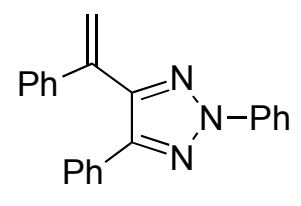

1.2.5a

2,4-diphenyl-5-(1-phenylvinyl)-2H-1,2,3-triazole 1.2.5a: yield 87\%; oil; ${ }^{1} \mathrm{H}$ NMR (600 MHz, $\left.\mathrm{CDCl}_{3}\right): \delta 8.17(\mathrm{~d}, J=8.4 \mathrm{~Hz}, 2 \mathrm{H}), 7.69(\mathrm{~d}, J=8.4 \mathrm{~Hz}, 2 \mathrm{H}), 7.47(\mathrm{t}, J=7.8 \mathrm{~Hz}, 2 \mathrm{H}), 7.40(\mathrm{~d}, J$ $=8.4 \mathrm{~Hz}, 2 \mathrm{H}), 7.33(\mathrm{t}, J=7.2 \mathrm{~Hz}, 1 \mathrm{H}), 7.30-7.20(\mathrm{~m}, 6 \mathrm{H}), 5.93(\mathrm{~s}, 1 \mathrm{H}), 5.64(\mathrm{~s}, 1 \mathrm{H}) ;{ }^{13} \mathrm{C} \mathrm{NMR}$ $\left(125 \mathrm{MHz}, \mathrm{CDCl}_{3}\right): \delta 146.6,145.9,139.7,139.2,138.5,130.2,129.2,128.35,128.28,128.13$, 127.7, 127.4, 126.8, 118.9, 118.8; UV (MeOH) $\lambda_{\max } 297(\varepsilon=36300), 266(\varepsilon=76900)$; HRMS Calculated for $\left[\mathrm{C}_{22} \mathrm{H}_{17} \mathrm{~N}_{3}+\mathrm{H}\right]^{+}: 324.14952$, Found: 324.14964 .

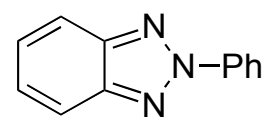

$1.2 .5 b$

2-phenyl-2H-benzo[d][1,2,3]triazole $1.2 .5 b$ : yield 8\%; m.p. $109-110{ }^{\circ} \mathrm{C} ;{ }^{1} \mathrm{H}$ NMR $(600 \mathrm{MHz}$, $\left.\mathrm{CDCl}_{3}\right): \delta 8.36(\mathrm{~d}, J=7.2 \mathrm{~Hz}, 2 \mathrm{H}), 7.95-7.92(\mathrm{~m}, 2 \mathrm{H}), 7.55(\mathrm{t}, J=7.2 \mathrm{~Hz}, 2 \mathrm{H}), 7.45(\mathrm{t}, J=7.2$ $\mathrm{Hz}, 1 \mathrm{H}), 7.44-7.40(\mathrm{~m}, 2 \mathrm{H}) ;{ }^{13} \mathrm{C}$ NMR $\left(125 \mathrm{MHz}, \mathrm{CDCl}_{3}\right): \delta 145.0,140.4,129.4,128.9,127.2$, 120.6, 118.4; UV (MeOH) $\lambda_{\max } 222(\varepsilon=48800), 310(\varepsilon=29600)$; HRMS Calculated for $\left[\mathrm{C}_{12} \mathrm{H}_{9} \mathrm{~N}_{3}+\mathrm{H}\right]^{+}:$196.08692, Found: 196.08691. 


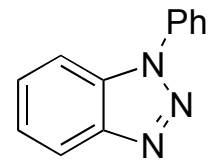

$\mathrm{N}-1-1.2 .5 b$

1-phenyl-1H-benzo $[\boldsymbol{d}][\mathbf{1 , 2 , 3}]$ triazole $\mathbf{N}-1-1.2 .5 b$ : yield $60 \%$; m.p. $85-87{ }^{\circ} \mathrm{C} ;{ }^{1} \mathrm{H}$ NMR $(600$ $\left.\mathrm{MHz}, \mathrm{CDCl}_{3}\right): \delta 8.14(\mathrm{~d}, J=8.4 \mathrm{~Hz}, 1 \mathrm{H}), 7.78(\mathrm{~d}, J=7.8 \mathrm{~Hz}, 2 \mathrm{H}), 7.74(\mathrm{~d}, J=8.4 \mathrm{~Hz}, 1 \mathrm{H}), 7.60$ $(\mathrm{d}, J=7.8 \mathrm{~Hz}, 2 \mathrm{H}), 7.53(\mathrm{t}, J=7.2 \mathrm{~Hz}, 1 \mathrm{H}), 7.49(\mathrm{t}, J=7.8 \mathrm{~Hz}, 1 \mathrm{H}) ; 7.42(\mathrm{t}, J=7.8 \mathrm{~Hz}, 1 \mathrm{H}) ;{ }^{13} \mathrm{C}$ NMR (125 MHz, $\left.\mathrm{CDCl}_{3}\right): \delta 146.4,136.9,132.2,129.8,128.6,128.2,124.3,122.8,120.2,110.3$; UV (MeOH) $\lambda_{\max } 222(\varepsilon=41400)$; HRMS Calculated for $\left[\mathrm{C}_{12} \mathrm{H}_{9} \mathrm{~N}_{3}+\mathrm{H}\right]^{+}:$196.08692, Found: 196.08688.

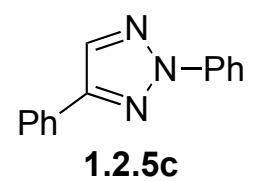

2,4-diphenyl-2H-1,2,3-triazole 1.2.5c: yield 68\%; m.p. $43-45^{\circ} \mathrm{C} ;{ }^{1} \mathrm{H} \mathrm{NMR}\left(600 \mathrm{MHz}, \mathrm{CDCl}_{3}\right): \delta$ $8.13(\mathrm{~d}, J=8.4 \mathrm{~Hz}, 2 \mathrm{H}), 8.04(\mathrm{~s}, 1 \mathrm{H}), 7.89(\mathrm{~d}, J=7.2 \mathrm{~Hz}, 2 \mathrm{H}), 7.50-7.43(\mathrm{~m}, 4 \mathrm{H}), 7.37(\mathrm{t}, J=$ $7.2 \mathrm{~Hz}, 1 \mathrm{H}), 7.33(\mathrm{t}, J=7.2 \mathrm{~Hz}, 1 \mathrm{H}) ;{ }^{13} \mathrm{C} \mathrm{NMR}\left(125 \mathrm{MHz}, \mathrm{CDCl}_{3}\right): \delta 148.8,139.9,130.5,130.0$, 129.2, 128.9, 128.8, 127.4, 126.1, 118.8; UV (MeOH) $\lambda_{\max } 221(\varepsilon=44700), 294(\varepsilon=34700)$; HRMS Calculated for $\left[\mathrm{C}_{14} \mathrm{H}_{11} \mathrm{~N}_{3}+\mathrm{H}\right]^{+}:$222.10257, Found: 222.10262. 


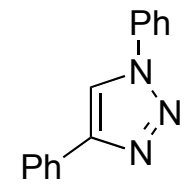

$\mathrm{N}-1-1.2 .5 \mathrm{c}$

1,4-diphenyl-1H-1,2,3-triazole N-1-1.2.5c: yield 11\%; m.p. $186-187^{\circ} \mathrm{C} ;{ }^{1} \mathrm{H} \mathrm{NMR}(600 \mathrm{MHz}$, $\left.\mathrm{CDCl}_{3}\right): \delta 8.19(\mathrm{~s}, 1 \mathrm{H}), 7.93-7.90(\mathrm{~m}, 2 \mathrm{H}), 7.82-7.78(\mathrm{~m}, 2 \mathrm{H}), 7.50-7.43(\mathrm{~m}, 2 \mathrm{H}), 7.48-7.44(\mathrm{~m}$ 3H), $7.37(\mathrm{t}, J=7.2 \mathrm{~Hz}, 1 \mathrm{H}) ;{ }^{13} \mathrm{C}$ NMR (125 MHz, $\left.\mathrm{CDCl}_{3}\right): \delta$ 148.4, 137.1, 130.3, 129.8, 128.9, 128.8, 128.4, 125.8, 120.5, 117.6; UV (MeOH) $\lambda_{\max } 222(\varepsilon=5100)$; HRMS Calculated for $\left[\mathrm{C}_{14} \mathrm{H}_{11} \mathrm{~N}_{3}+\mathrm{H}\right]^{+}:$222.10257, Found: 222.10269.

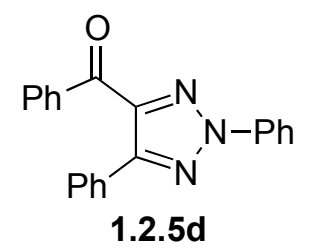

Phenyl(2,5-diphenyl-2H-1,2,3-triazol-4-yl)methanone 1.2.5d: yield 85\%; m.p. $115-117{ }^{\circ} \mathrm{C} ;{ }^{1} \mathrm{H}$ NMR (600 MHz, d6-DMSO): $\delta$ 8.14-8.10 (m, 4H), 7.87-7.83 (m, 2H), $7.74(\mathrm{t}, J=7.8 \mathrm{~Hz}, 1 \mathrm{H})$, $7.63(\mathrm{t}, J=7.8 \mathrm{~Hz}, 2 \mathrm{H}), 7.60(\mathrm{t}, J=7.8 \mathrm{~Hz}, 2 \mathrm{H}), 7.55-7.48(\mathrm{~m}, 4 \mathrm{H}),{ }^{13} \mathrm{C} \mathrm{NMR}(125 \mathrm{MHz}, d 6-$ DMSO): $\delta 188.1,150.3,143.4,139.3,137.3,134.6,130.9,130.5,130.1,129.6,129.5,129.3$, 129.19, 129.18, 119.9; UV (MeOH) $\lambda_{\max } 210(\varepsilon=45200), 263(\varepsilon=30600)$; HRMS Calculated for $\left[\mathrm{C}_{21} \mathrm{H}_{15} \mathrm{~N}_{3} \mathrm{O}+\mathrm{H}\right]^{+}: 326.12879$, Found: 326.12876 . 


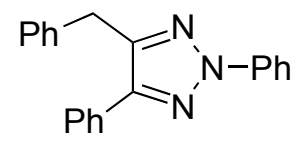

$1.2 .5 \mathrm{e}$

4-benzyl-2,5-diphenyl-2H-1,2,3-triazole 1.2.5e: yield 85\%; m.p. 93-95 ${ }^{\circ} \mathrm{C} ;{ }^{1} \mathrm{H}$ NMR (600 MHz, $\left.\mathrm{CDCl}_{3}\right): \delta 8.14-8.11(\mathrm{~m}, 2 \mathrm{H}), 7.69-7.65(\mathrm{~m}, 2 \mathrm{H}), 7.50-7.45(\mathrm{~m}, 2 \mathrm{H}), 7.42-7.24(\mathrm{~m}, 8 \mathrm{H}), 7.23-$

$7.18(\mathrm{~m}, 1 \mathrm{H}), 4.31(\mathrm{~s}, 2 \mathrm{H}) ;{ }^{13} \mathrm{C} \mathrm{NMR}\left(125 \mathrm{MHz}, \mathrm{CDCl}_{3}\right): \delta 146.9,145.0,139.8,138.4,130.6$, $129.2,128.7,128.6,128.5,128.4,127.7,127.1,126.5,118.6,31.7 ; \mathrm{UV}(\mathrm{MeOH}) \lambda_{\max } 221(\varepsilon=$ 37700), $290(\varepsilon=29900)$; HRMS Calculated for $\left[\mathrm{C}_{21} \mathrm{H}_{17} \mathrm{~N}_{3}+\mathrm{H}\right]^{+}: 312.14952$, Found: 312.14965.

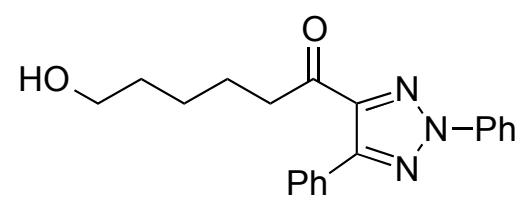

1.2.5f

6-hydroxy-1-(2,5-diphenyl-2H-1,2,3-triazol-4-yl)hexan-1-one 1.2.5f: yield 60\%; m.p. 73-75 ${ }^{\circ} \mathrm{C}$;

${ }^{1} \mathrm{H}$ NMR $\left(600 \mathrm{MHz}, \mathrm{CDCl}_{3}\right): \delta 8.18(\mathrm{~d}, J=8.4 \mathrm{~Hz}, 2 \mathrm{H}), 8.01(\mathrm{~d}, J=7.2 \mathrm{~Hz}, 2 \mathrm{H}), 7.53(\mathrm{t}, J=7.2$ $\mathrm{Hz}, 2 \mathrm{H}), 7.50-7.40(\mathrm{~m}, 4 \mathrm{H}), 3.67(\mathrm{t}, J=6.6 \mathrm{~Hz}, 2 \mathrm{H}), 3.21(\mathrm{t}, J=7.2 \mathrm{~Hz}, 2 \mathrm{H}), 1.84-1.77(\mathrm{~m}, 2 \mathrm{H})$, 1.66-1.61 (m, 2H), 1.61-1.52 (br, 1H), 1.52-1.45 (m, 2H); ${ }^{13} \mathrm{C}$ NMR (125 MHz, $\left.\mathrm{CDCl}_{3}\right): \delta 195.3$, $149.5,143.2,139.2,129.5,129.43,129.40,129.3,128.5,128.2,119.3,62.7,40.6,32.5,25.4$ 23.6; UV (MeOH) $\lambda_{\max } 221(\varepsilon=40200)$; HRMS Calculated for $\left[\mathrm{C}_{20} \mathrm{H}_{21} \mathrm{~N}_{3} \mathrm{O}_{2}+\mathrm{H}\right]^{+}: 336.17065$, Found: 336.17086. 


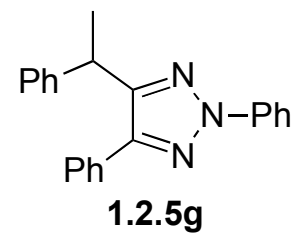

2,4-diphenyl-5-(1-phenylethyl)-2H-1,2,3-triazole 1.2.5g: yield 85\%; oil; ${ }^{1} \mathrm{H}$ NMR (600 MHz, $\left.\mathrm{CDCl}_{3}\right): \delta 8.17(\mathrm{~d}, J=8.4 \mathrm{~Hz}, 2 \mathrm{H}), 7.56(\mathrm{~d}, J=7.2 \mathrm{~Hz}, 2 \mathrm{H}), 7.49(\mathrm{t}, J=8.4 \mathrm{~Hz}, 2 \mathrm{H}), 7.41-7.27$ $(\mathrm{m}, 8 \mathrm{H}), 7.20(\mathrm{t}, J=7.2 \mathrm{~Hz}, 1 \mathrm{H}), 4.43(\mathrm{q}, J=7.2 \mathrm{~Hz}, 1 \mathrm{H}), 1.76(\mathrm{~d}, J=7.2 \mathrm{~Hz}, 3 \mathrm{H}) ;{ }^{13} \mathrm{C} \mathrm{NMR}$ $\left(125 \mathrm{MHz}, \mathrm{CDCl}_{3}\right): \delta 149.9,146.7,145.0,140.2,131.0,129.4,128.8,128.75,128.5,128.4$, 127.8, 127.2, 126.6, 118.9, 37.2, 23.3; UV (MeOH) $\lambda_{\max } 221(\varepsilon=48400), 285(\varepsilon=34800)$; HRMS Calculated for $\left[\mathrm{C}_{22} \mathrm{H}_{19} \mathrm{~N}_{3}+\mathrm{H}\right]^{+}: 326.16517$, Found: 326.16535 .

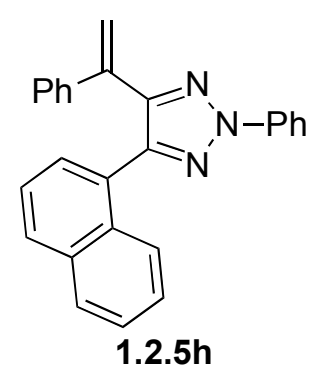

4-(naphthalen-1-yl)-2-phenyl-5-(1-phenylvinyl)-2H-1,2,3-triazole 1.2.5h: yield 87\%; oil; ${ }^{1} \mathrm{H}$ NMR (600 MHz, $\left.\mathrm{CDCl}_{3}\right): \delta 8.19(\mathrm{~d}, J=7.2 \mathrm{~Hz}, 2 \mathrm{H}), 7.96(\mathrm{~d}, J=7.8 \mathrm{~Hz}, 1 \mathrm{H}), 7.82-7.76(\mathrm{~m}, 2 \mathrm{H})$, 7.51-7.40 (m, 5H), 7.40-7.32 (m, 2H); 7.32-7.27 (m, 2H), 7.12-7.08 (m, 3H), $5.53(\mathrm{~s}, 1 \mathrm{H}), 5.48(\mathrm{~s}$, $1 \mathrm{H}) ;{ }^{13} \mathrm{C}$ NMR $\left(125 \mathrm{MHz}, \mathrm{CDCl}_{3}\right): \delta 147.6,146.3,139.8,139.0,138.9,133.6,131.6,129.2$, $129.1,128.3,128.2,128.1,127.8,127.7,127.4,127.3,126.4,125.9,125.7,125.0,118.8,118.7$; UV (MeOH) $\lambda_{\max } 221(\varepsilon=96900), 295(\varepsilon=34600)$; HRMS Calculated for $\left[\mathrm{C}_{26} \mathrm{H}_{19} \mathrm{~N}_{3}+\mathrm{H}\right]^{+}$: 374.16517, Found: 374.16535. 


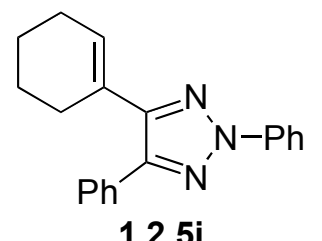

$1.2 .5 i$

4-cyclohexenyl-2,5-diphenyl-2H-1,2,3-triazole $1.2 .5 i$ : yield $80 \%$; oil; ${ }^{1} \mathrm{H}$ NMR (600 MHz, $\left.\mathrm{CDCl}_{3}\right): \delta 8.11(\mathrm{~d}, J=7.8 \mathrm{~Hz}, 2 \mathrm{H}), 7.74(\mathrm{~d}, J=6.6 \mathrm{~Hz}, 2 \mathrm{H}), 7.48-7.41(\mathrm{~m}, 4 \mathrm{H}), 7.40-7.35(\mathrm{~m}$, $1 \mathrm{H}), 7.31(\mathrm{t}, J=7.2 \mathrm{~Hz}, 1 \mathrm{H}), 6.12-6.11(\mathrm{~m}, 1 \mathrm{H}), 2.43-2.39(\mathrm{~m}, 2 \mathrm{H}), 2.19-2.14(\mathrm{~m}, 2 \mathrm{H}), 1.80-1.74$ (m, 2H), 1.73-1.65 (m, 2H); ${ }^{13} \mathrm{C}$ NMR (125 MHz, $\left.\mathrm{CDCl}_{3}\right): \delta 147.9,145.4,139.8,131.5,130.1$, $129.1,128.6,128.5,128.3,128.2,127.0,118.6,27.6,25.5,22.7,21.9 ; \mathrm{UV}(\mathrm{MeOH}) \lambda_{\max } 221(\varepsilon$ $=70800), 290(\varepsilon=47300)$; HRMS Calculated for $\left[\mathrm{C}_{20} \mathrm{H}_{19} \mathrm{~N}_{3}-\mathrm{Ph}+2 \mathrm{H}\right]^{+}:$226.13387, Found: 226.13388 .

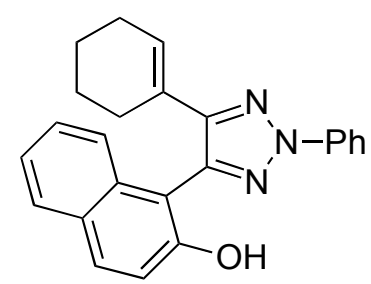

1.2.5j

1-(5-cyclohexenyl-2-phenyl-2H-1,2,3-triazol-4-yl)naphthalen-2-ol $\quad$ 1.2.5j: yield $45 \%$;.p. 124-126 ${ }^{\circ} \mathrm{C} ;{ }^{1} \mathrm{H}$ NMR $\left(600 \mathrm{MHz}, \mathrm{CDCl}_{3}\right): \delta 8.13(\mathrm{~d}, J=7.8 \mathrm{~Hz}, 2 \mathrm{H}), 7.84(\mathrm{~d}, J=9.0 \mathrm{~Hz}, 1 \mathrm{H})$, $7.79(\mathrm{~d}, J=7.8 \mathrm{~Hz}, 1 \mathrm{H}), 7.51-7.46(\mathrm{~m}, 3 \mathrm{H}), 7.40-7.30(\mathrm{~m}, 3 \mathrm{H}), 7.28(\mathrm{~d}, J=9.0 \mathrm{~Hz}, 1 \mathrm{H}) ; 6.55(\mathrm{~s}$ $1 \mathrm{H}), 5.83(\mathrm{~s}, 1 \mathrm{H}), 2.40-2.36(\mathrm{~m}, 2 \mathrm{H}), 1.88-1.84(\mathrm{~m}, 2 \mathrm{H}), 1.64-1.58(\mathrm{~m}, 2 \mathrm{H}), 1.53-1.48(\mathrm{~m}, 2 \mathrm{H})$; ${ }^{13} \mathrm{C}$ NMR $\left(125 \mathrm{MHz}, \mathrm{CDCl}_{3}\right): \delta 152.4,149.7,139.5,139.4,132.3,131.1,130.1,129.3,128.9$, 
$128.2,127.7,127.5,126.7,124.7,123.6,118.6,117.9,110.2,26.5,25.5,22.4,21.7 ; \mathrm{UV}(\mathrm{MeOH})$ $\lambda_{\max } 227(\varepsilon=103000), 292(\varepsilon=37400)$; HRMS Calculated for $\left[\mathrm{C}_{24} \mathrm{H}_{21} \mathrm{~N}_{3} \mathrm{O}+\mathrm{H}\right]^{+}: 368.17574$, Found: 368.17578 .

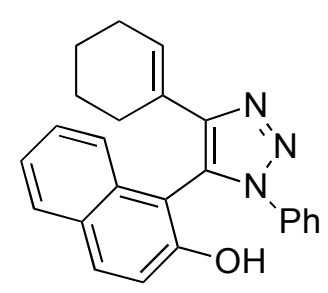

N-3-1.2.5j

4-cyclohexenyl-5-(2-phenoxynaphthalen-1-yl)-2H-1,2,3-triazole $\mathbf{N - 3 - 1 . 2 . 5 j : ~ y i e l d ~ 4 0 \% ; ~ m . p . ~}$ 313-315 ${ }^{\circ} \mathrm{C} ;{ }^{1} \mathrm{H}$ NMR (600 MHz, $\left.d 6-\mathrm{DMSO}\right): \delta 10.23(\mathrm{~s}, 1 \mathrm{H}), 7.82(\mathrm{~d}, J=9.0 \mathrm{~Hz}, 1 \mathrm{H}), 7.74(\mathrm{~d}, J$ $=7.8 \mathrm{~Hz}, 1 \mathrm{H}), 7.32-7.17(\mathrm{~m}, 8 \mathrm{H}), 7.00(\mathrm{~d}, J=8.4 \mathrm{~Hz}, 1 \mathrm{H}), 5.91(\mathrm{~s}, 1 \mathrm{H}), 2.24-2.11(\mathrm{~m}, 2 \mathrm{H}), 1.88-$ $1.70(\mathrm{~m}, 2 \mathrm{H}), 1.45-1.34(\mathrm{~m}, 4 \mathrm{H}) ;{ }^{13} \mathrm{C}$ NMR (125 MHz, $\left.d 6-\mathrm{DMSO}\right): \delta 155.2,147.2,137.2,133.9$, 132.3, 129.6, 129.5, 129.0, 128.8, 128.4, 128.2, 128.1, 125.4, 124.6, 123.8, 123.3, 118.7, 107.6, 26.3, 25.4, 22.7, 22.2; HRMS Calculated for $\left[\mathrm{C}_{24} \mathrm{H}_{21} \mathrm{~N}_{3} \mathrm{O}+\mathrm{H}\right]^{+}: 368.17574$, Found: 368.17588 .

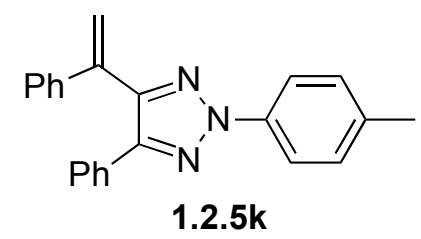

4-phenyl-5-(1-phenylvinyl)-2-p-tolyl-2H-1,2,3-triazole 1.2.5k: yield 86\%; 70-72 ${ }^{\circ} \mathrm{C} ;{ }^{1} \mathrm{H}$ NMR $\left(600 \mathrm{MHz}, \mathrm{CDCl}_{3}\right): \delta 8.04(\mathrm{~d}, J=7.8 \mathrm{~Hz}, 2 \mathrm{H}), 7.69-7.67(\mathrm{~m}, 2 \mathrm{H}), 7.41-7.38(\mathrm{~m}, 2 \mathrm{H}), 7.28-7.20$ (m, 8H), $5.92(\mathrm{~s}, 1 \mathrm{H}), 5.63(\mathrm{~s}, 1 \mathrm{H}), 2.38(\mathrm{~s}, 3 \mathrm{H}) ;{ }^{13} \mathrm{C} \mathrm{NMR}\left(125 \mathrm{MHz}, \mathrm{CDCl}_{3}\right): \delta$ 146.3, 145.6, 
139.3, 138.6, 137.6, 137.3, 130.3, 129.7, 128.3, 128.26, 128.2, 128.1, 127.7, 126.8, 118.8, 118.7, 21.0; UV (MeOH) $\lambda_{\max } 296(\varepsilon=21700)$; HRMS Calculated for $\left[\mathrm{C}_{23} \mathrm{H}_{19} \mathrm{~N}_{3} \mathrm{O}+\mathrm{H}\right]^{+}: 338.14517$, Found: 338.16510 .<smiles>O=C(c1ccccc1)c1nn(-c2ccc([N+](=O)[O-])cc2)nc1-c1ccccc1</smiles>

(2-(4-nitrophenyl)-5-phenyl-2H-1,2,3-triazol-4-yl)(phenyl)methanone 1.2.5l: yield 88\%; m.p. 151-153 ${ }^{\circ} \mathrm{C} ;{ }^{1} \mathrm{H}$ NMR (600 MHz, $\left.d 6-\mathrm{DMSO}\right): \delta 8.46(\mathrm{~d}, J=9.0 \mathrm{~Hz}, 2 \mathrm{H}), 8.34(\mathrm{~d}, J=9.0 \mathrm{~Hz}, 2 \mathrm{H})$, $8.11(\mathrm{~d}, J=8.4 \mathrm{~Hz}, 2 \mathrm{H}), 7.86-7.81(\mathrm{~m}, 2 \mathrm{H}), 7.75(\mathrm{t}, J=7.2 \mathrm{~Hz}, 1 \mathrm{H}), 7.61(\mathrm{t}, J=7.8 \mathrm{~Hz}, 2 \mathrm{H})$, 7.52-7.48 (m, 3H); ${ }^{13} \mathrm{C}$ NMR (125 MHz, d6-DMSO): $\delta$ 187.2, 150.4, 146.6, 143.9, 142.5, 136.3, $134.2,130.3,129.8,128.7,128.6,128.5,128.4,125.6,119.9 ; \mathrm{UV}(\mathrm{MeOH}) \lambda_{\max } 222(\varepsilon=48300)$, $322(\varepsilon=31300)$; HRMS Calculated for $\left[\mathrm{C}_{21} \mathrm{H}_{14} \mathrm{~N}_{4} \mathrm{O}_{3}+\mathrm{H}\right]^{+}: 371.11387$, Found: 371.11409 .

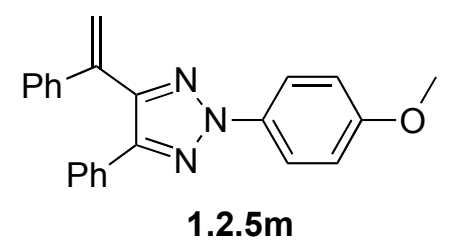

2-(4-methoxyphenyl)-4-phenyl-5-(1-phenylvinyl)-2H-1,2,3-triazole 1.2.5m: yield 74\%; oil; ${ }^{1} \mathrm{H}$ NMR (600 MHz, $\left.\mathrm{CDCl}_{3}\right): \delta 8.07(\mathrm{~d}, J=9.0 \mathrm{~Hz}, 2 \mathrm{H}), 7.69-7.66(\mathrm{~m}, 2 \mathrm{H}), 7.41-7.39(\mathrm{~m}, 2 \mathrm{H})$, 7.28-7.20 (m, 6H), $7.98(\mathrm{~d}, J=9.0 \mathrm{~Hz}, 2 \mathrm{H}), 5.92(\mathrm{~s}, 1 \mathrm{H}), 5.63(\mathrm{~s}, 1 \mathrm{H}), 3.84(\mathrm{~s}, 3 \mathrm{H}) ;{ }^{13} \mathrm{C}$ NMR (125 MHz, $\left.\mathrm{CDCl}_{3}\right): \delta 158.9,146.1,145.4,139.2,138.6,133.5,130.3,128.32,128.25,128.13$ 
$128.08,127.6,126.8,120.2,118.8,114.3,55.5$; HRMS Calculated for $\left[\mathrm{C}_{23} \mathrm{H}_{19} \mathrm{~N}_{3} \mathrm{O}+\mathrm{H}\right]^{+}$: 354.16009, Found: 354.16012.

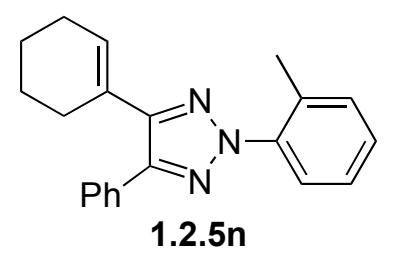

4-cyclohexenyl-5-phenyl-2-o-tolyl-2H-1,2,3-triazole $1.2 .5 n$ : yield $70 \%$; oil; ${ }^{1} \mathrm{H}$ NMR $(600$ $\left.\mathrm{MHz}, \mathrm{CDCl}_{3}\right): \delta$ 7.78-7.72 (m, 2H), 7.66-7.64 (m, 1H), 7.45-7.40 (m, 2H), 7.40-7.35 (m, 1H), 7.34-7.27 (m, 3H), 6.13-6.10 (m, 1H), 2.47 (s, 3H), 2.45-2.39 (m, 2H), 2.18-2.13 (m, 2H), 1.79$1.74(\mathrm{~m}, 2 \mathrm{H}), 1.71-1.66(\mathrm{~m}, 2 \mathrm{H}) ;{ }^{13} \mathrm{C} \mathrm{NMR}\left(125 \mathrm{MHz}, \mathrm{CDCl}_{3}\right): \delta 147.2,144.7,139.5,132.5$, $131.61,131.59,129.7,128.5,128.4,128.3,128.1$ (may 2$), 126.5,125.0,27.6,25.5,22.7,21.9$, 19.2; UV $(\mathrm{MeOH}) \lambda_{\max } 221(\varepsilon=51700)$; HRMS Calculated for $\left[\mathrm{C}_{21} \mathrm{H}_{21} \mathrm{~N}_{3}+\mathrm{H}\right]^{+}: 316.18082$, Found: 316.18098 .

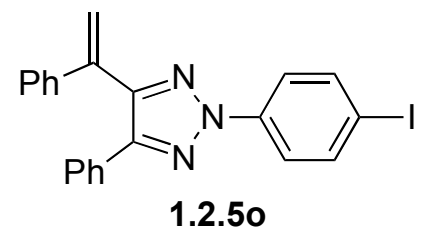

2-(4-iodophenyl)-4-phenyl-5-(1-phenylvinyl)-2H-1,2,3-triazole 1.2 .50 : yield $67 \%$; oil; ${ }^{1} \mathrm{H}$ NMR (600 MHz, $\left.\mathrm{CDCl}_{3}\right): \delta 7.91(\mathrm{~d}, J=9.0 \mathrm{~Hz}, 2 \mathrm{H}), 7.78(\mathrm{~d}, J=9.0 \mathrm{~Hz}, 2 \mathrm{H}), 7.69(\mathrm{dd}, J=7.8$ $\mathrm{Hz}, 1.8 \mathrm{~Hz}, 2 \mathrm{H}), 7.38$ (dd, $J=7.8 \mathrm{~Hz}, 1.8 \mathrm{~Hz}, 2 \mathrm{H}), 7.29-7.10$ (m, 6H), 5.93 (s, 1H), 5.63 (s, 1H); ${ }^{13} \mathrm{C}$ NMR (125 MHz, $\left.\mathrm{CDCl}_{3}\right): \delta 147.0,146.3,139.4,139.1,138.4,138.3,129.9,128.5,128.4$ 
$128.3,128.2,127.7,126.8,120.5,119.1,91.9 ; \mathrm{UV}(\mathrm{MeOH}) \lambda_{\max } 220(\varepsilon=66200), 304(\varepsilon=$ 49900); HRMS Calculated for $\left[\mathrm{C}_{22} \mathrm{H}_{16} \mathrm{IN}_{3}+\mathrm{H}\right]^{+}: 450.04617$, Found: 450.04614.

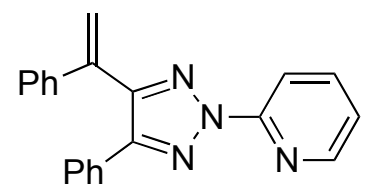

$1.2 .5 p$

2-(4-phenyl-5-(1-phenylvinyl)-2H-1,2,3-triazol-2-yl)pyridine 1.2.5p: yield 88\%; m.p. 103-105 ${ }^{\circ} \mathrm{C} ;{ }^{1} \mathrm{H}$ NMR $\left(600 \mathrm{MHz}, \mathrm{CDCl}_{3}\right): \delta 8.64(\mathrm{~s}, 1 \mathrm{H}), 8.16(\mathrm{~d}, J=7.2 \mathrm{~Hz}, 1 \mathrm{H}), 7.88(\mathrm{t}, J=7.8 \mathrm{~Hz}, 1 \mathrm{H})$, 7.76-7.70 (m, 2H), 7.40-7.36 (m, 2H), $7.32(\mathrm{br}, 1 \mathrm{H}), 7.28-7.20(\mathrm{~m}, 6 \mathrm{H}), 5.96(\mathrm{~s}, 1 \mathrm{H}), 5.70(\mathrm{~s}$, $1 \mathrm{H}) ;{ }^{13} \mathrm{C}$ NMR $\left(125 \mathrm{MHz}, \mathrm{CDCl}_{3}\right): \delta 150.7,148.9,147.8,147.2,138.9,138.8,128.3,129.6$, 128.5, 128.3, 128.2, 128.1, 127.9, 126.8, 122.8, 119.4, 113.8; HRMS Calculated for $\left[\mathrm{C}_{21} \mathrm{H}_{16} \mathrm{~N}_{4}+\mathrm{H}\right]^{+}:$325.14477, Found: 325.14470.

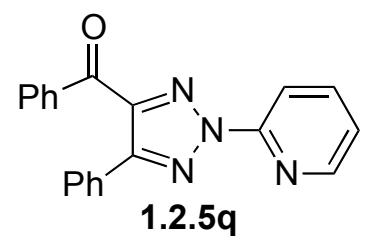

Phenyl(5-phenyl-2-(pyridin-2-yl)-2H-1,2,3-triazol-4-yl)methanone 1.2.5q: yield 83\%; m.p. 133-135 ${ }^{\circ} \mathrm{C} ;{ }^{1} \mathrm{H} \mathrm{NMR}\left(600 \mathrm{MHz}, \mathrm{CDCl}_{3}\right): \delta 8.64(\mathrm{dd}, J=4.8 \mathrm{~Hz}, 1.2 \mathrm{~Hz}, 1 \mathrm{H}), 8.17(\mathrm{~d}, J=8.4 \mathrm{~Hz}$, 1H), $8.12(\mathrm{~d}, J=8.4 \mathrm{~Hz}, 2 \mathrm{H}), 7.94-7.89(\mathrm{~m}, 3 \mathrm{H}), 7.62(\mathrm{t}, J=7.8 \mathrm{~Hz}, 1 \mathrm{H}), 7.48(\mathrm{t}, J=7.8 \mathrm{~Hz}$, 2H), 7.42-7.40 (m, 3H), $7.39(\mathrm{dd}, J=7.2 \mathrm{~Hz}, 4.8 \mathrm{~Hz}, 1 \mathrm{H}) ;{ }^{13} \mathrm{C} \mathrm{NMR}\left(125 \mathrm{MHz}, \mathrm{CDCl}_{3}\right): \delta 188.0$, $150.8,150.6,149.1,144.0,139.0,136.9,133.7,130.5,129.5,128.9,128.8,128.5,128.4,123.7$, 
114.4; UV (MeOH) $\lambda_{\max } 221(\varepsilon=39200), 289(\varepsilon=29900)$; HRMS Calculated for $\left[\mathrm{C}_{20} \mathrm{H}_{14} \mathrm{~N}_{4} \mathrm{O}+\mathrm{H}\right]^{+}:$327.12404, Found: 327.12404 .

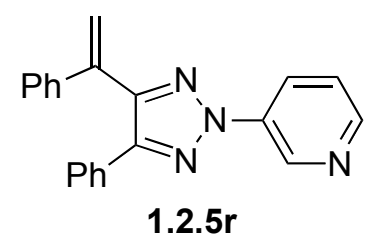

3-(4-phenyl-5-(1-phenylvinyl)-2H-1,2,3-triazol-2-yl)pyridine $1.2 .5 \mathrm{r}$ : yield 70\%; 99-101 ${ }^{\circ} \mathrm{C} ;{ }^{1} \mathrm{H}$ NMR (600 MHz, $\left.\mathrm{CDCl}_{3}\right): \delta 9.47(\mathrm{~s}, 1 \mathrm{H}), 8.61(\mathrm{~s}, 1 \mathrm{H}), 8.45-8.42(\mathrm{~m}, 1 \mathrm{H}), 7.71-7.67(\mathrm{~m}, 2 \mathrm{H})$, $7.43(\mathrm{dd}, J=7.8 \mathrm{~Hz}, 4.8 \mathrm{~Hz}, 1 \mathrm{H}), 7.41-7.37$ (m, 2H), 7.31-7.23 (m, 6H), $5.96(\mathrm{~s}, 1 \mathrm{H}), 5.66(\mathrm{~s}$, $1 \mathrm{H}) ;{ }^{13} \mathrm{C}$ NMR $\left(125 \mathrm{MHz}, \mathrm{CDCl}_{3}\right): \delta 148.2,147.5,146.8,140.4,138.9,138.2,136.1,129.7$, $128.5,128.4,128.35,128.25,127.7,126.8,125.8,123.7,119.3$; UV $(\mathrm{MeOH}) \lambda_{\max } 221(\varepsilon=$ 68500), $301(\varepsilon=28400)$; HRMS Calculated for $\left[\mathrm{C}_{21} \mathrm{H}_{16} \mathrm{~N}_{4}+\mathrm{H}\right]^{+}: 325.14477$, Found: 325.14474 .

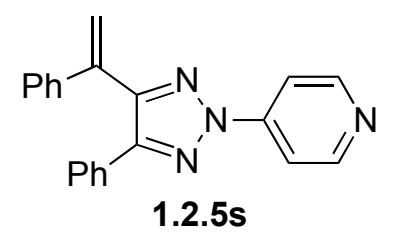

4-(4-phenyl-5-(1-phenylvinyl)-2H-1,2,3-triazol-2-yl)pyridine 1.2.5s: yield 70\%; m.p. 107$108^{\circ} \mathrm{C} ;{ }^{1} \mathrm{H}$ NMR (600 MHz, $\left.d 6-\mathrm{DMSO}\right): \delta 8.80$ (bs, 2H), 8.06 (br, 2H), 7.70-7.66 (m, 2H), 7.437.27 (m, 8H), 6.14 (s, 1H), 5.67 (s, 1H); ${ }^{13} \mathrm{C}$ NMR (125 MHz, d6-DMSO): $\delta$ 151.4, 147.3, 147.1, $144.5,137.8,137.5,129.0,128.9,128.6,128.5,128.4,127.3,126.4,120.0,112.3$; HRMS Calculated for $\left[\mathrm{C}_{21} \mathrm{H}_{16} \mathrm{~N}_{4}+\mathrm{H}\right]^{+}: 325.14477$, Found: 325.14495 . 


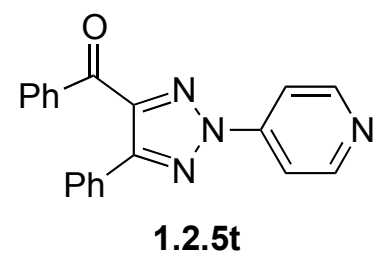

Phenyl(5-phenyl-2-(pyridin-4-yl)-2H-1,2,3-triazol-4-yl)methanone 1.2.5t: yield 60\%; m.p. $119-121{ }^{\circ} \mathrm{C} ;{ }^{1} \mathrm{H}$ NMR $\left(600 \mathrm{MHz}, \mathrm{CDCl}_{3}\right): \delta 8.83(\mathrm{br}, 2 \mathrm{H}), 8.10(\mathrm{~d}, J=8.4 \mathrm{~Hz}, 4 \mathrm{H}), 7.90-7.84(\mathrm{~m}$, 2H), $7.63(\mathrm{t}, J=7.8 \mathrm{~Hz}, 1 \mathrm{H}), 7.50(\mathrm{t}, J=7.8 \mathrm{~Hz}, 2 \mathrm{H}), 7.46-7.41(\mathrm{~m}, 3 \mathrm{H}) ;{ }^{13} \mathrm{C}$ NMR $(125 \mathrm{MHz}$, $\left.\mathrm{CDCl}_{3}\right): \delta 187.5,151.3,151.1,145.2,144.3,136.7,133.9,130.5,129.8,128.7,128.67,128.57$ 128.52, 113.3; UV (MeOH) $\lambda_{\max } 221(\varepsilon=64300)$; HRMS Calculated for $\left[\mathrm{C}_{20} \mathrm{H}_{14} \mathrm{~N}_{4} \mathrm{O}+\mathrm{H}\right]^{+}$: 327.12404, Found: 327.12402 .

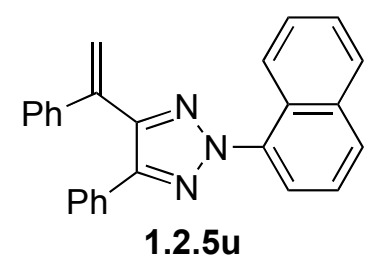

2-(naphthalen-1-yl)-4-phenyl-5-(1-phenylvinyl)-2H-1,2,3-triazole 1.2.5u: yield 70\%; m.p. 96$98^{\circ} \mathrm{C} ;{ }^{1} \mathrm{H}$ NMR $\left(600 \mathrm{MHz}, \mathrm{CDCl}_{3}\right): \delta 8.44(\mathrm{~d}, J=8.4 \mathrm{~Hz}, 1 \mathrm{H}), 7.98-7.90(\mathrm{~m}, 3 \mathrm{H}), 7.74(\mathrm{dt}, J=$ $6.6 \mathrm{~Hz}, 1.8 \mathrm{~Hz}, 2 \mathrm{H}), 7.60-7.52(\mathrm{~m}, 3 \mathrm{H}), 7.46(\mathrm{dt}, J=6.6 \mathrm{~Hz}, 1.8 \mathrm{~Hz}, 2 \mathrm{H}), 7.30-7.22(\mathrm{~m}, 6 \mathrm{H})$, $5.96(\mathrm{~s}, 1 \mathrm{H}), 5.72(\mathrm{~s}, 1 \mathrm{H}) ;{ }^{13} \mathrm{C}$ NMR $\left(125 \mathrm{MHz}, \mathrm{CDCl}_{3}\right): \delta$ 146.6, 145.9, 139.2, 138.7, 136.6, $134.5,130.2,129.5,128.4,128.33,128.31,128.2,128.16,127.8,127.43,127.38,126.9,126.6$, 125.0, 123.7, 122.7, 119.1; UV $(\mathrm{MeOH}) \lambda_{\max } 222(\varepsilon=75300)$; HRMS Calculated for $\left[\mathrm{C}_{26} \mathrm{H}_{19} \mathrm{~N}_{3}+\mathrm{H}\right]^{+}:$374.16517, Found: 374.16534 . 
<smiles>[Y16]c1nc(-c2ccccc2)n(-c2ccc(-n3nc(C(=C)c4ccccc4)c(-c4ccccc4)n3)cc2)n1</smiles>

4-phenyl-2-(4-(4-phenyl-5-(1-phenylvinyl)-2H-1,2,3-triazol-2-yl)phenyl)-5-(1-phenylvinyl)2H-1,2,3-triazole 1.2.5v: yield $10 \%$; m.p. $177-179^{\circ} \mathrm{C} ;{ }^{1} \mathrm{H} \mathrm{NMR}\left(600 \mathrm{MHz}, \mathrm{CDCl}_{3}\right): \delta 8.31(\mathrm{~s}$, 4H), $7.70(\mathrm{dd}, J=7.8 \mathrm{~Hz}, 1.8 \mathrm{~Hz}, 4 \mathrm{H}), 7.42(\mathrm{dd}, J=7.8 \mathrm{~Hz}, 1.8 \mathrm{~Hz}, 4 \mathrm{H}), 7.31-7.24(\mathrm{~m}, 12 \mathrm{H})$, $5.97(\mathrm{~s}, 2 \mathrm{H}), 5.68(\mathrm{~s}, 2 \mathrm{H}) ;{ }^{13} \mathrm{C} \mathrm{NMR}\left(125 \mathrm{MHz}, \mathrm{CDCl}_{3}\right): \delta 147.0,146.3,139.1,138.5,138.4$, $130.0,128.5,128.42,128.36,128.2,127.7,126.9,119.5,119.2 ; \mathrm{UV}(\mathrm{MeOH}) \lambda_{\max } 332(\varepsilon=$ 63800); HRMS Calculated for $\left[\mathrm{C}_{38} \mathrm{H}_{28} \mathrm{~N}_{6}+\mathrm{H}\right]^{+}$: 569.24482, Found: 569.24498 .

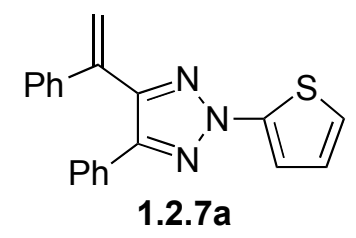

4-phenyl-5-(1-phenylvinyl)-2-(thiophen-2-yl)-2H-1,2,3-triazole 1.2.7a: yield 40\%; m.p. 83$85^{\circ} \mathrm{C} ;{ }^{1} \mathrm{H}$ NMR $\left(600 \mathrm{MHz}, \mathrm{CDCl}_{3}\right): \delta 7.67-7.65(\mathrm{~m}, 2 \mathrm{H}), 7.51(\mathrm{dd}, J=3.6 \mathrm{~Hz}, 1.8 \mathrm{~Hz}, 1 \mathrm{H}), 7.40-$ $7.38(\mathrm{~m}, 2 \mathrm{H}), 7.28-7.22(\mathrm{~m}, 6 \mathrm{H}), 7.09(\mathrm{dd}, J=5.4 \mathrm{~Hz}, 1.8 \mathrm{~Hz}, 1 \mathrm{H}), 7.00(\mathrm{dd}, J=5.4 \mathrm{~Hz}, 3.6 \mathrm{~Hz}$, 1H), $5.94(\mathrm{~s}, 1 \mathrm{H}), 5.65(\mathrm{~s}, 1 \mathrm{H}) ;{ }^{13} \mathrm{C} \mathrm{NMR}\left(125 \mathrm{MHz}, \mathrm{CDCl}_{3}\right): \delta 146.7,146.0,142.6,138.8,138.3$, 129.7, 128.4, 128.36, 128.3, 128.2, 127.7, 126.8, 126.4, 121.0, 119.2, 115.9; HRMS Calculated for $\left[\mathrm{C}_{20} \mathrm{H}_{15} \mathrm{~N}_{3} \mathrm{~S}+\mathrm{H}\right]^{+}:$330.10594, Found: 330.10602 . 


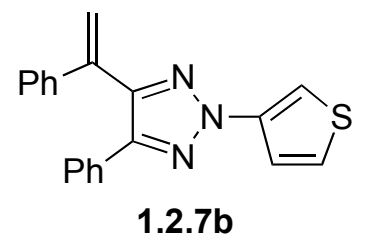

4-phenyl-5-(1-phenylvinyl)-2-(thiophen-3-yl)-2H-1,2,3-triazole $1.2 .7 \mathrm{~b}$ : yield $60 \%$; oil; ${ }^{1} \mathrm{H}$ NMR (600 MHz, $\left.\mathrm{CDCl}_{3}\right): \delta 7.73(\mathrm{dd}, J=3.0 \mathrm{~Hz}, 1.2 \mathrm{~Hz}, 1 \mathrm{H}), 7.71(\mathrm{dd}, J=4.8 \mathrm{~Hz}, 1.2 \mathrm{~Hz}, 1 \mathrm{H})$, 7.67-7.64 (m, 2H), 7.40-7.37 (m, 2H), 7.34 (dd, $J=5.4 \mathrm{~Hz}, 3.6 \mathrm{~Hz}, 1 \mathrm{H}), 7.27-7.19$ (m, 6H), 5.92 (s, 1H), $5.62(\mathrm{~s}, 1 \mathrm{H}) ;{ }^{13} \mathrm{C}$ NMR (125 MHz, $\left.\mathrm{CDCl}_{3}\right): \delta 146.1,145.4,139.3,139.1,138.4,130.0$ $128.33,128.27,128.1,127.6,126.8,126.3,120.5,119.0,112.2$; HRMS Calculated for $\left[\mathrm{C}_{20} \mathrm{H}_{15} \mathrm{~N}_{3} \mathrm{~S}+\mathrm{H}\right]^{+}: 330.10594$, Found: 330.10600 .

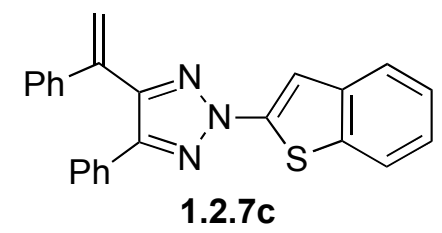

2-(benzo[b]thiophen-2-yl)-4-phenyl-5-(1-phenylvinyl)-2H-1,2,3-triazole $1.2 .7 \mathrm{c}$ : yield $50 \%$; 259-261 ${ }^{\circ} \mathrm{C} ;{ }^{1} \mathrm{H}$ NMR (600 MHz, $\left.\mathrm{CDCl}_{3}\right): \delta 7.80(\mathrm{~d}, J=8.4 \mathrm{~Hz}, 1 \mathrm{H}), 7.77(\mathrm{~d}, J=7.2 \mathrm{~Hz}, 1 \mathrm{H}), 7$. $74(\mathrm{~s}, 1 \mathrm{H}), 7.68-7.66(\mathrm{~m}, 2 \mathrm{H}), 7.41-7.32(\mathrm{~m}, 4 \mathrm{H}), 7.29-7.22(\mathrm{~m}, 6 \mathrm{H}), 5.96(\mathrm{~s}, 1 \mathrm{H}), 5.68(\mathrm{~s}, 1 \mathrm{H})$ ${ }^{13} \mathrm{C}$ NMR $\left(125 \mathrm{MHz}, \mathrm{CDCl}_{3}\right): \delta 147.3,146.7,141.6,138.7,138.5,138.2,136.2,129.5,128.6$, 128.4, 128.35, 128.26, 127.8, 126.8, 125.1, 124.8, 124.0, 122.3, 119.5, 111.4; HRMS Calculated for $\left[\mathrm{C}_{24} \mathrm{H}_{17} \mathrm{~N}_{3} \mathrm{~S}+\mathrm{H}\right]^{+}: 380.12159$, Found: 380.12166 . 


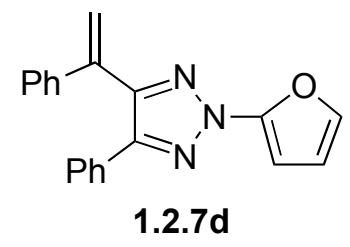

2-(furan-2-yl)-4-phenyl-5-(1-phenylvinyl)-2H-1,2,3-triazole 1.2.7d: yield 30\%; oil; ${ }^{1} \mathrm{H}$ NMR $\left(600 \mathrm{MHz}, \mathrm{CDCl}_{3}\right): \delta$ 7.59-7.57 (m, 2H), 7.32-7.28 (m, 3H), 7.20-7.15 (m, 6H), $6.56(\mathrm{dd}, J=3.6$ $\mathrm{Hz}, 1.2 \mathrm{~Hz}, 1 \mathrm{H}), 6.48(\mathrm{dd}, J=3.0 \mathrm{~Hz}, 1.8 \mathrm{~Hz}, 1 \mathrm{H}), 5.87(\mathrm{~s}, 1 \mathrm{H}), 5.58(\mathrm{~s}, 1 \mathrm{H}) ;{ }^{13} \mathrm{C} \mathrm{NMR}(125$ $\left.\mathrm{MHz}, \mathrm{CDCl}_{3}\right): \delta 147.1,146.4,139.6,138.7,138.3,132.2,129.5,128.6,128.4,128.3,128.2$, 127.8, 126.8, 119.4, 111.9, 97.7; HRMS Calculated for $\left[\mathrm{C}_{20} \mathrm{H}_{15} \mathrm{~N}_{3} \mathrm{O}+\mathrm{H}\right]^{+}:$314.12879, Found: 314.12884.

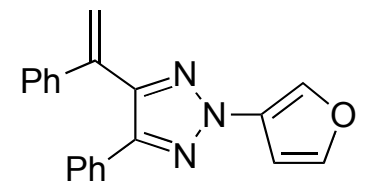

1.2.7e

2-(furan-3-yl)-4-phenyl-5-(1-phenylvinyl)-2H-1,2,3-triazole $1.2 .7 \mathrm{e}$ : yield $50 \%$; oil; ${ }^{1} \mathrm{H}$ NMR $\left(600 \mathrm{MHz}, \mathrm{CDCl}_{3}\right): \delta 8.06(\mathrm{~s}, 1 \mathrm{H}), 7.64(\mathrm{dd}, J=7.8 \mathrm{~Hz}, 1.8 \mathrm{~Hz}, 2 \mathrm{H}), 7.45(\mathrm{t}, J=1.2 \mathrm{~Hz}, 1 \mathrm{H})$, 7.40-7.36 (m, 2H), 7.28-7.21 (m, 6H), $7.04(\mathrm{~d}, J=1.2 \mathrm{~Hz}, 1 \mathrm{H}), 5.92(\mathrm{~s}, 1 \mathrm{H}), 5.62(\mathrm{~s}, 1 \mathrm{H}) ;{ }^{13} \mathrm{C}$ NMR $\left(125 \mathrm{MHz}, \mathrm{CDCl}_{3}\right): \delta 146.3,145.5,143.4,139.0,138.4,132.8,130.1,129.9,128.4,128.3$, 128.2, 127.7, 126.8, 119.0, 104.8; HRMS Calculated for $\left[\mathrm{C}_{20} \mathrm{H}_{15} \mathrm{~N}_{3} \mathrm{O}+\mathrm{H}\right]^{+}:$314.12879, Found: 314.12882. 


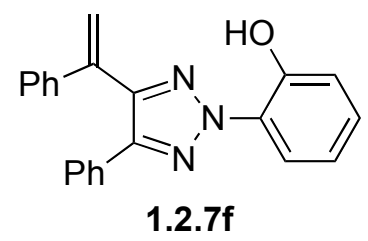

2-(4-phenyl-5-(1-phenylvinyl)-2H-1,2,3-triazol-2-yl)phenol 1.2.7f: yield 82\%; oil; ${ }^{1} \mathrm{H}$ NMR $\left(270 \mathrm{MHz}, \mathrm{CDCl}_{3}\right): \delta 10.70(\mathrm{~s}, 1 \mathrm{H}), 8.21(\mathrm{~d}, J=7.6 \mathrm{~Hz}, 1 \mathrm{H}), 7.72-7.65(\mathrm{~m}, 2 \mathrm{H}), 7.44-7.37(\mathrm{~m}$, 2H), 7.35-7.23 (m, 7H), $7.17(\mathrm{~d}, J=7.6 \mathrm{~Hz}, 1 \mathrm{H}), 7.02(\mathrm{t}, J=7.6 \mathrm{~Hz}, 1 \mathrm{H}), 5.94(\mathrm{~s}, 1 \mathrm{H}), 5.65(\mathrm{~s}$, $1 \mathrm{H}) ;{ }^{13} \mathrm{C} \mathrm{NMR}\left(67.5 \mathrm{MHz}, \mathrm{CDCl}_{3}\right): \delta 148.1,144.8,143.9,138.3,138.0,129.1,128.8,128.7$, 128.4, 128.3, 127.7, 126.7, 124.9, 119.9, 119.4, 118.6; HRMS Calculated for $\left[\mathrm{C}_{22} \mathrm{H}_{17} \mathrm{~N}_{3} \mathrm{O}+\mathrm{H}\right]^{+}$: 340.14444, Found: 340.14452. 


\section{ORTEP Drawing of the Crystal Structures}

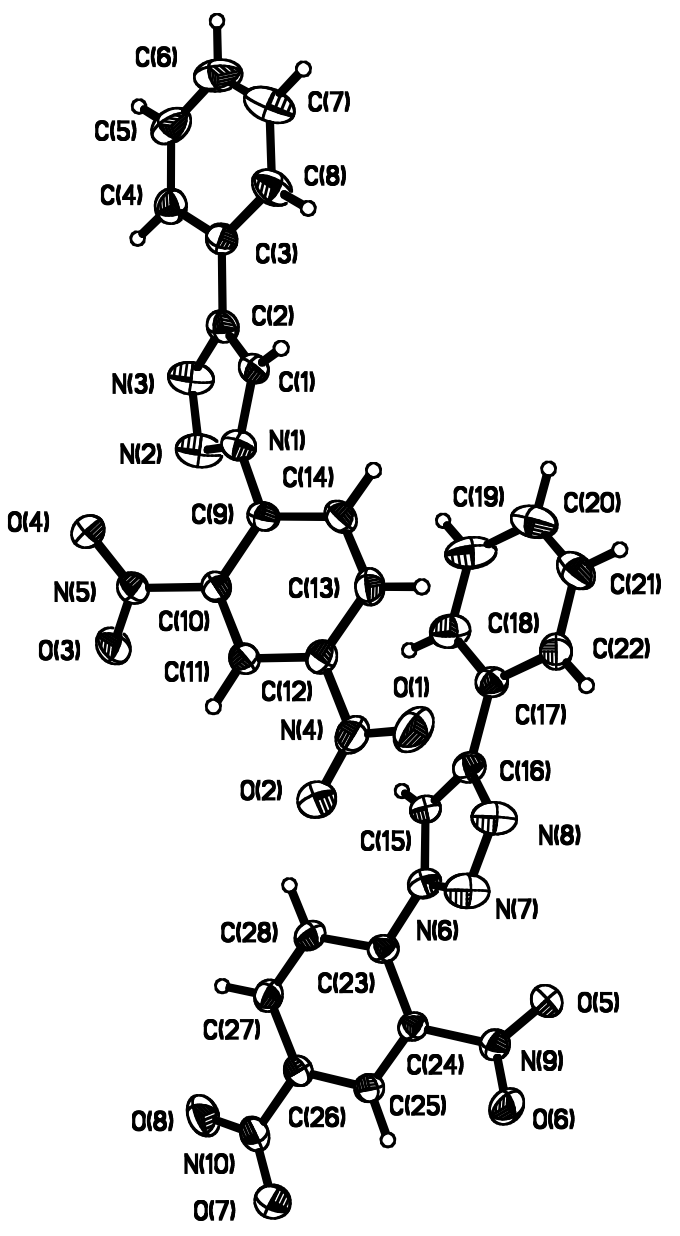

Figure S1.2-1.Perspective view of the molecular structures of the two independent molecules of N-1-1.2.3b $\left(\mathrm{C}_{14} \mathrm{H}_{9} \mathrm{~N}_{5} \mathrm{O}_{4}\right)$ with the atom-labeling scheme. The thermal ellipsoids are scaled to enclose $30 \%$ probability. CCDC number: 702805 


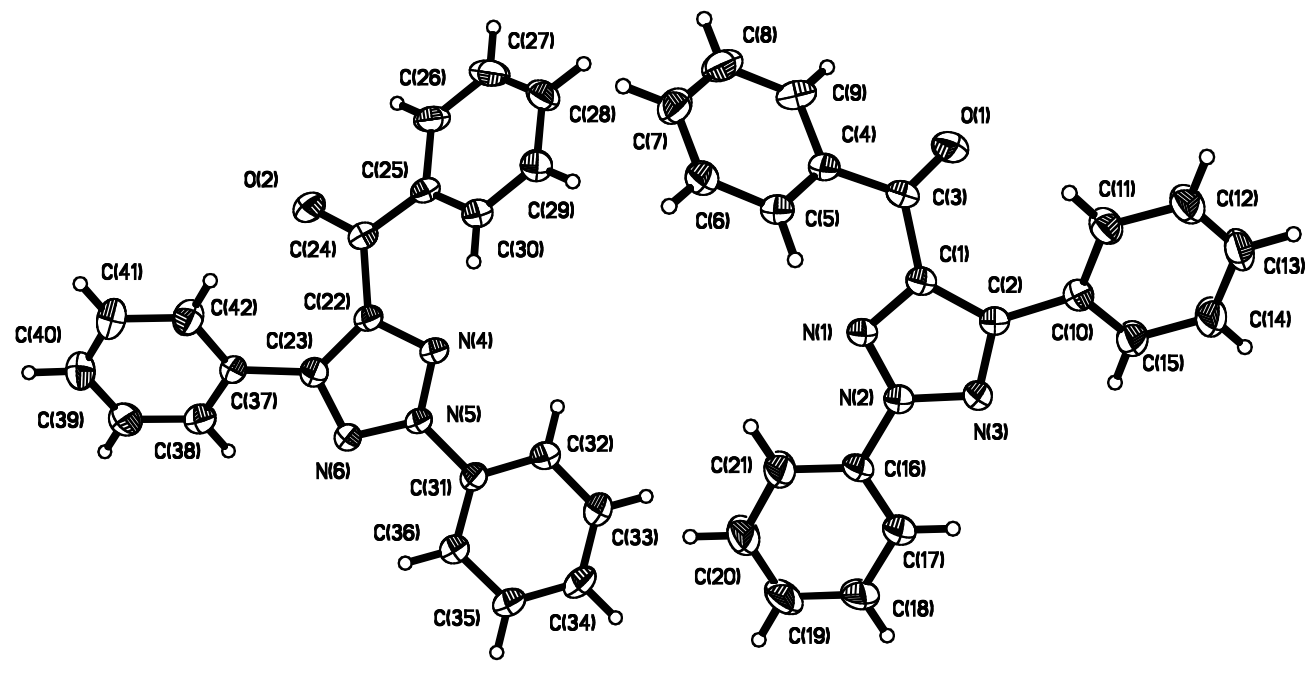

Figure S1.2-2.Perspective view of the molecular structures of the two independent molecules of 1.2.5d $\left(\mathrm{C}_{21} \mathrm{H}_{15} \mathrm{~N}_{3}\right)$ with the atom-labeling scheme. The thermal ellipsoids are scaled to enclose $30 \%$ probability. CCDC number: 702800 


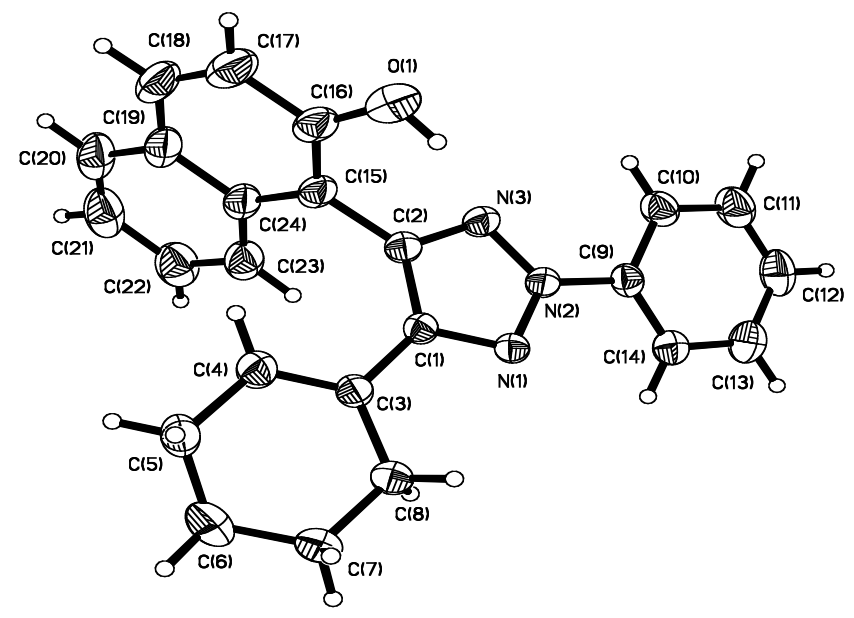

Figure S1.2-3.Perspective view of the molecular structure of 1.2.5 $\mathbf{j}\left(\mathrm{C}_{24} \mathrm{H}_{21} \mathrm{~N}_{3} \mathrm{O}\right)$ with the atomlabeling scheme. The thermal ellipsoids are scaled to enclose $30 \%$ probability. CCDC number: 702801 


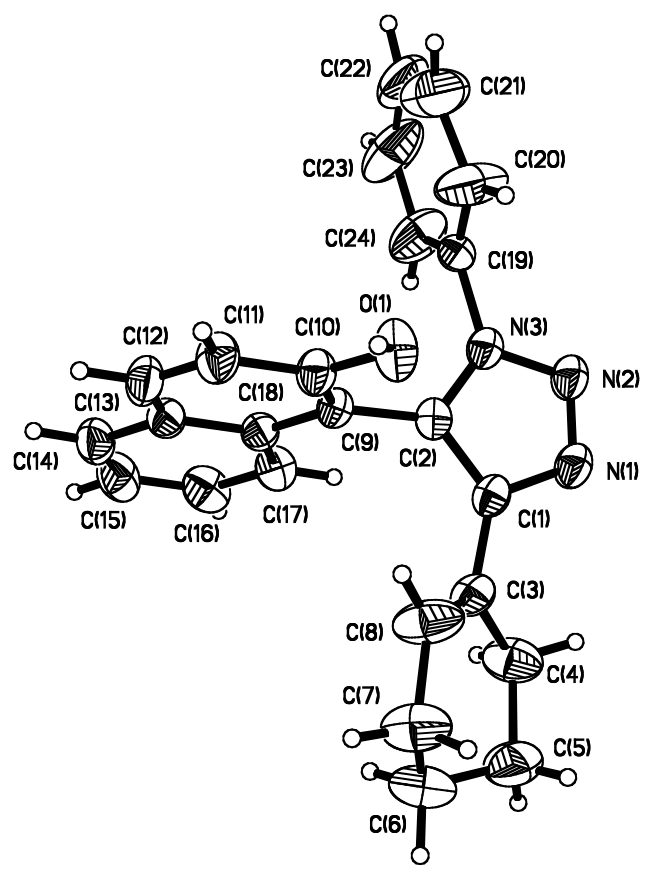

Figure S1.2-4:Perspective view of the molecular structure of $\mathbf{N - 3 - 1 . 2 . 5 j}\left(\mathrm{C}_{24} \mathrm{H}_{21} \mathrm{~N}_{3} \mathrm{O}\right)$ with the atom-labeling scheme. The thermal ellipsoids are scaled to enclose $30 \%$ probability. CCDC number: 702806. 


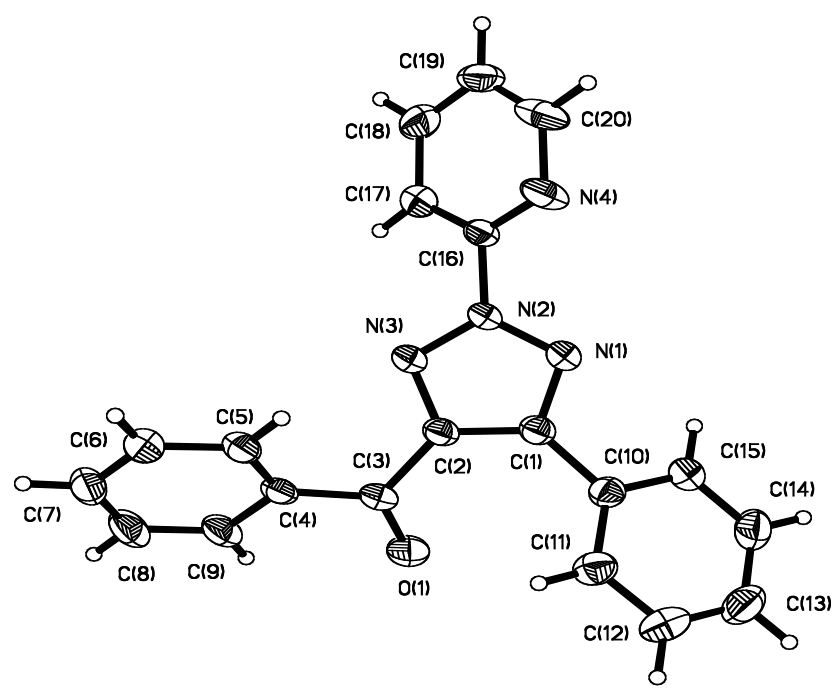

Figure S1.2-5: Perspective view of the molecular structure of 1.2.5q $\left(\mathrm{C}_{20} \mathrm{H}_{14} \mathrm{~N}_{4} \mathrm{O}\right)$ with the atomlabeling scheme. The thermal ellipsoids are scaled to enclose $30 \%$ probability. CCDC number: 702802 


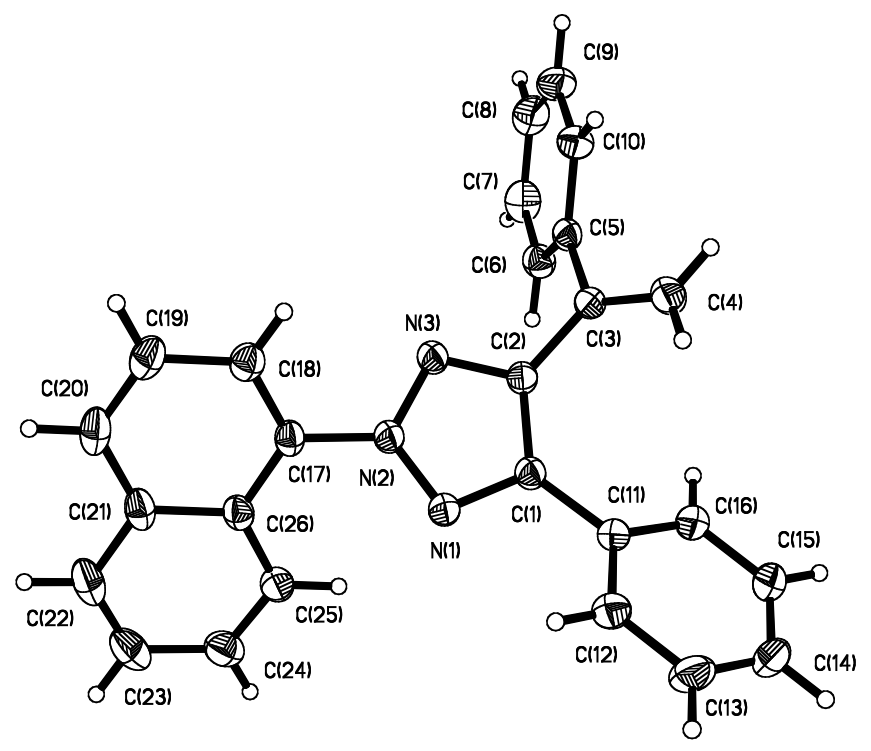

Figure S1.2-6.Perspective view of the molecular structure of 1.2.5u $\left(\mathrm{C}_{26} \mathrm{H}_{19} \mathrm{~N}_{3}\right)$ with the atomlabeling scheme. The thermal ellipsoids are scaled to enclose $30 \%$ probability. CCDC number: 702804 


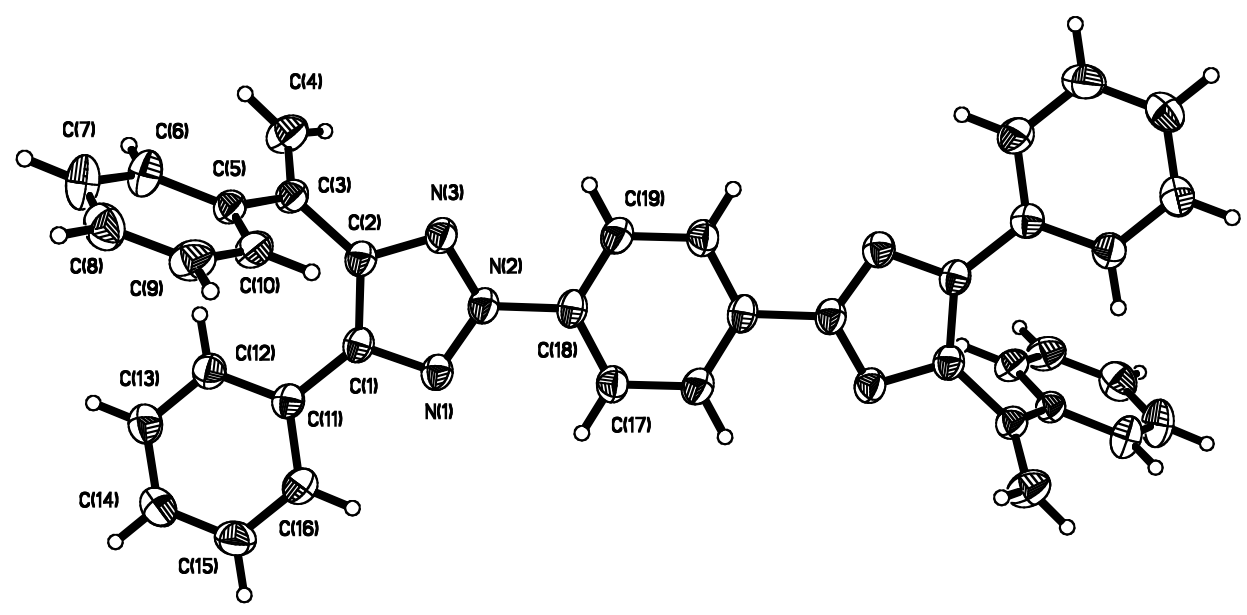

Figure S1.2-7.Perspective view of the molecular structure of $1.2 .5 \mathrm{v}\left(\mathrm{C}_{38} \mathrm{H}_{28} \mathrm{~N}_{6}\right)$ with the atomlabeling scheme. The thermal ellipsoids are scaled to enclose $30 \%$ probability. CCDC number: 702803 


\subsection{Highly efficient synthesis of vinyl substituted triazoles by Au(I) catalyzed alkyne activation}

\section{General Methods and materials}

Unless otherwise noted, all commercial reagents and solvents were obtained from the commercial provider and used without further purification. Air and/or moisture-sensitive reactions were carried out under an atmosphere of nitrogen using oven/flame-dried glassware and standard syringe/septa techniques. 1H-NMR and 13C-NMR spectra were recorded on Joel 270 and Varian $600 \mathrm{MHz}$ spectrometers. Chemical shifts were reported relative to internal tetramethylsilane $(\delta 0.00 \mathrm{ppm})$ or $\mathrm{CDCl}_{3}(\delta 7.26 \mathrm{ppm})$ or DMSO $(\delta 2.49 \mathrm{ppm})$ for ${ }^{1} \mathrm{H}$ and $\mathrm{CDCl}_{3}$

$(\delta 77.0 \mathrm{ppm})$ or DMSO ( $\delta 39.5 \mathrm{ppm})$ for ${ }^{13} \mathrm{C}$. Infrared (IR) spectra were obtained on a Prospect MIDAC FT-IR spectrometer. Flash column chromatography was performed on 230-430 mesh silica gel. Analytical thin layer chromatography was performed with precoated glass baked plates $(250 \mu)$ and visualized by fluorescence and by charring after treatment with potassium permanganate stain. $\mathrm{R}_{f}$ values were obtained by elution in the stated solvent ratios. 


\section{General Procedure for $\mathrm{Au}(\mathrm{I})$ catalyzed addition of triazoles to alkynes}

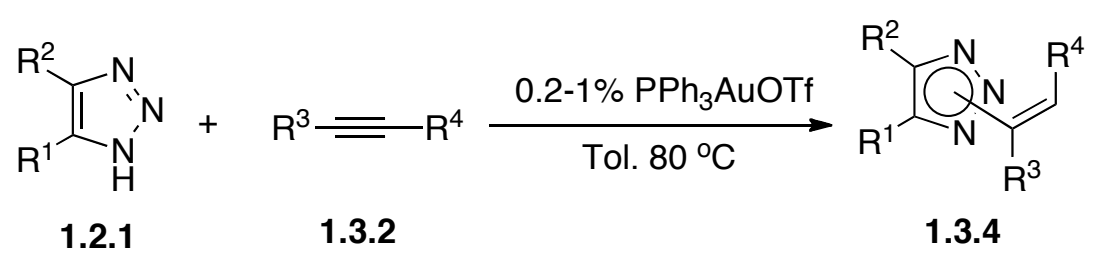

To a solution of benzotriazole $\mathbf{1 . 2 . 1}(59.5 \mathrm{mg}, 0.5 \mathrm{mmol})$ in $2.5 \mathrm{ml}$ of freshly distilled toluene, was added $\mathrm{PP}_{3} \mathrm{AuCl}(12.5 \mathrm{mg}, 0.025 \mathrm{mmol})$, AgOTf $(6.5 \mathrm{mg}, 0.025 \mathrm{mmol})$ and phenylacetylene $\mathbf{1 . 3 . 2}$ (76.5mg, $0.75 \mathrm{mmol}$ ). The reaction mixture was then heated at $80{ }^{\circ} \mathrm{C}$ for 12 hours. After heating, the reaction mixture was cooled, passed through a short silica plug and concentrated to remove toluene. The crude mixture was then purified by flash silica gel chromatography to give the vinyl triazole 1.3.4 as clear oil in quantitative yield. 


\section{Compound Characterization}

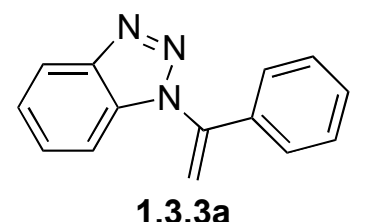

1-(1-phenylvinyl)-1H-benzo[d][1,2,3]triazole 1.3.3a: purified by flash chromatography (Hexane-EtOAc, v/v 5/1) as yellow oil with overall isolated yield: 92\%. ${ }^{1} \mathrm{H}-\mathrm{NMR}(600 \mathrm{MHz}$, $\left.\mathrm{CDCl}_{3}\right) \delta 8.05-8.09(\mathrm{~m}, 1 \mathrm{H}), 7.30-7.39(\mathrm{~m}, 5 \mathrm{H}), 7.25(\mathrm{~d}, J=7.8 \mathrm{~Hz}, 2 \mathrm{H}), 7.00-7.05(\mathrm{~m}, 1 \mathrm{H}), 5.76$ (s, 1H), $5.73(\mathrm{~s}, 1 \mathrm{H}) ;{ }^{13} \mathrm{C}-\mathrm{NMR}\left(67.5 \mathrm{MHz}, \mathrm{CDCl}_{3}\right) \delta 146.1,142.6,134.6,132.9,129.72,128.8$, 127.7, 126.8, 124.1, 120.0, 111.1, 111.0; HRMS Calculated for $\left[\mathrm{C}_{14} \mathrm{H}_{11} \mathrm{~N}_{3}\right]^{+}: 221.2572$, Found: 221.0953 .

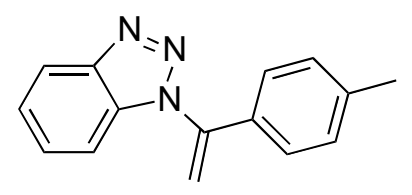

1.3.3b

1-(1-p-tolylvinyl)-1H-benzo[d][1,2,3]triazole $1.3 .3 \mathrm{~b}$ : purified by flash chromatography (Hexane-EtOAc, v/v 5/1) as yellow oil with overall isolated yield: 94\%. ${ }^{1} \mathrm{H}-\mathrm{NMR}(600 \mathrm{MHz}$, $\left.\mathrm{CDCl}_{3}\right) \delta 8.08-8.10(\mathrm{~m}, 1 \mathrm{H}), 7.33-7.36(\mathrm{~m}, 2 \mathrm{H}), 7.16(\mathrm{~s}, 4 \mathrm{H}), 7.05-7.08(\mathrm{~m}, 1 \mathrm{H}), 5.73(\mathrm{~s}, 1 \mathrm{H})$, $5.70(\mathrm{~s}, 1 \mathrm{H}), 2.37(\mathrm{~s}, 3 \mathrm{H}) ;{ }^{13} \mathrm{C}-\mathrm{NMR}\left(67.5 \mathrm{MHz}, \mathrm{CDCl}_{3}\right) \delta$ 146.4, 142.9, 140.2, 133.2, 132.0, 129.7, 127.9, 127.0, 124.3, 120.3, 111.5, 110.4, 21.5; HRMS Calculated for $\left[\mathrm{C}_{15} \mathrm{H}_{13} \mathrm{~N}_{3}\right]^{+}$: 235.2838, Found: 235.1109 . 


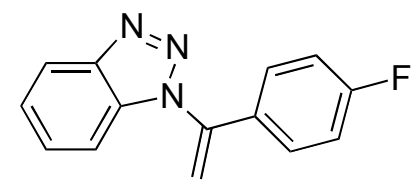

1.3.3c

1-(1-(4-fluorophenyl)vinyl)-1H-benzo[d][1,2,3]triazole $1.3 .3 \mathrm{c}:$ purified by flash chromatography (Hexane-EtOAc, v/v 5/1) as yellow oil with overall isolated yield: 96\%. ${ }^{1} \mathrm{H}-$ NMR (600 MHz, $\left.\mathrm{CDCl}_{3}\right) \delta 8.08(\mathrm{~d}, J=6.6 \mathrm{~Hz}, 1 \mathrm{H}), 7.34-7.39(\mathrm{~m}, 2 \mathrm{H}), 7.24-7.24(\mathrm{~m}, 2 \mathrm{H}), 7.08$ $(\mathrm{d}, J=7.2 \mathrm{~Hz}, 1 \mathrm{H}), 7.04(\mathrm{t}, J=8.4 \mathrm{~Hz}, 2 \mathrm{H}), 5.72(\mathrm{~d}, J=4.2 \mathrm{~Hz}, 2 \mathrm{H}) ;{ }^{13} \mathrm{C}-\mathrm{NMR}\left(67.5 \mathrm{MHz}, \mathrm{CDCl}_{3}\right)$ $\delta 164.6,163.0,146.3,141.9,133.1,131.1,129.1,128.2,124.5,120.4,116.2,111.3$; HRMS Calculated for $\left[\mathrm{C}_{14} \mathrm{H}_{10} \mathrm{FN}_{3}\right]^{+}: 239.2477$, Found: 239.0859 .

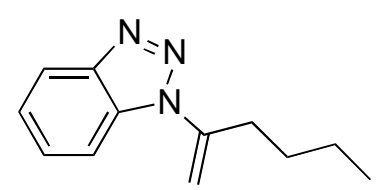

1.3.3d

1-(hex-1-en-2-yl)-1H-benzo[d][1,2,3]triazole 1.3.3d: purified by flash chromatography (Hexane-EtOAc, v/v 5/1) as yellow oil with overall isolated yield: 90\%. ${ }^{1} \mathrm{H}-\mathrm{NMR}(600 \mathrm{MHz}$, $\left.\mathrm{CDCl}_{3}\right) \delta 8.05(\mathrm{~d}, J=7.8 \mathrm{~Hz}, 1 \mathrm{H}), 7.64(\mathrm{~d}, J=8.4 \mathrm{~Hz}, 1 \mathrm{H}), 7.48(\mathrm{t}, J=7.2 \mathrm{~Hz}, 1 \mathrm{H}), 7.36(\mathrm{t}, J=$ $7.8 \mathrm{~Hz}, 1 \mathrm{H}), 5.38(\mathrm{~s}, 1 \mathrm{H}), 5.24(\mathrm{~s}, 1 \mathrm{H}), 2.89(\mathrm{t}, J=7.2 \mathrm{~Hz}, 2 \mathrm{H}), 1.43-1.48(\mathrm{~m}, 2 \mathrm{H}), 1.32-14.38(\mathrm{~m}$, 2H), $0.86(\mathrm{t}, J=7.2 \mathrm{~Hz}, 3 \mathrm{H}) ;{ }^{13} \mathrm{C}-\mathrm{NMR}\left(67.5 \mathrm{MHz}, \mathrm{CDCl}_{3}\right) \delta 146.4,144.6,132.5,128.1,124.4$, 120.3, 111.3, 106.3, 34.31, 29.3, 22.2, 13.9; HRMS Calculated for $\left[\mathrm{C}_{12} \mathrm{H}_{15} \mathrm{~N}_{3}\right]^{+}:$201.2676, Found: 201.1266. 


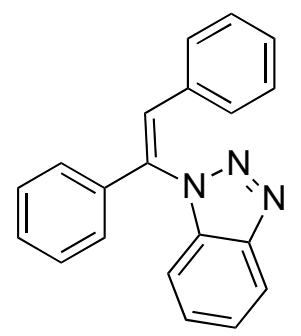

$1.3 .3 \mathrm{e}$

(Z)-1-(1,2-diphenylvinyl)-1H-benzo[d][1,2,3]triazole 1.3.3e: purified by flash chromatography (Hexane-EtOAc, v/v 5/1) as yellow oil with overall isolated yield: 70\%. ${ }^{1} \mathrm{H}-\mathrm{NMR}(600 \mathrm{MHz}$, $\left.\mathrm{CDCl}_{3}\right) \delta 8.14(\mathrm{~d}, J=8.4 \mathrm{~Hz}, 1 \mathrm{H}), 7.34-7.41(\mathrm{~m}, 6 \mathrm{H}), 7.22(\mathrm{~d}, J=9.0 \mathrm{~Hz}, 2 \mathrm{H}), 7.06-7.13(\mathrm{~m}, 4 \mathrm{H})$, $6.75(\mathrm{~d}, J=7.8 \mathrm{~Hz}, 2 \mathrm{H}) ;{ }^{13} \mathrm{C}-\mathrm{NMR}\left(67.5 \mathrm{MHz}, \mathrm{CDCl}_{3}\right) \delta 145.8,136.6,133.6,133.5,133.1,129.3$, $128.9,128.6,128.5,128.2,128.0,127.6,125.9,124.2,120.0,110.5 ;$ HRMS Calculated for $\left[\mathrm{C}_{20} \mathrm{H}_{15} \mathrm{~N}_{3}\right]^{+}$: 297.3532, Found: 297.1266.

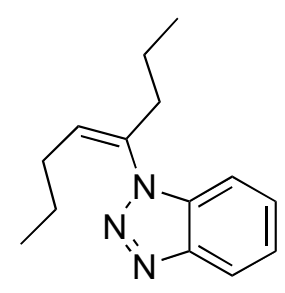

1.3.3f

(Z)-1-(oct-4-en-4-yl)-1H-benzo[d][1,2,3]triazole 1.3.3f: purified by flash chromatography (Hexane-EtOAc, v/v 5/1) as yellow oil with overall isolated yield: 83\%. ${ }^{1} \mathrm{H}-\mathrm{NMR}(600 \mathrm{MHz}$, $\left.\mathrm{CDCl}_{3}\right) \delta 8.04(\mathrm{~d}, J=8.4 \mathrm{~Hz}, 1 \mathrm{H}), 7.44(\mathrm{t}, J=7.8 \mathrm{~Hz}, 1 \mathrm{H}), 7.33(\mathrm{q}, J=8.4 \mathrm{~Hz}, 16.2 \mathrm{~Hz}, 2 \mathrm{H}), 5.79$ $(\mathrm{t}, J=7.2 \mathrm{~Hz}, 1 \mathrm{H}), 2.55(\mathrm{t}, J=7.8 \mathrm{~Hz}, 2 \mathrm{H}), 1.68(\mathrm{q}, J=7.2 \mathrm{~Hz}, 14.4 \mathrm{~Hz}, 2 \mathrm{H}), 1.26-1.34(\mathrm{~m}, 4 \mathrm{H})$, $0.86(\mathrm{t}, J=7.2 \mathrm{~Hz}, 3 \mathrm{H}), 0.74(\mathrm{t}, J=7.8 \mathrm{~Hz}, 3 \mathrm{H}),{ }^{13} \mathrm{C}-\mathrm{NMR}\left(67.5 \mathrm{MHz}, \mathrm{CDCl}_{3}\right) \delta 145.5,135.2$, 
133.5, 129.7, 127.7, 124.0, 120.1, 110.3, 38.4, 29.6, 22.5, 20.5, 13.8, 13.5; HRMS Calculated for $\left[\mathrm{C}_{14} \mathrm{H}_{19} \mathrm{~N}_{3}\right]^{+}:$229.3208, Found: 229.1579 .

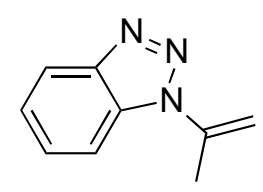

$1.3 .3 \mathrm{~g}$

1-(prop-1-en-2-yl)-1H-benzo[d][1,2,3]triazole 1.3.3g: purified by flash chromatography (Hexane-EtOAc, v/v 5/1) as yellow oil with overall isolated yield: 82\%. ${ }^{1} \mathrm{H}-\mathrm{NMR}(600 \mathrm{MHz}$, $\left.\mathrm{CDCl}_{3}\right) \delta 8.08(\mathrm{~d}, J=8.4 \mathrm{~Hz}, 1 \mathrm{H}), 7.72(\mathrm{~d}, J=8.4 \mathrm{~Hz}, 1 \mathrm{H}), 7.51(\mathrm{t}, J=7.2 \mathrm{~Hz}, 1 \mathrm{H}), 7.39(\mathrm{t}, J=$ $7.8 \mathrm{~Hz}, 1 \mathrm{H}), 5.44(\mathrm{~s}, 1 \mathrm{H}), 5.21(\mathrm{~s}, 1 \mathrm{H}), 2.55(\mathrm{~s}, 3 \mathrm{H}) ;{ }^{13} \mathrm{C}-\mathrm{NMR}\left(67.5 \mathrm{MHz}, \mathrm{CDCl}_{3}\right) \delta$ 146.6, 140.6, 132.0, 128.2, 124.5, 120.5, 111.6, 105.5, 21.3; HRMS Calculated for $\left[\mathrm{C}_{9} \mathrm{H}_{9} \mathrm{~N}_{3}\right]^{+}: 159.1879$, Found: 159.0796 .

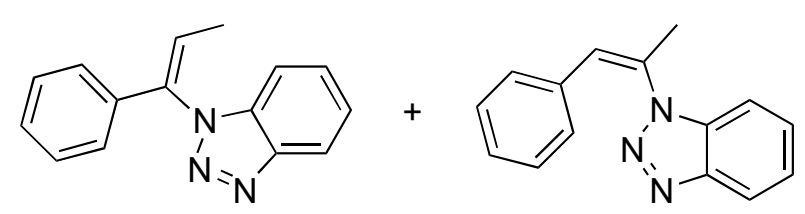

1.3.3h

1-((Z)-1-phenylprop-1-enyl)-1H-benzo[d][1,2,3]triazole vs 1-((Z)-1-phenylprop-1-en-2-yl)1H-benzo[d][1,2,3]triazole 1.3.3h: purified by flash chromatography (Hexane-EtOAc, v/v 5/1) as yellow oil with overall isolated yield: $84 \% .{ }^{1} \mathrm{H}-\mathrm{NMR}\left(600 \mathrm{MHz}, \mathrm{CDCl}_{3}\right) \delta 8.12(\mathrm{~d}, J=7.8 \mathrm{~Hz}$, $1 \mathrm{H}), 8.02(\mathrm{~d}, J=8.4 \mathrm{~Hz}, 1 \mathrm{H}), 7.36-7.41(\mathrm{~m}, 2 \mathrm{H}), 7.16-7.28(\mathrm{~m}, 6 \mathrm{H}), 7.06-7.08(\mathrm{~m}, 2 \mathrm{H}), 6.97-7.02$ 
(m, 3H), $6.73(\mathrm{~s}, 1 \mathrm{H}), 6.68(\mathrm{~d}, J=7.2 \mathrm{~Hz}, 2 \mathrm{H}), 6.59(\mathrm{q}, J=7.2 \mathrm{~Hz}, 13.8 \mathrm{~Hz}, 1 \mathrm{H}), 2.48(\mathrm{~s}, 2 \mathrm{H})$, $1.67(\mathrm{~d}, J=7.2 \mathrm{~Hz}, 3 \mathrm{H}) ;{ }^{13} \mathrm{C}-\mathrm{NMR}\left(67.5 \mathrm{MHz}, \mathrm{CDCl}_{3}\right) \delta 145.9,145.8,136.2,135.9,134.0,133.8$, $131.8,131.5,129.0,129.0,128.9,128.6,128.2,128.1,128.0,127.7,127.1,126.1,126.0,125.6$ 124.3, 124.1, 120.3, 120.0, 110.9, 110.6, 23.8, 14.1; HRMS Calculated for $\left[\mathrm{C}_{15} \mathrm{H}_{13} \mathrm{~N}_{3}\right]^{+}$: 235.2838, Found: 235.1109 .

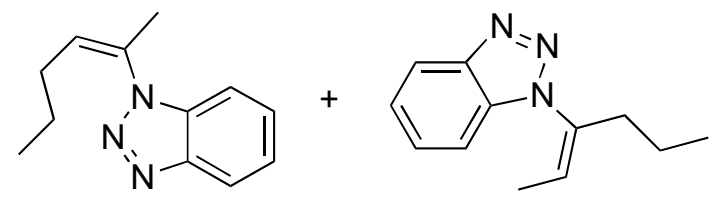

1.3.3i

benzo[d][1,2,3]triazole 1.3.3i: purified by flash chromatography (Hexane-EtOAc, v/v 5/1) as yellow oil with overall isolated yield: $81 \% .{ }^{1} \mathrm{H}-\mathrm{NMR}\left(600 \mathrm{MHz}, \mathrm{CDCl}_{3}\right) \delta 8.05(\mathrm{~d}, J=9.0 \mathrm{~Hz}$, 2H), $7.45(\mathrm{t}, J=7.8 \mathrm{~Hz}, 2 \mathrm{H}), 7.32-7.37(\mathrm{~m}, 4 \mathrm{H}), 5.86(\mathrm{q}, J=7.2 \mathrm{~Hz}, 13.8 \mathrm{~Hz}, 1 \mathrm{H}), 5.80(\mathrm{t}, J=$ $7.8 \mathrm{~Hz}, 1 \mathrm{H}), 2.57(\mathrm{t}, J=7.2 \mathrm{~Hz}, 2 \mathrm{H}), 2.23(\mathrm{~s}, 3 \mathrm{H}), 1.71(\mathrm{q}, J=7.2 \mathrm{~Hz}, 14.4 \mathrm{~Hz}, 2 \mathrm{H}), 1.40(\mathrm{~d}, J=$ $7.2 \mathrm{~Hz}, 3 \mathrm{H}), 1.23-1.35(\mathrm{~m}, 4 \mathrm{H}), 0.84(\mathrm{t}, J=7.2 \mathrm{~Hz}, 3 \mathrm{H}), 0.74(\mathrm{t}, J=7.2 \mathrm{~Hz}, 3 \mathrm{H}) ;{ }^{13} \mathrm{C}-\mathrm{NMR}(67.5$ $\left.\mathrm{MHz}, \mathrm{CDCl}_{3}\right) \delta 145.6,145.5,136.3,133.4,133.0,131.1,130.0,127.8,127.8,124.1,124.0$, 123.7, 120.2, 120.1, 110.4, 110.3; HRMS Calculated for $\left[\mathrm{C}_{12} \mathrm{H}_{15} \mathrm{~N}_{3}\right]^{+}:$201.2676, Found: 201.1266 . 


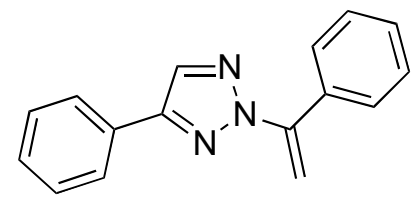

\subsection{4a(N2)}

4-phenyl-2-(1-phenylvinyl)-2H-1,2,3-triazole 1.3.4a(N2): purified by flash chromatography (Hexane-EtOAc, v/v 5/1) as yellow oil with overall isolated yield: 91\%. ${ }^{1} \mathrm{H}-\mathrm{NMR}(600 \mathrm{MHz}$, $\left.\mathrm{CDCl}_{3}\right) \delta 8.02(\mathrm{~s}, 1 \mathrm{H}), 7.85-7.86(\mathrm{~m}, 2 \mathrm{H}), 7.36-7.48(\mathrm{~m}, 8 \mathrm{H}), 5.97(\mathrm{~s}, 1 \mathrm{H}), 5.41(\mathrm{~s}, 1 \mathrm{H}){ }^{13} \mathrm{C}-$ $\operatorname{NMR}\left(67.5 \mathrm{MHz}, \mathrm{CDCl}_{3}\right) \delta 148.9,146.4,135.0,132.7,130.1,129.5,129.2,129.1,128.6,128.5$, 126.4, 107.6; HRMS Calculated for $\left[\mathrm{C}_{16} \mathrm{H}_{13} \mathrm{~N}_{3}\right]^{+}:$247.2954, Found: 247.1109.

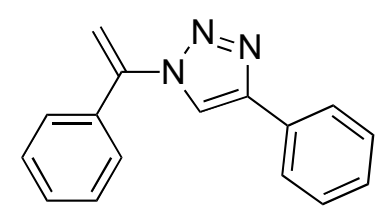

\subsection{4a(N1)}

4-phenyl-1-(1-phenylvinyl)-1H-1,2,3-triazole 1.3.4a(N1): purified by flash chromatography (Hexane-EtOAc, v/v 5/1) as yellow oil with overall isolated yield: 91\%. ${ }^{1} \mathrm{H}-\mathrm{NMR}(600 \mathrm{MHz}$, $\left.\mathrm{CDCl}_{3}\right) \delta$ 7.83-7.85 (m, 2H), $7.79(\mathrm{~s}, 1 \mathrm{H}), 7.32-7.46(\mathrm{~m}, 8 \mathrm{H}), 5.85(\mathrm{~s}, 1 \mathrm{H}), 5.54(\mathrm{~s}, 1 \mathrm{H}) ;{ }^{13} \mathrm{C}-$ NMR $\left(67.5 \mathrm{MHz}, \mathrm{CDCl}_{3}\right) \delta 147.6,142.9,134.6,129.9,128.9,128.8,128.7,128.3,127.3,125.8$, 119.8, 109.4; HRMS Calculated for $\left[\mathrm{C}_{16} \mathrm{H}_{13} \mathrm{~N}_{3}\right]^{+}: 247.2954$, Found: 247.1109. 


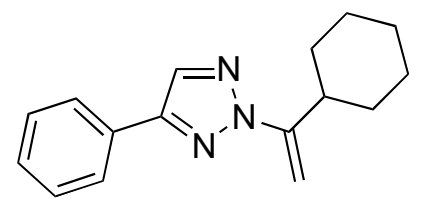

1.3.4b(N2)

2-(1-cyclohexylvinyl)-4-phenyl-2H-1,2,3-triazole $\quad 1.3 .4 b(\mathbf{N} 2)$ : purified by flash chromatography (Hexane-EtOAc, v/v 5/1) as yellow oil with overall isolated yield: $87 \% .{ }^{1} \mathrm{H}-$ NMR (600 MHz, $\left.\mathrm{CDCl}_{3}\right) \delta 7.93(\mathrm{~s}, 1 \mathrm{H}), 7.82-7.84(\mathrm{~m}, 2 \mathrm{H}), 7.42-7.44(\mathrm{~m}, 2 \mathrm{H}), 7.34-7.37(\mathrm{~m}$, $1 \mathrm{H}), 5.83(\mathrm{~s}, 1 \mathrm{H}), 4.90(\mathrm{~s}, 1 \mathrm{H}), 3.07-3.12(\mathrm{~m}, 1 \mathrm{H}), 2.01(\mathrm{~d}, J=12.0 \mathrm{~Hz}, 2 \mathrm{H}) 1.74-1.84(\mathrm{~m}, 3 \mathrm{H})$, 1.40-1.47 (m, 2H), 1.22-1.34 (m, 3H), $;^{13} \mathrm{C}-\mathrm{NMR}\left(67.5 \mathrm{MHz}, \mathrm{CDCl}_{3}\right) \delta 151.6,148.2,131.8$, 130.5, 129.0, 128.8, 126.3, 101.1, 39.6, 32.2, 26.7, 26.6; HRMS Calculated for $\left[\mathrm{C}_{16} \mathrm{H}_{19} \mathrm{~N}_{3}\right]^{+}$: 253.3422, Found: 253.1579.

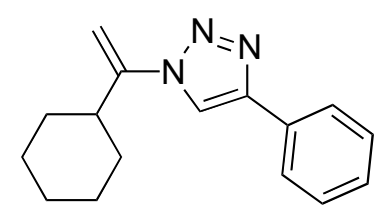

\subsection{4b(N1)}

1-(1-cyclohexylvinyl)-4-phenyl-1H-1,2,3-triazole $\quad 1.3 .4 b(N 1)$ : purified by flash chromatography (Hexane-EtOAc, v/v 5/1) as yellow oil with overall isolated yield: $87 \% .{ }^{1} \mathrm{H}-$ NMR (600 MHz, $\left.\mathrm{CDCl}_{3}\right) \delta 7.90(\mathrm{~s}, 1 \mathrm{H}), 7.85(\mathrm{~d}, J=7.2 \mathrm{~Hz}, 2 \mathrm{H}), 7.39-7.43(\mathrm{~m}, 2 \mathrm{H}), 7.31-$ 7.34(m, 1H), $5.36(\mathrm{~s}, 1 \mathrm{H}), 5.00(\mathrm{~s}, 1 \mathrm{H}), 2.90(\mathrm{t}, J=9.0,1 \mathrm{H}), 1.91(\mathrm{~d}, J=12.6 \mathrm{~Hz}, 2 \mathrm{H}), 1.80(\mathrm{~d}, J$ $=14.4 \mathrm{~Hz}, 2 \mathrm{H}), 1.74(\mathrm{~d}, J=13.8 \mathrm{~Hz}, 2 \mathrm{H}), 1.19-1.41(\mathrm{~m}, 4 \mathrm{H}) ;{ }^{13} \mathrm{C}-\mathrm{NMR}\left(67.5 \mathrm{MHz}, \mathrm{CDCl}_{3}\right) \delta$ 149.7, 131.8, 130.6, 129.0, 128.7, 126.3, 118.4, 104.2, 40.9, 31.6, 26.4, 26.3; HRMS Calculated for $\left[\mathrm{C}_{16} \mathrm{H}_{19} \mathrm{~N}_{3}\right]^{+}: 253.3422$, Found: 253.1579 . 


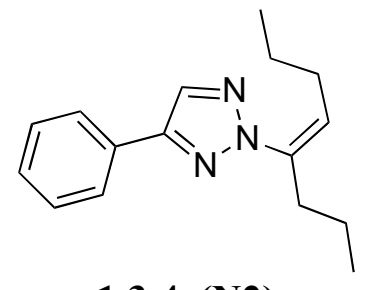

$1.3 .4 c(N 2)$

2-((Z)-oct-4-en-4-yl)-4-phenyl-2H-1,2,3-triazole 1.3.4c(N2): purified by flash chromatography (Hexane-EtOAc, v/v 5/1) as yellow oil with overall isolated yield: $81 \% .{ }^{1} \mathrm{H}-\mathrm{NMR}(600 \mathrm{MHz}$, $\left.\mathrm{CDCl}_{3}\right) \delta 7.93(\mathrm{~s}, 1 \mathrm{H}), 7.82(\mathrm{~d}, J=7.2 \mathrm{~Hz}, 2 \mathrm{H}), 7.43(\mathrm{t}, J=7.2 \mathrm{~Hz}, 2 \mathrm{H}), 7.35(\mathrm{t}, J=7.2 \mathrm{~Hz}, 1 \mathrm{H})$, $5.49(\mathrm{t}, J=7.2 \mathrm{~Hz}, 1 \mathrm{H}), 2.62(\mathrm{t}, J=7.2 \mathrm{~Hz}, 2 \mathrm{H}), 2.21(\mathrm{q}, J=7.2 \mathrm{~Hz}, 9.0 \mathrm{~Hz}, 2 \mathrm{H}), 1.41-1.47(\mathrm{~m}, 4 \mathrm{H})$, 0.89-0.93 (m, 6H), ${ }^{13} \mathrm{C}-\mathrm{NMR}\left(67.5 \mathrm{MHz}, \mathrm{CDCl}_{3}\right) \delta 147.5,138.8,131.1,130.7,129.1,128.6$, 126.2, 125.2, 37.51, 29.7, 22.9, 20.7, 14.0, 13.6; HRMS Calculated for $\left[\mathrm{C}_{16} \mathrm{H}_{21} \mathrm{~N}_{3}\right]^{+}: 255.3580$, Found: 255.1735 .

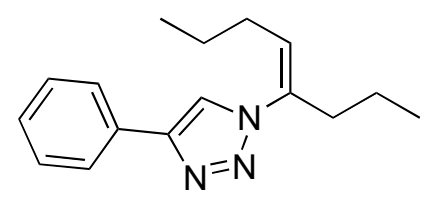

$1.3 .4 \mathrm{c}(\mathrm{N} 1)$

1-((Z)-oct-4-en-4-yl)-4-phenyl-1H-1,2,3-triazole 1.3.4c(N1): purified by flash chromatography (Hexane-EtOAc, v/v 5/1) as yellow oil with overall isolated yield: 81\%. ${ }^{1} \mathrm{H}-\mathrm{NMR}(600 \mathrm{MHz}$, $\left.\mathrm{CDCl}_{3}\right) \delta 7.87(\mathrm{~d}, J=7.2 \mathrm{~Hz}, 2 \mathrm{H}), 7.73(\mathrm{~s}, 1 \mathrm{H}), 7.42(\mathrm{t}, J=7.2 \mathrm{~Hz}, 2 \mathrm{H}), 7.33(\mathrm{t}, J=7.8 \mathrm{~Hz}, 1 \mathrm{H})$, $5.60(\mathrm{t}, J=7.2 \mathrm{~Hz}, 1 \mathrm{H}), 2.52(\mathrm{t}, J=7.8 \mathrm{~Hz}, 2 \mathrm{H}), 1.95(\mathrm{q}, J=7.2 \mathrm{~Hz}, 14.4 \mathrm{~Hz}, 2 \mathrm{H}), 1.37-1.43(\mathrm{~m}$, 4H), 0.90-0.93 (m, 3H), 0.85-0.88 (m, 3H); ${ }^{13} \mathrm{C}-\mathrm{NMR}\left(67.5 \mathrm{MHz}, \mathrm{CDCl}_{3}\right) \delta 147.1,136.3,130.8$, 
129.1, 128.4, 127.6, 125.9, 120.5, 38.4, 29.4, 22.7, 20.4, 13.9, 13.5; HRMS Calculated for $\left[\mathrm{C}_{16} \mathrm{H}_{21} \mathrm{~N}_{3}\right]^{+}: 255.3580$, Found: 255.1735 .

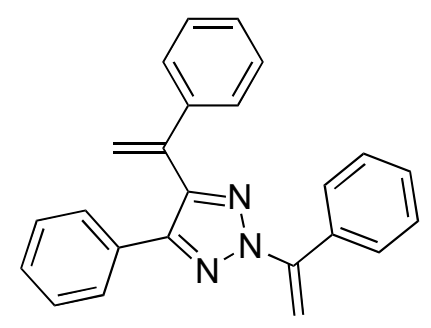

\subsection{4d(N2)}

4-phenyl-2,5-bis(1-phenylvinyl)-2H-1,2,3-triazole $\quad 1.3 .4 d(\mathbf{N} 2)$ : purified by flash chromatography (Hexane-EtOAc, v/v 5/1) as yellow oil with overall isolated yield: $81 \% .{ }^{1} \mathrm{H}-$ NMR (600 MHz, $\left.\mathrm{CDCl}_{3}\right) \delta$ 7.67-7.69 (m, 2H), 7.56-7.58 (m, 2H), 7.42-7.45 (m, 5H), 7.25-7.30 (m, 6H), $6.04(\mathrm{~s}, 1 \mathrm{H}), 5.95(\mathrm{~s}, 1 \mathrm{H}), 5.66(\mathrm{~s}, 1 \mathrm{H}), 5.46(\mathrm{~s}, 1 \mathrm{H}) ;{ }^{13} \mathrm{C}-\mathrm{NMR}\left(67.5 \mathrm{MHz}, \mathrm{CDCl}_{3}\right) \delta$ $146.7,146.3,146.0,139.4,138.8,135.0,130.3,129.5,128.62,128.61,128.58,128.56,128.52$, 128.4, 128.0, 127.1, 119.4, 107.8; HRMS Calculated for $\left[\mathrm{C}_{24} \mathrm{H}_{19} \mathrm{~N}_{3}\right]^{+}:$: 349.4728, Found: 349.1579.

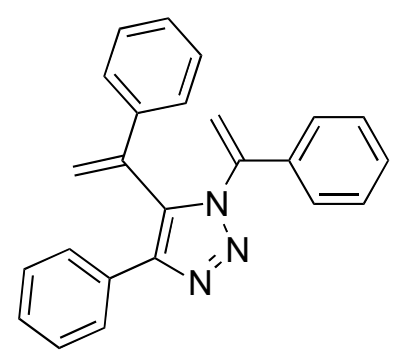

\subsection{4d(N1)}


chromatography (Hexane-EtOAc, v/v 5/1) as yellow oil with overall isolated yield: $90 \% .{ }^{1} \mathrm{H}-$ NMR (600 MHz, $\left.\mathrm{CDCl}_{3}\right) \delta$ 7.86-7.88 (m, 2H), 7.30-7.33 (m, 2H), 7.24-7.28 (m, 2H), 7.16-7.22 (m, 5H), 7.05-7.09 (m, 4H), $5.82(\mathrm{~s}, 1 \mathrm{H}), 5.64(\mathrm{~s}, 1 \mathrm{H}), 5.41(\mathrm{~s}, 1 \mathrm{H}), 5.29(\mathrm{~s}, 1 \mathrm{H}){ }^{13} \mathrm{C}-\mathrm{NMR}(67.5$ $\left.\mathrm{MHz}, \mathrm{CDCl}_{3}\right) \delta 145.3,143.0,137.4,136.4,135.3,133.9,130.9,129.5,128.8,128.7,128.6$, 128.5, 128.2, 127.1, 126.5, 126.4, 121.4, 114.5; HRMS Calculated for $\left[\mathrm{C}_{24} \mathrm{H}_{19} \mathrm{~N}_{3}\right]^{+}: 349.4728$, Found: 349.1579.

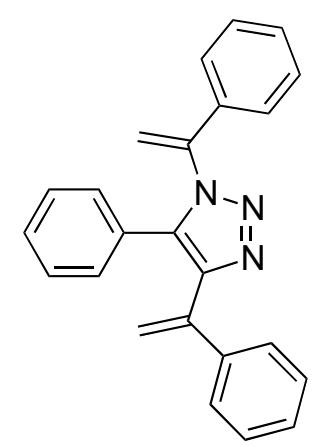

\subsection{4d(N3)}

5-phenyl-1,4-bis(1-phenylvinyl)-1H-1,2,3-triazole $1.3 .4 \mathrm{~d}(\mathrm{~N} 3): \quad$ purified $\quad$ by flash chromatography (Hexane-EtOAc, v/v 5/1) as yellow oil with overall isolated yield: $90 \% .{ }^{1} \mathrm{H}-$ NMR (600 MHz, $\left.\mathrm{CDCl}_{3}\right) \delta$ 7.29-7.31 (m, 2H), 7.21-7.25 (m, 3H), 7.14-7.18 (m, 3H), 7.07-7.12 (m, 3H), 7.00-7.05 (m, 4H), $5.82(\mathrm{~s}, 1 \mathrm{H}), 5.64(\mathrm{~s}, 1 \mathrm{H}), 5.63(\mathrm{~s}, 1 \mathrm{H}), 5.55(\mathrm{~s}, 1 \mathrm{H}),{ }^{13} \mathrm{C}-\mathrm{NMR}(67.5$ $\left.\mathrm{MHz}, \mathrm{CDCl}_{3}\right) \delta 145.0,143.0,139.4,139.3,135.9,135.3,129.5,129.4,129.0,128.8,128.7$, 128.2, 127.9, 127.6, 127.1, 126.1, 118.5, 115.0; HRMS Calculated for $\left[\mathrm{C}_{24} \mathrm{H}_{19} \mathrm{~N}_{3}\right]^{+}: 349.4728$, Found: 349.1579. 


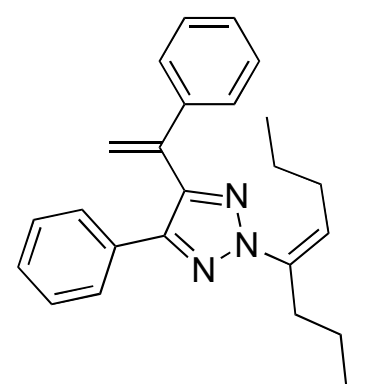

\subsection{4e(N2)}

2-((Z)-oct-4-en-4-yl)-4-phenyl-5-(1-phenylvinyl)-2H-1,2,3-triazole $1.3 .4 \mathrm{e}(\mathrm{N} 2)$ : purified by flash chromatography (Hexane-EtOAc, v/v 5/1) as yellow oil with overall isolated yield: $85 \%$. ${ }^{1} \mathrm{H}-\mathrm{NMR}\left(600 \mathrm{MHz}, \mathrm{CDCl}_{3}\right) \delta 7.63(\mathrm{~d}, J=8.4 \mathrm{~Hz}, 2 \mathrm{H}), 7.36(\mathrm{~d}, J=8.4 \mathrm{~Hz}, 2 \mathrm{H}), 7.22-7.25(\mathrm{~m}$, $6 \mathrm{H}), 5.88(\mathrm{~s}, 1 \mathrm{H}), 5.57(\mathrm{~s}, 1 \mathrm{H}), 5.50(\mathrm{t}, J=7.2 \mathrm{~Hz}, 1 \mathrm{H}), 2.65(\mathrm{t}, J=7.2 \mathrm{~Hz}, 2 \mathrm{H}), 2.27(\mathrm{q}, J=$ 7.2Hz, 14.4Hz, 2H), 1.45-1.51 (m, 4H), 0.90-0.96 (m, 6H); ${ }^{13} \mathrm{C}-\mathrm{NMR}\left(67.5 \mathrm{MHz}, \mathrm{CDCl}_{3}\right) \delta$ $145.1,144.3,139.6,139.1,138.7,130.8,128.6,128.4,128.2,128.1,127.8,127.0,125.0,118.8$ 37.4, 29.9, 22.9, 20.8, 14.1, 13.6; HRMS Calculated for $\left[\mathrm{C}_{24} \mathrm{H}_{27} \mathrm{~N}_{3}\right]^{+}$: 357.4913, Found: 357.2205 .

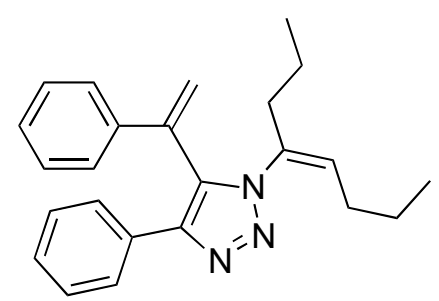

1.3.4e(N1)

1-((Z)-oct-4-en-4-yl)-4-phenyl-5-(1-phenylvinyl)-1H-1,2,3-triazole $1.3 .4 \mathrm{e}(\mathrm{N} 1)$ : purified by flash chromatography (Hexane-EtOAc, v/v 5/1) as yellow oil with overall isolated yield: $85 \%$. ${ }^{1} \mathrm{H}-\mathrm{NMR}\left(600 \mathrm{MHz}, \mathrm{CDCl}_{3}\right) \delta 7.85(\mathrm{~d}, J=7.2 \mathrm{~Hz}, 2 \mathrm{H}), 7.24-7.31(\mathrm{~m}, 8 \mathrm{H}), 6.01(\mathrm{~s}, 1 \mathrm{H}), 5.51(\mathrm{t}$, 
$J=7.2 \mathrm{~Hz}, 1 \mathrm{H}), 5.44(\mathrm{~s}, 1 \mathrm{H}), 2.06(\mathrm{t}, J=7.2 \mathrm{~Hz}, 2 \mathrm{H}), 1.69(\mathrm{q}, J=7.2 \mathrm{~Hz}, 15.0 \mathrm{~Hz}, 2 \mathrm{H}), 1.23-1.32$ (m, 4H), 0.77-0.82 (m, 6H); ${ }^{13} \mathrm{C}-\mathrm{NMR}\left(67.5 \mathrm{MHz}, \mathrm{CDCl}_{3}\right) \delta 144.3,137.5,136.7,135.2,132.8$, $130.8,130.5,128.7,128.6,128.4,127.7,126.6,126.2,120.5,37.5,29.7,22.1,19.8,13.7,13.2$; HRMS Calculated for $\left[\mathrm{C}_{24} \mathrm{H}_{27} \mathrm{~N}_{3}\right]^{+}: 357.4913$, Found: 357.2205 .

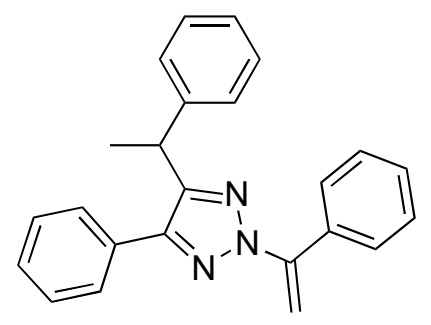

1.3.4f(N2)

4-phenyl-5-(1-phenylethyl)-2-(1-phenylvinyl)-2H-1,2,3-triazole 1.3.4f(N2): purified by flash chromatography (Hexane-EtOAc, v/v 5/1) as yellow oil with overall isolated yield: $88 \% .{ }^{1} \mathrm{H}-$ NMR $\left(600 \mathrm{MHz}, \mathrm{CDCl}_{3}\right) \delta$ 7.48-7.52 (m, 4H), 7.39-7.42 (m, 3H), 7.27-7.36 (m, 8H), $5.95(\mathrm{~s}$, 1H), $5.40(\mathrm{~s}, 1 \mathrm{H}), 4.39$ (q, $J=7.2 \mathrm{~Hz}, 14.4 \mathrm{~Hz}, 1 \mathrm{H}), 1.68(\mathrm{~d}, J=7.2 \mathrm{~Hz}, 3 \mathrm{H}), ;{ }^{13} \mathrm{C}-\mathrm{NMR}(67.5$ $\left.\mathrm{MHz}, \mathrm{CDCl}_{3}\right) \delta 149.4,146.3,146.2,144.7,135.0,130.6,129.1,128.6,128.5,128.4,128.3$, 128.2, 128.1, 127.6, 126.4, 106.9, 37.0, 23.1; HRMS Calculated for $\left[\mathrm{C}_{24} \mathrm{H}_{21} \mathrm{~N}_{3}\right]^{+}: 351.4436$, Found: 351.1735. 


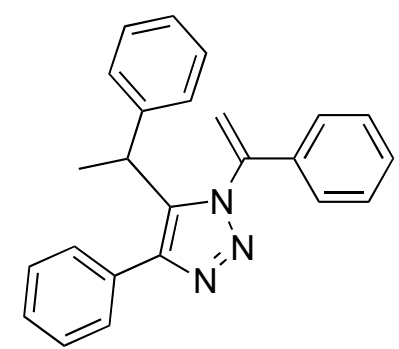

\subsection{4f(N1)}

4-phenyl-5-(1-phenylethyl)-1-(1-phenylvinyl)-1H-1,2,3-triazole 1.3.4f(N1): purified by flash chromatography (Hexane-EtOAc, v/v 5/1) as yellow oil with overall isolated yield: $88 \% .{ }^{1} \mathrm{H}-$ $\operatorname{NMR}\left(600 \mathrm{MHz}, \mathrm{CDCl}_{3}\right) \delta 7.34(\mathrm{~d}, J=7.8 \mathrm{~Hz}, 4 \mathrm{H}), 7.25-7.29(\mathrm{~m}, 2 \mathrm{H}), 7.16-7.24(\mathrm{~m}, 7 \mathrm{H}), 7.04$ $(\mathrm{d}, J=7.8 \mathrm{~Hz}, 2 \mathrm{H}), 6.98(\mathrm{~d}, J=7.2 \mathrm{~Hz}, 2 \mathrm{H}), 5.74(\mathrm{~s}, 1 \mathrm{H}), 5.48(\mathrm{~s}, 1 \mathrm{H}), 4.14(\mathrm{q}, J=7.2 \mathrm{~Hz}, 14.4 \mathrm{~Hz}$, 1H), $1.76(\mathrm{~d}, J=7.2 \mathrm{~Hz}, 3 \mathrm{H}) ;{ }^{13} \mathrm{C}-\mathrm{NMR}\left(67.5 \mathrm{MHz}, \mathrm{CDCl}_{3}\right) \delta 148.5,145.7,143.1,135.4,134.6$, 129.6, 129.4, 129.0, 128.7, 128.6, 128.5, 127.7, 127.5, 126.47, 126.2, 114.5, 36.7, 22.7; HRMS Calculated for $\left[\mathrm{C}_{24} \mathrm{H}_{21} \mathrm{~N}_{3}\right]^{+}: 351.4436$, Found: 351.1735 .

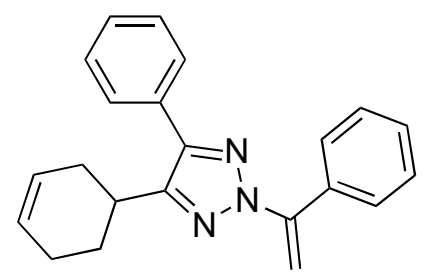

\section{$1.3 .4 \mathrm{~g}(\mathrm{~N} 2)$}

4-cyclohexenyl-5-phenyl-2-(1-phenylvinyl)-2H-1,2,3-triazole $1.3 .4 \mathrm{~g}(\mathrm{~N} 2)$ : was purified by flash chromatography (Hexane-EtOAc, v/v 5/1) as yellow oil with overall isolated yield: $87 \%$. ${ }^{1} \mathrm{H}-\mathrm{NMR}\left(600 \mathrm{MHz}, \mathrm{CDCl}_{3}\right) \delta$ 7.67-7.69 (m, 2H), 7.48-7.50 (m, 2H), 7.38-7.41 (m, 5H), 7.34$7.36(\mathrm{~m}, 1 \mathrm{H}), 6.06-6.09(\mathrm{~m}, 1 \mathrm{H}), 5.90(\mathrm{~s}, 1 \mathrm{H}), 5.36(\mathrm{~s}, 1 \mathrm{H}), 2.32-2.36(\mathrm{~m}, 2 \mathrm{H}), 2.11-2.16(\mathrm{~m}$, 2H), 1.70-1.76 (m, 2H), 1.63-1.69 (m, 2H); ${ }^{13} \mathrm{C}-\mathrm{NMR}\left(67.5 \mathrm{MHz}, \mathrm{CDCl}_{3}\right) \delta 147.9,146.2,145.4$, 
$135.1,131.6,130.4,129.3,128.7,128.6,128.53,128.50,128.42,128.37,107.1,27.8,25.7,22.8$ 22.1; HRMS Calculated for $\left[\mathrm{C}_{22} \mathrm{H}_{21} \mathrm{~N}_{3}\right]^{+}:$327.4222, Found: 327.1735 .

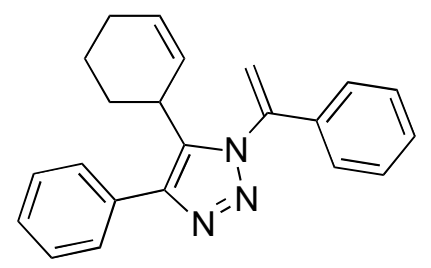

\section{$1.3 .4 \mathrm{~g}(\mathrm{~N} 1)$}

5-cyclohexenyl-4-phenyl-1-(1-phenylvinyl)-1H-1,2,3-triazole 1.3.4g(N1): purified by flash chromatography (Hexane-EtOAc, v/v 5/1) as yellow oil with overall isolated yield: $87 \% .{ }^{1} \mathrm{H}-$ NMR $\left(600 \mathrm{MHz}, \mathrm{CDCl}_{3}\right) \delta 7.18-7.27(\mathrm{~m}, 6 \mathrm{H}), 7.13(\mathrm{~d}, J=7.2 \mathrm{~Hz}, 2 \mathrm{H}), 7.04(\mathrm{~d}, J=7.8 \mathrm{~Hz}, 2 \mathrm{H})$, 6.05-6.16 (m, 1H), $5.75(\mathrm{~s}, 1 \mathrm{H}), 5.46(\mathrm{~s}, 1 \mathrm{H}), 2.29-2.36(\mathrm{~m}, 2 \mathrm{H}), 2.03-2.10(\mathrm{~m}, 2 \mathrm{H}), 1.65-1.71$ (m, 2H), 1.52-1.63 (m, 2H); ${ }^{13} \mathrm{C}-\mathrm{NMR}\left(67.5 \mathrm{MHz}, \mathrm{CDCl}_{3}\right) \delta 147.7,146.4,143.0,135.7,133.5$, $130.0,129.4,129.0,128.6,128.4,128.3,127.9,126.1,114.7,27.3,25.7,22.9,22.2$; HRMS Calculated for $\left[\mathrm{C}_{22} \mathrm{H}_{21} \mathrm{~N}_{3}\right]^{+}$: 327.4222 , Found: 327.1735 .

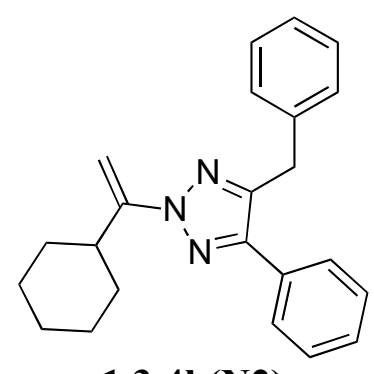

\subsection{4h(N2)}


4-benzyl-2-(1-cyclohexylvinyl)-5-phenyl-2H-1,2,3-triazole $1.3 .4 h(\mathbf{N 2})$ : purified by flash chromatography (Hexane-EtOAc, v/v 5/1) as yellow oil with overall isolated yield: 93\%. ${ }^{1} \mathrm{H}-$ NMR $\left(600 \mathrm{MHz}, \mathrm{CDCl}_{3}\right) \delta 7.54(\mathrm{~d}, J=7.2 \mathrm{~Hz}, 2 \mathrm{H}), 7.26-7.32(\mathrm{~m}, 3 \mathrm{H}), 7.21(\mathrm{t}, J=7.8 \mathrm{~Hz}, 2 \mathrm{H})$, $7.14(\mathrm{t}, J=7.8 \mathrm{~Hz}, 3 \mathrm{H}), 5.72(\mathrm{~s}, 1 \mathrm{H}), 4.79(\mathrm{~s}, 1 \mathrm{H}), 4.18(\mathrm{~s}, 2 \mathrm{H}), 3.03(\mathrm{t}, J=11.4 \mathrm{~Hz}, 1 \mathrm{H}), 1.95(\mathrm{~d}$, $J=12.6 \mathrm{~Hz}, 2 \mathrm{H}), 1.75(\mathrm{~d}, J=13.8 \mathrm{~Hz}, 2 \mathrm{H}), 1.34(\mathrm{~d}, J=13.2 \mathrm{~Hz}, 2 \mathrm{H}), 1.17-1.27(\mathrm{~m}, 4 \mathrm{H}) ;{ }^{13} \mathrm{C}-\mathrm{NMR}$ $\left(67.5 \mathrm{MHz}, \mathrm{CDCl}_{3}\right) \delta 151.5,144.2,138.8,131.0,128.9,128.8,128.7,128.6,128.4,127.9,126.6$, 100.6, 39.5, 32.2, 31.8, 26.7, 26.6; HRMS Calculated for $\left[\mathrm{C}_{23} \mathrm{H}_{25} \mathrm{~N}_{3}\right]^{+}$: 343.4647, Found: 343.2048 .

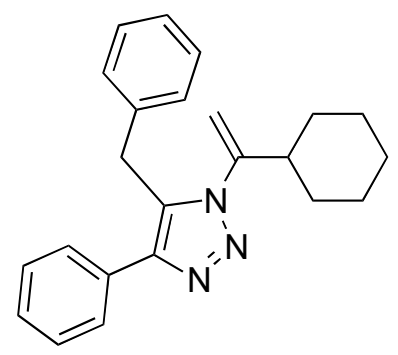

\subsection{4h(N1)}

5-benzyl-1-(1-cyclohexylvinyl)-4-phenyl-1H-1,2,3-triazole 1.3 .4 h(N1): purified by flash chromatography (Hexane-EtOAc, v/v 5/1) as yellow oil with overall isolated yield: 93\%. ${ }^{1} \mathrm{H}-$ NMR $\left(600 \mathrm{MHz}, \mathrm{CDCl}_{3}\right) \delta 7.73(\mathrm{~d}, J=7.8 \mathrm{~Hz}, 2 \mathrm{H}), 7.37(\mathrm{t}, J=7.2 \mathrm{~Hz} 2 \mathrm{H}), 7.25-7.32(\mathrm{~m}, 3 \mathrm{H})$, $7.21(\mathrm{t}, J=7.2 \mathrm{~Hz} 1 \mathrm{H}), 7.03(\mathrm{~d}, J=7.8 \mathrm{~Hz}, 2 \mathrm{H}), 5.15(\mathrm{~s}, 1 \mathrm{H}), 4.96(\mathrm{~s}, 1 \mathrm{H}), 4.24(\mathrm{~s}, 2 \mathrm{H}), 2.47(\mathrm{t}, J=$ $10.8 \mathrm{~Hz} 1 \mathrm{H}), 1.63-1.71(\mathrm{~m}, 5 \mathrm{H}), 1.03-1.17(\mathrm{~m}, 5 \mathrm{H}) ;{ }^{13} \mathrm{C}-\mathrm{NMR}\left(67.5 \mathrm{MHz}, \mathrm{CDCl}_{3}\right) \delta 148.7$, $145.1,137.1,131.2,131.1,128.9,128.7,127.9,127.8,127.0,126.9,111.0,42.7,30.8,29.3,26.0$, 25.9; HRMS Calculated for $\left[\mathrm{C}_{23} \mathrm{H}_{25} \mathrm{~N}_{3}\right]^{+}: 343.4647$, Found: 343.2048 . 


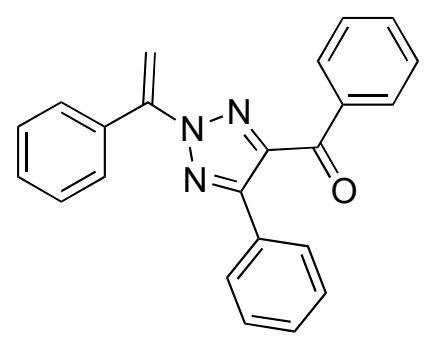

1.3.4i(N2)

Phenyl(5-phenyl-2-(1-phenylvinyl)-2H-1,2,3-triazol-4-yl)methanone 1.3.4i(N2): purified by flash chromatography (Hexane-EtOAc, v/v 5/1) as yellow oil with overall isolated yield: 84\%. ${ }^{1} \mathrm{H}-\mathrm{NMR}\left(600 \mathrm{MHz}, \mathrm{CDCl}_{3}\right) \delta 8.09(\mathrm{~d}, J=7.2 \mathrm{~Hz}, 2 \mathrm{H}), 7.84-7.85(\mathrm{~m}, 2 \mathrm{H}), 7.57(\mathrm{t}, J=7.2 \mathrm{~Hz}, 1 \mathrm{H})$, 7.49-7.51(m, 2H), 7.38-7.46 (m, 8H), $6.06(\mathrm{~s}, 1 \mathrm{H}), 5.54(\mathrm{~s}, 1 \mathrm{H}) ;{ }^{13} \mathrm{C}-\mathrm{NMR}\left(67.5 \mathrm{MHz}, \mathrm{CDCl}_{3}\right) \delta$ $188.0,150.3,146.2,143.1,137.2,134.3,133.7,130.8,129.7,129.5,129.1,128.9,128.8,128.6$, 128.5, 128.4, 109.5; HRMS Calculated for $\left[\mathrm{C}_{23} \mathrm{H}_{17} \mathrm{~N}_{3} \mathrm{O}\right]^{+}: 351.4006$, Found: 351.1372. 


\subsection{Iron catalyzed C-O bond activation for the synthesis of propargyl-1,2,3-triazoles and 1,1-bis-triazoles}

\section{General Methods and materials}

All of the reactions dealing with air and/or moisture-sensitive reactions were carried out under an atmosphere of nitrogen using oven/flame-dried glassware and standard syringe/septa techniques. Unless otherwise noted, all commercial reagents and solvents were obtained from the commercial provider and used without further purification. ${ }^{1} \mathrm{H}$ NMR and ${ }^{13} \mathrm{C}$ NMR spectra were recorded on Varian $600 \mathrm{MHz}$ spectrometers. Chemical shifts were reported relative to internal tetramethylsilane $(\delta 0.00 \mathrm{ppm})$ or $\mathrm{CDCl}_{3}(\delta 7.26 \mathrm{ppm})$ for ${ }^{1} \mathrm{H}$ and $\mathrm{CDCl}_{3}(\delta 77.0 \mathrm{ppm})$ for ${ }^{13} \mathrm{C}$. Flash column chromatography was performed on 230-430 mesh silica gel. Analytical thin layer chromatography was performed with precoated glass baked plates $(250 \mu)$ and visualized by fluorescence and by charring after treatment with potassium permanganate stain. Melting points were measured on a Mel-Temp 1001D apparatus and uncorrected. HRMS were recorded on LTQ-FTUHRA spectrometer.

Substrates 1.4.2b, 1.4.4 were synthesized according to the literatures as below:

1. Yu, M.; Zhang, G.; Zhang, L. Org. Lett. 2007, 9, 2147-2150.

2. Marion, N.; Carlqvist, P.; Gealageas, R.; Fremont, P.; Maseras, F.; Nolan, S. P. Chem. Eur. J. 2007, 13, 6437-6451.

3. Wang, D.; Ye, X.; Shi, X. Org. Lett. 2010, 12, 2088-2091. 


\section{Representative procedure for the preparation of propargylic acetate 1.4.2a}

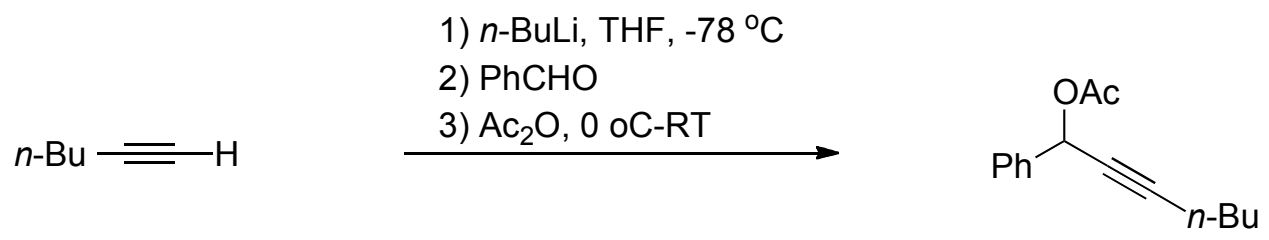

To a solution of hex-1-yne $(39 \mathrm{mmol})$ in anhydrous THF $(50 \mathrm{~mL})$ at $-78{ }^{\circ} \mathrm{C}$ under $\mathrm{N}_{2}$ atmosphere was added $n$-BuLi (1.6 M solution in hexanes, $20.6 \mathrm{~mL}, 33 \mathrm{mmol})$. The reaction was stirred at this temperature for $20 \mathrm{~min}$ then at room temperature for one hour. After cooling to at $-78{ }^{\circ} \mathrm{C}$, benzaldehyde $(30 \mathrm{mmol})$ was added to the mixture and was allowed to warm to room temperature gradually and stirred for an additional hour. After addition of acetate anhydrous (60 mmol) at $0{ }^{\circ} \mathrm{C}$, the reaction mixture was warmed to room temperature and stirred for $2 \mathrm{~h}$ before quenched with aqueous $\mathrm{NH}_{4} \mathrm{Cl}$. The mixture was extracted with EtOAc $(3 \times 20 \mathrm{~mL})$, and the combined organic phases were washed with water and brine, dried with anhydrous $\mathrm{MgSO}_{4}$, and filtered. The filtrate was concentrated under reduced pressure and the residue was purified by flash chromatography on silica gel (hexanes/ethyl acetate, V/V, 20:1) to produce the desired acetate as yellow oil in quantitative yield. 


\section{$\underline{\text { Representative procedure for the preparation of propargylic alcohol 1.4.4 }}$}

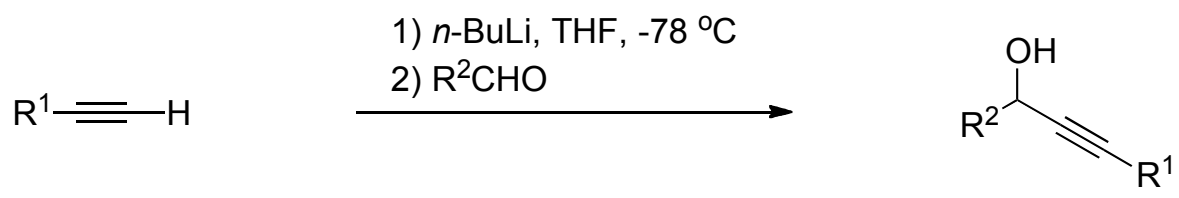

To a solution of alkyne $(39 \mathrm{mmol})$ in anhydrous THF $(50 \mathrm{~mL})$ at $-78{ }^{\circ} \mathrm{C}$ under $\mathrm{N}_{2}$ atmosphere was added $n$-BuLi (1.6 M solution in hexanes, $20.6 \mathrm{~mL}, 33 \mathrm{mmol})$. The reaction was stirred at this temperature for $20 \mathrm{~min}$ then at room temperature for $1 \mathrm{~h}$. After cooling to at $-78{ }^{\circ} \mathrm{C}$, aldehyde (30 mmol) was added to the mixture and was allowed to warm to room temperature gradually and stirred for an additional hour before quenched with aqueous $\mathrm{NH}_{4} \mathrm{Cl}$. The mixture was extracted with EtOAc $(3 \times 20 \mathrm{~mL})$, and the combined organic phases were washed with water and brine, dried with anhydrous $\mathrm{MgSO}_{4}$, and filtered. The filtrate was concentrated under reduced pressure and the residue was purified by flash chromatography on silica gel (hexanes/ethyl acetate, $\mathrm{V} / \mathrm{V}, 15: 1$ ) to produce the desired alcohol as yellow oil in quantitative yield. 


\section{Representative procedure for propargylation of 1.4 .4 with triazoles}
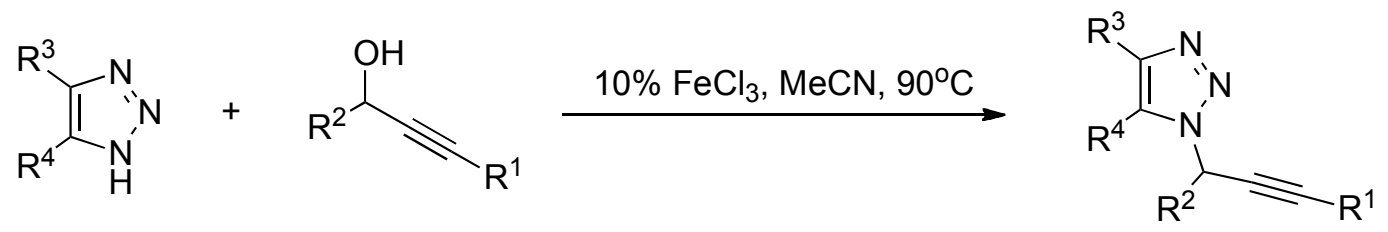

A solution of propargylic alcohol 1.4.4 $(1 \mathrm{mmol})$ and triazole $(1.2 \mathrm{mmol})$ with catalyst iron(III) chloride $(0.1 \mathrm{mmol})$ in acetonitrile $(10 \mathrm{ml})$ was stirred at 90 degree in the presence of water condensor for $5 \mathrm{~h}$. The reaction mixture was quenched with $10 \mathrm{ml}$ distilled water and then extracted with EtOAc $(3 \times 10 \mathrm{~mL})$. The combined organic phases were washed with water and brine, dried with anhydrous $\mathrm{MgSO}_{4}$, and filtered. The filtrate was concentrated under reduced pressure and the residue was purified by flash chromatography on silica gel (hexanes/ethyl acetate, $\mathrm{V} / \mathrm{V}, 15: 1)$ to produce the desired product as yellow oil except for 1.4.3f. 


\section{Compounds Characterization}

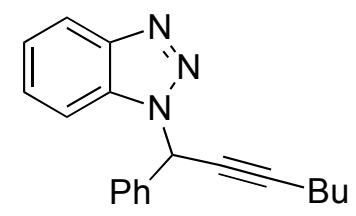

1.4.3a (N-1)

1-(1-phenylhept-2-ynyl)-1H-benzo[d][1,2,3]triazole $\quad(1.4 .3 \mathrm{a} \quad(\mathbf{N}-1)):{ }^{1} \mathrm{H}-\mathrm{NMR} \quad(600 \mathrm{MHz}$, $\left.\mathrm{CDCl}_{3}\right) \delta 8.04(\mathrm{~d}, J=8.4 \mathrm{~Hz}, 1 \mathrm{H}), 7.54-7.51(\mathrm{~m}, 3 \mathrm{H}), 7.37-7.29(\mathrm{~m}, 5 \mathrm{H}), 7.14(\mathrm{~s}, 1 \mathrm{H}), 2.34(\mathrm{dt}, J$ $=7.2 \mathrm{~Hz}, 2.4 \mathrm{~Hz}, 2 \mathrm{H}), 1.56$ (quintet, $J=7.2 \mathrm{~Hz}, 2 \mathrm{H}), 1.42$ (hextet, $J=7.2 \mathrm{~Hz}, 2 \mathrm{H}), 0.90(\mathrm{t}, J=$ $7.2 \mathrm{~Hz}, 3 \mathrm{H}) ;{ }^{13} \mathrm{C}-\mathrm{NMR}\left(150 \mathrm{MHz}, \mathrm{CDCl}_{3}\right) \delta 146.7,135.9,131.3,128.7,128.6,127.1,123.9$, 120.0, 111.1, 90.3, 73.6, 55.5, 30.4, 21.9, 18.4, 13.4; HRMS Calculated for $\mathrm{C}_{19} \mathrm{H}_{20} \mathrm{~N}_{3}[\mathrm{M}+\mathrm{H}]^{+}$: 290.16572, Found: 290.16517.

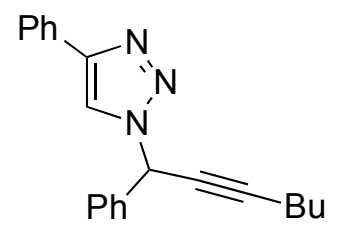

1.4.3b (N-1)

4-phenyl-1-(1-phenylhept-2-ynyl)-1H-1,2,3-triazole $\quad(1.4 .3 b \quad(\mathbf{N}-1)):{ }^{1} \mathrm{H}-\mathrm{NMR} \quad(600 \mathrm{MHz}$, $\left.\mathrm{CDCl}_{3}\right) \delta 7.89(\mathrm{~s}, 1 \mathrm{H}), 7.82(\mathrm{~d}, J=7.8 \mathrm{~Hz}, 2 \mathrm{H}), 7.52(\mathrm{~d}, J=7.2 \mathrm{~Hz}, 2 \mathrm{H}), 7.41-7.30(\mathrm{~m}, 6 \mathrm{H}), 6.73$ (s, 1H), 2.37 (dt, $J=7.2 \mathrm{~Hz}, 1.8 \mathrm{~Hz} .2 \mathrm{H}$ ), 1.60 (quintet, $J=7.2 \mathrm{~Hz}, 2 \mathrm{H}$ ), 1.47 (hextet, $J=7.2 \mathrm{~Hz}$, 2H), $0.95(\mathrm{t}, J=7.2 \mathrm{~Hz}, 3 \mathrm{H}) ;{ }^{13} \mathrm{C}-\mathrm{NMR}\left(150 \mathrm{MHz}, \mathrm{CDCl}_{3}\right) \delta 148.0,136.7,130.5,129.0,128.8$, 
128.7, 128.1, 127.1, 125.7, 118.0, 90.1, 74.4, 56.3, 30.4, 22.0, 18.5, 13.5; HRMS Calculated for $\mathrm{C}_{21} \mathrm{H}_{22} \mathrm{~N}_{3}[\mathrm{M}+\mathrm{H}]^{+}: 316.18137$, Found: 316.18082 .

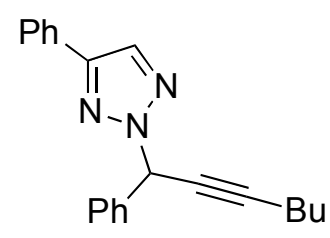

1.4.3b (N-2)

4-phenyl-2-(1-phenylhept-2-ynyl)-2H-1,2,3-triazole (1.4.3b (N-2)): ${ }^{1} \mathrm{H}-\mathrm{NMR} \quad(600 \mathrm{MHz}$, $\left.\mathrm{CDCl}_{3}\right) \delta 7.89(\mathrm{~s}, 1 \mathrm{H}), 7.81(\mathrm{~d}, J=7.2 \mathrm{~Hz}, 2 \mathrm{H}), 7.59(\mathrm{~d}, J=7.2 \mathrm{~Hz}, 2 \mathrm{H}), 7.42(\mathrm{t}, J=7.2 \mathrm{~Hz}, 2 \mathrm{H})$, 7.39-7.32 (m, 5H), 2.37 (dt, $J=7.2 \mathrm{~Hz}, 1.8 \mathrm{~Hz}, 2 \mathrm{H}), 1.59$ (quintet, $J=7.2 \mathrm{~Hz}, 2 \mathrm{H}$ ), 1.47 (hextet, $J=7.2 \mathrm{~Hz}, 2 \mathrm{H}), 0.94(\mathrm{t}, J=7.2 \mathrm{~Hz}, 3 \mathrm{H}) ;{ }^{13} \mathrm{C}-\mathrm{NMR}\left(150 \mathrm{MHz}, \mathrm{CDCl}_{3}\right) \delta 147.9,137.0,131.7$, $130.3,128.7,128.6,128.5,128.4,127.4,126.0,89.3,74.7,60.8,30.4,21.9,18.6,13.5$; HRMS Calculated for $\mathrm{C}_{21} \mathrm{H}_{22} \mathrm{~N}_{3}[\mathrm{M}+\mathrm{H}]^{+}: 316.18137$, Found: 316.18082 .

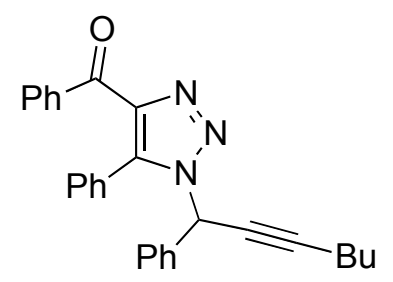

1.4.3c (N-1)

phenyl(5-phenyl-1-(1-phenylhept-2-ynyl)-1H-1,2,3-triazol-4-yl)methanone $\quad(1.4 .3 \mathrm{c} \quad(\mathrm{N}-1))$ : ${ }^{1} \mathrm{H}-\mathrm{NMR}\left(600 \mathrm{MHz}, \mathrm{CDCl}_{3}\right) \delta 8.26(\mathrm{~d}, J=7.2 \mathrm{~Hz}, 2 \mathrm{H}), 7.56(\mathrm{~d}, J=7.2 \mathrm{~Hz}, 1 \mathrm{H}), 7.47(\mathrm{t}, J=7.2$ Hz, 2H), 7.38-7.29 (m, 4H), 7.25-7.23 (m, 4H), 7.05 (dd, $J=7.2 \mathrm{~Hz}, 2.4 \mathrm{~Hz}, 2 \mathrm{H}), 6.27$ (t, $J=3.6$ 
$\mathrm{Hz}, 1 \mathrm{H}$ ), 2.71 (dt, $J=7.2 \mathrm{~Hz}, 1.8 \mathrm{~Hz}, 2 \mathrm{H}$ ), 1.54 (quintet, $J=7.2 \mathrm{~Hz}, 2 \mathrm{H}$ ), 1.28 (hextet, $J=7.2$ $\mathrm{Hz}, 2 \mathrm{H}), 0.89$ (t, $J=7.2 \mathrm{~Hz}, 3 \mathrm{H}) ;{ }^{13} \mathrm{C}-\mathrm{NMR}\left(150 \mathrm{MHz}, \mathrm{CDCl}_{3}\right) \delta 186.4,141.9,141.5,137.1$, $133.0,131.9,130.7,129.8,129.6,128.7,128.5,128.4,128.2,127.8,127.4,126.8,111.8,103.4$, 32.3, 28.7, 22.0, 13.7; HRMS Calculated for $\mathrm{C}_{28} \mathrm{H}_{26} \mathrm{~N}_{3} \mathrm{O}[\mathrm{M}+\mathrm{H}]^{+}: 420.20759$, Found: 420.20704 .

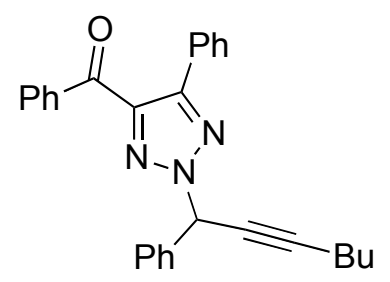

1.4.3c (N-2)

phenyl(5-phenyl-2-(1-phenylhept-2-ynyl)-2H-1,2,3-triazol-4-yl)methanone $\quad(1.4 .3 \mathrm{c} \quad(\mathrm{N}-2))$ : ${ }^{1} \mathrm{H}-\mathrm{NMR}\left(600 \mathrm{MHz}, \mathrm{CDCl}_{3}\right) \delta 8.08(\mathrm{~d}, J=8.4 \mathrm{~Hz}, 2 \mathrm{H}), 7.83(\mathrm{dd}, J=7.2 \mathrm{~Hz}, 2.4 \mathrm{~Hz}, 2 \mathrm{H}), 7.63$ (d, $J=7.2 \mathrm{~Hz}, 2 \mathrm{H}), 7.59(\mathrm{t}, J=7.8 \mathrm{~Hz}, 1 \mathrm{H}), 7.46(\mathrm{t}, J=7.8 \mathrm{~Hz}, 2 \mathrm{H}), 7.41-7.37(\mathrm{~m}, 6 \mathrm{H}), 6.69(\mathrm{t}$, $J=2.4 \mathrm{~Hz}, 1 \mathrm{H}$ ), 2.37 (dt, $J=7.2 \mathrm{~Hz}, 2.4 \mathrm{~Hz}, 2 \mathrm{H}$ ), 1.60 (quintet, $J=7.2 \mathrm{~Hz}, 2 \mathrm{H}$ ), 1.48 (hextet, $J$ $=7.8 \mathrm{~Hz}, 2 \mathrm{H}), 0.94(\mathrm{t}, J=7.2 \mathrm{~Hz}, 3 \mathrm{H}) ;{ }^{13} \mathrm{C}-\mathrm{NMR}\left(150 \mathrm{MHz}, \mathrm{CDCl}_{3}\right) \delta$ 187.7, 149.9, 142.1, 137.2, 136.4, 133.2, 130.6, 129.7, 129.0, 128.9, 128.8, 128.7, 128.2, 128.1, 127.6, 90.0, 74.3, 61.5, 30.4, 21.9, 18.6, 13.5; HRMS Calculated for $\mathrm{C}_{28} \mathrm{H}_{26} \mathrm{~N}_{3} \mathrm{O}[\mathrm{M}+\mathrm{H}]^{+}: 420.20759$, Found: 420.20704 .

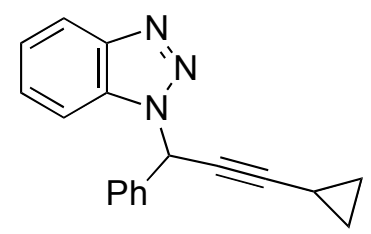

1.4.3d (N-1) 
1-(3-cyclopropyl-1-phenylprop-2-ynyl)-1H-benzo[d][1,2,3]triazole $(1.4 .3 \mathrm{~d}(\mathrm{~N}-1))$ : ${ }^{1} \mathrm{H}-\mathrm{NMR}$ $\left(600 \mathrm{MHz}, \mathrm{CDCl}_{3}\right) \delta 8.05(\mathrm{~d}, J=8.4 \mathrm{~Hz}, 1 \mathrm{H}), 7.52(\mathrm{~d}, J=7.8 \mathrm{~Hz}, 1 \mathrm{H}), 7.49(\mathrm{~d} J=7.2 \mathrm{~Hz}, 2 \mathrm{H})$, 7.38-7.29 (m, 5H), $7.10(\mathrm{~s}, 1 \mathrm{H}), 1.40-1.37(\mathrm{~m}, 1 \mathrm{H}), 0.86-0.83(\mathrm{~m}, 2 \mathrm{H}), 0.78-0.75(\mathrm{~m}, 2 \mathrm{H}) ;{ }^{13} \mathrm{C}-$ NMR $\left(150 \mathrm{MHz}, \mathrm{CDCl}_{3}\right) \delta 143.4,136.0,134.6,131.8,128.8,128.7,127.2,127.0,123.9,120.0$, 111.1, 93.2, 68.7, 55.5, 8.4; HRMS Calculated for $\mathrm{C}_{18} \mathrm{H}_{16} \mathrm{~N}_{3}[\mathrm{M}+\mathrm{H}]^{+}$: 274.13442, Found: 274.13423.

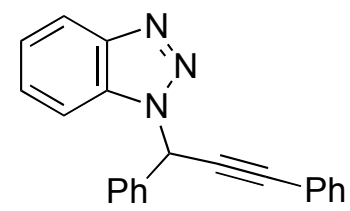

1.4.3e (N-1)

1-(1,3-diphenylprop-2-ynyl)-1H-benzo[d][1,2,3]triazole (1.4.3e (N-1)): ${ }^{1} \mathrm{H}-\mathrm{NMR}(600 \mathrm{MHz}$, $\left.\mathrm{CDCl}_{3}\right) \delta 8.08(\mathrm{~d}, J=7.2 \mathrm{~Hz}, 1 \mathrm{H}), 7.60-7.64(\mathrm{~m}, 3 \mathrm{H}), 7.52(\mathrm{~d}, J=7.8 \mathrm{~Hz}, 2 \mathrm{H}), 7.40-7.33(\mathrm{~m}$, 9H); ${ }^{13} \mathrm{C}-\mathrm{NMR}\left(150 \mathrm{MHz}, \mathrm{CDCl}_{3}\right) \delta 146.8,135.4,131.8,131.4,129.2,128.9,128.8,128.4$, $127.4,127.0,124.0,121.5,120.1,111.0,89.1,82.4$, 55.7; HRMS Calculated for $\mathrm{C}_{21} \mathrm{H}_{16} \mathrm{~N}_{3}$ $[\mathrm{M}+\mathrm{H}]^{+}:$310.13442, Found: 310.13387.

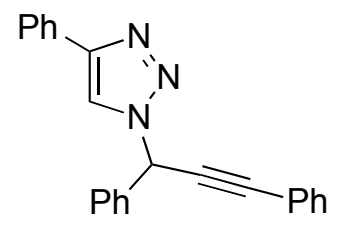

1.4.3f (N-1) 
1-(1,3-diphenylprop-2-ynyl)-4-phenyl-1H-1,2,3-triazole (1.4.3f (N-1)): ${ }^{1} \mathrm{H}-\mathrm{NMR}(600 \mathrm{MHz}$, $\left.\mathrm{CDCl}_{3}\right) \delta 7.96(\mathrm{~s}, 1 \mathrm{H}), 7.83(\mathrm{~d}, J=8.4 \mathrm{~Hz}, 2 \mathrm{H}), 7.61(\mathrm{~d}, J=7.2 \mathrm{~Hz}, 2 \mathrm{H}), 7.55(\mathrm{~d}, J=7.2 \mathrm{~Hz}$, 2H), 7.44-7.37 (m, 8H), 7.33-7.31 (m, 1H), $6.99(\mathrm{~s}, 1 \mathrm{H}) ;{ }^{13} \mathrm{C}-\mathrm{NMR}\left(150 \mathrm{MHz}, \mathrm{CDCl}_{3}\right) \delta 148.3$, $136.2,131.9,130.5,129.3,129.2,129.1,128.8,128.5,128.2,127.2,125.8,121.4,118.1,88.8$, 83.1, 56.7; HRMS Calculated for $\mathrm{C}_{23} \mathrm{H}_{18} \mathrm{~N}_{3}[\mathrm{M}+\mathrm{H}]^{+}: 336.15007$, Found: 336.14992 .

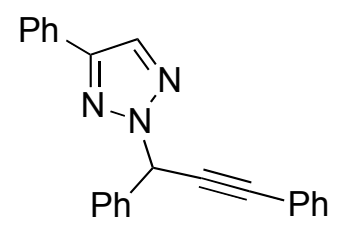

1.4.3f (N-2)

2-(1,3-diphenylprop-2-ynyl)-4-phenyl-2H-1,2,3-triazole (1.4.3f (N-2)): ${ }^{1} \mathrm{H}-\mathrm{NMR}(600 \mathrm{MHz}$, $\left.\mathrm{CDCl}_{3}\right) \delta 7.95(\mathrm{~s}, 1 \mathrm{H}), 7.86(\mathrm{~d}, J=7.2 \mathrm{~Hz}, 2 \mathrm{H}), 7.72(\mathrm{~d}, J=7.2 \mathrm{~Hz}, 2 \mathrm{H}), 7.59(\mathrm{dd}, J=7.2 \mathrm{~Hz}$, $2.4 \mathrm{~Hz}, 2 \mathrm{H}), 7.45(\mathrm{q}, J=7.8 \mathrm{~Hz}, 4 \mathrm{H}), 7.40-7.35(\mathrm{~m}, 5 \mathrm{H}), 6.94(\mathrm{~s}, 1 \mathrm{H}) ;{ }^{13} \mathrm{C}-\mathrm{NMR}(150 \mathrm{MHz}$, $\left.\mathrm{CDCl}_{3}\right) \delta 148.2,136.4,131.9,131.8,130.2,128.9,128.8,128.7,128.5,128.2,127.5,126.0$ 122.0, 88.0, 83.7, 61.0; HRMS Calculated for $\mathrm{C}_{23} \mathrm{H}_{18} \mathrm{~N}_{3}[\mathrm{M}+\mathrm{H}]^{+}: 336.15007$, Found: 336.14992.

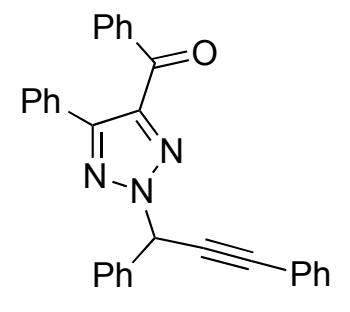

\section{$1.4 .3 g(\mathrm{~N}-2)$}


(2-(1,3-diphenylprop-2-ynyl)-5-phenyl-2H-1,2,3-triazol-4-yl)(phenyl)methanone (1.4.3g (N2)): ${ }^{1} \mathrm{H}-\mathrm{NMR}\left(600 \mathrm{MHz}, \mathrm{CDCl}_{3}\right) \delta 8.10(\mathrm{~d}, J=8.4 \mathrm{~Hz}, 2 \mathrm{H}), 7.86(\mathrm{~d}, J=7.8 \mathrm{~Hz}, 2 \mathrm{H}), 7.72(\mathrm{~d}, J$ $=7.2 \mathrm{~Hz}, 2 \mathrm{H}), 7.60(\mathrm{t}, J=7.8 \mathrm{~Hz}, 1 \mathrm{H}), 7.56(\mathrm{~d}, J=7.8 \mathrm{~Hz}, 2 \mathrm{H}), 7.47-7.34(\mathrm{~m}, 11 \mathrm{H}), 6.94(\mathrm{~s}$, $1 \mathrm{H}) ;{ }^{13} \mathrm{C}-\mathrm{NMR}\left(150 \mathrm{MHz}, \mathrm{CDCl}_{3}\right) \delta 187.6,150.1,142.3,137.2,135.8,133.3,131.9,130.6$, $129.6,129.2,129.1,129.0,128.9,128.8,128.4,128.3,128.2,127.8,121.8,88.7,83.1,61.8$; HRMS Calculated for $\mathrm{C}_{30} \mathrm{H}_{22} \mathrm{~N}_{3} \mathrm{O}[\mathrm{M}+\mathrm{H}]^{+}:$440.17629, Found: 440.17679 .

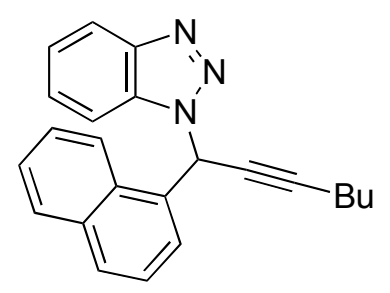

1.4.3h (N-1)

1-(1-(naphthalen-1-yl)hept-2-ynyl)-1H-benzo[d][1,2,3]triazole (1.4.3h (N-1)): ${ }^{1} \mathrm{H}-\mathrm{NMR}(600$ $\left.\mathrm{MHz}, \mathrm{CDCl}_{3}\right) \delta 8.25(\mathrm{~d}, J=9.0 \mathrm{~Hz}, 1 \mathrm{H}), 8.19(\mathrm{~d}, J=7.2 \mathrm{~Hz}, 1 \mathrm{H}), 7.99(\mathrm{~d}, J=8.4 \mathrm{~Hz}, 1 \mathrm{H}), 7.87$ $(\mathrm{d}, J=8.4 \mathrm{~Hz}, 1 \mathrm{H}), 7.82(\mathrm{~d}, J=9.0 \mathrm{~Hz}, 2 \mathrm{H}), 7.55(\mathrm{t}, J=7.2 \mathrm{~Hz}, 1 \mathrm{H}), 7.48-7.41(\mathrm{~m}, 3 \mathrm{H}), 7.30-$ $7.23(\mathrm{~m}, 2 \mathrm{H}), 2.32$ (dt, $J=7.2 \mathrm{~Hz}, 2.4 \mathrm{~Hz}, 2 \mathrm{H}), 1.54$ (quintet, $J=7.2 \mathrm{~Hz}, 2 \mathrm{H}$ ), 1.39 (hextet, $J=$ $7.2 \mathrm{~Hz}, 2 \mathrm{H}), 0.88(\mathrm{t}, J=7.2 \mathrm{~Hz}, 3 \mathrm{H}) ;{ }^{13} \mathrm{C}-\mathrm{NMR}\left(150 \mathrm{MHz}, \mathrm{CDCl}_{3}\right) \delta 146.6,134.0,131.7,130.6$, $130.5,130.3,128.8,127.1,127.0,126.5,126.1,124.8,123.7,122.9,120.0,111.1,90.5,74.3$, 53.7, 30.4, 21.9, 18.5, 13.5; HRMS Calculated for $\mathrm{C}_{23} \mathrm{H}_{22} \mathrm{~N}_{3}[\mathrm{M}+\mathrm{H}]^{+}:$340.18137, Found: 340.18082 . 


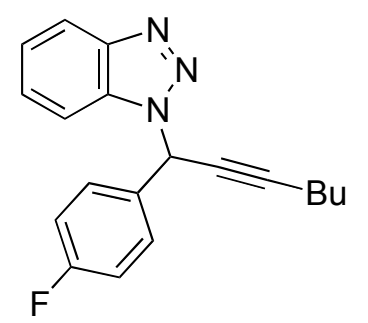

1.4.3i (N-1)

1-(1-(4-fluorophenyl)hept-2-ynyl)-1H-benzo[d][1,2,3]triazole (1.4.3i (N-1)): ${ }^{1} \mathrm{H}-\mathrm{NMR}(600$ $\left.\mathrm{MHz}, \mathrm{CDCl}_{3}\right) \delta 8.05(\mathrm{~d}, J=8.4 \mathrm{~Hz}, 1 \mathrm{H}), 7.53-7.49(\mathrm{~m}, 3 \mathrm{H}), 7.37(\mathrm{t}, J=7.2 \mathrm{~Hz}, 1 \mathrm{H}), 7.33(\mathrm{t}, J=$ $7.2 \mathrm{~Hz}, 1 \mathrm{H}), 7.11(\mathrm{~s}, 1 \mathrm{H}), 7.02(\mathrm{t}, J=8.4 \mathrm{~Hz}, 2 \mathrm{H}), 2.34(\mathrm{dt}, J=7.2 \mathrm{~Hz}, 2.4 \mathrm{~Hz}, 2 \mathrm{H}), 1.56$ (quintet, $J=7.2 \mathrm{~Hz}, 2 \mathrm{H}), 1.41$ (hextet, $J=7.2 \mathrm{~Hz}, 2 \mathrm{H}), 0.90(\mathrm{t}, J=7.2 \mathrm{~Hz}, 3 \mathrm{H}) ;{ }^{13} \mathrm{C}-\mathrm{NMR}(150$ $\left.\mathrm{MHz}, \mathrm{CDCl}_{3}\right) \delta 163.6,162.0,146.8,131.9,131.8,131.2,129.0,128.9,127.2,124.0,120.1$, 115.8, 115.6, 110.9, 90.6, 73.5, 54.8, 30.3, 21.9, 18.4, 13.4; HRMS Calculated for $\mathrm{C}_{19} \mathrm{H}_{19} \mathrm{FN}_{3}$ $[\mathrm{M}+\mathrm{H}]^{+}:$308.15630, Found: 308.15588 .

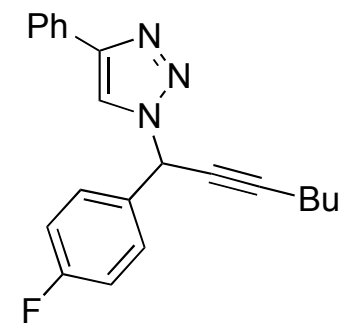

1.4.3j (N-1)

1-(1-(4-fluorophenyl)hept-2-ynyl)-4-phenyl-1H-1,2,3-triazole (1.4.3j (N-1)): ${ }^{1} \mathrm{H}-\mathrm{NMR}$ (600 $\left.\mathrm{MHz}, \mathrm{CDCl}_{3}\right) \delta 7.89(\mathrm{~s}, 1 \mathrm{H}), 7.82(\mathrm{~d}, J=7.2 \mathrm{~Hz}, 2 \mathrm{H}), 7.51-7.49(\mathrm{~m}, 2 \mathrm{H}), 7.38(\mathrm{t}, J=7.8 \mathrm{~Hz}$, 2H), $7.30(\mathrm{t}, J=7.2 \mathrm{~Hz}, 1 \mathrm{H}), 7.04(\mathrm{t}, J=7.8 \mathrm{~Hz}, 2 \mathrm{H}), 6.68(\mathrm{~s}, 1 \mathrm{H}), 2.35(\mathrm{dt}, J=7.2 \mathrm{~Hz}, 2.4 \mathrm{~Hz}$, 2H), 1.58 (quintet, $J=7.2 \mathrm{~Hz}, 2 \mathrm{H}), 1.45$ (hextet, $J=7.2 \mathrm{~Hz}, 2 \mathrm{H}), 0.93(\mathrm{t}, J=7.2 \mathrm{~Hz}, 3 \mathrm{H})$; ${ }^{13} \mathrm{C}-$ NMR $\left(150 \mathrm{MHz}, \mathrm{CDCl}_{3}\right) \delta 163.7,162.1,148.0,132.6,132.5,130.3,129.0,128.9,128.7,128.2$, 
125.7, 117.9, 115.9, 115.7, 90.4, 74.2, 55.7, 30.3, 22.0, 18.4, 13.5; HRMS Calculated for $\mathrm{C}_{21} \mathrm{H}_{21} \mathrm{FN}_{3}[\mathrm{M}+\mathrm{H}]^{+}: 334.17195$, Found: 334.17140 .

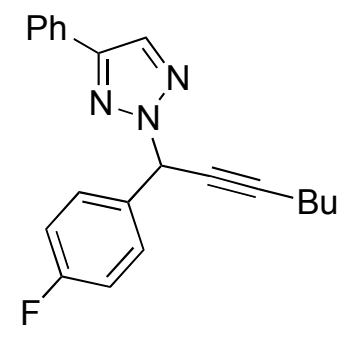

1.4.3j (N-2)

2-(1-(4-fluorophenyl)hept-2-ynyl)-4-phenyl-2H-1,2,3-triazole (1.4.3j (N-2)): ${ }^{1} \mathrm{H}-\mathrm{NMR}(600$ $\left.\mathrm{MHz}, \mathrm{CDCl}_{3}\right) \delta 7.88(\mathrm{~s}, 1 \mathrm{H}), 7.79(\mathrm{~d}, J=7.2 \mathrm{~Hz}, 2 \mathrm{H}), 7.57(\mathrm{q}, J=4.8 \mathrm{~Hz}, 2 \mathrm{H}), 7.42(\mathrm{t}, J=7.8$ $\mathrm{Hz}, 2 \mathrm{H}), 7.36-7.33(\mathrm{~m}, 1 \mathrm{H}), 7.05$ (t, $J=7.8 \mathrm{~Hz}, 2 \mathrm{H}), 6.64(\mathrm{~s}, 1 \mathrm{H}), 2.36$ (dt, $J=7.2 \mathrm{~Hz}, 2.4 \mathrm{~Hz}$, 2H), 1.58 (quintet, $J=7.2 \mathrm{~Hz}, 2 \mathrm{H}$ ), 1.47 (hextet, $J=7.2 \mathrm{~Hz}, 2 \mathrm{H}$ ), $0.93\left(\mathrm{t}, J=7.2 \mathrm{~Hz}, 3 \mathrm{H}\right.$ ); ${ }^{13} \mathrm{C}$ NMR (150 MHz, $\left.\mathrm{CDCl}_{3}\right) \delta 163.9,162.3,148.3,133.1,133.0,132.0,130.5,129.7,129.6,129.0$, $128.7,126.3,115.9,115.7,89.9,74.9,60.3,30.7,22.2,18.8,13.8$; HRMS Calculated for $\mathrm{C}_{21} \mathrm{H}_{21} \mathrm{FN}_{3}[\mathrm{M}+\mathrm{H}]^{+}: 334.17195$, Found: 334.17140 .

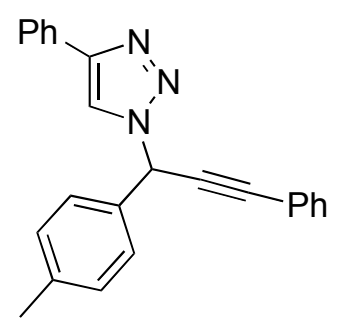

1.4.3k (N-1) 
4-phenyl-1-(3-phenyl-1-p-tolylprop-2-ynyl)-1H-1,2,3-triazole (1.4.3k (N-1)): ${ }^{1} \mathrm{H}-\mathrm{NMR}(600$ $\left.\mathrm{MHz}, \mathrm{CDCl}_{3}\right) \delta 7.94(\mathrm{~s}, 1 \mathrm{H}), 7.82(\mathrm{~d}, J=7.2 \mathrm{~Hz}, 2 \mathrm{H}), 7.54(\mathrm{~d}, J=7.2 \mathrm{~Hz}, 2 \mathrm{H}), 7.50(\mathrm{~d}, J=8.4$ $\mathrm{Hz}, 2 \mathrm{H}), 7.41-7.36(\mathrm{~m}, 5 \mathrm{H}), 7.31(\mathrm{t}, J=7.2 \mathrm{~Hz}, 1 \mathrm{H}), 7.23(\mathrm{~d}, J=8.4 \mathrm{~Hz}, 2 \mathrm{H}), 6.94(\mathrm{~s}, 1 \mathrm{H}), 2.37$ $(\mathrm{s}, 3 \mathrm{H}) ;{ }^{13} \mathrm{C}-\mathrm{NMR}\left(150 \mathrm{MHz}, \mathrm{CDCl}_{3}\right) \delta 148.2,139.3,133.2,131.9,130.5,129.7,129.3,128.7$, $128.5,128.2,127.2,125.7,121.5,118.1,88.6,83.3,56.5,21.1$; HRMS Calculated for $\mathrm{C}_{24} \mathrm{H}_{20} \mathrm{~N}_{3}$ $[\mathrm{M}+\mathrm{H}]^{+}:$350.16572, Found: 350.16517.

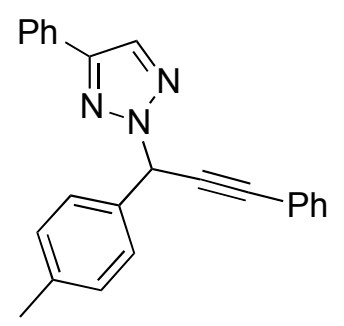

1.4.3k (N-2)

4-phenyl-2-(3-phenyl-1-p-tolylprop-2-ynyl)-2H-1,2,3-triazole (1.4.3k (N-2)): ${ }^{1} \mathrm{H}-\mathrm{NMR}(600$ $\left.\mathrm{MHz}, \mathrm{CDCl}_{3}\right) \delta 7.92(\mathrm{~s}, 1 \mathrm{H}), 7.83(\mathrm{~d}, J=7.2 \mathrm{~Hz}, 2 \mathrm{H}), 7.59-7.55(\mathrm{~m}, 4 \mathrm{H}), 7.44(\mathrm{t}, J=7.2 \mathrm{~Hz}$, 2H), 7.37-7.33 (m, 4H), $7.23(\mathrm{~d}, J=7.8 \mathrm{~Hz}, 2 \mathrm{H}), 6.89(\mathrm{~s}, 1 \mathrm{H}), 2.37(\mathrm{~s}, 3 \mathrm{H}) ;{ }^{13} \mathrm{C}-\mathrm{NMR}(150$ $\left.\mathrm{MHz}, \mathrm{CDCl}_{3}\right) \delta 148.1,138.8,133.5,132.0,131.8,130.3,129.4,128.8,128.7,128.4,128.2$ 127.5, 126.0, 122.1, 87.8, 83.9, 60.8, 21.1; HRMS Calculated for $\mathrm{C}_{24} \mathrm{H}_{20} \mathrm{~N}_{3}[\mathrm{M}+\mathrm{H}]^{+}: 350.16572$, Found: 350.16517. 


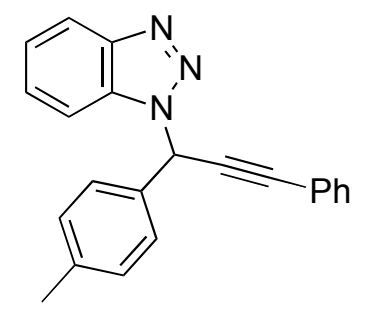

1.4.3I (N-1)

1-(3-phenyl-1-p-tolylprop-2-ynyl)-1H-benzo[d][1,2,3]triazole (1.4.3I (N-1)): ${ }^{1} \mathrm{H}-\mathrm{NMR}(600$ $\left.\mathrm{MHz}, \mathrm{CDCl}_{3}\right) \delta 8.07(\mathrm{~d}, J=8.4 \mathrm{~Hz}, 1 \mathrm{H}), 7.62(\mathrm{~d}, J=8.4 \mathrm{~Hz}, 1 \mathrm{H}), 7.51-7.48(\mathrm{~m}, 4 \mathrm{H}), 7.40-7.32$ $(\mathrm{m}, 6 \mathrm{H}), 7.18(\mathrm{~d}, J=7.8 \mathrm{~Hz}, 2 \mathrm{H}), 2.33(\mathrm{~s}, 3 \mathrm{H}) ;{ }^{13} \mathrm{C}-\mathrm{NMR}\left(150 \mathrm{MHz}, \mathrm{CDCl}_{3}\right) \delta 146.8,138.9$, $132.5,131.9,131.4,129.6,129.1,128.4,127.3,127.0,124.0,121.7,120.1,111.1,88.9,82.7$, 55.6, 21.1; HRMS Calculated for $\mathrm{C}_{22} \mathrm{H}_{18} \mathrm{~N}_{3}[\mathrm{M}+\mathrm{H}]^{+}$: 324.15007 , Found: 324.14952 .

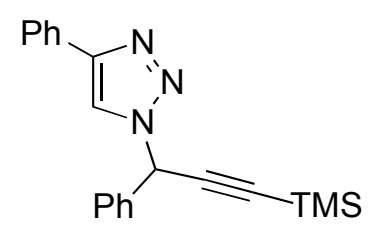

\subsection{3m (N-1)}

4-phenyl-1-(1-phenyl-3-(trimethylsilyl)prop-2-ynyl)-1H-1,2,3-triazole $\quad(1.4 .3 \mathrm{~m} \quad(\mathrm{~N}-1)):{ }^{1} \mathrm{H}-$ NMR $\left(600 \mathrm{MHz}, \mathrm{CDCl}_{3}\right) \delta 7.87(\mathrm{~s}, 1 \mathrm{H}), 7.81(\mathrm{~d}, J=8.4 \mathrm{~Hz}, 2 \mathrm{H}), 7.52(\mathrm{~d}, J=8.4 \mathrm{~Hz}, 2 \mathrm{H}), 7.42-$ $7.37(\mathrm{~m}, 5 \mathrm{H}), 7.32(\mathrm{t}, J=8.4 \mathrm{~Hz}, 1 \mathrm{H}), 6.75(\mathrm{~s}, 1 \mathrm{H}), 0.26(\mathrm{~s}, 9 \mathrm{H}) ;{ }^{13} \mathrm{C}-\mathrm{NMR}\left(150 \mathrm{MHz}, \mathrm{CDCl}_{3}\right) \delta$ $148.2,135.9,130.5,129.2,129.0,128.8,128.2,127.2,125.7,118.0,98.8,94.8,56.6,-0.3$; HRMS Calculated for $\mathrm{C}_{20} \mathrm{H}_{22} \mathrm{~N}_{3} \mathrm{Si}[\mathrm{M}+\mathrm{H}]^{+}: 332.15830$, Found: 332.15790 . 


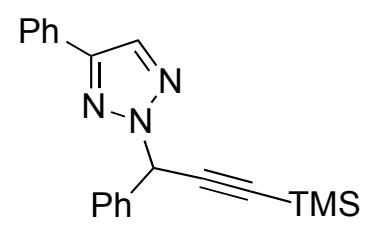

$1.4 .3 \mathrm{~m}(\mathrm{~N}-2)$

4-phenyl-2-(1-phenyl-3-(trimethylsilyl)prop-2-ynyl)-2H-1,2,3-triazole (1.4.3m (N-2)): ${ }^{1} \mathrm{H}-$ $\operatorname{NMR}\left(600 \mathrm{MHz}, \mathrm{CDCl}_{3}\right) \delta 7.88(\mathrm{~s}, 1 \mathrm{H}), 7.81(\mathrm{~d}, J=7.2 \mathrm{~Hz}, 2 \mathrm{H}), 7.58(\mathrm{~d}, J=7.8 \mathrm{~Hz}, 2 \mathrm{H}), 7.43-$ $7.33(\mathrm{~m}, 6 \mathrm{H}), 6.69(\mathrm{~s}, 1 \mathrm{H}), 0.25(\mathrm{~s}, 9 \mathrm{H}) ;{ }^{13} \mathrm{C}-\mathrm{NMR}\left(150 \mathrm{MHz}, \mathrm{CDCl}_{3}\right) \delta 148.1,136.1,131.9$, 130.2, 128.8, 128.7, 128.6, 128.5, 127.5, 126.0, 99.1, 93.6, 61.0, -0.3; HRMS Calculated for $\mathrm{C}_{20} \mathrm{H}_{22} \mathrm{~N}_{3} \mathrm{Si}[\mathrm{M}+\mathrm{H}]^{+}: 332.15830$, Found: 332.15790 .

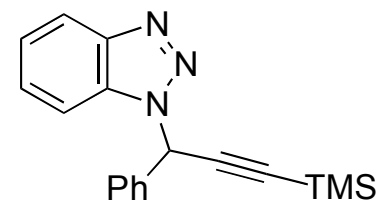

1.4.3n (N-1)

1-(1-phenyl-3-(trimethylsilyl)prop-2-ynyl)-1H-benzo[d][1,2,3]triazole $\quad(1.4 .3 \mathrm{n} \quad(\mathrm{N}-1)):{ }^{1} \mathrm{H}-$ NMR $\left(600 \mathrm{MHz}, \mathrm{CDCl}_{3}\right) \delta 8.04(\mathrm{~d}, J=8.4 \mathrm{~Hz}, 1 \mathrm{H}), 7.57(\mathrm{~d}, J=8.4 \mathrm{~Hz}, 1 \mathrm{H}), 7.51(\mathrm{~d}, J=7.2$ $\mathrm{Hz}, 2 \mathrm{H}), 7.38-7.28(\mathrm{~m}, 5 \mathrm{H}), 7.17(\mathrm{~s}, 1 \mathrm{H}), 0.23(\mathrm{~s}, 9 \mathrm{H}) ;{ }^{13} \mathrm{C}-\mathrm{NMR}\left(150 \mathrm{MHz}, \mathrm{CDCl}_{3}\right) \delta 146.7$, $135.1,131.3,128.8,128.7,127.1,126.9,123.9,120.0,111.1,98.0,95.0,55.7,-0.4$; HRMS Calculated for $\mathrm{C}_{18} \mathrm{H}_{20} \mathrm{~N}_{3} \mathrm{Si}[\mathrm{M}+\mathrm{H}]^{+}: 306.14265$, Found: 306.14210 . 


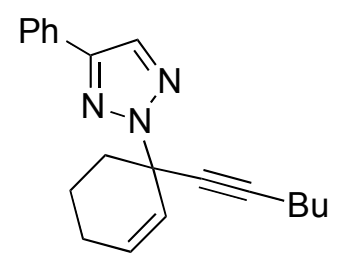

$1.4 .30(\mathrm{~N}-2)$

2-(1-(hex-1-ynyl)cyclohex-2-enyl)-4-phenyl-2H-1,2,3-triazole (1.4.3o (N-2)): ${ }^{1} \mathrm{H}-\mathrm{NMR}$ (600 $\left.\mathrm{MHz}, \mathrm{CDCl}_{3}\right) \delta 7.83(\mathrm{~s}, 1 \mathrm{H}), 7.79(\mathrm{~d}, J=7.2 \mathrm{~Hz}, 2 \mathrm{H}), 7.42(\mathrm{t}, J=7.8 \mathrm{~Hz}, 2 \mathrm{H}), 7.33(\mathrm{t}, J=7.8$ $\mathrm{Hz}, 1 \mathrm{H}), 6.14(\mathrm{~s}, 1 \mathrm{H}), 5.31-5.27(\mathrm{~m}, 1 \mathrm{H}), 2.35-2.30(\mathrm{~m}, 3 \mathrm{H}), 2.23-2.14(\mathrm{~m}, 3 \mathrm{H}), 1.99-1.95(\mathrm{~m}$, $1 \mathrm{H}), 1.77-1.72(\mathrm{~m}, 1 \mathrm{H}), 1.51$ (quintet, $J=7.2 \mathrm{~Hz}, 2 \mathrm{H}), 1.42$ (sextet, $J=7.2 \mathrm{~Hz}, 2 \mathrm{H}), 0.92(\mathrm{t}, J=$ $7.2 \mathrm{~Hz}, 3 \mathrm{H}) ;{ }^{13} \mathrm{C}-\mathrm{NMR}\left(150 \mathrm{MHz}, \mathrm{CDCl}_{3}\right) \delta 147.7,131.0,130.8,129.3,129.0,128.5,126.2$, 126.1, 90.8, 81.1, 61.0, 31.0, 29.7, 29.0, 22.2, 20.1, 19.2, 13.8; HRMS Calculated for $\mathrm{C}_{20} \mathrm{H}_{24} \mathrm{~N}_{3}$ $[\mathrm{M}+\mathrm{H}]^{+}:$306.19702, Found: 306.19678.

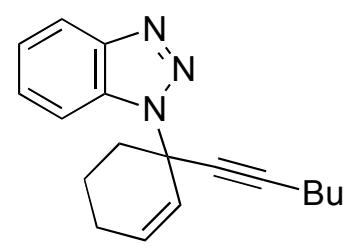

1.4.3p (N-1)

1-(1-(hex-1-ynyl)cyclohex-2-enyl)-1H-benzo[d][1,2,3]triazole (1.4.3p (N-1)): ${ }^{1} \mathrm{H}-\mathrm{NMR}(600$ $\left.\mathrm{MHz}, \mathrm{CDCl}_{3}\right) \delta 8.06(\mathrm{~d}, J=7.8 \mathrm{~Hz}, 1 \mathrm{H}), 7.57(\mathrm{~d}, J=8.4 \mathrm{~Hz}, 1 \mathrm{H}), 7.45(\mathrm{t}, J=7.2 \mathrm{~Hz}, 1 \mathrm{H}), 7.35$ (t, $7.2 \mathrm{~Hz}, 1 \mathrm{H}), 6.10(\mathrm{~s}, 1 \mathrm{H}), 5.65-5.68(\mathrm{~m}, 1 \mathrm{H}), 2.43-2.37(\mathrm{~m}, 1 \mathrm{H}), 2.32(\mathrm{t}, J=7.2 \mathrm{~Hz}, 3 \mathrm{H})$, 2.29-2.22 (m, 1H), 2.16-2.08 (m, 1H), 2.00-1.96 (m, 1H), 1.86-1.81 (m, 1H), 1.52 (quintet, $J=$ $7.2 \mathrm{~Hz}, 2 \mathrm{H}), 1.42$ (sextet, $J=7.2 \mathrm{~Hz}, 2 \mathrm{H}), 0.92(\mathrm{t}, J=7.2 \mathrm{~Hz}, 3 \mathrm{H}) ;{ }^{13} \mathrm{C}-\mathrm{NMR}\left(150 \mathrm{MHz}, \mathrm{CDCl}_{3}\right)$ 
$\delta 146.5,132.1,128.8,127.0,126.9,123.7,120.1,110.4,91.3,80.5,56.4,30.7,29.5,28.9,21.9$, 20.6, 19.0, 13.6; HRMS Calculated for $\mathrm{C}_{18} \mathrm{H}_{22} \mathrm{~N}_{3}[\mathrm{M}+\mathrm{H}]^{+}: 280.18137$, Found: 280.18082 .

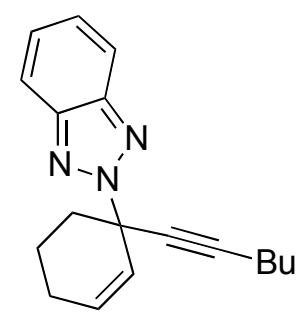

\subsection{3p (N-2)}

2-(1-(hex-1-ynyl)cyclohex-2-enyl)-2H-benzo[d][1,2,3]triazole (1.4.3p (N-2)): ${ }^{1} \mathrm{H}-\mathrm{NMR}(600$ $\left.\mathrm{MHz}, \mathrm{CDCl}_{3}\right) \delta 7.87(\mathrm{dd}, J=6.6 \mathrm{~Hz}, 3.0 \mathrm{~Hz}, 2 \mathrm{H}), 7.36(\mathrm{dd}, J=6.6 \mathrm{~Hz}, 3.0 \mathrm{~Hz}, 2 \mathrm{H})$, ), 6.19 (s, 1H), 5.55-5.58 (m, $1 \mathrm{H}), 2.39-2.21(\mathrm{~m}, 6 \mathrm{H}), 2.02-1.97(\mathrm{~m}, 1 \mathrm{H}), 1.80-1.76(\mathrm{~m}, 1 \mathrm{H}), 1.50$ (quintet, $J=7.2 \mathrm{~Hz}, 2 \mathrm{H}), 1.41$ (sextet, $J=7.2 \mathrm{~Hz}, 2 \mathrm{H}), 0.90$ (t, $J=7.2 \mathrm{~Hz}, 3 \mathrm{H}) ;{ }^{13} \mathrm{C}-\mathrm{NMR}(150 \mathrm{MHz}$, $\left.\mathrm{CDCl}_{3}\right) \delta 144.4,128.8,126.7,126.4,118.3,91.3,81.0,62.7,30.9,29.7,29.6,22.1,20.1,19.2$ 13.8; HRMS Calculated for $\mathrm{C}_{18} \mathrm{H}_{22} \mathrm{~N}_{3}[\mathrm{M}+\mathrm{H}]^{+}: 280.18137$, Found: 280.18082 .

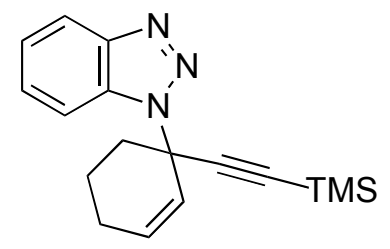

1.4.3q (N-1)

1-(1-((trimethylsilyl)ethynyl)cyclohex-2-enyl)-1H-benzo[d][1,2,3]triazole $(1.4 .3 q(\mathrm{~N}-1)):{ }^{1} \mathrm{H}-$ NMR $\left(600 \mathrm{MHz}, \mathrm{CDCl}_{3}\right) \delta 8.05(\mathrm{~d}, J=7.2 \mathrm{~Hz}, 1 \mathrm{H}), 7.54(\mathrm{~d}, J=8.4 \mathrm{~Hz}, 1 \mathrm{H}), 7.44(\mathrm{t}, J=7.2$ $\mathrm{Hz}, 1 \mathrm{H}), 7.34$ (t, $J=7.2 \mathrm{~Hz}, 1 \mathrm{H}), 6.23(\mathrm{~s}, 1 \mathrm{H}), 5.65-5.61(\mathrm{~m}, 1 \mathrm{H}), 2.44-2.37$ (m, 1H), 2.33-2.30 
(m, $1 \mathrm{H}), 2.25-2.20(\mathrm{~m}, 1 \mathrm{H}), 2.14-2.08(\mathrm{~m}, 1 \mathrm{H}), 2.01-1.95(\mathrm{~m}, 1 \mathrm{H}), 1.85-1.78(\mathrm{~m}, 1 \mathrm{H}), 0.17(\mathrm{~s}$ 9H); ${ }^{13} \mathrm{C}-\mathrm{NMR}\left(150 \mathrm{MHz}, \mathrm{CDCl}_{3}\right) \delta 146.4,132.1,131.4,127.1,126.3,123.8,120.1,110.2$, 104.7, 95.0, 56.1, 29.0, 28.7, 20.5, -0.2; HRMS Calculated for $\mathrm{C}_{17} \mathrm{H}_{22} \mathrm{~N}_{3} \mathrm{Si}[\mathrm{M}+\mathrm{H}]^{+}$: 290.15830, Found: 290.15786.

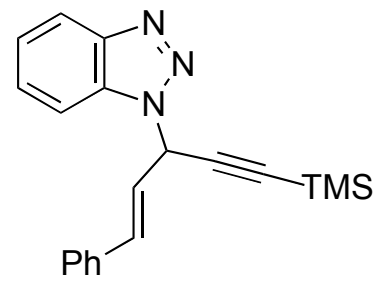

1.4.3r (N-1)

(E)-1-(1-phenyl-5-(trimethylsilyl)pent-1-en-4-yn-3-yl)-1H-benzo[d][1,2,3] triazole (1.4.3r $(\mathrm{N}-$

1)): ${ }^{1} \mathrm{H}-\mathrm{NMR}\left(600 \mathrm{MHz}, \mathrm{CDCl}_{3}\right) \delta 8.07(\mathrm{~d}, J=7.8 \mathrm{~Hz}, 1 \mathrm{H}), 7.36-7.32(\mathrm{~m}, 5 \mathrm{H}), 7.27-7.24(\mathrm{~m}$, 3H), $6.92(\mathrm{dd}, J=16.2 \mathrm{~Hz}, 6.6 \mathrm{~Hz}, 1 \mathrm{H}), 6.63(\mathrm{~d}, J=7.2 \mathrm{~Hz}, 1 \mathrm{H}), 5.65(\mathrm{dd}, J=16.2 \mathrm{~Hz}, 1.8 \mathrm{~Hz}$, 1H), $0.18(\mathrm{~s}, 9 \mathrm{H}) ;{ }^{13} \mathrm{C}-\mathrm{NMR}\left(150 \mathrm{MHz}, \mathrm{CDCl}_{3}\right) \delta 146.4,139.0,136.6,132.2,129.0,128.8$, $127.4,127.3,124.0,120.2,114.8,110.2,101.7,97.8,64.8,-0.3$; HRMS Calculated for $\mathrm{C}_{20} \mathrm{H}_{22} \mathrm{~N}_{3} \mathrm{Si}[\mathrm{M}+\mathrm{H}]^{+}:$332.15830, Found: 332.15883 .

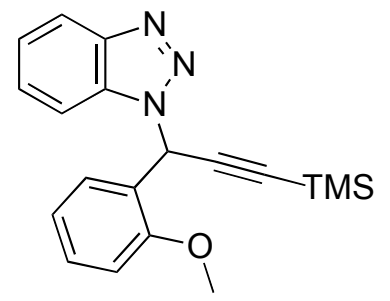

1.4.3s (N-1) 
(N-1)): ${ }^{1} \mathrm{H}-\mathrm{NMR}\left(600 \mathrm{MHz}, \mathrm{CDCl}_{3}\right) \delta 8.02(\mathrm{~d}, J=8.4 \mathrm{~Hz}, 1 \mathrm{H}), 7.97(\mathrm{dd}, J=7.8 \mathrm{~Hz}, 1.8 \mathrm{~Hz}, 1 \mathrm{H})$, $7.60(\mathrm{~d}, J=8.4 \mathrm{~Hz}, 1 \mathrm{H}), 7.39(\mathrm{t}, J=7.2 \mathrm{~Hz}, 1 \mathrm{H}), 7.34-7.28(\mathrm{~m}, 3 \mathrm{H}), 7.05(\mathrm{t}, J=7.8 \mathrm{~Hz}, 1 \mathrm{H})$, $6.80(\mathrm{~d}, J=8.4 \mathrm{~Hz}, 1 \mathrm{H}), 3.66(\mathrm{~s}, 3 \mathrm{H}), 0.19(\mathrm{~s}, 9 \mathrm{H}) ;{ }^{13} \mathrm{C}-\mathrm{NMR}\left(150 \mathrm{MHz}, \mathrm{CDCl}_{3}\right) \delta 156.8,146.0$, $131.9,130.6,129.2,126.8,123.5,123.4,120.5,119.8,110.9,110.7,99.1,93.3,55.4,50.3,-0.4$; HRMS Calculated for $\mathrm{C}_{19} \mathrm{H}_{22} \mathrm{~N}_{3} \mathrm{OSi}[\mathrm{M}+\mathrm{H}]^{+}: 336.15321$, Found: 336.15266 .

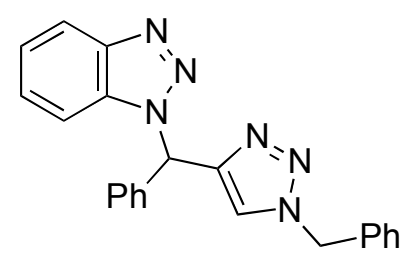

\subsection{5a}

1-((1-benzyl-1H-1,2,3-triazol-4-yl)(phenyl)methyl)-1H-benzo[d][1,2,3]triazole $(1.4 .5 a):{ }^{1} \mathrm{H}-$ NMR $\left(600 \mathrm{MHz}, \mathrm{CDCl}_{3}\right) \delta 8.05(\mathrm{~d}, J=8.4 \mathrm{~Hz}, 1 \mathrm{H}), 7.65(\mathrm{~s}, 1 \mathrm{H}), 7.49(\mathrm{~d}, J=8.4 \mathrm{~Hz}, 1 \mathrm{H}), 7.45$ (s, 1H), $7.41(\mathrm{t}, J=7.2 \mathrm{~Hz}, 1 \mathrm{H}), 7.38-7.33(\mathrm{~m}, 4 \mathrm{H}), 7.31-7.28(\mathrm{~m}, 3 \mathrm{H}), 7.25-7.24(\mathrm{~m}, 4 \mathrm{H}), 5.52$ $(\mathrm{s}, 2 \mathrm{H}) ;{ }^{13} \mathrm{C}-\mathrm{NMR}\left(150 \mathrm{MHz}, \mathrm{CDCl}_{3}\right) \delta 146.1,145.8,137.3,134.2,132.7,129.2,128.9,128.8$, 128.6, 128.0, 127.6, 127.2, 124.1, 123.8, 120.0, 110.3, 59.5, 54.4; HRMS Calculated for $\mathrm{C}_{22} \mathrm{H}_{19} \mathrm{~N}_{6}[\mathrm{M}+\mathrm{H}]^{+}:$367.16712, Found: 367.16789 . 


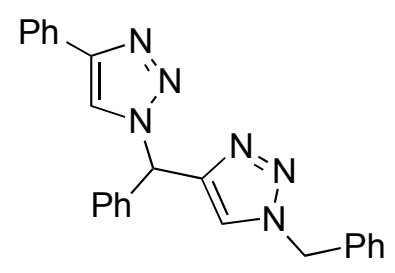

1.4.5b

1-benzyl-4-(phenyl(4-phenyl-1H-1,2,3-triazol-1-yl)methyl)-1H-1,2,3-triazole $\quad(1.4 .5 b):{ }^{1} \mathrm{H}-$ $\operatorname{NMR}\left(600 \mathrm{MHz}, \mathrm{CDCl}_{3}\right) \delta 7.87(\mathrm{~s}, 1 \mathrm{H}), 7.74(\mathrm{~d}, J=7.2 \mathrm{~Hz}, 2 \mathrm{H}), 7.57(\mathrm{~s}, 1 \mathrm{H}), 7.40(\mathrm{t}, J=7.8$ $\mathrm{Hz}, 2 \mathrm{H}), 7.37-7.24(\mathrm{~m}, 12 \mathrm{H}), 5.53(\mathrm{~s}, 2 \mathrm{H}) ;{ }^{13} \mathrm{C}-\mathrm{NMR}\left(150 \mathrm{MHz}, \mathrm{CDCl}_{3}\right) \delta 138.1,134.5,131.6$, $130.2,129.1,128.8,128.7,128.6,128.5,128.4,128.0,127.2,126.0,123.6,65.5,54.3$; HRMS Calculated for $\mathrm{C}_{24} \mathrm{H}_{21} \mathrm{~N}_{6}[\mathrm{M}+\mathrm{H}]^{+}$: 393.18277, Found: 393.18342 .

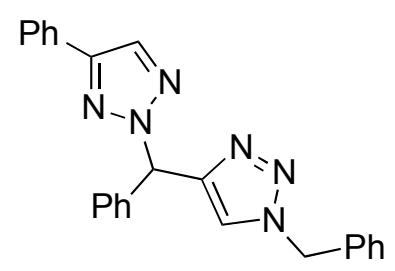

$1.4 .5 \mathrm{c}$

1-benzyl-4-(phenyl(4-phenyl-2H-1,2,3-triazol-2-yl)methyl)-1H-1,2,3-triazole $\quad(1.4 .5 c): \quad{ }^{1} \mathrm{H}-$ NMR (600 MHz, $\left.\mathrm{CDCl}_{3}\right) \delta 8.02(\mathrm{~s}, 1 \mathrm{H}), 7.81(\mathrm{~d}, J=7.8 \mathrm{~Hz}, 2 \mathrm{H}), 7.56(\mathrm{~s}, 1 \mathrm{H}), 7.41-7.27(\mathrm{~m}$, 13H), $7.17(\mathrm{~s}, 1 \mathrm{H}), 5.53(\mathrm{~s}, 2 \mathrm{H}) ;{ }^{13} \mathrm{C}-\mathrm{NMR}\left(150 \mathrm{MHz}, \mathrm{CDCl}_{3}\right) \delta$ 137.3, 134.0, 130.5, 129.3, $129.1,129.0,128.8,128.7,128.4,128.2,127.5,125.7,123.4,119.9,60.7,54.5 ;$ HRMS Calculated for $\mathrm{C}_{24} \mathrm{H}_{21} \mathrm{~N}_{6}[\mathrm{M}+\mathrm{H}]^{+}$: 393.18277, Found: 393.18342 . 


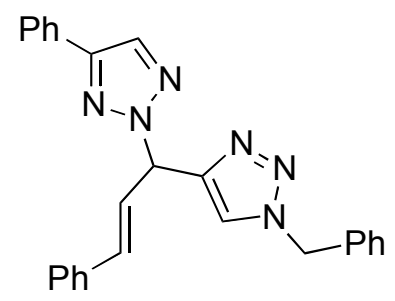

$1.4 .5 d$

(E)-1-benzyl-4-(3-phenyl-1-(4-phenyl-2H-1,2,3-triazol-2-yl)allyl)-1H-1,2,3-triazole (1.4.5d):

${ }^{1} \mathrm{H}-\mathrm{NMR}\left(600 \mathrm{MHz}, \mathrm{CDCl}_{3}\right) \delta 7.87(\mathrm{~s}, 1 \mathrm{H}), 7.78(\mathrm{~d}, J=7.2 \mathrm{~Hz}, 2 \mathrm{H}), 7.50(\mathrm{~d}, J=9.0 \mathrm{~Hz}, 1 \mathrm{H})$, $7.45(\mathrm{~s}, 1 \mathrm{H}), 7.43-7.39(\mathrm{~m}, 4 \mathrm{H}), 7.37-7.26(\mathrm{~m}, 9 \mathrm{H}), 6.67-6.60(\mathrm{~m}, 2 \mathrm{H}), 5.51(\mathrm{~s}, 2 \mathrm{H}) ;{ }^{13} \mathrm{C}-\mathrm{NMR}$ $\left(150 \mathrm{MHz}, \mathrm{CDCl}_{3}\right) \delta 147.6,139.3,134.4,131.1,130.5,129.1,129.0,128.8,128.7,128.6,128.3$, 128.1, 128.0, 127.0, 126.0, 122.8, 119.9, 66.2, 54.1; HRMS Calculated for $\mathrm{C}_{26} \mathrm{H}_{23} \mathrm{~N}_{6}[\mathrm{M}+\mathrm{H}]^{+}$: 419.19842, Found: 419.19793.

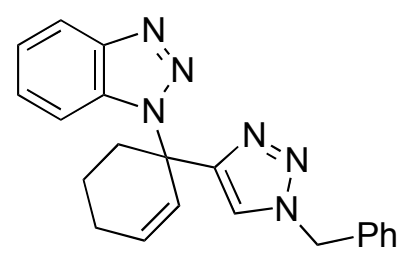

$1.4 .5 \mathrm{e}$

1-(1-(1-benzyl-1H-1,2,3-triazol-4-yl)cyclohex-2-enyl)-1H-benzo[d][1,2,3]triazole

(1.4.5e):

${ }^{1} \mathrm{H}-\mathrm{NMR}\left(600 \mathrm{MHz}, \mathrm{CDCl}_{3}\right) \delta 8.05(\mathrm{~d}, J=8.4 \mathrm{~Hz}, 1 \mathrm{H}), 7.55(\mathrm{~d}, J=8.4 \mathrm{~Hz}, 1 \mathrm{H}), 7.45(\mathrm{~s}, 1 \mathrm{H})$, 7.41-7.32 (m, 5H), 7.28-7.27 (m, 2H), $6.55(\mathrm{~s}, 1 \mathrm{H}), 5.77-5.74(\mathrm{~m}, 1 \mathrm{H}), 5.52(\mathrm{~s}, 2 \mathrm{H}), 2.68-2.66$ (m, 2H), 2.34-2.30 (m, 1H), 2.25-2.19 (m, 1H), 2.14-2.08 (m, 1H), 1.97-1.90 (m, 1H); ${ }^{13} \mathrm{C}-\mathrm{NMR}$ $\left(150 \mathrm{MHz}, \mathrm{CDCl}_{3}\right) \delta 148.1,146.4,134.5,132.9,132.2,129.1,128.8,128.1,127.0,123.7,121.4$ 
120.1, 119.5, 110.3, 56.3, 54.2, 29.4, 25.9, 20.4; HRMS Calculated for $\mathrm{C}_{21} \mathrm{H}_{21} \mathrm{~N}_{6}[\mathrm{M}+\mathrm{H}]^{+}$: 357.18277, Found: 357.18226.

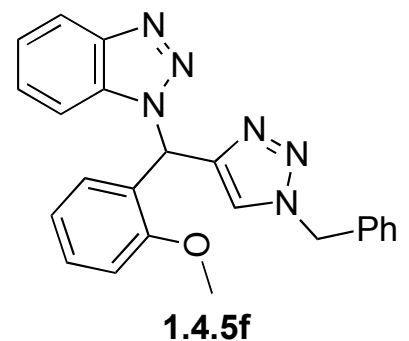

1-((1-benzyl-1H-1,2,3-triazol-4-yl)(2-methoxyphenyl)methyl)-1H-benzo[d][1,2,3]triazole

(1.4.5f): ${ }^{1} \mathrm{H}-\mathrm{NMR}\left(600 \mathrm{MHz}, \mathrm{CDCl}_{3}\right) \delta 8.02(\mathrm{~d}, J=8.4 \mathrm{~Hz}, 1 \mathrm{H}), 7.84(\mathrm{~s}, 1 \mathrm{H}), 7.59$ (s, 1H), 7.53 (d, $J=8.4 \mathrm{~Hz}, 1 \mathrm{H}), 7.40(\mathrm{t}, J=7.8 \mathrm{~Hz}, 1 \mathrm{H}), 7.36-7.30(\mathrm{~m}, 4 \mathrm{H}), 7.28-7.23(\mathrm{~m}, 4 \mathrm{H}), 6.89-6.85(\mathrm{~m}$, 2H), $5.50(\mathrm{~s}, 2 \mathrm{H}), 3.74(\mathrm{~s}, 3 \mathrm{H}) ;{ }^{13} \mathrm{C}-\mathrm{NMR}\left(150 \mathrm{MHz}, \mathrm{CDCl}_{3}\right) \delta$ 156.5, 145.9, 145.6, 134.4, 132.9, 133.0, 129.1, 128.8, 128.7, 127.9, 127.2, 125.5, 123.8, 123.7, 120.8, 119.7, 110.8, 110.3, 55.5, 54.2, 53.2; HRMS Calculated for $\mathrm{C}_{23} \mathrm{H}_{21} \mathrm{~N}_{6} \mathrm{O}[\mathrm{M}+\mathrm{H}]^{+}: 397.17768$, Found: 397.17698 .

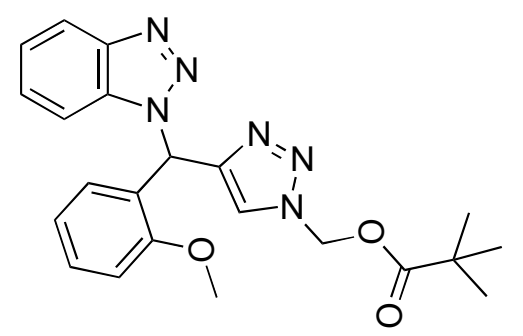

$1.4 .5 \mathrm{~g}$

(4-((1H-benzo[d][1,2,3] triazol-1-yl)(2-methoxyphenyl)methyl)-1H-1,2,3-triazol-1-yl)methyl pivalate (1.4.5g): ${ }^{1} \mathrm{H}-\mathrm{NMR}\left(600 \mathrm{MHz}, \mathrm{CDCl}_{3}\right) \delta 8.04(\mathrm{~d}, J=8.4 \mathrm{~Hz}, 1 \mathrm{H}), 7.84(\mathrm{~s}, 2 \mathrm{H}), 7.49(\mathrm{~d}$, 
$J=8.4 \mathrm{~Hz}, 1 \mathrm{H}), 7.40$ (t, $J=7.2 \mathrm{~Hz}, 1 \mathrm{H}), 7.31$ (quintet, $J=7.2 \mathrm{~Hz}, 2 \mathrm{H}), 7.22(\mathrm{~d}, J=7.8 \mathrm{~Hz}, 1 \mathrm{H})$, 6.92-6.89 (m, 2H), $6.22(\mathrm{~s}, 2 \mathrm{H}), 3.78(\mathrm{~s}, 3 \mathrm{H}), 1.17(\mathrm{~s}, 9 \mathrm{H}) ;{ }^{13} \mathrm{C}-\mathrm{NMR}\left(150 \mathrm{MHz}, \mathrm{CDCl}_{3}\right) \delta$ 177.5, $156.5,146.1,145.8,133.0,130.2,128.6,127.3,125.2,125.1,123.9,120.9,119.9,110.9,110.2$ 69.8, 55.6, 53.2, 38.8, 26.7; HRMS Calculated for $\mathrm{C}_{22} \mathrm{H}_{25} \mathrm{~N}_{6} \mathrm{O}_{3}[\mathrm{M}+\mathrm{H}]^{+}$: 421.19881, Found: 421.19816.

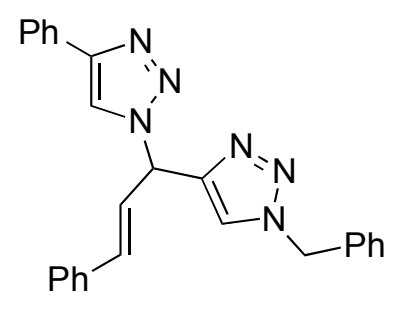

1.4.5h

(E)-1-benzyl-4-(3-phenyl-1-(4-phenyl-1H-1,2,3-triazol-1-yl)allyl)-1H-1,2,3-triazole (1.4.5h):

${ }^{1} \mathrm{H}-\mathrm{NMR}\left(600 \mathrm{MHz}, \mathrm{CDCl}_{3}\right) \delta 7.89(\mathrm{~s}, 1 \mathrm{H}), 7.79(\mathrm{~d}, J=7.2 \mathrm{~Hz}, 2 \mathrm{H}), 7.44(\mathrm{~s}, 1 \mathrm{H}), 7.79(\mathrm{t}, J=$ $7.8 \mathrm{~Hz}, 3 \mathrm{H}), 7.39-7.30(\mathrm{~m}, 9 \mathrm{H}), 7.25(\mathrm{~d}, J=5.4 \mathrm{~Hz}, 1 \mathrm{H}), 7.04$ (dd, $J=15.6 \mathrm{~Hz}, 5.4 \mathrm{~Hz}, 1 \mathrm{H})$, $6.54(\mathrm{~d}, J=15.6 \mathrm{~Hz}, 1 \mathrm{H}), 6.47(\mathrm{~d}, J=7.2 \mathrm{~Hz}, 1 \mathrm{H}), 5.50(\mathrm{~s}, 2 \mathrm{H}) ;{ }^{13} \mathrm{C}-\mathrm{NMR}\left(150 \mathrm{MHz}, \mathrm{CDCl}_{3}\right) \delta$ $147.9,138.1,134.4,131.3,130.3,129.1,129.0,128.9,128.8,128.7,128.6,128.4,128.3,128.1$, 127.4, 126.0, 122.4, 120.7, 70.5, 54.2; HRMS Calculated for $\mathrm{C}_{26} \mathrm{H}_{23} \mathrm{~N}_{6}[\mathrm{M}+\mathrm{H}]^{+}$: 419.19842, Found: 419.19793 


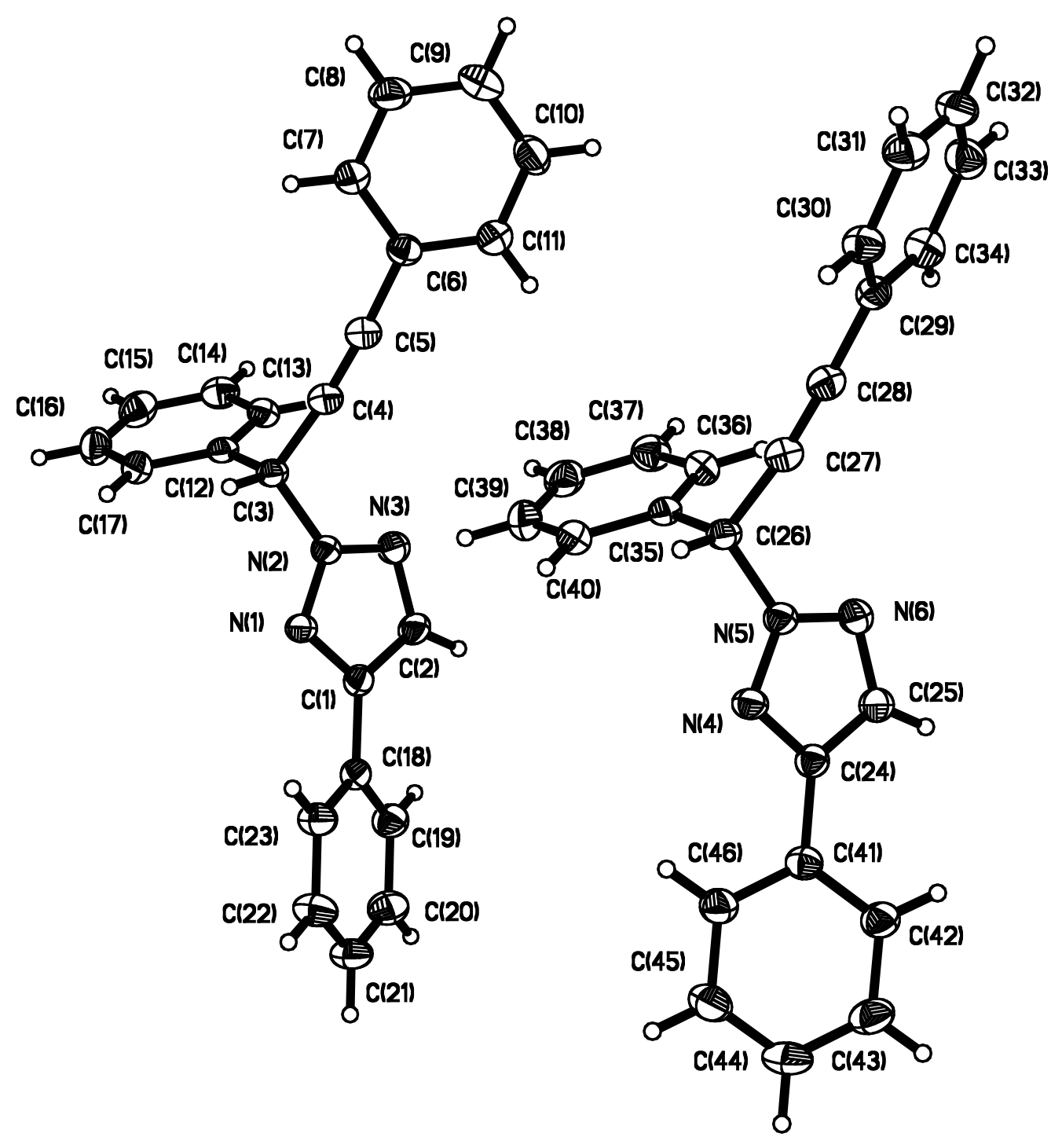

Figure S1.4-1.Perspective view of the molecular structure of $\mathrm{C}_{22} \mathrm{H}_{24} \mathrm{~N}_{6} \mathrm{O}_{3}$ (1.4.3f (N-2)) with the atom labeling scheme. The thermal ellipsoids are scaled to enclose $30 \%$ probability. CCDC number: 775267

For details regarding the crystal structure, refer to supporting information for paper: Yan, W.; Wang, Q.; Chen, Y.; Petersen, J. L.; Shi, X. Org. Lett. 2010, 12, 3308-3311. 


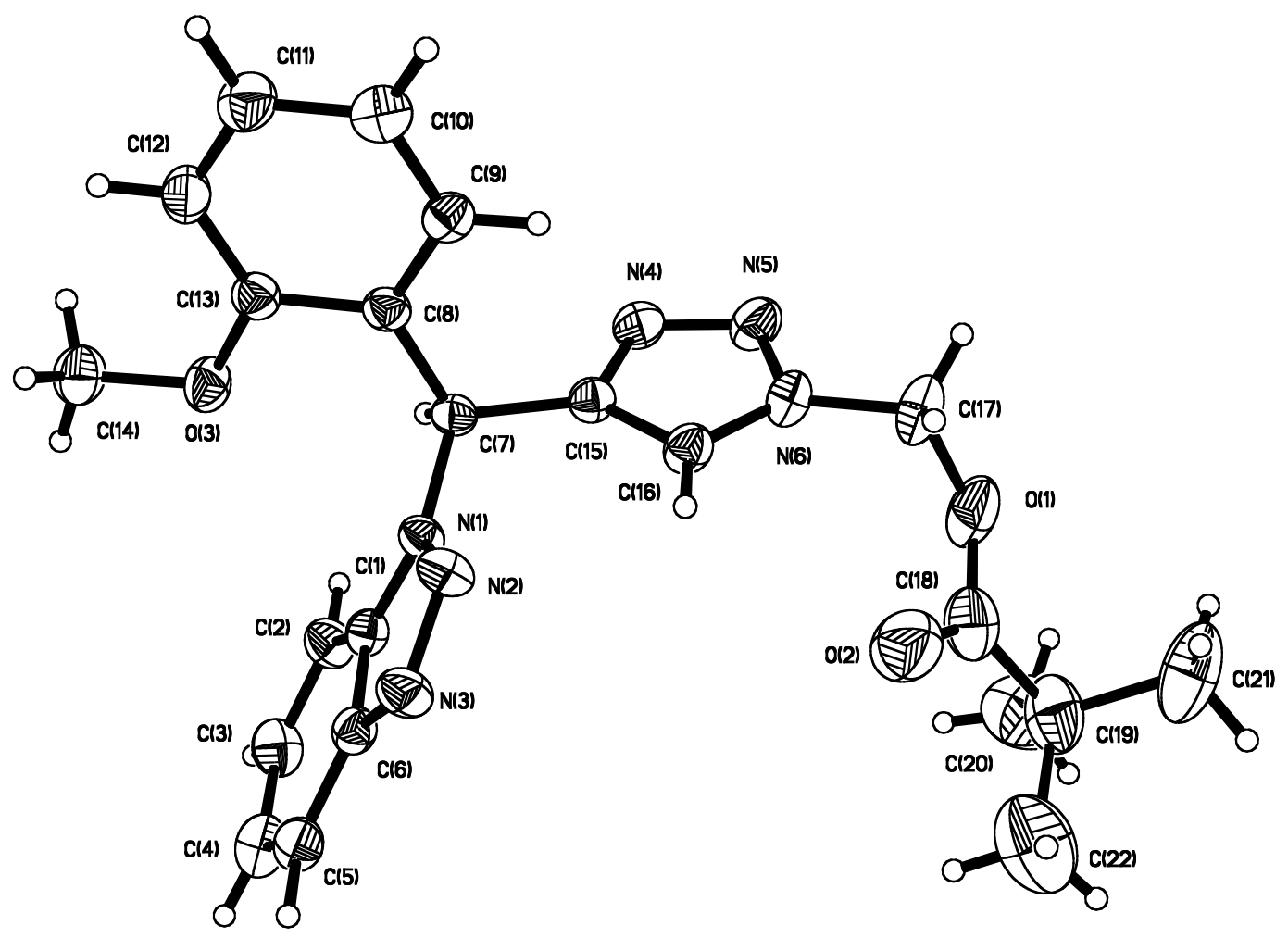

Figure S1.4-2.Perspective view of the molecular structure of $\mathrm{C}_{22} \mathrm{H}_{24} \mathrm{~N}_{6} \mathrm{O}_{3}$ (1.4.5g) with the atom labeling scheme. The thermal ellipsoids are scaled to enclose $30 \%$ probability. CCDC number: 775268 


\subsection{Synthesis of allene triazole through iron catalyzed regioselective addition to propargyl alcohols}

\section{$\underline{\text { General Methods and Materials }}$}

All of the reactions dealing with air and/or moisture-sensitive reactions were carried out under an atmosphere of nitrogen using oven/flame-dried glassware and standard syringe/septa techniques. Unless otherwise noted, all commercial reagents and solvents were obtained from the commercial provider and used without further purification. ${ }^{1} \mathrm{H}$ NMR and ${ }^{13} \mathrm{C}$ NMR spectra were recorded on Varian $600 \mathrm{MHz}$ spectrometers. Chemical shifts were reported relative to internal tetramethylsilane $(\delta 0.00 \mathrm{ppm})$ or $\mathrm{CDCl}_{3}(\delta 7.26 \mathrm{ppm})$ for ${ }^{1} \mathrm{H}$ and $\mathrm{CDCl}_{3}(\delta 77.0 \mathrm{ppm})$ for ${ }^{13} \mathrm{C}$. Melting points were measured on a Mel-Temp 1001D apparatus and uncorrected. Infrared (IR) spectra were obtained on a FT-IR spectrometer.Flash column chromatography was performed on 230-430 mesh silica gel. Analytical thin layer chromatography was performed with precoated glass baked plates $(250 \mu)$ and visualized by fluorescence and by charring after treatment with $p$ anisaldehyde or potassium permanganate stain. HRMS were recorded on LTQ-FTUHRA spectrometer. UV-visible spectra were obtained on a Shimadzu UV-2550.

Propargylic alcohols 1.5.3 were synthesized according to the literatures as below:

Yan, W.; Wang, Q.; Chen, Y.; Petersen, J.; Shi, X. Org. Lett. 2010, 12, 3308-3311. 


\section{Representative procedure for propargylation of 1.5.4 with triazoles}

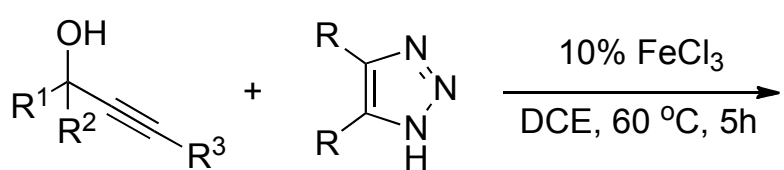

1.5 .3

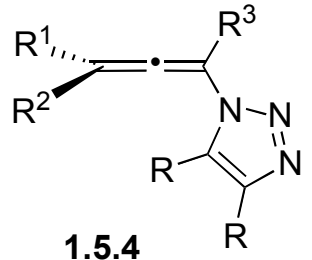

1.5 .4

A solution of triazole $(1.3 \mathrm{mmol})$ with catalyst iron(III) chloride $(0.1 \mathrm{mmol})$ in $1,2-$ dichlorethane(DCE) $(5 \mathrm{ml})$ was stirred at $60{ }^{\circ} \mathrm{C}$ for one hour, then propargyl alcohol $\mathbf{1 . 5 . 3}(1.0$ mmol) was added. The reaction mixture was quenched with $10 \mathrm{ml}$ distilled water and then extracted with EtOAc $(3 \times 10 \mathrm{~mL})$ after 5 hours. The combined organic phases were washed with water and brine, dried with anhydrous $\mathrm{MgSO}_{4}$, and filtered. The filtrate was concentrated under reduced pressure and the residue was purified by flash chromatography on silica gel (hexanes/ethyl acetate, V/V, 20/1) to produce the desired product. 


\section{Compounds Characterization}

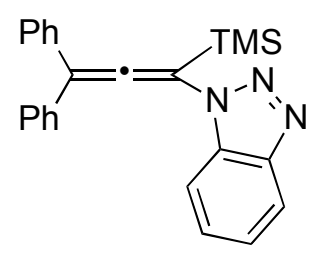

1.5.4a

1-(1-(trimethylsilyl)-3,3-diphenylpropa-1,2-dienyl)-1H-benzo[d][1,2,3]triazole $(1.5 .4 a)$ was purified by flash chromatography (Hexane-EtOAc, v/v 20/1) as white solid. Isolated yield 84\%; m. p. $127-129{ }^{\circ} \mathrm{C} ; \mathrm{R} f$ (hexanes/ethyl acetate, V/V, 10/1) = 0.49; UV absorption: 232(0.182), 263(0.134), 295(0.126); IR (neat), 2957, 1598, 1489, 1447, 1285, 1245, 1062, 936, 833, 767, 694; ${ }^{1} \mathrm{H}-\mathrm{NMR}\left(600 \mathrm{MHz}, \mathrm{CDCl}_{3}\right) \delta 8.09(\mathrm{~d}, J=7.8 \mathrm{~Hz}, 1 \mathrm{H}), 7.82(\mathrm{~d}, J=7.8 \mathrm{~Hz}, 1 \mathrm{H}), 7.50-7.47$ $(\mathrm{m}, 4 \mathrm{H}), 7.43-7.38(\mathrm{~m}, 5 \mathrm{H}), 7.37-7.32(\mathrm{~m}, 3 \mathrm{H}), 0.50(\mathrm{~s}, 9 \mathrm{H}) ;{ }^{13} \mathrm{C}-\mathrm{NMR}\left(150 \mathrm{MHz}, \mathrm{CDCl}_{3}\right) \delta$ $200.4(\mathrm{C}), 146.2(\mathrm{C}), 135.8(\mathrm{C}), 132.5(\mathrm{C}), 128.7(\mathrm{CH}), 128.4(\mathrm{CH}), 128.1(\mathrm{CH}), 127.9(\mathrm{CH})$, $124.3(\mathrm{CH}), 118.0(\mathrm{CH}), 115.3(\mathrm{C}), 111.9(\mathrm{CH}), 108.8(\mathrm{C}),-0.5\left(\mathrm{CH}_{3}\right)$; HRMS Calculated for $\mathrm{C}_{24} \mathrm{H}_{24} \mathrm{~N}_{3} \mathrm{Si}[\mathrm{M}+\mathrm{H}]^{+}:$382.1734, Found: 382.1735 .

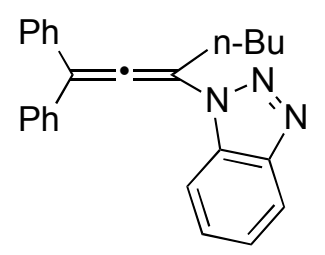

$1.5 .4 b$

1-(1,1-diphenylhepta-1,2-dien-3-yl)-1H-benzo[d $[$ [1,2,3]triazole $(1.5 .4 b)$ was purified by flash chromatography (Hexane-EtOAc, v/v 20/1) as yellow liquid. Isolated yield 74\%; $\mathrm{R} f$ 
(hexanes/ethyl acetate, V/V, 10/1) $=0.45$; UV absorption: 233(0.160), 259(0.129), 295(0.100); IR (neat), 2935, 1603, 1485, 1447, 1281, 1244, 1056, 957, 785, 744, 691; ${ }^{1} \mathrm{H}-\mathrm{NMR}$ (600 MHz, $\left.\mathrm{CDCl}_{3}\right) \delta 8.08(\mathrm{~d}, J=7.8 \mathrm{~Hz}, 1 \mathrm{H}), 7.54(\mathrm{~d}, J=7.8 \mathrm{~Hz}, 1 \mathrm{H}), 7.46-7.43(\mathrm{~m}, 4 \mathrm{H}), 7.42-7.30(\mathrm{~m}$, 8H), 3.22 (t, $J=7.8 \mathrm{~Hz}, 2 \mathrm{H}), 1.75$ (quin, $J=7.8 \mathrm{~Hz}, 2 \mathrm{H}), 1.47$ (sex, $J=7.8 \mathrm{~Hz}, 2 \mathrm{H}), 0.93$ (t, $J=$ $7.8 \mathrm{~Hz}, 3 \mathrm{H}) ;{ }^{13} \mathrm{C}-\mathrm{NMR}\left(150 \mathrm{MHz}, \mathrm{CDCl}_{3}\right) \delta 197.0(\mathrm{C}), 146.3$ (C), 135.9 (C), 132.1 (C), 128.7 $(\mathrm{CH}), 128.6(\mathrm{CH}), 128.4(\mathrm{CH}), 127.9(\mathrm{CH}), 124.3(\mathrm{CH}), 120.0(\mathrm{CH}), 119.8(\mathrm{C}), 113.5(\mathrm{C}), 111.6$ (CH), $31.2\left(\mathrm{CH}_{2}\right), 29.2\left(\mathrm{CH}_{2}\right), 22.3\left(\mathrm{CH}_{2}\right), 13.8\left(\mathrm{CH}_{3}\right)$; HRMS Calculated for $\mathrm{C}_{25} \mathrm{H}_{24} \mathrm{~N}_{3}[\mathrm{M}+\mathrm{H}]^{+}$: 366.1965, Found: 366.1964.

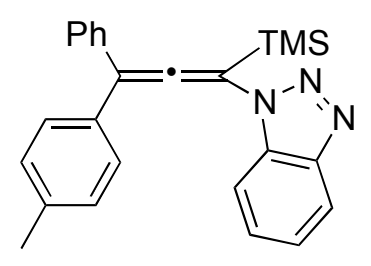

$1.5 .4 \mathrm{c}$

\section{1-(1-(trimethylsilyl)-3-phenyl-3-p-tolylpropa-1,2-dienyl)-1H-benzo $[d][1,2,3]$ triazole $(1.5 .4 c)$}

was purified by flash chromatography (Hexane-EtOAc, v/v 20/1) as yellow liquid. Isolated yield $80 \%$; $\mathrm{R} f$ (hexanes/ethyl acetate, $\mathrm{V} / \mathrm{V}, 10 / 1)=0.51$; UV absorption: 234(0.301), 263(0.209), 299(0.195); IR (neat), 2930, 1600, 1485, 1449, 1281, 1246, 1068, 935, 838, 764, 693; ${ }^{1} \mathrm{H}-\mathrm{NMR}$ $\left(600 \mathrm{MHz}, \mathrm{CDCl}_{3}\right) \delta 8.07(\mathrm{~d}, J=7.8 \mathrm{~Hz}, 1 \mathrm{H}), 7.79(\mathrm{~d}, J=7.8 \mathrm{~Hz}, 1 \mathrm{H}), 7.44(\mathrm{~d}, J=7.8 \mathrm{~Hz}, 2 \mathrm{H})$, 7.40-7.36 (m, 4H), 7.35-7.32 (m, 3H), 7.20 (d, $J=7.8 \mathrm{~Hz}, 2 \mathrm{H}), 2.38$ (s, 3H), $0.46(\mathrm{~s}, 9 \mathrm{H}) ;{ }^{13} \mathrm{C}-$ NMR (150 MHz, $\left.\mathrm{CDCl}_{3}\right) \delta 200.4$ (C), 146.2 (C), 138.0 (C), 136.0 (C), 132.8 (C), 132.5 (C), $129.5(\mathrm{CH}), 128.7(\mathrm{CH}), 128.5(\mathrm{CH}), 128.4(\mathrm{CH}), 128.0(\mathrm{CH}), 127.8(\mathrm{CH}), 124.3(\mathrm{CH}), 120.0$ $(\mathrm{CH}), 115.2(\mathrm{C}), 112.0(\mathrm{CH}), 108.7(\mathrm{C}), 21.2\left(\mathrm{CH}_{3}\right),-0.5\left(\mathrm{CH}_{3}\right)$; HRMS Calculated for $\mathrm{C}_{25} \mathrm{H}_{26} \mathrm{~N}_{3} \mathrm{Si}[\mathrm{M}+\mathrm{H}]^{+}: 396.1887$, Found: 396.1883 . 


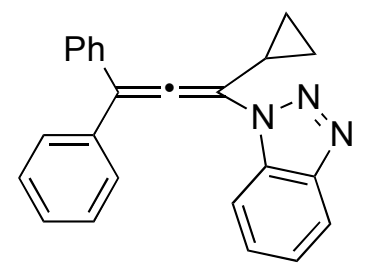

$1.5 .4 d$

1-(1-cyclopropyl-3,3-diphenylpropa-1,2-dienyl)-1H-benzo[d][1,2,3]triazole $\quad(1.5 .4 d) \quad$ was purified by flash chromatography (Hexane-EtOAc, v/v 20/1) as white solid. Isolated yield 82\%; m. p. $107-109{ }^{\circ} \mathrm{C} ; \mathrm{R} f$ (hexanes/ethyl acetate, $\left.\mathrm{V} / \mathrm{V}, 10 / 1\right)=0.48 ; \mathrm{UV}$ absorption: $229(0.205)$, 260(0.146), 295(0.102); IR (neat), 3008, 1598, 1490, 1449, 1278, 1161, 1078, 977, 853, 747, 692; ${ }^{1} \mathrm{H}-\mathrm{NMR}\left(600 \mathrm{MHz}, \mathrm{CDCl}_{3}\right) \delta$ 8.09-8.07 (m, 1H), 7.54-7.51 (m, 1H), 7.41-7.32 (m, 12H), 2.51-2.45 (m, 1H), 1.13-1.09 (m, 2H), 0.85-0.81 (m, 2H); ${ }^{13} \mathrm{C}-\mathrm{NMR}\left(150 \mathrm{MHz}, \mathrm{CDCl}_{3}\right) \delta 196.4$ (C), $146.4(\mathrm{C}), 135.7(\mathrm{C}), 132.0(\mathrm{C}), 128.7(\mathrm{CH}), 128.6(\mathrm{CH}), 128.5(\mathrm{CH}), 127.9(\mathrm{CH}), 124.3$ (CH), $120.9(\mathrm{C}), 120.0(\mathrm{CH}), 116.6(\mathrm{C}), 111.4(\mathrm{CH}), 11.8(\mathrm{CH}), 7.7\left(\mathrm{CH}_{2}\right)$; HRMS Calculated for $\mathrm{C}_{24} \mathrm{H}_{20} \mathrm{~N}_{3}[\mathrm{M}+\mathrm{H}]^{+}: 350.1652$, Found: 350.1653 .

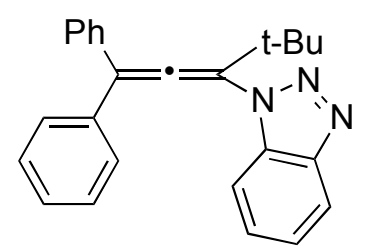

$1.5 .4 \mathrm{e}$

1-(4,4-dimethyl-1,1-diphenylpenta-1,2-dien-3-yl)-1H-benzo[d][1,2,3]triazole $\quad(1.5 .4 \mathrm{e})$ was purified by flash chromatography (Hexane-EtOAc, v/v 20/1) as yellow liquid. Isolated yield 91\%; m. p. $81-83{ }^{\circ} \mathrm{C} ; \mathrm{R} f($ hexanes/ethyl acetate, $\mathrm{V} / \mathrm{V}, 10 / 1)=0.47$; UV absorption: $236(0.193)$, 
265(0.215); IR (neat), 2972, 1589, 1490, 1451, 1274, 1231, 1062, 947, 810, 766, 694; ${ }^{1} \mathrm{H}-\mathrm{NMR}$ $\left(600 \mathrm{MHz}, \mathrm{CDCl}_{3}\right) \delta$ 8.06-8.02 (m, 1H), 7.39-7.33 (m, 10H), 7.31-7.28 (m, 2H), 7.18-7.16 (m, 1H), $1.38(\mathrm{~s}, 9 \mathrm{H}) ;{ }^{13} \mathrm{C}-\mathrm{NMR}\left(150 \mathrm{MHz}, \mathrm{CDCl}_{3}\right) \delta 200.6(\mathrm{C}), 145.0(\mathrm{C}), 135.2(\mathrm{C}), 134.3(\mathrm{C})$, 128.6 (CH), $128.5(\mathrm{CH}), 128.3(\mathrm{CH}), 127.5(\mathrm{CH}), 123.8(\mathrm{CH}), 119.7(\mathrm{CH}), 119.1(\mathrm{C}), 117.2(\mathrm{C})$, 110.6 (CH), $37.2(\mathrm{C}), 29.1\left(\mathrm{CH}_{3}\right)$; HRMS Calculated for $\mathrm{C}_{25} \mathrm{H}_{24} \mathrm{~N}_{3}[\mathrm{M}+\mathrm{H}]^{+}$: 366.1965 , Found: 366.1966.

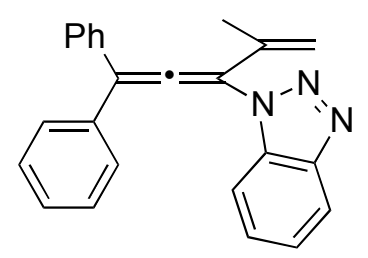

$1.5 .4 \mathrm{f}$

1-(4-methyl-1,1-diphenylpenta-1,2,4-trien-3-yl)-1H-benzo $[d][1,2,3]$ triazole $\quad(1.5 .4 f) \quad$ was purified by flash chromatography (Hexane-EtOAc, v/v 20/1) as yellow liquid. Isolated yield $86 \% ; \mathrm{R} f$ (hexanes/ethyl acetate, $\mathrm{V} / \mathrm{V}, 10 / 1)=0.52 ; \mathrm{UV}$ absorption: $235(0.165), 265(0.096)$, 297(0.062); IR (neat), 3057, 1597, 1490, 1448, 1278, 1160, 1065, 1002, 908, 743, 695; ${ }^{1} \mathrm{H}-\mathrm{NMR}$ $\left(600 \mathrm{MHz}, \mathrm{CDCl}_{3}\right) \delta 8.08(\mathrm{~d}, J=7.8 \mathrm{~Hz}, 1 \mathrm{H}), 7.45-7.34(\mathrm{~m}, 12 \mathrm{H}), 7.29(\mathrm{~d}, J=7.8 \mathrm{~Hz}, 1 \mathrm{H}), 5.17$ (s, 1H), 4.66 (s, 1H), 2.15 (s, 3H); ${ }^{13} \mathrm{C}-\mathrm{NMR}\left(150 \mathrm{MHz}, \mathrm{CDCl}_{3}\right) \delta 204.6$ (C), $145.4(\mathrm{C}), 136.0$ (C), $134.6(\mathrm{C}), 133.7(\mathrm{C}), 128.9(\mathrm{CH}), 128.8(\mathrm{CH}), 128.7(\mathrm{CH}), 127.7(\mathrm{CH}), 124.0(\mathrm{CH}), 120.0$ $(\mathrm{CH}), 118.7(\mathrm{C}), 115.4(\mathrm{CH}), 113.4(\mathrm{C}), 110.4\left(\mathrm{CH}_{2}\right), 20.5\left(\mathrm{CH}_{3}\right)$; HRMS Calculated for $\mathrm{C}_{24} \mathrm{H}_{20} \mathrm{~N}_{3}[\mathrm{M}+\mathrm{H}]^{+}: 350.1651$, Found: 350.1650 . 


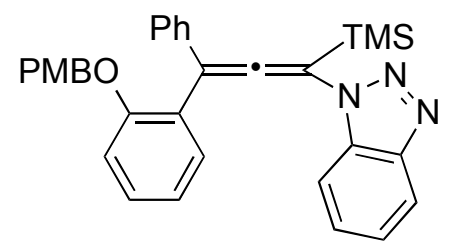

$1.5 .4 \mathrm{~g}$

\section{1-(3-(2-(4-methoxybenzyloxy)phenyl)-1-(trimethylsilyl)-3-phenylpropa-1,2-dienyl)-1H-}

benzo $[\boldsymbol{d}][1,2,3]$ triazole $(\mathbf{1 . 5 . 4 g})$ was purified by flash chromatography (Hexane-EtOAc, v/v 20/1) as yellow liquid. Isolated yield 90\%; $\mathrm{R} f$ (hexanes/ethyl acetate, V/V, 6/1) = 0.58; UV absorption: 231(0.186), 263(0.145), 299(0.128); IR (neat), 2976, 1583, 1493, 1450, 1286, 1240, 1063, 938, 831, 757, 692; ${ }^{1} \mathrm{H}-\mathrm{NMR}\left(600 \mathrm{MHz}, \mathrm{CDCl}_{3}\right) \delta 8.05(\mathrm{~d}, J=8.4 \mathrm{~Hz}, 1 \mathrm{H}), 7.98(\mathrm{~d}, J=$ $8.4 \mathrm{~Hz}, 1 \mathrm{H}), 7.42-7.31(\mathrm{~m}, 7 \mathrm{H}), 7.24(\mathrm{t}, J=8.4 \mathrm{~Hz}, 1 \mathrm{H}), 7.07-7.03(\mathrm{~m}, 2 \mathrm{H}), 6.85(\mathrm{~d}, J=8.4 \mathrm{~Hz}$, 2H), $6.81(\mathrm{~s}, 1 \mathrm{H}), 6.69(\mathrm{~d}, J=8.4 \mathrm{~Hz}, 2 \mathrm{H}), 4.93(\mathrm{q}, J=11.4 \mathrm{~Hz}, 2 \mathrm{H}), 3.78(\mathrm{~s}, 3 \mathrm{H}), 0.5(\mathrm{~s}, 9 \mathrm{H})$;

${ }^{13} \mathrm{C}-\mathrm{NMR}\left(150 \mathrm{MHz}, \mathrm{CDCl}_{3}\right) \delta 200.0$ (C), 159.0 (C), 156.1 (C), 145.9 (C), 136.4 (C), 132.4 (C), $130.8(\mathrm{C}), 129.5(\mathrm{C}), 128.6(\mathrm{CH}), 128.5(\mathrm{CH}), 128.4(\mathrm{CH}), 127.5(\mathrm{CH}), 127.4(\mathrm{CH}), 127.0(\mathrm{CH})$, $125.0(\mathrm{CH}), 124.1(\mathrm{CH}), 121.0(\mathrm{CH}), 119.4(\mathrm{CH}), 113.5(\mathrm{CH}), 112.8(\mathrm{CH}), 112.3(\mathrm{CH}), 111.8$ (C), $108.4(\mathrm{C}), 69.5\left(\mathrm{CH}_{2}\right), 55.2\left(\mathrm{CH}_{3}\right),-0.4\left(\mathrm{CH}_{3}\right)$; HRMS Calculated for $\mathrm{C}_{32} \mathrm{H}_{32} \mathrm{~N}_{3} \mathrm{O}_{2} \mathrm{Si}$ $[\mathrm{M}+\mathrm{H}]^{+}:$517.2179, Found: 517.5180 .

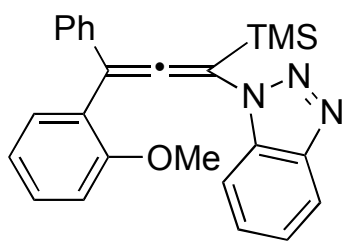

$1.5 .4 \mathrm{~h}$

\section{1-(3-(2-methoxyphenyl)-1-(trimethylsilyl)-3-phenylpropa-1,2-dienyl)-1 H-}

benzo $[\boldsymbol{d}][1,2,3]$ triazole $(\mathbf{1 . 5 . 4 h})$ was purified by flash chromatography (Hexane-EtOAc, v/v 
20/1) as white solid. Isolated yield $90 \%$; m. p. $131-133{ }^{\circ} \mathrm{C}$; $\mathrm{R} f($ hexanes/ethyl acetate, $\mathrm{V} / \mathrm{V}, 6 / 1)=$ 0.60; UV absorption: 230(0.200), 264(0.210), 295(0.173); IR (neat), 2957, 1599, 1490, 1453, 1286, 1245, 1066, 939, 829, 740, 691; ${ }^{1} \mathrm{H}-\mathrm{NMR}\left(600 \mathrm{MHz}, \mathrm{CDCl}_{3}\right) \delta 8.15(\mathrm{~d}, J=7.8 \mathrm{~Hz}, 1 \mathrm{H})$, $8.06(\mathrm{~d}, J=7.8 \mathrm{~Hz}, 1 \mathrm{H}), 7.42-7.32(\mathrm{~m}, 8 \mathrm{H}), 7.28(\mathrm{t}, J=7.8 \mathrm{~Hz}, 1 \mathrm{H}), 7.03(\mathrm{t}, J=7.8 \mathrm{~Hz}, 1 \mathrm{H})$, $6.98(\mathrm{~d}, J=7.8 \mathrm{~Hz}, 1 \mathrm{H}), 3.71(\mathrm{~s}, 3 \mathrm{H}), 0.51(\mathrm{~s}, 9 \mathrm{H}) ;{ }^{13} \mathrm{C}-\mathrm{NMR}\left(150 \mathrm{MHz}, \mathrm{CDCl}_{3}\right) \delta 200.0(\mathrm{C})$, $157.1(\mathrm{C}), 146.1(\mathrm{C}), 136.0(\mathrm{C}), 132.5(\mathrm{C}), 130.8(\mathrm{CH}), 129.5(\mathrm{CH}), 128.5(\mathrm{CH}), 127.5(\mathrm{CH})$, $127.4(\mathrm{CH}), 127.0(\mathrm{CH}), 124.5(\mathrm{CH}), 124.2(\mathrm{CH}), 120.8(\mathrm{CH}), 119.6(\mathrm{CH}), 112.7(\mathrm{C}), 111.3(\mathrm{C})$, $111.2(\mathrm{CH}), 108.3(\mathrm{C}), 55.1\left(\mathrm{CH}_{3}\right),-0.4\left(\mathrm{CH}_{3}\right)$; HRMS Calculated for $\mathrm{C}_{25} \mathrm{H}_{26} \mathrm{~N}_{3} \mathrm{OSi}[\mathrm{M}+\mathrm{H}]^{+}$: 412.1840, Found: 412.1841.

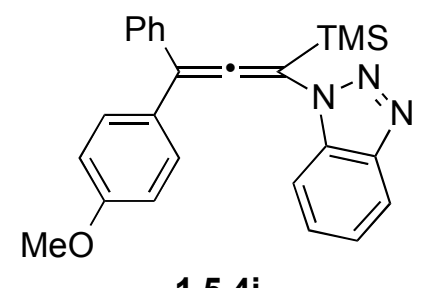

$1.5 .4 \mathrm{i}$

\section{1-(3-(4-methoxyphenyl)-1-(trimethylsilyl)-3-phenylpropa-1,2-dienyl)-1 H-}

benzo $[d][1,2,3]$ triazole $(1.5 .4 i)$ was purified by flash chromatography (Hexane-EtOAc, $\mathrm{v} / \mathrm{v}$ 20/1) as yellow liquid. Isolated yield $91 \%$; $R f$ (hexanes/ethyl acetate, $\mathrm{V} / \mathrm{V}, 6 / 1)=0.64 ; \mathrm{UV}$ absorption: 231(0.197), 262(0.184), 296(0.155); IR (neat), 2963, 1603, 1498, 1466, 1270, 1235, 1055, 930, 821, 747, 695; ${ }^{1} \mathrm{H}-\mathrm{NMR}\left(600 \mathrm{MHz}, \mathrm{CDCl}_{3}\right) \delta 8.08(\mathrm{~d}, J=7.2 \mathrm{~Hz}, 1 \mathrm{H}), 7.80(\mathrm{~d}, J=$ $7.2 \mathrm{~Hz}, 1 \mathrm{H}), 7.47(\mathrm{~d}, J=9.0 \mathrm{~Hz}, 2 \mathrm{H}), 7.41-7.32(\mathrm{~m}, 7 \mathrm{H}), 6.94(\mathrm{~d}, J=9.0 \mathrm{~Hz}, 2 \mathrm{H}), 3.84(\mathrm{~s}, 3 \mathrm{H})$, 0.47 (s, 9H); ${ }^{13} \mathrm{C}-\mathrm{NMR}\left(150 \mathrm{MHz}, \mathrm{CDCl}_{3}\right) \delta 200.6$ (C), 159.8 (C), 146.4 (C), 136.3 (C), 132.7 (C), $129.9(\mathrm{CH}), 129.0(\mathrm{CH}), 128.7(\mathrm{CH}), 128.3(\mathrm{CH}), 128.1(\mathrm{CH}), 124.6(\mathrm{CH}), 120.2(\mathrm{CH})$, 
$115.3(\mathrm{C}), 114.5(\mathrm{CH}), 112.2(\mathrm{CH}), 108.9(\mathrm{C}), 55.6\left(\mathrm{CH}_{3}\right),-0.22\left(\mathrm{CH}_{3}\right)$; HRMS Calculated for $\mathrm{C}_{25} \mathrm{H}_{26} \mathrm{~N}_{3} \mathrm{OSi}[\mathrm{M}+\mathrm{H}]^{+}:$412.1838, Found: 412.1840.

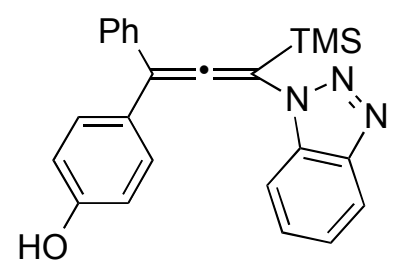

$1.5 .4 \mathrm{j}$

\section{4-(3-(1H-benzo $[d][1,2,3]$ triazol-1-yl)-3-(trimethylsilyl)-1-phenylpropa-1,2-dienyl)phenol}

(1.5.4j) was purified by flash chromatography (Hexane-EtOAc, v/v 20/1) as yellow liquid. Isolated yield 80\%; Rf(hexanes/ethyl acetate, $\mathrm{V} / \mathrm{V}, 4 / 1)=0.44 ; \mathrm{UV}$ absorption: $233(0.217)$, 257(0.166), 271(0.187), 300(0.134); IR (neat), 3363, 2988, 1620, 1476, 1433, 1271, 1212, 1055 , 938, 811, 745, 698; ${ }^{1} \mathrm{H}-\mathrm{NMR}\left(600 \mathrm{MHz}, \mathrm{CDCl}_{3}\right) \delta 8.06(\mathrm{~d}, J=7.2 \mathrm{~Hz}, 1 \mathrm{H}), 7.80(\mathrm{~d}, J=7.2 \mathrm{~Hz}$, 1H), $7.45(\mathrm{~d}, J=7.2 \mathrm{~Hz}, 2 \mathrm{H}), 7.41-7.37(\mathrm{~m}, 4 \mathrm{H}), 7.36-7.30(\mathrm{~m}, 3 \mathrm{H}), 6.95(\mathrm{~d}, J=7.2 \mathrm{~Hz}, 2 \mathrm{H})$, 0.45 (s, 9H); ${ }^{13} \mathrm{C}-\mathrm{NMR}\left(150 \mathrm{MHz}, \mathrm{CDCl}_{3}\right) \delta 200.5$ (C), 156.4 (C), 145.7 (C), $136.0(\mathrm{C}), 132.5$ (C), $129.8(\mathrm{CH}), 128.7(\mathrm{CH}), 128.5(\mathrm{CH}), 128.0(\mathrm{CH}), 127.3(\mathrm{CH}), 124.7(\mathrm{CH}), 119.6(\mathrm{CH})$, $115.9(\mathrm{CH}), 115.3(\mathrm{C}), 112.2(\mathrm{CH}), 108.6(\mathrm{C}),-0.51\left(\mathrm{CH}_{3}\right)$; HRMS Calculated for $\mathrm{C}_{24} \mathrm{H}_{24} \mathrm{~N}_{3} \mathrm{OSi}$ $[\mathrm{M}+\mathrm{H}]^{+}:$398.1681, Found: 398.1682.

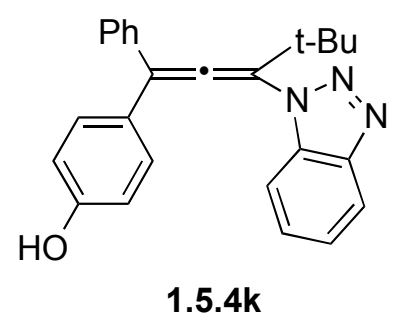


was purified by flash chromatography (Hexane-EtOAc, v/v 20/1) as yellow liquid. Isolated yield 83\%; Rf (hexanes/ethyl acetate, $\mathrm{V} / \mathrm{V}, 4 / 1)=0.43$; UV absorption: 231(0.233), 270(0.209), 298(0.174); IR (neat), 3345, 2995, 1605, 1455, 1445, 1287, 1225, 1043, 936, 814, 748, 695; ${ }^{1} \mathrm{H}-$ $\operatorname{NMR}\left(600 \mathrm{MHz}, \mathrm{CDCl}_{3}\right) \delta 8.06-8.03(\mathrm{~m}, 1 \mathrm{H}), 7.40-7.35(\mathrm{~m}, 5 \mathrm{H}), 7.33-7.30(\mathrm{~m}, 2 \mathrm{H}), 7.24(\mathrm{~d}, J=$ $8.4 \mathrm{~Hz}, 2 \mathrm{H}), 7.20-7.17(\mathrm{~m}, 1 \mathrm{H}), 6.93(\mathrm{~d}, J=8.4 \mathrm{~Hz}, 2 \mathrm{H}), 1.36(\mathrm{~s}, 9 \mathrm{H}) ;{ }^{13} \mathrm{C}-\mathrm{NMR}(150 \mathrm{MHz}$, $\left.\mathrm{CDCl}_{3}\right) \delta 200.4(\mathrm{C}), 156.5(\mathrm{C}), 144.5(\mathrm{C}), 135.5(\mathrm{C}), 134.4(\mathrm{C}), 132.9(\mathrm{CH}), 130.0(\mathrm{CH}), 128.7$ $(\mathrm{CH}), 128.6(\mathrm{CH}), 128.3(\mathrm{CH}), 128.2(\mathrm{CH}), 127.7(\mathrm{CH}), 124.1(\mathrm{CH}), 119.4(\mathrm{CH}), 115.7(\mathrm{CH})$, $115.3(\mathrm{C}), 110.8(\mathrm{C}), 37.2(\mathrm{C}), 29.2\left(\mathrm{CH}_{3}\right)$; HRMS Calculated for $\mathrm{C}_{25} \mathrm{H}_{24} \mathrm{~N}_{3} \mathrm{O}[\mathrm{M}+\mathrm{H}]^{+}: 382.1912$, Found: 382.1910 .

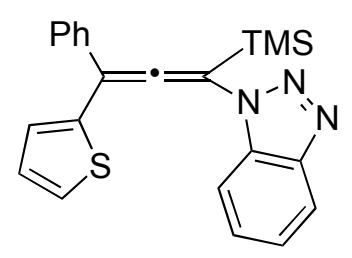

$1.5 .4 I$

\section{1-(4,4-dimethyl-1-phenyl-1-(thiophen-2-yl)penta-1,2-dien-3-yl)-1H-benzo $[d][1,2,3]$ triazole}

(1.5.4l) was purified by flash chromatography (Hexane-EtOAc, v/v 20/1) as yellow liquid. Isolated yield 95\%; Rf(hexanes/ethyl acetate, $\mathrm{V} / \mathrm{V}, 6 / 1)=0.46 ; \mathrm{UV}$ absorption: 236(0.189), 260(0.237), 289(0.125); IR (neat), 2988, 1621, 1490, 1454, 1268, 1221, 1034, 951, 830, 745, 688; ${ }^{1} \mathrm{H}-\mathrm{NMR}\left(600 \mathrm{MHz}, \mathrm{CDCl}_{3}\right) \delta 8.09(\mathrm{~d}, J=8.4 \mathrm{~Hz}, 1 \mathrm{H}), 7.86(\mathrm{~d}, J=8.4 \mathrm{~Hz}, 1 \mathrm{H}), 7.57(\mathrm{~d}, J$ $=7.2 \mathrm{~Hz}, 2 \mathrm{H}), 7.45-7.36(\mathrm{~m}, 5 \mathrm{H}), 7.34(\mathrm{dd}, J=5.4 \mathrm{~Hz}, 1.2 \mathrm{~Hz}, 1 \mathrm{H}), 7.12(\mathrm{dd}, J=3.6 \mathrm{~Hz}, 1.2 \mathrm{~Hz}$, 1H), $7.08(\mathrm{dd}, J=5.4 \mathrm{~Hz}, 3.6 \mathrm{~Hz}, 1 \mathrm{H}), 0.50(\mathrm{~s}, 9 \mathrm{H}) ;{ }^{13} \mathrm{C}-\mathrm{NMR}\left(150 \mathrm{MHz}, \mathrm{CDCl}_{3}\right) \delta 199.9(\mathrm{C})$, 
$146.2(\mathrm{C}), 139.4(\mathrm{C}), 135.5(\mathrm{C}), 132.4(\mathrm{C}), 128.8(\mathrm{CH}), 128.4(\mathrm{CH}), 128.2(\mathrm{CH}), 128.0(\mathrm{CH})$, 127.8 (CH), $127.0(\mathrm{CH}), 126.4(\mathrm{CH}), 124.4(\mathrm{CH}), 120.0(\mathrm{CH}), 111.9(\mathrm{CH}), 110.5(\mathrm{C}), 109.0(\mathrm{C})$, $-0.57\left(\mathrm{CH}_{3}\right)$; HRMS Calculated for $\mathrm{C}_{23} \mathrm{H}_{22} \mathrm{~N}_{3} \mathrm{~S}[\mathrm{M}+\mathrm{H}]^{+}: 372.1528$, Found: 372.1527 .

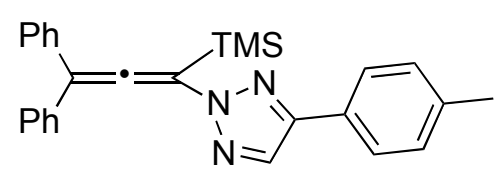

$\mathrm{N}-2-1.5 .4 m$

2-(1-(trimethylsilyl)-3,3-diphenylpropa-1,2-dienyl)-4-p-tolyl-2H-1,2,3-triazole $(\mathrm{N}-2-1.5 .4 \mathrm{~m})$ was purified by flash chromatography (Hexane-EtOAc, v/v 20/1) as yellow liquid. Isolated yield 60\%; Rf (hexanes/ethyl acetate, V/V, 10/1) $=0.62$; UV absorption: $237(0.202), 264(0.153)$, 299(0.135); IR (neat), 2923, 1633, 1487, 1446, 1281, 1243, 1039, 931, 827, 779, 692; ${ }^{1} \mathrm{H}-\mathrm{NMR}$ $\left(600 \mathrm{MHz}, \mathrm{CDCl}_{3}\right) \delta 8.01(\mathrm{~s}, 1 \mathrm{H}), 7.77(\mathrm{~d}, J=7.8 \mathrm{~Hz}, 2 \mathrm{H}), 7.45-7.40(\mathrm{~m}, 8 \mathrm{H}), 7.39-7.36(\mathrm{~m}$, 2H), $7.24(\mathrm{~d}, J=7.8 \mathrm{~Hz}, 2 \mathrm{H}), 2.39$ (s, 3H), $0.47(\mathrm{~s}, 9 \mathrm{H}) ;{ }^{13} \mathrm{C}-\mathrm{NMR}\left(150 \mathrm{MHz}, \mathrm{CDCl}_{3}\right) \delta 201.3$ (C), $147.6(\mathrm{C}), 138.0(\mathrm{C}), 135.2(\mathrm{C}), 129.4(\mathrm{CH}), 128.7(\mathrm{CH}), 128.4(\mathrm{CH}), 128.3(\mathrm{CH}), 127.5$ (C), $125.6(\mathrm{CH}), 117.0(\mathrm{CH}), 116.1(\mathrm{C}), 109.3(\mathrm{C}), 21.2\left(\mathrm{CH}_{3}\right),-0.62\left(\mathrm{CH}_{3}\right)$; HRMS Calculated for $\mathrm{C}_{27} \mathrm{H}_{28} \mathrm{~N}_{3} \mathrm{Si}[\mathrm{M}+\mathrm{H}]^{+}: 422.2046$, Found: 422.2044 .

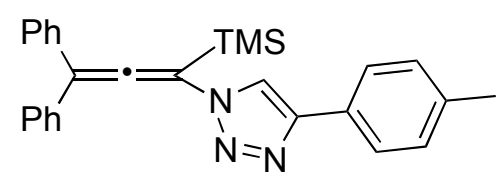

$\mathrm{N}-1-1.5 .4 \mathrm{~m}$ 


\section{1-(1-(trimethylsilyl)-3,3-diphenylpropa-1,2-dienyl)-4-p-tolyl-1H-1,2,3-triazole $(\mathrm{N}-1-1.5 .4 \mathrm{~m})$}

was purified by flash chromatography (Hexane-EtOAc, v/v 20/1) as yellow liquid. Isolated yield 12\%; $\mathrm{R} f$ (hexanes/ethyl acetate, V/V, 10/1) = 0.29; UV absorption: 236(0.179), 265(0.144), 297(0.114); IR (neat), 2928, 1635, 1469, 1433, 1287, 1222, 1032, 930, 816, 770, 694; ${ }^{1} \mathrm{H}-\mathrm{NMR}$ $\left(600 \mathrm{MHz}, \mathrm{CDCl}_{3}\right) \delta 7.63(\mathrm{~s}, 1 \mathrm{H}), 7.30-7.27(\mathrm{~m}, 6 \mathrm{H}), 7.07(\mathrm{~d}, J=7.8 \mathrm{~Hz}, 2 \mathrm{H}), 7.05-7.03(\mathrm{~m}$, 4H), $6.73(\mathrm{~d}, J=7.8 \mathrm{~Hz}, 2 \mathrm{H}), 2.14(\mathrm{~s}, 3 \mathrm{H}), 0.32(\mathrm{~s}, 9 \mathrm{H}) ;{ }^{13} \mathrm{C}-\mathrm{NMR}\left(150 \mathrm{MHz}, \mathrm{CDCl}_{3}\right) \delta 203.8$ (C), $138.8(\mathrm{C}), 137.8(\mathrm{C}), 135.0(\mathrm{C}), 133.3(\mathrm{C}), 128.9(\mathrm{CH}), 128.7(\mathrm{CH}), 128.5(\mathrm{CH}), 128.3$ $(\mathrm{CH}), 127.8(\mathrm{CH}), 124.3(\mathrm{CH}), 114.2(\mathrm{C}), 107.3(\mathrm{C}), 21.2\left(\mathrm{CH}_{3}\right),-0.66\left(\mathrm{CH}_{3}\right)$; HRMS Calculated for $\mathrm{C}_{27} \mathrm{H}_{28} \mathrm{~N}_{3} \mathrm{Si}[\mathrm{M}+\mathrm{H}]^{+}$: 422.2046, Found: 422.2045 .

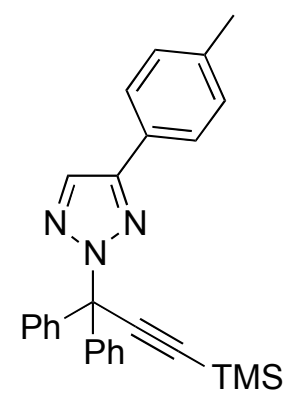

N-2-1.5.4m'

2-(3-(trimethylsilyl)-1,1-diphenylprop-2-ynyl)-4-p-tolyl-2H-1,2,3-triazole (N-2-1.5.4m') was purified by flash chromatography (Hexane-EtOAc, v/v 20/1) as yellow liquid. Isolated yield 13\%; R $f$ (hexanes/ethyl acetate, $\mathrm{V} / \mathrm{V}, 10 / 1)=0.48 ; \mathrm{UV}$ absorption: $244(0.408), 255(0.418)$; IR (neat), 3108, 2960, 1490, 1456, 1354, 1296, 1071, 974, 833, 764, 734, 687; ${ }^{1} \mathrm{H}-\mathrm{NMR}(600 \mathrm{MHz}$, $\left.\mathrm{CDCl}_{3}\right) \delta 7.91(\mathrm{~s}, 1 \mathrm{H}), 7.68(\mathrm{~d}, J=7.8 \mathrm{~Hz}, 2 \mathrm{H}), 7.36-7.33(\mathrm{~m}, 6 \mathrm{H}), 7.32-7.29(\mathrm{~m}, 4 \mathrm{H}), 7.21(\mathrm{~d}, J$ $=7.8 \mathrm{~Hz}, 2 \mathrm{H}), 2.37(\mathrm{~s}, 3 \mathrm{H}), 0.28(\mathrm{~s}, 9 \mathrm{H}) ;{ }^{13} \mathrm{C}-\mathrm{NMR}\left(150 \mathrm{MHz}, \mathrm{CDCl}_{3}\right) \delta 148.0(\mathrm{C}), 141.2(\mathrm{C})$, $138.3(\mathrm{C}), 131.2(\mathrm{CH}), 129.4(\mathrm{CH}), 128.3(\mathrm{CH}), 128.2(\mathrm{CH}), 128.1(\mathrm{CH}), 127.5(\mathrm{C}), 126.0(\mathrm{CH})$, 
104.4 (C), $94.2(\mathrm{C}), 73.8(\mathrm{C}), 21.3\left(\mathrm{CH}_{3}\right),-0.28\left(\mathrm{CH}_{3}\right)$; HRMS Calculated for $\mathrm{C}_{27} \mathrm{H}_{28} \mathrm{~N}_{3} \mathrm{Si}$ $[\mathrm{M}+\mathrm{H}]^{+}:$422.2046, Found: 422.2047.

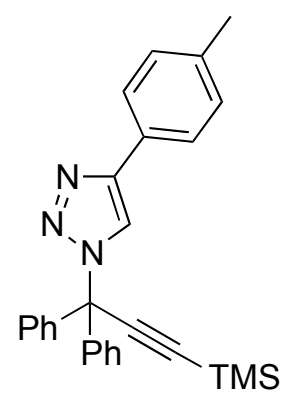

$\mathrm{N}-1-1.5 .4 \mathrm{~m}^{\prime}$

1-(3-(trimethylsilyl)-1,1-diphenylprop-2-ynyl)-4-p-tolyl-2H-1,2,3-triazole (N-1-1.5.4m') was purified by flash chromatography (Hexane-EtOAc, v/v 20/1) as yellow liquid. Isolated yield 5\%; $\mathrm{R} f$ (hexanes/ethyl acetate, $\mathrm{V} / \mathrm{V}, 10 / 1)=0.24$; UV absorption: 243(0.314), 253(0.298); IR (neat), 3083, 2936, 1490, 1456, 1425, 1156, 1073, 976, 833, 751, 730, 686; ${ }^{1} \mathrm{H}-\mathrm{NMR}$ (600 MHz, $\left.\mathrm{CDCl}_{3}\right) \delta 7.97(\mathrm{~s}, 1 \mathrm{H}), 7.73(\mathrm{~d}, J=7.8 \mathrm{~Hz}, 2 \mathrm{H}), 7.37-7.35(\mathrm{~m}, 6 \mathrm{H}), 7.32-7.29(\mathrm{~m}, 4 \mathrm{H}), 7.22(\mathrm{~d}, J$ $=7.8 \mathrm{~Hz}, 2 \mathrm{H}), 2.38(\mathrm{~s}, 3 \mathrm{H}), 0.29(\mathrm{~s}, 9 \mathrm{H}) ;{ }^{13} \mathrm{C}-\mathrm{NMR}\left(150 \mathrm{MHz}, \mathrm{CDCl}_{3}\right) \delta 146.8(\mathrm{C}), 140.6(\mathrm{C})$, $137.9(\mathrm{C}), 129.4(\mathrm{CH}), 128.7(\mathrm{CH}), 128.4(\mathrm{CH}), 128.0(\mathrm{CH}), 127.7(\mathrm{C}), 125.6(\mathrm{CH}), 120.2(\mathrm{CH})$, 104.1 (C), 94.9 (C), $70.0(\mathrm{C}), 21.2\left(\mathrm{CH}_{3}\right),-0.37\left(\mathrm{CH}_{3}\right)$; HRMS Calculated for $\mathrm{C}_{27} \mathrm{H}_{28} \mathrm{~N}_{3} \mathrm{Si}$ $[\mathrm{M}+\mathrm{H}]^{+}:$422.2046, Found: 422.2049 . 


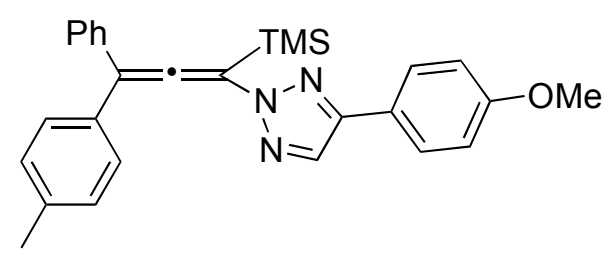

$\mathrm{N}-2-1.5 .4 n$

\section{4-(4-methoxyphenyl)-2-(1-(trimethylsilyl)-3-phenyl-3-p-tolylpropa-1,2-dienyl)-2H-1,2,3-}

triazole (N-2-1.5.4n) was purified by flash chromatography (Hexane-EtOAc, v/v 20/1) as yellow liquid. $\mathrm{R} f$ (hexanes/ethyl acetate, $\mathrm{V} / \mathrm{V}, 6 / 1$ ) $=0.66$; Isolated yield 70\%; UV absorption: 244(0.223), 271(0.189), 298(0.154); IR (neat), 2987, 1621, 1503, 1454, 1268, 1237, 1067, 941, 832, 754, 687; ${ }^{1} \mathrm{H}-\mathrm{NMR}\left(600 \mathrm{MHz}, \mathrm{CDCl}_{3}\right) \delta 7.92(\mathrm{~s}, 1 \mathrm{H}), 7.77(\mathrm{~d}, J=9.0 \mathrm{~Hz}, 2 \mathrm{H}), 7.39(\mathrm{~d}, J=$ $9.0 \mathrm{~Hz}, 4 \mathrm{H}), 7.28$ (d, $J=7.8 \mathrm{~Hz}, 2 \mathrm{H}), 7.21(\mathrm{~d}, J=7.8 \mathrm{~Hz}, 2 \mathrm{H}), 6.94$ (d, $J=9.0 \mathrm{~Hz}, 2 \mathrm{H}), 3.84$ (s, 3H), 2.39 (s, 3H), 0.42 (s, 9H); ${ }^{13} \mathrm{C}-\mathrm{NMR}\left(150 \mathrm{MHz}, \mathrm{CDCl}_{3}\right) \delta 201.3$ (C), 159.7 (C), $147.4(\mathrm{C})$ $138.3(\mathrm{C}), 135.4(\mathrm{C}), 132.2(\mathrm{C}), 129.5(\mathrm{CH}), 128.7(\mathrm{CH}), 128.4(\mathrm{CH}), 128.3(\mathrm{CH}), 128.2(\mathrm{CH})$, 127.1 (CH), $123.1(\mathrm{C}), 116.6(\mathrm{CH}), 116.0(\mathrm{C}), 114.2(\mathrm{CH}), 109.2(\mathrm{C}), 55.3\left(\mathrm{CH}_{3}\right), 21.2\left(\mathrm{CH}_{3}\right),-$ $0.59\left(\mathrm{CH}_{3}\right)$; HRMS Calculated for $\mathrm{C}_{28} \mathrm{H}_{30} \mathrm{~N}_{3} \mathrm{OSi}[\mathrm{M}+\mathrm{H}]^{+}:$452.2153, Found: 452.2156 .

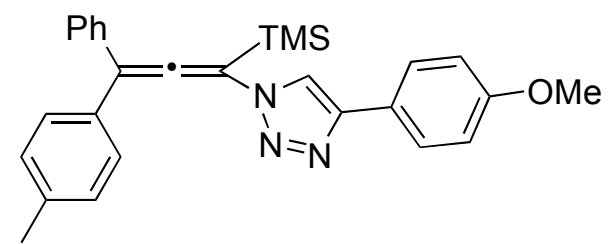

$\mathrm{N}-1-1.5 .4 n$

\section{4-(4-methoxyphenyl)-1-(1-(trimethylsilyl)-3-phenyl-3-p-tolylpropa-1,2-dienyl)-1 H-1,2,3-}

triazole (N-1-1.5.4n) was purified by flash chromatography (Hexane-EtOAc, v/v 20/1) as yellow liquid. Isolated yield 14\%; $\mathrm{R} f$ (hexanes/ethyl acetate, $\mathrm{V} / \mathrm{V}, 6 / 1$ ) $=0.30 ; \mathrm{UV}$ absorption: 244(0.165), 273(0.132), 299(0.103); IR (neat), 2960, 1611, 1494, 1469, 1284, 1232, 1049, 938, 
819, 732, 691; ${ }^{1} \mathrm{H}-\mathrm{NMR}\left(600 \mathrm{MHz}, \mathrm{CDCl}_{3}\right) \delta 7.62(\mathrm{~s}, 1 \mathrm{H}), 7.36-7.33(\mathrm{~m}, 2 \mathrm{H}), 7.20-7.14(\mathrm{~m}$, 3H), $7.10(\mathrm{~d}, J=9.0 \mathrm{~Hz}, 4 \mathrm{H}), 7.07-7.05(\mathrm{~m}, 2 \mathrm{H}), 6.46(\mathrm{~d}, J=7.8 \mathrm{~Hz}, 2 \mathrm{H}), 3.64(\mathrm{~s}, 3 \mathrm{H}), 2.36(\mathrm{~s}$, 3H), 0.31 (s, 9H); ${ }^{13} \mathrm{C}-\mathrm{NMR}\left(150 \mathrm{MHz}, \mathrm{CDCl}_{3}\right) \delta 204.0$ (C), 160.2 (C), 142.9 (C), 137.9 (C), $135.4(\mathrm{C}), 132.3(\mathrm{C}), 130.5(\mathrm{CH}), 129.3(\mathrm{CH}), 128.8(\mathrm{CH}), 128.5(\mathrm{CH}), 128.3(\mathrm{CH}), 128.2(\mathrm{CH})$, $128.0(\mathrm{C}), 127.9(\mathrm{CH}), 120.0(\mathrm{C}), 113.9(\mathrm{CH}), 107.4(\mathrm{C}), 55.2\left(\mathrm{CH}_{3}\right), 21.3\left(\mathrm{CH}_{3}\right),-0.45\left(\mathrm{CH}_{3}\right)$; HRMS Calculated for $\mathrm{C}_{28} \mathrm{H}_{30} \mathrm{~N}_{3} \mathrm{OSi}[\mathrm{M}+\mathrm{H}]^{+}$: 452.2153, Found: 452.2150 .

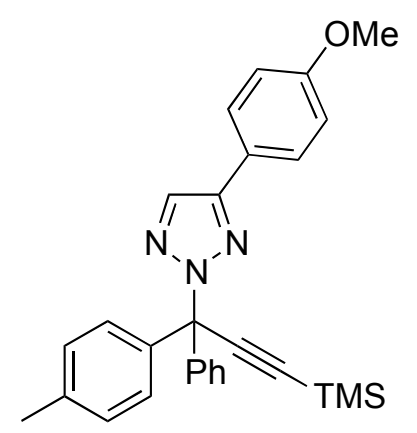

N-2-1.5.4n'

\section{4-(4-methoxyphenyl)-2-(3-(trimethylsilyl)-1-phenyl-1-p-tolylprop-2-ynyl)-2H-1,2,3-triazole}

(N-2-1.5.4n') was purified by flash chromatography (Hexane-EtOAc, v/v 20/1) as yellow liquid. Isolated yield 10\%; $\mathrm{R} f$ (hexanes/ethyl acetate, $\mathrm{V} / \mathrm{V}, 6 / 1)=0.51$; $\mathrm{UV}$ absorption: $256(0.413)$, 269(0.424); IR (neat), 3096, 2943, 1482, 1423, 1368, 1299, 1065, 975, 830, 751, 724, 689; ${ }^{1} \mathrm{H}-$ NMR (600 MHz, $\left.\mathrm{CDCl}_{3}\right) \delta 7.83(\mathrm{~s}, 1 \mathrm{H}), 7.70(\mathrm{~d}, J=8.4 \mathrm{~Hz}, 2 \mathrm{H}), 7.32-7.29(\mathrm{~m}, 2 \mathrm{H}), 7.25-7.23$ (m, 2H), $7.18(\mathrm{~d}, J=8.4 \mathrm{~Hz}, 2 \mathrm{H}), 7.12(\mathrm{t}, J=8.4 \mathrm{~Hz}, 3 \mathrm{H}), 6.91(\mathrm{t}, J=8.4 \mathrm{~Hz}, 2 \mathrm{H}), 3.81(\mathrm{~s}, 3 \mathrm{H})$, 2.34 (s, 3H), 0.26 (s, 9H); ${ }^{13} \mathrm{C}-\mathrm{NMR}\left(150 \mathrm{MHz}, \mathrm{CDCl}_{3}\right) \delta 159.8$ (C), 147.7 (C), 141.5 (C), 138.3 (C), $137.4(\mathrm{C}), 130.8(\mathrm{CH}), 128.8(\mathrm{CH}), 128.2(\mathrm{CH}), 128.1(\mathrm{CH}), 128.0(\mathrm{CH}), 127.9(\mathrm{CH}), 127.4$ $(\mathrm{CH}), 123.1(\mathrm{C}), 114.1(\mathrm{CH}), 104.6(\mathrm{C}), 93.9(\mathrm{C}), 73.5(\mathrm{C}), 55.2\left(\mathrm{CH}_{3}\right), 21.1\left(\mathrm{CH}_{3}\right),-0.26\left(\mathrm{CH}_{3}\right)$; HRMS Calculated for $\mathrm{C}_{28} \mathrm{H}_{30} \mathrm{~N}_{3} \mathrm{OSi}[\mathrm{M}+\mathrm{H}]^{+}$: 452.2153, Found: 452.2150 . 


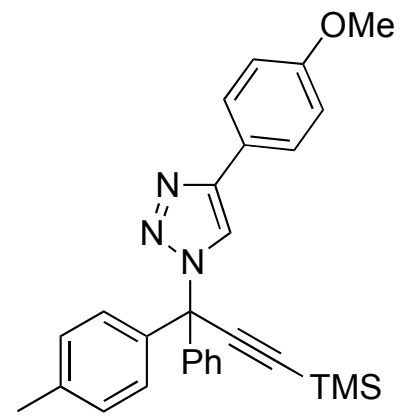

$\mathrm{N}-1-1.5 .4 n^{\prime}$

\section{4-(4-methoxyphenyl)-1-(3-(trimethylsilyl)-1-phenyl-1-p-tolylprop-2-ynyl)-1H-1,2,3-triazole}

(N-1-1.5.4n') was purified by flash chromatography (Hexane-EtOAc, v/v 20/1) as yellow liquid. Isolated yield 4\%; $\mathrm{R} f$ (hexanes/ethyl acetate, $\mathrm{V} / \mathrm{V}, 6 / 1)=0.33 ; \mathrm{UV}$ absorption: 256(0.335), 266(0.356); IR (neat), 3090, 2955, 1498, 1453, 1434, 1149, 1068, 956, 837, 750, 721, 680; ${ }^{1} \mathrm{H}-$ NMR (600 MHz, $\left.\mathrm{CDCl}_{3}\right) \delta 7.90(\mathrm{~s}, 1 \mathrm{H}), 7.75(\mathrm{~d}, J=9.0 \mathrm{~Hz}, 2 \mathrm{H}), 7.36-7.34(\mathrm{~m}, 3 \mathrm{H}), 7.29-7.25$ (m, 2H), $7.19(\mathrm{~d}, J=8.4 \mathrm{~Hz}, 2 \mathrm{H}), 7.15(\mathrm{~d}, J=8.4 \mathrm{~Hz}, 2 \mathrm{H}), 6.94(\mathrm{~d}, J=9.0 \mathrm{~Hz}, 2 \mathrm{H}), 3.84(\mathrm{~s}, 3 \mathrm{H})$, $2.36(\mathrm{~s}, 3 \mathrm{H}), 0.27(\mathrm{~s}, 9 \mathrm{H}) ;{ }^{13} \mathrm{C}-\mathrm{NMR}\left(150 \mathrm{MHz}, \mathrm{CDCl}_{3}\right) \delta 159.6(\mathrm{C}), 146.6(\mathrm{C}), 140.8(\mathrm{C}), 138.6$ (C), $137.6(\mathrm{C}), 129.0(\mathrm{CH}), 128.6(\mathrm{CH}), 128.4(\mathrm{CH}), 128.0(\mathrm{CH}), 127.9(\mathrm{CH}), 127.0(\mathrm{CH}), 123.4$ (C), $119.7(\mathrm{CH}), 114.2(\mathrm{CH}), 104.3(\mathrm{C}), 94.6(\mathrm{C}), 69.8(\mathrm{C}), 55.3\left(\mathrm{CH}_{3}\right), 21.1\left(\mathrm{CH}_{3}\right),-0.35\left(\mathrm{CH}_{3}\right)$; HRMS Calculated for $\mathrm{C}_{28} \mathrm{H}_{30} \mathrm{~N}_{3} \mathrm{OSi}[\mathrm{M}+\mathrm{H}]^{+}:$452.2153, Found: 452.2151 .

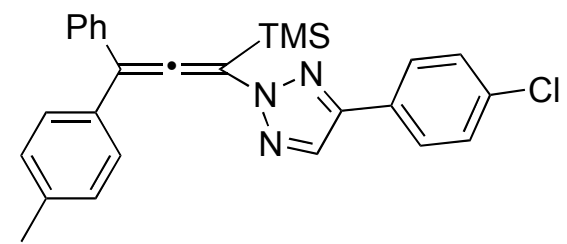

$\mathrm{N}-2-1.5 .40$ 


\section{4-(4-chlorophenyl)-2-(1-(trimethylsilyl)-3-phenyl-3-p-tolylpropa-1,2-dienyl)-2H-1,2,3-}

triazole (N-2-1.5.40) was purified by flash chromatography (Hexane-EtOAc, v/v 20/1) as yellow liquid. Isolated yield 57\%; $\mathrm{R} f$ (hexanes/ethyl acetate, $\mathrm{V} / \mathrm{V}, 10 / 1)=0.57$; UV absorption: 238(0.329), 269(0.254), 301(0.216); IR (neat), 2937, 1623, 1477, 1450, 1276, 1235, 1063, 932, 818, 745, 696; ${ }^{1} \mathrm{H}-\mathrm{NMR}\left(600 \mathrm{MHz}, \mathrm{CDCl}_{3}\right) \delta 7.99$ (s, 1H), 7.78 (d, $\left.J=8.4 \mathrm{~Hz}, 2 \mathrm{H}\right)$, 7.40-7.38 (m, 5H), 7.37-7.35 (m, 2H), $7.28(\mathrm{~d}, J=7.8 \mathrm{~Hz}, 2 \mathrm{H}), 7.21(\mathrm{~d}, J=7.8 \mathrm{~Hz}, 2 \mathrm{H}), 2.39(\mathrm{~s}, 3 \mathrm{H}), 0.43$ (s, 9H); ${ }^{13} \mathrm{C}-\mathrm{NMR}\left(150 \mathrm{MHz}, \mathrm{CDCl}_{3}\right) \delta 201.3(\mathrm{C}), 146.5$ (C), 138.4 (C), 135.3 (C), 134.0 (C), $132.1(\mathrm{C}), 129.5(\mathrm{CH}), 129.0(\mathrm{CH}), 128.9(\mathrm{CH}), 128.8(\mathrm{CH}), 128.4(\mathrm{CH}), 128.3(\mathrm{CH}), 128.2(\mathrm{C})$, $127.0(\mathrm{CH}), 117.5(\mathrm{CH}), 116.2(\mathrm{C}), 109.2(\mathrm{C}), 21.2\left(\mathrm{CH}_{3}\right),-0.62\left(\mathrm{CH}_{3}\right)$; HRMS Calculated for $\mathrm{C}_{27} \mathrm{H}_{27} \mathrm{ClN}_{3} \mathrm{Si}[\mathrm{M}+\mathrm{H}]^{+}:$456.1655, Found: 456.1652 .

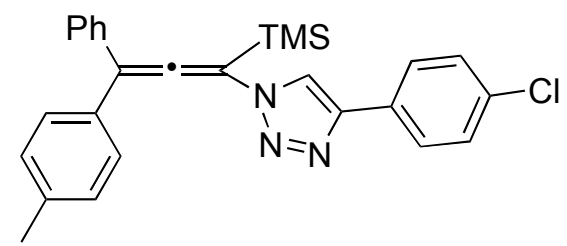

$\mathrm{N}-1-1.5 .40$

\section{4-(4-chlorophenyl)-1-(1-(trimethylsilyl)-3-phenyl-3-p-tolylpropa-1,2-dienyl)-1H-1,2,3-}

triazole (N-1-1.5.4o) was purified by flash chromatography (Hexane-EtOAc, v/v 20/1) as yellow liquid. Isolated yield 15\%; $\mathrm{R} f$ (hexanes/ethyl acetate, $\mathrm{V} / \mathrm{V}, 10 / 1)=0.29$; UV absorption: 242(0.308), 261(0.249), 300(0.233); IR (neat), 2946, 1589, 1480, 1441, 1292, 1231, 1063, 939, 825, 756, 694; ${ }^{1} \mathrm{H}-\mathrm{NMR}\left(600 \mathrm{MHz}, \mathrm{CDCl}_{3}\right) \delta 7.65(\mathrm{~s}, 1 \mathrm{H}), 7.32-7.28(\mathrm{~m}, 3 \mathrm{H}), 7.11(\mathrm{~d}, J=8.4$ $\mathrm{Hz}, 2 \mathrm{H}), 7.07$ (d, $J=8.4 \mathrm{~Hz}, 2 \mathrm{H}), 7.05-7.02(\mathrm{~m}, 2 \mathrm{H}), 6.92(\mathrm{~d}, J=8.4 \mathrm{~Hz}, 2 \mathrm{H}), 6.85(\mathrm{~d}, J=8.4$ $\mathrm{Hz}, 2 \mathrm{H}), 2.38(\mathrm{~s}, 3 \mathrm{H}), 0.34(\mathrm{~s}, 9 \mathrm{H}) ;{ }^{13} \mathrm{C}-\mathrm{NMR}\left(150 \mathrm{MHz}, \mathrm{CDCl}_{3}\right) \delta 204.0(\mathrm{C}), 138.0(\mathrm{C}), 136.6$ (C), $135.0(\mathrm{C}), 134.8(\mathrm{C}), 133.5(\mathrm{C}), 131.6(\mathrm{C}), 129.9(\mathrm{CH}), 129.2(\mathrm{CH}), 128.5(\mathrm{CH}), 128.4$ 
$(\mathrm{CH}), 128.3(\mathrm{CH}), 128.2(\mathrm{CH}), 128.0(\mathrm{CH}), 125.8(\mathrm{CH}), 114.5(\mathrm{C}), 107.2(\mathrm{C}), 21.2\left(\mathrm{CH}_{3}\right),-0.67$

$\left(\mathrm{CH}_{3}\right)$; HRMS Calculated for $\mathrm{C}_{27} \mathrm{H}_{27} \mathrm{ClN}_{3} \mathrm{Si}[\mathrm{M}+\mathrm{H}]^{+}: 456.1655$, Found: 456.1654 .

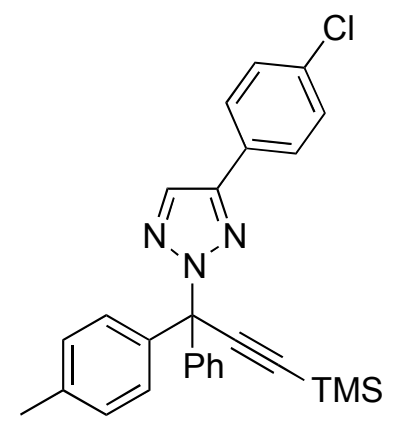

$\mathrm{N}-2-1.5 .40^{\prime}$

\section{4-(4-chlorophenyl)-2-(3-(trimethylsilyl)-1-phenyl-1-p-tolylprop-2-ynyl)-2H-1,2,3-triazole}

(N-2-1.5.40') was purified by flash chromatography (Hexane-EtOAc, v/v 20/1) as yellow liquid. Isolated yield 19\%; $\mathrm{R} f$ (hexanes/ethyl acetate, $\mathrm{V} / \mathrm{V}, 10 / 1)=0.47$; $\mathrm{UV}$ absorption: $250(0.378)$, 265(0.345); IR (neat), 3100, 2947, 1498, 1434, 1367, 1292, 1049, 967, 854, 788, 730, 691; ${ }^{1} \mathrm{H}-$ NMR (600 MHz, $\left.\mathrm{CDCl}_{3}\right) \delta 7.90(\mathrm{~s}, 1 \mathrm{H}), 7.71(\mathrm{~d}, J=8.4 \mathrm{~Hz}, 2 \mathrm{H}), 7.36(\mathrm{~d}, J=8.4 \mathrm{~Hz}, 2 \mathrm{H}), 7.35-$ $7.33(\mathrm{~m}, 3 \mathrm{H}), 7.27-7.24(\mathrm{~m}, 2 \mathrm{H}), 7.18(\mathrm{~d}, J=8.4 \mathrm{~Hz}, 2 \mathrm{H}), 7.15(\mathrm{t}, J=8.4 \mathrm{~Hz}, 2 \mathrm{H}), 2.36(\mathrm{~s}, 3 \mathrm{H})$, 0.27 (s, 9H); ${ }^{13} \mathrm{C}-\mathrm{NMR}\left(150 \mathrm{MHz}, \mathrm{CDCl}_{3}\right) \delta 146.9$ (C), 141.3 (C), 138.3 (C), 138.1 (C), 134.2 (C), $131.2(\mathrm{CH}), 128.9(\mathrm{CH}), 128.8(\mathrm{CH}), 128.3(\mathrm{CH}), 128.2(\mathrm{CH}), 128.1(\mathrm{CH}), 128.0(\mathrm{CH})$, $127.4(\mathrm{CH}), 104.4(\mathrm{C}), 94.2(\mathrm{C}), 73.8(\mathrm{C}), 21.1\left(\mathrm{CH}_{3}\right),-0.28\left(\mathrm{CH}_{3}\right)$; HRMS Calculated for $\mathrm{C}_{27} \mathrm{H}_{27} \mathrm{ClN}_{3} \mathrm{Si}[\mathrm{M}+\mathrm{H}]^{+}:$456.1655, Found: 456.1654 . 


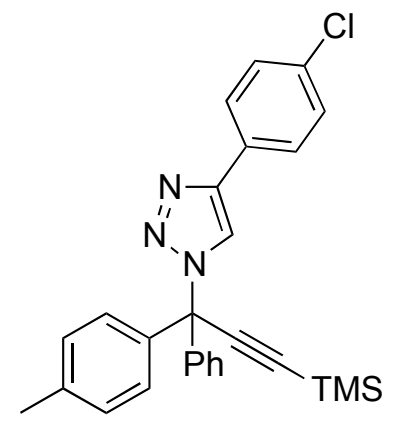

N-1-1.5.4o'

\section{4-(4-chlorophenyl)-1-(3-(trimethylsilyl)-1-phenyl-1-p-tolylprop-2-ynyl)-2H-1,2,3-triazole}

(N-1-1.5.40') was purified by flash chromatography (Hexane-EtOAc, v/v 20/1) as yellow liquid. Isolated yield 5\%; Rf(hexanes/ethyl acetate, $\mathrm{V} / \mathrm{V}, 10 / 1)=0.26 ; \mathrm{UV}$ absorption: 248(0.268), 263(0.247); IR (neat), 3054, 2921, 1495, 1458, 1421, 1150, 1054, 971, 827, 749, 720, 688; ${ }^{1} \mathrm{H}-$ NMR $\left(600 \mathrm{MHz}, \mathrm{CDCl}_{3}\right) \delta 7.95(\mathrm{~s}, 1 \mathrm{H}), 7.75(\mathrm{~d}, J=8.4 \mathrm{~Hz}, 2 \mathrm{H}), 7.38-7.33(\mathrm{~m}, 5 \mathrm{H}), 7.28-7.25$ (m, 2H), $7.18(\mathrm{~d}, J=9.0 \mathrm{~Hz}, 2 \mathrm{H}), 7.15(\mathrm{~d}, J=9.0 \mathrm{~Hz}, 2 \mathrm{H}), 2.35$ (s, 3H), $0.27(\mathrm{~s}, 9 \mathrm{H}) ;{ }^{13} \mathrm{C}-\mathrm{NMR}$ (150 MHz, $\left.\mathrm{CDCl}_{3}\right) \delta 145.9(\mathrm{C}), 140.8(\mathrm{C}), 139.0(\mathrm{C}), 137.7$ (C), $134.0(\mathrm{C}), 129.4(\mathrm{CH}), 129.2$ $(\mathrm{CH}), 128.9(\mathrm{CH}), 128.6(\mathrm{CH}), 128.2(\mathrm{CH}), 128.1(\mathrm{CH}), 127.2(\mathrm{CH}), 120.8(\mathrm{CH}), 104.3(\mathrm{C}), 95.1$ (C), $70.3(\mathrm{C}), 21.3\left(\mathrm{CH}_{3}\right),-0.13\left(\mathrm{CH}_{3}\right)$; HRMS Calculated for $\mathrm{C}_{27} \mathrm{H}_{27} \mathrm{ClN}_{3} \mathrm{Si}[\mathrm{M}+\mathrm{H}]^{+}$: 456.1655 , Found: 456.1655.

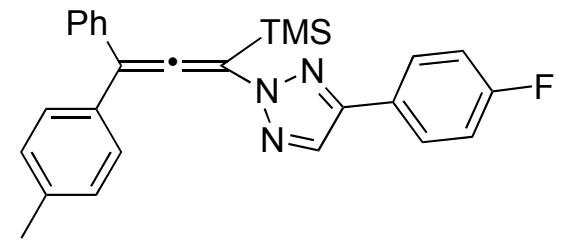

N-2-1.5.4p

\section{4-(4-fluorophenyl)-2-(1-(trimethylsilyl)-3-phenyl-3-p-tolylpropa-1,2-dienyl)-2H-1,2,3-}

triazole (N-2-1.5.4p) was purified by flash chromatography (Hexane-EtOAc, v/v 20/1) as yellow 
liquid. Isolated yield 62\%; $\mathrm{R} f$ (hexanes/ethyl acetate, $\mathrm{V} / \mathrm{V}, 10 / 1)=0.55 ; \mathrm{UV}$ absorption: 257(0.405), 263(0.353), 311(0.252); IR (neat), 2966, 1586, 1493, 1444, 1282, 1234, 1039, 933, 854, 756, 682; ${ }^{1} \mathrm{H}-\mathrm{NMR}\left(600 \mathrm{MHz}, \mathrm{CDCl}_{3}\right) \delta 7.97(\mathrm{~s}, 1 \mathrm{H}), 7.84-7.81(\mathrm{~m}, 2 \mathrm{H}), 7.42-7.40(\mathrm{~m}$, 4H), 7.38-7.35 (m, 1H), 7.30 (d, $J=7.8 \mathrm{~Hz}, 2 \mathrm{H}), 7.22(\mathrm{~d}, J=7.8 \mathrm{~Hz}, 2 \mathrm{H}), 7.11$ (t, $J=7.8 \mathrm{~Hz}$, 2H), 2.40 (s, 3H), 0.44 (s, 9H); ${ }^{13} \mathrm{C}-\mathrm{NMR}\left(150 \mathrm{MHz}, \mathrm{CDCl}_{3}\right) \delta 201.3$ (C), 163.5 (C), $161.9(\mathrm{C})$, $146.6(\mathrm{C}), 138.4(\mathrm{C}), 135.3(\mathrm{C}), 132.1(\mathrm{C}), 129.5(\mathrm{CH}), 128.7(\mathrm{CH}), 128.4(\mathrm{CH}), 128.3(\mathrm{CH})$, $128.2(\mathrm{CH}), 127.5(\mathrm{CH}), 127.4(\mathrm{CH}), 126.7(\mathrm{C}), 126.6(\mathrm{C}), 117.2(\mathrm{CH}), 116.1(\mathrm{C}), 115.8(\mathrm{CH})$, $115.7(\mathrm{CH}), 109.2(\mathrm{C}), 21.2\left(\mathrm{CH}_{3}\right),-0.63\left(\mathrm{CH}_{3}\right)$; HRMS Calculated for $\mathrm{C}_{27} \mathrm{H}_{27} \mathrm{FN}_{3} \mathrm{Si}[\mathrm{M}+\mathrm{H}]^{+}$: 440.1951, Found: 440.1950.

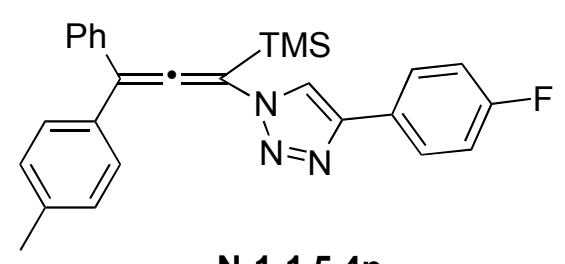

N-1-1.5.4p

\section{4-(4-fluorophenyl)-1-(1-(trimethylsilyl)-3-phenyl-3-p-tolylpropa-1,2-dienyl)-1H-1,2,3-}

triazole (N-1-1.5.4p) was purified by flash chromatography (Hexane-EtOAc, v/v 20/1) as yellow liquid. Isolated yield 14\%; $\mathrm{R} f$ (hexanes/ethyl acetate, $\mathrm{V} / \mathrm{V}, 10 / 1)=0.25 ; \mathrm{UV}$ absorption: 253(0.333), 261(0.279), 313(0.223); IR (neat), 2987, 1618, 1464, 1426, 1289, 1235, 1052, 946, 823, 745, 688; ${ }^{1} \mathrm{H}-\mathrm{NMR}\left(600 \mathrm{MHz}, \mathrm{CDCl}_{3}\right) \delta 7.64(\mathrm{~s}, 1 \mathrm{H}), 7.31-7.28(\mathrm{~m}, 3 \mathrm{H}), 7.14-7.11(\mathrm{~m}$, 2H), $7.10(\mathrm{~d}, J=7.8 \mathrm{~Hz}, 2 \mathrm{H}), 7.06-7.03(\mathrm{~m}, 2 \mathrm{H}), 6.94(\mathrm{~d}, J=7.8 \mathrm{~Hz}, 2 \mathrm{H}), 6.59(\mathrm{t}, J=7.8 \mathrm{~Hz}$, 2H), 2.37 (s, 3H), 0.32 (s, 9H); ${ }^{13} \mathrm{C}-\mathrm{NMR}\left(150 \mathrm{MHz}, \mathrm{CDCl}_{3}\right) \delta 203.8(\mathrm{C}), 163.7$ (C), $163.0(\mathrm{C})$, $162.0(\mathrm{C}), 138.0(\mathrm{C}), 136.8(\mathrm{C}), 134.9(\mathrm{C}), 133.5(\mathrm{CH}), 131.7(\mathrm{CH}), 130.8(\mathrm{C}), 130.7(\mathrm{C}), 129.2$ $(\mathrm{CH}), 128.4(\mathrm{CH}), 128.4(\mathrm{CH}), 128.3(\mathrm{CH}), 128.0(\mathrm{CH}), 123.3(\mathrm{CH}), 115.4(\mathrm{CH}), 115.3(\mathrm{CH})$, 
$114.4(\mathrm{C}), 107.2(\mathrm{C}), 21.2\left(\mathrm{CH}_{3}\right),-0.67\left(\mathrm{CH}_{3}\right)$; HRMS Calculated for $\mathrm{C}_{27} \mathrm{H}_{27} \mathrm{FN}_{3} \mathrm{Si}[\mathrm{M}+\mathrm{H}]^{+}$: 440.1951, Found: 440.1954.

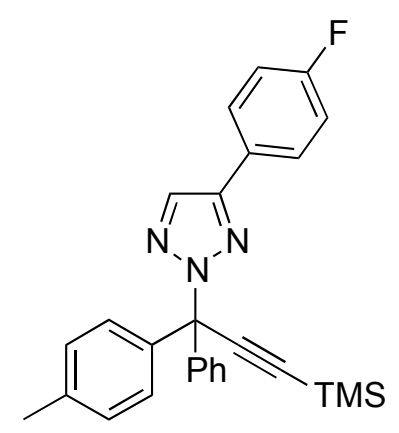

$\mathrm{N}-2-1.5 .4 p^{\prime}$

\section{4-(4-fluorophenyl)-2-(3-(trimethylsilyl)-1-phenyl-1-p-tolylprop-2-ynyl)-2H-1,2,3-triazole}

(N-2-1.5.4p') was purified by flash chromatography (Hexane-EtOAc, v/v 20/1) as yellow liquid. Isolated yield 15\%; $\mathrm{R} f$ (hexanes/ethyl acetate, $\mathrm{V} / \mathrm{V}, 10 / 1)=0.45 ; \mathrm{UV}$ absorption: $271(0.513)$, 285(0.446); IR (neat), 3118, 2937, 1476, 1424, 1375, 1299, 1055, 963, 850, 781, 727, 690; ${ }^{1} \mathrm{H}-$ NMR (600 MHz, $\left.\mathrm{CDCl}_{3}\right) \delta 7.87(\mathrm{~s}, 1 \mathrm{H}), 7.77-7.74(\mathrm{~m}, 2 \mathrm{H}), 7.35-7.32(\mathrm{~m}, 3 \mathrm{H}), 7.28-7.24(\mathrm{~m}$, 2H), $7.19(\mathrm{~d}, J=8.4 \mathrm{~Hz}, 2 \mathrm{H}), 7.15(\mathrm{~d}, J=8.4 \mathrm{~Hz}, 2 \mathrm{H}), 7.08(\mathrm{t}, J=8.4 \mathrm{~Hz}, 2 \mathrm{H}), 2.36(\mathrm{~s}, 3 \mathrm{H})$, $0.27(\mathrm{~s}, 9 \mathrm{H}) ;{ }^{13} \mathrm{C}-\mathrm{NMR}\left(150 \mathrm{MHz}, \mathrm{CDCl}_{3}\right) \delta 163.7$ (C), 162.0 (C), $147.0(\mathrm{C}), 141.4(\mathrm{C}), 138.3$ (C), $138.2(\mathrm{C}), 131.0(\mathrm{CH}), 128.9(\mathrm{CH}), 128.3(\mathrm{CH}), 128.2(\mathrm{CH}), 128.1(\mathrm{CH}), 128.0(\mathrm{CH}), 127.9$ $(\mathrm{CH}), 127.8(\mathrm{CH}), 126.7(\mathrm{C}), 126.6(\mathrm{C}), 115.8(\mathrm{CH}), 115.6(\mathrm{CH}), 104.4(\mathrm{C}), 94.1(\mathrm{C}), 73.7(\mathrm{C})$, $21.1\left(\mathrm{CH}_{3}\right),-0.27\left(\mathrm{CH}_{3}\right)$; HRMS Calculated for $\mathrm{C}_{27} \mathrm{H}_{27} \mathrm{FN}_{3} \mathrm{Si}[\mathrm{M}+\mathrm{H}]^{+}$: 440.1951, Found: 440.1948. 


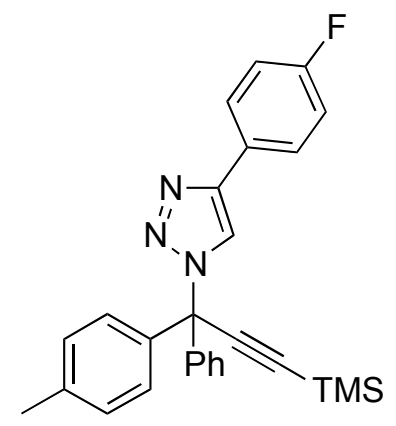

$\mathrm{N}-1-1.5 .4 p^{\prime}$

\section{4-(4-fluorophenyl)-1-(3-(trimethylsilyl)-1-phenyl-1-p-tolylprop-2-ynyl)-2H-1,2,3-triazole}

(N-1-1.5.4p') was purified by flash chromatography (Hexane-EtOAc, v/v 20/1) as yellow liquid. Isolated yield 4\%; $\mathrm{R} f$ (hexanes/ethyl acetate, $\mathrm{V} / \mathrm{V}, 10 / 1)=0.28$; UV absorption: $270(0.436)$, 283(0.389); IR (neat), 3089, 2931, 1488, 1433, 1363, 1281, 1042, 965, 864, 774, 735, 695; ${ }^{1} \mathrm{H}-$ NMR (600 MHz, $\left.\mathrm{CDCl}_{3}\right) \delta 7.94(\mathrm{~s}, 1 \mathrm{H}), 7.81-7.78(\mathrm{~m}, 2 \mathrm{H}), 7.36-7.34(\mathrm{~m}, 3 \mathrm{H}), 7.29-7.27(\mathrm{~m}$, 2H), $7.18(\mathrm{~d}, J=8.4 \mathrm{~Hz}, 2 \mathrm{H}), 7.16(\mathrm{~d}, J=8.4 \mathrm{~Hz}, 2 \mathrm{H}), 7.10(\mathrm{t}, J=8.4 \mathrm{~Hz}, 2 \mathrm{H}), 2.36(\mathrm{~s}, 3 \mathrm{H})$, 0.28 (s, 9H); ${ }^{13} \mathrm{C}-\mathrm{NMR}\left(150 \mathrm{MHz}, \mathrm{CDCl}_{3}\right) \delta 163.5$ (C), 161.8 (C), 145.9 (C), 140.6 (C), 138.7 (C), $137.5(\mathrm{C}), 129.1(\mathrm{CH}), 128.7(\mathrm{CH}), 128.4(\mathrm{CH}), 128.0(\mathrm{CH}), 127.9(\mathrm{CH}), 127.5(\mathrm{CH}), 127.4$ $(\mathrm{CH}), 125.9(\mathrm{C}), 125.8(\mathrm{C}), 120.3(\mathrm{CH}), 115.8(\mathrm{CH}), 115.7(\mathrm{CH}), 104.1(\mathrm{C}), 94.8(\mathrm{C}), 70.0(\mathrm{C})$, $21.0\left(\mathrm{CH}_{3}\right),-0.36\left(\mathrm{CH}_{3}\right)$; HRMS Calculated for $\mathrm{C}_{27} \mathrm{H}_{27} \mathrm{FN}_{3} \mathrm{Si}[\mathrm{M}+\mathrm{H}]^{+}:$440.1951, Found: 440.1953.

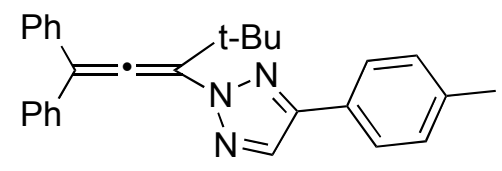

$\mathrm{N}-2-1.5 .4 \mathrm{q}$ 
was purified by flash chromatography (Hexane-EtOAc, v/v 20/1) as yellow liquid. Isolated yield $65 \%$; $\mathrm{R} f$ (hexanes/ethyl acetate, $\mathrm{V} / \mathrm{V}, 10 / 1)=0.60 ; \mathrm{UV}$ absorption: $240(0.214), 258(0.182)$, 294(0.195); IR (neat), 2956, 1588, 1497, 1441, 1264, 1245, 1072, 958, 815, 760, 675; ${ }^{1} \mathrm{H}-\mathrm{NMR}$ $\left(600 \mathrm{MHz}, \mathrm{CDCl}_{3}\right) \delta 7.77(\mathrm{~s}, 1 \mathrm{H}), 7.74(\mathrm{~d}, J=7.8 \mathrm{~Hz}, 2 \mathrm{H}), 7.44-7.39(\mathrm{~m}, 8 \mathrm{H}), 7.38-7.34(\mathrm{~m}$, 2H), $7.22(\mathrm{~d}, J=7.8 \mathrm{~Hz}, 2 \mathrm{H}), 2.37$ (s, 3H), $1.39(\mathrm{~s}, 9 \mathrm{H}) ;{ }^{13} \mathrm{C}-\mathrm{NMR}\left(150 \mathrm{MHz}, \mathrm{CDCl}_{3}\right) \delta 199.4$ (C), $146.8(\mathrm{C}), 138.0(\mathrm{C}), 135.2(\mathrm{C}), 129.4(\mathrm{CH}), 128.7(\mathrm{CH}), 128.5(\mathrm{CH}), 128.4(\mathrm{CH}), 127.6$ (C), $125.6(\mathrm{CH}), 121.0(\mathrm{C}), 120.6(\mathrm{CH}), 117.6(\mathrm{C}), 36.3(\mathrm{C}), 29.1\left(\mathrm{CH}_{3}\right), 21.3\left(\mathrm{CH}_{3}\right)$; HRMS Calculated for $\mathrm{C}_{28} \mathrm{H}_{28} \mathrm{~N}_{3}[\mathrm{M}+\mathrm{H}]^{+}: 406.2280$, Found: 406.2280 .

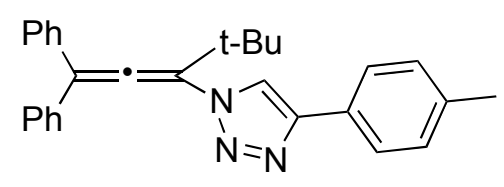

$\mathrm{N}-1-1.5 .4 q$

1-(4,4-dimethyl-1,1-diphenylpenta-1,2-dien-3-yl)-4-p-tolyl-2H-1,2,3-triazole

$(\mathbf{N}-1-1.5 .4 q)$ was purified by flash chromatography (Hexane-EtOAc, v/v 20/1) as yellow liquid. Isolated yield $11 \%$; $\mathrm{R} f$ (hexanes/ethyl acetate, $\mathrm{V} / \mathrm{V}, 10 / 1)=0.28 ; \mathrm{UV}$ absorption: 243(0.253), 259(0.298), 297(0.203); IR (neat), 2934, 1576, 1501, 1433, 1237, 1216, 1066, 939, 811, 773, 683; ${ }^{1} \mathrm{H}-\mathrm{NMR}$ $\left(600 \mathrm{MHz}, \mathrm{CDCl}_{3}\right) \delta 7.69(\mathrm{~s}, 1 \mathrm{H}), 7.40-7.33(\mathrm{~m}, 10 \mathrm{H}), 7.02(\mathrm{~d}, J=7.8 \mathrm{~Hz}, 2 \mathrm{H}), 6.84(\mathrm{~d}, J=7.8$ $\mathrm{Hz}, 2 \mathrm{H}), 2.29$ (s, 3H), 1.11 (s, 9H); ${ }^{13} \mathrm{C}-\mathrm{NMR}\left(150 \mathrm{MHz}, \mathrm{CDCl}_{3}\right) \delta 202.6$ (C), $163.0(\mathrm{C}), 138.9$ (C), $134.7(\mathrm{C}), 132.1(\mathrm{CH}), 129.1(\mathrm{CH}), 128.9(\mathrm{CH}), 128.7(\mathrm{CH}), 128.6(\mathrm{CH}), 128.3(\mathrm{CH}), 124.2$ (C), 119.1 (C), $117.1(\mathrm{C}), 37.1(\mathrm{C}), 29.1\left(\mathrm{CH}_{3}\right), 21.3\left(\mathrm{CH}_{3}\right)$; HRMS Calculated for $\mathrm{C}_{28} \mathrm{H}_{28} \mathrm{~N}_{3}$ $[\mathrm{M}+\mathrm{H}]^{+}:$406.2280, Found: 406.2285 . 


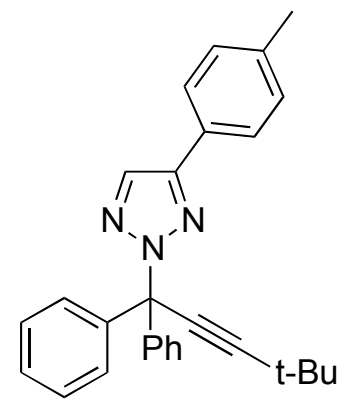

$\mathrm{N}-2-1.5 .4 q^{\prime}$

2-(4,4-dimethyl-1,1-diphenylpent-2-ynyl)-4-p-tolyl-2H-1,2,3-triazole $\quad\left(\mathrm{N}-2-1.5 .4 q^{\prime}\right) \quad$ was purified by flash chromatography (Hexane-EtOAc, v/v 20/1) as yellow liquid. Isolated yield 15\%; $\mathrm{R} f$ (hexanes/ethyl acetate, $\mathrm{V} / \mathrm{V}, 10 / 1)=0.45$; UV absorption: $240(0.267), 253(0.305)$; IR (neat), 3123, 2956, 1500, 1431, 1362, 1280, 1040, 968, 842, 781, 733, 701; ${ }^{1} \mathrm{H}-\mathrm{NMR}(600 \mathrm{MHz}$, $\left.\mathrm{CDCl}_{3}\right) \delta 7.88(\mathrm{~s}, 1 \mathrm{H}), 7.67(\mathrm{~d}, J=7.8 \mathrm{~Hz}, 2 \mathrm{H}), 7.35-7.31(\mathrm{~m}, 6 \mathrm{H}), 7.30-7.27(\mathrm{~m}, 4 \mathrm{H}), 7.20(\mathrm{~d}, J$ $=7.8 \mathrm{~Hz}, 2 \mathrm{H}), 2.37(\mathrm{~s}, 3 \mathrm{H}), 1.35(\mathrm{~s}, 9 \mathrm{H}) ;{ }^{13} \mathrm{C}-\mathrm{NMR}\left(150 \mathrm{MHz}, \mathrm{CDCl}_{3}\right) \delta 147.7(\mathrm{C}), 142.1(\mathrm{C})$, $138.1(\mathrm{C}), 131.0(\mathrm{CH}), 129.4(\mathrm{CH}), 128.1(\mathrm{CH}), 128.1(\mathrm{CH}), 128.0(\mathrm{CH}), 127.7(\mathrm{C}), 126.0(\mathrm{CH})$, 97.7 (C), $79.0(\mathrm{C}), 73.4(\mathrm{C}), 30.6\left(\mathrm{CH}_{3}\right), 27.8(\mathrm{C}), 21.3\left(\mathrm{CH}_{3}\right)$; HRMS Calculated for $\mathrm{C}_{28} \mathrm{H}_{28} \mathrm{~N}_{3}$ $[\mathrm{M}+\mathrm{H}]^{+}:$406.2280, Found: 406.2284 .

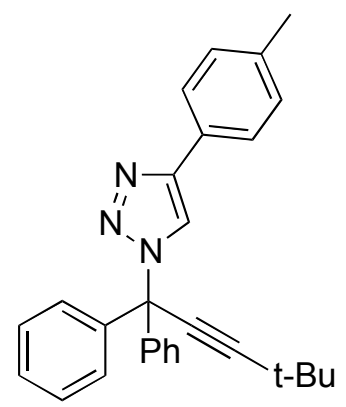

$\mathbf{N}-1-1.5 .4 q^{\prime}$ 
1-(4,4-dimethyl-1,1-diphenylpent-2-ynyl)-4-p-tolyl-2H-1,2,3-triazole $\quad\left(\mathbf{N}-1-1.5 .4 q^{\prime}\right) \quad$ was purified by flash chromatography (Hexane-EtOAc, v/v 20/1) as yellow liquid. Isolated yield 4\%; $\mathrm{R} f$ (hexanes/ethyl acetate, $\mathrm{V} / \mathrm{V}, 10 / 1)=0.25 ; \mathrm{UV}$ absorption: 242(0.253), 254(0.236); IR (neat), 3076, 2927, 1463, 1448, 1354, 1290, 1033, 985, 855, 793, 721, 698; ${ }^{1} \mathrm{H}-\mathrm{NMR}$ (600 MHz, $\left.\mathrm{CDCl}_{3}\right) \delta 7.96(\mathrm{~s}, 1 \mathrm{H}), 7.71(\mathrm{~d}, J=7.8 \mathrm{~Hz}, 2 \mathrm{H}), 7.36-7.33(\mathrm{~m}, 6 \mathrm{H}), 7.31-7.27(\mathrm{~m}, 4 \mathrm{H}), 7.21(\mathrm{~d}, J$ $=7.8 \mathrm{~Hz}, 2 \mathrm{H}), 2.37(\mathrm{~s}, 3 \mathrm{H}), 1.35(\mathrm{~s}, 9 \mathrm{H}) ;{ }^{13} \mathrm{C}-\mathrm{NMR}\left(150 \mathrm{MHz}, \mathrm{CDCl}_{3}\right) \delta 163.0(\mathrm{C}), 146.7(\mathrm{C})$, $141.3(\mathrm{C}), 137.9(\mathrm{C}), 129.4(\mathrm{CH}), 128.5(\mathrm{CH}), 128.3(\mathrm{CH}), 128.0(\mathrm{CH}), 125.6(\mathrm{CH}), 120.2(\mathrm{CH})$, 98.3 (C), 78.9 (C), 69.7 (C), $30.6\left(\mathrm{CH}_{3}\right), 27.9$ (C), $21.3\left(\mathrm{CH}_{3}\right)$; HRMS Calculated for $\mathrm{C}_{28} \mathrm{H}_{28} \mathrm{~N}_{3}$ $[\mathrm{M}+\mathrm{H}]^{+}:$406.2280, Found: 406.2279 .

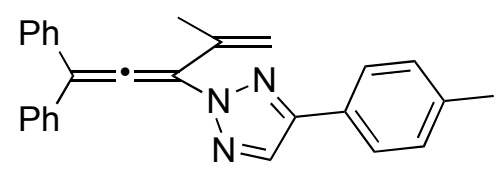

N-2-1.5.4r

2-(4-methyl-1,1-diphenylpenta-1,2,4-trien-3-yl)-4-p-tolyl-2H-1,2,3-triazole (N-2-1.5.4r) was purified by flash chromatography (Hexane-EtOAc, v/v 20/1) as yellow liquid. Isolated yield 70\%; Rf (hexanes/ethyl acetate, V/V, 10/1) = 0.64; UV absorption: 231(0.405), 263(0.353), 311(0.252); IR (neat), 3033, 1586, 1495, 1438, 1266, 1153, 1071, 992, 903, 734, 694; ${ }^{1} \mathrm{H}-\mathrm{NMR}$ $\left(600 \mathrm{MHz}, \mathrm{CDCl}_{3}\right) \delta 7.86(\mathrm{~s}, 1 \mathrm{H}), 7.74(\mathrm{~d}, J=8.4 \mathrm{~Hz}, 2 \mathrm{H}), 7.47-7.45(\mathrm{~m}, 4 \mathrm{H}), 7.43-7.35(\mathrm{~m}$, $6 \mathrm{H}), 7.23(\mathrm{~d}, J=8.4 \mathrm{~Hz}, 2 \mathrm{H}), 5.21(\mathrm{~s}, 1 \mathrm{H}), 4.88(\mathrm{~s}, 1 \mathrm{H}), 2.38(\mathrm{~s}, 3 \mathrm{H}), 2.09(\mathrm{~s}, 3 \mathrm{H}) ;{ }^{13} \mathrm{C}-\mathrm{NMR}$ $\left(150 \mathrm{MHz}, \mathrm{CDCl}_{3}\right) \delta 203.5(\mathrm{C}), 147.3(\mathrm{C}), 138.1(\mathrm{C}), 136.2$ (C), $134.6(\mathrm{C}), 129.5(\mathrm{CH}), 128.9$ $(\mathrm{CH}), 128.7(\mathrm{CH}), 128.7(\mathrm{CH}), 127.5(\mathrm{C}), 125.7(\mathrm{CH}), 120.8(\mathrm{CH}), 119.0(\mathrm{C}), 115.4\left(\mathrm{CH}_{2}\right)$, 
$114.8(\mathrm{C}), 21.3\left(\mathrm{CH}_{3}\right), 20.7\left(\mathrm{CH}_{3}\right)$; HRMS Calculated for $\mathrm{C}_{27} \mathrm{H}_{24} \mathrm{~N}_{3}[\mathrm{M}+\mathrm{H}]^{+}: 390.1960$, Found: 390.1962.

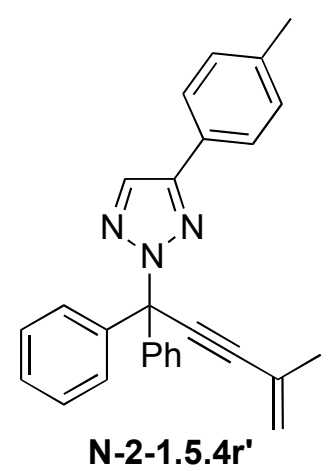

2-(4-methyl-1,1-diphenylpent-4-en-2-ynyl)-4-p-tolyl-2H-1,2,3-triazole $\quad\left(\mathrm{N}-2-1.5 .4 r^{\prime}\right) \quad$ was purified by flash chromatography (Hexane-EtOAc, v/v 20/1) as yellow liquid. Isolated yield $10 \%$; R $f$ (hexanes/ethyl acetate, $\mathrm{V} / \mathrm{V}, 10 / 1)=0.50 ; \mathrm{UV}$ absorption: 248(0.231), 259(0.210); IR (neat), 3110, 2930, 1471, 1423, 1373, 1297, 1053, 960, 838, 779, 718, 679; ${ }^{1} \mathrm{H}-\mathrm{NMR}$ (600 MHz, $\left.\mathrm{CDCl}_{3}\right) \delta 7.91(\mathrm{~s}, 1 \mathrm{H}), 7.67(\mathrm{~d}, J=7.8 \mathrm{~Hz}, 2 \mathrm{H}), 7.36-7.33(\mathrm{~m}, 6 \mathrm{H}), 7.31-7.28(\mathrm{~m}, 4 \mathrm{H}), 7.20(\mathrm{~d}, J$ $=7.8 \mathrm{~Hz}, 2 \mathrm{H}), 5.48(\mathrm{~s}, 1 \mathrm{H}), 5.35$ (quin, $J=1.8 \mathrm{~Hz}, 1 \mathrm{H}), 2.37(\mathrm{~s}, 3 \mathrm{H}), 2.01(\mathrm{~s}, 3 \mathrm{H}) ;{ }^{13} \mathrm{C}-\mathrm{NMR}$ $\left(150 \mathrm{MHz}, \mathrm{CDCl}_{3}\right) \delta 148.0(\mathrm{C}), 141.5(\mathrm{C}), 138.4(\mathrm{C}), 131.2(\mathrm{CH}), 129.4(\mathrm{CH}), 128.3(\mathrm{CH})$, $128.2(\mathrm{CH}), 128.1(\mathrm{CH}), 127.5(\mathrm{C}), 126.1(\mathrm{C}), 126.0(\mathrm{CH}), 123.1\left(\mathrm{CH}_{2}\right), 90.2(\mathrm{C}), 88.0(\mathrm{C}), 73.8$ (C), $23.2\left(\mathrm{CH}_{3}\right), 21.3\left(\mathrm{CH}_{3}\right)$; HRMS Calculated for $\mathrm{C}_{27} \mathrm{H}_{24} \mathrm{~N}_{3}[\mathrm{M}+\mathrm{H}]^{+}: 390.1960$, Found: 390.1962. 


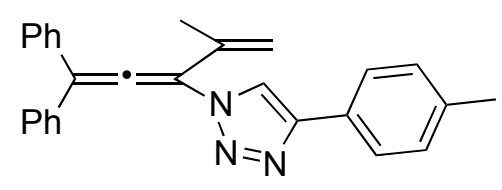

$\mathrm{N}-1-1.5 .4 r$

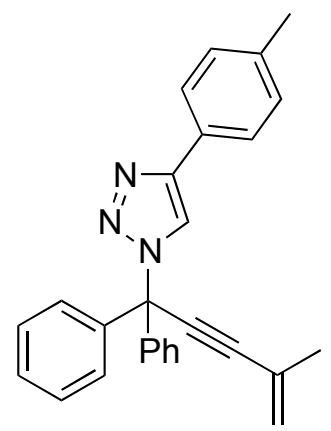

$\mathbf{N}-1-1.5 .4 r^{\prime}$

Mixture of 1-(4-methyl-1,1-diphenylpenta-1,2,4-trien-3-yl)-4-p-tolyl-2H-1,2,3-triazole (N-21.5.4r) and 1-(4-methyl-1,1-diphenylpent-4-en-2-ynyl)-4-p-tolyl-2H-1,2,3-triazole (N-11.5.4r') couldn't separated by flash chromatography with different developing solvent systems which were Hexane-EtOAc (v/v: 20/1) and Hexane-ethylene chloride (v/v: 5/1) as yellow liquid. $\mathrm{R} f($ hexanes/ethyl acetate, $\mathrm{V} / \mathrm{V}, 10 / 1)=0.29$; Isolated yield 11\%; UV absorption: 233(0.333), 245(0.200), 261(0.257), 313(0.163); IR (neat), 3066, 2935, 1589, 1477, 1448, 1350, 1284, 1165, 1049, 997, 913, 857 732, 688; ${ }^{1} \mathrm{H}-\mathrm{NMR}\left(600 \mathrm{MHz}, \mathrm{CDCl}_{3}\right) \delta 7.88(\mathrm{~s}, 1.00 \mathrm{H})$, 7.57-7.54 (m, 3.47H), 7.38-7.34 (m, 3.88H), 7.33-7.28 (m, 7.77H), 7.25-7.23 (m, 4.18H), 7.19 (d, J=7.8 Hz, 2.20H), $7.01(\mathrm{~d}, J=7.8 \mathrm{~Hz}, 2.10 \mathrm{H}), 2.41(\mathrm{~s}, 2.62 \mathrm{H}), 2.34(\mathrm{~s}, 3.00 \mathrm{H}), 2.01(\mathrm{~s}, 3.03 \mathrm{H}) ;{ }^{13} \mathrm{C}-\mathrm{NMR}$ (150 MHz, $\left.\mathrm{CDCl}_{3}\right) \delta 205.3(\mathrm{C}), 163.0(\mathrm{C}), 143.2(\mathrm{C}), 139.2(\mathrm{C}), 138.8(\mathrm{C}), 137.0(\mathrm{C}), 134.0(\mathrm{C})$, $131.7(\mathrm{C}), 129.4(\mathrm{CH}), 128.9(\mathrm{CH}), 128.6(\mathrm{CH}), 128.5(\mathrm{CH}), 128.5(\mathrm{CH}), 128.3(\mathrm{CH}), 127.9$ $(\mathrm{CH}), 126.0(\mathrm{CH}), 123.7\left(\mathrm{CH}_{2}\right), 118.8(\mathrm{C}), 115.0\left(\mathrm{CH}_{2}\right), 113.9(\mathrm{C}), 92.4(\mathrm{C}), 85.8(\mathrm{C}), 74.5(\mathrm{C})$, 32.7 $\left(\mathrm{CH}_{3}\right), 21.3\left(\mathrm{CH}_{3}\right), 20.2\left(\mathrm{CH}_{3}\right)$; HRMS Calculated for $\mathrm{C}_{27} \mathrm{H}_{24} \mathrm{~N}_{3}[\mathrm{M}+\mathrm{H}]^{+}:$390.1960, Found: 390.1962. 


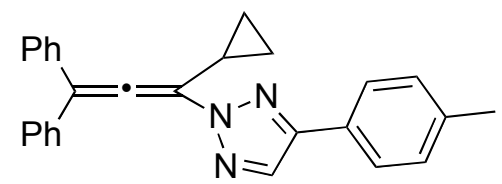

$\mathrm{N}-2-1.5 .4 \mathrm{~s}$

2-(1-cyclopropyl-3,3-diphenylpropa-1,2-dienyl)-4-p-tolyl-2H-1,2,3-triazole (N-2-1.5.4s) was purified by flash chromatography (Hexane-EtOAc, v/v 20/1) as yellow liquid. Isolated yield 87\%; Rf (hexanes/ethyl acetate, V/V, 10/1) = 0.61; UV absorption: 226(0.242), 257(0.166), 299(0.129); IR (neat), 2962, 1583, 1480, 1423, 1262, 1151, 1067, 956, 862, 742, 690; ${ }^{1} \mathrm{H}-\mathrm{NMR}$ $\left(600 \mathrm{MHz}, \mathrm{CDCl}_{3}\right) \delta 7.97(\mathrm{~s}, 1 \mathrm{H}), 7.74(\mathrm{~d}, J=7.8 \mathrm{~Hz}, 2 \mathrm{H}), 7.42-7.36(\mathrm{~m}, 10 \mathrm{H}), 7.23(\mathrm{~d}, J=7.8$ $\mathrm{Hz}, 2 \mathrm{H}), 2.38$ (s, 3H), 2.29-2.24 (m, 1H), 1.11-1.07 (m, 2H), 0.83-0.79 (m, 2H); ${ }^{13} \mathrm{C}-\mathrm{NMR}(150$ $\left.\mathrm{MHz}, \mathrm{CDCl}_{3}\right) \delta 196.7(\mathrm{C}), 147.8(\mathrm{C}), 138.1(\mathrm{C}), 135.4(\mathrm{C}), 129.5(\mathrm{CH}), 128.7(\mathrm{CH}), 128.6(\mathrm{CH})$, 128.6 (CH), $127.6(\mathrm{C}), 125.7(\mathrm{CH}), 121.2(\mathrm{C}), 117.4(\mathrm{CH}), 116.6(\mathrm{C}), 21.3\left(\mathrm{CH}_{3}\right), 10.8(\mathrm{CH}), 7.5$ $\left(\mathrm{CH}_{2}\right)$; HRMS Calculated for $\mathrm{C}_{27} \mathrm{H}_{24} \mathrm{~N}_{3}[\mathrm{M}+\mathrm{H}]^{+}: 390.1960$, Found: 390.1963 .

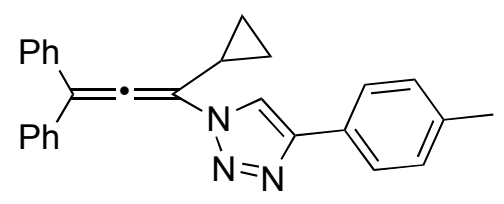

$\mathrm{N}-1-1.5 .4 \mathrm{~s}$

1-(1-cyclopropyl-3,3-diphenylpropa-1,2-dienyl)-4-p-tolyl-1H-1,2,3-triazole (N-1-1.5.4s) was purified by flash chromatography (Hexane-EtOAc, v/v 20/1) as yellow liquid. Isolated yield 9\%; $\mathrm{R} f$ (hexanes/ethyl acetate, $\mathrm{V} / \mathrm{V}, 10 / 1)=0.30$; UV absorption: 229(0.199), 258(0.113), 298(0.098); IR (neat), 3001, 1597, 1474, 1444, 1273, 1175, 1073, 976, 849, 737, 694; ${ }^{1} \mathrm{H}-\mathrm{NMR}$ $\left(600 \mathrm{MHz}, \mathrm{CDCl}_{3}\right) \delta 7.69(\mathrm{~s}, 1 \mathrm{H}), 7.32-7.27(\mathrm{~m}, 6 \mathrm{H}), 7.17-7.13(\mathrm{~m}, 6 \mathrm{H}), 6.88(\mathrm{~d}, J=7.8 \mathrm{~Hz}$ 
2H), $2.25(\mathrm{~s}, 3 \mathrm{H}), 1.94-1.88(\mathrm{~m}, 1 \mathrm{H}), 0.91-0.86(\mathrm{~m}, 2 \mathrm{H}), 0.69-0.65(\mathrm{~m}, 2 \mathrm{H}) ;{ }^{13} \mathrm{C}-\mathrm{NMR}(150$ $\left.\mathrm{MHz}, \mathrm{CDCl}_{3}\right) \delta 200.6(\mathrm{C}), 138.9(\mathrm{C}), 137.8(\mathrm{C}), 134.8(\mathrm{C}), 132.8(\mathrm{CH}), 129.2(\mathrm{CH}), 128.8(\mathrm{CH})$, $128.4(\mathrm{CH}), 128.3(\mathrm{CH}), 128.2(\mathrm{CH}), 123.9(\mathrm{C}), 119.1(\mathrm{C}), 114.1(\mathrm{C}), 21.3\left(\mathrm{CH}_{3}\right), 13.3(\mathrm{CH}), 7.3$ $\left(\mathrm{CH}_{2}\right)$; HRMS Calculated for $\mathrm{C}_{27} \mathrm{H}_{24} \mathrm{~N}_{3}[\mathrm{M}+\mathrm{H}]^{+}: 390.1960$, Found: 390.1959 .

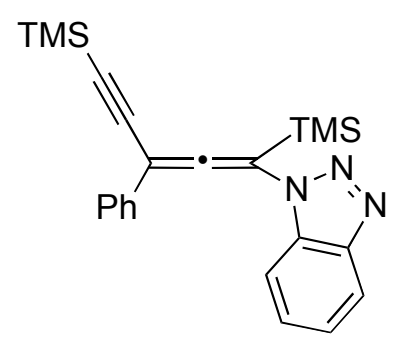

$1.5 .4 \mathrm{t}$

\section{1-(1,5-bis(trimethylsilyl)-3-phenylpenta-1,2-dien-4-ynyl)-1 $H$-benzo $[d][1,2,3]$ triazole $(1.5 .4 t)$}

was purified by flash chromatography (Hexane-EtOAc, v/v 20/1) as yellow liquid. Isolated yield 76\%; Rf (hexanes/ethyl acetate, V/V, 10/1) = 0.46; UV absorption: 234(0.093), 250(0.077), 296(0.054); IR (neat), 3057, 2950, 1587, 1476, 1418, 1256, 1160, 1079, 987, 813, 710, 685; ${ }^{1} \mathrm{H}-$ NMR $\left(600 \mathrm{MHz}, \mathrm{CDCl}_{3}\right) \delta 8.07(\mathrm{~d}, J=8.4 \mathrm{~Hz}, 1 \mathrm{H}), 7.79(\mathrm{~d}, J=8.4 \mathrm{~Hz}, 1 \mathrm{H}), 7.64(\mathrm{~d}, J=8.4$ $\mathrm{Hz}, 2 \mathrm{H}), 7.44$ (t, $J=8.4 \mathrm{~Hz}, 1 \mathrm{H}), 7.39$ (t, $J=8.4 \mathrm{~Hz}, 3 \mathrm{H}), 7.32$ (t, $J=8.4 \mathrm{~Hz}, 1 \mathrm{H}), 0.48(\mathrm{~s}, 9 \mathrm{H})$, 0.29 (s, 9H); ${ }^{13} \mathrm{C}-\mathrm{NMR}\left(150 \mathrm{MHz}, \mathrm{CDCl}_{3}\right) \delta 205.8$ (C), 146.0 (C), 132.8 (C), $132.4(\mathrm{C}), 128.8$ $(\mathrm{CH}), 128.4(\mathrm{CH}), 128.2(\mathrm{CH}), 126.3(\mathrm{CH}), 124.6(\mathrm{CH}), 119.9(\mathrm{CH}), 111.8(\mathrm{CH}), 110.2(\mathrm{C})$, 102.1 (C), 99.1 (C), 96.9 (C), -0.10 $\left(\mathrm{CH}_{3}\right),-0.63\left(\mathrm{CH}_{3}\right)$; HRMS Calculated for $\mathrm{C}_{23} \mathrm{H}_{28} \mathrm{~N}_{3} \mathrm{Si}_{2}$ $[\mathrm{M}+\mathrm{H}]^{+}:$402.1820, Found: 402.1819 . 


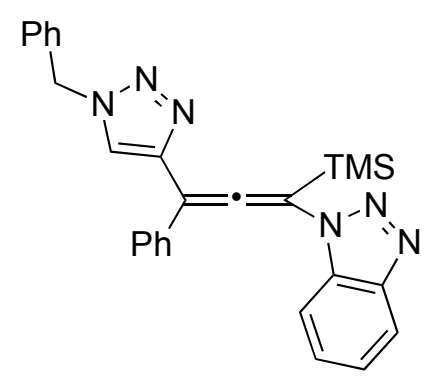

$1.5 .4 \mathrm{u}$

\section{1-(3-(1-benzyl-1H-1,2,3-triazol-4-yl)-1-(trimethylsilyl)-3-phenylpropa-1,2-dienyl)-1H-}

benzo $[d][1,2,3]$ triazole $(1.5 .4 u)$ was purified by flash chromatography (Hexane-EtOAc, v/v 20/1) as white solid. R $f$ (hexanes/ethyl acetate, V/V, 2/1) $=0.45$; UV absorption: 233(0.081), 253(0.06), 295(0.071); IR (neat), 3150, 3030, 2951, 1448, 1286, 1241, 1108, 1048, 938, 835, 766, 697; Isolated yield 58\%; m. p. $155-157{ }^{\circ} \mathrm{C} ;{ }^{1} \mathrm{H}-\mathrm{NMR}\left(600 \mathrm{MHz}, \mathrm{CDCl}_{3}\right) \delta 8.05(\mathrm{~d}, J=7.2$ $\mathrm{Hz}, 1 \mathrm{H}), 7.87$ (d, J = 7.2 Hz, 1H), 7.59-7.56 (m, 2H), $7.48(\mathrm{~s}, 1 \mathrm{H}), 7.41-7.29(\mathrm{~m}, 10 \mathrm{H}), 5.57$ (s, 2H), $0.44(\mathrm{~s}, 9 \mathrm{H}) ;{ }^{13} \mathrm{C}-\mathrm{NMR}\left(150 \mathrm{MHz}, \mathrm{CDCl}_{3}\right) \delta 200.9(\mathrm{C}), 146.1$ (C), 143.6 (C), 134.4 (C), $134.3(\mathrm{C}), 132.6(\mathrm{C}), 129.2(\mathrm{CH}), 128.9(\mathrm{CH}), 128.4(\mathrm{CH}), 128.1(\mathrm{CH}), 128.0(\mathrm{CH}), 127.8(\mathrm{CH})$, 124.4 (CH), $122.4(\mathrm{CH}), 119.9(\mathrm{CH}), 112.1(\mathrm{CH}), 109.4(\mathrm{C}), 106.0(\mathrm{C}), 54.4\left(\mathrm{CH}_{2}\right),-0.55\left(\mathrm{CH}_{3}\right)$; HRMS Calculated for $\mathrm{C}_{27} \mathrm{H}_{27} \mathrm{~N}_{6} \mathrm{Si}[\mathrm{M}+\mathrm{H}]^{+}:$463.2056, Found: 46.04059.

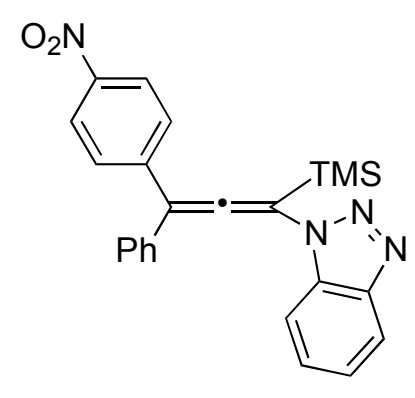

1.5.4v 


\section{1-(1-(trimethylsilyl)-3-(4-nitrophenyl)-3-phenylpropa-1,2-dienyl)-1H-}

benzo $[\boldsymbol{d}][1,2,3]$ triazole $(\mathbf{1 . 5 . 4 v})$ was purified by flash chromatography (Hexane-EtOAc, v/v 20/1) as yellow liquid. Isolated yield 71\%; $\mathrm{R} f$ (hexanes/ethyl acetate, $\mathrm{V} / \mathrm{V}, 6 / 1)=0.48$; UV absorption: 231(0.078), 256(0.059), 299(0.041); IR (neat), 2967, 1634, 1523, 1438, 1299, 1235 , 1157, 1045, 933, 847, 722; ${ }^{1} \mathrm{H}-\mathrm{NMR}\left(600 \mathrm{MHz}, \mathrm{CDCl}_{3}\right) \delta 8.24(\mathrm{~d}, J=8.4 \mathrm{~Hz}, 2 \mathrm{H}), 8.10(\mathrm{~d}, J=$ $7.2 \mathrm{~Hz}, 1 \mathrm{H}), 7.70(\mathrm{~d}, J=7.2 \mathrm{~Hz}, 1 \mathrm{H}), 7.61(\mathrm{~d}, J=8.4 \mathrm{~Hz}, 2 \mathrm{H}), 7.46-7.38(\mathrm{~m}, 7 \mathrm{H}), 0.48(\mathrm{~s}, 9 \mathrm{H})$;

${ }^{13} \mathrm{C}-\mathrm{NMR}\left(150 \mathrm{MHz}, \mathrm{CDCl}_{3}\right) \delta 200.9$ (C), 147.4 (C), 146.3 (C), 143.0 (C), 134.6 (C), 132.4 (C), $129.2(\mathrm{CH}), 129.0(\mathrm{CH}), 128.7(\mathrm{CH}), 128.3(\mathrm{CH}), 128.2(\mathrm{CH}), 124.6(\mathrm{CH}), 124.1(\mathrm{CH}), 120.3$ $(\mathrm{CH}), 114.0(\mathrm{C}), 111.4(\mathrm{CH}), 109.7(\mathrm{C}),-0.45\left(\mathrm{CH}_{3}\right)$; HRMS Calculated for $\mathrm{C}_{24} \mathrm{H}_{23} \mathrm{~N}_{4} \mathrm{O}_{2} \mathrm{Si}$ $[\mathrm{M}+\mathrm{H}]^{+}:$427.1593, Found: 427.1590.

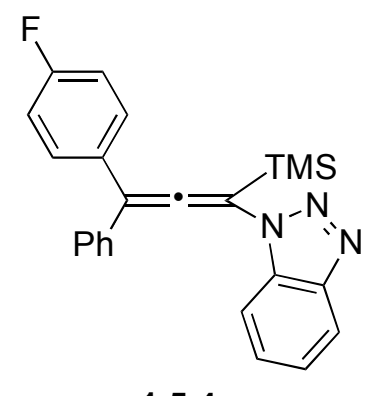

$1.5 .4 \mathrm{w}$

\section{1-(3-(4-fluorophenyl)-1-(trimethylsilyl)-3-phenylpropa-1,2-dienyl)-1H-}

benzo $[d][1,2,3]$ triazole $(1.5 .4 w)$ was purified by flash chromatography (Hexane-EtOAc, v/v 20/1) as white solid. Isolated yield $88 \%$; m. p. $114-116^{\circ} \mathrm{C}$; $\mathrm{R} f$ (hexanes/ethyl acetate, V/V, 10/1) $=0.46$; UV absorption: 234(0.203), 262(0.160), 300(0.141); IR (neat), 2955, 1603, 1504, 1447, 1285, 1248, 1155, 1066, 936, 833, 744, 692; ${ }^{1} \mathrm{H}-\mathrm{NMR}\left(600 \mathrm{MHz}, \mathrm{CDCl}_{3}\right) \delta 8.08(\mathrm{~d}, J=7.2 \mathrm{~Hz}$, 1H), $7.77(\mathrm{~d}, J=7.2 \mathrm{~Hz}, 1 \mathrm{H}), 7.46-7.34(\mathrm{~m}, 9 \mathrm{H}), 7.12-7.06(\mathrm{~m}, 2 \mathrm{H}), 0.47(\mathrm{~s}, 9 \mathrm{H}) ;{ }^{13} \mathrm{C}-\mathrm{NMR}(150$ 
$\left.\mathrm{MHz}, \mathrm{CDCl}_{3}\right) \delta 200.2(\mathrm{C}), 163.4(\mathrm{C}), 161.7$ (C), $146.2(\mathrm{C}), 135.7$ (C), 132.4 (C), $131.82(\mathrm{C})$, $131.80(\mathrm{C}), 130.1(\mathrm{CH}), 130.0(\mathrm{CH}), 128.8(\mathrm{CH}), 128.3(\mathrm{CH}), 128.2(\mathrm{CH}), 128.0(\mathrm{CH}), 124.4$ $(\mathrm{CH}), 120.0(\mathrm{CH}), 115.9(\mathrm{CH}), 115.7(\mathrm{CH}), 114.5(\mathrm{C}), 111.8(\mathrm{C}), 109.0(\mathrm{CH}),-0.49\left(\mathrm{CH}_{3}\right)$; HRMS Calculated for $\mathrm{C}_{24} \mathrm{H}_{23} \mathrm{FN}_{3} \mathrm{Si}[\mathrm{M}+\mathrm{H}]^{+}: 400.1655$, Found: 400.1658 .

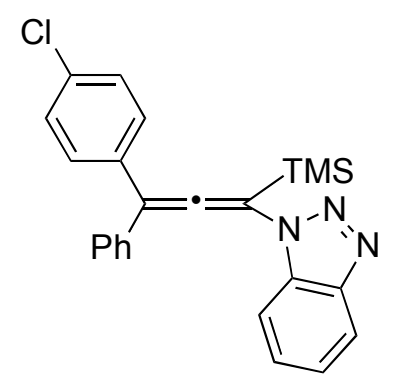

$1.5 .4 x$

\section{1-(3-(4-chlorophenyl)-1-(trimethylsilyl)-3-phenylpropa-1,2-dienyl)-1H-}

benzo $[d][1,2,3]$ triazole $(1.5 .4 x)$ was purified by flash chromatography (Hexane-EtOAc, $v / v$ 20/1) as white solid. Isolated yield $72 \%$; m. p. $139-141{ }^{\circ} \mathrm{C} ; \mathrm{R} f$ (hexanes/ethyl acetate, V/V, 10/1) $=0.47$; UV absorption: 238(0.229), 264(0.167), 299(0.156); IR (neat), 2957, 1485, 1448, 1285, 1248, 1156, 1069, 937, 876, 831, 743, 692; ${ }^{1} \mathrm{H}-\mathrm{NMR}\left(600 \mathrm{MHz}, \mathrm{CDCl}_{3}\right) \delta 8.09$ (d, $J=7.8 \mathrm{~Hz}$, $1 \mathrm{H}), 7.76(\mathrm{~d}, J=7.8 \mathrm{~Hz}, 1 \mathrm{H}), 7.46-7.35(\mathrm{~m}, 11 \mathrm{H}), 0.49(\mathrm{~s}, 9 \mathrm{H}) ;{ }^{13} \mathrm{C}-\mathrm{NMR}\left(150 \mathrm{MHz}, \mathrm{CDCl}_{3}\right) \delta$ 200.2 (C), $146.2(\mathrm{C}), 135.4$ (C), $134.4(\mathrm{C}), 134.0(\mathrm{C}), 132.4(\mathrm{C}), 129.6(\mathrm{CH}), 129.0(\mathrm{CH}), 128.9$ $(\mathrm{CH}), 128.3(\mathrm{CH}), 128.2(\mathrm{CH}), 128.0(\mathrm{CH}), 124.4(\mathrm{CH}), 120.0(\mathrm{CH}), 114.4(\mathrm{C}), 111.7(\mathrm{CH})$, 109.1 (C), $-0.49\left(\mathrm{CH}_{3}\right)$; HRMS Calculated for $\mathrm{C}_{24} \mathrm{H}_{23} \mathrm{ClN}_{3} \mathrm{Si}[\mathrm{M}+\mathrm{H}]^{+}$: 416.1344, Found: 416.1346. 


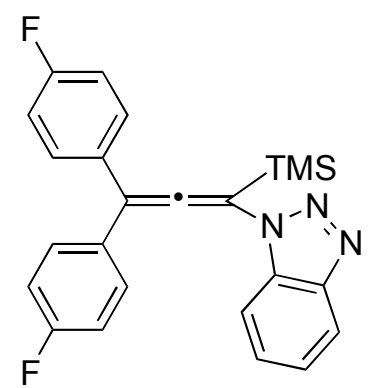

1.5.4y

\section{1-(3,3-bis(4-fluorophenyl)-1-(trimethylsilyl)propa-1,2-dienyl)-1H-benzo[d] $[1,2,3]$ triazole}

(1.5.4y) was purified by flash chromatography (Hexane-EtOAc, v/v 20/1) as white solid. Isolated yield $84 \%$; m. p. $118-120{ }^{\circ} \mathrm{C}$; $\mathrm{R} f$ (hexanes/ethyl acetate, $\mathrm{V} / \mathrm{V}, 10 / 1$ ) $=0.43$; UV absorption: 235(0.175), 261(0.151), 299(0.127); IR (neat), 2964, 1602, 1504, 1450, 1282, 1223, 1157, 1069, 937, 836, 752; ${ }^{1} \mathrm{H}-\mathrm{NMR}\left(600 \mathrm{MHz}, \mathrm{CDCl}_{3}\right) \delta 8.08(\mathrm{~d}, J=7.8 \mathrm{~Hz}, 1 \mathrm{H}), 7.75(\mathrm{~d}, J=7.8 \mathrm{~Hz}, 1 \mathrm{H})$, 7.45-7.40 (m, 5H), $7.38(\mathrm{t}, J=7.8 \mathrm{~Hz}, 1 \mathrm{H}), 7.13-7.08(\mathrm{~m}, 4 \mathrm{H}), 0.48(\mathrm{~s}, 9 \mathrm{H}) ;{ }^{13} \mathrm{C}-\mathrm{NMR}(150$ $\left.\mathrm{MHz}, \mathrm{CDCl}_{3}\right) \delta 200.0(\mathrm{C}), 163.4(\mathrm{C}), 161.7$ (C), 146.2 (C), 132.4 (C), 131.7 (C), 131.6 (C), $130.0(\mathrm{CH}), 129.9(\mathrm{CH}), 128.0(\mathrm{CH}), 124.4(\mathrm{CH}), 120.1(\mathrm{CH}), 116.0(\mathrm{CH}), 115.8(\mathrm{CH}), 113.6$ (C), $111.6(\mathrm{CH}), 109.1(\mathrm{C}),-0.53\left(\mathrm{CH}_{3}\right)$; HRMS Calculated for $\mathrm{C}_{24} \mathrm{H}_{22} \mathrm{~F}_{2} \mathrm{~N}_{3} \mathrm{Si}[\mathrm{M}+\mathrm{H}]^{+}: 418.1546$, Found: 418.1550 .

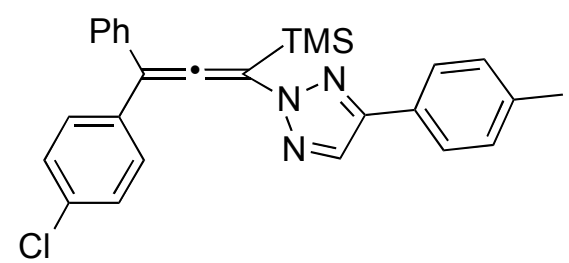

N-2-1.5.4z

\section{2-(3-(4-chlorophenyl)-1-(trimethylsilyl)-3-phenylpropa-1,2-dienyl)-4-p-tolyl-2H-1,2,3-}

triazole (N-2-1.5.4z) was purified by flash chromatography (Hexane-EtOAc, v/v 20/1) as yellow 
liquid. Isolated yield 59\%; $\mathrm{R} f$ (hexanes/ethyl acetate, $\mathrm{V} / \mathrm{V}, 10 / 1)=0.60 ; \mathrm{UV}$ absorption: 243(0.318), 270(0.267), 295(0.116); IR (neat), 2917, 1613, 1457, 1443, 1278, 1233, 1073, 929, 816, 738, 699; ${ }^{1} \mathrm{H}-\mathrm{NMR}\left(600 \mathrm{MHz}, \mathrm{CDCl}_{3}\right) \delta 7.94(\mathrm{~s}, 1 \mathrm{H}), 7.74(\mathrm{~d}, J=8.4 \mathrm{~Hz}, 2 \mathrm{H}), 7.41(\mathrm{~d}, J=$ $7.8 \mathrm{~Hz}, 2 \mathrm{H}), 7.39-7.36(\mathrm{~m}, 5 \mathrm{H}), 7.34(\mathrm{~d}, J=7.8 \mathrm{~Hz}, 2 \mathrm{H}), 7.22(\mathrm{~d}, J=8.4 \mathrm{~Hz}, 2 \mathrm{H}), 2.16(\mathrm{~s}, 3 \mathrm{H})$, 0.43 (s, 9H); ${ }^{13} \mathrm{C}-\mathrm{NMR}\left(150 \mathrm{MHz}, \mathrm{CDCl}_{3}\right) \delta 201.2(\mathrm{C}), 147.7$ (C), 138.2 (C), 134.8 (C), 134.3 (C), $133.8(\mathrm{C}), 129.6(\mathrm{CH}), 129.5(\mathrm{CH}), 129.0(\mathrm{CH}), 128.9(\mathrm{CH}), 128.5(\mathrm{CH}), 128.3(\mathrm{CH}), 127.4$ $(\mathrm{CH}), 125.7(\mathrm{CH}), 117.0(\mathrm{C}), 115.2(\mathrm{CH}), 109.7(\mathrm{C}), 21.3\left(\mathrm{CH}_{3}\right),-0.60\left(\mathrm{CH}_{3}\right)$; HRMS Calculated for $\mathrm{C}_{27} \mathrm{H}_{27} \mathrm{ClN}_{3} \mathrm{Si}[\mathrm{M}+\mathrm{H}]^{+}: 456.1655$, Found: 456.1652 .

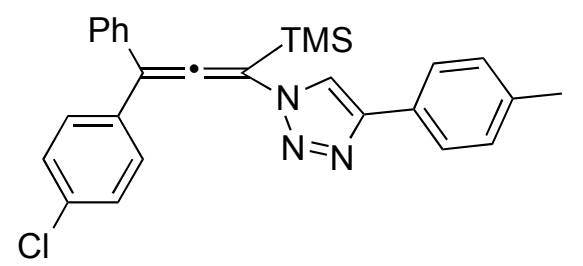

$\mathrm{N}-1-1.5 .4 \mathrm{z}$

\section{1-(3-(4-chlorophenyl)-1-(trimethylsilyl)-3-phenylpropa-1,2-dienyl)-4-p-tolyl-2H-1,2,3-}

triazole (N-1-1.5.4z) was purified by flash chromatography (Hexane-EtOAc, v/v 20/1) as yellow liquid. Isolated yield 15\%; $\mathrm{R} f$ (hexanes/ethyl acetate, $\mathrm{V} / \mathrm{V}, 10 / 1)=0.33$; $\mathrm{UV}$ absorption: 244(0.295), 264(0.244), 299(0.138); IR (neat), 2936, 1588, 1477, 1422, 1278, 1213, 1034, 932, 843, 771, 687; ${ }^{1} \mathrm{H}-\mathrm{NMR}\left(600 \mathrm{MHz}, \mathrm{CDCl}_{3}\right) \delta 7.63(\mathrm{~s}, 1 \mathrm{H}), 7.30-7.28(\mathrm{~m}, 3 \mathrm{H}), 7.24(\mathrm{~d}, J=8.4$ $\mathrm{Hz}, 2 \mathrm{H}), 7.05(\mathrm{~d}, J=7.8 \mathrm{~Hz}, 2 \mathrm{H}), 7.03-7.01(\mathrm{~m}, 2 \mathrm{H}), 6.96(\mathrm{~d}, J=8.4 \mathrm{~Hz}, 2 \mathrm{H}), 6.76(\mathrm{~d}, J=7.8$ $\mathrm{Hz}, 2 \mathrm{H}), 2.16$ (s, 3H), $0.32(\mathrm{~s}, 9 \mathrm{H}) ;{ }^{13} \mathrm{C}-\mathrm{NMR}\left(150 \mathrm{MHz}, \mathrm{CDCl}_{3}\right) \delta 203.6(\mathrm{C}), 138.9(\mathrm{C}), 137.8$ (C), $134.6(\mathrm{C}), 133.7(\mathrm{C}), 133.6(\mathrm{C}), 133.4(\mathrm{C}), 129.6(\mathrm{CH}), 129.0(\mathrm{CH}), 128.7(\mathrm{CH}), 128.5$ 
$(\mathrm{CH}), 128.4(\mathrm{CH}), 128.3(\mathrm{CH}), 128.0(\mathrm{CH}), 124.3(\mathrm{C}), 113.2(\mathrm{CH}), 107.6(\mathrm{C}), 21.2\left(\mathrm{CH}_{3}\right),-0.68$

$\left(\mathrm{CH}_{3}\right)$; HRMS Calculated for $\mathrm{C}_{27} \mathrm{H}_{27} \mathrm{ClN}_{3} \mathrm{Si}[\mathrm{M}+\mathrm{H}]^{+}: 456.1655$, Found: 456.1659 .

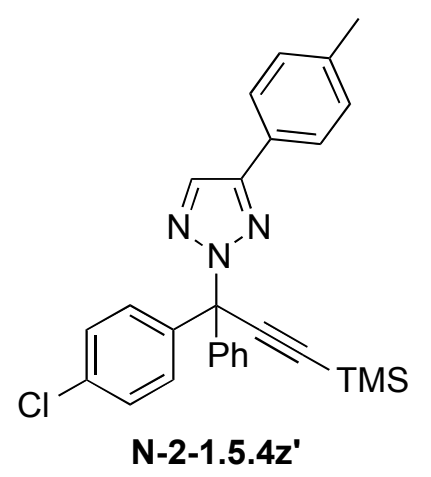

2-(1-(4-chlorophenyl)-3-(trimethylsilyl)-1-phenylprop-2-ynyl)-4-p-tolyl-2H-1,2,3-triazole

(N-2-1.5.4z') was purified by flash chromatography (Hexane-EtOAc, v/v 20/1) as yellow liquid. Isolated yield 15\%; $\mathrm{R} f$ (hexanes/ethyl acetate, $\mathrm{V} / \mathrm{V}, 10 / 1)=0.49$; $\mathrm{UV}$ absorption: 256(0.408), 271(0.393); IR (neat), 3077, 2934, 1483, 1446, 1343, 1297, 1041, 961, 852, 777, 739, 695; ${ }^{1} \mathrm{H}-$ $\operatorname{NMR}\left(600 \mathrm{MHz}, \mathrm{CDCl}_{3}\right) \delta 7.91(\mathrm{~s}, 1 \mathrm{H}), 7.67(\mathrm{~d}, J=7.8 \mathrm{~Hz}, 2 \mathrm{H}), 7.37-7.34(\mathrm{~m}, 3 \mathrm{H})$, 7.33-7.31 (m, 2H), 7.29-7.26 (m, 4H), $7.21(\mathrm{~d}, J=7.8 \mathrm{~Hz}, 2 \mathrm{H}), 2.38$ (s, 3H), 0.29 (s, 9H); ${ }^{13} \mathrm{C}-\mathrm{NMR}(150$ $\left.\mathrm{MHz}, \mathrm{CDCl}_{3}\right) \delta 148.1(\mathrm{C}), 140.9(\mathrm{C}), 139.8(\mathrm{C}), 138.5(\mathrm{C}), 134.4(\mathrm{C}), 131.3(\mathrm{CH}), 129.8(\mathrm{CH})$, $129.4(\mathrm{CH}), 128.5(\mathrm{CH}), 128.3(\mathrm{CH}), 128.2(\mathrm{CH}), 127.9(\mathrm{CH}), 127.4(\mathrm{C}), 126.0(\mathrm{CH}), 103.8(\mathrm{C})$, $94.8(\mathrm{C}), 73.2(\mathrm{C}), 21.3\left(\mathrm{CH}_{3}\right),-0.32\left(\mathrm{CH}_{3}\right)$; HRMS Calculated for $\mathrm{C}_{27} \mathrm{H}_{27} \mathrm{ClN}_{3} \mathrm{Si}[\mathrm{M}+\mathrm{H}]^{+}$: 456.1655, Found: 456.1652. 


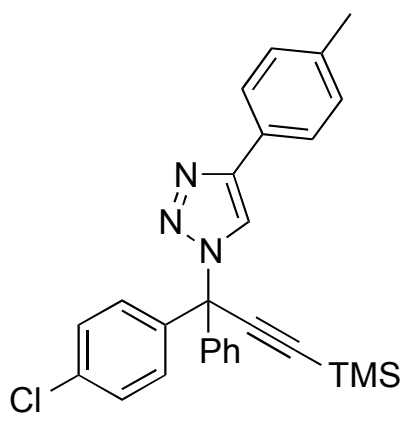

$\mathrm{N}-1-1.5 .4 z^{\prime}$

\section{1-(1-(4-chlorophenyl)-3-(trimethylsilyl)-1-phenylprop-2-ynyl)-4-p-tolyl-2H-1,2,3-triazole}

(N-1-1.5.4z') was purified by flash chromatography (Hexane-EtOAc, v/v 20/1) as yellow liquid. Isolated yield 4\%; Rf (hexanes/ethyl acetate, $\mathrm{V} / \mathrm{V}, 10 / 1)=0.30 ; \mathrm{UV}$ absorption: 255(0.358), 273(0.367); IR (neat), 3089, 2928, 1487, 1455, 1433, 1148, 1052, 989, 820, 734, 709, 679; ${ }^{1} \mathrm{H}-$ $\operatorname{NMR}\left(600 \mathrm{MHz}, \mathrm{CDCl}_{3}\right) \delta 8.01(\mathrm{~s}, 1 \mathrm{H}), 7.73(\mathrm{~d}, J=7.8 \mathrm{~Hz}, 2 \mathrm{H}), 7.39-7.35(\mathrm{~m}, 3 \mathrm{H}), 7.33(\mathrm{~d}, J=$ 8.4 Hz, 2H), 7.29-7.27 (m, 2H), $7.25(\mathrm{~d}, J=8.4 \mathrm{~Hz}, 2 \mathrm{H}), 7.23(\mathrm{~d}, J=7.8 \mathrm{~Hz}, 2 \mathrm{H}), 2.38(\mathrm{~s}, 3 \mathrm{H})$, $0.29(\mathrm{~s}, 9 \mathrm{H}) ;{ }^{13} \mathrm{C}-\mathrm{NMR}\left(150 \mathrm{MHz}, \mathrm{CDCl}_{3}\right) \delta 147.0(\mathrm{C}), 140.2(\mathrm{C}), 139.1(\mathrm{C}), 138.0(\mathrm{C}), 134.8$ (C), $129.6(\mathrm{CH}), 129.5(\mathrm{CH}), 128.9(\mathrm{CH}), 128.5(\mathrm{CH}), 128.3(\mathrm{CH}), 127.8(\mathrm{CH}), 127.6(\mathrm{C}), 125.6$ $(\mathrm{CH}), 120.1(\mathrm{CH}), 103.5(\mathrm{C}), 95.6(\mathrm{C}), 69.5(\mathrm{C}), 21.3\left(\mathrm{CH}_{3}\right),-0.41\left(\mathrm{CH}_{3}\right)$; HRMS Calculated for $\mathrm{C}_{27} \mathrm{H}_{27} \mathrm{ClN}_{3} \mathrm{Si}[\mathrm{M}+\mathrm{H}]^{+}:$456.1655, Found: 456.1650 .

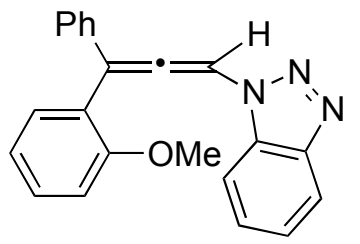

$1.5 .7 a$

1-(3-(2-methoxyphenyl)-3-phenylpropa-1,2-dienyl)-1 $H$-benzo $[d][1,2,3]$ triazole $(1.5 .7$ a) was purified by flash chromatography (Hexane-EtOAc, v/v 20/1) as white solid. Isolated yield 98\%; 
m. p. $173-175{ }^{\circ} \mathrm{C} ; \mathrm{R} f$ (hexanes/ethyl acetate, $\left.\mathrm{V} / \mathrm{V}, 6 / 1\right)=0.42$; UV absorption: $235(0.327$ ), 299(0.206); IR (neat), 3056, 1596, 1491, 1443, 1278, 1172, 1061, 741, 694; ${ }^{1} \mathrm{H}-\mathrm{NMR}$ (600 MHz, $\left.\mathrm{CDCl}_{3}\right) \delta 8.26(\mathrm{~s}, 1 \mathrm{H}), 8.07(\mathrm{~d}, J=8.4 \mathrm{~Hz}, 1 \mathrm{H}), 8.00(\mathrm{~d}, J=8.4 \mathrm{~Hz}, 1 \mathrm{H}), 7.44-7.27(\mathrm{~m}, 9 \mathrm{H}), 7.01$ $(\mathrm{t}, J=7.8 \mathrm{~Hz}, 1 \mathrm{H}), 6.96(\mathrm{~d}, J=8.4 \mathrm{~Hz}, 1 \mathrm{H}), 3.68(\mathrm{~s}, 3 \mathrm{H}) ;{ }^{13} \mathrm{C}-\mathrm{NMR}\left(150 \mathrm{MHz}, \mathrm{CDCl}_{3}\right) \delta 196.6$ (C), $157.0(\mathrm{C}), 146.6(\mathrm{C}), 135.3(\mathrm{C}), 131.4(\mathrm{C}), 130.9(\mathrm{CH}), 130.0(\mathrm{CH}), 128.5(\mathrm{CH}), 128.4$ $(\mathrm{CH}), 127.9(\mathrm{CH}), 127.6(\mathrm{CH}), 124.5(\mathrm{CH}), 124.1(\mathrm{CH}), 120.8(\mathrm{CH}), 119.9(\mathrm{CH}), 117.8(\mathrm{C})$, $111.9(\mathrm{CH}), 111.3(\mathrm{C}), 99.4(\mathrm{CH}), 55.3\left(\mathrm{CH}_{3}\right)$; HRMS Calculated for $\mathrm{C}_{22} \mathrm{H}_{18} \mathrm{~N}_{3} \mathrm{O}[\mathrm{M}+\mathrm{H}]^{+}$: 340.1458, Found: 340.1455. 


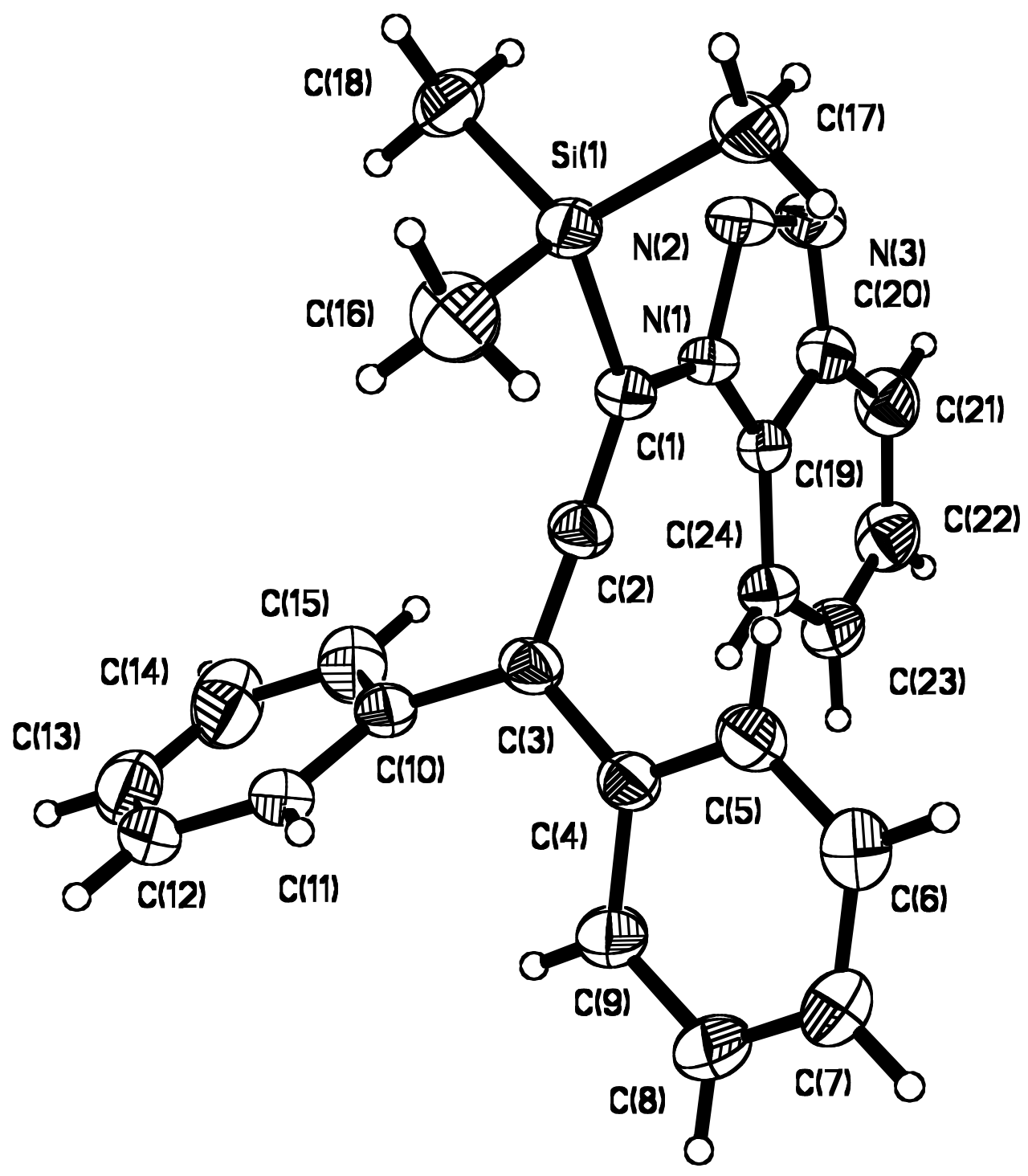

Figure S1.5-1.Perspective view of the molecular structure of 1.5.4a $\left(\mathrm{C}_{24} \mathrm{H}_{23} \mathrm{~N}_{3} \mathrm{Si}\right)$ with the atom labeling scheme. The thermal ellipsoids are scaled to enclose $30 \%$ probability. CCDC number: 851290. 


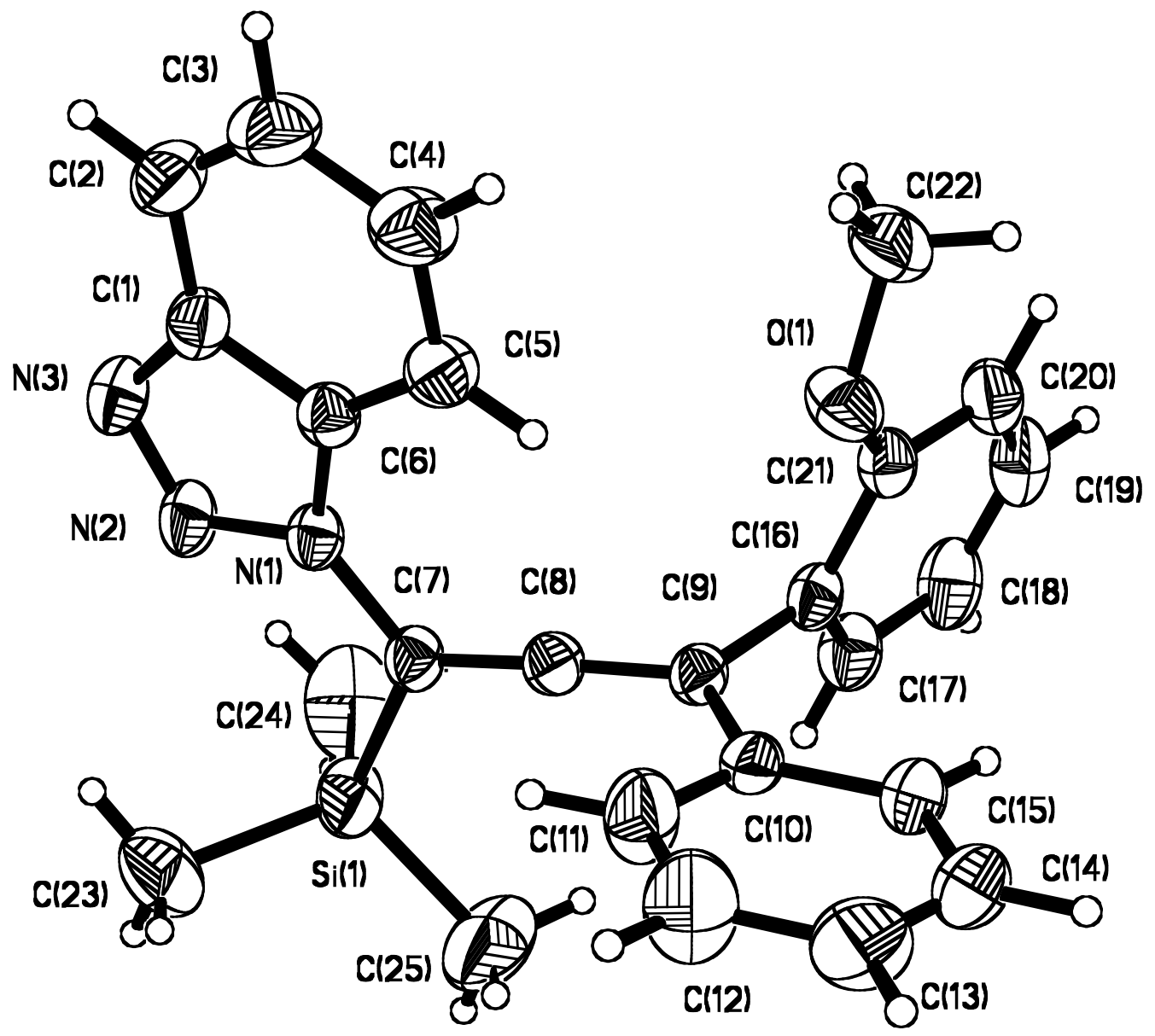

Figure S1.5-2.Perspective view of the molecular structure of $1.5 .4 \mathrm{~g}\left(\mathrm{C}_{25} \mathrm{H}_{25} \mathrm{~N}_{3} \mathrm{SiO}\right)$ with the atom labeling scheme. The thermal ellipsoids are scaled to enclose $30 \%$ probability. CCDC number: 851291. 


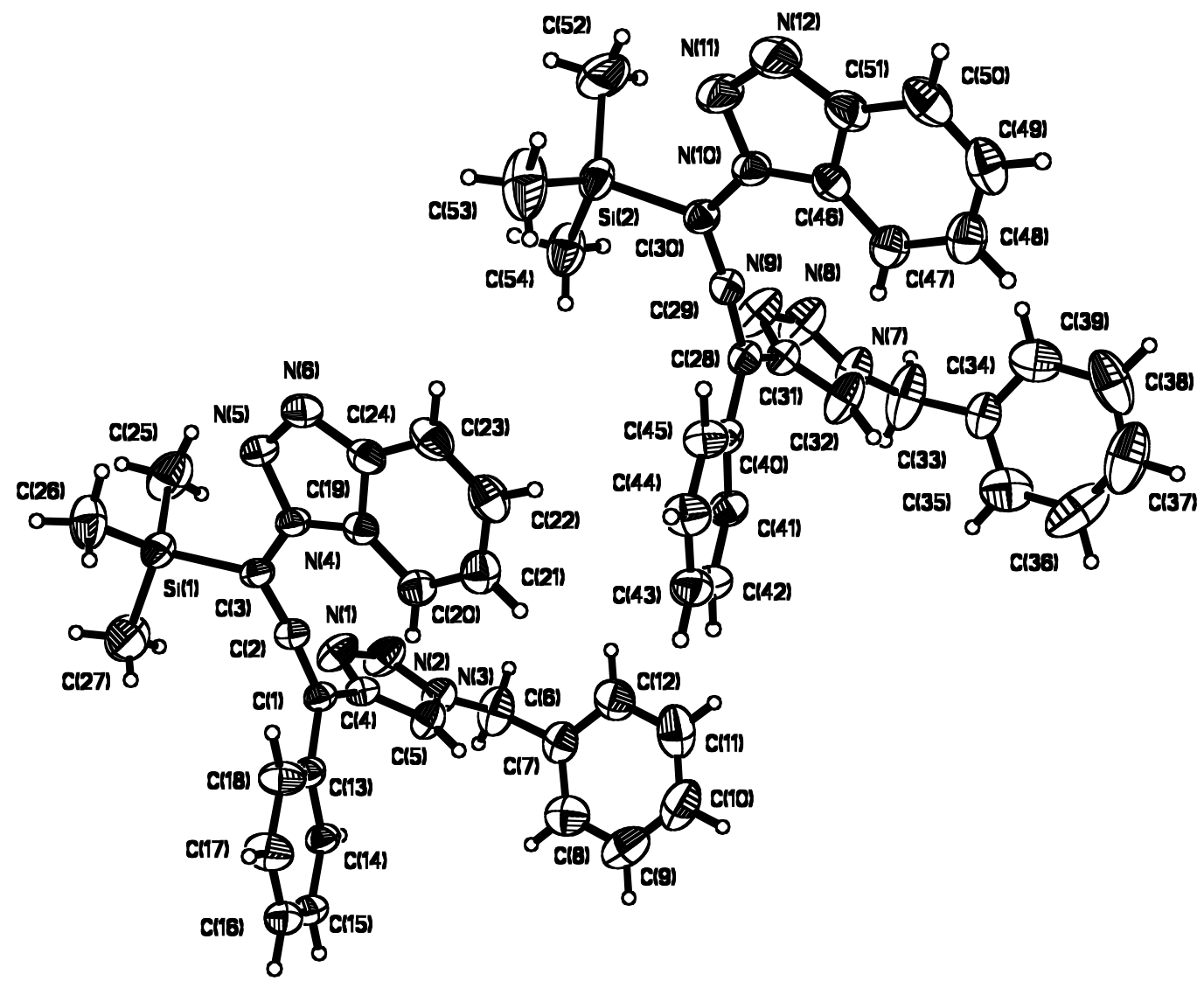

Figure S1.5-3.Perspective view of the molecular structure of 1.5.4u $\left(\mathrm{C}_{27} \mathrm{H}_{26} \mathrm{~N}_{6} \mathrm{Si}\right)$ with the atom labeling scheme. The thermal ellipsoids are scaled to enclose $30 \%$ probability. CCDC number: 851293. 


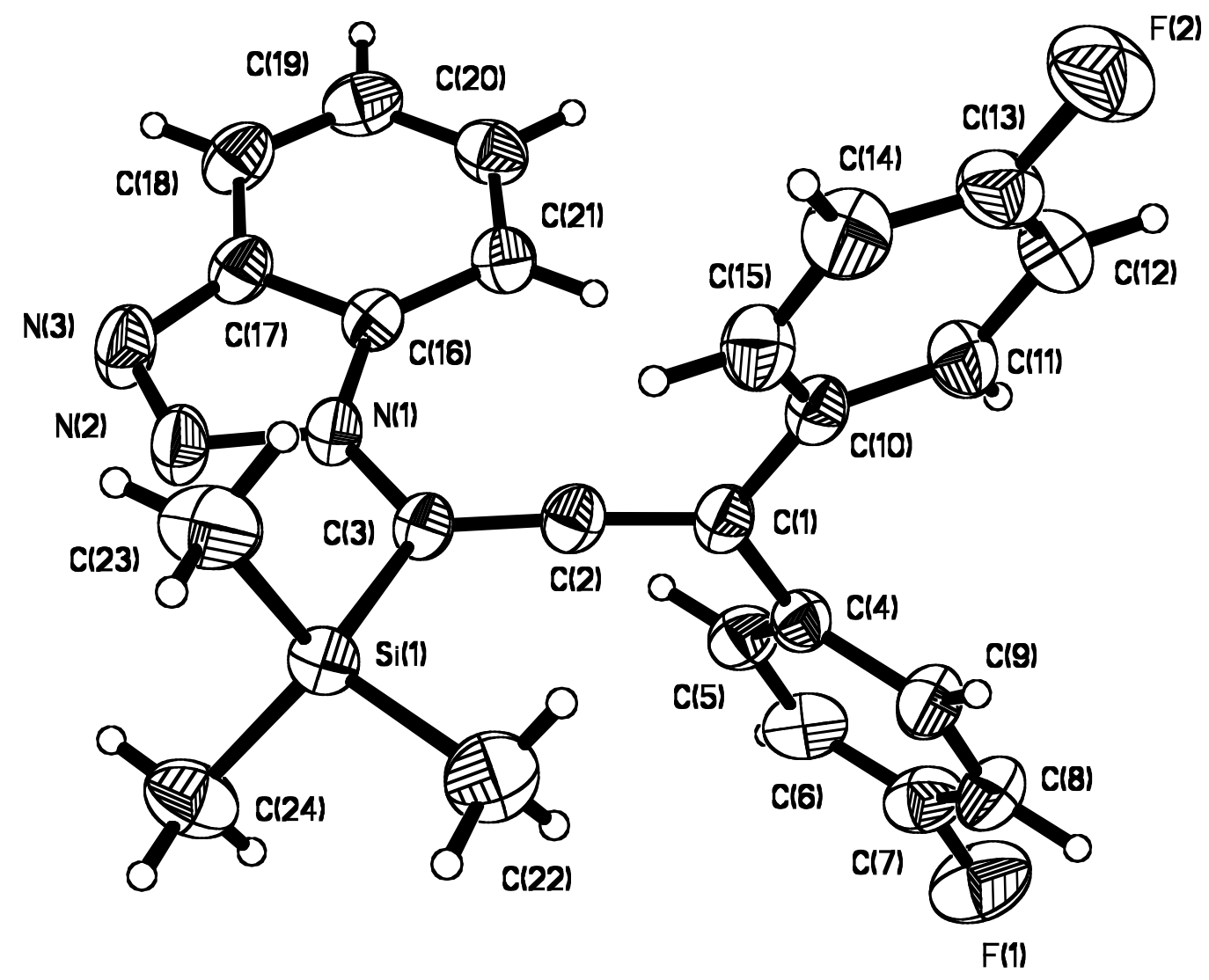

Figure S1.5-4.Perspective view of the molecular structure of $\mathbf{1 . 5 . 4 y}\left(\mathrm{C}_{24} \mathrm{H}_{21} \mathrm{~F}_{2} \mathrm{~N}_{3} \mathrm{Si}\right)$ with the atom labeling scheme. The thermal ellipsoids are scaled to enclose $30 \%$ probability. CCDC number: 851294. 


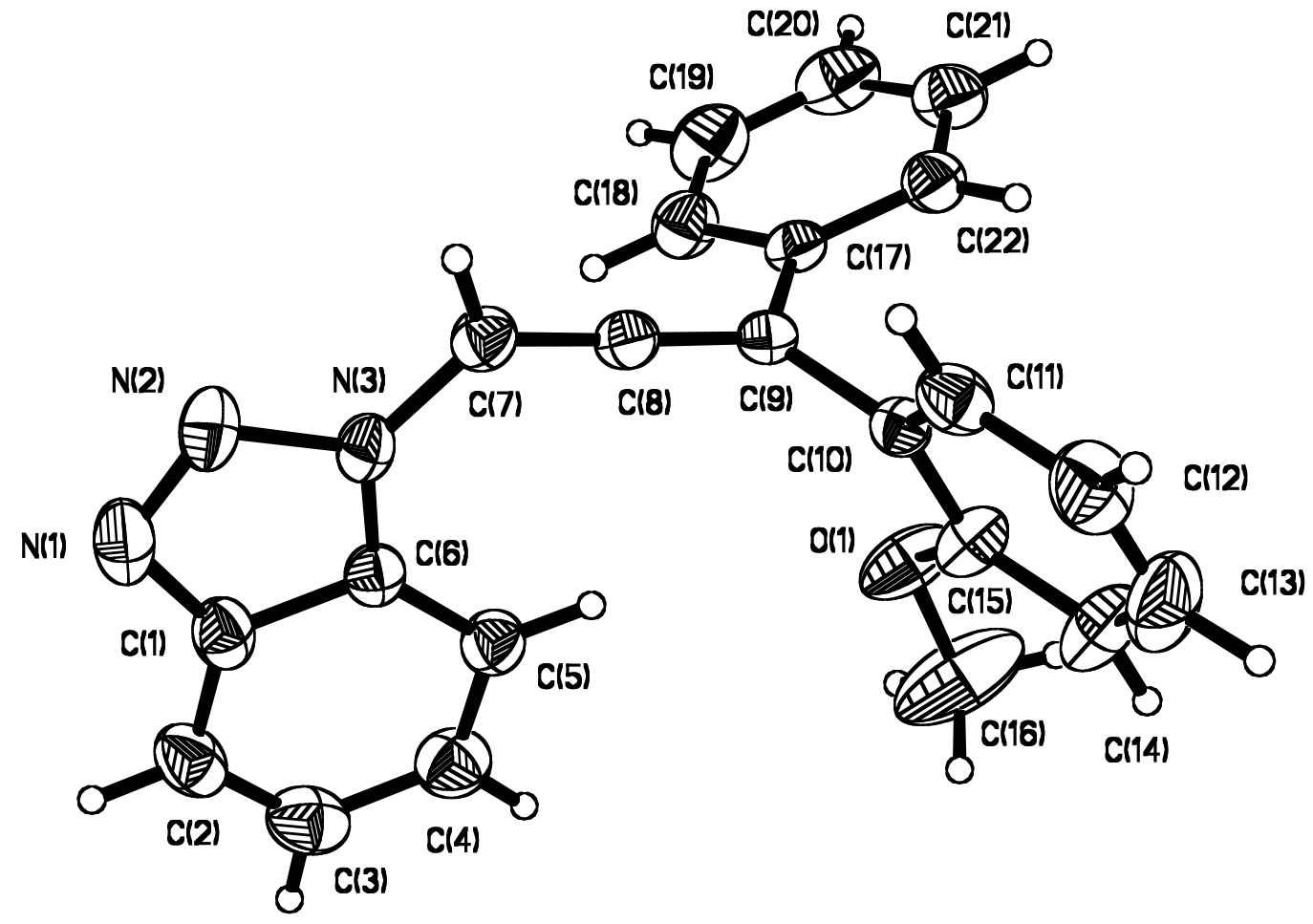

Figure S1.5-5.Perspective view of the molecular structure of $1.5 .7 \mathbf{a}\left(\mathrm{C}_{22} \mathrm{H}_{17} \mathrm{~N}_{3} \mathrm{O}\right)$ with the atom labeling scheme. The thermal ellipsoids are scaled to enclose $30 \%$ probability. CCDC number: 851292. 


\subsection{Mitsunobu reaction of 1,2,3- $\mathrm{NH}$-triazoles: regio- and stereo-selective synthesis of triazole derivatives}

\section{General Methods and Materials}

All of the reactions dealing with air and/or moisture-sensitive reactions were carried out under an atmosphere of nitrogen using oven/flame-dried glassware and standard syringe/septa techniques. Unless otherwise noted, all commercial reagents and solvents were obtained from the commercial provider and used without further purification. ${ }^{1} \mathrm{H}$ NMR and ${ }^{13} \mathrm{C}$ NMR spectra were recorded on Varian $600 \mathrm{MHz}$ spectrometers. Chemical shifts were reported relative to internal tetramethylsilane $(\delta 0.00 \mathrm{ppm})$ or $\mathrm{CDCl}_{3}(\delta 7.26 \mathrm{ppm})$ for ${ }^{1} \mathrm{H}$ and $\mathrm{CDCl}_{3}(\delta 77.0 \mathrm{ppm})$ for ${ }^{13} \mathrm{C}$. Flash column chromatography was performed on 230-430 mesh silica gel. Analytical thin layer chromatography was performed with precoated glass baked plates $(250 \mu)$ and visualized by fluorescence and by charring after treatment with potassium permanganate stain. HRMS were recorded on LTQ-FTUHRA spectrometer. Optical rotations were measured with a digital polarimeter in the solvent specified. Enantiomeric excess was determined by HPLC analysis, using chiral column described below in detail. The configuration of products was determined by comparison of rotation sign with the literature data or by analogue.

Substrates 1.6.4g, 1.6.4h, 1.6.4i, 1.6.4m were reported the literature as below:

Chen, Y.; Liu, Y.; Petersen, J. L.; Shi, X. Chem. Commun. 2008, 3254-3256. 


\section{General Procedure for Mitsunobu reaction of 1,2,3-NH-triazole with alcohol.}

A $25 \mathrm{~mL}$ round-bottomed flask is equipped with a stirring bar, nitrogen inlet, rubber septum. The flask is charged with alcohol $(3.0 \mathrm{mmol})$, NH-triazole $(3.6 \mathrm{mmol})$, triphenylphosphine $\left(\mathrm{PPh}_{3}\right)$ (3.6 mmol), and $12 \mathrm{~mL}$ of distilled tetrahydrofuran (THF). The flask is immersed in an ice bath, and diisopropyl azodicarboxylate $(3.6 \mathrm{mmol})$ is added dropwise at a rate such that the temperature of the reaction mixture is maintained below $10{ }^{\circ} \mathrm{C}$. Upon completion of the addition, the flask is removed from the ice bath and the solution is allowed to stir at room temperature for 3 hours, and monitored by TLC. After the reaction is completed, $30 \mathrm{ml}$ of water is added to quench the reaction. The mixture is extracted three times with $20 \mathrm{~mL}$ portions of ethyl acetate. The combined organic layers are washed with $20 \mathrm{ml}$ brine twice and dried over anhydrous sodium sulfate $\left(\mathrm{Na}_{2} \mathrm{SO}_{4}\right)$. Excess solvent and other volatile reaction components are completely removed under reduced pressure initially on a rotary evaporator. The residue is applied to a flash silica gel chromatography column (hexane/ethyl acetate $=10: 1$ ) to give the products. 


\section{Compounds Characterization}

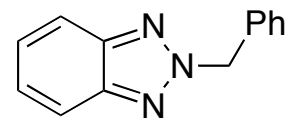

$\mathrm{N}-2-1.6 .3 a$

2-benzyl-2H-benzo[d][1,2,3]triazole (N-2-1.6.3a) was purified by flash chromatography (Hexane-EtOAc, v/v 10/1) as yellow liquid. ${ }^{1} \mathrm{H}-\mathrm{NMR}\left(600 \mathrm{MHz}, \mathrm{CDCl}_{3}\right) \delta$ 7.87-7.85 (m, 2H), 7.42-7.40 (m, 2H), 7.37-7.30 (m, 5H), $5.87(\mathrm{~s}, 2 \mathrm{H}) ;{ }^{13} \mathrm{C}-\mathrm{NMR}\left(150 \mathrm{MHz}, \mathrm{CDCl}_{3}\right) \delta$ 144.9, 134.9, 129.1, 128.8, 128.6, 126.6, 118.4, 60.6; HRMS Calculated for $\mathrm{C}_{13} \mathrm{H}_{12} \mathrm{~N}_{3}[\mathrm{M}+\mathrm{H}]^{+}:$210.1031, Found: 210.1025.

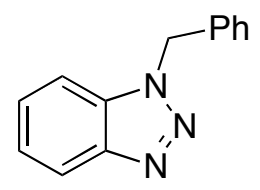

$\mathrm{N}-1-1.6 .3 a$

1-benzyl-1H-benzo[d][1,2,3]triazole (N-1-1.6.3a) was purified by flash chromatography (Hexane-EtOAc, v/v 10/1) as yellow liquid. ${ }^{1} \mathrm{H}-\mathrm{NMR}\left(600 \mathrm{MHz}, \mathrm{CDCl}_{3}\right) \delta$ 8.05-8.03 (m, 1H), 7.39-7.23 (m, 8H), $5.82(\mathrm{~s}, 2 \mathrm{H}) ;{ }^{13} \mathrm{C}-\mathrm{NMR}\left(150 \mathrm{MHz}, \mathrm{CDCl}_{3}\right) \delta 146.6,135.0,133.0,129.2$, 128.7, 127.8, 127.6, 124.1, 120.3, 109.9, 52.4; HRMS Calculated for $\mathrm{C}_{13} \mathrm{H}_{12} \mathrm{~N}_{3}[\mathrm{M}+\mathrm{H}]^{+}$: 210.1031, Found: 210.1036. 


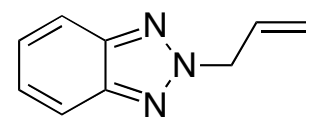

$\mathrm{N}-2-1.6 .3 b$

2-allyl-2H-benzo[d][1,2,3]triazole $(\mathbf{N}-2-1.6 .3 b)$ was purified by flash chromatography (Hexane-EtOAc, v/v 10/1) as yellow liquid. ${ }^{1} \mathrm{H}-\mathrm{NMR}\left(600 \mathrm{MHz}, \mathrm{CDCl}_{3}\right) \delta$ 7.87-7.84 (m, 2H), 7.38-7.34 (m, 2H), 6.23-6.15 (m, 1H), $5.38(\mathrm{~d}, J=10.8 \mathrm{~Hz}, 1 \mathrm{H}), 7.37-7.35(\mathrm{~m}, 1 \mathrm{H}), 5.32(\mathrm{~d}, J=$ $6.6 \mathrm{~Hz}, 2 \mathrm{H}) ;{ }^{13} \mathrm{C}-\mathrm{NMR}\left(150 \mathrm{MHz}, \mathrm{CDCl}_{3}\right) \delta 144.7,131.3,126.6,120.4,118.2,59.1$; HRMS Calculated for $\mathrm{C}_{9} \mathrm{H}_{10} \mathrm{~N}_{3}[\mathrm{M}+\mathrm{H}]^{+}: 160.0875$, Found: 160.0870 .

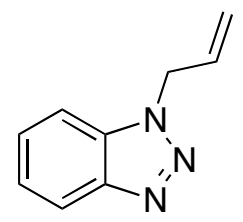

$\mathrm{N}-1-1.6 .3 b$

1-allyl-1H-benzo[d][1,2,3]triazole (N-1-1.6.3b) was purified by flash chromatography (Hexane-EtOAc, v/v 10/1) as yellow liquid. ${ }^{1} \mathrm{H}-\mathrm{NMR}\left(600 \mathrm{MHz}, \mathrm{CDCl}_{3}\right) \delta 8.03(\mathrm{~d}, J=8.4 \mathrm{~Hz}$, 1H), 7.50-7.48 (m, 1H), 7.46-7.42 (m, 1H), 7.35-7.32 (m, 1H), 6.07-6.00 (m, 1H), $5.29(\mathrm{~d}, J=$ $10.2 \mathrm{~Hz}, 1 \mathrm{H}), 5.26-5.21(\mathrm{~m}, 3 \mathrm{H}) ;{ }^{13} \mathrm{C}-\mathrm{NMR}\left(150 \mathrm{MHz}, \mathrm{CDCl}_{3}\right) \delta 146.4,133.1,131.4,127.5$, 124.1, 120.2, 119.4, 109.9, 51.0; HRMS Calculated for $\mathrm{C}_{9} \mathrm{H}_{10} \mathrm{~N}_{3}[\mathrm{M}+\mathrm{H}]^{+}: 160.0875$, Found: 160.0879 .

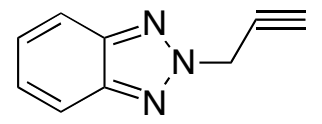

N-2-1.6.3c 
2-(prop-2-ynyl)-2H-benzo[d][1,2,3]triazole (N-2-1.6.3c) was purified by flash chromatography (Hexane-EtOAc, v/v 10/1) as yellow liquid. ${ }^{1} \mathrm{H}-\mathrm{NMR}\left(600 \mathrm{MHz}, \mathrm{CDCl}_{3}\right) \delta$ 7.89-7.86 (m, 2H), 7.40-7.37 (m, 2H), $5.52(\mathrm{~d}, J=3.0 \mathrm{~Hz}, 2 \mathrm{H}), 2.61(\mathrm{t}, J=3.0 \mathrm{~Hz}, 1 \mathrm{H}) ;{ }^{13} \mathrm{C}-\mathrm{NMR}(150 \mathrm{MHz}$ $\left.\mathrm{CDCl}_{3}\right) \delta 144.9,127.0,118.4,75.9,75.4,46.2$; HRMS Calculated for $\mathrm{C}_{9} \mathrm{H}_{8} \mathrm{~N}_{3}[\mathrm{M}+\mathrm{H}]^{+}$: 158.0718, Found: 158.0711.

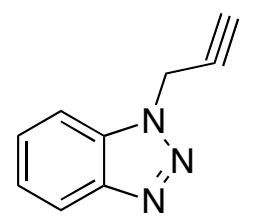

N-1-1.6.3c

1-(prop-2-ynyl)-1H-benzo[d][1,2,3]triazole (N-1-1.6.3c) was purified by flash chromatography (Hexane-EtOAc, v/v 10/1) as yellow liquid. ${ }^{1} \mathrm{H}-\mathrm{NMR}\left(600 \mathrm{MHz}, \mathrm{CDCl}_{3}\right) \delta 8.03(\mathrm{~d}, J=8.4 \mathrm{~Hz}$, 1H), $7.68(\mathrm{~d}, J=8.4 \mathrm{~Hz}, 1 \mathrm{H}), 7.49$ (t, $J=7.8 \mathrm{~Hz}, 1 \mathrm{H}), 7.36(\mathrm{t}, J=7.8 \mathrm{~Hz}, 1 \mathrm{H}), 5.42$ (d, $J=2.4$ $\mathrm{Hz}, 2 \mathrm{H}), 2.48(\mathrm{t}, J=2.4 \mathrm{~Hz}, 1 \mathrm{H}) ;{ }^{13} \mathrm{C}-\mathrm{NMR}\left(150 \mathrm{MHz}, \mathrm{CDCl}_{3}\right) \delta 146.5,132.7,128.0,124.4$, 120.3, 110.0, 75.5, 75.3, 38.2; HRMS Calculated for $\mathrm{C}_{9} \mathrm{H}_{8} \mathrm{~N}_{3}[\mathrm{M}+\mathrm{H}]^{+}$: 158.0718, Found: 158.0725 .

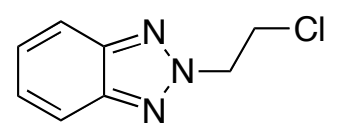

N-2-1.6.3d

2-(2-chloroethyl)-2H-benzo[d][1,2,3]triazole $\quad(\mathbf{N}-2-1.6 .3 d) \quad$ was purified by flash chromatography (Hexane-EtOAc, v/v 10/1) as yellow liquid. ${ }^{1} \mathrm{H}-\mathrm{NMR}\left(600 \mathrm{MHz}, \mathrm{CDCl}_{3}\right) \delta$ 
7.86-7.83 (m, 2H), 7.36-7.33 (m, 2H), $4.99(\mathrm{t}, J=6.0 \mathrm{~Hz}, 2 \mathrm{H}), 4.11(\mathrm{t}, J=6.0 \mathrm{~Hz}, 2 \mathrm{H}) ;{ }^{13} \mathrm{C}-$ NMR $\left(150 \mathrm{MHz}, \mathrm{CDCl}_{3}\right) \delta 144.3,126.5,117.9,57.3,41.4$; HRMS Calculated for $\mathrm{C}_{8} \mathrm{H}_{9} \mathrm{ClN}_{3}$ $[\mathrm{M}+\mathrm{H}]^{+}: 182.0485$, Found: 182.0480 .

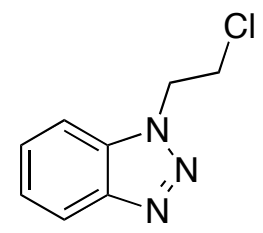

N-1-1.6.3d

1-(2-chloroethyl)-1H-benzo[d][1,2,3]triazole $\quad(\mathbf{N}-1-1.6 .3 d) \quad$ was purified by flash chromatography (Hexane-EtOAc, v/v 10/1) as yellow liquid. ${ }^{1} \mathrm{H}-\mathrm{NMR}\left(600 \mathrm{MHz}, \mathrm{CDCl}_{3}\right) \delta 8.08$ $(\mathrm{d}, J=8.4 \mathrm{~Hz}, 1 \mathrm{H}), 7.59(\mathrm{~d}, J=8.4 \mathrm{~Hz}, 1 \mathrm{H}), 7.52(\mathrm{t}, J=7.8 \mathrm{~Hz}, 1 \mathrm{H}), 7.39$ (t, $J=7.8 \mathrm{~Hz}, 1 \mathrm{H})$, $4.96(\mathrm{t}, J=6.0 \mathrm{~Hz}, 2 \mathrm{H}), 4.05(\mathrm{t}, J=6.0 \mathrm{~Hz}, 2 \mathrm{H}) ;{ }^{13} \mathrm{C}-\mathrm{NMR}\left(150 \mathrm{MHz}, \mathrm{CDCl}_{3}\right) \delta 145.8,133.5$, 127.7, 124.1, 120.1, 109.3, 49.4, 42.2; HRMS Calculated for $\mathrm{C}_{8} \mathrm{H}_{9} \mathrm{ClN}_{3}[\mathrm{M}+\mathrm{H}]^{+}:$182.0485, Found: 182.0492 .

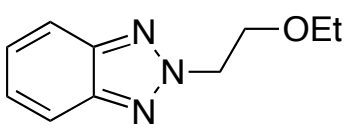

N-2-1.6.3e

2-(2-ethoxyethyl)-2H-benzo[d][1,2,3]triazole $\quad(\mathbf{N}-2-1.6 .3 e)$ was purified by flash chromatography (Hexane-EtOAc, v/v 10/1) as yellow liquid. ${ }^{1} \mathrm{H}-\mathrm{NMR}\left(600 \mathrm{MHz}, \mathrm{CDCl}_{3}\right) \delta$ 7.85-7.82 (m, 2H), 7.35-7.32 (m, 2H), $4.86(\mathrm{t}, J=6.0 \mathrm{~Hz}, 2 \mathrm{H}), 4.05(\mathrm{t}, J=6.0 \mathrm{~Hz}, 2 \mathrm{H}), 3.46(\mathrm{q}, J$ 
$=7.2 \mathrm{~Hz}, 2 \mathrm{H}), 1.09(\mathrm{t}, J=7.2 \mathrm{~Hz}, 3 \mathrm{H}) ;{ }^{13} \mathrm{C}-\mathrm{NMR}\left(150 \mathrm{MHz}, \mathrm{CDCl}_{3}\right) \delta$ 144.3, 126.1, 117.9, 68.3, 66.5, 56.1, 14.8; HRMS Calculated for $\mathrm{C}_{10} \mathrm{H}_{14} \mathrm{~N}_{3} \mathrm{O}[\mathrm{M}+\mathrm{H}]^{+}$: 192.1137, Found: 192.1126 .

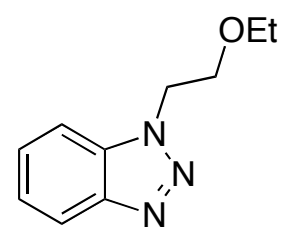

$\mathrm{N}-1-1.6 .3 e$

1-(2-ethoxyethyl)-1H-benzo[d][1,2,3]triazole $\quad(\mathbf{N}-1-1.6 .3 e) \quad$ was purified by flash chromatography (Hexane-EtOAc, v/v 10/1) as yellow liquid. ${ }^{1} \mathrm{H}-\mathrm{NMR}\left(600 \mathrm{MHz}, \mathrm{CDCl}_{3}\right) \delta 8.01$ $(\mathrm{d}, J=8.4 \mathrm{~Hz}, 1 \mathrm{H}), 7.62(\mathrm{~d}, J=8.4 \mathrm{~Hz}, 1 \mathrm{H}), 7.44(\mathrm{t}, J=7.8 \mathrm{~Hz}, 1 \mathrm{H}), 7.32(\mathrm{t}, J=7.8 \mathrm{~Hz}, 1 \mathrm{H})$, $4.78(\mathrm{t}, J=6.0 \mathrm{~Hz}, 2 \mathrm{H}), 3.88(\mathrm{t}, J=6.0 \mathrm{~Hz}, 2 \mathrm{H}), 3.40(\mathrm{q}, J=7.2 \mathrm{~Hz}, 2 \mathrm{H}), 1.06(\mathrm{t}, J=7.2 \mathrm{~Hz}$, $3 \mathrm{H}) ;{ }^{13} \mathrm{C}-\mathrm{NMR}\left(150 \mathrm{MHz}, \mathrm{CDCl}_{3}\right) \delta 145.8,133.5,127.7,124.1,120.1,109.3,49.4,42.2 ; \mathrm{HRMS}$ Calculated for $\mathrm{C}_{10} \mathrm{H}_{14} \mathrm{~N}_{3} \mathrm{O}[\mathrm{M}+\mathrm{H}]^{+}:$192.1137, Found: 192.1131 .

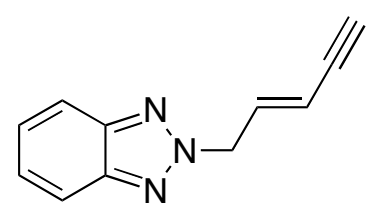

N-2-1.6.3f

(E)-2-(pent-2-en-4-ynyl)-2H-benzo[d][1,2,3]triazole $(\mathbf{N}-2-1.6 .3 f)$ was purified by flash chromatography (Hexane-EtOAc, v/v 10/1) as yellow liquid. ${ }^{1} \mathrm{H}-\mathrm{NMR}\left(600 \mathrm{MHz}, \mathrm{CDCl}_{3}\right) \delta$ 7.87-7.83 (m, 2H), 7.40-7.36 (m, 2H), $6.52(\mathrm{dt}, J=15.6 \mathrm{~Hz}, 6.0 \mathrm{~Hz}, 1 \mathrm{H}), 5.76(\mathrm{dq}, J=15.6 \mathrm{~Hz}$, $1.8 \mathrm{~Hz}, 1 \mathrm{H}), 5.36(\mathrm{dd}, J=6.6 \mathrm{~Hz}, 1.2 \mathrm{~Hz}, 2 \mathrm{H}), 2.95(\mathrm{~d}, J=1.2 \mathrm{~Hz}, 1 \mathrm{H}) ;{ }^{13} \mathrm{C}-\mathrm{NMR}(150 \mathrm{MHz}$, 
$\left.\mathrm{CDCl}_{3}\right) \delta 144.5,136.7,126.5,118.0,114.1,80.4,79.5,57.5$; HRMS Calculated for $\mathrm{C}_{11} \mathrm{H}_{10} \mathrm{~N}_{3}$ $[\mathrm{M}+\mathrm{H}]^{+}:$184.0875, Found: 184.0868 .

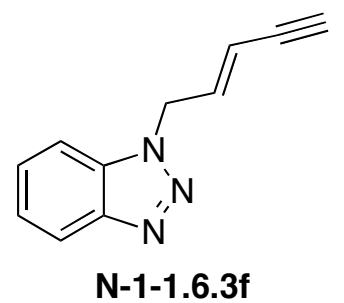

(E)-1-(pent-2-en-4-ynyl)-1H-benzo[d][1,2,3]triazole $(\mathbf{N}-1-1.6 .3 f)$ was purified by flash chromatography (Hexane-EtOAc, v/v 10/1) as yellow liquid. ${ }^{1} \mathrm{H}-\mathrm{NMR}\left(600 \mathrm{MHz}, \mathrm{CDCl}_{3}\right) \delta 8.01$ (d, $J=8.4 \mathrm{~Hz}, 1 \mathrm{H}), 7.46-7.41(\mathrm{~m}, 2 \mathrm{H}), 7.34-7.31(\mathrm{~m}, 1 \mathrm{H}), 6.35(\mathrm{dt}, J=15.6 \mathrm{~Hz}, 6.0 \mathrm{~Hz}, 1 \mathrm{H})$, 5.59 (dq, $J=15.6 \mathrm{~Hz}, 1.8 \mathrm{~Hz}, 1 \mathrm{H}), 5.28(\mathrm{dd}, J=6.6 \mathrm{~Hz}, 1.2 \mathrm{~Hz}, 2 \mathrm{H}), 2.91(\mathrm{~d}, J=1.2 \mathrm{~Hz}, 1 \mathrm{H})$; ${ }^{13} \mathrm{C}-\mathrm{NMR}\left(150 \mathrm{MHz}, \mathrm{CDCl}_{3}\right) \delta 145.9,137.0,132.6,127.5,123.9,119.9,113.0,109.3,80.1$, 79.5, 49.3; HRMS Calculated for $\mathrm{C}_{11} \mathrm{H}_{10} \mathrm{~N}_{3}[\mathrm{M}+\mathrm{H}]^{+}: 184.0875$, Found: 184.0882 .

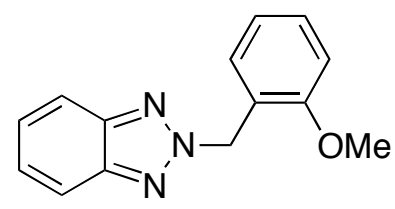

$\mathrm{N}-2-1.6 .3 \mathrm{~g}$

(E)-2-(pent-2-en-4-ynyl)-2H-benzo[d][1,2,3]triazole $(\mathbf{N}-2-1.6 .3 g)$ was purified by flash chromatography (Hexane-EtOAc, v/v 10/1) as yellow liquid. ${ }^{1} \mathrm{H}-\mathrm{NMR}\left(600 \mathrm{MHz}, \mathrm{CDCl}_{3}\right) \delta$ 7.89-7.83 (m, 2H), 7.37-7.32 (m, 2H), 7.30 (dt, $J=7.8 \mathrm{~Hz}, 1.8 \mathrm{~Hz}, 1 \mathrm{H}), 7.11$ (dd, $J=7.8 \mathrm{~Hz}, 1.8$ $\mathrm{Hz}, 1 \mathrm{H}), 6.90(\mathrm{t}, J=7.8 \mathrm{~Hz}, 2 \mathrm{H}), 5.94(\mathrm{~s}, 2 \mathrm{H}), 3.85(\mathrm{~s}, 3 \mathrm{H}) ;{ }^{13} \mathrm{C}-\mathrm{NMR}\left(150 \mathrm{MHz}, \mathrm{CDCl}_{3}\right) \delta$ 
157.3, 144.7, 130.1, 129.9, 126.4, 123.4, 121.0, 118.4, 111.0, 55.8, 55.2; HRMS Calculated for $\mathrm{C}_{14} \mathrm{H}_{14} \mathrm{~N}_{3} \mathrm{O}[\mathrm{M}+\mathrm{H}]^{+}: 240.1137$, Found: 240.1127.

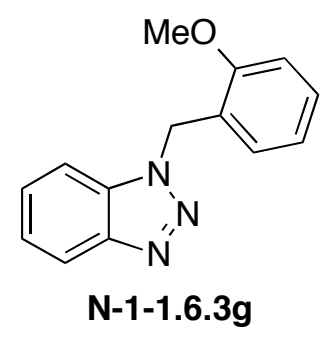

(E)-1-(pent-2-en-4-ynyl)-1H-benzo[d][1,2,3]triazole $(\mathbf{N}-1-1.6 .3 g)$ was purified by flash chromatography (Hexane-EtOAc, v/v 10/1) as yellow liquid. ${ }^{1} \mathrm{H}-\mathrm{NMR}\left(600 \mathrm{MHz}, \mathrm{CDCl}_{3}\right) \delta 8.05$ $(\mathrm{d}, J=8.4 \mathrm{~Hz}, 1 \mathrm{H}), 7.48(\mathrm{~d}, J=8.4 \mathrm{~Hz}, 1 \mathrm{H}), 7.41(\mathrm{t}, J=7.2 \mathrm{~Hz}, 1 \mathrm{H}), 7.33(\mathrm{t}, J=7.2 \mathrm{~Hz}, 1 \mathrm{H})$, $7.29(\mathrm{~d}, J=8.4 \mathrm{~Hz}, 1 \mathrm{H}), 7.07(\mathrm{~d}, J=7.2 \mathrm{~Hz}, 1 \mathrm{H}), 6.91(\mathrm{~d}, J=8.4 \mathrm{~Hz}, 1 \mathrm{H}), 6.88(\mathrm{t}, J=7.2 \mathrm{~Hz}$, 1H), $5.86(\mathrm{~s}, \quad 2 \mathrm{H}), 3.88(\mathrm{~s}, 3 \mathrm{H}) ;{ }^{13} \mathrm{C}-\mathrm{NMR}\left(150 \mathrm{MHz}, \mathrm{CDCl}_{3}\right) \delta 156.9,146.1,133.1,129.8$, 129.4, 127.1, 123.7, 123.2, 120.9, 119.9, 110.6, 110.0, 55.4, 46.8; HRMS Calculated for $\mathrm{C}_{14} \mathrm{H}_{14} \mathrm{~N}_{3} \mathrm{O}[\mathrm{M}+\mathrm{H}]^{+}:$240.1137, Found: 240.1135 .

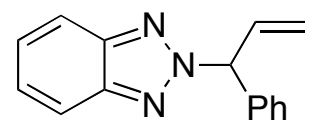

N-2-1.6.3h

2-(1-phenylallyl)-2H-benzo[d][1,2,3]triazole $\quad(\mathbf{N}-2-1.6 .3 h) \quad$ was purified by flash chromatography (Hexane-EtOAc, v/v 10/1) as yellow liquid. ${ }^{1} \mathrm{H}-\mathrm{NMR}\left(600 \mathrm{MHz}, \mathrm{CDCl}_{3}\right) \delta$ 7.88-7.85 (m, 2H), 7.40-7.36 (m, 4H), 7.31-7.27 (m, 2H), 7.25-7.21 (m, 1H), $6.76(\mathrm{~d}, J=15.6$ $\mathrm{Hz}, 1 \mathrm{H}), 6.53(\mathrm{dt}, J=15.6 \mathrm{~Hz}, 6.6 \mathrm{~Hz}, 1 \mathrm{H}), 5.47(\mathrm{~d}, J=6.6 \mathrm{~Hz}, 2 \mathrm{H}) ;{ }^{13} \mathrm{C}-\mathrm{NMR}(150 \mathrm{MHz}$, 
$\left.\mathrm{CDCl}_{3}\right) \delta 144.8,136.0,135.5,128.8,128.5,127.0,126.6,122.2,118.3,58.8$; HRMS Calculated for $\mathrm{C}_{15} \mathrm{H}_{14} \mathrm{~N}_{3}[\mathrm{M}+\mathrm{H}]^{+}: 236.1188$, Found: 236.1180 .

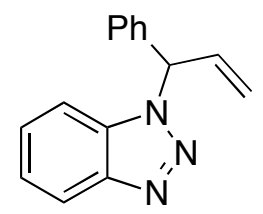

$\mathrm{N}-1-1.6 .3 \mathrm{~h}$

1-(1-phenylallyl)-1H-benzo[d][1,2,3]triazole $\quad(\mathbf{N}-1-1.6 .3 h)$ was purified by flash chromatography (Hexane-EtOAc, v/v 10/1) as yellow liquid. ${ }^{1} \mathrm{H}-\mathrm{NMR}\left(600 \mathrm{MHz}, \mathrm{CDCl}_{3}\right) \delta 8.06$ $(\mathrm{d}, J=8.4 \mathrm{~Hz}, 1 \mathrm{H}), 7.55(\mathrm{~d}, J=8.4 \mathrm{~Hz}, 1 \mathrm{H}), 7.44(\mathrm{t}, J=7.2 \mathrm{~Hz}, 1 \mathrm{H}), 7.37-7.32(\mathrm{~m}, 3 \mathrm{H}), 7.31-$ $7.27(\mathrm{~m}, 2 \mathrm{H}), 7.26-7.22(\mathrm{~m}, 1 \mathrm{H}), 6.66(\mathrm{~d}, J=8.4 \mathrm{~Hz}, 1 \mathrm{H}), 6.38(\mathrm{dt}, J=15.6 \mathrm{~Hz}, 6.0 \mathrm{~Hz}, 1 \mathrm{H})$, $5.42(\mathrm{dd}, J=15.6 \mathrm{~Hz}, 6.0 \mathrm{~Hz}, 2 \mathrm{H}) ;{ }^{13} \mathrm{C}-\mathrm{NMR}\left(150 \mathrm{MHz}, \mathrm{CDCl}_{3}\right) \delta 146.5,135.8,134.7,133.1$, 128.9, 128.6, 127.6, 126.9, 124.2, 122.4, 120.3, 110.0, 50.8; HRMS Calculated for $\mathrm{C}_{15} \mathrm{H}_{14} \mathrm{~N}_{3}$ $[\mathrm{M}+\mathrm{H}]^{+}: 236.1188$, Found: 236.1178 .

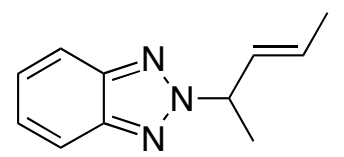

N-2-1.6.3i

(E)-2-(pent-3-en-2-yl)-2H-benzo[d][1,2,3]triazole (N-2-3i) was purified by flash chromatography (Hexane-EtOAc, v/v 10/1) as yellow liquid. ${ }^{1} \mathrm{H}-\mathrm{NMR}\left(600 \mathrm{MHz}, \mathrm{CDCl}_{3}\right) \delta$ 7.90-7.85 (m, 2H), 7.40-7.35 (m, 2H), 5.90 (qq, $J=7.2 \mathrm{~Hz}, 1.2 \mathrm{~Hz}, 1 \mathrm{H}), 5.80$ (dqd, $J=15.6 \mathrm{~Hz}$, $7.2 \mathrm{~Hz}, 1.2 \mathrm{~Hz}, 1 \mathrm{H}$ ), 5.50 (quin, $J=7.2 \mathrm{~Hz}, 1 \mathrm{H}), 1.81$ (d, $J=7.2 \mathrm{~Hz}, 3 \mathrm{H}$ ), 1.73 (dq, $J=7.2 \mathrm{~Hz}$, 
$1.2 \mathrm{~Hz}, 3 \mathrm{H}) ;{ }^{13} \mathrm{C}-\mathrm{NMR}\left(150 \mathrm{MHz}, \mathrm{CDCl}_{3}\right) \delta 144.3,130.4,129.4,126.3,118.3,64.9,21.3,17.9$; HRMS Calculated for $\mathrm{C}_{11} \mathrm{H}_{14} \mathrm{~N}_{3}[\mathrm{M}+\mathrm{H}]^{+}$: 188.118, Found: 188.1183 .

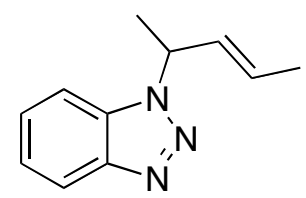

N-1-1.6.3i

(E)-1-(pent-3-en-2-yl)-1H-benzo[d][1,2,3]triazole $\quad(\mathbf{N}-1-1.6 .3 i) \quad$ was purified by flash chromatography (Hexane-EtOAc, v/v 10/1) as yellow liquid. ${ }^{1} \mathrm{H}-\mathrm{NMR}\left(600 \mathrm{MHz}, \mathrm{CDCl}_{3}\right) \delta 8.03$ $(\mathrm{d}, J=8.4 \mathrm{~Hz}, 1 \mathrm{H}), 7.52(\mathrm{~d}, J=8.4 \mathrm{~Hz}, 1 \mathrm{H}), 7.42(\mathrm{t}, J=7.2 \mathrm{~Hz}, 1 \mathrm{H}), 7.32(\mathrm{t}, J=7.2 \mathrm{~Hz}, 1 \mathrm{H})$, $5.81(\mathrm{qq}, J=7.2 \mathrm{~Hz}, 1.2 \mathrm{~Hz}, 1 \mathrm{H}), 5.71(\mathrm{dqd}, J=15.6 \mathrm{~Hz}, 7.2 \mathrm{~Hz}, 1.2 \mathrm{~Hz}, 1 \mathrm{H}$ ), 5.47 (quin, $J=7.2$ $\mathrm{Hz}, 1 \mathrm{H}), 1.81(\mathrm{~d}, J=7.2 \mathrm{~Hz}, 3 \mathrm{H}), 1.70(\mathrm{~d}, J=7.2 \mathrm{~Hz}, 3 \mathrm{H}) ;{ }^{13} \mathrm{C}-\mathrm{NMR}\left(150 \mathrm{MHz}, \mathrm{CDCl}_{3}\right) \delta$ 146.6, 132.4 130.2, 128.8, 127.0, 123.9, 120.2, 110.4, 57.6, 20.3, 17.8; HRMS Calculated for $\mathrm{C}_{11} \mathrm{H}_{14} \mathrm{~N}_{3}[\mathrm{M}+\mathrm{H}]^{+}:$188.118, Found: 188.1189.

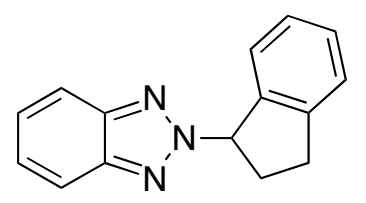

N-2-1.6.3j

2-(2,3-dihydro-1H-inden-2-yl)-2H-benzo[d][1,2,3]triazole $(\mathbf{N}-2-1.6 .3 j)$ was purified by flash chromatography (Hexane-EtOAc, v/v 10/1) as yellow liquid. ${ }^{1} \mathrm{H}-\mathrm{NMR}\left(600 \mathrm{MHz}, \mathrm{CDCl}_{3}\right) \delta$ 7.87-7.83 (m, 2H), 7.37-7.32 (m, 3H), $7.30(\mathrm{t}, J=7.2 \mathrm{~Hz}, 1 \mathrm{H}), 7.21-7.15(\mathrm{~m}, 2 \mathrm{H}), 6.47(\mathrm{t}, J=7.2$ $\mathrm{Hz}, 1 \mathrm{H}), 3.47-3.41(\mathrm{~m}, 1 \mathrm{H}), 3.13-3.07(\mathrm{~m}, 1 \mathrm{H}), 2.90-2.82(\mathrm{~m}, 2 \mathrm{H}) ;{ }^{13} \mathrm{C}-\mathrm{NMR}\left(150 \mathrm{MHz}, \mathrm{CDCl}_{3}\right)$ 
$\delta 144.6,144.2,140.7,129.3,127.2,126.4,125.4,125.0,118.4,71.5,33.0,31.2$; HRMS Calculated for $\mathrm{C}_{16} \mathrm{H}_{16} \mathrm{~N}_{3}[\mathrm{M}+\mathrm{H}]^{+}:$250.1344, Found: 250.1349 .

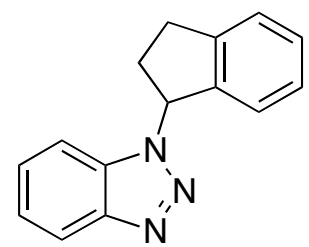

$\mathrm{N}-1-1.6 .3 j$

2-(2,3-dihydro-1H-inden-2-yl)-2H-benzo[d][1,2,3]triazole $(\mathbf{N}-1-1.6 .3 j)$ was purified by flash chromatography (Hexane-EtOAc, v/v 10/1) as yellow liquid. ${ }^{1} \mathrm{H}-\mathrm{NMR}\left(600 \mathrm{MHz}, \mathrm{CDCl}_{3}\right) \delta 8.04$ (d, $J=7.2 \mathrm{~Hz}, 1 \mathrm{H}), 7.40(\mathrm{~d}, J=7.2 \mathrm{~Hz}, 1 \mathrm{H}), 7.33(\mathrm{t}, J=7.2 \mathrm{~Hz}, 1 \mathrm{H}), 7.31-7.24(\mathrm{~m}, 2 \mathrm{H}), 7.14(\mathrm{t}$, $J=7.2 \mathrm{~Hz}, 1 \mathrm{H}), 6.98(\mathrm{~d}, J=7.2 \mathrm{~Hz}, 1 \mathrm{H}), 6.91(\mathrm{~d}, J=7.2 \mathrm{~Hz}, 1 \mathrm{H}), 6.63(\mathrm{t}, J=7.2 \mathrm{~Hz}, 1 \mathrm{H}), 3.31$ (ddd, $J=15.6 \mathrm{~Hz}, 9.0 \mathrm{~Hz}, 4.2 \mathrm{~Hz}, 1 \mathrm{H}$ ), 3.13 (quin, $J=7.2 \mathrm{~Hz}, 1 \mathrm{H}$ ), 2.89-2.82 (m, 1H), 2.56-2.49 $(\mathrm{m}, 1 \mathrm{H}) ;{ }^{13} \mathrm{C}-\mathrm{NMR}\left(150 \mathrm{MHz}, \mathrm{CDCl}_{3}\right) \delta 147.0,143.7,139.5,131.9,129.3,127.4,127.2,125.5$, 125.0, 124.0, 120.4, 110.6, 65.1, 32.8, 31.0; HRMS Calculated for $\mathrm{C}_{16} \mathrm{H}_{16} \mathrm{~N}_{3}[\mathrm{M}+\mathrm{H}]^{+}: 250.1344$, Found: 250.1343

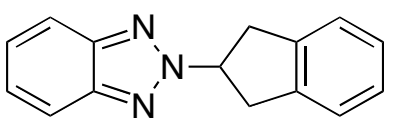

N-2-1.6.3k

2-(2,3-dihydro-1H-inden-2-yl)-2H-benzo[d][1,2,3]triazole (N-2-1.6.3k) was purified by flash chromatography (Hexane-EtOAc, v/v 10/1) as yellow liquid. ${ }^{1} \mathrm{H}-\mathrm{NMR}\left(600 \mathrm{MHz}, \mathrm{CDCl}_{3}\right) \delta$ 7.89-7.84 (m, 2H), 7.40-7.35 (m, 2H), 7.32-7.29 (m, 2H), 7.27-7.23 (m, 2H), $5.80(\mathrm{tt}, J=8.4 \mathrm{~Hz}$ 
$6.6 \mathrm{~Hz}, 1 \mathrm{H}), 3.84(\mathrm{~d}, J=6.6 \mathrm{~Hz}, 1 \mathrm{H}), 3.82(\mathrm{~d}, J=6.6 \mathrm{~Hz}, 1 \mathrm{H}), 3.71(\mathrm{~d}, J=8.4 \mathrm{~Hz}, 1 \mathrm{H}), 3.68(\mathrm{~d}$, $J=8.4 \mathrm{~Hz}, 1 \mathrm{H}) ;{ }^{13} \mathrm{C}-\mathrm{NMR}\left(150 \mathrm{MHz}, \mathrm{CDCl}_{3}\right) \delta 144.1,139.8,127.2,126.2,124.5,118.0,66.2$, 39.9; HRMS Calculated for $\mathrm{C}_{16} \mathrm{H}_{16} \mathrm{~N}_{3}[\mathrm{M}+\mathrm{H}]^{+}:$:250.1344, Found: 250.1351 .

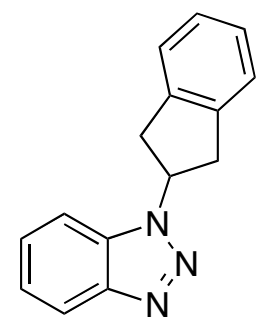

N-1-1.6.3k

2-(2,3-dihydro-1H-inden-2-yl)-2H-benzo[d][1,2,3]triazole $(\mathbf{N}-1-1.6 .3 k)$ was purified by flash chromatography (Hexane-EtOAc, v/v 10/1) as yellow liquid. ${ }^{1} \mathrm{H}-\mathrm{NMR}\left(600 \mathrm{MHz}, \mathrm{CDCl}_{3}\right) \delta 8.07$ (d, $J=8.4 \mathrm{~Hz}, 1 \mathrm{H}), 7.42-7.38(\mathrm{~m}, 1 \mathrm{H}), 7.37-7.27(\mathrm{~m}, 6 \mathrm{H}), 5.82$ (quin, $J=7.2 \mathrm{~Hz}, 1 \mathrm{H}), 3.69(\mathrm{~s}$, 2H), $3.67(\mathrm{~s}, 2 \mathrm{H}) ;{ }^{13} \mathrm{C}-\mathrm{NMR}\left(150 \mathrm{MHz}, \mathrm{CDCl}_{3}\right) \delta 146.6,139.9,132.0,127.4,127.1,124.6$, 123.8, 120.2, 109.9, 59.4, 38.9; HRMS Calculated for $\mathrm{C}_{16} \mathrm{H}_{16} \mathrm{~N}_{3}[\mathrm{M}+\mathrm{H}]^{+}:$250.1344, Found: 250.1353 .

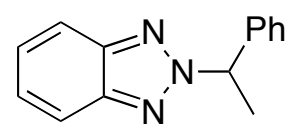

N-2-1.6.3I

2-(1-phenylethyl)-2H-benzo[d][1,2,3]triazole $\quad(\mathbf{N}-2-1.6 .31)$ was purified by flash chromatography (Hexane-EtOAc, v/v 10/1) as yellow liquid. ${ }^{1} \mathrm{H}-\mathrm{NMR}\left(600 \mathrm{MHz}, \mathrm{CDCl}_{3}\right) \delta$ 7.88-7.82 (m, 2H), 7.40-7.38 (m, 2H), 7.37-7.25 (m, 5H), 6.14 (q, $J=7.2 \mathrm{~Hz}, 1 \mathrm{H}), 2.13$ (d, $J=$ 
$7.2 \mathrm{~Hz}, 3 \mathrm{H}) ;{ }^{13} \mathrm{C}-\mathrm{NMR}\left(150 \mathrm{MHz}, \mathrm{CDCl}_{3}\right) \delta 144.4,140.4,129.0,128.5,126.8,126.4,118.4$, 66.4, 21.6; HRMS Calculated for $\mathrm{C}_{14} \mathrm{H}_{14} \mathrm{~N}_{3}[\mathrm{M}+\mathrm{H}]^{+}: 224.1188$, Found: 224.1189 .

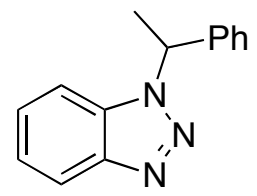

$\mathrm{N}-1-1.6 .31$

1-(1-phenylethyl)-1H-benzo[d][1,2,3]triazole $\quad(\mathbf{N}-1-1.6 .31) \quad$ was purified by flash chromatography (Hexane-EtOAc, v/v 10/1) as yellow liquid. ${ }^{1} \mathrm{H}-\mathrm{NMR}\left(600 \mathrm{MHz}, \mathrm{CDCl}_{3}\right) \delta 8.03$ $(\mathrm{d}, J=7.2 \mathrm{~Hz}, 1 \mathrm{H}), 7.33-7.22(\mathrm{~m}, 8 \mathrm{H}), 6.02(\mathrm{q}, J=7.2 \mathrm{~Hz}, 1 \mathrm{H}), 2.16(\mathrm{~d}, J=7.2 \mathrm{~Hz}, 3 \mathrm{H}) ;{ }^{13} \mathrm{C}-$ NMR $\left(150 \mathrm{MHz}, \mathrm{CDCl}_{3}\right) \delta 146.4,140.1,132.4,128.9,128.2,127.0,126.2,123.8,119.9,110.1$, 59.0, 21.1; HRMS Calculated for $\mathrm{C}_{14} \mathrm{H}_{14} \mathrm{~N}_{3}[\mathrm{M}+\mathrm{H}]^{+}$: 224.1188, Found: 224.1191 .

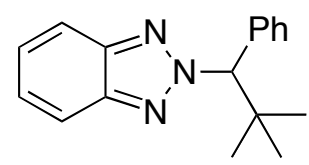

N-2-1.6.3m

2-(2,2-dimethyl-1-phenylpropyl)-2H-benzo[d][1,2,3]triazole $(\mathbf{N}-2-1.6 .3 m)$ was purified by flash chromatography (Hexane-EtOAc, v/v 10/1) as yellow liquid. ${ }^{1} \mathrm{H}-\mathrm{NMR}\left(600 \mathrm{MHz}, \mathrm{CDCl}_{3}\right)$ $\delta$ 7.91-7.86 (m, 2H), 7.77-7.73 (m, 2H), 7.38-7.28 (m, 5H), $5.82(\mathrm{~s}, 1 \mathrm{H}), 1.11(\mathrm{~s}, 9 \mathrm{H}) ;{ }^{13} \mathrm{C}-\mathrm{NMR}$ $\left(150 \mathrm{MHz}, \mathrm{CDCl}_{3}\right) \delta 143.7,135.8,129.6,128.0,127.8,126.0,118.2,80.5,36.9,27.1$; HRMS Calculated for $\mathrm{C}_{17} \mathrm{H}_{20} \mathrm{~N}_{3}[\mathrm{M}+\mathrm{H}]^{+}$: 266.1657, Found: 266.1663 . 


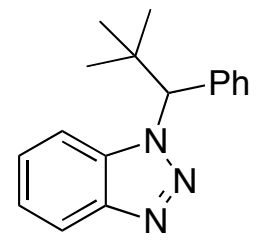

$\mathrm{N}-1-1.6 .3 \mathrm{~m}$

2-(2,2-dimethyl-1-phenylpropyl)-2H-benzo[d][1,2,3]triazole $(\mathbf{N}-1-1.6 .3 m)$ was purified by flash chromatography (Hexane-EtOAc, v/v 10/1) as yellow liquid. ${ }^{1} \mathrm{H}-\mathrm{NMR}\left(600 \mathrm{MHz}, \mathrm{CDCl}_{3}\right)$ $\delta 8.03(\mathrm{~d}, J=8.4 \mathrm{~Hz}, 1 \mathrm{H}), 7.64-7.61(\mathrm{~m}, 2 \mathrm{H}), 7.49(\mathrm{~d}, J=8.4 \mathrm{~Hz}, 1 \mathrm{H}), 7.41(\mathrm{t}, J=7.2 \mathrm{~Hz}, 1 \mathrm{H})$, 7.32-7.23 (m, 4H), $5.43(\mathrm{~s}, 1 \mathrm{H}), 1.13(\mathrm{~s}, 9 \mathrm{H}) ;{ }^{13} \mathrm{C}-\mathrm{NMR}\left(150 \mathrm{MHz}, \mathrm{CDCl}_{3}\right) \delta$ 144.7, 136.4, 134.3, 129.4, 127.9, 127.0, 123.7, 119.8, 109.4, 72.5, 37.2, 27.6; HRMS Calculated for $\mathrm{C}_{17} \mathrm{H}_{20} \mathrm{~N}_{3}$ $[\mathrm{M}+\mathrm{H}]^{+}:$266.1657, Found: 266.1648.

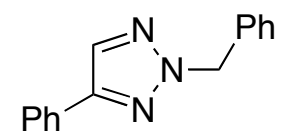

$\mathrm{N}-2-1.6 .4 a$

2-benzyl-4-phenyl-2H-1,2,3-triazole (N-2-1.6.4a) was purified by flash chromatography (Hexane-EtOAc, v/v 10/1) as yellow liquid. ${ }^{1} \mathrm{H}-\mathrm{NMR}\left(600 \mathrm{MHz}, \mathrm{CDCl}_{3}\right) \delta 7.93(\mathrm{~s}, 1 \mathrm{H}), 7.87$ $7.84(\mathrm{~m}, 2 \mathrm{H}), 7.46(\mathrm{t}, J=8.4 \mathrm{~Hz}, 2 \mathrm{H}), 7.43-7.33(\mathrm{~m}, 6 \mathrm{H}), 5.67(\mathrm{~s}, 2 \mathrm{H}) ;{ }^{13} \mathrm{C}-\mathrm{NMR}(150 \mathrm{MHz}$, $\left.\mathrm{CDCl}_{3}\right) \delta 147.9,135.2,131.3,130.2,128.7,128.6,128.3,128.1,127.8,125.8,58.5$; HRMS Calculated for $\mathrm{C}_{15} \mathrm{H}_{14} \mathrm{~N}_{3}[\mathrm{M}+\mathrm{H}]^{+}:$236.1188, Found: 236.1178 . 


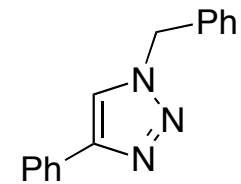

$\mathrm{N}-1-1.6 .4 a$

1-benzyl-4-phenyl-1H-1,2,3-triazole (N-1-1.6.4a) was purified by flash chromatography (Hexane-EtOAc, v/v 10/1) as yellow liquid. ${ }^{1} \mathrm{H}-\mathrm{NMR}\left(600 \mathrm{MHz}, \mathrm{CDCl}_{3}\right) \delta 7.81-7.79(\mathrm{~m}, 2 \mathrm{H})$, $7.66(\mathrm{~s}, 1 \mathrm{H}), 7.41-7.35(\mathrm{~m}, 5 \mathrm{H}), 7.33-7.29(\mathrm{~m}, 3 \mathrm{H}), 5.57(\mathrm{~s}, 2 \mathrm{H}) ;{ }^{13} \mathrm{C}-\mathrm{NMR}\left(150 \mathrm{MHz}, \mathrm{CDCl}_{3}\right) \delta$ 148.2, 134.7, 130.5, 129.1, 128.8, 128.1, 128.0, 125.7, 119.4, 54.2; HRMS Calculated for $\mathrm{C}_{15} \mathrm{H}_{14} \mathrm{~N}_{3}[\mathrm{M}+\mathrm{H}]^{+}:$236.1188, Found: 236.1178 .

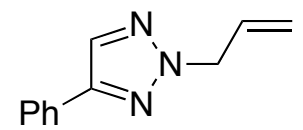

$\mathrm{N}-2-1.6 .4 b$

2-allyl-4-phenyl-2H-1,2,3-triazole $(\mathbf{N}-2-1.6 .4 b)$ was purified by flash chromatography (Hexane-EtOAc, v/v 10/1) as yellow liquid. ${ }^{1} \mathrm{H}-\mathrm{NMR}\left(600 \mathrm{MHz}, \mathrm{CDCl}_{3}\right) \delta 7.86(\mathrm{~s}, 1 \mathrm{H}), 7.81$ $7.78(\mathrm{~m}, 2 \mathrm{H}), 7.40(\mathrm{t}, J=7.8 \mathrm{~Hz}, 2 \mathrm{H}), 7.34-7.30(\mathrm{~m}, 1 \mathrm{H}), 6.15-6.07(\mathrm{~m}, 1 \mathrm{H}), 5.31-5.26(\mathrm{~m}, 2 \mathrm{H})$, $5.05(\mathrm{dt}, J=6.0 \mathrm{~Hz}, 1.2 \mathrm{~Hz}, 2 \mathrm{H}) ;{ }^{13} \mathrm{C}-\mathrm{NMR}\left(150 \mathrm{MHz}, \mathrm{CDCl}_{3}\right) \delta 147.6,131.5,130.9,130.1$, 128.6, 128.1, 125.6, 118.9, 57.1; HRMS Calculated for $\mathrm{C}_{11} \mathrm{H}_{12} \mathrm{~N}_{3}[\mathrm{M}+\mathrm{H}]^{+}$: 186.1031, Found: 186.1032. 


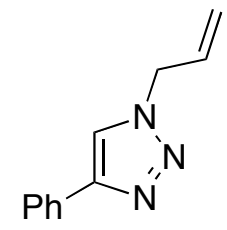

N-1-1.6.4b

1-allyl-4-phenyl-1H-1,2,3-triazole (N-1-1.6.4b) was purified by flash chromatography (Hexane-EtOAc, v/v 10/1) as yellow liquid. ${ }^{1} \mathrm{H}-\mathrm{NMR}\left(600 \mathrm{MHz}, \mathrm{CDCl}_{3}\right) \delta$ 7.84-7.82 (m, 2H), $7.76(\mathrm{~s}, 1 \mathrm{H}), 7.44-7.40(\mathrm{~m}, 2 \mathrm{H}), 7.35-7.31(\mathrm{~m}, 1 \mathrm{H}), 6.10-6.03(\mathrm{~m}, 1 \mathrm{H}), 5.40-5.36(\mathrm{~m}, 1 \mathrm{H})$, 5.34$5.33(\mathrm{~m}, 1 \mathrm{H}), 5.03(\mathrm{dt}, J=6.0 \mathrm{~Hz}, 1.2 \mathrm{~Hz}, 2 \mathrm{H}) ;{ }^{13} \mathrm{C}-\mathrm{NMR}\left(150 \mathrm{MHz}, \mathrm{CDCl}_{3}\right) \delta 148.0,131.3$, 130.6, 128.8, 128.1, 125.7, 120.2, 119.4, 93.0, 52.8; HRMS Calculated for $\mathrm{C}_{11} \mathrm{H}_{12} \mathrm{~N}_{3}[\mathrm{M}+\mathrm{H}]^{+}$: 186.1031, Found: 186.1043.

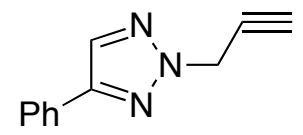

$\mathrm{N}-2-1.6 .4 \mathrm{c}$

4-phenyl-2-(prop-2-ynyl)-2H-1,2,3-triazole (N-2-1.6.4c) was purified by flash chromatography (Hexane-EtOAc, v/v 10/1) as yellow liquid. ${ }^{1} \mathrm{H}-\mathrm{NMR}\left(600 \mathrm{MHz}, \mathrm{CDCl}_{3}\right) \delta 7.89(\mathrm{~s}, 1 \mathrm{H}), 7.80-$ $7.78(\mathrm{~m}, 2 \mathrm{H}), 7.44-7.40(\mathrm{~m}, 2 \mathrm{H}), 7.37-7.33(\mathrm{~m}, 1 \mathrm{H}), 5.25(\mathrm{~d}, J=2.4 \mathrm{~Hz}, 2 \mathrm{H}), 2.54(\mathrm{t}, J=2.4$ $\mathrm{Hz}, 1 \mathrm{H}) ;{ }^{13} \mathrm{C}-\mathrm{NMR}\left(150 \mathrm{MHz}, \mathrm{CDCl}_{3}\right) \delta 148.5,131.7,129.9,128.8,128.5,125.9,75.7,74.8$, 44.4; HRMS Calculated for $\mathrm{C}_{11} \mathrm{H}_{10} \mathrm{~N}_{3}[\mathrm{M}+\mathrm{H}]^{+}:$184.0875, Found: 184.0879 . 


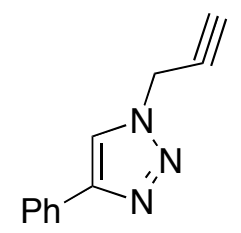

$\mathrm{N}-1-1.6 .4 \mathrm{c}$

4-phenyl-1-(prop-2-ynyl)-1H-1,2,3-triazole (N-1-1.6.4c) was purified by flash chromatography (Hexane-EtOAc, v/v 10/1) as yellow liquid. ${ }^{1} \mathrm{H}-\mathrm{NMR}\left(600 \mathrm{MHz}, \mathrm{CDCl}_{3}\right) \delta 7.97(\mathrm{~s}, 1 \mathrm{H}), 7.83$ $7.81(\mathrm{~m}, 2 \mathrm{H}), 7.42-7.39(\mathrm{~m}, 2 \mathrm{H}), 7.34-7.30(\mathrm{~m}, 1 \mathrm{H}), 5.18(\mathrm{~d}, J=2.4 \mathrm{~Hz}, 2 \mathrm{H}), 2.59(\mathrm{t}, J=2.4 \mathrm{~Hz}$ $1 \mathrm{H}) ;{ }^{13} \mathrm{C}-\mathrm{NMR}\left(150 \mathrm{MHz}, \mathrm{CDCl}_{3}\right) \delta 148.0,130.2,128.7,128.2,125.6,119.3,75.7,75.1,39.7$; HRMS Calculated for $\mathrm{C}_{11} \mathrm{H}_{10} \mathrm{~N}_{3}[\mathrm{M}+\mathrm{H}]^{+}:$184.0875, Found: 184.0885.

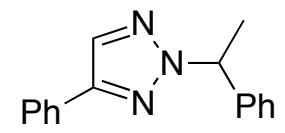

N-2-1.6.4d

4-phenyl-2-(1-phenylethyl)-2H-1,2,3-triazole (N-2-1.6.4d) was purified by flash chromatography (Hexane-EtOAc, v/v 10/1) as yellow liquid. ${ }^{1} \mathrm{H}-\mathrm{NMR}\left(600 \mathrm{MHz}, \mathrm{CDCl}_{3}\right) \delta 7.87$ $(\mathrm{s}, 1 \mathrm{H}), 7.82-7.80(\mathrm{~m}, 2 \mathrm{H}), 7.44-7.41(\mathrm{~m}, 2 \mathrm{H}), 7.37-7.32(\mathrm{~m}, 5 \mathrm{H}), 7.31-7.27(\mathrm{~m}, 1 \mathrm{H}), 5.89(\mathrm{q}, J=$ $7.2 \mathrm{~Hz}, 1 \mathrm{H}), 2.04(\mathrm{~d}, J=7.2 \mathrm{~Hz}, 3 \mathrm{H}) ;{ }^{13} \mathrm{C}-\mathrm{NMR}\left(150 \mathrm{MHz}, \mathrm{CDCl}_{3}\right) \delta 147.4,140.9,130.9$, 130.6, 128.8, 128.6, 128.3, 128.0, 126.4, 125.9, 64.5, 21.3; HRMS Calculated for $\mathrm{C}_{16} \mathrm{H}_{16} \mathrm{~N}_{3}$ $[\mathrm{M}+\mathrm{H}]^{+}: 250.1344$, Found: 250.1343. 


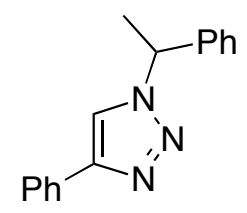

N-1-1.6.4d

4-phenyl-1-(1-phenylethyl)-1H-1,2,3-triazole $\quad(\mathbf{N}-1-1.6 .4 d) \quad$ was purified by flash chromatography (Hexane-EtOAc, v/v 10/1) as yellow liquid. ${ }^{1} \mathrm{H}-\mathrm{NMR}\left(600 \mathrm{MHz}, \mathrm{CDCl}_{3}\right) \delta$ 7.82-7.79 (m, 2H), $7.66(\mathrm{~s}, 1 \mathrm{H}), 7.41-7.28(\mathrm{~m}, 8 \mathrm{H}), 5.86(\mathrm{q}, J=7.2 \mathrm{~Hz}, 1 \mathrm{H}), 2.02(\mathrm{~d}, J=7.2 \mathrm{~Hz}$ $3 \mathrm{H}) ;{ }^{13} \mathrm{C}-\mathrm{NMR}\left(150 \mathrm{MHz}, \mathrm{CDCl}_{3}\right) \delta 147.7,139.9,130.6,129.0,128.7,128.5,128.0,126.4$, 125.6, 118.3, 60.2, 21.2; HRMS Calculated for $\mathrm{C}_{16} \mathrm{H}_{16} \mathrm{~N}_{3}[\mathrm{M}+\mathrm{H}]^{+}$: 250.1344, Found: 250.1352.

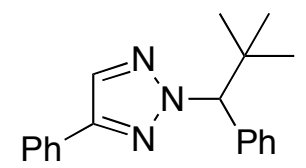

$\mathrm{N}-2-1.6 .4 \mathrm{e}$

2-(2,2-dimethyl-1-phenylpropyl)-4-phenyl-2H-1,2,3-triazole (N-2-1.6.4e) was purified by flash chromatography (Hexane-EtOAc, v/v 10/1) as yellow liquid. ${ }^{1} \mathrm{H}-\mathrm{NMR}\left(600 \mathrm{MHz}, \mathrm{CDCl}_{3}\right)$ $\delta 7.85(\mathrm{~s}, 1 \mathrm{H}), 7.84-7.82(\mathrm{~m}, 2 \mathrm{H}), 7.70-7.67(\mathrm{~m}, 2 \mathrm{H}), 7.45-7.41(\mathrm{~m}, 2 \mathrm{H}), 7.36-7.27(\mathrm{~m}, 4 \mathrm{H}), 5.55$ (s, 1H), $1.08(\mathrm{~s}, 9 \mathrm{H}) ;{ }^{13} \mathrm{C}-\mathrm{NMR}\left(150 \mathrm{MHz}, \mathrm{CDCl}_{3}\right) \delta 146.9,136.3,130.7,130.1,129.5,128.8$, 128.2, 127.7, 125.9, 78.8, 36.8, 27.1; HRMS Calculated for $\mathrm{C}_{19} \mathrm{H}_{22} \mathrm{~N}_{3}[\mathrm{M}+\mathrm{H}]^{+}:$292.1814, Found: 292.1810. 


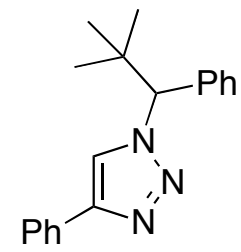

$\mathrm{N}-1-1.6 .4 e$

2-(2,2-dimethyl-1-phenylpropyl)-4-phenyl-2H-1,2,3-triazole (N-1-1.6.4e) was purified by flash chromatography (Hexane-EtOAc, v/v 10/1) as yellow liquid. ${ }^{1} \mathrm{H}-\mathrm{NMR}\left(600 \mathrm{MHz}, \mathrm{CDCl}_{3}\right)$ $\delta 7.85(\mathrm{~s}, 1 \mathrm{H}), 7.84-7.82(\mathrm{~m}, 2 \mathrm{H}), 7.60-7.57(\mathrm{~m}, 2 \mathrm{H}), 7.41(\mathrm{t}, J=7.8 \mathrm{~Hz}, 2 \mathrm{H}), 7.37-7.31(\mathrm{~m}$, 4H), $5.30(\mathrm{~s}, 1 \mathrm{H}), 1.11(\mathrm{~s}, 9 \mathrm{H}) ;{ }^{13} \mathrm{C}-\mathrm{NMR}\left(150 \mathrm{MHz}, \mathrm{CDCl}_{3}\right) \delta 136.3,130.7,129.3,128.8,128.1$, 128.0, 125.6, 120.6, 75.8, 36.6, 27.5; HRMS Calculated for $\mathrm{C}_{19} \mathrm{H}_{22} \mathrm{~N}_{3}[\mathrm{M}+\mathrm{H}]^{+}:$292.1814, Found: 292.1810.

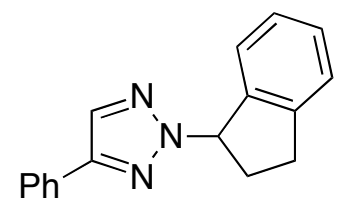

N-2-1.6.4f

2-(2,3-dihydro-1H-inden-1-yl)-4-phenyl-2H-1,2,3-triazole (N-2-1.6.4f) was purified by flash chromatography (Hexane-EtOAc, v/v 10/1) as yellow liquid. ${ }^{1} \mathrm{H}-\mathrm{NMR}\left(600 \mathrm{MHz}, \mathrm{CDCl}_{3}\right) \delta 7.86$ $(\mathrm{s}, 1 \mathrm{H}), 7.79(\mathrm{~d}, J=7.8 \mathrm{~Hz}, 2 \mathrm{H}), 7.42(\mathrm{t}, J=7.8 \mathrm{~Hz}, 2 \mathrm{H}), 7.35-7.32(\mathrm{~m}, 2 \mathrm{H}), 7.30(\mathrm{t}, J=7.8 \mathrm{~Hz}$ 1H), $7.24(\mathrm{~d}, J=7.8 \mathrm{~Hz}, 1 \mathrm{H}), 7.20(\mathrm{t}, J=7.8 \mathrm{~Hz}, 1 \mathrm{H}), 6.22(\mathrm{t}, J=7.8 \mathrm{~Hz}, 1 \mathrm{H}), 3.39-3.33(\mathrm{~m}$, 1H), 3.08-3.02 (m, 1H), 2.82-2.72 (m, 2H); ${ }^{13} \mathrm{C}-\mathrm{NMR}\left(150 \mathrm{MHz}, \mathrm{CDCl}_{3}\right) \delta 147.5,143.7,140.7$, 131.0, 130.5, 128.7, 128.2, 126.8, 125.9, 125.0, 124.7, 69.6, 32.2, 30.7; HRMS Calculated for $\mathrm{C}_{18} \mathrm{H}_{18} \mathrm{~N}_{3}[\mathrm{M}+\mathrm{H}]^{+}:$276.1501, Found: 276.1498 . 


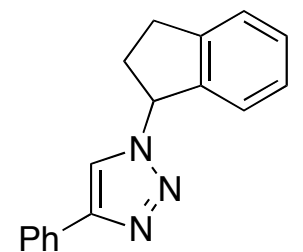

N-1-1.6.4f

1-(2,3-dihydro-1H-inden-1-yl)-4-phenyl-1H-1,2,3-triazole (N-1-1.6.4f) was purified by flash chromatography (Hexane-EtOAc, v/v 10/1) as yellow liquid. ${ }^{1} \mathrm{H}-\mathrm{NMR}\left(600 \mathrm{MHz}, \mathrm{CDCl}_{3}\right) \delta$ 7.80-7.77 (m, 2H), $7.48(\mathrm{~s}, 1 \mathrm{H}), 7.40-7.35(\mathrm{~m}, 4 \mathrm{H}), 7.32-7.28(\mathrm{~m}, 2 \mathrm{H}), 7.28-7.21(\mathrm{~m}, 1 \mathrm{H}), 6.31-$ $6.28(\mathrm{~m}, 1 \mathrm{H}), 3.25-3.19(\mathrm{~m}, 1 \mathrm{H}), 3.10-3.04(\mathrm{~m}, 1 \mathrm{H}), 2.90-2.83(\mathrm{~m}, 1 \mathrm{H}), 2.45-2.39(\mathrm{~m}, 1 \mathrm{H}) ;{ }^{13} \mathrm{C}-$ NMR $\left(150 \mathrm{MHz}, \mathrm{CDCl}_{3}\right) \delta 147.9,143.9,139.8,130.7,129.4,128.7,128.1,127.4,125.7,125.3$, 124.9, 117.6, 65.5, 34.2, 30.4; HRMS Calculated for $\mathrm{C}_{18} \mathrm{H}_{18} \mathrm{~N}_{3}[\mathrm{M}+\mathrm{H}]^{+}$: 276.1501, Found: 276.1495 .

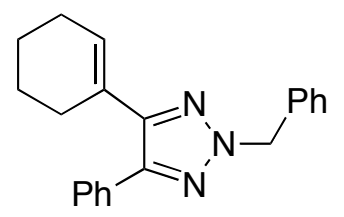

N-2-1.6.4j

2-benzyl-4-cyclohexenyl-5-phenyl-2H-1,2,3-triazole (N-2-1.6.4j) was purified by flash chromatography (Hexane-EtOAc, v/v 10/1) as yellow liquid. ${ }^{1} \mathrm{H}-\mathrm{NMR}\left(600 \mathrm{MHz}, \mathrm{CDCl}_{3}\right) \delta$ 7.71-7.68 (m, 2H), 7.43-7.40 (m, 4H), 7.38-7.35 (m, 3H), 7.34-7.30 (m, 1H), 6.07 (sep, $J=1.8$ $\mathrm{Hz}, 1 \mathrm{H}), 5.59(\mathrm{~s}, 2 \mathrm{H}), 7.38-7.34(\mathrm{~m}, 2 \mathrm{H}), 2.18-2.14(\mathrm{~m}, 2 \mathrm{H}), 1.79-1.73(\mathrm{~m}, 2 \mathrm{H}), 1.72-1.67(\mathrm{~m}$, $2 \mathrm{H}) ;{ }^{13} \mathrm{C}-\mathrm{NMR}\left(150 \mathrm{MHz}, \mathrm{CDCl}_{3}\right) \delta 146.6,144.0,135.4,131.6,129.2,128.6,128.5,128.3$, 128.0, 127.9, 127.8, 127.7, 58.3, 27.6, 25.3, 22.6, 21.8,; HRMS Calculated for $\mathrm{C}_{21} \mathrm{H}_{22} \mathrm{~N}_{3}$ $[\mathrm{M}+\mathrm{H}]^{+}:$316.1814, Found: 316.1821 . 


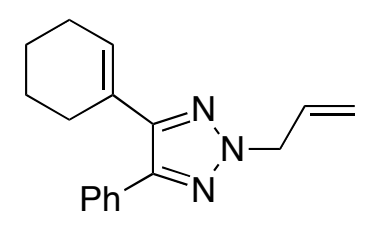

N-2-1.6.4k

2-allyl-4-cyclohexenyl-5-phenyl-2H-1,2,3-triazole $\quad(\mathbf{N}-2-1.6 .4 k)$ was purified by flash chromatography (Hexane-EtOAc, v/v 10/1) as yellow liquid. ${ }^{1} \mathrm{H}-\mathrm{NMR}\left(600 \mathrm{MHz}, \mathrm{CDCl}_{3}\right) \delta$ 7.66-7.64 (m, 2H), 7.38 (t, $J=7.2 \mathrm{~Hz}, 2 \mathrm{H}), 7.34-7.30$ (m, 1H), 6.16-6.08 (m, 1H), 6.03 (sep, $J=$ $1.8 \mathrm{~Hz}, 1 \mathrm{H}), 5.33-5.28(\mathrm{~m}, 2 \mathrm{H}), 5.01(\mathrm{~d}, J=7.2 \mathrm{~Hz}, 2 \mathrm{H}), 2.34-2.30(\mathrm{~m}, 2 \mathrm{H}), 2.15-2.11(\mathrm{~m}, 2 \mathrm{H})$, $1.75-1.70(\mathrm{~m}, 2 \mathrm{H}), 1.68-1.63(\mathrm{~m}, 2 \mathrm{H}) ;{ }^{13} \mathrm{C}-\mathrm{NMR}\left(150 \mathrm{MHz}, \mathrm{CDCl}_{3}\right) \delta 146.5,143.9,131.7$, 131.6, 129.2, 128.5, 128.3, 127.8, 119.0, 57.1, 27.5, 25.3, 22.5, 21.8; HRMS Calculated for $\mathrm{C}_{17} \mathrm{H}_{20} \mathrm{~N}_{3}[\mathrm{M}+\mathrm{H}]^{+}:$266.1657, Found: 266.1650 .

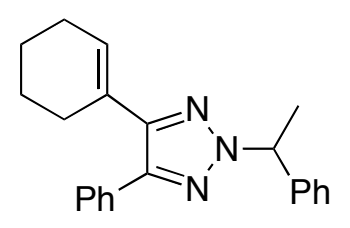

N-2-1.6.4

4-cyclohexenyl-5-phenyl-2-(1-phenylethyl)-2H-1,2,3-triazole (N-2-1.6.4l) was purified by flash chromatography (Hexane-EtOAc, v/v 10/1) as yellow liquid. ${ }^{1} \mathrm{H}-\mathrm{NMR}\left(600 \mathrm{MHz}, \mathrm{CDCl}_{3}\right)$ $\delta$ 7.78-7.76 (m, 2H), $7.45(\mathrm{t}, J=7.2 \mathrm{~Hz}, 4 \mathrm{H}), 7.40-7.36(\mathrm{~m}, 3 \mathrm{H}), 7.32(\mathrm{tt}, J=7.2 \mathrm{~Hz}, 1.2 \mathrm{~Hz}$, 1H), $6.13(\mathrm{sep}, J=1.8 \mathrm{~Hz}, 1 \mathrm{H}), 5.91(\mathrm{q}, J=7.2 \mathrm{~Hz}, 1 \mathrm{H}), 2.46-2.41(\mathrm{~m}, 2 \mathrm{H}), 2.22-2.18(\mathrm{~m}, 2 \mathrm{H})$, $2.07(\mathrm{~d}, J=7.2 \mathrm{~Hz}, 3 \mathrm{H}), 1.83-1.78(\mathrm{~m}, 2 \mathrm{H}), 1.76-1.71(\mathrm{~m}, 2 \mathrm{H}) ;{ }^{13} \mathrm{C}-\mathrm{NMR}\left(150 \mathrm{MHz}, \mathrm{CDCl}_{3}\right) \delta$ 
$145.9,143.4,141.0,131.8,128.9,128.7,128.4,128.2,127.8,127.7,127.6,126.3,64.1,27.5$, 25.3, 22.5, 21.7, 21.3; HRMS Calculated for $\mathrm{C}_{22} \mathrm{H}_{24} \mathrm{~N}_{3}[\mathrm{M}+\mathrm{H}]^{+}: 330.1970$, Found: 330.1965 .

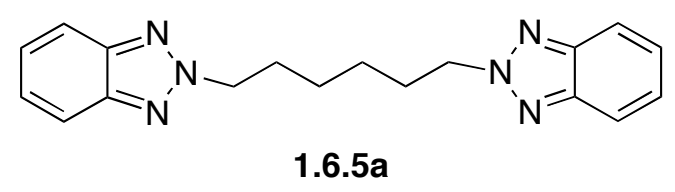

1,6-di(2H-benzo[d][1,2,3]triazol-2-yl)hexane (1.6.5a) was purified by flash chromatography (Hexane-EtOAc, v/v 10/1) as yellow liquid. ${ }^{1} \mathrm{H}-\mathrm{NMR}\left(600 \mathrm{MHz}, \mathrm{CDCl}_{3}\right) \delta$ 7.86-7.82 (m, 4H), 7.39-7.35 (m, 4H), $4.71(\mathrm{t}, J=7.2 \mathrm{~Hz}, 4 \mathrm{H}), 2.14-2.09(\mathrm{~m}, 4 \mathrm{H}), 1.43-1.40(\mathrm{~m}, 4 \mathrm{H}) ;{ }^{13} \mathrm{C}-\mathrm{NMR}$ $\left(150 \mathrm{MHz}, \mathrm{CDCl}_{3}\right) \delta 144.3,126.2,117.9,56.3,29.8,26.0$; HRMS Calculated for $\mathrm{C}_{18} \mathrm{H}_{21} \mathrm{~N}_{6}$ $[\mathrm{M}+\mathrm{H}]^{+}:$321.1828, Found: 321.1820 .

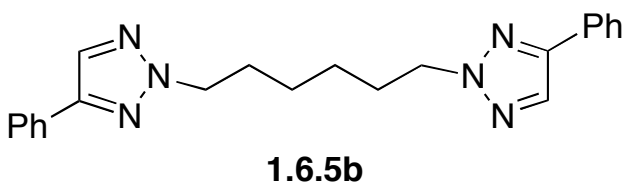

1,6-bis(4-phenyl-2H-1,2,3-triazol-2-yl)hexane (1.6.5b) was purified by flash chromatography (Hexane-EtOAc, v/v 10/1) as yellow liquid. ${ }^{1} \mathrm{H}-\mathrm{NMR}\left(600 \mathrm{MHz}, \mathrm{CDCl}_{3}\right) \delta 7.80$ (s, 2H), 7.787.76 (m, 4H), 7.42 (t, $J=7.2 \mathrm{~Hz}, 4 \mathrm{H}), 7.34$ (tt, $J=7.2 \mathrm{~Hz}, 1.2 \mathrm{~Hz}, 2 \mathrm{H}), 7.34$ (t, $J=7.2 \mathrm{~Hz}, 4 \mathrm{H})$, 2.03-1.98 (m, 4H), 1.42-1.39 (m, 4H); ${ }^{13} \mathrm{C}-\mathrm{NMR}\left(150 \mathrm{MHz}, \mathrm{CDCl}_{3}\right) \delta 147.7,130.6,130.5$, 128.8, 128.3, 125.8, 54.8, 29.5, 26.0; HRMS Calculated for $\mathrm{C}_{22} \mathrm{H}_{25} \mathrm{~N}_{6}[\mathrm{M}+\mathrm{H}]^{+}: 373.2141$, Found: 373.2150 . 


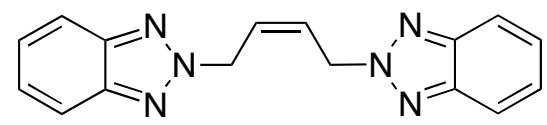

$1.6 .5 c$

(Z)-1,4-bis(4-phenyl-2H-1,2,3-triazol-2-yl)but-2-ene $\quad(\mathbf{1 . 6 . 5 c})$ was purified by flash chromatography (Hexane-EtOAc, v/v 10/1) as yellow liquid. ${ }^{1} \mathrm{H}-\mathrm{NMR}\left(600 \mathrm{MHz}, \mathrm{CDCl}_{3}\right) \delta$ 7.88-7.85 (m, 4H), 7.40-7.36 (m, 4H), $6.27(\mathrm{t}, J=6.0 \mathrm{~Hz}, 2 \mathrm{H}), 5.62(\mathrm{~d}, J=6.0 \mathrm{~Hz}, 4 \mathrm{H}) ;{ }^{13} \mathrm{C}-$ NMR $\left(150 \mathrm{MHz}, \mathrm{CDCl}_{3}\right) \delta 144.6,127.8,126.5,118.0,52.9$; HRMS Calculated for $\mathrm{C}_{16} \mathrm{H}_{15} \mathrm{~N}_{6}$ $[\mathrm{M}+\mathrm{H}]^{+}:$291.1358, Found: 291.1350 .

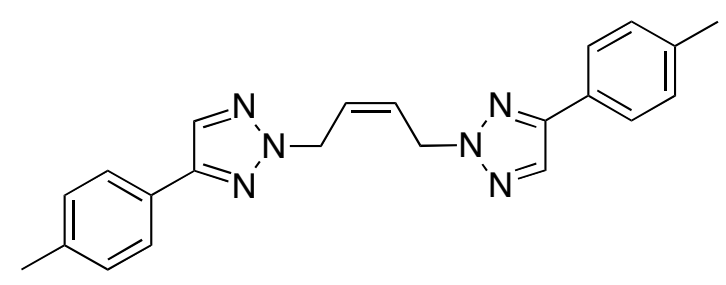

1.6.5d

(Z)-1,4-bis(4-p-tolyl-2H-1,2,3-triazol-2-yl)but-2-ene $\quad(\mathbf{1 . 6 . 5 d})$ was purified by flash chromatography (Hexane-EtOAc, v/v 10/1) as yellow liquid. ${ }^{1} \mathrm{H}-\mathrm{NMR}\left(600 \mathrm{MHz}, \mathrm{CDCl}_{3}\right) \delta 7.83$ $(\mathrm{s}, 2 \mathrm{H}), 7.68(\mathrm{~d}, J=7.8 \mathrm{~Hz}, 4 \mathrm{H}), 7.23(\mathrm{~d}, J=7.8 \mathrm{~Hz}, 4 \mathrm{H}), 6.13(\mathrm{t}, J=4.8 \mathrm{~Hz}, 2 \mathrm{H}), 5.29(\mathrm{~d}, J=$ $4.8 \mathrm{~Hz}, 4 \mathrm{H}), 2.39(\mathrm{~s}, 6 \mathrm{H}) ;{ }^{13} \mathrm{C}-\mathrm{NMR}\left(150 \mathrm{MHz}, \mathrm{CDCl}_{3}\right) \delta 148.2,138.3,131.0,129.5,127.6$, 127.4, 125.8, 51.5, 21.2; HRMS Calculated for $\mathrm{C}_{22} \mathrm{H}_{23} \mathrm{~N}_{6}[\mathrm{M}+\mathrm{H}]^{+}: 371.1984$, Found: 371.1989 .

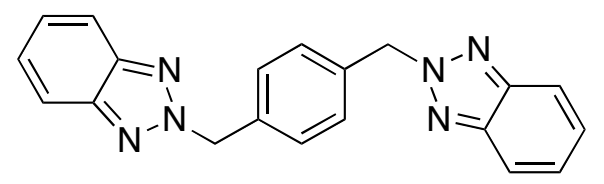

1.6.5e 
1,4-bis((2H-benzo[d][1,2,3]triazol-2-yl)methyl)benzene (1.6.5e) was purified by flash chromatography (Hexane-EtOAc, v/v 10/1) as yellow liquid. ${ }^{1} \mathrm{H}-\mathrm{NMR}\left(600 \mathrm{MHz}, \mathrm{CDCl}_{3}\right) \delta$ 7.85-7.82 (m, 4H), $7.41(\mathrm{~s}, 4 \mathrm{H}), 7.37-7.34(\mathrm{~m}, 4 \mathrm{H}), 5.84(\mathrm{~s}, 4 \mathrm{H}) ;{ }^{13} \mathrm{C}-\mathrm{NMR}\left(150 \mathrm{MHz}, \mathrm{CDCl}_{3}\right) \delta$ 144.6, 135.1, 128.9, 126.4, 118.1, 59.9; HRMS Calculated for $\mathrm{C}_{20} \mathrm{H}_{17} \mathrm{~N}_{6}[\mathrm{M}+\mathrm{H}]^{+}$: 341.1515, Found: 341.1519 .

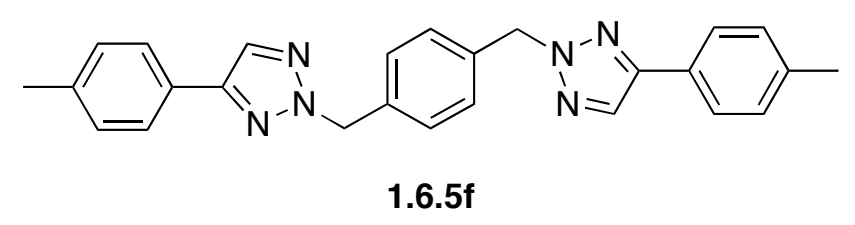

1,4-bis((4-p-tolyl-2H-1,2,3-triazol-2-yl)methyl)benzene (1.6.5f) was purified by flash chromatography (Hexane-EtOAc, v/v 10/1) as yellow liquid. ${ }^{1} \mathrm{H}-\mathrm{NMR}\left(600 \mathrm{MHz}, \mathrm{CDCl}_{3}\right) \delta 7.79$ (s, 2H), 7.63 (d, $J=7.8 \mathrm{~Hz}, 4 \mathrm{H}), 7.31(\mathrm{~s}, 4 \mathrm{H}), 7.20$ (d, $J=7.8 \mathrm{~Hz}, 4 \mathrm{H}), 5.57$ (s, 4H), 2.36 (s, $6 \mathrm{H}) ;{ }^{13} \mathrm{C}-\mathrm{NMR}\left(150 \mathrm{MHz}, \mathrm{CDCl}_{3}\right) \delta$ 148.2, 138.3, 135.5, 131.3, 129.5, 128.4, 127.4, 125.8, 58.2, 21.3; HRMS Calculated for $\mathrm{C}_{26} \mathrm{H}_{25} \mathrm{~N}_{6}[\mathrm{M}+\mathrm{H}]^{+}$: 421.2141, Found: 421.2137 .

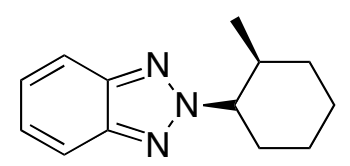

N-2-1.6.6a

cis-2-(2-methylcyclohexyl)-2H-benzo[d][1,2,3]triazole (N-2-1.6.6a) was purified by flash chromatography (Hexane-EtOAc, v/v 10/1) as yellow liquid. ${ }^{1} \mathrm{H}-\mathrm{NMR}\left(600 \mathrm{MHz}, \mathrm{CDCl}_{3}\right) \delta$ 7.89-7.85 (m, 2H), 7.37-7.33 (m, 2H), 4.97 (quin, $J=4.8 \mathrm{~Hz}, 1 \mathrm{H}), 2.58-2.53(\mathrm{~m}, 1 \mathrm{H}), 2.44-2.36$ (m, 1H), 2.17-2.03 (m, 2H), 1.89-1.83 (m, 1H), 1.76-1.64 (m, 2H), 1.55-1.46 (m, 2H), $0.70(\mathrm{~d}, J$ 
$=7.2 \mathrm{~Hz}, 3 \mathrm{H}) ;{ }^{13} \mathrm{C}-\mathrm{NMR}\left(150 \mathrm{MHz}, \mathrm{CDCl}_{3}\right) \delta 143.7,125.8,118.0,67.9,34.8,30.8,26.7,24.1$, 21.2, 13.5; HRMS Calculated for $\mathrm{C}_{13} \mathrm{H}_{18} \mathrm{~N}_{3}[\mathrm{M}+\mathrm{H}]^{+}: 216.1501$, Found: 216.1498 .

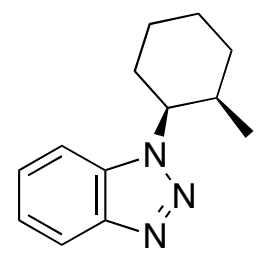

N-1-1.6.6a

cis-1-(2-methylcyclohexyl)-1H-benzo[d][1,2,3]triazole (N-1-1.6.6a) was purified by flash chromatography (Hexane-EtOAc, v/v 10/1) as yellow liquid. ${ }^{1} \mathrm{H}-\mathrm{NMR}\left(600 \mathrm{MHz}, \mathrm{CDCl}_{3}\right) \delta 8.06$ (d, $J=8.4 \mathrm{~Hz}, 1 \mathrm{H}), 7.53(\mathrm{~d}, J=8.4 \mathrm{~Hz}, 1 \mathrm{H}), 7.44$ (t, $J=8.4 \mathrm{~Hz}, 1 \mathrm{H}), 7.34(\mathrm{t}, J=8.4 \mathrm{~Hz}, 1 \mathrm{H})$, 4.82 (quin, $J=4.8 \mathrm{~Hz}, 1 \mathrm{H}), 2.55-2.48(\mathrm{~m}, 1 \mathrm{H}), 2.43-2.36(\mathrm{~m}, 1 \mathrm{H}), 2.12-2.04(\mathrm{~m}, 2 \mathrm{H}), 1.92-1.88$ (m, 1H), 1.78-1.69 (m, 2H), 1.59-1.50 (m, 2H), $0.72(\mathrm{~d}, J=7.2 \mathrm{~Hz}, 3 \mathrm{H}) ;{ }^{13} \mathrm{C}-\mathrm{NMR}(150 \mathrm{MHz}$, $\left.\mathrm{CDCl}_{3}\right) \delta 145.7,133.2,126.7,123.6,120.1,109.7,60.8,34.2,30.9,27.0,24.4,21.4,14.0$ HRMS Calculated for $\mathrm{C}_{13} \mathrm{H}_{18} \mathrm{~N}_{3}[\mathrm{M}+\mathrm{H}]^{+}:$216.1501, Found: 216.1495.

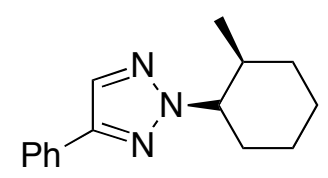

$\mathrm{N}-2-1.6 .6 b$

cis-2-(2-methylcyclohexyl)-4-phenyl-2H-1,2,3-triazole (N-2-1.6.6b) was purified by flash chromatography (Hexane-EtOAc, v/v 10/1) as yellow liquid. ${ }^{1} \mathrm{H}-\mathrm{NMR}\left(600 \mathrm{MHz}, \mathrm{CDCl}_{3}\right) \delta 7.83$ (s, 1H), 7.80 (d, $J=7.2 \mathrm{~Hz}, 2 \mathrm{H}), 7.42$ (t, $J=7.2 \mathrm{~Hz}, 2 \mathrm{H}), 7.33$ (tt, $J=7.2 \mathrm{~Hz}, 1.2 \mathrm{~Hz}, 1 \mathrm{H}), 4.71$ 
(quin, $J=4.8 \mathrm{~Hz}, 1 \mathrm{H}), 2.46-2.39(\mathrm{~m}, 1 \mathrm{H}), 2.31-2.23(\mathrm{~m}, 1 \mathrm{H}), 2.06-1.99(\mathrm{~m}, 2 \mathrm{H}), 1.86-1.78(\mathrm{~m}$, $1 \mathrm{H}), 1.71-1.62(\mathrm{~m}, 2 \mathrm{H}), 1.52-1.44(\mathrm{~m}, 2 \mathrm{H}), 0.75(\mathrm{~d}, J=7.2 \mathrm{~Hz}, 3 \mathrm{H}) ;{ }^{13} \mathrm{C}-\mathrm{NMR}(150 \mathrm{MHz}$, $\left.\mathrm{CDCl}_{3}\right) \delta 146.7,130.9,129.9,128.7,128.0,125.8,66.1,34.5,30.5,26.8,23.8,21.5,13.9$; HRMS Calculated for $\mathrm{C}_{15} \mathrm{H}_{20} \mathrm{~N}_{3}[\mathrm{M}+\mathrm{H}]^{+}: 242.1657$, Found: 242.1655 .

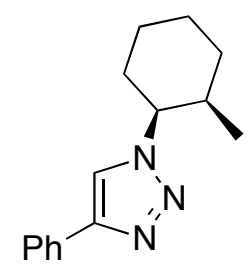

$\mathrm{N}-1-1.6 .6 b$

cis-1-(2-methylcyclohexyl)-4-phenyl-1H-1,2,3-triazole (N-1-1.6.6b) was purified by flash chromatography (Hexane-EtOAc, v/v 10/1) as yellow liquid. ${ }^{1} \mathrm{H}-\mathrm{NMR}\left(600 \mathrm{MHz}, \mathrm{CDCl}_{3}\right) \delta 7.84$ (d, $J=7.2 \mathrm{~Hz}, 2 \mathrm{H}), 7.75(\mathrm{~s}, 1 \mathrm{H}), 7.42$ (t, $J=7.2 \mathrm{~Hz}, 2 \mathrm{H}), 7.32$ (tt, $J=7.2 \mathrm{~Hz}, 1.2 \mathrm{~Hz}, 1 \mathrm{H}), 4.82$ (dt, $J=10.8 \mathrm{~Hz}, 4.8 \mathrm{~Hz}, 1 \mathrm{H}), 2.47-2.43(\mathrm{~m}, 1 \mathrm{H}), 2.17-2.09$ (m, 1H), 2.04-1.95 (m, 2H), 1.75$1.69(\mathrm{~m}, 2 \mathrm{H}), 1.68-1.57(\mathrm{~m}, 1 \mathrm{H}), 1.56-1.48(\mathrm{~m}, 2 \mathrm{H}), 0.78(\mathrm{~d}, J=7.2 \mathrm{~Hz}, 3 \mathrm{H}) ;{ }^{13} \mathrm{C}-\mathrm{NMR}(150$ $\left.\mathrm{MHz}, \mathrm{CDCl}_{3}\right) \delta 130.9,128.8,127.9,125.6,118.5,62.4,34.2,30.8,26.5,24.6,20.7,13.1 ;$ HRMS Calculated for $\mathrm{C}_{15} \mathrm{H}_{20} \mathrm{~N}_{3}[\mathrm{M}+\mathrm{H}]^{+}: 242.1657$, Found: 242.1655 .

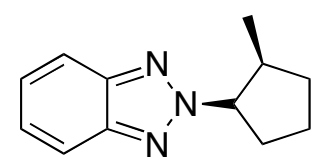

N-2-1.6.6c 
cis-2-(2-methylcyclopentyl)-2H-benzo[d][1,2,3] triazole (N-2-1.6.6c) was purified by flash chromatography (Hexane-EtOAc, v/v 10/1) as yellow liquid. ${ }^{1} \mathrm{H}-\mathrm{NMR}\left(600 \mathrm{MHz}, \mathrm{CDCl}_{3}\right) \delta$ 7.88-7.84 (m, 2H), 7.34-7.31 (m, 2H), 5.31-5.27 (m, 1H), 2.62-2.55 (m, 1H), 2.48-2.33 (m, 2H), 2.23-2.16 (m, 1H), 1.93-1.87 (m, 1H), 1.81-1.71 (m, 2H), $0.55(\mathrm{~d}, J=7.2 \mathrm{~Hz}, 3 \mathrm{H}),{ }^{13} \mathrm{C}-\mathrm{NMR}$ $\left(150 \mathrm{MHz}, \mathrm{CDCl}_{3}\right) \delta 143.8,125.8,117.9,70.8,40.5,32.5,31.5,23.6,13.8$; HRMS Calculated for $\mathrm{C}_{12} \mathrm{H}_{16} \mathrm{~N}_{3}[\mathrm{M}+\mathrm{H}]^{+}:$202.1344, Found: 202.1340 .

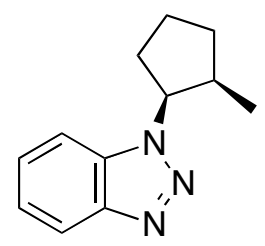

$\mathrm{N}-1-1.6 .6 \mathrm{c}$

cis-1-(2-methylcyclopentyl)-1H-benzo[d][1,2,3]triazole (N-1-1.6.6c) was purified by flash chromatography (Hexane-EtOAc, v/v 10/1) as yellow liquid. ${ }^{1} \mathrm{H}-\mathrm{NMR}\left(600 \mathrm{MHz}, \mathrm{CDCl}_{3}\right) \delta 8.06$ $(\mathrm{d}, J=7.2 \mathrm{~Hz}, 1 \mathrm{H}), 7.53(\mathrm{~d}, J=7.2 \mathrm{~Hz}, 1 \mathrm{H}), 7.45(\mathrm{t}, J=7.2 \mathrm{~Hz}, 1 \mathrm{H}), 7.34(\mathrm{t}, J=7.2 \mathrm{~Hz}, 1 \mathrm{H})$ 5.11-5.07 (m, 1H), 2.68-2.63 (m, 1H), 2.51-2.45 (m, 1H), 2.44-2.37 (m, 1H), 2.25-2.19 (m, 1H), 1.99-1.94 (m, 1H), 1.84-1.73 (m, 2H), $0.53(\mathrm{~d}, J=7.2 \mathrm{~Hz}, 3 \mathrm{H}) ;{ }^{13} \mathrm{C}-\mathrm{NMR}\left(150 \mathrm{MHz}, \mathrm{CDCl}_{3}\right) \delta$ 145.4, 134.0, 126.8, 123.6, 119.9, 109.6, 62.9, 39.8, 32.6, 31.0, 23.3, 14.3; HRMS Calculated for $\mathrm{C}_{12} \mathrm{H}_{16} \mathrm{~N}_{3}[\mathrm{M}+\mathrm{H}]^{+}:$202.1344, Found: 202.1337.

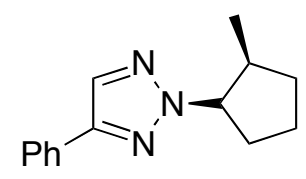

$\mathrm{N}-2-1.6 .6 \mathrm{~d}$ 
cis-2-(2-methylcyclopentyl)-4-phenyl-2H-1,2,3-triazole (N-2-1.6.6d) was purified by flash chromatography (Hexane-EtOAc, v/v 10/1) as yellow liquid. ${ }^{1} \mathrm{H}-\mathrm{NMR}\left(600 \mathrm{MHz}, \mathrm{CDCl}_{3}\right) \delta 7.82$ (s, 1H), $7.81(\mathrm{~d}, J=7.2 \mathrm{~Hz}, 2 \mathrm{H}), 7.42(\mathrm{t}, J=7.2 \mathrm{~Hz}, 2 \mathrm{H}), 7.33$ (tt, $J=7.2 \mathrm{~Hz}, 1.2 \mathrm{~Hz}, 1 \mathrm{H}), 5.06-$ $5.02(\mathrm{~m}, 1 \mathrm{H}), 2.49-2.43(\mathrm{~m}, 1 \mathrm{H}), 2.39-2.26(\mathrm{~m}, 2 \mathrm{H}), 2.17-2.11(\mathrm{~m}, 1 \mathrm{H}), 1.92-1.86(\mathrm{~m}, 1 \mathrm{H}), 1.74-$ $1.67(\mathrm{~m}, 2 \mathrm{H}), 0.65(\mathrm{~d}, J=7.2 \mathrm{~Hz}, 3 \mathrm{H}) ;{ }^{13} \mathrm{C}-\mathrm{NMR}\left(150 \mathrm{MHz}, \mathrm{CDCl}_{3}\right) \delta 146.8,130.9,130.0$ 128.7, 128.1, 125.8, 69.2, 40.3, 32.4, 31.1, 23.5, 14.0; HRMS Calculated for $\mathrm{C}_{14} \mathrm{H}_{18} \mathrm{~N}_{3}[\mathrm{M}+\mathrm{H}]^{+}$: 228.1501, Found: 228.1496.

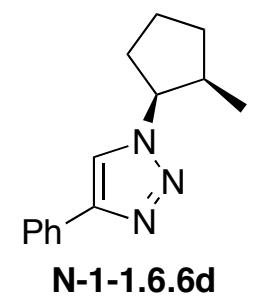

cis-1-(2-methylcyclopentyl)-4-phenyl-1H-1,2,3-triazole (N-1-1.6.6d) was purified by flash chromatography (Hexane-EtOAc, v/v 10/1) as yellow liquid. ${ }^{1} \mathrm{H}-\mathrm{NMR}\left(600 \mathrm{MHz}, \mathrm{CDCl}_{3}\right) \delta 7.85$ (d, $J=7.2 \mathrm{~Hz}, 2 \mathrm{H}), 7.68(\mathrm{~s}, 1 \mathrm{H}), 7.42(\mathrm{t}, J=7.2 \mathrm{~Hz}, 2 \mathrm{H}), 7.32(\mathrm{t}, J=7.2 \mathrm{~Hz}, 1 \mathrm{H}), 4.98-4.94(\mathrm{~m}$, 1H), 2.42-2.33 (m, 2H), 2.32-2.26 (m, 1H), 2.14-2.08 (m, 1H), 2.00-1.93 (m, 1H), 1.84-1.77 (m, 1H), 1.61-1.54 (m, 1H), $0.70(\mathrm{~d}, J=7.2 \mathrm{~Hz}, 3 \mathrm{H}) ;{ }^{13} \mathrm{C}-\mathrm{NMR}\left(150 \mathrm{MHz}, \mathrm{CDCl}_{3}\right) \delta$ 147.1, 130.9, 128.8, 127.9, 125.6, 119.3, 65.3, 39.4, 32.1, 31.2, 22.9, 14.2; HRMS Calculated for $\mathrm{C}_{14} \mathrm{H}_{18} \mathrm{~N}_{3}$ $[\mathrm{M}+\mathrm{H}]^{+}: 228.1501$, Found: 228.1506. 


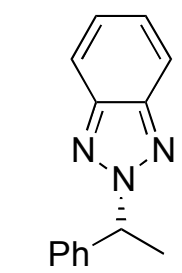

$(R)-\mathrm{N}-2-1.6 .7 \mathrm{a}$

(R)-2-(1-phenylethyl)-2H-benzo[d][1,2,3]triazole $((R)-\mathbf{N}-2-1.6 .7 a)$ was purified by flash chromatography (Hexane-EtOAc, v/v 10/1) as yellow liquid. ${ }^{1} \mathrm{H}-\mathrm{NMR}\left(600 \mathrm{MHz}, \mathrm{CDCl}_{3}\right) \delta$ 7.91-7.87 (m, 2H), 7.43-7.40 (m, 2H), 7.39-7.32 (m, 4H), 7.29 (tt, J=7.2 Hz, 1.2 Hz, 1H), 6.16 $(\mathrm{q}, J=7.2 \mathrm{~Hz}, 1 \mathrm{H}), 2.16(\mathrm{~d}, J=7.2 \mathrm{~Hz}, 3 \mathrm{H}) ;{ }^{13} \mathrm{C}-\mathrm{NMR}\left(150 \mathrm{MHz}, \mathrm{CDCl}_{3}\right) \delta 144.1,140.2,128.7$, 128.3, 126.5, 126.2, 118.1, 66.1, 21.3; HRMS Calculated for $\mathrm{C}_{14} \mathrm{H}_{14} \mathrm{~N}_{3}[\mathrm{M}+\mathrm{H}]^{+}:$224.1188, Found: 224.1179. Enantiomeric excess was determined by HPLC with a Chiralcel OD-H column; $\lambda=254 \mathrm{~nm}$; eluent: Hexane/Isopropanol = 95/5; Flow rate: $1.0 \mathrm{~mL} / \mathrm{min} ; \mathrm{t}_{\mathrm{R}}=6.8 \mathrm{~min}, \mathrm{t}_{\mathrm{R}}$ $=11.4 \mathrm{~min} ; 87 \%$ ee.

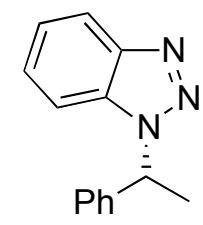

(R)-N-1-1.6.7a

(R)-1-(1-phenylethyl)-1H-benzo[d][1,2,3]triazole $((R)-N-1-1.6 .7 a)$ was purified by flash chromatography (Hexane-EtOAc, v/v 10/1) as yellow liquid. ${ }^{1} \mathrm{H}-\mathrm{NMR}\left(600 \mathrm{MHz}, \mathrm{CDCl}_{3}\right) \delta 8.02$ $(\mathrm{d}, J=7.2 \mathrm{~Hz}, 1 \mathrm{H}), 7.32-7.22(\mathrm{~m}, 8 \mathrm{H}), 6.02(\mathrm{q}, J=7.2 \mathrm{~Hz}, 1 \mathrm{H}), 2.15(\mathrm{~d}, J=7.2 \mathrm{~Hz}, 3 \mathrm{H}) ;{ }^{13} \mathrm{C}-$ NMR $\left(150 \mathrm{MHz}, \mathrm{CDCl}_{3}\right) \delta 146.7,140.4,132.7,129.1,128.4,127.2,126.5,124.0,120.1,110.4$, 59.3, 21.4; HRMS Calculated for $\mathrm{C}_{14} \mathrm{H}_{14} \mathrm{~N}_{3}[\mathrm{M}+\mathrm{H}]^{+}:$224.1188, Found: 224.1190. Enantiomeric 
excess was determined by HPLC with a Chiralcel OD-H column; $\lambda=254 \mathrm{~nm}$; eluent: Hexane/Isopropanol $=95 / 5$; Flow rate: $1.0 \mathrm{~mL} / \mathrm{min} ; \mathrm{t}_{\mathrm{R}}=6.8 \mathrm{~min}, \mathrm{t}_{\mathrm{R}}=11.4 \mathrm{~min} ; 87 \%$ ee.

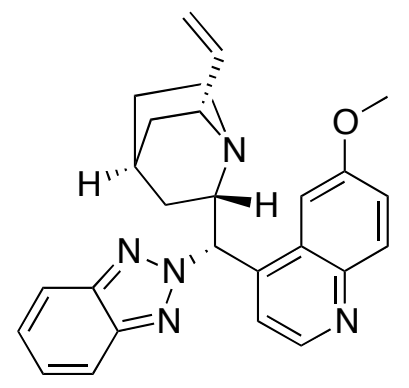

$\mathrm{N}-2-1.6 .7 \mathrm{~b}$

\section{$(2 S, 4 S, 7 R)-2-((S)-(2 H$-benzo[d][1,2,3]triazol-2-yl)(6-methoxyquinolin-4-yl)methyl)-7-}

vinylquinuclidine (N-2-1.6.7b) was purified by flash chromatography (Hexane-EtOAc, v/v 10/1) as white solid. [ $]^{25}{ }_{\mathrm{D}}=-243.20\left(\mathrm{CH}_{2} \mathrm{Cl}_{2}, c 1.0\right) ;{ }^{1} \mathrm{H}-\mathrm{NMR}\left(600 \mathrm{MHz}, \mathrm{CDCl}_{3}\right) \delta 8.83(\mathrm{~d}, J$ $=4.8 \mathrm{~Hz}, 1 \mathrm{H}), 8.01(\mathrm{~d}, J=9.0 \mathrm{~Hz}, 1 \mathrm{H}), 7.84(\mathrm{~d}, J=4.2 \mathrm{~Hz}, 1 \mathrm{H}), 7.83-7.79(\mathrm{~m}, 2 \mathrm{H}), 7.77(\mathrm{~s}, 1 \mathrm{H})$, $7.36(\mathrm{dd}, J=9.0 \mathrm{~Hz}, 3.0 \mathrm{~Hz}, 1 \mathrm{H}), 7.31-7.27(\mathrm{~m}, 2 \mathrm{H}), 6.74(\mathrm{~d}, J=11.4 \mathrm{~Hz}, 1 \mathrm{H}), 5.99-5.92(\mathrm{~m}$, 1H), 5.12-5.10 (m, 1H), $5.08(\mathrm{~s}, 1 \mathrm{H}), 4.45(\mathrm{q}, J=8.4 \mathrm{~Hz}, 1 \mathrm{H}), 4.02(\mathrm{~s}, 3 \mathrm{H}), 3.57-3.51(\mathrm{~m}, 1 \mathrm{H})$, $3.14(\mathrm{dd}, J=13.8 \mathrm{~Hz}, 10.2 \mathrm{~Hz}, 1 \mathrm{H}), 2.84(\mathrm{~d}, J=14.4 \mathrm{~Hz}, 1 \mathrm{H}), 2.79-2.73(\mathrm{~m}, 1 \mathrm{H}), 2.31(\mathrm{~s}, 1 \mathrm{H})$, $1.90(\mathrm{t}, J=12.0 \mathrm{~Hz}, 1 \mathrm{H}), 1.76(\mathrm{sex}, J=3.0 \mathrm{~Hz}, 1 \mathrm{H}), 1.69-1.57(\mathrm{~m}, 2 \mathrm{H}), 0.93(\mathrm{dd}, J=13.2 \mathrm{~Hz}$, $7.2 \mathrm{~Hz}, 1 \mathrm{H}) ;{ }^{13} \mathrm{C}-\mathrm{NMR}\left(150 \mathrm{MHz}, \mathrm{CDCl}_{3}\right) \delta 158.4,148.1,147.6,147.0,144.9,144.1,141.4$, 139.0, 128.3, 126.2, 122.0, 118.3, 118.1, 114.7, 101.1, 58.6, 55.8, 55.7, 41.1, 39.3, 27.8, 27.7, 26.8; HRMS Calculated for $\mathrm{C}_{26} \mathrm{H}_{28} \mathrm{~N}_{5} \mathrm{O}[\mathrm{M}+\mathrm{H}]^{+}$: 426.2294, Found: 426.2290 . 


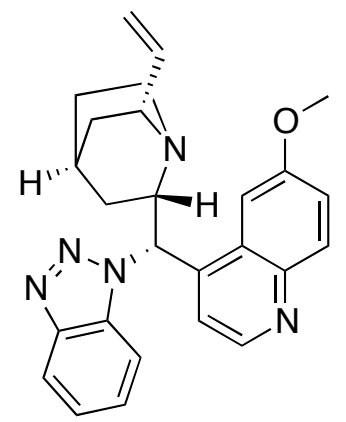

$\mathrm{N}-1-1.6 .7 b$

\section{$(2 S, 4 S, 7 R)-2-((S)-(1 H$-benzo $[d][1,2,3]$ triazol-1-yl)(6-methoxyquinolin-4-yl)methyl)-7-}

vinylquinuclidine (N-1-1.6.7b) was purified by flash chromatography (Hexane-EtOAc, v/v 10/1) as white solid. [ $]_{\mathrm{D}}^{25}=-96.20\left(\mathrm{CH}_{2} \mathrm{Cl}_{2}, c 1.0\right) ;{ }^{1} \mathrm{H}-\mathrm{NMR}\left(600 \mathrm{MHz}, \mathrm{CDCl}_{3}\right) \delta 8.84(\mathrm{~d}, J=$ $4.8 \mathrm{~Hz}, 1 \mathrm{H}), 7.98(\mathrm{dd}, J=11.4 \mathrm{~Hz}, 9.0 \mathrm{~Hz}, 2 \mathrm{H}), 7.72(\mathrm{~d}, J=4.2 \mathrm{~Hz}, 1 \mathrm{H}), 7.66(\mathrm{~d}, J=2.4 \mathrm{~Hz}$, 1H), $7.41(\mathrm{~d}, J=8.4 \mathrm{~Hz}, 1 \mathrm{H}), 7.33$ (dd, $J=9.0 \mathrm{~Hz}, 2.4 \mathrm{~Hz}, 1 \mathrm{H}), 7.30$ (tt, $J=7.8 \mathrm{~Hz}, 1.2 \mathrm{~Hz}, 1 \mathrm{H})$, $7.23(\mathrm{tt}, J=7.8 \mathrm{~Hz}, 1.2 \mathrm{~Hz}, 1 \mathrm{H}), 6.70(\mathrm{~d}, J=11.4 \mathrm{~Hz}, 1 \mathrm{H}), 5.99-5.93(\mathrm{~m}, 1 \mathrm{H}), 5.10(\mathrm{tt}, J=9.0$ Hz, $1.2 \mathrm{~Hz}, 2 \mathrm{H}), 4.39$ (s, 1H), 3.95 (s, 3H), 3.51-3.45 (m, 1H), 3.07 (dd, $J=13.8 \mathrm{~Hz}, 9.0 \mathrm{~Hz}$, 1H), $2.73(\mathrm{~d}, J=12.6 \mathrm{~Hz}, 1 \mathrm{H}), 2.70-2.65(\mathrm{~m}, 1 \mathrm{H}), 2.31(\mathrm{~s}, 1 \mathrm{H}), 2.07-2.02(\mathrm{~m}, 1 \mathrm{H}), 1.81(\mathrm{sex}, J=$ $3.0 \mathrm{~Hz}, 1 \mathrm{H}), 1.70-1.64(\mathrm{~m}, 1 \mathrm{H}), 1.61-1.55(\mathrm{~m}, 1 \mathrm{H}), 1.04(\mathrm{dd}, J=13.2 \mathrm{~Hz}, 7.2 \mathrm{~Hz}, 1 \mathrm{H}) ;{ }^{13} \mathrm{C}-\mathrm{NMR}$ $\left(150 \mathrm{MHz}, \mathrm{CDCl}_{3}\right) \delta 158.5,147.1,146.3,145.1,141.5,138.2,132.2,132.0,128.4,127.2,123.6$ 122.2, 120.4, 114.7, 109.7, 101.0, 56.4, 55.9, 55.8, 41.0, 39.2, 27.8, 27.7; HRMS Calculated for $\mathrm{C}_{26} \mathrm{H}_{28} \mathrm{~N}_{5} \mathrm{O}[\mathrm{M}+\mathrm{H}]^{+}:$426.2294, Found: 426.2302 . 


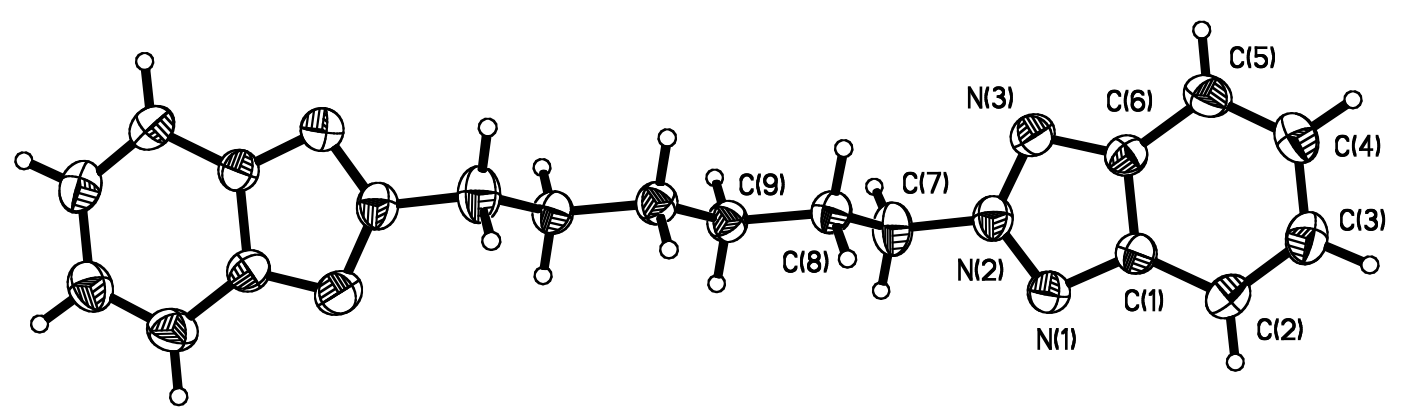

Figure S1.6-1.Perspective view of the molecular structure of 1.6.5a $\left(\mathrm{C}_{18} \mathrm{H}_{20} \mathrm{~N}_{6}\right)$ with the atom labeling scheme. The thermal ellipsoids are scaled to enclose $30 \%$ probability. CCDC number: 775283 


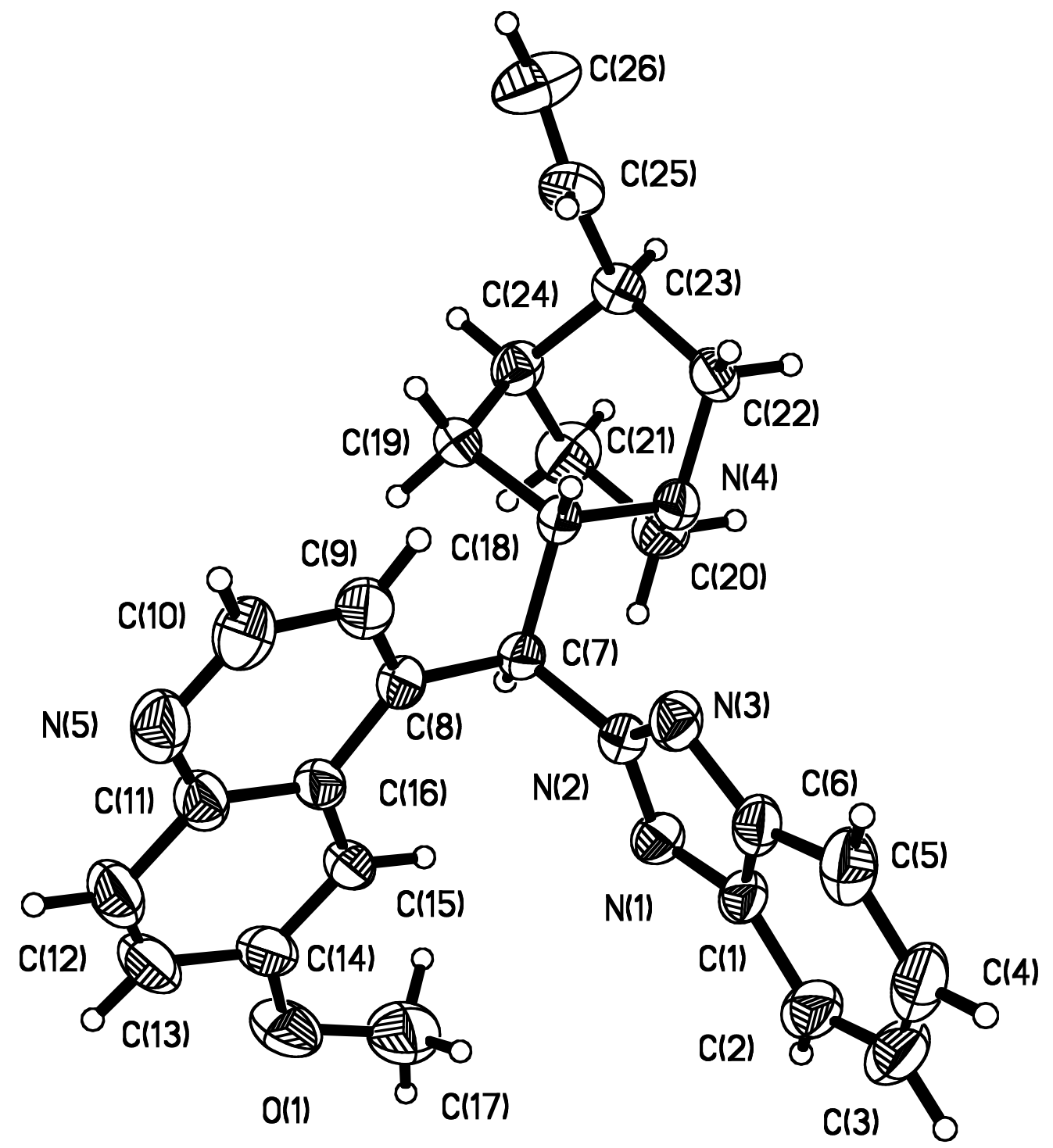

Figure S1.6-2.Perspective view of the molecular structure of $\mathbf{N}-2-1.6 .7 \mathbf{b}\left(\mathrm{C}_{26} \mathrm{H}_{27} \mathrm{~N}_{5} \mathrm{O}\right)$ with the atom labeling scheme. The thermal ellipsoids are scaled to enclose $30 \%$ probability. CCDC number: 775283 


\section{Chapter Two: 1,2,3-trizazoles applied as ligands in transition metal catalysis}

\subsection{Synthesis of $\left[\operatorname{Ir}\left(L^{\wedge} X\right)(A r T r i a z)_{2}\right]$ and $\left[\operatorname{Ir}(b b p y)(A r T r i a z)_{2}\right] C l$ complexes and their application in photoredox catalysis}

\section{$\underline{\text { General Methods and Materials }}$}

All of the reactions dealing with air and/or moisture-sensitive reactions were carried out under an atmosphere of nitrogen using oven/flame-dried glassware and standard syringe/septa techniques. Unless otherwise noted, all commercial reagents and solvents were obtained from the commercial provider and used without further purification. ${ }^{1} \mathrm{H}$ NMR and ${ }^{13} \mathrm{C}$ NMR spectra were recorded on Varian $600 \mathrm{MHz}$ spectrometers. Chemical shifts were reported relative to internal tetramethylsilane $(\delta 0.00 \mathrm{ppm})$ or $\mathrm{CDCl}_{3}(\delta 7.26 \mathrm{ppm})$ for ${ }^{1} \mathrm{H}$ and $\mathrm{CDCl}_{3}(\delta 77.0 \mathrm{ppm})$ for ${ }^{13} \mathrm{C}$. Melting points were measured on a Mel-Temp 1001D apparatus and uncorrected. Flash column chromatography was performed on 230-430 mesh silica gel. Analytical thin layer chromatography was performed with precoated glass baked plates $(250 \mu)$ and visualized by fluorescence and by charring after treatment with potassium permanganate stain. HRMS were recorded on LTQ-FTUHRA spectrometer. 


\section{Procedure for preparation of 2-Phenyl-1,2,3-triazole (2.2.1)}

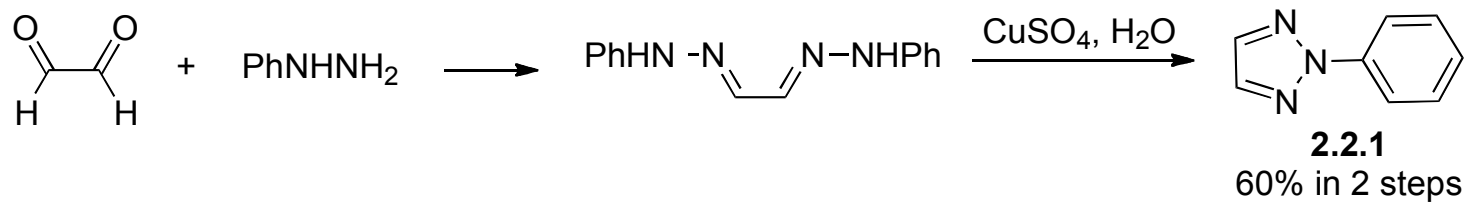

To a $40 \%$ aqueous solution of glyoxal $(50 \mathrm{mmol})$ was added phenylhydrazine $(100 \mathrm{mmol})$ with stirring. Immediately a yellow solid was formed. To the reaction solution was added methanol $(150 \mathrm{~mL})$, and the yellow suspension was stirred for $0.5 \mathrm{~h}$ at room temperature and filtered to yield the resulting products Glyoxalbis( $N$-phenyl)osazone which was pure enough for running the next step.

A mixture of glyoxal phenylosazone from previous step and $\mathrm{Cu}(\mathrm{OTf})_{2}(0.2$ equiv.) was heated in water to reflux at $100{ }^{\circ} \mathrm{C}$ for 2 hours. The reaction mixture then purified by vacuum distillation to give the mixture products 2-phenyl-1,2,3-triazole and aniline at $120^{\circ} \mathrm{C}$. For the isolation of the target product, $100 \mathrm{ml}$ of ether have been added in the distillate. The solution then was treated in succession with $1 \mathrm{~N} \mathrm{HCl}(3 \times 10 \mathrm{ml})$ until $\mathrm{PH}=6$. The extract was dried with potassium carbonate, followed by the removal of ether to give the final products 2.2.1 as yellow liquid (60\% yield in two steps). 


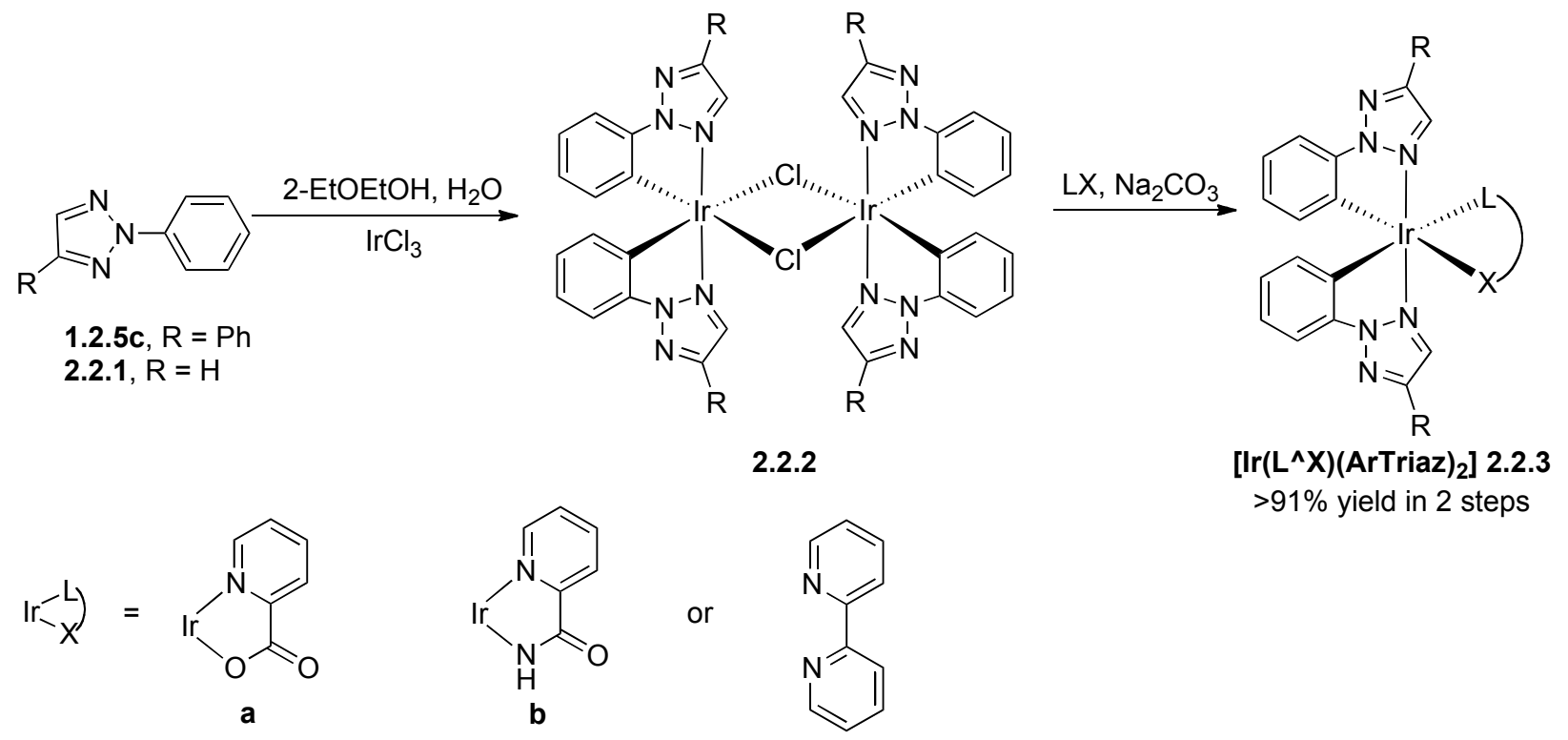

[(ArTriaz) $\left.)_{2} \operatorname{Ir}(\mu-\mathbf{C l})\right]_{2}: \mathrm{IrCl}_{3} \cdot \mathrm{H}_{2} \mathrm{O}(1 \mathrm{mmol})$ and $\mathrm{N}$-2-aryl-1,2,3-triazole (4 mmol) were dissolved in $40 \mathrm{~mL}$ mixed solution of 2-EtOEtOH and $\mathrm{H}_{2} \mathrm{O}(\mathrm{V} / \mathrm{V}: 3 / 1)$, and refluxed at $140{ }^{\circ} \mathrm{C}$ for 24 hours. After cooling, yellow precipitate was filtered and washed with $40 \mathrm{~mL}$ water. The washed product was dried in air to give yellow-green solids.

$\left[\operatorname{Ir}\left(\mathbf{L}^{\wedge} \mathbf{X}\right)(\text { ArTriaz })_{2}\right]:\left[(\text { ArTriaz })_{2} \operatorname{Ir}(\mu-C l)\right]_{2}$ complex $(1 \mathrm{mmol})$, sodium corbonate $(10 \mathrm{mmol})$ and ancilaary ligand $(2.5 \mathrm{mmol})$ were dissolved in $80 \mathrm{~mL}$ of 2-EtOEtOH under the nitrogen environment. The reaction mixture was heated up to $140{ }^{\circ} \mathrm{C}$ and stirred for 12 hours. Then cooled reaction mixture was poured to ethyl acetate $(200 \mathrm{~mL})$ and washed with water to remove 2EtOEtOH. Silica column purification with dichloromethane gave the pure products. 


\section{General procedure for $1,2,3,4$-tetrahydroisoquinoline substrates}

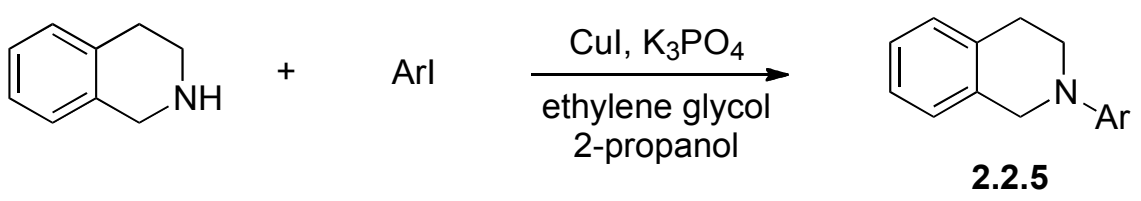

Copper(I) iodide $(0.1 \mathrm{mmol})$ and potassium phosphate $(2.0 \mathrm{mmol})$ were put into a $100 \mathrm{~mL}$ round-bottom flask. The flask was evacuated and refilled with nitrogen. 2-Propanol (10 $\mathrm{mL})$, ethylene glycol (2.0 mmol), 1,2,3,4-tetrahydro-isoquinoline (1.0 mmol) and iodobenzene (1.2 mmol) were added successively using a syringe at room temperature. The reaction mixture was heated at $90{ }^{\circ} \mathrm{C}$ and kept for $24 \mathrm{~h}$ and then allowed to cool down to room temperature. Diethyl ether $(20 \mathrm{~mL})$ and water $(20 \mathrm{~mL})$ were then added to the reaction mixture. The organic layer was extracted by diethyl ether $(2 \times 20 \mathrm{~mL})$. The combined organic phases were washed with brine and dried over magnesium sulfate. The solvent was removed by rotary evaporation and purified by column chromatography on silica gel (hexane/ethyl acetate=20:1) to give the desired product 2.2.5. 


\section{General procedure for reaction of photoredox catalysis}

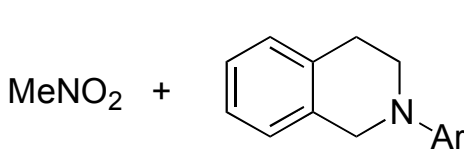

2.2.5

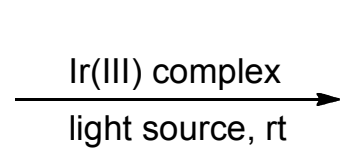

light source, rt

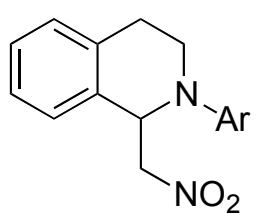

2.2.6

To a $20 \mathrm{~mL}$ white cap vial equipped with a magnetic stir bar were added [Ir(bbpy)(ArTriaz) $)_{2} \mathrm{Cl}(0.005 \mathrm{mmol})$, a 1,2,3,4- tetrahydroisoquinoline derivative $(0.5 \mathrm{mmol})$, and nitromethane $(2.0 \mathrm{~mL})$. The reaction stirred open to air at room temperature at a distance of $\sim 10 \mathrm{~cm}$ from a blue LED light. After the reaction completed (monitored by TLC analysis), the solvent was removed in vacuo. The residue was purified by column chromatography on silica gel (hexane/ethyl acetate $=10: 1$ ) to yield pure 2-aryl-1-(nitromethyl)-1,2,3,4tetrahydroisoquinoline 2.2.6. 


\section{Compounds Characterization}

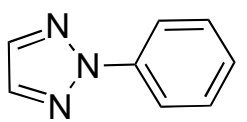

2.2.1

2-Phenyl-1,2,3-triazole (2.2.1) was purified by flash chromatography as yellow liquid. Isolated yield 60\%; ${ }^{1} \mathrm{H} \mathrm{NMR}\left(\mathrm{CDCl}_{3}, 600 \mathrm{MHz}\right) \delta 8.12-8.08(\mathrm{~m}, 2 \mathrm{H}), 7.79(\mathrm{~s}, 2 \mathrm{H}), 7.48-7.44(\mathrm{~m}, 2 \mathrm{H})$, 7.35-7.31 (m, 1H); ${ }^{13} \mathrm{C}-\mathrm{NMR}\left(150 \mathrm{MHz}, \mathrm{CDCl}_{3}\right) \delta 139.8,135.4,129.2,127.4,118.8$.

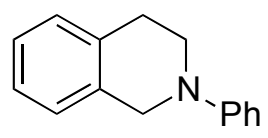

2.2.5a

1,2,3,4-Tetrahydro-2-phenylisoquinoline (2.2.5a) was purified by flash chromatography as brown solid. ${ }^{1} \mathrm{H} \mathrm{NMR}\left(\mathrm{CDCl}_{3}, 600 \mathrm{MHz}\right) \delta$ 7.36-7.33 (m, 2H), 7.25-7.19 (m, 4H), 7.04(d, $J=$ $7.8 \mathrm{~Hz}, 2 \mathrm{H}), 6.89(\mathrm{dd}, J=7.2,7.2 \mathrm{~Hz}, 1 \mathrm{H}), 4.46(\mathrm{~s}, 2 \mathrm{H}), 3.61(\mathrm{dd}, J=6.0,6.0 \mathrm{~Hz}, 2 \mathrm{H}), 3.04$ $(\mathrm{dd}, J=6.0,6.0 \mathrm{~Hz}, 2 \mathrm{H}) ;{ }^{13} \mathrm{C}-\mathrm{NMR}\left(150 \mathrm{MHz}, \mathrm{CDCl}_{3}\right) \delta 150.5,134.8,134.4,129.1,128.5$, $126.5,126.3,126.0,118.6,115.1,50.7,46.5,29.1$.

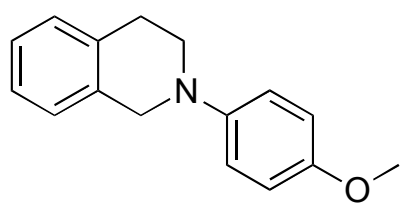

2.2.5b 
1,2,3,4-Tetrahydro-2-(4-methoxyphenyl)isoquinoline (2.2.5b) was purified by flash chromatography as brown solid. ${ }^{1} \mathrm{H}$ NMR $\left(\mathrm{CDCl}_{3}, 600 \mathrm{MHz}\right) \delta$ 7.19-7.12 (m, 4H), $6.99(\mathrm{~d}, J=$ $9.0 \mathrm{~Hz}, 2 \mathrm{H}), 6.88(\mathrm{~d}, J=9.0 \mathrm{~Hz}, 2 \mathrm{H}), 4.31(\mathrm{~s}, 2 \mathrm{H}), 3.79(\mathrm{~s}, 3 \mathrm{H}), 3.46(\mathrm{dd}, J=6.0,6.0 \mathrm{~Hz}, 2 \mathrm{H})$, $3.00(\mathrm{dd}, J=6.0,6.0 \mathrm{~Hz}, 2 \mathrm{H}) ;{ }^{13} \mathrm{C}-\mathrm{NMR}\left(150 \mathrm{MHz}, \mathrm{CDCl}_{3}\right) \delta 153.5,145.4,134.6,134.5,128.7$, $126.5,126.2,125.9,118.0,114.6,55.6,52.7,48.4,29.1$.

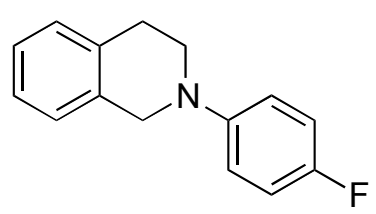

2.2.5c

1,2,3,4-Tetrahydro-2-(4-fluorophenyl)isoquinoline (2.2.5c) was purified by flash chromatography as brown solid. ${ }^{1} \mathrm{H}$ NMR $\left(\mathrm{CDCl}_{3}, 600 \mathrm{MHz}\right) \delta$ 7.21-7.14 (m, 4H), 7.02-6.98 (m, 2H), 6.98-6.94 (m, 2H), $4.35(\mathrm{~s}, 2 \mathrm{H}), 3.79(\mathrm{~s}, 3 \mathrm{H}), 3.50(\mathrm{dd}, J=6.0,6.0 \mathrm{~Hz}, 2 \mathrm{H}), 3.00(\mathrm{dd}, J=$ 6.0, $6.0 \mathrm{~Hz}, 2 \mathrm{H}) ;{ }^{13} \mathrm{C}-\mathrm{NMR}\left(150 \mathrm{MHz}, \mathrm{CDCl}_{3}\right) \delta 157.5,155.9,147.3,134.5,134.2,128.6,126.5$, $126.4,126.0,117.2,117.1,115.6,115.5,51.9,47.8,29.0$.<smiles>O=[N+]([O-])CC1c2ccccc2CCN1c1ccccc1</smiles>

2.2.6a

1-(Nitromethyl)-2-phenyl-1,2,3,4-tetrahydroisoquinoline $\mathbf{( 2 . 2 . 6 a )}$ purified by flash chromatography as yellow liquid. Reaction time about $12 \mathrm{~h}$; Isolated yield $75 \% ;{ }^{1} \mathrm{H}$ NMR 
$\left(\mathrm{CDCl}_{3}, 600 \mathrm{MHz}\right) \delta$ 7.28-7.22 (m, 3H), 7.21-7.17 (m, $\left.2 \mathrm{H}\right), 7.12(\mathrm{~d}, J=7.8 \mathrm{~Hz}, 1 \mathrm{H}), 6.97$ $(\mathrm{d}, J=8.4 \mathrm{~Hz}, 2 \mathrm{H}), 6.84(\mathrm{t}, J=7.2 \mathrm{~Hz}, 1 \mathrm{H}), 5.54(\mathrm{~d}, J=7.2 \mathrm{~Hz}, 1 \mathrm{H}), 4.86(\mathrm{dd}, J=12.6,7.8$ $\mathrm{Hz}, 1 \mathrm{H}), 4.41$ (dd, $J=12.0,6.6 \mathrm{~Hz}, 1 \mathrm{H}), 3.68-3.58$ (m, $2 \mathrm{H}), 3.08$ (ddd, $J=16.0,8.4,6.0$ $\mathrm{Hz}, 1 \mathrm{H}), 2.78(\mathrm{dt}, J=16.8,5.1,4.8 \mathrm{~Hz}, 1 \mathrm{H}) ;{ }^{13} \mathrm{C}-\mathrm{NMR}\left(150 \mathrm{MHz}, \mathrm{CDCl}_{3}\right) \delta 148.4,135.3$, 133.0, 129.5, 129.2, 128.1, 127.0, 126.7, 119.5, 115.1, 78.8, 58.2, 42.1, 26.5 .

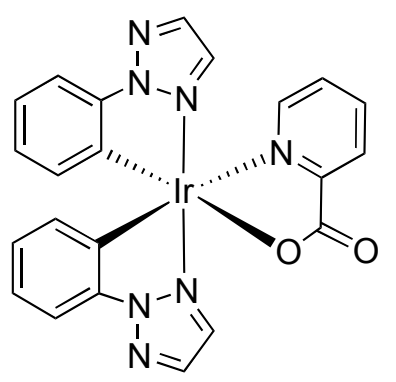

2.2.3e

[Ir(Flrpic)(PhTriaz) $)_{2}$ ( (2.2.3e) was purified by flash chromatography as yellow solid. ${ }^{1} \mathrm{H}$ NMR $\left(\mathrm{CDCl}_{3}, 600 \mathrm{MHz}\right) \delta 8.22(\mathrm{~d}, J=7.2 \mathrm{~Hz}, 1 \mathrm{H}), 7.92(\mathrm{~d}, J=4.8 \mathrm{~Hz}, 2 \mathrm{H}), 7.85(\mathrm{t}, J=7.8 \mathrm{~Hz}, 1$ H), $7.82(\mathrm{~d}, J=4.8 \mathrm{~Hz}, 1 \mathrm{H}), 7.79(\mathrm{~s}, 1 \mathrm{H}), 7.64(\mathrm{~d}, J=7.8 \mathrm{~Hz}, 1 \mathrm{H}), 7.54(\mathrm{~d}, J=7.8 \mathrm{~Hz}, 1 \mathrm{H})$, $7.27(\mathrm{~d}, J=6.0 \mathrm{~Hz}, 1 \mathrm{H}), 6.97(\mathrm{t}, J=7.2 \mathrm{~Hz}, 2 \mathrm{H}), 6.87(\mathrm{t}, J=7.2 \mathrm{~Hz}, 1 \mathrm{H}), 6.80(\mathrm{t}, J=7.2$ $\mathrm{Hz}, 1 \mathrm{H}), 6.73(\mathrm{t}, J=7.2 \mathrm{~Hz}, 1 \mathrm{H}), 6.28(\mathrm{~d}, J=7.8 \mathrm{~Hz}, 1 \mathrm{H}), 6.15(\mathrm{~d}, J=7.8 \mathrm{~Hz}, 1 \mathrm{H}) ;{ }^{13} \mathrm{C}-$ NMR $\left(150 \mathrm{MHz}, \mathrm{CDCl}_{3}\right) \delta 172.9,151.7,148.8,141.6,141.5,138.5,135.1,135.0,133.0,132.9$, $132.6,131.9,127.94,127.92,127.87,127.49,127.33,125.1,122.9,114.2,113.9$. 


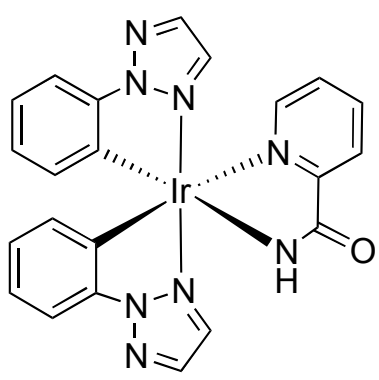

2.2.3f

[Ir(FIrpca)(PhTriaz) 2 ] (2.2.3f) was purified by flash chromatography as yellow solid. ${ }^{1} \mathrm{H}$ NMR $\left(\mathrm{CDCl}_{3}, 600 \mathrm{MHz}\right) \delta 8.24(\mathrm{~d}, J=7.2 \mathrm{~Hz}, 1 \mathrm{H}), 7.92(\mathrm{~d}, J=16.8 \mathrm{~Hz}, 2 \mathrm{H}), 7.89(\mathrm{~d}, J=6.0 \mathrm{~Hz}$, $2 \mathrm{H}), 7.87(\mathrm{t}, J=6.0 \mathrm{~Hz}, 1 \mathrm{H}), 7.71(\mathrm{~d}, J=7.8 \mathrm{~Hz}, 1 \mathrm{H}), 7.66(\mathrm{~d}, J=7.8 \mathrm{~Hz}, 1 \mathrm{H}), 7.25(\mathrm{t}, J=$ $6.6 \mathrm{~Hz}, 2 \mathrm{H}), 7.04(\mathrm{t}, J=7.2 \mathrm{~Hz}, 1 \mathrm{H}), 6.99(\mathrm{~s}, 1 \mathrm{H}), 6.97(\mathrm{t}, J=7.2 \mathrm{~Hz}, 1 \mathrm{H}), 6.80$ (t, $J=$ $7.2 \mathrm{~Hz}, 1 \mathrm{H}), 6.44(\mathrm{t}, J=7.2 \mathrm{~Hz}, 1 \mathrm{H}), 6.21(\mathrm{~d}, J=7.8 \mathrm{~Hz}, 1 \mathrm{H}), 5.89(\mathrm{br}, 1 \mathrm{H}) ;{ }^{13} \mathrm{C}-\mathrm{NMR}(150$ $\left.\mathrm{MHz}, \mathrm{CDCl}_{3}\right) \delta 173.8,156.3,149.7,142.1,141.2,138.1,135.18,135.09,135.00,133.3,133.2$, $132.7,131.7,128.3,128.1,127.6,126.9,126.4,122.8,122.5,114.3,114.1$. 


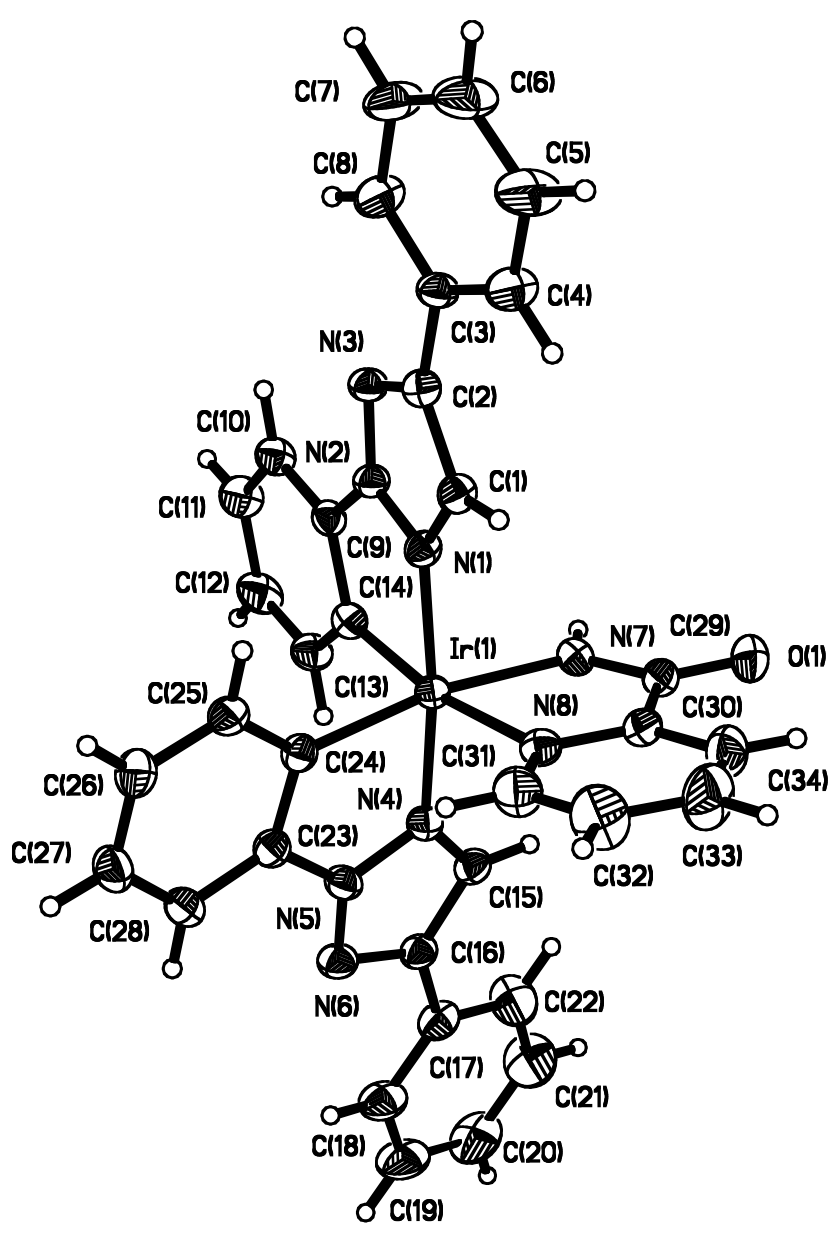

Figure S2.2-1.Perspective view of the molecular structure of 2.2.3b $\left(\mathrm{C}_{34} \mathrm{H}_{25} \mathrm{IrN}_{8} \mathrm{O}\right)$ with the atom labeling scheme. The thermal ellipsoids are scaled to enclose $30 \%$ probability. 


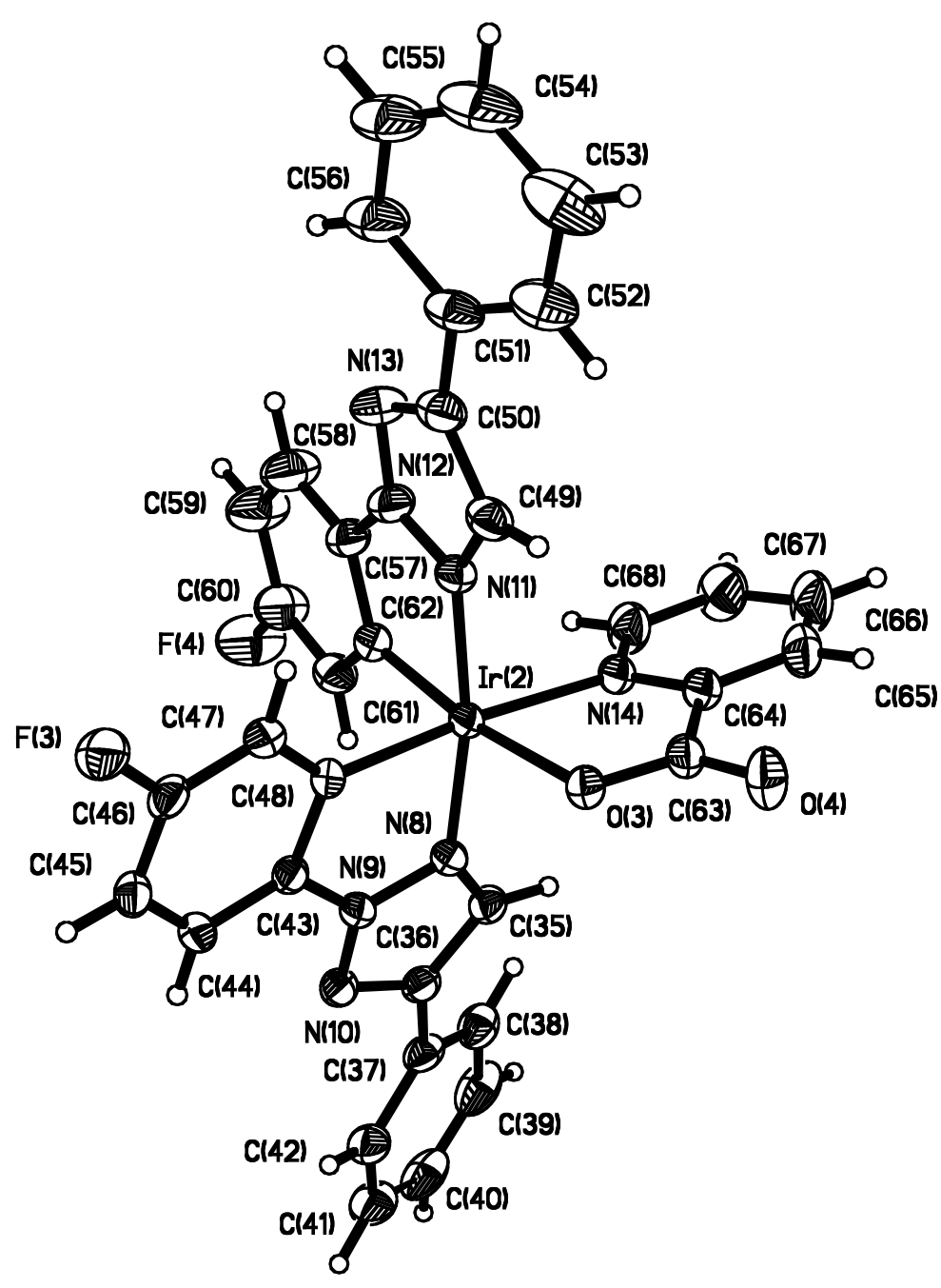

Figure S2.2-2.Perspective view of the molecular structure of 2.2.3c $\left(\mathrm{C}_{34} \mathrm{H}_{22} \mathrm{~F}_{2} \mathrm{IrN}_{7} \mathrm{O}_{2}\right)$ with the atom labeling scheme. The thermal ellipsoids are scaled to enclose $30 \%$ probability. 


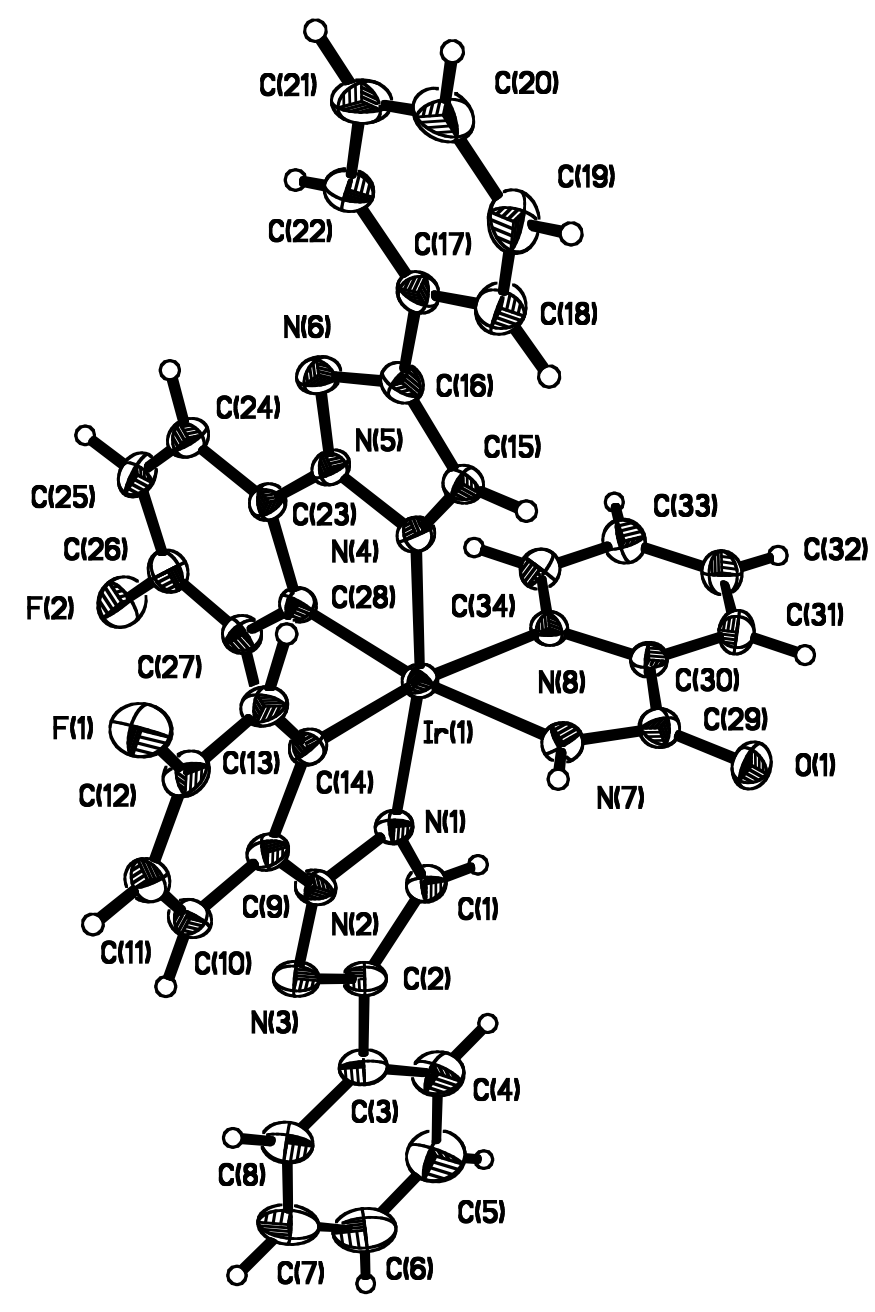

Figure S2.2-3.Perspective view of the molecular structure of 2.2.3d $\left(\mathrm{C}_{34} \mathrm{H}_{23} \mathrm{~F}_{2} \mathrm{IrN}_{8} \mathrm{O}\right)$ with the atom labeling scheme. The thermal ellipsoids are scaled to enclose $30 \%$ probability. 


\subsection{1,2,3-Triazole as Unique Ligand in Promoting Iron Catalyzed Propargyl Alcohol Dehydration for the Synthesis of Conjugated Enynes}

\section{General Methods and Materials}

All of the reactions dealing with air and/or moisture-sensitive reactions were carried out under an atmosphere of nitrogen using oven/flame-dried glassware and standard syringe/septa techniques. Unless otherwise noted, all commercial reagents and solvents were obtained from the commercial provider and used without further purification. ${ }^{1} \mathrm{H}$ NMR and ${ }^{13} \mathrm{C}$ NMR spectra were recorded on Varian $600 \mathrm{MHz}$ spectrometers. Chemical shifts were reported relative to internal tetramethylsilane $(\delta 0.00 \mathrm{ppm})$ or $\mathrm{CDCl}_{3}(\delta 7.26 \mathrm{ppm})$ for ${ }^{1} \mathrm{H}$ and $\mathrm{CDCl}_{3}(\delta 77.0 \mathrm{ppm})$ for ${ }^{13} \mathrm{C}$. Melting points were measured on a Mel-Temp 1001D apparatus and uncorrected. Flash column chromatography was performed on 230-430 mesh silica gel. Analytical thin layer chromatography was performed with precoated glass baked plates $(250 \mu)$ and visualized by fluorescence and by charring after treatment with potassium permanganate stain. HRMS were recorded on LTQ-FTUHRA spectrometer.

Propargylic alcohols 2.3.1 were synthesized according to the literatures as below:

Yan, W.; Wang, Q.; Chen, Y.; Petersen, J.; Shi, X. Org. Lett. 2010, 12, 3308-3311.

\section{$\underline{\text { Representative procedure for propargyl alcohol dehydration reaction }}$}




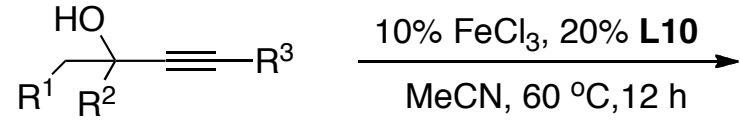

2.3.1
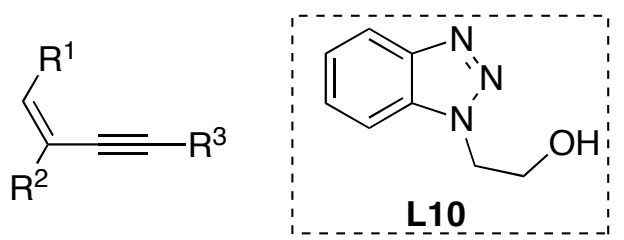

2.3.2

A $20 \mathrm{~mL}$ screw-cap vial was charged with the catalyst $\mathrm{FeCl}_{3}(16.2 \mathrm{mg}, 0.1 \mathrm{mmol})$ and the ligand 2-(1H-benzo[d][1,2,3]triazol-1-yl)ethanol $\mathbf{L 1 0}(32.4 \mathrm{mg}, 0.2 \mathrm{mmol})$ in $5 \mathrm{~mL} \mathrm{MeCN}$. The vial was placed in a preheated metal block at $60^{\circ} \mathrm{C}$ for $15 \mathrm{mins}$, and then propargyl alcohol $(1 \mathrm{mmol})$ was added. The reaction mixture was stirred at $60{ }^{\circ} \mathrm{C}$ and monitored by TLC. After the reaction was completed (about 2-7 h), the solvent was removed under reduced pressure and the residue was purified by flash chromatograpgy on silica gel (ethyl acetate/hexane $=1: 40, \mathrm{~V} / \mathrm{V}$ ) to give desired enyne product as yellow oil. 


\section{General procedure for NBS promoted enyne activation reaction.}

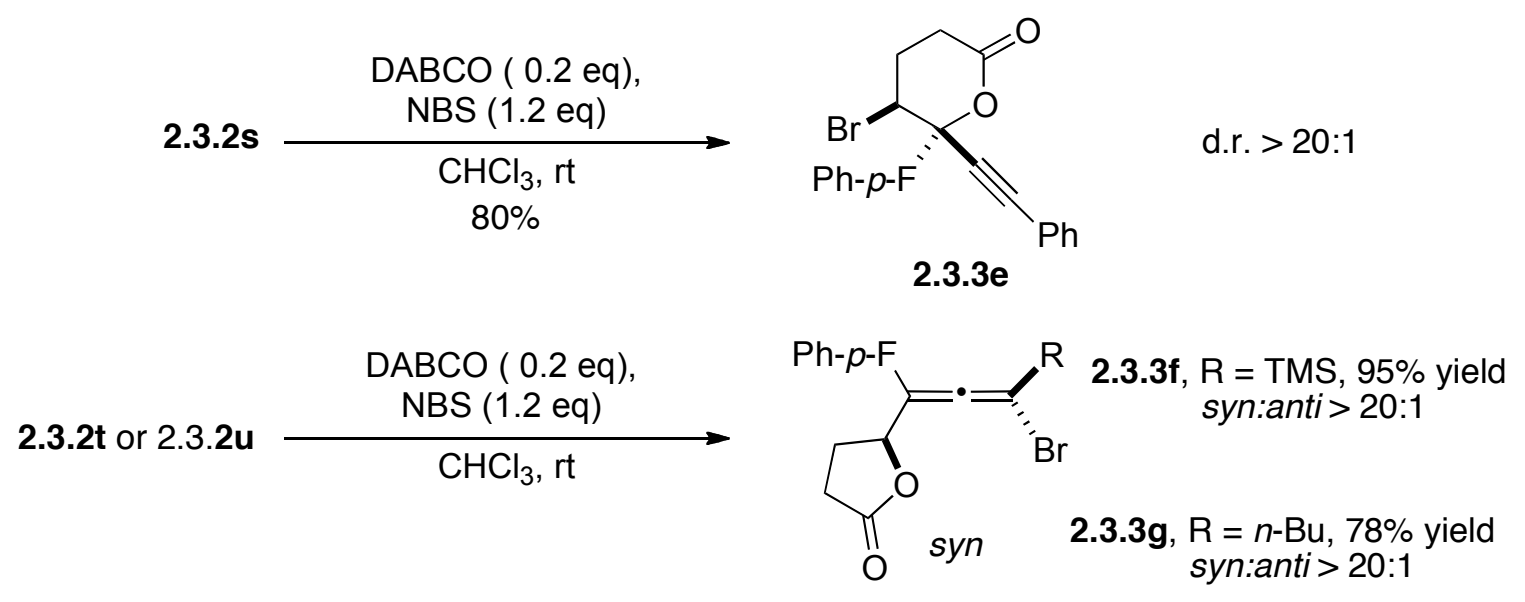

The enynyl acid (1 mmol), NBS (230 mg, $1.2 \mathrm{mmol})$, DABCO (2 mg, $0.02 \mathrm{mmol})$ and $5 \mathrm{~mL}$ chloroform $\mathrm{CHCl}_{3}$ were combined in a $20 \mathrm{~mL}$ vial. The reaction mixture was stirred ar rt until all the enynyl acid consumed as indicated by TLC. The reaction mixture was concentrated under reduced pressure and directly purified by flash chromatography on silica gel (ethyl acetate/hexane $=1: 20, \mathrm{~V} / \mathrm{V})$ to give product as yellow oil. 


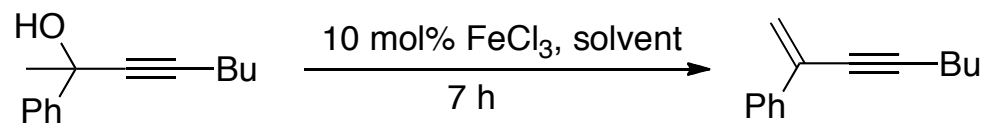

2.3.1a

2.3.2a

\begin{tabular}{|c|c|c|c|c|c|c|c|}
\hline & catalyst & loading (\%) & solv. & temp $\left({ }^{\circ} \mathrm{C}\right)$ & time $(\mathrm{h})$ & conv. $(\%)^{b}$ & yield $(\%)^{c}$ \\
\hline 18 & $\mathrm{FeCl}_{3}$ & 10 & $\mathrm{MeCN}$ & 60 & 7 & 95 & 29 \\
\hline 19 & $\mathrm{FeCl}_{3}$ & 10 & Acetone & 60 & 7 & 66 & 13 \\
\hline 20 & $\mathrm{FeCl}_{3}$ & 10 & THF & 60 & 7 & 73 & 20 \\
\hline 21 & $\mathrm{FeCl}_{3}$ & 10 & Toluene & 60 & 7 & 35 & $<5$ \\
\hline 22 & $\mathrm{FeCl}_{3}$ & 10 & $\mathrm{MeOH}$ & 60 & 7 & 65 & $<5$ \\
\hline 23 & $\mathrm{FeCl}_{3}$ & 10 & $\mathrm{MeNO}_{2}$ & 60 & 7 & 70 & 18 \\
\hline 24 & $\mathrm{FeCl}_{3}$ & 10 & DMSO & 60 & 7 & $<5$ & $<5$ \\
\hline 25 & $\mathrm{FeCl}_{3}$ & 10 & DMF & 60 & 7 & $<5$ & $<5$ \\
\hline 26 & $\mathrm{FeCl}_{3}$ & 10 & $\mathrm{CHCl}_{3}$ & 60 & 7 & 80 & 17 \\
\hline 27 & $\mathrm{FeCl}_{3}$ & 10 & EtOAc & 60 & 7 & 78 & 15 \\
\hline 28 & $\mathrm{FeCl}_{3}$ & 10 & $\mathrm{DCE}$ & 60 & 7 & 81 & 24 \\
\hline
\end{tabular}

${ }^{a}$ General reaction conditions: $1 \mathrm{a}(0.25 \mathrm{mmol}, 1.0$ equiv. $)$ and $\mathrm{FeCl}_{3}(10 \mathrm{~mol} \%)$ in solvent (5 $\mathrm{mL}) ;{ }^{b}$ Conversions were determined based on the consumption of propargyl alcohol; ${ }^{c}$ NMR yields of 2a with 1,3,5-trimethoxybenzene as internal standard. 


\section{Compounds Characterization}

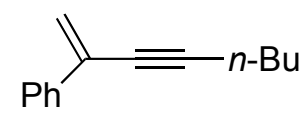

2.3.2a

Oct-1-en-3-yn-2-ylbenzene (2.3.2a) was purified by flash chromatography (Hexane-EtOAc, v/v $40 / 1)$ as yellow liquid. Reaction time about $10 \mathrm{~h}$; Isolated yield $91 \%$; ${ }^{1} \mathrm{H}-\mathrm{NMR}(600 \mathrm{MHz}$, $\left.\mathrm{CDCl}_{3}\right) \delta$ 7.67-7.64 (m, 2H), 7.37-7.33 (m, 2H), 7.31-7.28 (m, 1H), $5.84(\mathrm{~s}, 1 \mathrm{H}), 5.58(\mathrm{~s}, 1 \mathrm{H})$, $2.42(\mathrm{t}, J=7.2 \mathrm{~Hz}, 2 \mathrm{H}), 1.61$ (quin, $J=7.2 \mathrm{~Hz}, 2 \mathrm{H}), 1.49(\mathrm{sex}, J=7.2 \mathrm{~Hz}, 2 \mathrm{H}), 0.96(\mathrm{t}, J=7.2$ $\mathrm{Hz}, 3 \mathrm{H}) ;{ }^{13} \mathrm{C}-\mathrm{NMR}\left(150 \mathrm{MHz}, \mathrm{CDCl}_{3}\right) \delta 137.8,131.0,128.2,128.0,126.0,119.3,92.0,79.7$, 30.8, 22.0, 19.0, 13.6; HRMS Calculated for $\mathrm{C}_{14} \mathrm{H}_{17}[\mathrm{M}+\mathrm{H}]^{+}$: 185.1330, Found: 185.1333.

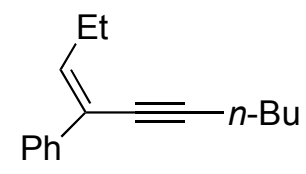

2.3.2b

(Z)-dec-3-en-5-yn-4-ylbenzene (2.3.2b) was purified by flash chromatography (Hexane-EtOAc, $\mathrm{v} / \mathrm{v} 40 / 1)$ as yellow liquid. Reaction time about $2 \mathrm{~h}$; Isolated yield 95\%; ${ }^{1} \mathrm{H}-\mathrm{NMR}(600 \mathrm{MHz}$, $\left.\mathrm{CDCl}_{3}\right) \delta 7.61-7.59(\mathrm{~m}, 2 \mathrm{H}), 7.34-7.31(\mathrm{~m}, 2 \mathrm{H}), 7.26-7.23(\mathrm{~m}, 1 \mathrm{H}), 6.33(\mathrm{t}, J=7.2 \mathrm{~Hz}, 1 \mathrm{H}), 2.49$ (quin, $J=7.2 \mathrm{~Hz}, 2 \mathrm{H}), 2.47(\mathrm{t}, J=7.2 \mathrm{~Hz}, 2 \mathrm{H}), 1.62$ (quin, $J=7.2 \mathrm{~Hz}, 2 \mathrm{H}), 1.51$ (sex, $J=7.2$ $\mathrm{Hz}, 2 \mathrm{H}), 1.11(\mathrm{t}, J=7.2 \mathrm{~Hz}, 3 \mathrm{H}), 0.97(\mathrm{t}, J=7.2 \mathrm{~Hz}, 3 \mathrm{H}) ;{ }^{13} \mathrm{C}-\mathrm{NMR}\left(150 \mathrm{MHz}, \mathrm{CDCl}_{3}\right) \delta 138.9$, 
$138.7,128.2,127.1,125.9,123.2,96.2,77.6,31.0,24.5,22.0,19.3,13.6,13.5$; HRMS Calculated for $\mathrm{C}_{16} \mathrm{H}_{21}[\mathrm{M}+\mathrm{H}]^{+}:$213.1643, Found: 213.1640.

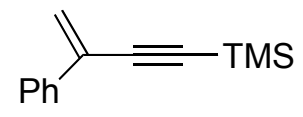

2.3.2c

Trimethyl(3-phenylbut-3-en-1-ynyl)silane (2.3.2c) was purified by flash chromatography (Hexane-EtOAc, v/v 40/1) as yellow liquid. Reaction time about $10 \mathrm{~h}$; Isolated yield $81 \% ;{ }^{1} \mathrm{H}$ NMR (600 MHz, $\left.\mathrm{CDCl}_{3}\right) \delta$ 7.67-7.64 (m, 2H), 7.38-7.35 (m, 2H), 7.33-7.30 (m, 1H), $5.94(\mathrm{~s}$, 1H), $5.72(\mathrm{~s}, 1 \mathrm{H}), 0.27(\mathrm{~s}, 9 \mathrm{H}) ;{ }^{13} \mathrm{C}-\mathrm{NMR}\left(150 \mathrm{MHz}, \mathrm{CDCl}_{3}\right) \delta$ 136.9, 130.6, 128.3, 128.2, 126.0, 121.4, 104.1, 95.9, -0.1; HRMS Calculated for $\mathrm{C}_{13} \mathrm{H}_{17} \mathrm{Si}[\mathrm{M}+\mathrm{H}]^{+}: 201.1100$, Found: 201.1093.

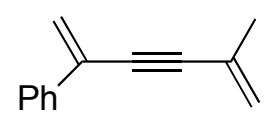

2.3.2d

(5-methylhexa-1,5-dien-3-yn-2-yl)benzene (2.3.2d) was purified by flash chromatography (Hexane-EtOAc, v/v 40/1) as yellow liquid. Reaction time about $10 \mathrm{~h}$; Isolated yield $90 \% ;{ }^{1} \mathrm{H}-$ NMR $\left(600 \mathrm{MHz}, \mathrm{CDCl}_{3}\right) \delta$ 7.67-7.64 (m, 2H), 7.38-7.34 (m, 2H), 7.33-7.29 (m, 1H), $5.92(\mathrm{~s}$, $1 \mathrm{H}), 5.66(\mathrm{~s}, 1 \mathrm{H}), 5.40(\mathrm{q}, J=1.2 \mathrm{~Hz}, 1 \mathrm{H}), 5.31$ (quin, $J=1.2 \mathrm{~Hz}, 1 \mathrm{H}), 1.99(\mathrm{t}, J=1.2 \mathrm{~Hz}, 3 \mathrm{H})$; ${ }^{13} \mathrm{C}-\mathrm{NMR}\left(150 \mathrm{MHz}, \mathrm{CDCl}_{3}\right) \delta 137.3,130.6,128.3,128.2,126.7,126.0,122.2,120.5,92.0$, 87.5, 23.4; HRMS Calculated for $\mathrm{C}_{13} \mathrm{H}_{13}[\mathrm{M}+\mathrm{H}]^{+}$: 169.1017, Found: 169.1006 . 


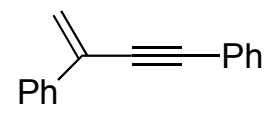

2.3.2e

But-3-en-1-yne-1,3-diyldibenzene (2.3.2e) was purified by flash chromatography (HexaneEtOAc, v/v 40/1) as yellow liquid. Reaction time about 10 h; Isolated yield 88\%; ${ }^{\mathrm{H}-\mathrm{NMR}}(600$ $\left.\mathrm{MHz}, \mathrm{CDCl}_{3}\right) \delta$ 7.74-7.72 (m, 2H), 7.55-7.53 (m, 2H), 7.41-7.36 (m, 2H), 7.35-7.33 (m, 4H), $5.98(\mathrm{~s}, 1 \mathrm{H}), 5.76(\mathrm{~s}, 1 \mathrm{H}) ;{ }^{13} \mathrm{C}-\mathrm{NMR}\left(150 \mathrm{MHz}, \mathrm{CDCl}_{3}\right) \delta 137.3,131.7,130.6,128.4,128.3$, 126.1, 123.1, 120.7, 90.7, 88.5; HRMS Calculated for $\mathrm{C}_{16} \mathrm{H}_{13}[\mathrm{M}+\mathrm{H}]^{+}$: 205.1017, Found: 205.1013.

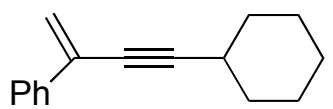

2.3.2f

(4-cyclohexylbut-1-en-3-yn-2-yl)benzene (2.3.2f) was purified by flash chromatography (Hexane-EtOAc, v/v 40/1) as yellow liquid. Reaction time about $10 \mathrm{~h}$; Isolated yield 85\%; ${ }^{1} \mathrm{H}$ NMR $\left(600 \mathrm{MHz}, \mathrm{CDCl}_{3}\right) \delta$ 7.68-7.66 (m, 2H), 7.37-7.33 (m, 2H), 7.31-7.28 (m, 1H), $5.84(\mathrm{~s}$, $1 \mathrm{H}), 5.59(\mathrm{~s}, 1 \mathrm{H}), 2.64-2.59(\mathrm{~m}, 1 \mathrm{H}), 1.91-1.88(\mathrm{~m}, 2 \mathrm{H}), 1.79-1.74(\mathrm{~m}, 2 \mathrm{H}), 1.56-1.54(\mathrm{~m}, 2 \mathrm{H})$, 1.40-1.34 (m, 4H); ${ }^{13} \mathrm{C}-\mathrm{NMR}\left(150 \mathrm{MHz}, \mathrm{CDCl}_{3}\right) \delta 137.8,130.9,128.2,128.0,126.0,119.1$, 96.0, 79.0, 32.7, 29.7, 25.9, 24.9; HRMS Calculated for $\mathrm{C}_{16} \mathrm{H}_{19}[\mathrm{M}+\mathrm{H}]^{+}:$211.1487, Found: 211.1480 . 


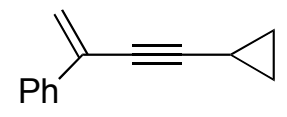

2.3.2g

(4-cyclopropylbut-1-en-3-yn-2-yl)benzene $\mathbf{( 2 . 3 . 2 g )}$ was purified by flash chromatography (Hexane-EtOAc, v/v 40/1) as yellow liquid. Reaction time about $10 \mathrm{~h}$; Isolated yield 85\%; ${ }^{1} \mathrm{H}$ NMR (600 MHz, $\left.\mathrm{CDCl}_{3}\right) \delta$ 7.63-7.61 (m, 2H), 7.36-7.32 (m, 2H), 7.31-7.27 (m, 1H), $5.82(\mathrm{~s}$, $1 \mathrm{H}), 5.56(\mathrm{~s}, 1 \mathrm{H}), 1.48-1.43(\mathrm{~m}, 1 \mathrm{H}), 0.89-0.85(\mathrm{~m}, 2 \mathrm{H}), 0.82-0.79(\mathrm{~m}, 2 \mathrm{H}) ;{ }^{13} \mathrm{C}-\mathrm{NMR}(150$ $\left.\mathrm{MHz}, \mathrm{CDCl}_{3}\right) \delta, 137.8,130.8,128.2,128.1,126.0,119.4,95.0,75.0,8.6,0.2 ;$ HRMS Calculated for $\mathrm{C}_{13} \mathrm{H}_{13}[\mathrm{M}+\mathrm{H}]^{+}:$169.1017, Found: 169.1000 .

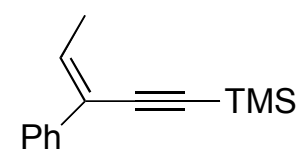

2.3.2h

(Z)-trimethyl(3-phenylpent-3-en-1-ynyl)silane (2.3.2h) was purified by flash chromatography (Hexane-EtOAc, v/v 40/1) as yellow liquid. Reaction time about 2 h; Isolated yield 70\%; ${ }^{1} \mathrm{H}-$ NMR (600 MHz, $\left.\mathrm{CDCl}_{3}\right) \delta$ 7.60-7.58 (m, 2H), 7.36-7.32 (m, 2H), 7.28-7.25 (m, 1H), $6.52(\mathrm{q}, J=$ $7.2 \mathrm{~Hz}, 1 \mathrm{H}), 2.10(\mathrm{~d}, J=7.2 \mathrm{~Hz}, 3 \mathrm{H}), 0.29(\mathrm{~s}, 9 \mathrm{H}) ;{ }^{13} \mathrm{C}-\mathrm{NMR}\left(150 \mathrm{MHz}, \mathrm{CDCl}_{3}\right) \delta 138.0,134.2$, 128.3, 127.3, 125.8, 124.6, 102.2, 100.7, 17.0, 0.12; HRMS Calculated for $\mathrm{C}_{14} \mathrm{H} 1{ }_{9} \mathrm{Si}[\mathrm{M}+\mathrm{H}]^{+}$: 215.1256, Found: 215.1250 . 


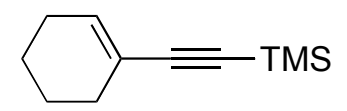

2.3.2i

(Cyclohexenylethynyl)trimethylsilane (2.3.2i) was purified by flash chromatography (HexaneEtOAc, v/v 40/1) as yellow liquid. Reaction time about 10 h; Isolated yield 46\% (conv. 50\%); ${ }^{1} \mathrm{H}-\mathrm{NMR}\left(600 \mathrm{MHz}, \mathrm{CDCl}_{3}\right) \delta$ 6.19-6.17 (m, 1H), 2.15-2.11 (m, 2H), 2.10-2.06 (m, 2H), 1.64$1.60(\mathrm{~m}, 2 \mathrm{H}), 1.58-1.54(\mathrm{~m}, 2 \mathrm{H}), 0.18(\mathrm{~s}, 9 \mathrm{H}) ;{ }^{13} \mathrm{C}-\mathrm{NMR}\left(150 \mathrm{MHz}, \mathrm{CDCl}_{3}\right) \delta 136.2,120.8$, 107.3, 90.8, 29.0, 25.6, 22.2, 21.4, 0.14; HRMS Calculated for $\mathrm{C}_{11} \mathrm{H}_{19} \mathrm{Si}[\mathrm{M}+\mathrm{H}]^{+}:$179.1256, Found: 179.1262 .

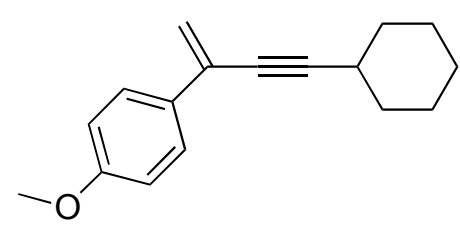

2.3.2j

1-(4-cyclohexylbut-1-en-3-yn-2-yl)-4-methoxybenzene $\mathbf{( 2 . 3 . 2 j )}$ was purified by flash chromatography (Hexane-EtOAc, v/v 40/1) as yellow liquid. Reaction time about 10 h; Isolated yield 81\%; ${ }^{1} \mathrm{H}-\mathrm{NMR}\left(600 \mathrm{MHz}, \mathrm{CDCl}_{3}\right) \delta 7.60(\mathrm{~d}, J=9.0 \mathrm{~Hz}, 2 \mathrm{H}), 6.87(\mathrm{~d}, J=9.0 \mathrm{~Hz}, 2 \mathrm{H})$, $5.73(\mathrm{~s}, 1 \mathrm{H}), 5.48(\mathrm{~s}, 1 \mathrm{H}), 2.63-2.59(\mathrm{~m}, 1 \mathrm{H}), 1.91-1.88(\mathrm{~m}, 2 \mathrm{H}), 1.79-1.73(\mathrm{~m}, 2 \mathrm{H}), 1.59-1.53$ (m, 2H), 1.40-1.32 (m, 4H); ${ }^{13} \mathrm{C}-\mathrm{NMR}\left(150 \mathrm{MHz}, \mathrm{CDCl}_{3}\right) \delta 159.8,130.7,130.4,127.5,117.5$, 113.7, 96.0, 80.0, 55.5, 32.9, 29.9, 26.1, 25.1; HRMS Calculated for $\mathrm{C}_{17} \mathrm{H}_{21} \mathrm{O}[\mathrm{M}+\mathrm{H}]^{+}: 241.1592$, Found: 241.1590 . 


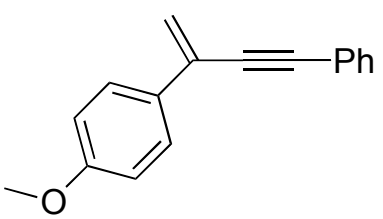

2.3.2k

1-methoxy-4-(4-phenylbut-1-en-3-yn-2-yl)benzene $\quad$ (2.3.2k) was purified by flash chromatography (Hexane-EtOAc, v/v 40/1) as yellow liquid. Reaction time about $10 \mathrm{~h}$; Isolated yield 75\%; ${ }^{1} \mathrm{H}-\mathrm{NMR}\left(600 \mathrm{MHz}, \mathrm{CDCl}_{3}\right) \delta 7.67(\mathrm{~d}, J=9.0 \mathrm{~Hz}, 2 \mathrm{H}), 7.55-7.52(\mathrm{~m}, 2 \mathrm{H}), 7.38-7.33$ $(\mathrm{m}, 3 \mathrm{H}), 6.91(\mathrm{~d}, J=9.0 \mathrm{~Hz}, 2 \mathrm{H}), 5.88(\mathrm{~s}, 1 \mathrm{H}), 5.66(\mathrm{~s}, 1 \mathrm{H}), 3.84(\mathrm{~s}, 3 \mathrm{H}) ;{ }^{13} \mathrm{C}-\mathrm{NMR}(150 \mathrm{MHz}$, $\left.\mathrm{CDCl}_{3}\right) \delta 159.8,131.6,129.9,128.3,127.3,123.2,118.7,113.7,102.8,90.5,88.8,55.3$; HRMS Calculated for $\mathrm{C}_{17} \mathrm{H}_{15} \mathrm{O}[\mathrm{M}+\mathrm{H}]^{+}:$235.1123, Found: 235.1122 .

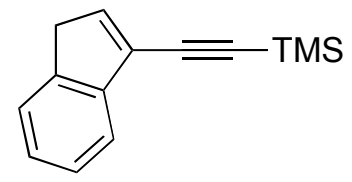

2.3.21

((1H-inden-3-yl)ethynyl)trimethylsilanetriazole (2.3.2l) was purified by flash chromatography (Hexane-EtOAc, v/v 40/1) as yellow liquid. Reaction time about 2 h; Isolated yield 80\%; ${ }^{1} \mathrm{H}-$ NMR $\left(600 \mathrm{MHz}, \mathrm{CDCl}_{3}\right) \delta 7.53(\mathrm{~d}, J=7.8 \mathrm{~Hz}, 1 \mathrm{H}), 7.44(\mathrm{~d}, J=7.8 \mathrm{~Hz}, 1 \mathrm{H}), 7.35(\mathrm{t}, J=7.2 \mathrm{~Hz}$, $1 \mathrm{H}), 7.24(\mathrm{t}, J=7.2 \mathrm{~Hz}, 1 \mathrm{H}), 6.79(\mathrm{t}, J=2.4 \mathrm{~Hz}, 1 \mathrm{H}), 3.46(\mathrm{~d}, J=2.4 \mathrm{~Hz}, 2 \mathrm{H}), 0.29(\mathrm{~s}, 9 \mathrm{H}) ;{ }^{13} \mathrm{C}-$ NMR $\left(150 \mathrm{MHz}, \mathrm{CDCl}_{3}\right) \delta 145.9,137.0,132.6,127.5,123.9,119.9,113.0,109.3,80.1,79.5$, 49.3; HRMS Calculated for $\mathrm{C}_{14} \mathrm{H}_{17} \mathrm{Si}[\mathrm{M}+\mathrm{H}]^{+}:$213.1100, Found: 213.1113. 


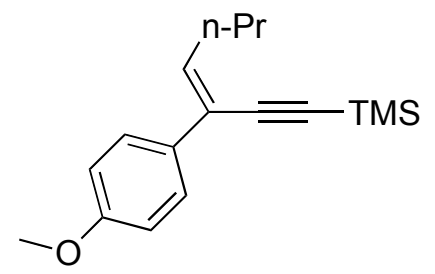

2.3.2m

(Z)-(3-(2-methoxyphenyl)hept-3-en-1-ynyl)trimethylsilane (2.3.2m) was purified by flash chromatography (Hexane-EtOAc, v/v 40/1) as yellow liquid. Reaction time about 2 h; Isolated yield 85\%; ${ }^{1} \mathrm{H}-\mathrm{NMR}\left(600 \mathrm{MHz}, \mathrm{CDCl}_{3}\right) \delta 7.39(\mathrm{dd}, J=7.2 \mathrm{~Hz}, 1.8 \mathrm{~Hz}, 1 \mathrm{H}), 7.23(\mathrm{dt}, J=7.2 \mathrm{~Hz}$, $1.8 \mathrm{~Hz}, 1 \mathrm{H}), 6.94(\mathrm{dt}, J=7.2 \mathrm{~Hz}, 1.2 \mathrm{~Hz}, 1 \mathrm{H}), 6.90(\mathrm{dd}, J=7.2 \mathrm{~Hz}, 1.2 \mathrm{~Hz}, 1 \mathrm{H}), 6.39(\mathrm{t}, J=7.8$ $\mathrm{Hz}, 1 \mathrm{H}), 3.84(\mathrm{~s}, 3 \mathrm{H}), 2.47$ (q, $J=7.8 \mathrm{~Hz}, 2 \mathrm{H}), 1.54(\mathrm{sex}, J=7.8 \mathrm{~Hz}, 2 \mathrm{H}), 0.99$ (t, $J=7.8 \mathrm{~Hz}$, 3H), $0.22(\mathrm{~s}, 9 \mathrm{H}) ;{ }^{13} \mathrm{C}-\mathrm{NMR}\left(150 \mathrm{MHz}, \mathrm{CDCl}_{3}\right) \delta 156.8,144.2,130.1,128.4,128.3,120.6$, 120.5, 111.6, 103.4, 98.1, 55.6, 33.3, 22.2, 13.9, 0.1, 55.2; HRMS Calculated for $\mathrm{C}_{17} \mathrm{H}_{25} \mathrm{OSi}$ $[\mathrm{M}+\mathrm{H}]^{+}:$273.1675, Found: 273.1671 .

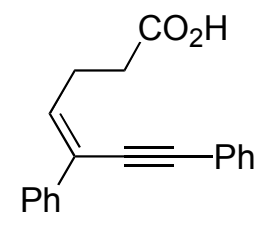

2.3.2n

(Z)-5,7-diphenylhept-4-en-6-ynoic acid (2.3.2n) was purified by flash chromatography (Hexane-EtOAc, v/v 10/1) as white solid. Reaction time about 0.5 h; Isolated yield $91 \% ;{ }^{1} \mathrm{H}-$ NMR (600 MHz, $\left.\mathrm{CDCl}_{3}\right) \delta$ 7.68-7.65 (m, 2H), 7.56-7.53 (m, 2H), 7.39-7.34 (m, 5H), 7.32-7.29 $(\mathrm{m}, 1 \mathrm{H}), 6.48(\mathrm{t}, J=7.2 \mathrm{~Hz}, 1 \mathrm{H}), 2.91(\mathrm{q}, J=7.2 \mathrm{~Hz}, 2 \mathrm{H}), 2.64(\mathrm{t}, J=7.2 \mathrm{~Hz}, 2 \mathrm{H}) ;{ }^{13} \mathrm{C}-\mathrm{NMR}$ $\left(150 \mathrm{MHz}, \mathrm{CDCl}_{3}\right) \delta 179.0,137.6,135.2,131.6,128.40,128.38,128.35,127.8,126.1,125.1$, 
123.2, 96.1, 86.1, 33.3, 26.3; HRMS Calculated for $\mathrm{C}_{19} \mathrm{H}_{17} \mathrm{O}_{2}[\mathrm{M}+\mathrm{H}]^{+}:$277.1229, Found: 277.1230.

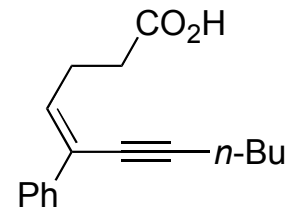

2.3 .20

(Z)-5-phenylundec-4-en-6-ynoic acid (2.3.20) was purified by flash chromatography (HexaneEtOAc, v/v 10/1) as yellow liquid. Reaction time about $0.5 \mathrm{~h}$; Isolated yield 89\%; ${ }^{\mathrm{H}-\mathrm{NMR}}(600$ $\left.\mathrm{MHz}, \mathrm{CDCl}_{3}\right) \delta$ 7.60-7.57 (m, 2H), 7.34-7.30 (m, 2H), 7.27-7.24 (m, 1H), $6.34(\mathrm{t}, J=7.2 \mathrm{~Hz}$ 1H), 2.79 (q, $J=7.2 \mathrm{~Hz}, 2 \mathrm{H}), 2.57$ (t, $J=7.2 \mathrm{~Hz}, 2 \mathrm{H}), 2.47$ (t, $J=7.2 \mathrm{~Hz}, 2 \mathrm{H}), 1.62$ (quin, $J=$ $7.2 \mathrm{~Hz}, 2 \mathrm{H}), 1.50(\mathrm{sex}, J=7.2 \mathrm{~Hz}, 2 \mathrm{H}), 0.95(\mathrm{t}, J=7.2 \mathrm{~Hz}, 3 \mathrm{H}) ;{ }^{13} \mathrm{C}-\mathrm{NMR}\left(150 \mathrm{MHz}, \mathrm{CDCl}_{3}\right) \delta$ $178.9,138.4,133.6,128.2,127.5,126.0,125.5,97.4,33.3,30.9,26.0,22.0,19.3,13.6$; HRMS Calculated for $\mathrm{C}_{17} \mathrm{H}_{21} \mathrm{O}_{2}[\mathrm{M}+\mathrm{H}]^{+}:$257.1542, Found: 257.1536 .

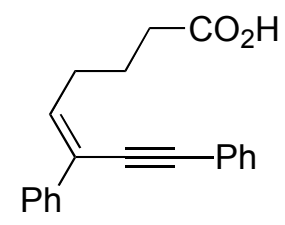

2.3.2p

(Z)-6,8-diphenyloct-5-en-7-ynoic acid (2.3.2p) was purified by flash chromatography (HexaneEtOAc, v/v 10/1) as yellow liquid. Reaction time about $0.5 \mathrm{~h}$; Isolated yield $92 \% ;{ }^{1} \mathrm{H}-\mathrm{NMR}(600$ $\left.\mathrm{MHz}, \mathrm{CDCl}_{3}\right) \delta$ 7.67-7.64 (m, 2H), 7.54-7.51 (m, 2H), 7.38-7.32 (m, 5H), 7.31-7.27 (m, 1H), 
$6.43(\mathrm{t}, J=7.2 \mathrm{~Hz}, 1 \mathrm{H}), 2.65(\mathrm{q}, J=7.2 \mathrm{~Hz}, 2 \mathrm{H}), 2.47$ (t, $J=7.2 \mathrm{~Hz}, 2 \mathrm{H}), 1.91$ (quin, $J=7.2 \mathrm{~Hz}$, $2 \mathrm{H}) ;{ }^{13} \mathrm{C}-\mathrm{NMR}\left(150 \mathrm{MHz}, \mathrm{CDCl}_{3}\right) \delta 179.0,137.9,136.7,131.5,128.4,128.3,128.2,127.7$, $126.0,124.8,123.3,95.5,86.5,33.2,30.5,24.0$; HRMS Calculated for $\mathrm{C}_{15} \mathrm{H}_{14} \mathrm{~N}_{3}[\mathrm{M}+\mathrm{H}]^{+}$: 236.1188, Found: 236.1178 .

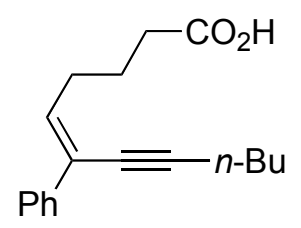

2.3.2q

(Z)-6-phenyldodec-5-en-7-ynoic acid (2.3.2q) was purified by flash chromatography (HexaneEtOAc, v/v 10/1) as yellow liquid. Reaction time about $0.5 \mathrm{~h}$; Isolated yield 88\%; ${ }^{1} \mathrm{H}-\mathrm{NMR}(600$ $\left.\mathrm{MHz}, \mathrm{CDCl}_{3}\right) \delta$ 7.60-7.57 (m, 2H), 7.34-7.30 (m, 2H), 7.27-7.23 (m, 1H), $6.29(\mathrm{t}, J=7.2 \mathrm{~Hz}$ 1H), 2.54 (q, $J=7.2 \mathrm{~Hz}, 2 \mathrm{H}), 2.46(\mathrm{t}, J=7.2 \mathrm{~Hz}, 2 \mathrm{H}), 2.44$ (t, $J=7.2 \mathrm{~Hz}, 2 \mathrm{H}$ ), 1.86 (quin, $J=$ $7.2 \mathrm{~Hz}, 2 \mathrm{H}), 1.61$ (quin, $J=7.2 \mathrm{~Hz}, 2 \mathrm{H}), 1.49(\mathrm{sex}, J=7.2 \mathrm{~Hz}, 2 \mathrm{H}), 0.96(\mathrm{t}, J=7.2 \mathrm{~Hz}, 3 \mathrm{H}) ;{ }^{13} \mathrm{C}-$ NMR $\left(150 \mathrm{MHz}, \mathrm{CDCl}_{3}\right) \delta 179.8,138.6,135.1,128.2,127.4,125.9,125.1,96.7,77.5,33.4$, 30.9, 30.3, 24.0, 22.0, 19.2, 13.6; HRMS Calculated for $\mathrm{C}_{18} \mathrm{H}_{23} \mathrm{O}_{2}[\mathrm{M}+\mathrm{H}]^{+}: 271.1698$, Found: 271.1704.

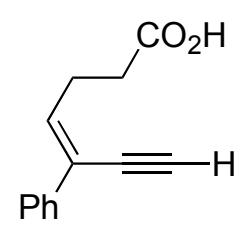

2.3.2r 
(E)-5-phenylhept-4-en-6-ynoic acid (2.3.2r) was purified by flash chromatography (HexaneEtOAc, v/v 10/1) as yellow liquid. Reaction time about $0.5 \mathrm{~h}$; Isolated yield $85 \% ;{ }^{1} \mathrm{H}-\mathrm{NMR}(600$ $\left.\mathrm{MHz}, \mathrm{CDCl}_{3}\right) \delta$ 7.60-7.58 (m, 2H), 7.36-7.32 (m, 2H), 7.29-7.27 (m, 1H), 7.32-7.29 (m, 1H), $6.50(\mathrm{t}, J=7.2 \mathrm{~Hz}, 1 \mathrm{H}), 3.38(\mathrm{~s}, 1 \mathrm{H}), 2.83(\mathrm{q}, J=7.2 \mathrm{~Hz}, 2 \mathrm{H}), 2.59(\mathrm{t}, J=7.2 \mathrm{~Hz}, 2 \mathrm{H}) ;{ }^{13} \mathrm{C}-\mathrm{NMR}$ $\left(150 \mathrm{MHz}, \mathrm{CDCl}_{3}\right) \delta 178.5,137.2,136.8,128.4,127.9,125.9,124.2,83.9,80.3,33.0,26.1 ;$ HRMS Calculated for $\mathrm{C}_{13} \mathrm{H}_{13} \mathrm{O}_{2}[\mathrm{M}+\mathrm{H}]^{+}:$201.0916, Found: 201.0912.

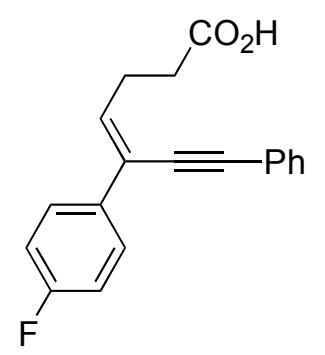

2.3.2s

(E)-5-(4-fluorophenyl)hept-4-en-6-ynoic acid (2.3.2s) was purified by flash chromatography (Hexane-EtOAc, v/v 10/1) as yellow liquid. Reaction time about $0.5 \mathrm{~h}$; Isolated yield $92 \% ;{ }^{1} \mathrm{H}-$ NMR $\left(600 \mathrm{MHz}, \mathrm{CDCl}_{3}\right) \delta$ 7.63-7.60 (m, 2H), 7.54-7.51 (m, 2H), 7.37-7.34 (m, 3H), 7.06-7.03 $(\mathrm{m}, 2 \mathrm{H}), 6.40(\mathrm{t}, J=7.2 \mathrm{~Hz}, 1 \mathrm{H}), 2.88(\mathrm{q}, J=7.2 \mathrm{~Hz}, 2 \mathrm{H}), 2.63(\mathrm{t}, J=7.2 \mathrm{~Hz}, 2 \mathrm{H}) ;{ }^{13} \mathrm{C}-\mathrm{NMR}$ $\left(150 \mathrm{MHz}, \mathrm{CDCl}_{3}\right) \delta 178.4,163.3,161.7,134.9,133.9,133.8,131.6,128.5,128.4,127.7,127.6$, 124.1, 123.0, 115.3, 115.2, 96.3, 85.8, 33.2, 26.3; HRMS Calculated for $\mathrm{C}_{13} \mathrm{H}_{12} \mathrm{FO}_{2}[\mathrm{M}+\mathrm{H}]^{+}$: 219.0821, Found: 219.0815. 


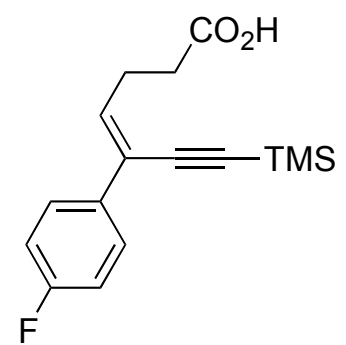

2.3.2t

(Z)-5-(4-fluorophenyl)-7-(trimethylsilyl)hept-4-en-6-ynoic acidtriazole (2.3.2t) was purified by flash chromatography (Hexane-EtOAc, v/v 10/1) as yellow liquid. Reaction time about 0.5 h; Isolated yield 88\%; ${ }^{1} \mathrm{H}-\mathrm{NMR}\left(600 \mathrm{MHz}, \mathrm{CDCl}_{3}\right) \delta$ 7.55-7.52 (m, 2H), 7.03-7.00 (m, 2H), $6.38(\mathrm{t}$, $J=7.2 \mathrm{~Hz}, 1 \mathrm{H}), 2.80(\mathrm{q}, J=7.2 \mathrm{~Hz}, 2 \mathrm{H}), 2.59(\mathrm{t}, J=7.2 \mathrm{~Hz}, 2 \mathrm{H}), 0.26(\mathrm{~s}, 9 \mathrm{H}) ;{ }^{13} \mathrm{C}-\mathrm{NMR}(150$ $\left.\mathrm{MHz}, \mathrm{CDCl}_{3}\right) \delta 178.9,163.3,161.7,135.9,133.5,133.4,127.6,127.5,124.2,115.2,115.1$, 101.9, 101.3, 33.0, 26.2, -0.05; HRMS Calculated for $\mathrm{C}_{16} \mathrm{H}_{20} \mathrm{FO}_{2} \mathrm{Si}[\mathrm{M}+\mathrm{H}]^{+}:$291.1217, Found: 291.1200 .

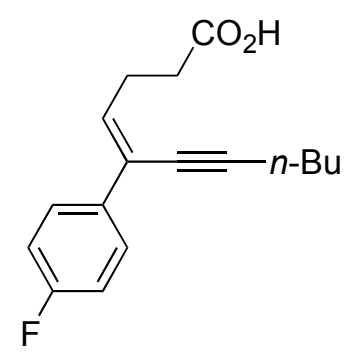

2.3.2u

(Z)-5-(4-fluorophenyl)undec-4-en-6-ynoic acid (2.3.2u) was purified by flash chromatography (Hexane-EtOAc, v/v 10/1) as yellow liquid. Reaction time about $0.5 \mathrm{~h}$; Isolated yield $83 \%$; ${ }^{1} \mathrm{H}-$ NMR (600 MHz, $\left.\mathrm{CDCl}_{3}\right) \delta$ 7.56-7.53 (m, 2H), 7.02-6.99 (m, 2H), $6.26(\mathrm{t}, J=7.2 \mathrm{~Hz}, 1 \mathrm{H}), 2.77$ (q, $J=7.2 \mathrm{~Hz}, 2 \mathrm{H}), 2.57(\mathrm{t}, J=7.2 \mathrm{~Hz}, 2 \mathrm{H}), 2.46(\mathrm{t}, J=7.2 \mathrm{~Hz}, 2 \mathrm{H}), 1.61$ (quin, $J=7.2 \mathrm{~Hz}, 2 \mathrm{H})$, 
$1.48(\operatorname{sex}, J=7.2 \mathrm{~Hz}, 2 \mathrm{H}), 0.95(\mathrm{t}, J=7.2 \mathrm{~Hz}, 3 \mathrm{H}) ;{ }^{13} \mathrm{C}-\mathrm{NMR}\left(150 \mathrm{MHz}, \mathrm{CDCl}_{3}\right) \delta 178.9,163.2$, 161.6, 134.5, 134.4, 133.3, 127.6, 127.5, 124.5, 115.1, 115.0, 97.7, 33.2, 30.9, 26.0, 22.0, 19.2, 13.6; HRMS Calculated for $\mathrm{C}_{17} \mathrm{H}_{20} \mathrm{FO}_{2}[\mathrm{M}+\mathrm{H}]^{+}:$275.1447, Found: 275.1441 .

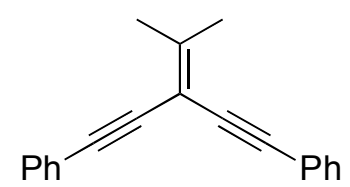

2.3.2v

(3-(propan-2-ylidene)penta-1,4-diyne-1,5-diyl)dibenzene (2.3.2v) was purified by flash chromatography (Hexane-EtOAc, v/v 40/1) as yellow liquid. Reaction time about $7 \mathrm{~h}$; Isolated yield 87\%; ${ }^{1} \mathrm{H}-\mathrm{NMR}\left(600 \mathrm{MHz}, \mathrm{CDCl}_{3}\right) \delta$ 7.53-7.50 (m, 4H), 7.36-7.31 (m, 6H), $2.18(\mathrm{~s}, 6 \mathrm{H})$; ${ }^{13} \mathrm{C}-\mathrm{NMR}\left(150 \mathrm{MHz}, \mathrm{CDCl}_{3}\right) \delta 154.2,131.4,128.2,128.0,123.4,101.3,91.1,86.4,22.8$; HRMS Calculated for $\mathrm{C}_{20} \mathrm{H}_{17}[\mathrm{M}+\mathrm{H}]^{+}$: 257.1330, Found: 257.1333.

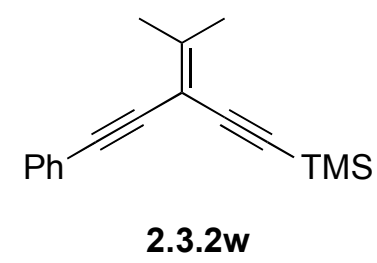

Trimethyl(4-methyl-3-(phenylethynyl)pent-3-en-1-ynyl)silane (2.3.2w) was purified by flash chromatography (Hexane-EtOAc, v/v 40/1) as yellow liquid. Reaction time about $7 \mathrm{~h}$; Isolated yield 90\%; ${ }^{1} \mathrm{H}-\mathrm{NMR}\left(600 \mathrm{MHz}, \mathrm{CDCl}_{3}\right) \delta$ 7.48-7.45 (m, 2H), 7.33-7.28 (m, 3H), $2.09(\mathrm{~s}, 3 \mathrm{H})$, $2.08(\mathrm{~s}, 3 \mathrm{H}), 0.23(\mathrm{~s}, 9 \mathrm{H}) ;{ }^{13} \mathrm{C}-\mathrm{NMR}\left(150 \mathrm{MHz}, \mathrm{CDCl}_{3}\right) \delta 155.6,131.4,128.2,128.0,123.4$, 
101.6, 101.5, 96.1, 91.3, 86.1, 22.8, 22.7, -0.03; HRMS Calculated for $\mathrm{C}_{17} \mathrm{H}_{21} \mathrm{Si}[\mathrm{M}+\mathrm{H}]^{+}$: 253.1413, Found: 253.1420 .

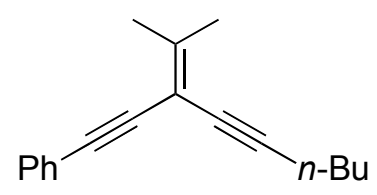

2.3.2x

(3-(propan-2-ylidene)nona-1,4-diynyl)benzene (2.3.2x) was purified by flash chromatography (Hexane-EtOAc, v/v 40/1) as yellow liquid. Reaction time about $7 \mathrm{~h}$; Isolated yield $79 \%$; ${ }^{1} \mathrm{H}-$ NMR $\left(600 \mathrm{MHz}, \mathrm{CDCl}_{3}\right) \delta$ 7.47-7.45 (m, 2H), 7.32-7.27 (m, 3H), $2.39(\mathrm{t}, J=7.2 \mathrm{~Hz}, 2 \mathrm{H}), 2.07$ (s, 3H), 2.05 (s, 3H), 1.57 (quin, $J=7.2 \mathrm{~Hz}, 2 \mathrm{H}), 1.47$ (sex, $J=7.2 \mathrm{~Hz}, 2 \mathrm{H}$ ), 0.94 (t, $J=7.2 \mathrm{~Hz}$, $3 \mathrm{H}) ;{ }^{13} \mathrm{C}-\mathrm{NMR}\left(150 \mathrm{MHz}, \mathrm{CDCl}_{3}\right) \delta 152.4,131.4,128.1,127.9,123.6,101.4,92.3,90.5,87.1$, 77.4, 30.9, 22.6, 22.5, 22.0, 19.2, 13.6; HRMS Calculated for $\mathrm{C}_{18} \mathrm{H}_{21}[\mathrm{M}+\mathrm{H}]^{+}:$237.1643, Found: 237.1635.

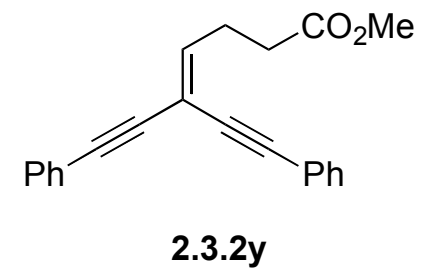

Methyl 7-phenyl-5-(phenylethynyl)hept-4-en-6-ynoate (2.3.2y) was purified by flash chromatography (Hexane-EtOAc, v/v 30/1) as yellow liquid. Reaction time about $0.5 \mathrm{~h}$; Isolated yield 93\%; ${ }^{1} \mathrm{H}-\mathrm{NMR}\left(600 \mathrm{MHz}, \mathrm{CDCl}_{3}\right) \delta$ 7.53-7.51 (m, 2H), 7.50-7.48 (m, 2H), 7.35-7.33 (m, 
3H), 7.33-7.31 (m, 3H), $6.46(\mathrm{t}, J=7.2 \mathrm{~Hz}, 1 \mathrm{H}), 3.71(\mathrm{~s}, 3 \mathrm{H}), 2.81(\mathrm{q}, J=7.2 \mathrm{~Hz}, 2 \mathrm{H}), 2.53(\mathrm{t}, J$ $=7.2 \mathrm{~Hz}, 2 \mathrm{H}) ;{ }^{13} \mathrm{C}-\mathrm{NMR}\left(150 \mathrm{MHz}, \mathrm{CDCl}_{3}\right) \delta 172.9,164.2,131.7,131.6,128.6,128.4,128.3$, 128.2, 122.8, 122.7, 107.1, 93.6, 87.2, 87.0, 84.3, 51.7, 32.8, 26.2; HRMS Calculated for $\mathrm{C}_{22} \mathrm{H}_{19} \mathrm{O}_{2}[\mathrm{M}+\mathrm{H}]^{+}: 315.1385$, Found: 315.1392 .

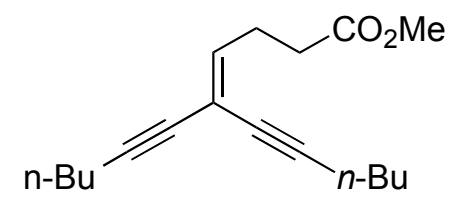

2.3.2z

Methyl 5-(hex-1-ynyl)undec-4-en-6-ynoate (2.3.2z) was purified by flash chromatography (Hexane-EtOAc, v/v 30/1) as yellow liquid. Reaction time about $0.5 \mathrm{~h}$; Isolated yield $90 \%$; ${ }^{1} \mathrm{H}-$ NMR (600 MHz, $\left.\mathrm{CDCl}_{3}\right) \delta 6.11(\mathrm{t}, J=7.2 \mathrm{~Hz}, 1 \mathrm{H}), 3.67(\mathrm{~s}, 3 \mathrm{H}), 2.60(\mathrm{q}, J=7.2 \mathrm{~Hz}, 2 \mathrm{H}), 2.40$ (t, $J=7.2 \mathrm{~Hz}, 2 \mathrm{H}), 2.35(\mathrm{t}, J=7.2 \mathrm{~Hz}, 2 \mathrm{H}), 2.28(\mathrm{t}, J=7.2 \mathrm{~Hz}, 2 \mathrm{H}$ ), 1.53 (quin, $J=7.2 \mathrm{~Hz}, 2 \mathrm{H}$ ), 1.50 (quin, $J=7.2 \mathrm{~Hz}, 2 \mathrm{H}), 1.42(\mathrm{sex}, J=7.2 \mathrm{~Hz}, 2 \mathrm{H}), 1.39(\mathrm{sex}, J=7.2 \mathrm{~Hz}, 2 \mathrm{H}), 0.91$ (t, $J=7.2$ $\mathrm{Hz}, 3 \mathrm{H}), 0.90(\mathrm{t}, J=7.2 \mathrm{~Hz}, 3 \mathrm{H}) ;{ }^{13} \mathrm{C}-\mathrm{NMR}\left(150 \mathrm{MHz}, \mathrm{CDCl}_{3}\right) \delta 173.1,143.4,107.3,94.5,87.6$, 78.8, 76.2, 51.6, 32.9, 30.6, 25.7, 22.0, 21.9, 19.1, 18.9, 13.6, 13.5; HRMS Calculated for $\mathrm{C}_{18} \mathrm{H}_{27} \mathrm{O}_{2}[\mathrm{M}+\mathrm{H}]^{+}:$275.2011, Found: 275.2023.

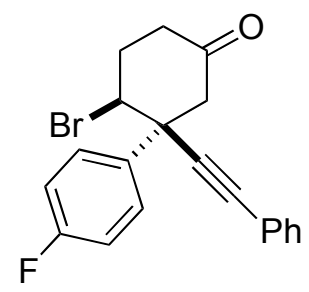

2.3.3e 
Syn-1-(2-bromo-1-(hex-1-ynyl)cyclohexyl)-4-fluorobenzene (2.3.3e) was purified by flash chromatography (Hexane-EtOAc, v/v 20/1) as yellow liquid. Check ${ }^{1} \mathrm{H},{ }^{13} \mathrm{C}-\mathrm{NMR}$ data in section (Full NMR characterizations of 3e, 3f, 3g); HRMS Calculated for $\mathrm{C}_{18} \mathrm{H}_{23} \mathrm{BrF}[\mathrm{M}+\mathrm{H}]^{+}$: 337.0967, Found: 337.0960.

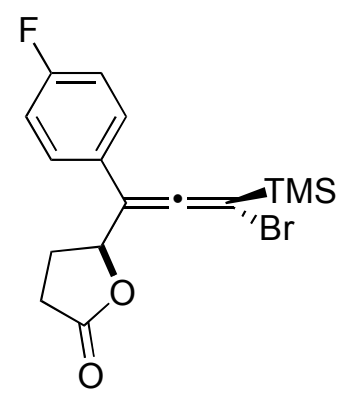

2.3.3f

Syn-5-(3-bromo-1-(4-fluorophenyl)-3-(trimethylsilyl)propa-1,2-dienyl)dihydrofuran-2(3H)one (2.3.3f) was purified by flash chromatography (Hexane-EtOAc, v/v 20/1) as yellow liquid. Check ${ }^{1} \mathrm{H},{ }^{13} \mathrm{C}-\mathrm{NMR}$ data in section (Full NMR characterizations of 3e, 3f, 3g); HRMS Calculated for $\mathrm{C}_{16} \mathrm{H}_{19} \mathrm{BrFO}_{2} \mathrm{Si}[\mathrm{M}+\mathrm{H}]^{+}: 369.0322$, Found: 369.0315 .

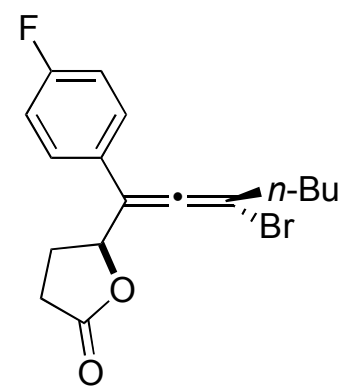

2.3.3g 
Syn-5-(3-bromo-1-(4-fluorophenyl)hepta-1,2-dienyl)dihydrofuran-2(3H)-one $\quad(2.3 .3 \mathrm{~g})$ was purified by flash chromatography (Hexane-EtOAc, v/v 20/1) as yellow liquid. Check ${ }^{1} \mathrm{H},{ }^{13} \mathrm{C}-$ NMR data in section (Full NMR characterizations of 3e, 3f, 3g); HRMS Calculated for $\mathrm{C}_{17} \mathrm{H}_{19} \mathrm{BrFO}_{2}[\mathrm{M}+\mathrm{H}]^{+}:$353.0552, Found: 353.0556.

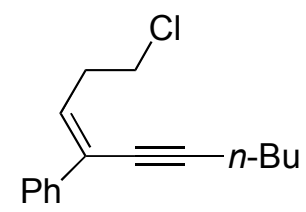

2.3.5a

(Z)-(1-chlorodec-3-en-5-yn-4-yl)benzene (2.3.5a) was purified by flash chromatography (Hexane-EtOAc, v/v 40/1) as yellow liquid. ${ }^{1} \mathrm{H}-\mathrm{NMR}\left(600 \mathrm{MHz}, \mathrm{CDCl}_{3}\right) \delta$ 7.62-7.59 (m, 2H), 7.35-7.32 (m, 2H), 7.29-7.25 (m, 1H), 6.36 (t, $J=7.2 \mathrm{~Hz}, 1 \mathrm{H}), 3.66$ (t, $J=7.2 \mathrm{~Hz}, 2 \mathrm{H}), 2.94(\mathrm{q}, J$ $=7.2 \mathrm{~Hz}, 2 \mathrm{H}), 2.47$ (t, $J=7.2 \mathrm{~Hz}, 2 \mathrm{H}), 1.62$ (quin, $J=7.2 \mathrm{~Hz}, 2 \mathrm{H}), 1.50($ sex, $J=7.2 \mathrm{~Hz}, 2 \mathrm{H})$, $0.97(\mathrm{t}, J=7.2 \mathrm{~Hz}, 3 \mathrm{H}) ;{ }^{13} \mathrm{C}-\mathrm{NMR}\left(150 \mathrm{MHz}, \mathrm{CDCl}_{3}\right) \delta 138.2,131.3,128.3,127.8,126.6,126.0$, 97.5, 77.3, 43.6, 34.2, 30.9, 22.0, 19.3, 13.6; HRMS Calculated for $\mathrm{C}_{16} \mathrm{H}_{20} \mathrm{Cl}[\mathrm{M}+\mathrm{H}]^{+}$: 247.1254, Found: 247.1250 .

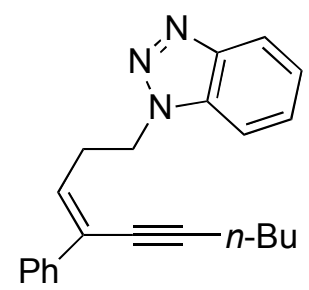

2.3.5b 
(Z)-1-(4-phenyldec-3-en-5-ynyl)-1H-benzo[d][1,2,3]triazole (2.3.5b) was purified by flash chromatography (Hexane-EtOAc, v/v 10/1) as yellow liquid. ${ }^{1} \mathrm{H}-\mathrm{NMR}\left(600 \mathrm{MHz}, \mathrm{CDCl}_{3}\right) \delta 8.07$ $(\mathrm{d}, J=8.4 \mathrm{~Hz}, 1 \mathrm{H}), 7.57(\mathrm{~d}, J=8.4 \mathrm{~Hz}, 1 \mathrm{H}), 7.50-7.48(\mathrm{~m}, 2 \mathrm{H}), 7.46(\mathrm{t}, J=7.8 \mathrm{~Hz}, 1 \mathrm{H}), 7.36(\mathrm{t}$, $J=7.8 \mathrm{~Hz}, 1 \mathrm{H}), 7.32-7.28(\mathrm{~m}, 2 \mathrm{H}), 7.27-7.23(\mathrm{~m}, 1 \mathrm{H}), 6.26(\mathrm{t}, J=7.2 \mathrm{~Hz}, 1 \mathrm{H}), 4.81(\mathrm{t}, J=7.2$ $\mathrm{Hz}, 2 \mathrm{H}), 3.15$ (q, $J=7.2 \mathrm{~Hz}, 2 \mathrm{H}), 2.41$ (t, $J=7.2 \mathrm{~Hz}, 2 \mathrm{H}), 1.57$ (quin, $J=7.2 \mathrm{~Hz}, 2 \mathrm{H}), 1.45$ (sex, $J=7.2 \mathrm{~Hz}, 2 \mathrm{H}), 0.94(\mathrm{t}, J=7.2 \mathrm{~Hz}, 3 \mathrm{H}) ;{ }^{13} \mathrm{C}-\mathrm{NMR}\left(150 \mathrm{MHz}, \mathrm{CDCl}_{3}\right) \delta 146.0,138.0,133.0$, $130.0,128.3,127.8,127.4,127.1,126.0,123.7,120.0,109.4,97.8,76.8,47.3,31.4,30.8,22.1$, 19.2, 13.6; HRMS Calculated for $\mathrm{C}_{22} \mathrm{H}_{24} \mathrm{~N}_{3}[\mathrm{M}+\mathrm{H}]^{+}: 330.1970$, Found: 330.1977 .

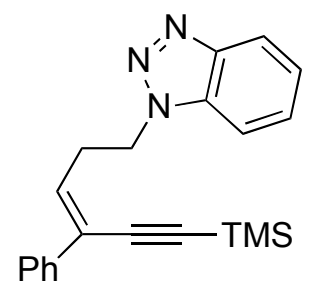

2.3.5c

(Z)-1-(4-phenyl-6-(trimethylsilyl)hex-3-en-5-ynyl)-1H-benzo[d][1,2,3]triazole $\quad(2.3 .5 c)$ was purified by flash chromatography (Hexane-EtOAc, v/v 10/1) as white solid. Isolated yield 75\%; ${ }^{1} \mathrm{H}-\mathrm{NMR}\left(600 \mathrm{MHz}, \mathrm{CDCl}_{3}\right) \delta 8.07(\mathrm{~d}, J=8.4 \mathrm{~Hz}, 1 \mathrm{H}), 7.58(\mathrm{~d}, J=8.4 \mathrm{~Hz}, 1 \mathrm{H}), 7.51-7.48(\mathrm{~m}$, 2H), $7.47(\mathrm{t}, J=7.8 \mathrm{~Hz}, 1 \mathrm{H}), 7.37(\mathrm{t}, J=7.8 \mathrm{~Hz}, 1 \mathrm{H}), 7.33-7.29(\mathrm{~m}, 2 \mathrm{H}), 7.28-7.25(\mathrm{~m}, 1 \mathrm{H}), 6.38$ (t, $J=7.2 \mathrm{~Hz}, 1 \mathrm{H}), 4.82(\mathrm{t}, J=7.2 \mathrm{~Hz}, 2 \mathrm{H}), 3.18(\mathrm{q}, J=7.2 \mathrm{~Hz}, 2 \mathrm{H}), 0.26(\mathrm{~s}, 9 \mathrm{H}) ;{ }^{13} \mathrm{C}-\mathrm{NMR}$ $\left(150 \mathrm{MHz}, \mathrm{CDCl}_{3}\right) \delta 146.0,137.0,133.0,132.4,128.3,128.0,127.1,127.0,126.0,123.8,120.0$ 109.3, 102.0, 101.2, 47.1, 31.6, -0.06; HRMS Calculated for $\mathrm{C}_{21} \mathrm{H}_{24} \mathrm{~N}_{3} \mathrm{Si}[\mathrm{M}+\mathrm{H}]^{+}: 346.1739$, Found: 346.1745. 


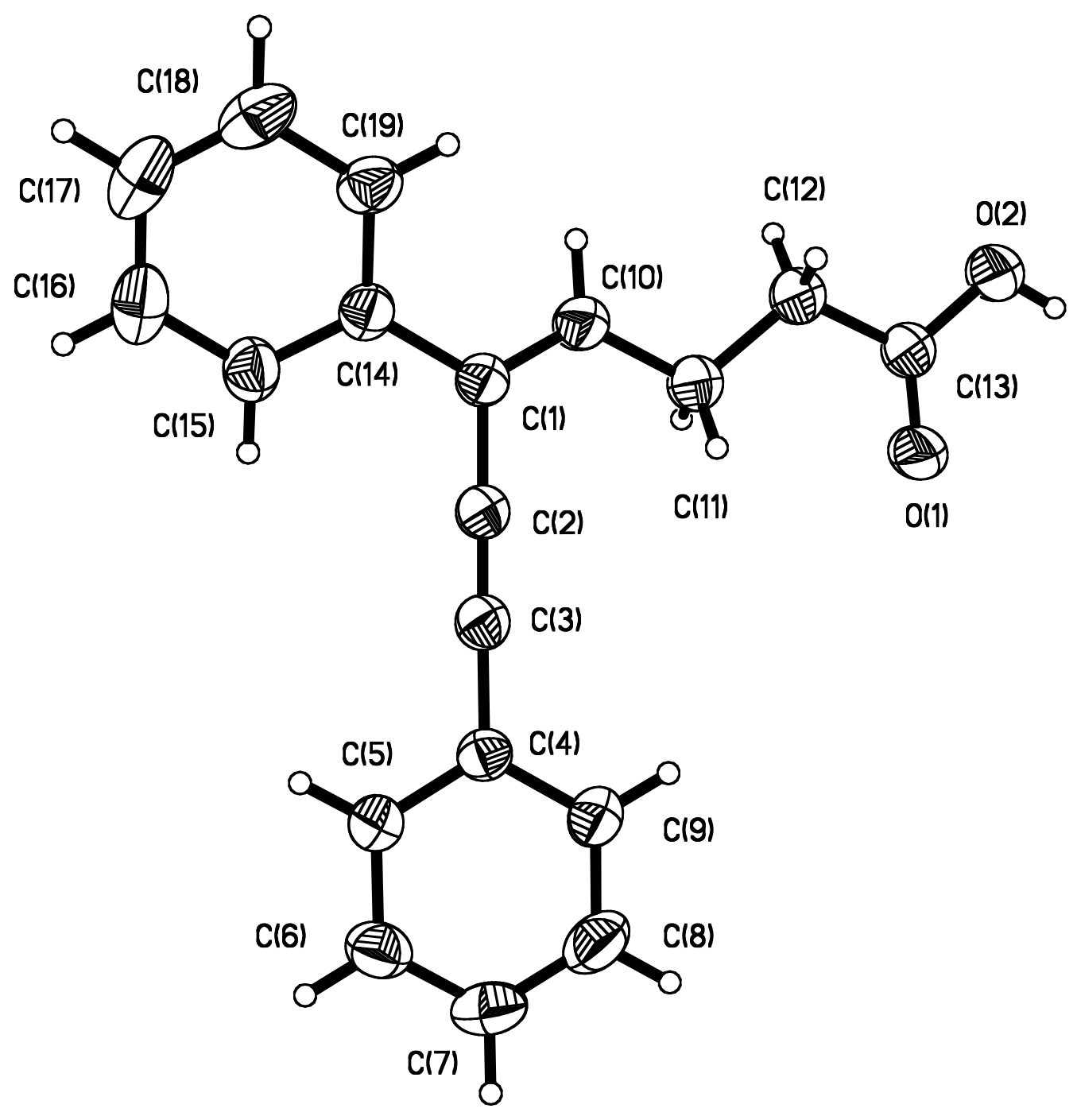

Figure S2.3-1.Perspective view of the molecular structure of 2.3.2n $\left(\mathrm{C}_{19} \mathrm{H}_{16} \mathrm{O}_{2}\right)$ with the atom labeling scheme. The thermal ellipsoids are scaled to enclose $30 \%$ probability. CCDC number: 861258 . 


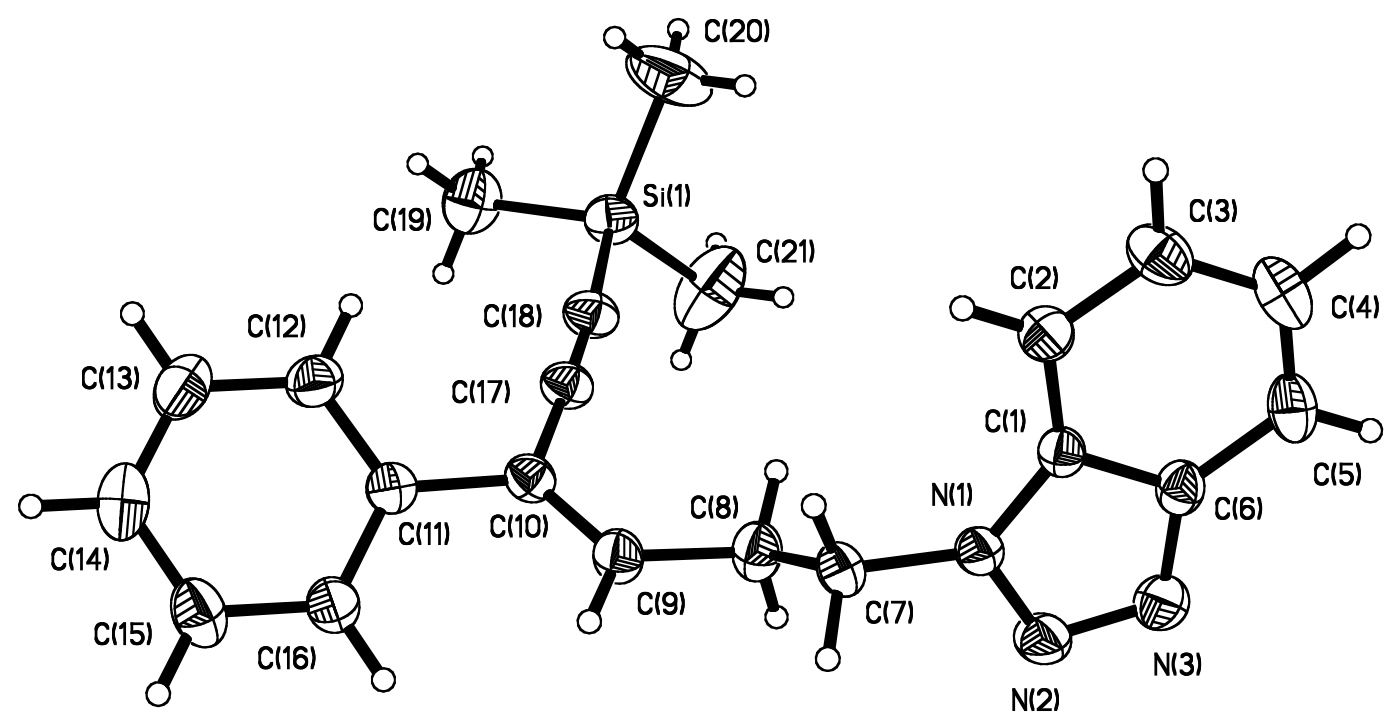

Figure S2.3-2.Perspective view of the molecular structure of 2.3.5b $\left(\mathrm{C}_{21} \mathrm{H}_{23} \mathrm{~N}_{3} \mathrm{Si}\right)$ with the atom labeling scheme. The thermal ellipsoids are scaled to enclose $30 \%$ probability. CCDC number: 861257. 


\section{${ }^{1} \mathrm{H}$ NMR spectrum of $2.3 .3 \mathrm{e}$ in $\mathrm{CDCl}_{3}$}
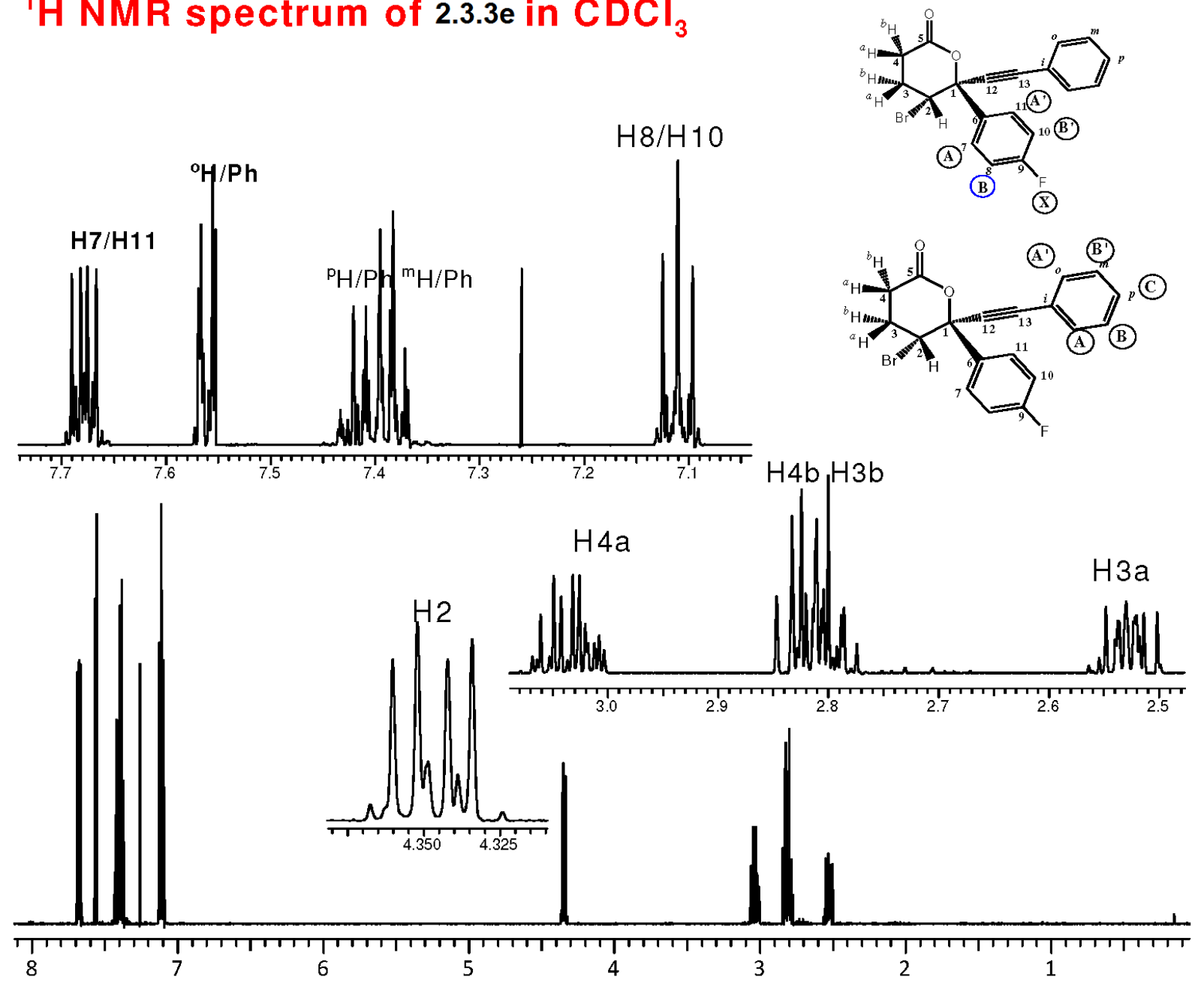
$\frac{\text { Virtual coupling of tetrahydropyrane-2-one protons in the }{ }^{\prime} H \text { NMR spectrum }}{\text { of 2.3.3e in } C D C l_{3}}$

\section{Experimental (a) and calculated (b) ${ }^{1} \mathrm{H}$ NMR spectrum}
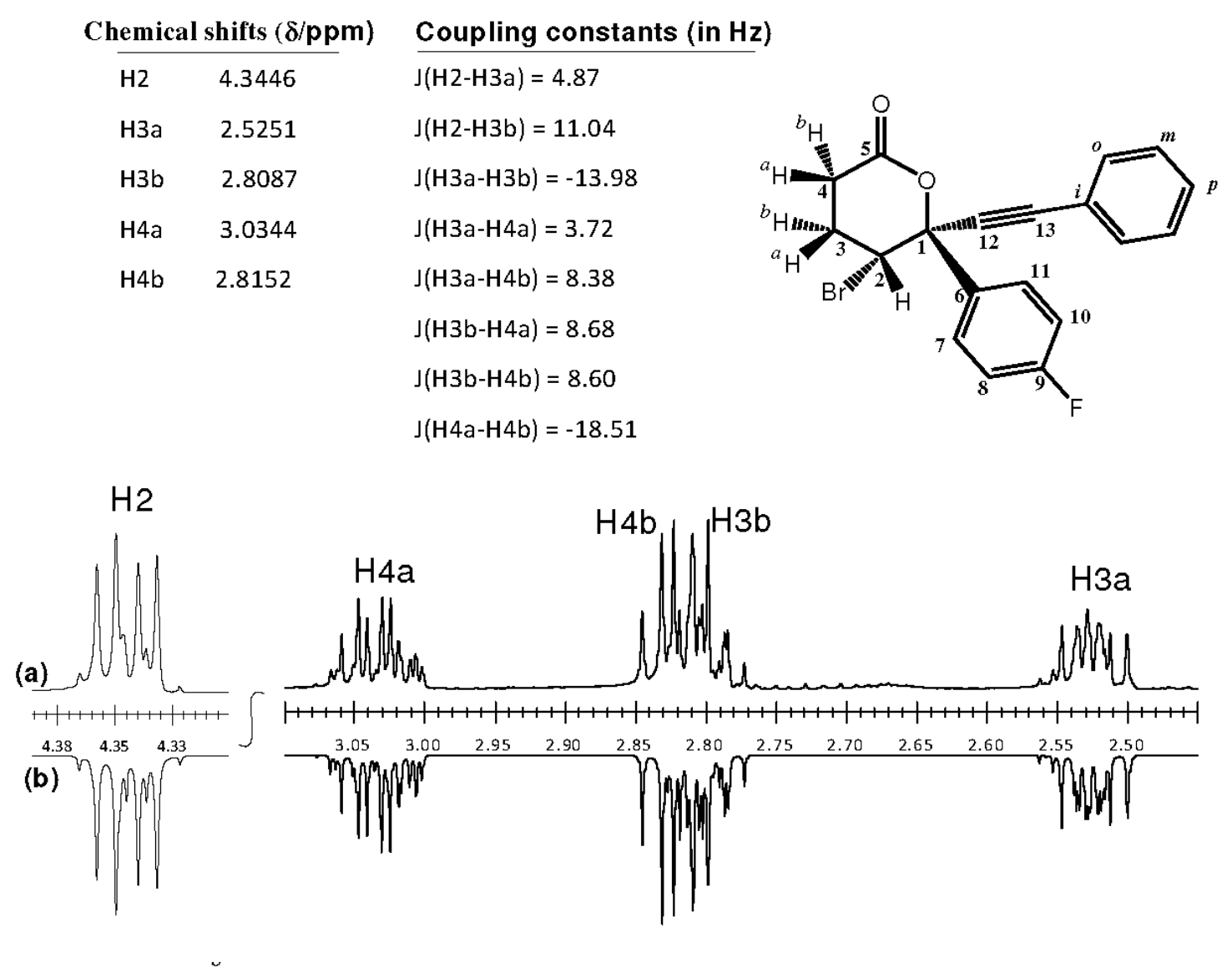

Expansion of splitting patterns
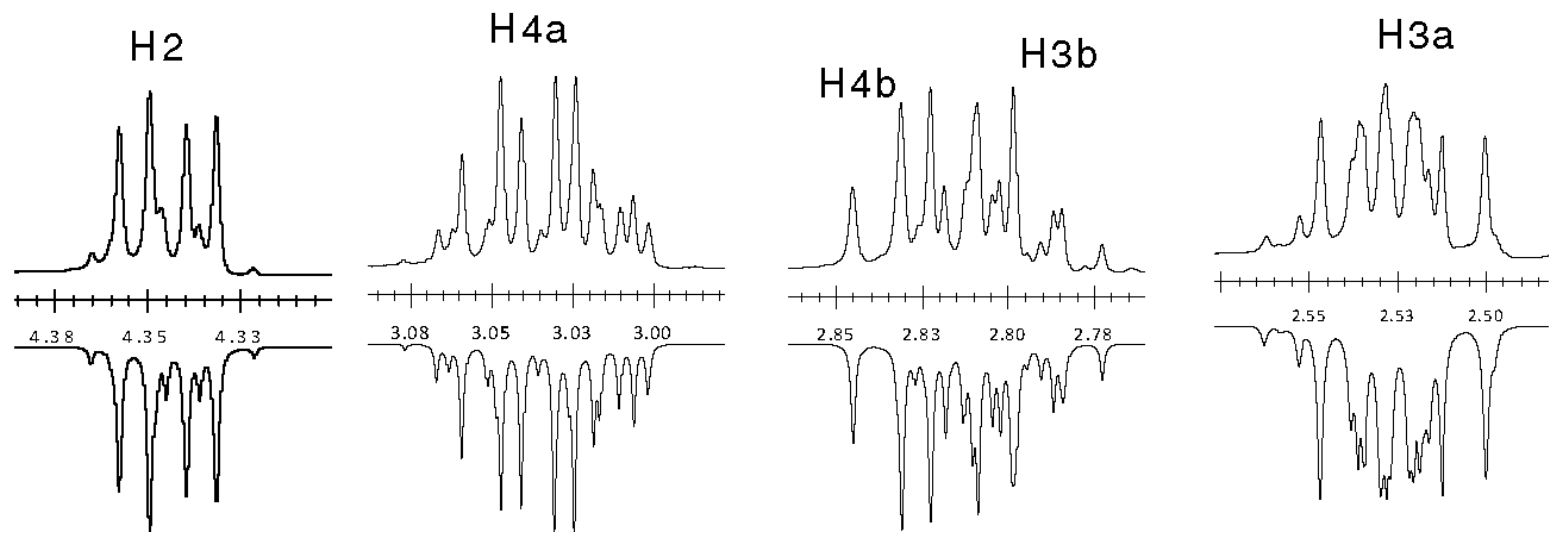
Expanded regions of the ${ }^{1} \mathrm{HNMR}$ spectrum of 2.3.3e in $\mathrm{CDCl}_{3}$ (a) and Toluene-d $\mathrm{d}_{\mathbf{8}}(\mathrm{b})$
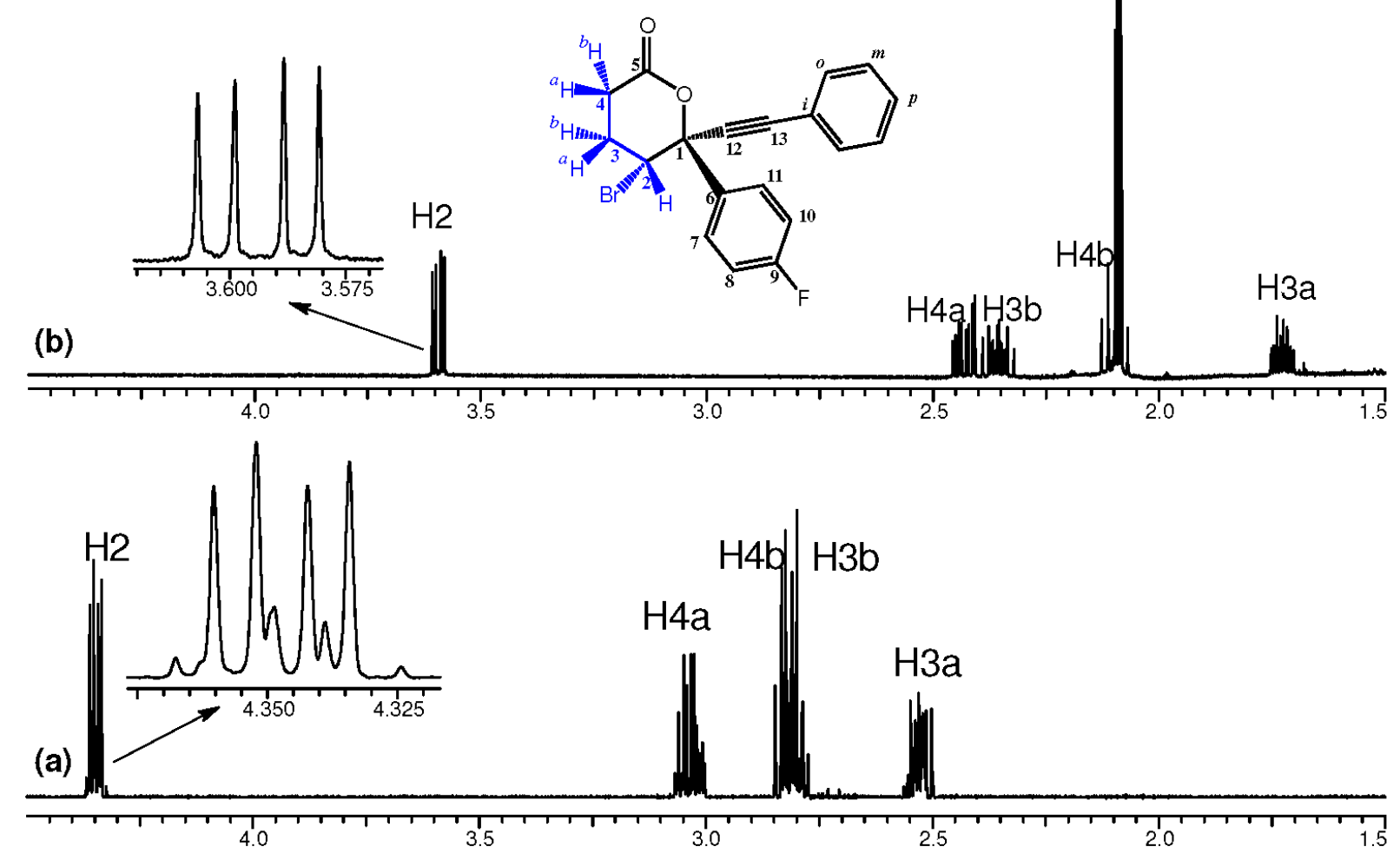
Experimental (a) and calculated (b) multiplicity patterns of Phenyl- and p-Fluoro-phenyl ring protons
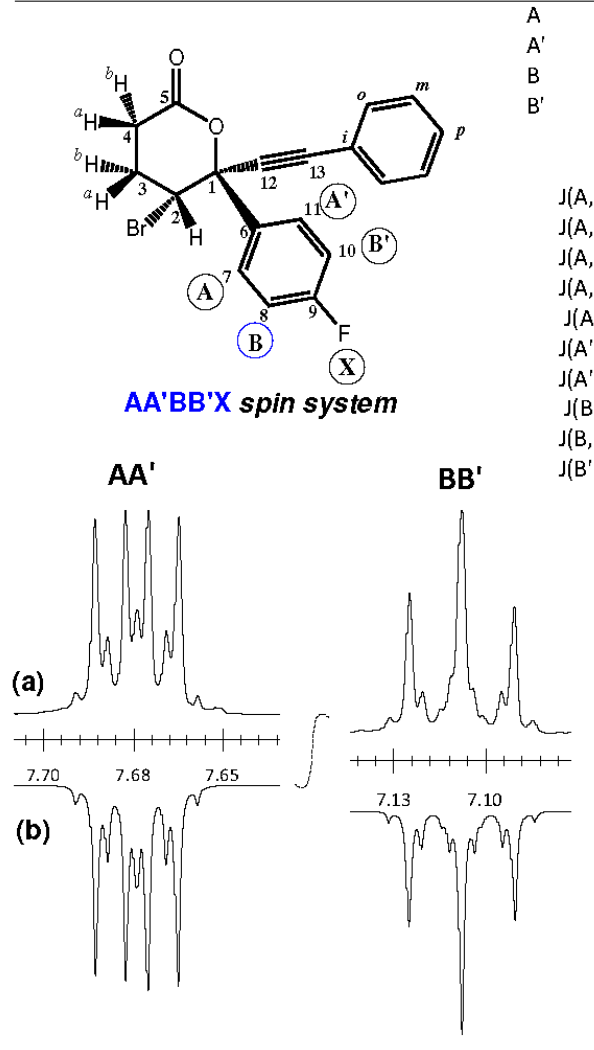

$\begin{array}{llll}\text { A } & 7.67 & \text { A } & 7.56 \\ \text { A }^{\prime} & 7.67 & \text { A }^{\prime} & 7.56\end{array}$

$\begin{array}{llll}\text { B } & 7.11 & \text { B } & 7.38\end{array}$

$\begin{array}{ll}\text { B } & 7.38 \\ \text { B' }^{\prime} & 7.38 \\ \text { C } & 7.41\end{array}$

\begin{tabular}{|c|c|c|c|}
\hline$J\left(A, A^{\prime}\right)$ & 2.46 & $J\left(A, A^{\prime}\right)$ & 1.93 \\
\hline$J(A, B)$ & 8.59 & $J(A, B)$ & 7.73 \\
\hline$J\left(A, B^{\prime}\right)$ & 0.22 & $J\left(A, B^{\prime}\right)$ & 0.66 \\
\hline$J(A, X)$ & 5.06 & $J(A, C)$ & 1.32 \\
\hline$J\left(A^{\prime}, B\right)$ & 0.22 & $J\left(A^{\prime}, B\right)$ & 0.66 \\
\hline$J\left(A^{\prime}, B^{\prime}\right)$ & 8.59 & $J\left(A^{\prime}, B^{\prime}\right)$ & 7.73 \\
\hline$J\left(A^{\prime}, X\right)$ & 5.06 & $J\left(A^{\prime}, C\right)$ & 1.32 \\
\hline$J\left(B, B^{\prime}\right)$ & 2.91 & $J\left(B, B^{\prime}\right)$ & 1.47 \\
\hline $\mathrm{J}(\mathrm{B}, \mathrm{X})$ & 8.91 & $J(B, C)$ & 7.54 \\
\hline$J^{\prime}\left(B^{\prime}, C\right)$ & 8.91 & $\mathrm{~J}\left(\mathrm{~B}^{\prime}, \mathrm{C}\right)$ & 7.54 \\
\hline
\end{tabular}
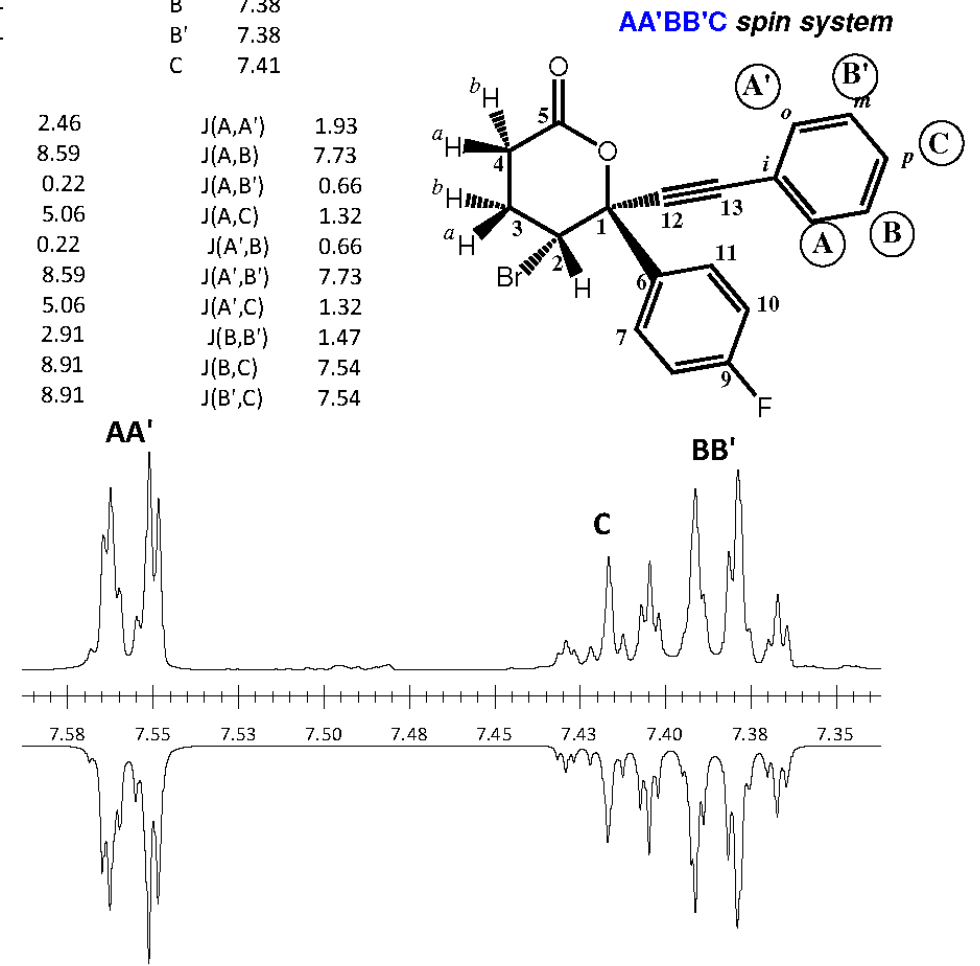


\section{$X$ part of $A B X$ spin system $(A=H 3 a, B=H 3 b, X=H 2)$}

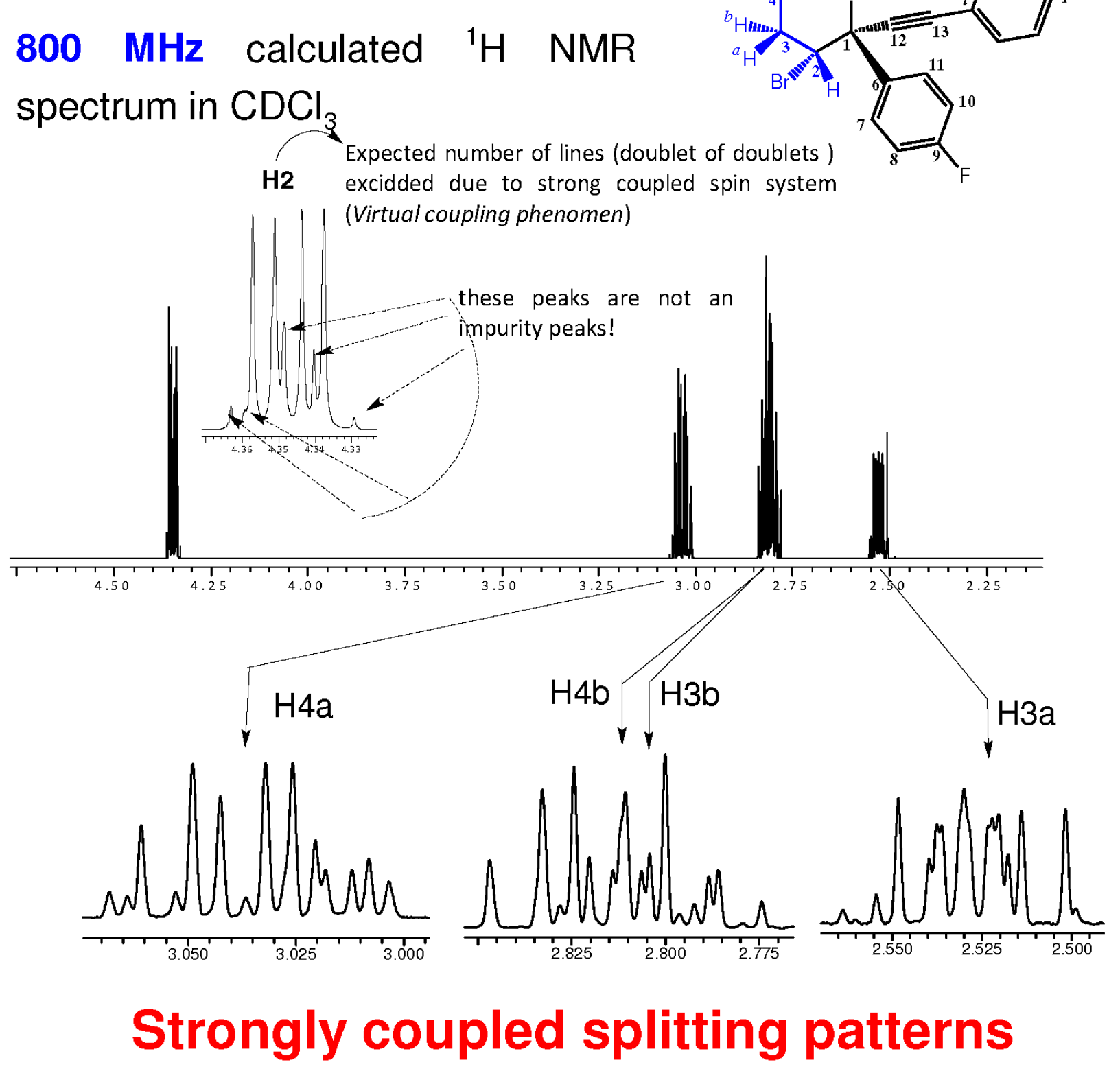


${ }^{1} \mathrm{H}$ NMR spectrum of 2.3.3e in Toluene- $d_{8}$
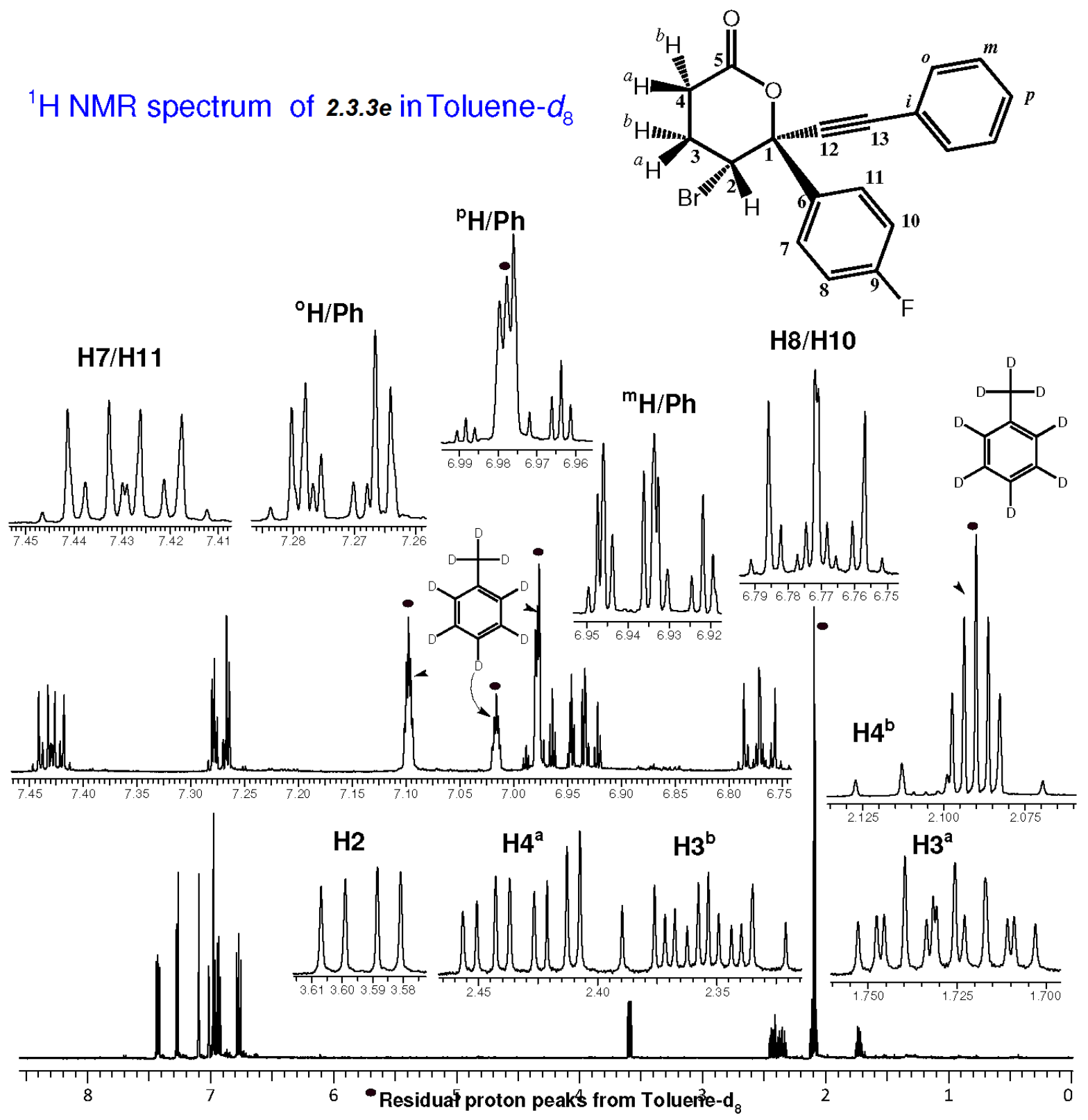
1D TOCSY spectrum (b); Control ${ }^{1} \mathrm{H}$ NMR spectrum (a).

Selective excitation of $\mathrm{H} 3 \mathrm{a}$ ( $\mathrm{mix}=250 \mathrm{~ms}$ )

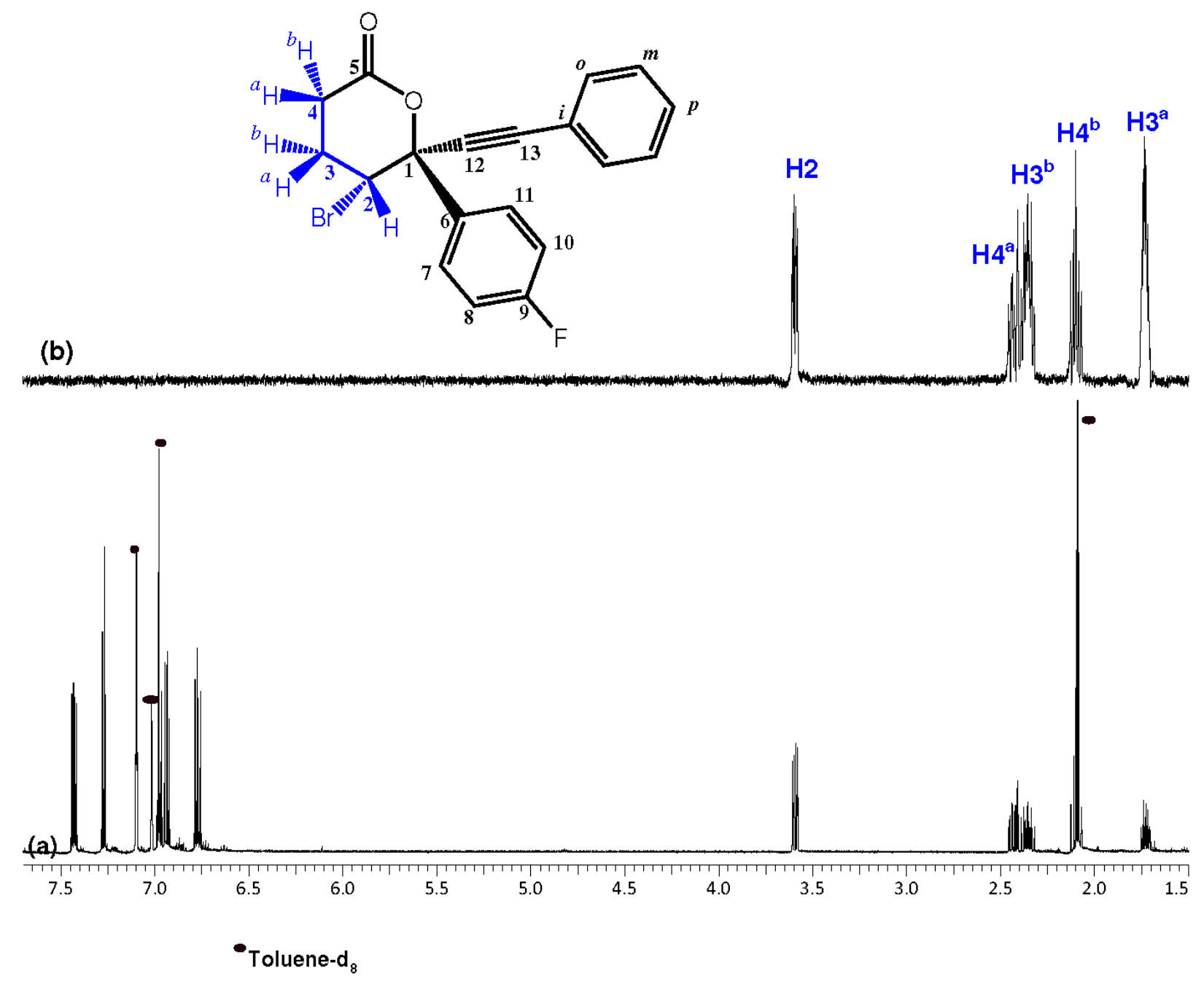


DPFGSNOE specta (b-c); Control ${ }^{1} \mathrm{H}$ NMR spectrum (a)

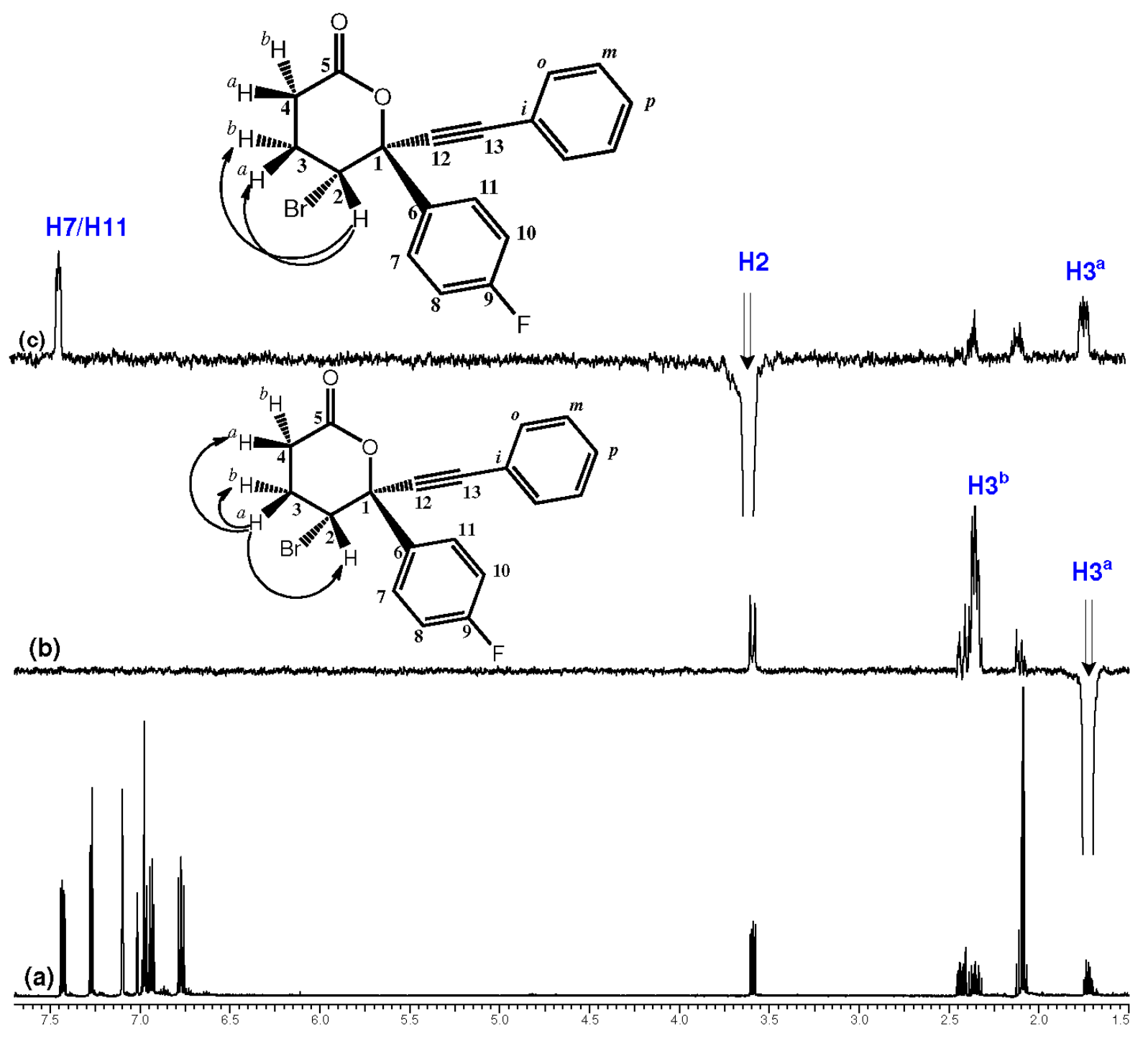




\section{Experiemntal (a) and calculated (b) multiplicity}

patterns of Phenyl-and p-Fluoro-phenyl ring protons

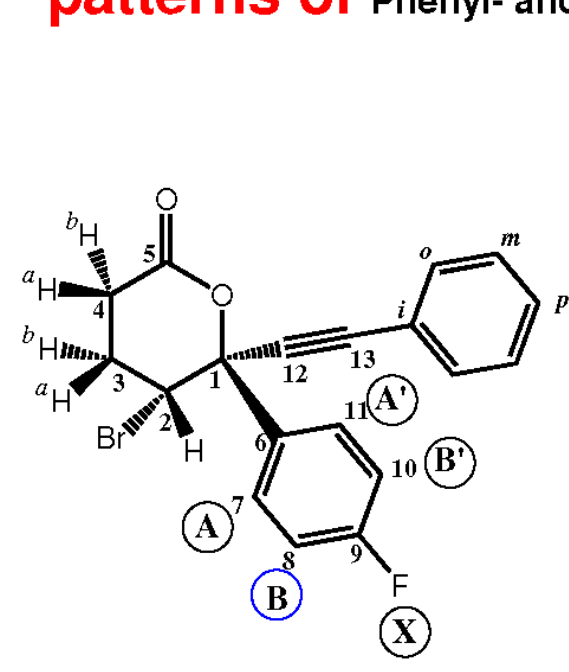

\section{AA'BB'X spin system}

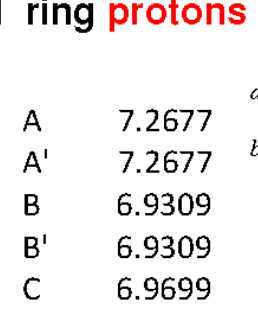

$J\left(A, A^{\prime}\right)$

$J(A, B)$

$J\left(A, B^{\prime}\right)$

$J(A, X)$

$J\left(A^{\prime}, B\right)$

$J\left(A^{\prime}, B^{\prime}\right)$

$J\left(A^{\prime}, X\right)$

$J\left(B, B^{\prime}\right)$

$J(B, X)$

$J\left(B^{\prime}, C\right)$

$\begin{array}{ccc}2.52 & \mathrm{~J}\left(A, A^{\prime}\right) & 1.71 \\ 8.51 & \mathrm{~J}(A, B) & 7.71 \\ 0.22 & \mathrm{~J}\left(A, B^{\prime}\right) & 0.61 \\ 5.15 & \mathrm{~J}(A, C) & 1.32 \\ 0.72 & \mathrm{~J}\left(\mathrm{~A}^{\prime}, \mathrm{B}^{\prime}\right) & 0.61 \\ 8.51 & \mathrm{~J}\left(\mathrm{~A}^{\prime}, \mathrm{B}^{\prime}\right) & 7.73 \\ 5.15 & \mathrm{~J}\left(\mathrm{~A}^{\prime}, \mathrm{C}\right) & 1.30 \\ 2.89 & \mathrm{~J}\left(\mathrm{~B}^{\prime}, \mathrm{B}^{\prime}\right) & 1.47 \\ 8.71 & \mathrm{~J}(\mathrm{~B}, \mathrm{C}) & 7.54 \\ 8.71 & \mathrm{~J}\left(\mathrm{~B}^{\prime}, C\right) & 7.54\end{array}$

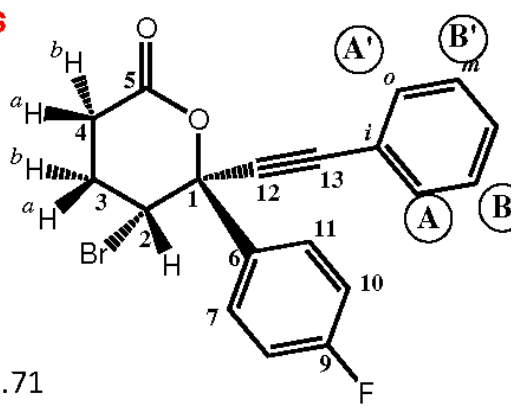

AA'BB'C spin system

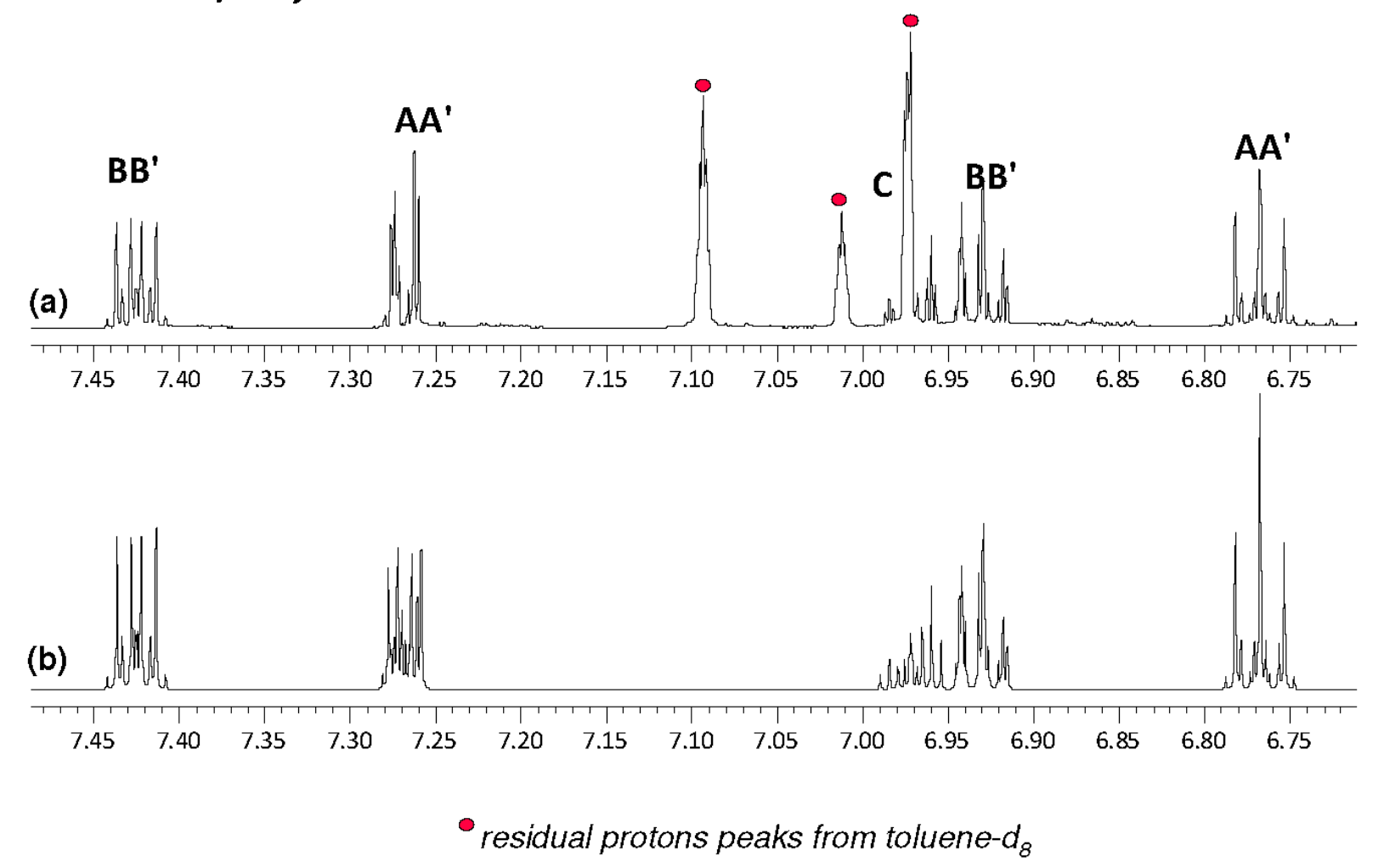


Experiemntal (a) and calculated (b) multiplicity patterns of Phenyl ring protons

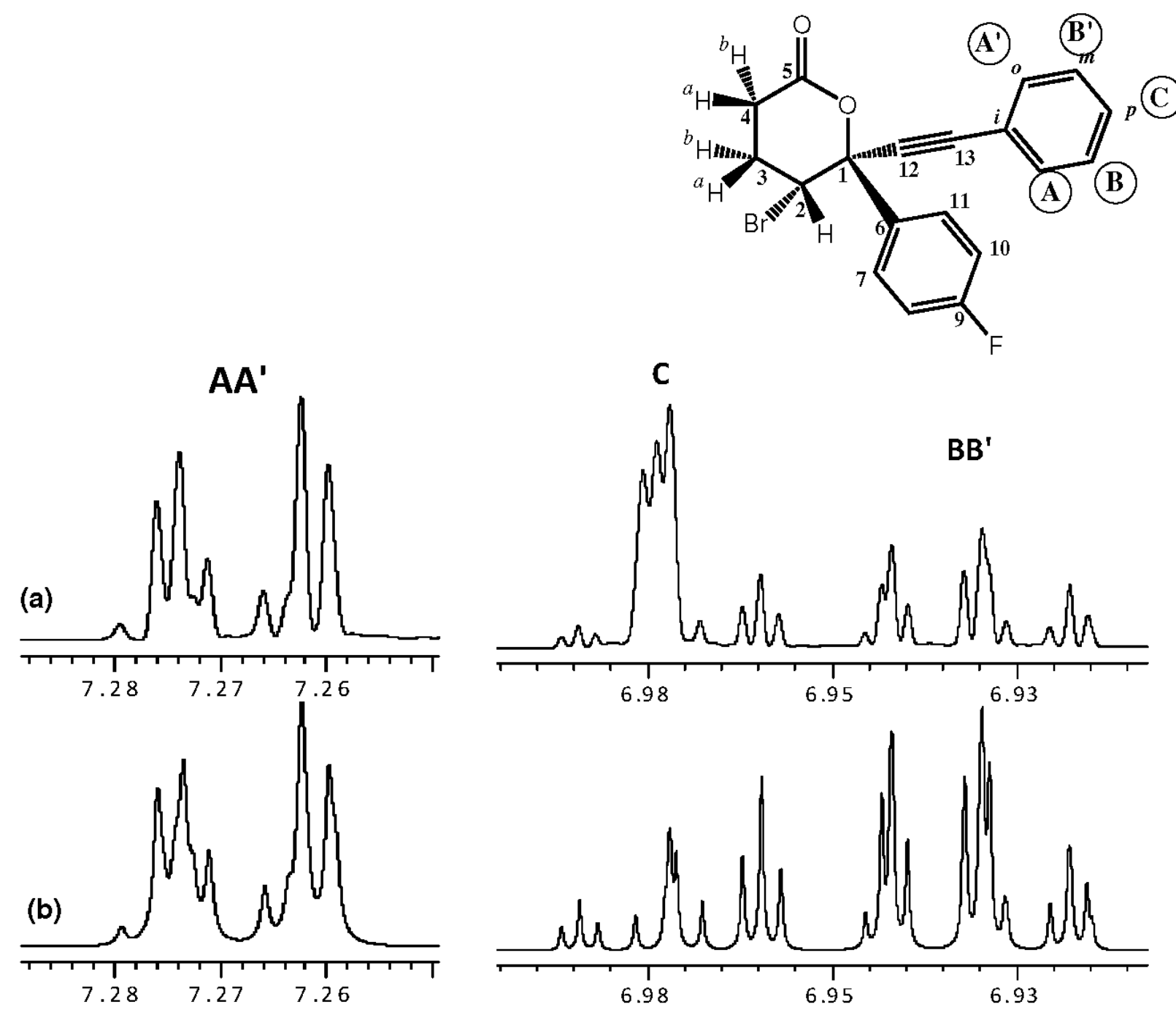


Expanded regions of the experimental (a) and calculated (b) ${ }^{1} H$ NMR spectrum of 2.3.3e in Toluene- $d_{s}$

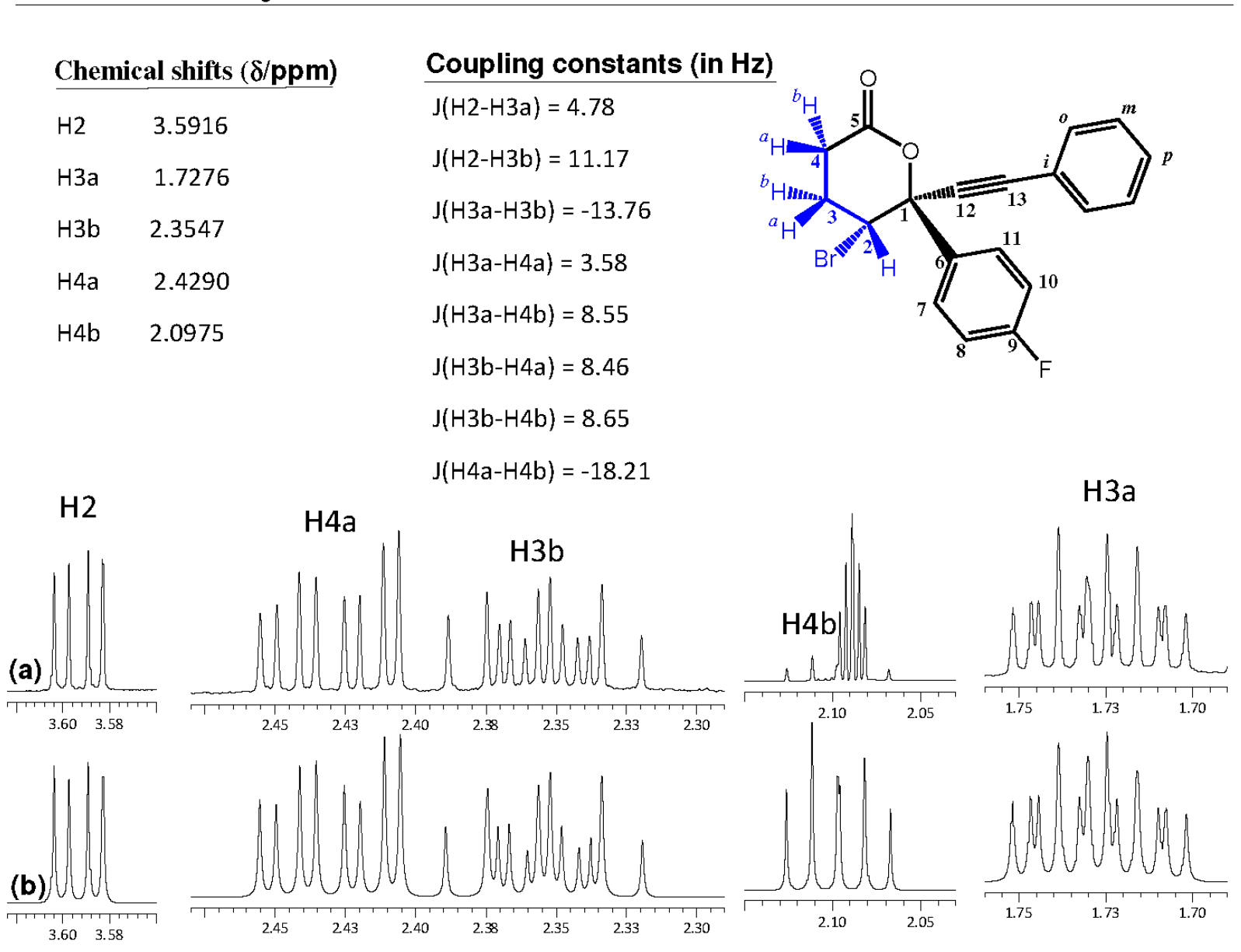




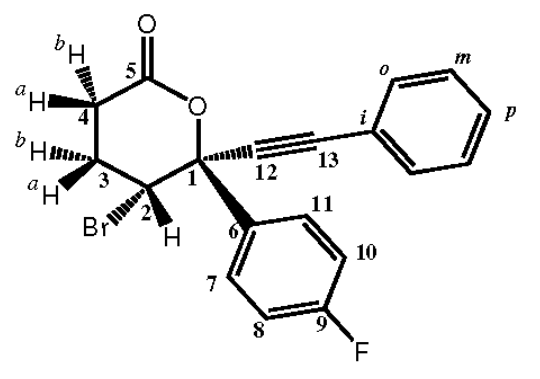

${ }^{13} \mathrm{C}$ NMR chemical shifts ( $\left.\delta / \mathrm{ppm}\right)$
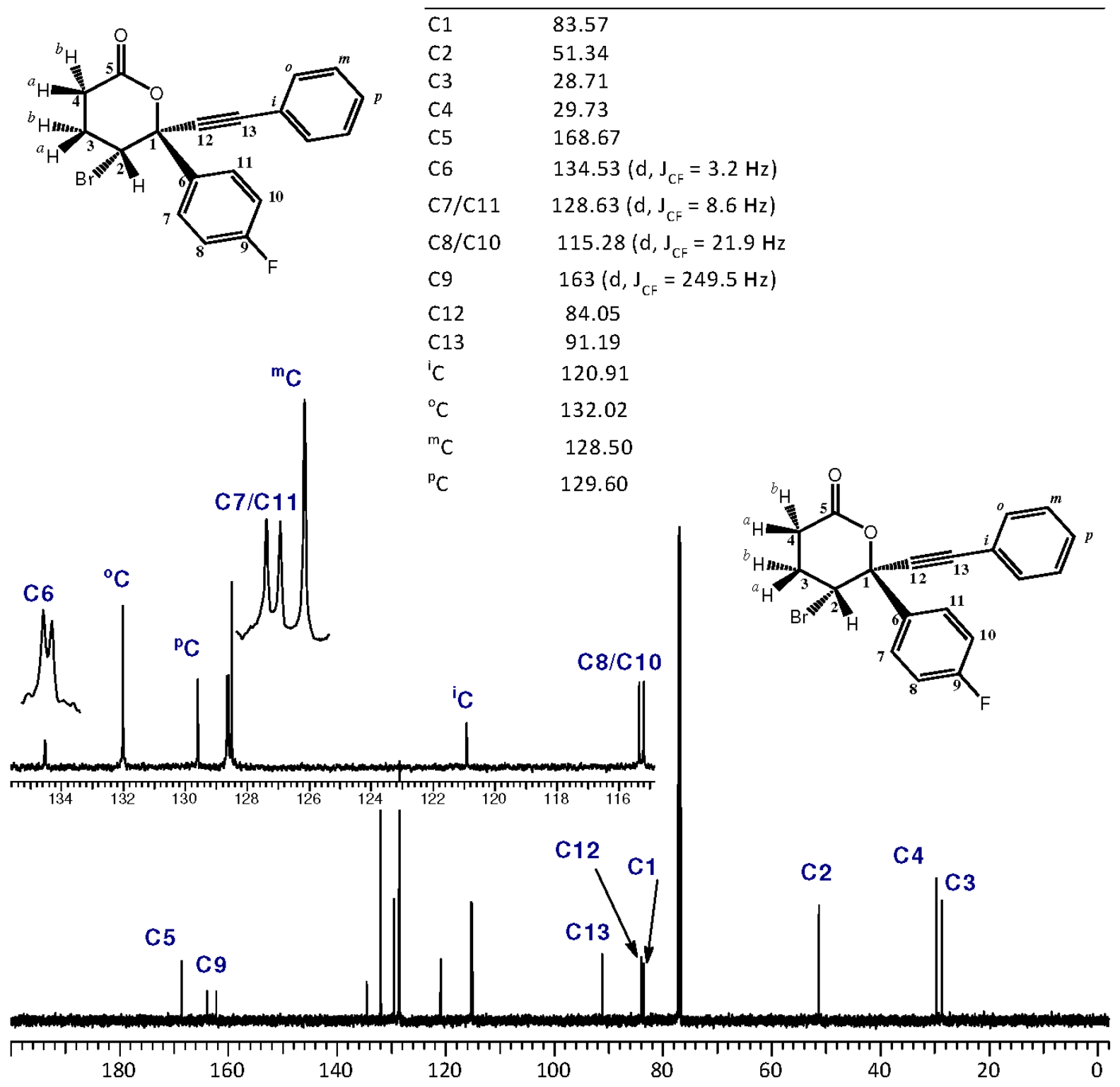


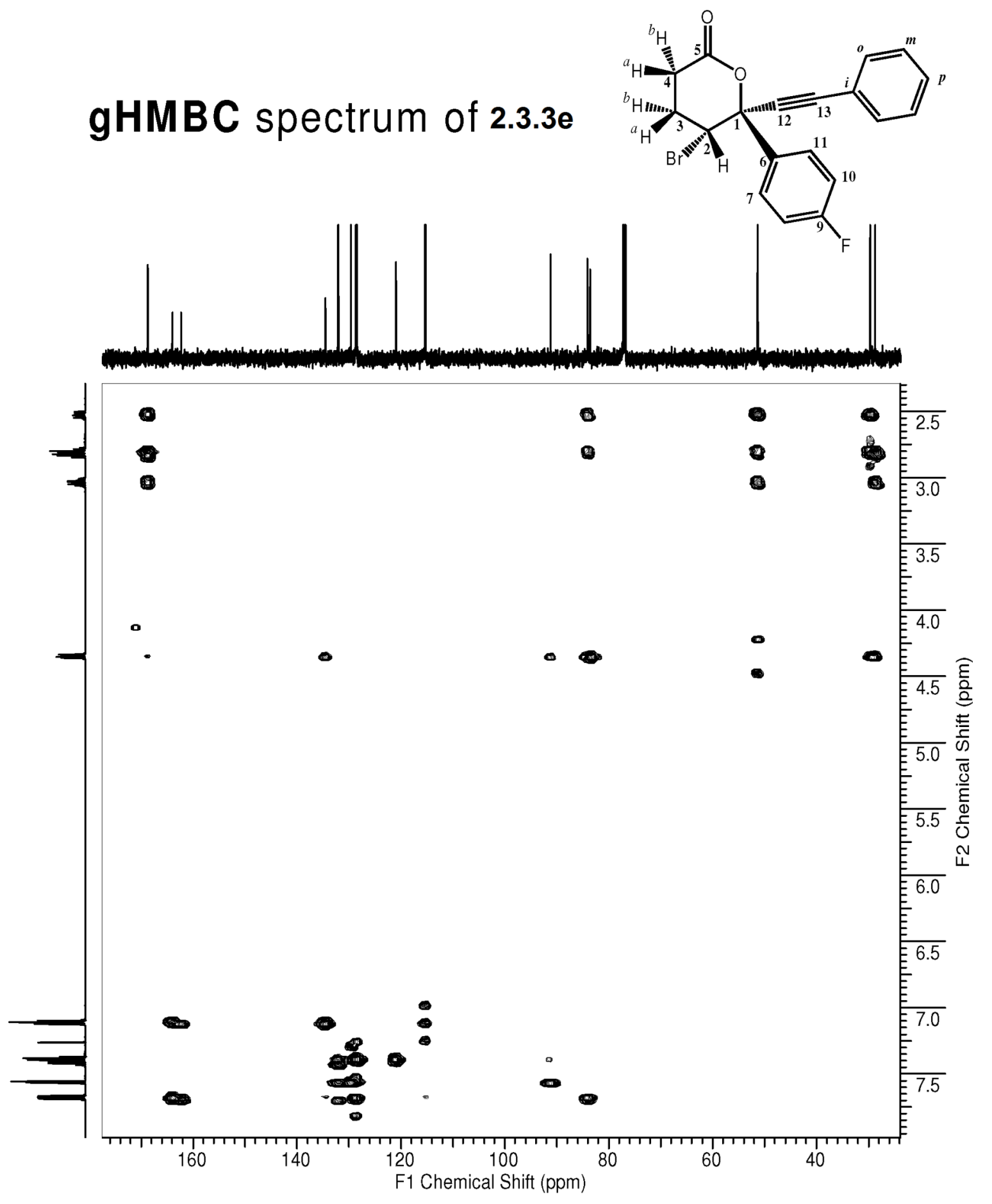




\section{Portion of the gHMBC spectrum of 2.3.3e}
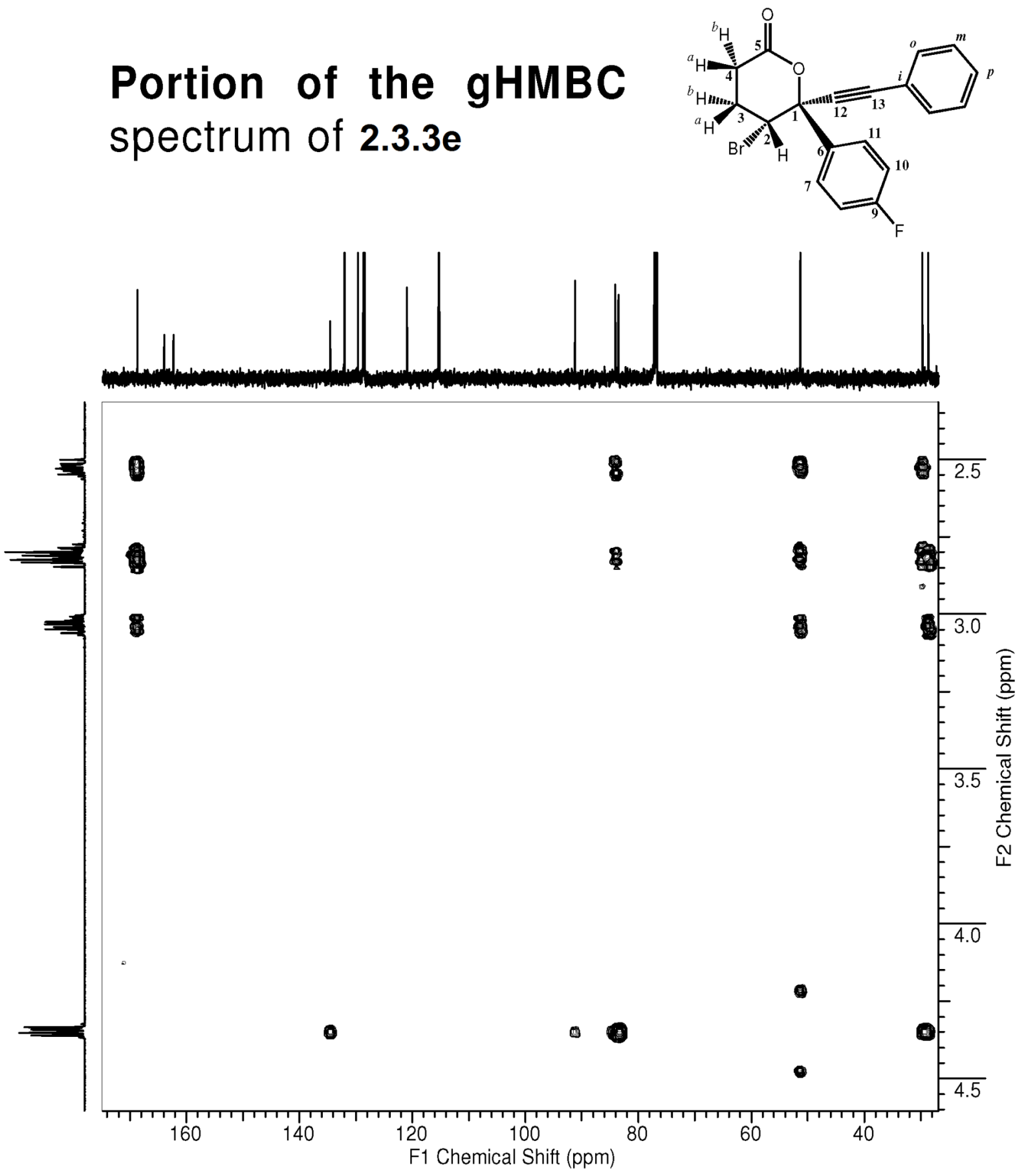


\section{NMR spectral parameters of 2.3.3f}

${ }^{1} \mathrm{H}$ and ${ }^{13} \mathrm{C}$ NMR chemical shifts $(\delta / \mathrm{ppm})$ and coupling constants $(\mathrm{J} / \mathrm{Hz})$

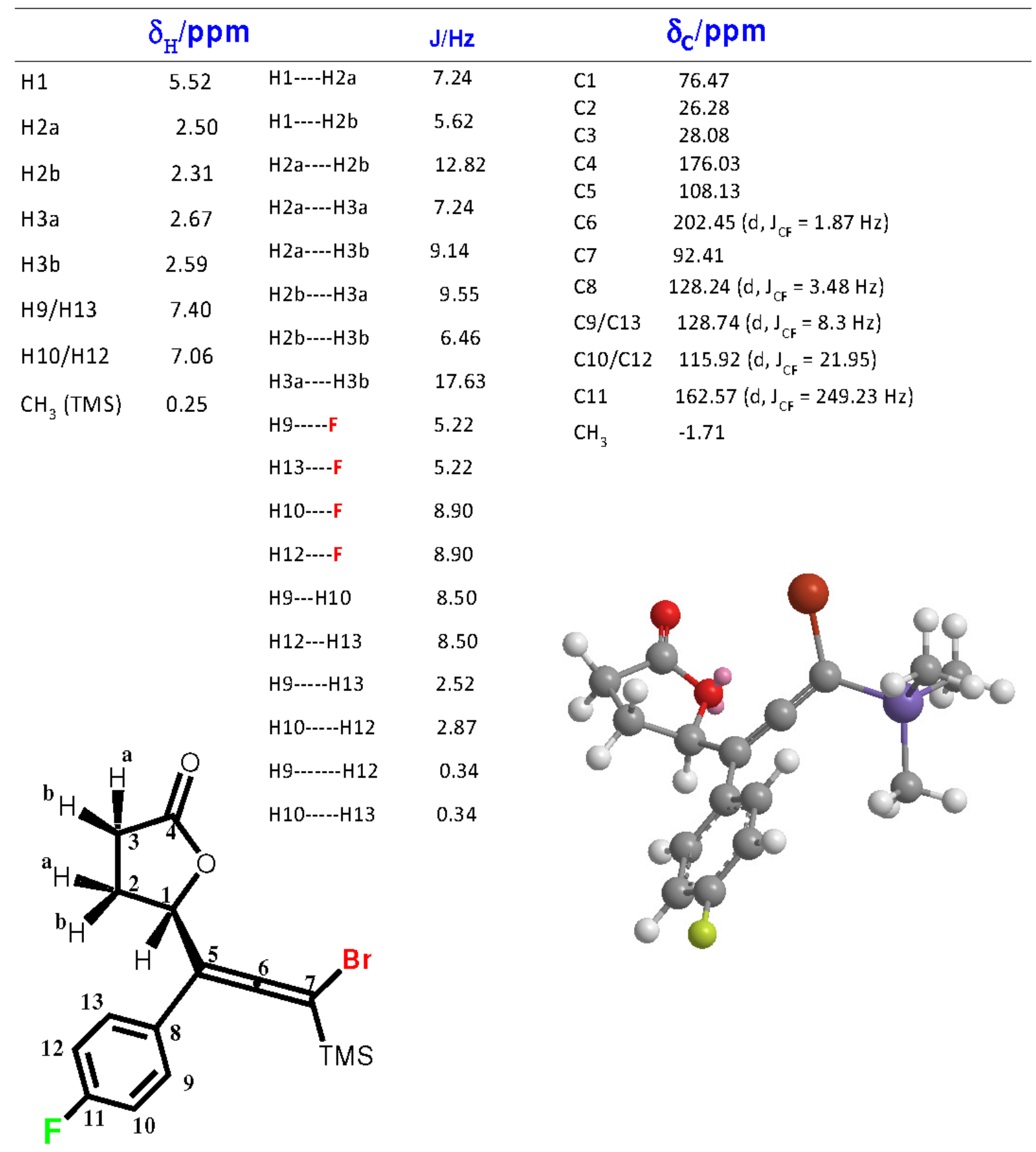




\section{Coupling constants $(\mathrm{J} / \mathrm{Hz})$}

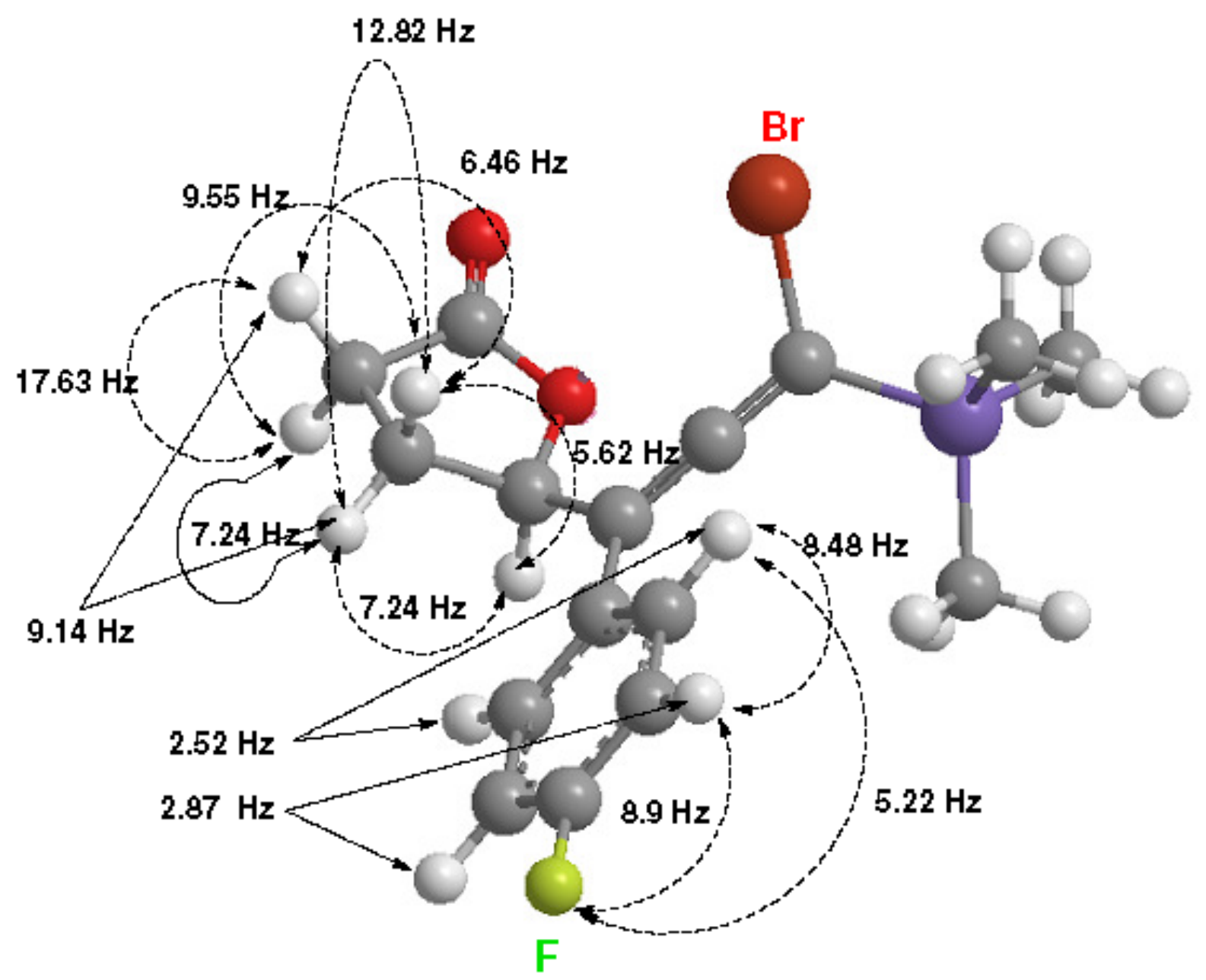




\section{Experimental (a) and calculated (b) ${ }^{1} \mathrm{H}$ NMR spectrum}

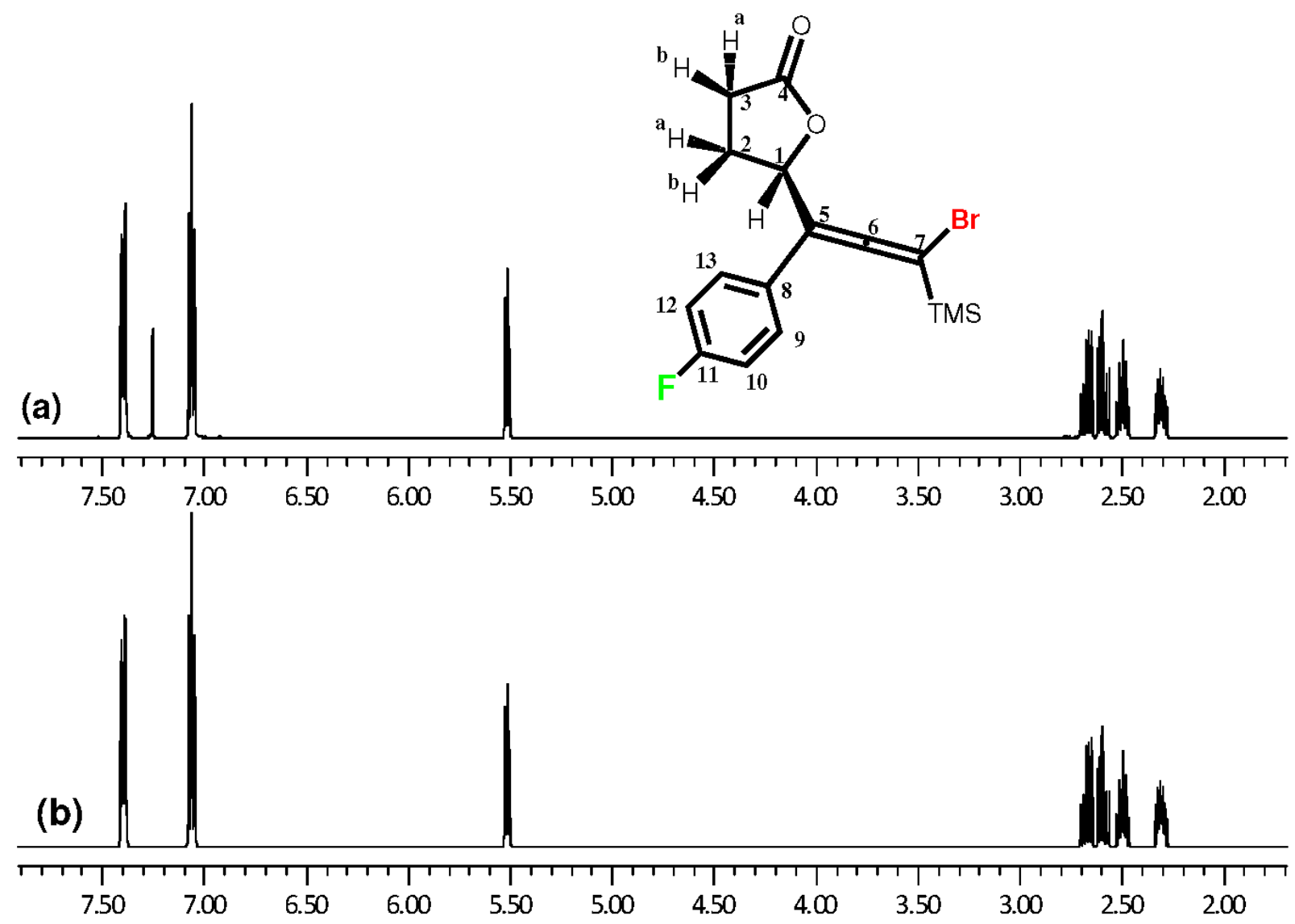




\section{Experimental (a) and calculated ${ }^{1}$ H NMR spectrum}
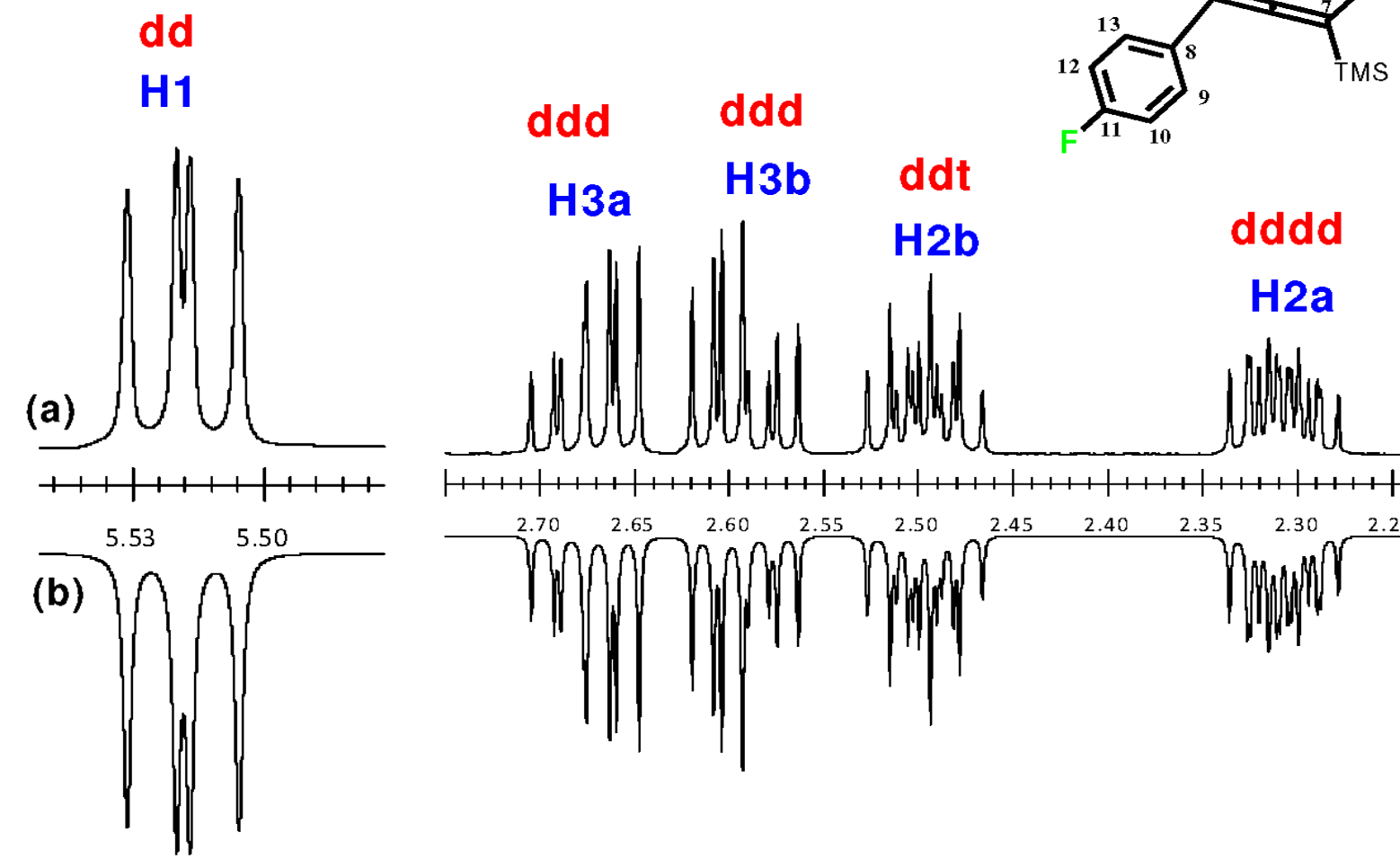

Experimental (a) and calculated (b) ${ }^{1} \mathrm{H}$ NMR spectrum

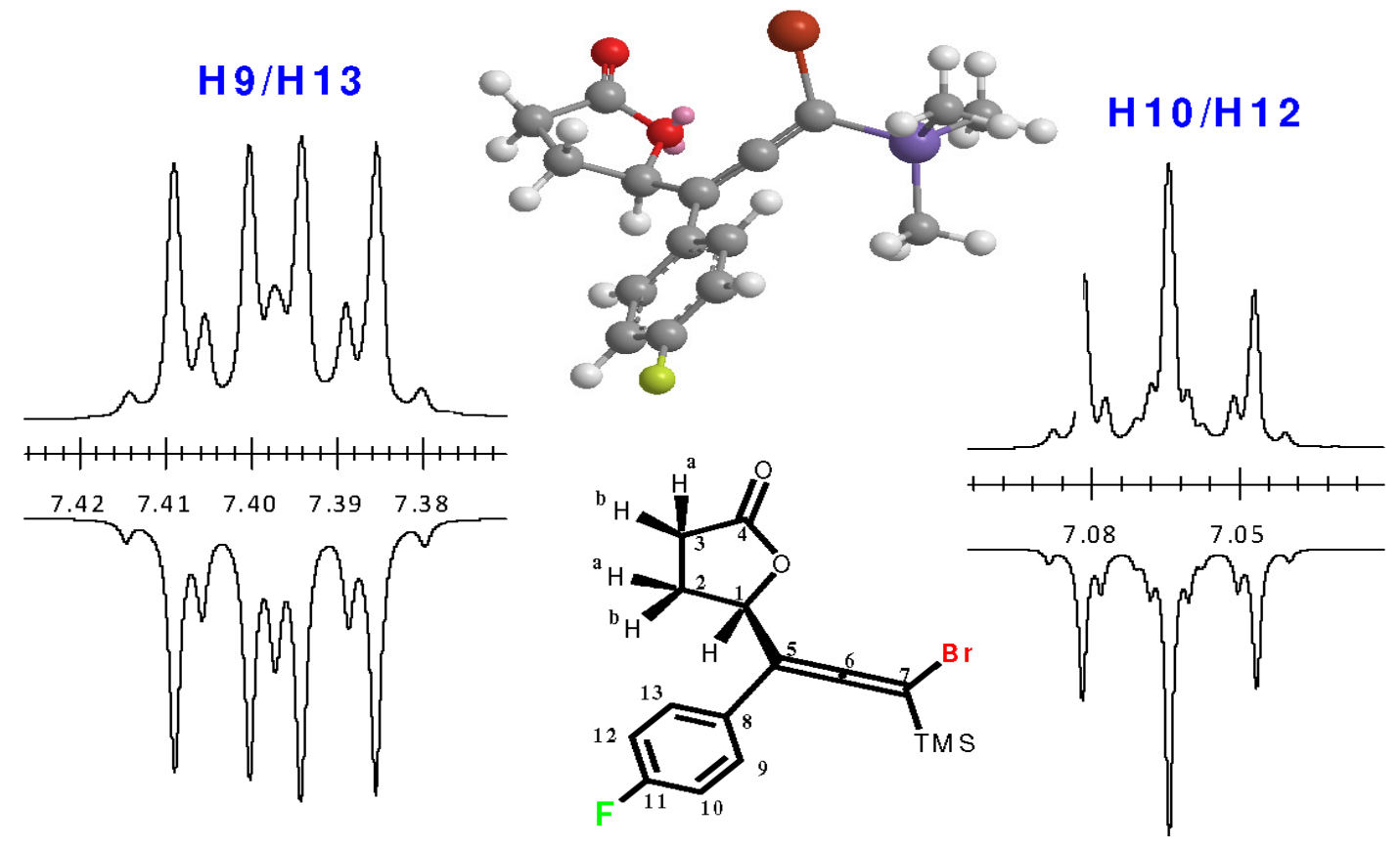




\section{$1 D$ NOESY \\ spectrum}
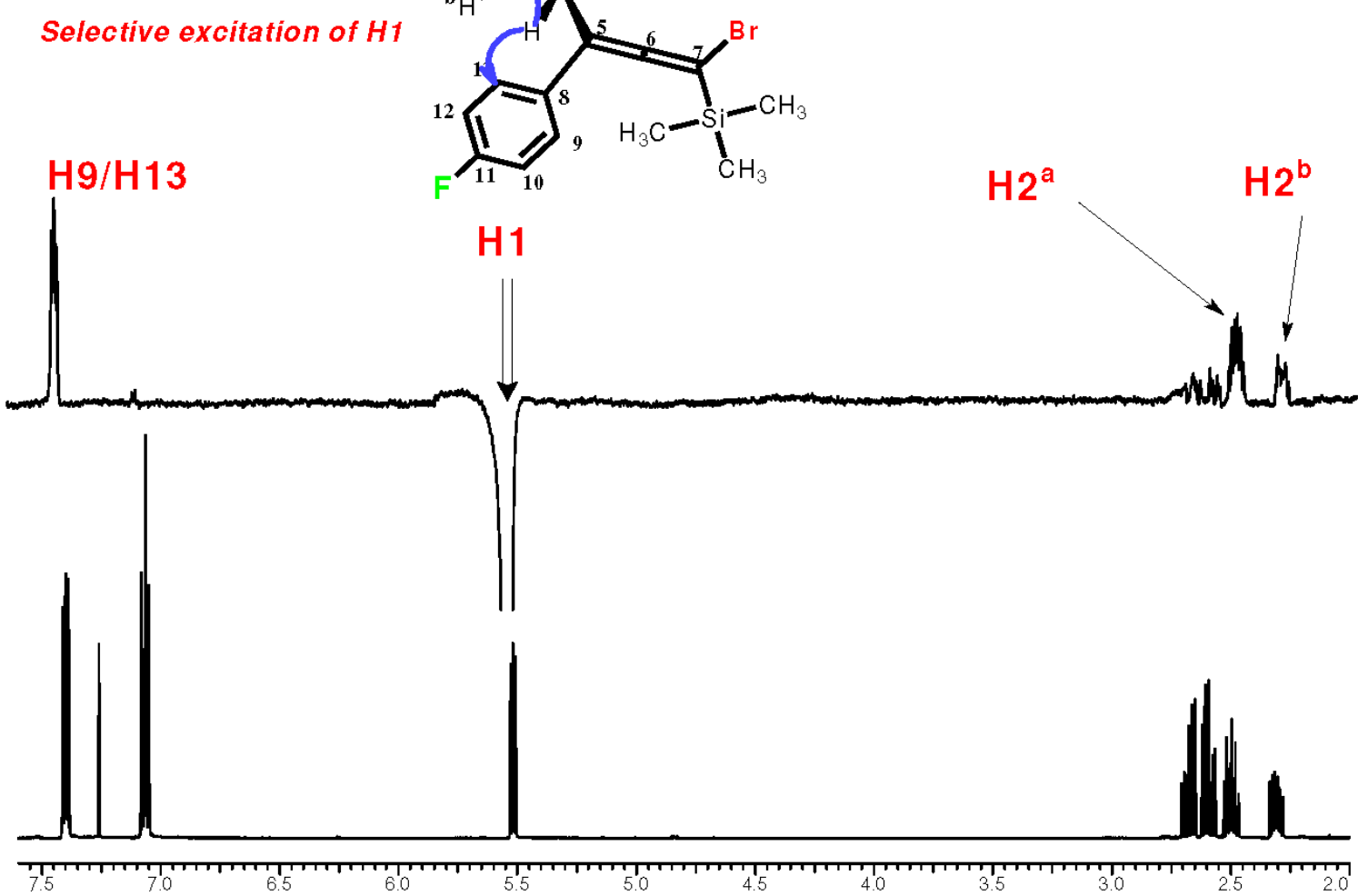

\section{D NOESY}

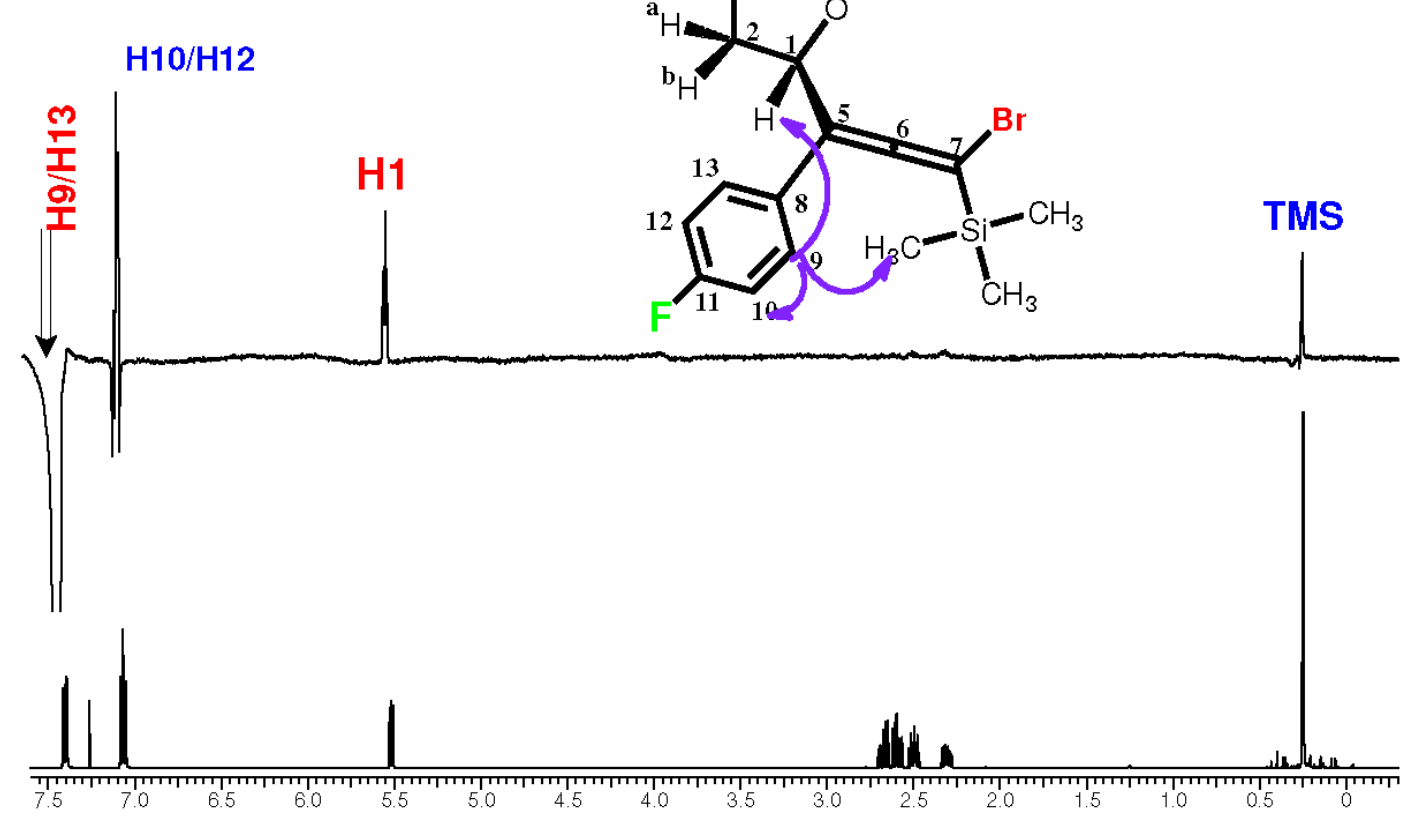




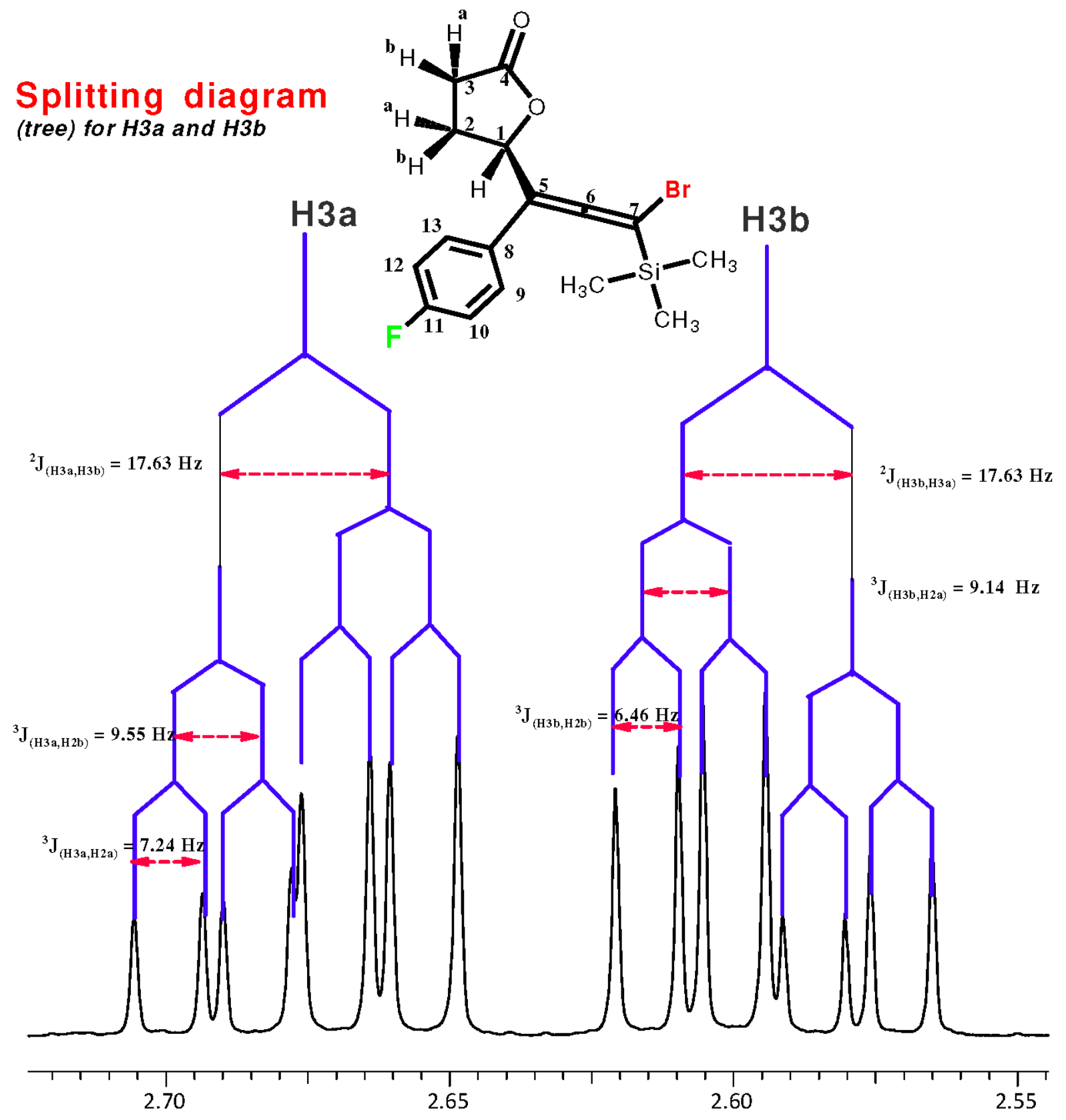




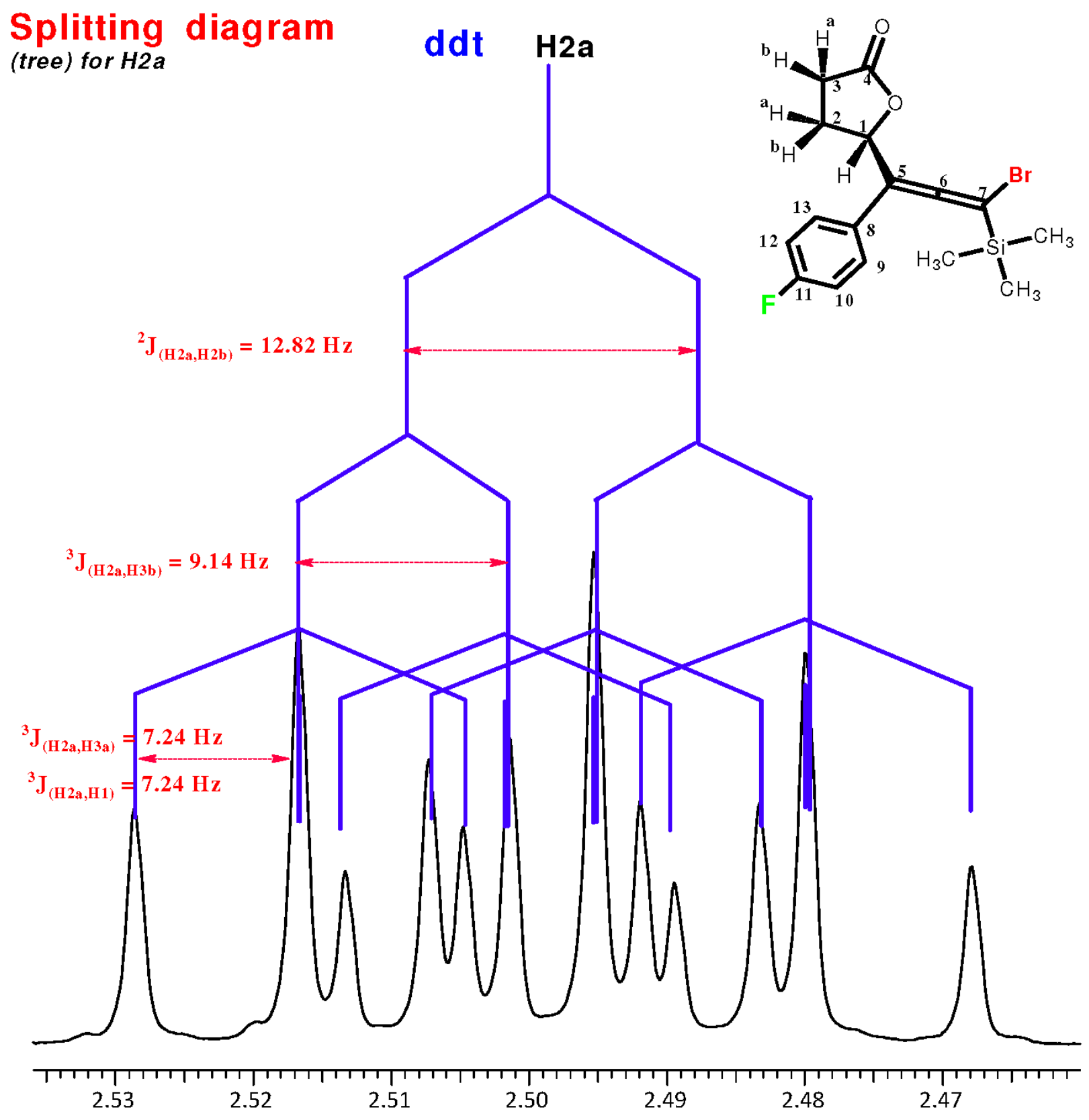




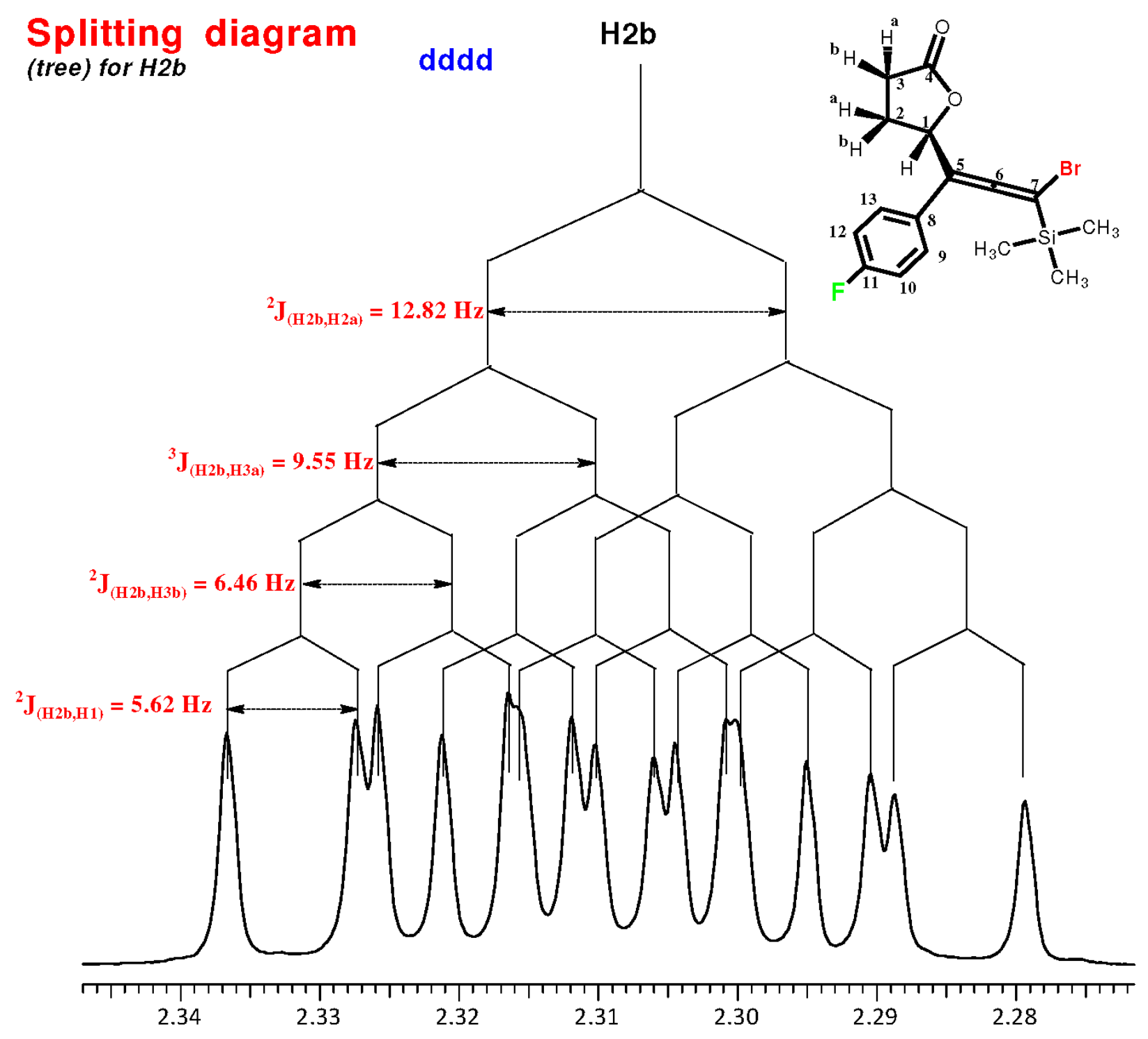




\section{$\underline{\cos Y}$}

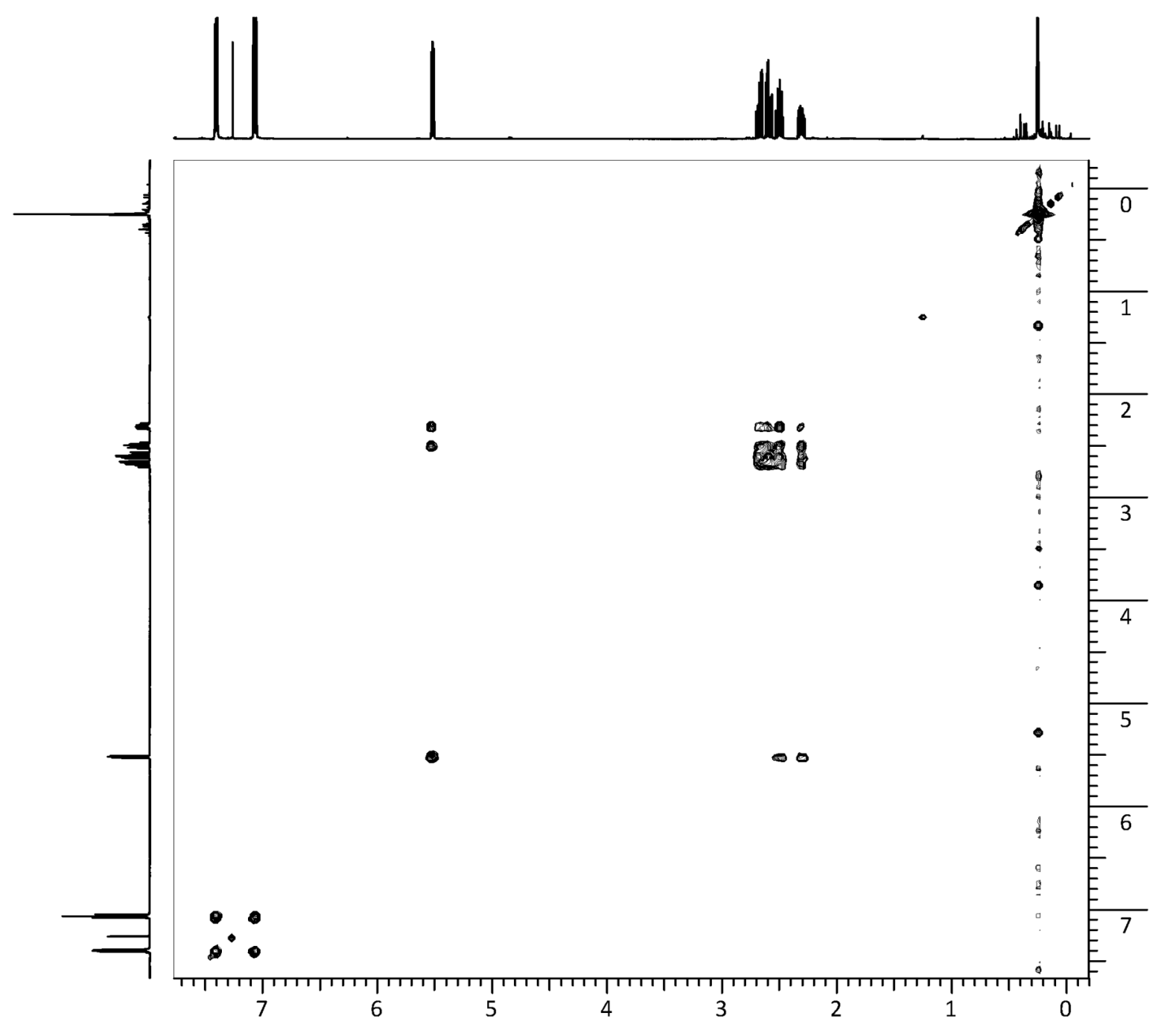




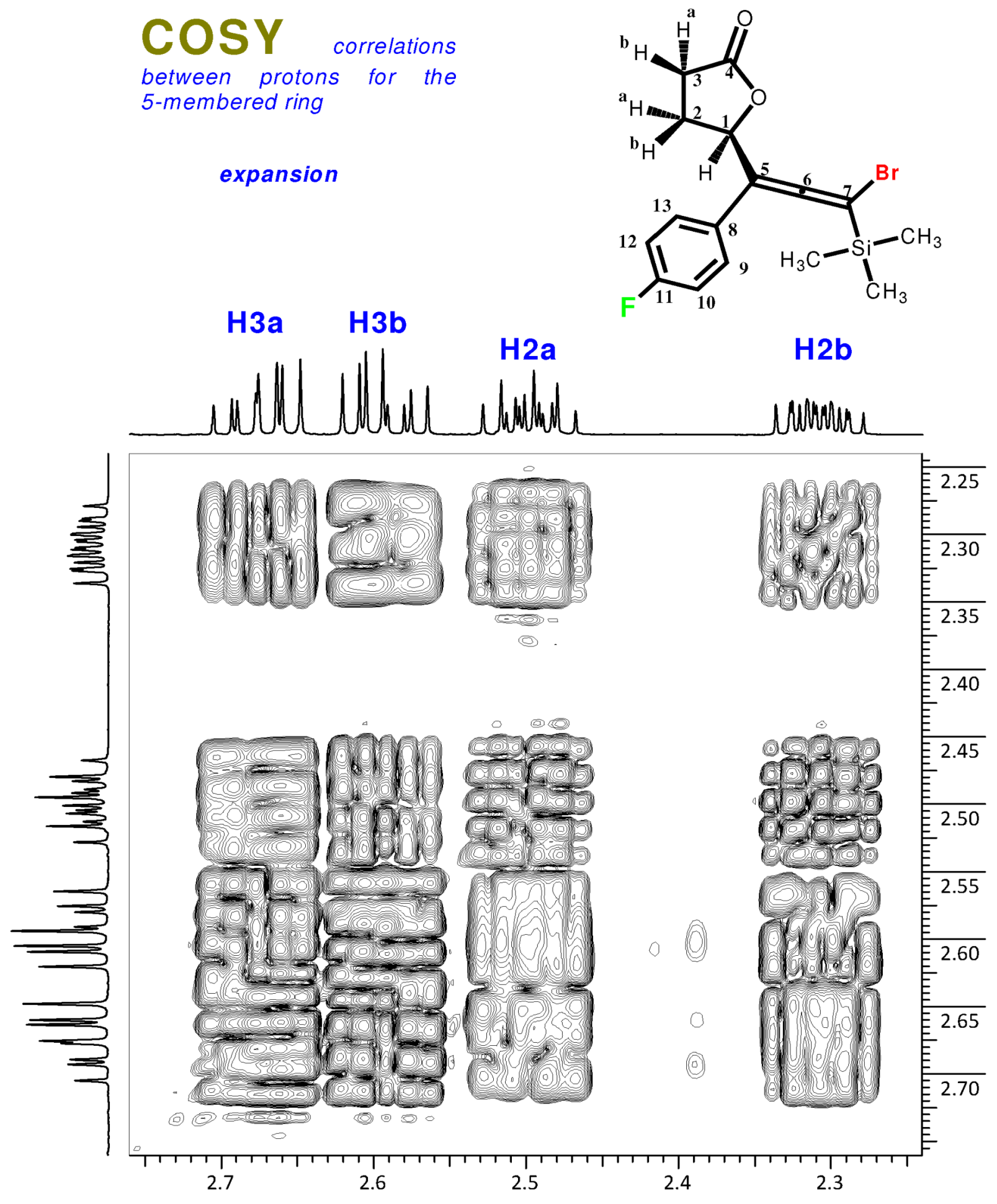




\section{${ }^{13} \mathrm{C}$ NMR spectrum of 2.3.3f in $\mathrm{CDCl}_{3}$}

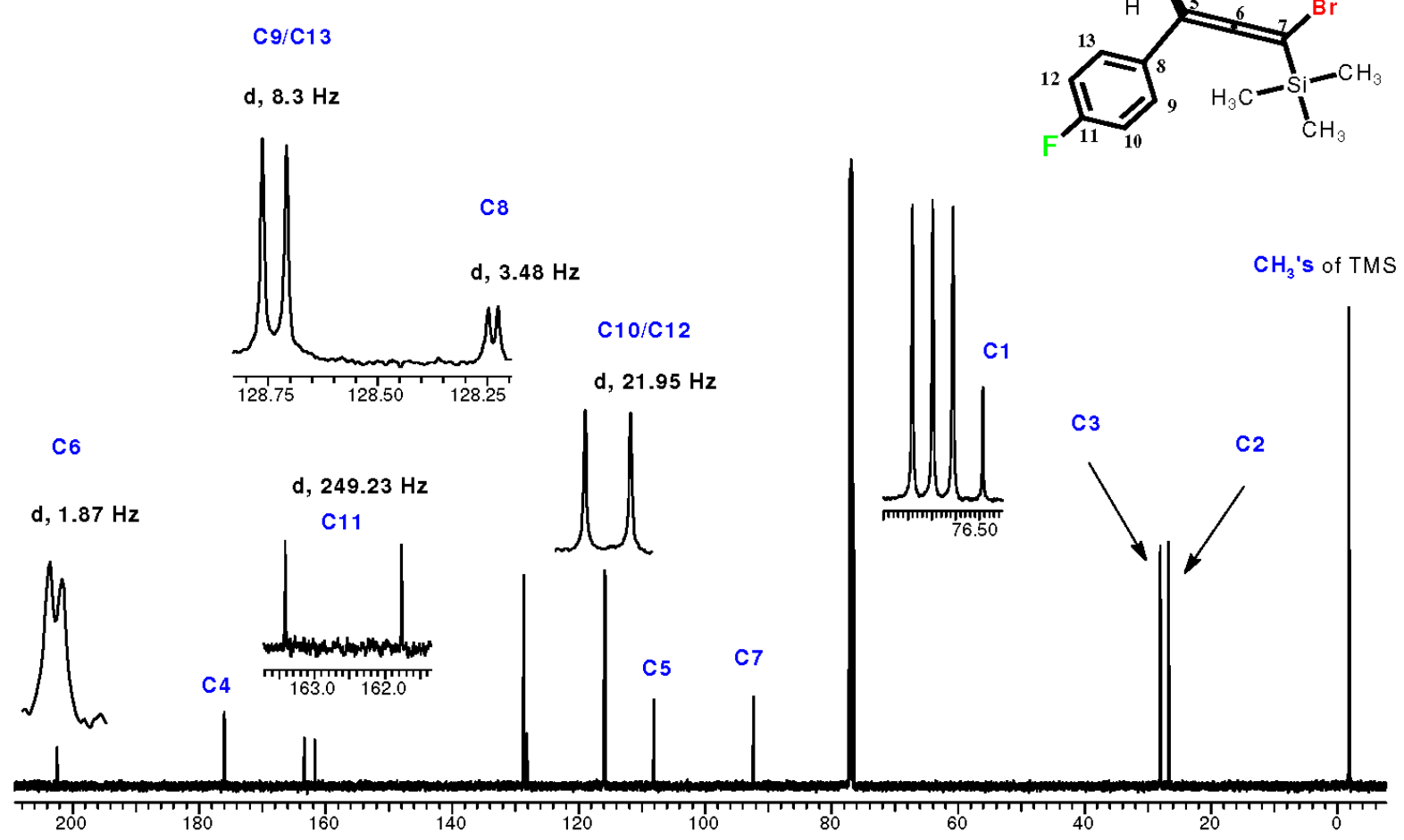




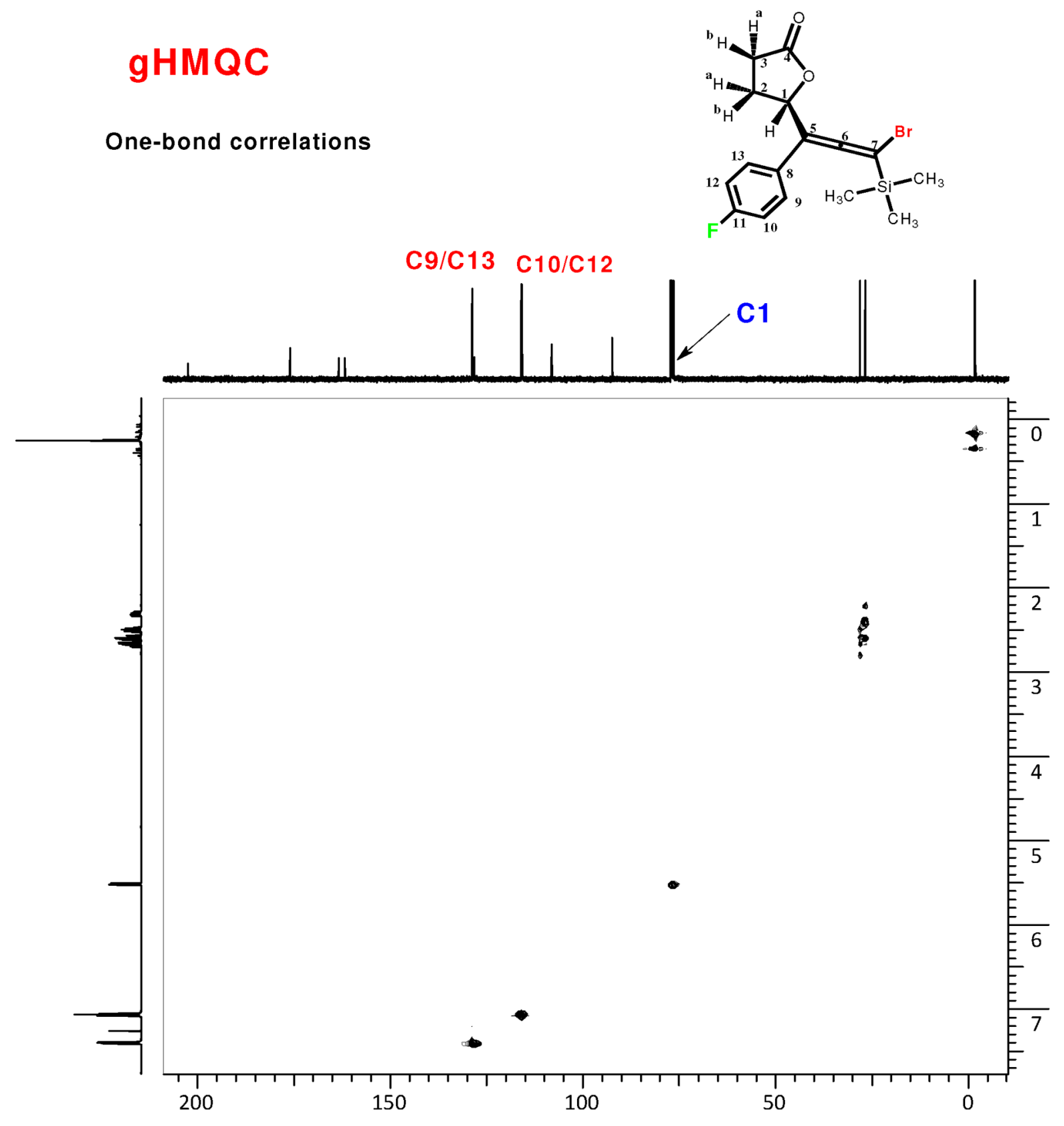




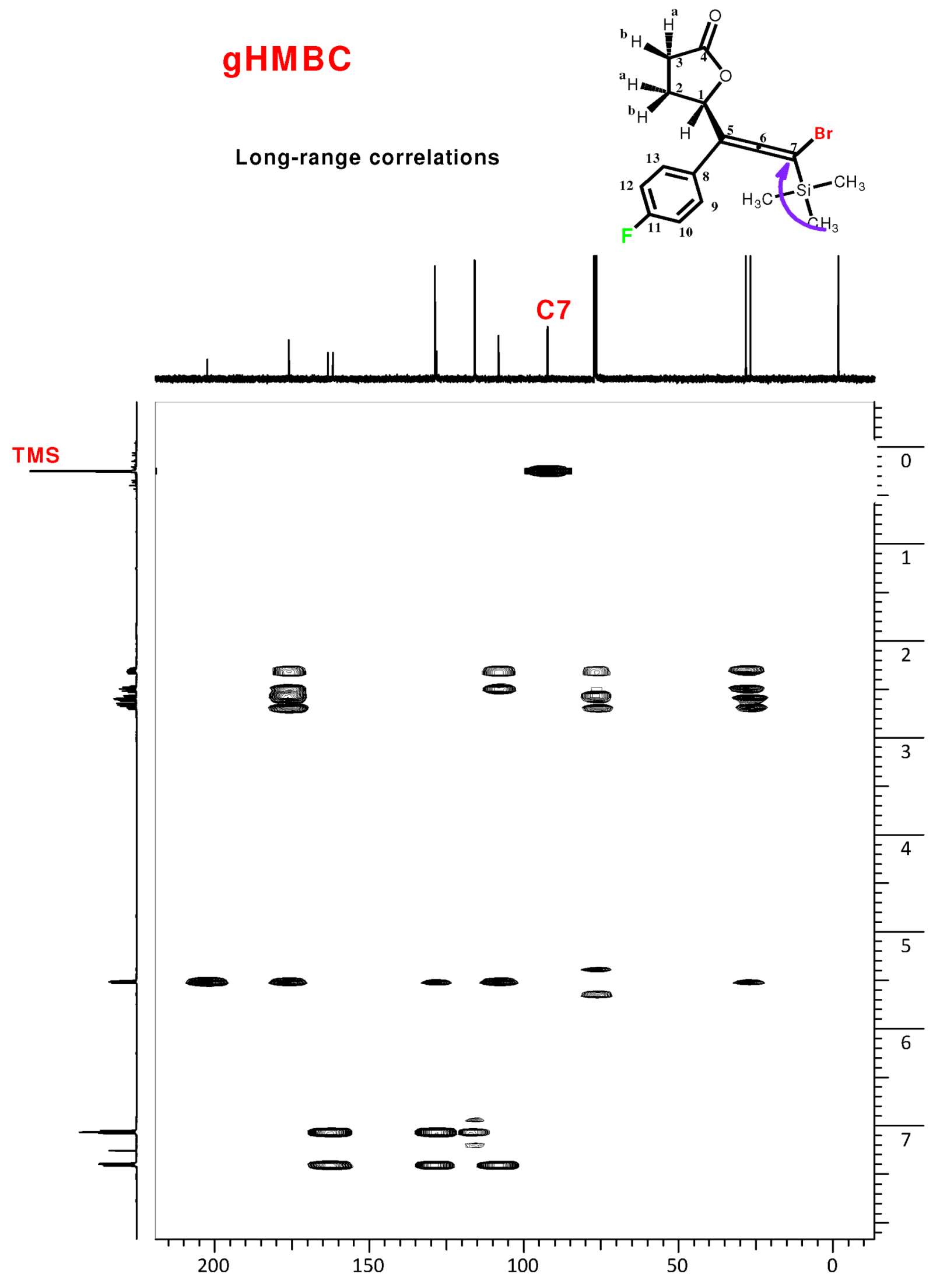



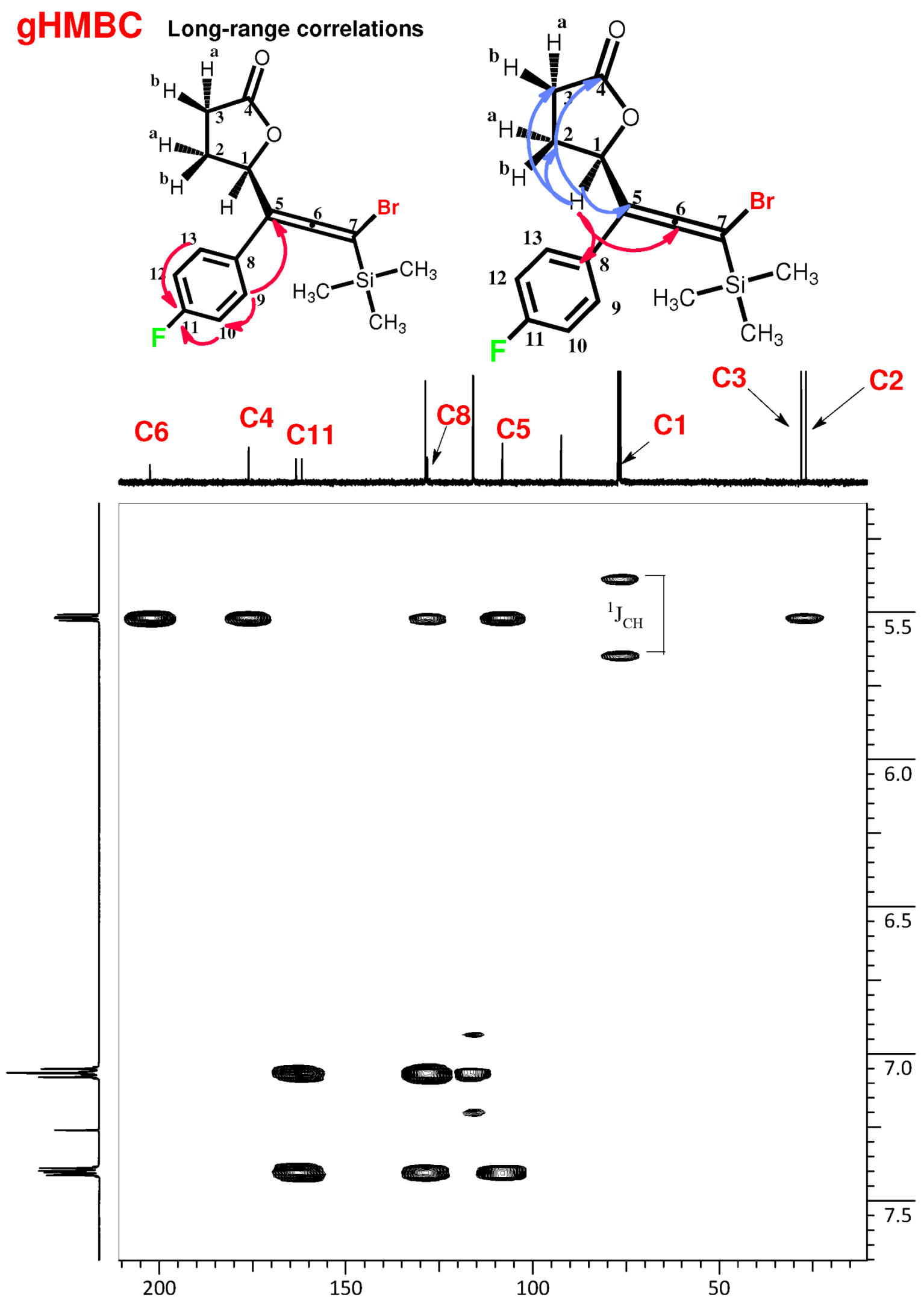


\section{gHMBC}

Long-range correlations
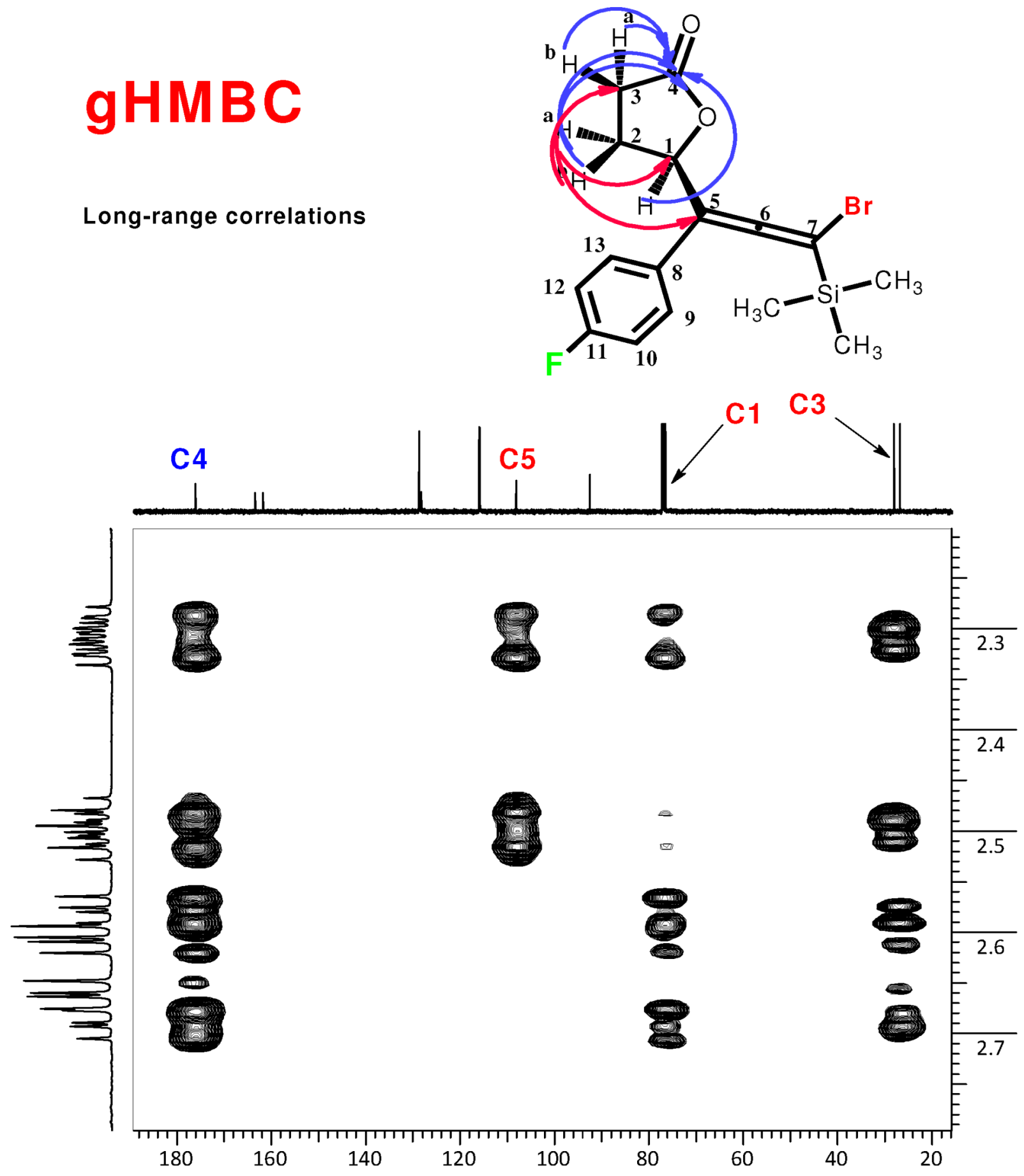


\begin{tabular}{|c|c|c|c|c|c|}
\hline & $\delta_{\mathrm{H}} / p p m$ & & $\mathbf{J} / \mathbf{H z}$ & & \\
\hline $\mathrm{H} 1$ & 5.52 & $\mathrm{H} 1---\mathrm{H} 2 \mathrm{a}$ & 7.19 & & \\
\hline $\mathrm{H} 2 \mathrm{a}$ & 2.51 & $\mathrm{H} 1----\mathrm{H} 2 b$ & 5.48 & & \\
\hline $\mathrm{H} 2 \mathrm{~b}$ & 2.35 & $\mathrm{H} 2 \mathrm{a}----\mathrm{H} 2 \mathrm{~b}$ & 12.82 & & \\
\hline H3а & 2.67 & H2a----H3a & 7.35 & & \\
\hline $\mathrm{H} 3 \mathrm{~b}$ & 2.59 & $\mathrm{H} 2 \mathrm{a}----\mathrm{H} 3 \mathrm{~b}$ & 9.14 & & \\
\hline $\mathrm{H} 9 / \mathrm{H} 13$ & 7.42 & $\mathrm{H} 2 \mathrm{~b}----\mathrm{H} 3 \mathrm{a}$ & 9.57 & & \\
\hline $\mathrm{H} 10 / \mathrm{H} 12$ & 7.07 & $\mathrm{H} 2 \mathrm{~b}----\mathrm{H} 3 \mathrm{~b}$ & 6.18 & & \\
\hline $14-\mathrm{CH}_{2}$ & 2.54 & $\begin{array}{l}\mathrm{H} 3 a----H 3 b \\
-19----F\end{array}$ & 17.47 & & $\delta_{c} / p p m$ \\
\hline $15-\mathrm{CH}_{2}$ & 1.54 & H13----F & 5.07 & $\begin{array}{l}\mathrm{C} 1 \\
\mathrm{C} 2\end{array}$ & $\begin{array}{l}76.62 \\
26.02\end{array}$ \\
\hline $16-\mathrm{CH}_{2}$ & 1.37 & H10----F & 8.86 & $\begin{array}{l}\mathrm{C} 3 \\
\mathrm{C} 4\end{array}$ & $\begin{array}{l}26.88 \\
176.09\end{array}$ \\
\hline \multirow{9}{*}{$16-\mathrm{CH}_{3}$} & 0.91 & H12----F & 8.86 & $\mathrm{C5}$ & 110.31 \\
\hline & & H9---H1O & 8.41 & C6 & $197.90\left(\mathrm{~d}, \mathrm{~J}_{\mathrm{CF}}=2.14 \mathrm{~Hz}\right)$ \\
\hline & & H12---H13 & 8.41 & $\begin{array}{l}\mathrm{C} 7 \\
\mathrm{C} 8\end{array}$ & $\begin{array}{l}98.66 \\
128.58\left(d, J_{C F}=3.48 \mathrm{~Hz}\right)\end{array}$ \\
\hline & & $\mathrm{H} 9----\mathrm{H} 13$ & 2.45 & $\mathrm{C} 9 / \mathrm{C} 13$ & $129.14\left(\mathrm{~d}, \mathrm{~J}_{\mathrm{CF}}=8.3 \mathrm{~Hz}\right)$ \\
\hline & & $\mathrm{H} 10-----\mathrm{H} 12$ & 2.95 & $\mathrm{C} 10 / \mathrm{C} 12$ & $115.68\left(d, J_{C F}=21.68\right)$ \\
\hline & & H9-------H12 & 0.24 & $\mathrm{C} 11$ & $162.75\left(\mathrm{~d}, \mathrm{~J}_{\mathrm{CF}}=249.23 \mathrm{~Hz}\right)$ \\
\hline & & H10-----H13 & 0.24 & $\begin{array}{l}\mathrm{C} 14 \\
\mathrm{C} 15\end{array}$ & $\begin{array}{l}37.65 \\
30.12\end{array}$ \\
\hline & & H15----H16 & 7.46 & C16 & 21.80 \\
\hline & & $\mathrm{H} 16----\mathrm{H} 17$ & 7.46 & $\mathrm{C} 17$ & 13.70 \\
\hline
\end{tabular}


${ }^{1} \mathrm{H}$ NMR spectrum of 2.3.3g in $\mathrm{CDCl}_{3}$

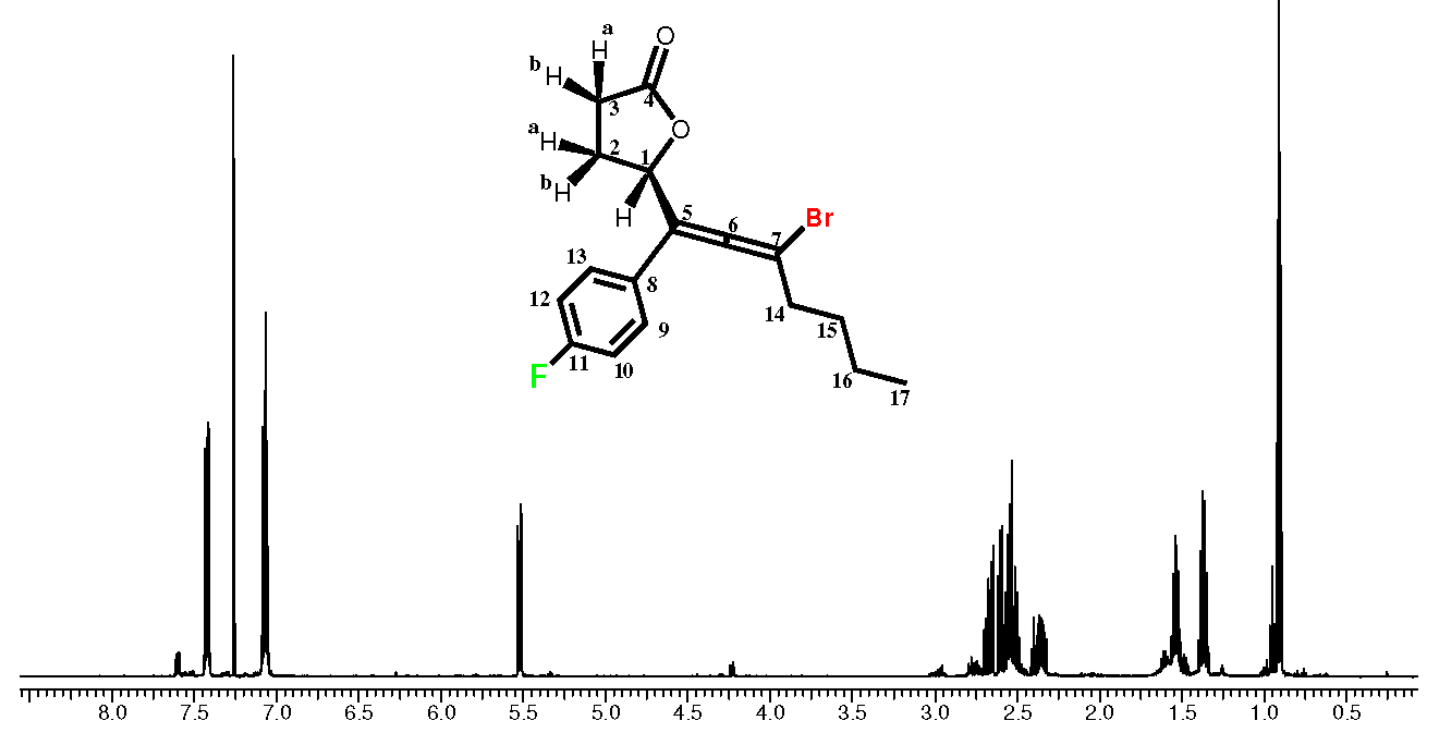

${ }^{13} \mathrm{C}$ NMR spectrum of $2.3 .3 \mathrm{~g}$ in $\mathrm{CDCl}_{3}$

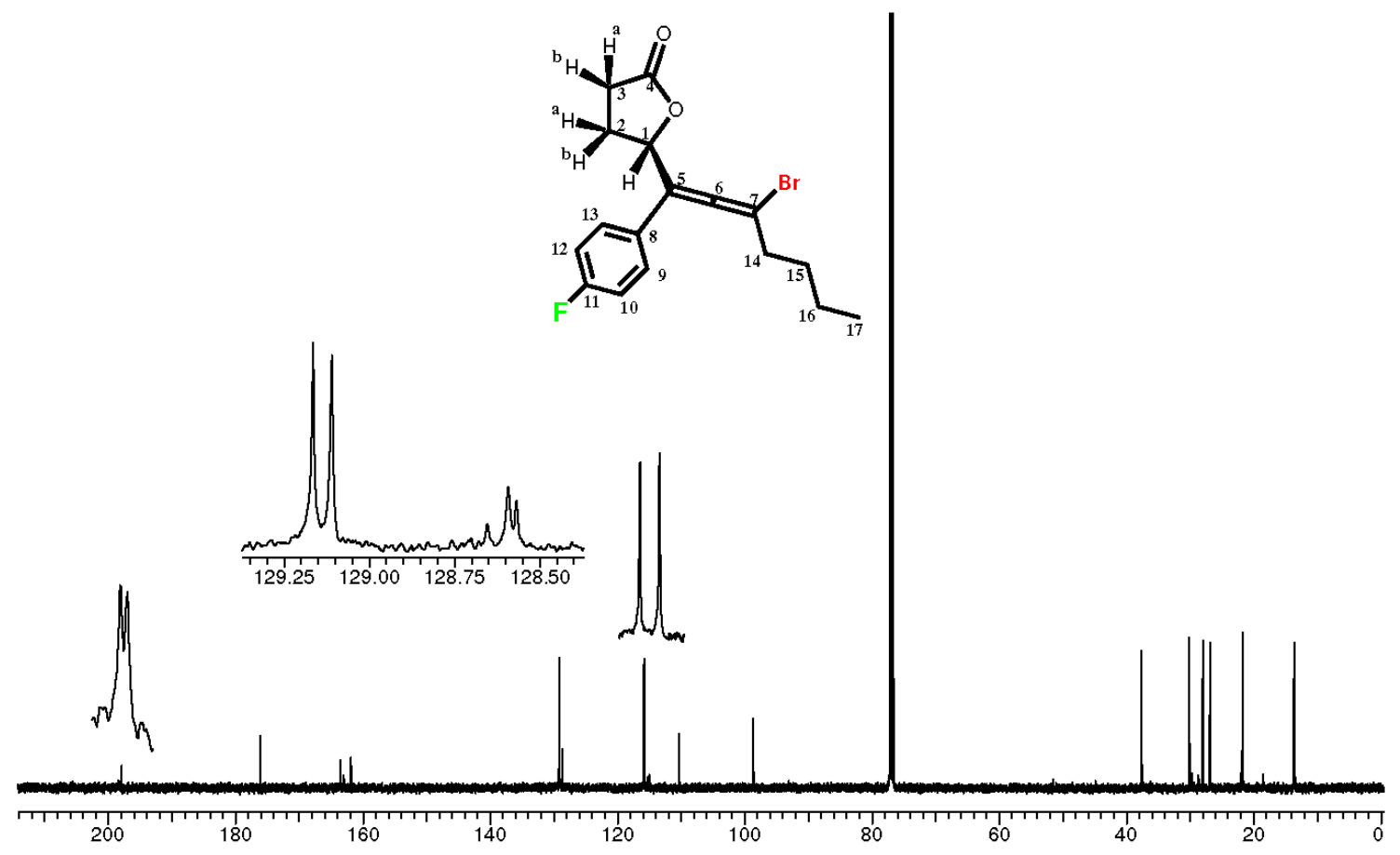


Experimental (a) and calculated (b) ${ }^{1} \mathrm{H}$ NMR spectra for the 5-membered ring protons

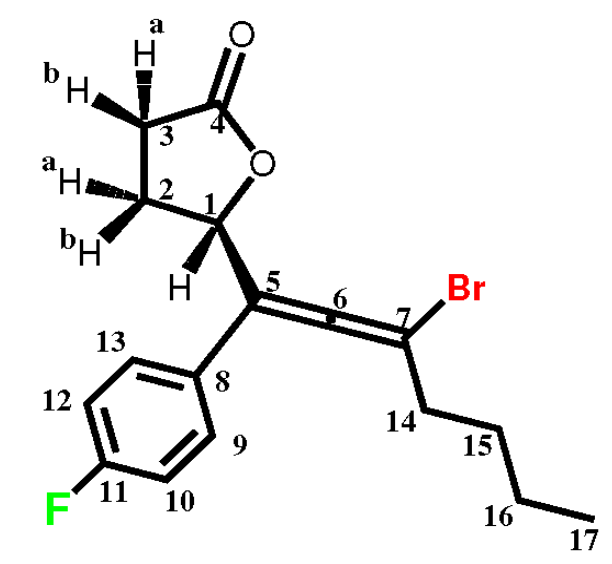

(a)
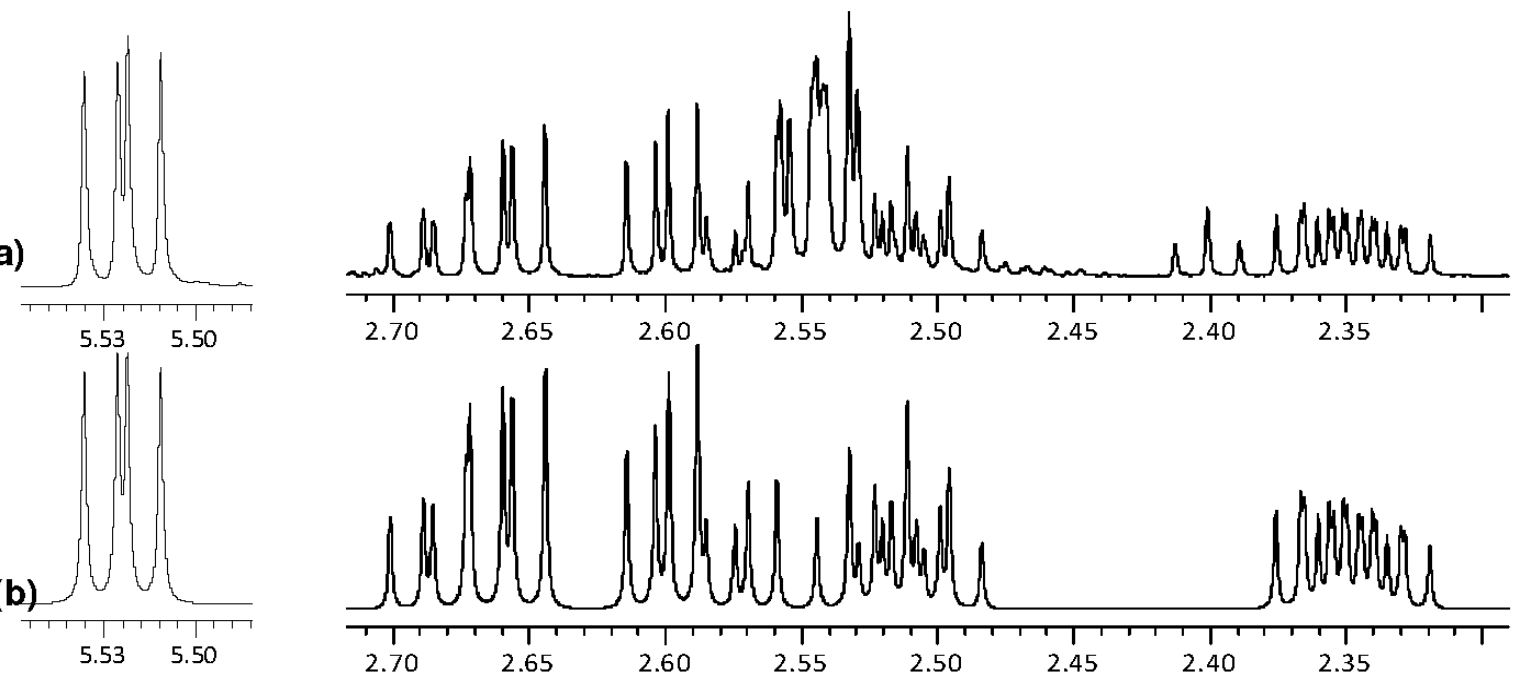


\section{$\mathrm{gHMBC}_{\text {spectrum of } \mathbf{2 . 3 . 3} \mathrm{g}}$}

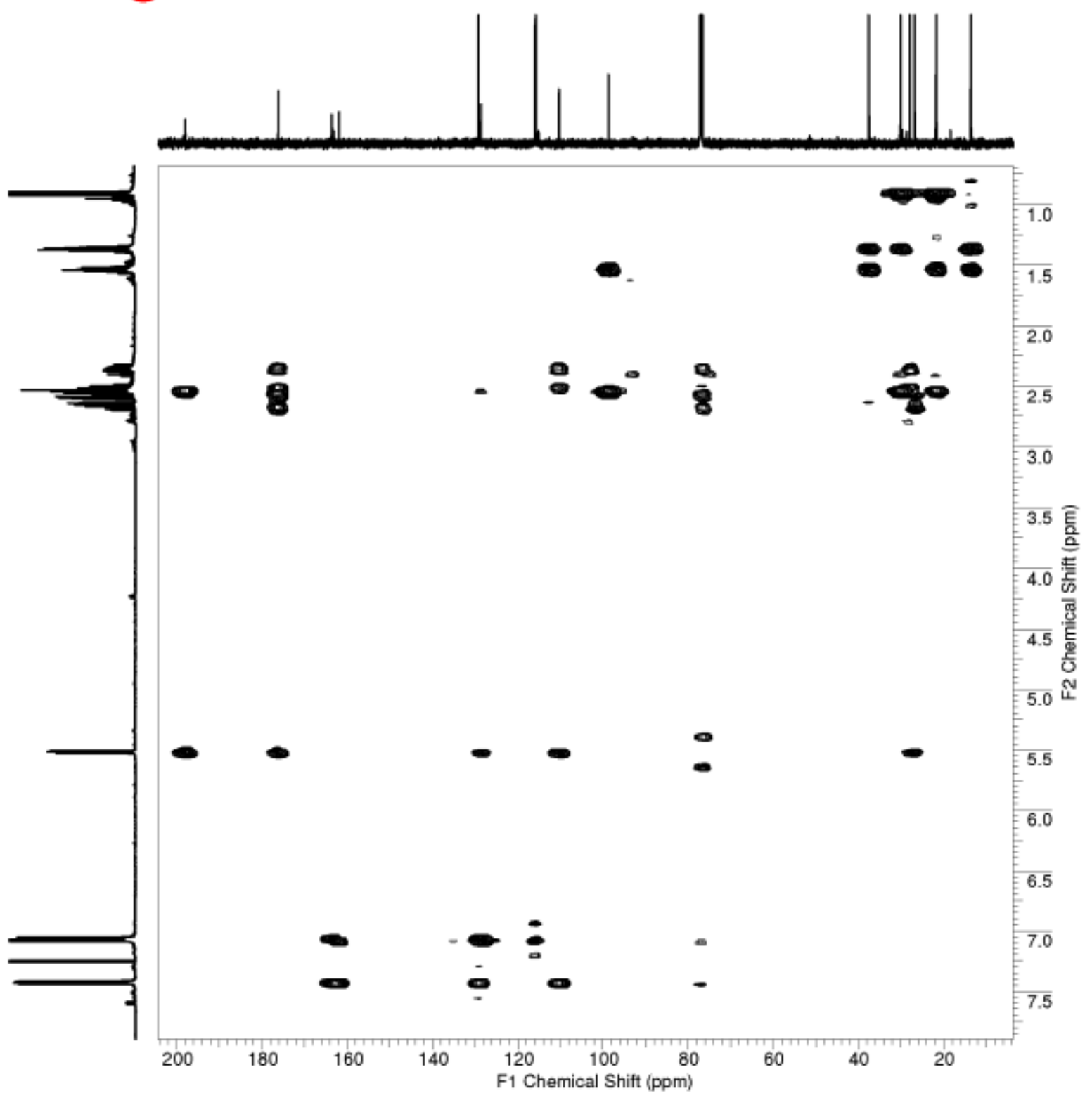




\section{Chapter Three: N-2-aryl-1,2,3-triazoles: a new class of UV-Blue emitting fluorophores through Planar-Intramolecular-Charge- Transfer (PICT)}

\section{General Methods and Materials}

All of the reactions dealing with air and/or moisture-sensitive reactions were carried out under an atmosphere of nitrogen using oven/flame-dried glassware and standard syringe/septa techniques. Unless otherwise noted, all commercial reagents and solvents were obtained from the commercial provider and used without further purification. ${ }^{1} \mathrm{H}$ NMR and ${ }^{13} \mathrm{C}$ NMR spectra were recorded on Varian $600 \mathrm{MHz}$ spectrometers. Chemical shifts were reported relative to internal tetramethylsilane $(\delta 0.00 \mathrm{ppm})$ or $\mathrm{CDCl}_{3}(\delta 7.26 \mathrm{ppm})$ for ${ }^{1} \mathrm{H}$ and $\mathrm{CDCl}_{3}(\delta 77.0 \mathrm{ppm})$ for ${ }^{13} \mathrm{C}$. Flash column chromatography was performed on 230-430 mesh silica gel. Analytical thin layer chromatography was performed with precoated glass baked plates $(250 \mu)$ and visualized by fluorescence and by charring after treatment with potassium permanganate stain. HRMS were recorded on LTQ-FTUHRA spectrometer. UV-visible spectra were obtained on a Shimadzu UV2500. Fluorescence spectra for emission and excitation were obtained on a Hitachi 7000.

All N-H triazoles were synthesized according to the literatures as below:

1. Quiclet-Sire, B.; Zard, S. Z.; Synthesis 2005, 19, 3319.

2. Sengupta, S.; Duan, H.; Lu, W.; Petersen, J. L.; Shi, X. Org. Lett. 2008, 10, 1493. 


\section{General Procedure for preparation of N-2-aryl-1,2,3-triazoles.}

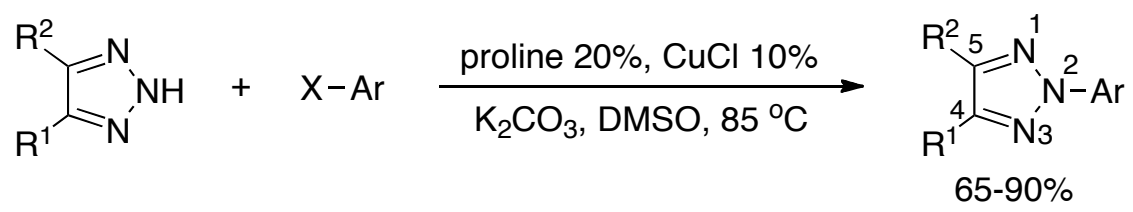

To a $25 \mathrm{~mL}$ round-bottom bottle was successively added $\mathrm{N}-\mathrm{H}$ triazole $(1 \mathrm{mmol})$, potassium carbonate (345 mg, $2.5 \mathrm{mmol})$, L-proline (23 mg, $0.2 \mathrm{mmol})$, copper (I) chloride (10 mg, 0.1 mmol) and iodo-substituted benzene $(1.2 \mathrm{mmol})$ in $10 \mathrm{~mL}$ DMSO under $\mathrm{N}_{2}$ protection. The reaction mixture was stirred and heated up to $85{ }^{\circ} \mathrm{C}$ for 4 hours, and monitored by TLC. After the reaction was completed, water $(20 \mathrm{~mL})$ was added. The mixture was extracted with ethyl acetate (3 $\times 30 \mathrm{~mL})$. The combined organic phases were washed with brine and dried with anhydrous $\mathrm{Na}_{2} \mathrm{SO}_{4}$. The solvent was removed under reduced pressure to get a residue, the residue was purified by flash silica gel chromatography (hexane/ethyl acetate $=20: 1$ ) to give the product. All yields were about $60 \%-75 \%(\mathrm{~N}-2$ isomer). 


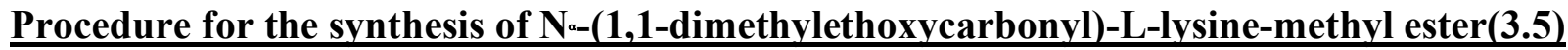

The $\mathrm{N}^{a}-t$ Boc-N $\mathrm{N}^{-}$-(carbobenzyloxy)-L-lysine 3.4 (1.14 g, $\left.3 \mathrm{mmol}\right)$, 4-dimethyl-aminopyridine (DMAP, $225 \mathrm{mg}, 1.83 \mathrm{mmol}$ ), and 1- ethyl-3-(3-dimethylaminopropyl)-carbodiimide (EDC, 1.15 g, $6 \mathrm{mmol})$ were added in dry $\mathrm{CH}_{2} \mathrm{Cl}_{2}(4 \mathrm{ml})$, and then dry $\mathrm{MeOH}(2 \mathrm{ml}, 33 \mathrm{mmol})$ was injected by syringe under $\mathrm{N}_{2}$ protection. After the reaction mixture was stirred at room temperature for 20 h, $20 \mathrm{ml} \mathrm{CH}_{2} \mathrm{Cl}_{2}$ was added. The mixture was washed with water and brine, dried with anhydrous $\mathrm{Na}_{2} \mathrm{SO}_{4}$ and concentrated under reduced pressure to give a yellow residue. The crude residue was purified by flash silica gel chromatography to produce the $\mathrm{N}^{\mathrm{c}}-\mathrm{B}$ Boc- $\mathrm{N}^{\mathrm{s}}$-(carbobenzyloxy)-Llysine-methyl ester as yellow oil.

The $\mathrm{N}^{a}-t$ Boc-N-(carbobenzyloxy)-L-lysine-methyl ester from last step was dissolved in $10 \mathrm{ml}$ of $\mathrm{MeOH}-\mathrm{CH}_{2} \mathrm{Cl}_{2}$ (1:1) with the addition of palladium-activated carbon $(0.157 \mathrm{~g}, 1.5 \mathrm{mmol})$. The mixture was stirred under high pressure hydrogen gas for $30 \mathrm{~min}$, and filtered by vacuum filtration to remove the palladium-activated carbon catalyst. The solvent was removed under reduced pressure to get a residue, the residue was purified by flash silica gel chromatography to afford product $3.5(0.663 \mathrm{~g}, 85 \%)$. 
Procedure for the synthesis of methyl 2-(tert-butoxycarbonylamino)-6-(2-(4iodophenyl)acetamido)hexanoate(3.7)

To a solution of 2-(4-iodophenyl)acetic acid $\mathbf{3 . 6}(1.44,5.5 \mathrm{mmol})$ and $\mathrm{N}$-(1,1dimethylethoxycarbonyl)-L-lysine-methyl ester $3.5(1.3,5 \mathrm{mmol})$ were dissolved in $20 \mathrm{~mL}$ $\mathrm{CH}_{2} \mathrm{Cl}_{2}$ was added 4-dimethyl-aminopyridine (DMAP, $62 \mathrm{mg}, 0.5 \mathrm{mmol}$ ), and 1- ethyl-3-(3dimethylaminopropyl)-carbodiimide (EDC, $1.15 \mathrm{~g}, 6 \mathrm{mmol}$ ). The reaction mixture was stirred under $\mathrm{N}_{2}$ protection for $12 \mathrm{~h}$ at room temperature, washed with water and brine, dried with anhydrous $\mathrm{Na}_{2} \mathrm{SO}_{4}$ and concentrated under reduced pressure to give the residue., the residue was purified by flash silica gel chromatography to give product $3.7(2.02 \mathrm{~g}, 80 \%)$. 


\section{UV absorption spectra:}

Absorption spectra were recorded on a Shimadzu UV-2500 spectrophotometer in $1 \mathrm{~cm}$-length quartz cell with a resolution of $0.2 \mathrm{~nm}$. All samples were measured from the $10^{-5} \mathrm{M}$ solution of $\mathrm{N}-2$-aryl-1,2,3-triazole in $\mathrm{MeOH}$ with the wavelength range between $200 \mathrm{~nm}$ and $800 \mathrm{~nm}$.

\section{Fluorescence excitation and emission spectra:}

Fluorescence excitation and emission spectra were measured on a Hitachi 7000 fluorophotometer with a slit width of $2.5 \mathrm{~nm}$. The photomultiplier voltage was $400 \mathrm{~V}$ and the scanning speed was $240 \mathrm{~nm} / \mathrm{min}$. The emission spectra were obtained from the $10^{-5} \mathrm{M}$ solution of N-2-aryl-1,2,3triazole in $\mathrm{MeOH}$ with an excitation wavelength at $254 \mathrm{~nm}$ or $310 \mathrm{~nm}$ and the excitation spectra were recorded with the emission wavelength $\lambda_{\max }$ which depended on different triazoles. 


\section{Quantum yield:}

Quantum yields were determined by using an integrated emission area and comparing that value to the area measured for the standard quinine sulfate dihydrate when excited at $365 \mathrm{~nm}\left(\Phi_{\text {reference }}\right.$ $=0.564$ ) in water. Quantum yields were calculated by using the following equation, where $\Phi$ is the quantum yield, Int is the area of emission peak, $A$ represents absorbance at the excitation wavelength and $n$ is reflective index of the solvent. The subscript reference is the respective values of the standard quinine sulfate dihydrate.

$$
\Phi=\Phi_{\text {reference }} \times \frac{\operatorname{Int} \times A_{\text {reference }} \times n^{2}}{\ln t_{\text {reference }} \times \mathrm{A} \times n_{\text {reference }}{ }^{2}}
$$




\section{Compounds Characterization}

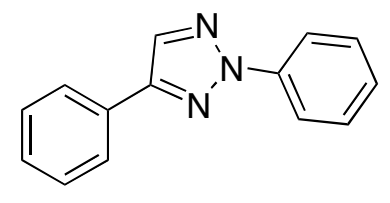

$3.1 \mathrm{e}$

2,4-diphenyl-2H-1,2,3-triazole (3.1e) was purified by flash chromatography (Hexane- EtOAc, v/v 10/1) as white solid. ${ }^{1} \mathrm{H}-\mathrm{NMR}\left(600 \mathrm{MHz}, \mathrm{CDCl}_{3}\right) \delta 8.15(\mathrm{~d}, J=7.8 \mathrm{~Hz}, 2 \mathrm{H}), 8.06(\mathrm{~m}, 1 \mathrm{H})$, $7.91(\mathrm{~d}, J=6.6 \mathrm{~Hz}, 2 \mathrm{H}), 7.52-7.46(\mathrm{~m}, 4 \mathrm{H}), 7.42-7.35(\mathrm{~m}, 2 \mathrm{H}) ;{ }^{13} \mathrm{C}-\mathrm{NMR}\left(150 \mathrm{MHz}, \mathrm{CDCl}_{3}\right) \delta$ $148.9,139.9,132.5,130.1,129.3,128.9,128.8,127.4,126.1,118.8$; HRMS Calculated for $\mathrm{C}_{14} \mathrm{H}_{11} \mathrm{~N}_{3}[\mathrm{M}+\mathrm{H}]^{+}:$222.1032, Found:222.1025.

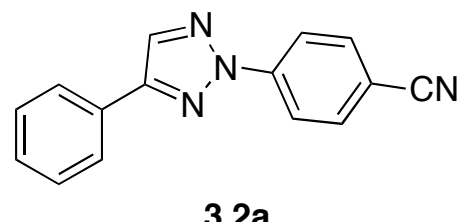

3.2a

4-(4-phenyl-2H-1,2,3-triazol-2-yl)benzonitrile (3.2a) was purified by flash chromatography (Hexane- EtOAc, v/v 10/1) as white solid. ${ }^{1} \mathrm{H}-\mathrm{NMR}\left(600 \mathrm{MHz}, \mathrm{CDCl}_{3}\right) \delta 8.28(\mathrm{~d}, J=9.0 \mathrm{~Hz}$, 2H), $8.12(\mathrm{~s}, 1 \mathrm{H}), 7.90(\mathrm{~d}, J=7.2 \mathrm{~Hz}, 2 \mathrm{H}), 7.80(\mathrm{~d}, J=9.0 \mathrm{~Hz}, 2 \mathrm{H}), 7.50-7.48(\mathrm{~m}, 2 \mathrm{H}), 7.45-$ $7.42(\mathrm{~m}, 1 \mathrm{H}) ;{ }^{13} \mathrm{C}-\mathrm{NMR}\left(150 \mathrm{MHz}, \mathrm{CDCl}_{3}\right) \delta 150.1,142.4,133.9,133.5,129.4,129.3,129.1$, 126.3, 119.0, 118.3, 110.7; HRMS Calculated for $\mathrm{C}_{15} \mathrm{H}_{10} \mathrm{~N}_{4}[\mathrm{M}+\mathrm{H}]^{+}$: 247.0984, Found: 247.0978 . 


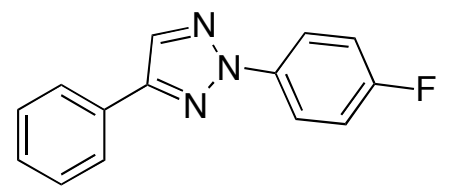

$3.2 b$

2-(4-fluorophenyl)-4-phenyl-2H-1,2,3-triazole (3.2b) was purified by flash chromatography (Hexane- EtOAc, v/v 10/1) as white solid. ${ }^{1} \mathrm{H}-\mathrm{NMR}\left(600 \mathrm{MHz}, \mathrm{CDCl}_{3}\right) \delta 8.13-8.11$ (m, $\left.2 \mathrm{H}\right)$, $8.04(\mathrm{~s}, 1 \mathrm{H}), 7.89(\mathrm{~d}, J=7.2 \mathrm{~Hz}, 2 \mathrm{H}), 7.48(\mathrm{t}, J=7.8 \mathrm{~Hz}, 2 \mathrm{H}), 7.40(\mathrm{t}, J=7.2 \mathrm{~Hz}, 1 \mathrm{H}), 7.19(\mathrm{t}, J$ $=8.4 \mathrm{~Hz}, 2 \mathrm{H}) ;{ }^{13} \mathrm{C}-\mathrm{NMR}\left(150 \mathrm{MHz}, \mathrm{CDCl}_{3}\right) \delta 162.6,161.0,149.0,136.2,132.6,130.0,129.0$, 128.9, 126.1, 120.6, 120.5, 116.2, 116.0; HRMS Calculated for $\mathrm{C}_{14} \mathrm{H}_{10} \mathrm{FN}_{3}[\mathrm{M}+\mathrm{H}]^{+}: 240.0938$, Found: 240.0931.

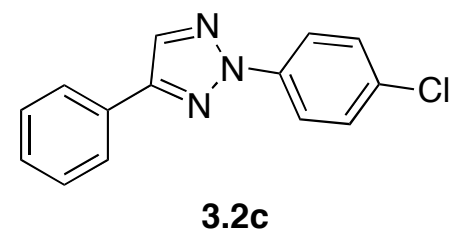

2-(4-chlorophenyl)-4-phenyl-2H-1,2,3-triazole (3.2c) was purified by flash chromatography (Hexane- EtOAc, v/v 10/1) as white solid. ${ }^{1} \mathrm{H}-\mathrm{NMR}\left(600 \mathrm{MHz}, \mathrm{CDCl}_{3}\right) \delta 8.09$ (d, $J=9.0 \mathrm{~Hz}$, 2H), $8.05(\mathrm{~s}, 1 \mathrm{H}), 7.89(\mathrm{~d}, J=7.8 \mathrm{~Hz}, 2 \mathrm{H}), 7.48(\mathrm{t}, J=8.4 \mathrm{~Hz}, 4 \mathrm{H}), 7.41(\mathrm{t}, J=7.2 \mathrm{~Hz}, 1 \mathrm{H}) ;{ }^{13} \mathrm{C}-$ NMR $\left(150 \mathrm{MHz}, \mathrm{CDCl}_{3}\right) \delta 149.2,138.4,133.0,132.8,129.8,129.4,129.0,126.2,120.0 ; \mathrm{HRMS}$ Calculated for $\mathrm{C}_{14} \mathrm{H}_{10} \mathrm{ClN}_{3}[\mathrm{M}+\mathrm{H}]^{+}:$256.0642, Found: 256.0636 . 


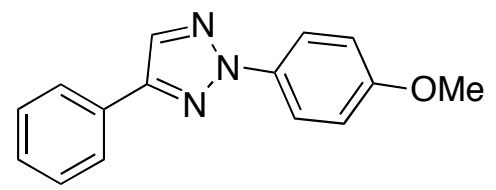

$3.2 d$

2-(4-methoxyphenyl)-4-phenyl-2H-1,2,3-triazole (3.2d) was purified by flash chromatography (Hexane- EtOAc, v/v 10/1) as white solid. ${ }^{1} \mathrm{H}-\mathrm{NMR}\left(600 \mathrm{MHz}, \mathrm{CDCl}_{3}\right) \delta 8.05(\mathrm{~d}, J=9.0 \mathrm{~Hz}$, 2H), $8.02(\mathrm{~s}, 1 \mathrm{H}), 7.89(\mathrm{~d}, J=6.6 \mathrm{~Hz}, 2 \mathrm{H}), 7.47$ (t, $J=7.8 \mathrm{~Hz}, 2 \mathrm{H}), 7.40-7.37(\mathrm{~m}, 1 \mathrm{H}), 7.01(\mathrm{t}, J$ $=9.0 \mathrm{~Hz}, 1 \mathrm{H}) ;{ }^{13} \mathrm{C}-\mathrm{NMR}\left(150 \mathrm{MHz}, \mathrm{CDCl}_{3}\right) \delta 159.2,148.7,134.0,132.3,130.4,129.1,128.9$, 126.3, 120.5, 114.6, 55.8; HRMS Calculated for $\mathrm{C}_{15} \mathrm{H}_{13} \mathrm{~N}_{3} \mathrm{O}[\mathrm{M}+\mathrm{H}]^{+}$: 252.1138, Found: 252.1170 .

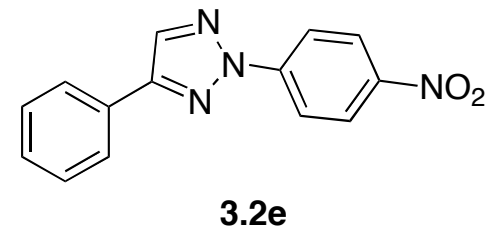

2-(4-nitrophenyl)-4-phenyl-2H-1,2,3-triazole (3.2e) was purified by flash chromatography (Hexane- EtOAc, v/v 10/1) as yellow solid. ${ }^{1} \mathrm{H}-\mathrm{NMR}\left(600 \mathrm{MHz}, \mathrm{CDCl}_{3}\right) \delta 8.38(\mathrm{~d}, J=9.0 \mathrm{~Hz}$, 2H), $8.32(\mathrm{~d}, J=9.0 \mathrm{~Hz}, 2 \mathrm{H}), 8.15(\mathrm{~s}, 1 \mathrm{H}), 7.92-7.88(\mathrm{~m}, 2 \mathrm{H}), 7.52-7.48(\mathrm{~m}, 2 \mathrm{H}), 7.46-7.43(\mathrm{~m}$, $1 \mathrm{H}) ;{ }^{13} \mathrm{C}-\mathrm{NMR}\left(150 \mathrm{MHz}, \mathrm{CDCl}_{3}\right) \delta 150.4,146.3,143.7,134.3,129.5,129.2,129.1,126.3$, 125.2, 118.8; HRMS Calculated for $\mathrm{C}_{14} \mathrm{H}_{11} \mathrm{~N}_{4} \mathrm{O}_{2}[\mathrm{M}+\mathrm{H}]^{+}:$267.0804, Found: 267.0836. 


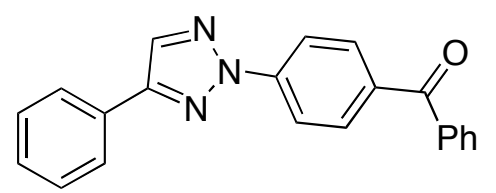

\section{$3.2 f$}

phenyl(4-(4-phenyl-2H-1,2,3-triazol-2-yl)phenyl)methanone (3.2f) was purified by flash chromatography (Hexane- EtOAc, v/v 10/1) as white solid. ${ }^{1} \mathrm{H}-\mathrm{NMR}\left(600 \mathrm{MHz}, \mathrm{CDCl}_{3}\right) \delta 8.27$ $(\mathrm{d}, J=9.0 \mathrm{~Hz}, 2 \mathrm{H}), 8.12(\mathrm{~s}, 1 \mathrm{H}), 7.97(\mathrm{~d}, J=9.0 \mathrm{~Hz}, 2 \mathrm{H}), 7.93-7.91(\mathrm{~m}, 2 \mathrm{H}), 7.84-7.82(\mathrm{~m}, 2 \mathrm{H})$, 7.64-7.60 (m, 1H), 7.53-7.47 (m, 4H), 7.44-7.40 (m, 1H); ${ }^{13} \mathrm{C}-\mathrm{NMR}\left(150 \mathrm{MHz}, \mathrm{CDCl}_{3}\right) \delta 195.5$, $149.7,142.4,137.5,136.1,133.5,132.5,131.5,129.9,129.6,129.1,129.0,128.4,126.2,118.3$; HRMS Calculated for $\mathrm{C}_{21} \mathrm{H}_{16} \mathrm{~N}_{3} \mathrm{O}[\mathrm{M}+\mathrm{H}]^{+}:$326.1215, Found: 326.1220 .

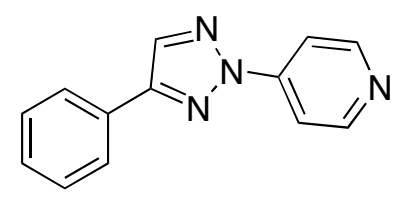

$3.2 \mathrm{~g}$

4-(4-phenyl-2H-1,2,3-triazol-2-yl)pyridine (3.2g) was purified by flash chromatography (Hexane- EtOAc, v/v 10/1) as white solid. ${ }^{1} \mathrm{H}-\mathrm{NMR}\left(600 \mathrm{MHz}, \mathrm{CDCl}_{3}\right) \delta 8.72(\mathrm{~d}, J=6.0 \mathrm{~Hz}$, 2H), $8.13(\mathrm{~s}, 1 \mathrm{H}), 8.05(\mathrm{~d}, J=6.0 \mathrm{~Hz}, 2 \mathrm{H}), 7.89$ (d, $J=7.2 \mathrm{~Hz}, 2 \mathrm{H}), 7.49(\mathrm{t}, J=7.8 \mathrm{~Hz}, 2 \mathrm{H})$, $7.43(\mathrm{t}, J=7.8 \mathrm{~Hz}, 1 \mathrm{H}) ;{ }^{13} \mathrm{C}-\mathrm{NMR}\left(150 \mathrm{MHz}, \mathrm{CDCl}_{3}\right) \delta 151.0,150.2,145.7,134.2,129.5,129.2$, 129.1, 126.3, 112.6; HRMS Calculated for $\mathrm{C}_{13} \mathrm{H}_{11} \mathrm{~N}_{4}[\mathrm{M}+\mathrm{H}]^{+}:$223.0905, Found: 223.0930. 


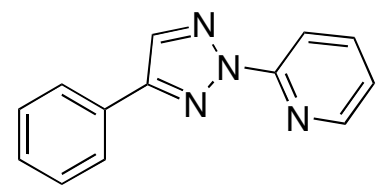

$3.2 \mathrm{~h}$

2-(4-phenyl-2H-1,2,3-triazol-2-yl)pyridine (3.2h) was purified by flash chromatography (Hexane- EtOAc, v/v 10/1) as white solid. ${ }^{1} \mathrm{H}-\mathrm{NMR}\left(600 \mathrm{MHz}, \mathrm{CDCl}_{3}\right) \delta 8.81(\mathrm{~s}, 1 \mathrm{H}), 8.52(\mathrm{~d}, J$ $=4.2 \mathrm{~Hz}, 1 \mathrm{H}), 8.25(\mathrm{~d}, J=8.4 \mathrm{~Hz}, 1 \mathrm{H}), 7.96-7.91(\mathrm{~m}, 3 \mathrm{H}), 7.47(\mathrm{t}, J=7.2 \mathrm{~Hz}, 2 \mathrm{H}), 7.39-7.34$ $(\mathrm{m}, 2 \mathrm{H}) ;{ }^{13} \mathrm{C}-\mathrm{NMR}\left(150 \mathrm{MHz}, \mathrm{CDCl}_{3}\right) \delta 149.2,148.5,148.0,139.1,130.2,128.9,128.4,125.9$, 123.5, 116.8, 113.8; HRMS Calculated for $\mathrm{C}_{13} \mathrm{H}_{11} \mathrm{~N}_{4}[\mathrm{M}+\mathrm{H}]^{+}:$223.0905, Found: 223.0930.

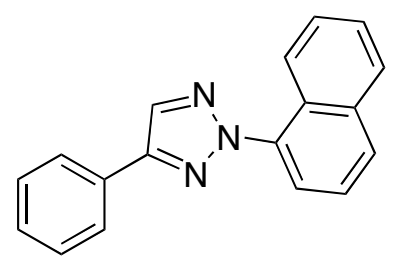

3.2i

2-(naphthalen-1-yl)-4-phenyl-2H-1,2,3-triazole (3.2i) was purified by flash chromatography (Hexane- EtOAc, v/v 10/1) as white solid. ${ }^{1} \mathrm{H}-\mathrm{NMR}\left(600 \mathrm{MHz}, \mathrm{CDCl}_{3}\right) \delta$ 8.29-8.26 (m, 1H), $8.21(\mathrm{~s}, 1 \mathrm{H}), 7.99-7.93(\mathrm{~m}, 4 \mathrm{H}), 7.87(\mathrm{~d}, J=7.2 \mathrm{~Hz}, 1 \mathrm{H}), 7.61-7.56(\mathrm{~m}, 3 \mathrm{H}), 7.49$ (t, $J=7.2 \mathrm{~Hz}$, 2H), $7.41(\mathrm{t}, J=7.2 \mathrm{~Hz}, 1 \mathrm{H}) ;{ }^{13} \mathrm{C}-\mathrm{NMR}\left(150 \mathrm{MHz}, \mathrm{CDCl}_{3}\right) \delta 148.9,136.9,134.4,132.5,130.1$, 129.6, 129.0, 128.8, 128.2, 127.6, 127.4, 126.6, 126.2, 125.0, 123.6, 122.8; HRMS Calculated for $\mathrm{C}_{18} \mathrm{H}_{14} \mathrm{~N}_{3}[\mathrm{M}+\mathrm{H}]^{+}:$272.1109, Found: 272.1125. 


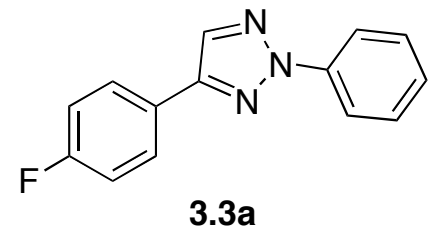

4-(4-fluorophenyl)-2-phenyl-2H-1,2,3-triazole (3.3a) was purified by flash chromatography (Hexane- EtOAc, v/v 10/1) as white solid. ${ }^{1} \mathrm{H}-\mathrm{NMR}\left(600 \mathrm{MHz}, \mathrm{CDCl}_{3}\right) \delta 8.13(\mathrm{~d}, J=7.8 \mathrm{~Hz}$, 2H), $8.01(\mathrm{~s}, 1 \mathrm{H}), 7.88(\mathrm{dd}, J=8.4 \mathrm{~Hz}, 5.4 \mathrm{~Hz}, 2 \mathrm{H}), 7.50(\mathrm{t}, J=7.8 \mathrm{~Hz}, 2 \mathrm{H}), 7.37$ (t, $J=7.2 \mathrm{~Hz}$, 1H), $7.16(\mathrm{t}, J=9.0 \mathrm{~Hz}, 2 \mathrm{H}) ;{ }^{13} \mathrm{C}-\mathrm{NMR}\left(150 \mathrm{MHz}, \mathrm{CDCl}_{3}\right) \delta 164.1,162.5,148.2,140.0,132.5$, $129.5,128.2,128.1,127.7,126.5,119.0,116.3,116.1$; HRMS Calculated for $\mathrm{C}_{14} \mathrm{H}_{10} \mathrm{FN}_{3}$ $[\mathrm{M}+\mathrm{H}]^{+}:$240.0938, Found: 240.0931 .

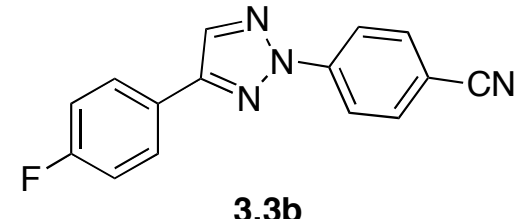

4-(4-(4-fluorophenyl)-2H-1,2,3-triazol-2-yl)benzonitrile (3.3b) was purified by flash chromatography (Hexane- EtOAc, v/v 10/1) as white solid. ${ }^{1} \mathrm{H}-\mathrm{NMR}\left(600 \mathrm{MHz}, \mathrm{CDCl}_{3}\right) \delta 8.26$ $(\mathrm{d}, J=8.4 \mathrm{~Hz}, 2 \mathrm{H}), 8.07(\mathrm{~s}, 1 \mathrm{H}), 7.88-7.86(\mathrm{~m}, 2 \mathrm{H}), 7.79(\mathrm{~d}, J=8.4 \mathrm{~Hz}, 2 \mathrm{H}), 7.17(\mathrm{t}, J=9.0 \mathrm{~Hz}$, $2 \mathrm{H}) ;{ }^{13} \mathrm{C}-\mathrm{NMR}\left(150 \mathrm{MHz}, \mathrm{CDCl}_{3}\right) \delta 149.2,142.4,133.7,133.5,128.2,128.1,125.5,119.0$, 118.3, 116.3, 116.1, 110.8, 29.7; HRMS Calculated for $\mathrm{C}_{15} \mathrm{H}_{9} \mathrm{FN}_{4}[\mathrm{M}+\mathrm{H}]^{+}:$265.0890, Found: 265.0884 . 


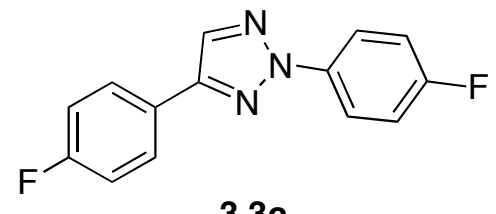

3.3c

2,4-bis(4-fluorophenyl)-2H-1,2,3-triazole (3.3c) was purified by flash chromatography (Hexane- EtOAc, v/v 10/1) as white solid. ${ }^{1} \mathrm{H}-\mathrm{NMR}\left(600 \mathrm{MHz}, \mathrm{CDCl}_{3}\right) \delta 8.10$ (q, $J=4.8 \mathrm{~Hz}$, 2H), $7.99(\mathrm{~s}, 1 \mathrm{H}), 7.86(\mathrm{dd}, J=8.4 \mathrm{~Hz}, 5.4 \mathrm{~Hz}, 2 \mathrm{H}), 7.20-7.15(\mathrm{~m}, 4 \mathrm{H}) ;{ }^{13} \mathrm{C}-\mathrm{NMR}(150 \mathrm{MHz}$, $\left.\mathrm{CDCl}_{3}\right) \delta 164.0,162.7,162.3,161.0,148.1,136.2,132.3,128.0,127.9,126.2,120.6,120.5$, 116.2, 116.1, 116.0, 115.9; HRMS Calculated for $\mathrm{C}_{14} \mathrm{H}_{9} \mathrm{~F}_{2} \mathrm{~N}_{3}[\mathrm{M}+\mathrm{H}]^{+}$: 258.0843, Found: 258.0837 .

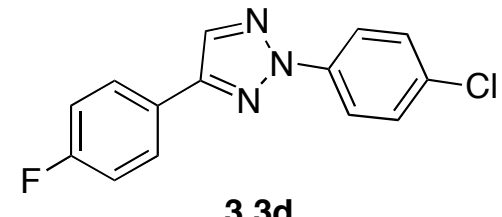

3.3d

2-(4-chlorophenyl)-4-(4-fluorophenyl)-2H-1,2,3-triazole (3.3d) was purified by flash chromatography (Hexane- EtOAc, v/v 10/1) as white solid. ${ }^{1} \mathrm{H}-\mathrm{NMR}\left(600 \mathrm{MHz}, \mathrm{CDCl}_{3}\right) \delta 8.07$ (d, $J=8.4 \mathrm{~Hz}, 2 \mathrm{H}), 8.00(\mathrm{~s}, 1 \mathrm{H}), 7.86(\mathrm{dd}, J=5.4 \mathrm{~Hz}, 8.4 \mathrm{~Hz}, 2 \mathrm{H}), 7.47$ (d, $J=9.0 \mathrm{~Hz}, 2 \mathrm{H}), 7.16$ $(\mathrm{t}, J=8.4 \mathrm{~Hz}, 2 \mathrm{H}) ;{ }^{13} \mathrm{C}-\mathrm{NMR}\left(150 \mathrm{MHz}, \mathrm{CDCl}_{3}\right) \delta 164.0,162.4,148.3,138.3,133.1,132.6$, 129.4, 128.0, 127.9, 126.0, 120.0, 116.1, 116.0; HRMS Calculated for $\mathrm{C}_{14} \mathrm{H}_{9} \mathrm{ClFN}_{3}[\mathrm{M}+\mathrm{H}]^{+}$: 274.0548, Found: 274.0541. 


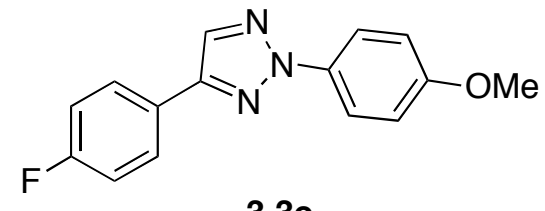

3.3e

4-(4-fluorophenyl)-2-(4-methoxyphenyl)-2H-1,2,3-triazole (3.3e) was purified by flash chromatography (Hexane- EtOAc, v/v 10/1) as white solid. ${ }^{1} \mathrm{H}-\mathrm{NMR}\left(600 \mathrm{MHz}, \mathrm{CDCl}_{3}\right) \delta 8.03$ $(\mathrm{d}, J=9.0 \mathrm{~Hz}, 2 \mathrm{H}), 7.96(\mathrm{~s}, 1 \mathrm{H}), 7.86(\mathrm{dd}, J=8.4 \mathrm{~Hz}, 5.4 \mathrm{~Hz}, 2 \mathrm{H}), 7.15(\mathrm{t}, J=8.4 \mathrm{~Hz}, 2 \mathrm{H}), 7.10$ $(\mathrm{d}, J=9.0 \mathrm{~Hz}, 2 \mathrm{H}) ;{ }^{13} \mathrm{C}-\mathrm{NMR}\left(150 \mathrm{MHz}, \mathrm{CDCl}_{3}\right) \delta 163.8,162.2,159.0,147.6,133.7,131.8$, 127.9, 127.8, 126.4, 120.3, 116.0, 115.9, 114.4, 55.6; HRMS Calculated for $\mathrm{C}_{15} \mathrm{H}_{12} \mathrm{FN}_{3} \mathrm{O}$ $[\mathrm{M}+\mathrm{H}]^{+}: 270.1043$, Found: 270.1037.

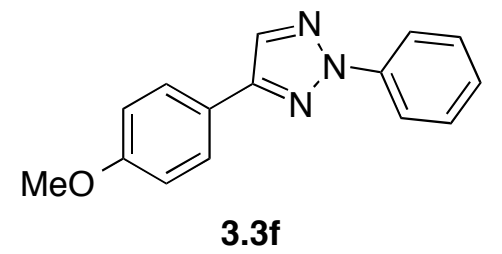

4-(4-methoxyphenyl)-2-phenyl-2H-1,2,3-triazole (3.3f) was purified by flash chromatography (Hexane- EtOAc, v/v 10/1) as white solid. ${ }^{1} \mathrm{H}-\mathrm{NMR}\left(600 \mathrm{MHz}, \mathrm{CDCl}_{3}\right) \delta 8.13(\mathrm{~d}, J=7.2 \mathrm{~Hz}$, 2H), $7.99(\mathrm{~s}, 1 \mathrm{H}), 7.83(\mathrm{~d}, J=8.4 \mathrm{~Hz}, 2 \mathrm{H}), 7.50(\mathrm{t}, J=7.8 \mathrm{~Hz}, 2 \mathrm{H}), 7.35(\mathrm{t}, J=7.2 \mathrm{~Hz}, 1 \mathrm{H}), 6.50$ $(\mathrm{d}, J=7.8 \mathrm{~Hz}, 2 \mathrm{H}) ;{ }^{13} \mathrm{C}-\mathrm{NMR}\left(150 \mathrm{MHz}, \mathrm{CDCl}_{3}\right) \delta 160.2,148.7,139.9,132.1,129.2,127.5$, 127.2, 122.7, 118.7, 114.4, 55.4; HRMS Calculated for $\mathrm{C}_{15} \mathrm{H}_{13} \mathrm{~N}_{3} \mathrm{O}[\mathrm{M}+\mathrm{H}]^{+}:$252.1138, Found: 252.1131. 


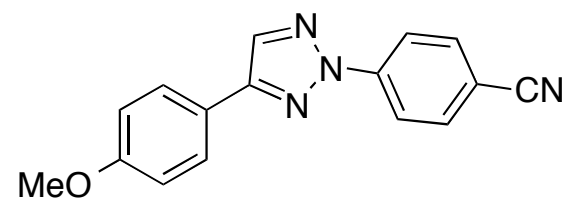

$3.3 \mathrm{~g}$

4-(4-(4-methoxyphenyl)-2H-1,2,3-triazol-2-yl)benzonitrile (3.3g) was purified by flash chromatography (Hexane- EtOAc, v/v 10/1) as white solid. ${ }^{1} \mathrm{H}-\mathrm{NMR}\left(600 \mathrm{MHz}, \mathrm{CDCl}_{3}\right) \delta 8.24$ $(\mathrm{d}, J=8.4 \mathrm{~Hz}, 2 \mathrm{H}), 8.04(\mathrm{~s}, 1 \mathrm{H}), 7.82(\mathrm{~d}, J=8.4 \mathrm{~Hz}, 2 \mathrm{H}), 7.78(\mathrm{~d}, J=9.0 \mathrm{~Hz}, 2 \mathrm{H}), 6.51(\mathrm{~d}, J=$ $9.0 \mathrm{~Hz}, 2 \mathrm{H}) ;{ }^{13} \mathrm{C}-\mathrm{NMR}\left(150 \mathrm{MHz}, \mathrm{CDCl}_{3}\right) \delta 160.6,149.9,142.4,133.5,133.4,127.6,121.9$, 118.9, 118.4, 114.5, 110.4, 55.4; HRMS Calculated for $\mathrm{C}_{16} \mathrm{H}_{12} \mathrm{~N}_{4} \mathrm{O}[\mathrm{M}+\mathrm{H}]^{+}$: 277.1090, Found: 277.1083.

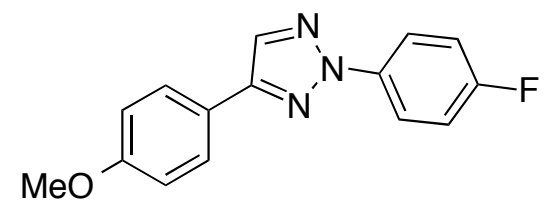

3.3h

2-(4-fluorophenyl)-4-(4-methoxyphenyl)-2H-1,2,3-triazole (3.3h) was purified by flash chromatography (Hexane- EtOAc, v/v 10/1) as white solid. ${ }^{1} \mathrm{H}-\mathrm{NMR}\left(600 \mathrm{MHz}, \mathrm{CDCl}_{3}\right) \delta 8.08$ $(\mathrm{q}, J=4.8 \mathrm{~Hz}, 2 \mathrm{H}), 7.95(\mathrm{~s}, 1 \mathrm{H}), 7.80(\mathrm{~d}, J=8.4 \mathrm{~Hz}, 2 \mathrm{H}), 7.16(\mathrm{t}, J=8.4 \mathrm{~Hz}, 2 \mathrm{H}), 6.98(\mathrm{~d}, J=$ $8.4 \mathrm{~Hz}, 2 \mathrm{H}) ;{ }^{13} \mathrm{C}-\mathrm{NMR}\left(150 \mathrm{MHz}, \mathrm{CDCl}_{3}\right) \delta 136.3,132.1,127.4,122.5,120.4,120.3,116.1$, 116.0, 114.4, 55.3; HRMS Calculated for $\mathrm{C}_{15} \mathrm{H}_{12} \mathrm{FN}_{3} \mathrm{O}[\mathrm{M}+\mathrm{H}]^{+}:$270.1043, Found: 270.1037 . 


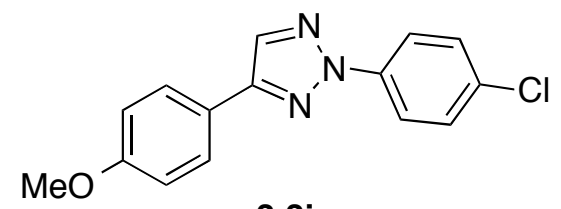

3.3i

2-(4-chlorophenyl)-4-(4-methoxyphenyl)-2H-1,2,3-triazole (3.3i) was purified by flash chromatography (Hexane- EtOAc, v/v 10/1) as white solid. ${ }^{1} \mathrm{H}-\mathrm{NMR}\left(600 \mathrm{MHz}, \mathrm{CDCl}_{3}\right) \delta 8.07$ $(\mathrm{d}, J=9.0 \mathrm{~Hz}, 2 \mathrm{H}), 7.97(\mathrm{~s}, 1 \mathrm{H}), 7.81(\mathrm{~d}, J=8.4 \mathrm{~Hz}, 2 \mathrm{H}), 7.45(\mathrm{~d}, J=8.4 \mathrm{~Hz}, 2 \mathrm{H}), 6.99(\mathrm{~d}, J=$ $8.4 \mathrm{~Hz}, 2 \mathrm{H}) ;{ }^{13} \mathrm{C}-\mathrm{NMR}\left(150 \mathrm{MHz}, \mathrm{CDCl}_{3}\right) \delta 160.3,149.0,138.4,132.8,132.4,129.3,127.5$, 122.4, 119.8, 114.4, 55.3; HRMS Calculated for $\mathrm{C}_{15} \mathrm{H}_{13} \mathrm{ClN}_{3} \mathrm{O}[\mathrm{M}+\mathrm{H}]^{+}$: 286.0748, Found: 286.0776 .

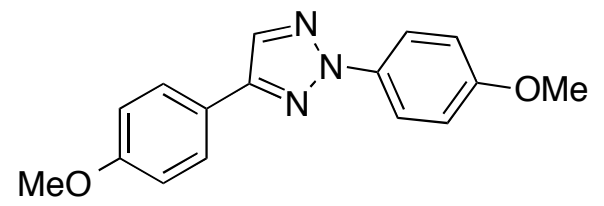

3.3j

2,4-bis(4-methoxyphenyl)-2H-1,2,3-triazole (3.3j) was purified by flash chromatography (Hexane- EtOAc, v/v 10/1) as white solid. ${ }^{1} \mathrm{H}-\mathrm{NMR}\left(600 \mathrm{MHz}, \mathrm{CDCl}_{3}\right) \delta 8.03(\mathrm{~d}, J=9.0 \mathrm{~Hz}$, 2H), $7.94(\mathrm{~s}, 1 \mathrm{H}), 7.81(\mathrm{~d}, J=9.0 \mathrm{~Hz}, 2 \mathrm{H}), 7.00(\mathrm{dd}, J=7.8 \mathrm{~Hz}, 1.8 \mathrm{~Hz}, 4 \mathrm{H}), 3.86(\mathrm{~d}, J=1.8 \mathrm{~Hz}$, $6 \mathrm{H}) ;{ }^{13} \mathrm{C}-\mathrm{NMR}\left(150 \mathrm{MHz}, \mathrm{CDCl}_{3}\right) \delta 160.0,158.8,148.3,133.8,131.5,127.4,122.9,120.2$, 114.3, 55.6, 55.3; HRMS Calculated for $\mathrm{C}_{16} \mathrm{H}_{15} \mathrm{~N}_{3} \mathrm{O}_{2}[\mathrm{M}+\mathrm{H}]^{+}:$282.1243, Found: 282.1237 . 


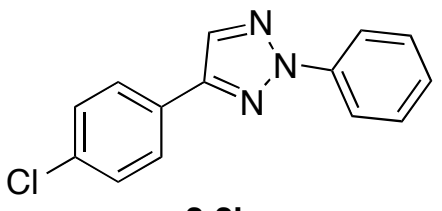

3.3k

4-(4-chlorophenyl)-2-phenyl-2H-1,2,3-triazole (3.3k) was purified by flash chromatography (Hexane- EtOAc, v/v 10/1) as white solid. ${ }^{1} \mathrm{H}-\mathrm{NMR}\left(600 \mathrm{MHz}, \mathrm{CDCl}_{3}\right) \delta 8.13(\mathrm{~d}, J=7.8 \mathrm{~Hz}$, 2H), $8.02(\mathrm{~s}, 1 \mathrm{H}), 7.83(\mathrm{~d}, J=8.4 \mathrm{~Hz}, 2 \mathrm{H}), 7.50(\mathrm{t}, J=7.8 \mathrm{~Hz}, 2 \mathrm{H}), 7.44(\mathrm{~d}, J=8.4 \mathrm{~Hz}, 2 \mathrm{H})$, $7.34(\mathrm{t}, J=7.8 \mathrm{~Hz}, 1 \mathrm{H}) ;{ }^{13} \mathrm{C}-\mathrm{NMR}\left(150 \mathrm{MHz}, \mathrm{CDCl}_{3}\right) \delta 148.0,140.0,134.9,132.7,129.5,129.4$, 128.8, 127.8, 127.6, 119.0; HRMS Calculated for $\mathrm{C}_{14} \mathrm{H}_{10} \mathrm{ClN}_{3}[\mathrm{M}+\mathrm{H}]^{+}$: 256.0642, Found: 256.0636.

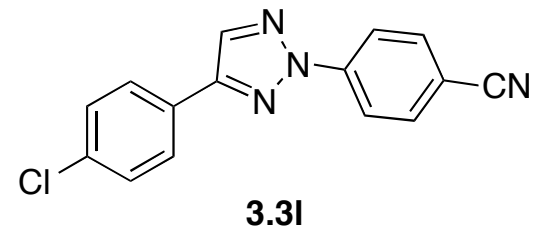

4-(4-(4-chlorophenyl)-2H-1,2,3-triazol-2-yl)benzonitrile (3.3l) was purified by flash chromatography (Hexane- EtOAc, v/v 10/1) as white solid. ${ }^{1} \mathrm{H}-\mathrm{NMR}\left(600 \mathrm{MHz}, \mathrm{CDCl}_{3}\right) \delta 8.27$ $(\mathrm{d}, J=9.0 \mathrm{~Hz}, 2 \mathrm{H}), 8.10(\mathrm{~s}, 1 \mathrm{H}), 7.84(\mathrm{~d}, J=8.4 \mathrm{~Hz}, 2 \mathrm{H}), 7.81(\mathrm{~d}, J=8.4 \mathrm{~Hz}, 2 \mathrm{H}), 7.47(\mathrm{~d}, J=$ $8.4 \mathrm{~Hz}, 2 \mathrm{H}) ;{ }^{13} \mathrm{C}-\mathrm{NMR}\left(150 \mathrm{MHz}, \mathrm{CDCl}_{3}\right) \delta 149.0,142.3,135.4,133.8,133.5,129.3,127.8$, 127.5, 119.1, 118.3, 111.0; HRMS Calculated for $\mathrm{C}_{15} \mathrm{H}_{9} \mathrm{ClN}_{4}[\mathrm{M}+\mathrm{H}]^{+}$: 281.0595, Found: 281.0588 . 


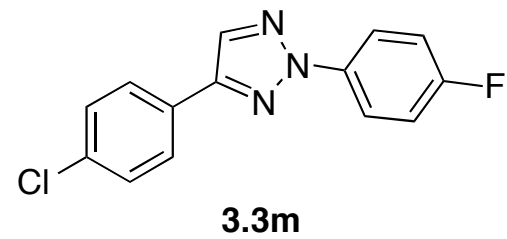

4-(4-chlorophenyl)-2-(4-fluorophenyl)-2H-1,2,3-triazole $\mathbf{( 3 . 3 m )}$ was purified by flash chromatography (Hexane- EtOAc, v/v 10/1) as white solid. ${ }^{1} \mathrm{H}-\mathrm{NMR}\left(600 \mathrm{MHz}, \mathrm{CDCl}_{3}\right) \delta 8.10$ $(\mathrm{q}, J=4.8 \mathrm{~Hz}, 2 \mathrm{H}), 8.01(\mathrm{~s}, 1 \mathrm{H}), 7.81(\mathrm{~d}, J=8.4 \mathrm{~Hz}, 2 \mathrm{H}), 7.44(\mathrm{~d}, J=8.0 \mathrm{~Hz}, 2 \mathrm{H}), 7.19(\mathrm{t}, J=$ $8.4 \mathrm{~Hz}, 2 \mathrm{H}) ;{ }^{13} \mathrm{C}-\mathrm{NMR}\left(150 \mathrm{MHz}, \mathrm{CDCl}_{3}\right) \delta 162.7,161.0,147.9,136.1,134.7,132.5,129.2$, 128.4, 127.3, 120.6, 120.5, 116.2, 116.1; HRMS Calculated for $\mathrm{C}_{14} \mathrm{H}_{9} \mathrm{ClFN}_{3}[\mathrm{M}+\mathrm{H}]^{+}: 274.0548$, Found: 274.0541.

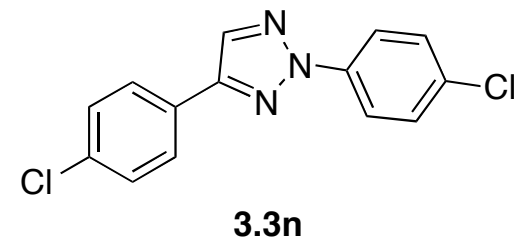

2,4-bis(4-chlorophenyl)-2H-1,2,3-triazole (3.3n) was purified by flash chromatography (Hexane- EtOAc, v/v 10/1) as white solid. ${ }^{1} \mathrm{H}-\mathrm{NMR}\left(600 \mathrm{MHz}, \mathrm{CDCl}_{3}\right) \delta 8.07(\mathrm{~d}, J=8.4 \mathrm{~Hz}$, 2H), $8.02(\mathrm{~s}, 1 \mathrm{H}), 7.82(\mathrm{~d}, J=8.4 \mathrm{~Hz}, 2 \mathrm{H}), 7.45(\mathrm{dd}, J=14.4 \mathrm{~Hz}, 9.0 \mathrm{~Hz}, 4 \mathrm{H}) ;{ }^{13} \mathrm{C}-\mathrm{NMR}(150$ $\left.\mathrm{MHz}, \mathrm{CDCl}_{3}\right) \delta 148.1,138.3,134.9,133.2,132.7,129.4,129.2,128.3,127.4,120.0 ;$ HRMS Calculated for $\mathrm{C}_{14} \mathrm{H}_{9} \mathrm{Cl}_{2} \mathrm{~N}_{3}[\mathrm{M}+\mathrm{H}]^{+}:$290.0252, Found: 290.0246 . 


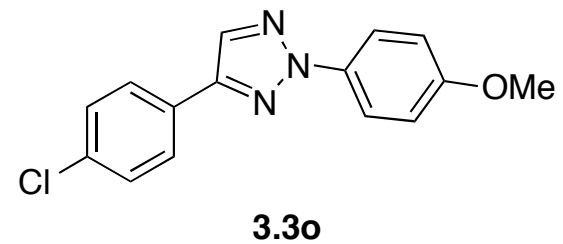

4-(4-chlorophenyl)-2-(4-methoxyphenyl)-2H-1,2,3-triazole (3.3o) was purified by flash chromatography (Hexane- EtOAc, v/v 10/1) as white solid. ${ }^{1} \mathrm{H}-\mathrm{NMR}\left(600 \mathrm{MHz}, \mathrm{CDCl}_{3}\right) \delta 8.03$ $(\mathrm{d}, J=9.0 \mathrm{~Hz}, 2 \mathrm{H}), 7.99(\mathrm{~s}, 1 \mathrm{H}), 7.82(\mathrm{~d}, J=7.8 \mathrm{~Hz}, 2 \mathrm{H}), 7.43(\mathrm{~d}, J=7.8 \mathrm{~Hz}, 2 \mathrm{H}), 7.01(\mathrm{~d}, J=$ $8.4 \mathrm{~Hz}, 2 \mathrm{H}) ;{ }^{13} \mathrm{C}-\mathrm{NMR}\left(150 \mathrm{MHz}, \mathrm{CDCl}_{3}\right) \delta 159.1,147.4,134.5,133.6,132.0,129.1,128.7$, 127.3, 120.3, 114.4, 55.6; HRMS Calculated for $\mathrm{C}_{15} \mathrm{H}_{12} \mathrm{ClN}_{3} \mathrm{O}[\mathrm{M}+\mathrm{H}]^{+}:$286.0748, Found: 286.0741.

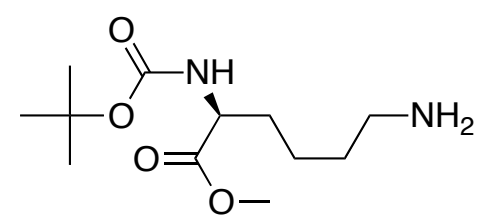

3.5

$\mathbf{N}^{a}$-(1,1-dimethylethoxycarbonyl)-L-lysine-methyl ester (3.5). ${ }^{1} \mathrm{H} \mathrm{NMR}\left(600 \mathrm{MHz}, \mathrm{CDCl}_{3}\right): \delta$ $5.18(\mathrm{br}, 1 \mathrm{H}), 4.23(\mathrm{br}, 1 \mathrm{H}), 3.68(\mathrm{~s}, 3 \mathrm{H}), 1.81-1.70(\mathrm{~m}, 1 \mathrm{H}), 1.62-1.56(\mathrm{~m}, 1 \mathrm{H}), 1.49-1.40(\mathrm{~m}$, 1H), $1.38(\mathrm{~s}, 9 \mathrm{H}), 1.37-1.30(\mathrm{~m}, 3 \mathrm{H}) .{ }^{13} \mathrm{C} \mathrm{NMR}\left(150 \mathrm{MHz}, \mathrm{CDCl}_{3}\right): \delta 173.5,155.6,79.9,53.5$, 52.4, 41.7, 32.6, 28.5, 22.8. HRMS Calculated for $\mathrm{C}_{12} \mathrm{H}_{25} \mathrm{~N}_{2} \mathrm{O}_{4}[\mathrm{M}+\mathrm{H}]^{+}:$261.1736, Found: 261.1773. 
<smiles>COC(=O)C(CCCCNC(=O)Cc1ccc(I)cc1)NC(=O)OC(C)(C)C</smiles>

3.7

Methyl 2-(tert-butoxycarbonylamino)-6-(2-(4-iodophenyl)acetamido)hexanoate (3.7). ${ }^{1} \mathrm{H}$ NMR (600 MHz, $\left.\mathrm{CDCl}_{3}\right): \delta 7.58(\mathrm{~d}, J=8.4 \mathrm{~Hz}, 2 \mathrm{H}), 6.95(\mathrm{~d}, J=8.4 \mathrm{~Hz}, 2 \mathrm{H}), 5.98(\mathrm{~s}, 1 \mathrm{H}), 5.16$ (br, 1H), $4.18(\mathrm{br}, 1 \mathrm{H}), 3.66(\mathrm{~s}, 3 \mathrm{H}), 3.39$ (s, 2H), 3.15-3.10 (m, 2H), 1.72-1.69 (m, 1H), 1.59$1.54(\mathrm{~m}, 1 \mathrm{H}), 1.43-1.40(\mathrm{~m}, 2 \mathrm{H}), 1.38(\mathrm{~s}, 9 \mathrm{H}), 1.30-1.23(\mathrm{~m}, 2 \mathrm{H}) .{ }^{13} \mathrm{C}$ NMR $\left(150 \mathrm{MHz}, \mathrm{CDCl}_{3}\right)$ : $\delta 173.0,170.2,155.4,137.6,134.7,131.1,92.4,79.7,53.0,52.1,42.8,39.1,32.0,28.7,28.2$, 22.4. HRMS Calculated for $\mathrm{C}_{20} \mathrm{H}_{30} \mathrm{IN}_{2} \mathrm{O}_{5}[\mathrm{M}+\mathrm{H}]^{+}: 505.1121$, Found: 505.1153 .

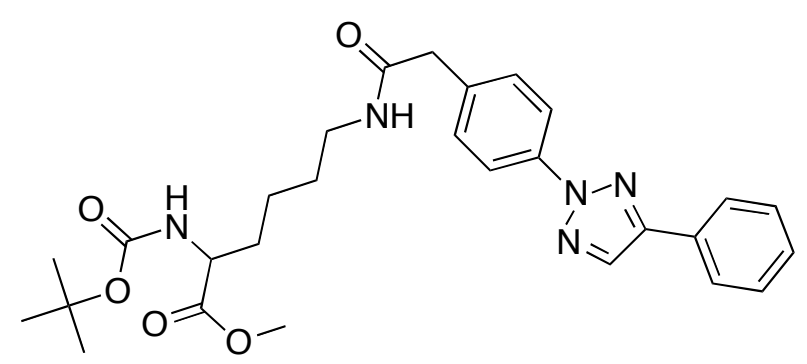

$3.4 b$

Methyl 2-(tert-butoxycarbonylamino)-6-(2-(4-(4-phenyl-2H-1,2,3-triazol-2yl)phenyl)acetamido)hexanoate (3.4b) was prepared from 4-phenyl-2H-1,2,3-triazole and iodosubstituted benzene by using the previously reported procedure of arylation. ${ }^{1} \mathrm{H}$ NMR $(600 \mathrm{MHz}$, 
$\left.\mathrm{CDCl}_{3}\right): \delta 8.13(\mathrm{~d}, J=8.4 \mathrm{~Hz}, 2 \mathrm{H}), 8.05(\mathrm{~s}, 1 \mathrm{H}), 7.90(\mathrm{~d}, J=8.4 \mathrm{~Hz}, 2 \mathrm{H}), 7.49-7.45(\mathrm{~m}, 2 \mathrm{H})$, 7.41-7.38 (m, 3H), $5.55(\mathrm{br}, 1 \mathrm{H}), 5.06(\mathrm{br}, 1 \mathrm{H}), 4.26(\mathrm{br}, 1 \mathrm{H}), 3.72(\mathrm{~s}, 3 \mathrm{H}), 3.62(\mathrm{~s}, 2 \mathrm{H}), 3.25-$ $3.20(\mathrm{~m}, 2 \mathrm{H}), 1.84-1.72(\mathrm{~m}, 2 \mathrm{H}), 1.65-1.58(\mathrm{~m}, 1 \mathrm{H}), 1.51-1.45(\mathrm{~m}, 2 \mathrm{H}), 1.43(\mathrm{~s}, 9 \mathrm{H}), 1.36-1.28$ (m, 3H). ${ }^{13} \mathrm{C}$ NMR (150 MHz, $\left.\mathrm{CDCl}_{3}\right): \delta 187.1,173.1,170.5,149.0,139.1,134.2,132.7,130.3$, 129.9, 129.0, 128.9, 126.1, 119.3, 79.9, 53.1, 52.3, 43.3, 39.3, 32.4, 28.9, 28.3, 22.5. HRMS Calculated for $\mathrm{C}_{28} \mathrm{H}_{36} \mathrm{~N}_{5} \mathrm{O}_{5}[\mathrm{M}+\mathrm{H}]^{+}:$522.2638, Found: 522.2680. 


\section{ORTEP Drawing of the Crystal Structure}

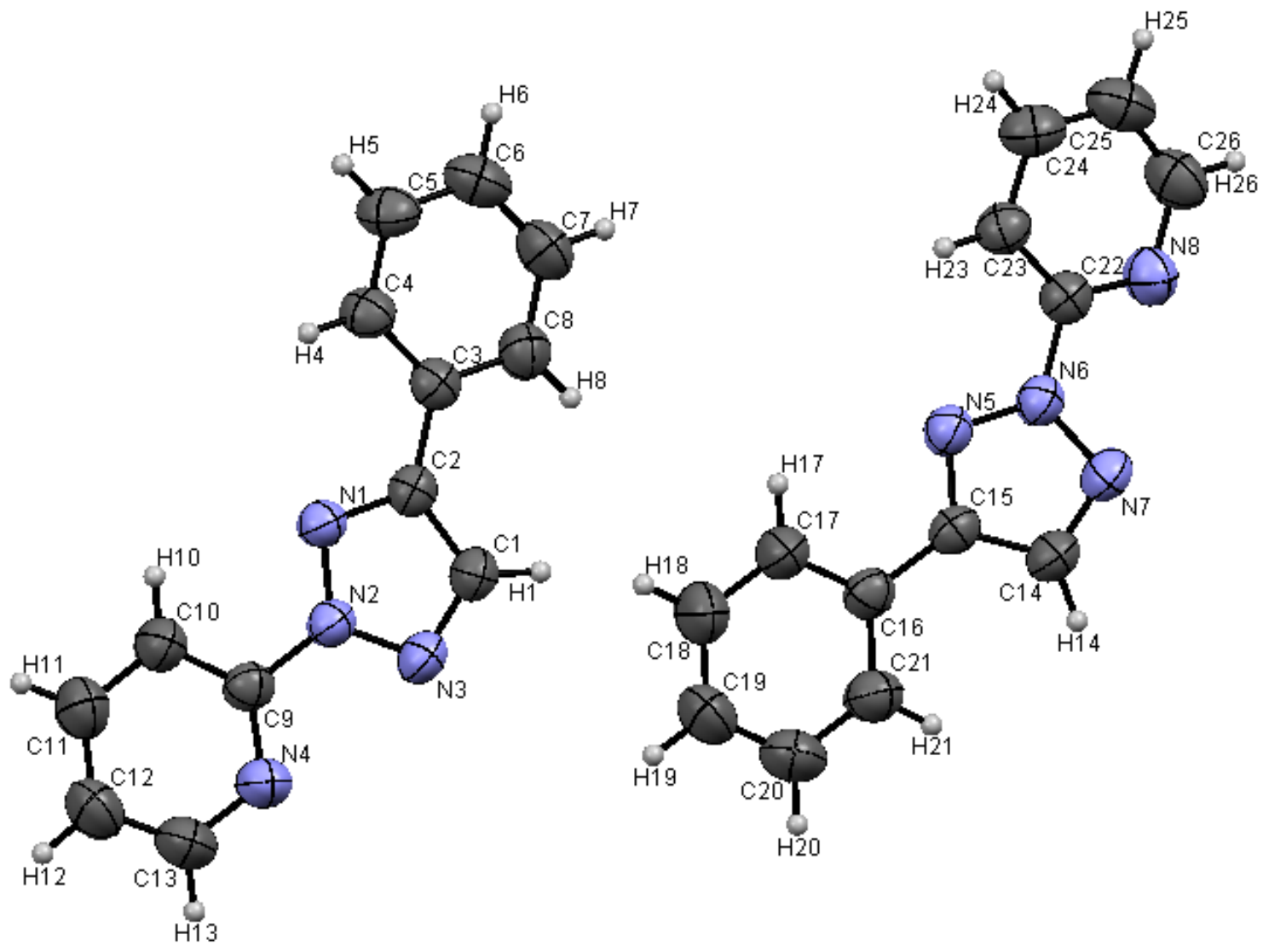

Figure S3-1. Perspective view of the molecular structure of 3.2h $\left(\mathrm{C}_{13} \mathrm{H}_{10} \mathrm{~N}_{4}\right)$ with the atom labeling scheme. The thermal ellipsoids are scaled to enclose $30 \%$ probability. CCDC number: 775283.

\section{Reported crystal structures}

\section{Compound \#}

3.1b

3.1c

$3.2 \mathrm{~g}$

3.4a
CCDC

702800

702801

702802

702803 


\section{The Data of Fluorophores with Emission Spectra}

Sample preparation: To a $20 \mathrm{ml}$ white cap vial was added the $\mathrm{N}$-2-aryl-1,2,3-triazoles $(0.01$ $\mathrm{mmol}$ ) in $10 \mathrm{ml} \mathrm{MeOH}$. The solution was shook several times. After triazoles was absolutely dissolved, the $100 \mu \mathrm{l}$ triazole solution has been transferred to another new $20 \mathrm{ml}$ white cap vial by using Socorex wheaton single-channel pipetter $(50-200 \mu \mathrm{l})$, and was then diluted with additional $9.9 \mathrm{ml} \mathrm{MeOH}$.

Integrated emission area: All the areas were integrated from the original spectra by FL Solution 2.0 software at the wavelength range which was $260 \mathrm{~nm}$ to $500 \mathrm{~nm}$ with an excitation wavelength at $254 \mathrm{~nm}$ or $320 \mathrm{~nm}$ to $500 \mathrm{~nm}$ with an excitation wavelength at $310 \mathrm{~nm}$.

Quantum yield determination: All the quantum yields of samples were determined based on $1.0 \times 10^{-5} \mathrm{~mol} / \mathrm{L}$ Quinine sulfate dihydrate in $\mathrm{H}_{2} \mathrm{O}(\Phi=0.564)$. 
Figure S3-2. Photoemission of N-2-aryl-1,2,3-triazole: sample preparation: $1.0 \times 10^{-5} \mathrm{~mol} / \mathrm{L}$ in $\mathrm{MeOH}$, excitation at $254 \mathrm{~nm}$ with $5 \mathrm{~nm}$ slit, quantum yield of 3.1a $\Phi=0.154$, quantum yield of 3.19' $\Phi=0.009$

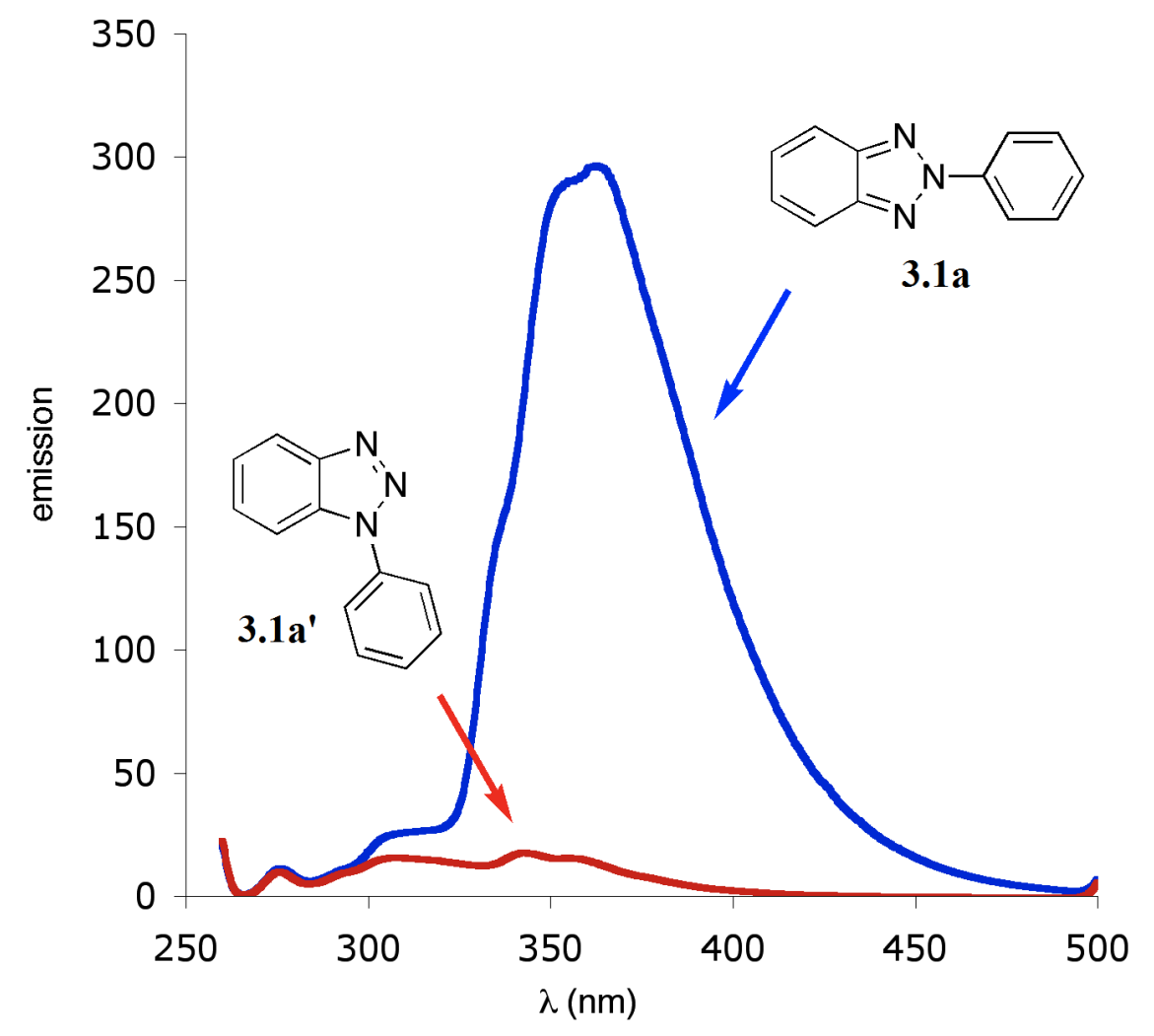

\begin{tabular}{ccccccc}
\hline Absorption(nm) & $\begin{array}{c}\text { Excitation } \\
\left(\boldsymbol{\lambda}_{\text {max }}\right)\end{array}$ & $\begin{array}{c}\text { Emission } \\
\left(\boldsymbol{\lambda}_{\text {max }}\right)\end{array}$ & $\begin{array}{c}\text { Stoke } \\
\text { Shift(nm) }\end{array}$ & $\boldsymbol{\Phi}$ & $\begin{array}{c}\text { Fluorescence } \\
\text { Intensity }\end{array}$ \\
\hline 3.1a & $222(0.127), 305(0.222)$ & 305 & 362 & 57 & 0.15 & 19576.0 \\
$3.1 a^{\prime}$ & $222(0.108), 292(0.116)$ & n.d. & 343 & n.d. & 0.01 & 1444.8 \\
\hline
\end{tabular}


Figure S3-3. Photoemission of N-2-aryl-1,2,3-triazole: sample information: $1.0 \times 10^{-5} \mathrm{~mol} / \mathrm{L}$ in $\mathrm{MeOH}$, excitation at $310 \mathrm{~nm}$ with $2.5 \mathrm{~nm}$ slit.<smiles>c1ccc(-n2nc3ccccc3n2)cc1</smiles>

3.1a<smiles>C=C(c1ccccc1)c1nn(-c2ccccc2)nc1-c1ccccc1</smiles>

3.1d<smiles>O=C(c1ccccc1)c1nn(-c2ccccc2)nc1-c1ccccc1</smiles>

$3.1 b$<smiles>Oc1ccc2ccccc2c1-c1nn(-c2ccccc2)nc1C1=CCCCC1</smiles>

3.1c<smiles>[R]c1nn(-c2ccccc2)nc1-c1ccccc1</smiles>

3.1e: $\mathrm{R}=\mathrm{H}$ 3.1f: $R=B n$
$3.1 \mathrm{~g}$<smiles>CC(c1ccccc1)c1nn(-c2ccccc2)nc1-c1ccccc1</smiles>

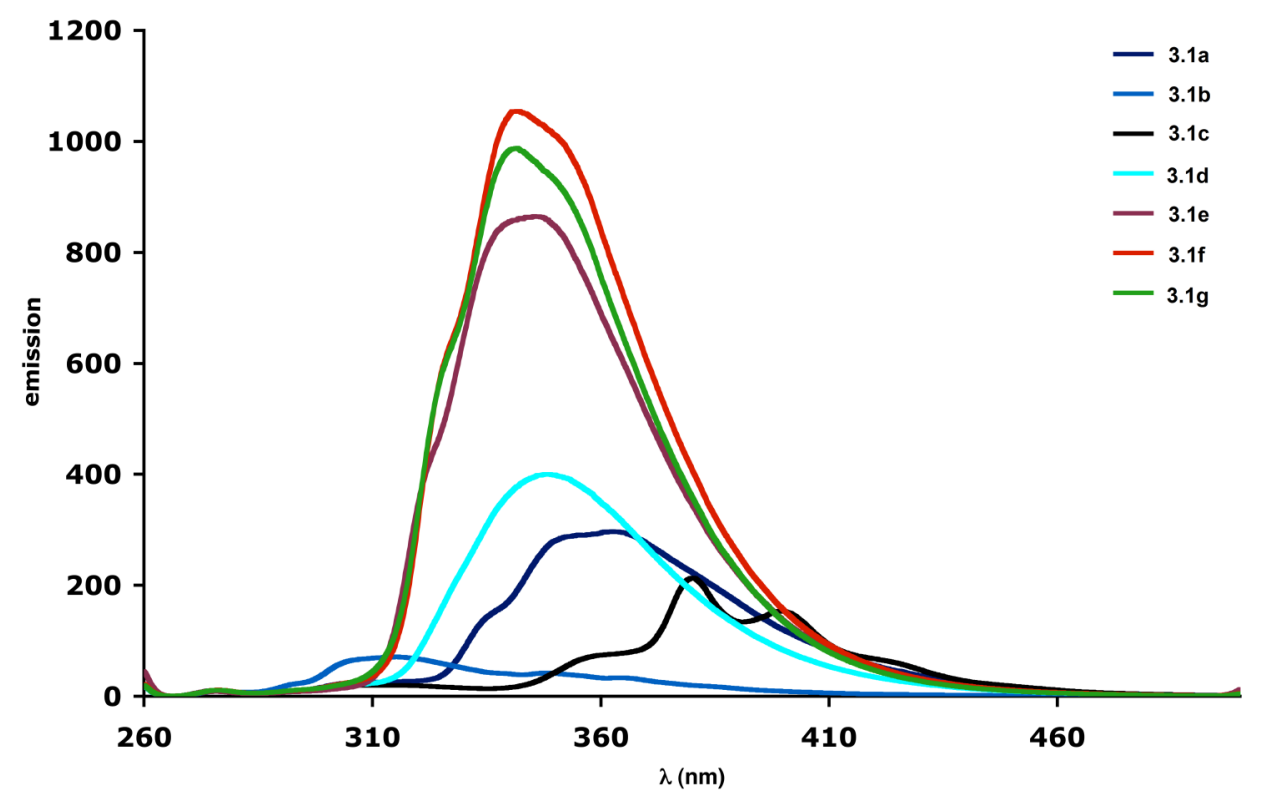




\begin{tabular}{|c|c|c|c|c|c|c|}
\hline & Absorption(nm) & $\begin{array}{c}\text { Excitation } \\
\left(\lambda_{\max }\right)\end{array}$ & $\begin{array}{c}\text { Emission } \\
\left(\lambda_{\max }\right)\end{array}$ & $\begin{array}{c}\text { Stoke } \\
\text { Shift(nm) } \\
\end{array}$ & $\Phi$ & $\begin{array}{c}\text { Fluorescence } \\
\text { Intensity } \\
\end{array}$ \\
\hline $3.1 \mathrm{a}$ & $222(0.127), 305(0.222)$ & 305 & 362 & 57 & 0.15 & 19576.0 \\
\hline $3.1 b$ & $206(0.128), 263(0.250)$ & n.d. & 316 & n.d. & 0.02 & 4662.9 \\
\hline $3.1 c$ & $227(0.257), 292(0.268)$ & n.d. & 380,400 & n.d. & 0.06 & 10822.5 \\
\hline $3.1 d$ & $220(0.146), 296(0.276)$ & n.d. & 348 & n.d. & 0.09 & 23036.8 \\
\hline $3.1 \mathrm{e}$ & $220(0.118), 294(0.263)$ & 298 & 347 & 49 & 0.35 & 48541.7 \\
\hline $3.1 f$ & $220(0.111), 290(0.253)$ & 284 & 342 & 58 & 0.45 & 56612.5 \\
\hline $3.1 \mathrm{~g}$ & $221(0.128), 285(0.265)$ & 285 & 342 & 57 & 0.30 & 52417.0 \\
\hline$\underset{1}{\text { STD- }}$ & $\begin{array}{c}207(0.452), 234(0.228), \\
349(0.710)\end{array}$ & 361 & 402,422 & 41,61 & 0.23 & 63090.6 \\
\hline $\begin{array}{l}\text { STD- } \\
\quad 2\end{array}$ & $234(0.423), 334(0.068)$ & n.d. & 365 & n.d. & 0.56 & 42012.4 \\
\hline
\end{tabular}

* STD-1 = Disodium 4,4'-bis(2-sulfostyryl)biphenyl (DSBP). STD-2 = Quinine sulfate dihydrate. 
Figure S3-4. Photoemission of N-2-aryl-1,2,3-triazole: sample information: $1.0 \times 10^{-5} \mathrm{~mol} / \mathrm{L}$ in $\mathrm{MeOH}$, excitation at $310 \mathrm{~nm}$ with $2.5 \mathrm{~nm}$ slit.
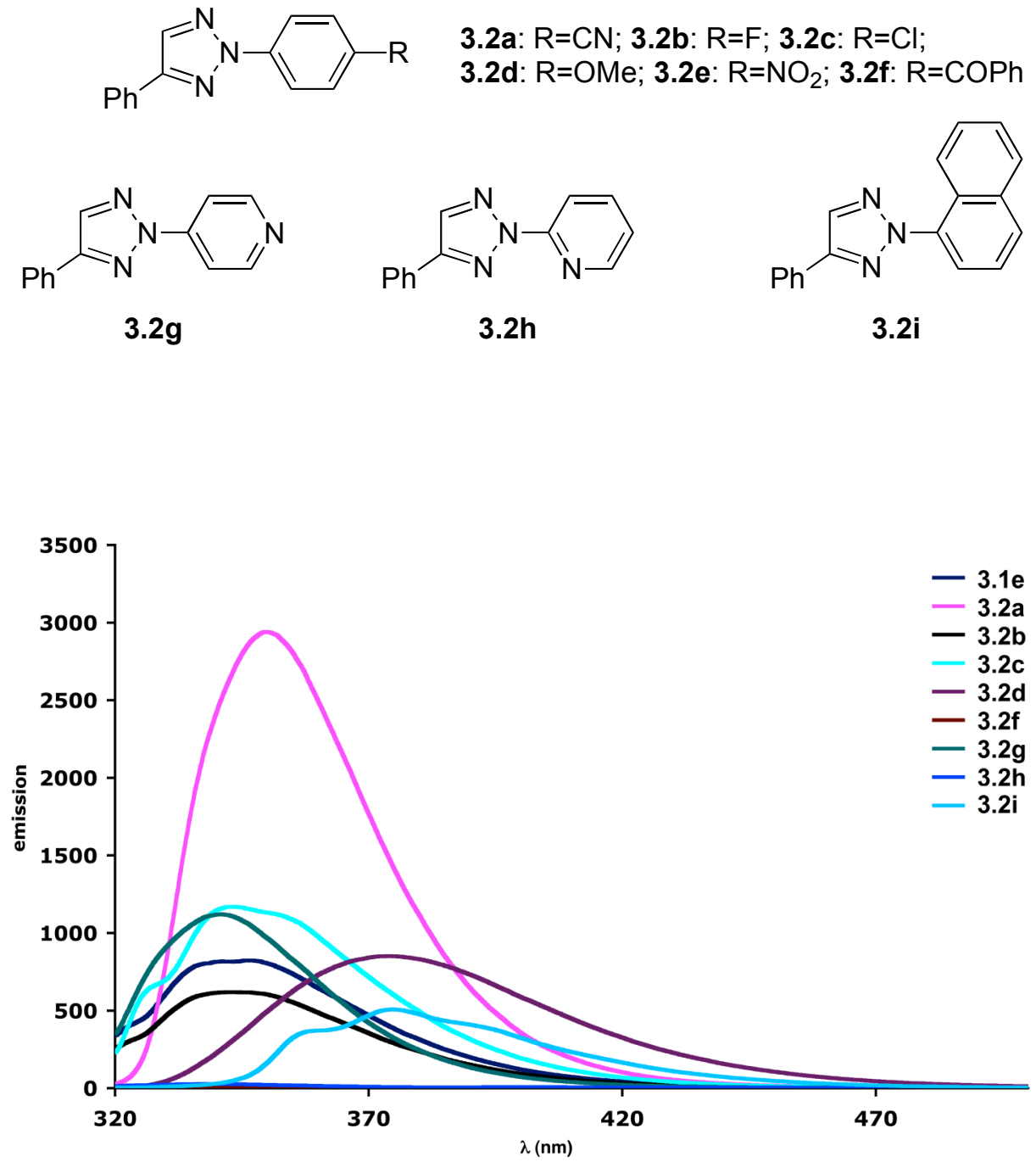


\begin{tabular}{ccccccc}
\hline & Absorption(nm) & $\begin{array}{c}\text { Excitation } \\
\left(\boldsymbol{\lambda}_{\text {max }}\right)\end{array}$ & $\begin{array}{c}\text { Emission } \\
\left(\boldsymbol{\lambda}_{\text {max }}\right)\end{array}$ & $\begin{array}{c}\text { Stoke } \\
\text { Shift(nm) }\end{array}$ & $\boldsymbol{\Phi}$ & $\begin{array}{c}\text { Fluorescence } \\
\text { Intensity }\end{array}$ \\
\hline 3.1e & $220(0.118), 294(0.263)$ & 298 & 347 & 49 & 0.35 & 46309.1 \\
3.2a & $224(0.154), 309(0.397)$ & 312 & 350 & 38 & 0.31 & 137679.5 \\
3.2b & $240(0.056), 294(0.212)$ & 298 & 343 & 45 & 0.34 & 34963.0 \\
3.2c & $298(0.274)$ & 292 & 344 & 52 & 0.35 & 72108.9 \\
3.2d & $245(0.041), 298(0.226)$ & 304 & 374 & 70 & 0.31 & 61629.7 \\
3.2e & $225(0.181), 336(0.205)$ & n.d. & 342 & n.d. & 0.003 & 443.5 \\
3.2f & $228(0.135), 250(0.14)$, & n.d. & 343 & n.d. & 0.001 & 269.2 \\
3.2g & $220.5(0.133), 300(0.242)$ & 302 & 341 & 39 & 0.28 & 52195.1 \\
3.2h & $238(0.156), 276(0.093)$ & 301 & 341 & 40 & 0.15 & 1684.2 \\
3.2i & $222(0.482), 260(0.115)$, & 289 & 375 & 86 & 0.27 & 29619.9 \\
\hline
\end{tabular}


Figure S3-5-1. Photoemission of N-2-aryl-1,2,3-triazole: sample information: $1.0 \times 10^{-5} \mathrm{~mol} / \mathrm{L}$ in $\mathrm{MeOH}$, excitation at $310 \mathrm{~nm}$ with $2.5 \mathrm{~nm}$ slit.

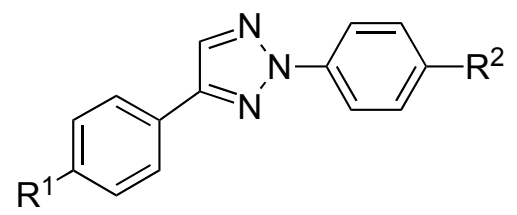

3.1e: $R^{1}=H, R^{2}=H$

3.2a: $R^{1}=H, R^{2}=C N$

3.2b: $R^{1}=H, R^{2}=F$

3.2c: $R^{1}=\mathrm{H}, \mathrm{R}^{2}=\mathrm{Cl}$

3.2d: $R^{1}=H, R^{2}=O M e$

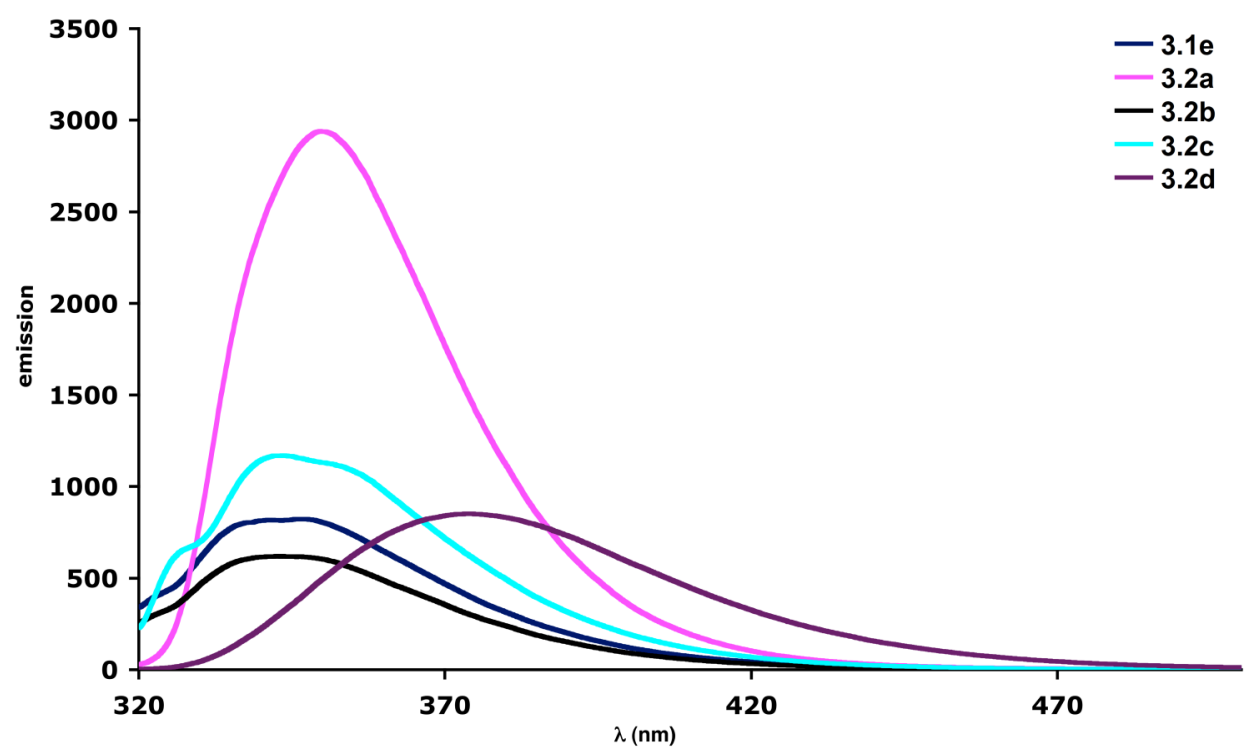

\begin{tabular}{ccccccc}
\hline Absorption(nm) & $\begin{array}{c}\text { Excitation } \\
\left(\boldsymbol{\lambda}_{\text {max }}\right)\end{array}$ & $\begin{array}{c}\text { Emission } \\
\left(\boldsymbol{\lambda}_{\text {max }}\right)\end{array}$ & $\begin{array}{c}\text { Stoke } \\
\text { Shift(nm) }\end{array}$ & $\boldsymbol{\Phi}$ & $\begin{array}{c}\text { Fluorescence } \\
\text { Intensity }\end{array}$ \\
\hline 3.1e & $220(0.118), 294(0.263)$ & 298 & 347 & 49 & 0.344 & 46309.1 \\
3.2a & $224(0.154), 309(0.397)$ & 312 & 350 & 38 & 0.311 & 137679.5 \\
3.2b & $240(0.056), 294(0.212)$ & 298 & 343 & 45 & 0.335 & 34963.0 \\
3.2c & $298(0.274)$ & 292 & 344 & 52 & 0.346 & 72108.9 \\
3.2d & $245(0.041), 298(0.226)$ & 304 & 374 & 70 & 0.311 & 61629.7 \\
\hline
\end{tabular}


Figure S3-5-2. Photoemission of N-2-aryl-1,2,3-triazole: sample information: $1.0 \times 10^{-5} \mathrm{~mol} / \mathrm{L}$ in $\mathrm{MeOH}$, excitation at $310 \mathrm{~nm}$ with $2.5 \mathrm{~nm}$ slit.

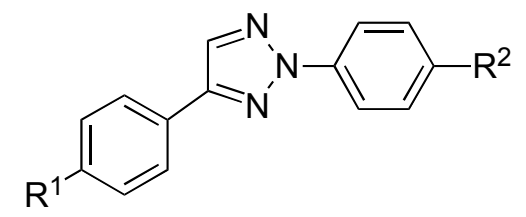

3.3a: $R^{1}=F, R^{2}=H$

3.3b: $R^{1}=F, R^{2}=C N$

3.3c: $R^{1}=F, R^{2}=F$

3.3d: $R^{1}=F, R^{2}=C l$

3.3e: $R^{1}=F, R^{2}=O M e$

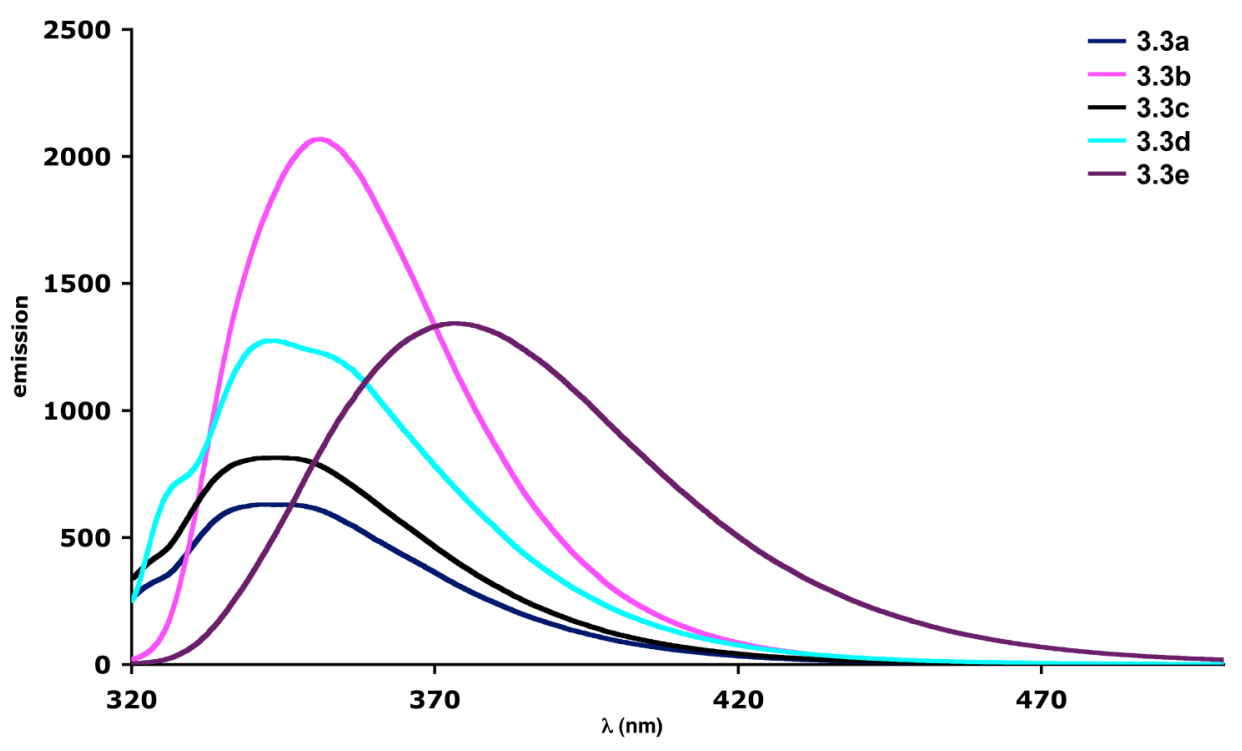

\begin{tabular}{ccccccc}
\hline Absorption(nm) & $\begin{array}{c}\text { Excitation } \\
\left(\boldsymbol{\lambda}_{\text {max }}\right)\end{array}$ & $\begin{array}{c}\text { Emission } \\
\left(\boldsymbol{\lambda}_{\text {max }}\right)\end{array}$ & $\begin{array}{c}\text { Stoke } \\
\text { Shift(nm) }\end{array}$ & $\boldsymbol{\Phi}$ & $\begin{array}{c}\text { Fluorescence } \\
\text { Intensity }\end{array}$ \\
\hline 3.3a & $279(0.147), 295(0.152)$ & 278 & 343 & 65 & 0.393 & 35187.2 \\
3.3b & $310(0.173)$ & 305 & 351 & 46 & 0.511 & 99110.2 \\
3.3c & $279(0.203), 295(0.202)$ & 277 & 343 & 66 & 0.398 & 45465.3 \\
3.3d & $281(0.328), 299(0.399)$ & 279 & 343 & 64 & 0.254 & 79606.4 \\
3.3e & $300(0.319)$ & 280 & 373 & 93 & 0.325 & 96251.2 \\
\hline
\end{tabular}


Figure S3-5-3. Photoemission of N-2-aryl-1,2,3-triazole: sample information: $1.0 \times 10^{-5} \mathrm{~mol} / \mathrm{L}$ in $\mathrm{MeOH}$, excitation at $310 \mathrm{~nm}$ with $2.5 \mathrm{~nm}$ slit.

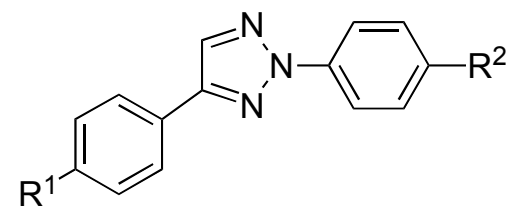

3.3f: $\mathrm{R}^{1}=\mathrm{OMe}, \mathrm{R}^{2}=\mathrm{H}$

3.3g: $R^{1}=\mathrm{OMe}, \mathrm{R}^{2}=\mathrm{CN}$

3.3h: $R^{1}=O M e, R^{2}=F$

3.3i: $\mathrm{R}^{1}=\mathrm{OMe}, \mathrm{R}^{2}=\mathrm{Cl}$

3.3j: $R^{1}=\mathrm{OMe}, \mathrm{R}^{2}=\mathrm{OMe}$

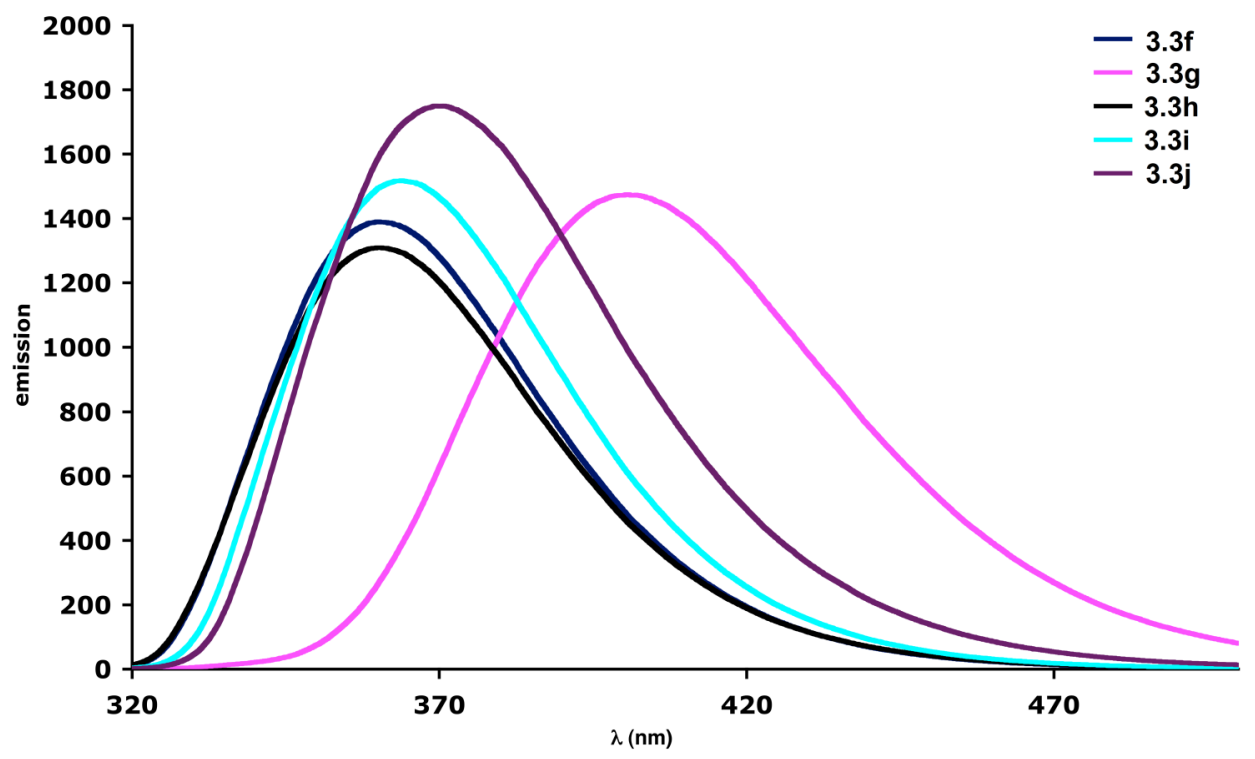

\begin{tabular}{|c|c|c|c|c|c|c|}
\hline & Absorption(nm) & $\begin{array}{c}\text { Excitation } \\
\left(\lambda_{\max }\right)\end{array}$ & $\begin{array}{c}\text { Emission } \\
\left(\lambda_{\max }\right)\end{array}$ & $\begin{array}{c}\text { Stoke } \\
\text { Shift(nm) }\end{array}$ & $\Phi$ & $\begin{array}{c}\text { Fluorescence } \\
\text { Intensity }\end{array}$ \\
\hline $3.3 f$ & $\begin{array}{c}245(0.067), 287(0.213) \\
305(0.198)\end{array}$ & 284 & 360 & 76 & 0.388 & 82234.7 \\
\hline $3.3 \mathrm{~g}$ & $250(0.162), 322(0.388)$ & 318 & 401 & 83 & 0.317 & 111193.8 \\
\hline $3.3 \mathrm{~h}$ & $\begin{array}{c}245(0.086), 287(0.241), \\
305(0.221)\end{array}$ & 283 & 360 & 77 & 0.337 & 78555.8 \\
\hline $3.3 \mathrm{i}$ & $\begin{array}{c}245(0.096), 289(0.208), \\
309(0.236)\end{array}$ & 287 & 364 & 77 & 0.341 & 89760.8 \\
\hline $\mathbf{3 . 3} \mathbf{j}$ & $\begin{array}{c}255(0.105), 289(0.292), \\
308(0.299)\end{array}$ & 284 & 369 & 85 & 0.344 & 114055.5 \\
\hline
\end{tabular}


Figure S3-5-4. Photoemission of N-2-aryl-1,2,3-triazole: sample information: $1.0 \times 10^{-5} \mathrm{~mol} / \mathrm{L}$ in $\mathrm{MeOH}$, excitation at $310 \mathrm{~nm}$ with $2.5 \mathrm{~nm}$ slit.

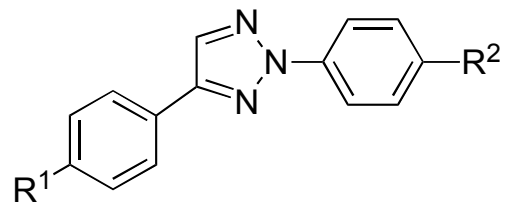

3.3k: $\mathrm{R}^{1}=\mathrm{Cl}, \mathrm{R}^{2}=\mathrm{H}$

3.3I: $\mathrm{R}^{1}=\mathrm{Cl}, \mathrm{R}^{2}=\mathrm{CN}$

3.3m: $R^{1}=\mathrm{Cl}, \mathrm{R}^{2}=\mathrm{F}$

3.3n: $\mathrm{R}^{1}=\mathrm{Cl}, \mathrm{R}^{2}=\mathrm{Cl}$

3.3o: $\mathrm{R}^{1}=\mathrm{Cl}, \mathrm{R}^{2}=\mathrm{OMe}$

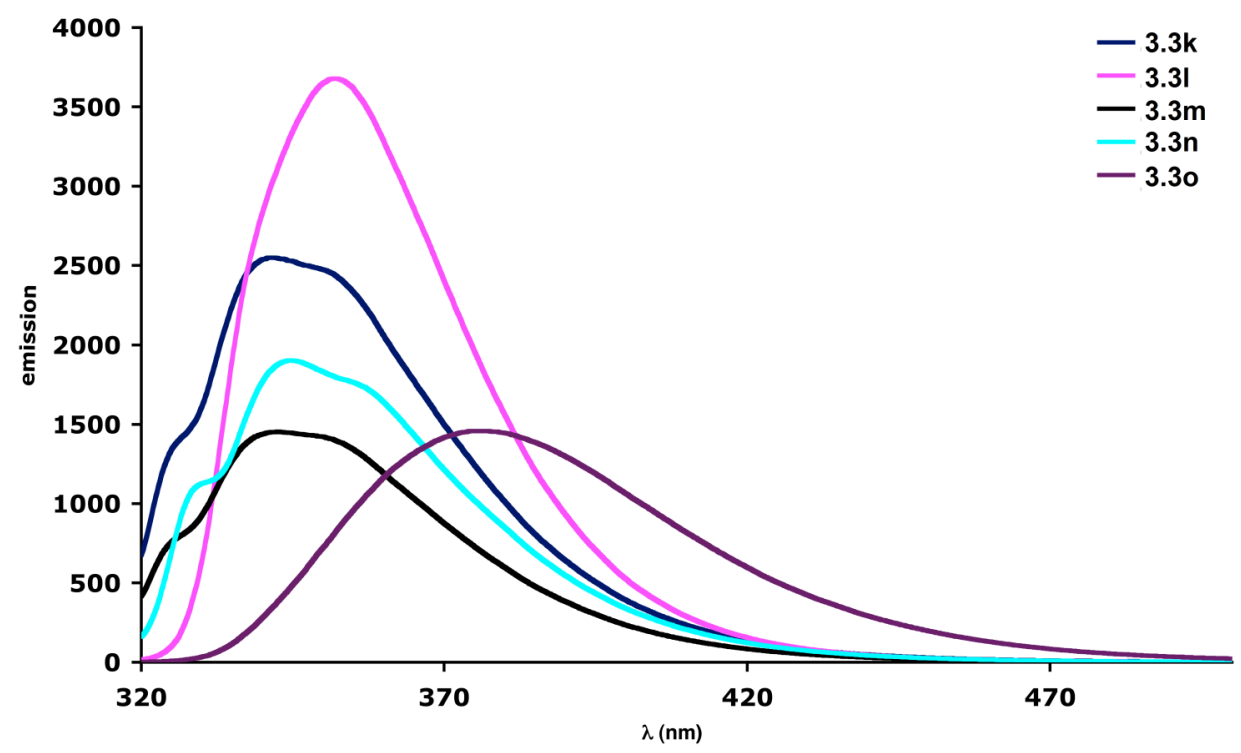

\begin{tabular}{|c|c|c|c|c|c|c|}
\hline & Absorption(nm) & $\begin{array}{c}\text { Excitation } \\
\left(\lambda_{\max }\right)\end{array}$ & $\begin{array}{c}\text { Emission } \\
\left(\lambda_{\max }\right)\end{array}$ & $\begin{array}{c}\text { Stoke } \\
\text { Shift(nm) }\end{array}$ & $\Phi$ & $\begin{array}{c}\text { Fluorescence } \\
\text { Intensity }\end{array}$ \\
\hline $3.3 k$ & $284(0.539), 298(0.532)$ & 280 & 341 & 61 & 0.345 & 133654.1 \\
\hline 3.31 & $\begin{array}{c}211(0.086), 245(0.075), \\
311(0.350)\end{array}$ & 306 & 352 & 46 & 0.446 & 173975.0 \\
\hline $3.3 \mathrm{~m}$ & $283(0.275)$ & 281 & 343 & 62 & 0.406 & 77275.4 \\
\hline $3.3 n$ & $245(0.059), 301(0.298)$ & 283 & 345 & 62 & 0.361 & 99041.4 \\
\hline 3.30 & $303(0.281)$ & 285 & 376 & 91 & 0.349 & 100999.4 \\
\hline
\end{tabular}


Figure S3-6. Comparison of photoluminescent spectra of 3.1e and 3.4b

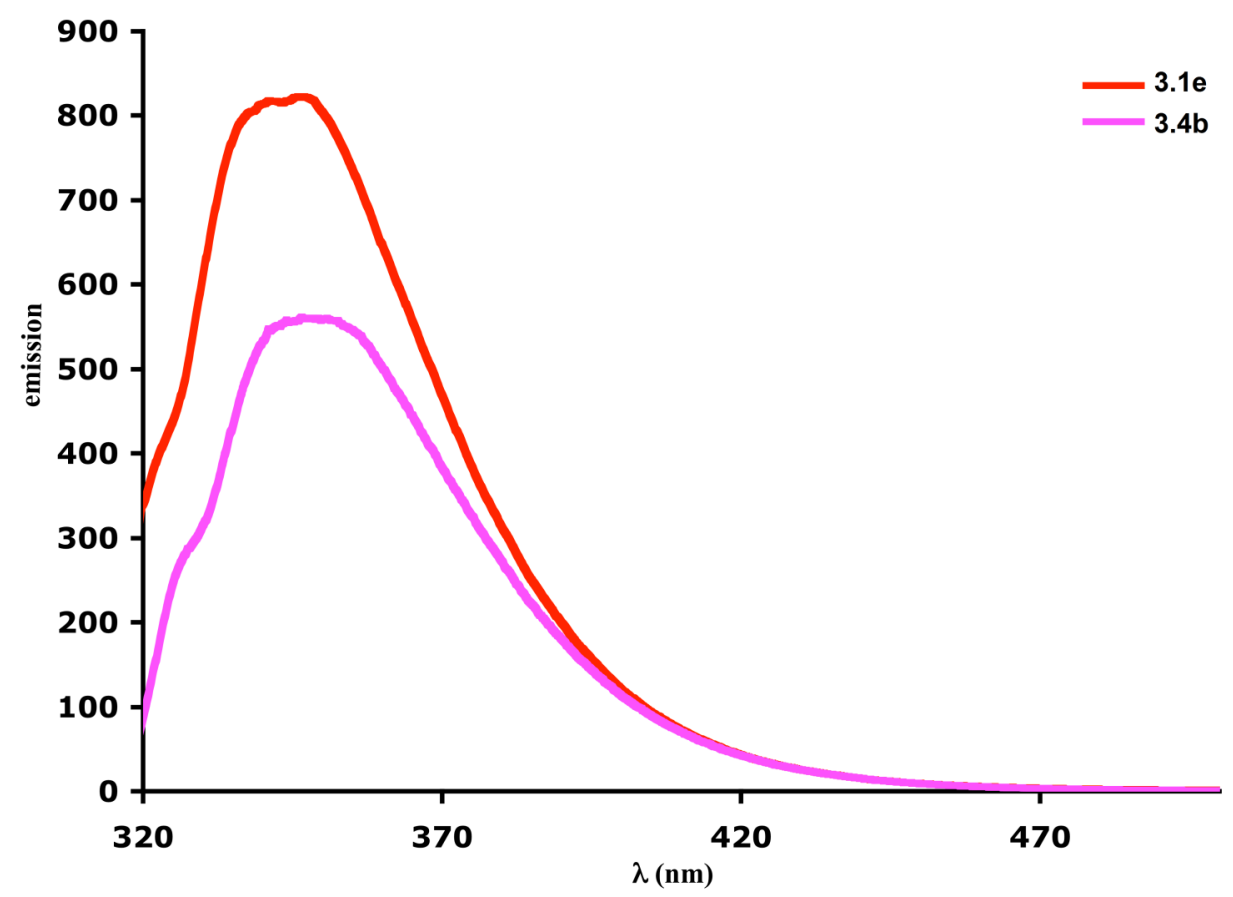

Sample preparation: $1.0 \times 10^{-5} \mathrm{~mol} / \mathrm{L}$ in $\mathrm{MeOH}$, excitation at $310 \mathrm{~nm}$, quantum yields of $\mathbf{1 e} \Phi$ $=0.344$, and $\mathbf{4 b} \Phi=0.346$. 


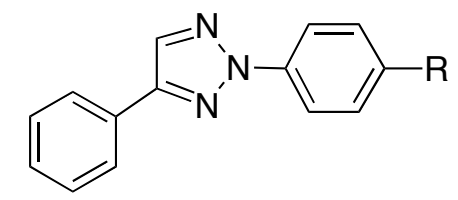

\begin{tabular}{ccccccccc}
\hline R & Solvent & $\begin{array}{c}\text { Excitation } \\
\left(\boldsymbol{\lambda}_{\text {max }}\right)\end{array}$ & $\begin{array}{c}\text { Emission } \\
\left(\boldsymbol{\lambda}_{\text {max }}\right)\end{array}$ & $\begin{array}{c}\text { Stoke } \\
\text { Shift }\end{array}$ & $\begin{array}{c}\text { A } 310 \\
\text { Fluorescence } \\
\text { Intensity }\end{array}$ & n & $\boldsymbol{\Phi}$ \\
\hline $\mathrm{CN}$ & $\mathrm{MeOH}$ & 312 & 350 & 38 & 0.395 & 137679.5 & 1.3314 & 0.311 \\
$\mathrm{H}$ & $\mathrm{MeOH}$ & 298 & 347 & 49 & 0.12 & 46309.1 & 1.3314 & 0.344 \\
$\mathrm{~F}$ & $\mathrm{MeOH}$ & 298 & 343 & 45 & 0.093 & 34963.0 & 1.3314 & 0.335 \\
$\mathrm{OMe}$ & $\mathrm{MeOH}$ & 304 & 374 & 70 & 0.177 & 61629.7 & 1.3314 & 0.311 \\
\hline $\mathrm{CN}$ & $\mathrm{DCM}$ & 313 & 351 & 38 & 0.276 & 101203.8 & 1.4242 & 0.374 \\
$\mathrm{H}$ & $\mathrm{DCM}$ & 298 & 345 & 47 & 0.118 & 41036.9 & 1.4242 & 0.355 \\
$\mathrm{~F}$ & $\mathrm{DCM}$ & 298 & 345 & 47 & 0.089 & 29776.1 & 1.4242 & 0.342 \\
$\mathrm{OMe}$ & $\mathrm{DCM}$ & 305 & 367 & 62 & 0.182 & 53109.8 & 1.4242 & 0.298 \\
\hline $\mathrm{CN}$ & $\mathrm{MeCN}$ & 311 & 350 & 39 & 0.281 & 103597.2 & 1.3460 & 0.336 \\
$\mathrm{H}$ & $\mathrm{MeCN}$ & 297 & 346 & 49 & 0.112 & 40844.0 & 1.3460 & 0.333 \\
$\mathrm{~F}$ & $\mathrm{MeCN}$ & 296 & 346 & 50 & 0.076 & 28906.5 & 1.3460 & 0.347 \\
$\mathrm{OMe}$ & $\mathrm{MeCN}$ & 304 & 369 & 65 & 0.158 & 51515.4 & 1.3460 & 0.297 \\
\hline $\mathrm{CN}$ & $\mathrm{DCE}$ & 314 & 352 & 38 & 0.363 & 96899.2 & 1.4448 & 0.281 \\
$\mathrm{H}$ & $\mathrm{DCE}$ & 298 & 346 & 48 & 0.157 & 38441.3 & 1.4448 & 0.257 \\
$\mathrm{~F}$ & $\mathrm{DCE}$ & 299 & 346 & 47 & 0.124 & 27725.3 & 1.4448 & 0.235 \\
$\mathrm{OMe}$ & $\mathrm{DCE}$ & 308 & 368 & 60 & 0.211 & 51679.6 & 1.4448 & 0.257 \\
\hline $\mathrm{CN}$ & $\mathrm{EtOAc}$ & 312 & 348 & 36 & 0.306 & 118760.7 & 1.3720 & 0.368 \\
$\mathrm{H}$ & $\mathrm{EtOAc}$ & 297 & 344 & 47 & 0.097 & 43464.0 & 1.3720 & 0.425 \\
$\mathrm{~F}$ & $\mathrm{EtOAc}$ & 297 & 343 & 46 & 0.081 & 39862.7 & 1.3720 & 0.466 \\
$\mathrm{OMe}$ & $\mathrm{EtOAc}$ & 305 & 365 & 60 & 0.214 & 66321.1 & 1.3720 & 0.294 \\
\hline $\mathrm{CN}$ & $\mathrm{THF}$ & 313 & 349 & 36 & 0.27 & 93017.5 & 1.4070 & 0.343 \\
$\mathrm{H}$ & $\mathrm{THF}$ & 300 & 344 & 44 & 0.134 & 51646.0 & 1.4070 & 0.384 \\
$\mathrm{~F}$ & $\mathrm{THF}$ & 302 & 343 & 41 & 0.154 & 54748.2 & 1.4070 & 0.354 \\
$\mathrm{OMe}$ & $\mathrm{THF}$ & 306 & 364 & 58 & 0.219 & 69518.7 & 1.4070 & 0.316 \\
\hline & & & & & & & & \\
\hline
\end{tabular}


Figure S3-7-1. Photoluminescent spectra of $2 \mathrm{a}$ in different solvents

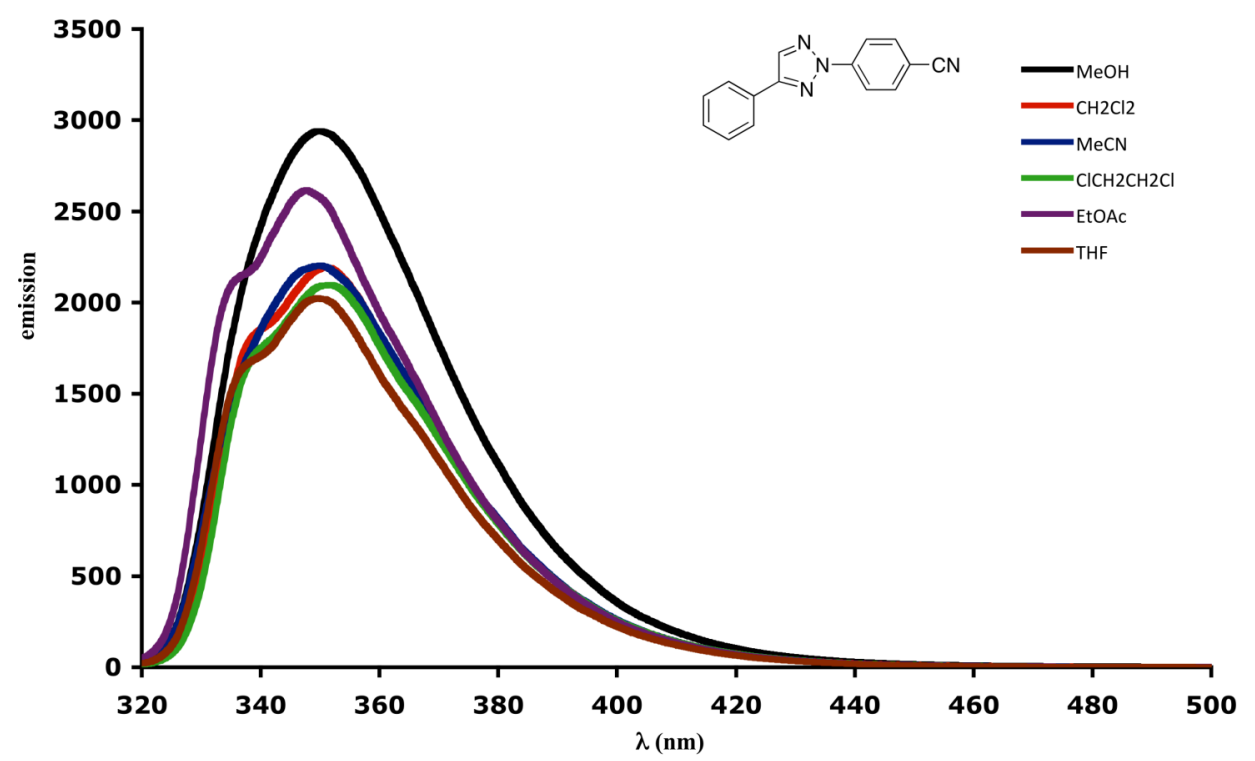

Figure S3-7-2. Photoluminescent spectra of 1e in different solvents

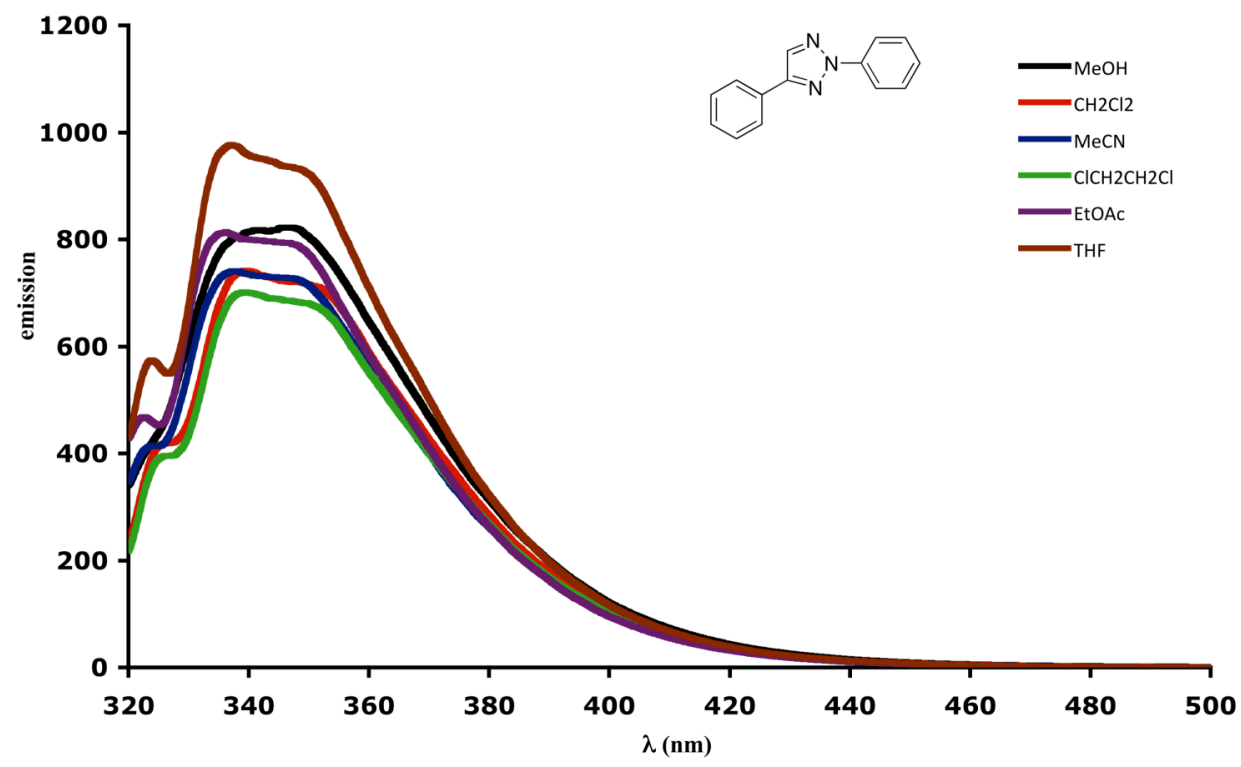


Figure S3-7-3. Photoluminescent spectra of $2 \mathrm{~b}$ in different solvents

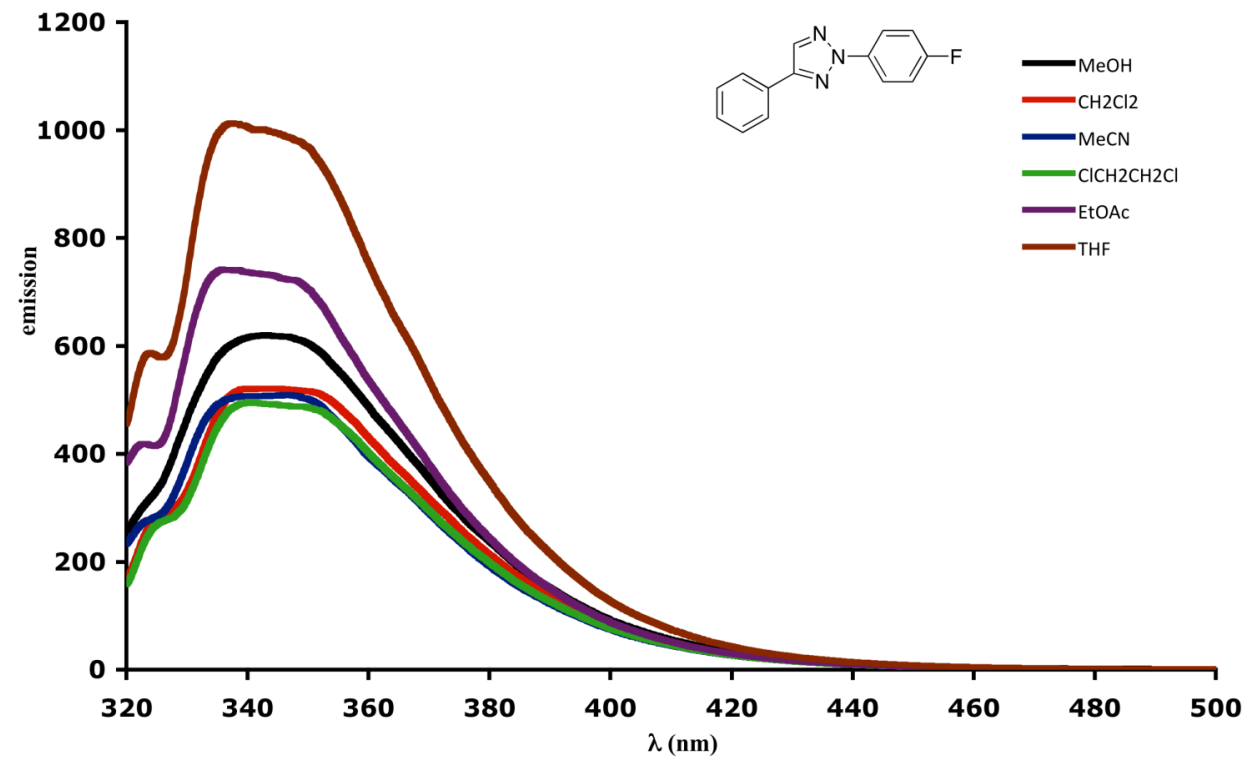

Figure S3-7-4. Photoluminescent spectra of $2 \mathrm{~d}$ in different solvents

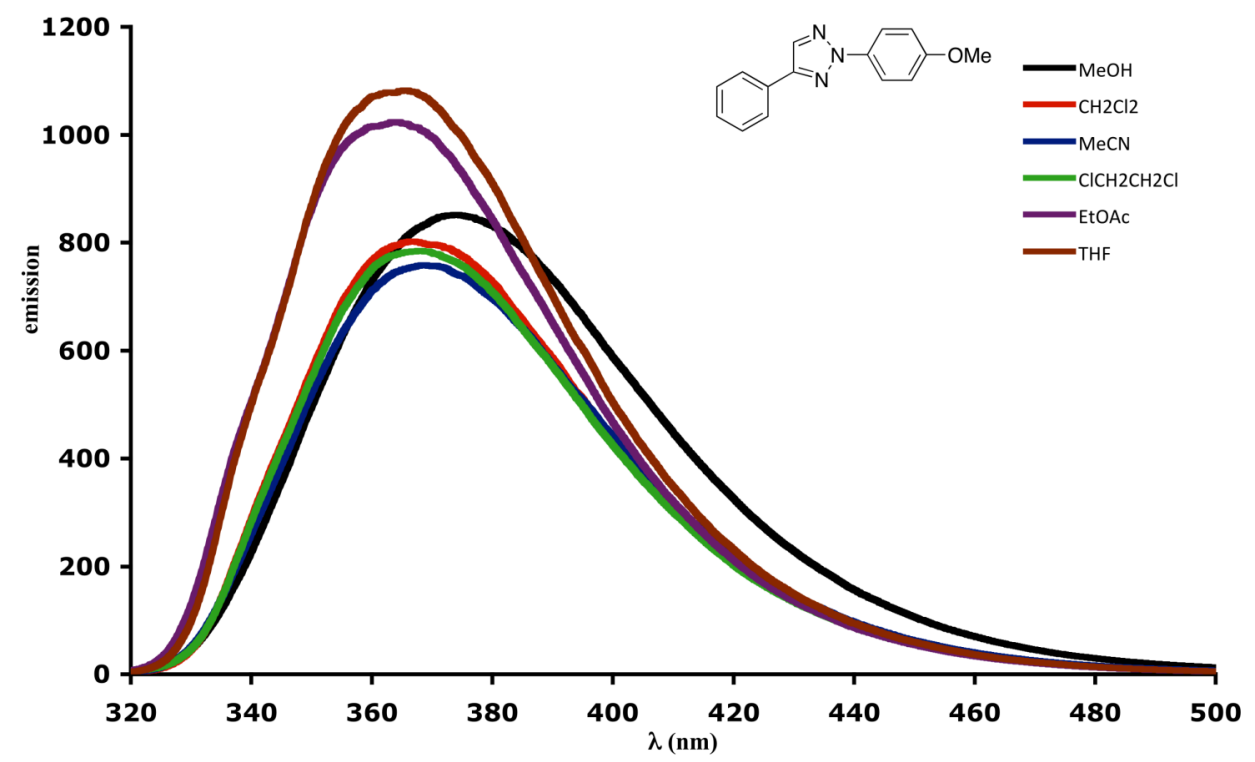


Figure S3-8. HOMO and LUMO orbitals of compounds 3.2d, 3.3g and N-1-3.1e'

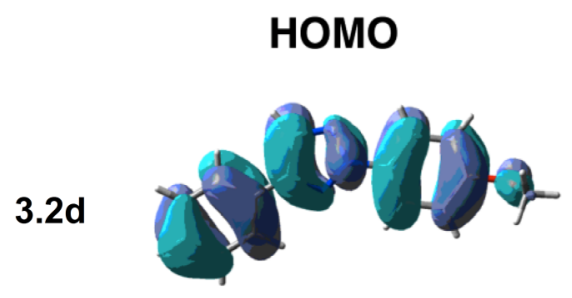

LUMO

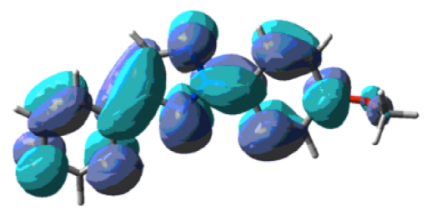

$3.2 \mathrm{~g}$

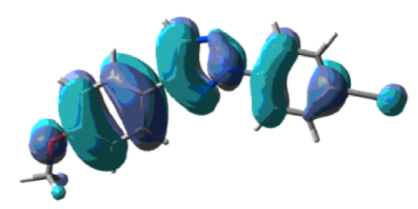

$\mathrm{N}-1-3.1 \mathrm{e}^{\prime}$

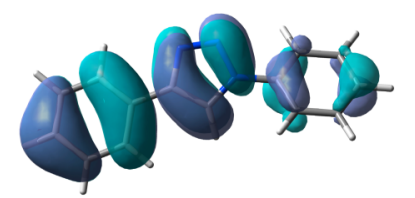

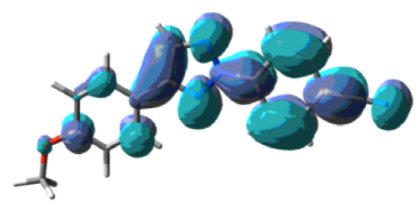

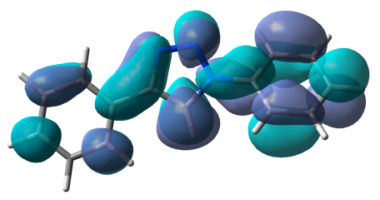


Calculated HOMO and LUMO energy gaps of 3.1e to 3.30

\begin{tabular}{cccccccc}
\hline & $\begin{array}{c}\text { excitation } \\
\left(\boldsymbol{\lambda}_{\text {max }}\right)\end{array}$ & $\begin{array}{c}\text { emission } \\
\left(\boldsymbol{\lambda}_{\text {max }}\right)\end{array}$ & $\boldsymbol{\Phi}$ & $\begin{array}{c}\text { Fluorescence } \\
\text { Intensity }\left(\mathbf{x ~ 1 0} \mathbf{~ 1 0}^{\mathbf{3}}\right)\end{array}$ & $\begin{array}{c}\text { Stoke shift } \\
(\mathbf{n m})\end{array}$ & $\mathbf{E g}(\mathbf{e V})$ & $\begin{array}{c}\text { LUMO- } \\
\text { HOMO (eV) }\end{array}$ \\
\hline $\mathbf{3 . 1 e}$ & 298 & 347 & 0.34 & 46.3 & 49 & 4.16 & 3.95 \\
$\mathbf{3 . 2 a}$ & 312 & 350 & 0.31 & 138 & 38 & 3.97 & 3.6 \\
$\mathbf{3 . 2 b}$ & 298 & 343 & 0.34 & 35 & 45 & 4.16 & 4.06 \\
$\mathbf{3 . 2 c}$ & 292 & 344 & 0.35 & 72.1 & 52 & 4.25 & 4.06 \\
$\mathbf{3 . 2 d}$ & 304 & 374 & 0.31 & 61.6 & 70 & 4.08 & 3.94 \\
\hline $\mathbf{3 . 3 a}$ & 278 & 343 & 0.39 & 35.2 & 65 & 4.46 & 4.29 \\
$\mathbf{3 . 3 b}$ & 305 & 351 & 0.51 & 99.1 & 46 & 4.07 & 3.94 \\
$\mathbf{3 . 3 c}$ & 277 & 343 & 0.4 & 45.5 & 66 & 4.48 & 4.78 \\
$\mathbf{3 . 3 d}$ & 279 & 343 & 0.25 & 79.6 & 64 & 4.44 & 4.77 \\
$\mathbf{3 . 3 e}$ & 280 & 373 & 0.33 & 96.3 & 93 & 4.43 & 4.21 \\
\hline $\mathbf{3 . 3 f}$ & 284 & 360 & 0.39 & 82.2 & 76 & 4.37 & 4.34 \\
$\mathbf{3 . 3 g}$ & 318 & 401 & 0.32 & 111 & 83 & 3.9 & 3.73 \\
$\mathbf{3 . 3 h}$ & 283 & 360 & 0.34 & 78.6 & 77 & 4.38 & 4.51 \\
$\mathbf{3 . 3 i}$ & 287 & 364 & 0.34 & 89.8 & 77 & 4.32 & 4.51 \\
$\mathbf{3 . 3 j}$ & 284 & 369 & 0.34 & 114 & 85 & 4.37 & 3.72 \\
\hline $\mathbf{3 . 3 k}$ & 280 & 341 & 0.35 & 134 & 61 & 4.43 & 4.27 \\
$\mathbf{3 . 3 1}$ & 306 & 352 & 0.45 & 174 & 46 & 4.05 & 3.9 \\
$\mathbf{3 . 3 m}$ & 281 & 343 & 0.41 & 77.3 & 62 & 4.41 & 4.04 \\
$\mathbf{3 . 3 n}$ & 283 & 345 & 0.36 & 99 & 62 & 4.38 & 4.03 \\
$\mathbf{3 . 3 0}$ & 285 & 376 & 0.35 & 101 & 91 & 4.35 & 4.16 \\
\hline
\end{tabular}


1.2. NMR Spectra Data

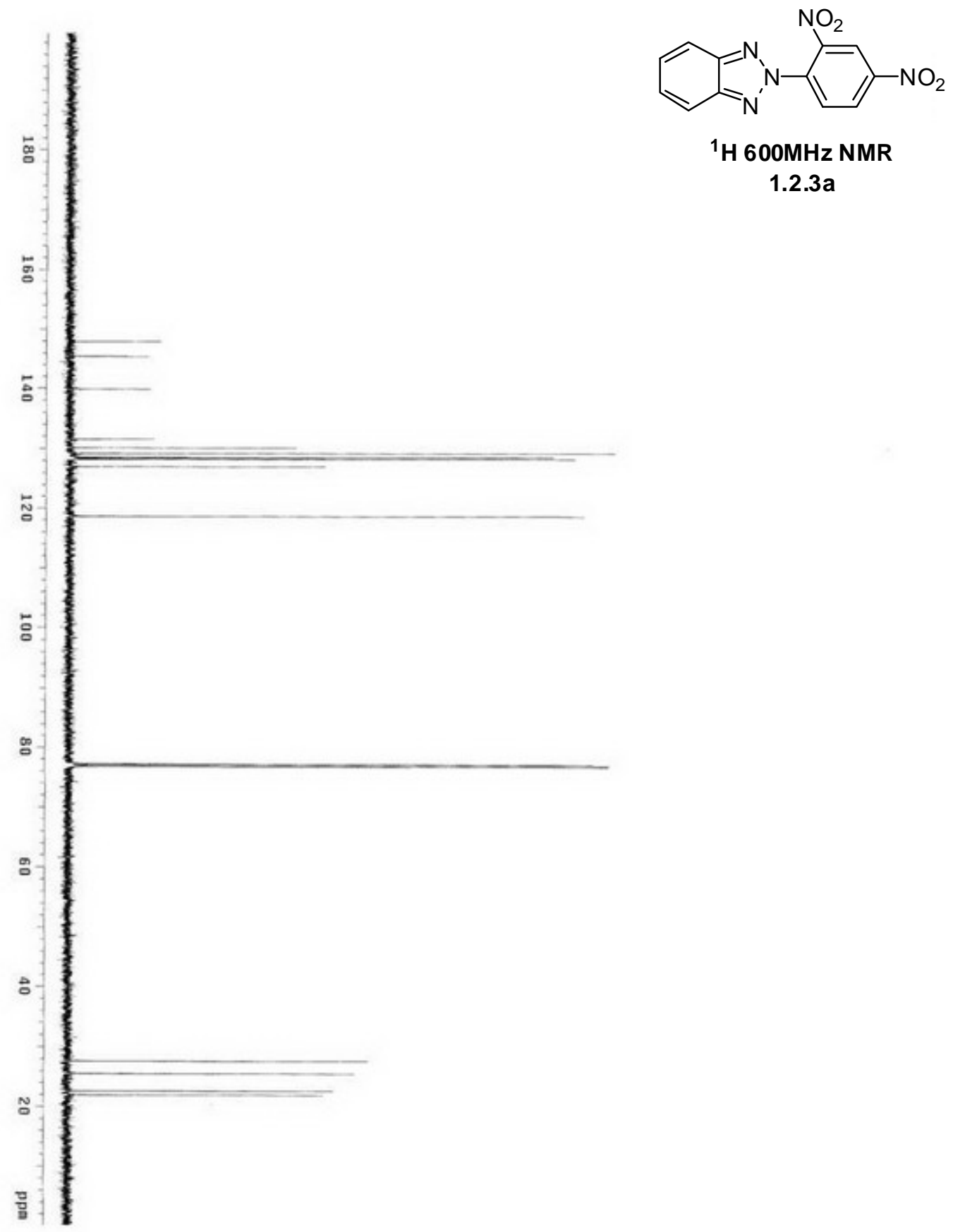




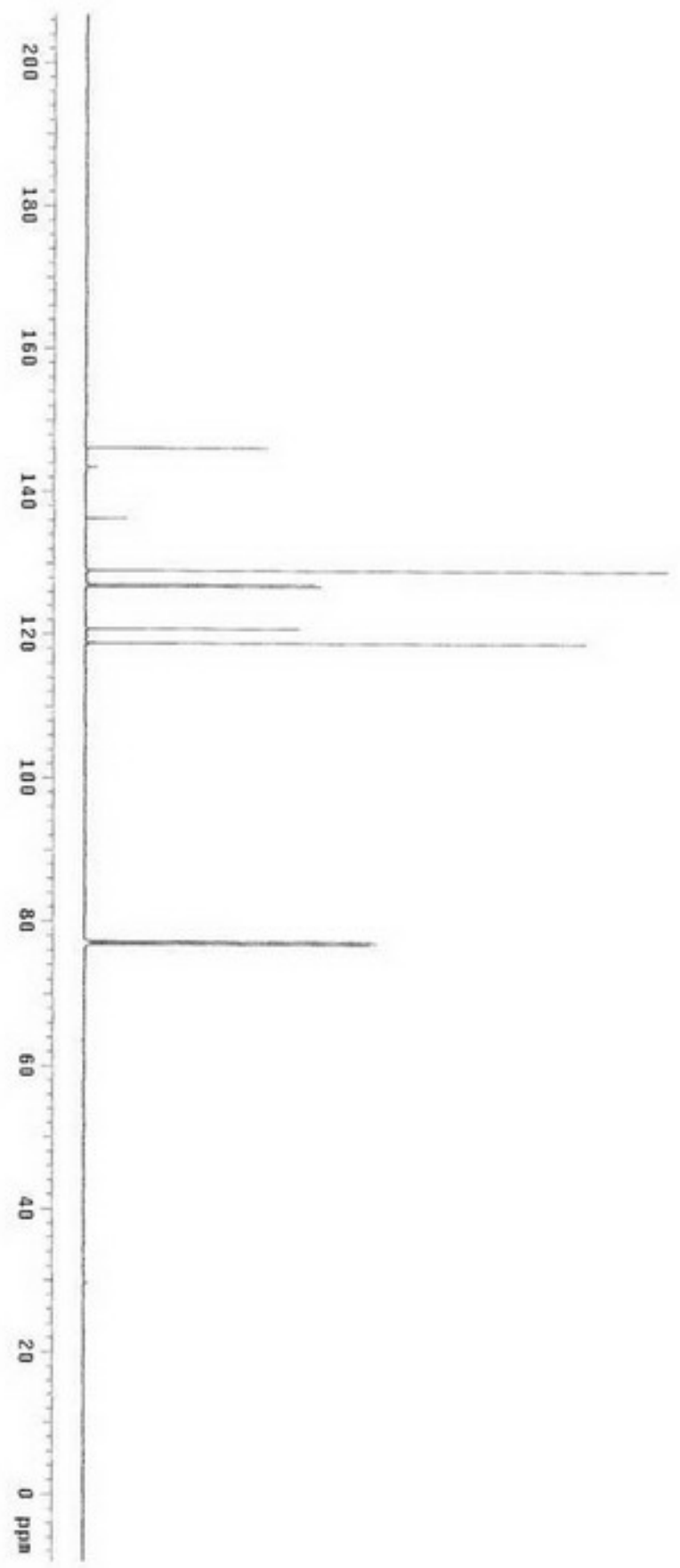

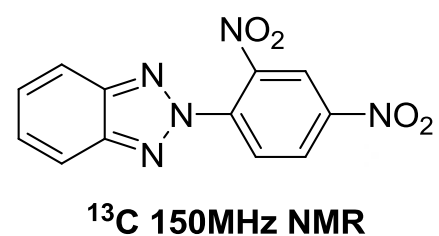

1.2.3a 

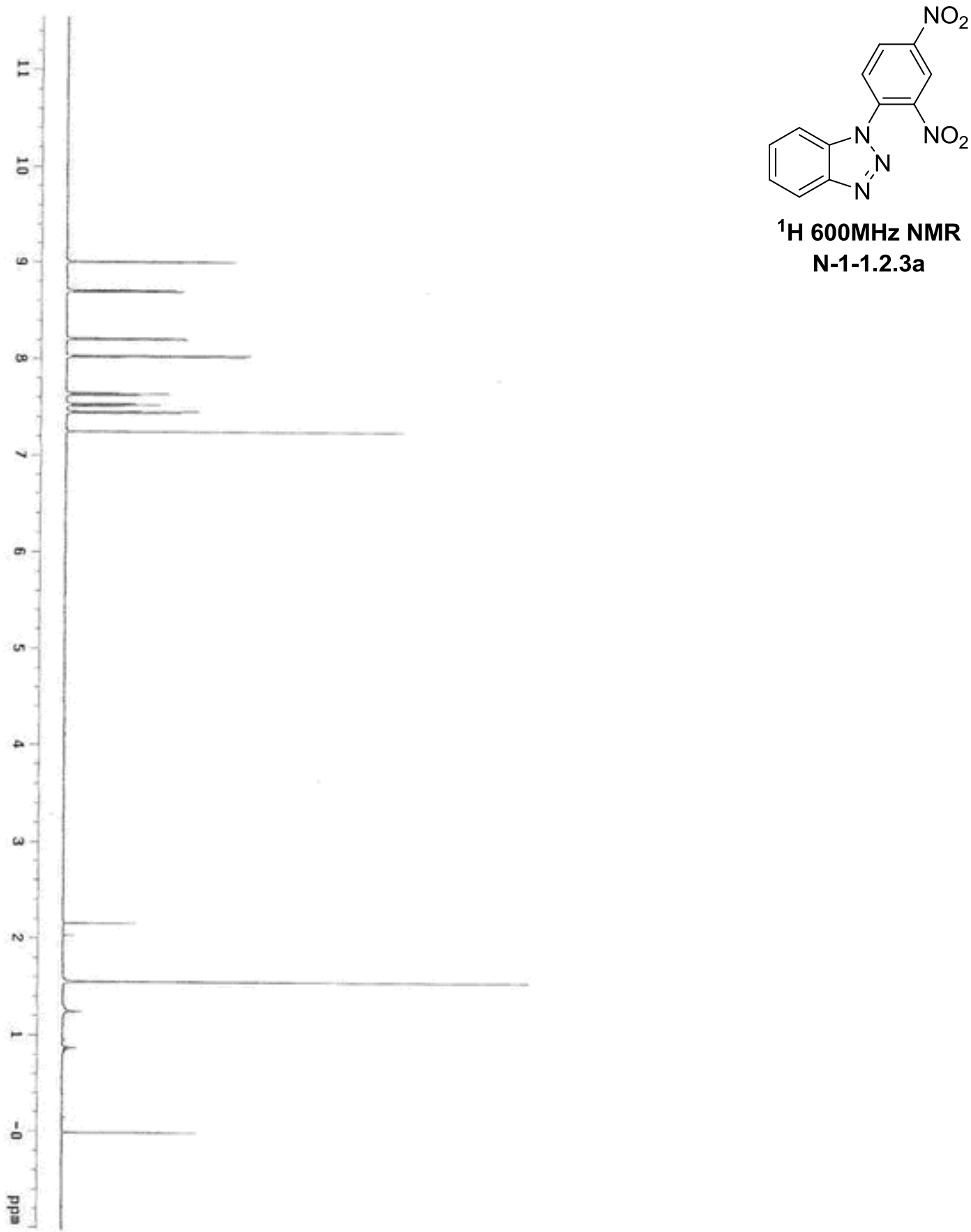

${ }^{1} \mathrm{H}$ 600MHz NMR N-1-1.2.3a 

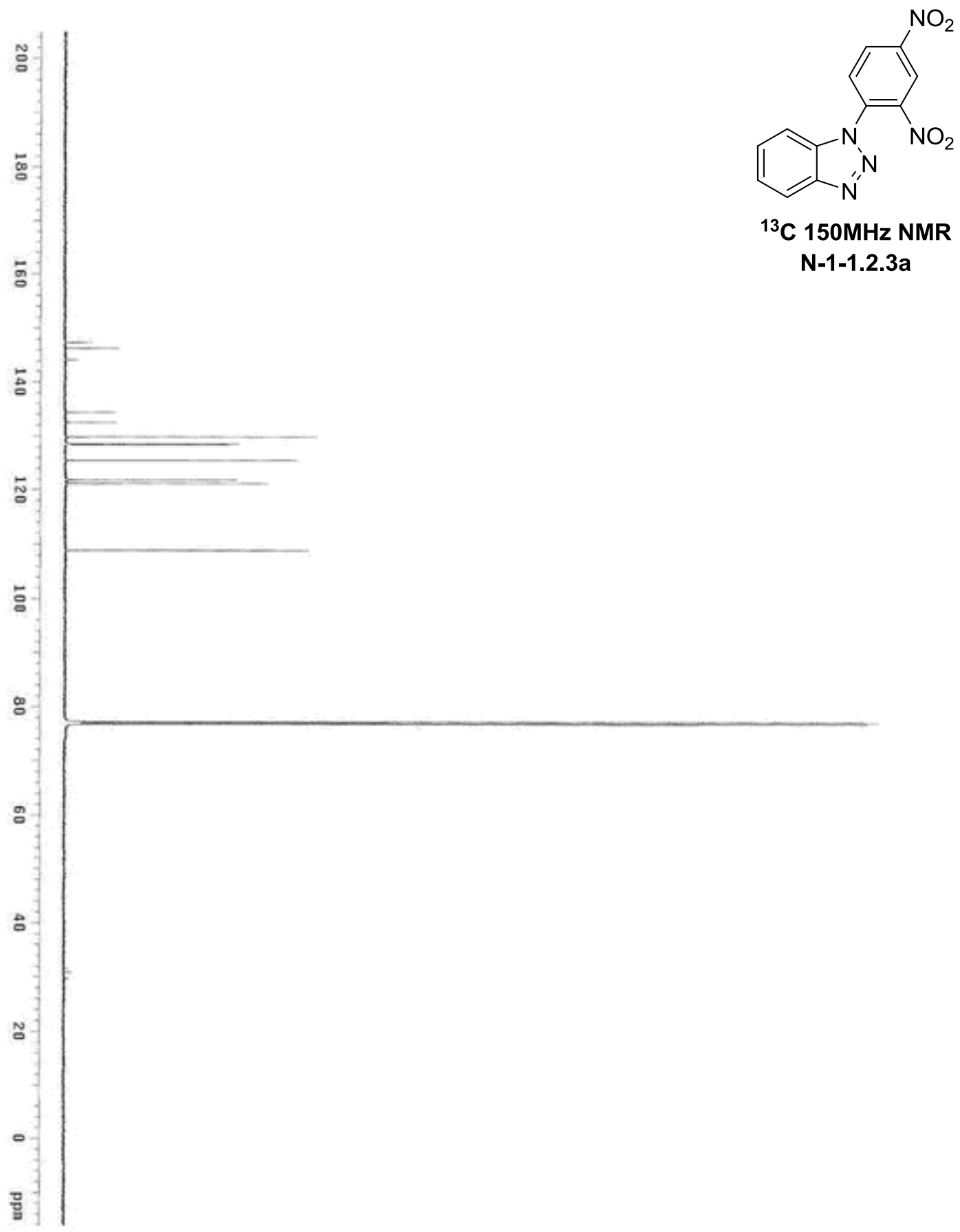

${ }^{13} \mathrm{C} 150 \mathrm{MHz}$ NMR

N-1-1.2.3a 


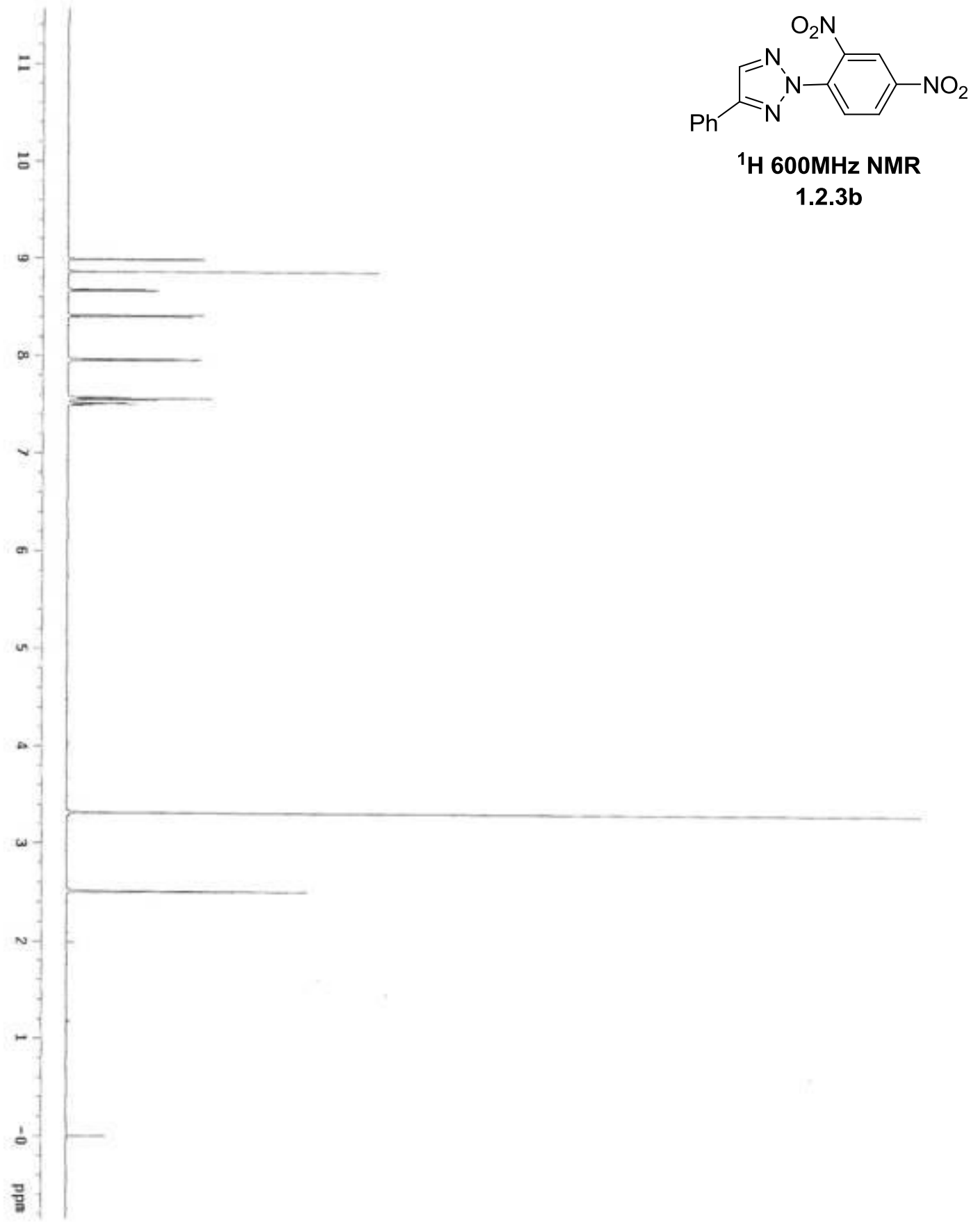




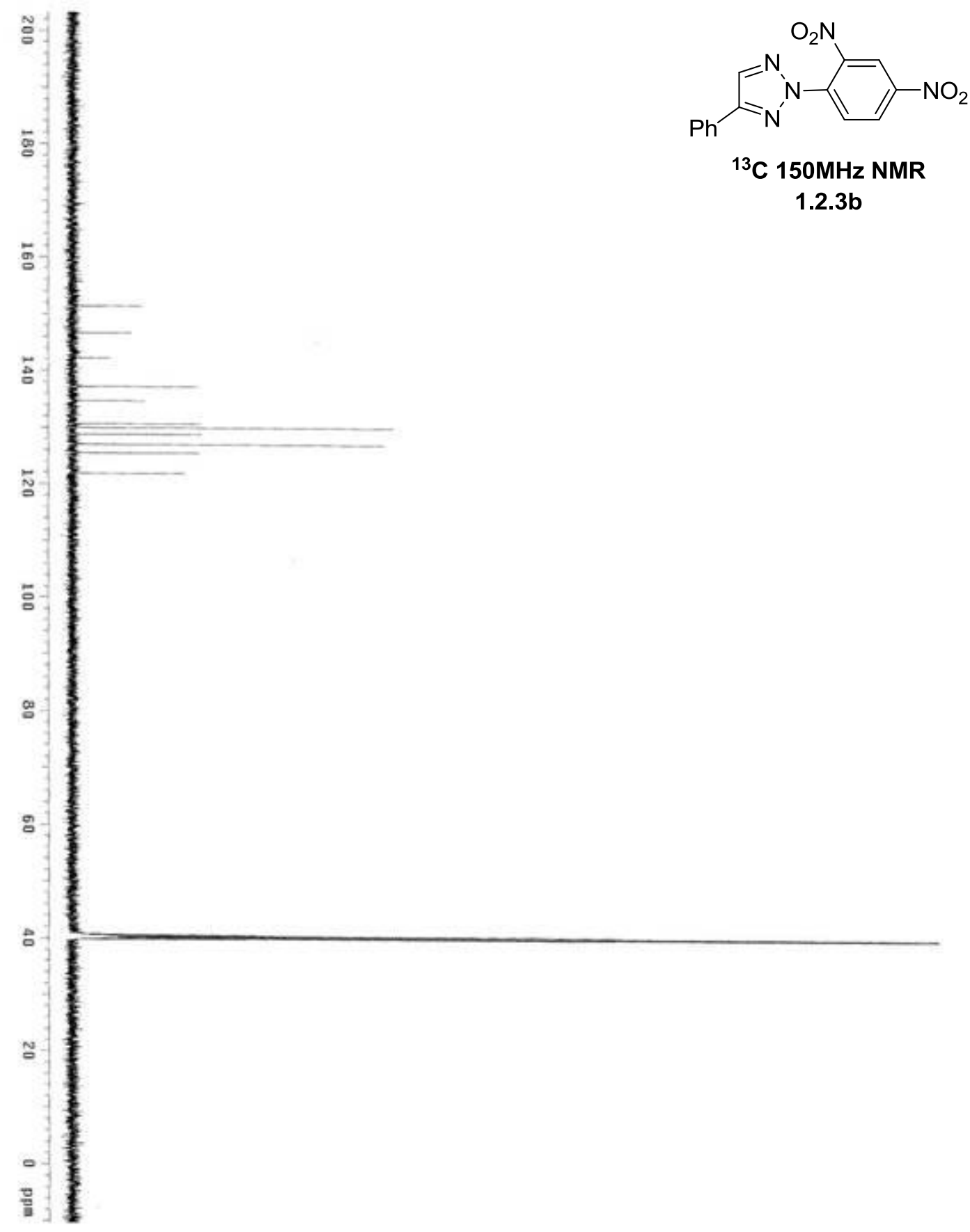




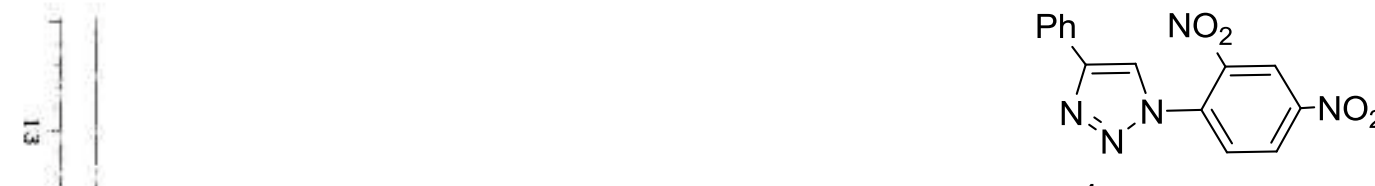

${ }^{1} \mathrm{H}$ 600MHz NMR

$\mathrm{N}-1-1.2 .3 b$

$=1$

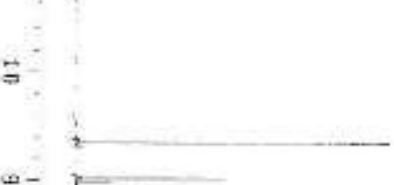

$\omega-$

$=$

$4=$

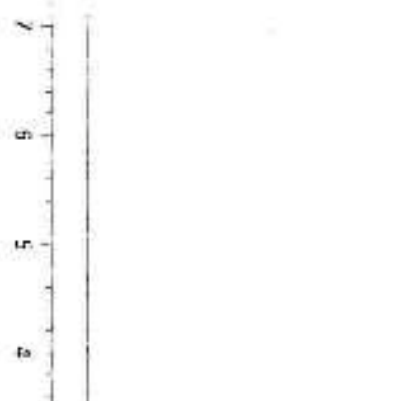

st

$n$

$-$

$-$

a

a

1

| 


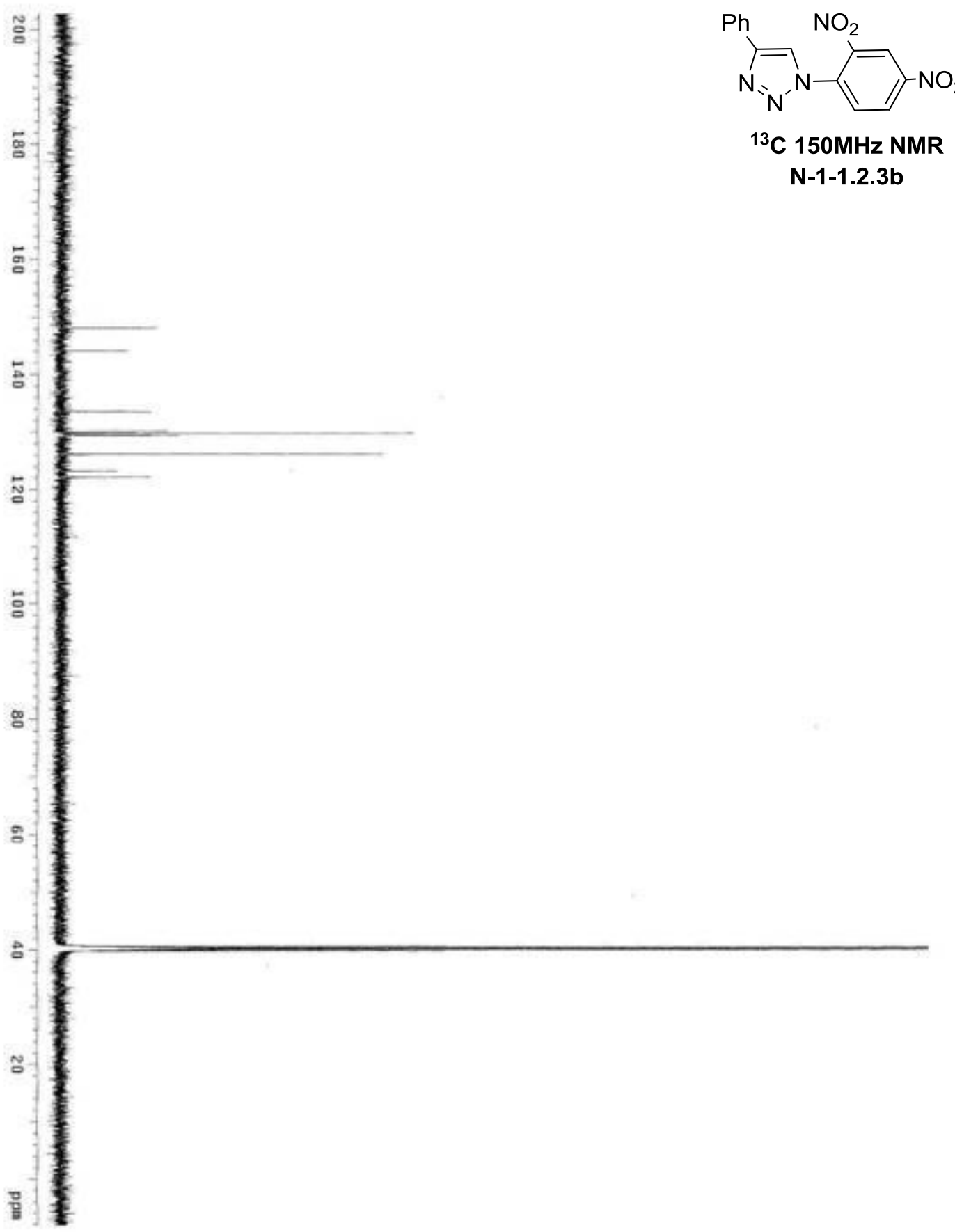




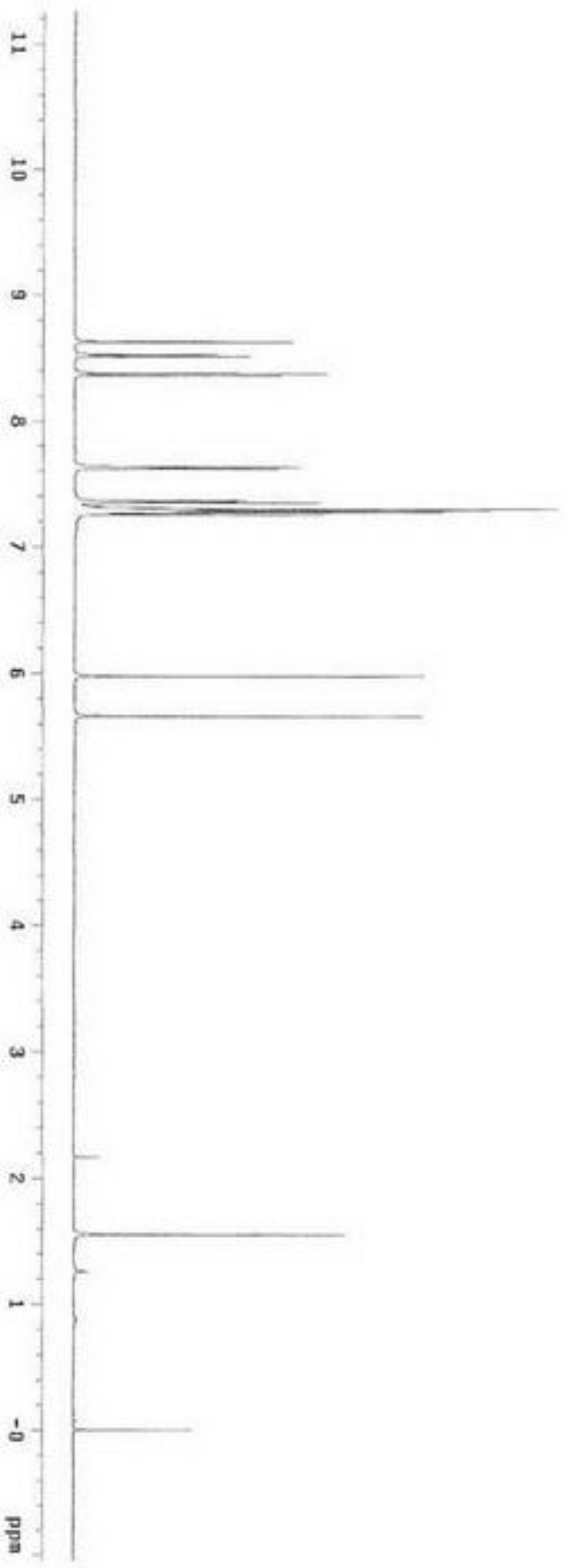

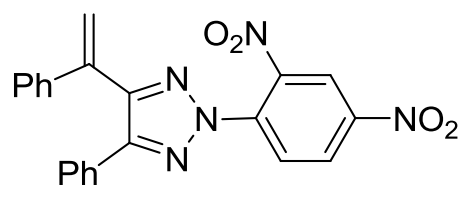

${ }^{1} \mathrm{H}$ 600MHz NMR

1.2.3c 


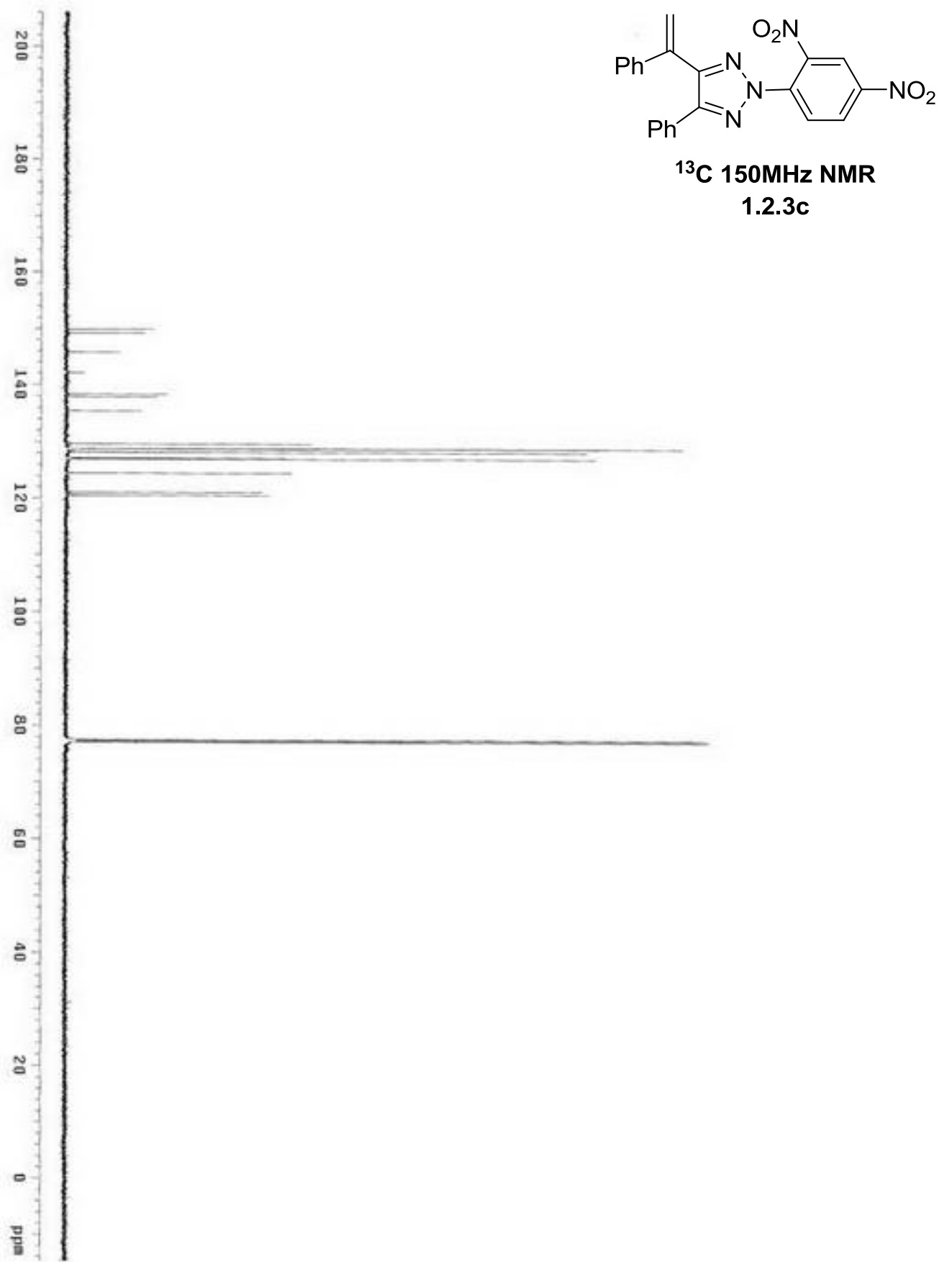




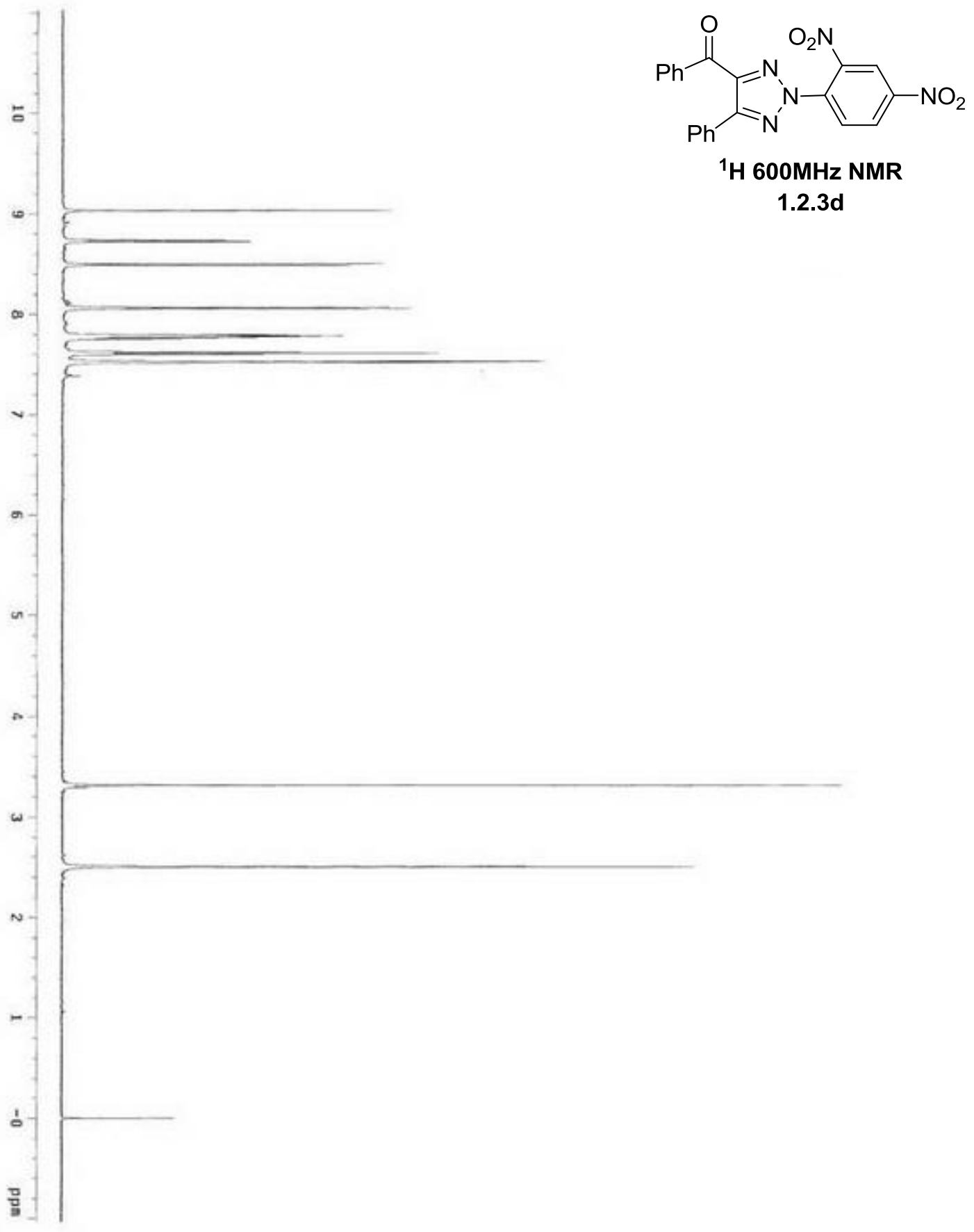




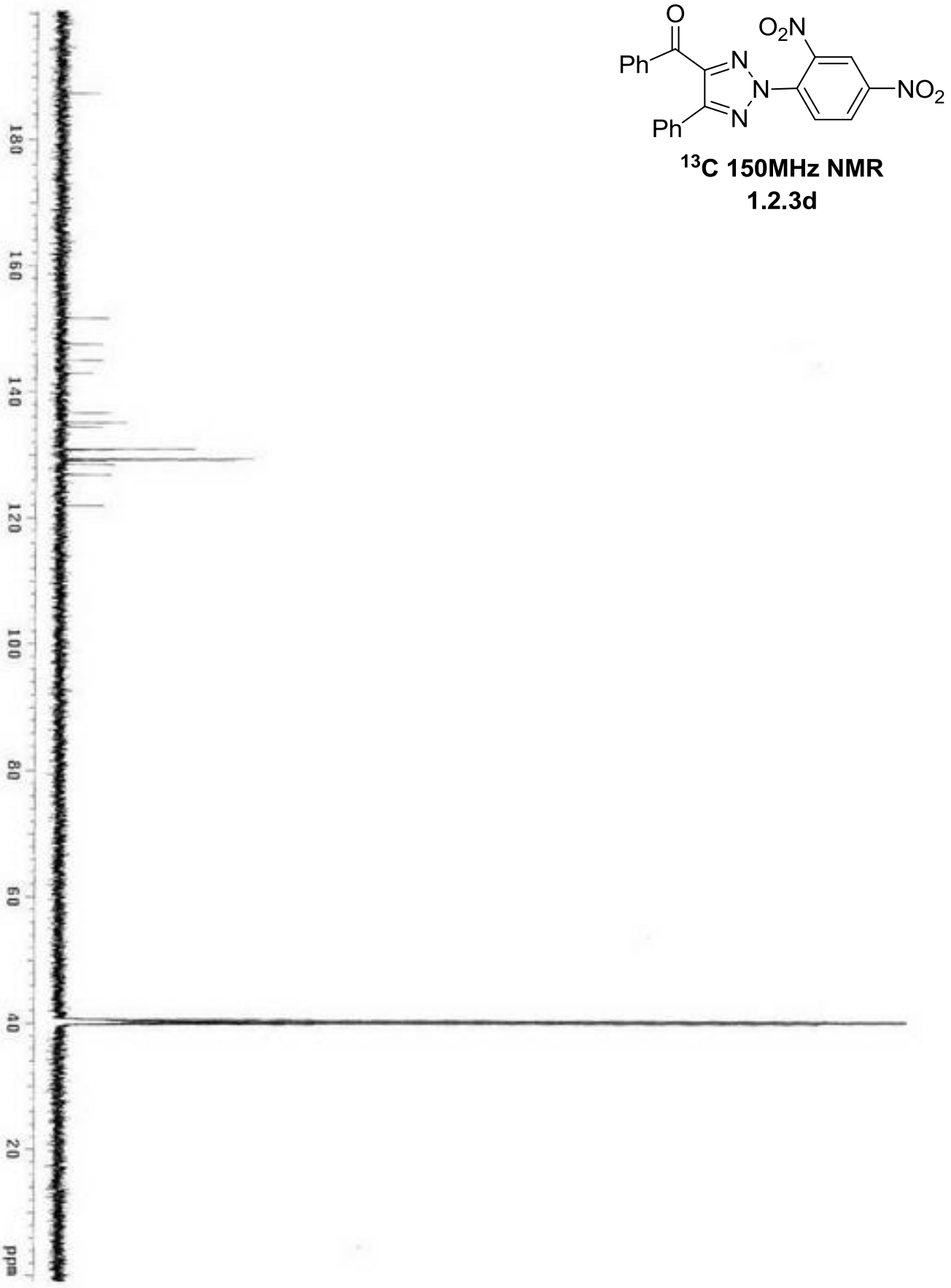




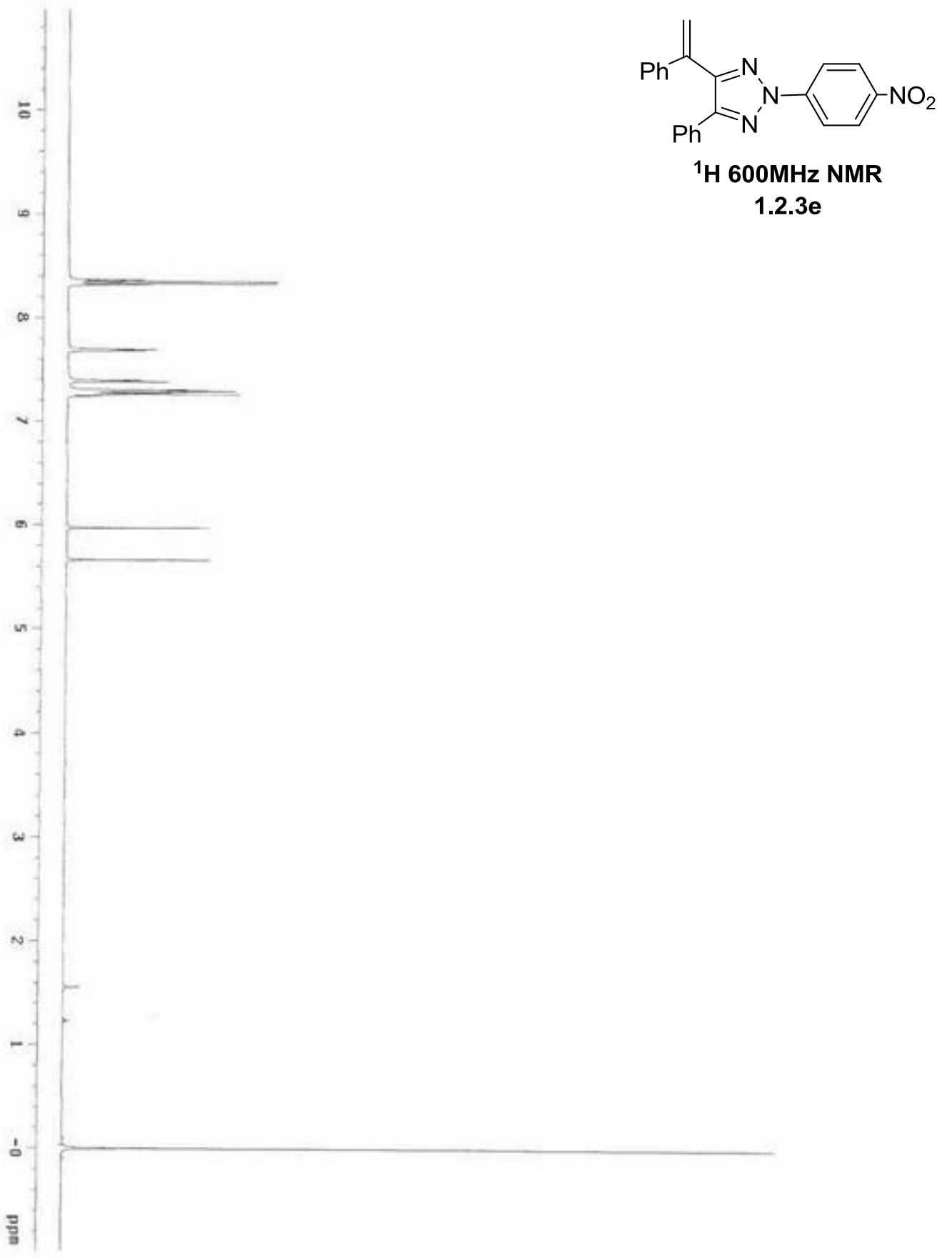




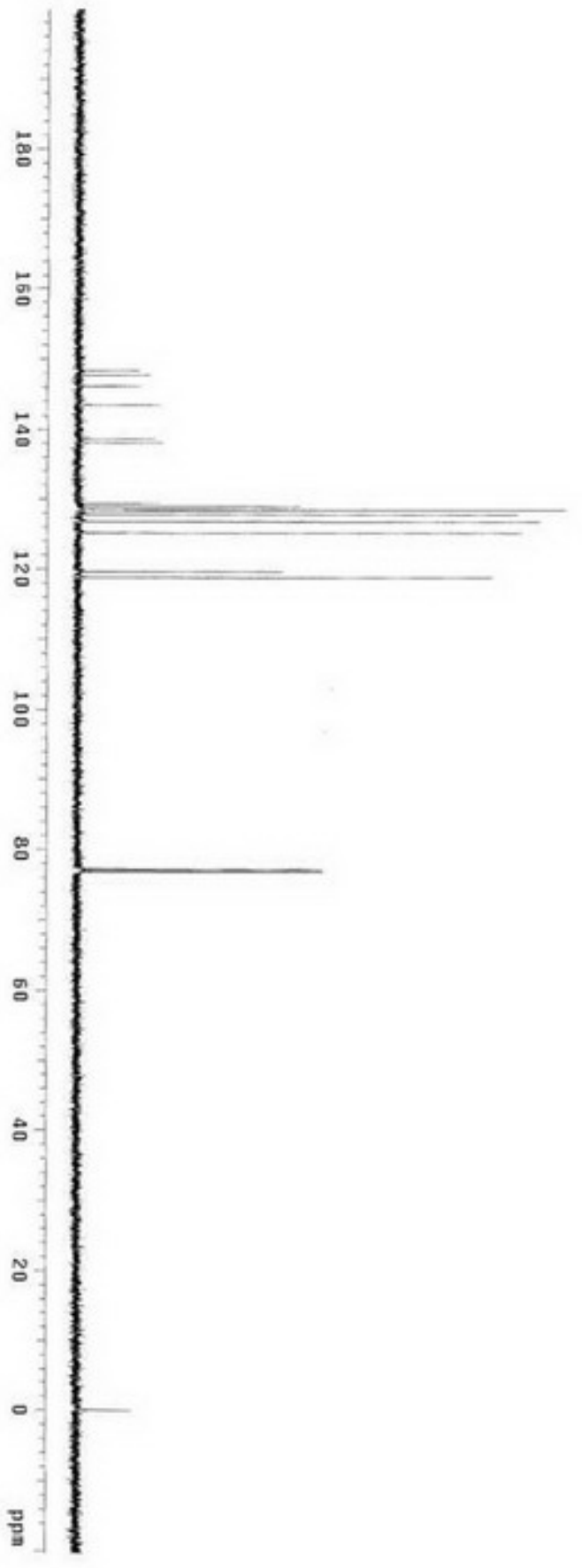

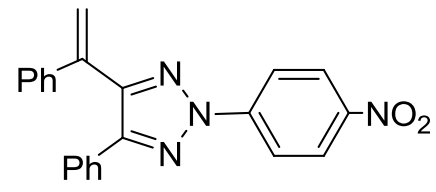

${ }^{13} \mathrm{C} 150 \mathrm{MHz}$ NMR

1.2.3e 

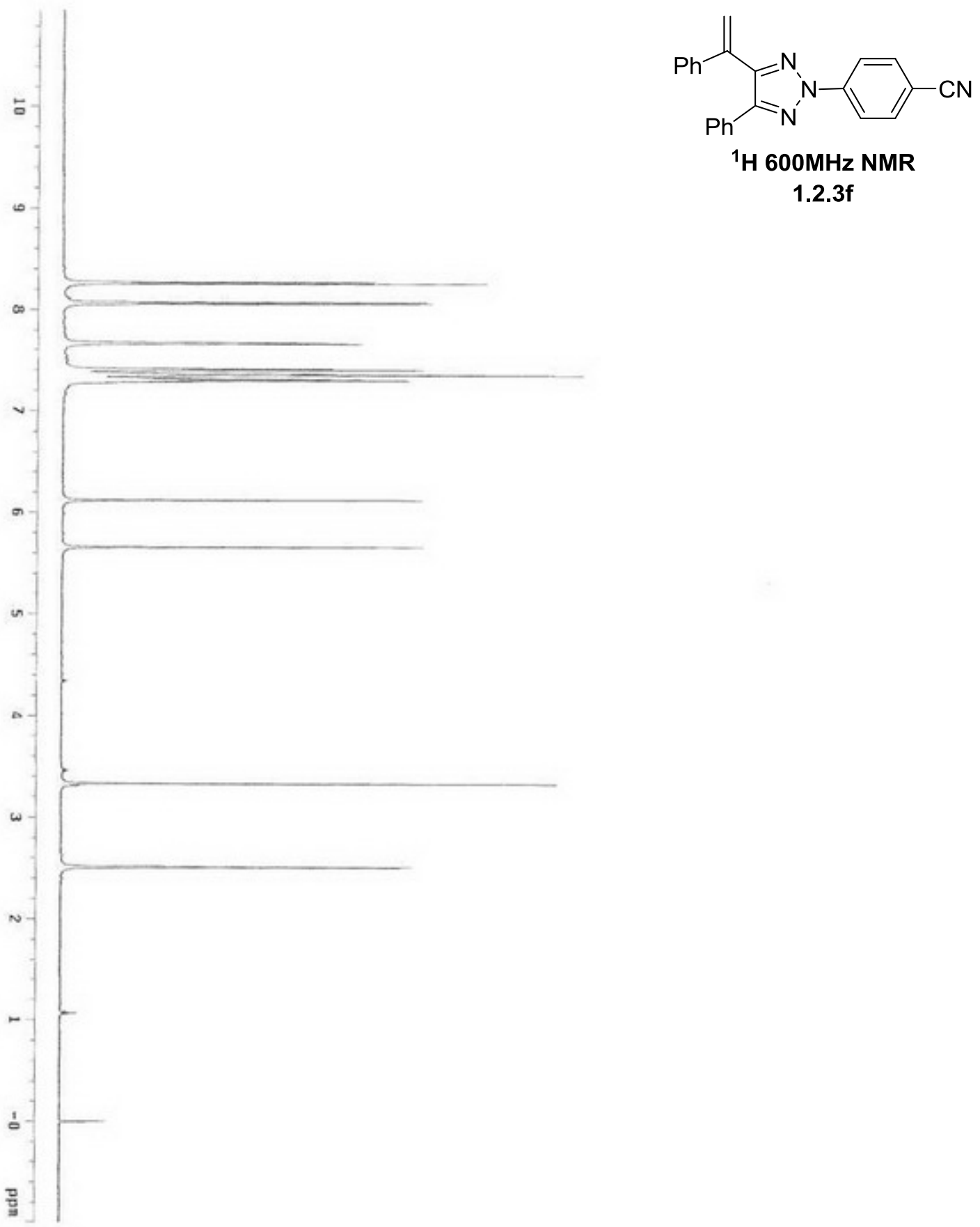

${ }^{1} \mathrm{H} 600 \mathrm{MHz}$ NMR

1.2.3f 


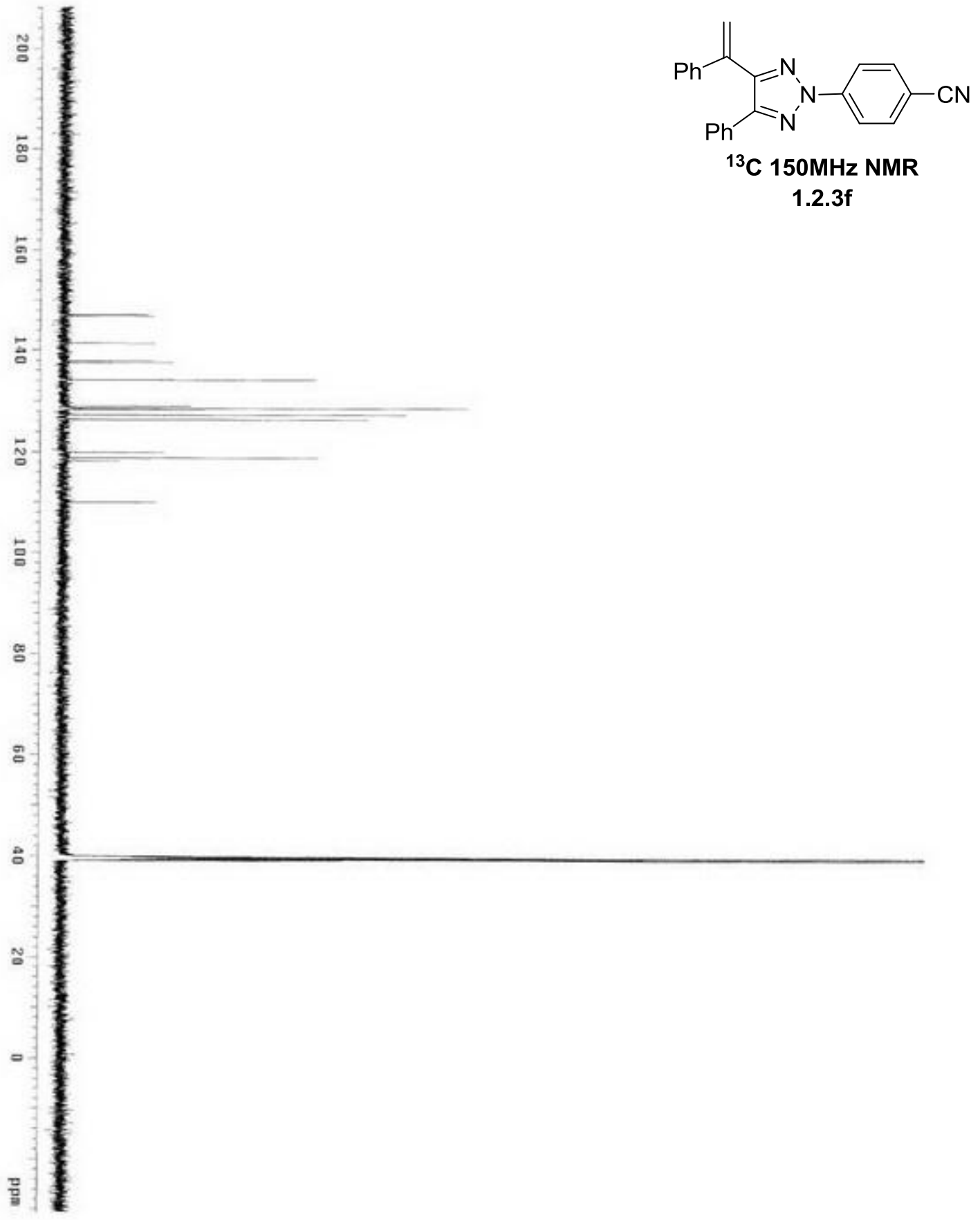



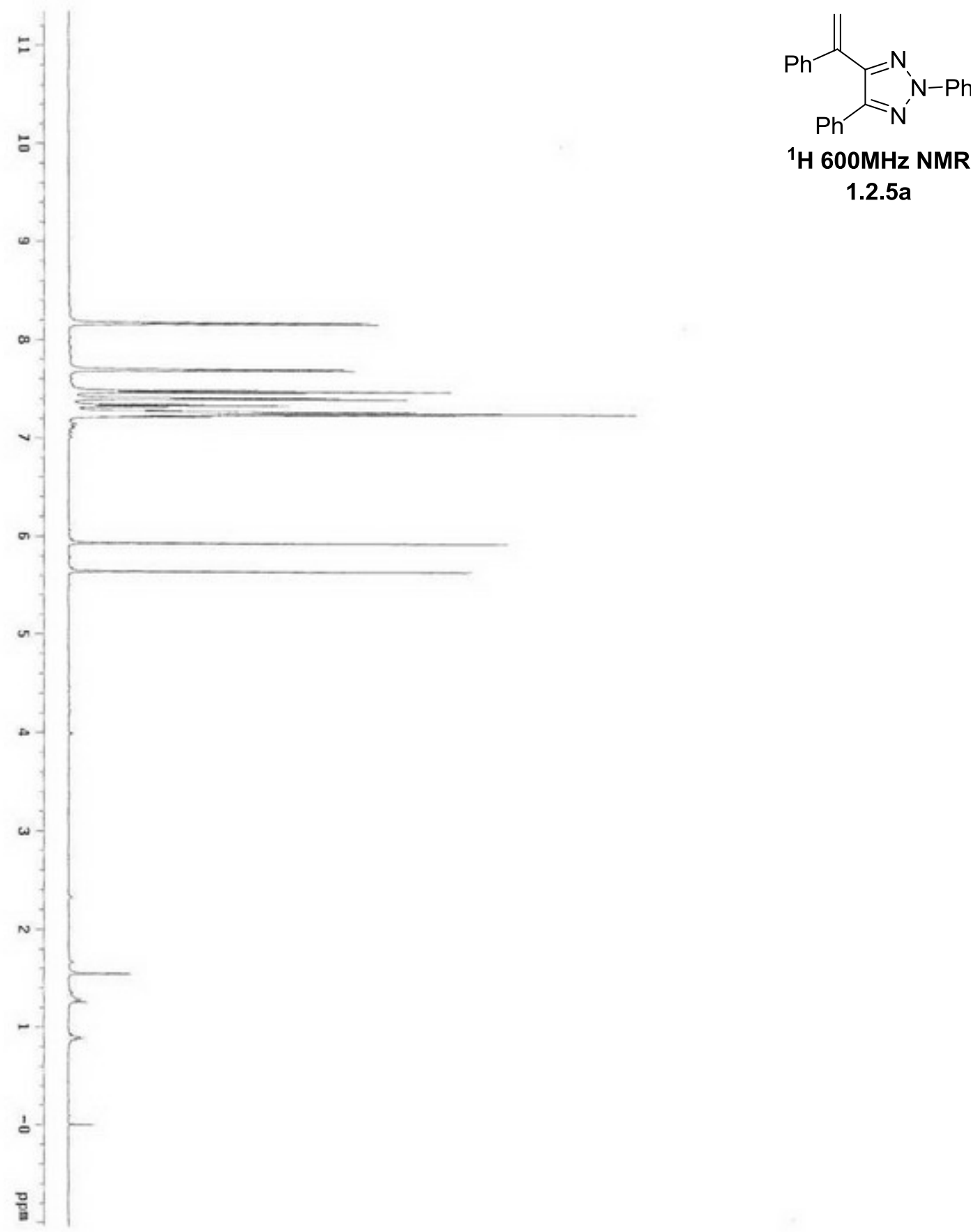

${ }^{1} \mathrm{H} 600 \mathrm{MHz}$ NMR

1.2.5a 

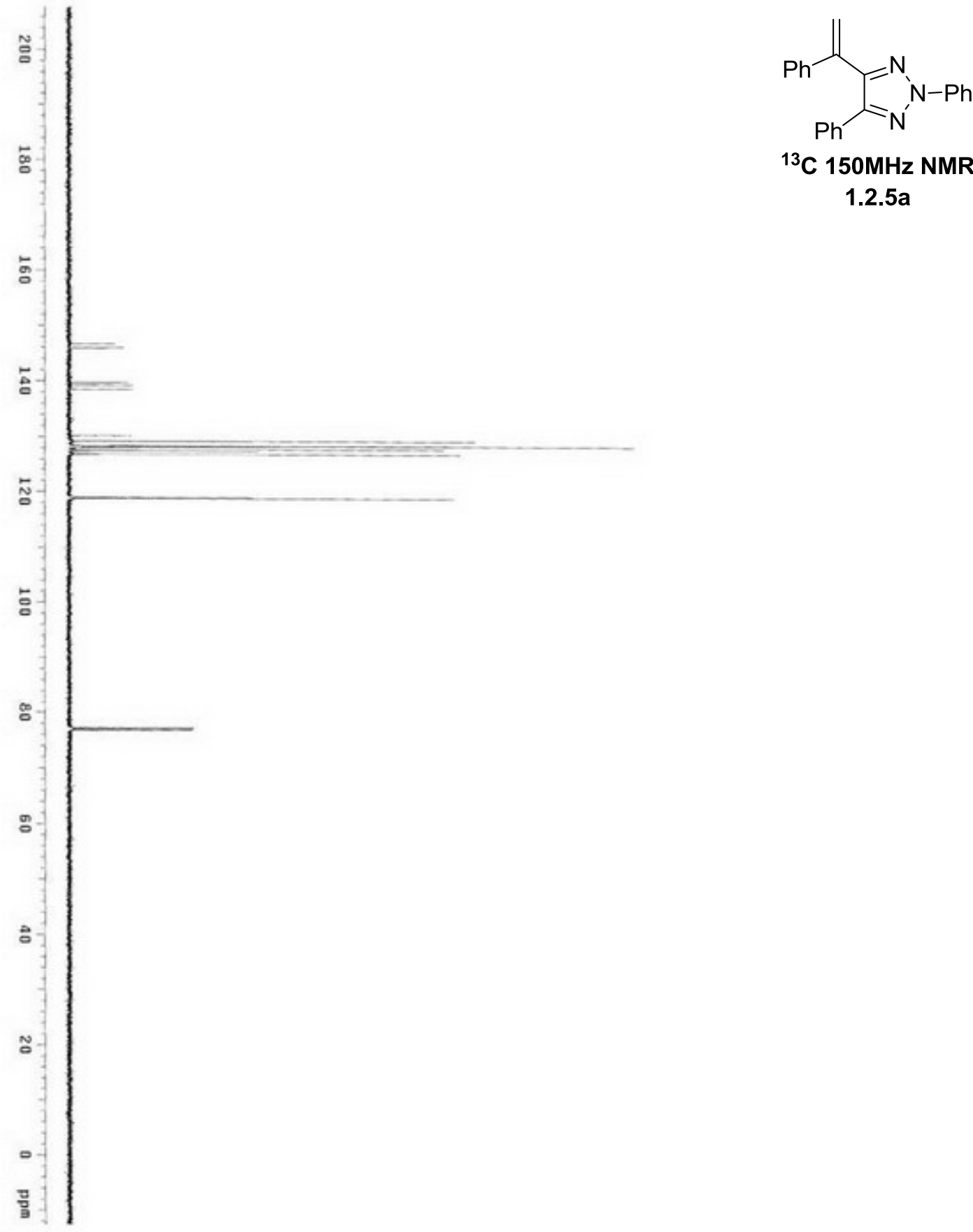

${ }^{13} \mathrm{C} 150 \mathrm{MHz}$ NMR

1.2.5a 


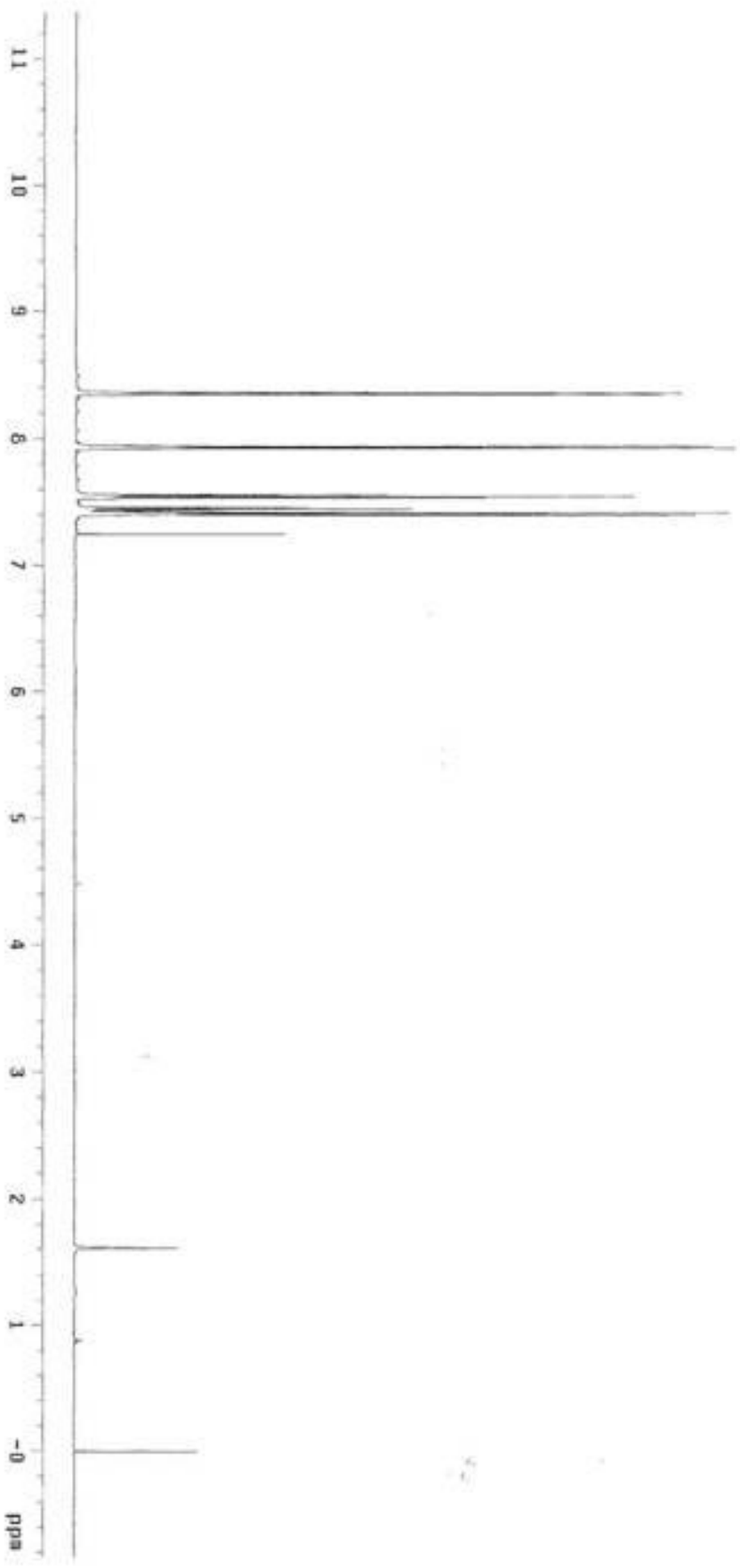

$C_{N}^{N}{ }_{N}^{N}-P h$

${ }^{1} \mathrm{H}$ 600MHz NMR

1.2.5b 

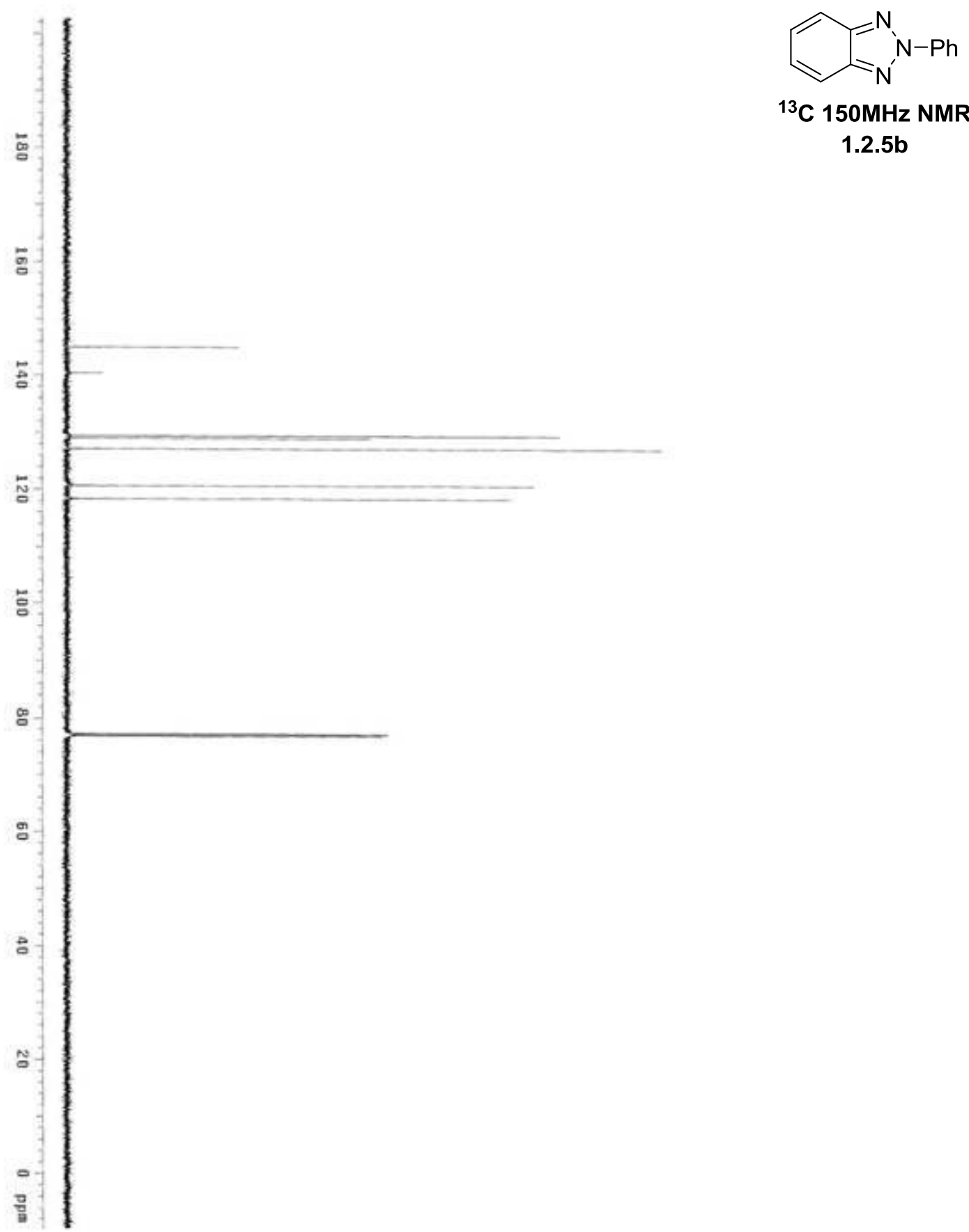

${ }^{13} \mathrm{C} 150 \mathrm{MHz}$ NMR 1.2.5b 

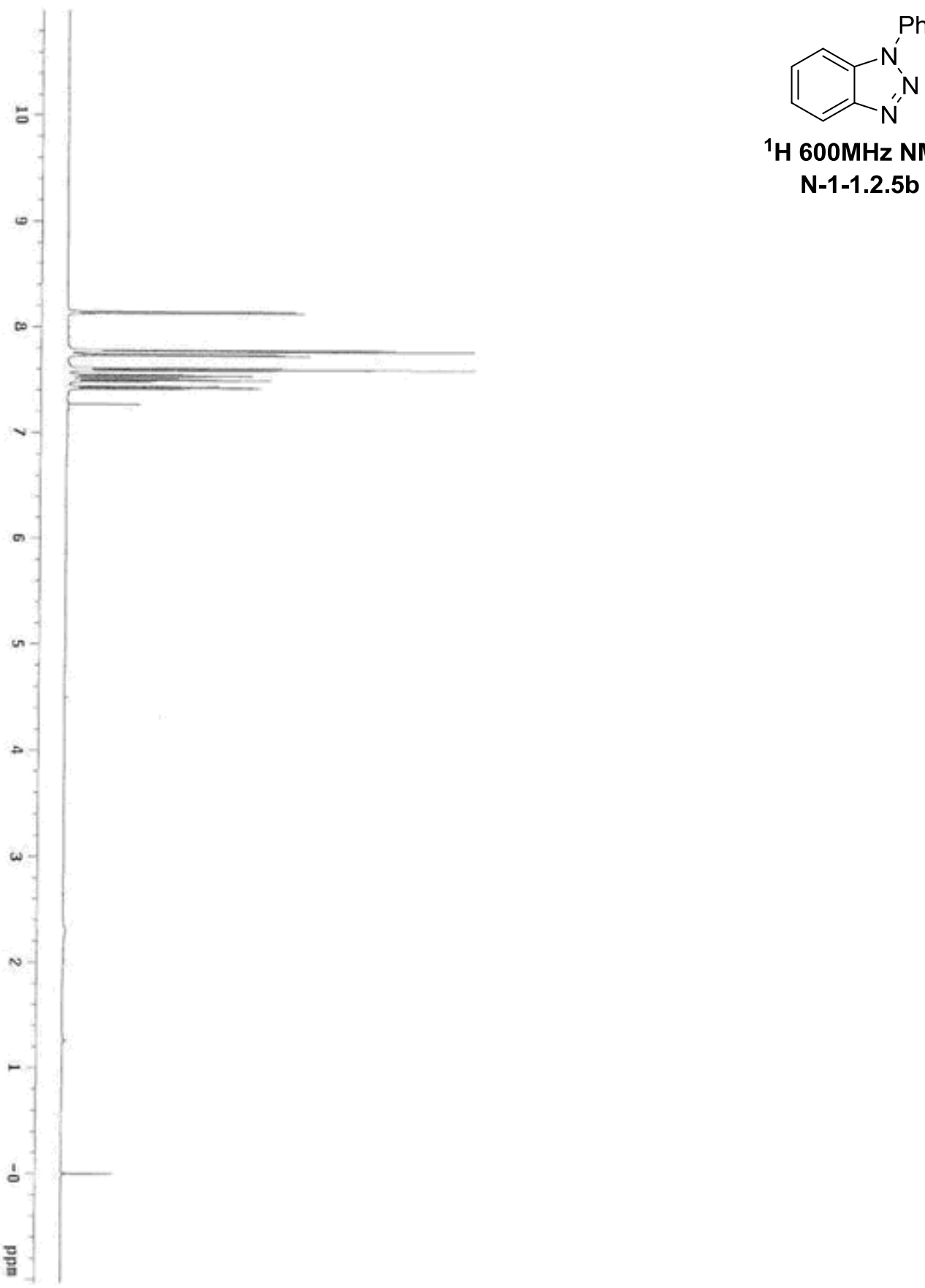

${ }^{1} \mathrm{H}$ 600MHz NMR $\mathrm{N}-1-1.2 .5 b$ 


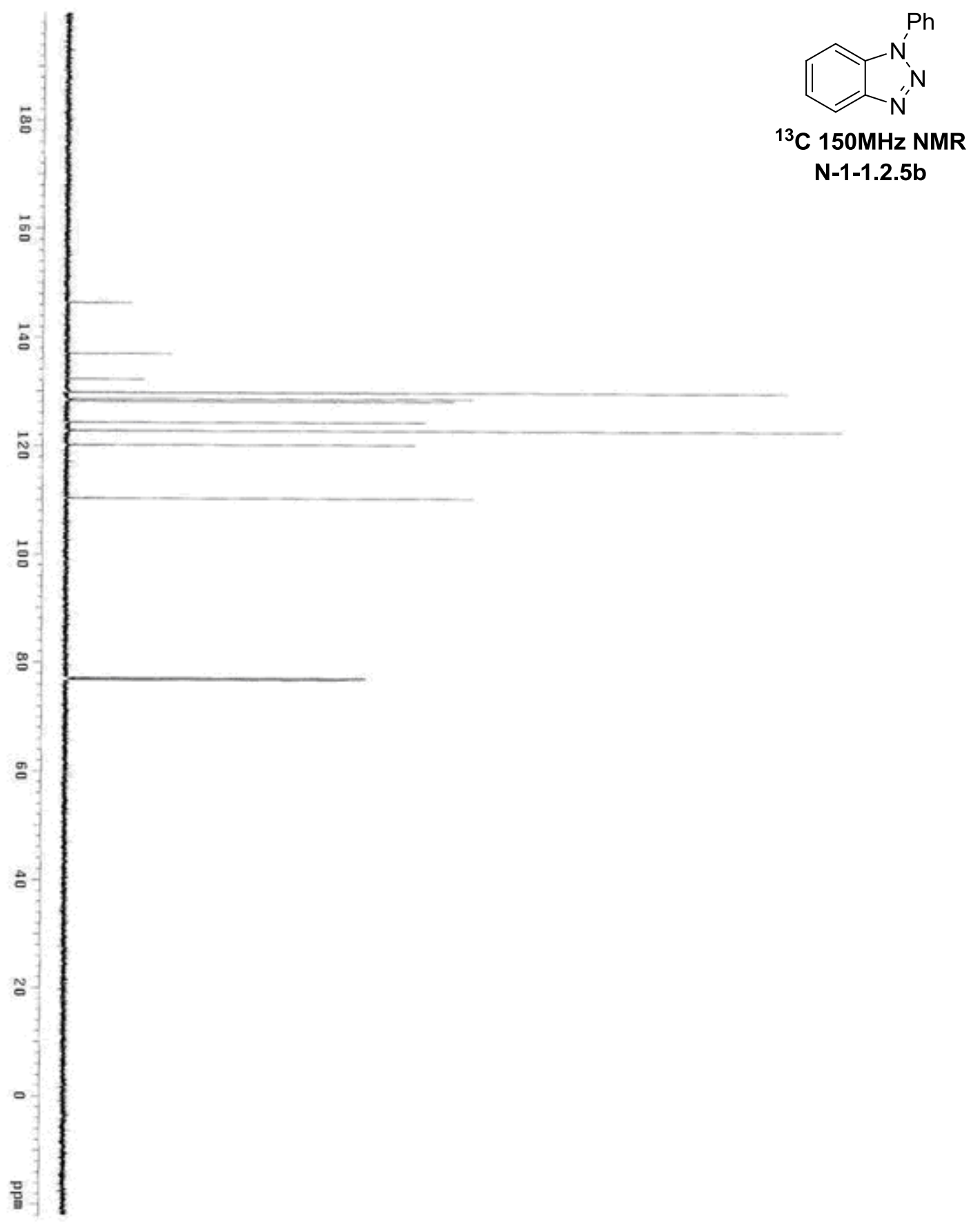



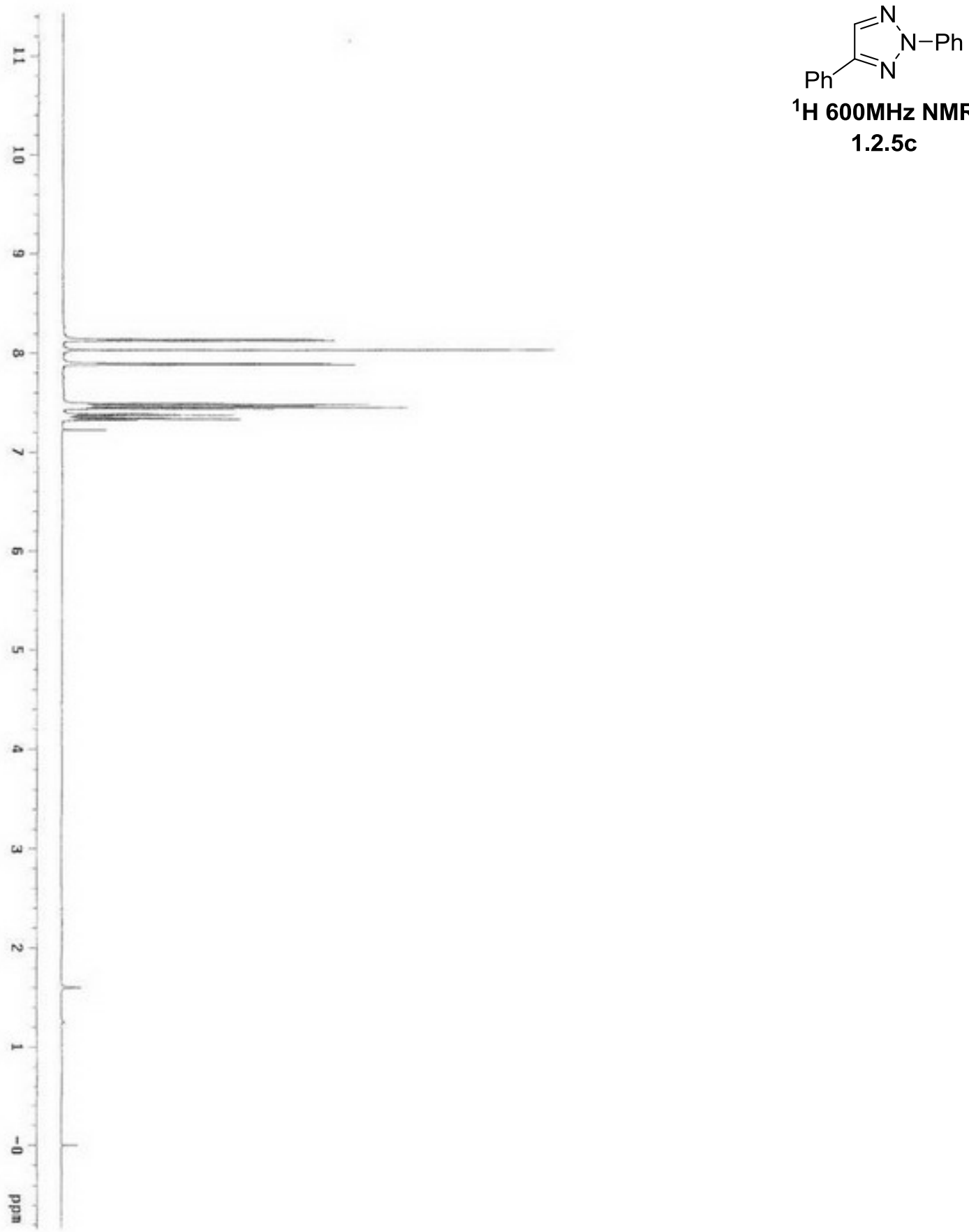

${ }^{1} \mathrm{H}$ 600MHz NMR

$1.2 .5 \mathrm{c}$ 


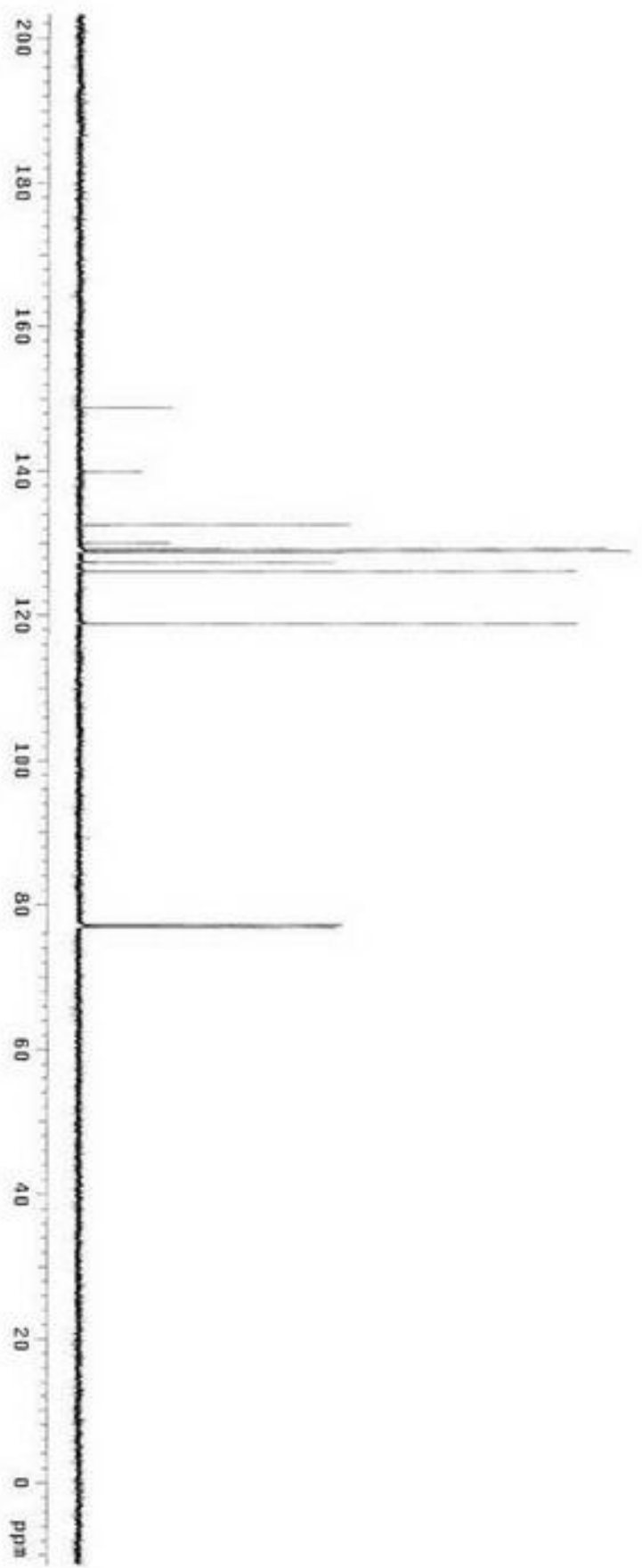

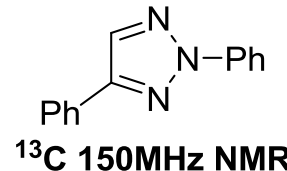

1.2.5c 

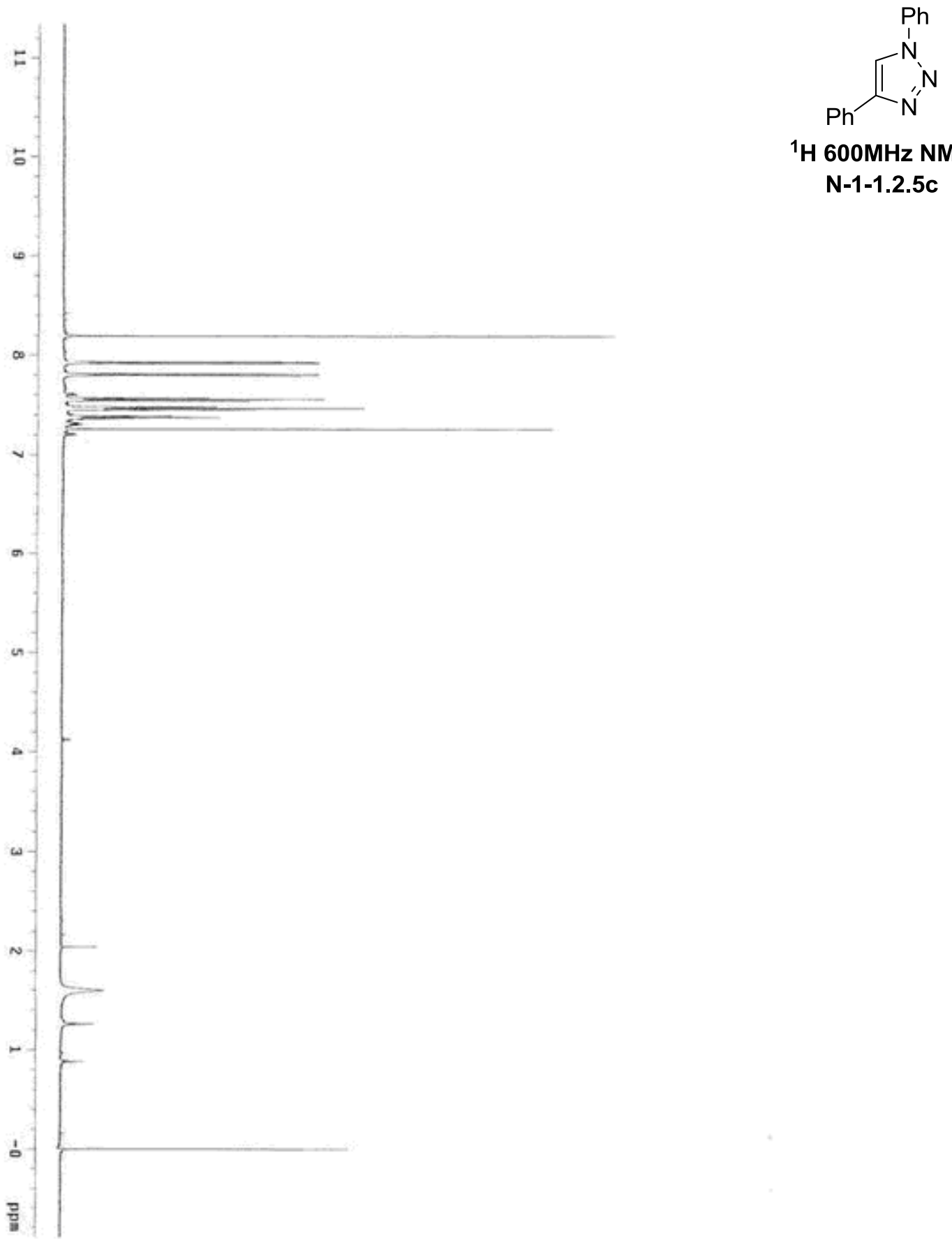

${ }^{1} \mathrm{H} 600 \mathrm{MHz}$ NMR

$\mathrm{N}-1-1.2 .5 \mathrm{C}$ 


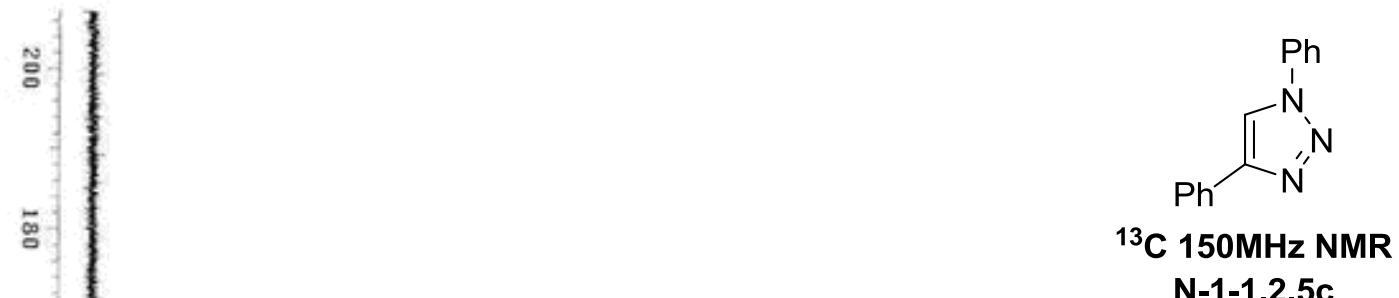
$\mathrm{N}-1-1.2 .5 \mathrm{C}$

g

g

a

음

맘 

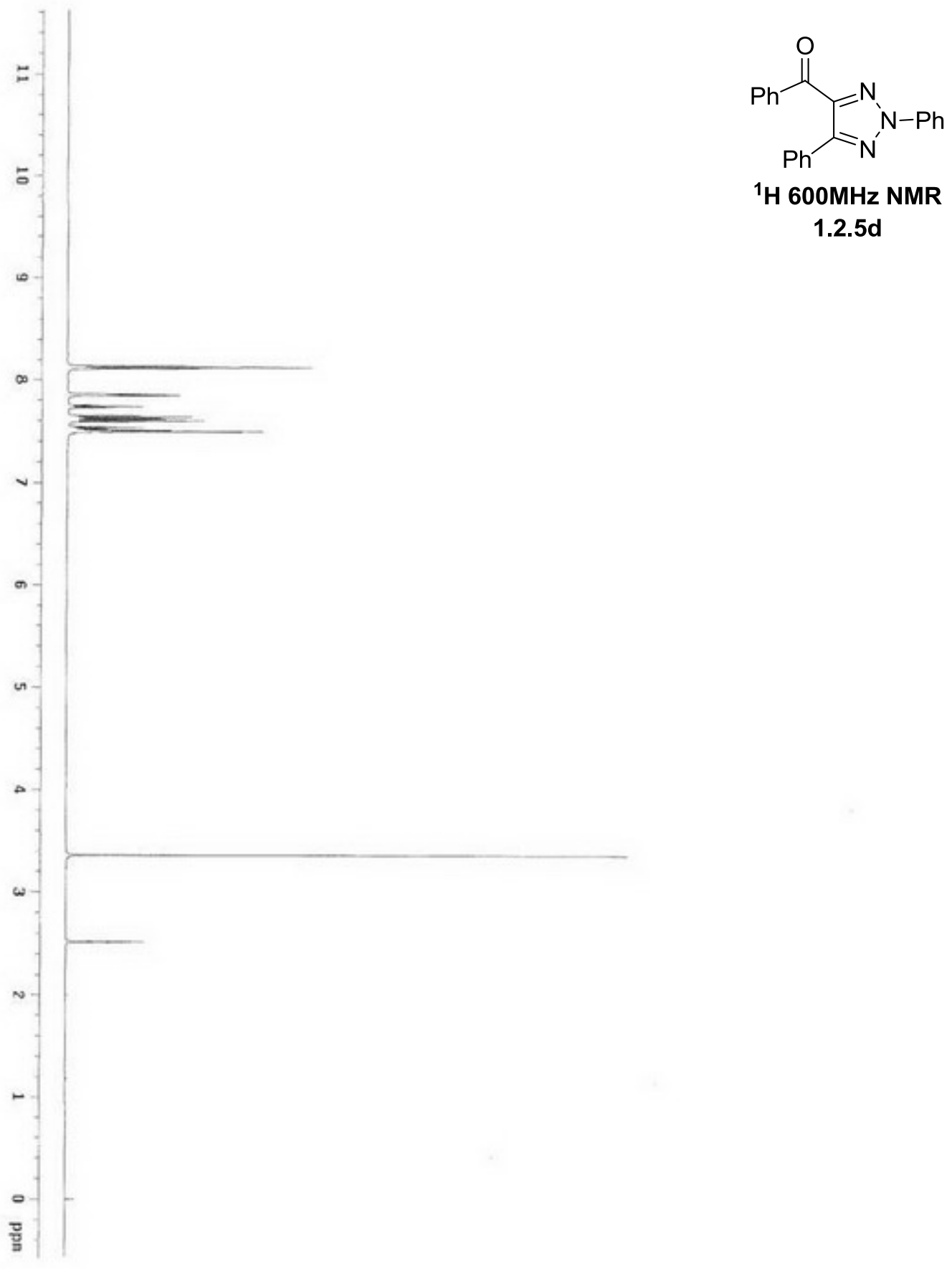

${ }^{1} \mathrm{H} 600 \mathrm{MHz}$ NMR

1.2.5d 

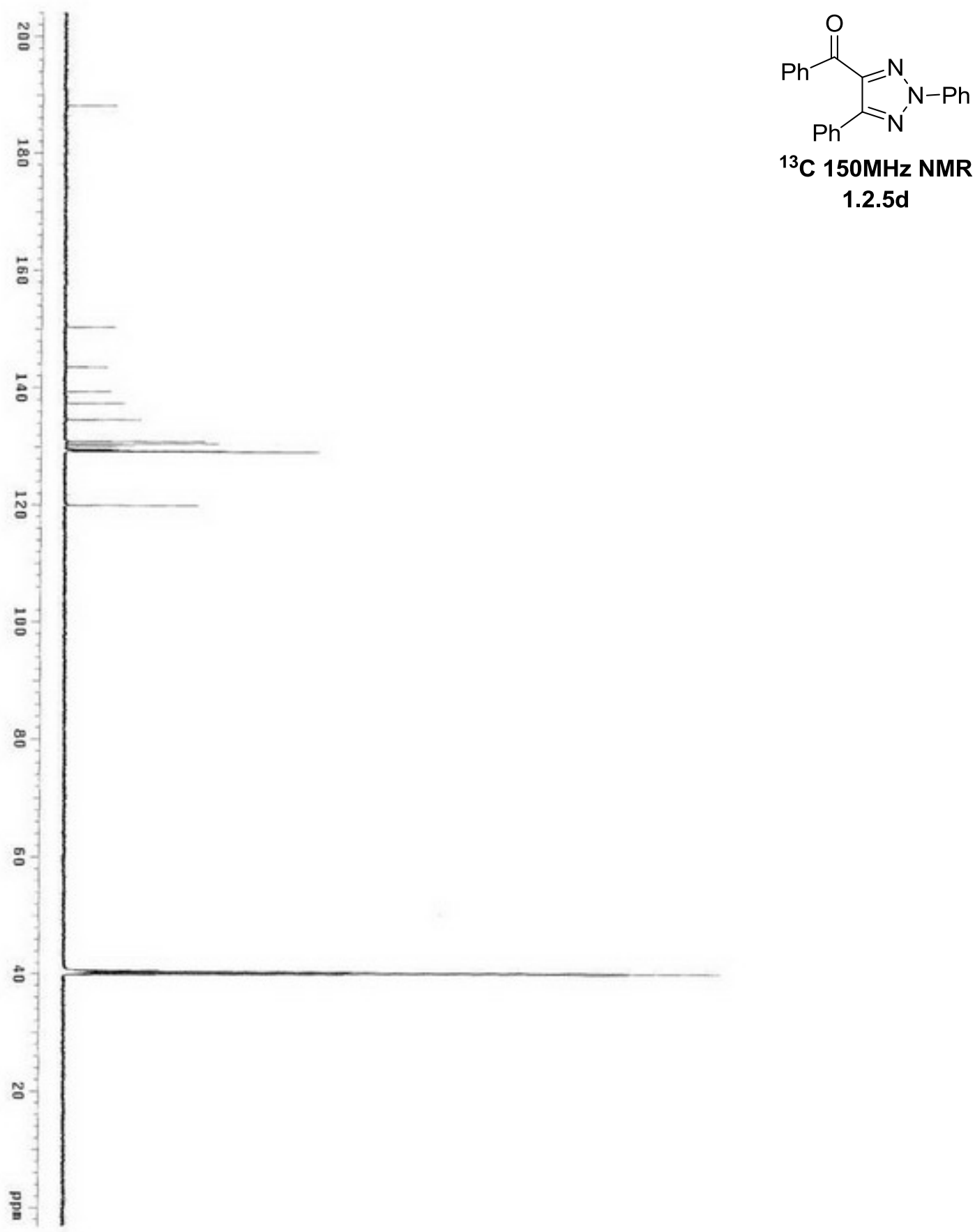

${ }^{13} \mathrm{C}$ 150MHz NMR

1.2.5d 


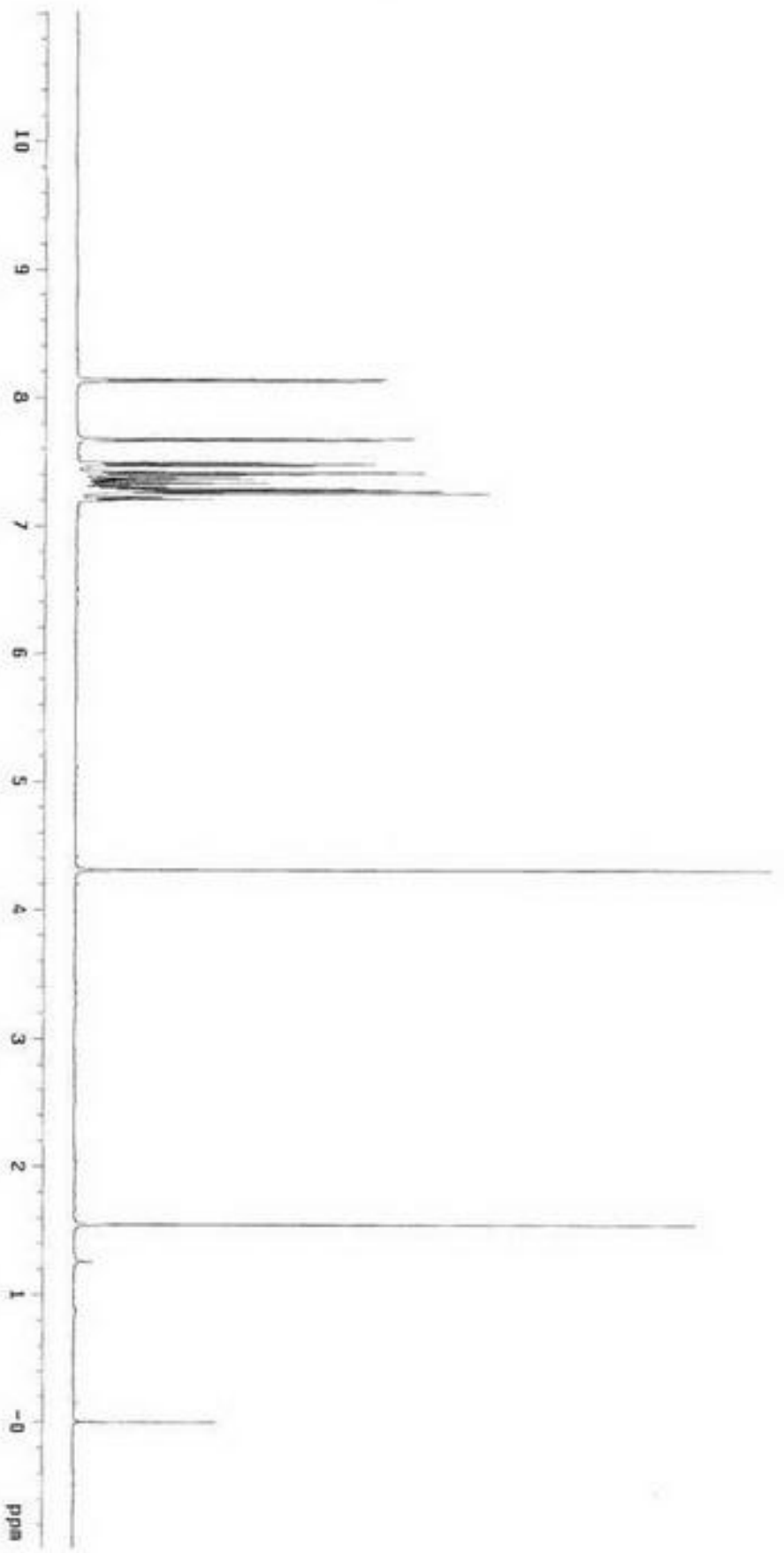

600MHz NMR

1.2.5e 

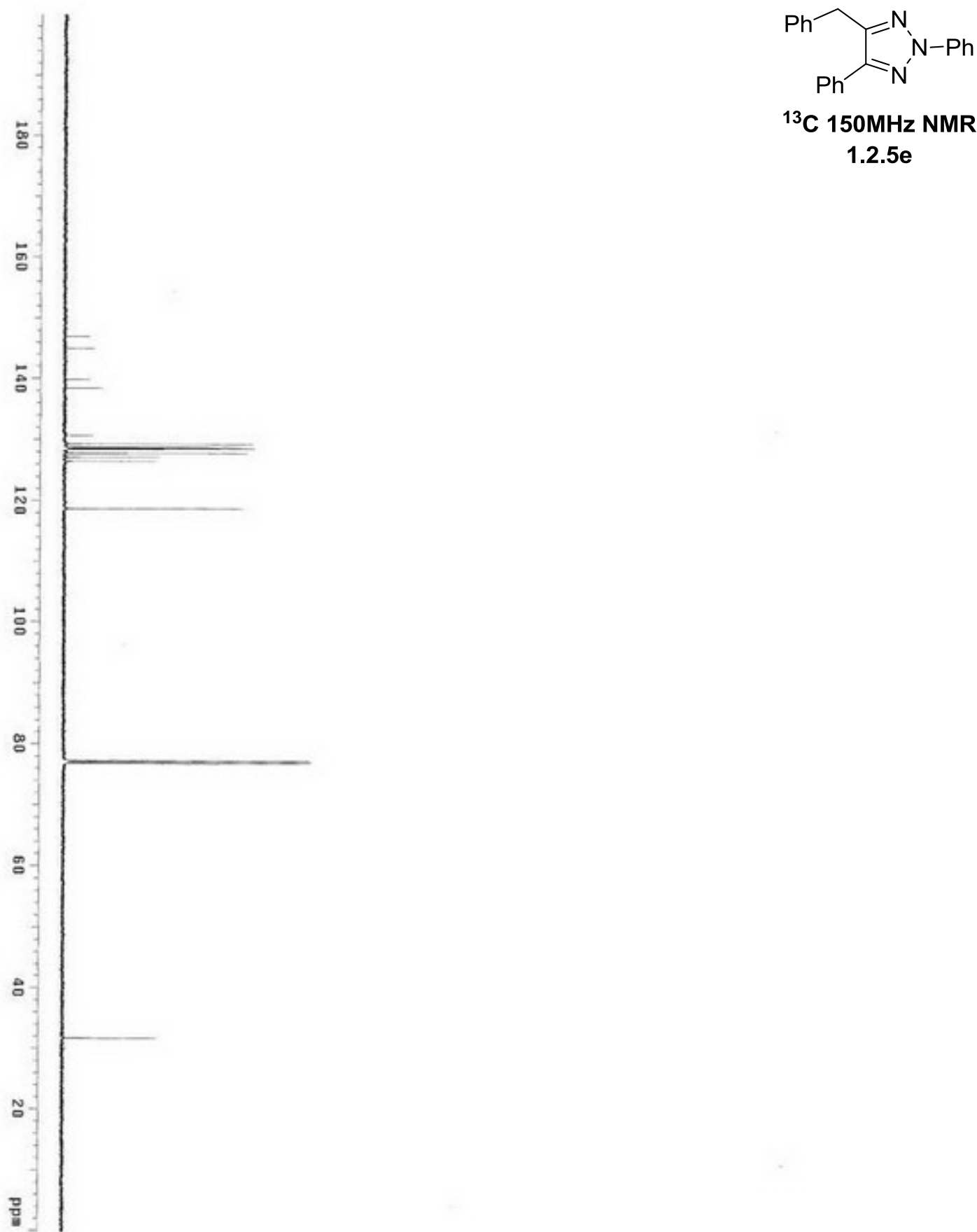

${ }^{13} \mathrm{C} 150 \mathrm{MHz}$ NMR

$1.2 .5 \mathrm{e}$ 


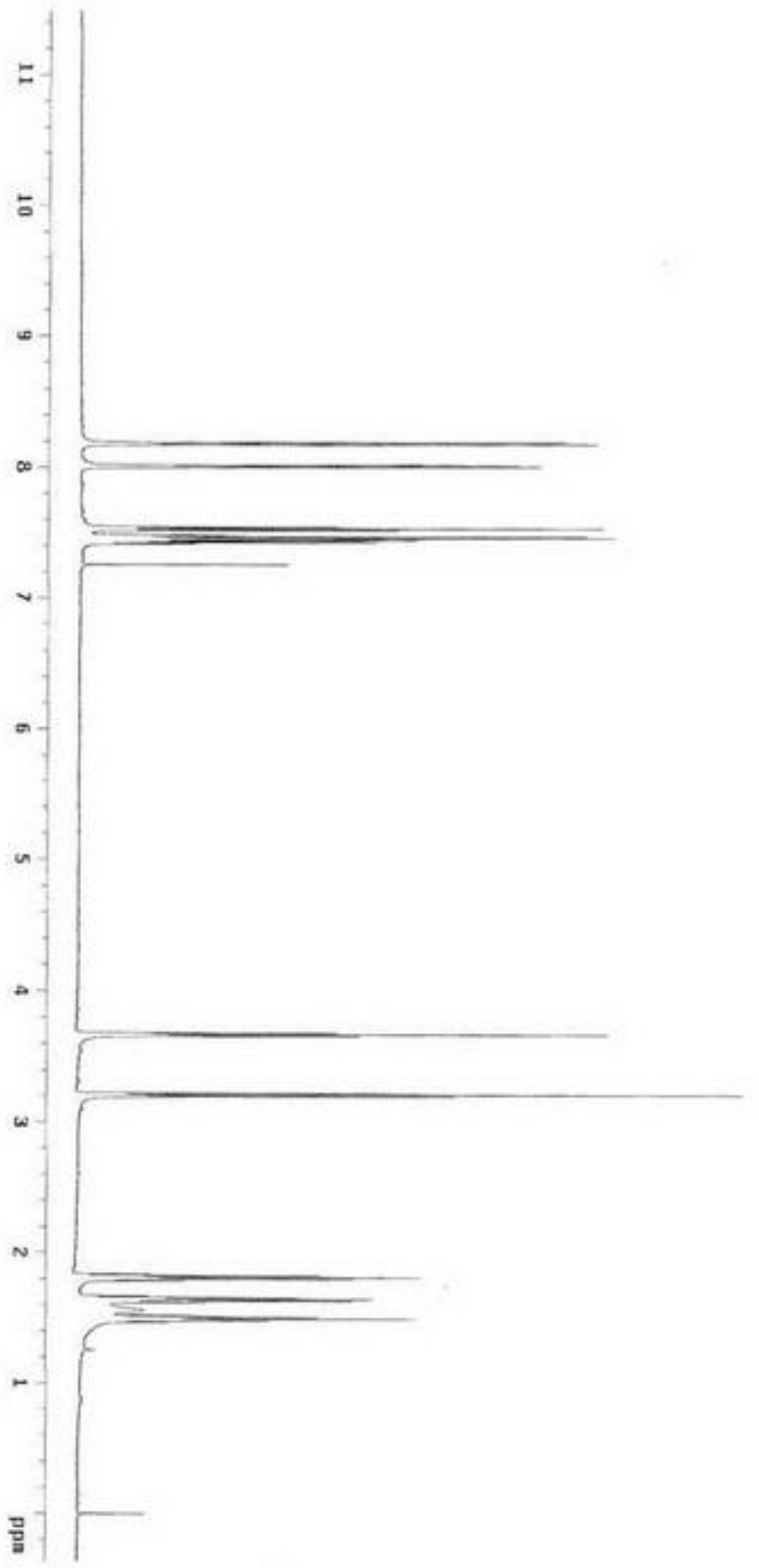

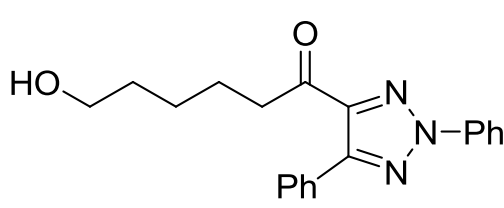

${ }^{1} \mathrm{H} 600 \mathrm{MHz}$ NMR

1.2.5f 


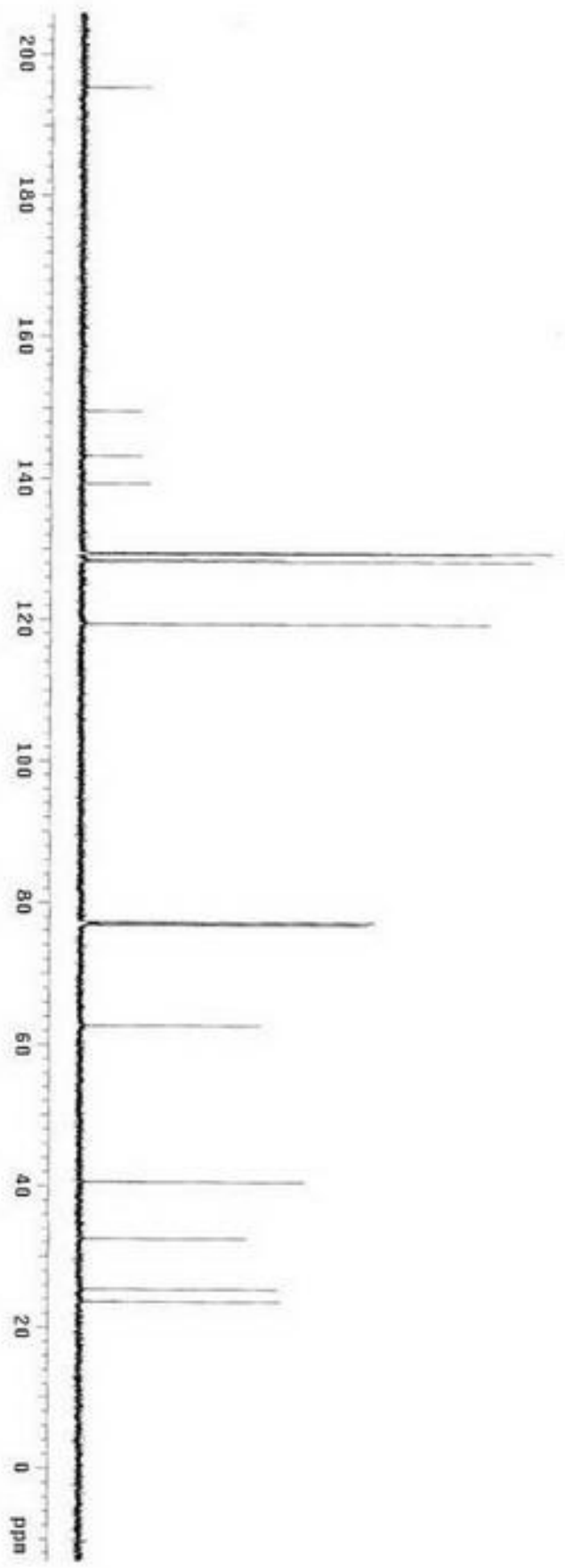

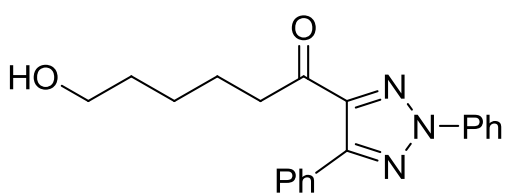

${ }^{13} \mathrm{C} 150 \mathrm{MHz}$ NMR

1.2.5f 

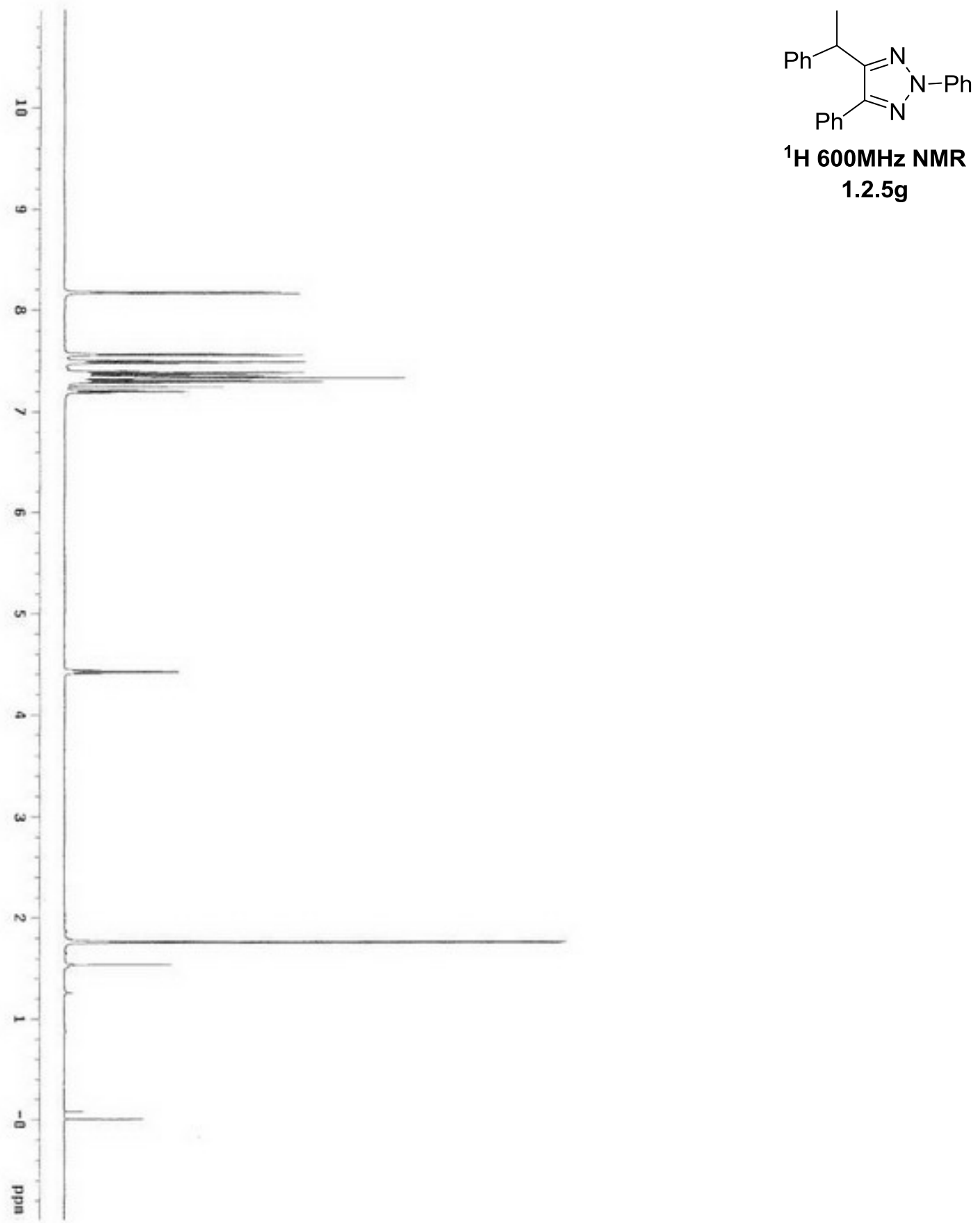

${ }^{1} \mathrm{H} 600 \mathrm{MHz}$ NMR

$1.2 .5 \mathrm{~g}$ 

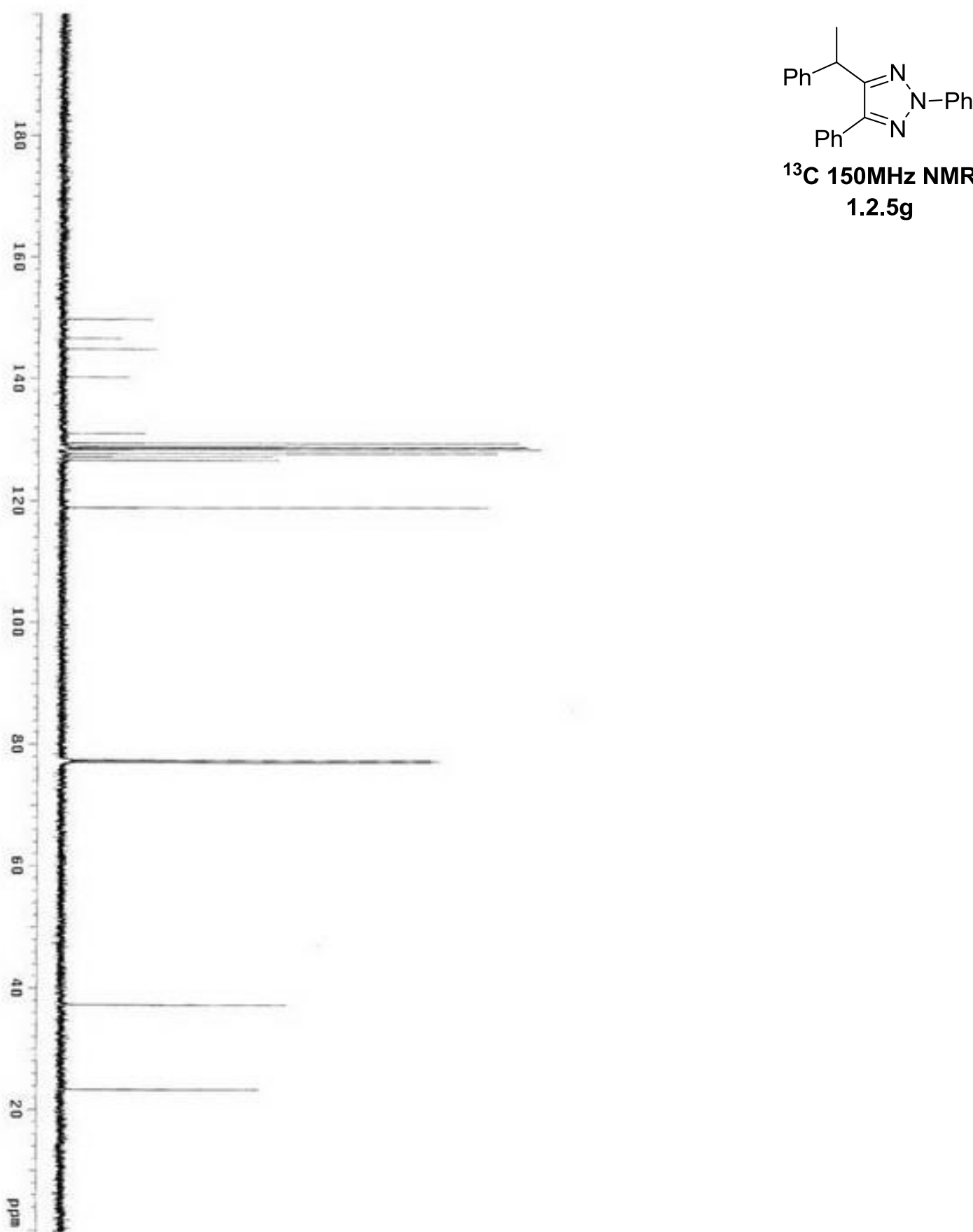

${ }^{13} \mathrm{C} 150 \mathrm{MHz}$ NMR

$1.2 .5 \mathrm{~g}$ 

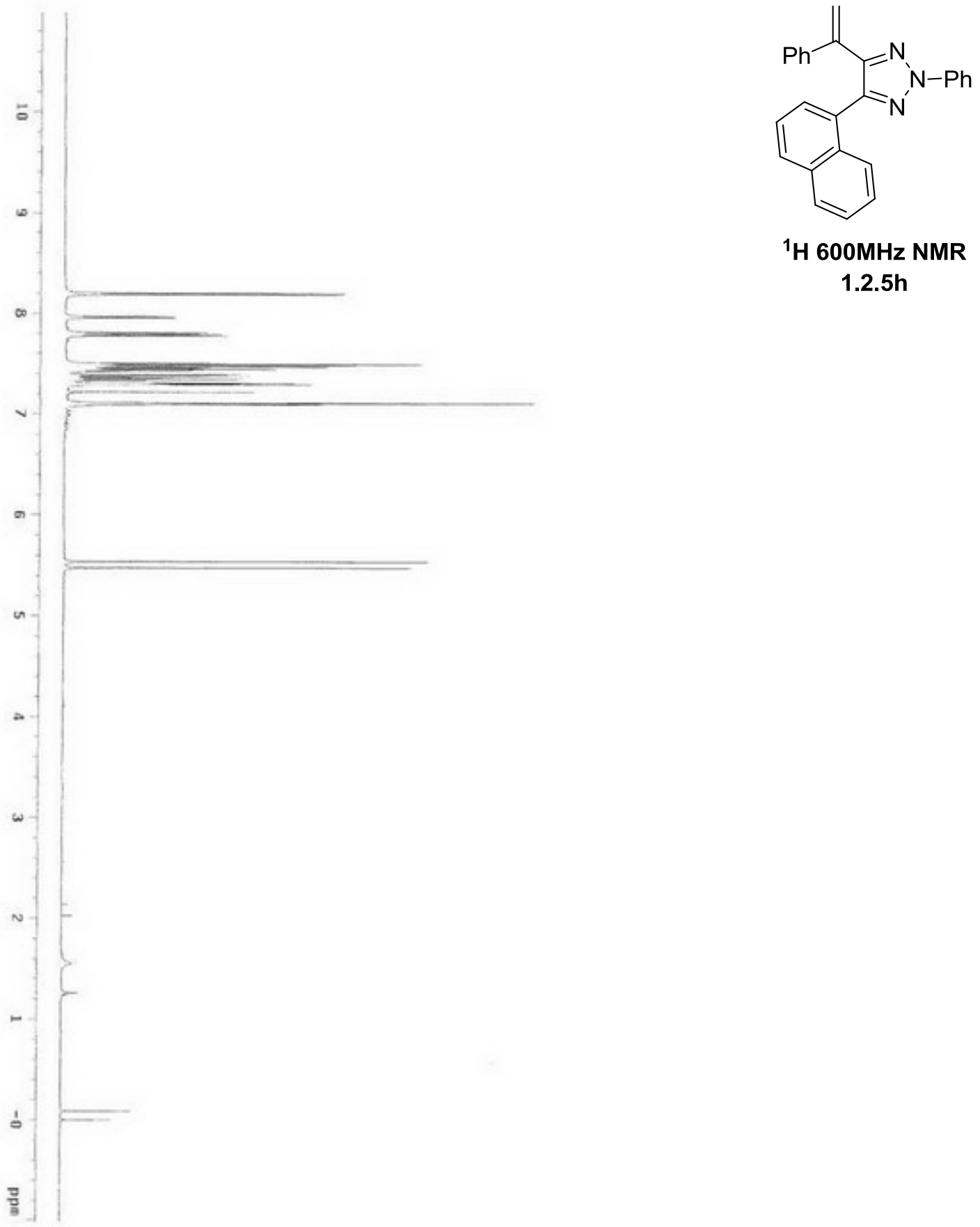

${ }^{1} \mathrm{H} 600 \mathrm{MHz}$ NMR 1.2.5h 

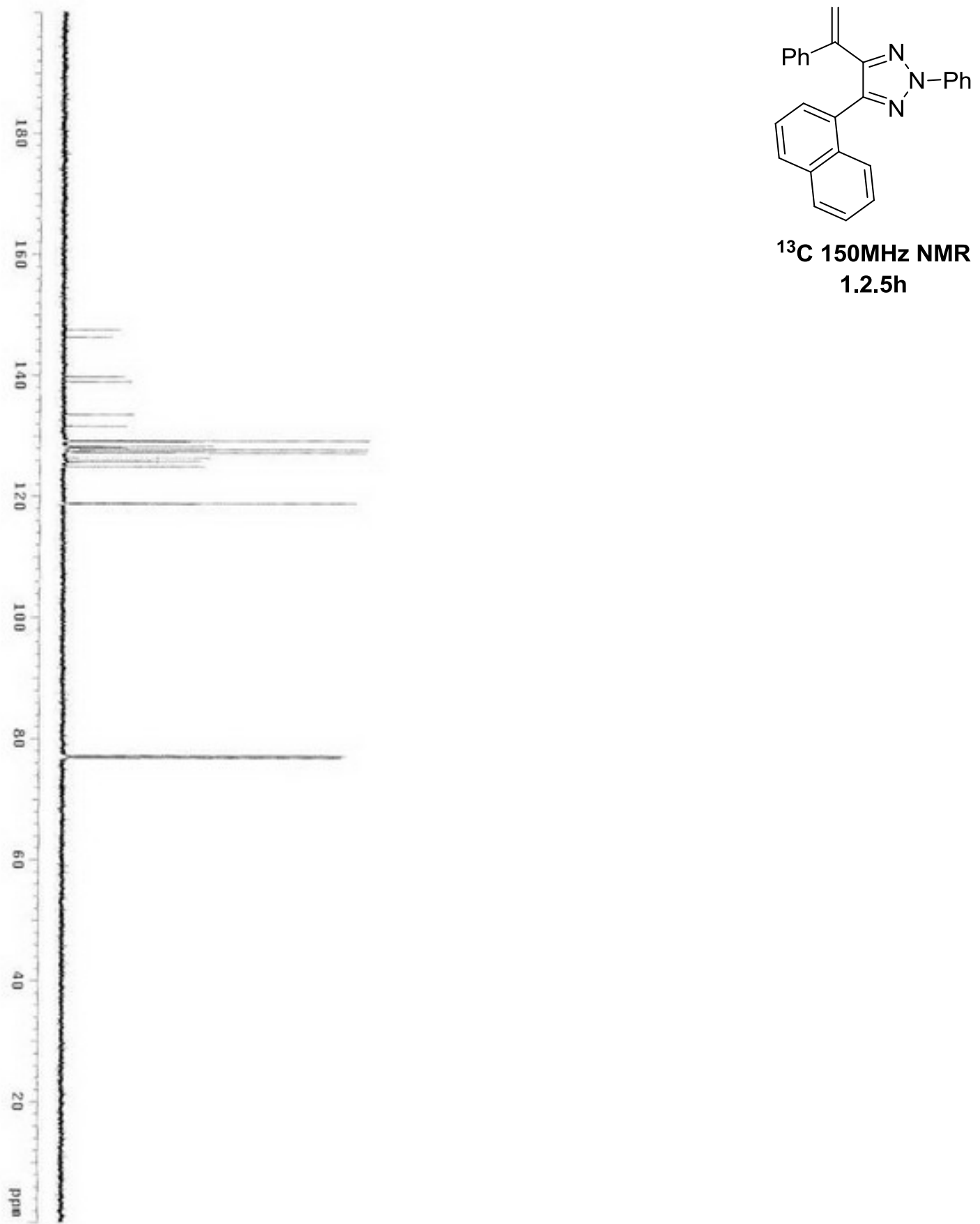

${ }^{13} \mathrm{C} 150 \mathrm{MHz}$ NMR 1.2.5h 


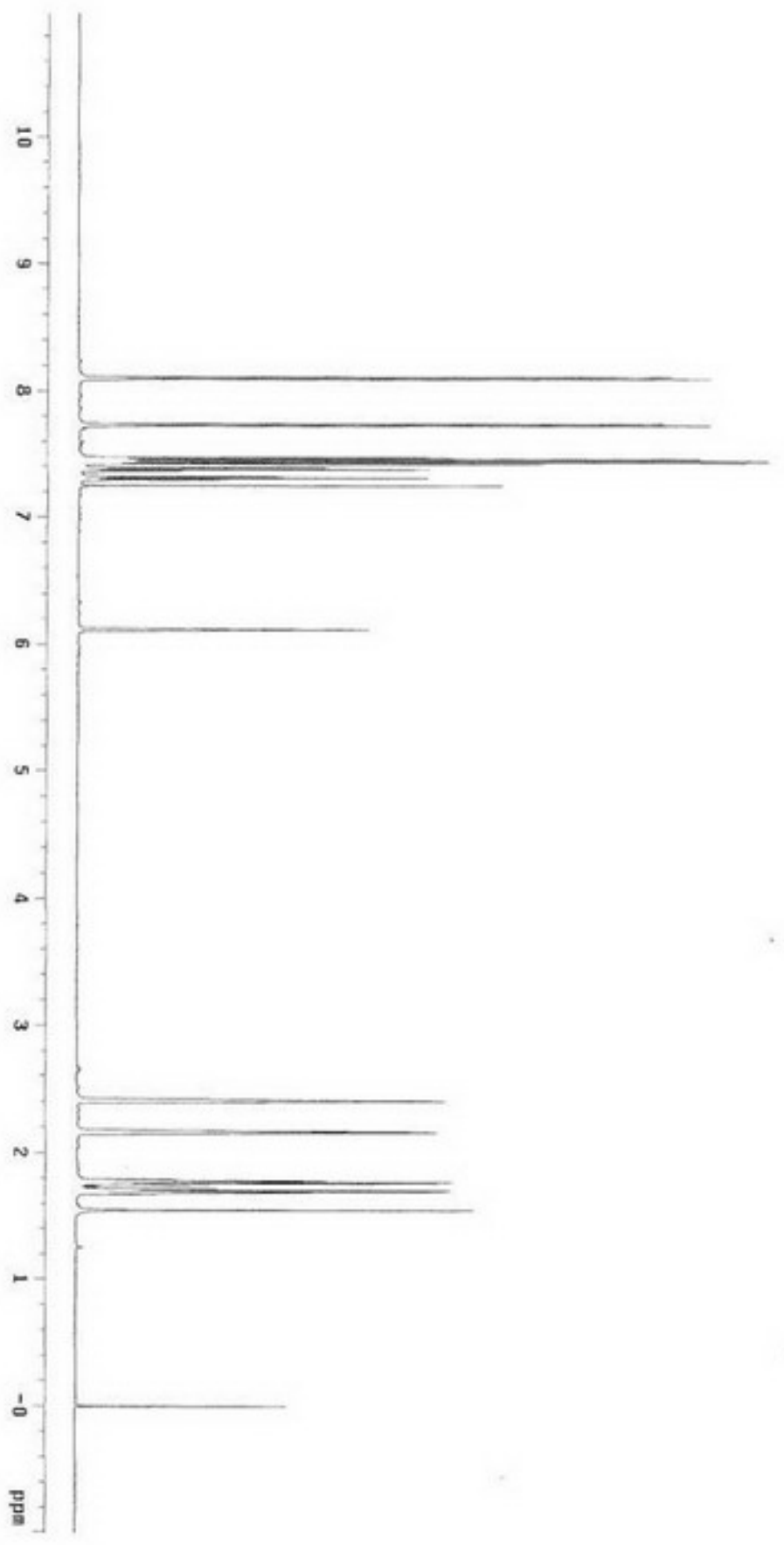

$\overbrace{N}^{N}=N_{N}^{N-P h}$

${ }^{1} \mathrm{H}$ 600MHz NMR

1.2.5i 

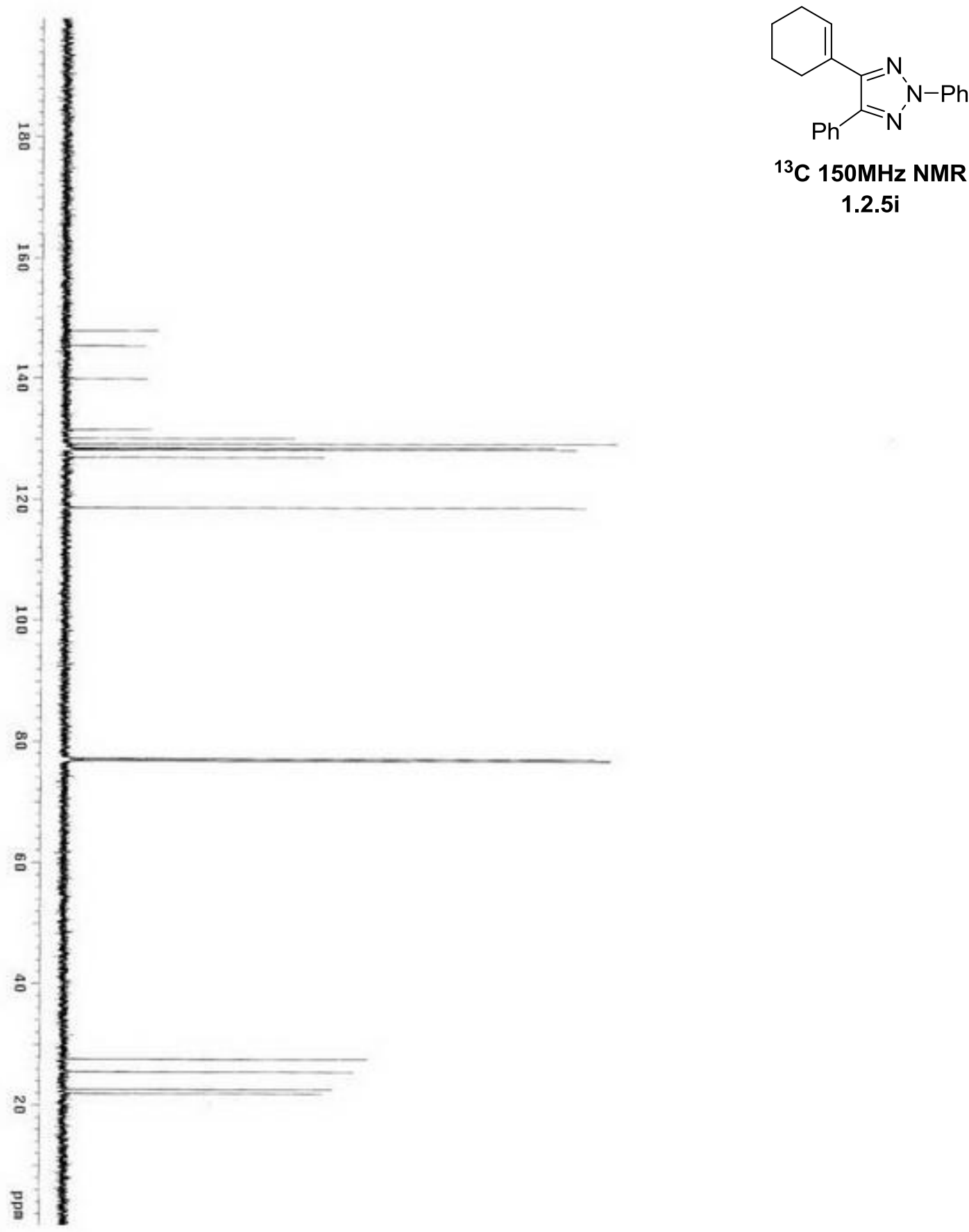

${ }^{13} \mathrm{C}$ 150MHz NMR

1.2.5i 


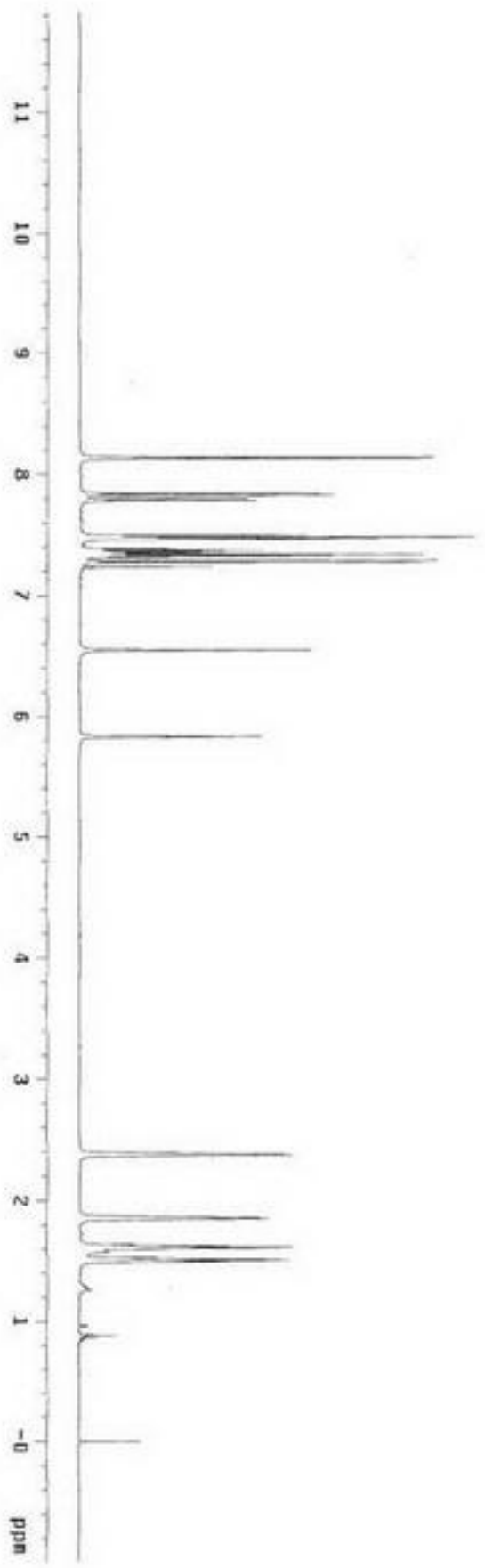

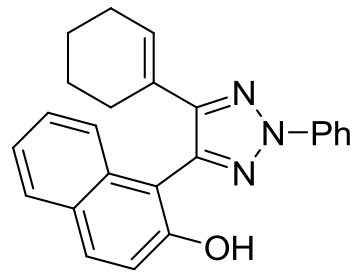

${ }^{1} \mathrm{H} 600 \mathrm{MHz}$ NMR 1.2.5j 

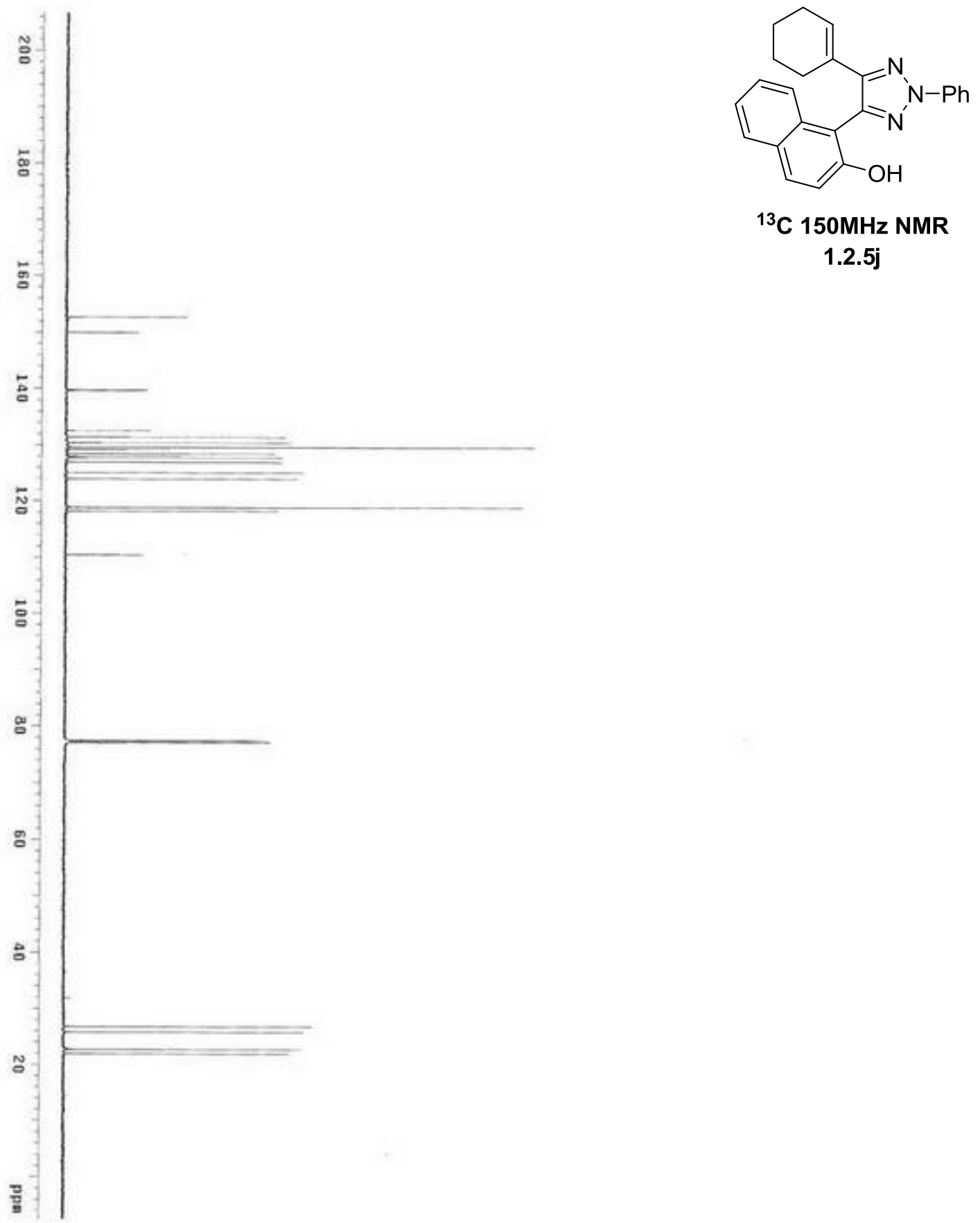

${ }^{13} \mathrm{C} 150 \mathrm{MHz}$ NMR

1.2.5j 


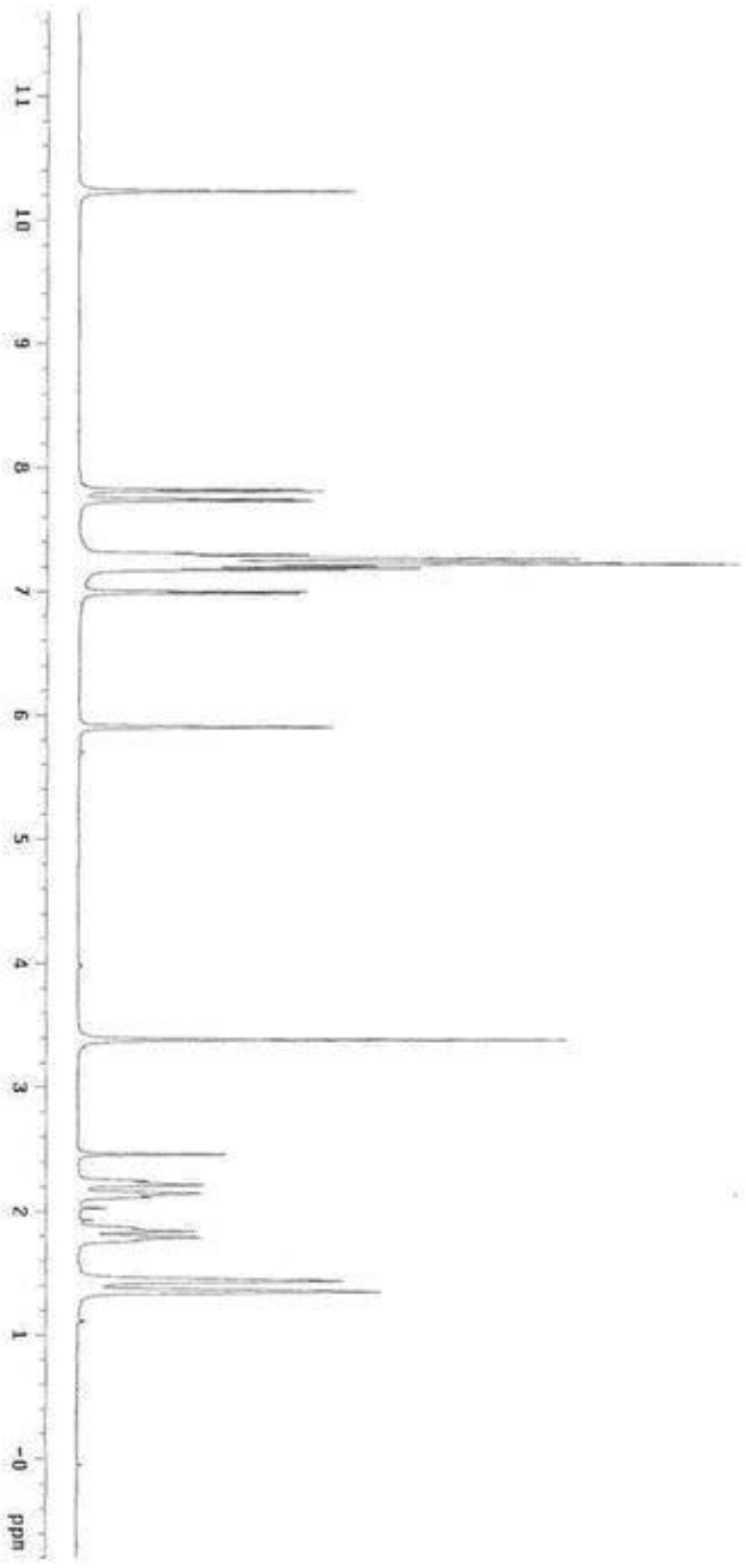

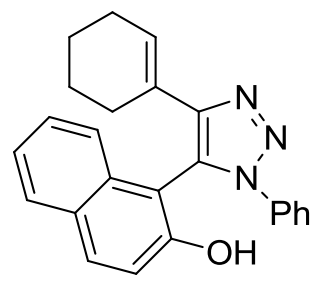

${ }^{1} \mathrm{H}$ 600MHz NMR

N-3-1.2.5j 

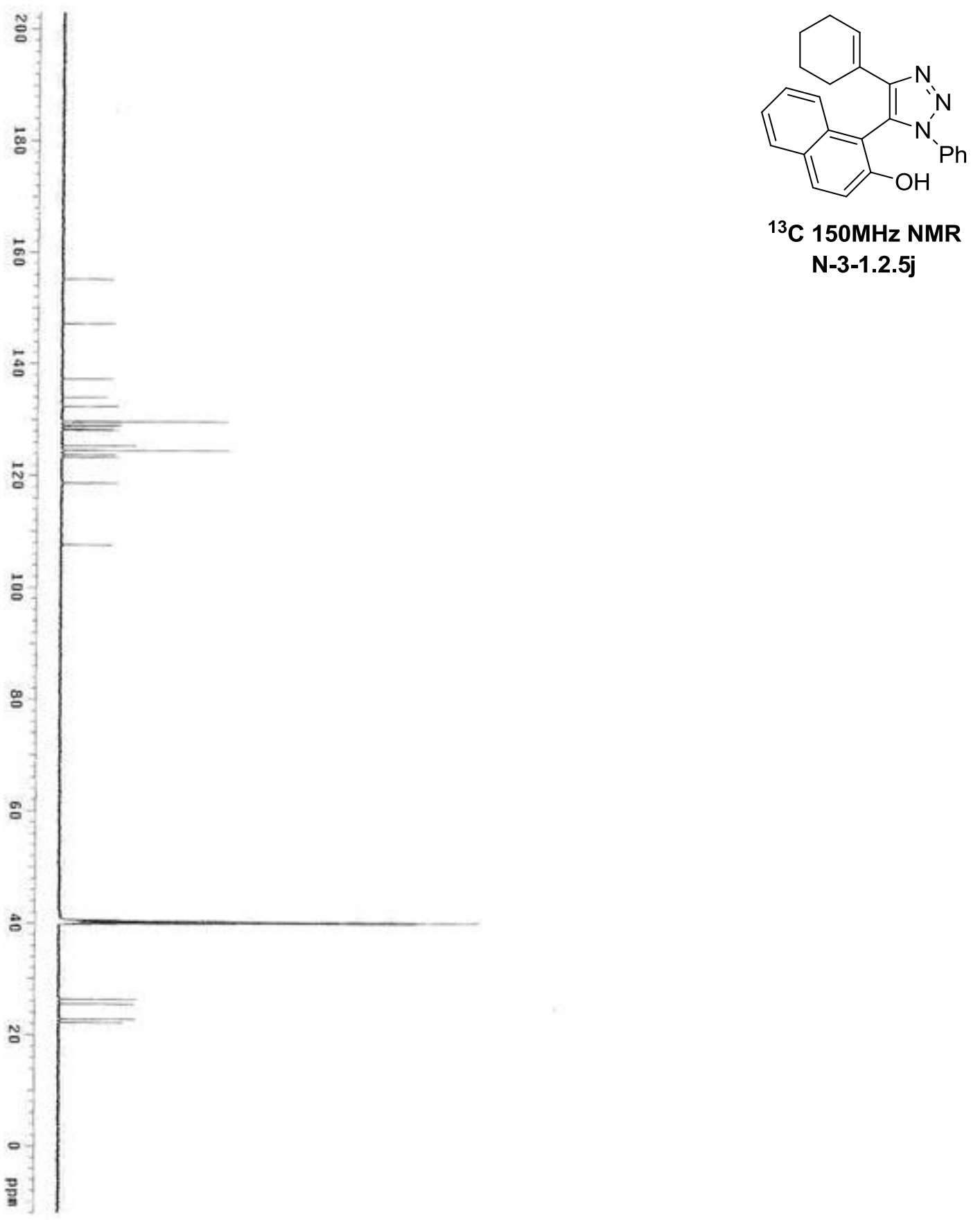

${ }^{13} \mathrm{C} 150 \mathrm{MHz}$ NMR N-3-1.2.5j 


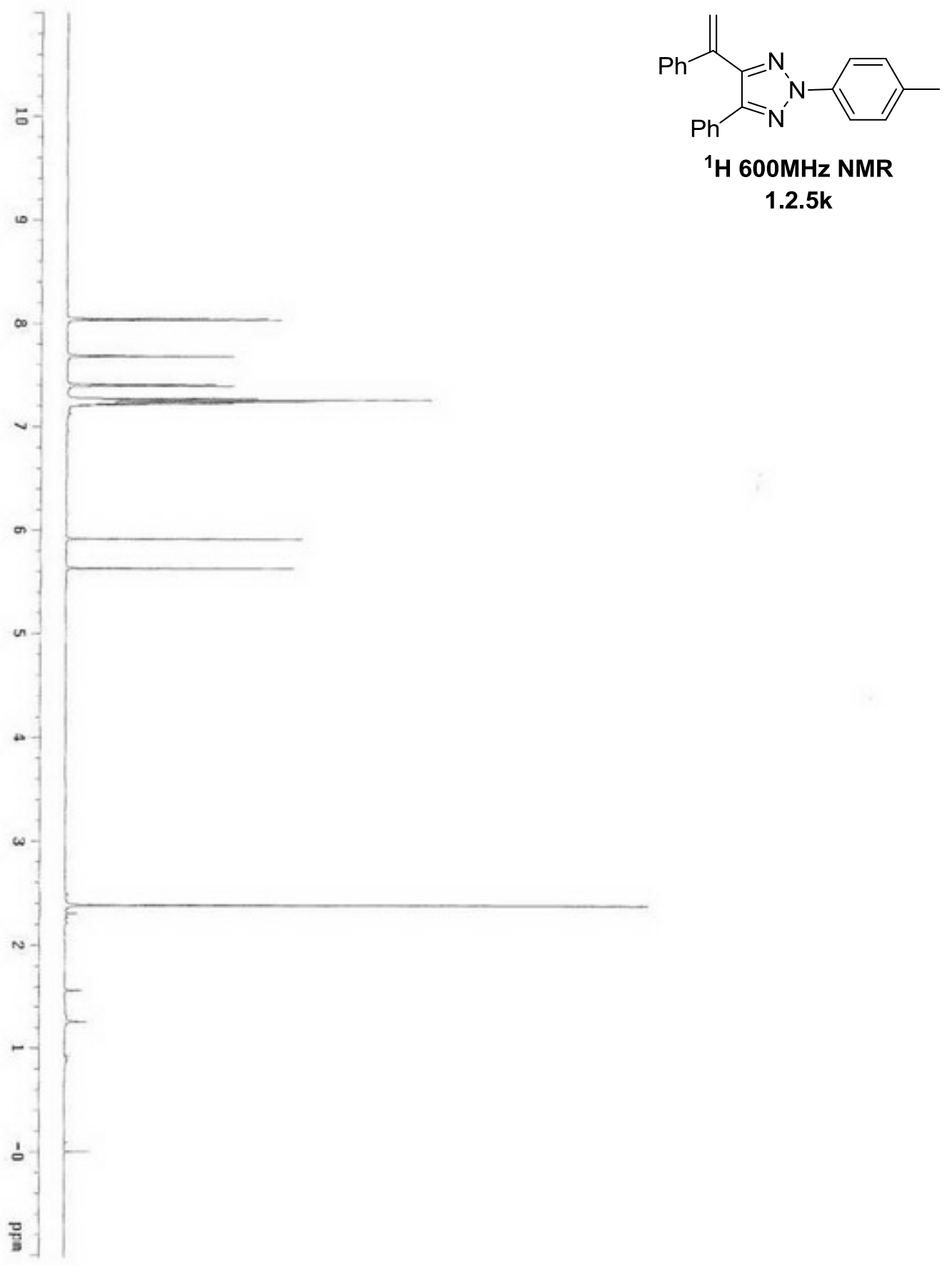




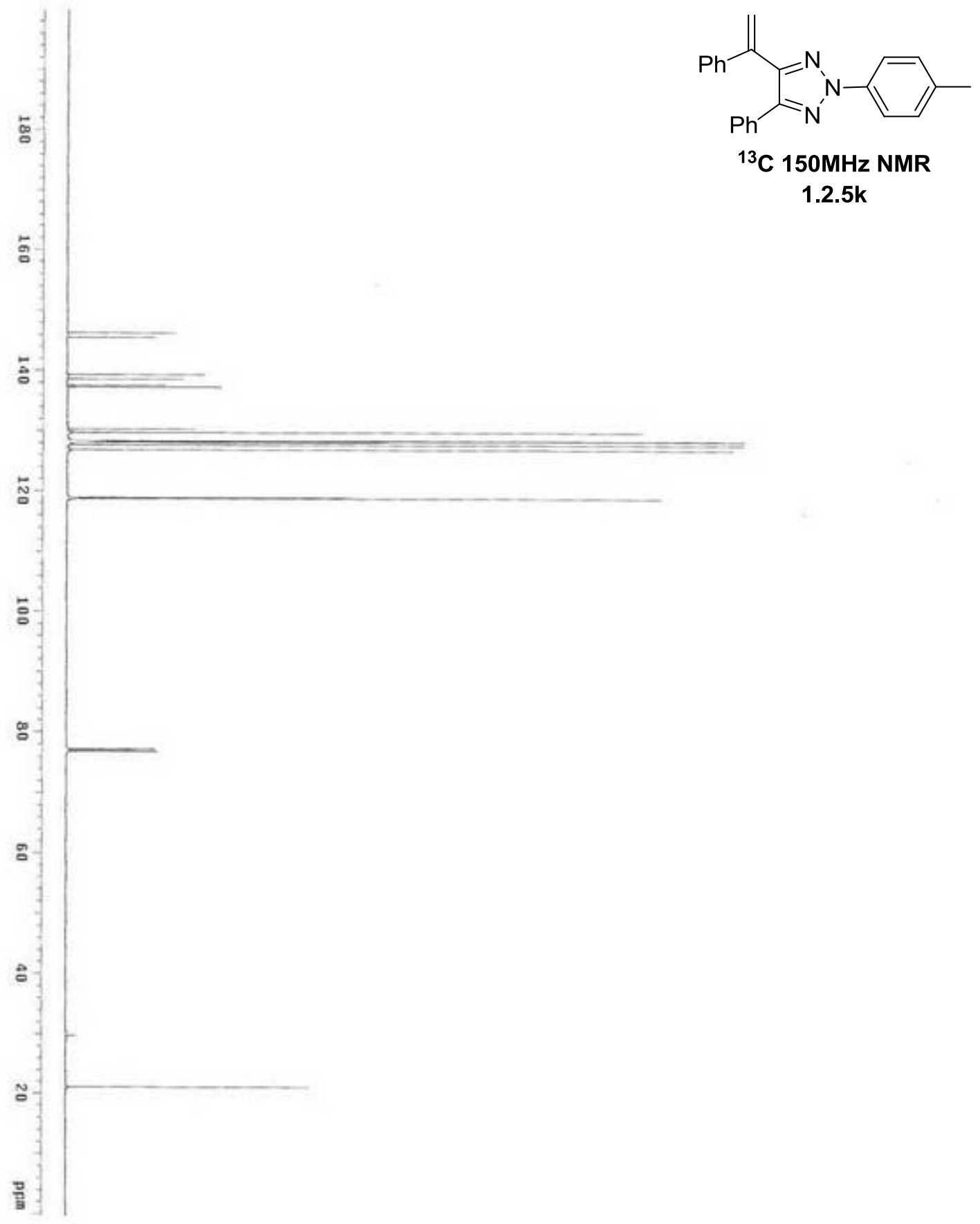




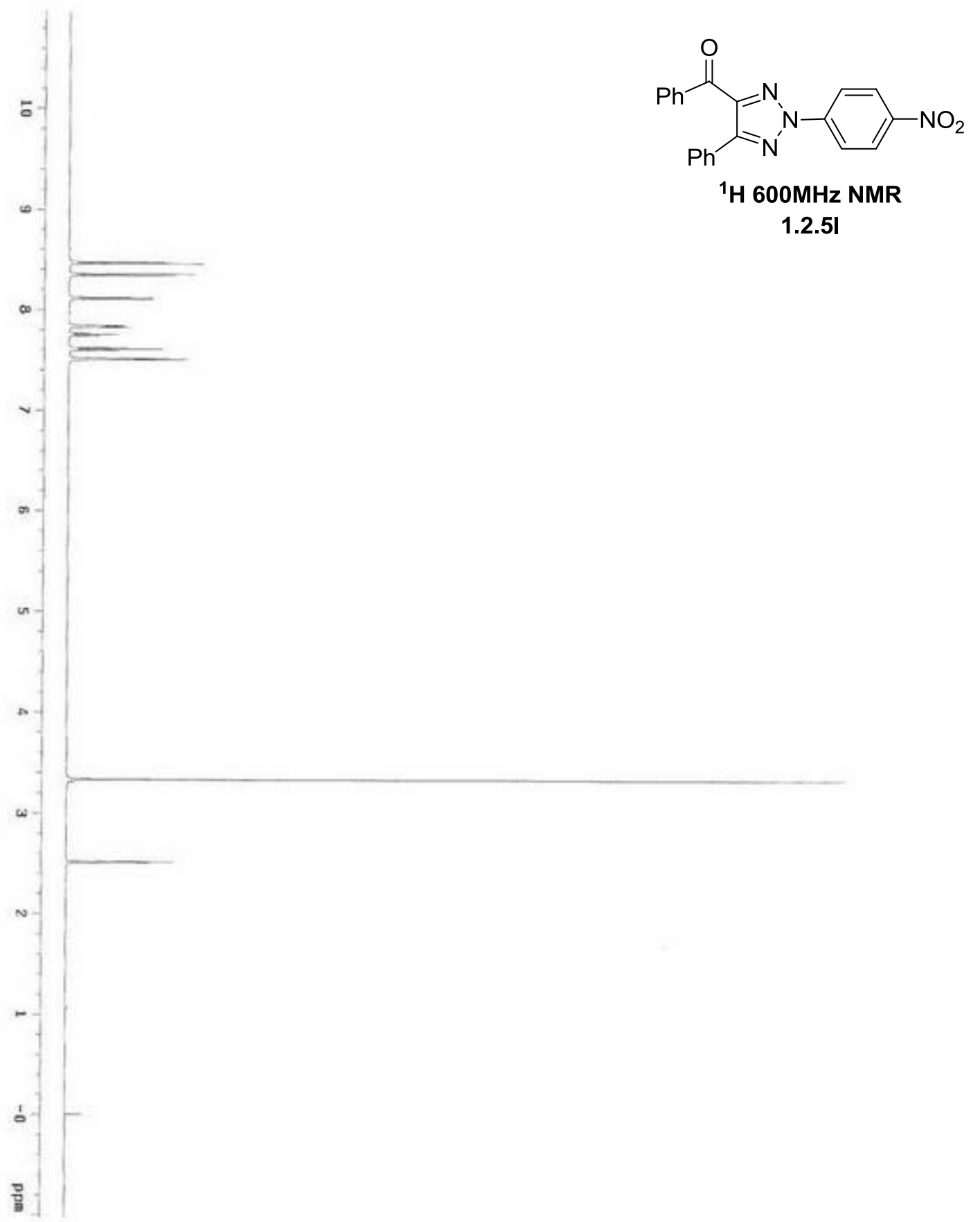




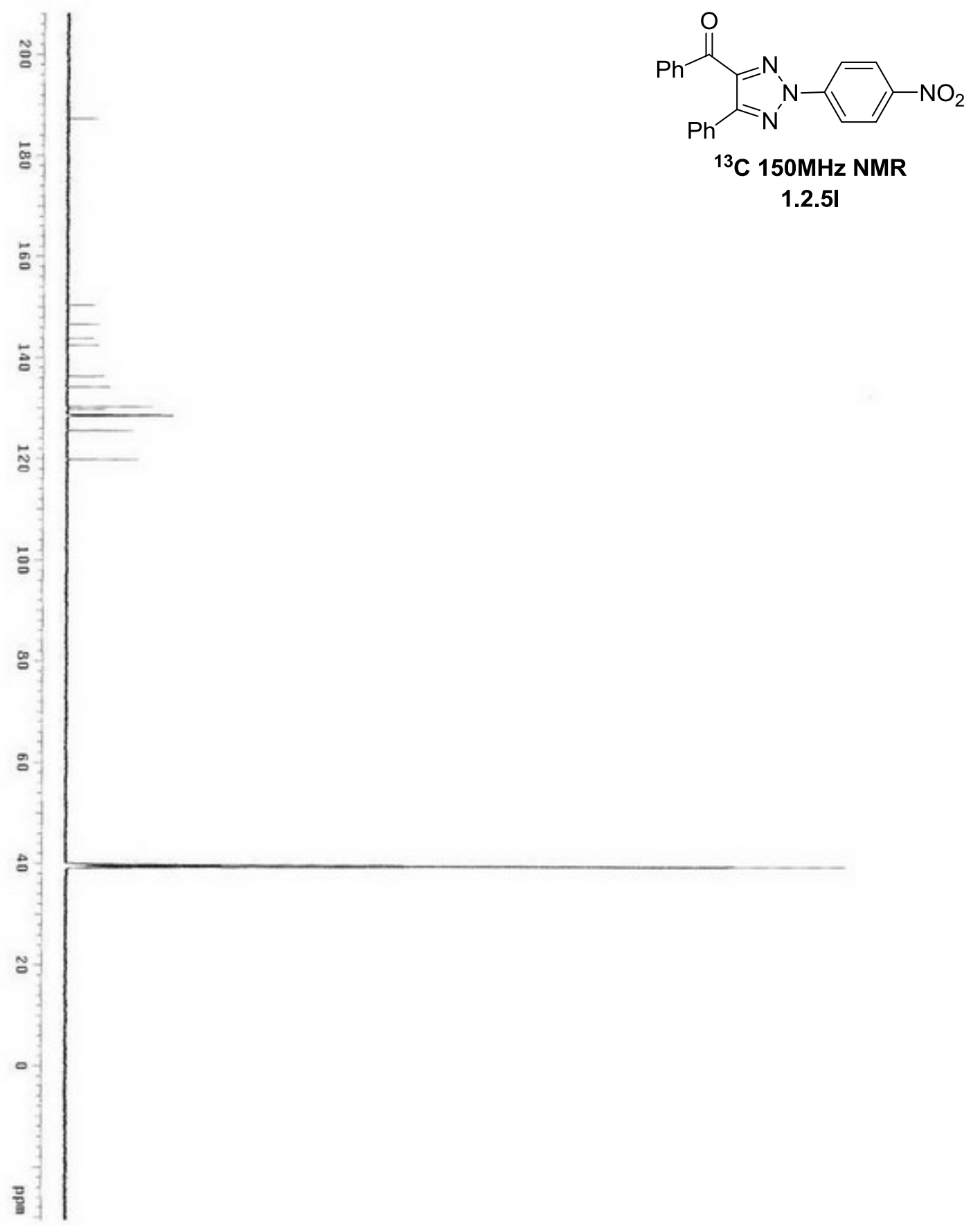




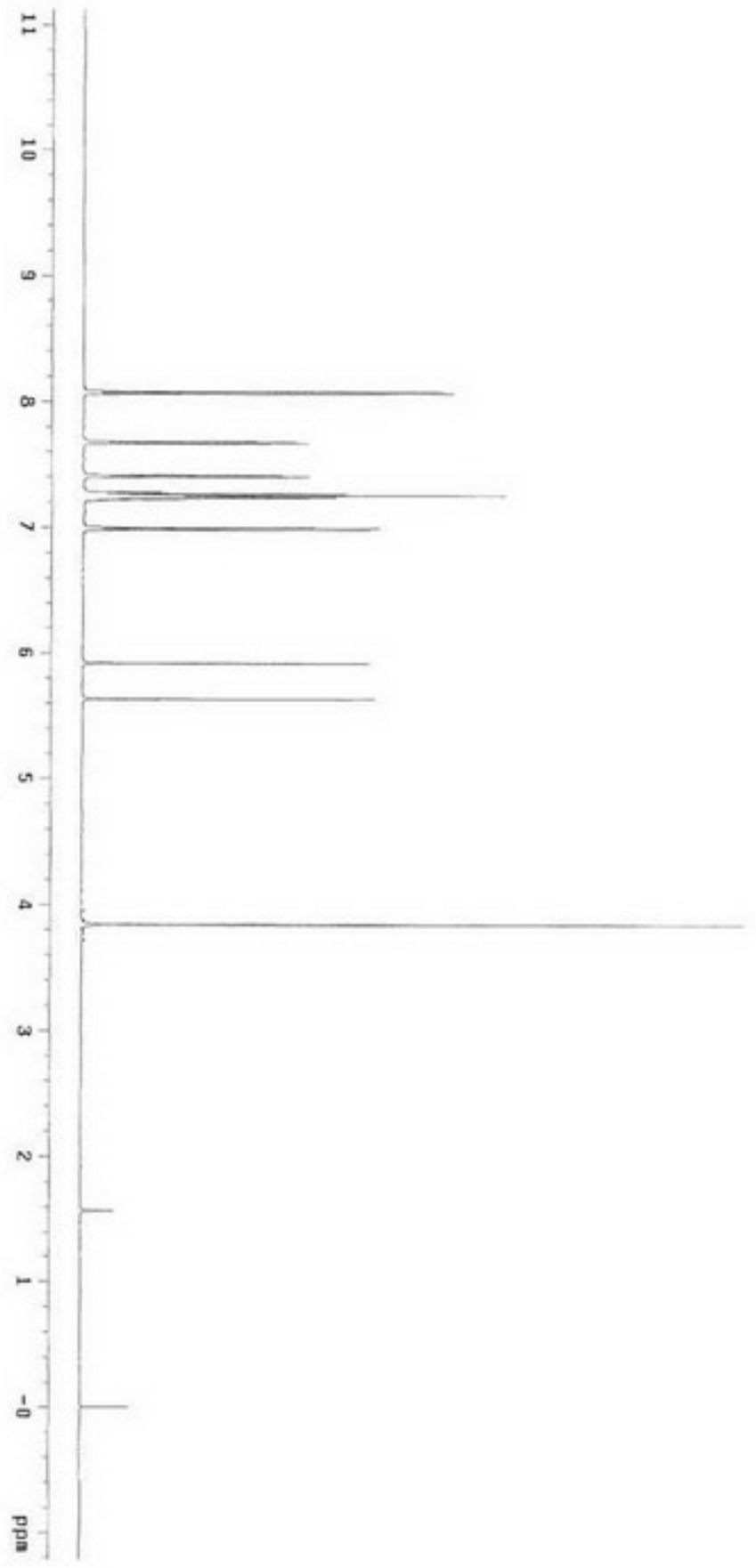

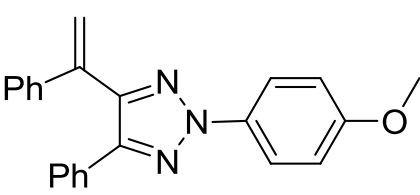

${ }^{1} \mathrm{H}$ 600MHz NMR

$1.2 .5 \mathrm{~m}$ 


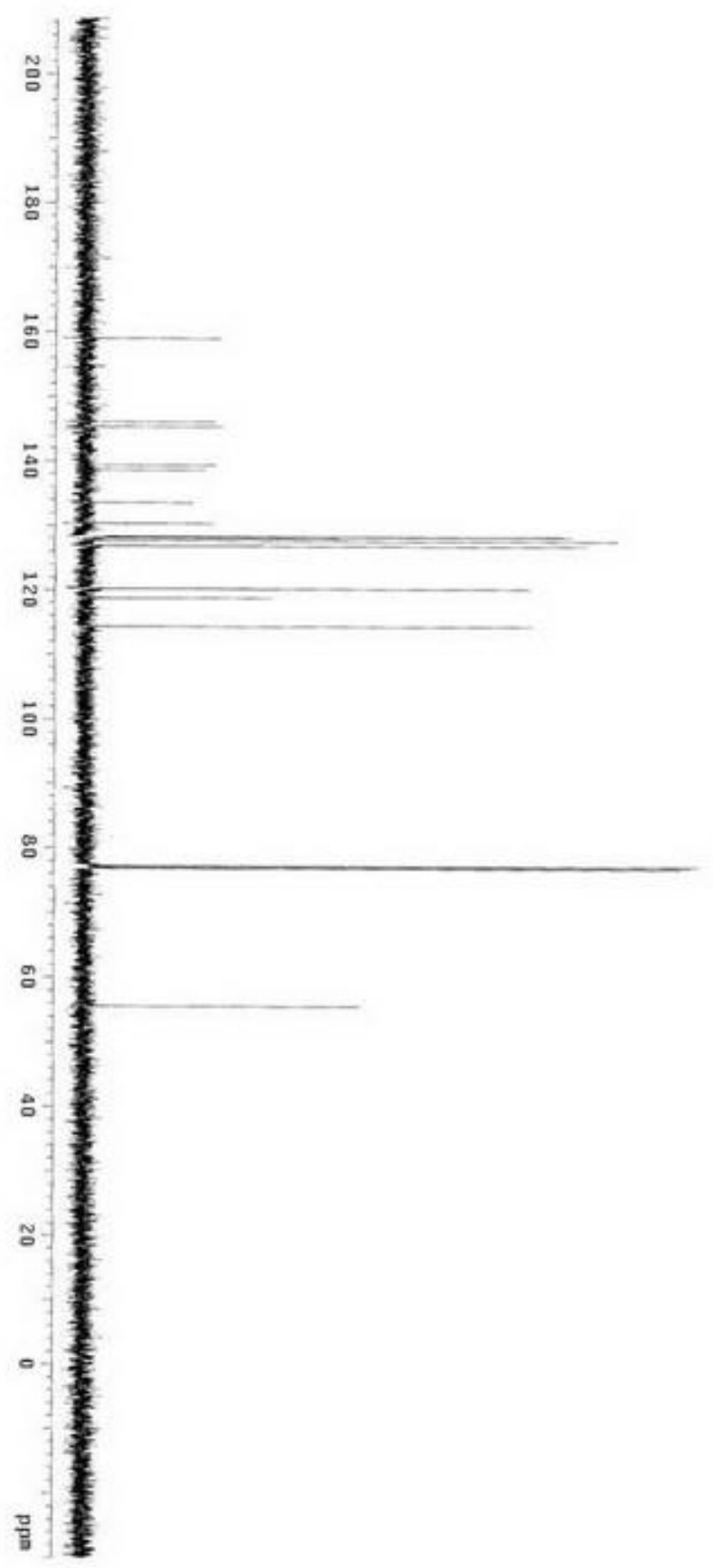

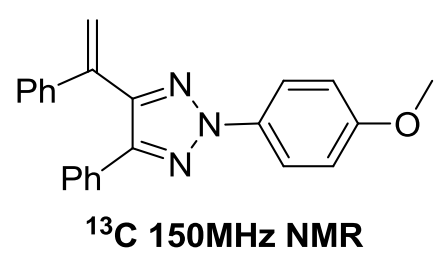

$1.2 .5 \mathrm{~m}$ 

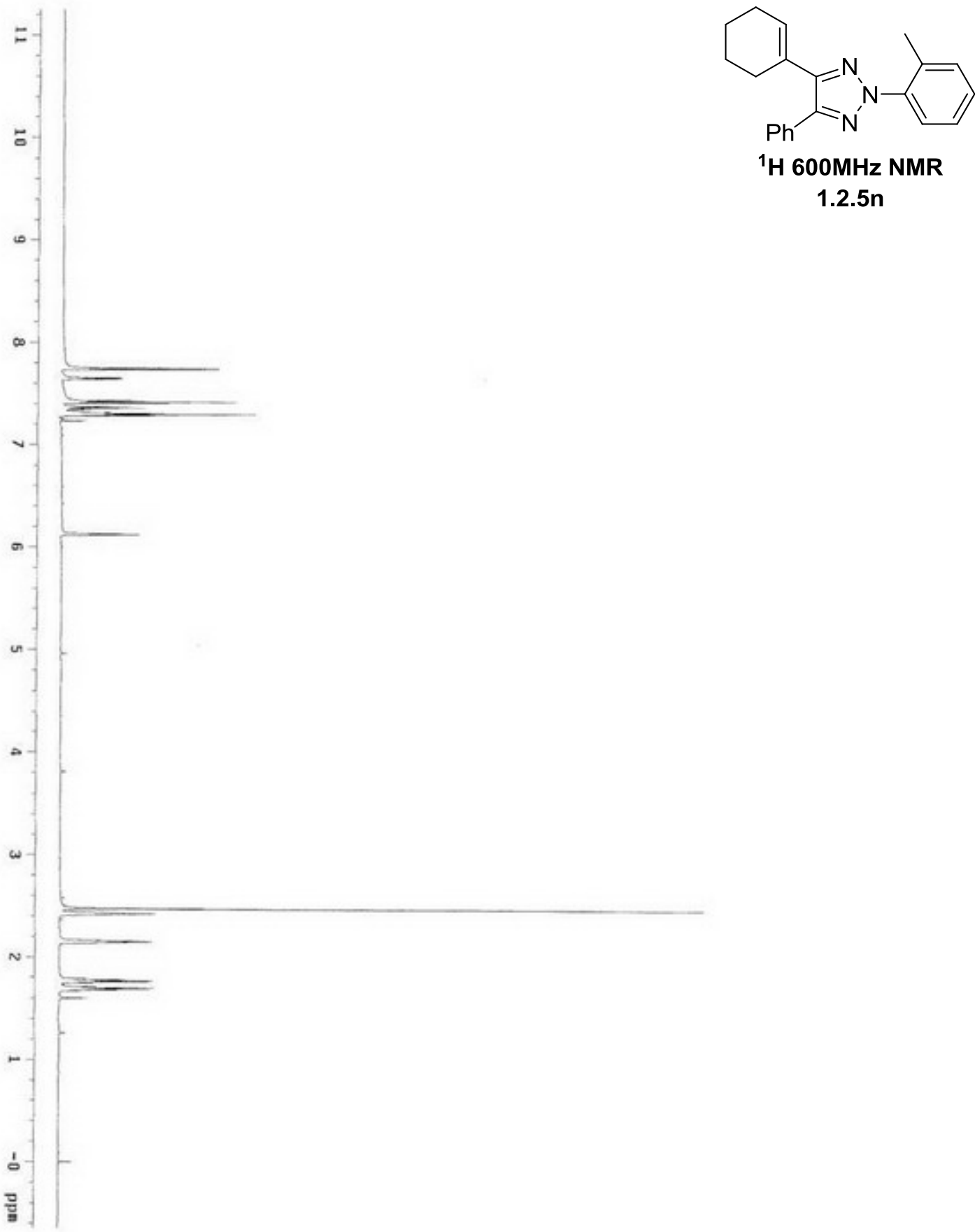

${ }^{1} \mathrm{H} 600 \mathrm{MHz}$ NMR

1.2.5n 

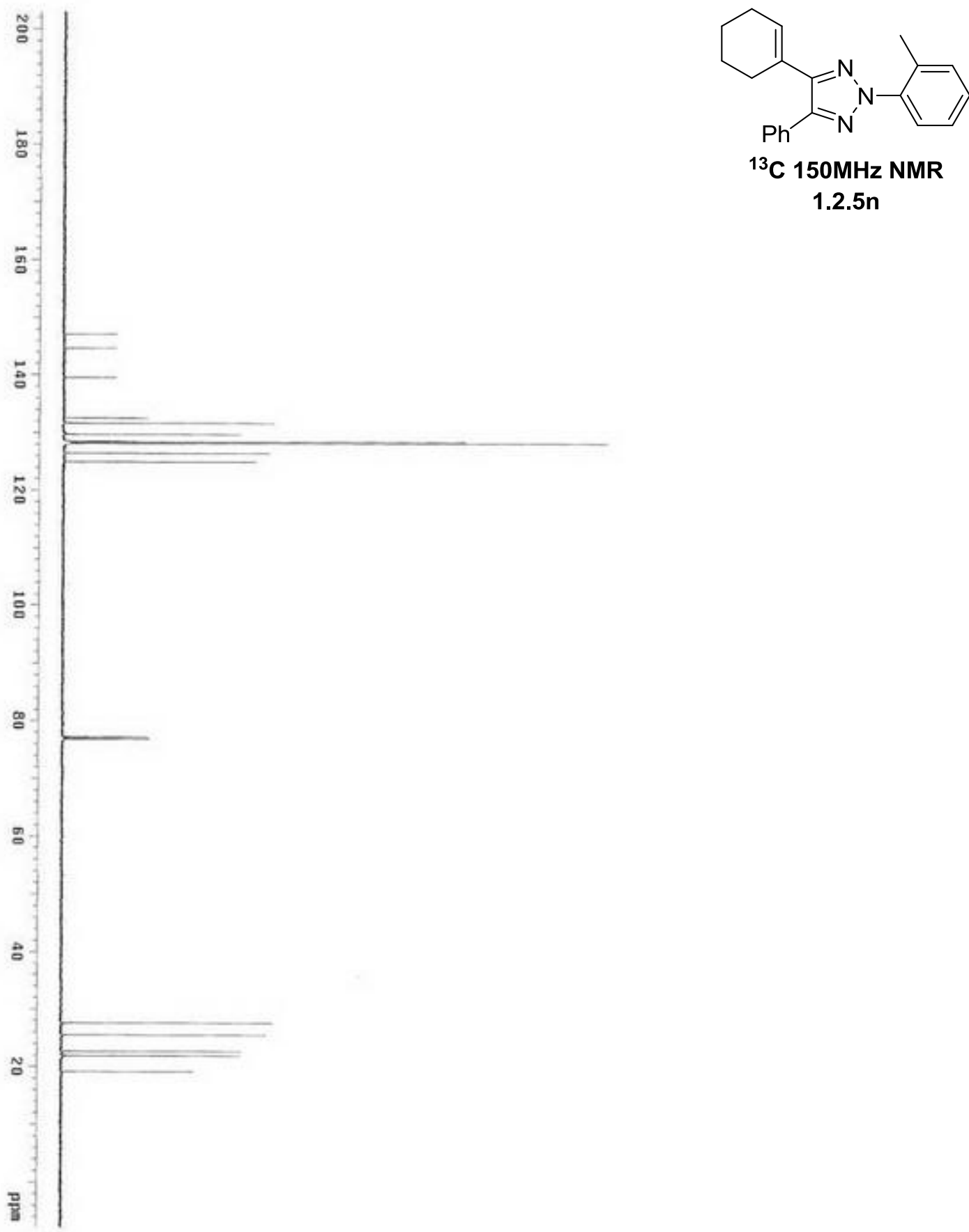

${ }^{13} \mathrm{C} 150 \mathrm{MHz}$ NMR

1.2.5n 


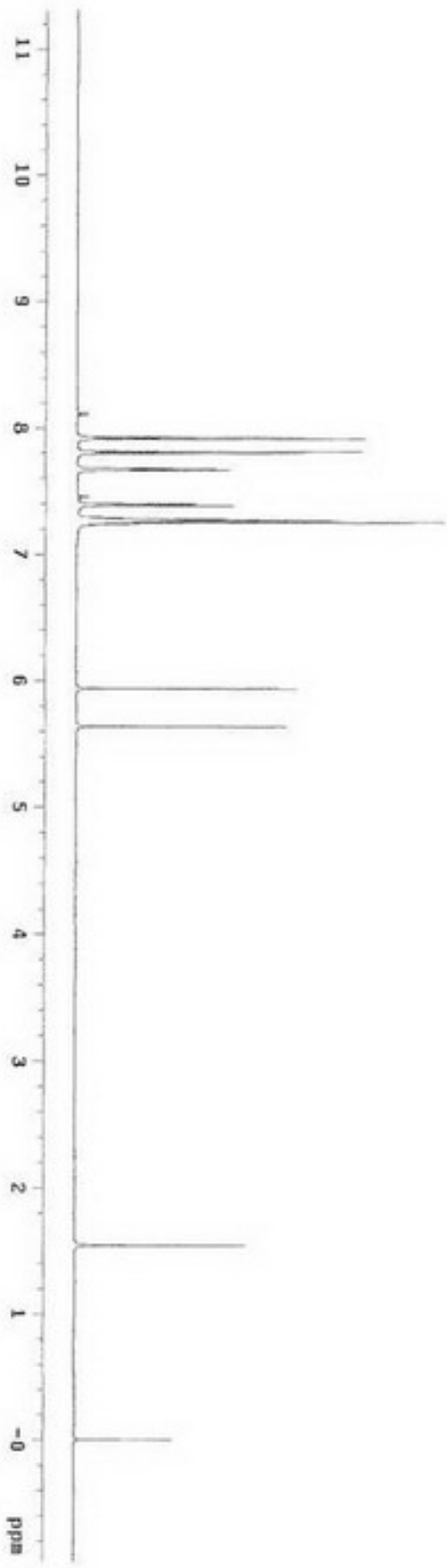

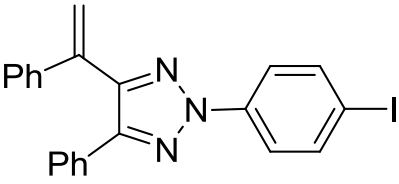

${ }^{1} \mathrm{H}$ 600MHz NMR

1.2 .50 


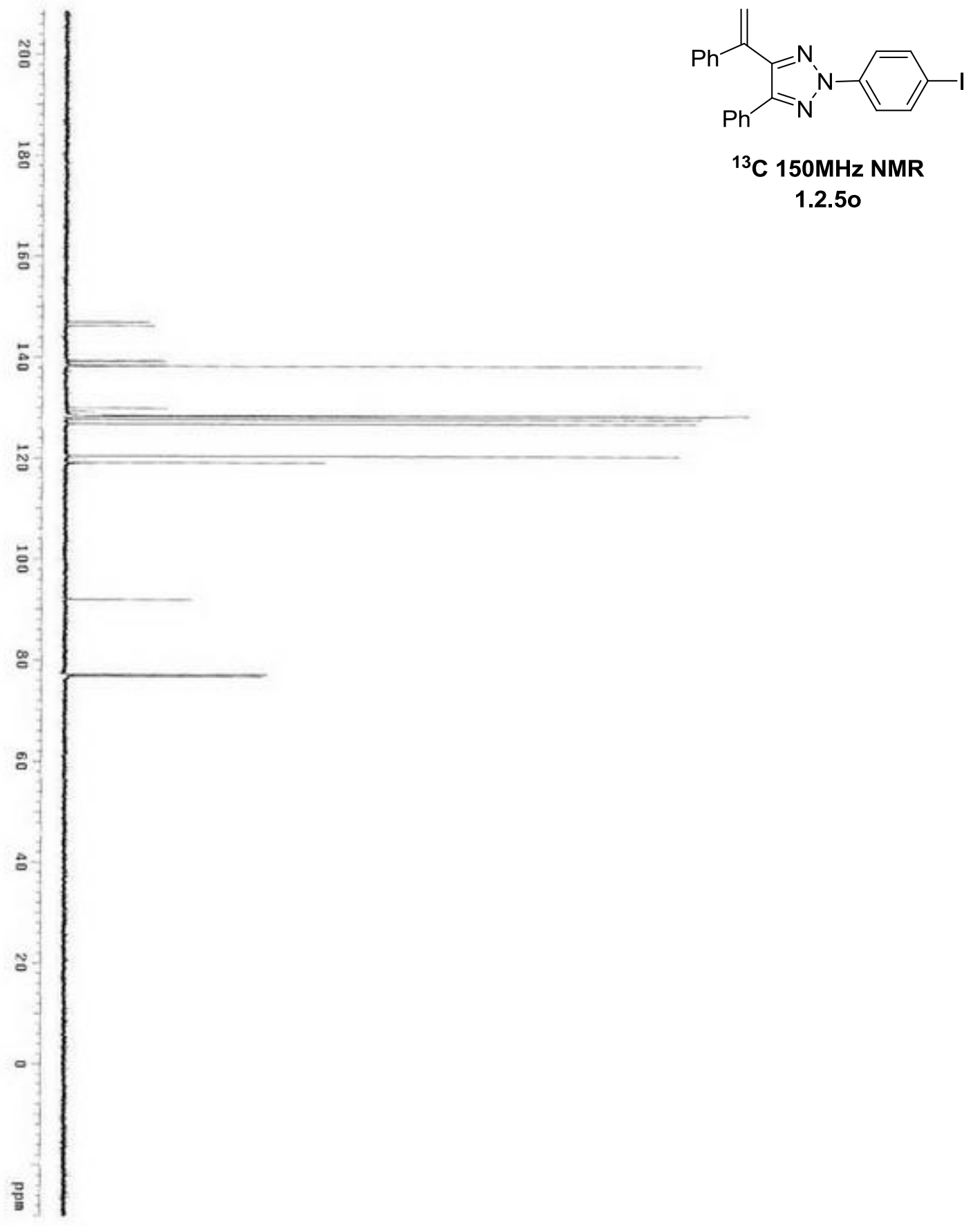



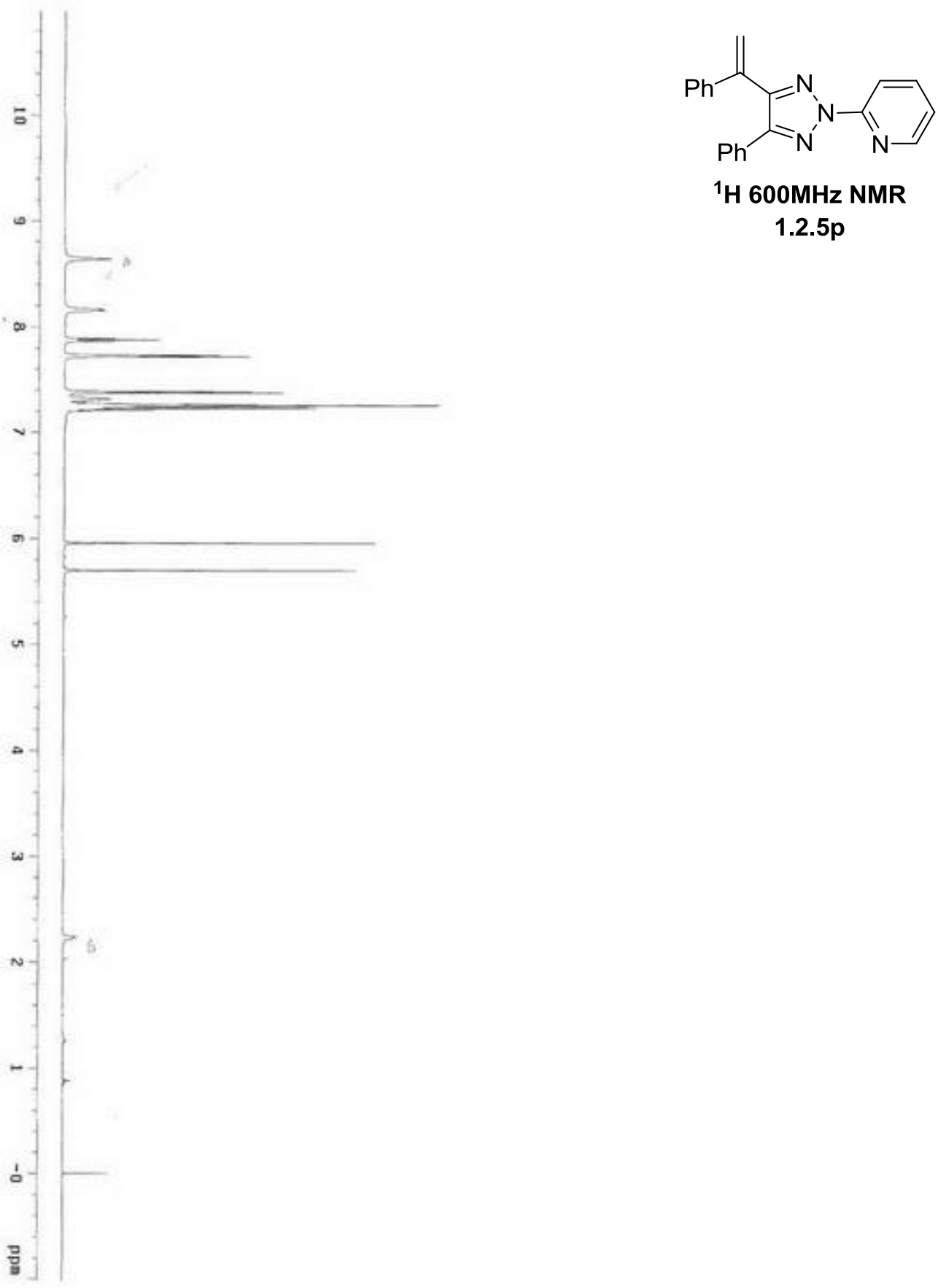

${ }^{1} \mathrm{H}$ 600MHz NMR

$1.2 .5 p$ 


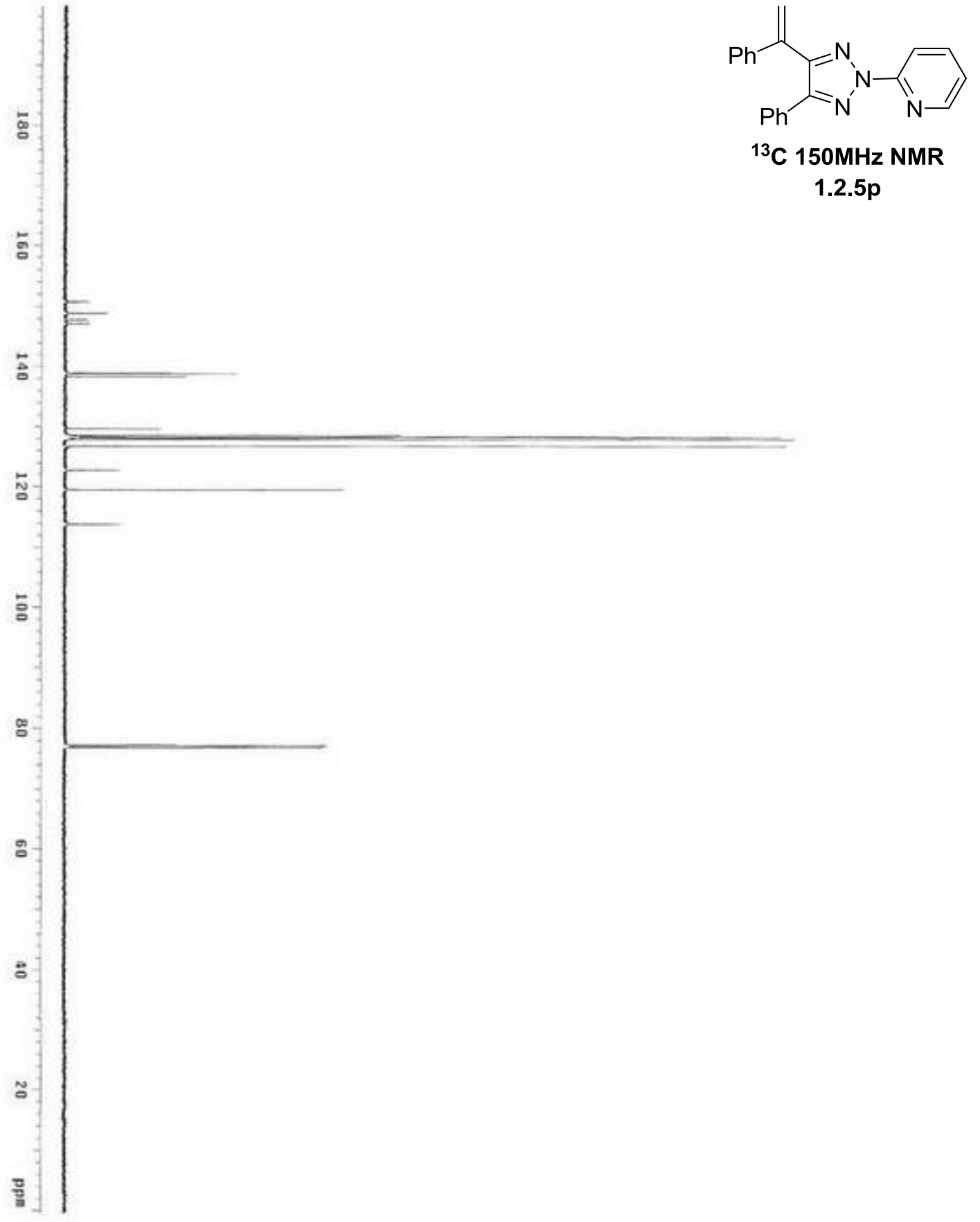




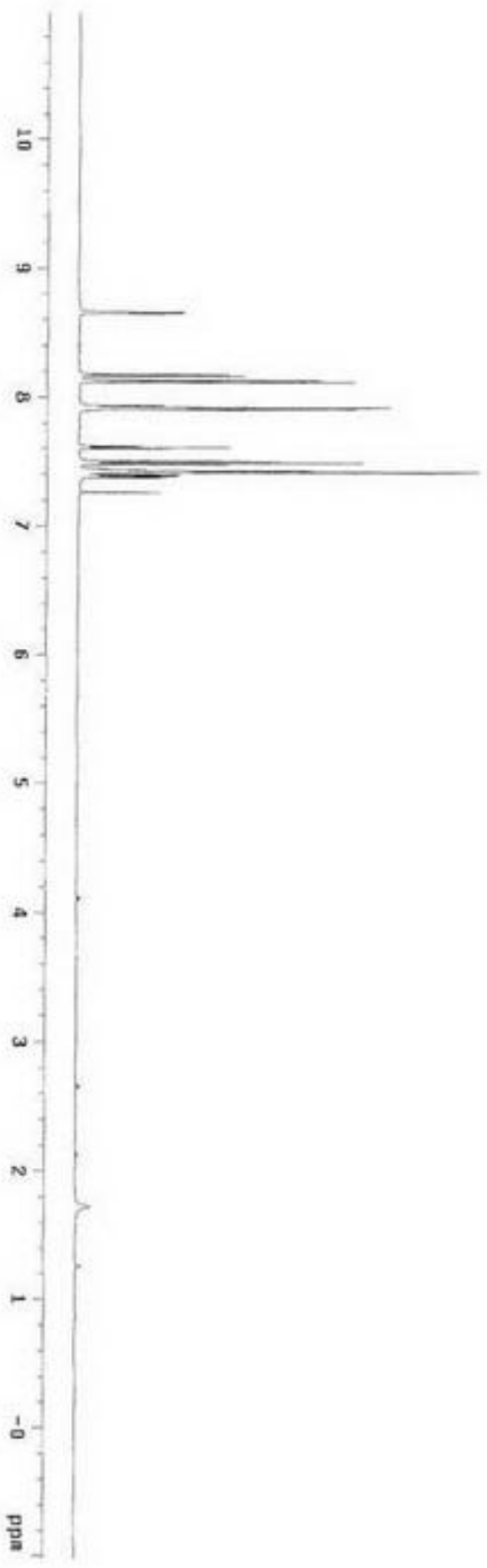

${ }^{1} \mathrm{H} 600 \mathrm{MHz}$ NMR

$1.2 .5 q$ 

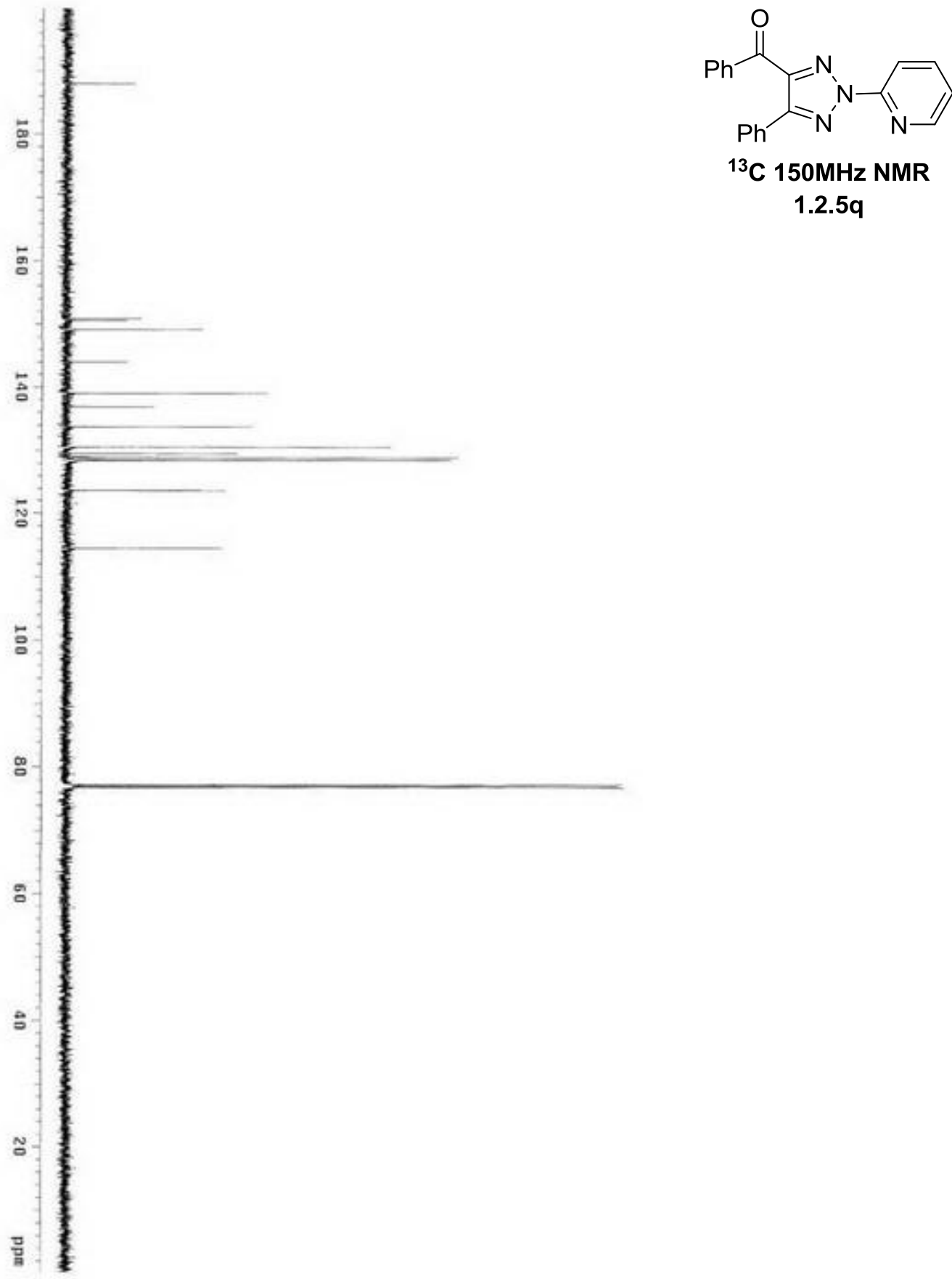

${ }^{13} \mathrm{C} 150 \mathrm{MHz}$ NMR

1.2.5q 

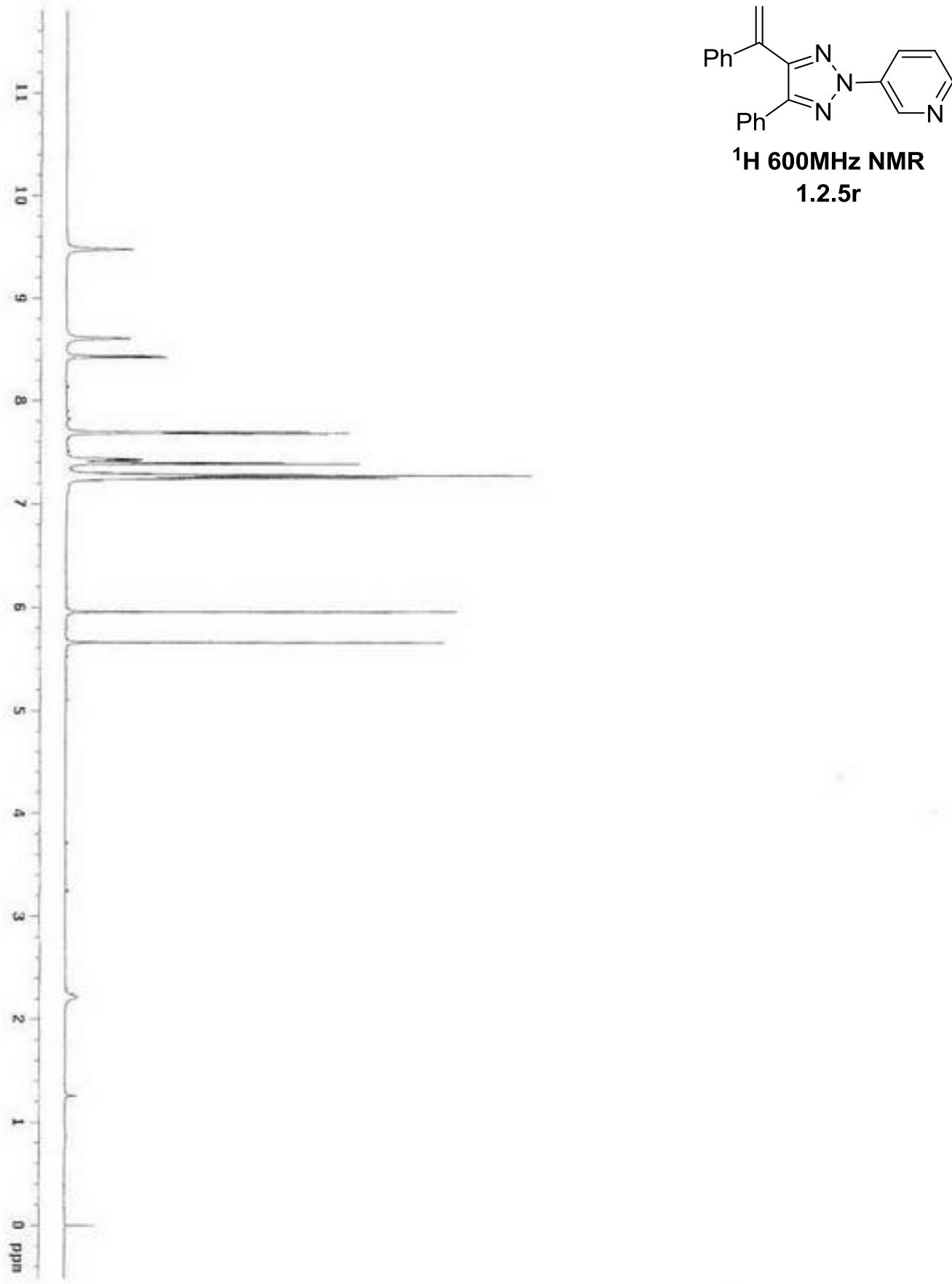

${ }^{1} \mathrm{H} 600 \mathrm{MHz}$ NMR $1.2 .5 r$ 

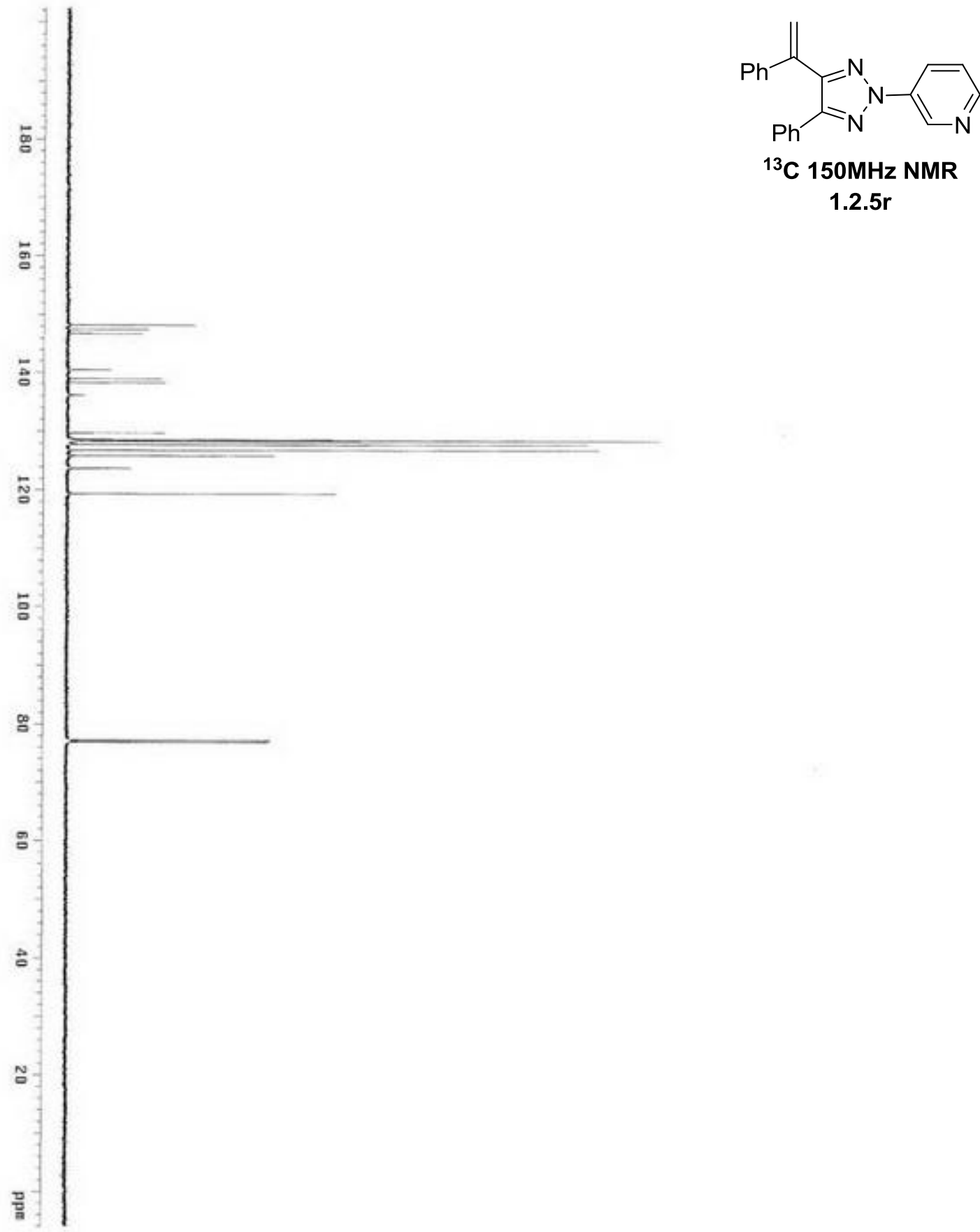

${ }^{13} \mathrm{C} 150 \mathrm{MHz}$ NMR

$1.2 .5 \mathrm{r}$ 

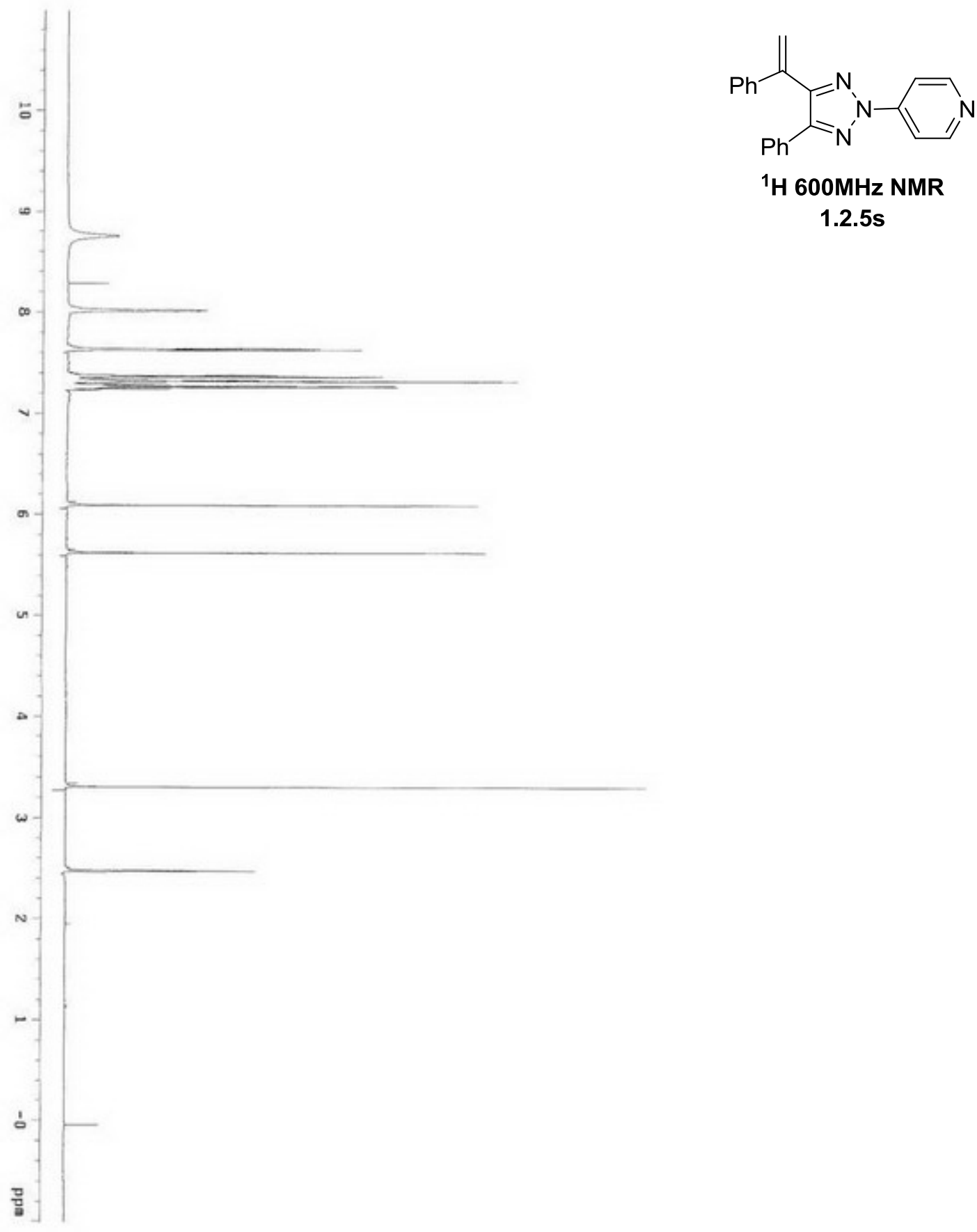

${ }^{1} \mathrm{H}$ 600MHz NMR

$1.2 .5 \mathrm{~s}$ 


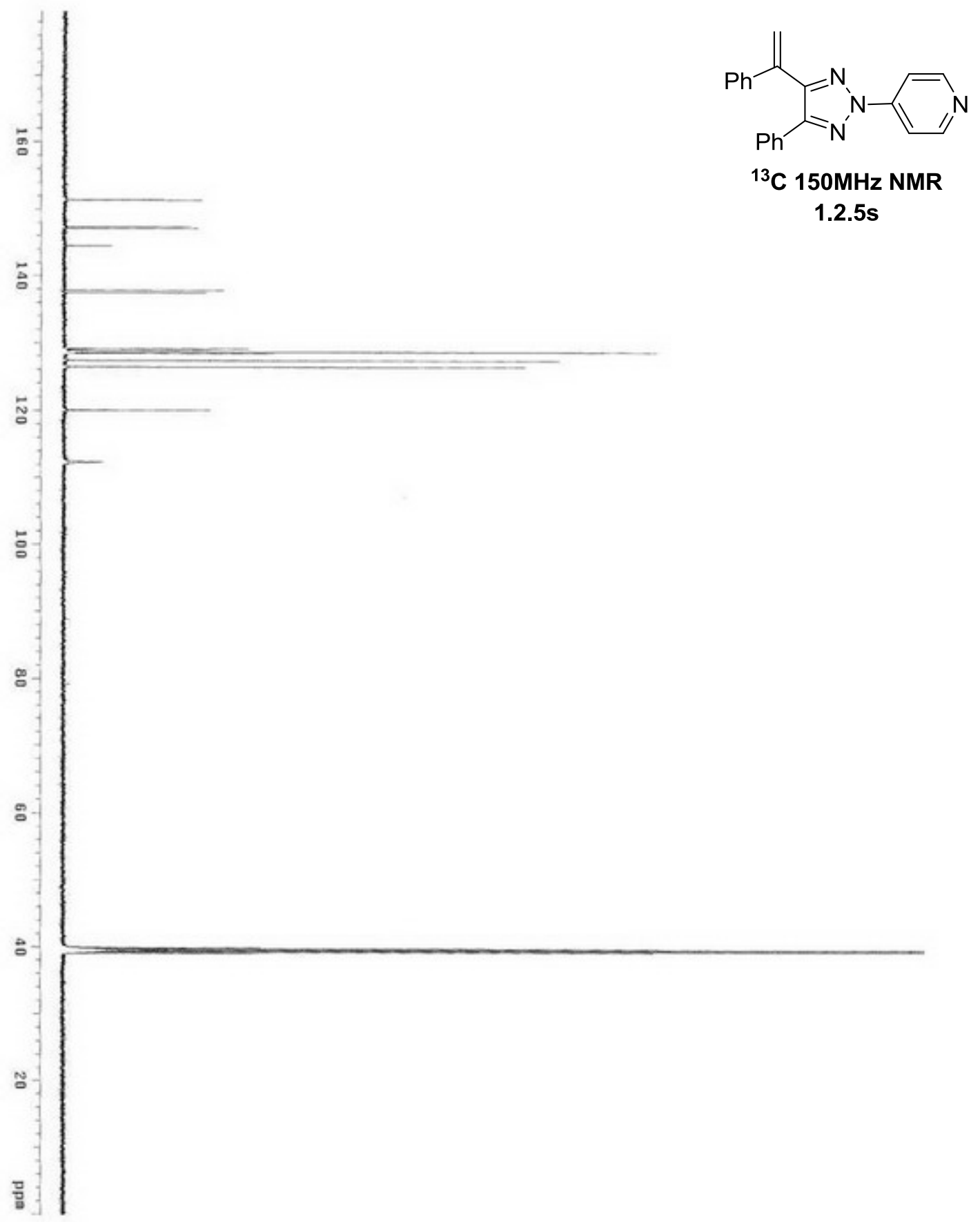




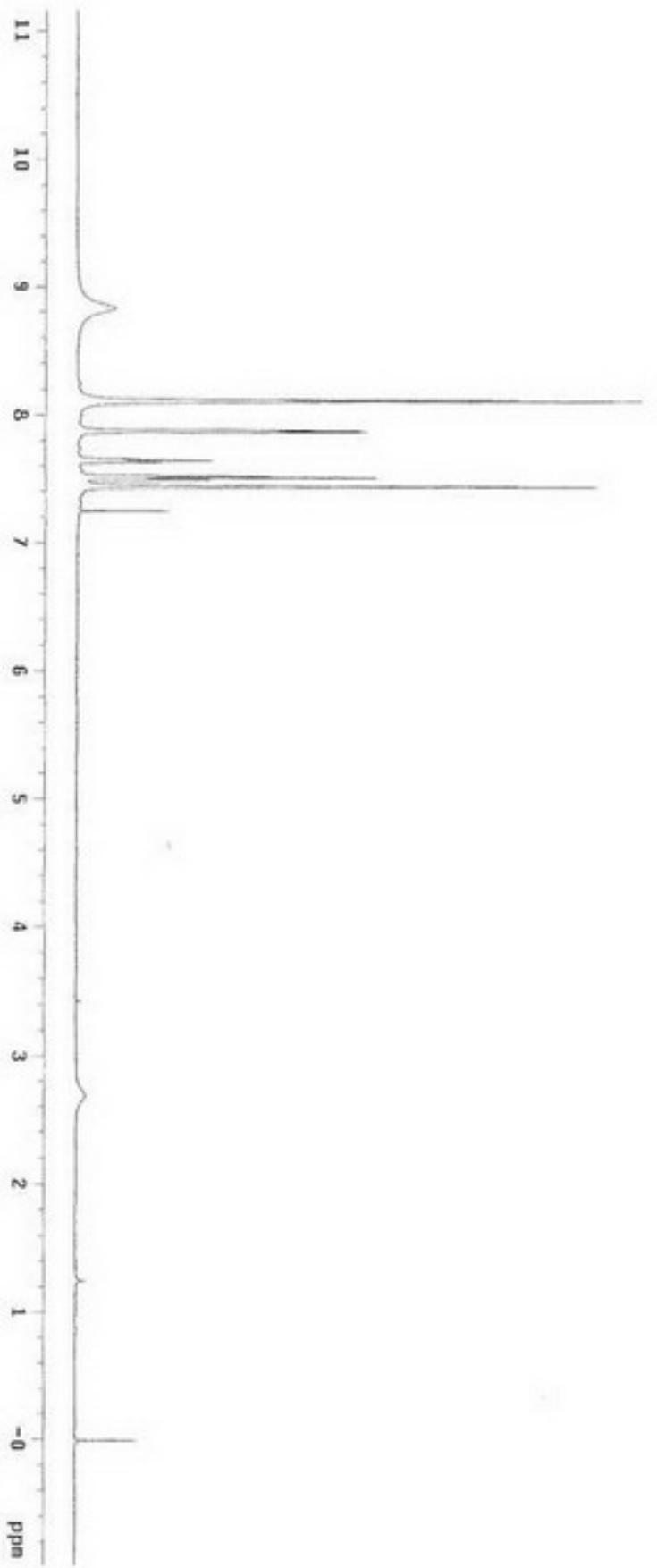

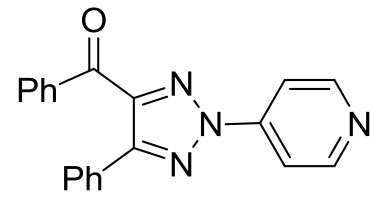

${ }^{1} \mathrm{H}$ 600MHz NMR

1.2.5t 


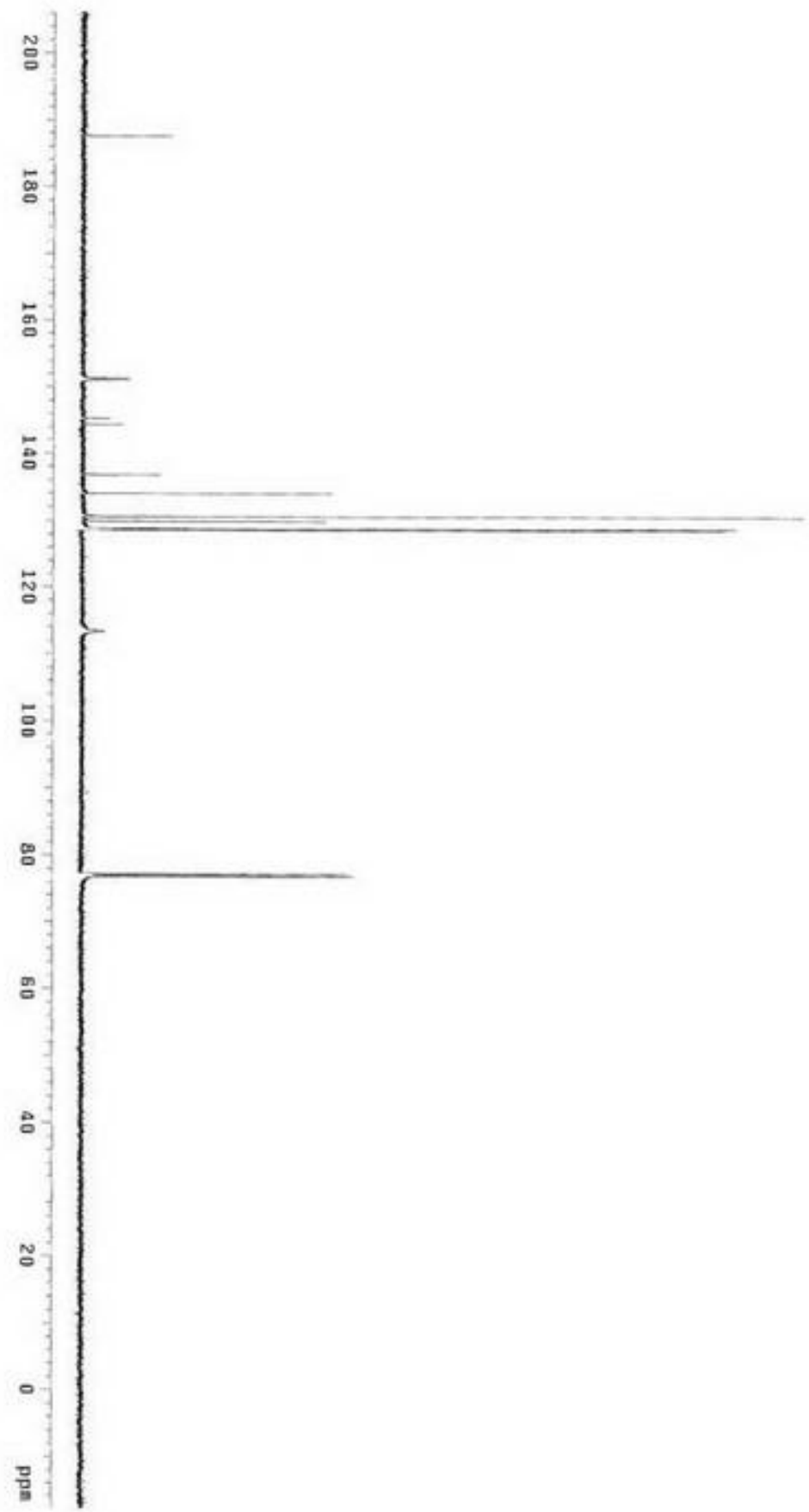

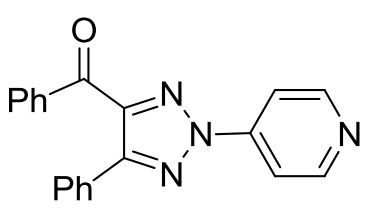

${ }^{13} \mathrm{C} 150 \mathrm{MHz}$ NMR

1.2.5t 


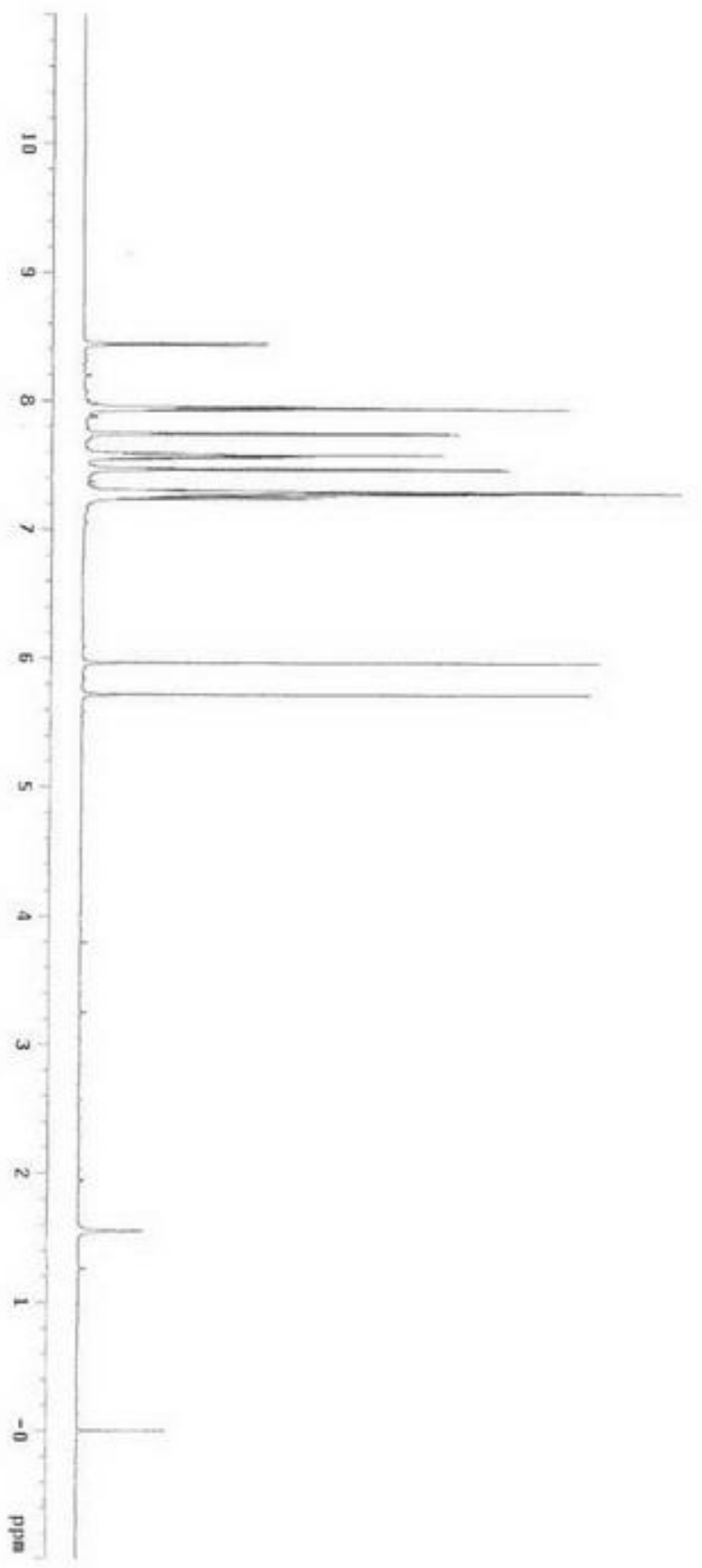

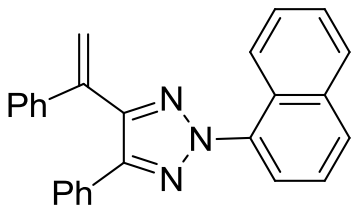

${ }^{1} \mathrm{H} 600 \mathrm{MHz}$ NMR

$1.2 .5 \mathrm{u}$ 

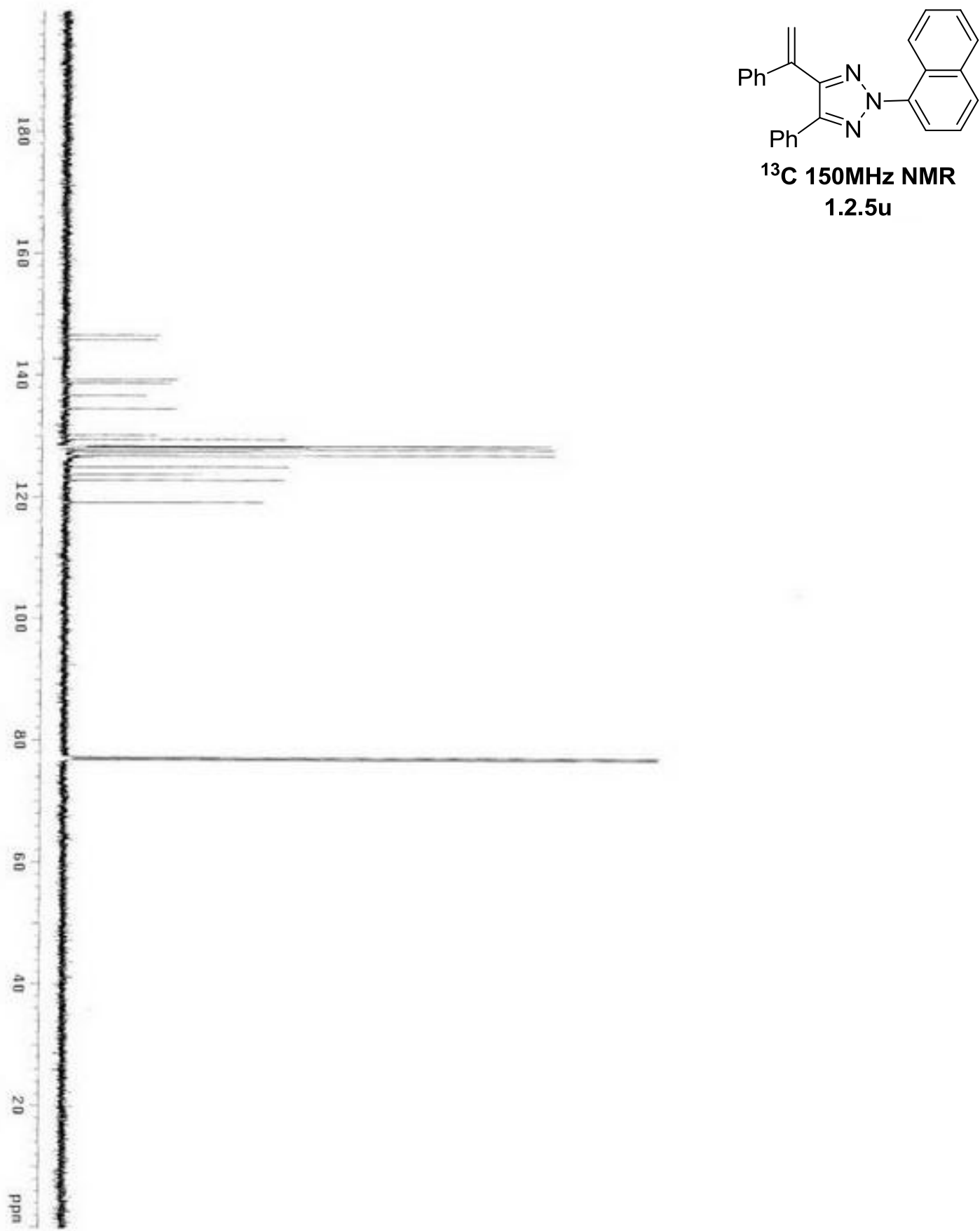

${ }^{13} \mathrm{C}$ 150MHz NMR

1.2.5u 


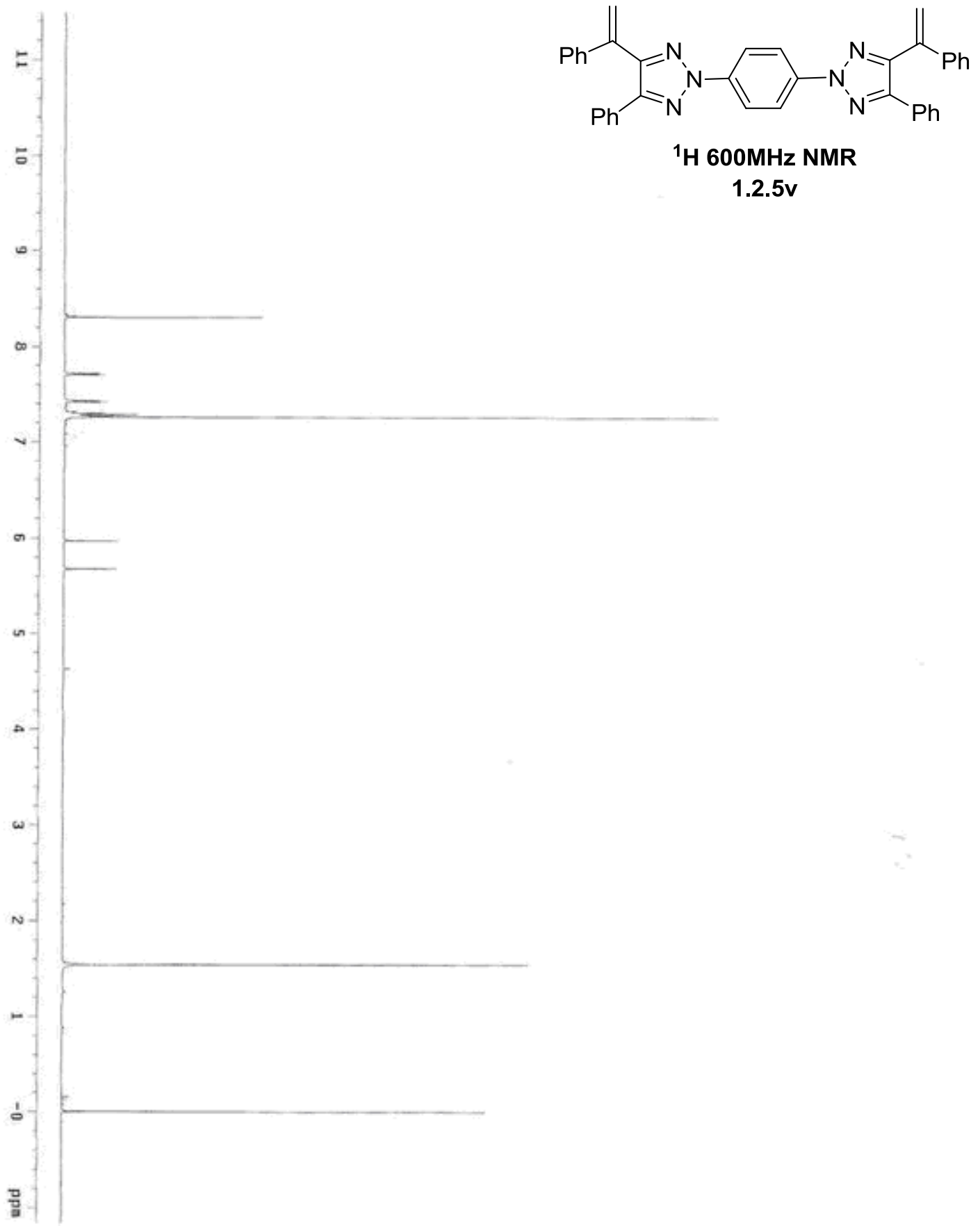



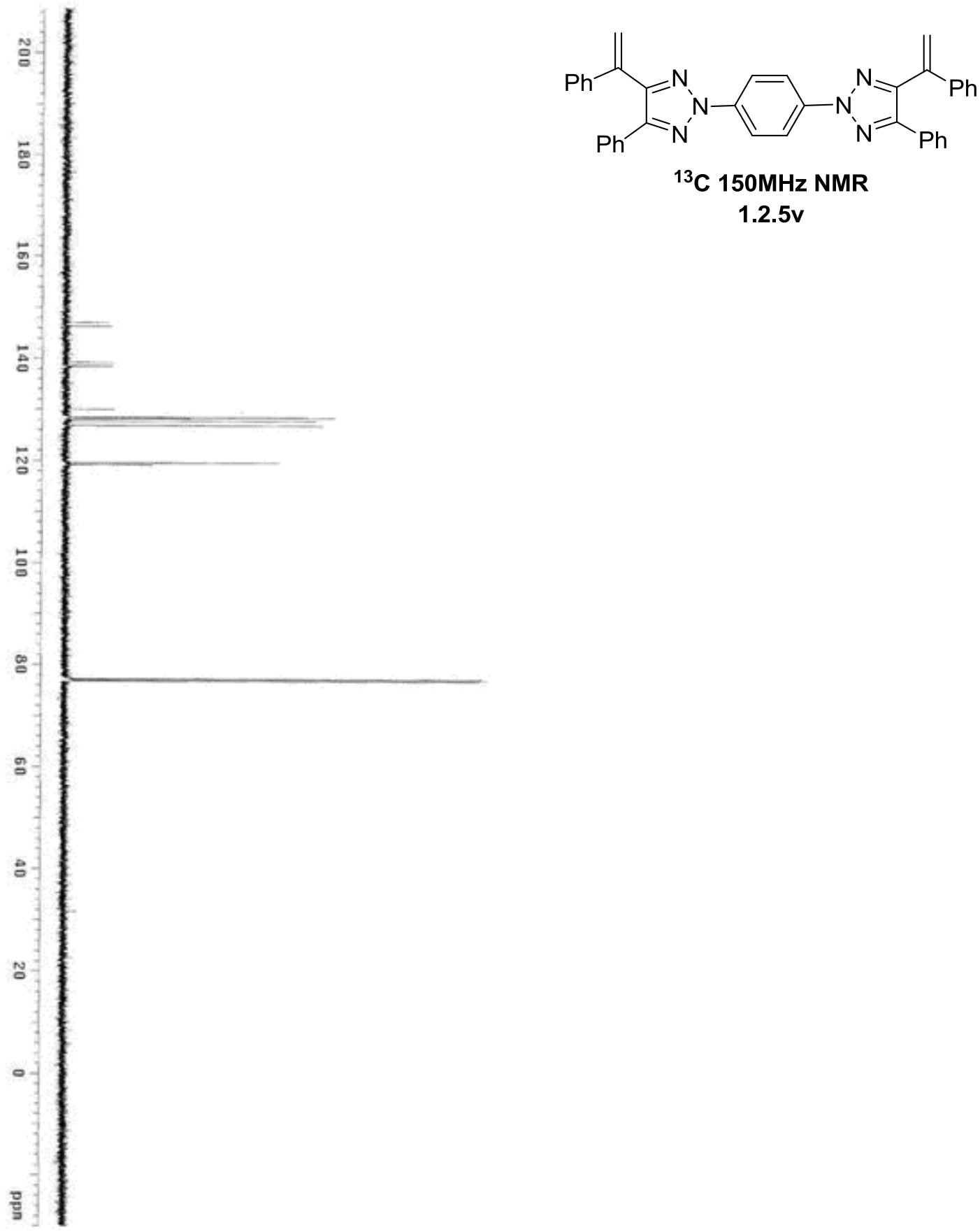

$1.2 .5 \mathrm{v}$ 

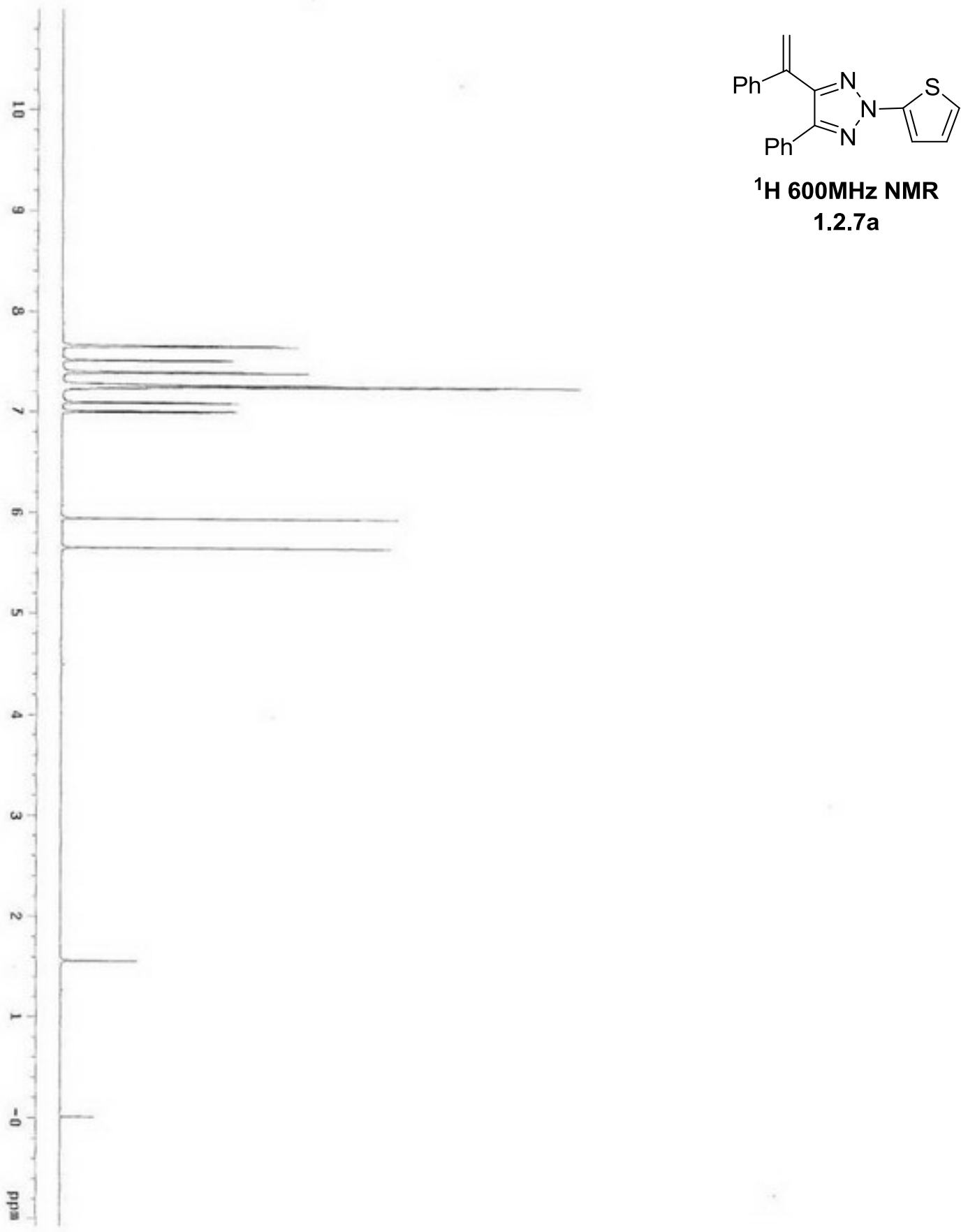

${ }^{1} \mathrm{H} 600 \mathrm{MHz}$ NMR

1.2.7a 

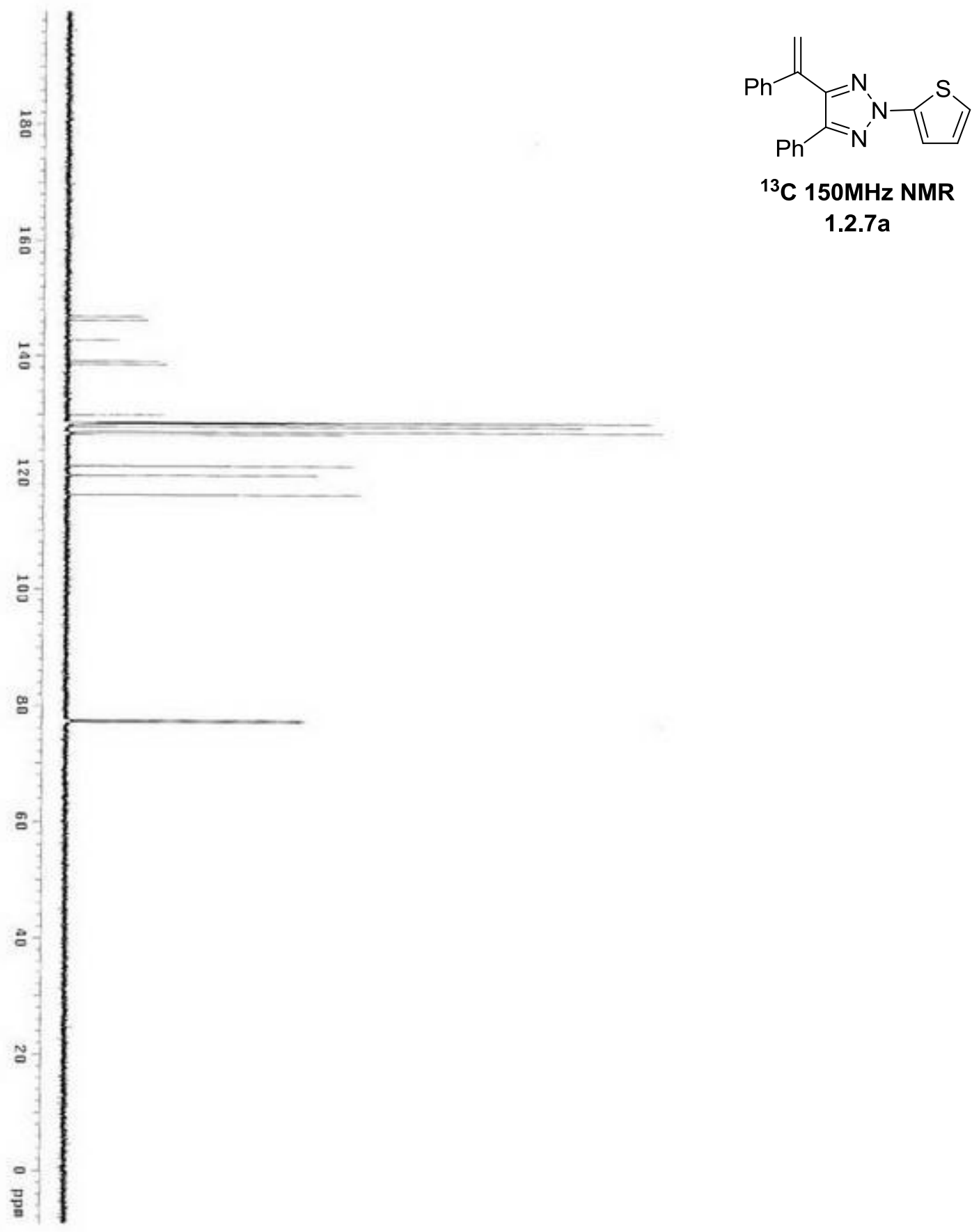

${ }^{13} \mathrm{C} 150 \mathrm{MHz}$ NMR

1.2.7a 


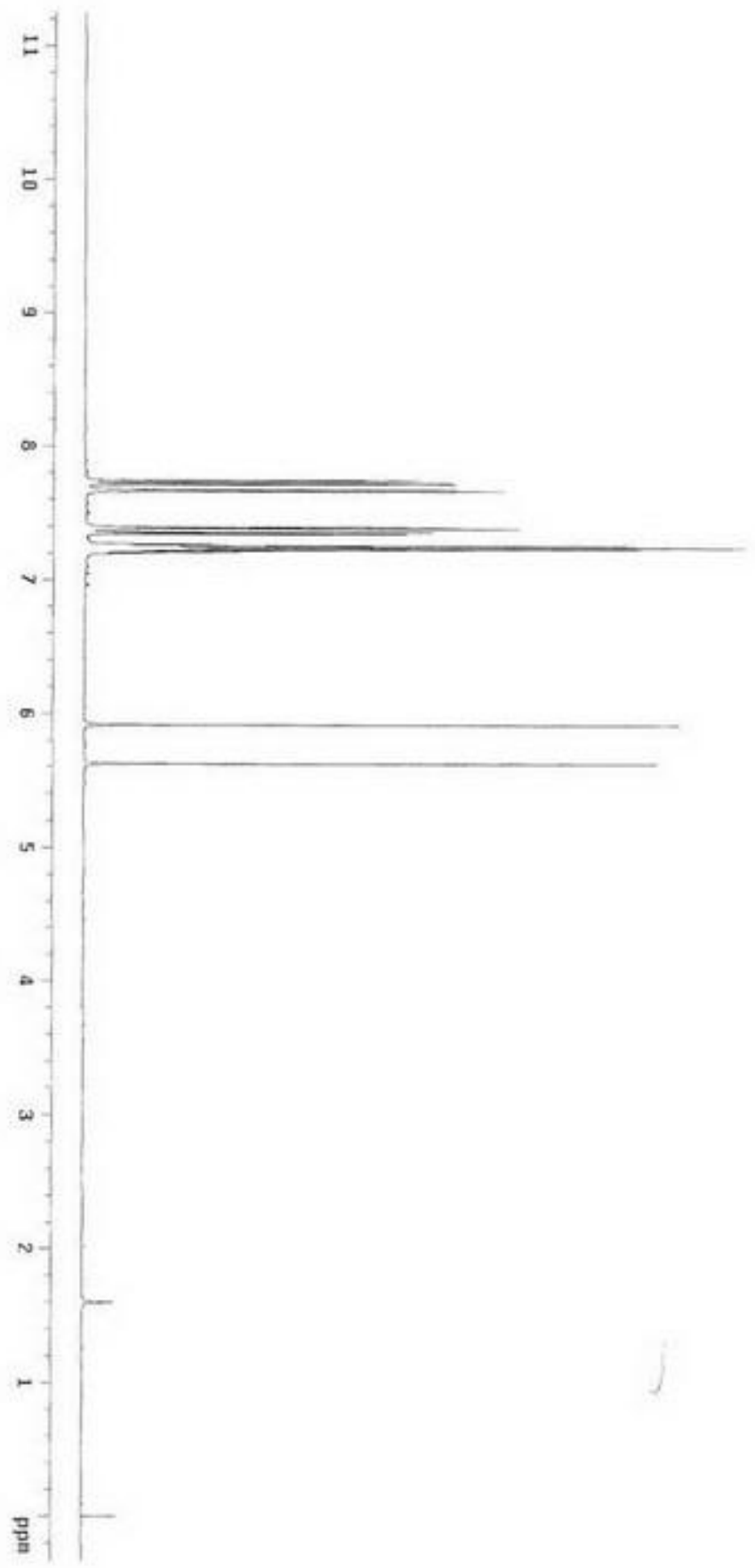

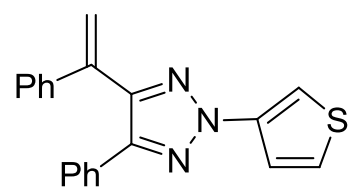

${ }^{1} \mathrm{H} 600 \mathrm{MHz}$ NMR

1.2.7b 

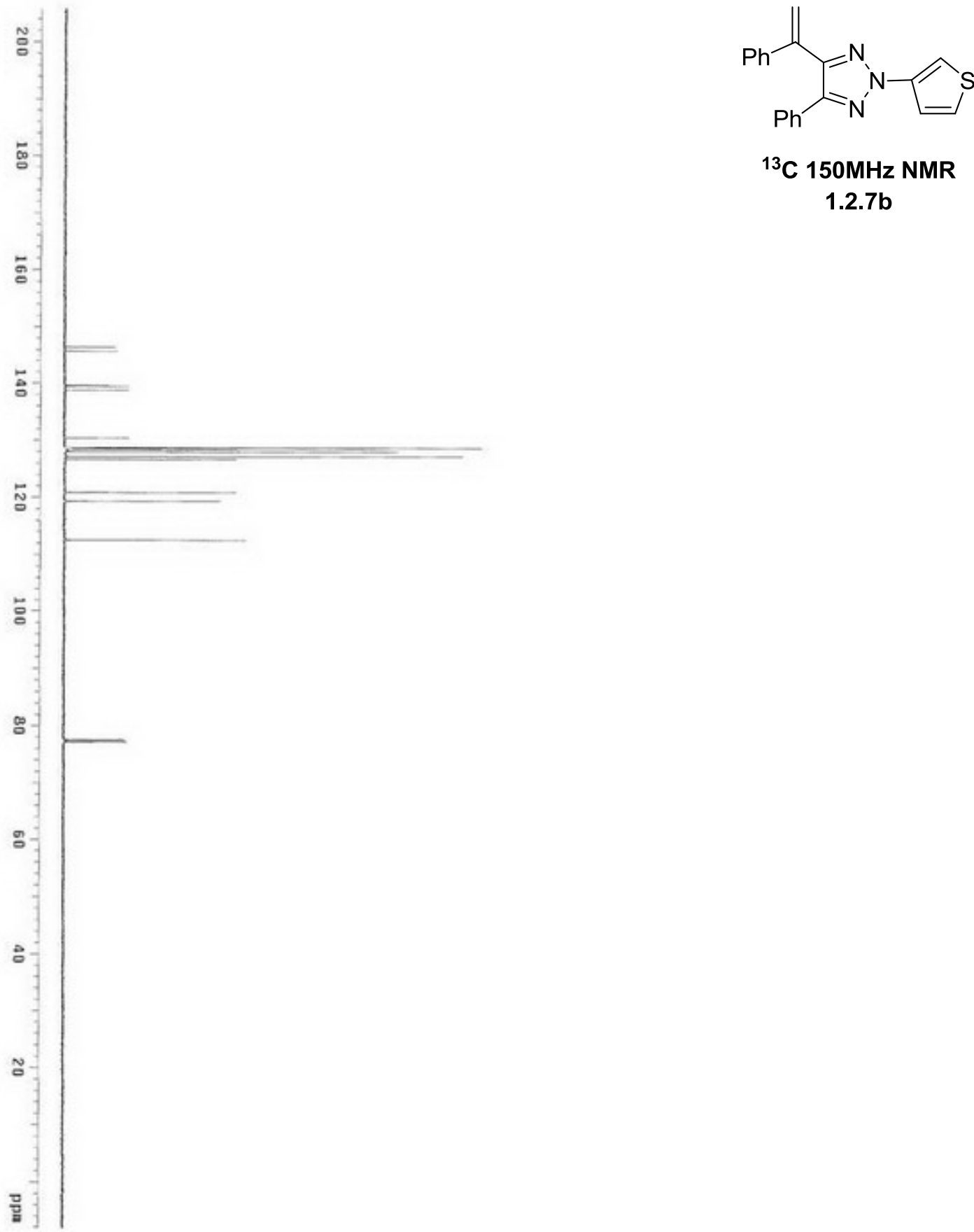

${ }^{13} \mathrm{C}$ 150MHz NMR

1.2.7b 


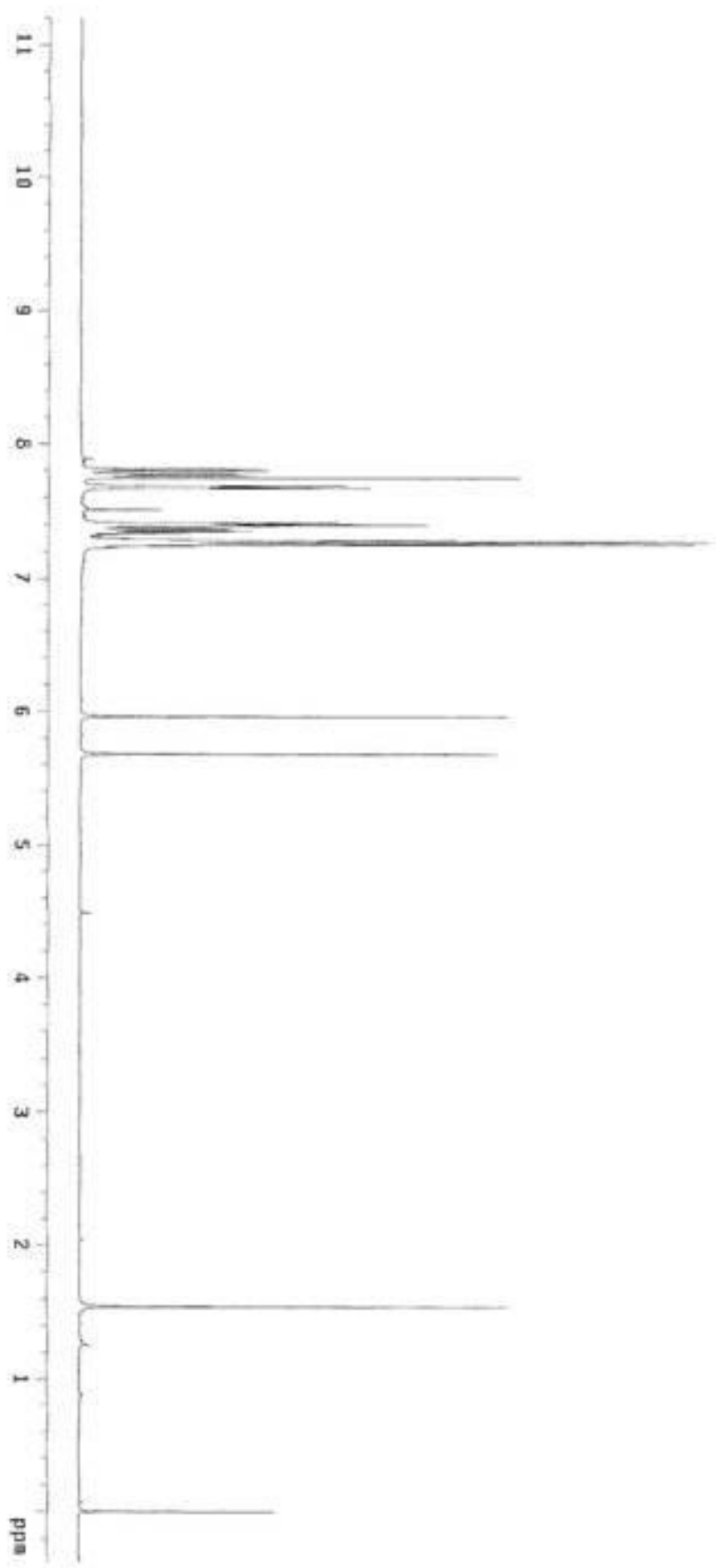

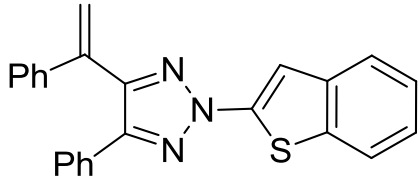

${ }^{1} \mathrm{H}$ 600MHz NMR

1.2.7c 

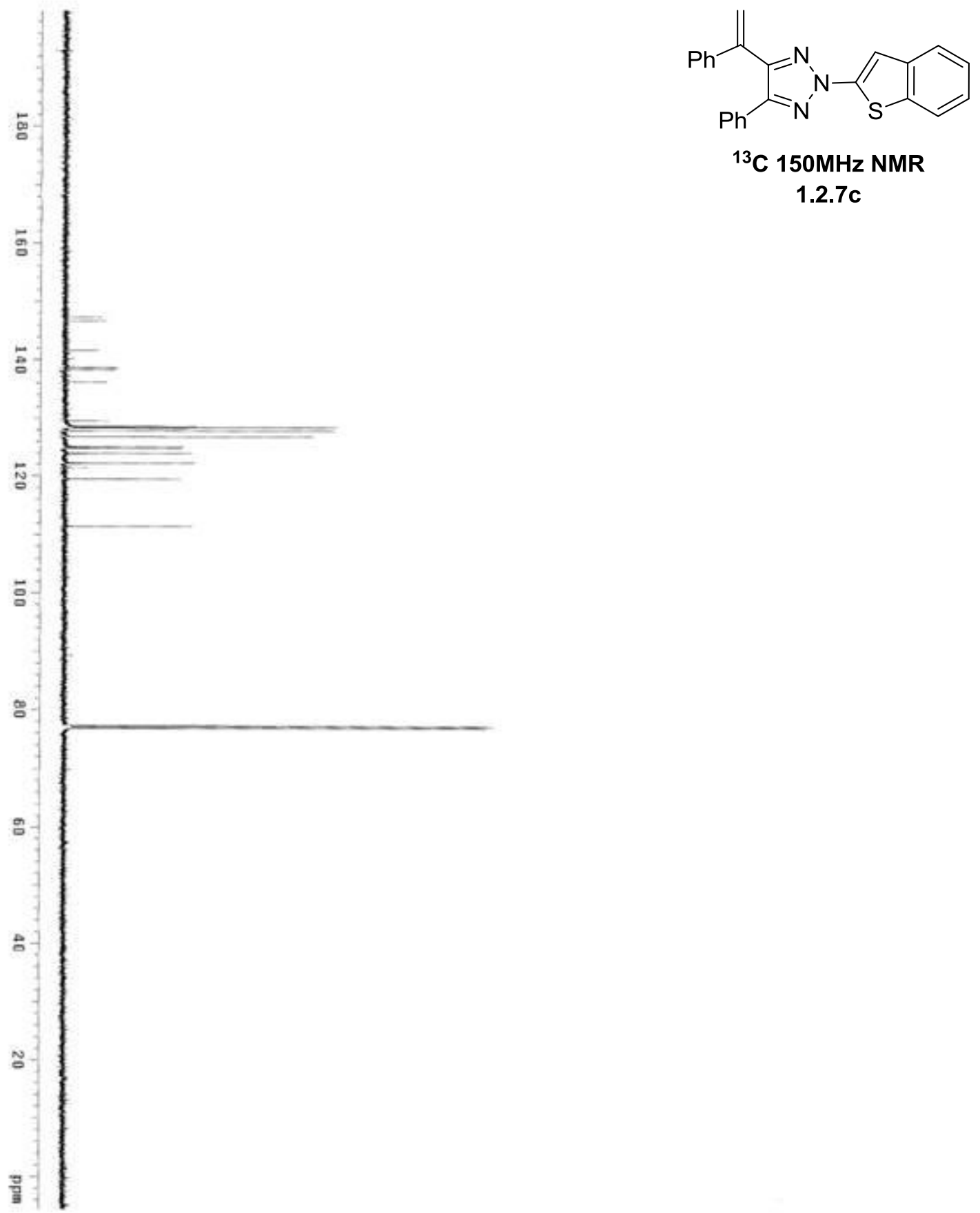


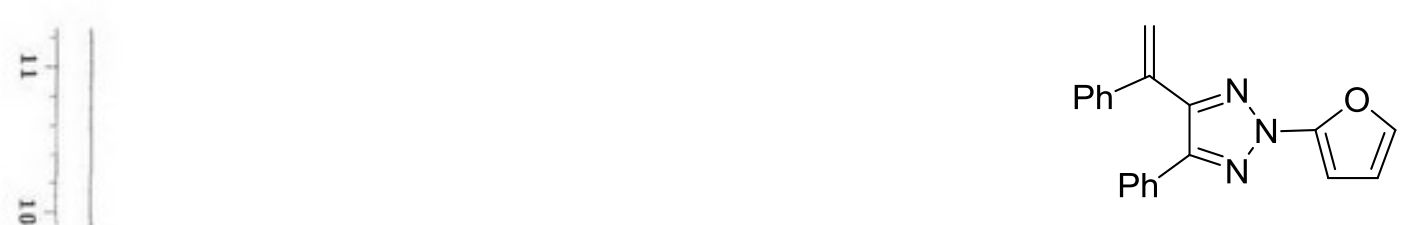

${ }^{1} \mathrm{H} 600 \mathrm{MHz}$ NMR

1.2.7d

$\infty$

$w$

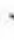

a

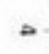

$\omega$

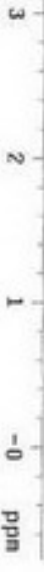

$N$

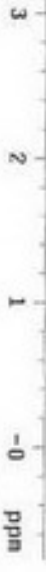

?

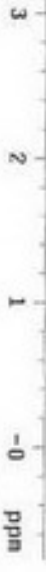
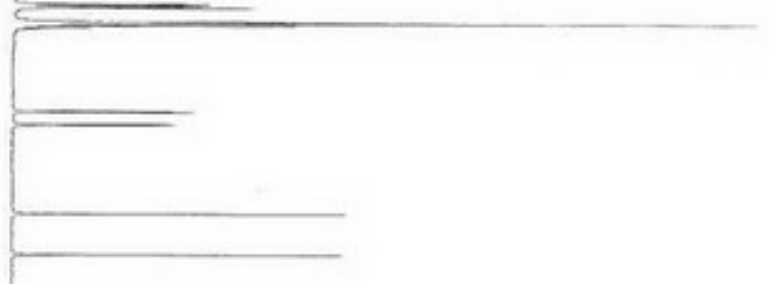

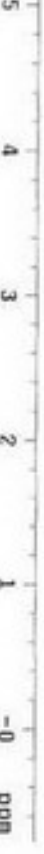



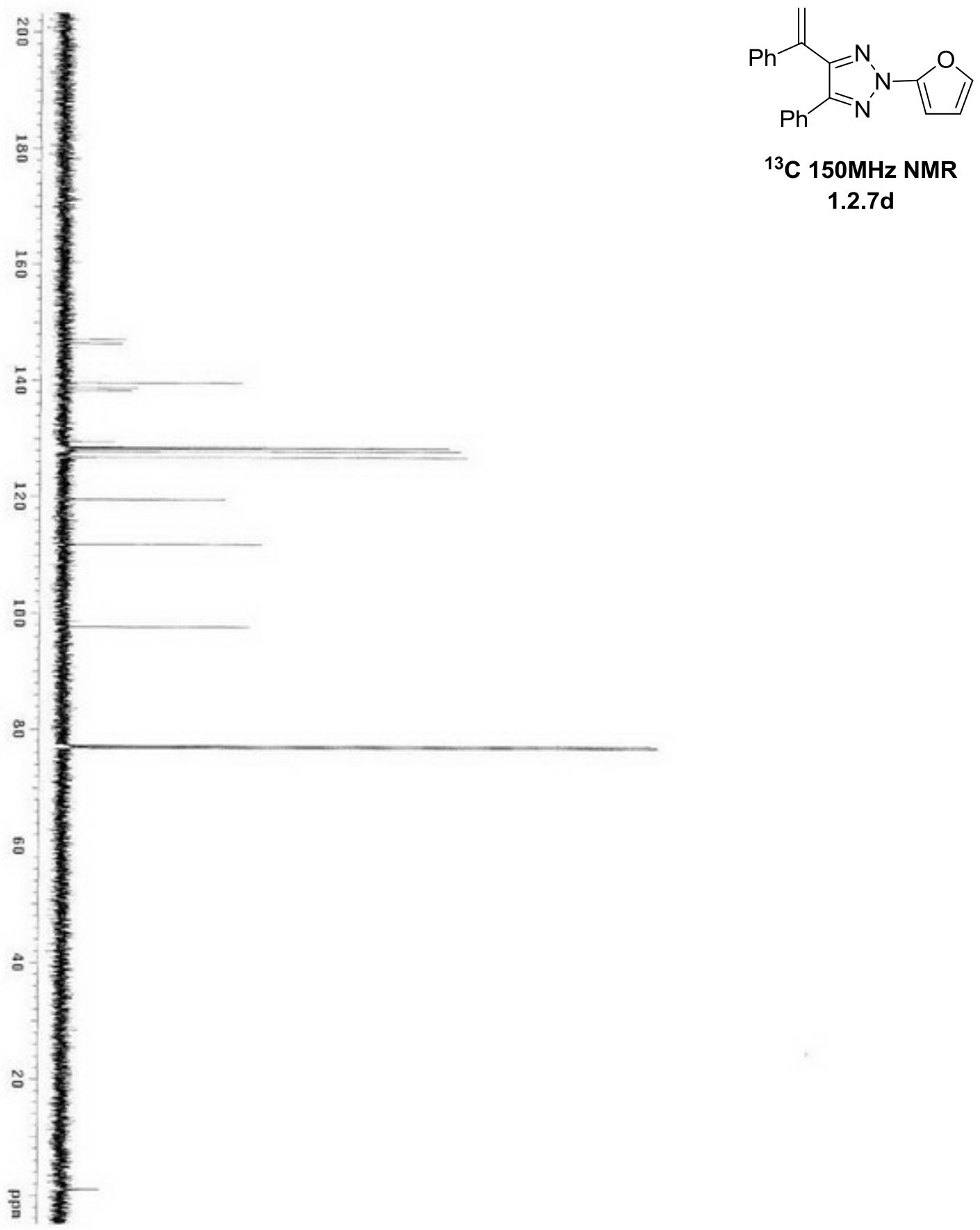

${ }^{13} \mathrm{C}$ 150MHz NMR

1.2.7d 


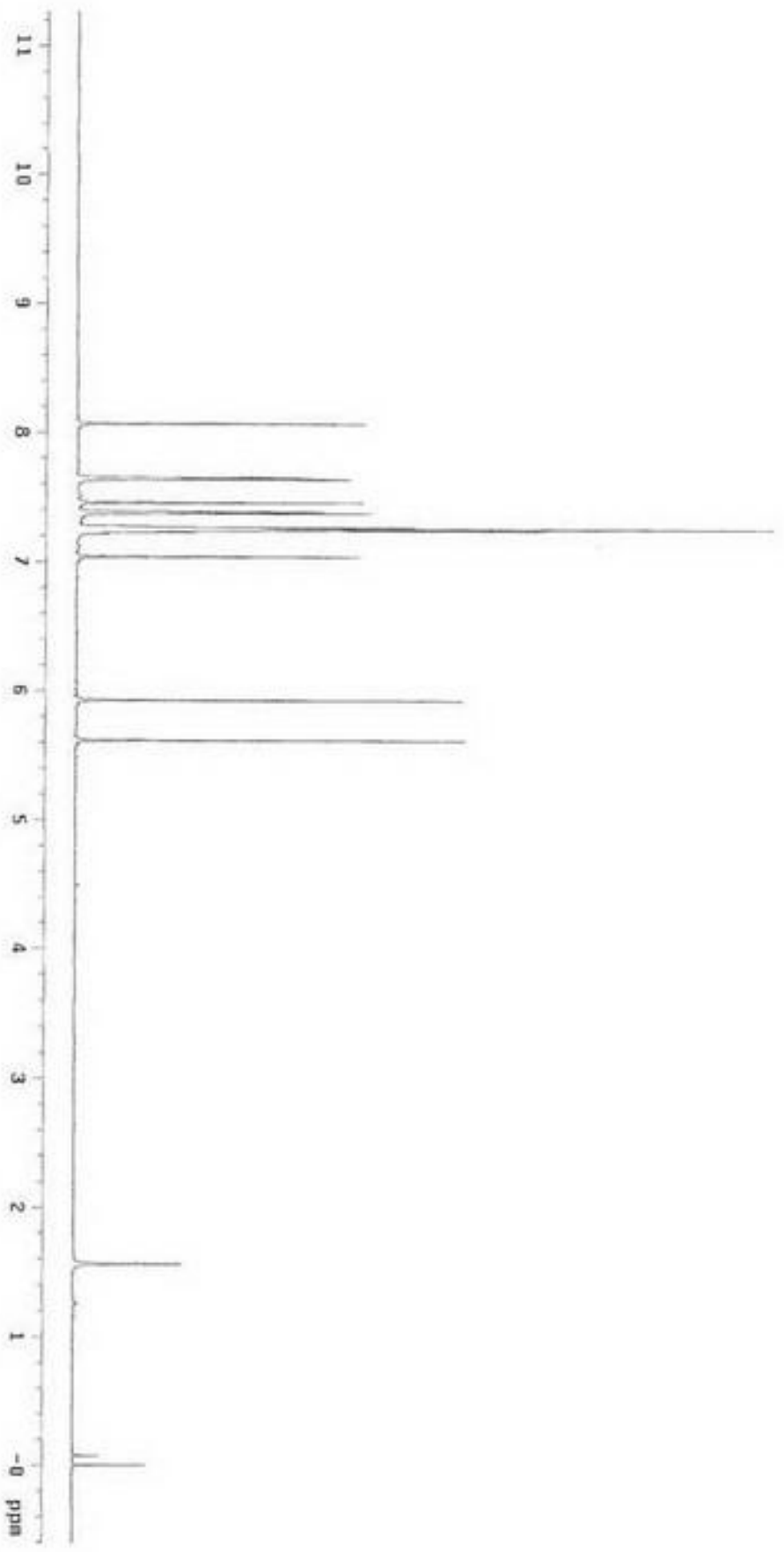

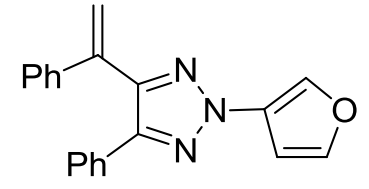

${ }^{1} \mathrm{H} 600 \mathrm{MHz}$ NMR

1.2.7e 

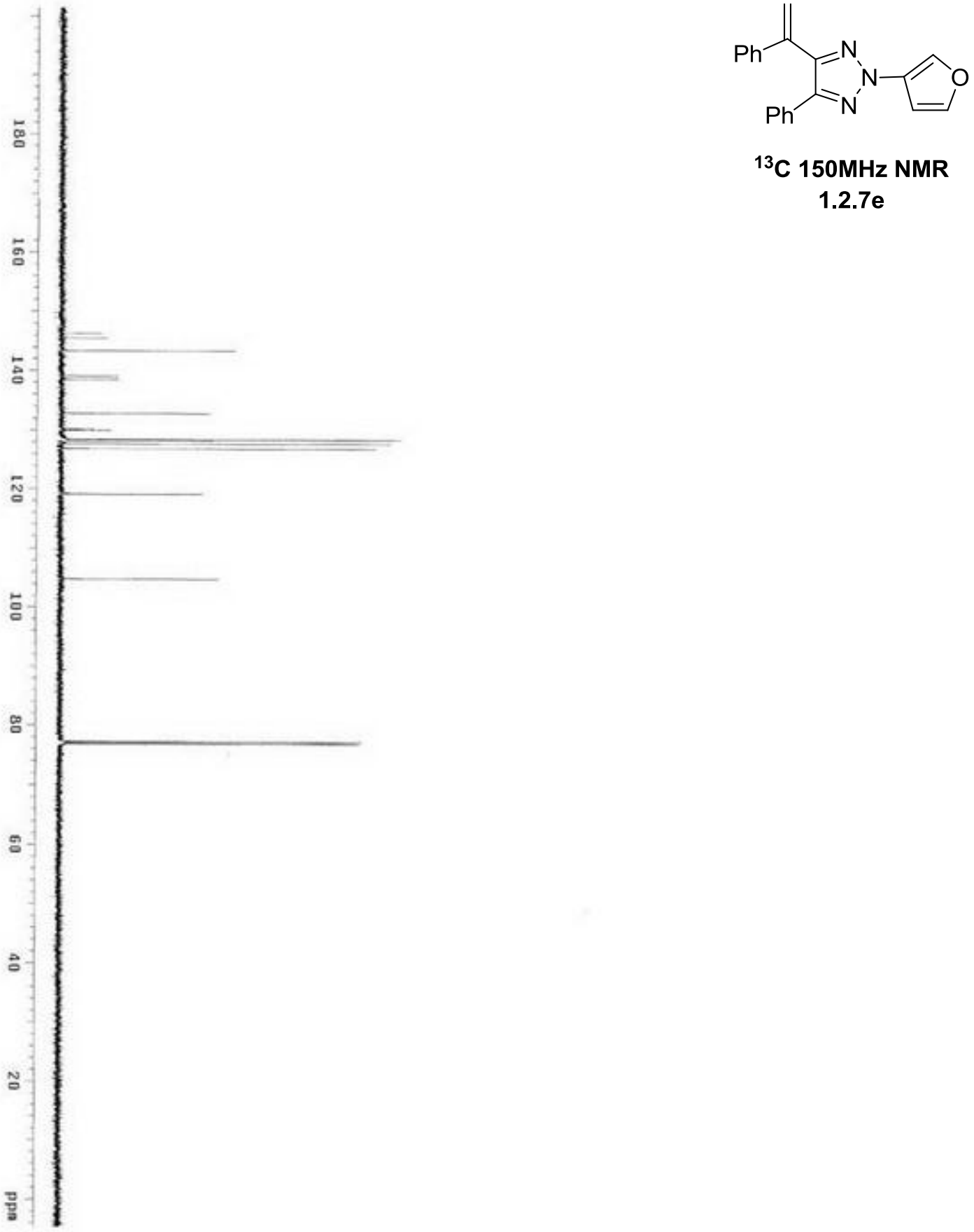

${ }^{13} \mathrm{C} 150 \mathrm{MHz}$ NMR

1.2.7e 


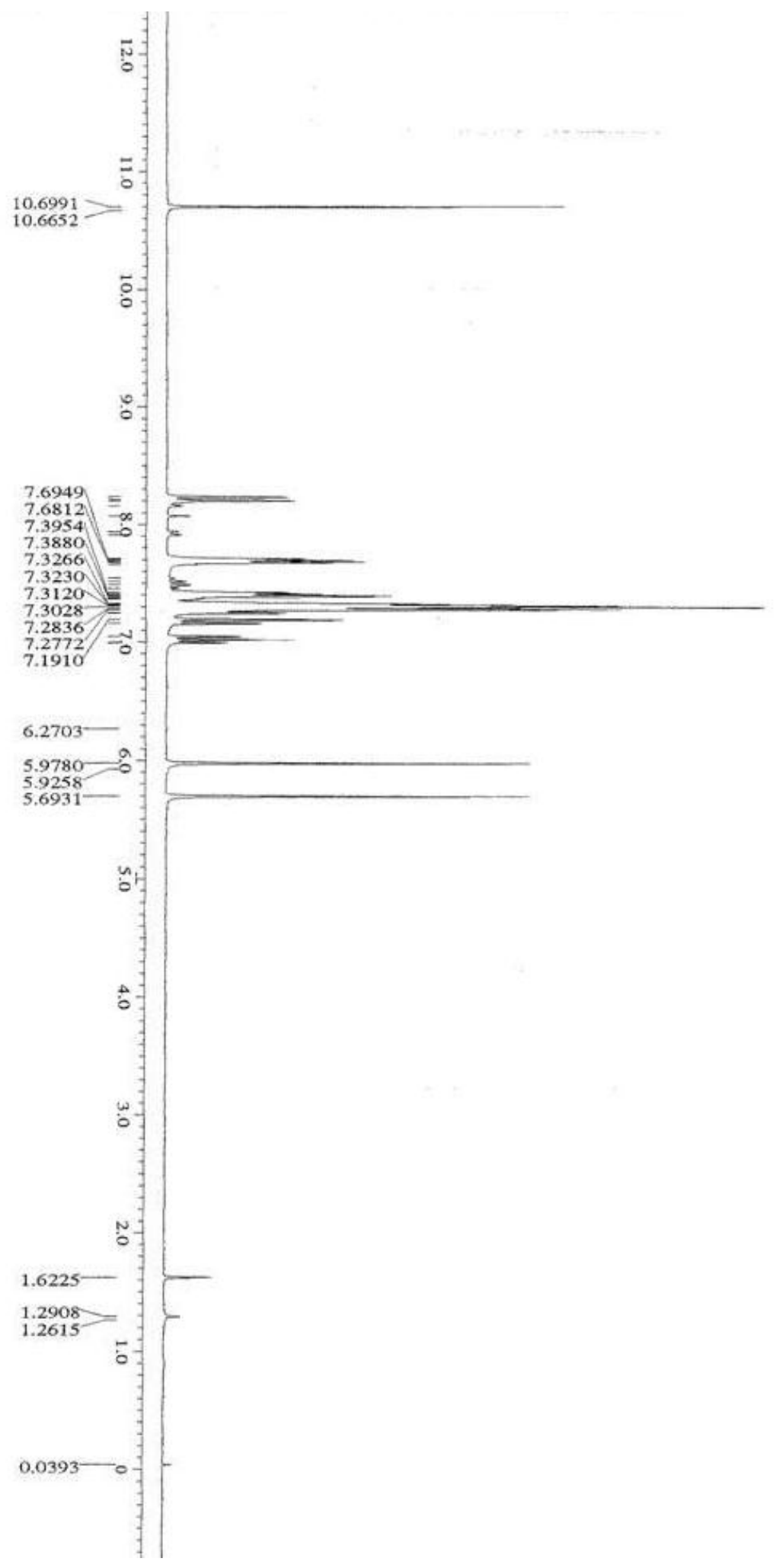

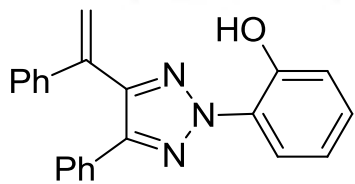

${ }^{1} \mathrm{H} 600 \mathrm{MHz}$ NMR

1.2.7f 


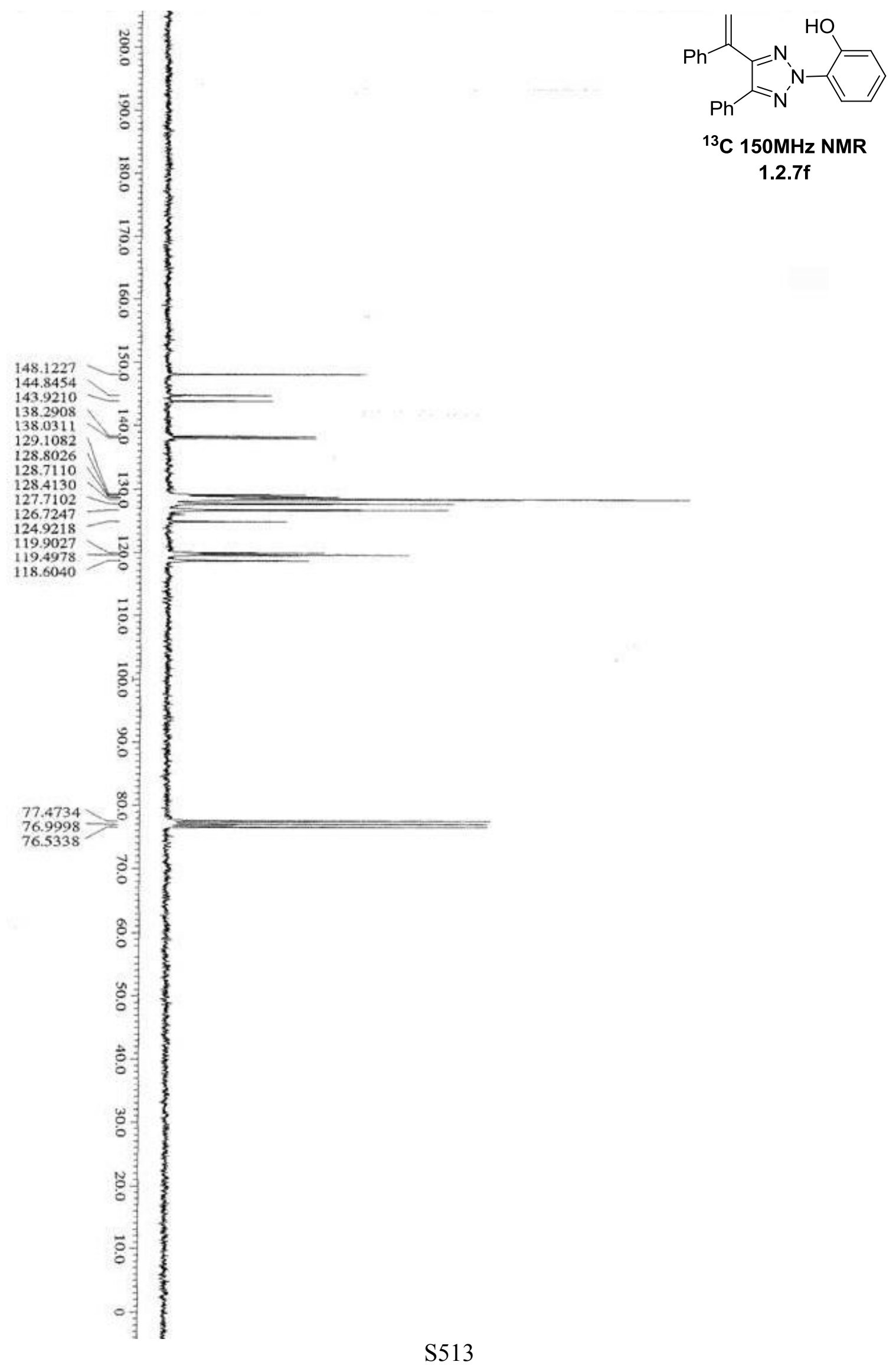


1.3. NMR Spectra Data

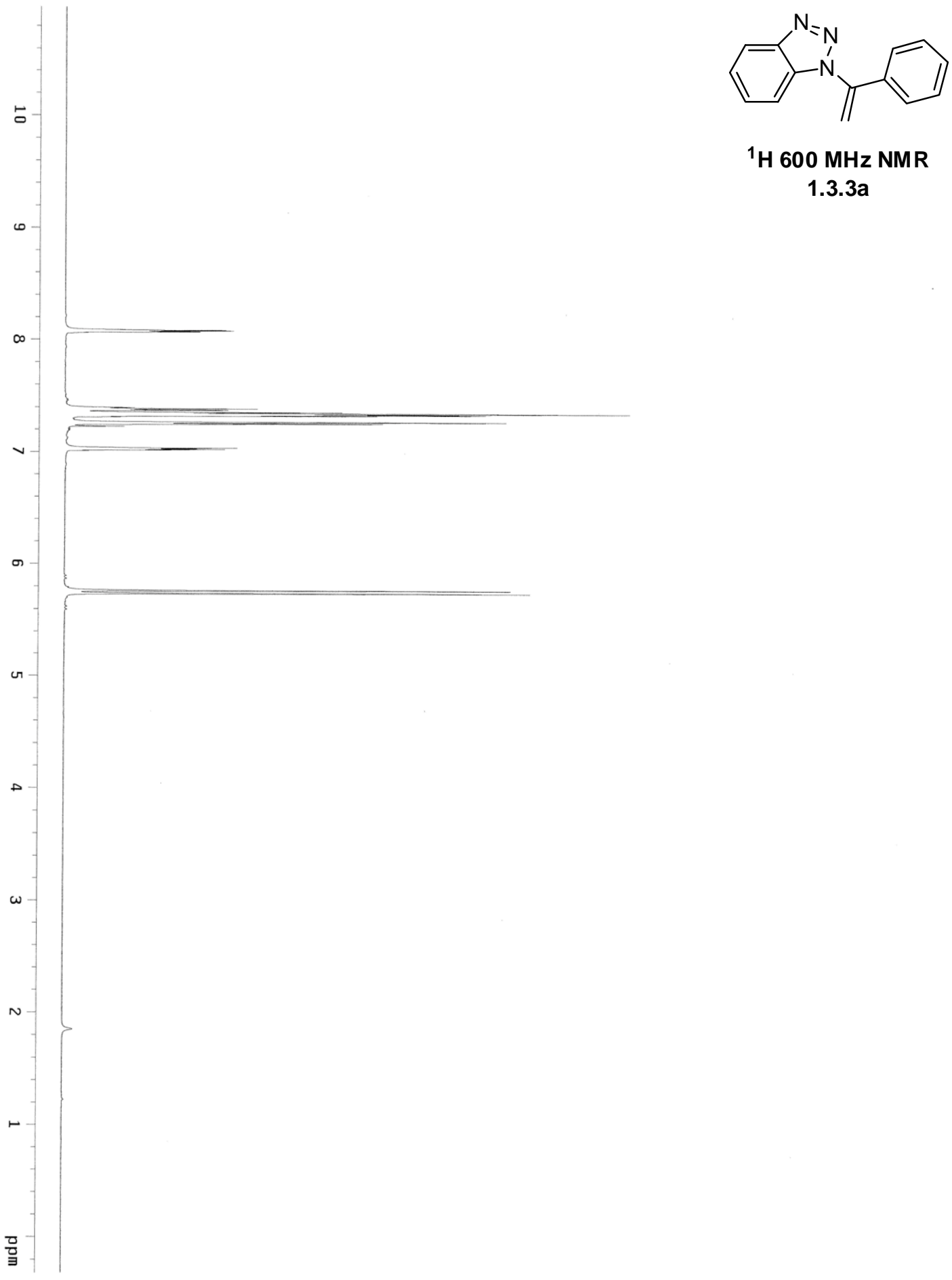




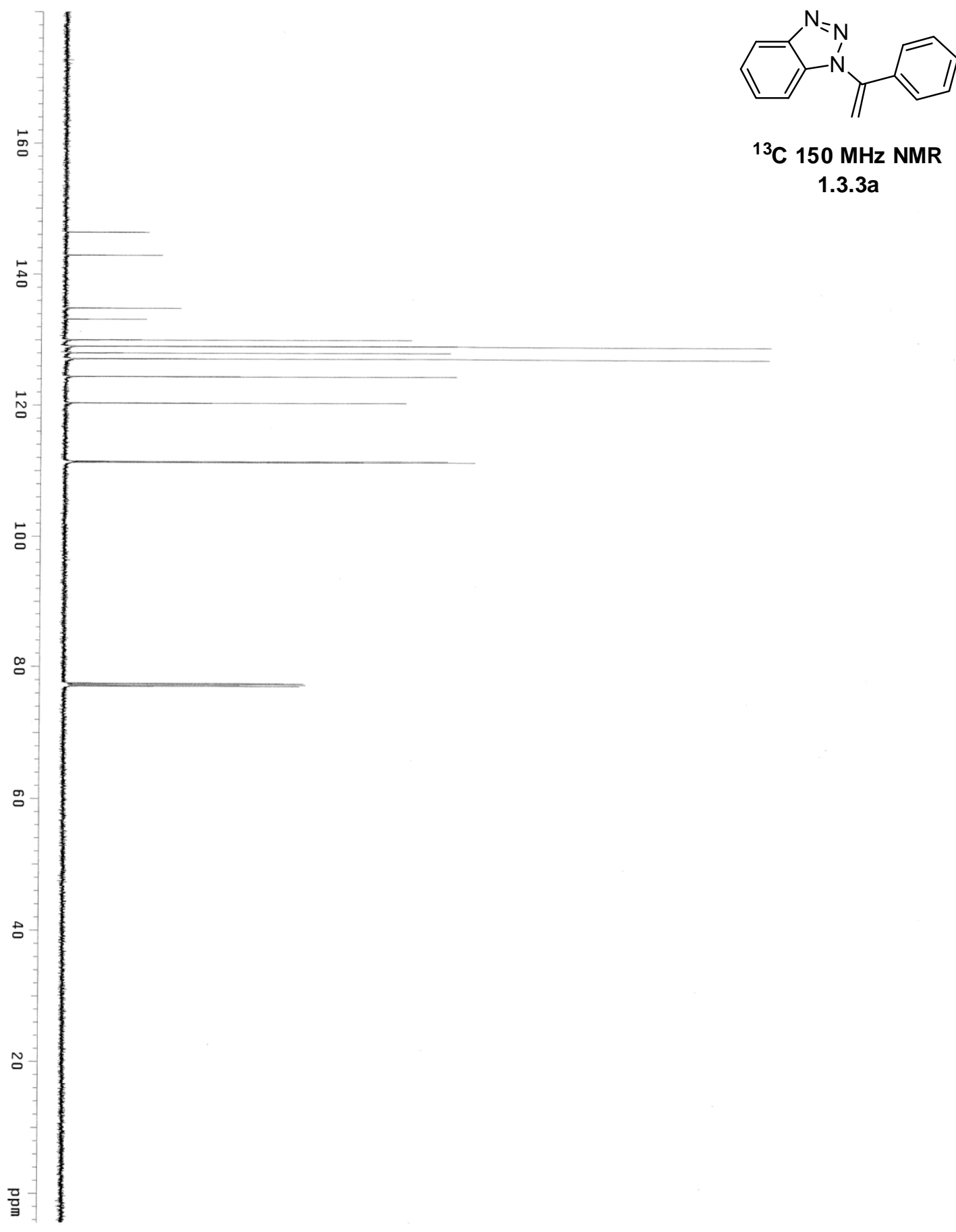




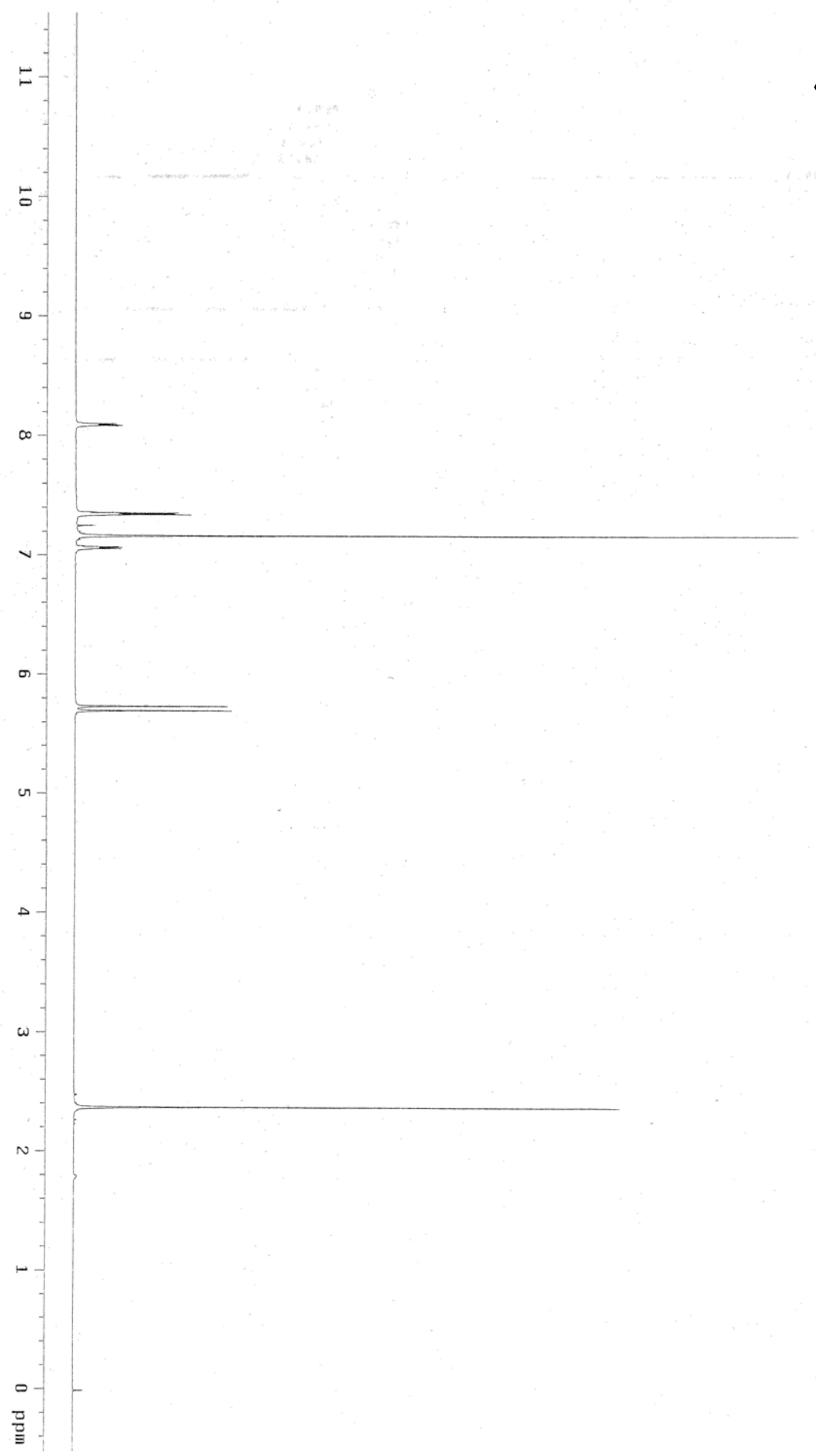

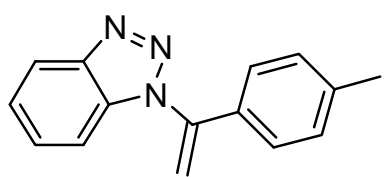

${ }^{1} \mathrm{H} 600 \mathrm{MHz}$ NMR

1.3.3b 


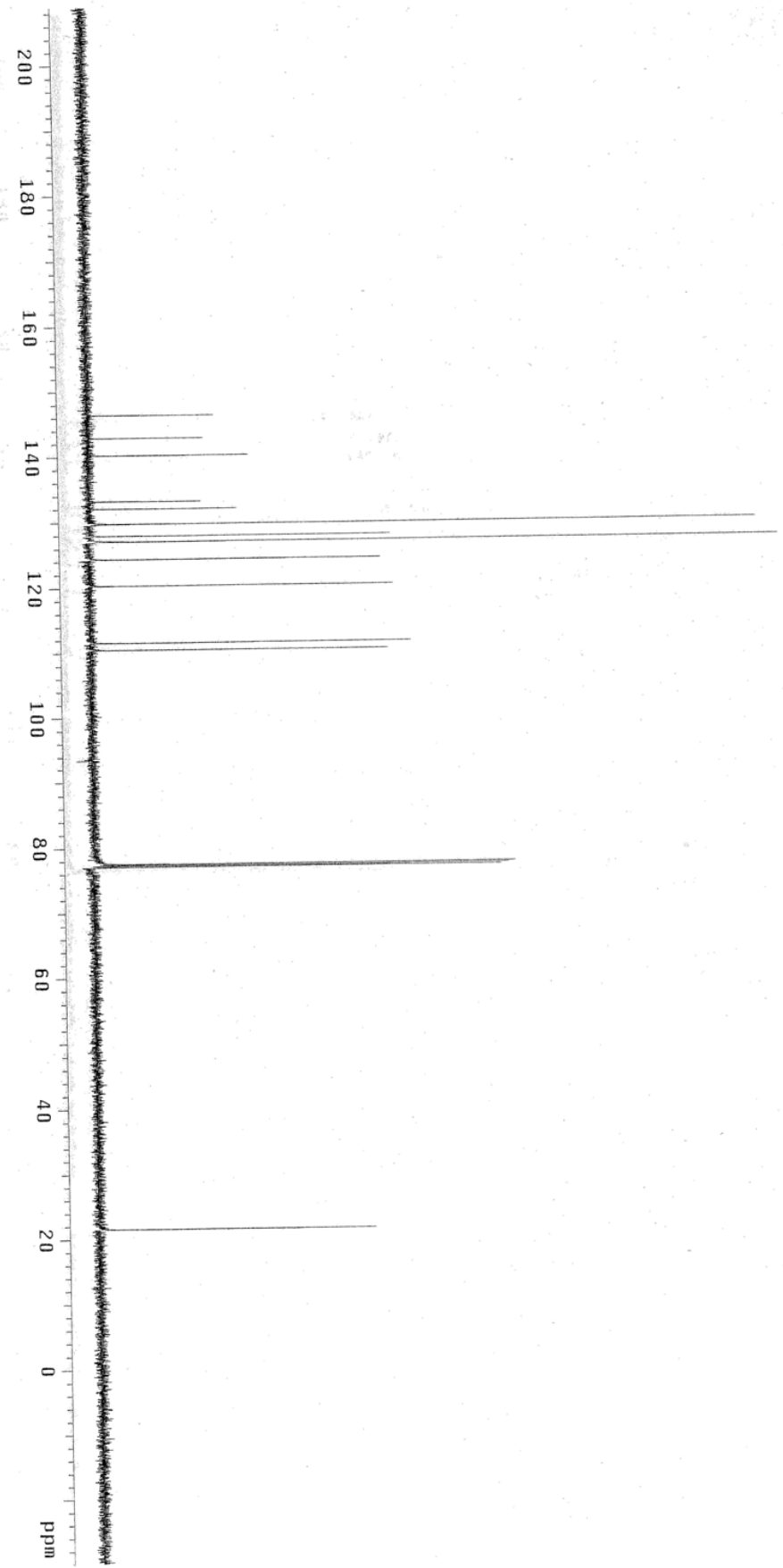

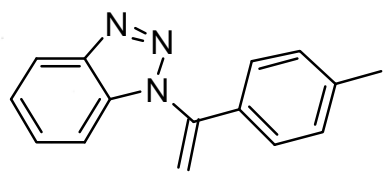

${ }^{13} \mathrm{C} 150 \mathrm{MHz}$ NMR 1.3.3b 


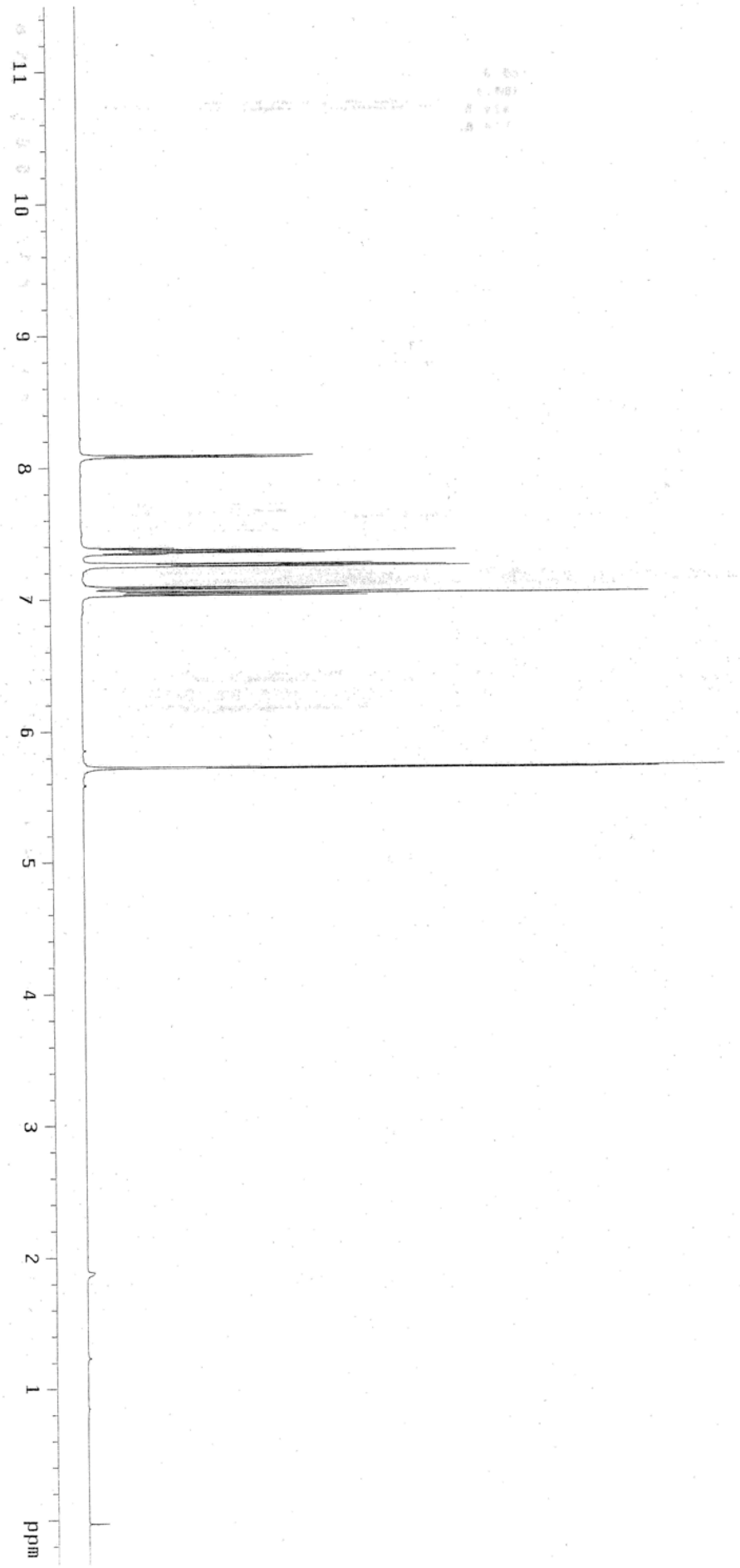

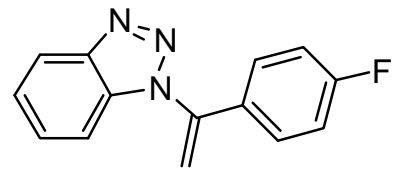

${ }^{1} \mathrm{H} 600 \mathrm{MHz}$ NMR 1.3.3c 

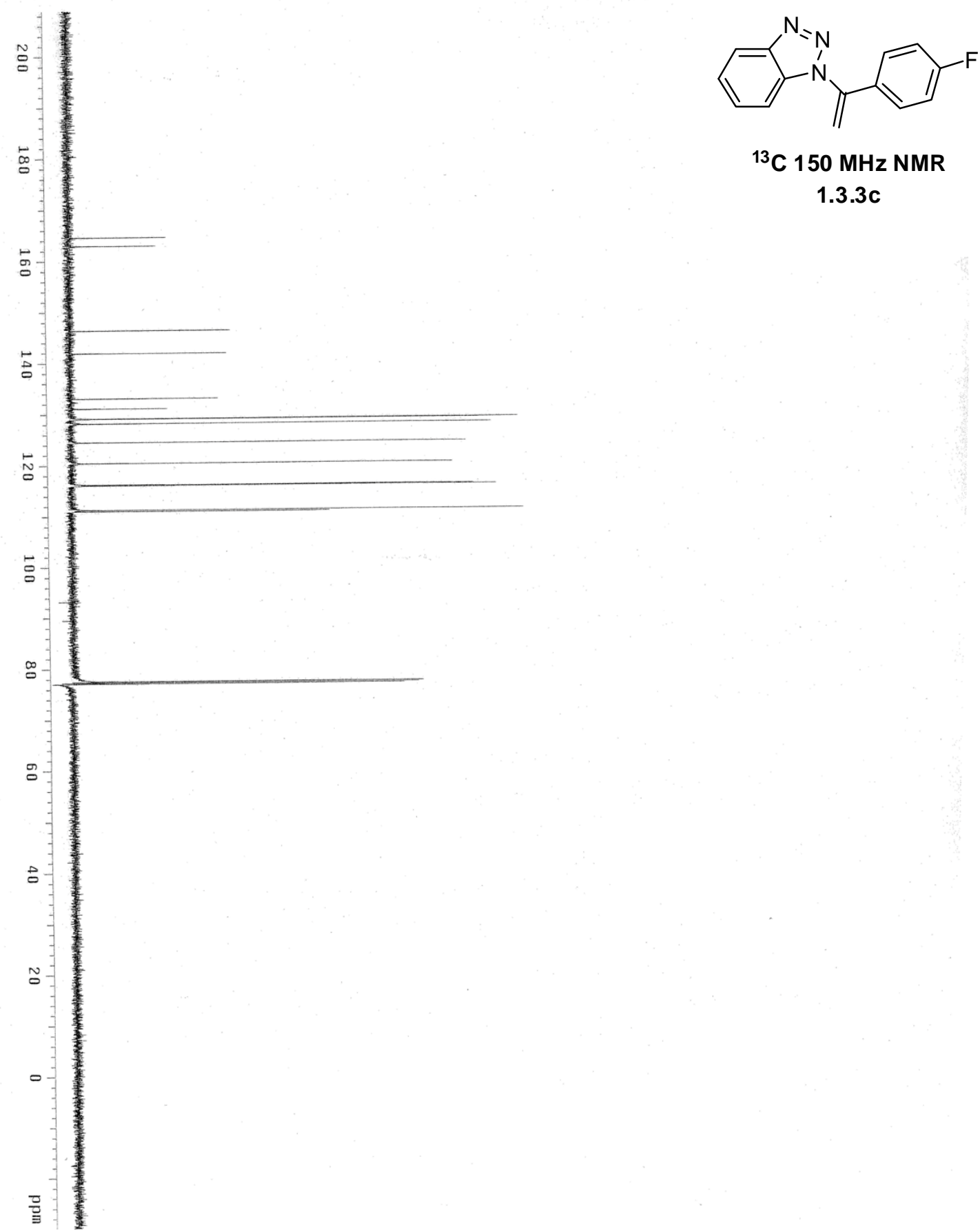

${ }^{13} \mathrm{C} 150 \mathrm{MHz}$ NMR 1.3.3c 


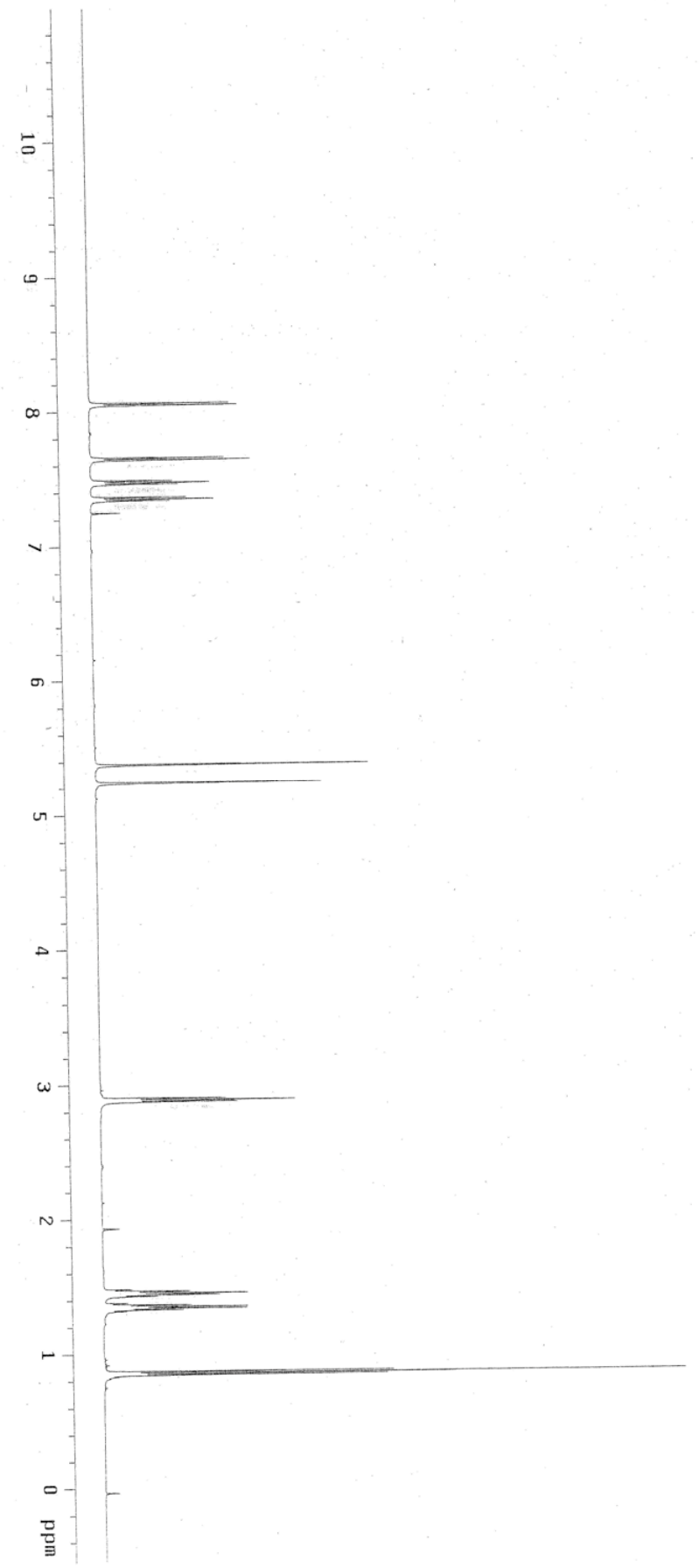

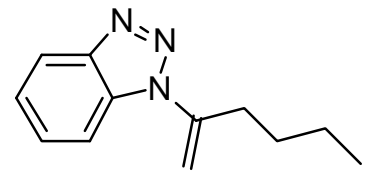

${ }^{1} \mathrm{H} 600 \mathrm{MHz}$ NMR

1.3.3d 


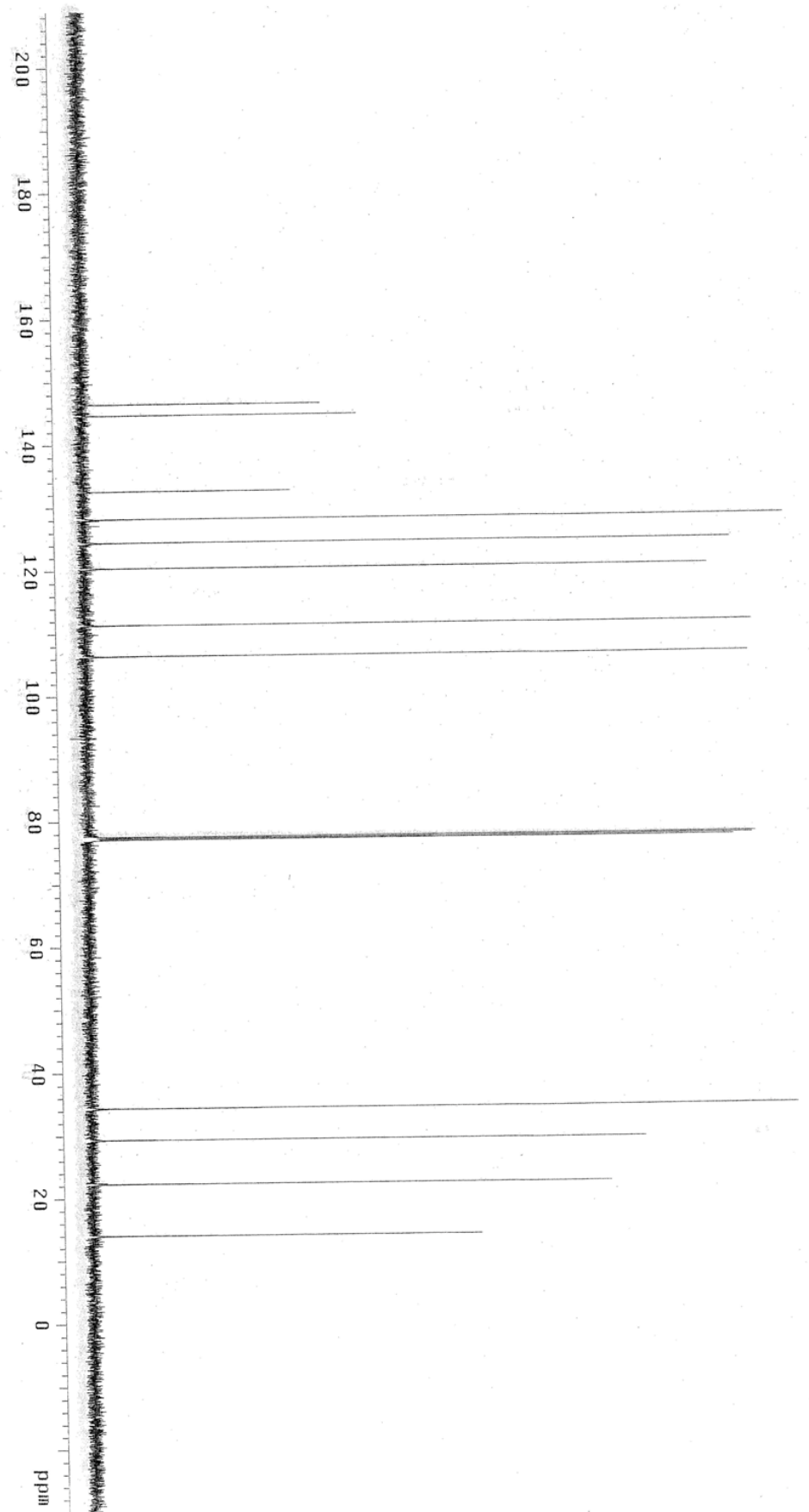

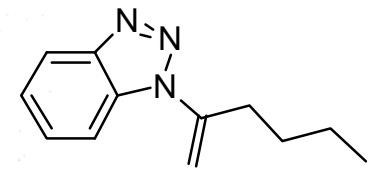

${ }^{13} \mathrm{C} 150 \mathrm{MHz}$ NMR 1.3.3d 


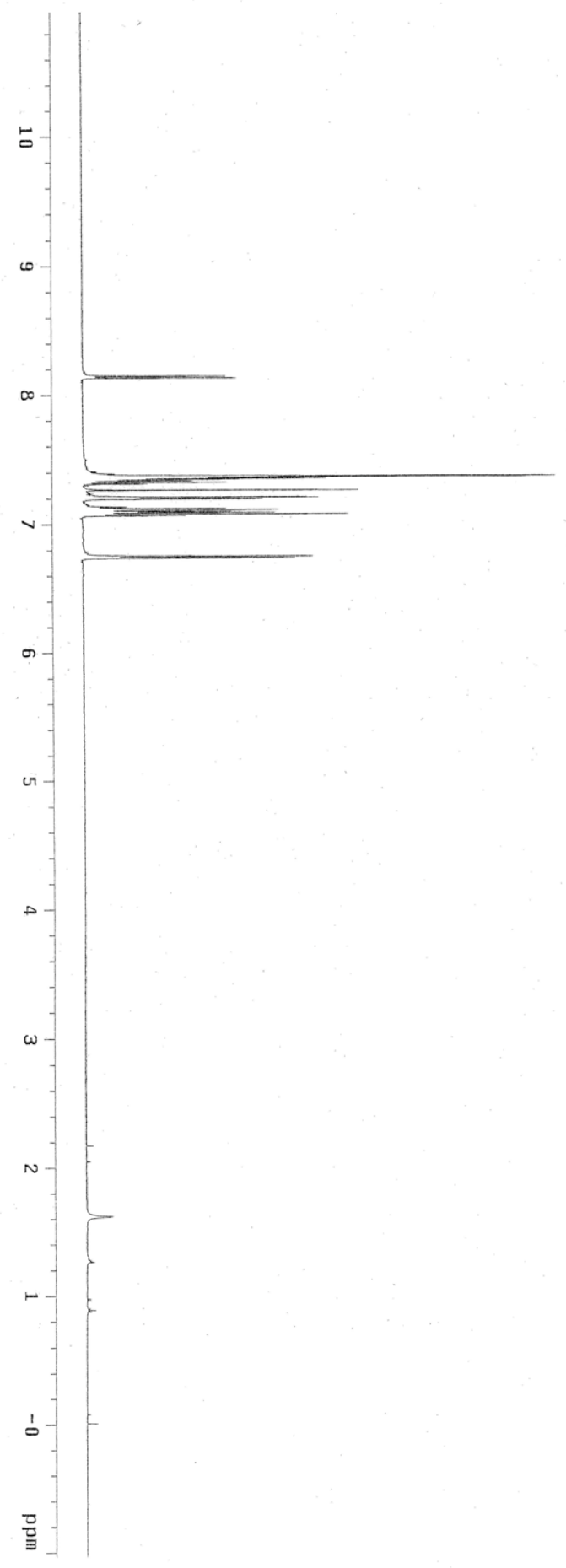

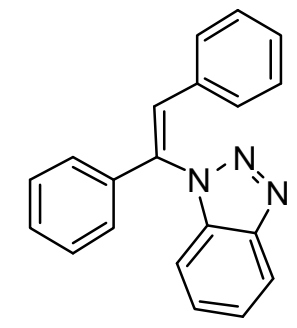

${ }^{1} \mathrm{H} 600 \mathrm{MHz}$ NMR 1.3.3e 


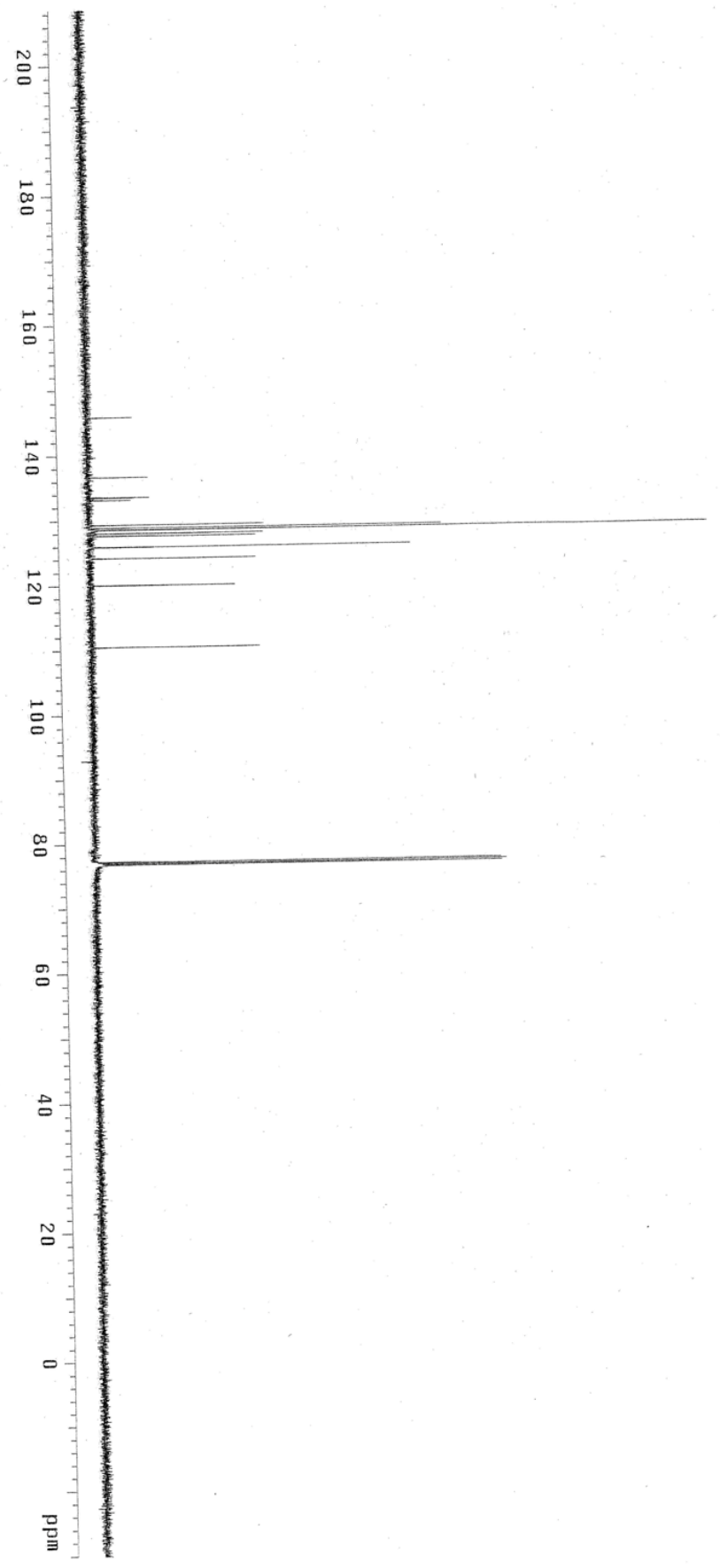

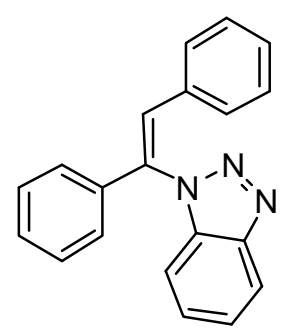

${ }^{13} \mathrm{C} 150 \mathrm{MHz}$ NMR 1.3.3e 

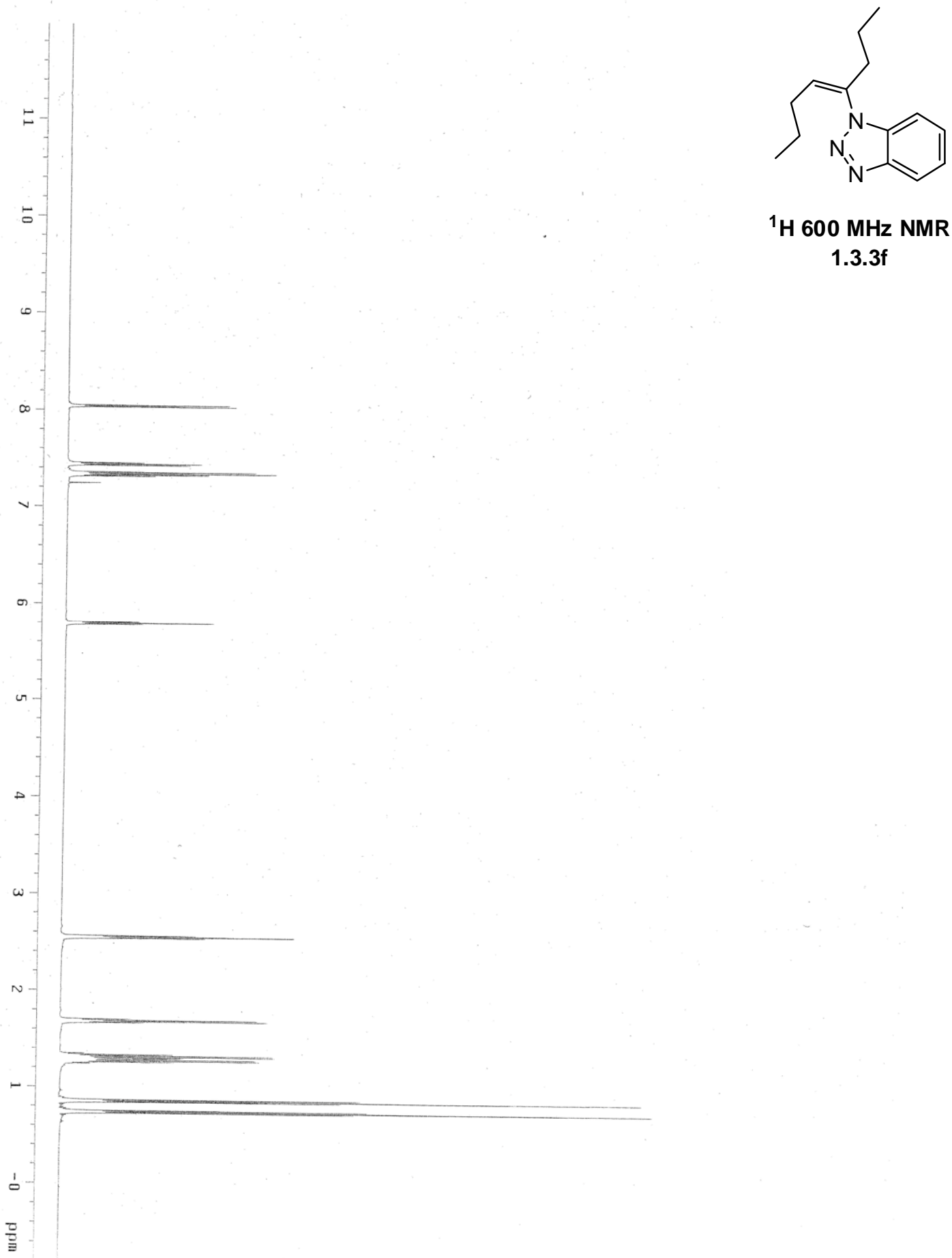

${ }^{1} \mathrm{H} 600 \mathrm{MHz}$ NMR

1.3.3f 

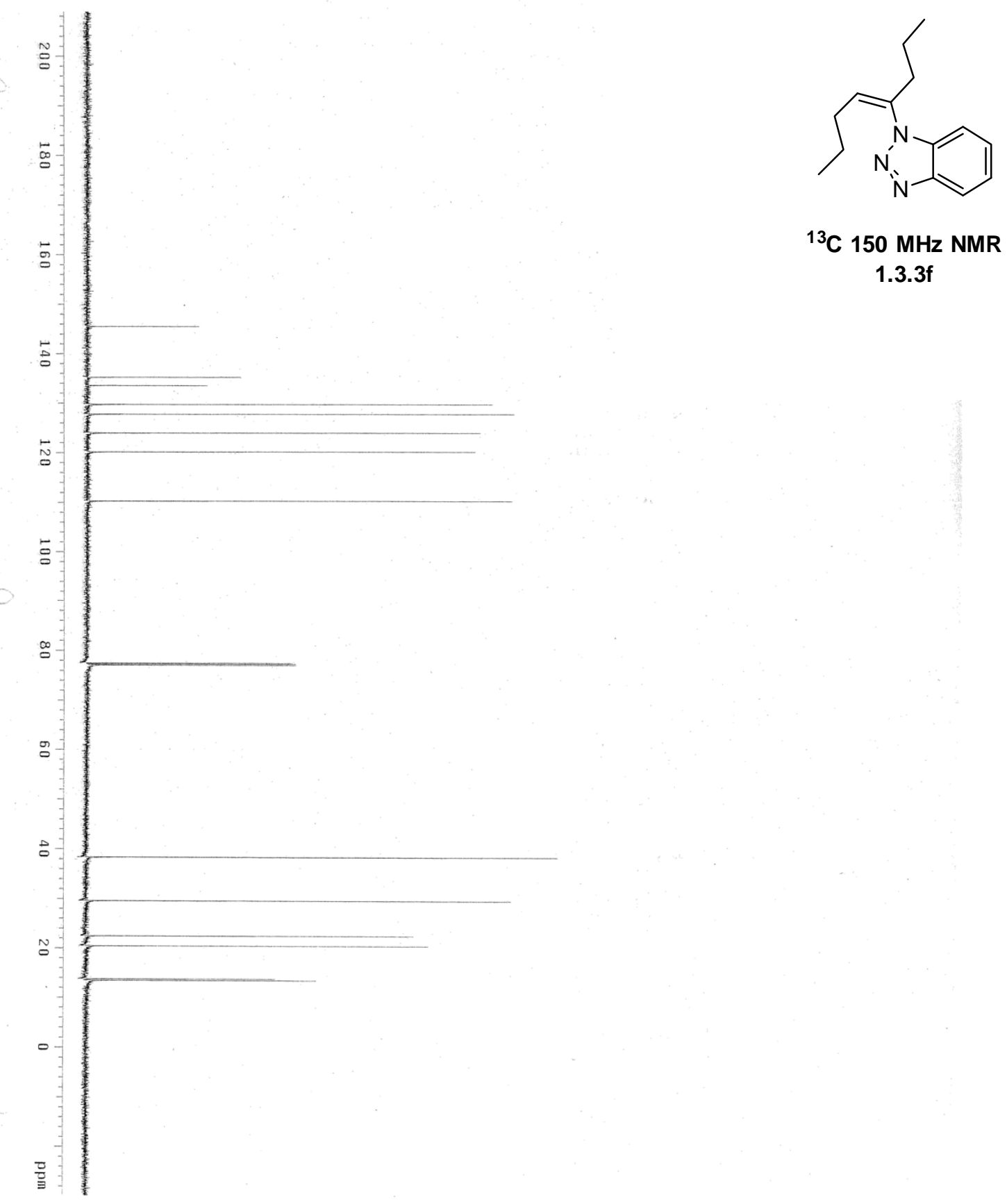

${ }^{13} \mathrm{C} 150 \mathrm{MHz}$ NMR

1.3.3f 

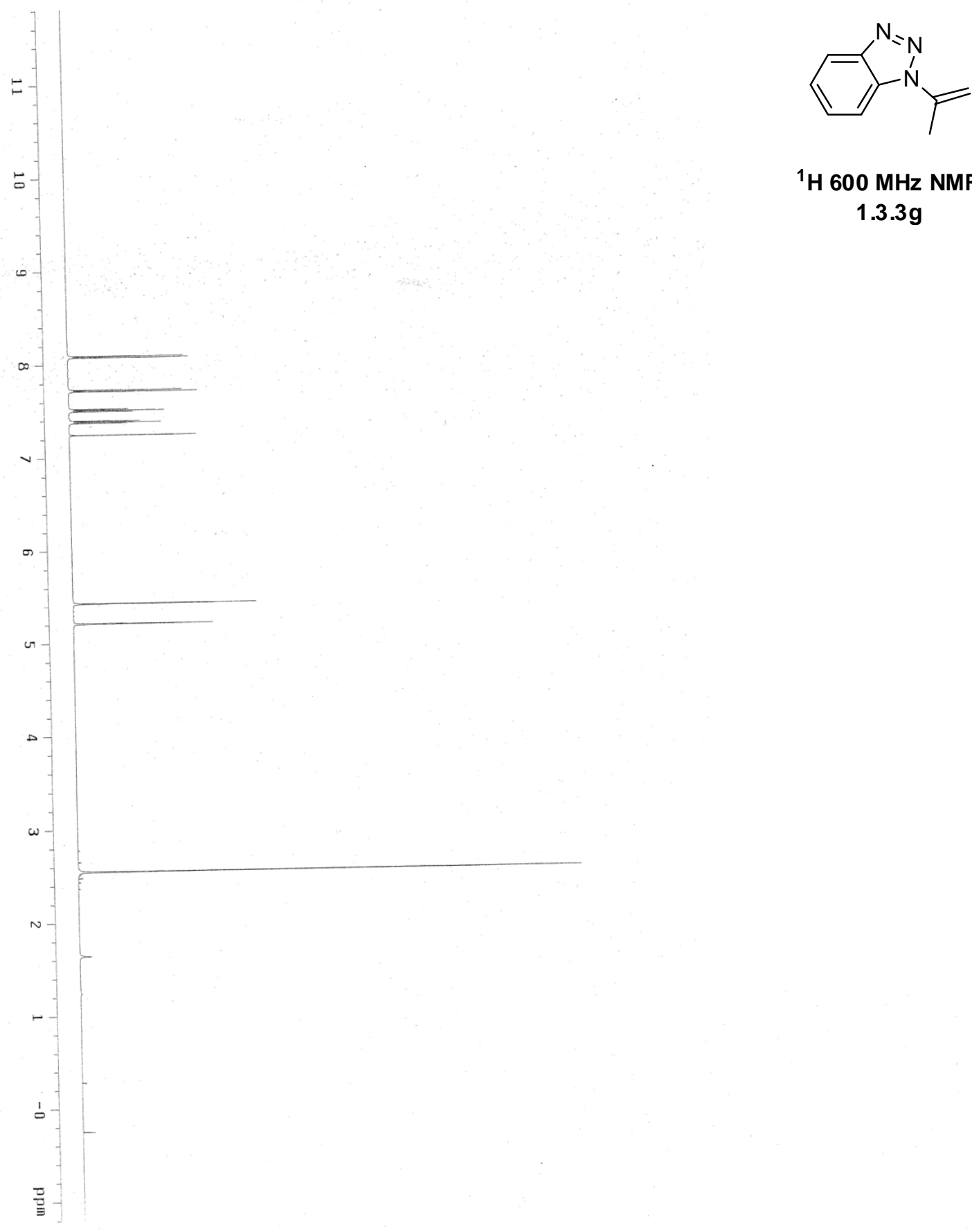

${ }^{1} \mathrm{H} 600 \mathrm{MHz}$ NMR 1.3.3g 

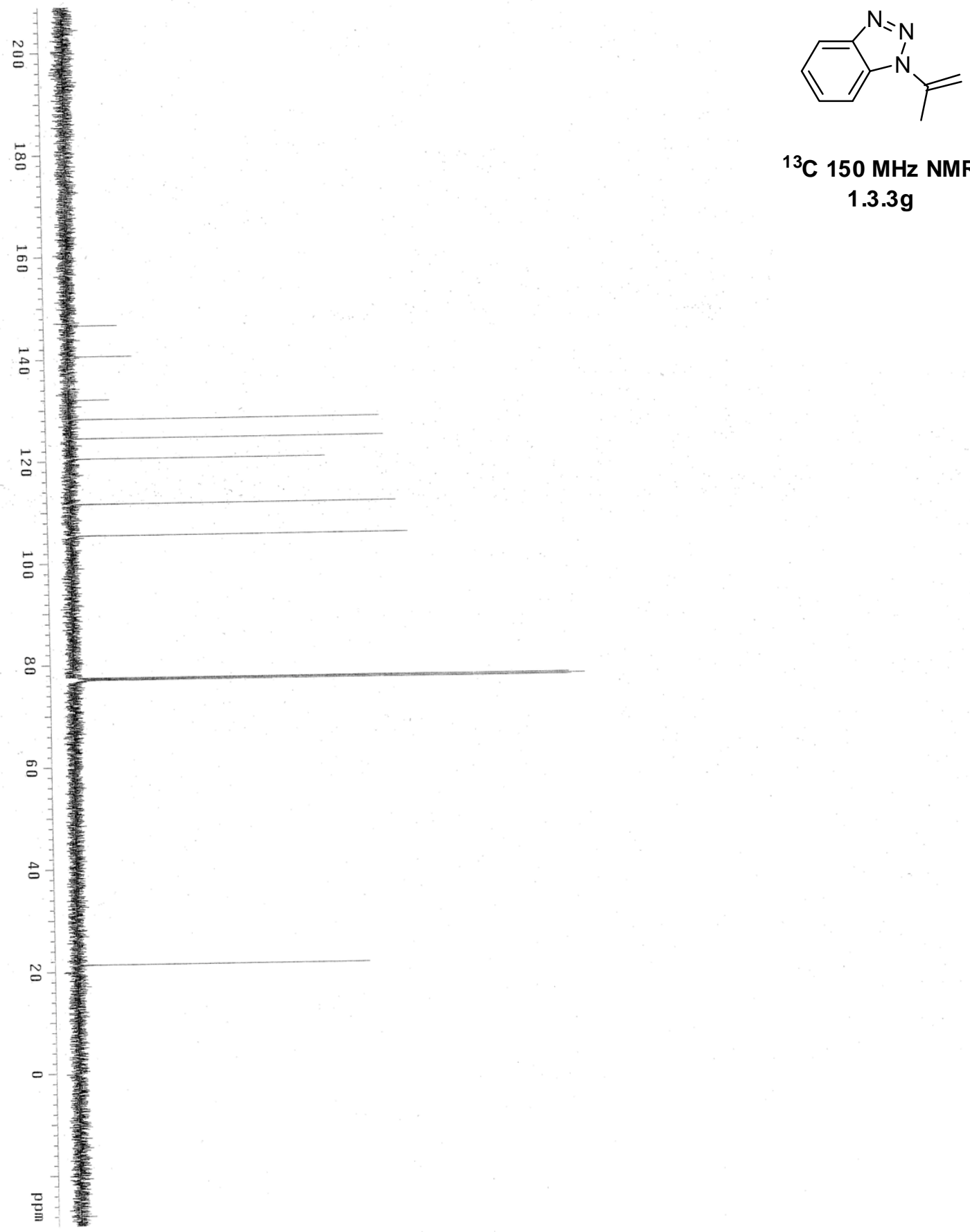

${ }^{13} \mathrm{C} 150 \mathrm{MHz}$ NMR 1.3.3g 


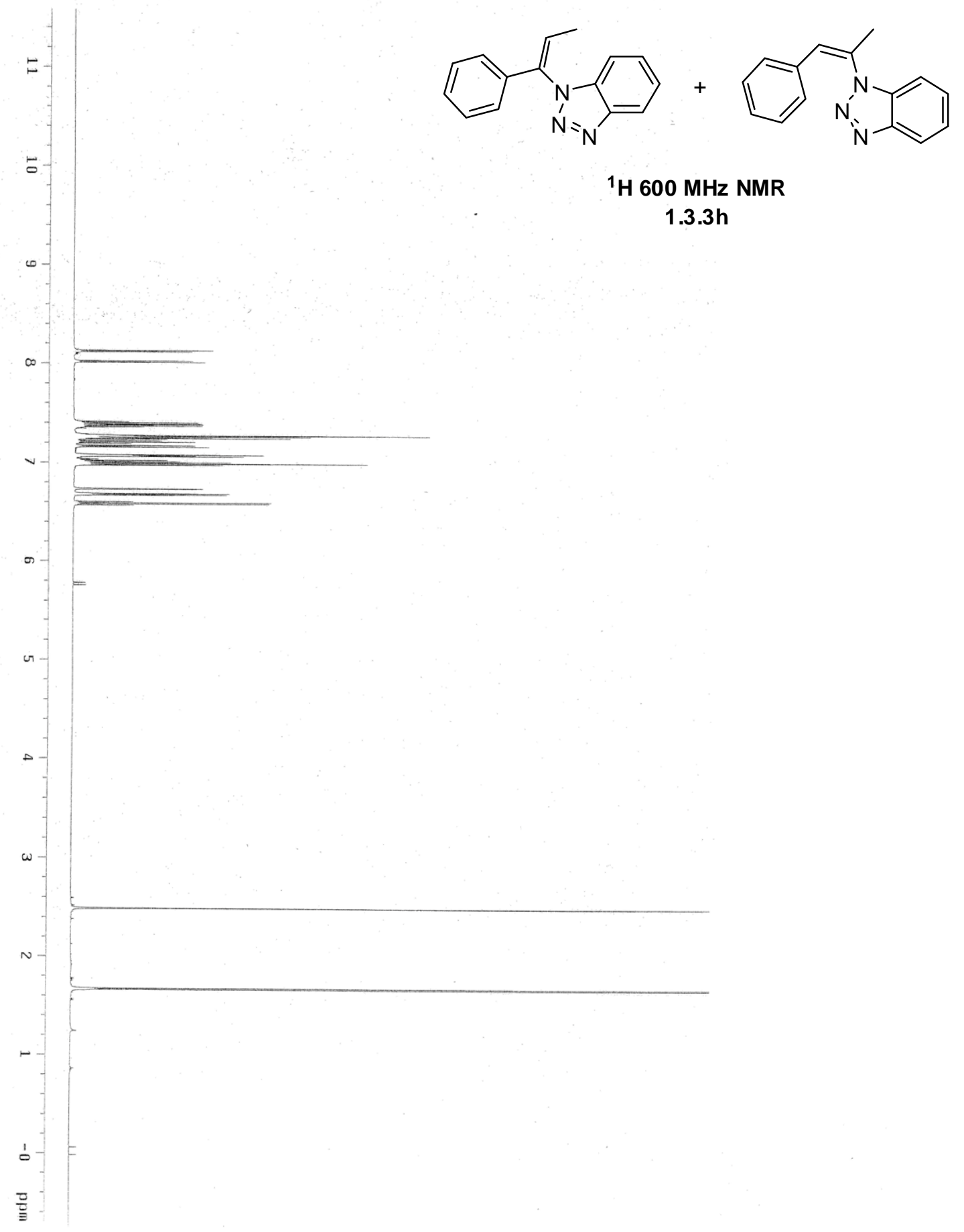




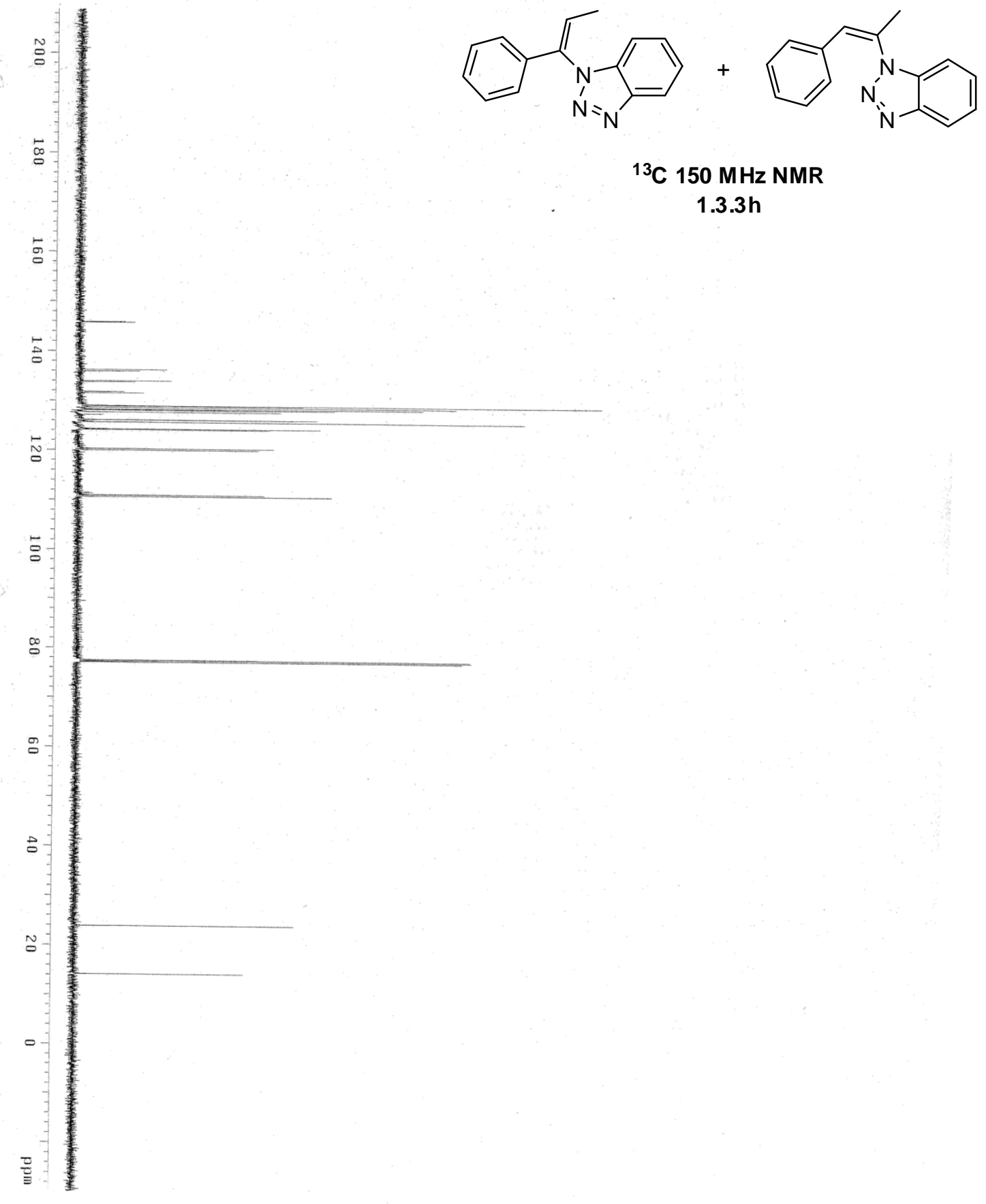




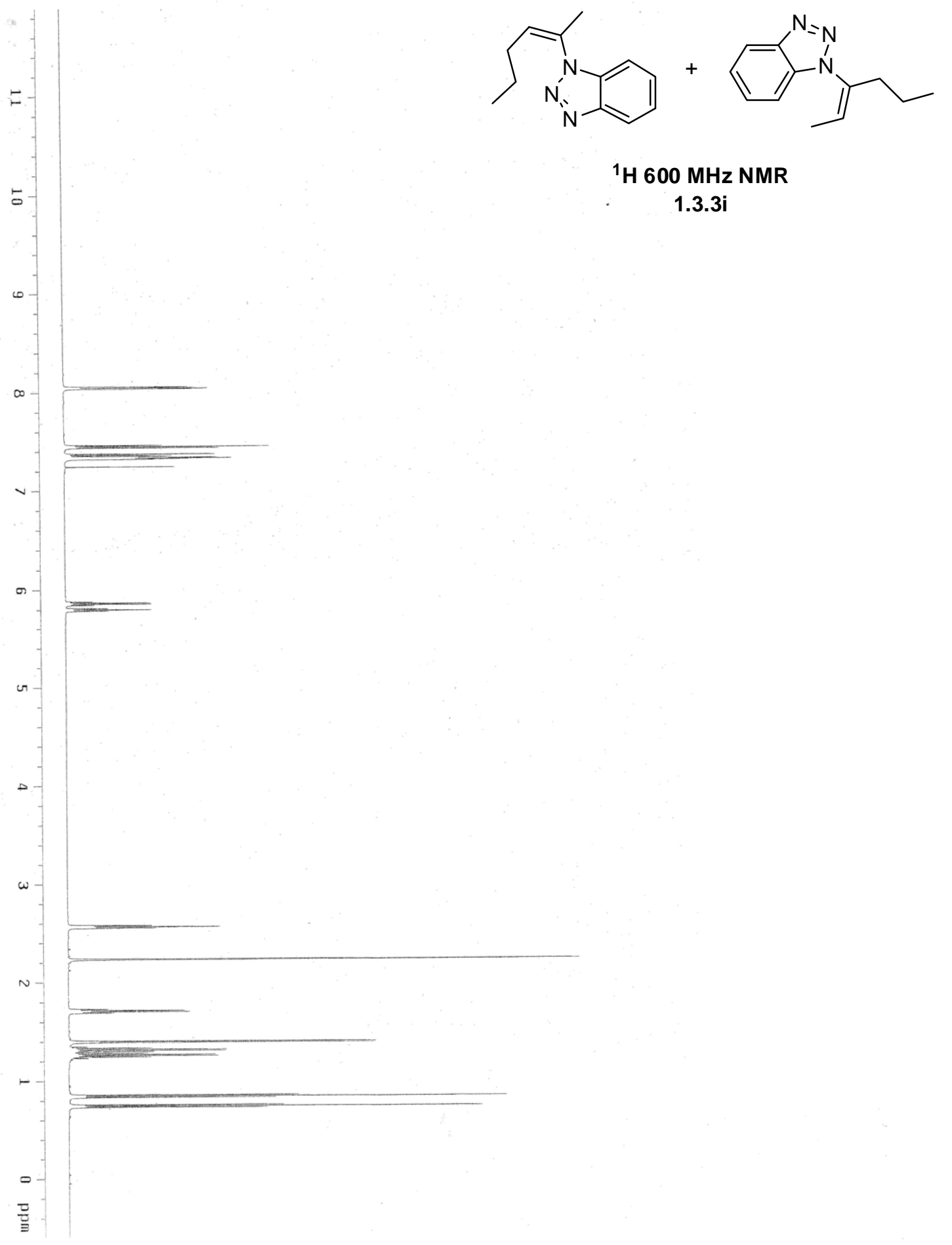



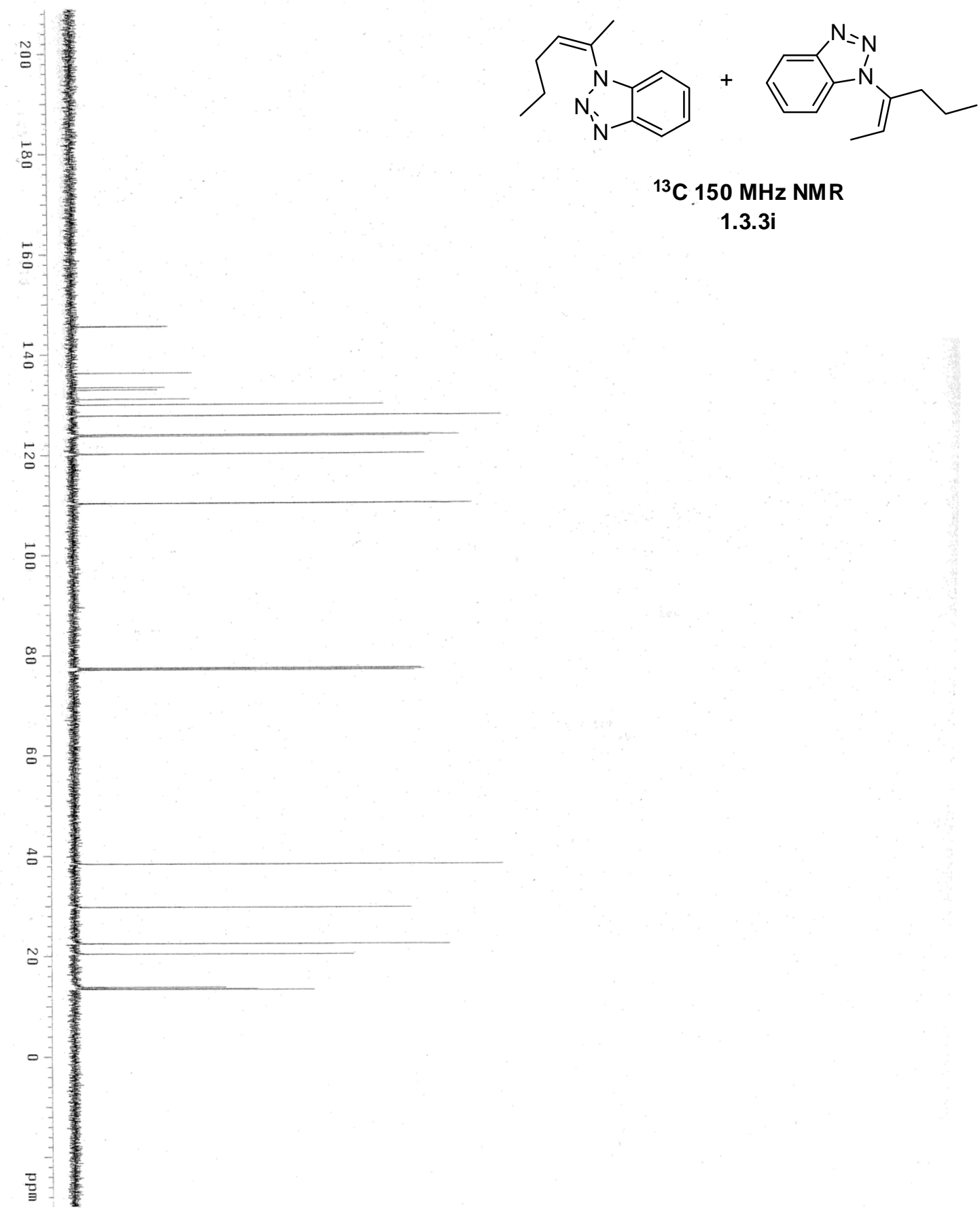

${ }^{13} \mathrm{C} 150 \mathrm{MHz}$ NMR

1.3.3i 

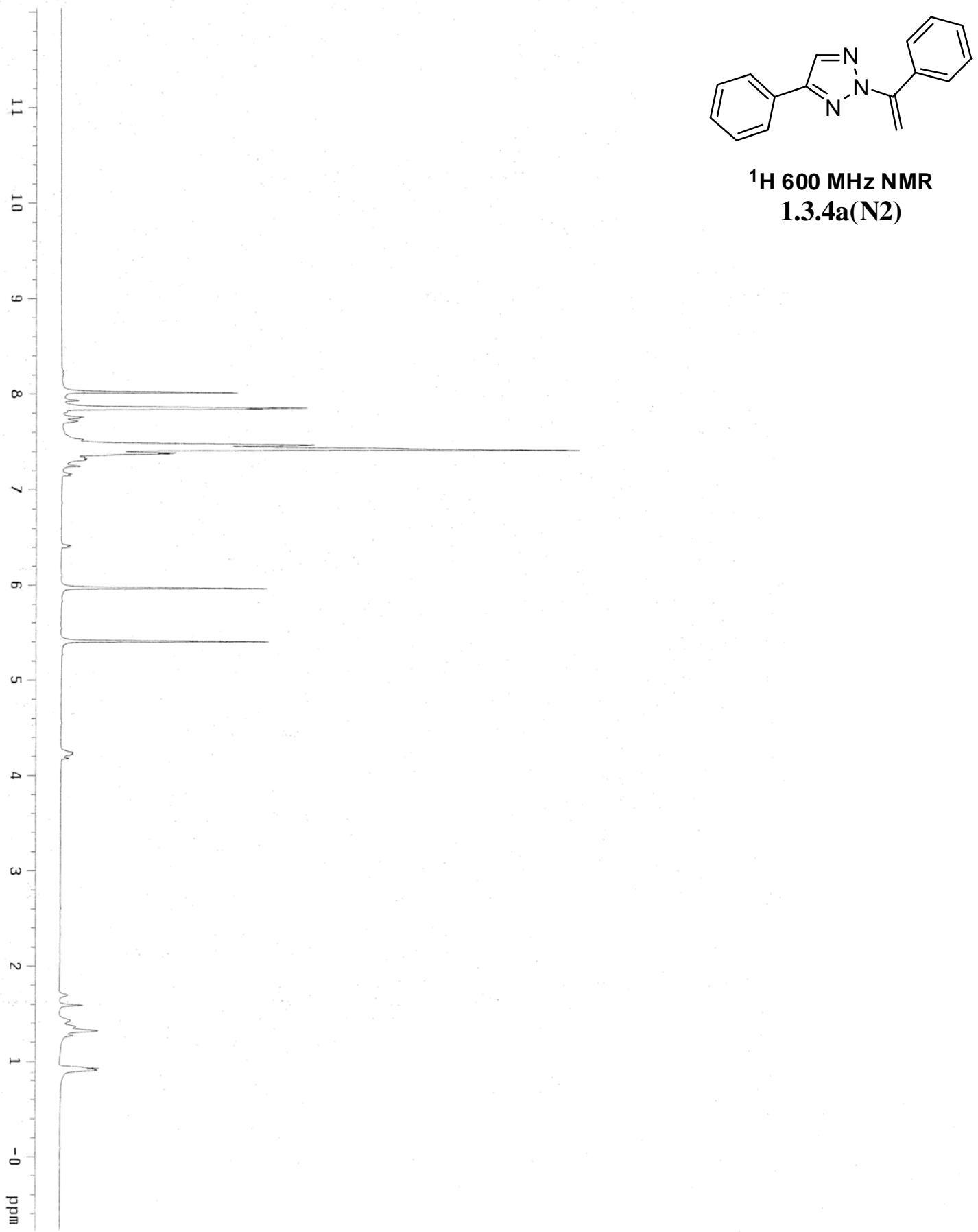

${ }^{1} \mathrm{H} 600 \mathrm{MHz}$ NMR

1.3.4a(N2) 


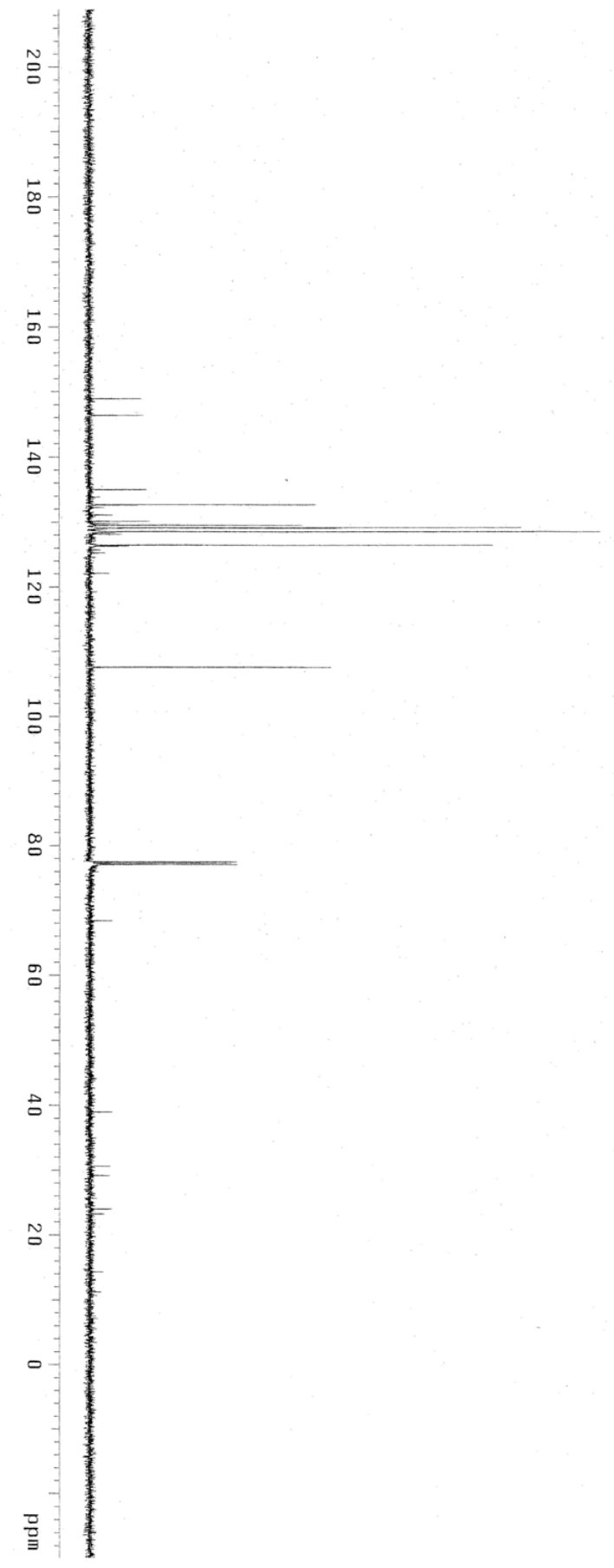

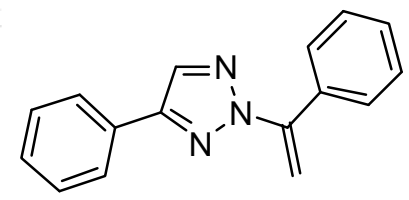

${ }^{13} \mathrm{C} 150 \mathrm{MHz}$ NMR

1.3.4a(N2) 


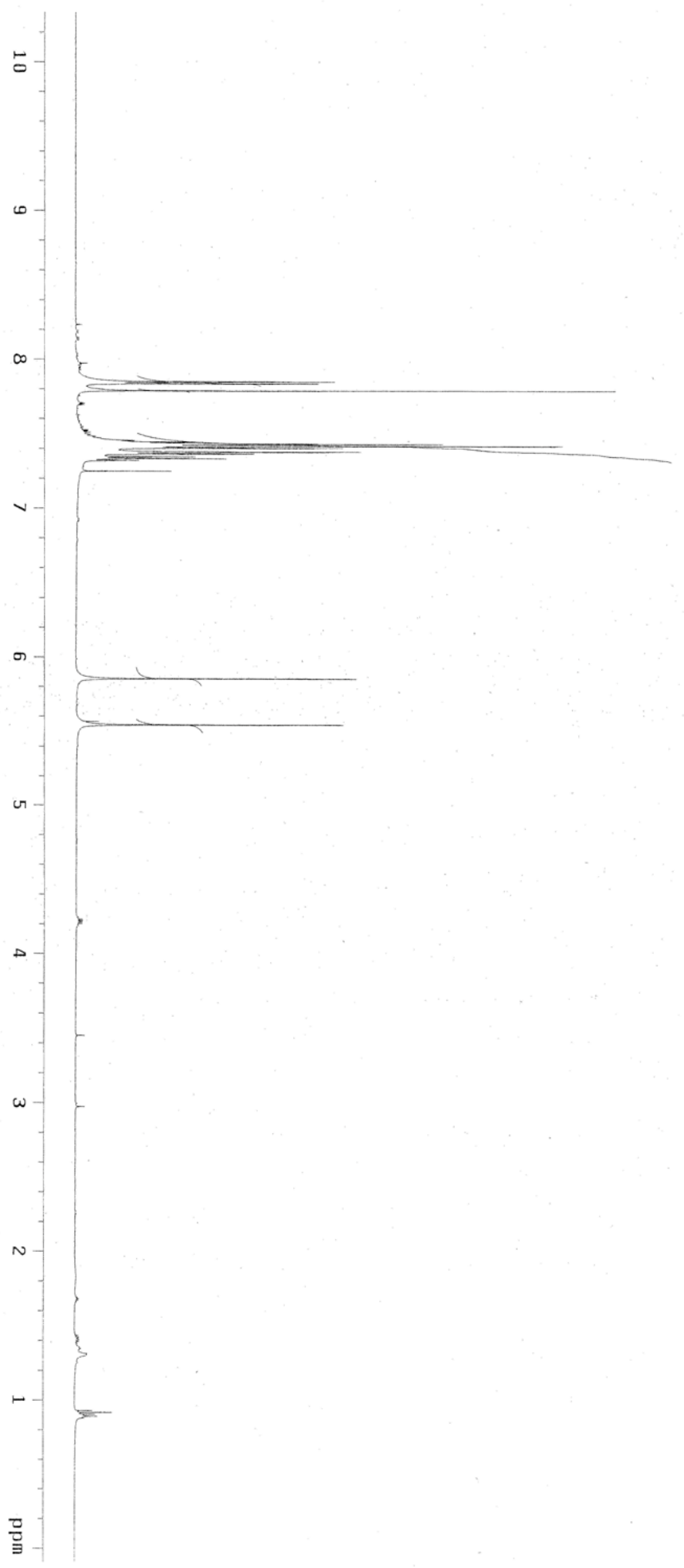

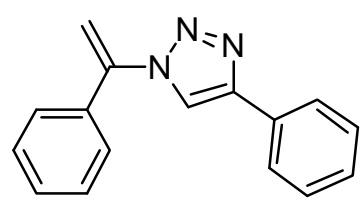

${ }^{1} \mathrm{H} 600 \mathrm{MHz}$ NMR 1.3.4a(N1) 

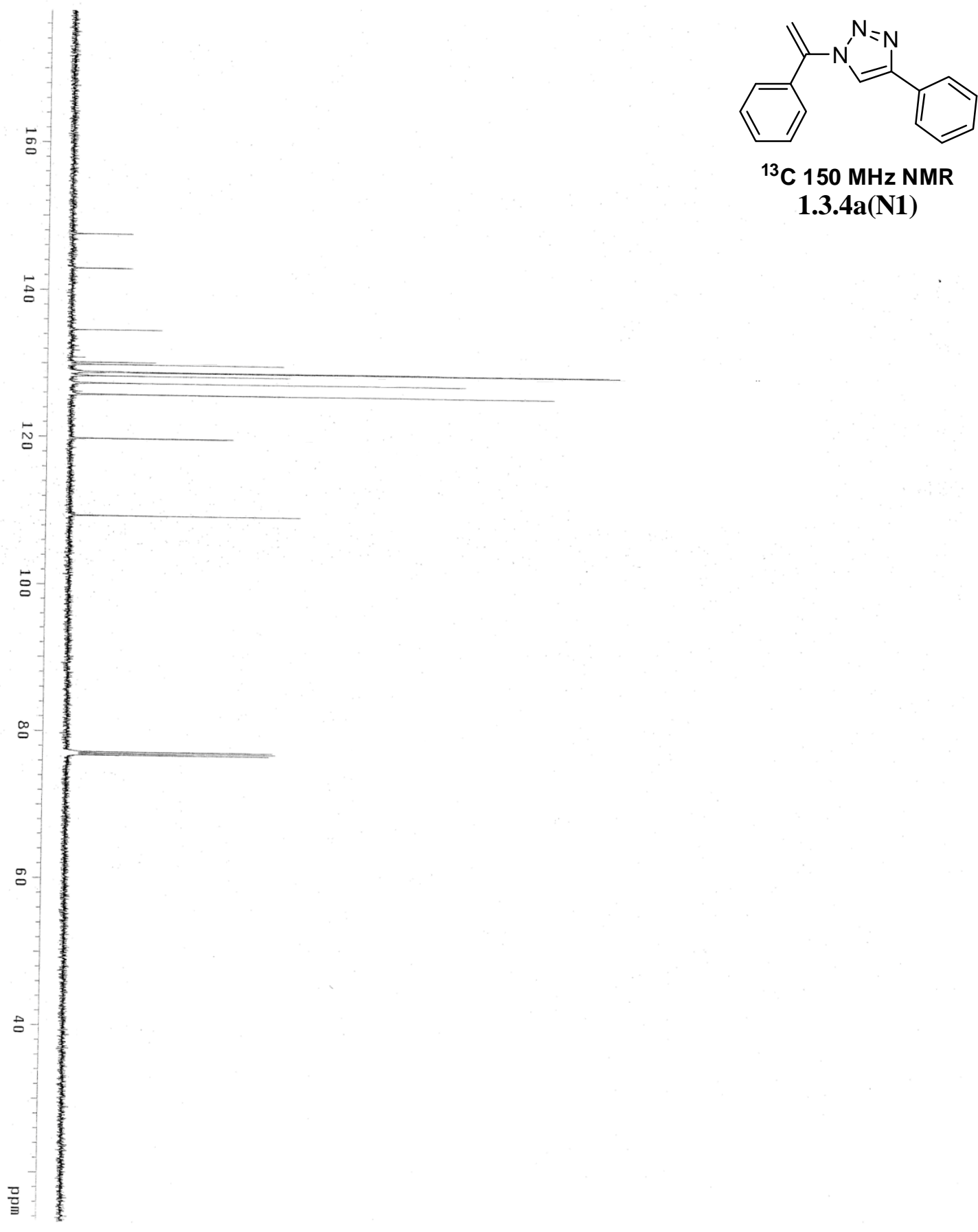

${ }^{13} \mathrm{C} 150 \mathrm{MHz}$ NMR

1.3.4a(N1) 

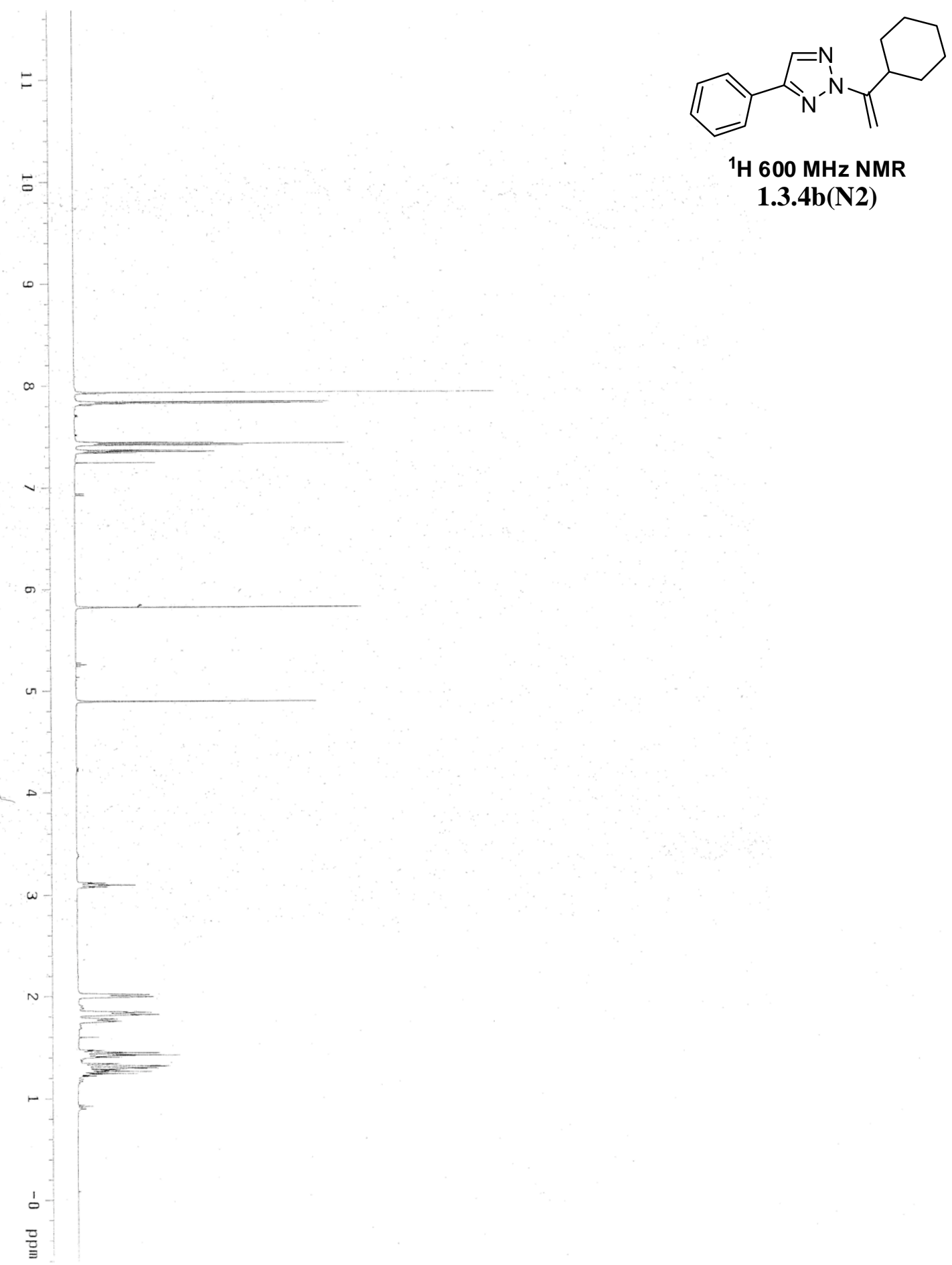

${ }^{1} \mathrm{H} 600 \mathrm{MHz}$ NMR

1.3.4b(N2) 


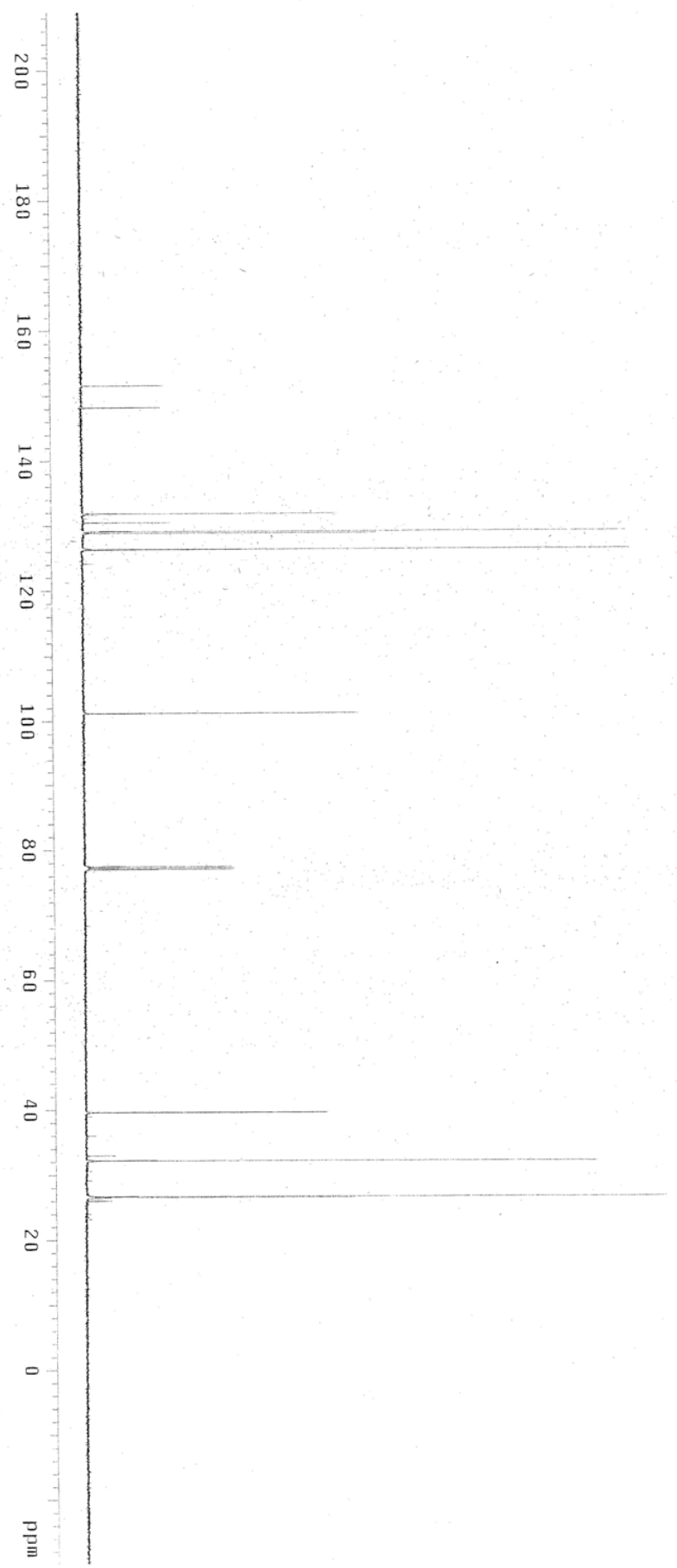

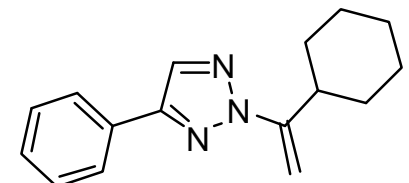

${ }^{13} \mathrm{C} 150 \mathrm{MHz}$ NMR 1.3.4b(N2) 


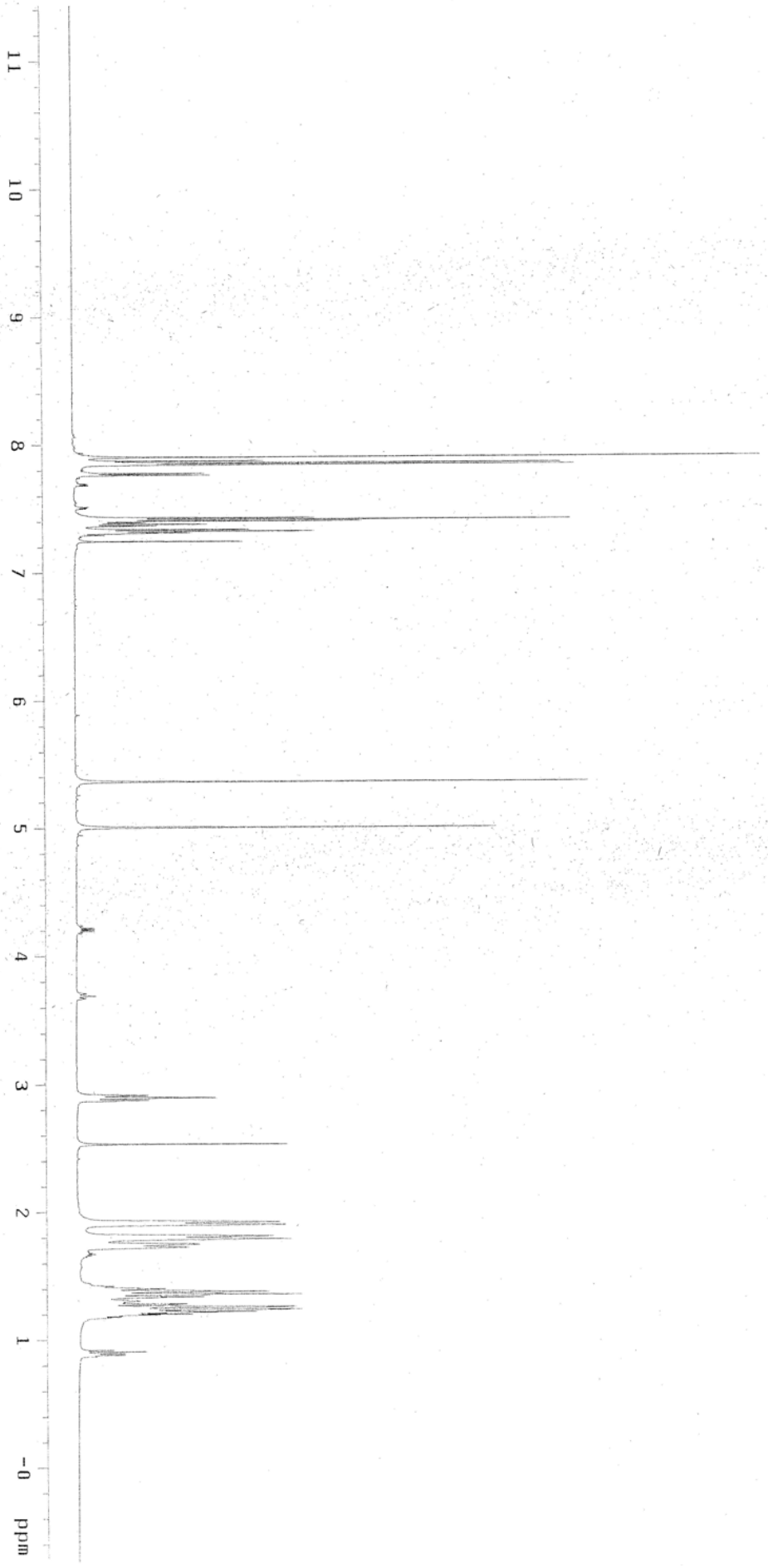

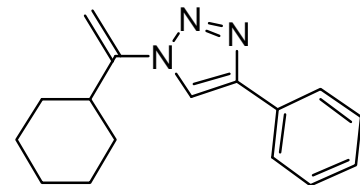

${ }^{1} \mathrm{H} 600 \mathrm{MHz}$ NMR 1.3.4b(N1) 


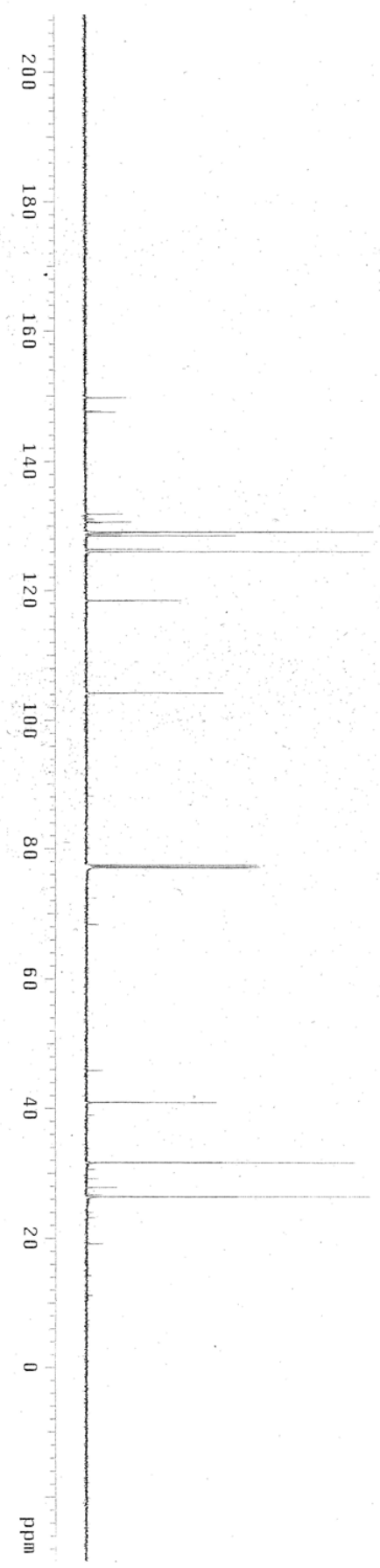

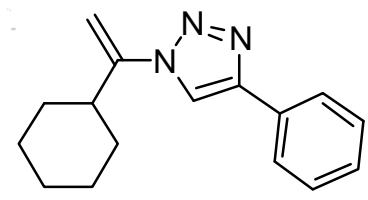

${ }^{13} \mathrm{C} 150 \mathrm{MHz}$ NMR

1.3.4b(N1) 

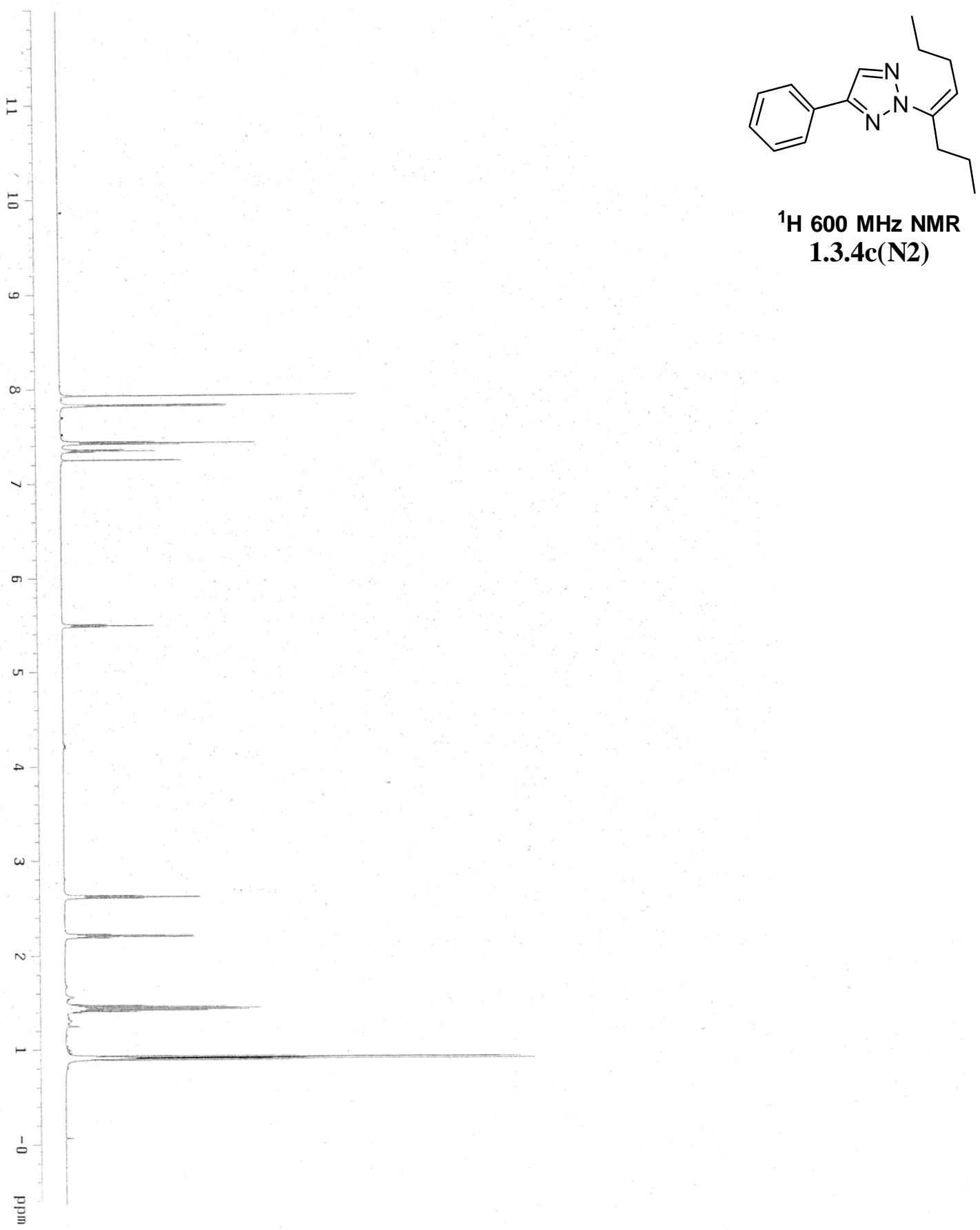

${ }^{1} \mathrm{H} 600 \mathrm{MHz}$ NMR

1.3.4c(N2) 


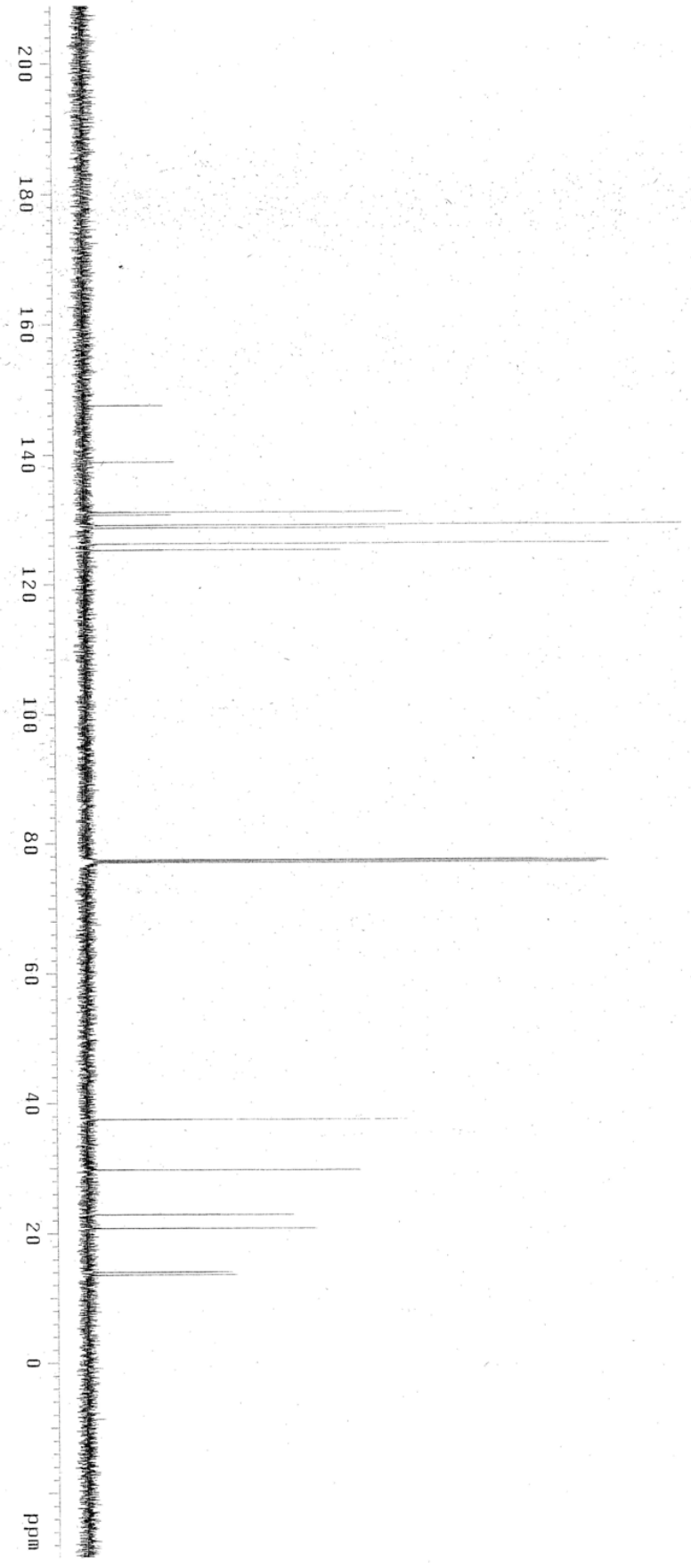

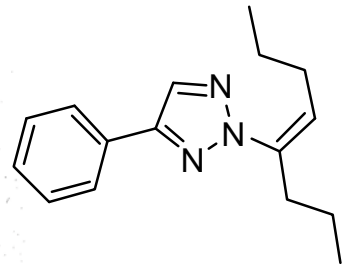

${ }^{13} \mathrm{C} 150 \mathrm{MHz}$ NMR

1.3.4c(N2) 


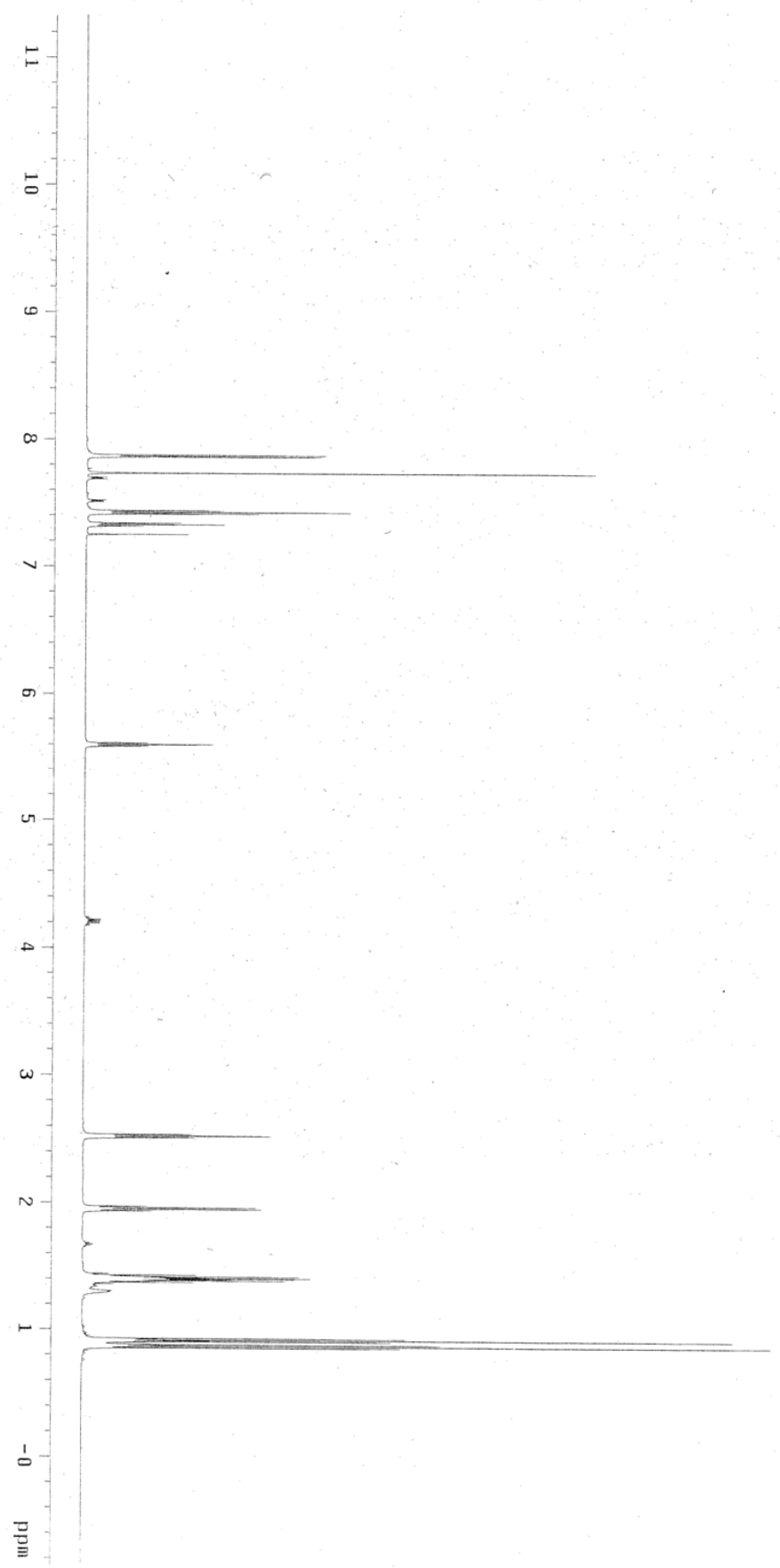

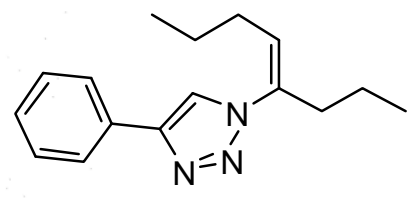

${ }^{1} \mathrm{H} 600 \mathrm{MHz}$ NMR

1.3.4c(N1) 

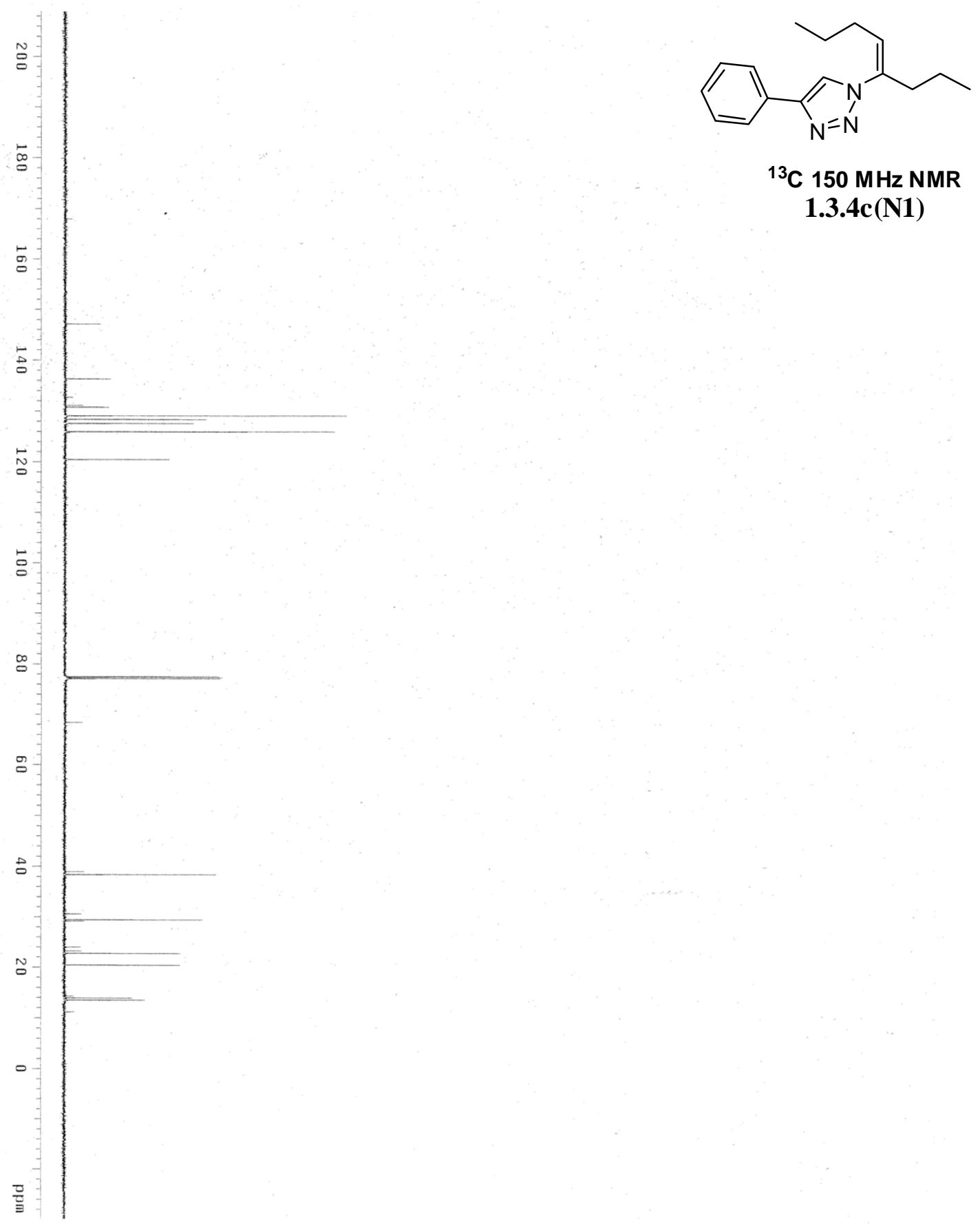

${ }^{13} \mathrm{C} 150 \mathrm{MHz}$ NMR 1.3.4c(N1) 


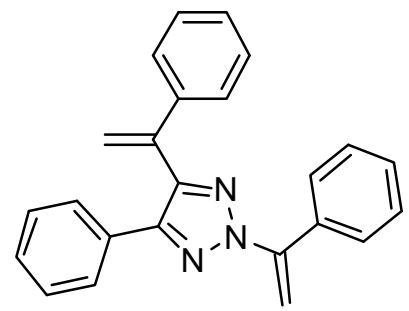

${ }^{1} \mathrm{H} 600 \mathrm{MHz}$ NMR

1.3.4d(N2)

$\infty$

믈 


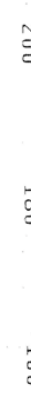

홍

辛

$\stackrel{\sim}{\sim}$

豆

$\stackrel{\infty}{\circ}$

$\stackrel{9}{\circ}$

b

$\cong$

$\circ$

플

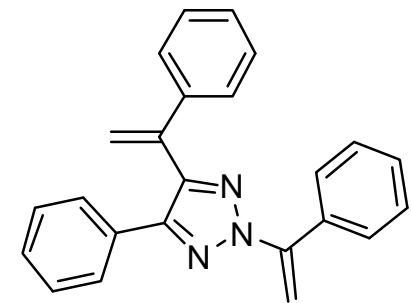

${ }^{13} \mathrm{C} 150 \mathrm{MHz}$ NMR

1.3.4d(N2) 


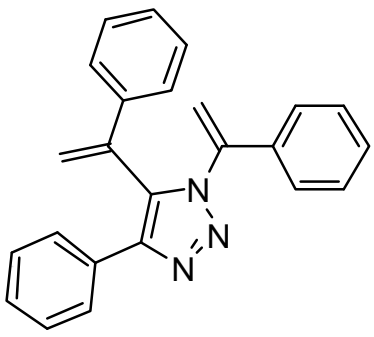

${ }^{1} \mathrm{H} 600 \mathrm{MHz}$ NMR

1.3.4d(N1)

$\infty$

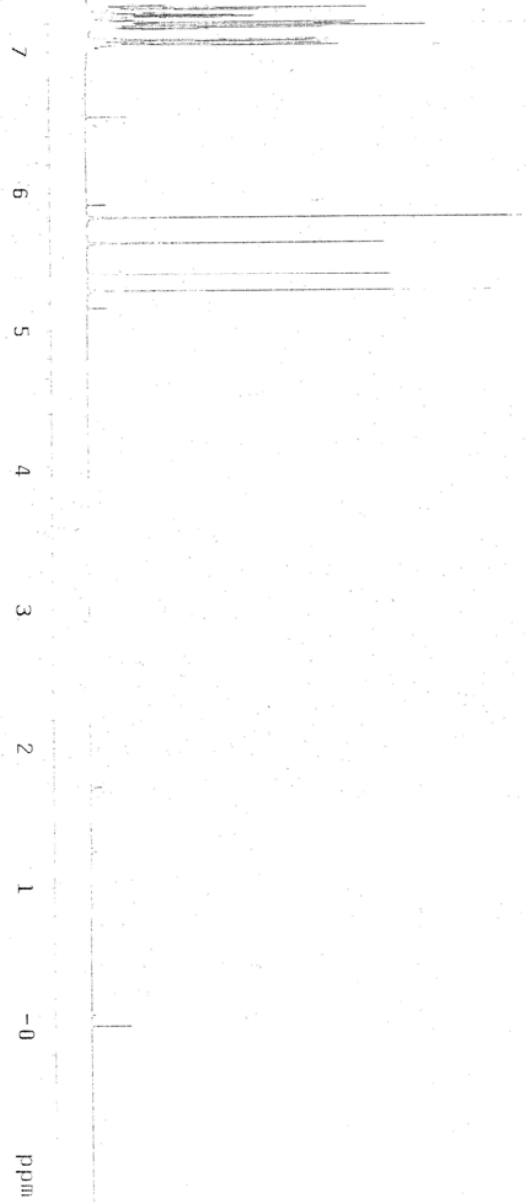



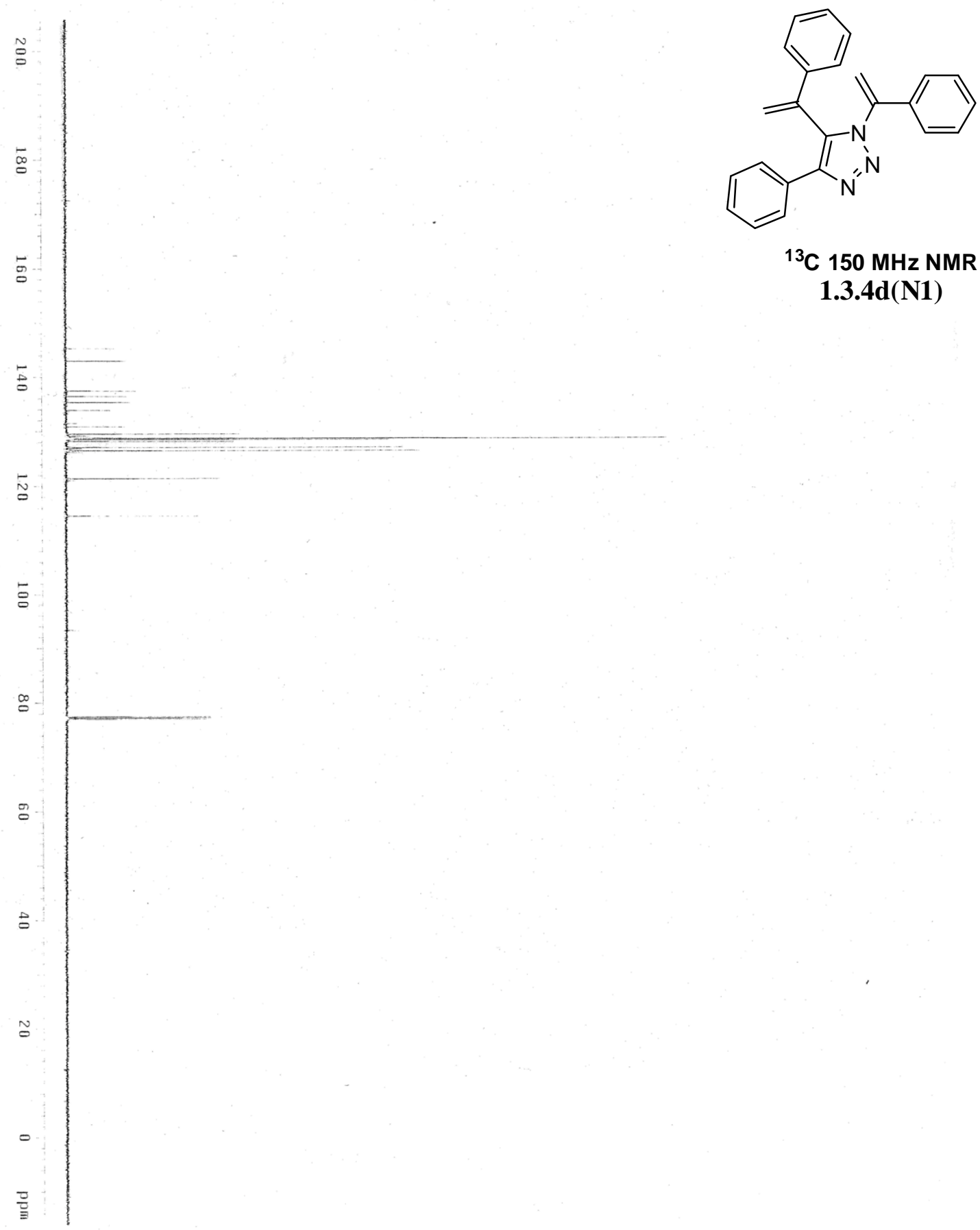

${ }^{13} \mathrm{C} 150 \mathrm{MHz}$ NMR 1.3.4d(N1) 


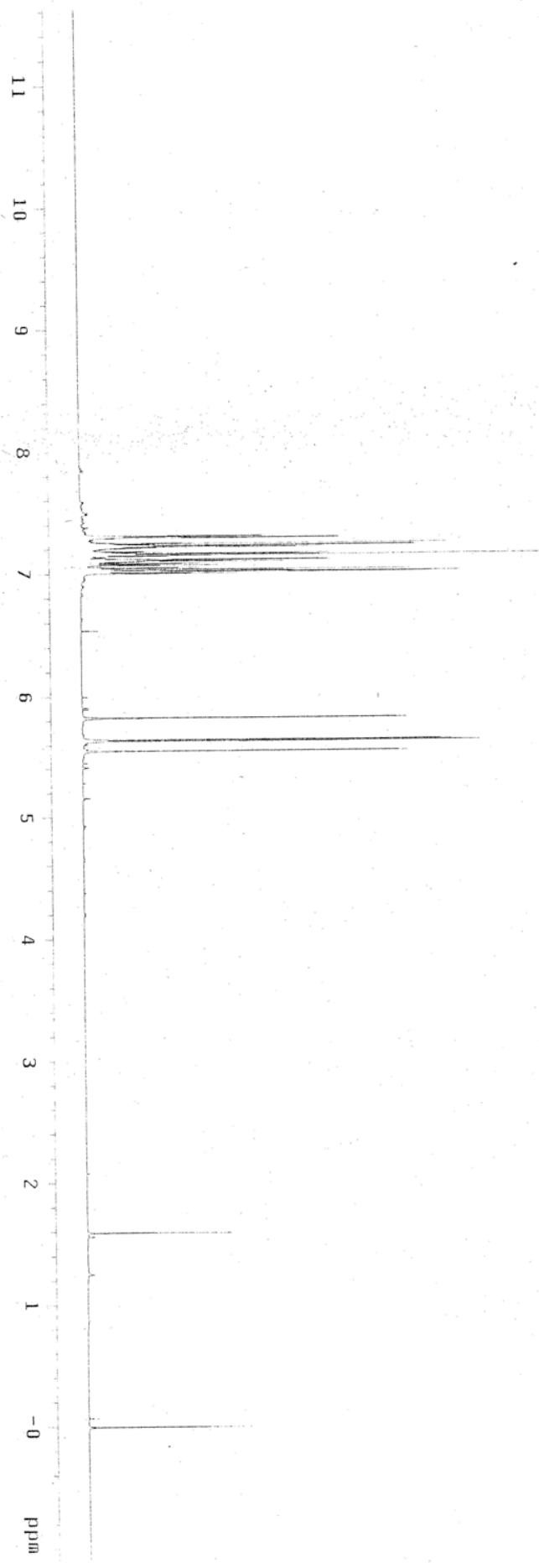

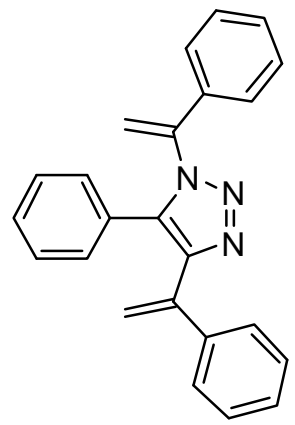

${ }^{1} \mathrm{H} 600 \mathrm{MHz}$ NMR 1.3.4d(N3) 


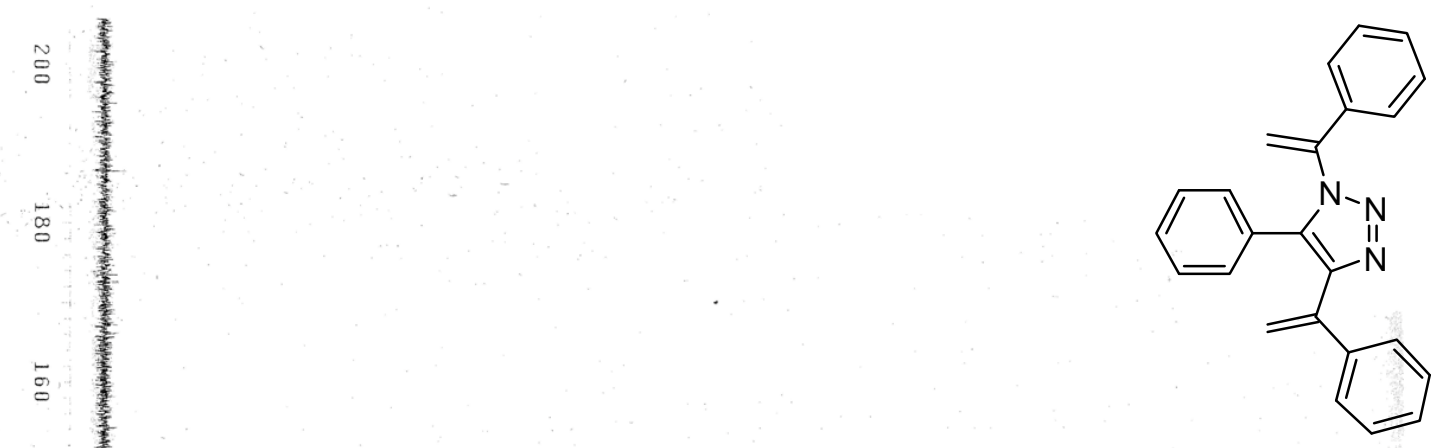

${ }^{13} \mathrm{C} 150 \mathrm{MHz}$ NMR 1.3.4d(N3) 


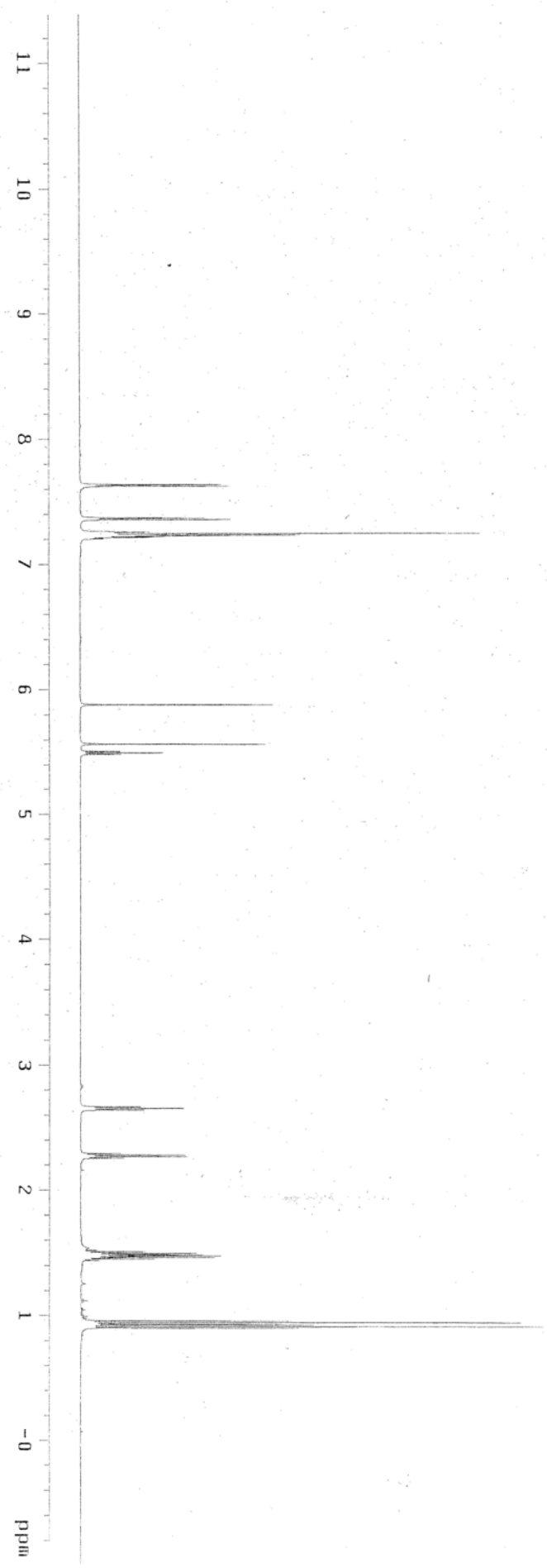

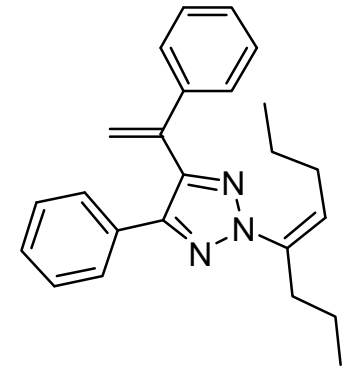

${ }^{1} \mathrm{H} 600 \mathrm{MHz}$ NMR

1.3.4e(N2) 


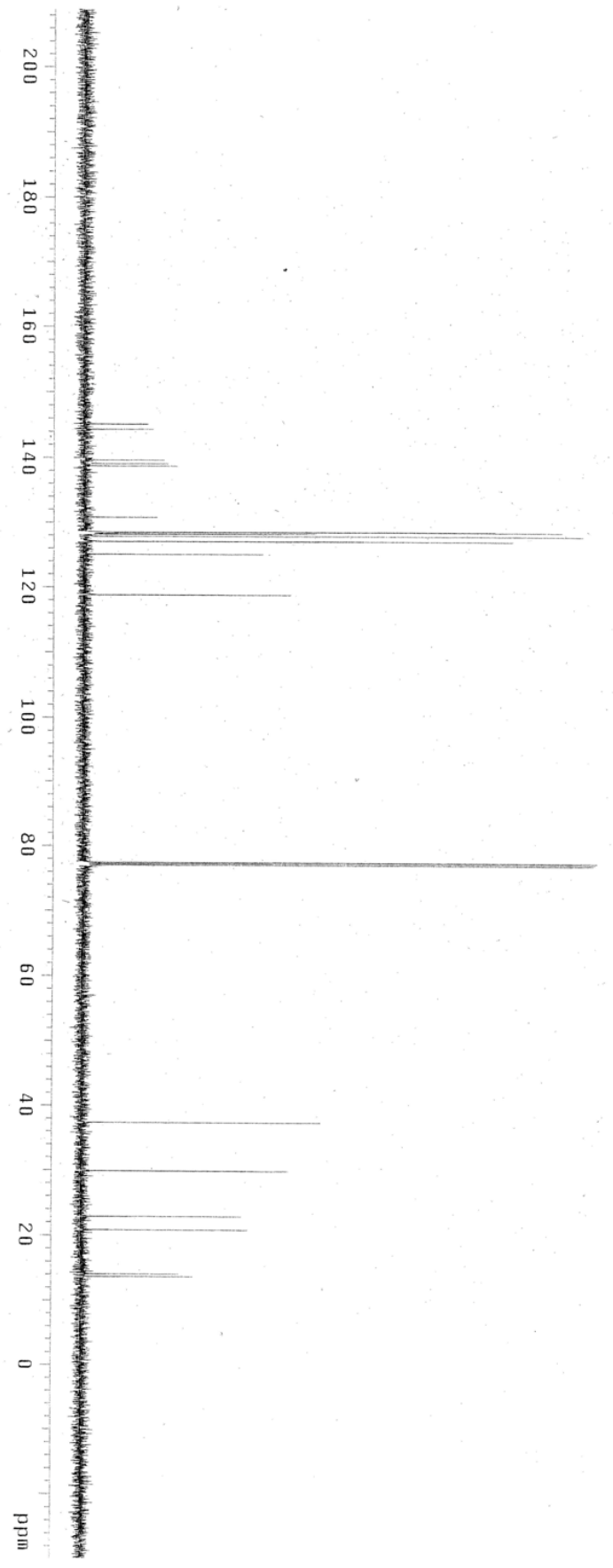

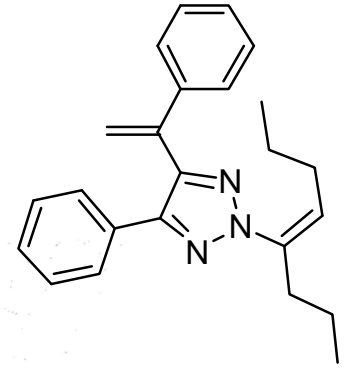

${ }^{13} \mathrm{C} 150 \mathrm{MHz}$ NMR 1.3.4e(N2) 

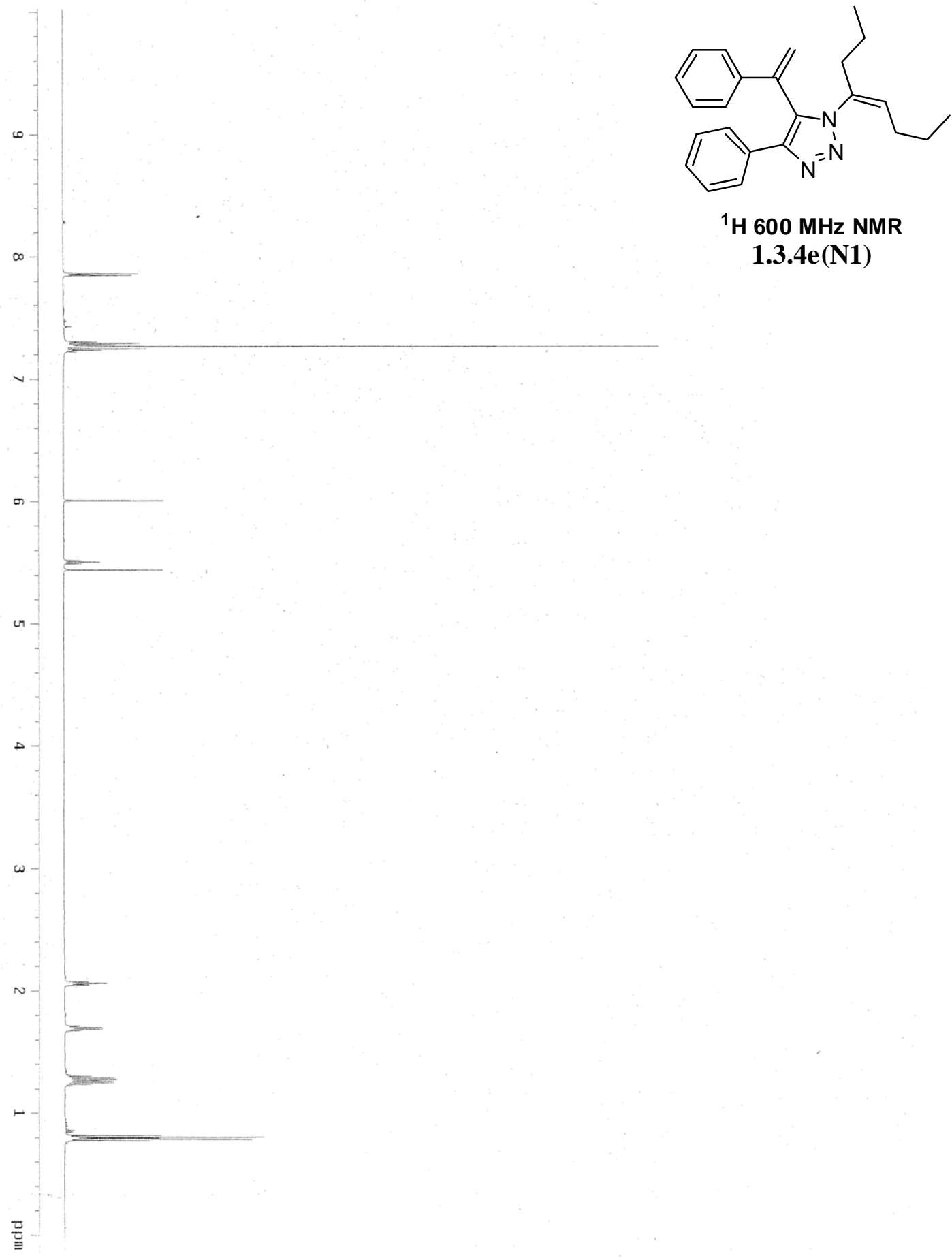

${ }^{1} \mathrm{H} 600 \mathrm{MHz}$ NMR

1.3.4e(N1) 

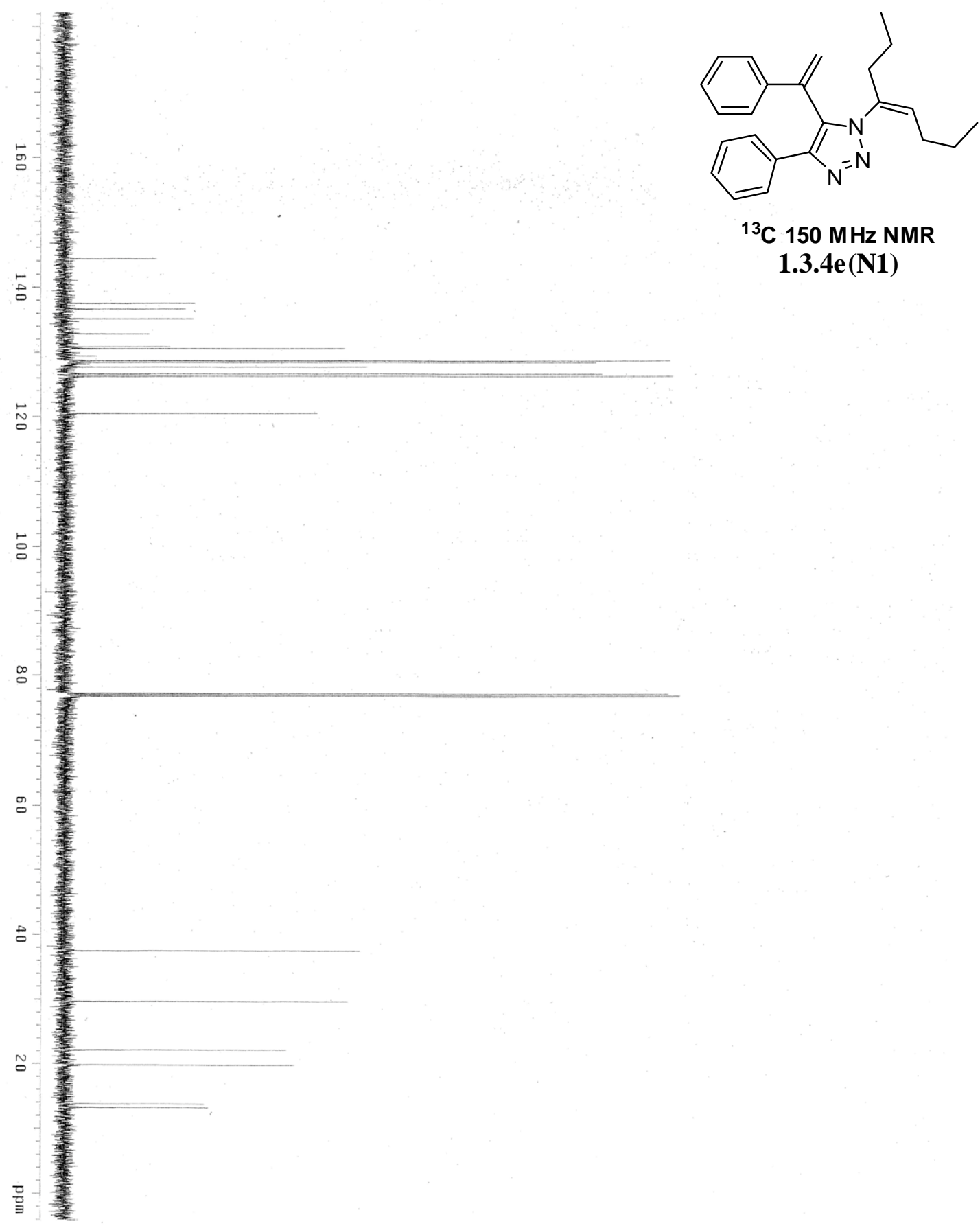

${ }^{13} \mathrm{C} 150 \mathrm{MHz}$ NMR

1.3.4e(N1) 


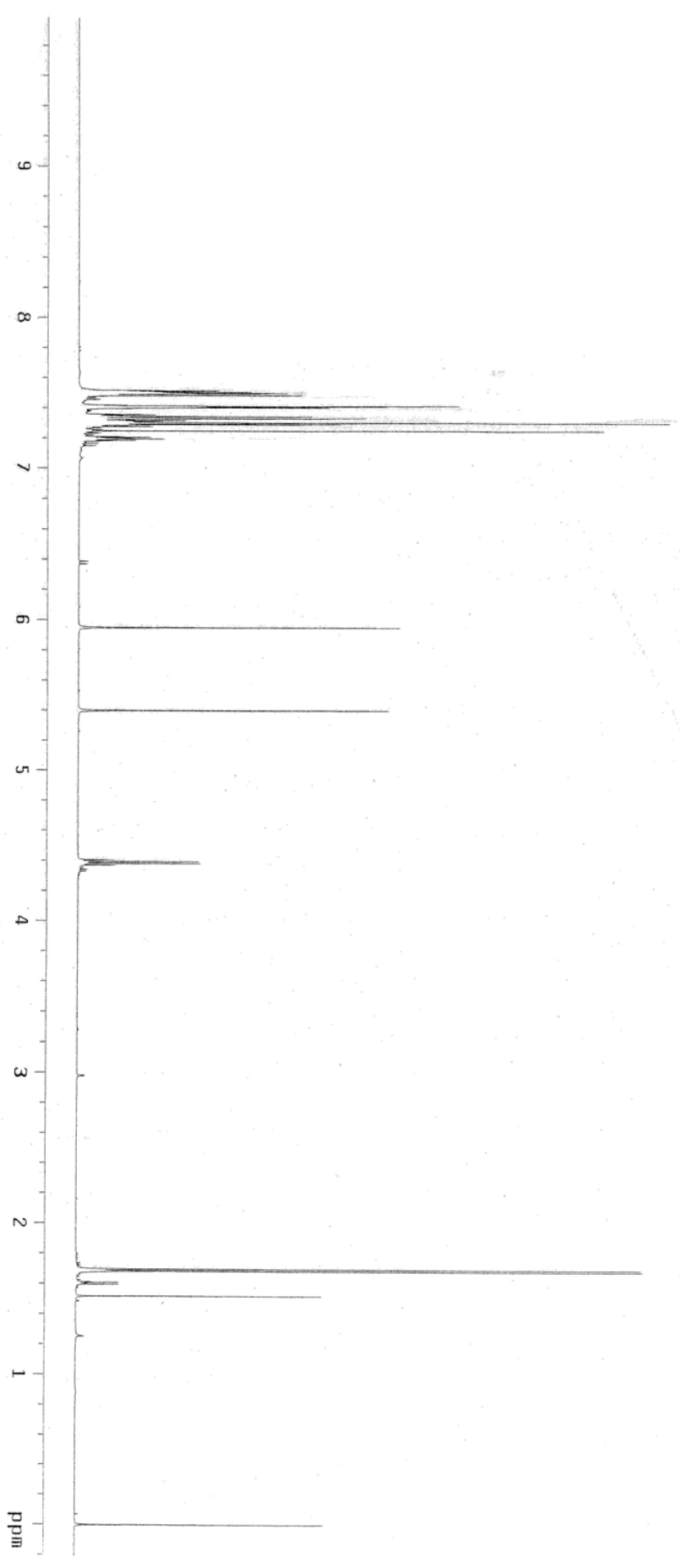

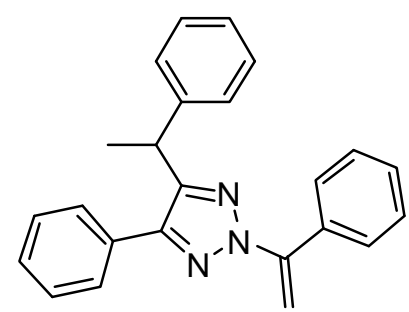

${ }^{1} \mathrm{H} 600 \mathrm{MHz}$ NMR

1.3.4f(N2) 


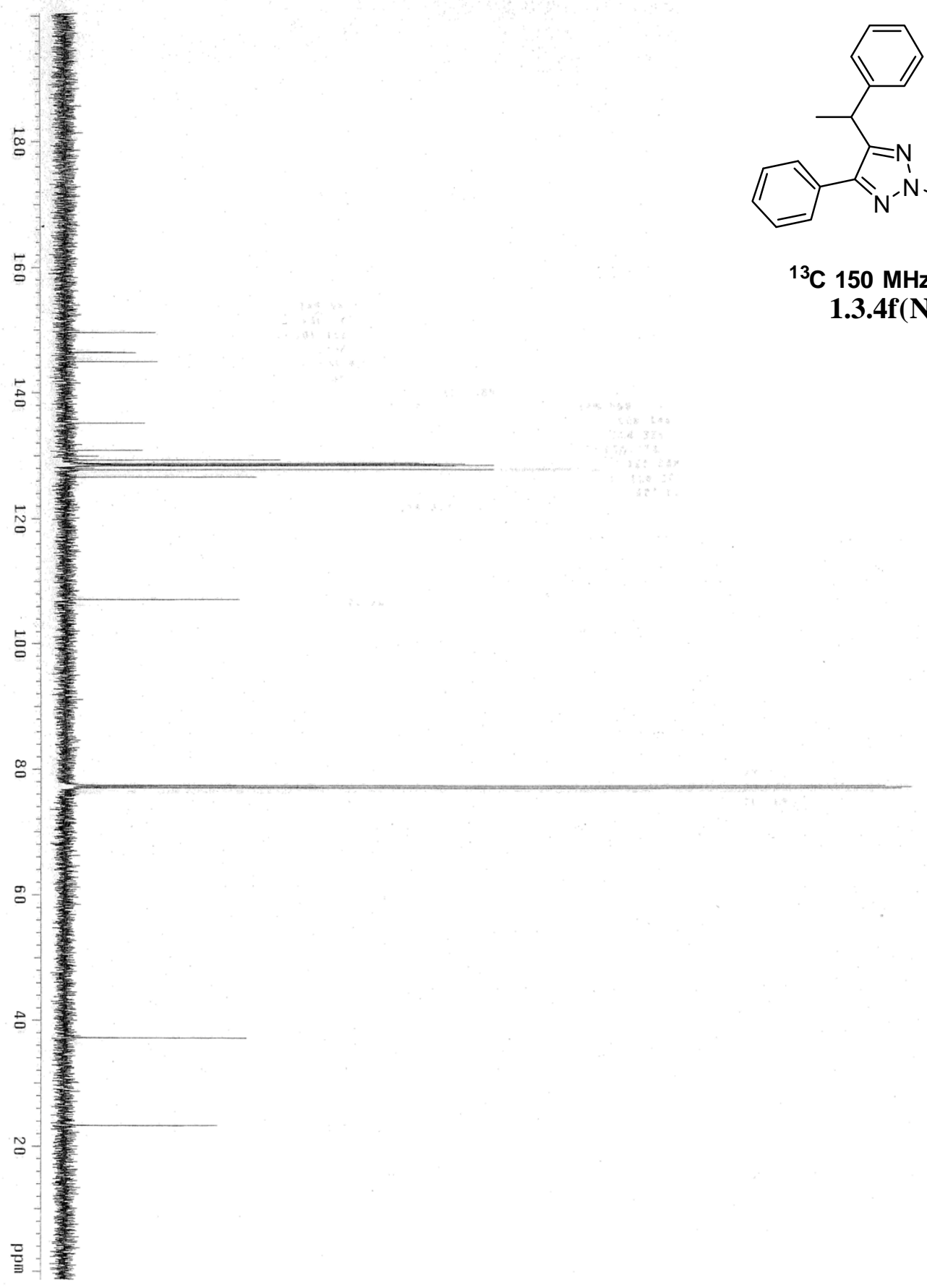



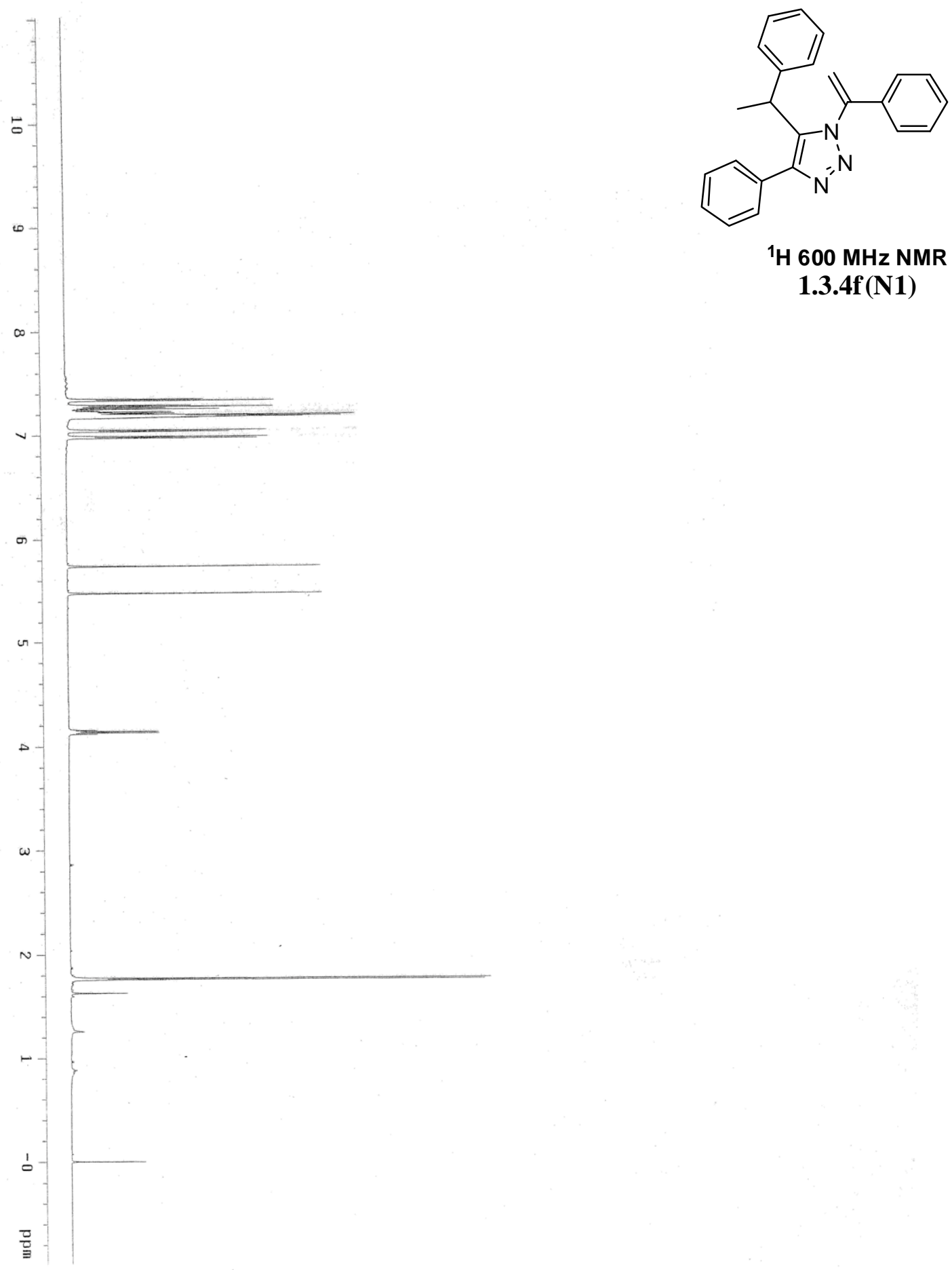

${ }^{1} \mathrm{H} 600 \mathrm{MHz}$ NMR

1.3.4f(N1) 

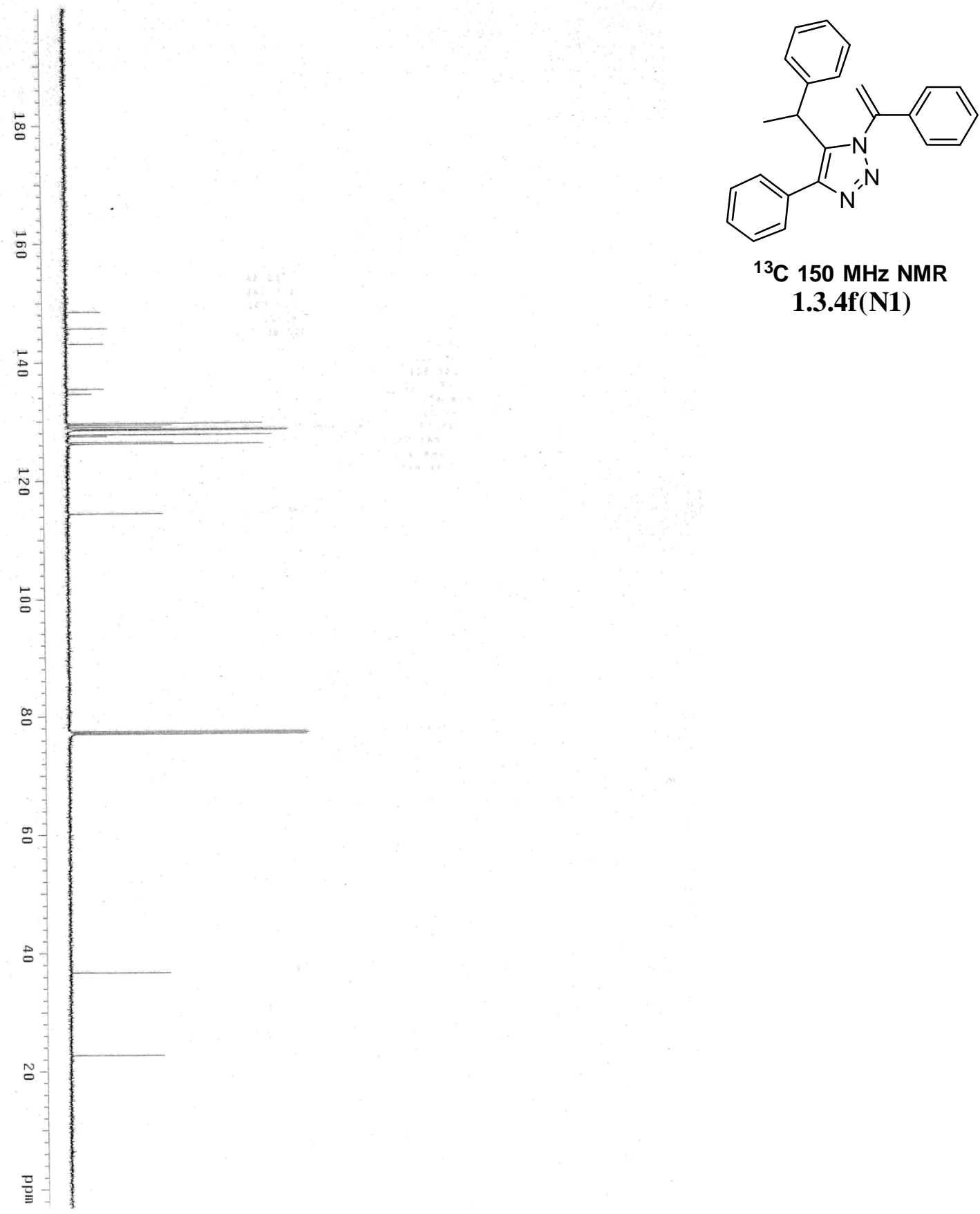

${ }^{13} \mathrm{C} 150 \mathrm{MHz}$ NMR 1.3.4f(N1) 


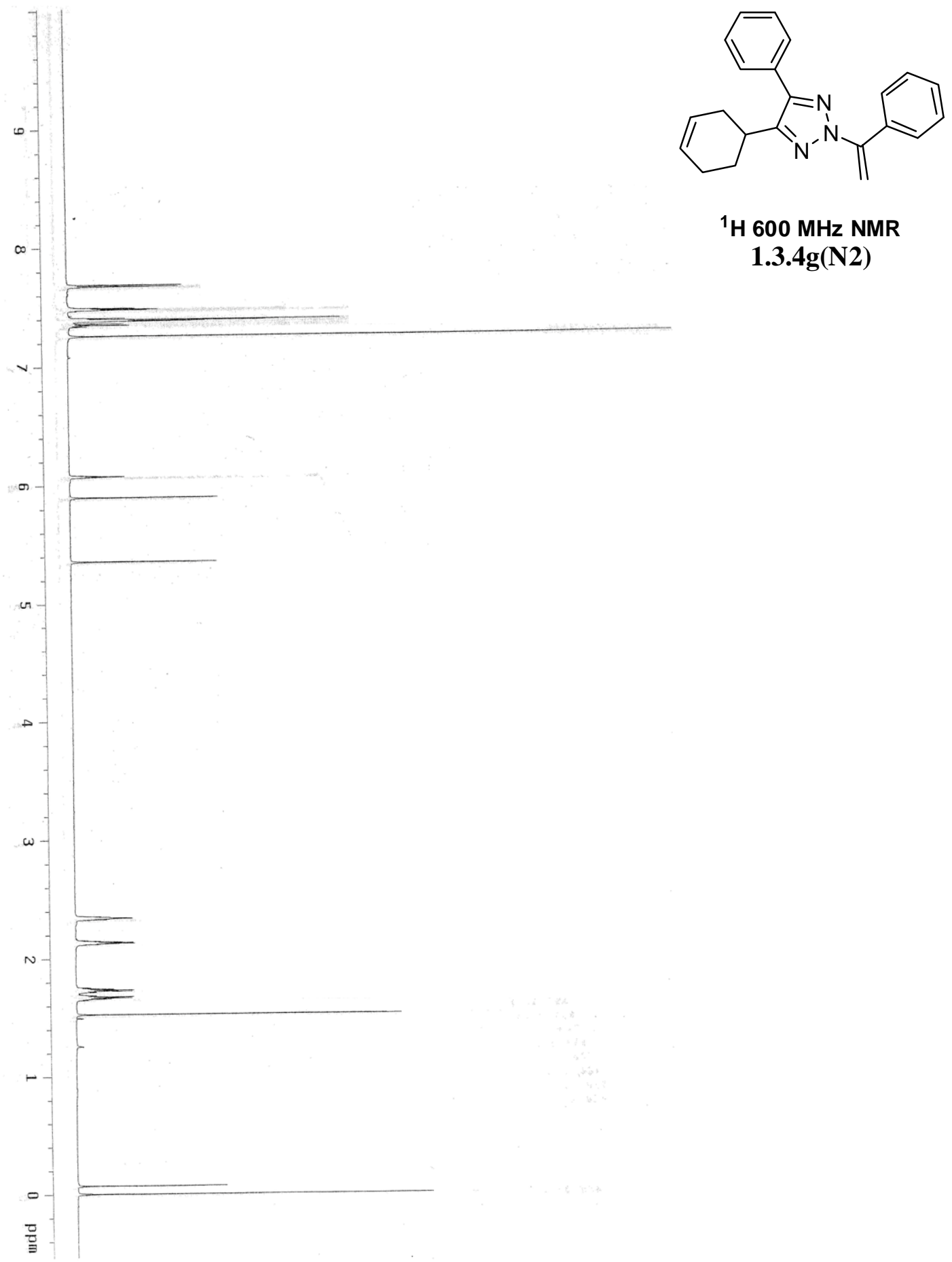




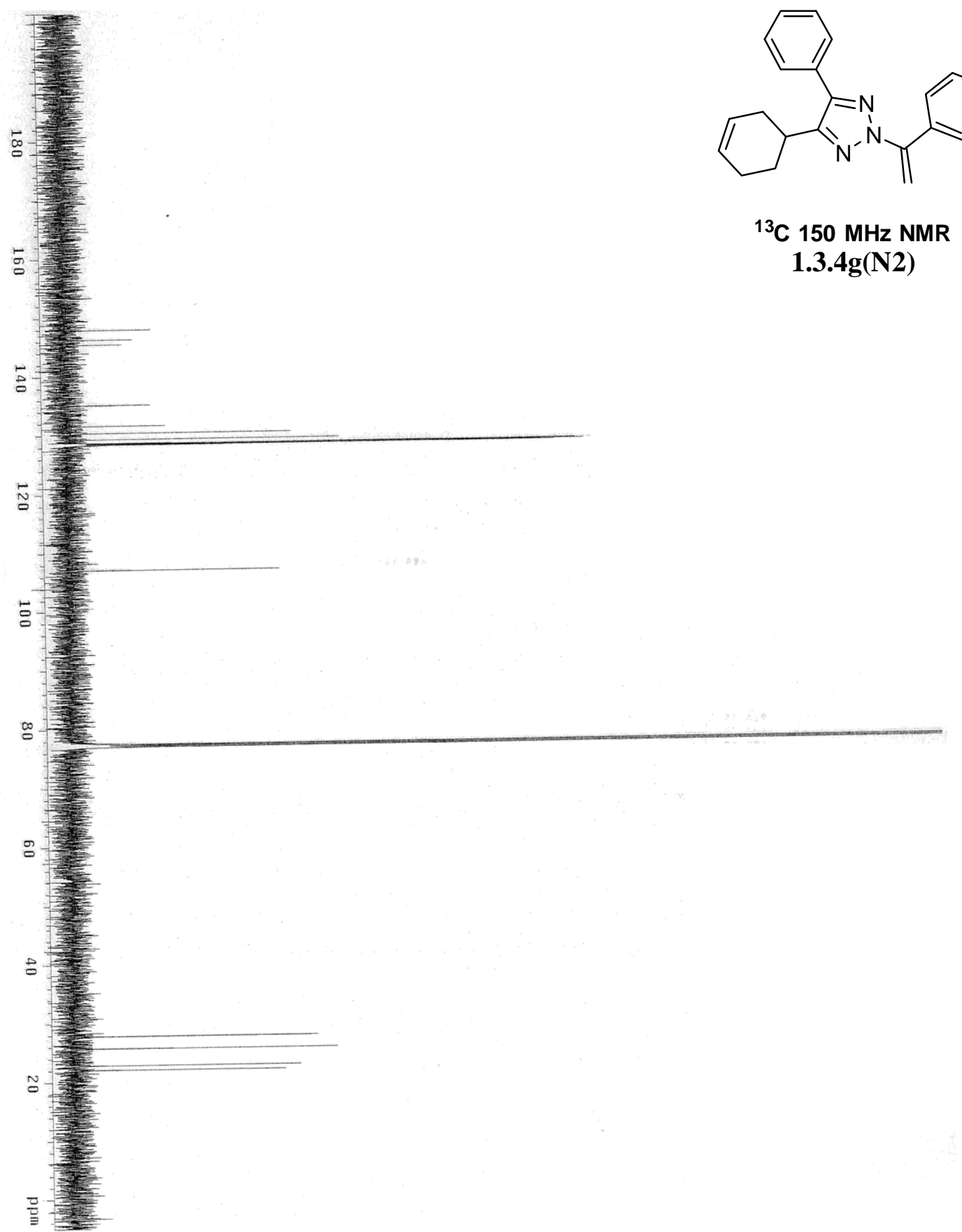



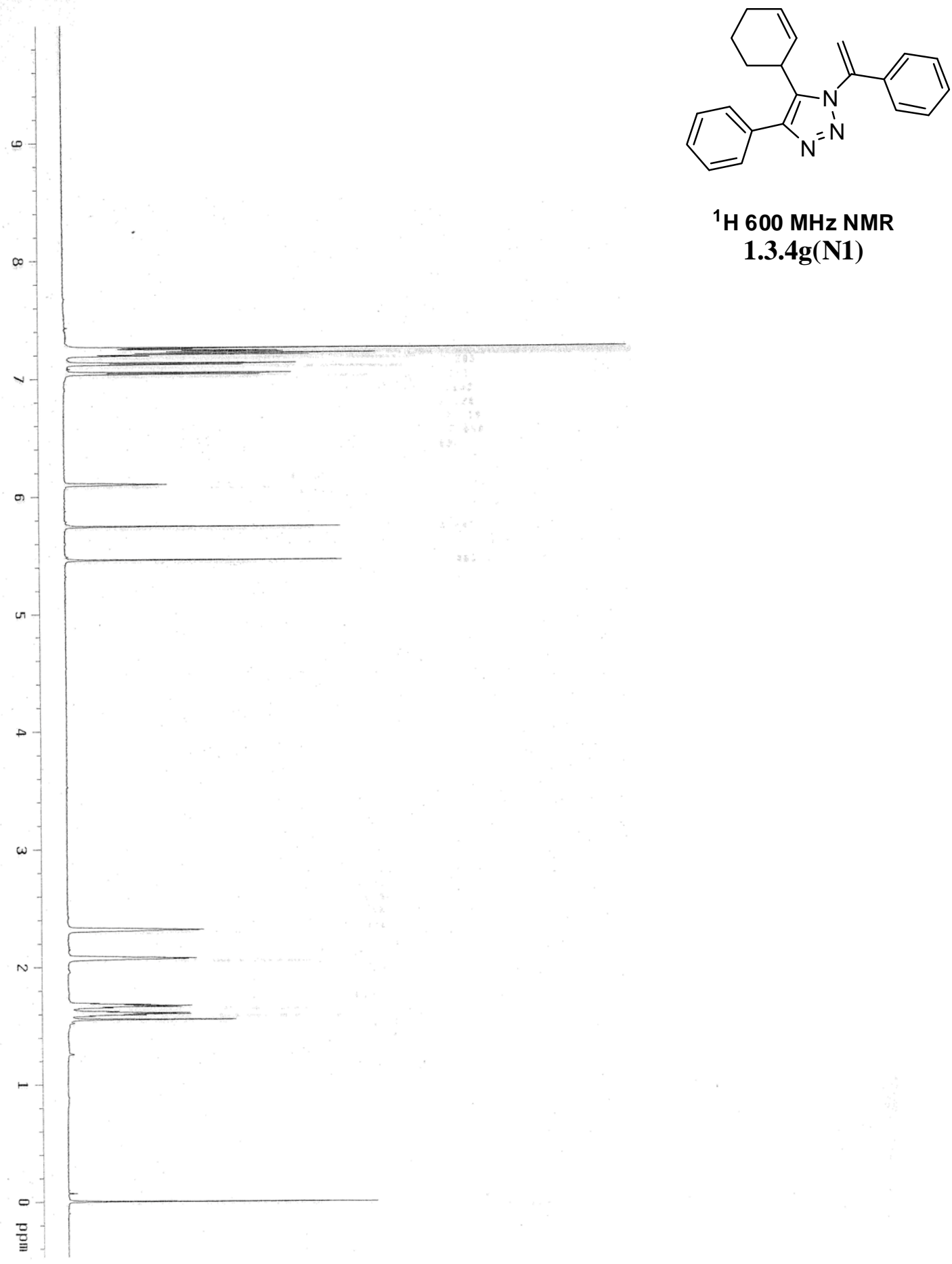

${ }^{1} \mathrm{H} 600 \mathrm{MHz}$ NMR 1.3.4g(N1) 

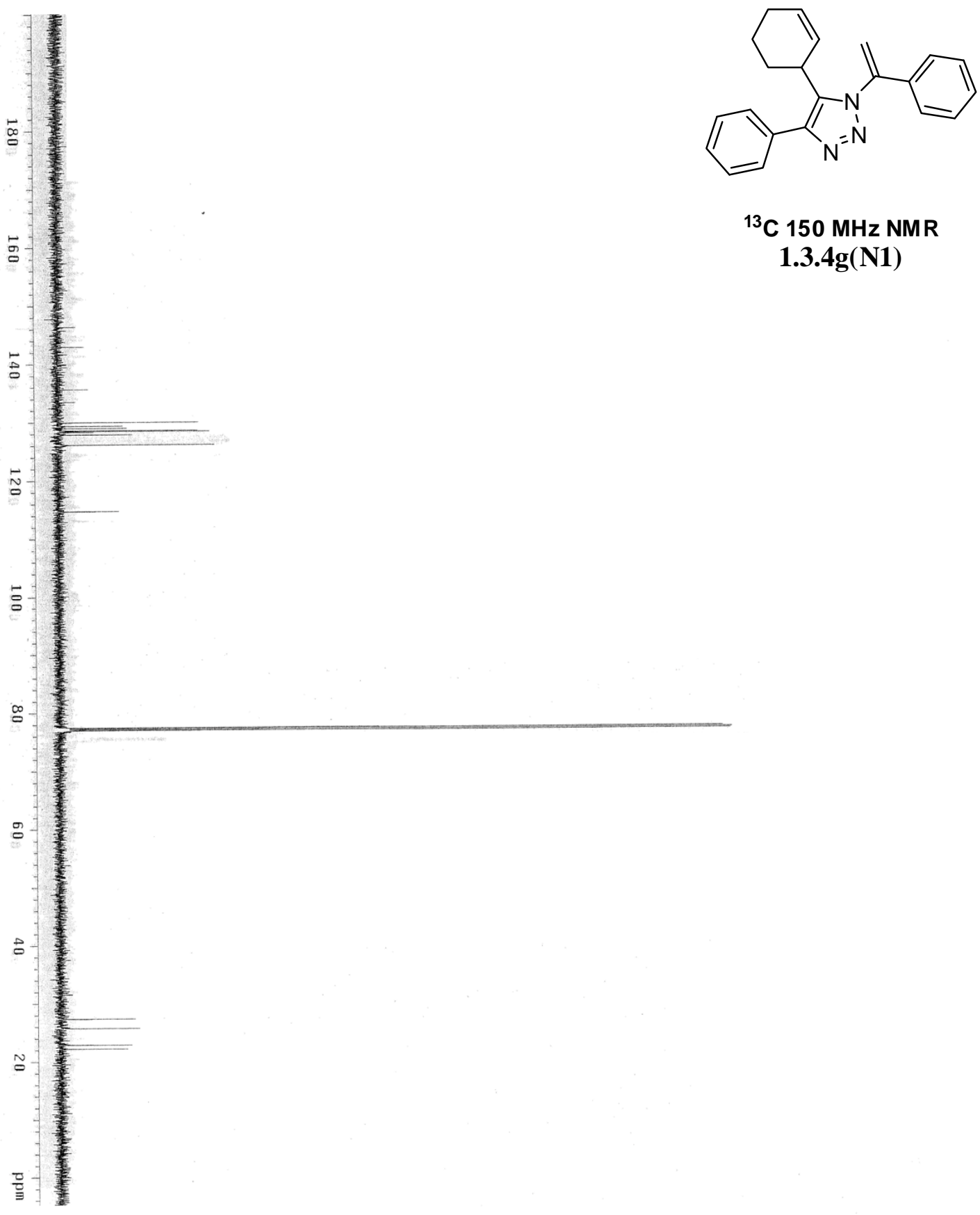

${ }^{13} \mathrm{C} 150 \mathrm{MHz}$ NMR

1.3.4g(N1) 


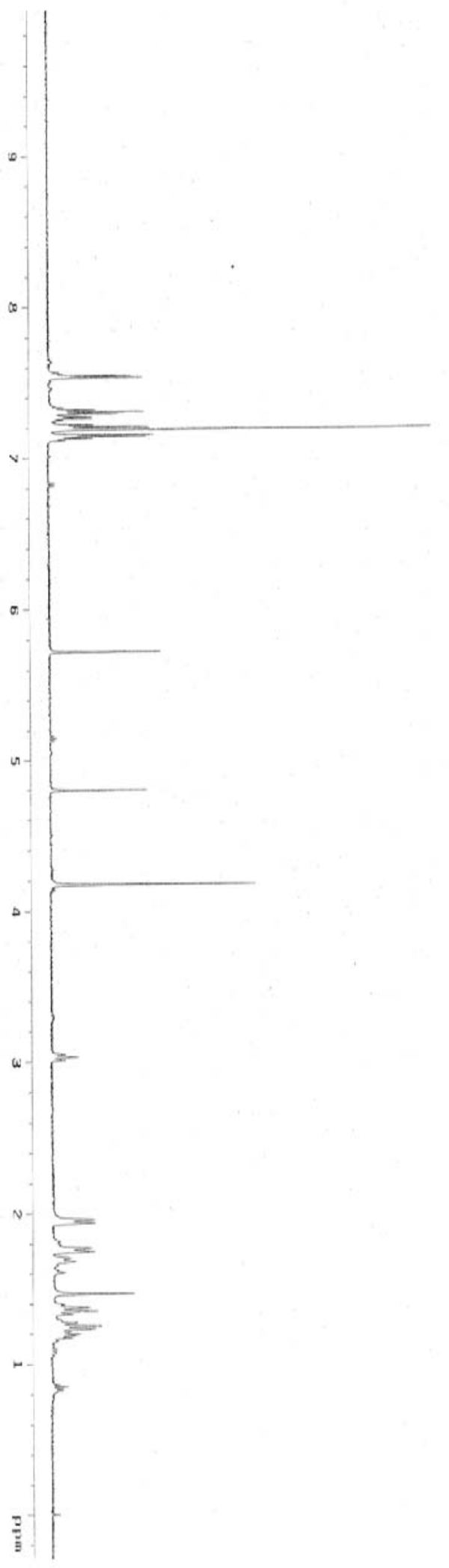

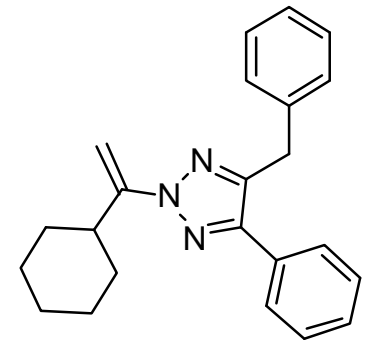

${ }^{1} \mathrm{H} 600 \mathrm{MHz}$ NMR

1.3.4h(N2) 


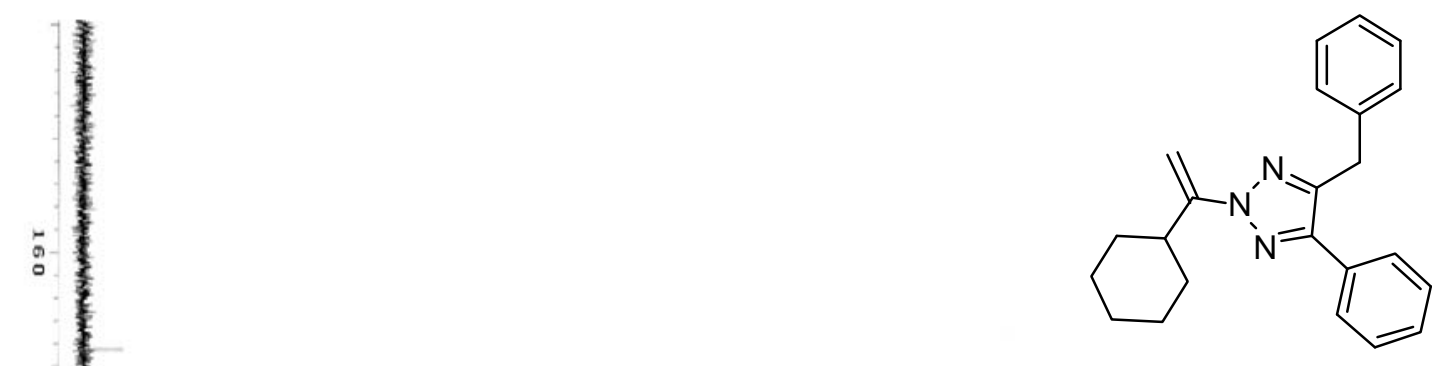

${ }^{13} \mathrm{C} 150 \mathrm{MHz}$ NMR

1.3.4h(N2)

$\stackrel{\circ}{=}$

:

ง.

:

:.

v.

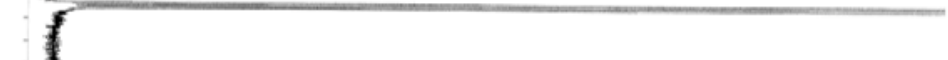

$\Delta$

N.

밈 

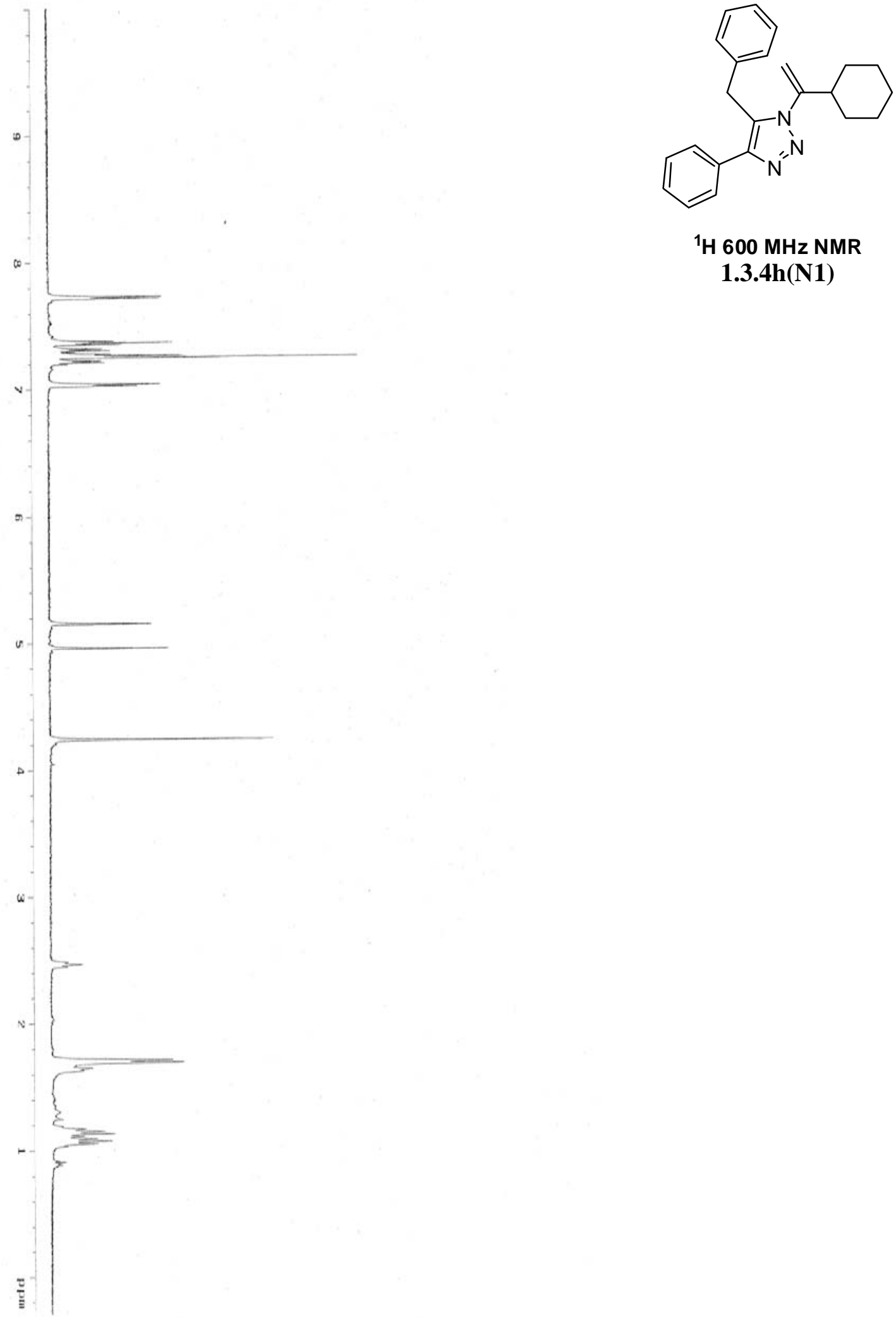

${ }^{1} \mathrm{H} 600 \mathrm{MHz}$ NMR 1.3.4h(N1) 

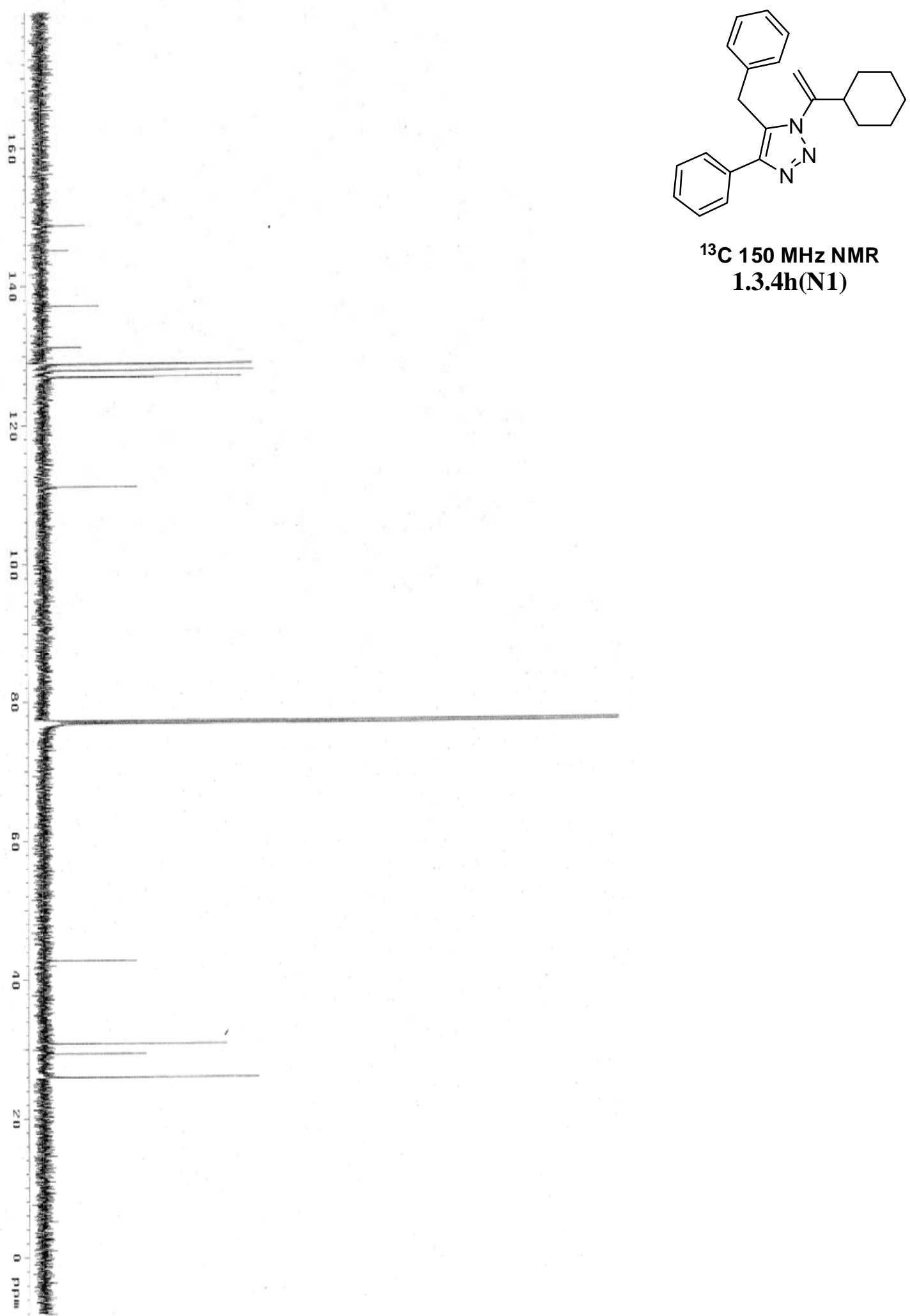

${ }^{13} \mathrm{C} 150 \mathrm{MHz}$ NMR

1.3.4h(N1) 


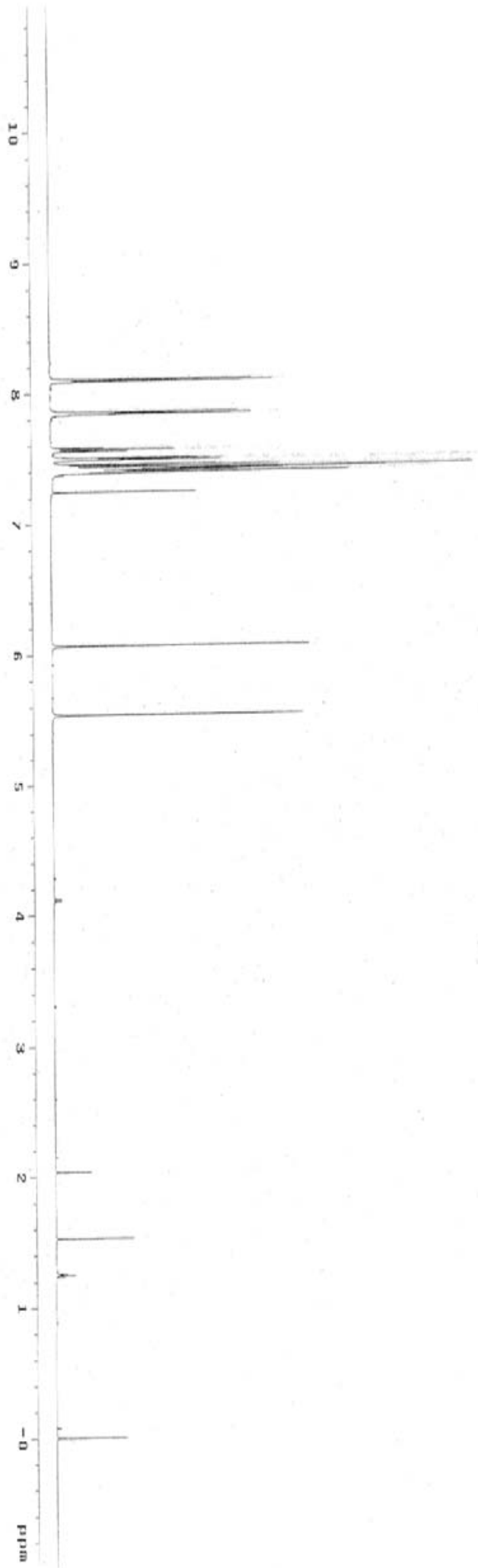

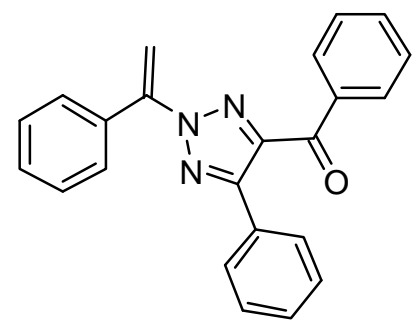

${ }^{1} \mathrm{H} 600 \mathrm{MHz}$ NMR

1.3.4i(N2) 


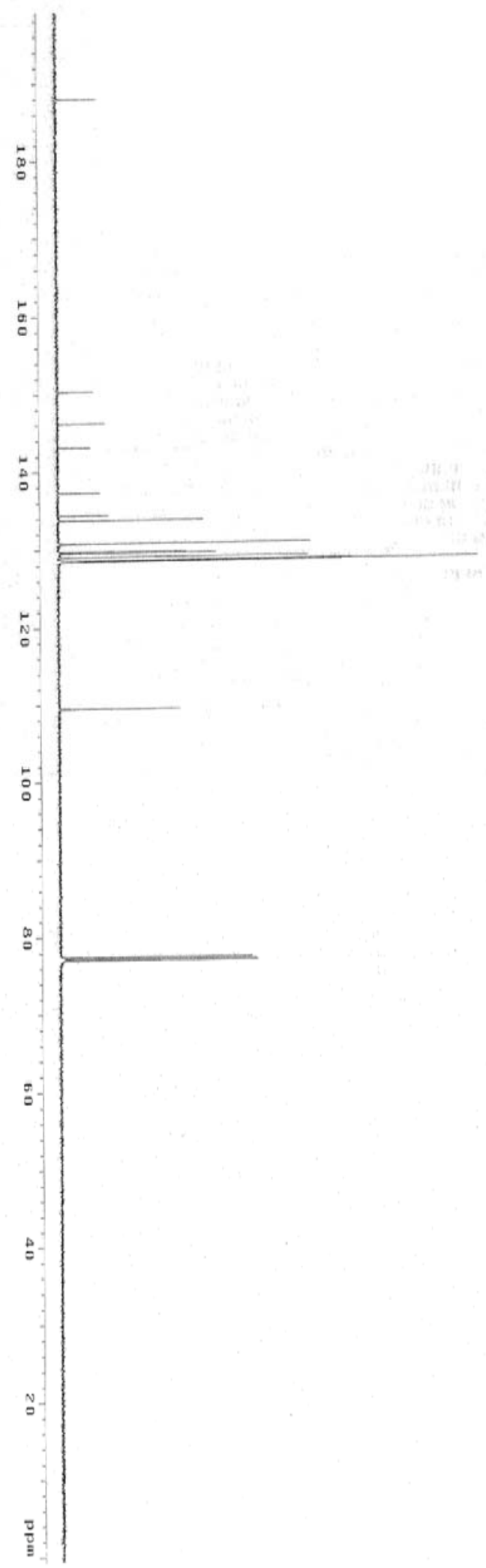

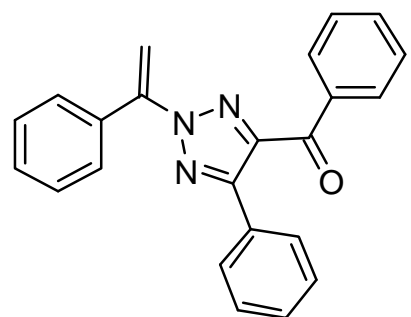

${ }^{13} \mathrm{C} 150 \mathrm{MHz}$ NMR

1.3.4i(N2) 


\subsection{NMR Spectra Data}

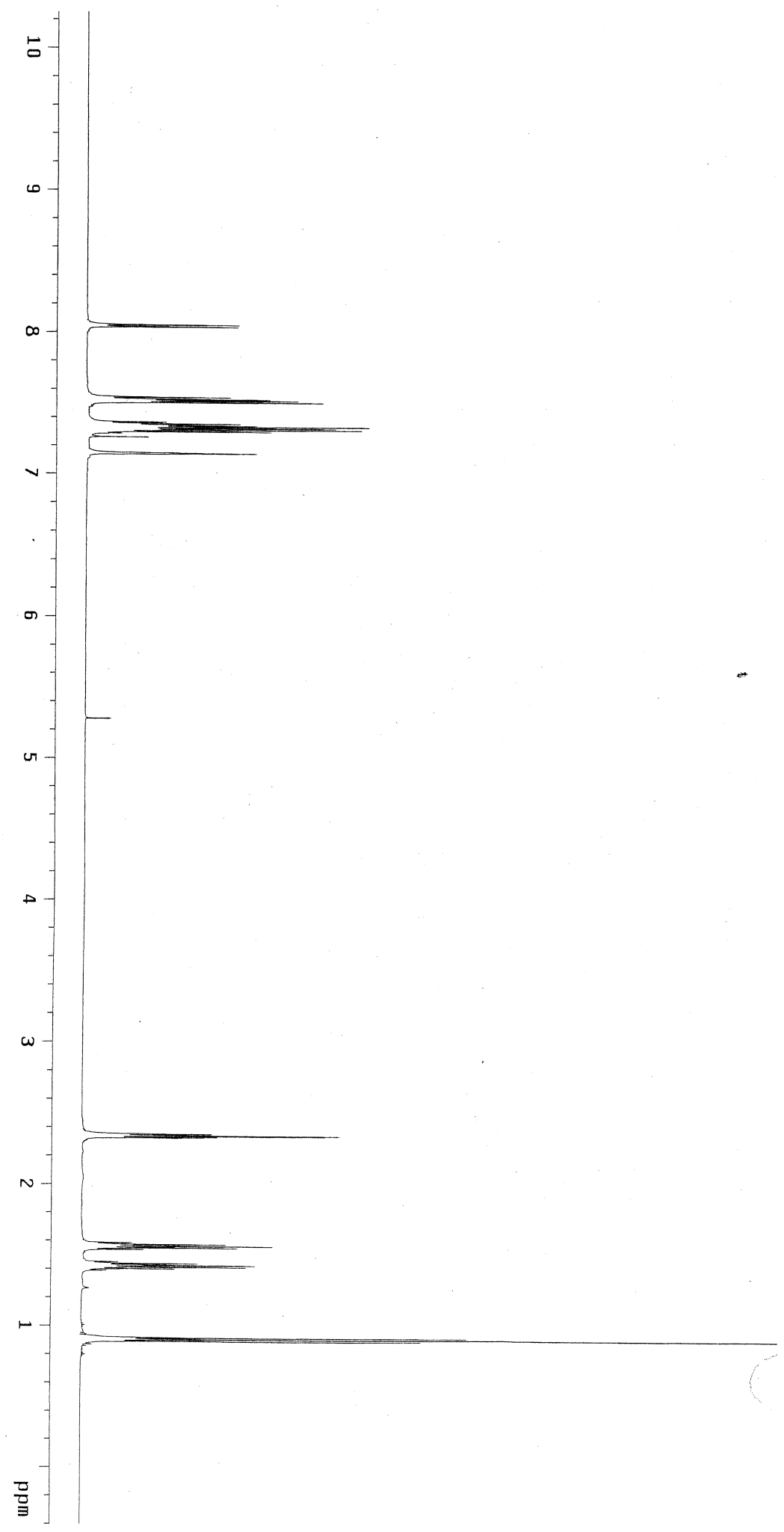

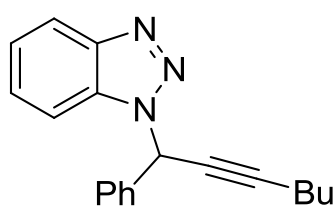

1.4.3a (N-1) $600 \mathrm{MHz}{ }^{1} \mathrm{H}$ NMR 

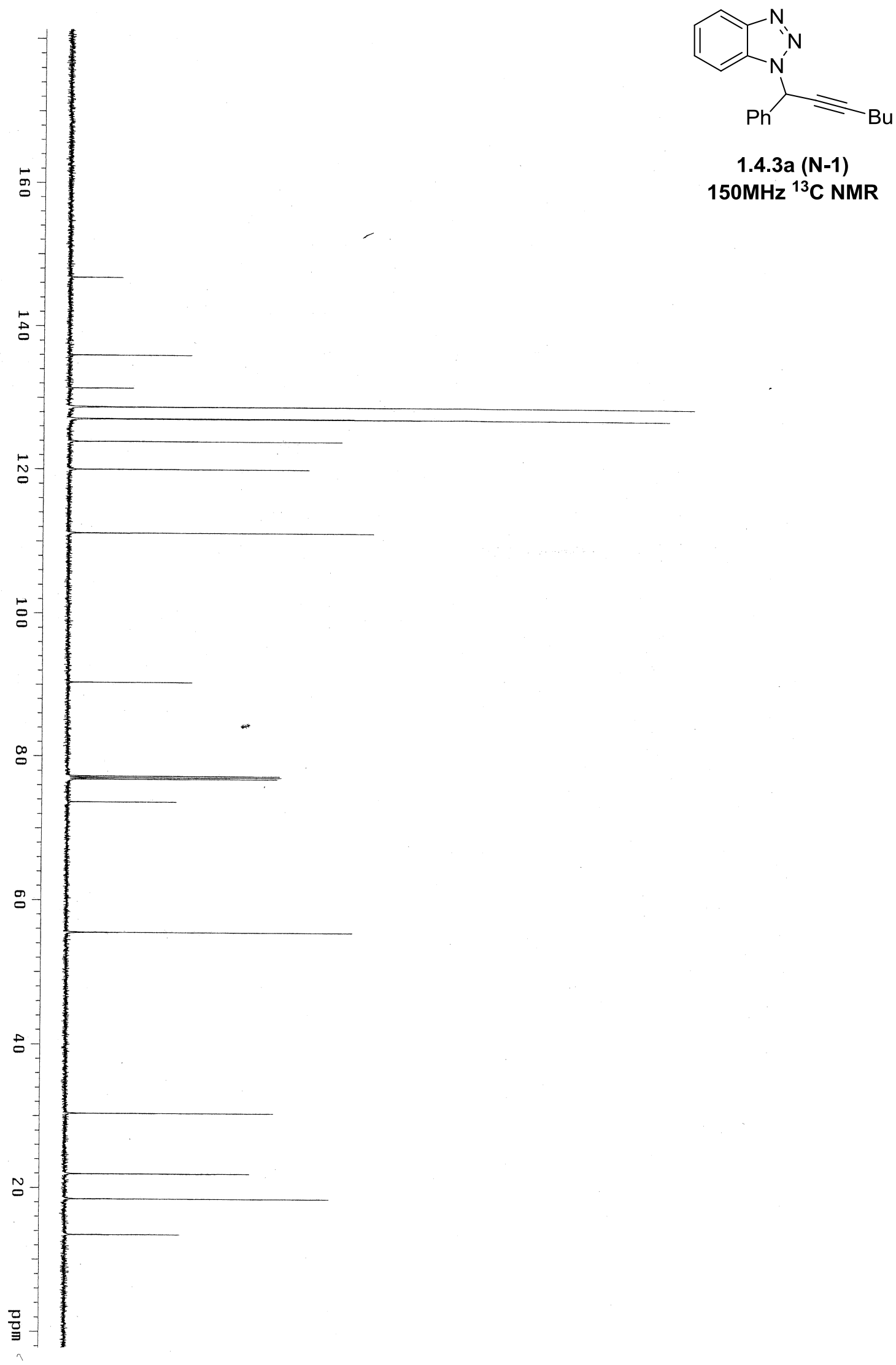

1.4.3a (N-1) $150 \mathrm{MHz}{ }^{13} \mathrm{C}$ NMR 

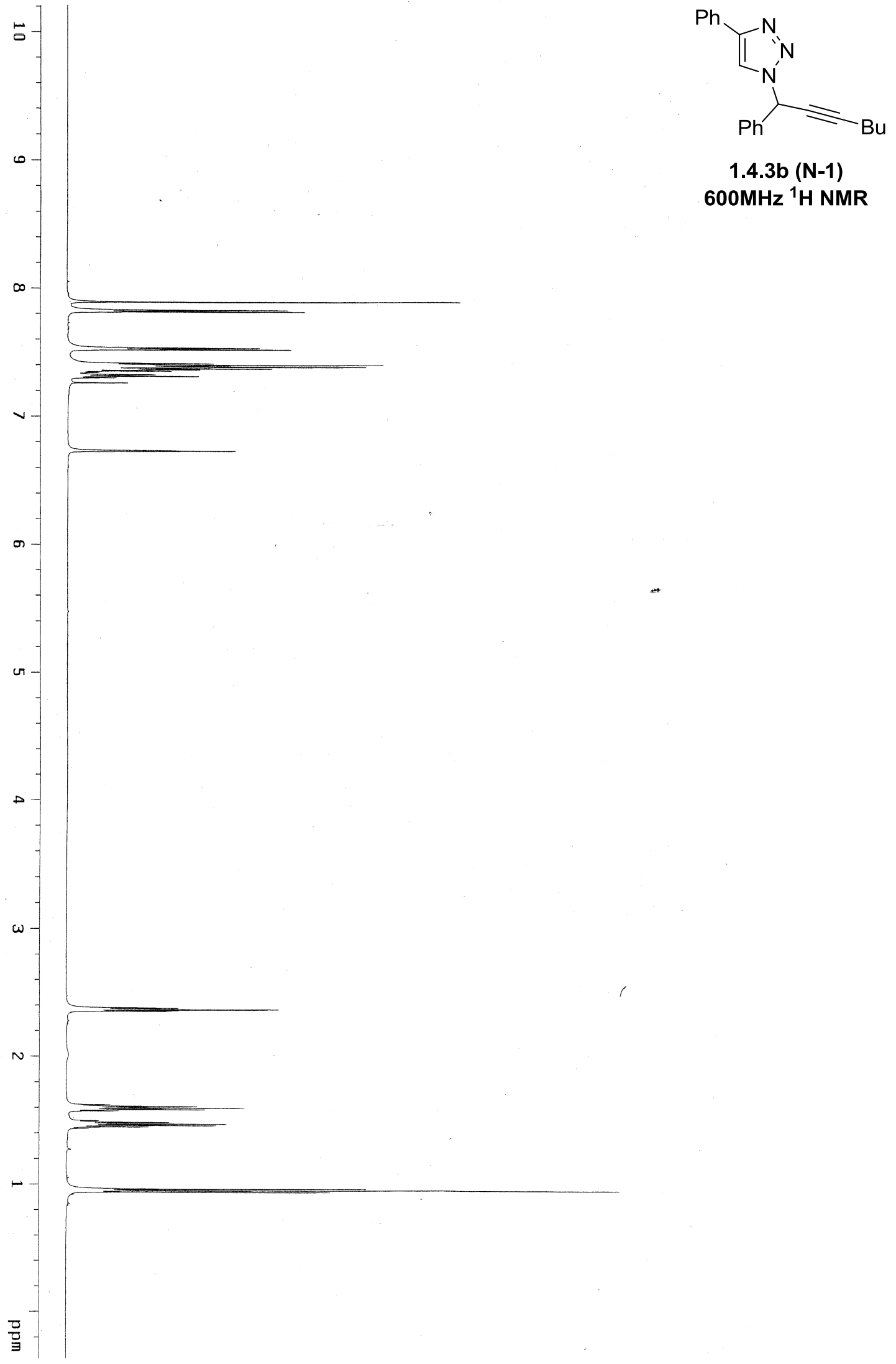

1.4.3b (N-1) $600 \mathrm{MHz}{ }^{1} \mathrm{H}$ NMR 


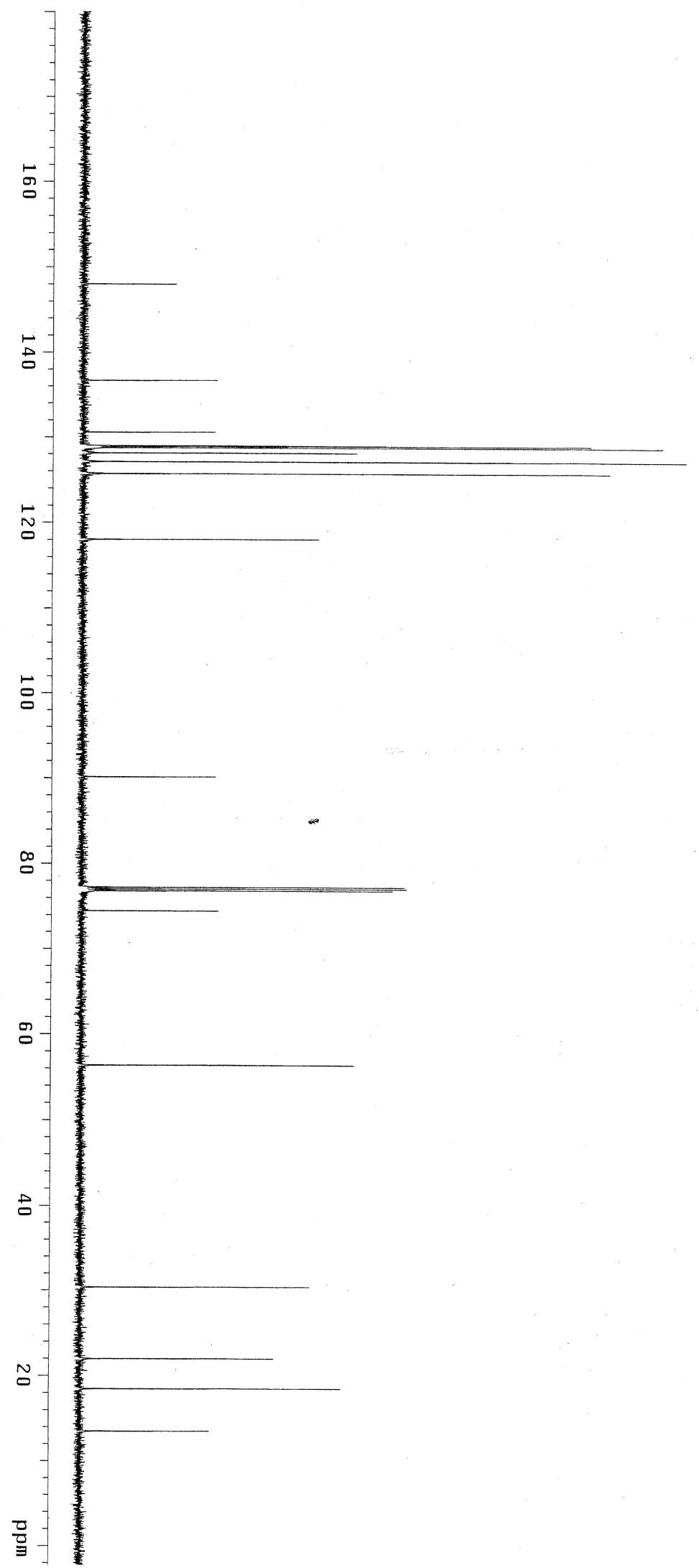

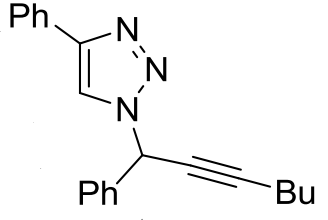

1.4.3b (N-1) $150 \mathrm{MHz}{ }^{13} \mathrm{C}$ NMR 


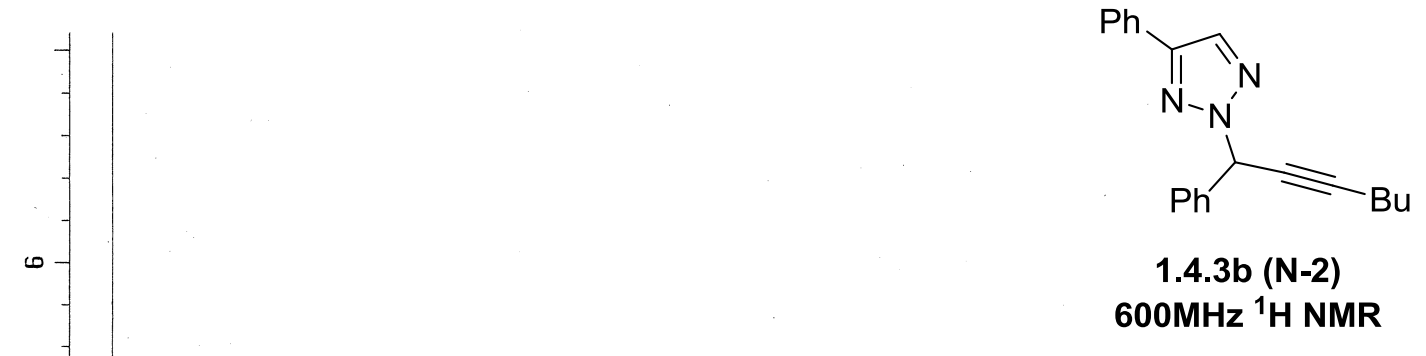

$\infty$

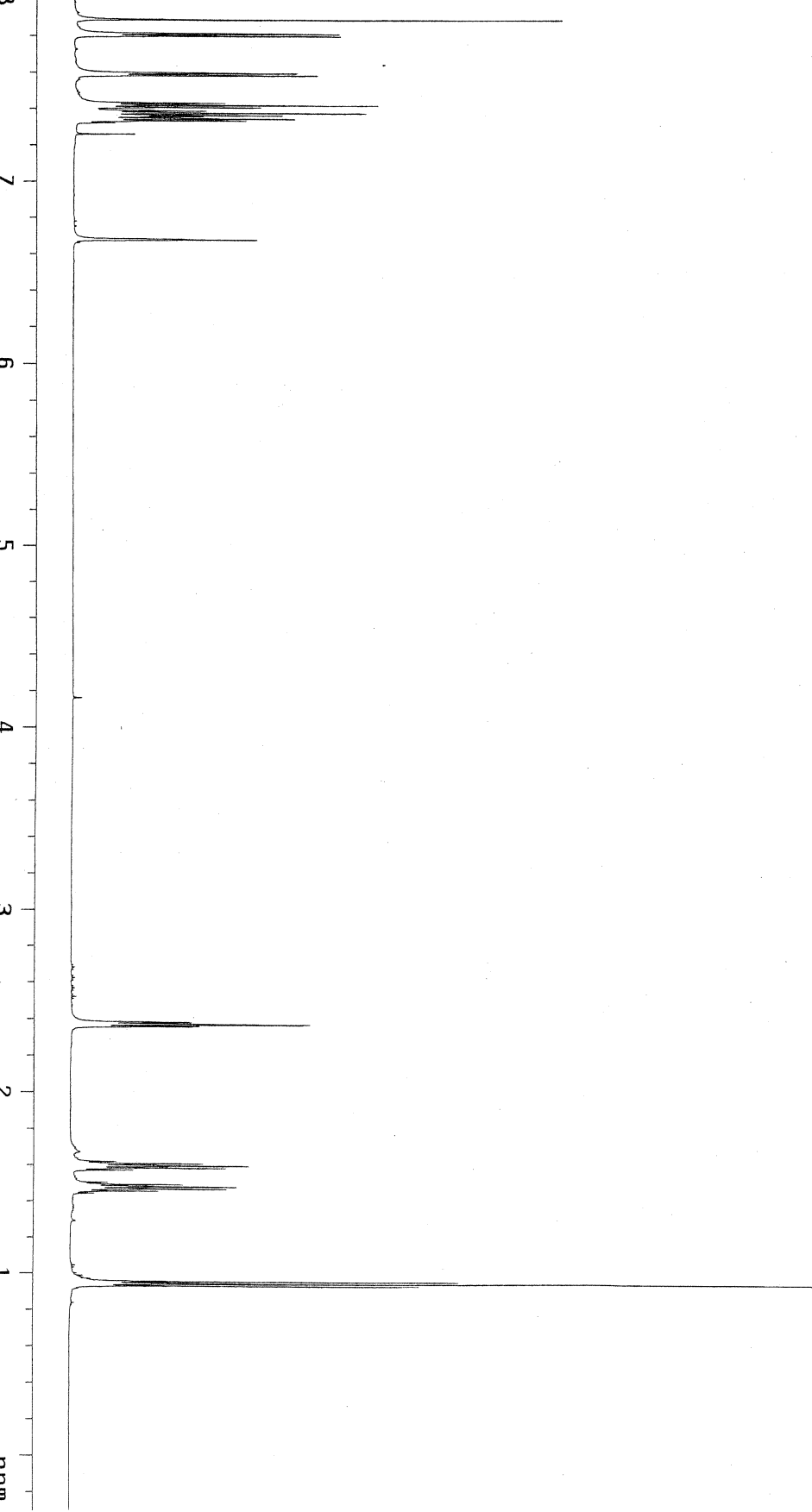




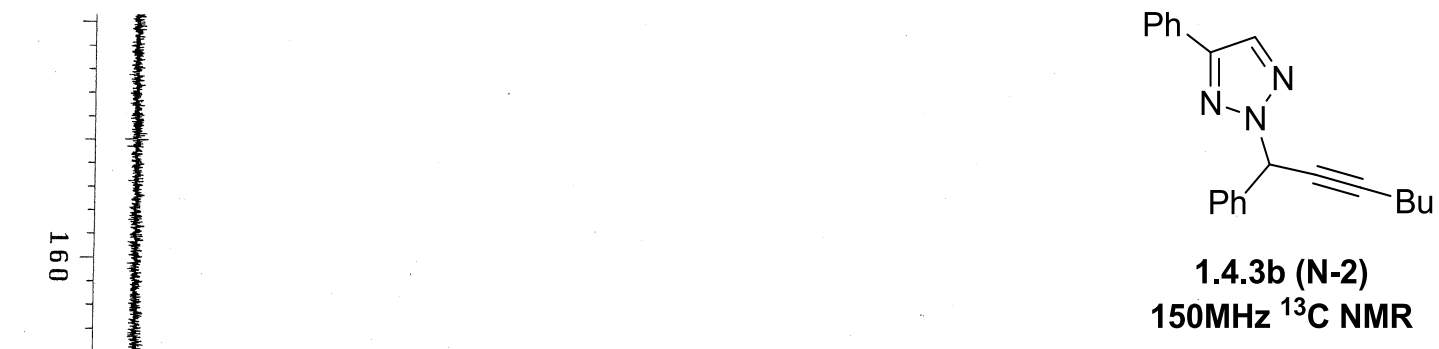

总
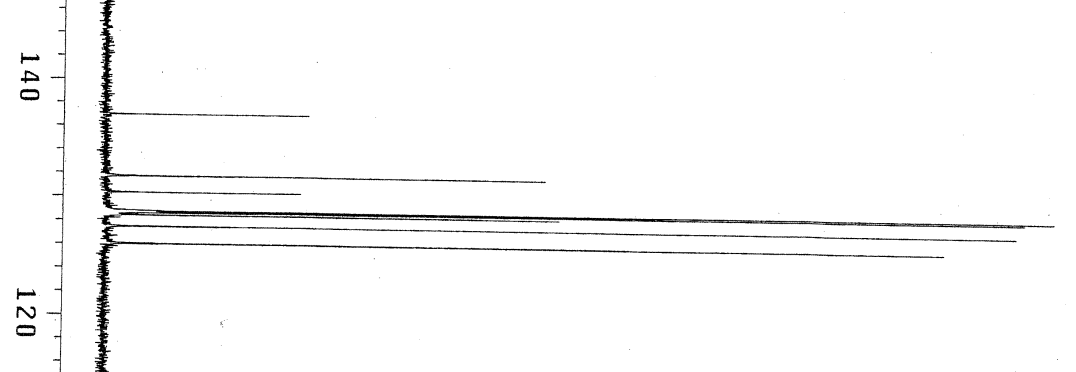

$\stackrel{\infty}{\circ}-$

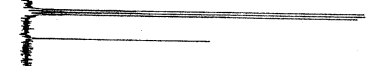

g

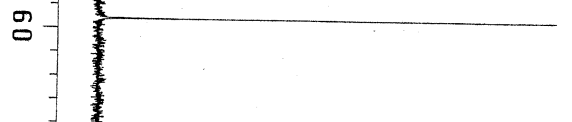

a

:

.

ㄱ.

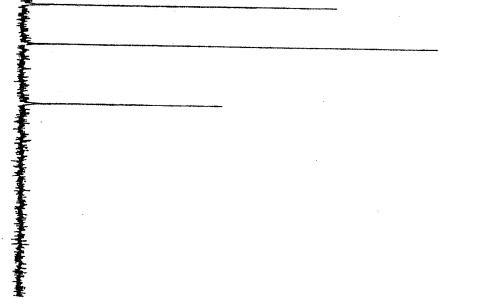




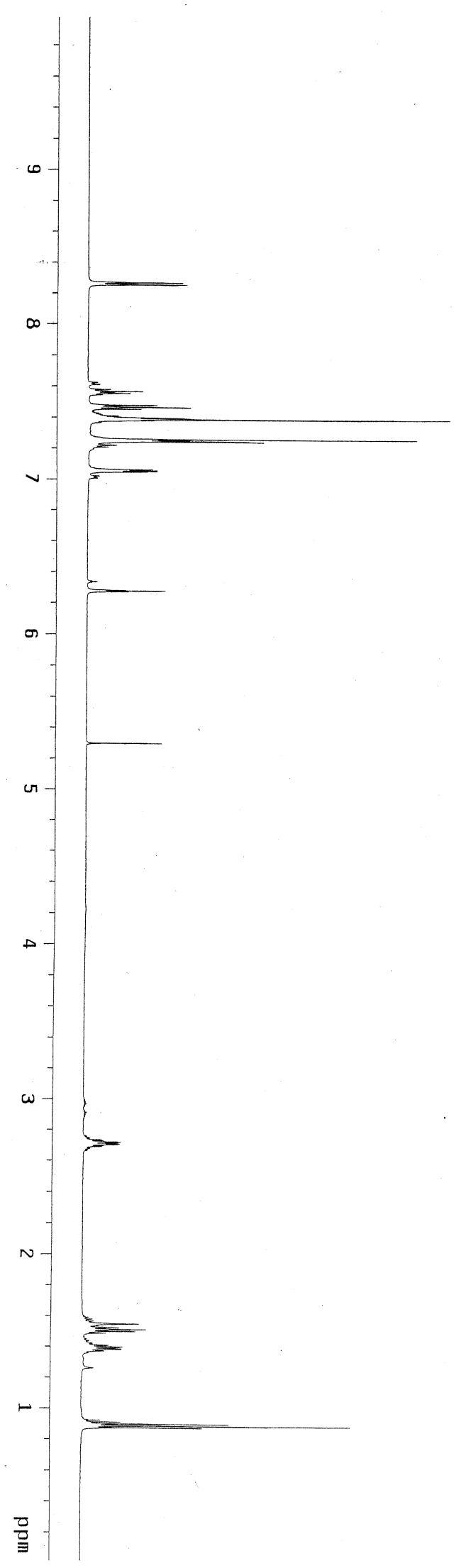

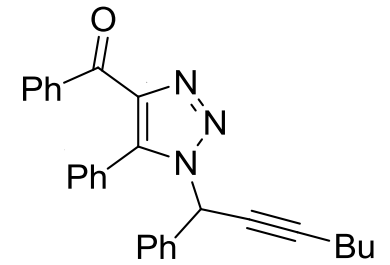

1.4.3c (N-1) $600 \mathrm{MHz}{ }^{1} \mathrm{H}$ NMR 


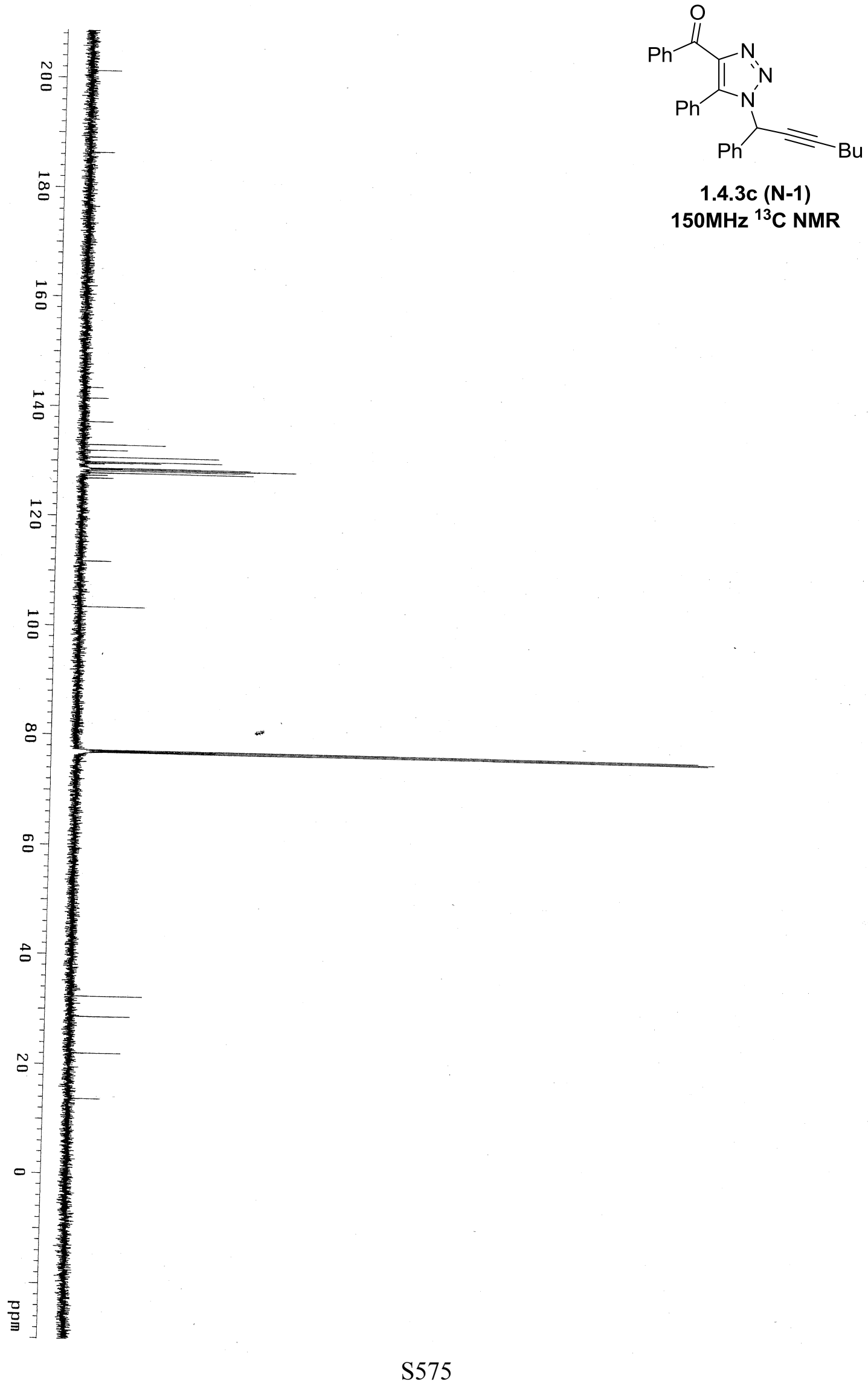



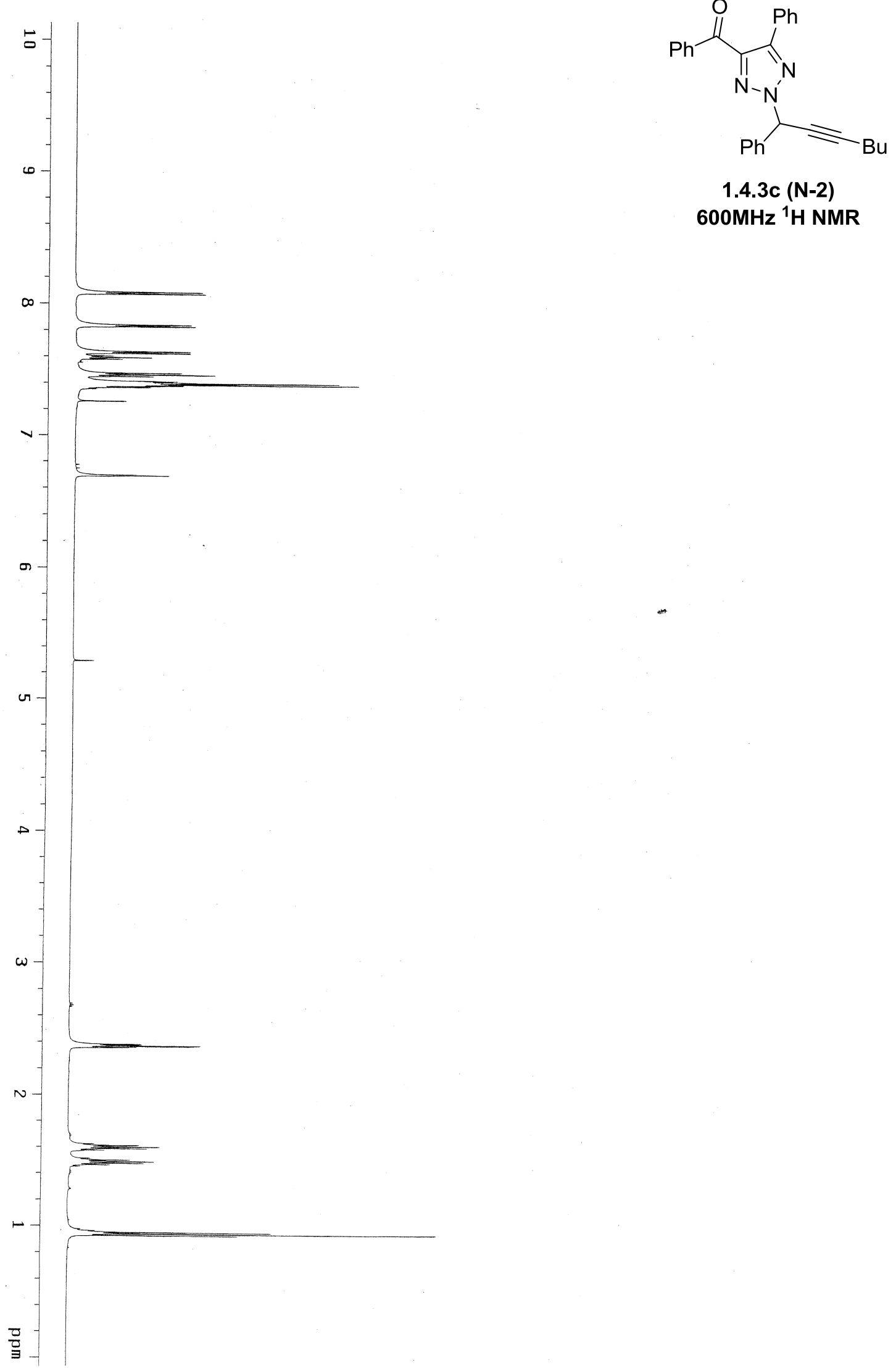


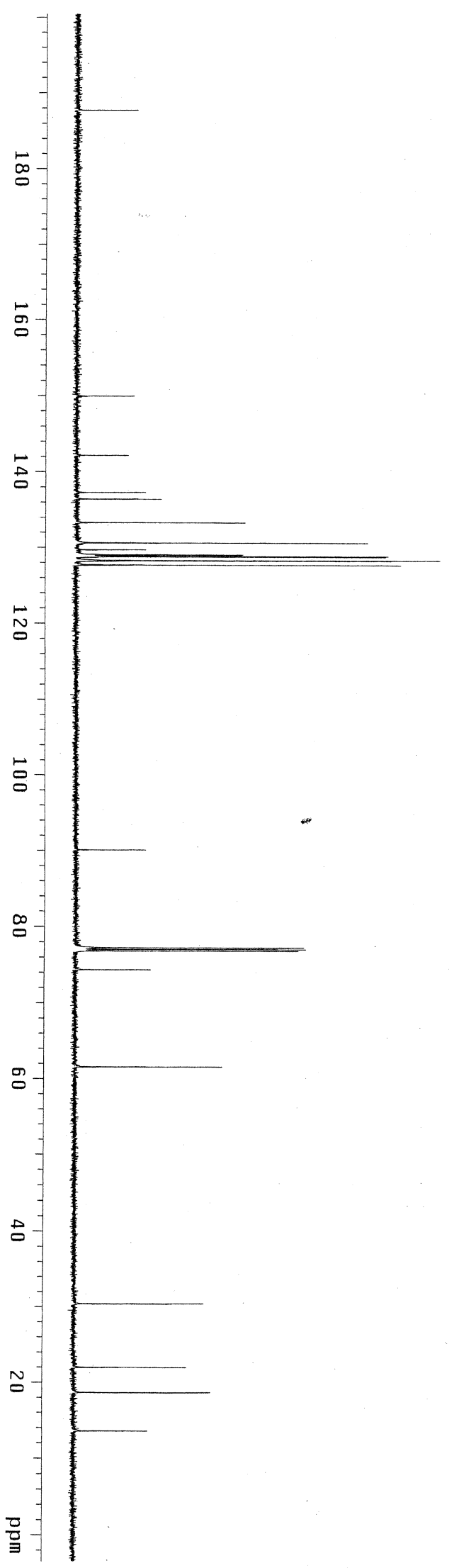

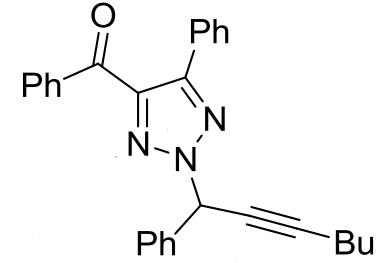

$1.4 .3 \mathrm{c}(\mathrm{N}-2)$ $150 \mathrm{MHz}{ }^{13} \mathrm{C}$ NMR 

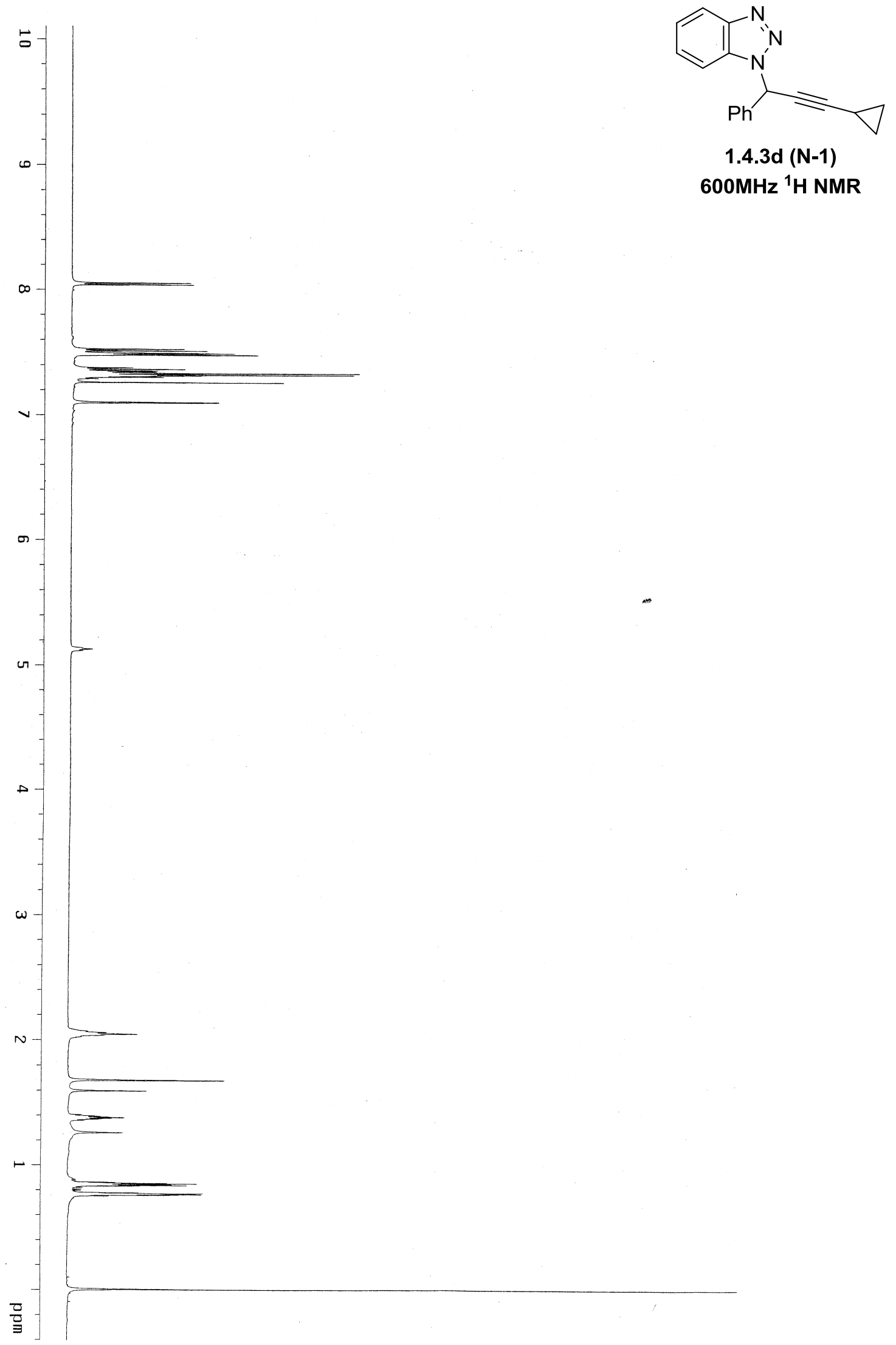


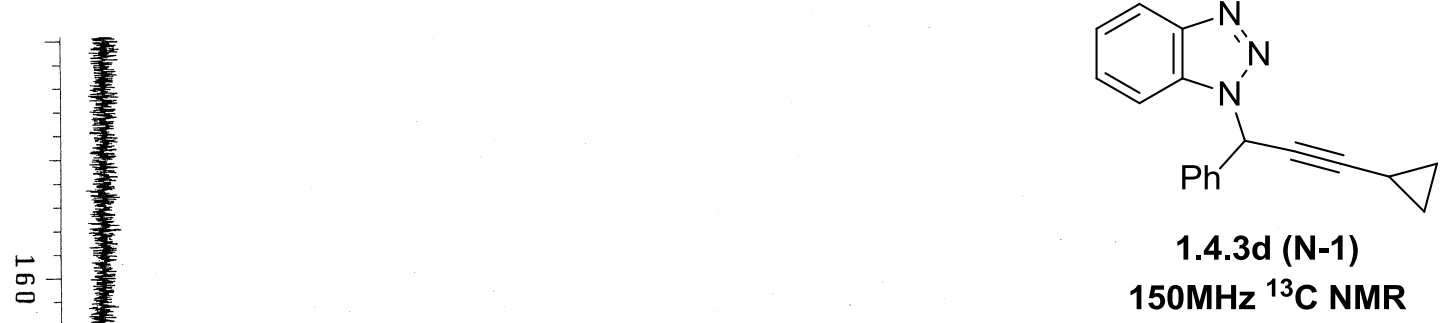

$\stackrel{\infty}{\circ}$

g

:-

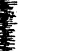

䇏

楚

ㄱ制

整

ㄱ.-
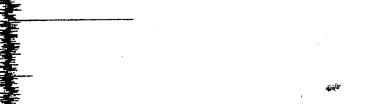

-

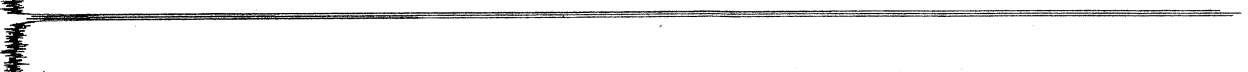

:-

.

¿-1

-1
-1
$j$
-1
-1
-1

읍-1毫 

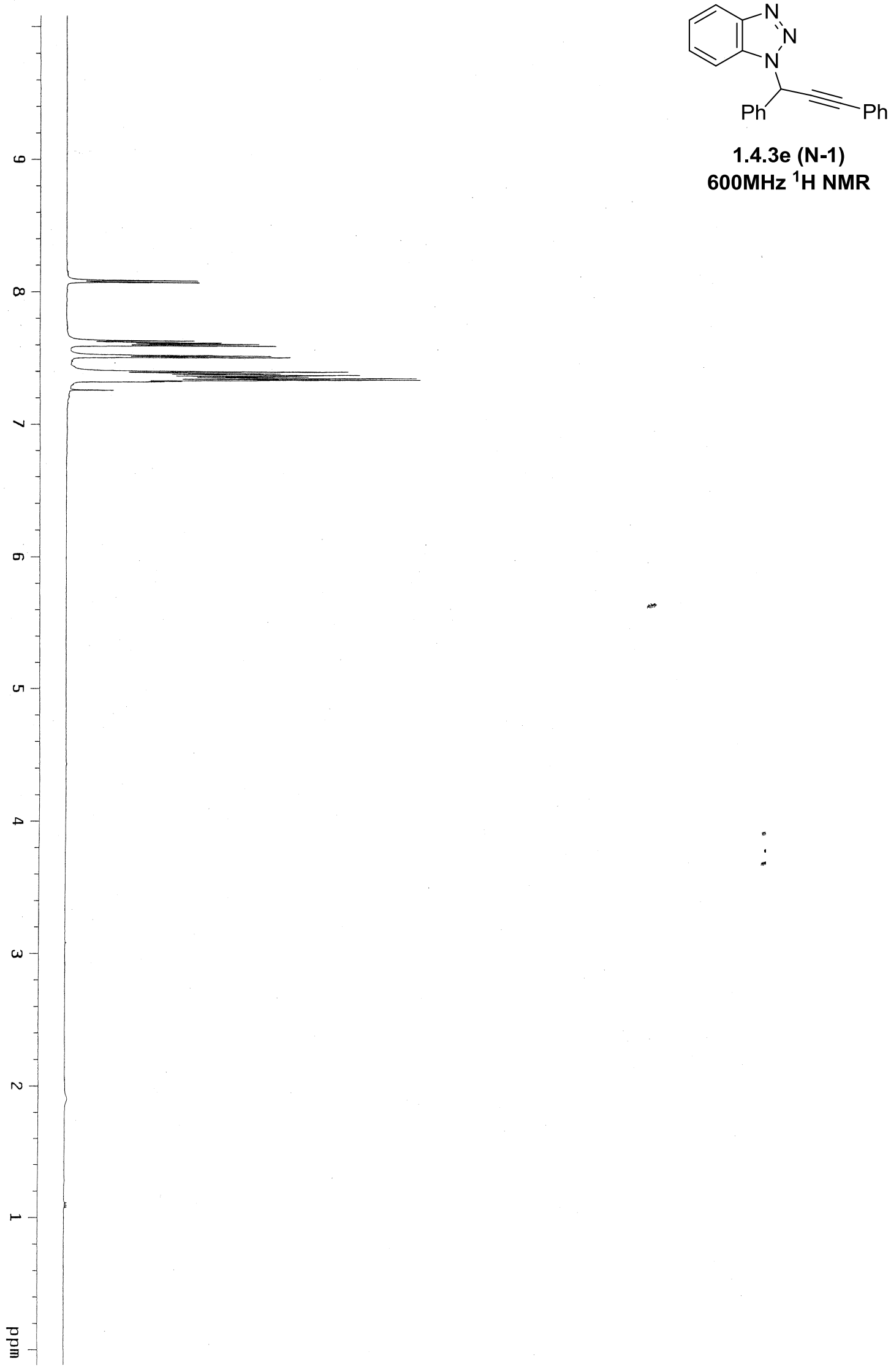

1.4.3e (N-1) $600 \mathrm{MHz}{ }^{1} \mathrm{H}$ NMR 

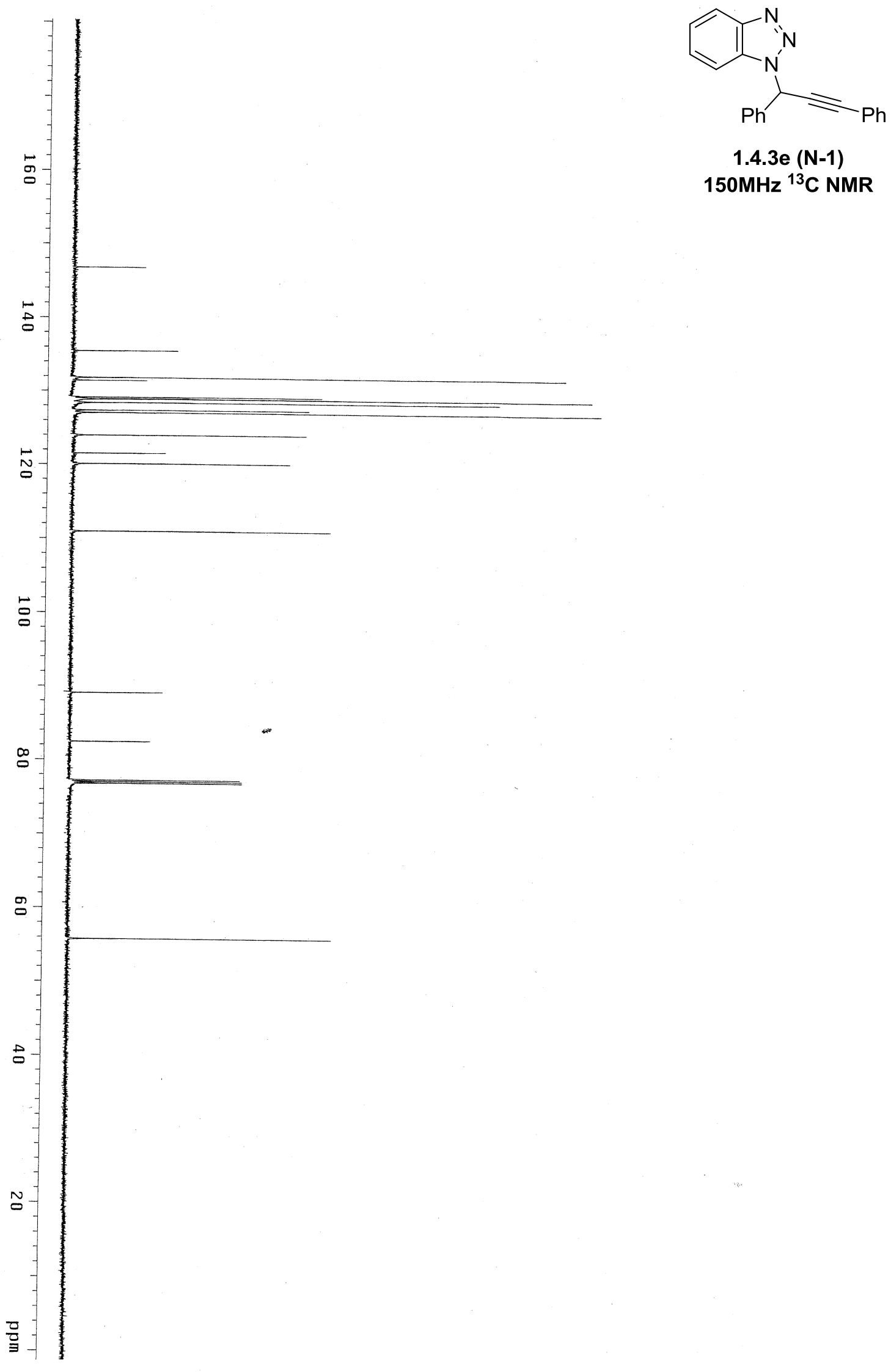

1.4.3e (N-1) $150 \mathrm{MHz}{ }^{13} \mathrm{C}$ NMR 


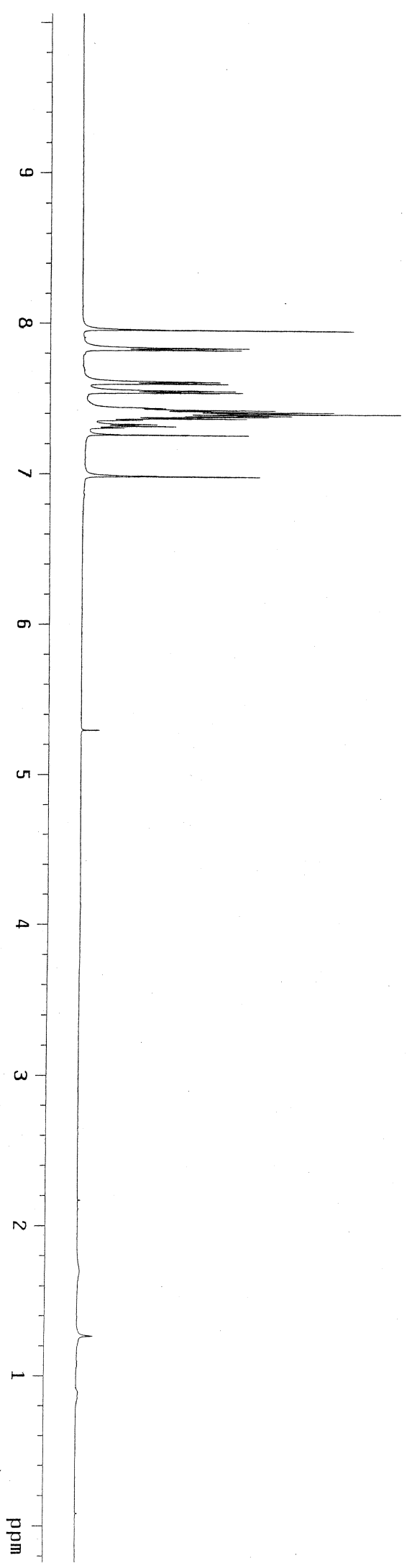

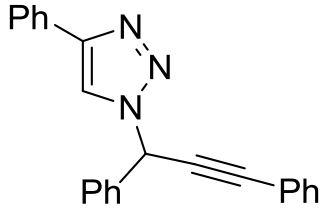

1.4.3f (N-1) $600 \mathrm{MHz}^{1} \mathrm{H}$ NMR 


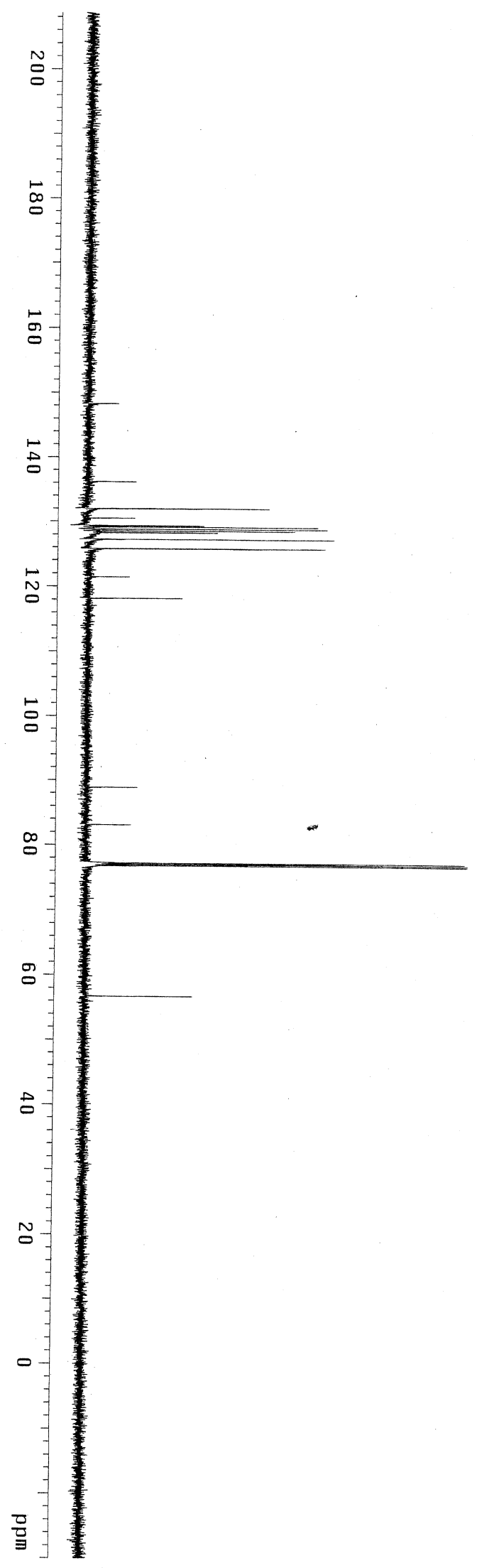

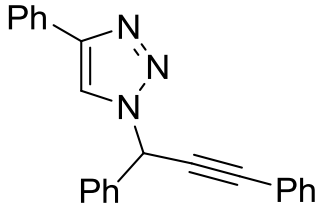

1.4.3f (N-1) $150 \mathrm{MHz}{ }^{13} \mathrm{C}$ NMR 


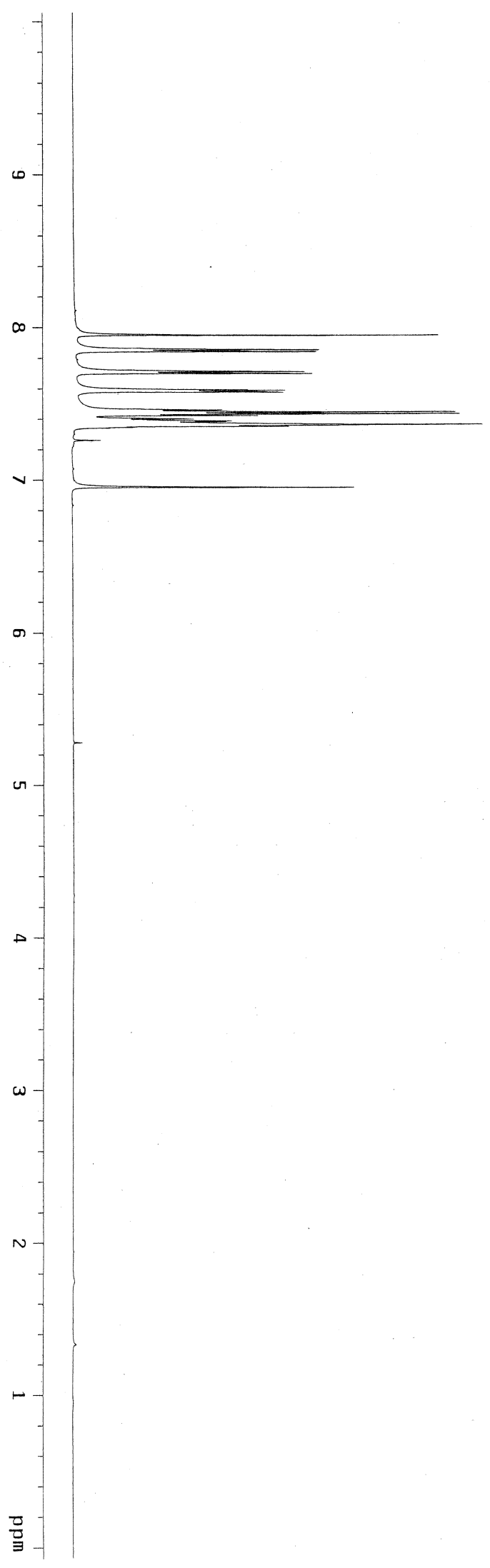

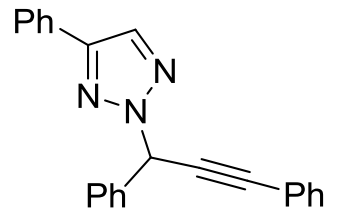

1.4.3f (N-2)

$600 \mathrm{MHz}{ }^{1} \mathrm{H}$ NMR 


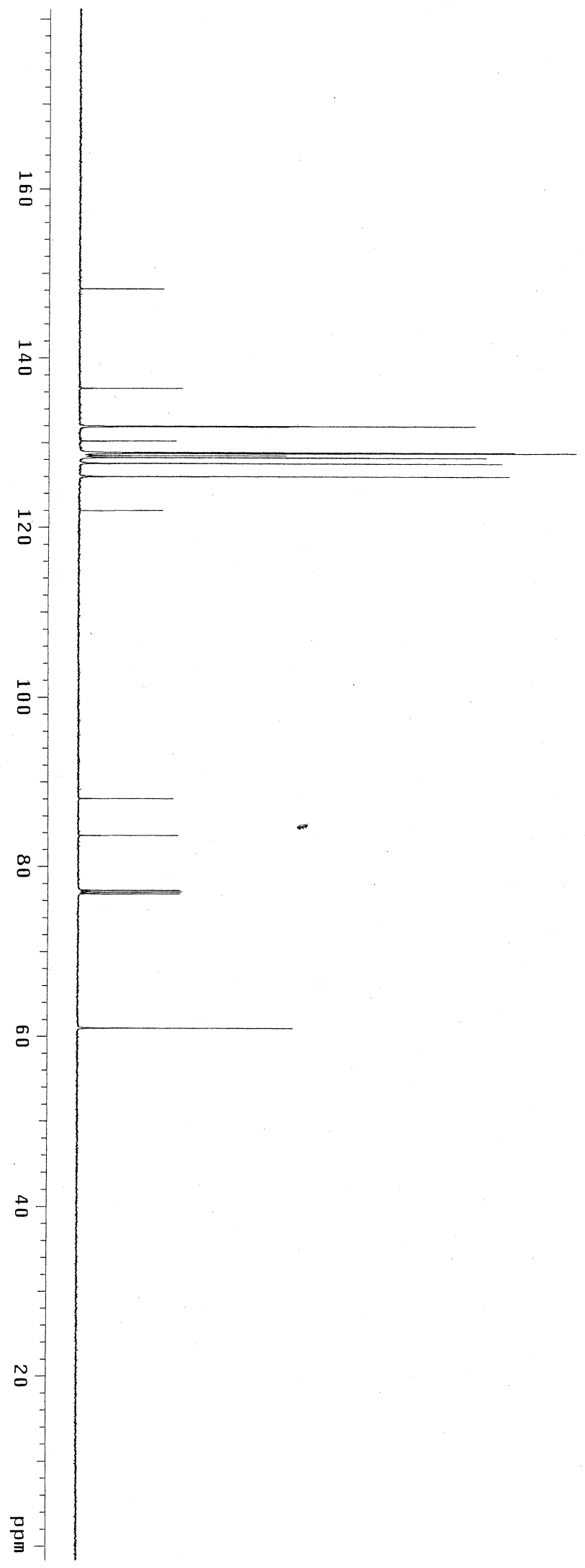

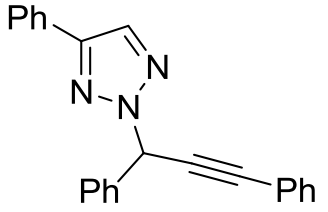

1.4.3f (N-2) $150 \mathrm{MHz}{ }^{13} \mathrm{C}$ NMR 


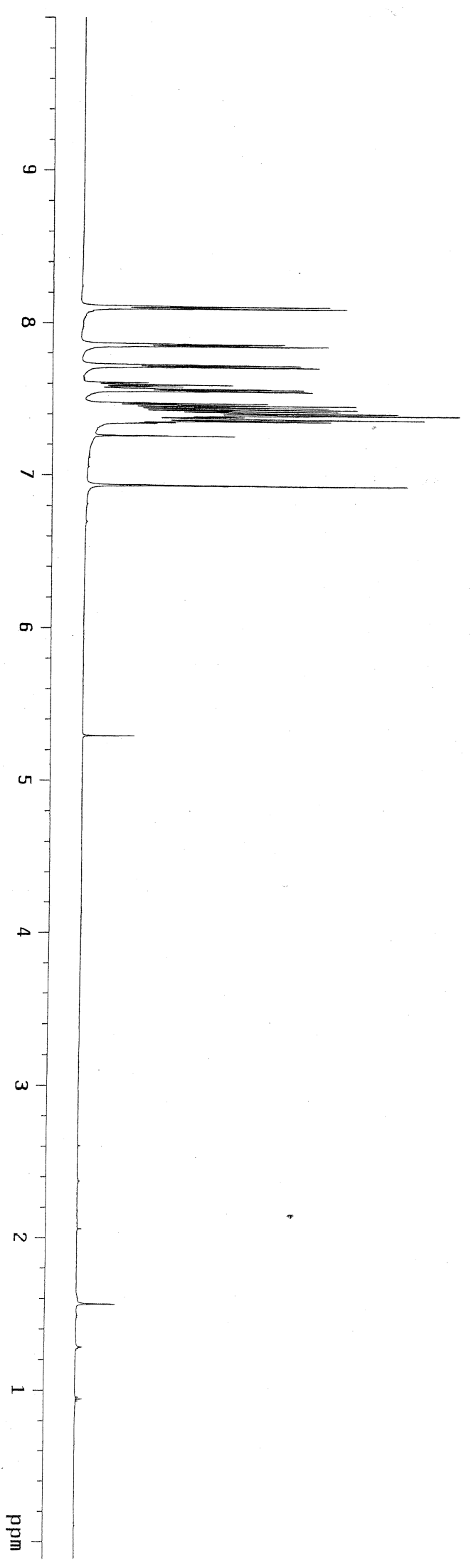

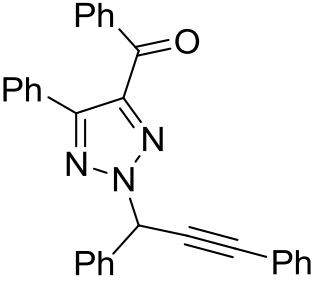

1.4.3g (N-2) $600 \mathrm{MHz}{ }^{1} \mathrm{H}$ NMR 

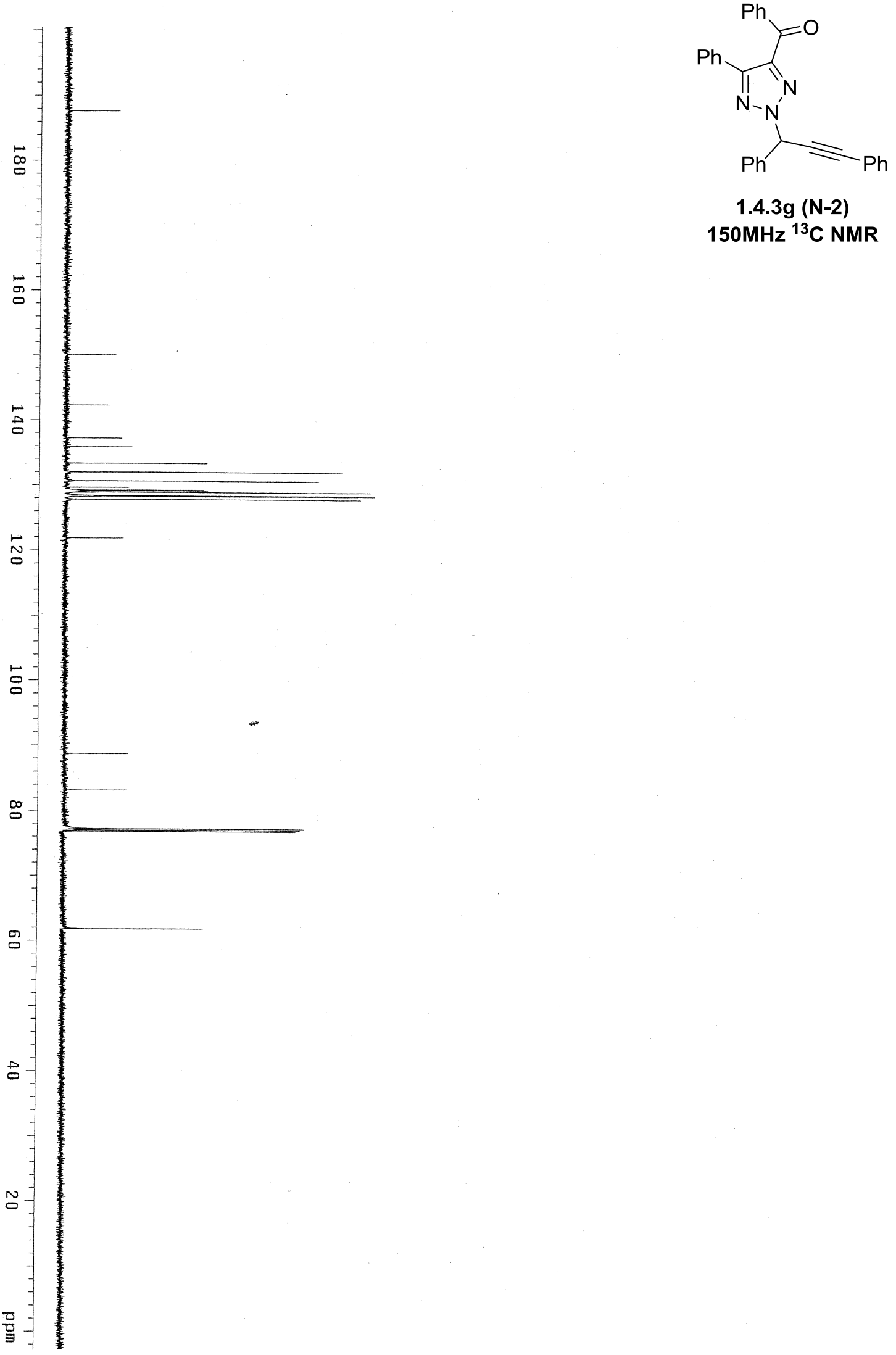

1.4.3g (N-2) $150 \mathrm{MHz}{ }^{13} \mathrm{C}$ NMR 


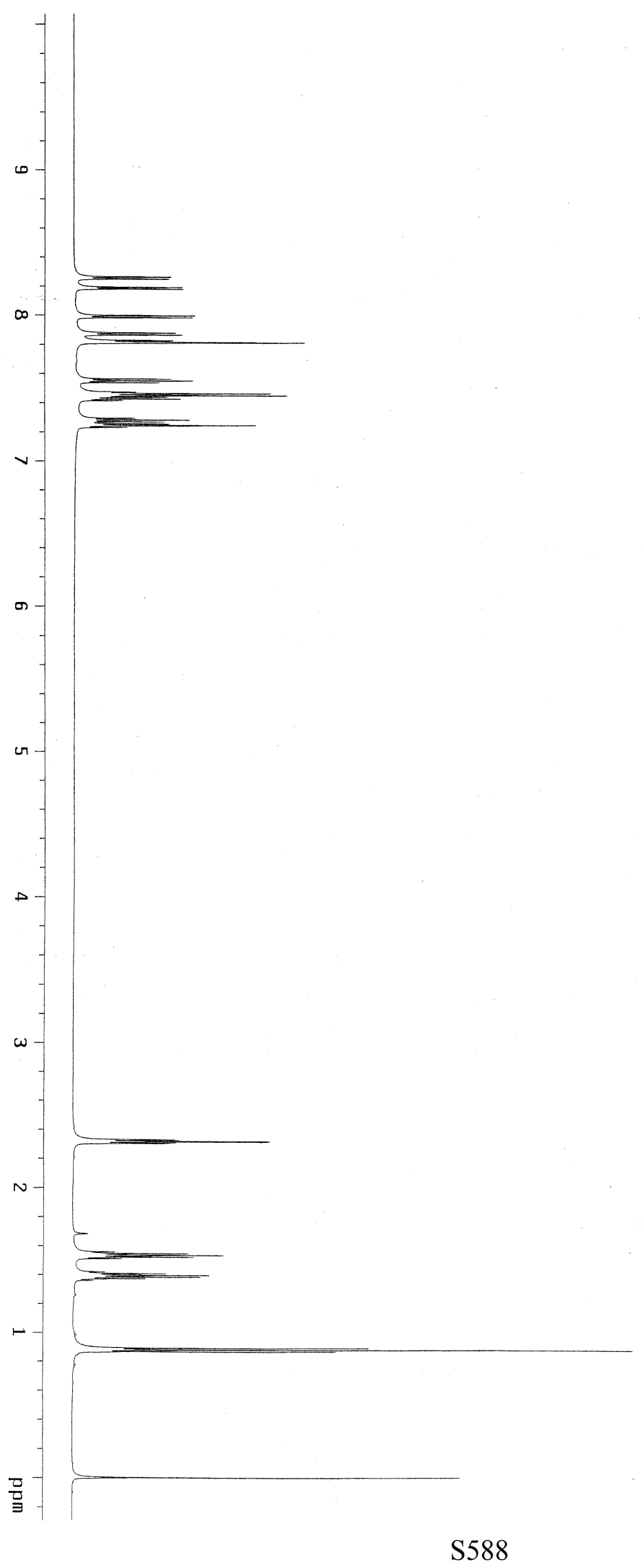

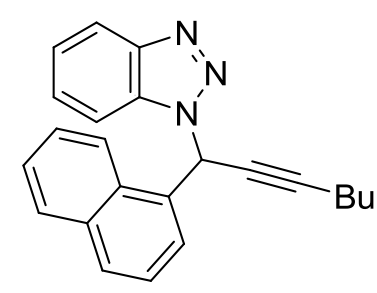

1.4.3h (N-1) $600 \mathrm{MHz}{ }^{1} \mathrm{H}$ NMR 

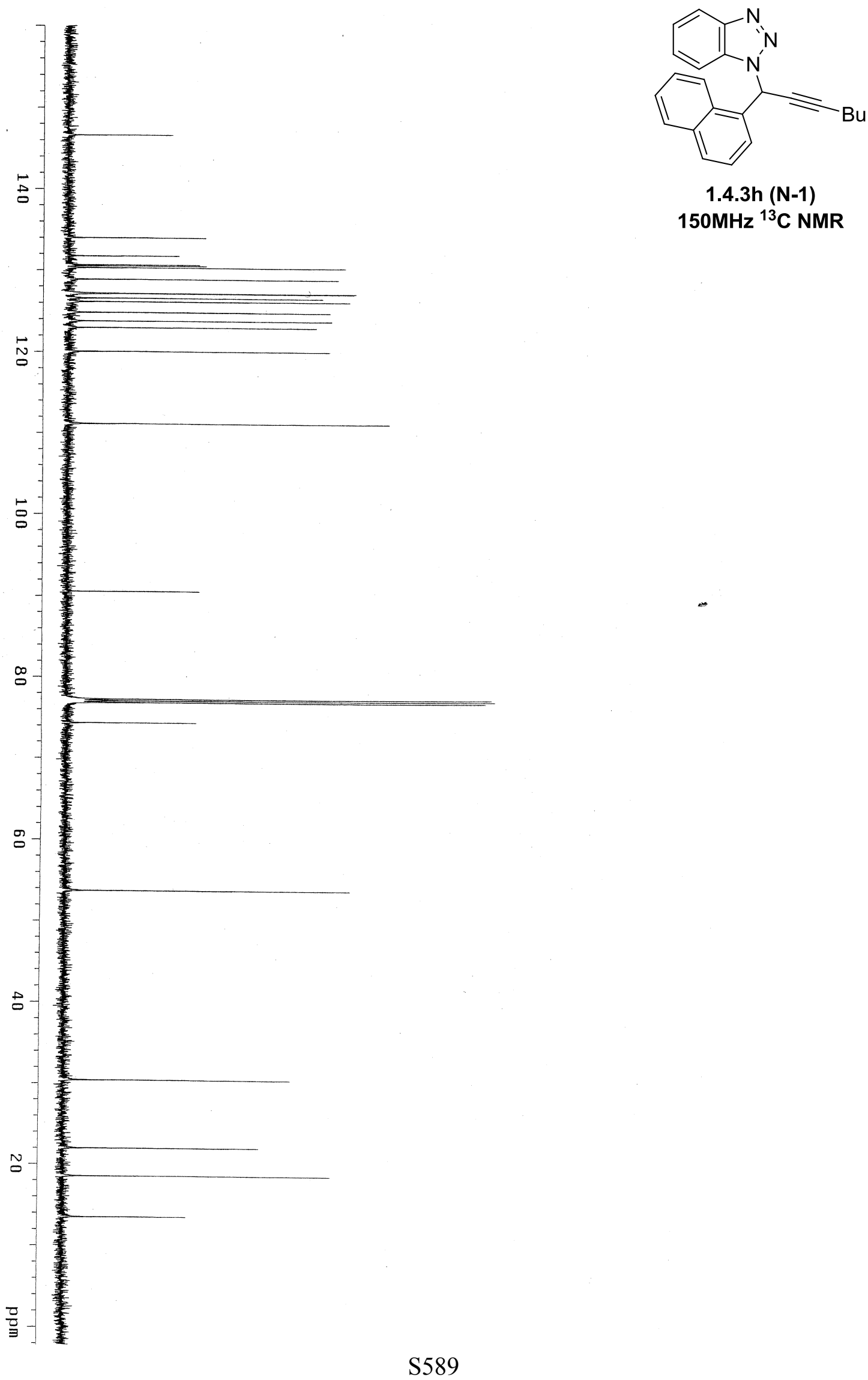

1.4.3h (N-1) $150 \mathrm{MHz}{ }^{13} \mathrm{C}$ NMR 

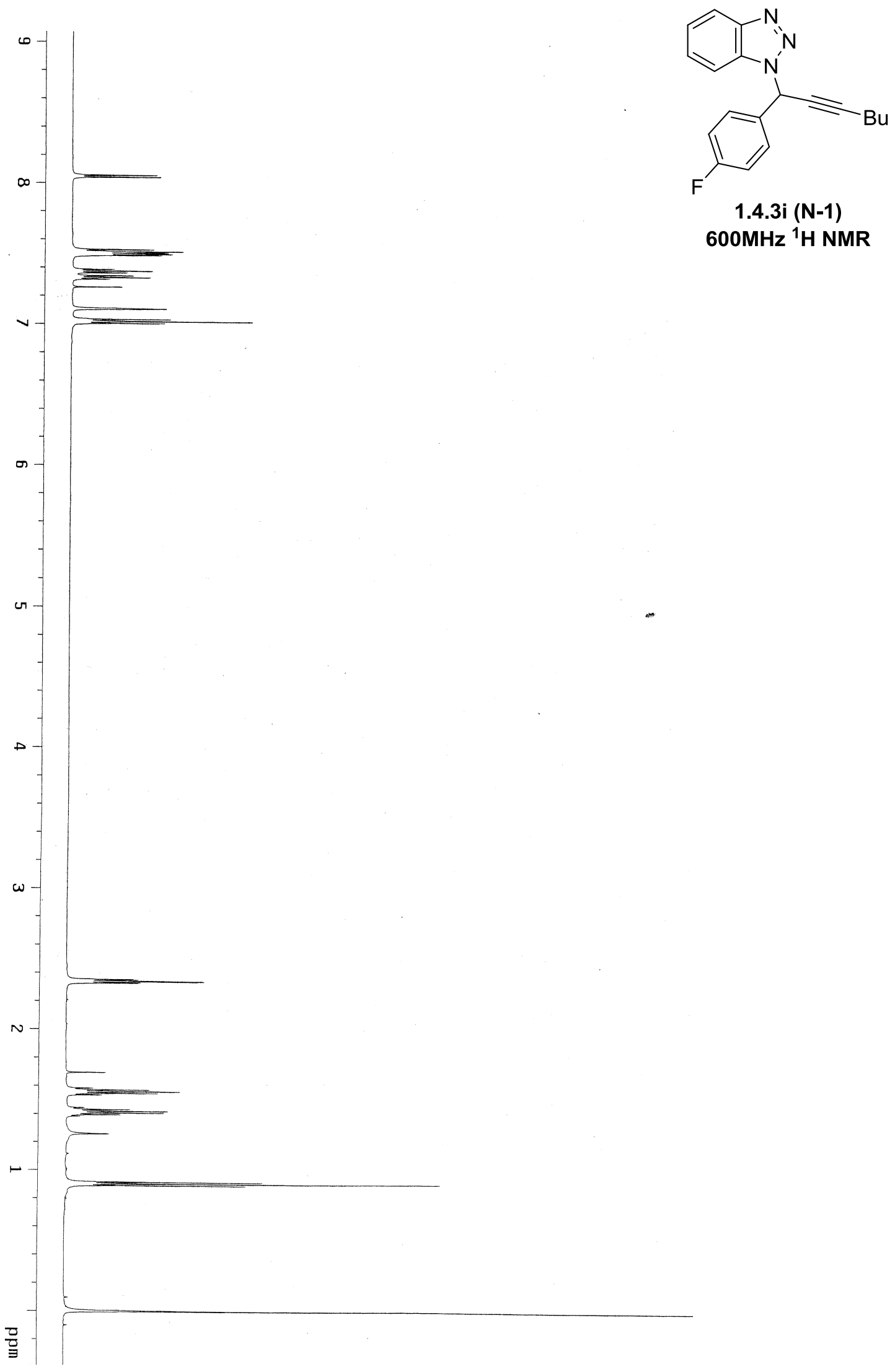

1.4.3i (N-1) $600 \mathrm{MHz}{ }^{1} \mathrm{H}$ NMR 

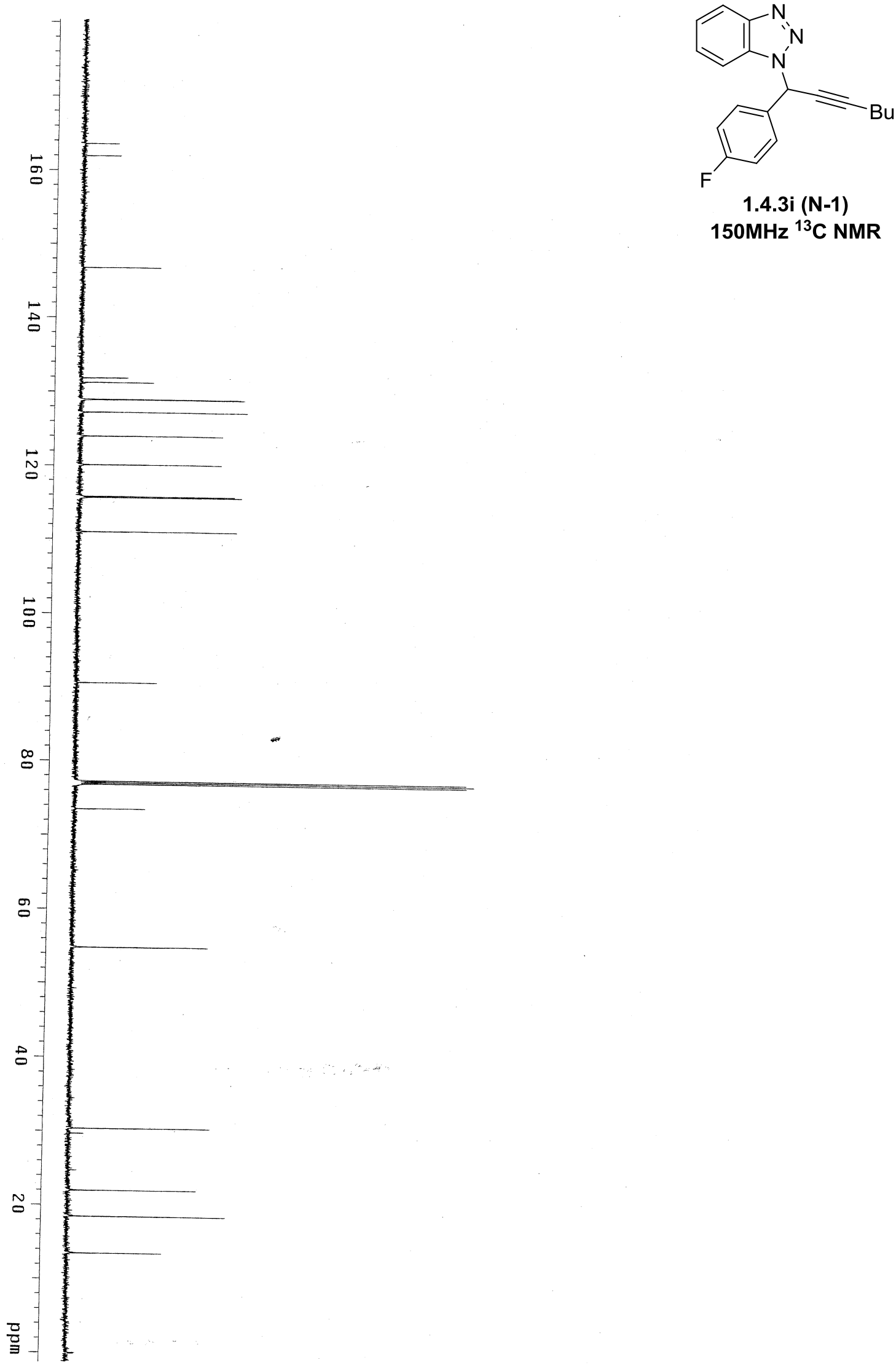

1.4.3i (N-1) $150 \mathrm{MHz}{ }^{13} \mathrm{C}$ NMR 

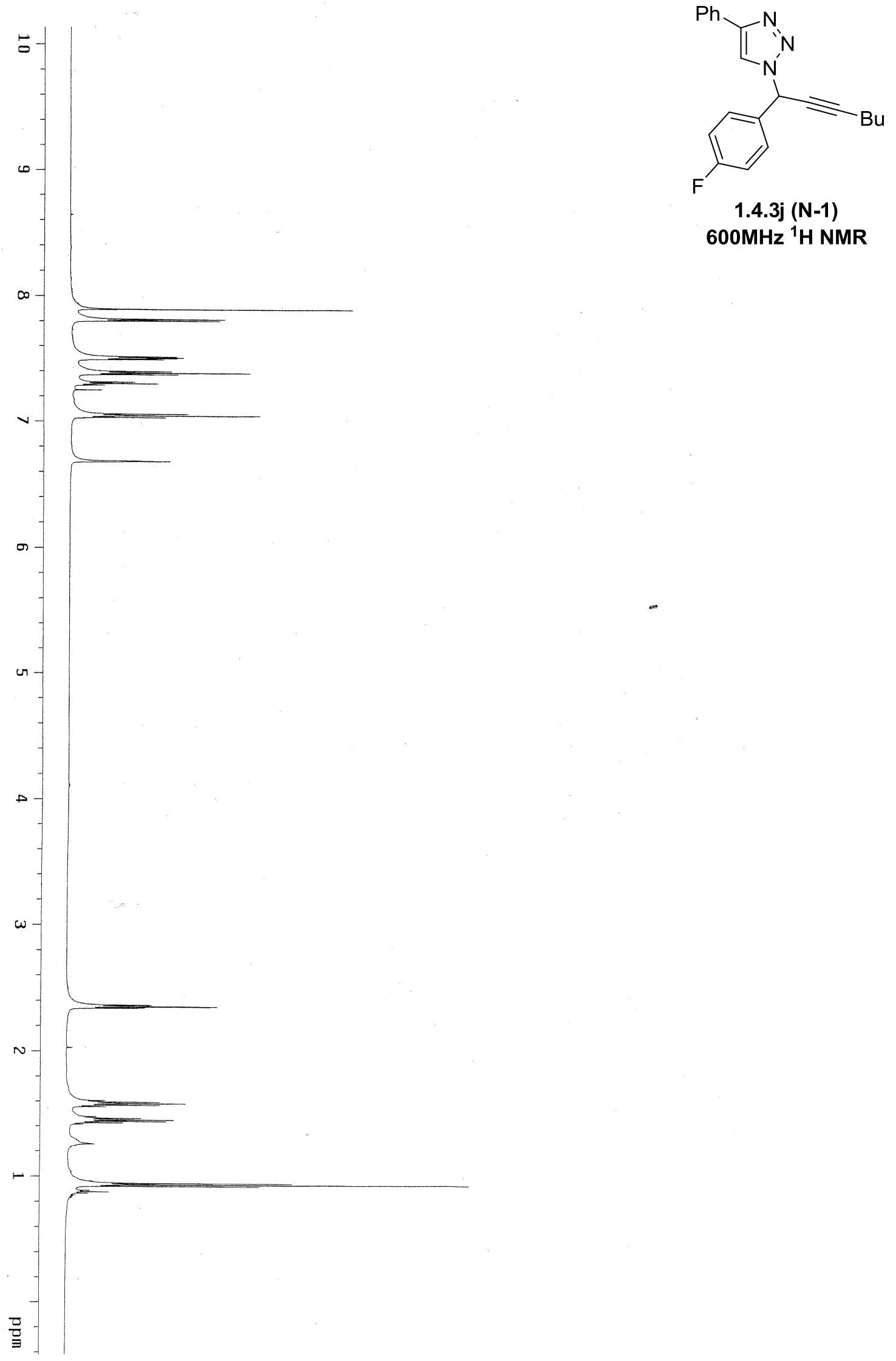

1.4.3j (N-1) $600 \mathrm{MHz}{ }^{1} \mathrm{H}$ NMR 

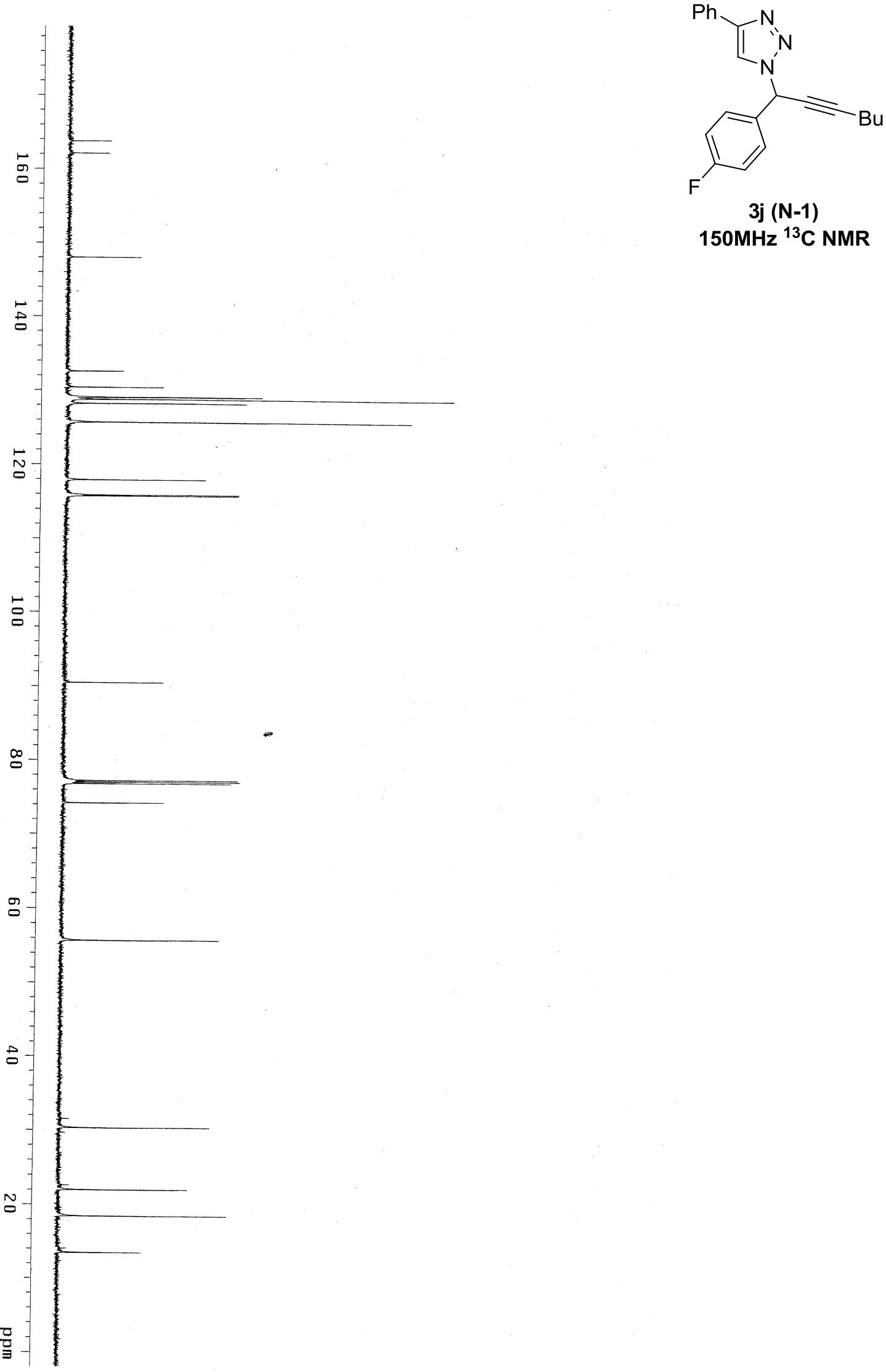


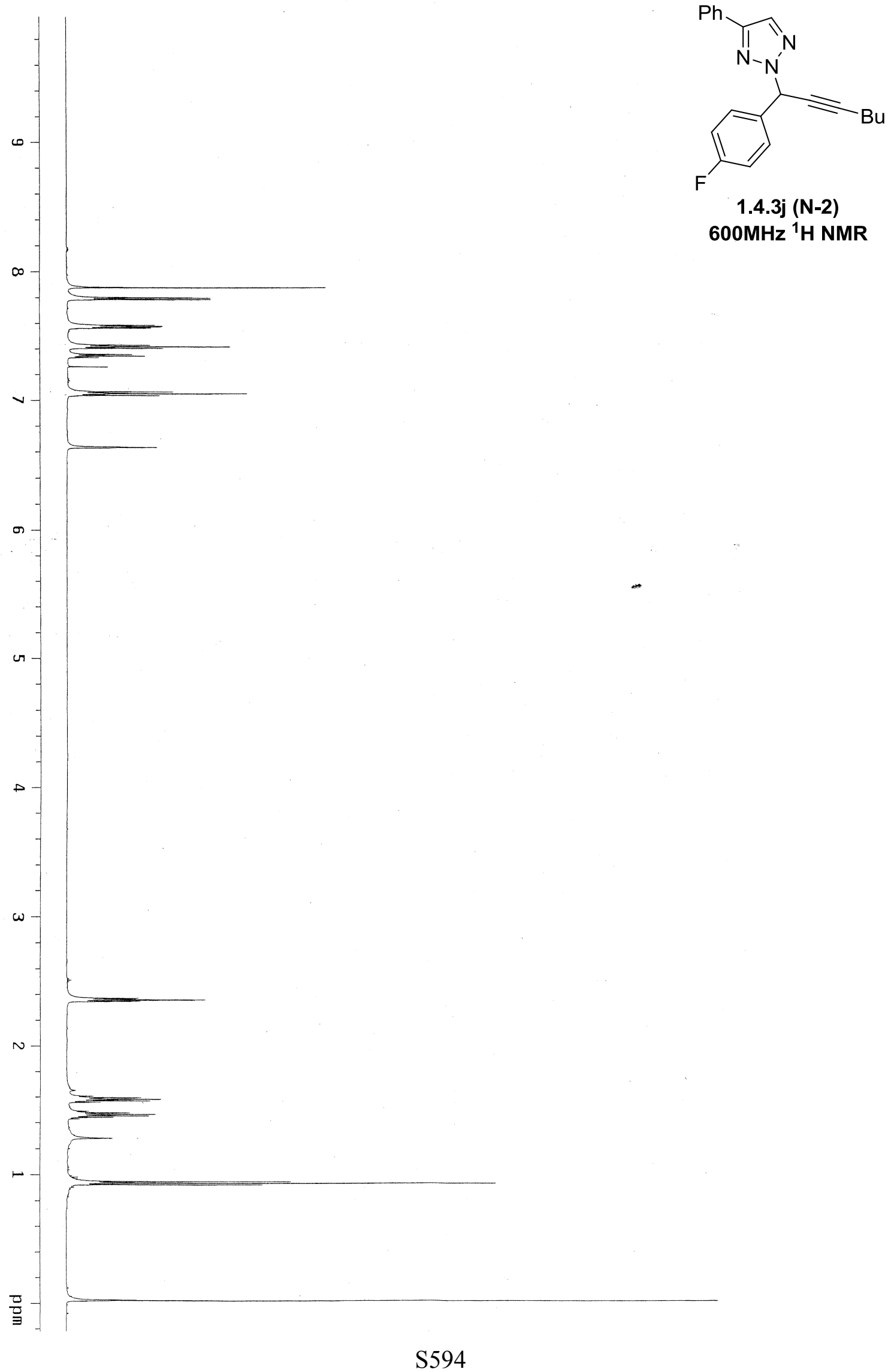



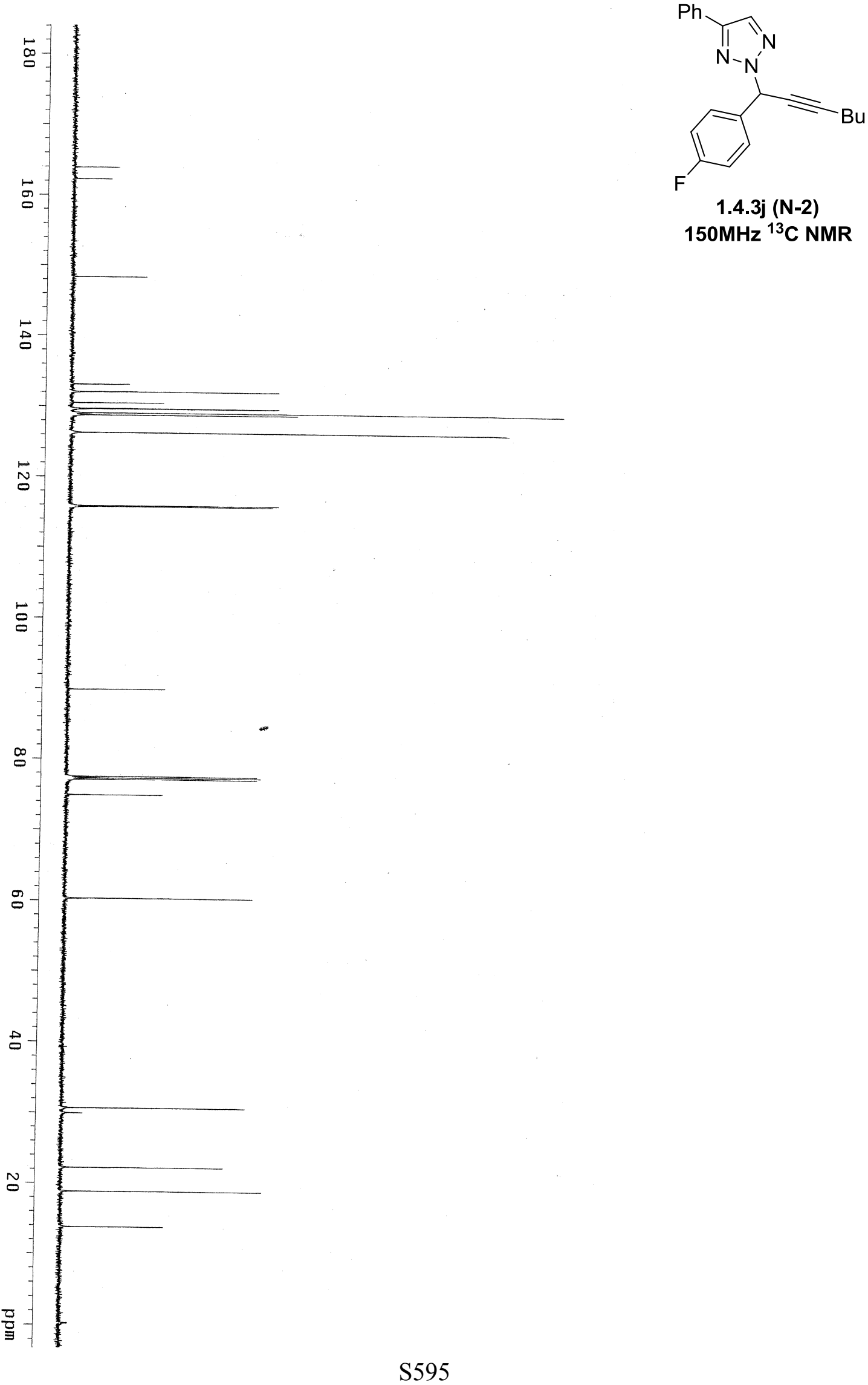


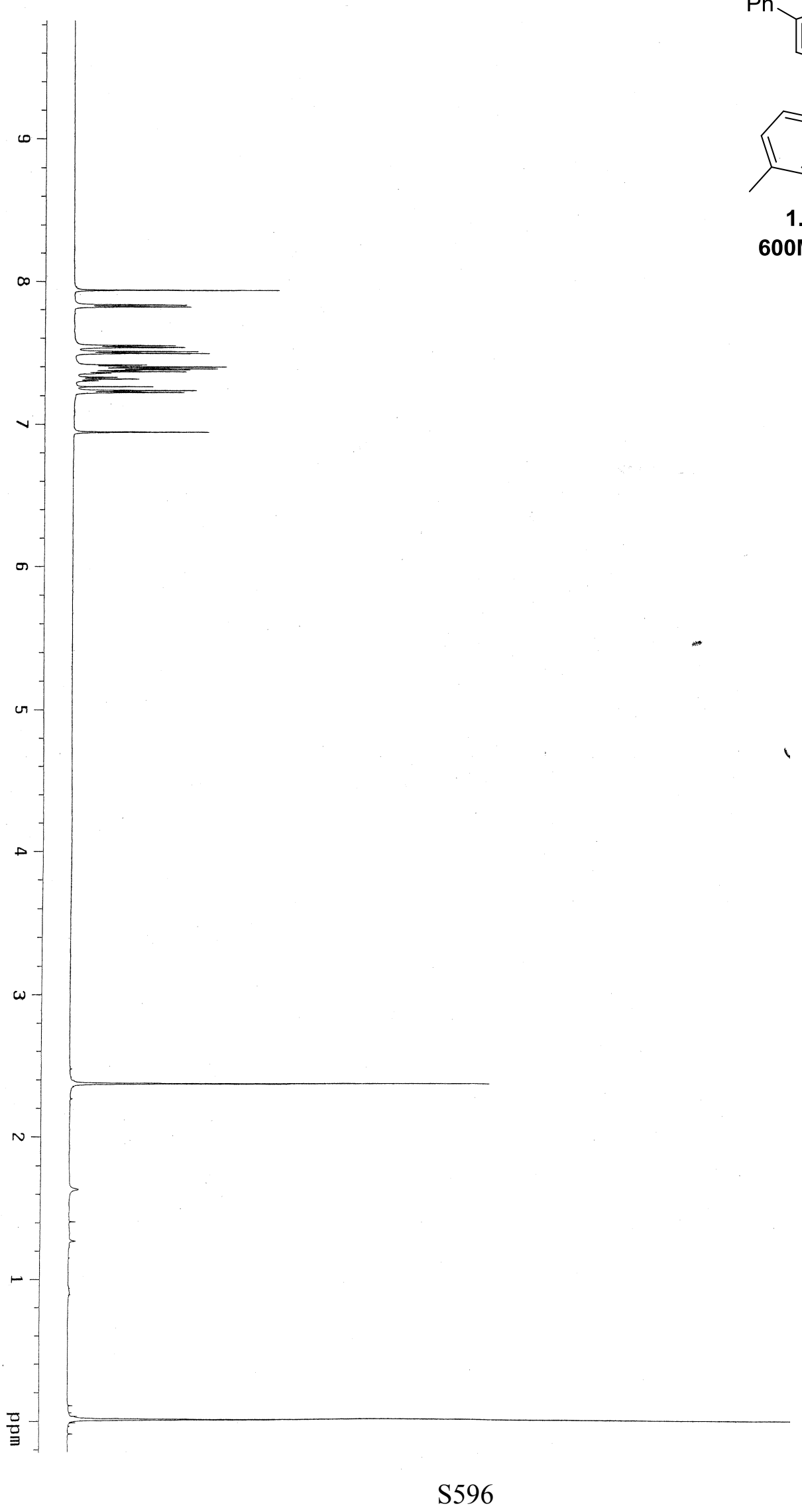



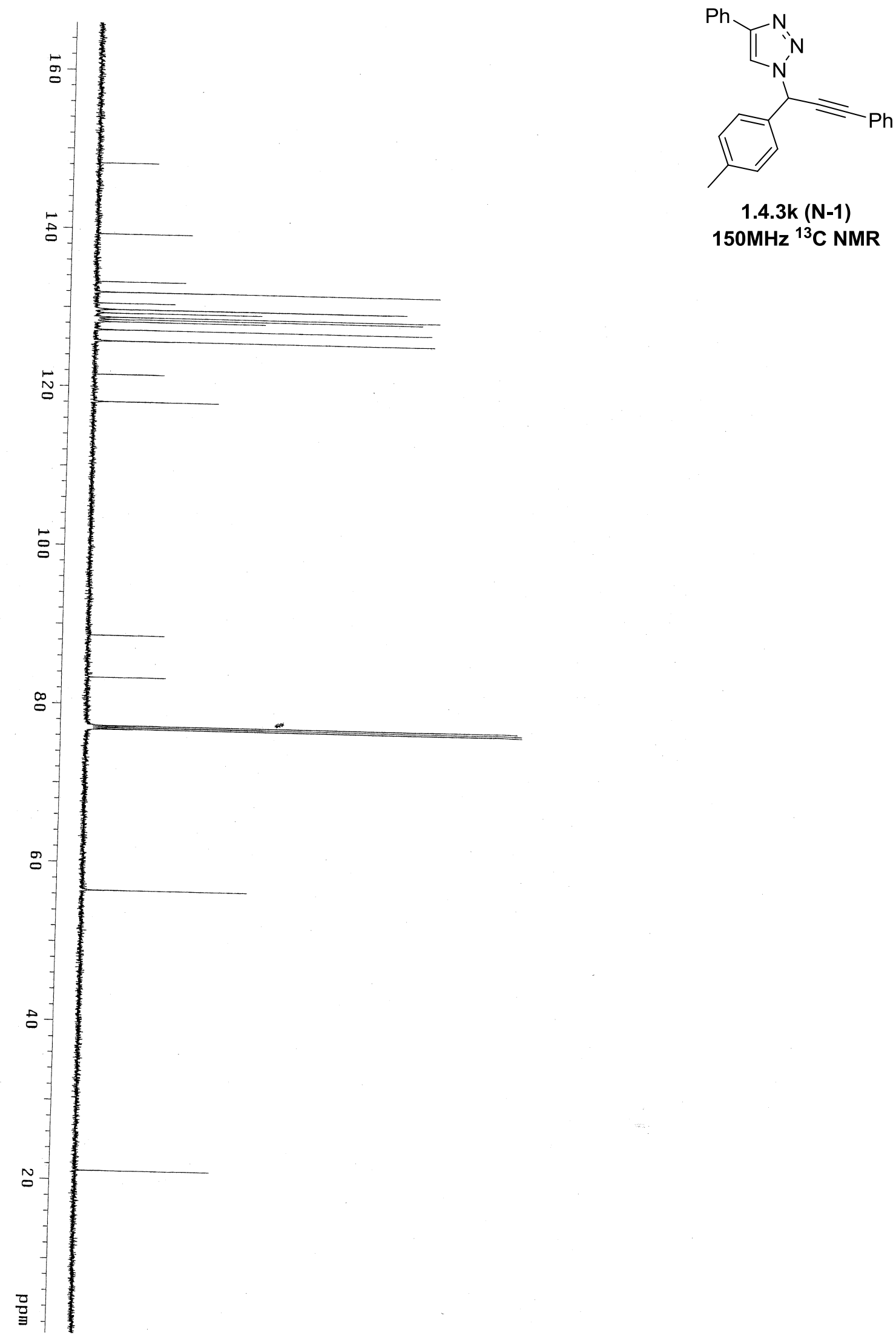


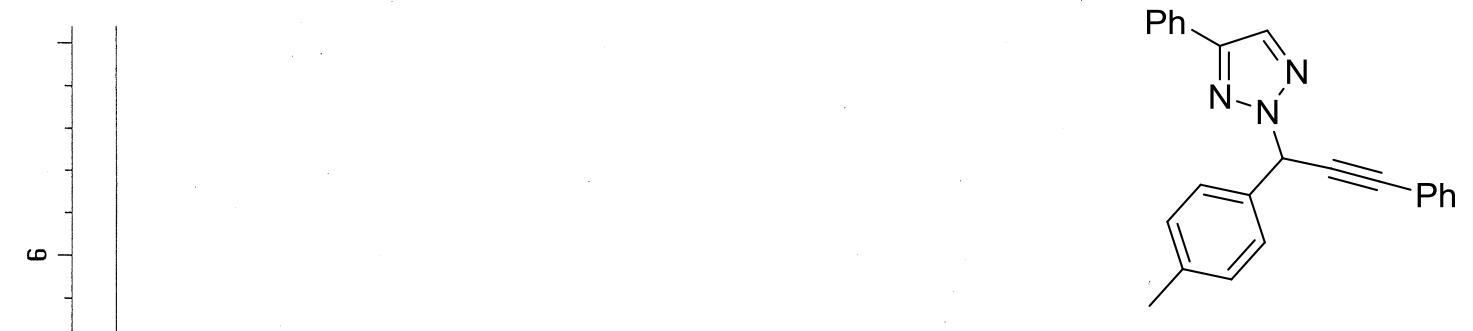

1.4.3k (N-2) $600 \mathrm{MHz}{ }^{1} \mathrm{H}$ NMR

$\infty$
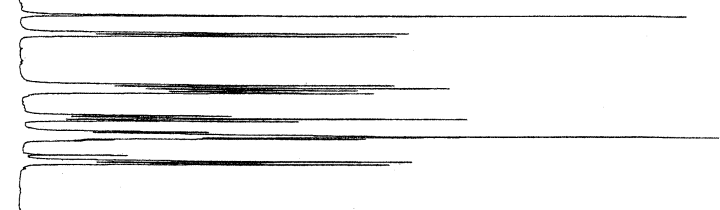

$\checkmark$

$\sigma$

$\triangle$

$\omega$

u

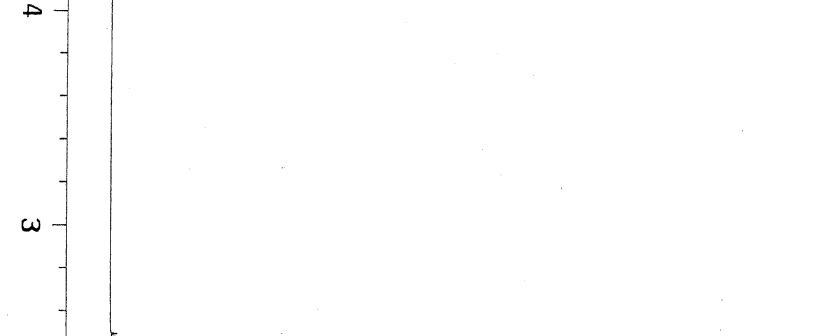

$N$

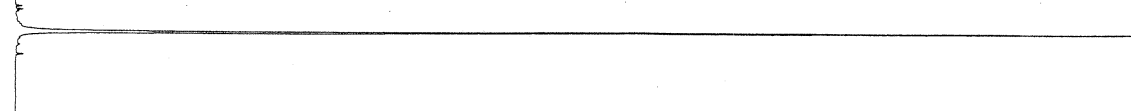

$-1\}$ 

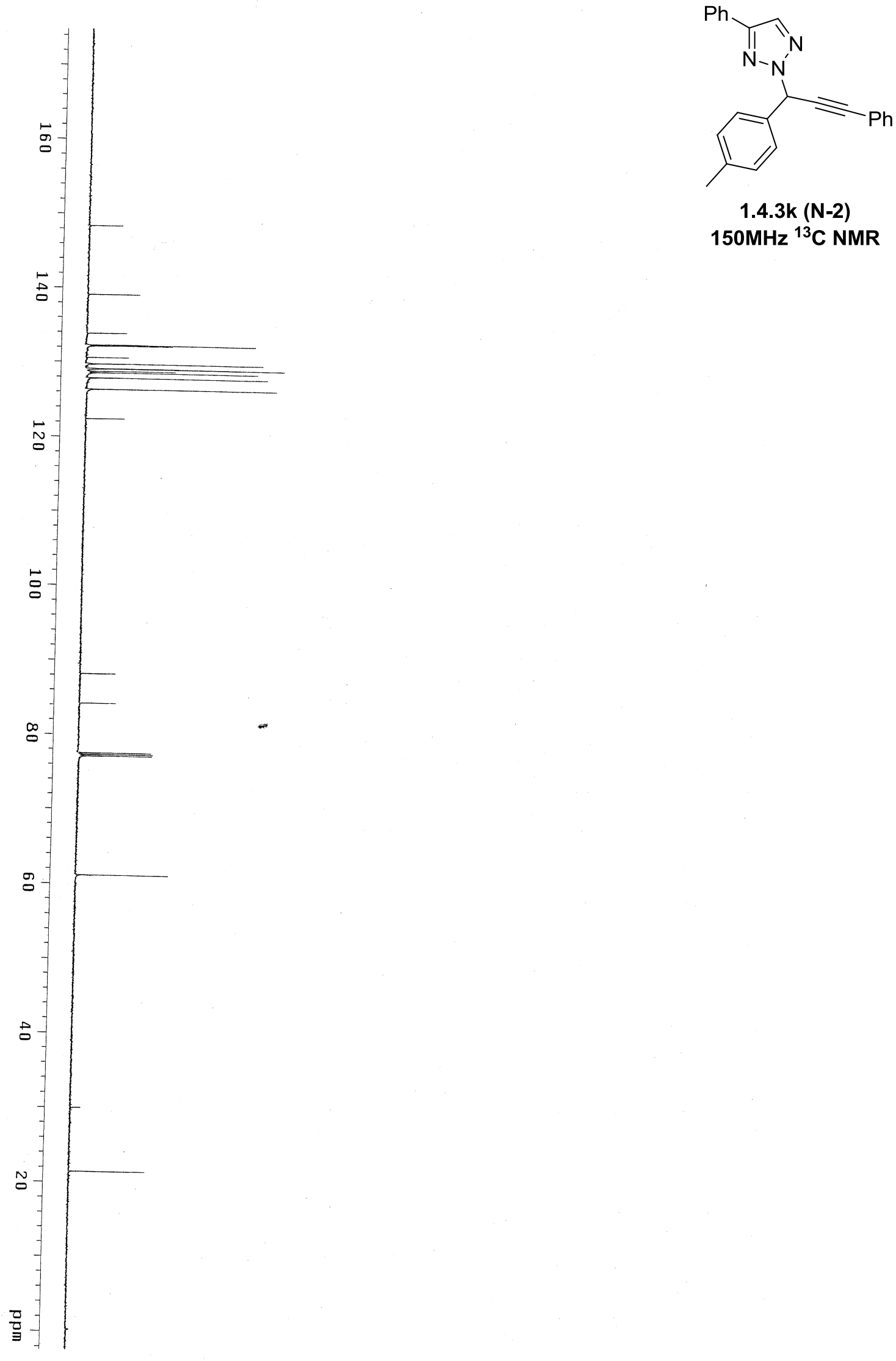

1.4.3k (N-2) $150 \mathrm{MHz}{ }^{13} \mathrm{C}$ NMR 


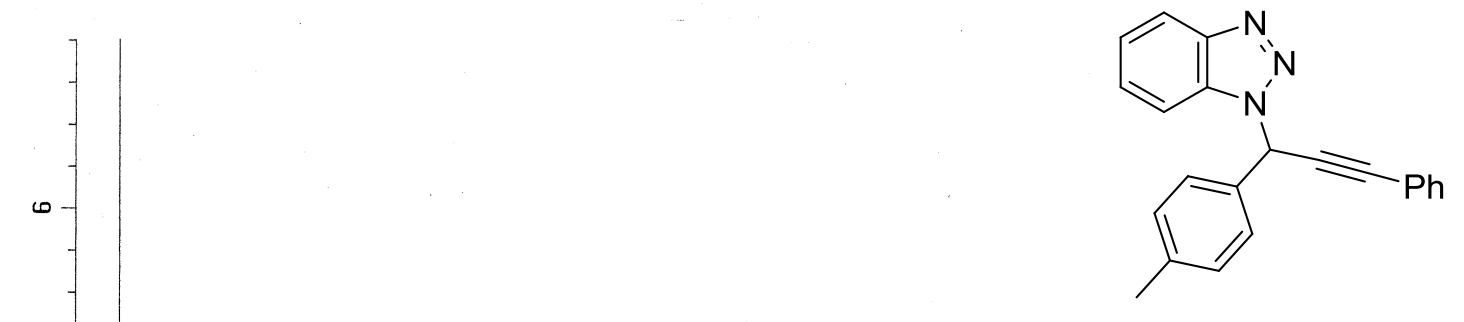

1.4.3I (N-1) $600 \mathrm{MHz}{ }^{1} \mathrm{H}$ NMR

0

$\omega$

or
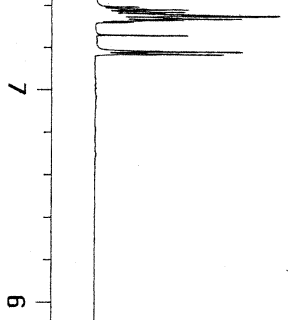

.

N

믑 

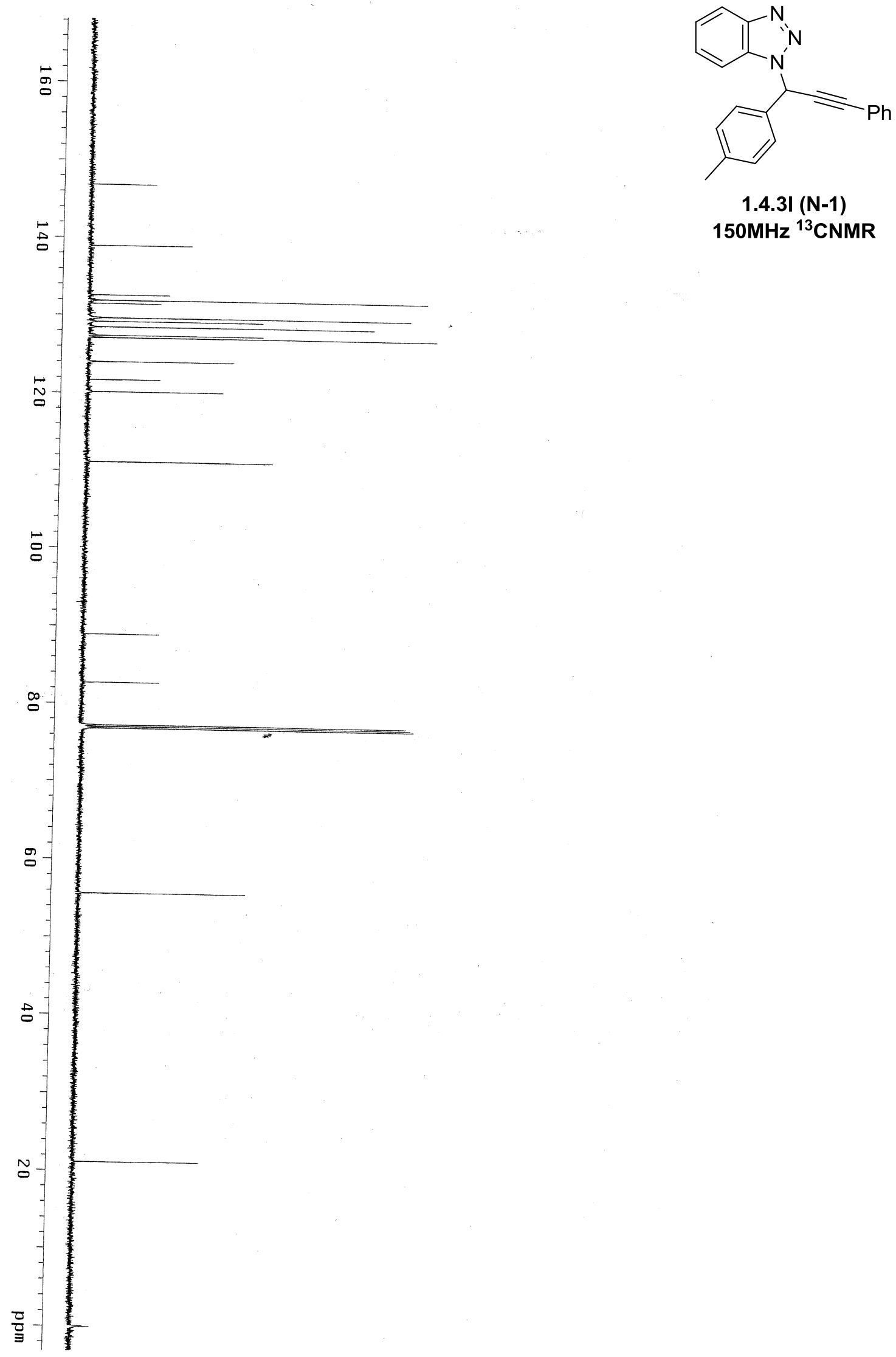

1.4.3I (N-1) 150MHz ${ }^{13} \mathrm{CNMR}$ 


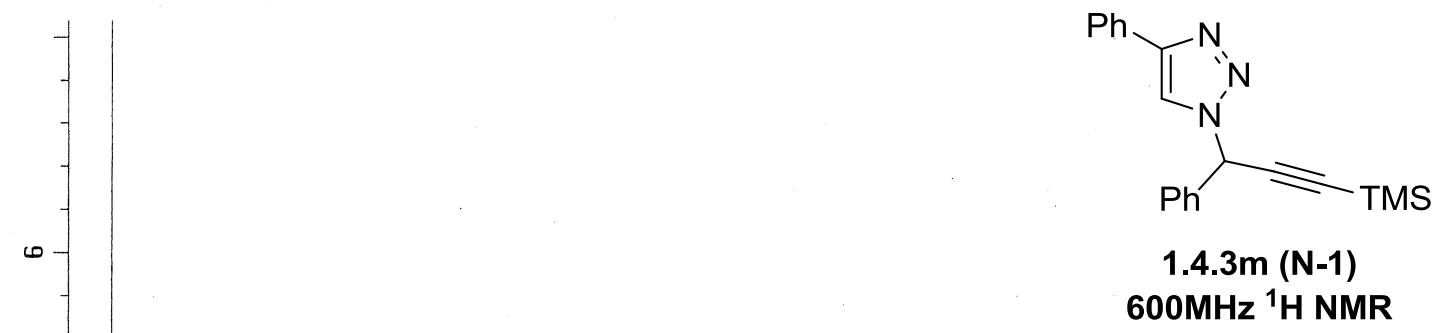

$\infty$

$\infty$

v

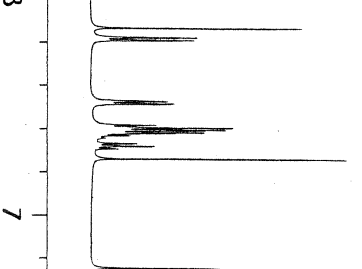

$\triangle$

$\omega$

릅

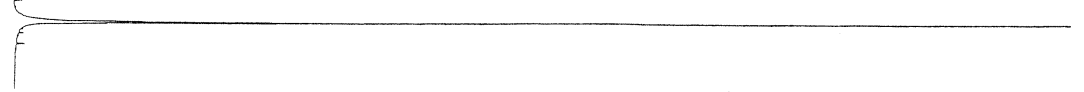



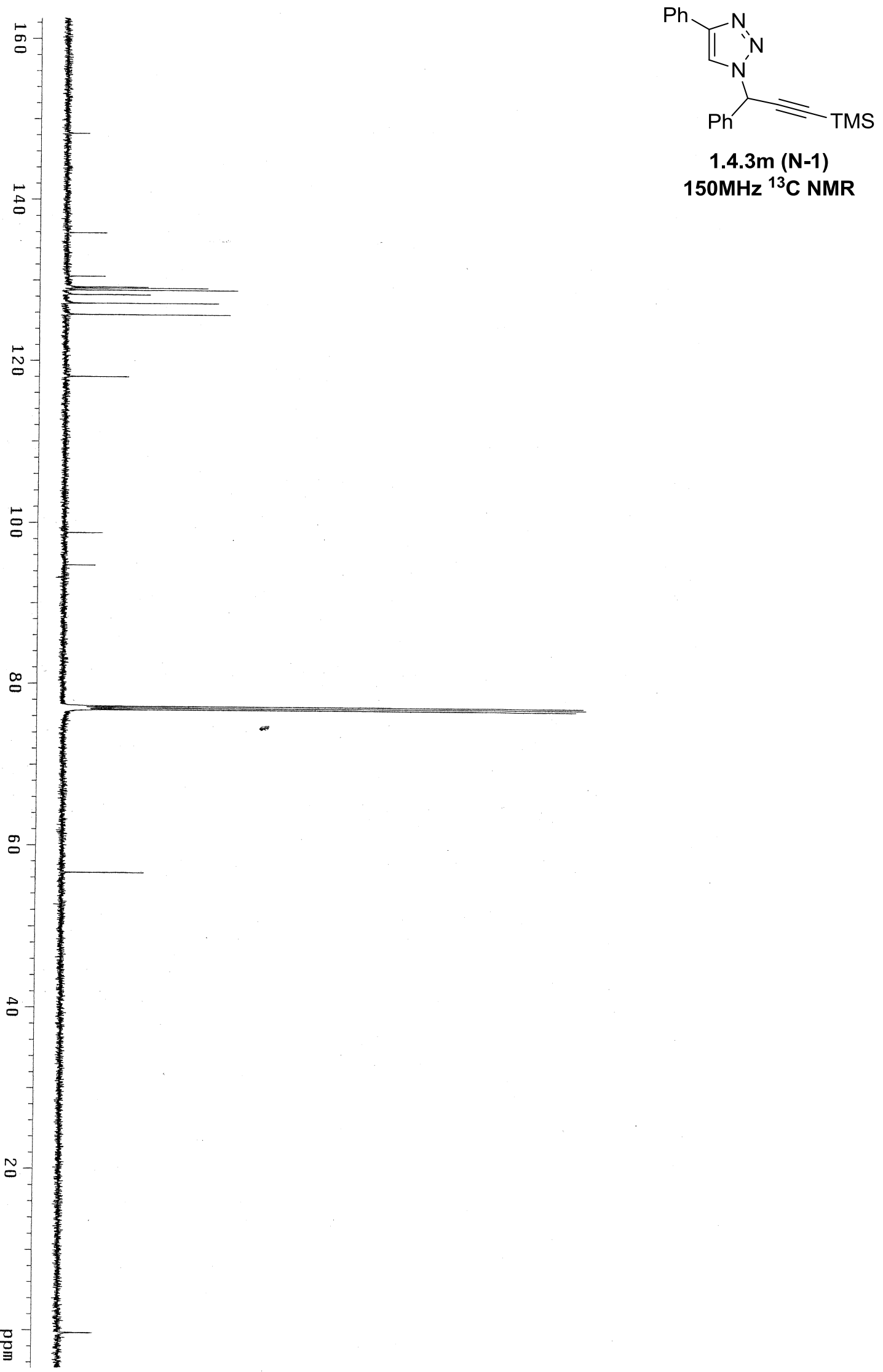

1.4.3m (N-1) $150 \mathrm{MHz}{ }^{13} \mathrm{C}$ NMR 

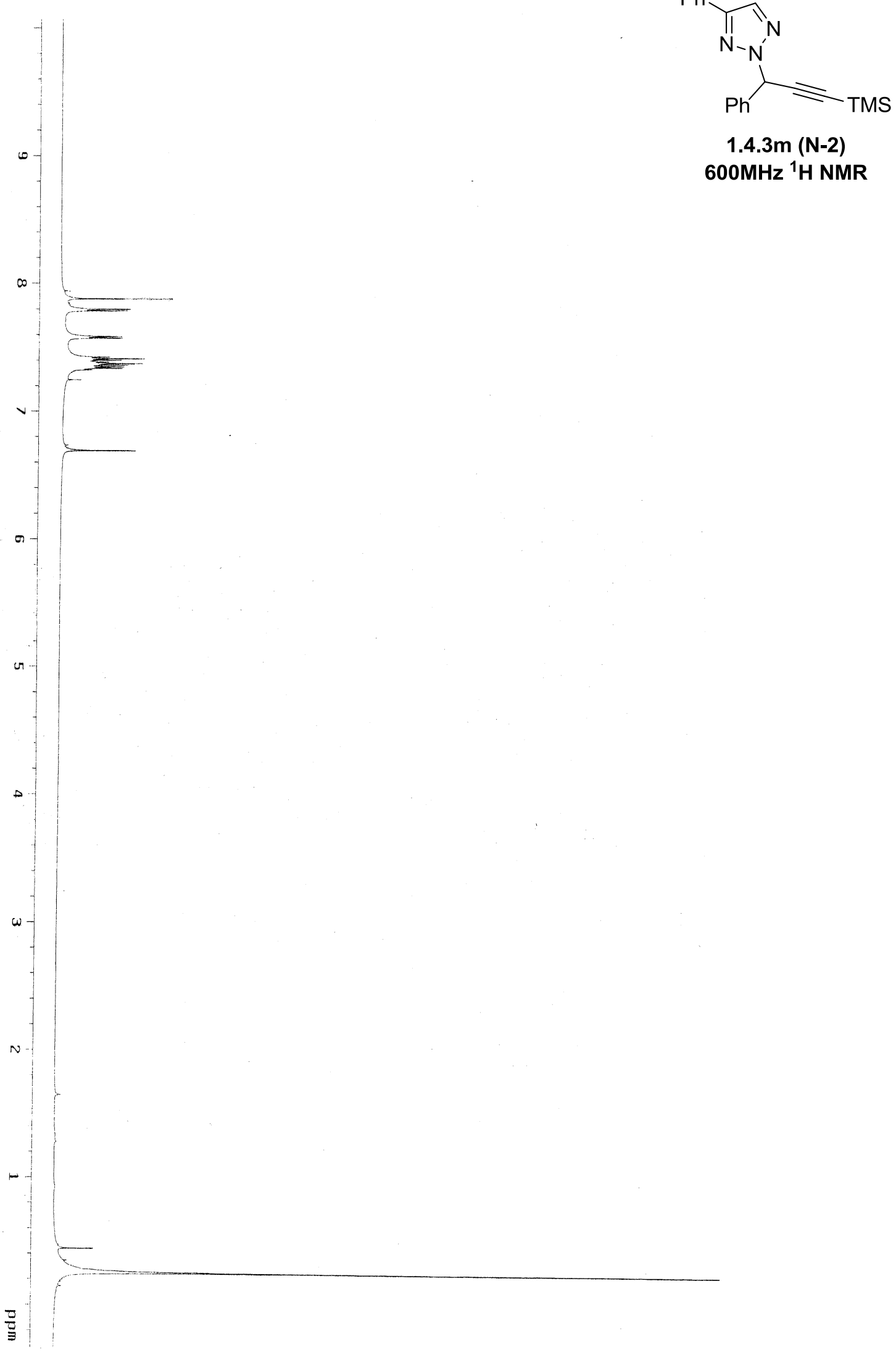

1.4.3m (N-2) $600 \mathrm{MHz}^{1} \mathrm{H}$ NMR 


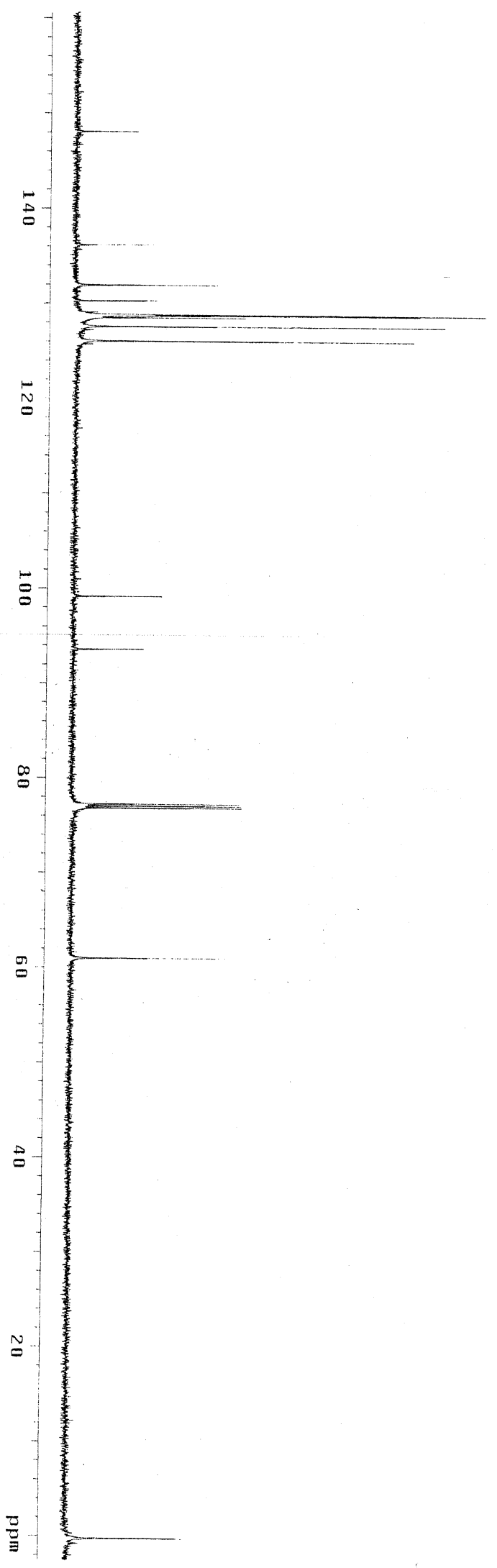

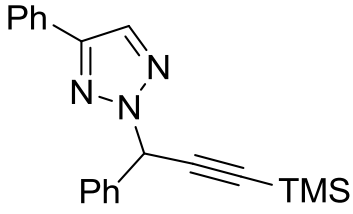

1.4.3m (N-2) $150 \mathrm{MHz}{ }^{13} \mathrm{C}$ NMR 

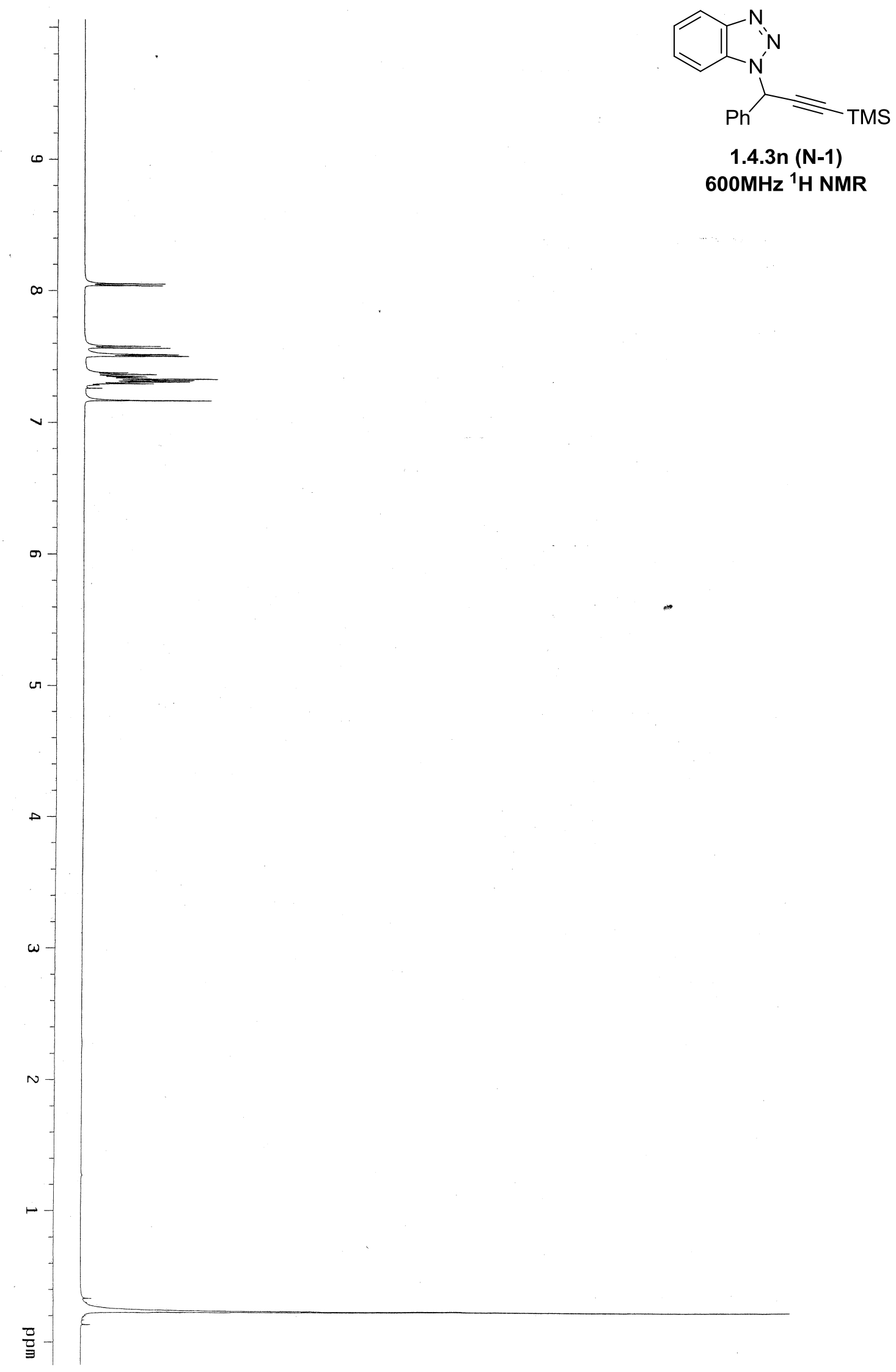


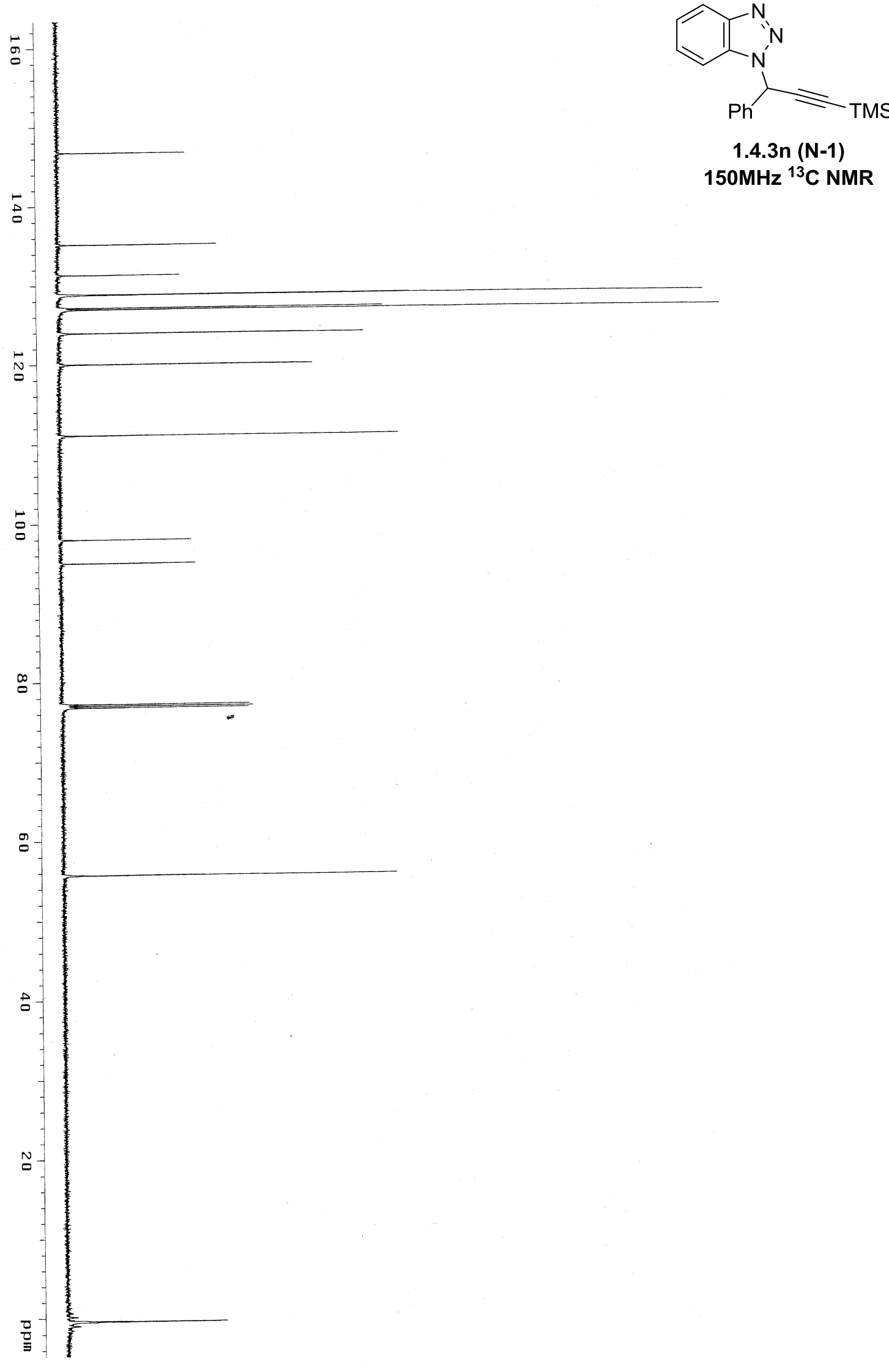




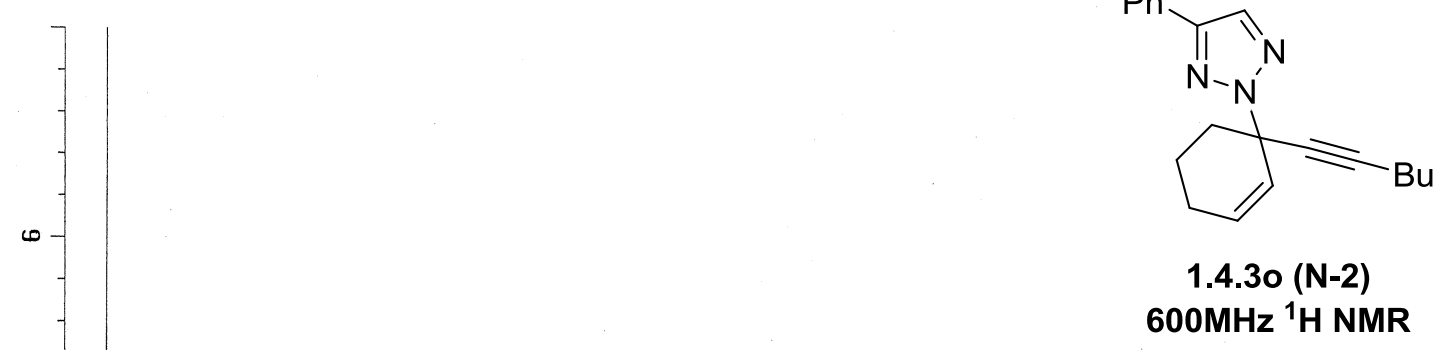

$\infty$
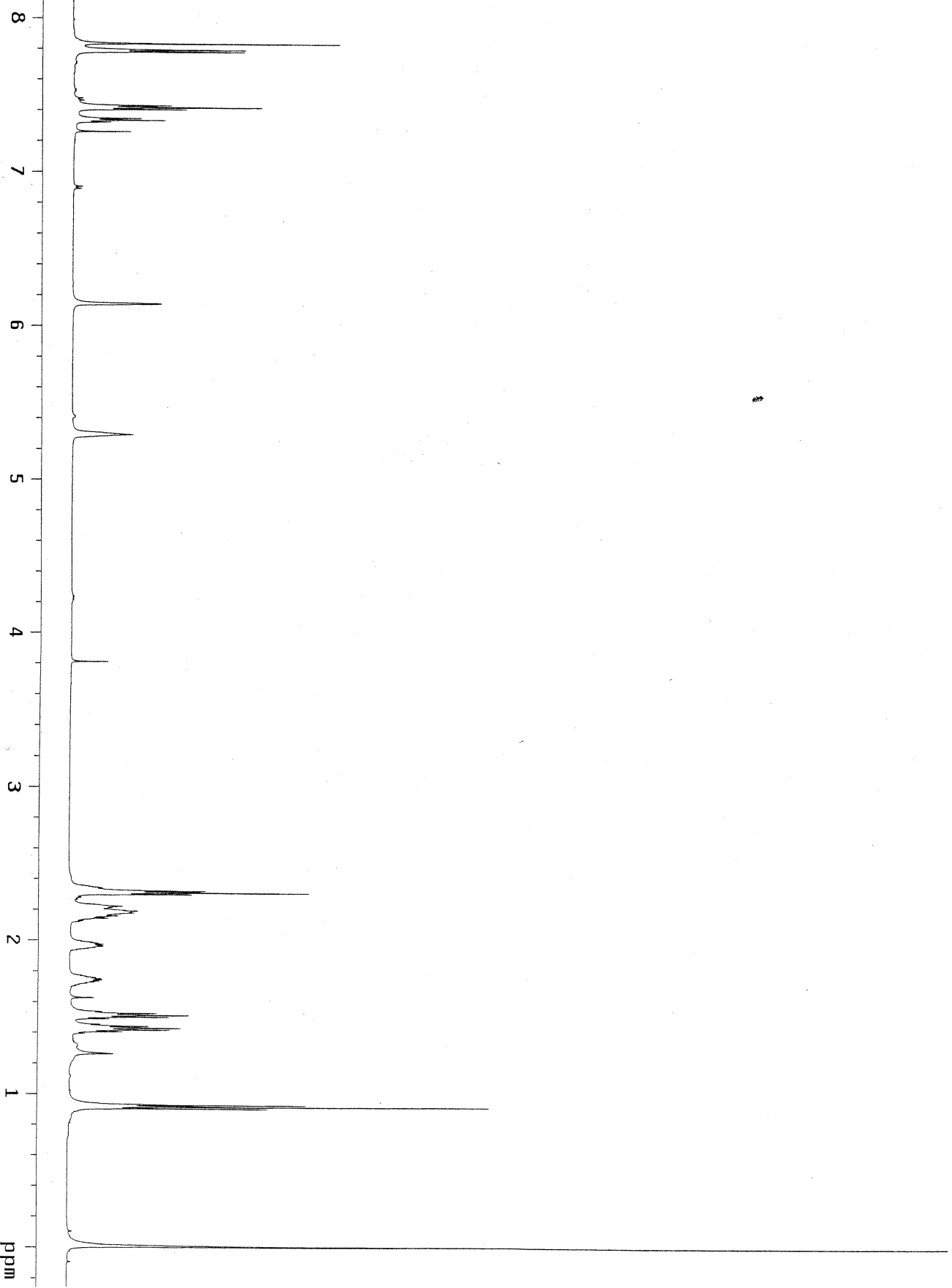


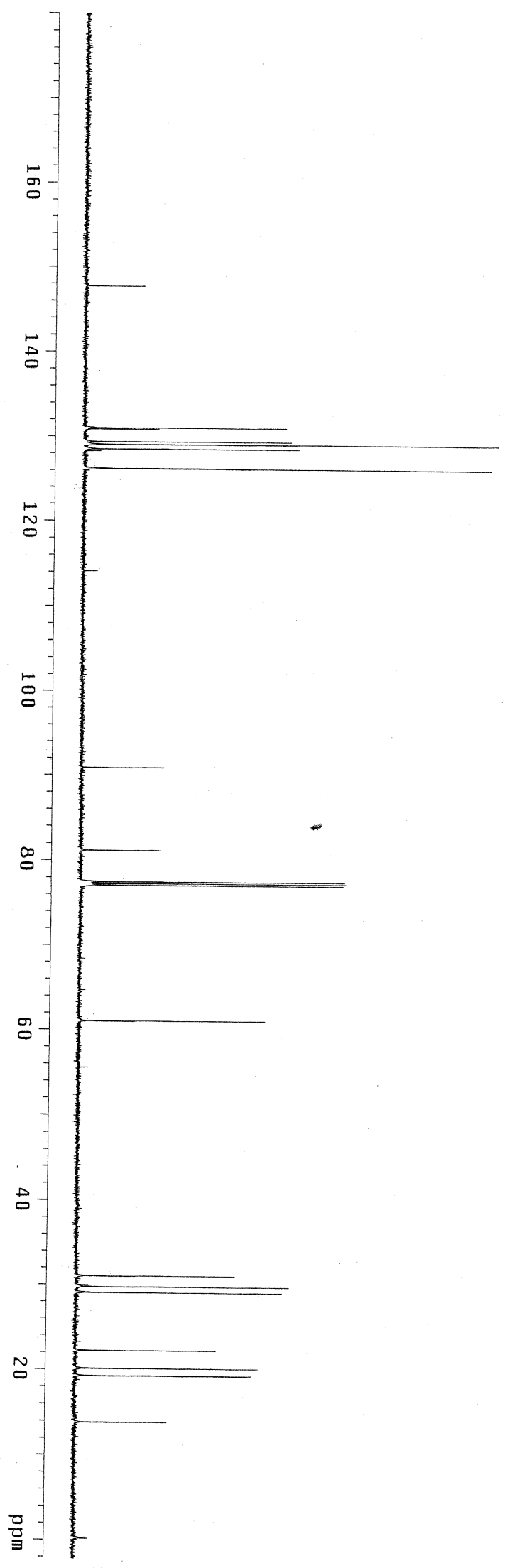

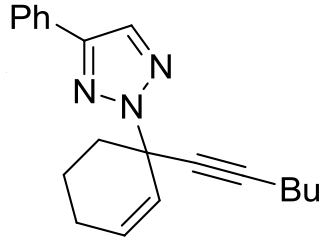

1.4.30 (N-2) $150 \mathrm{MHz}{ }^{13} \mathrm{C}$ NMR 


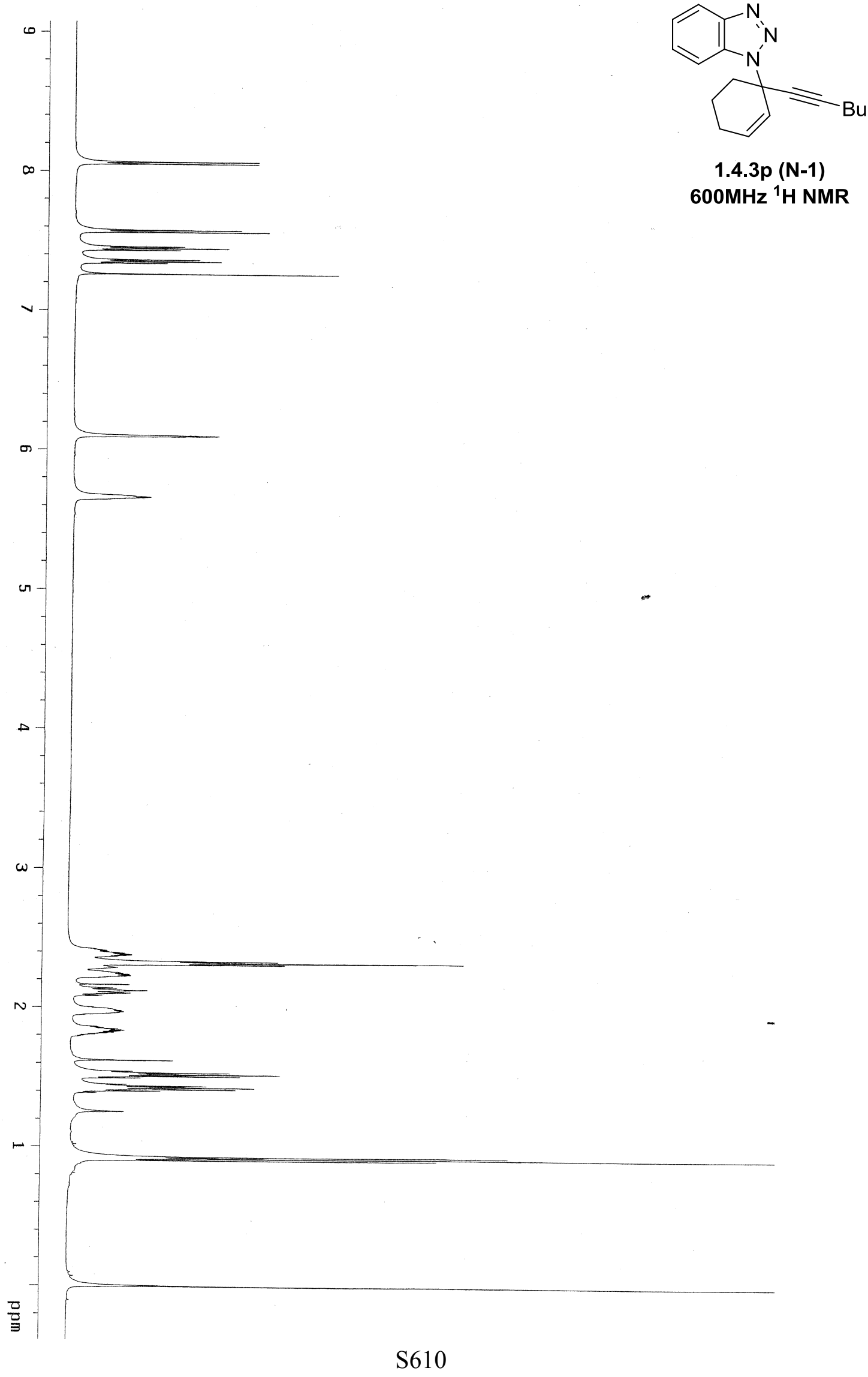



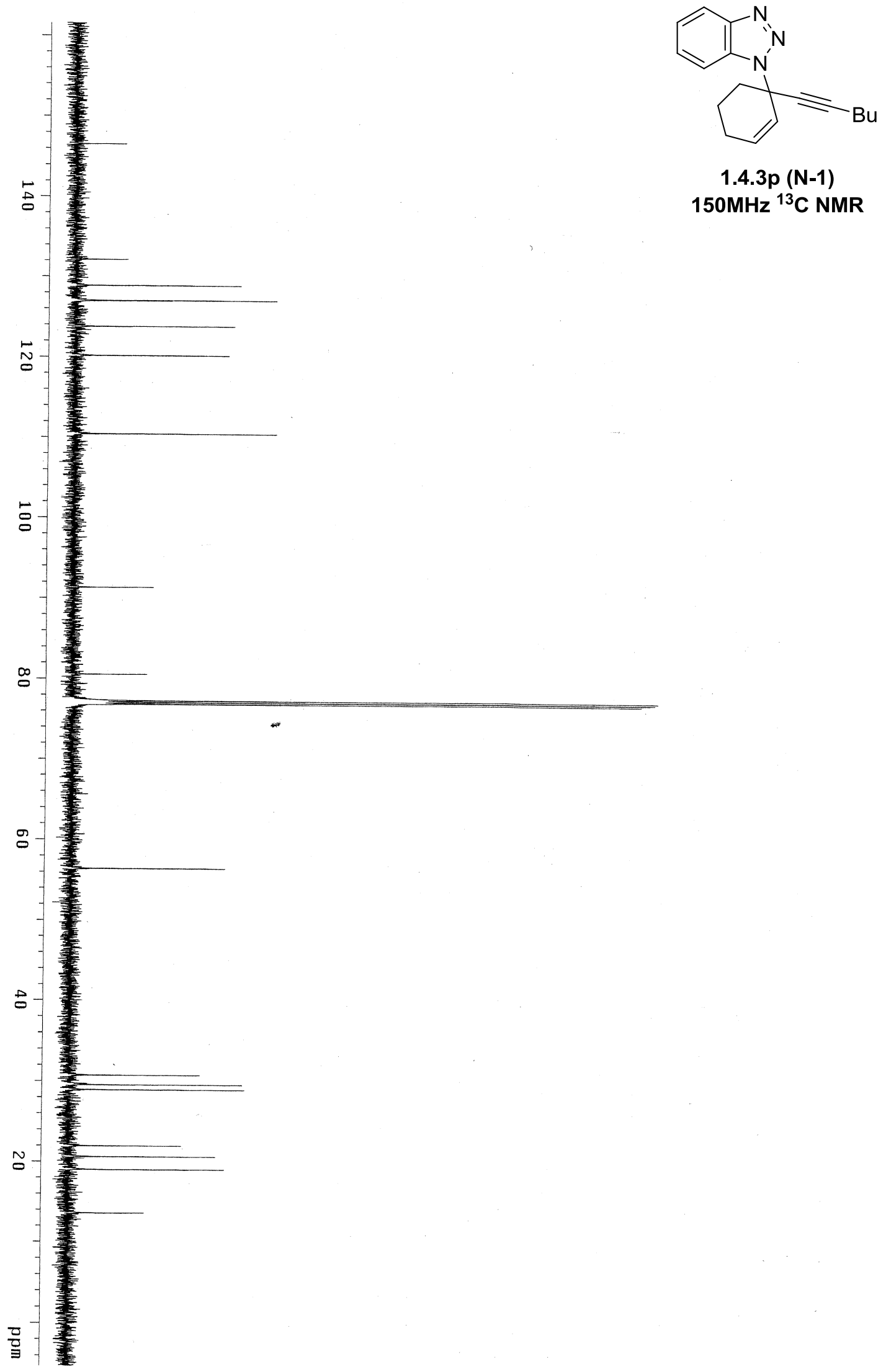

1.4.3p (N-1) $150 \mathrm{MHz}{ }^{13} \mathrm{C}$ NMR 


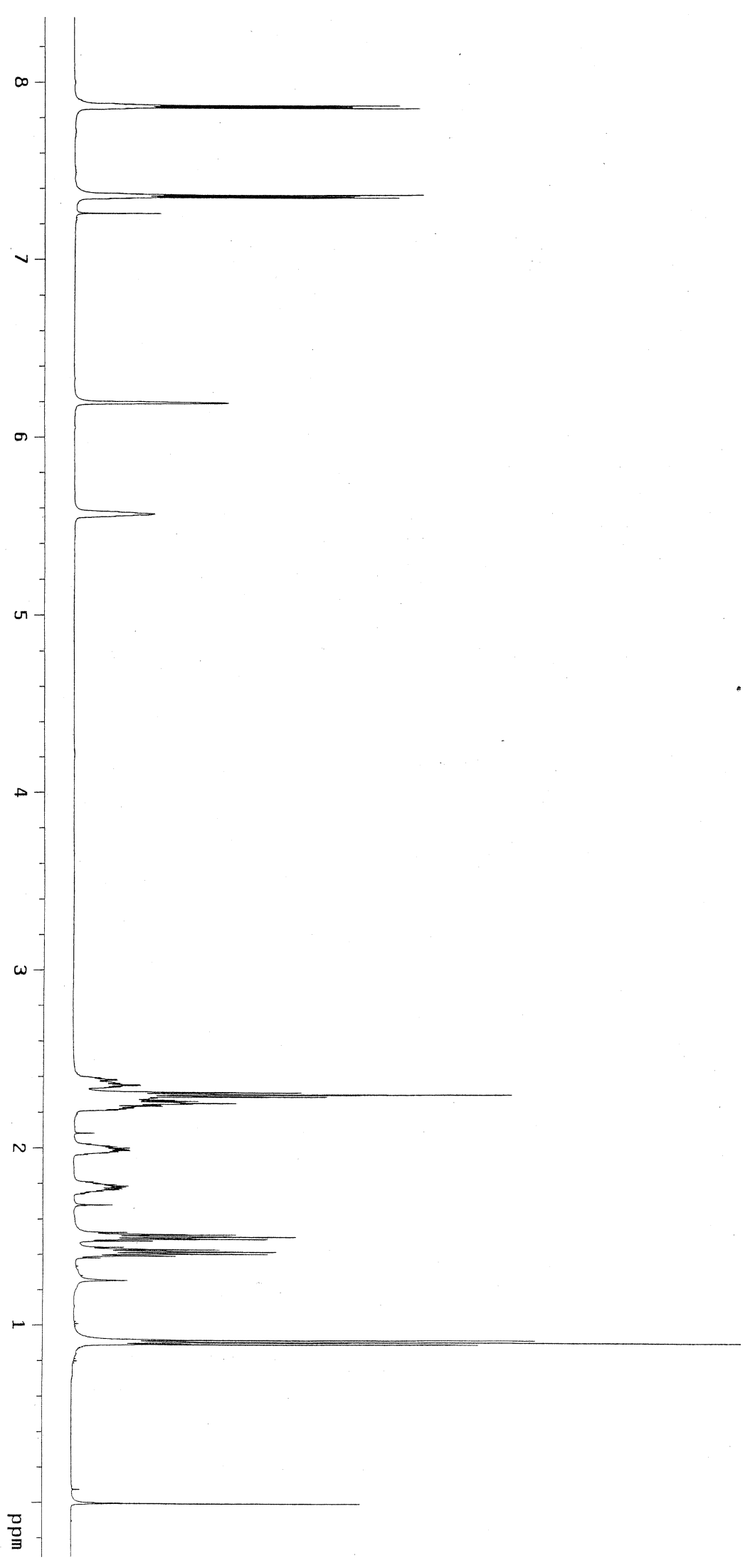

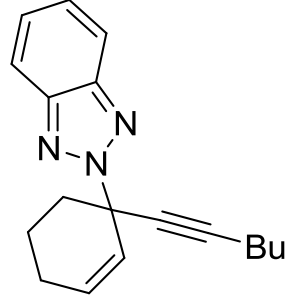

1.4.3p (N-2) $600 \mathrm{MHz}{ }^{1} \mathrm{H}$ NMR 


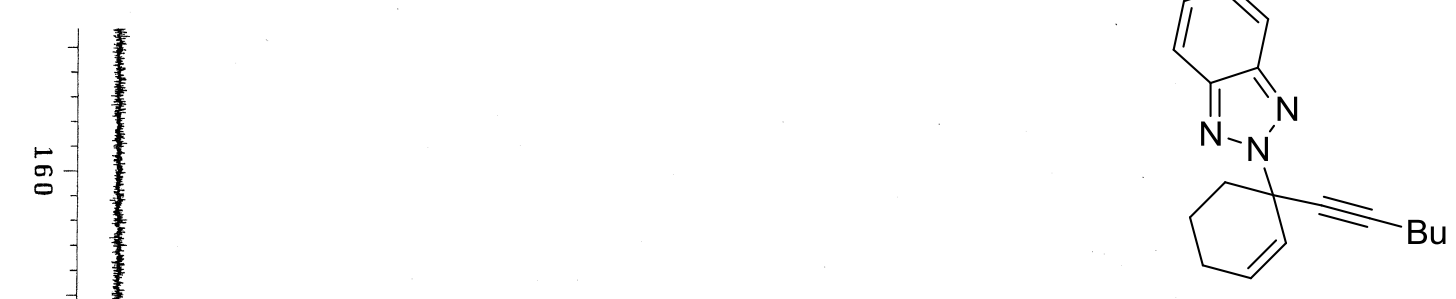

1.4.3p (N-2) $150 \mathrm{MHz}{ }^{13} \mathrm{C}$ NMR

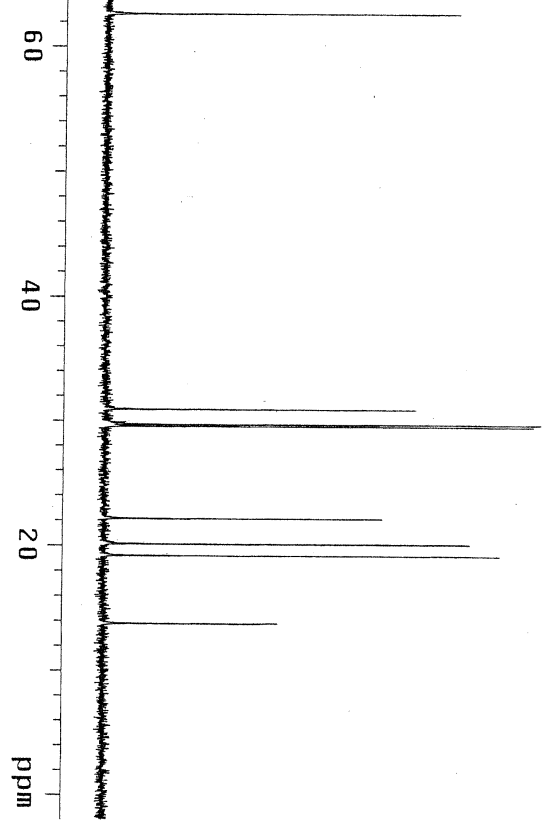



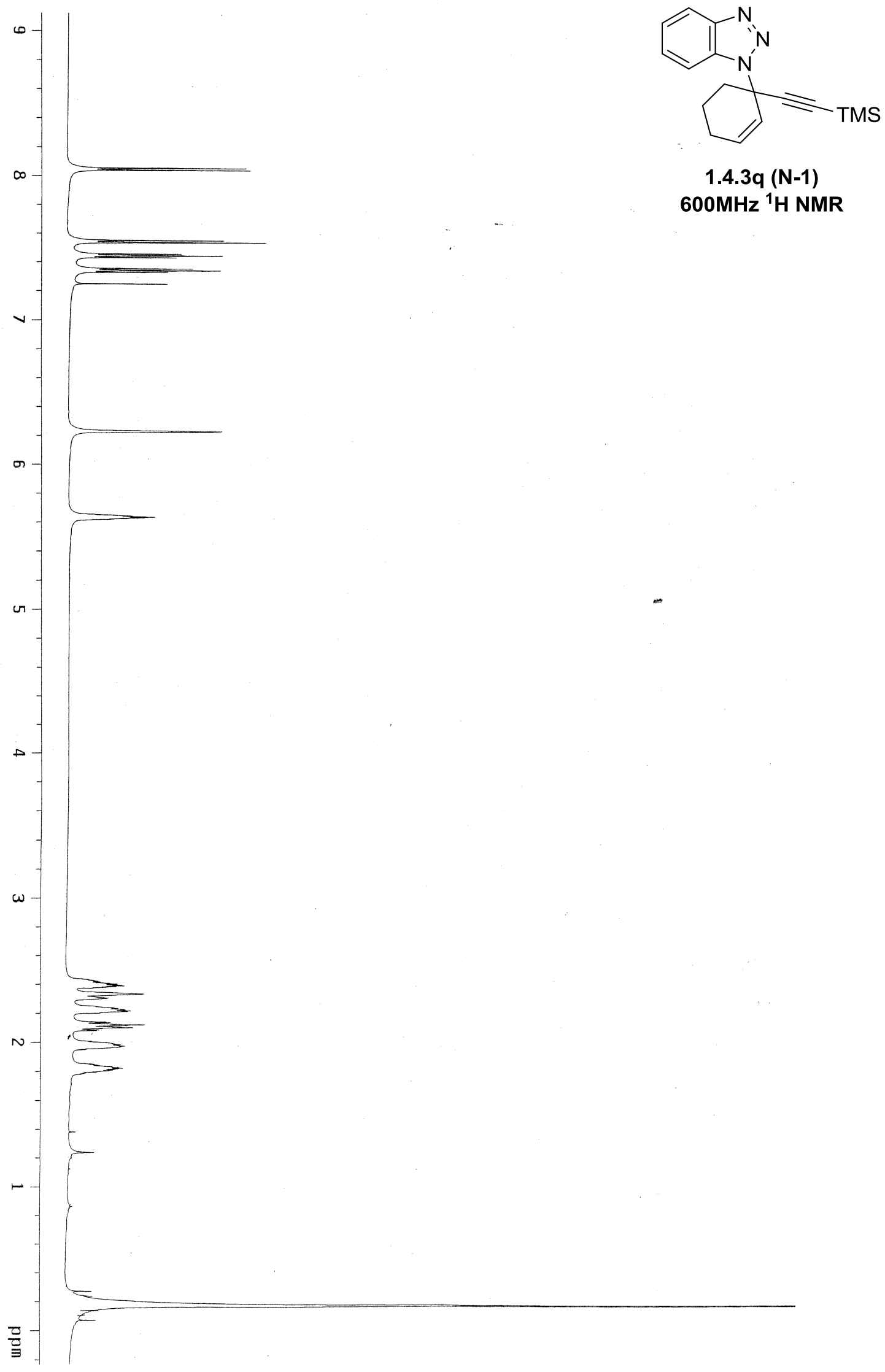

1.4.3q (N-1) $600 \mathrm{MHz}^{1} \mathrm{H}$ NMR 

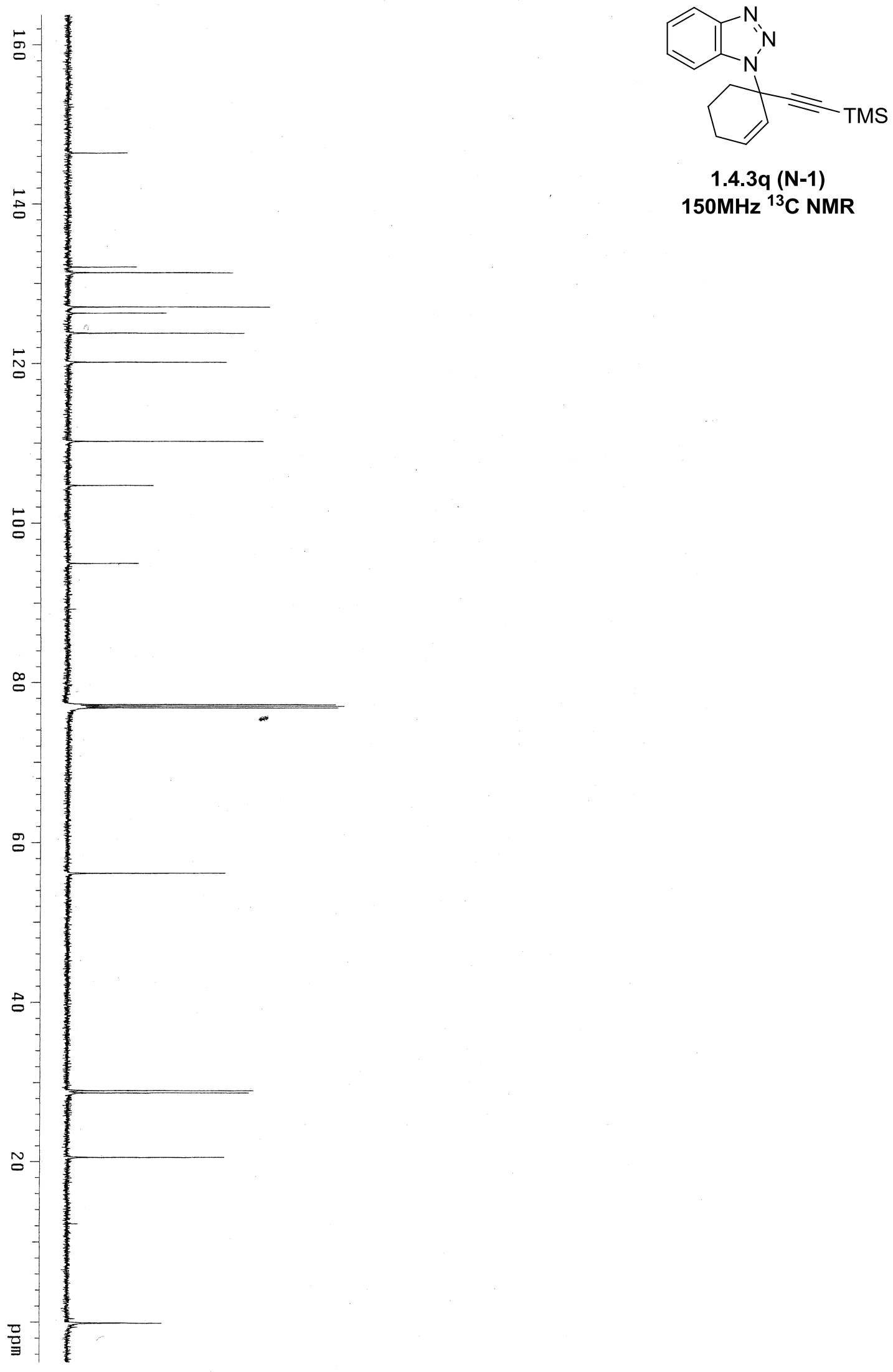

1.4.3q (N-1)

$150 \mathrm{MHz}{ }^{13} \mathrm{C}$ NMR 

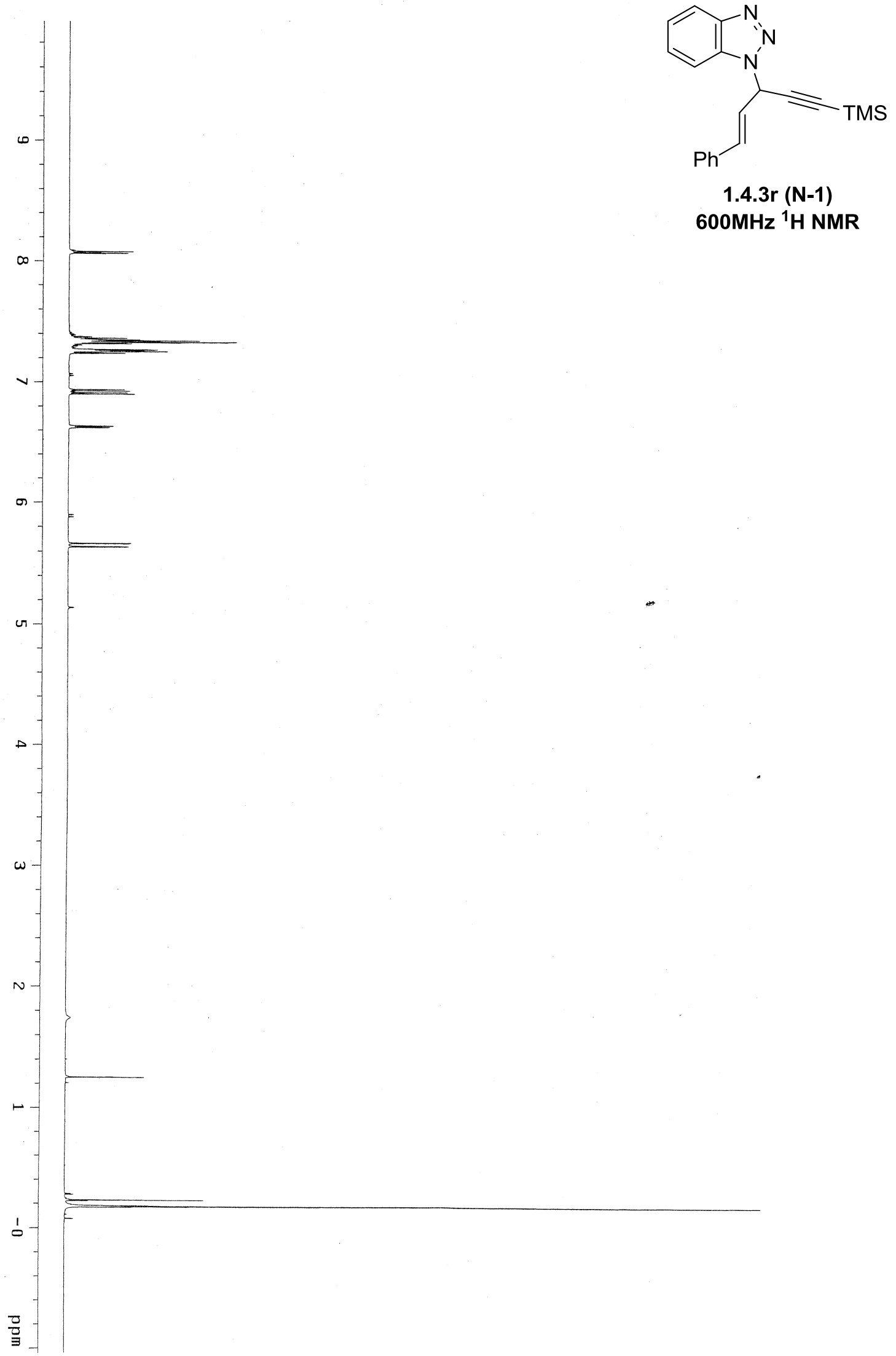


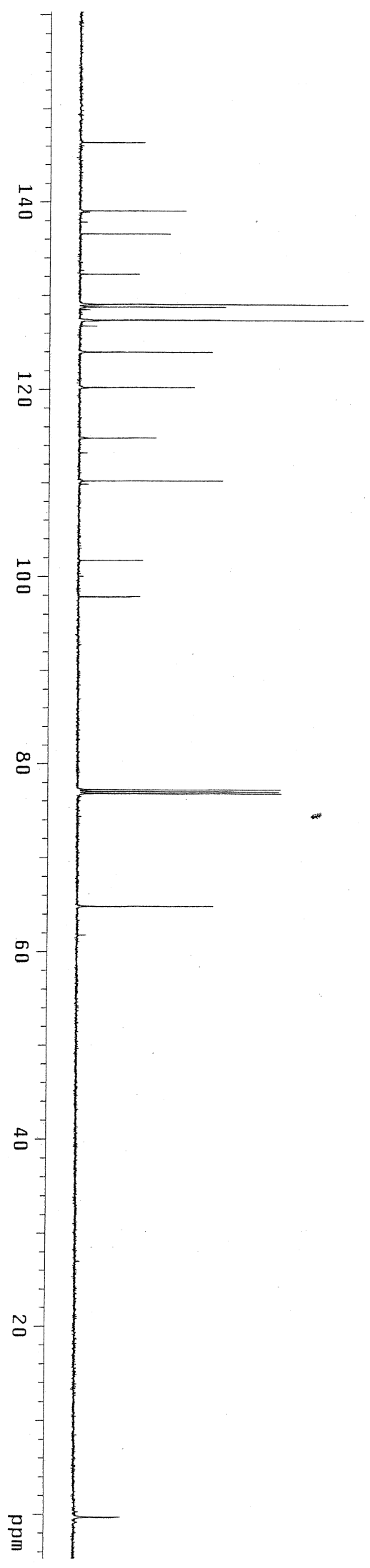

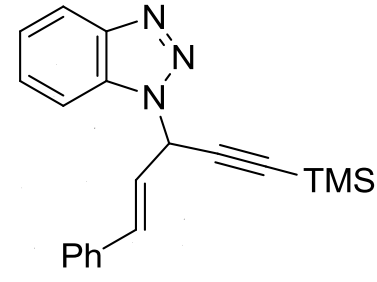

1.4.3r (N-1) $150 \mathrm{MHz}{ }^{13} \mathrm{C}$ NMR 


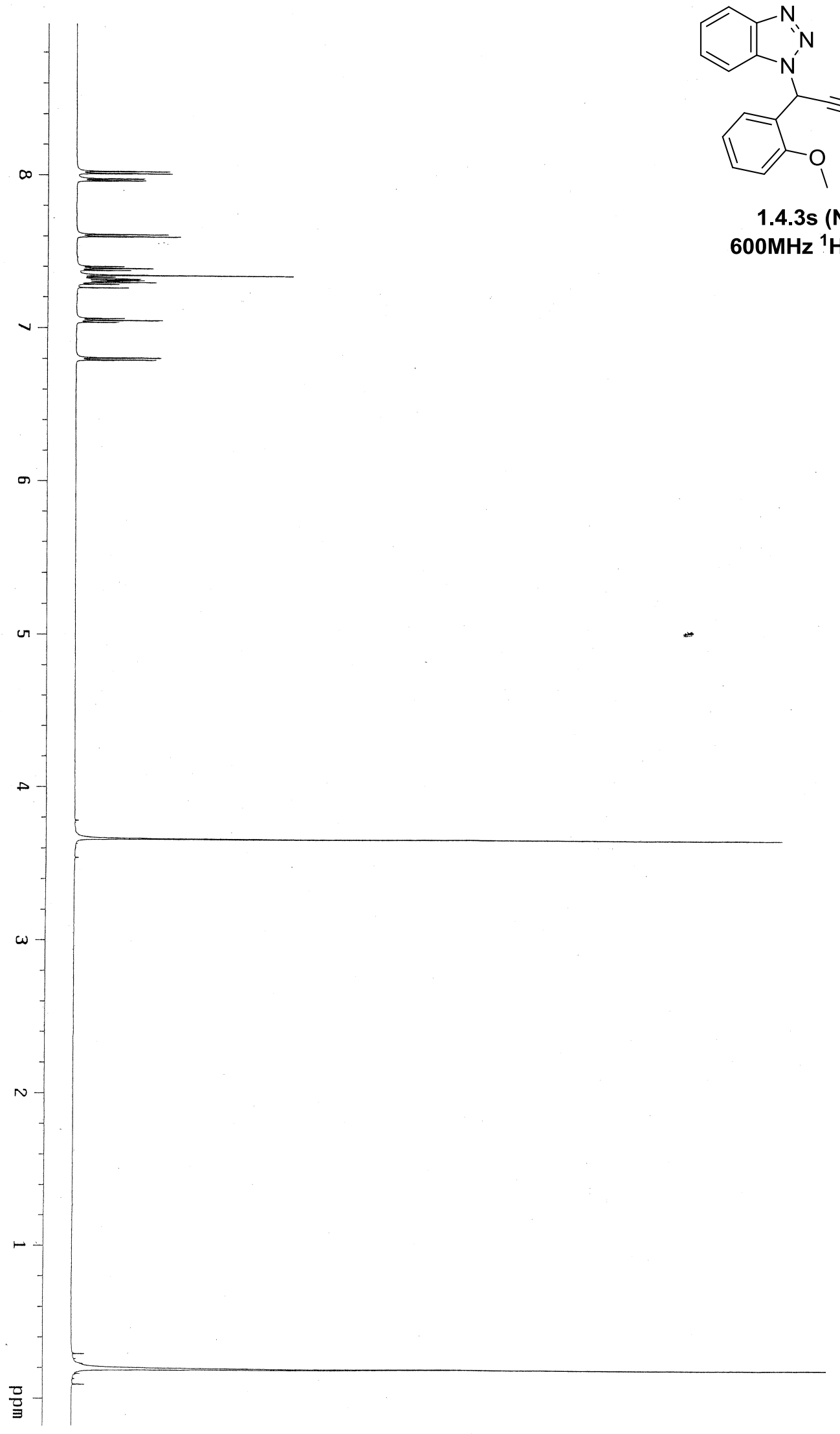




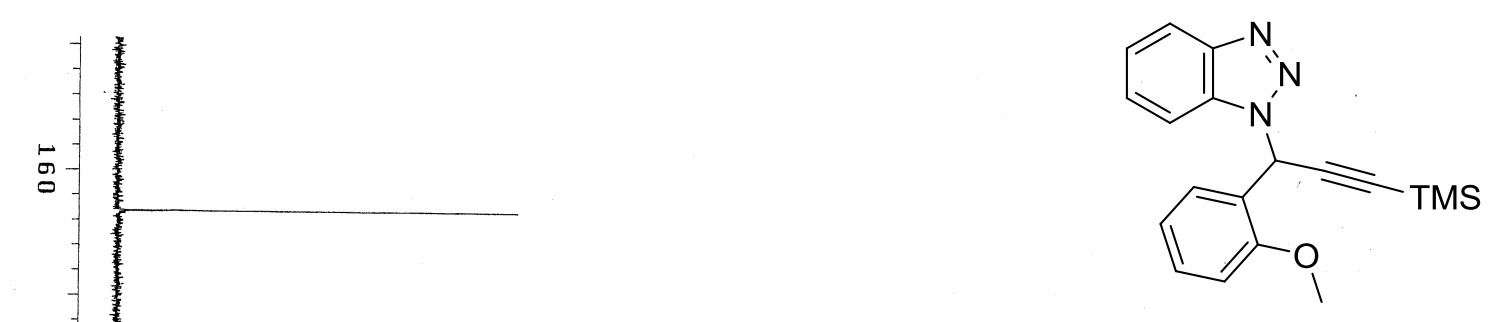

1.4.3s (N-1)

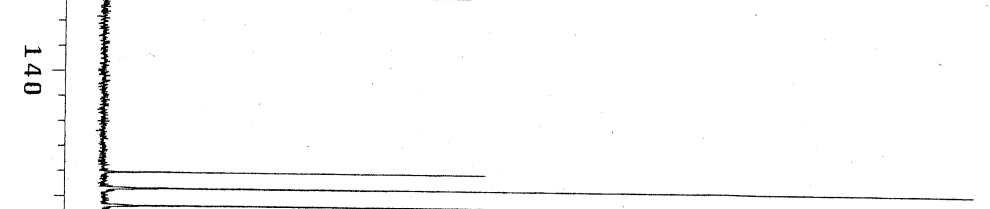
$150 \mathrm{MHz}{ }^{13} \mathrm{C}$ NMR

$\stackrel{\infty}{\circ}$

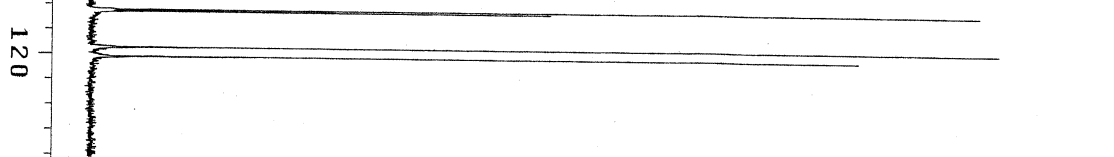

뭉

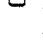

.

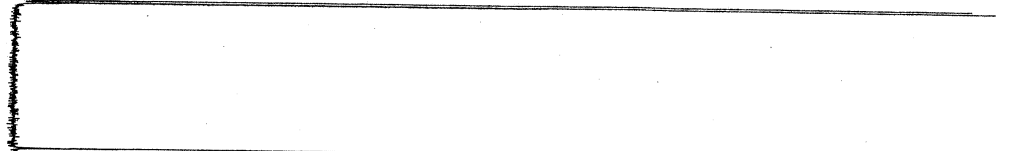

D

$-$

-

.

․-

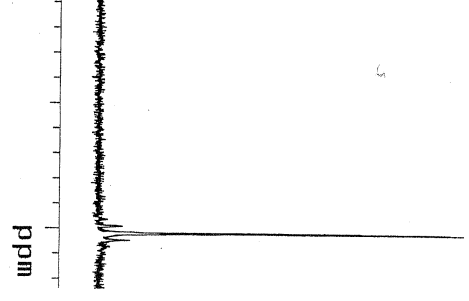



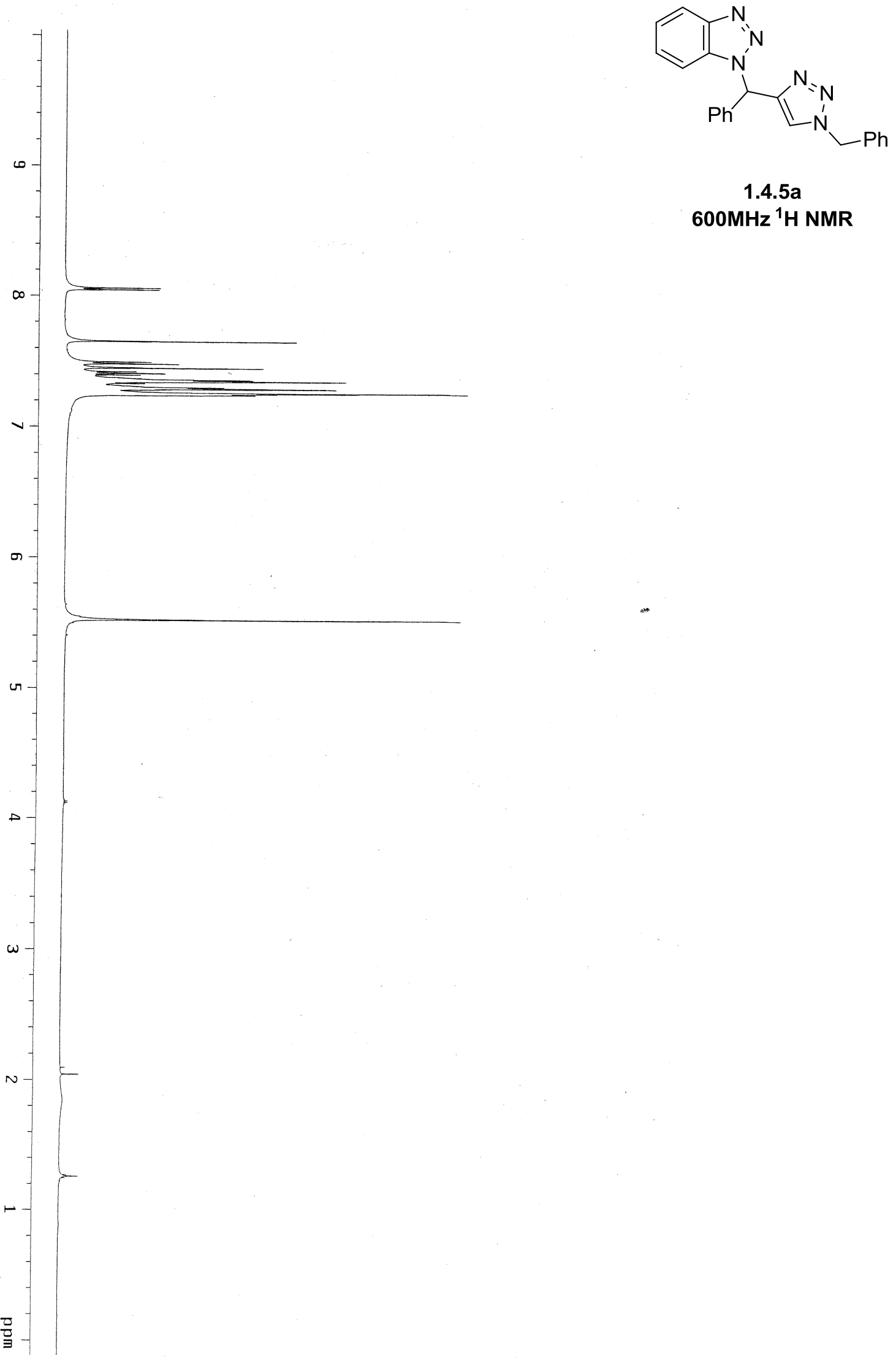

1.4.5a

$600 \mathrm{MHz}{ }^{1} \mathrm{H}$ NMR 


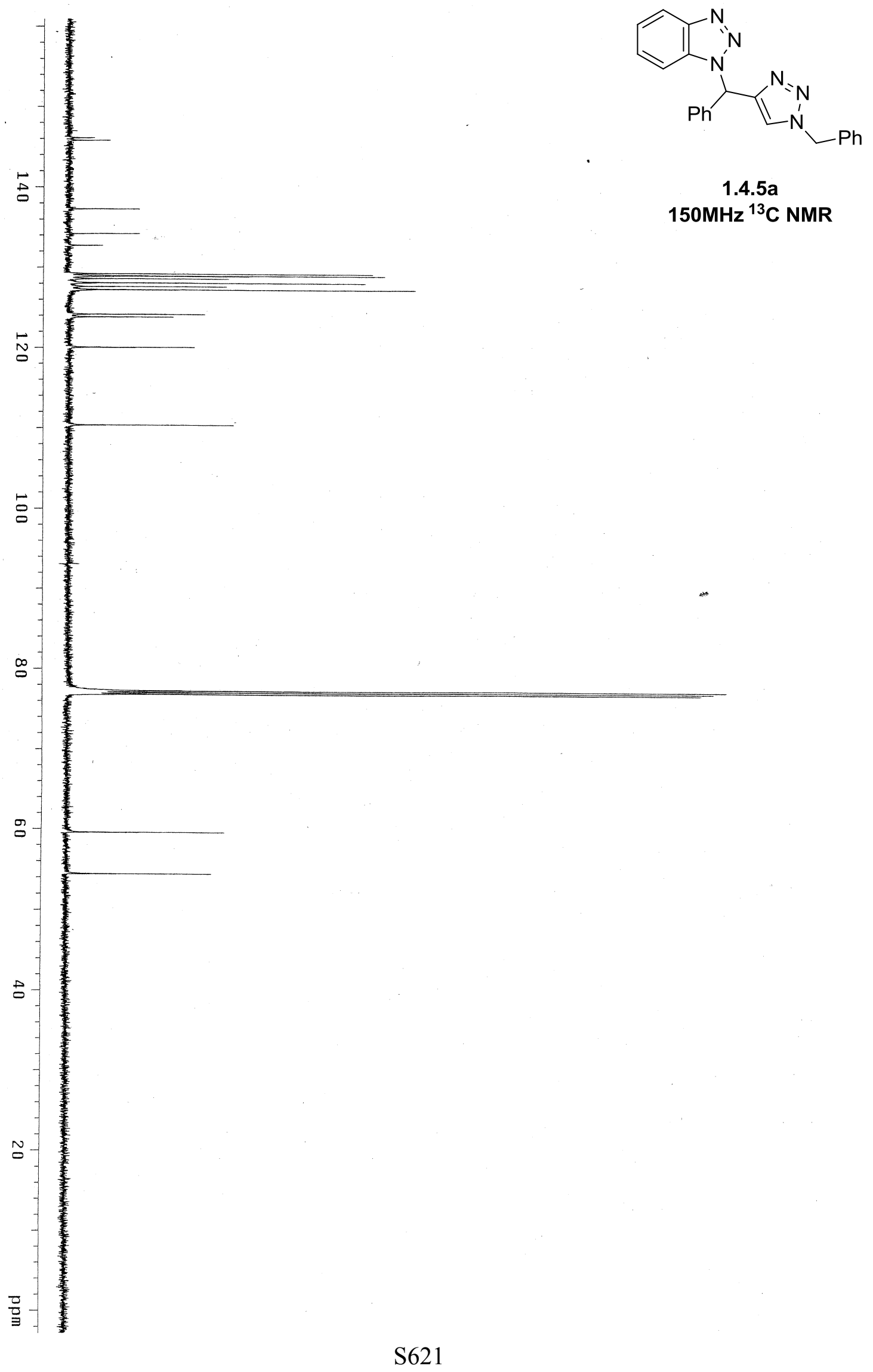




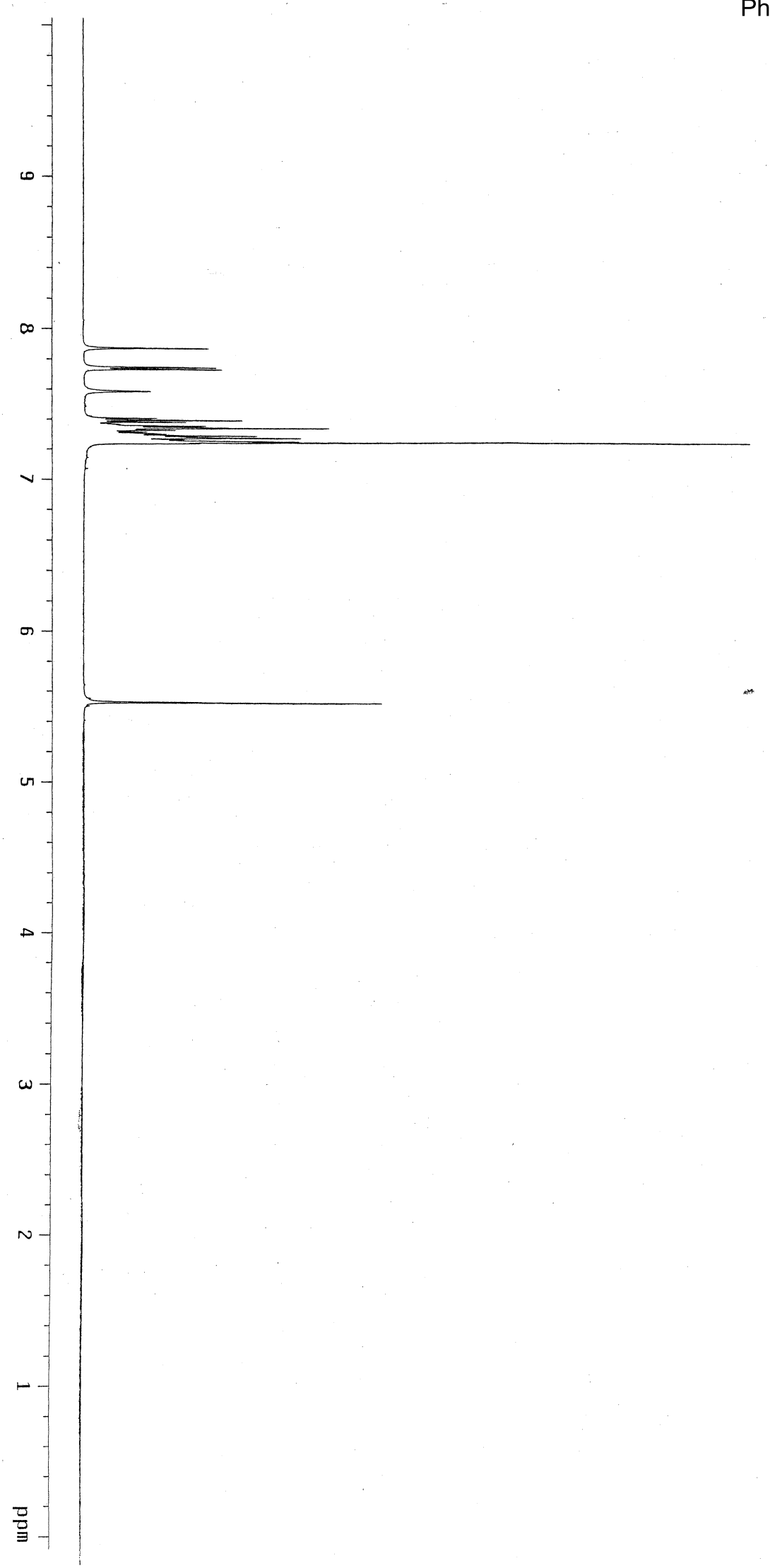

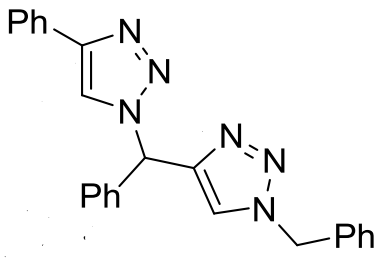

1.4.5b $600 \mathrm{MHz}{ }^{1} \mathrm{H}$ NMR 


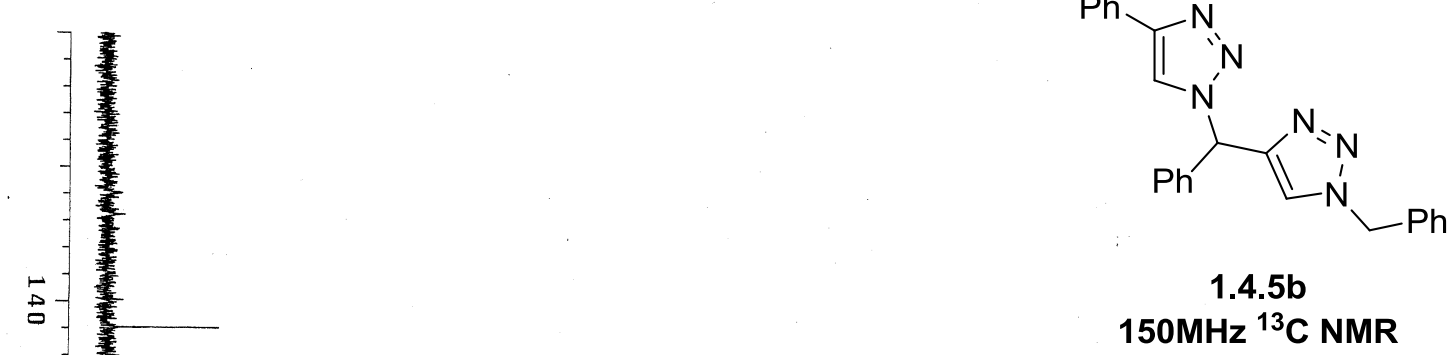

$\stackrel{\infty}{\circ}$

品.

-

N.

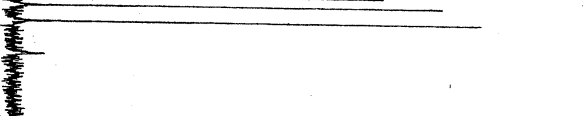

D-

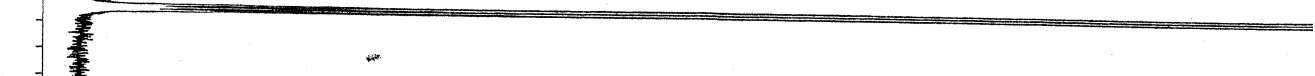

․-

a.

.

$-$

-

믈- 


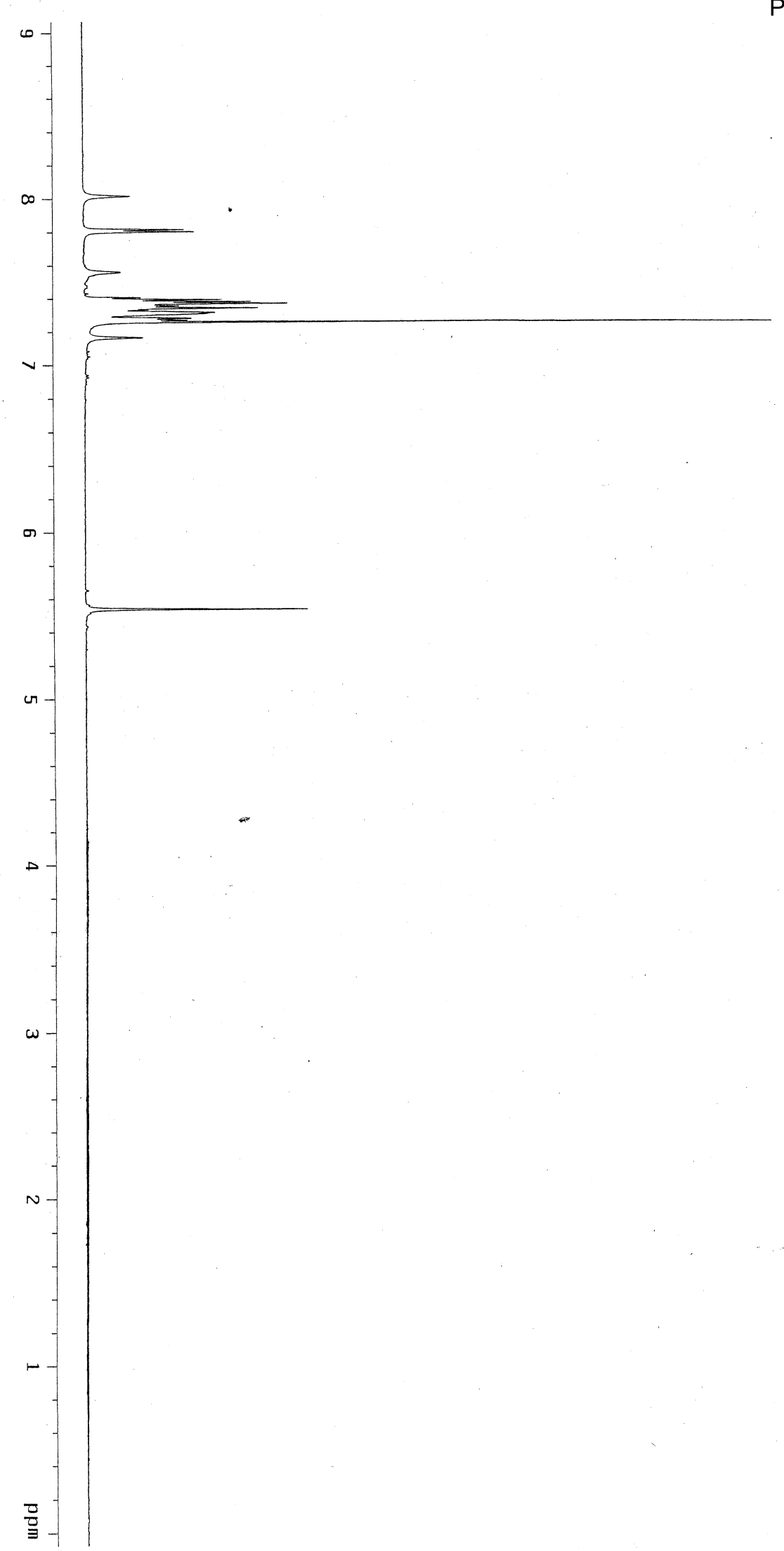

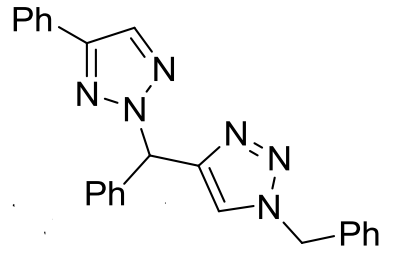

1.4.5c $600 \mathrm{MHz}{ }^{1} \mathrm{H}$ NMR 


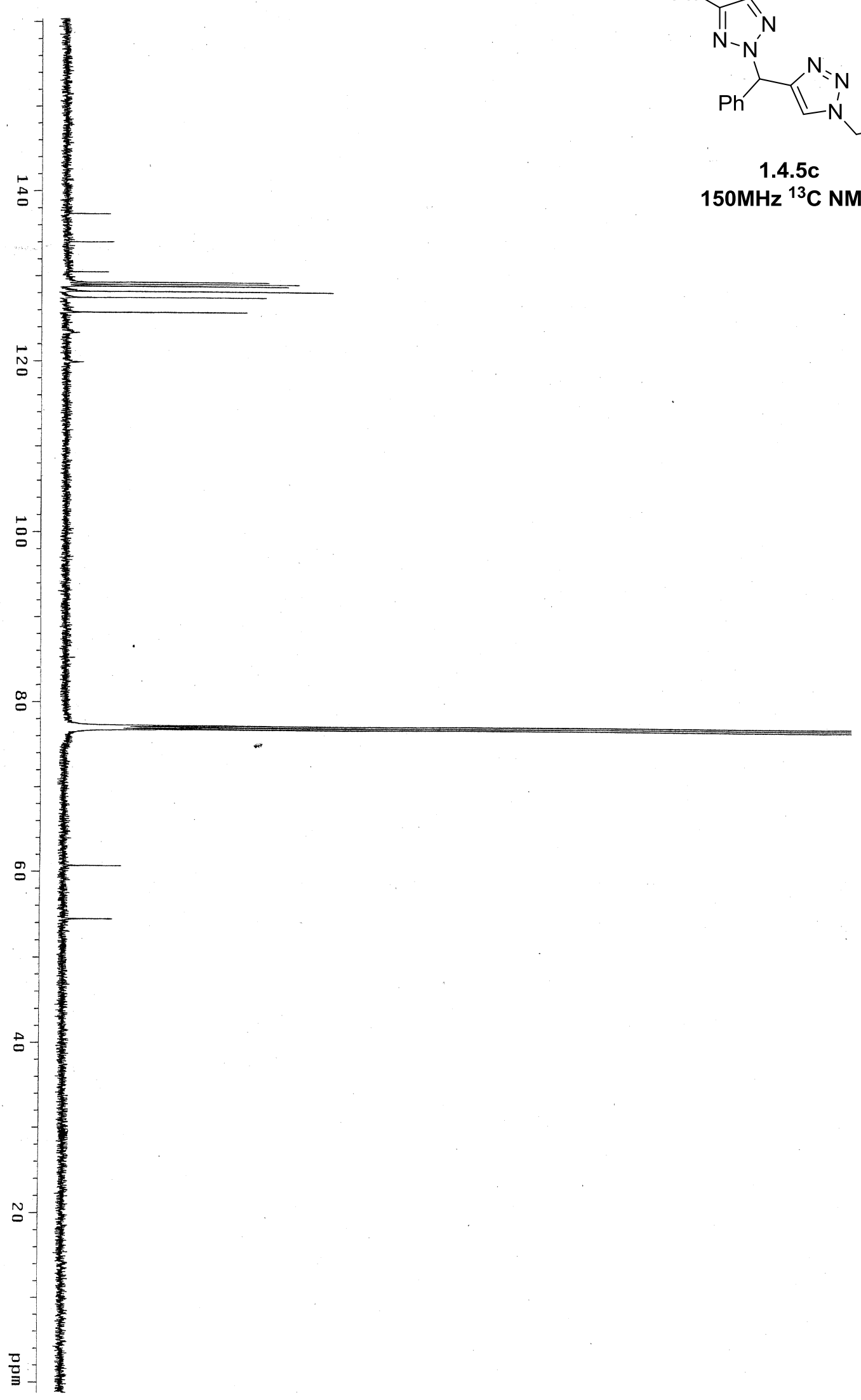




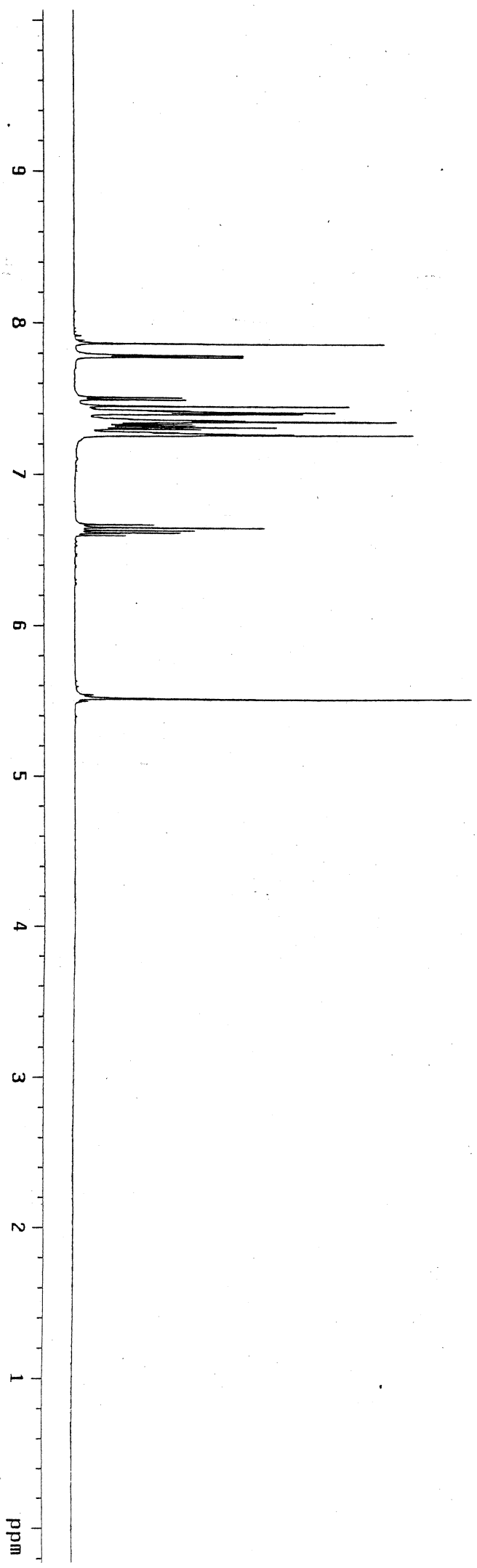

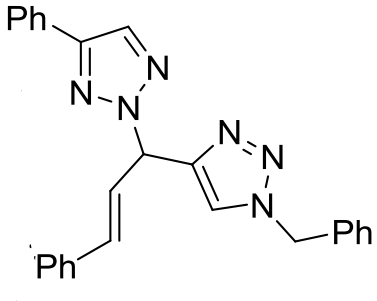

1.4.5d $600 \mathrm{MHz}{ }^{1} \mathrm{H}$ NMR 


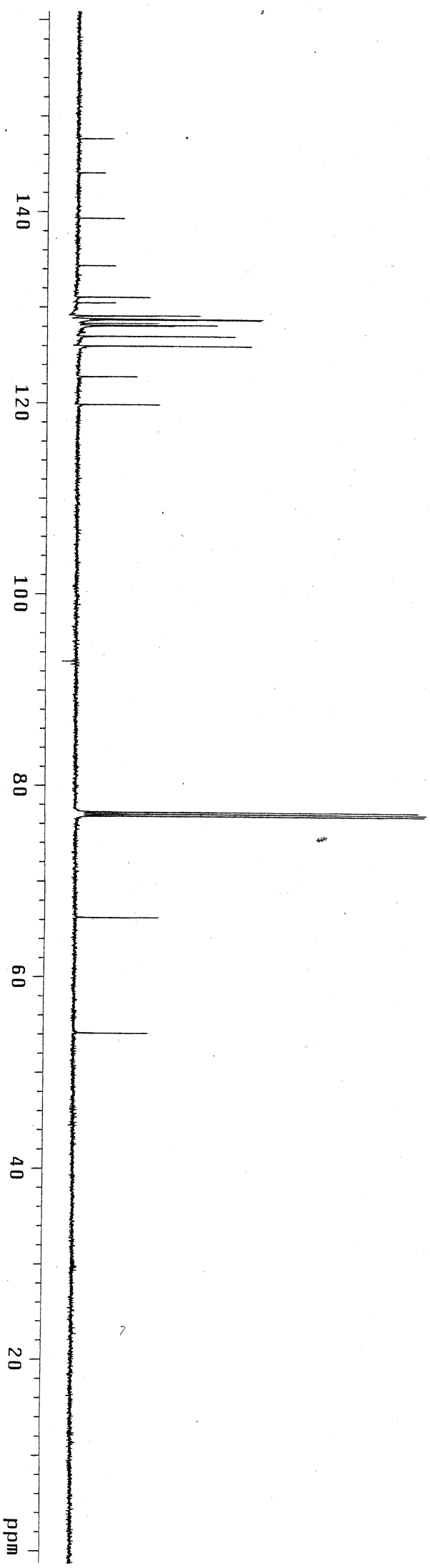

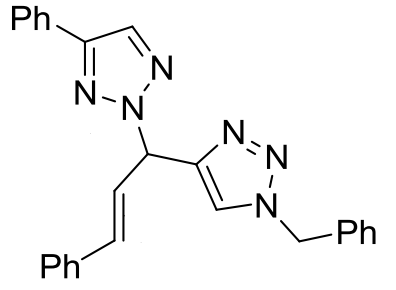

1.4.5d $150 \mathrm{MHz}{ }^{13} \mathrm{C}$ NMR 


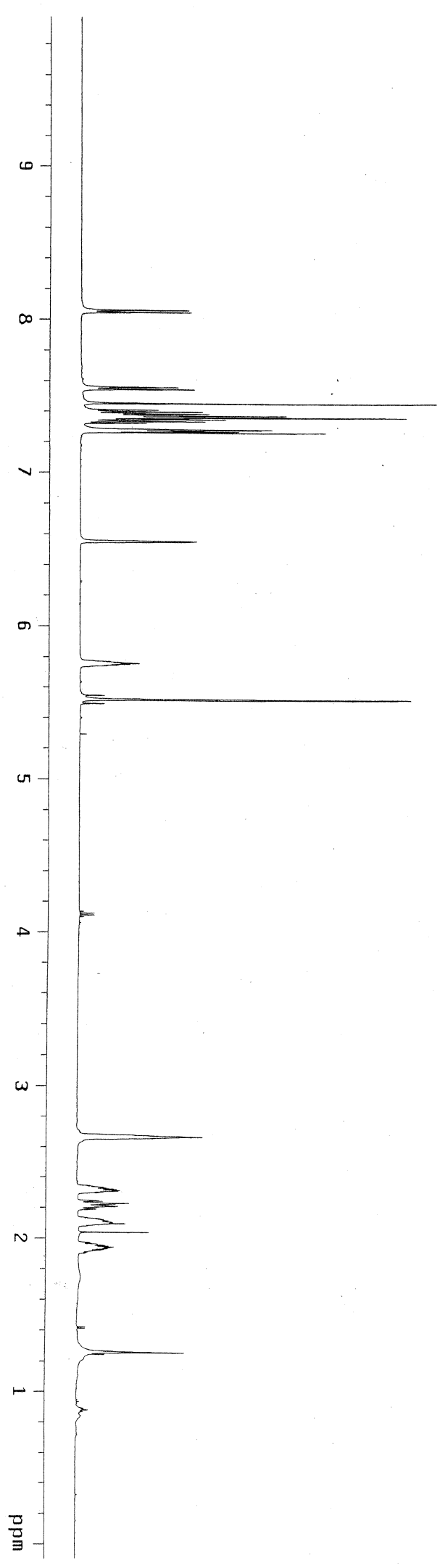

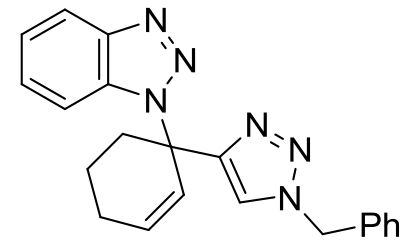

1.4.5e

$600 \mathrm{MHz}^{1} \mathrm{H}$ NMR 

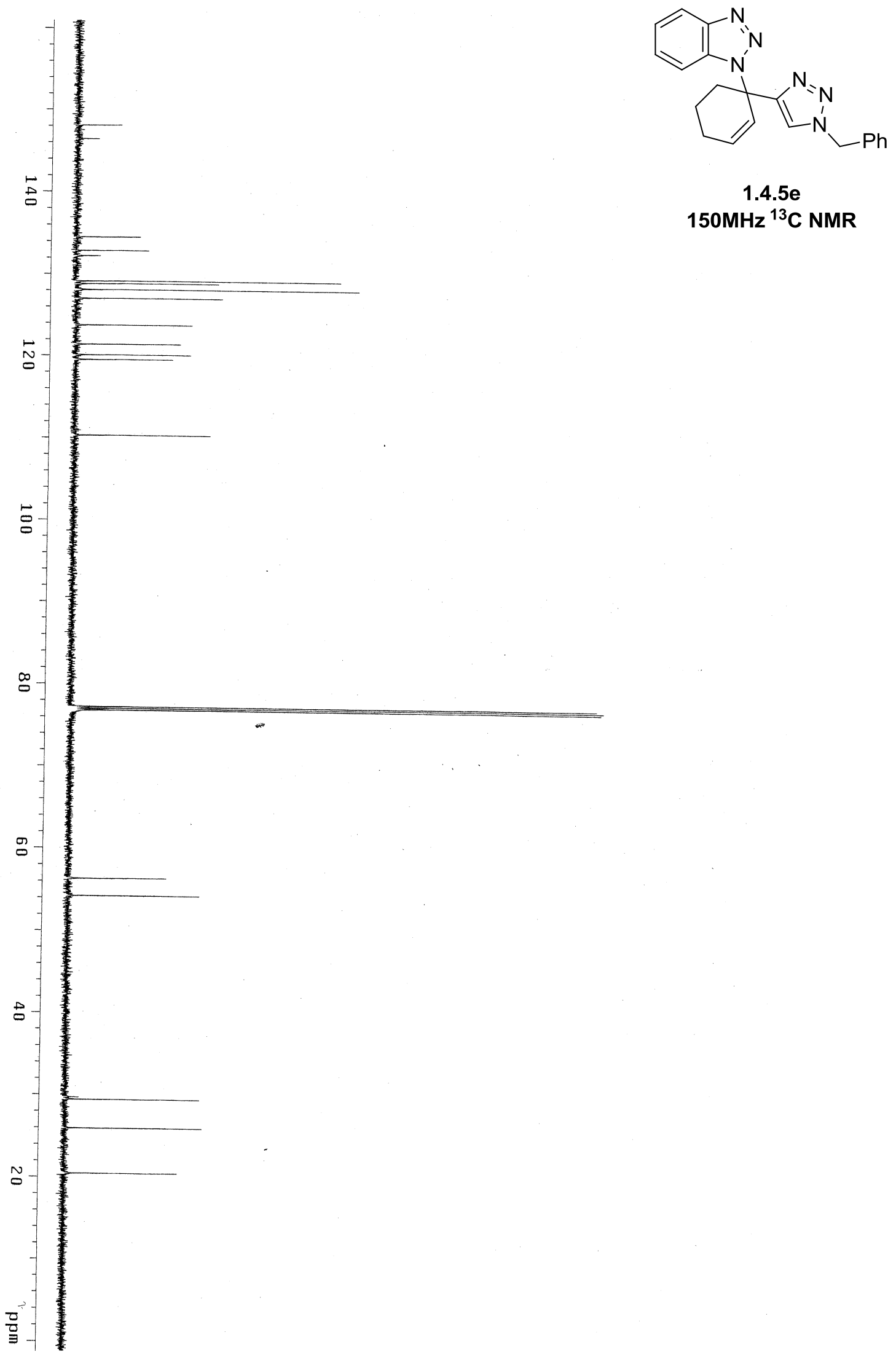

1.4.5e $150 \mathrm{MHz}{ }^{13} \mathrm{C}$ NMR 

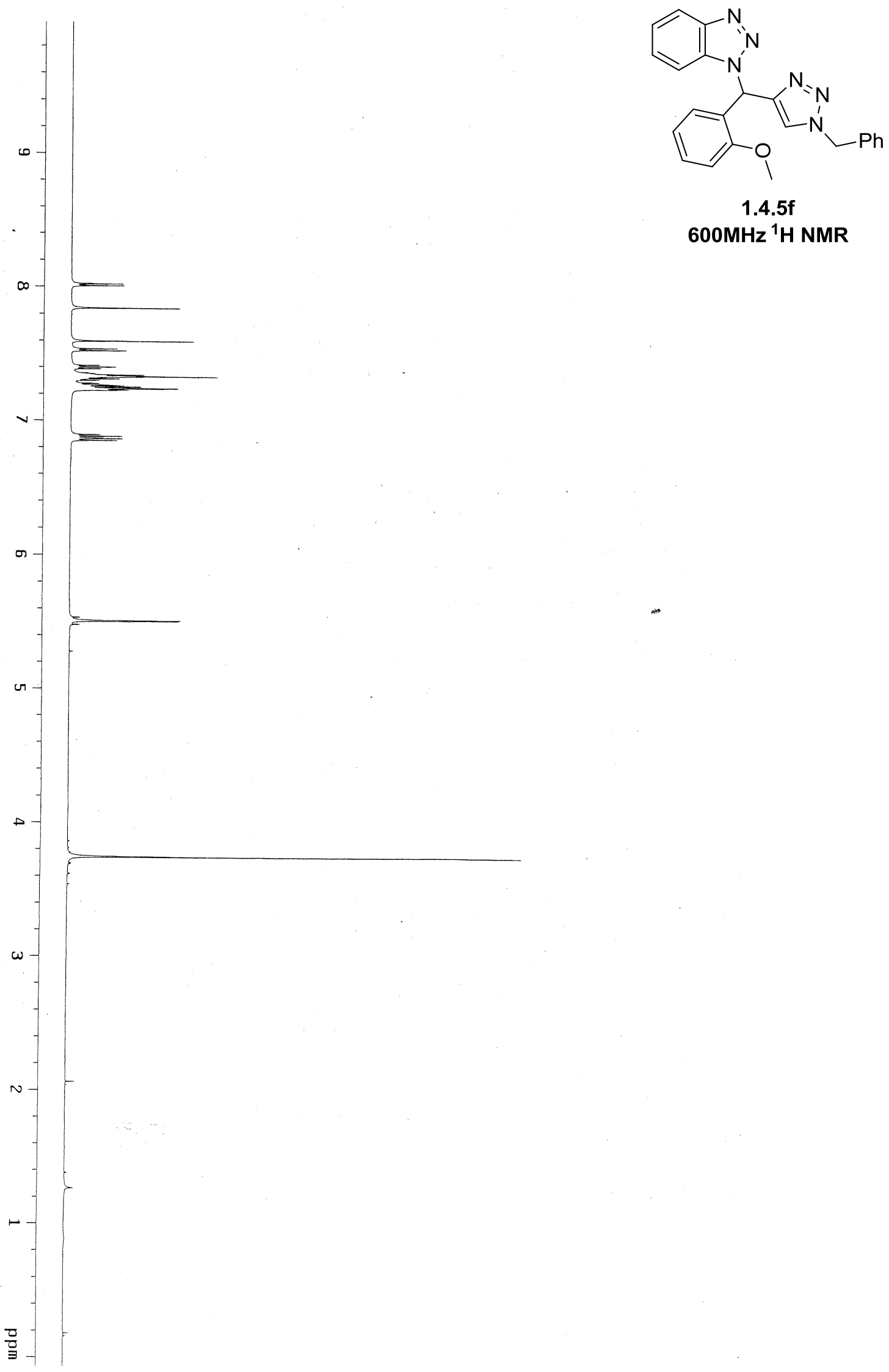

1.4.5f $600 \mathrm{MHz}{ }^{1} \mathrm{H}$ NMR 

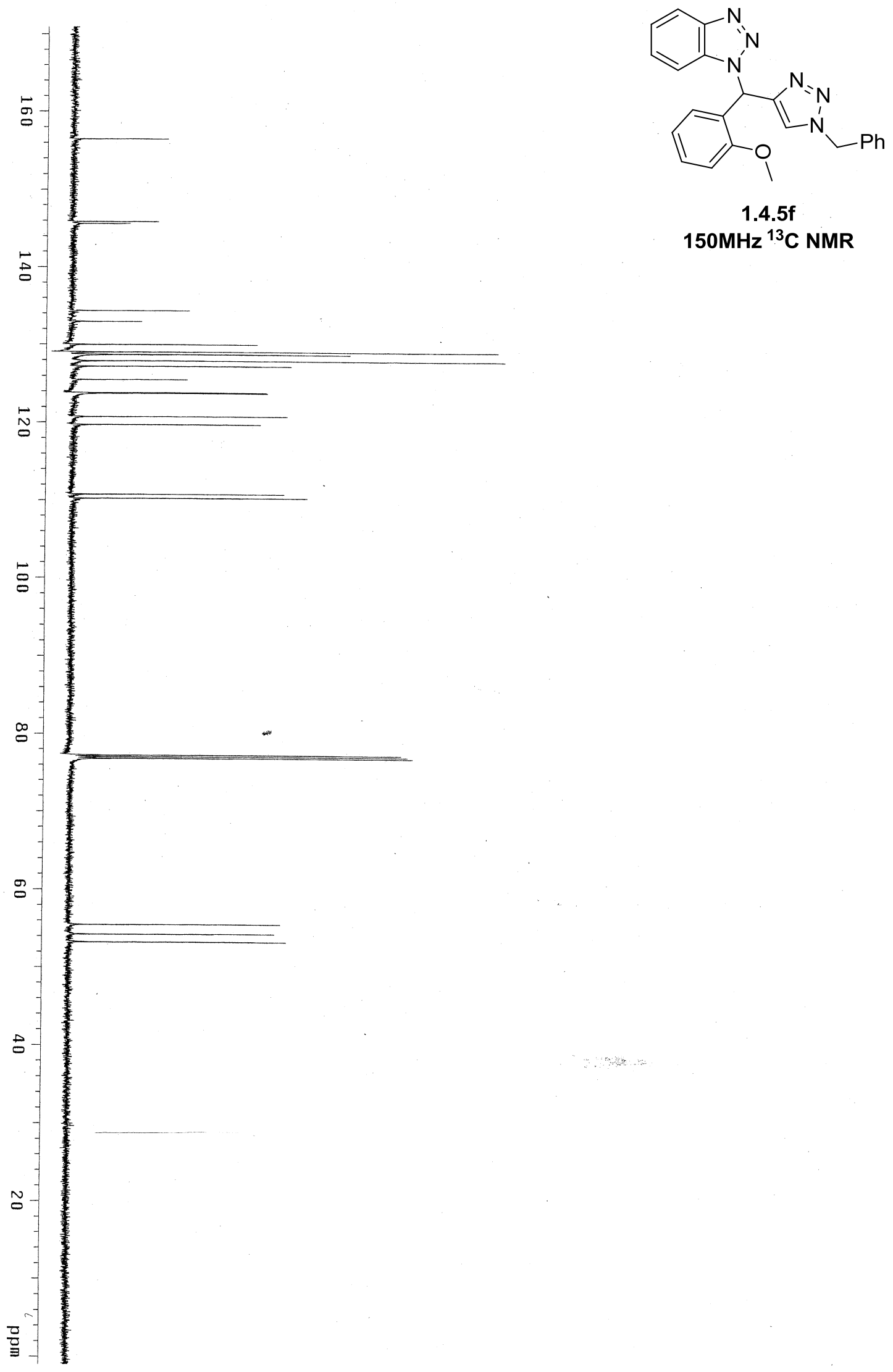

S631 

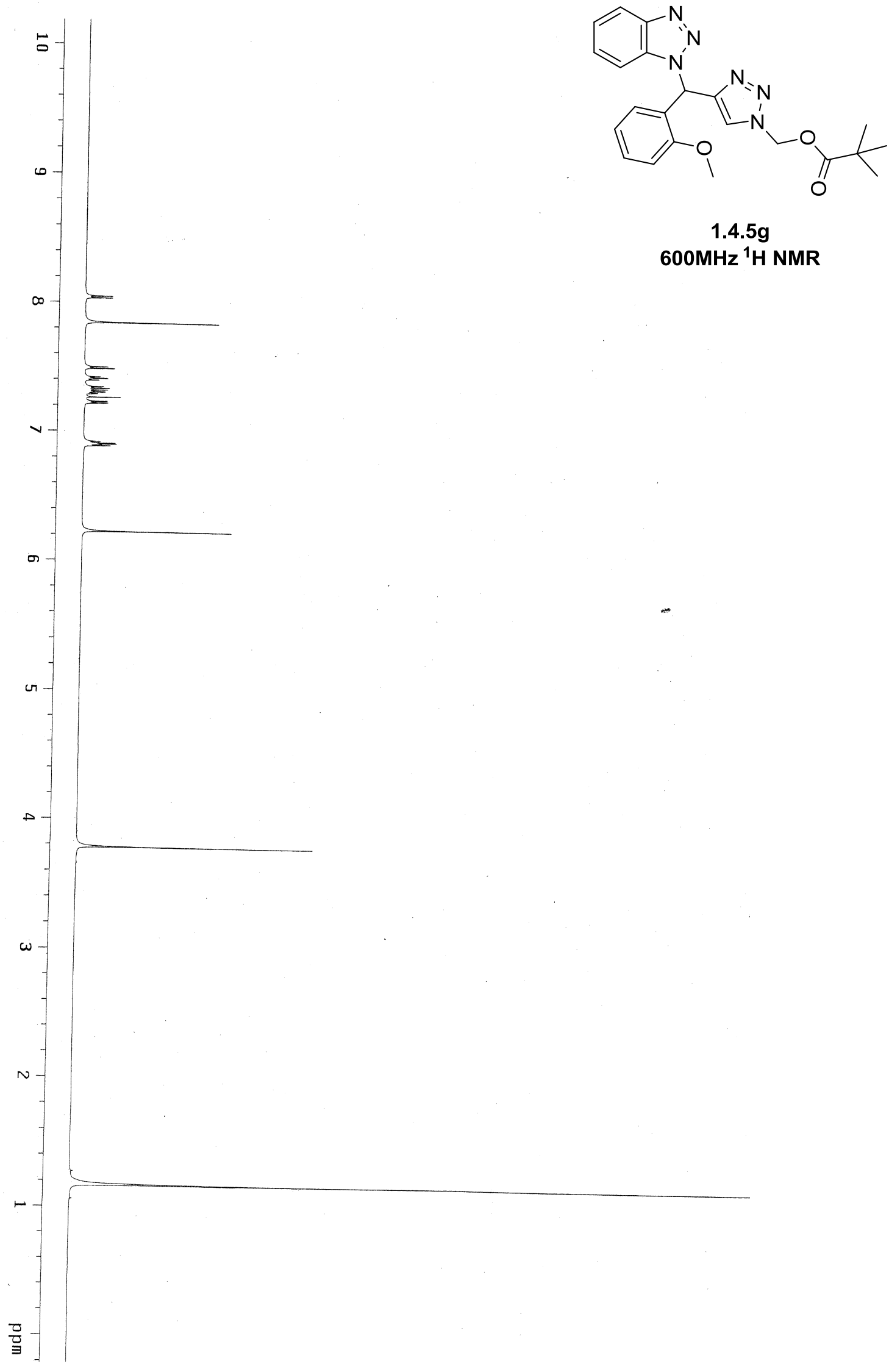

$1.4 .5 \mathrm{~g}$

$600 \mathrm{MHz}{ }^{1} \mathrm{H}$ NMR 


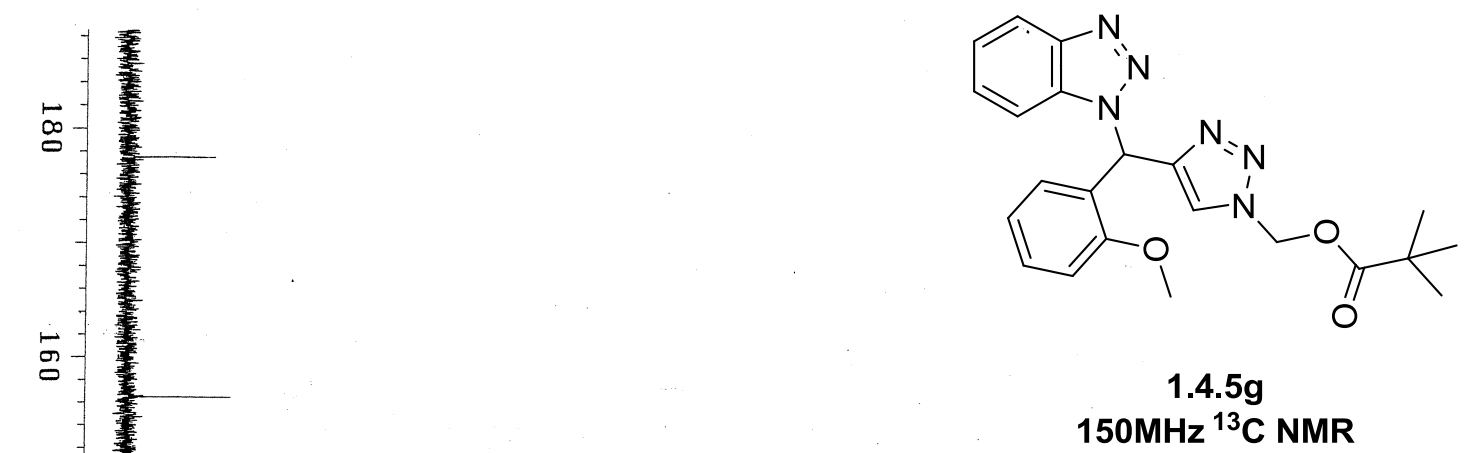

$150 \mathrm{MHz}{ }^{13} \mathrm{C}$ NMR 


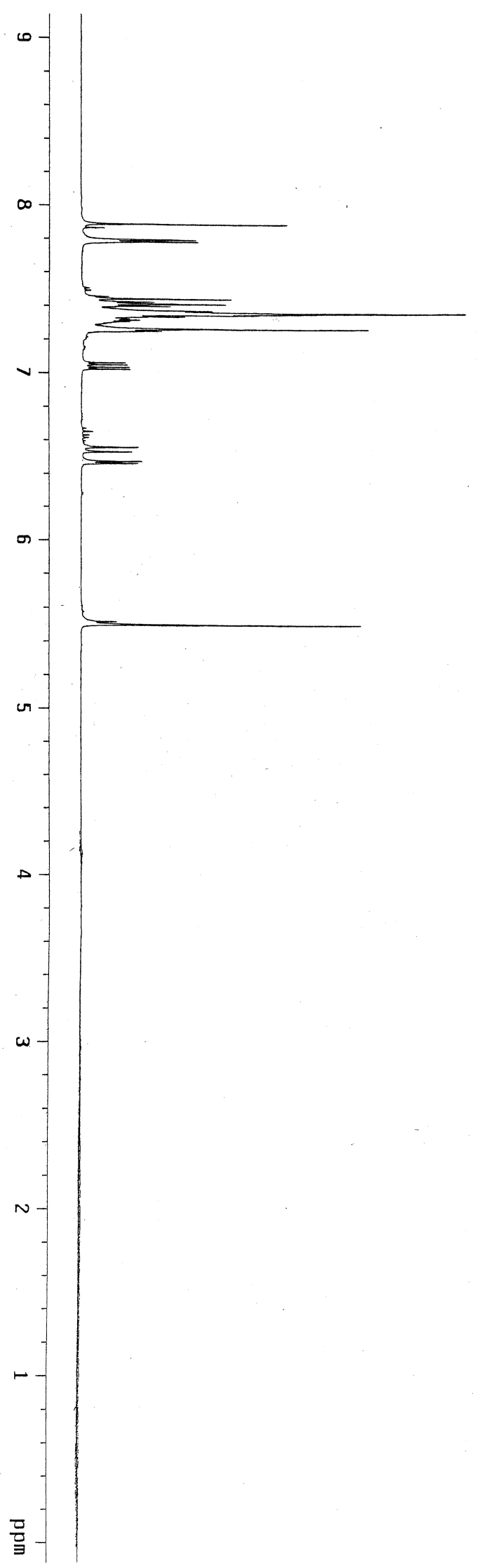

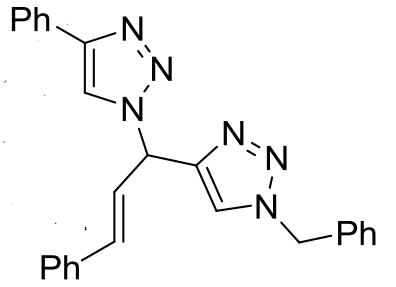

1.4.5h $600 \mathrm{MHz}{ }^{1} \mathrm{H}$ NMR 

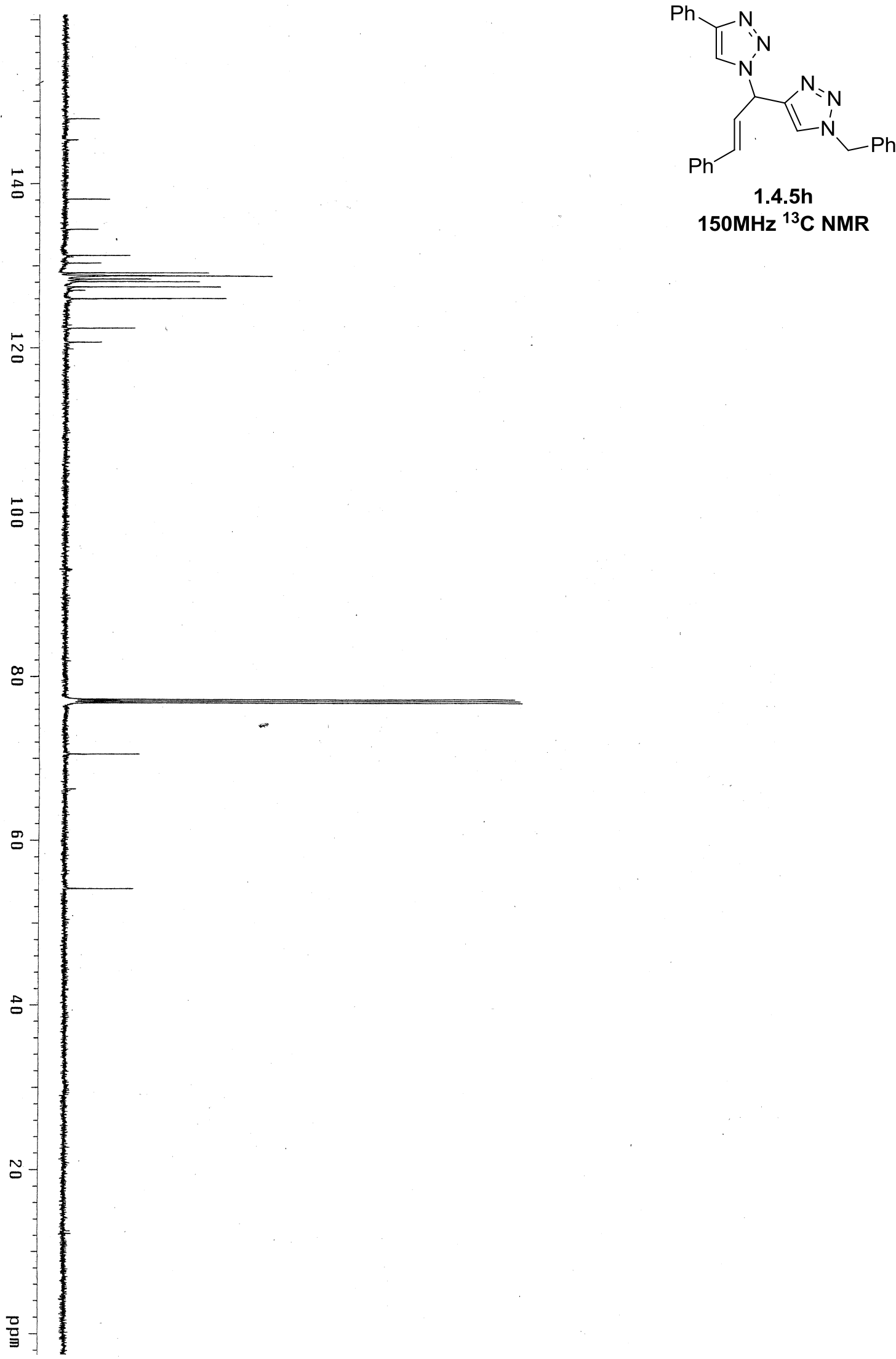


\subsection{NMR Spectra Data}

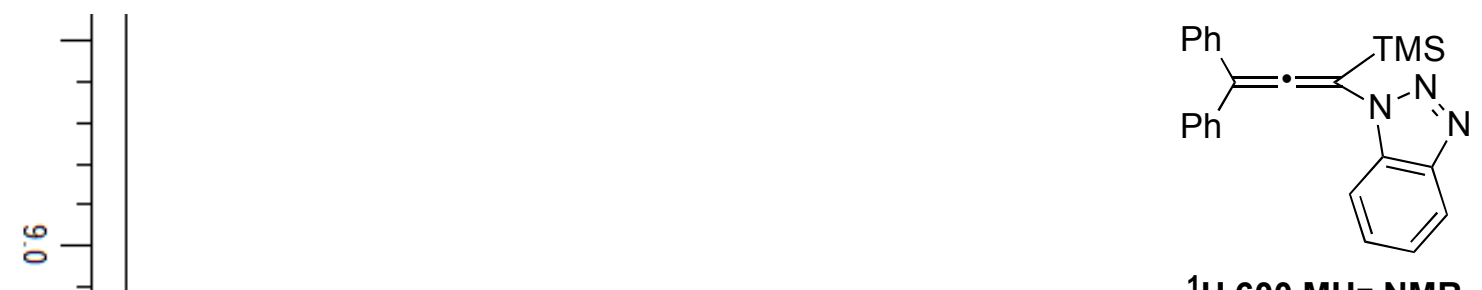

${ }^{1} \mathrm{H} 600 \mathrm{MHz}$ NMR

$1.5 .4 a$

(E)

궁

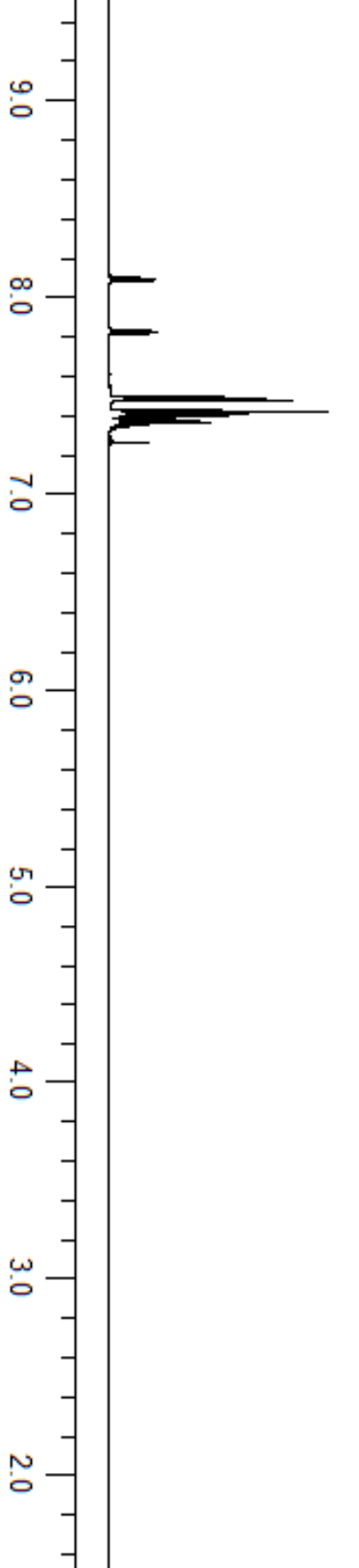

年

年

(E)

f-

$\overrightarrow{0}$

$\circ-1$ 


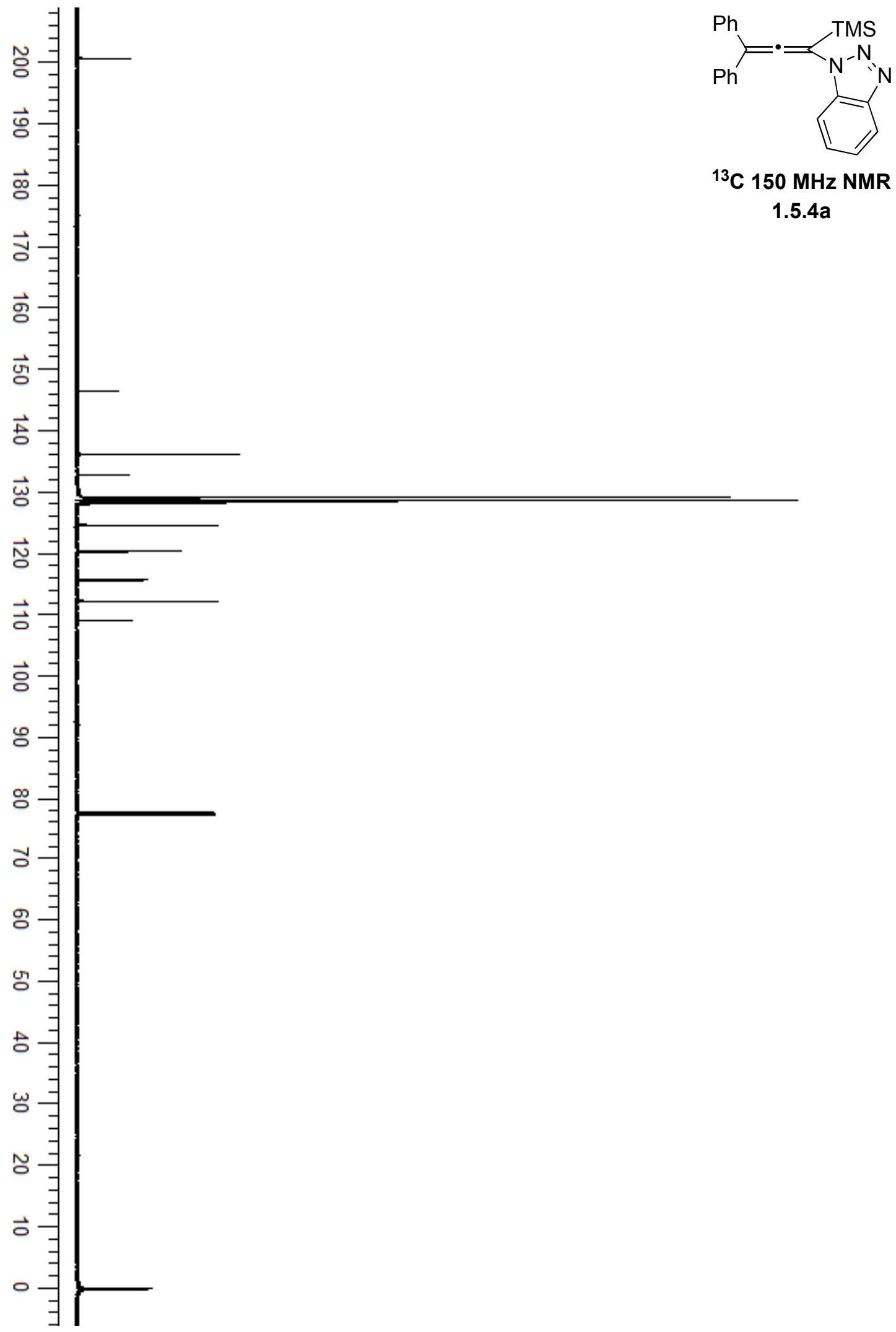



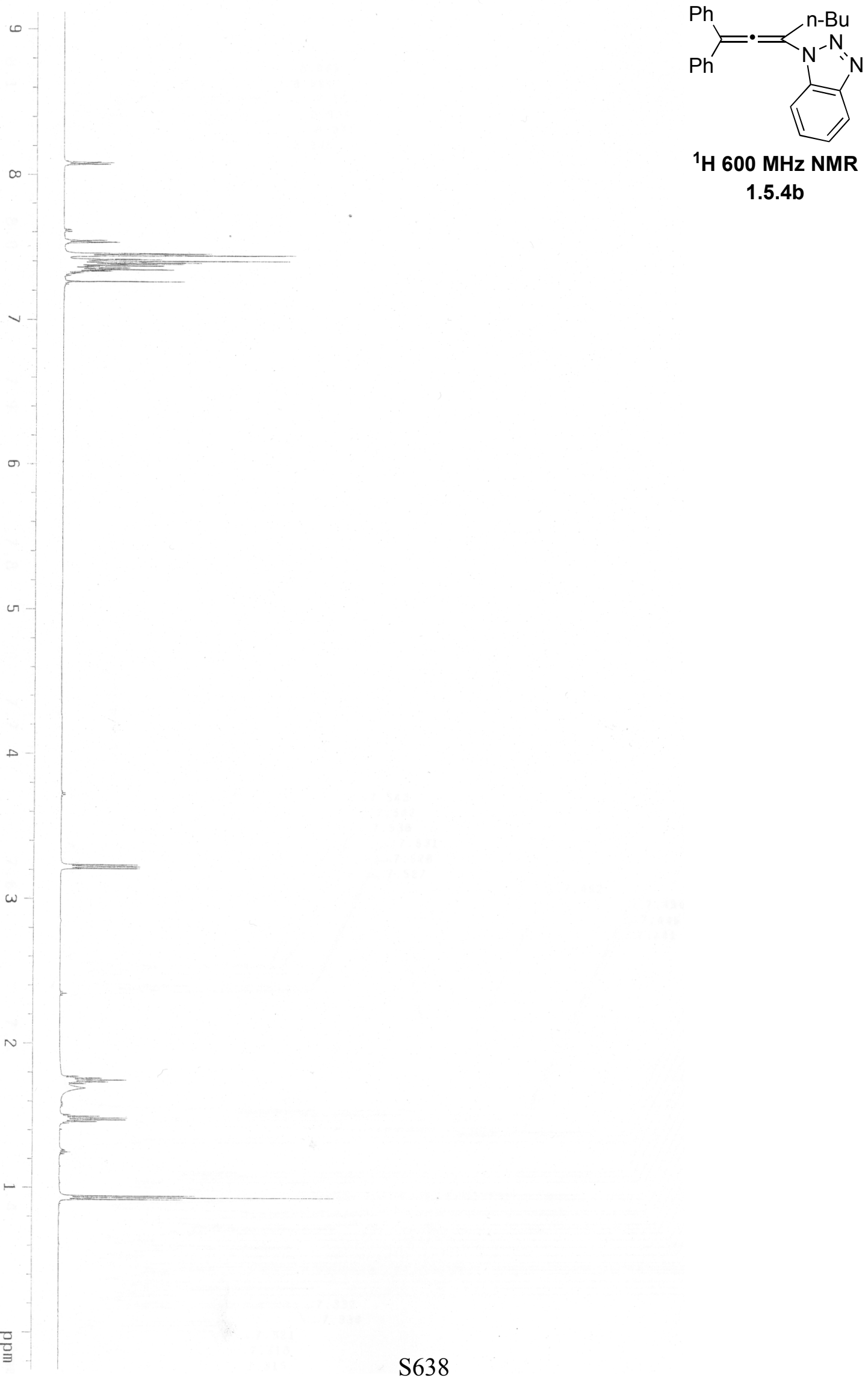

${ }^{1} \mathrm{H} 600 \mathrm{MHz}$ NMR $1.5 .4 \mathrm{~b}$ 


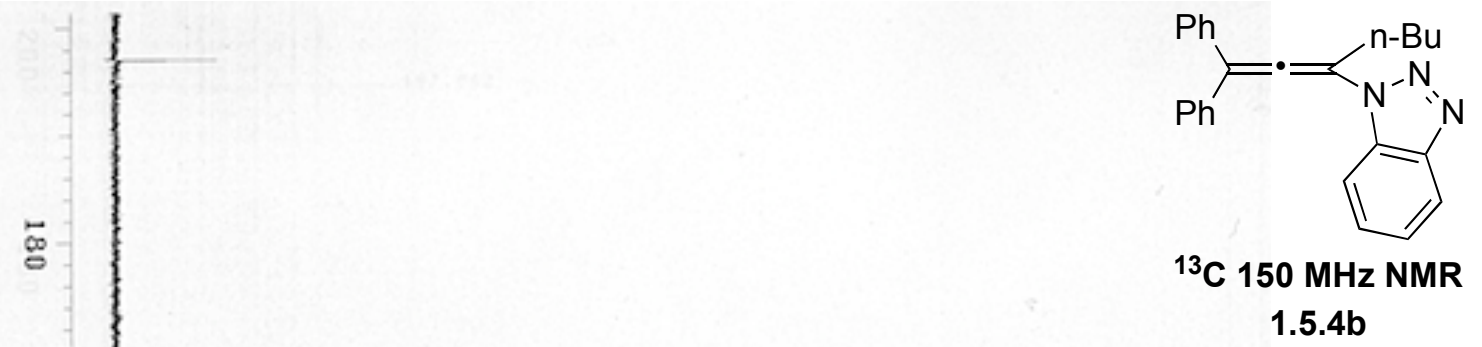

1.5.4b

뭉

宫

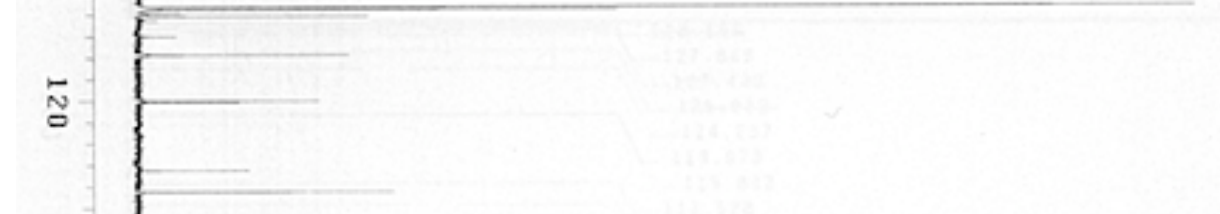

음

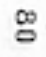

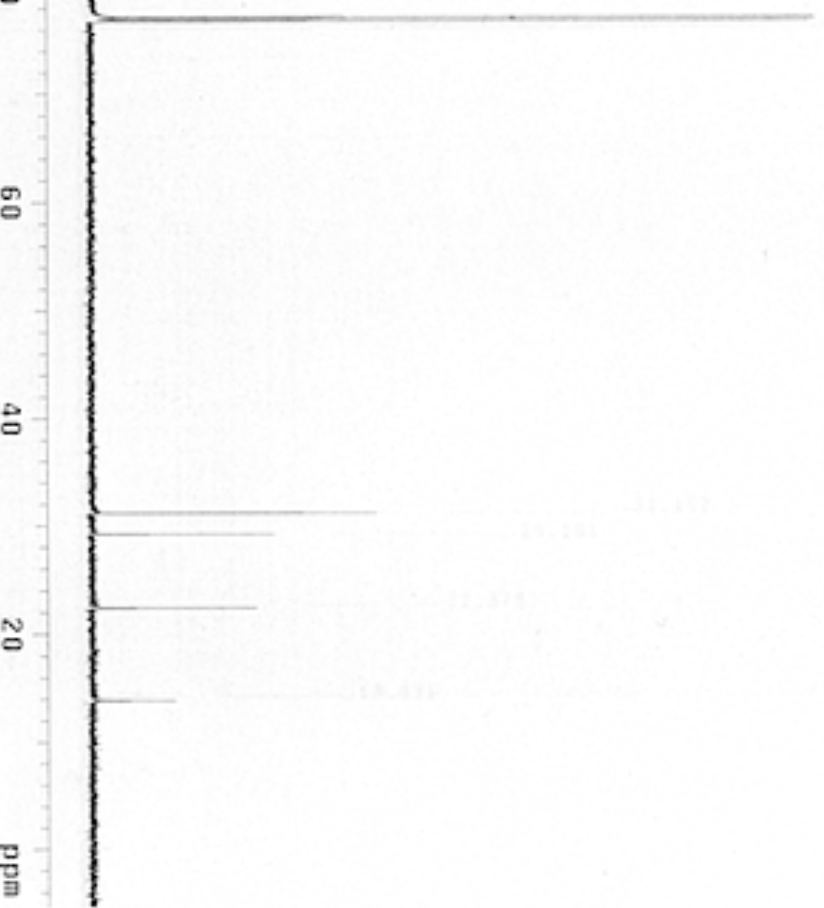




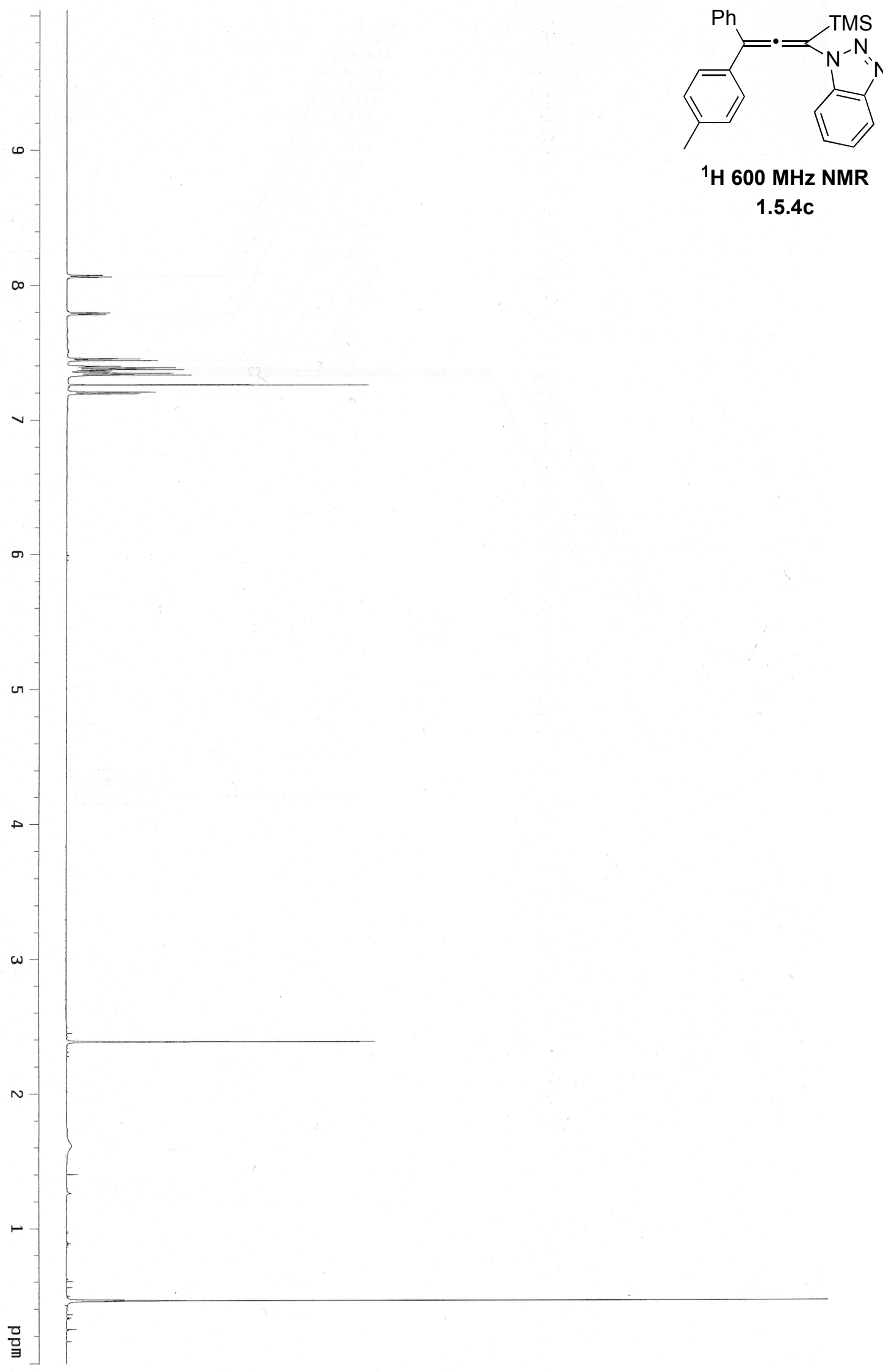




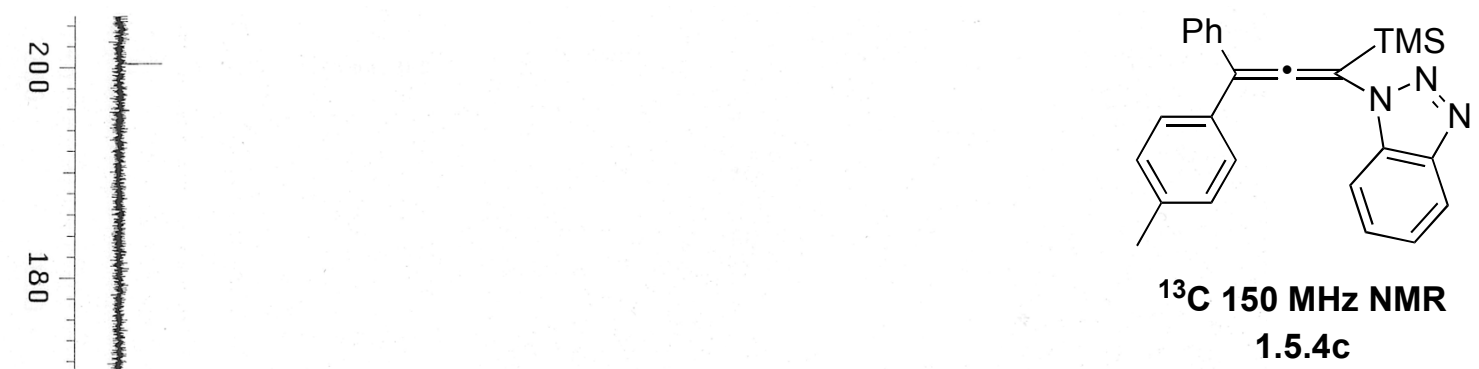

$\infty$

욤

뭉

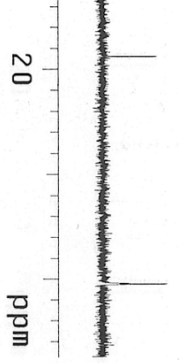



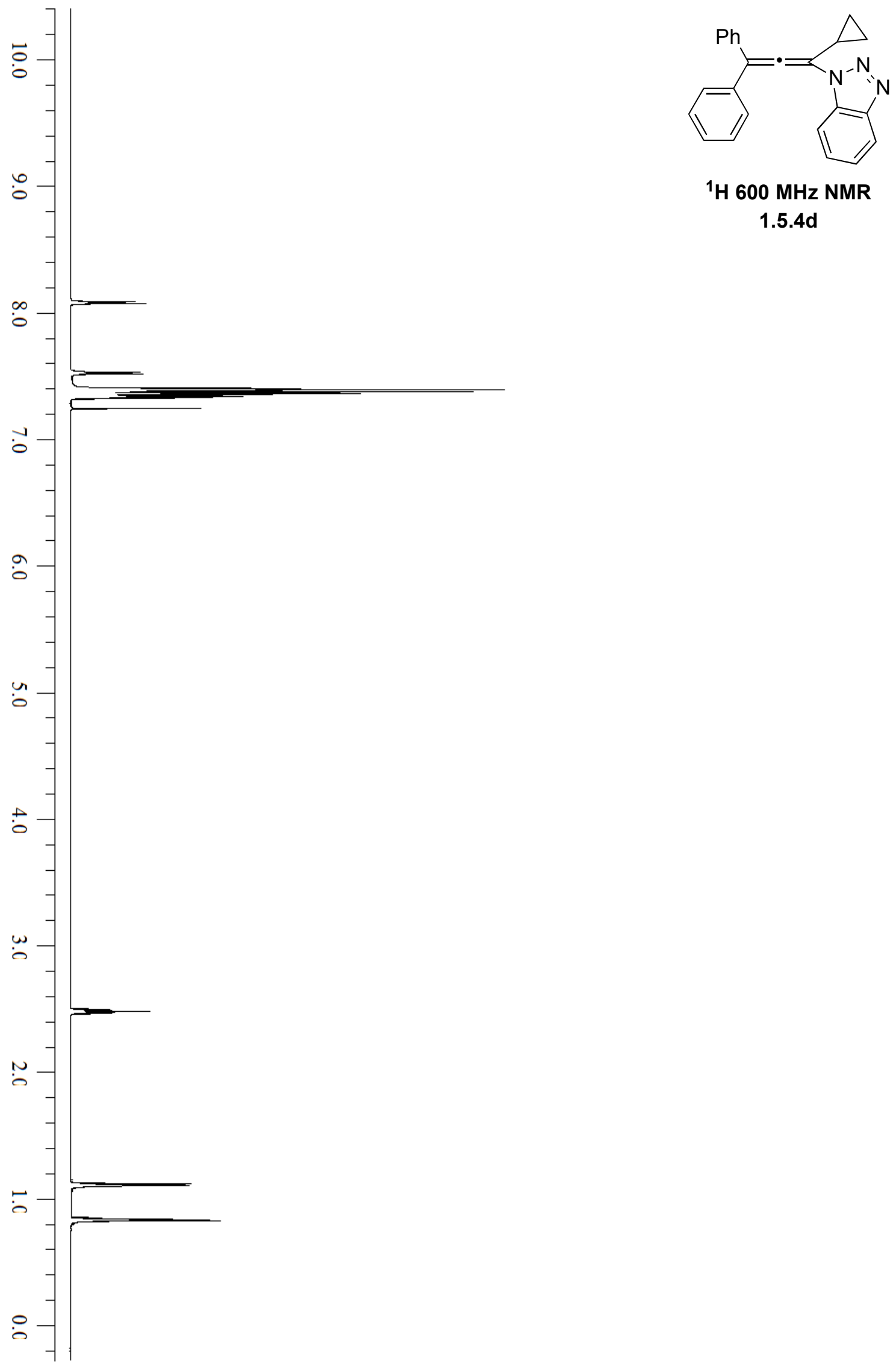

${ }^{1} \mathrm{H} 600 \mathrm{MHz}$ NMR

1.5.4d 


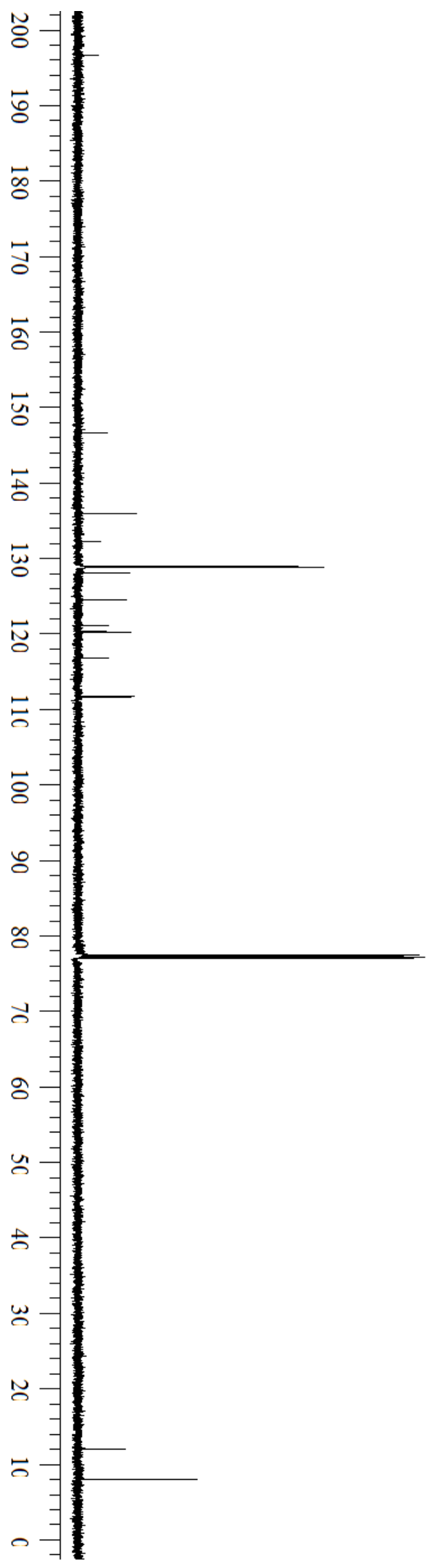

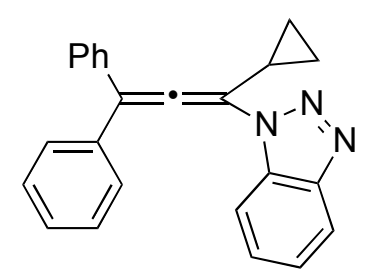

${ }^{13} \mathrm{C} 150 \mathrm{MHz}$ NMR

$1.5 .4 d$ 

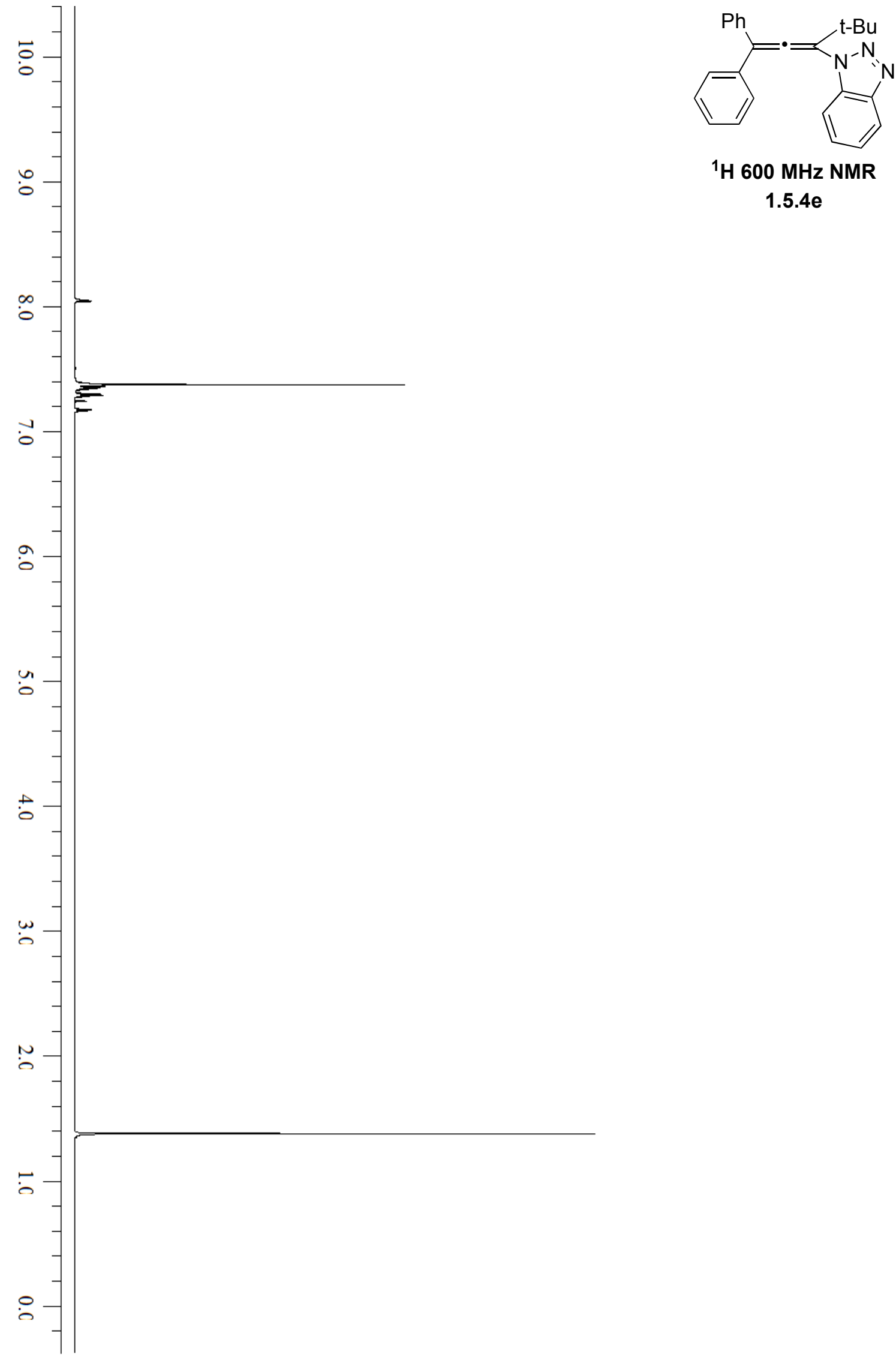

${ }^{1} \mathrm{H} 600 \mathrm{MHz}$ NMR

$1.5 .4 \mathrm{e}$ 

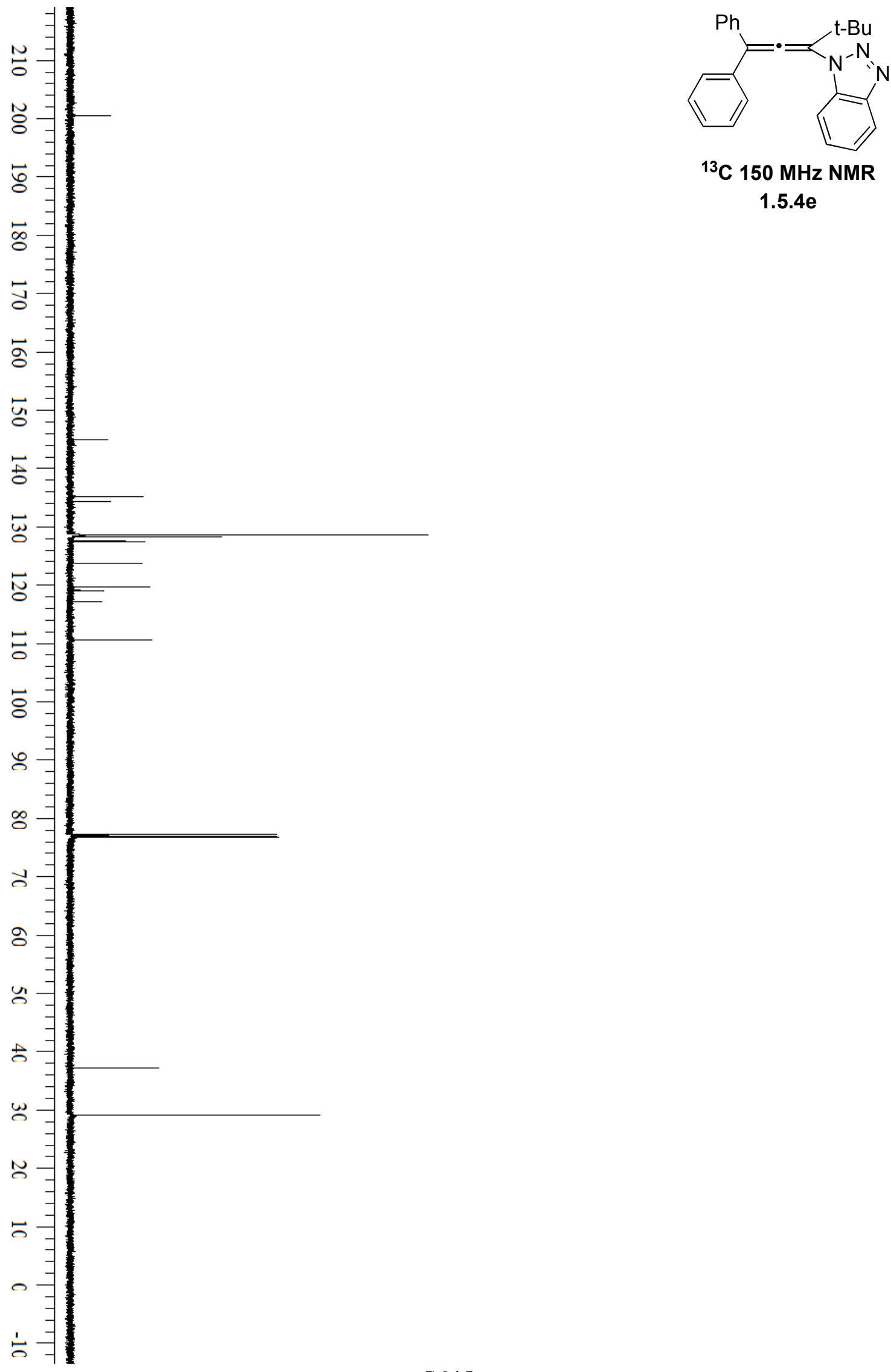

${ }^{13} \mathrm{C} 150 \mathrm{MHz}$ NMR

1.5 .4 e 

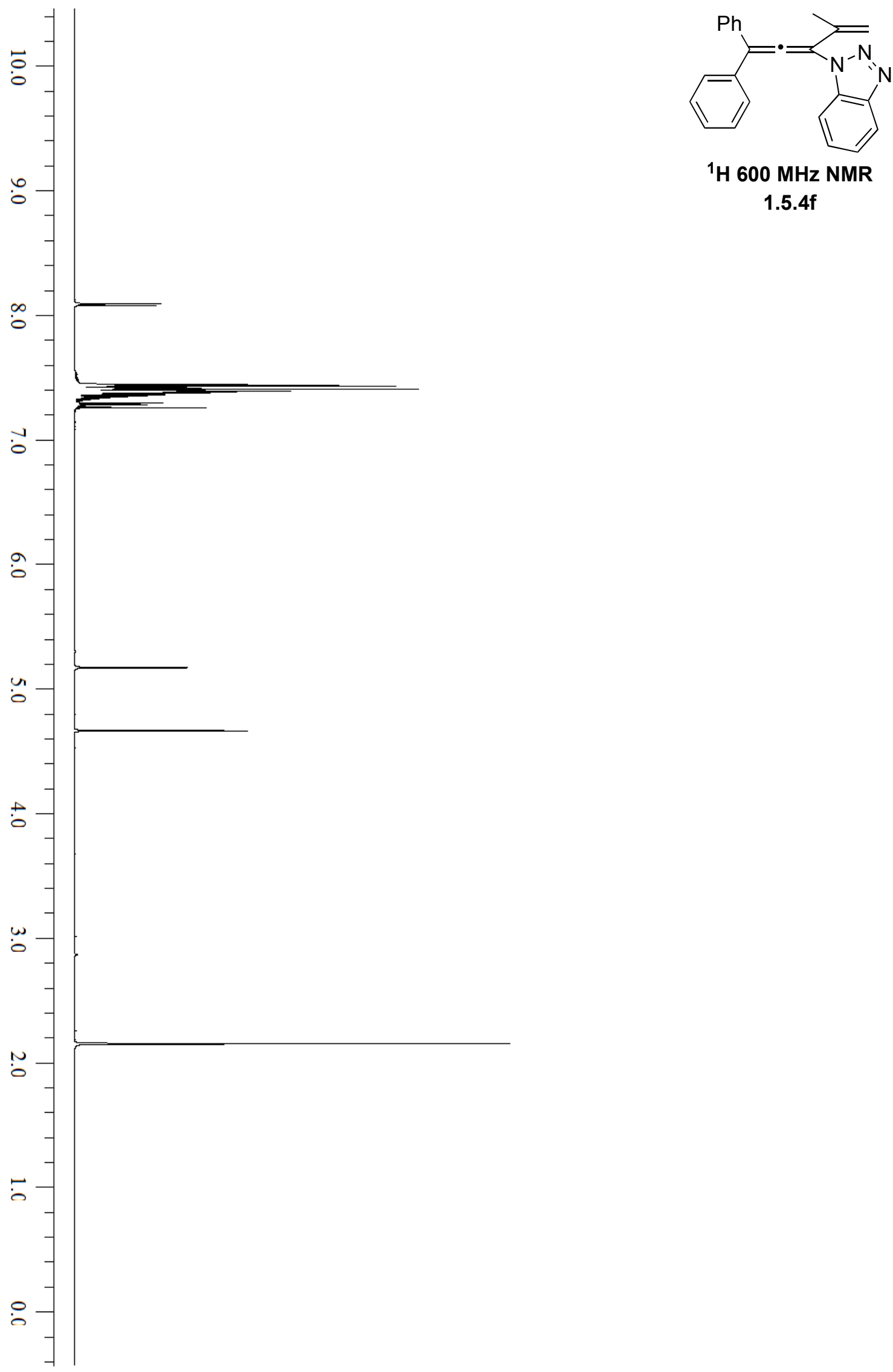

${ }^{1} \mathrm{H} 600 \mathrm{MHz}$ NMR

1.5.4f 

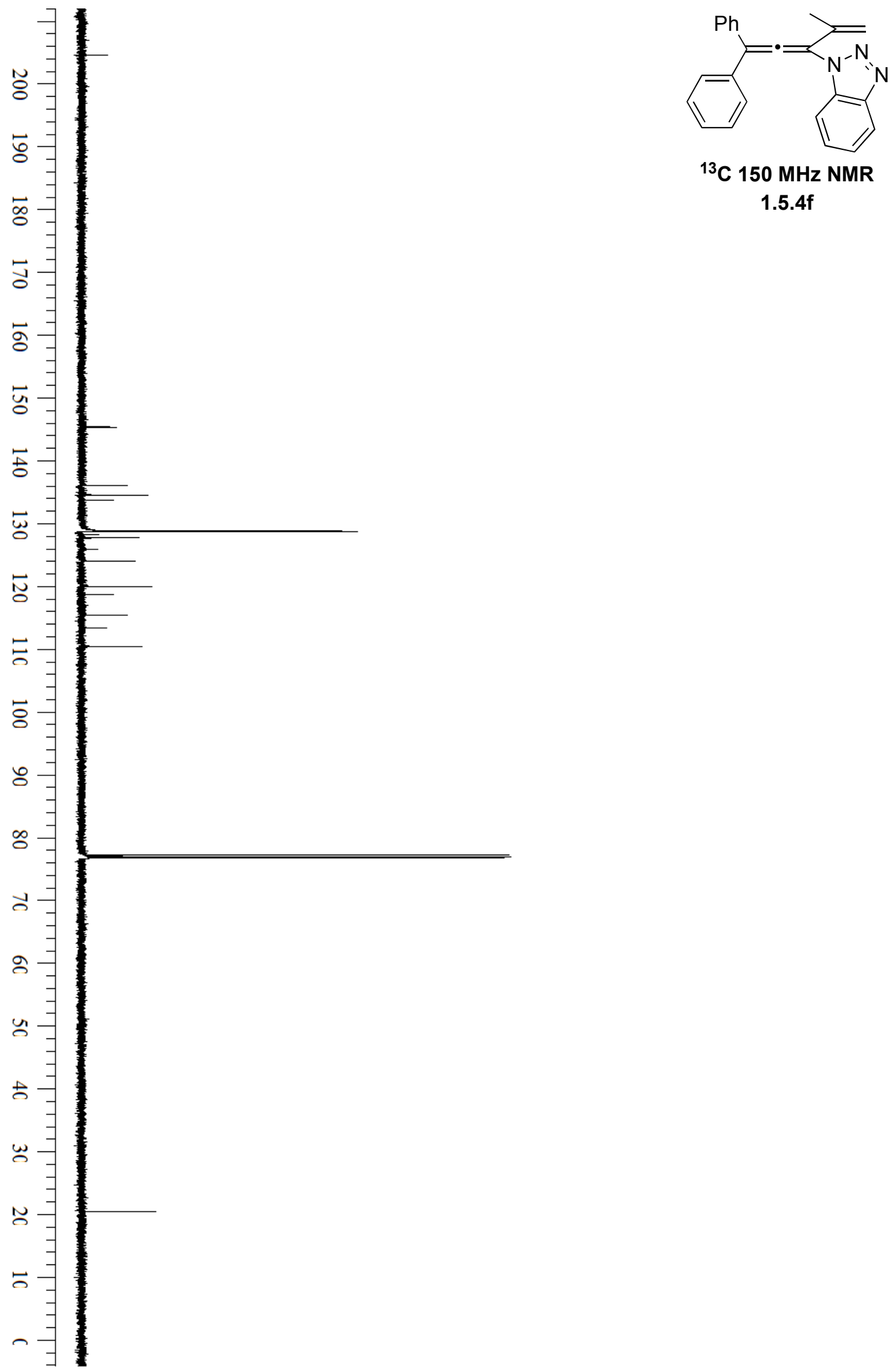

1.5.4f 

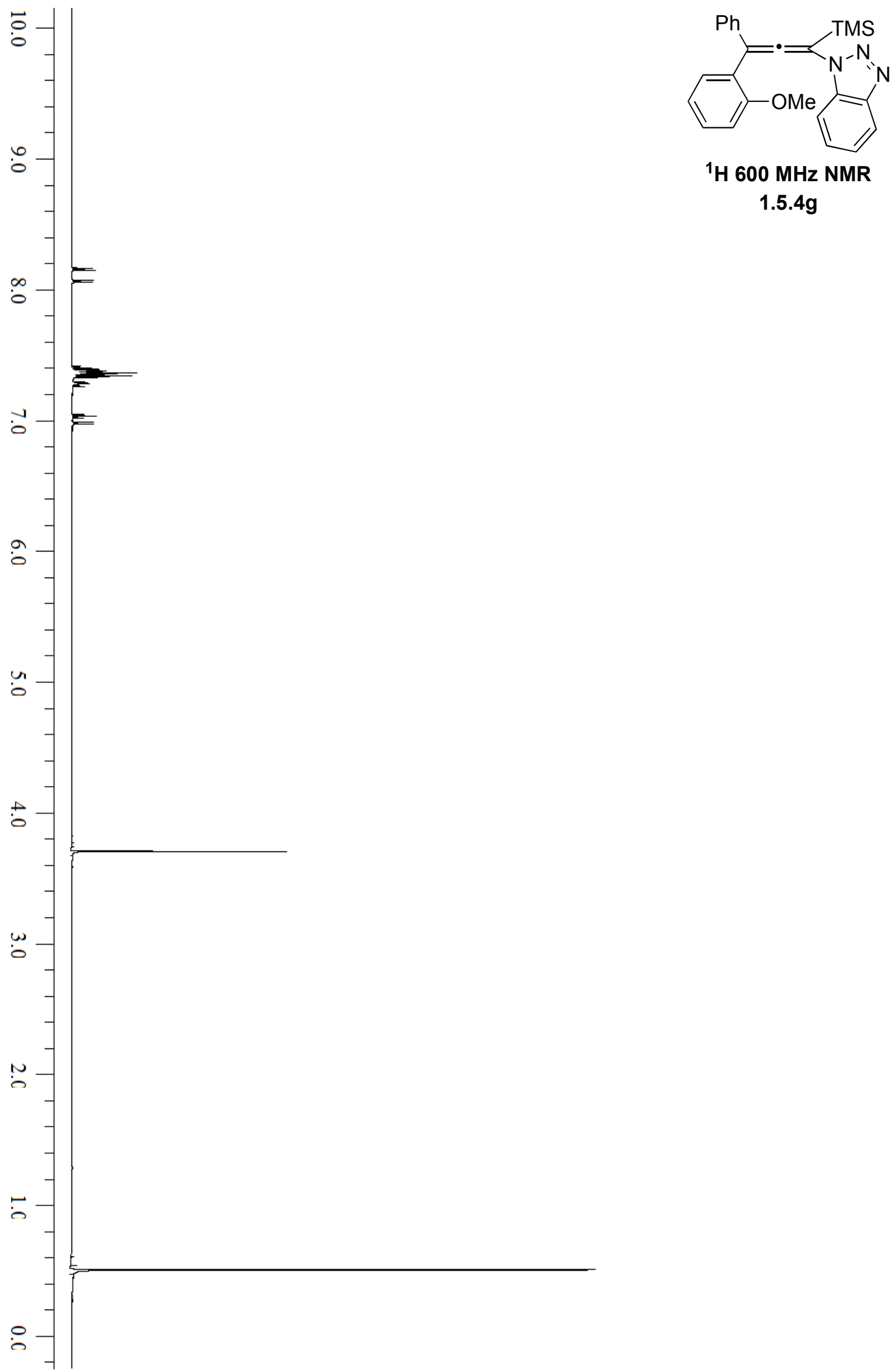

${ }^{1} \mathrm{H} 600 \mathrm{MHz}$ NMR

$1.5 .4 \mathrm{~g}$ 

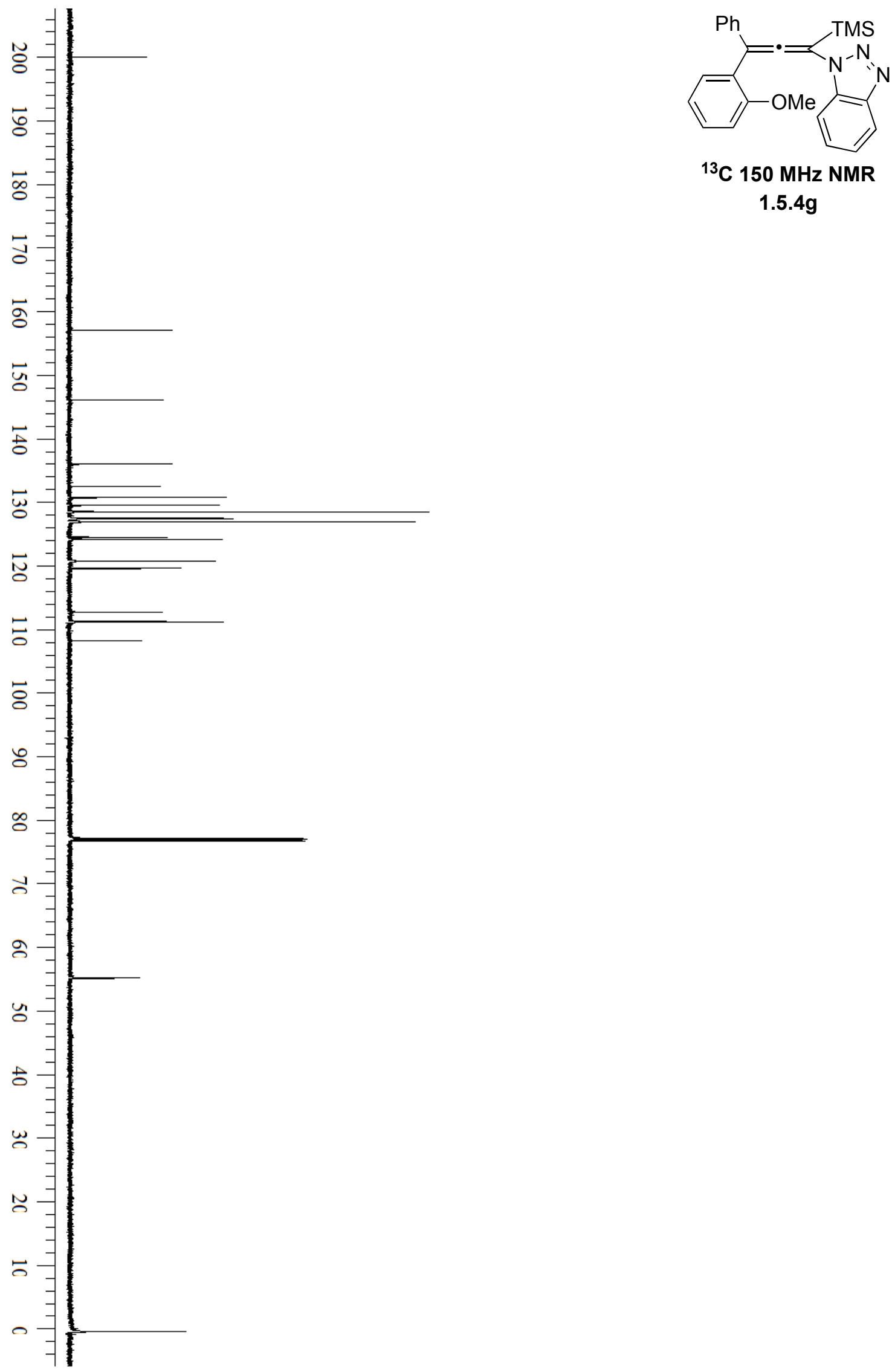

${ }^{13} \mathrm{C} 150 \mathrm{MHz}$ NMR

1.5.4g 


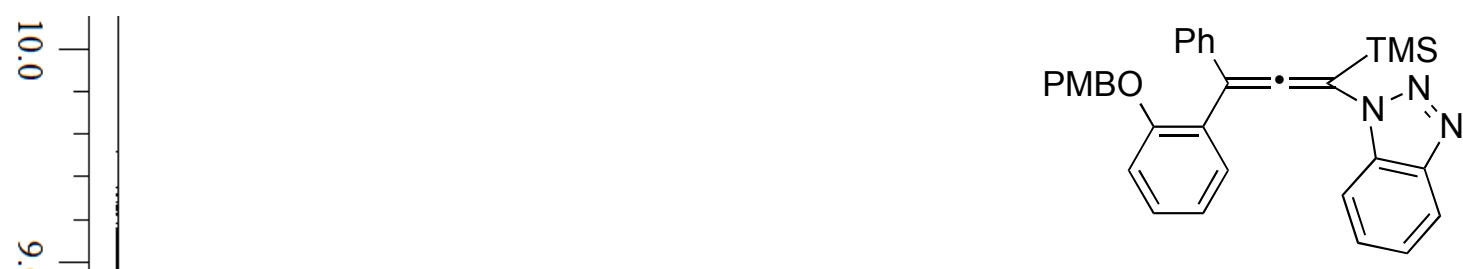

${ }^{1} \mathrm{H} 600 \mathrm{MHz}$ NMR

1.5.4h

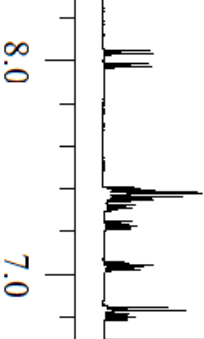

i

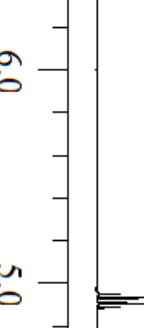

$+-$

w

$\stackrel{n}{\circ}$

w

-

$\stackrel{\circ}{\circ}$ 


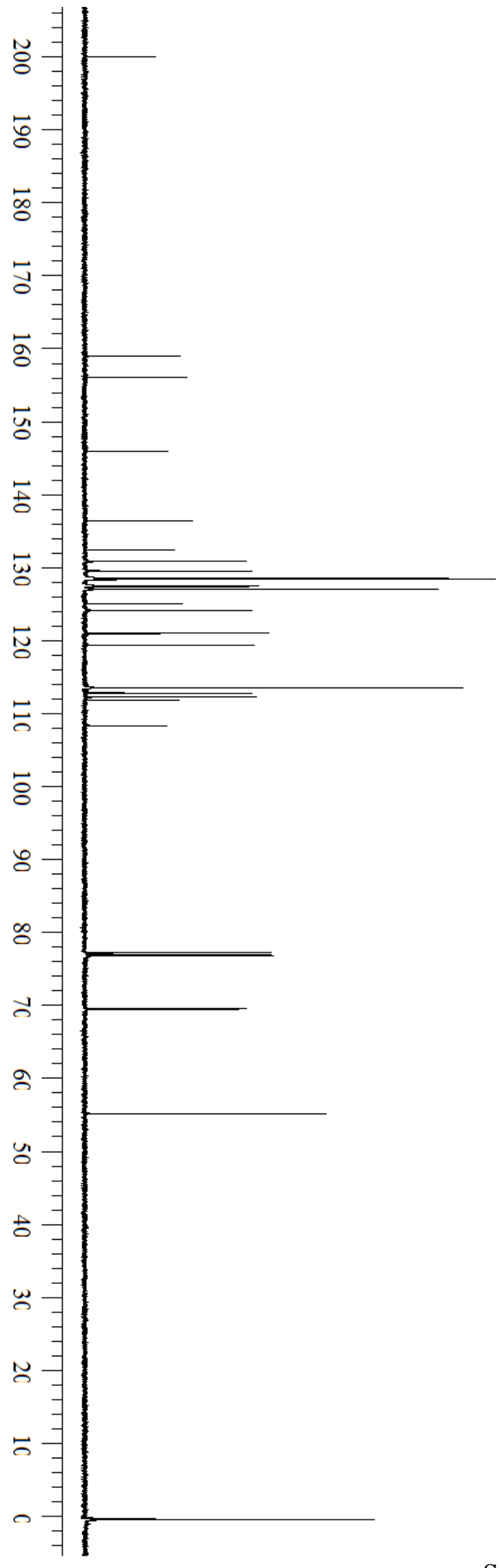

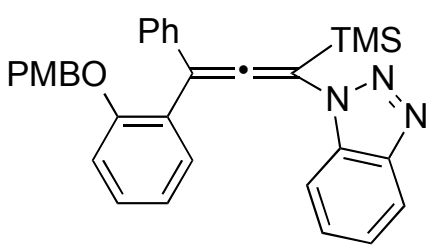

${ }^{13} \mathrm{C} 150 \mathrm{MHz}$ NMR

1.5.4h 


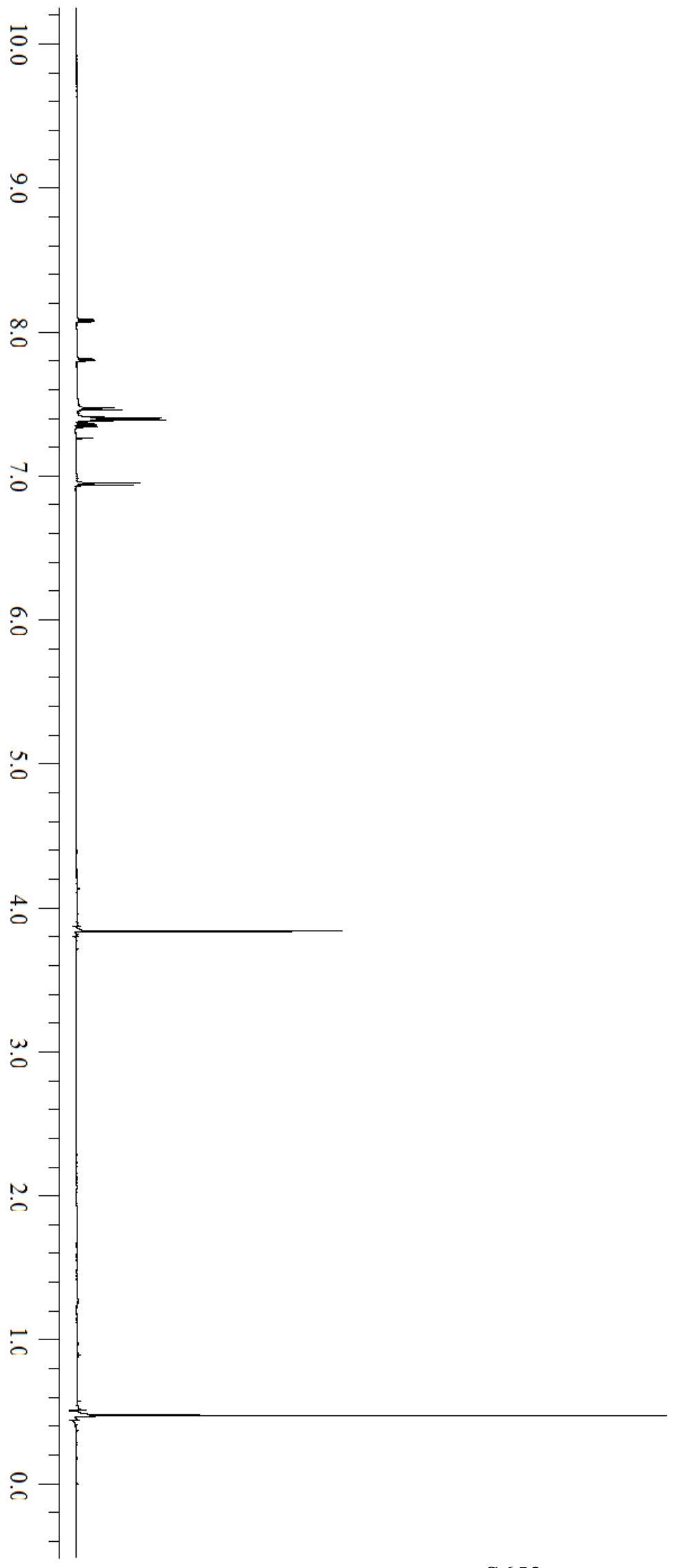

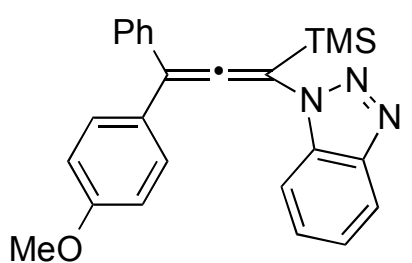

${ }^{1} \mathrm{H} 600 \mathrm{MHz}$ NMR

$1.5 .4 \mathrm{i}$ 

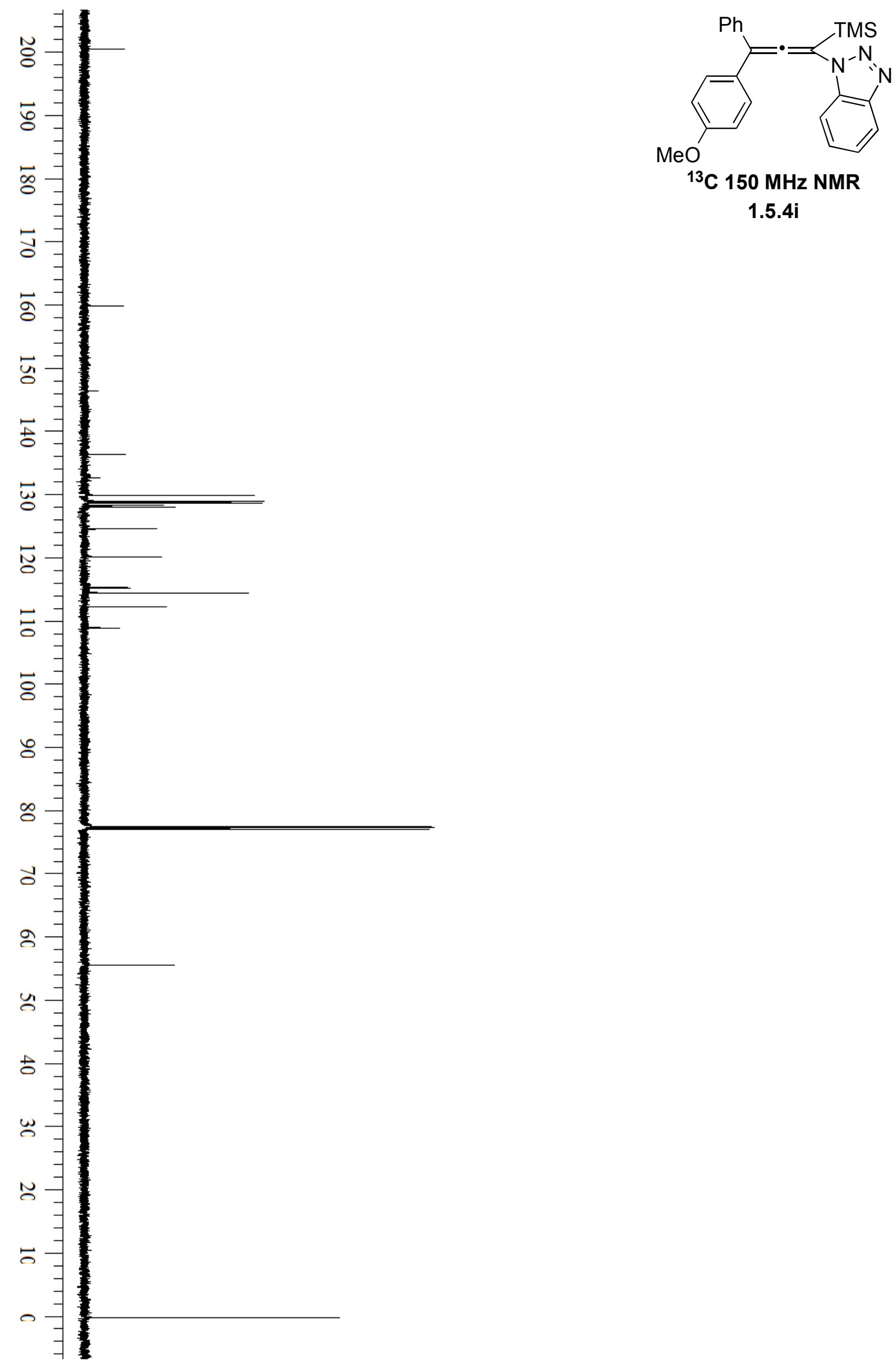

${ }^{13} \mathrm{C} 150 \mathrm{MHz}$ NMR

$1.5 .4 \mathrm{i}$ 


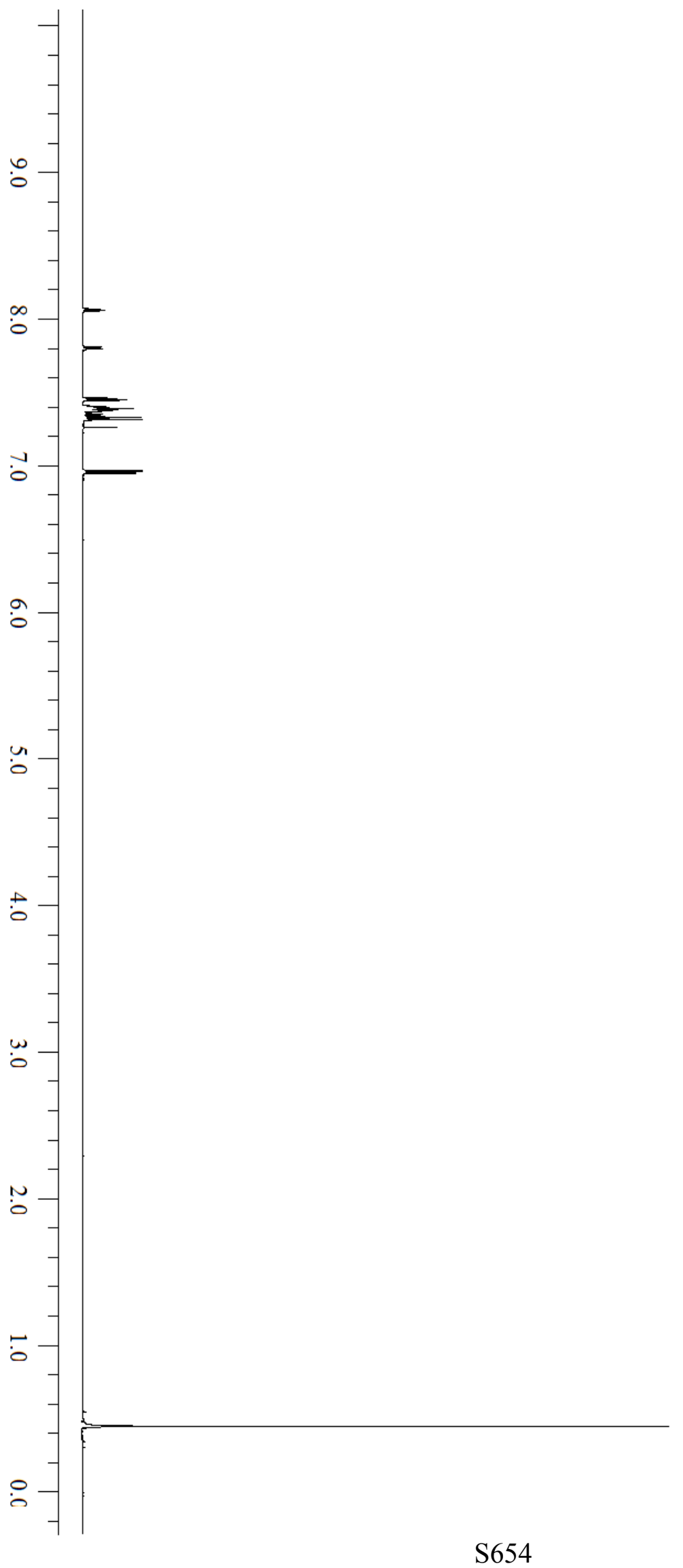

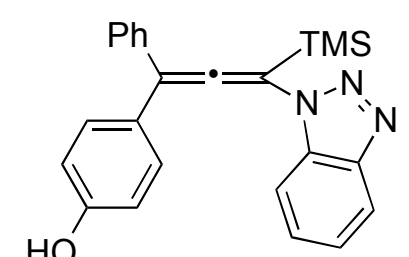

${ }^{1} \mathrm{H} 600 \mathrm{MHz}$ NMR

1.5.4j 

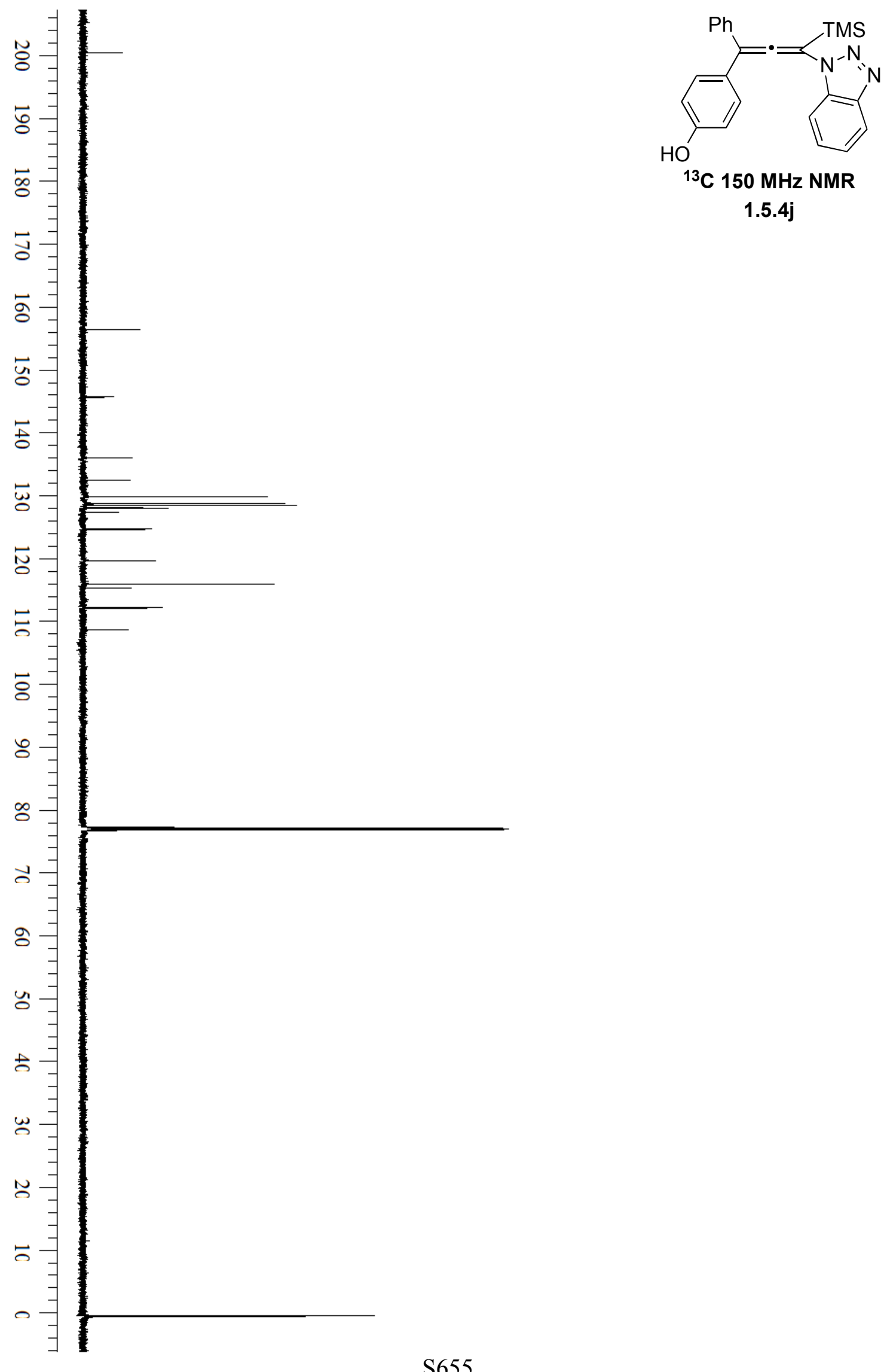

1.5.4j 


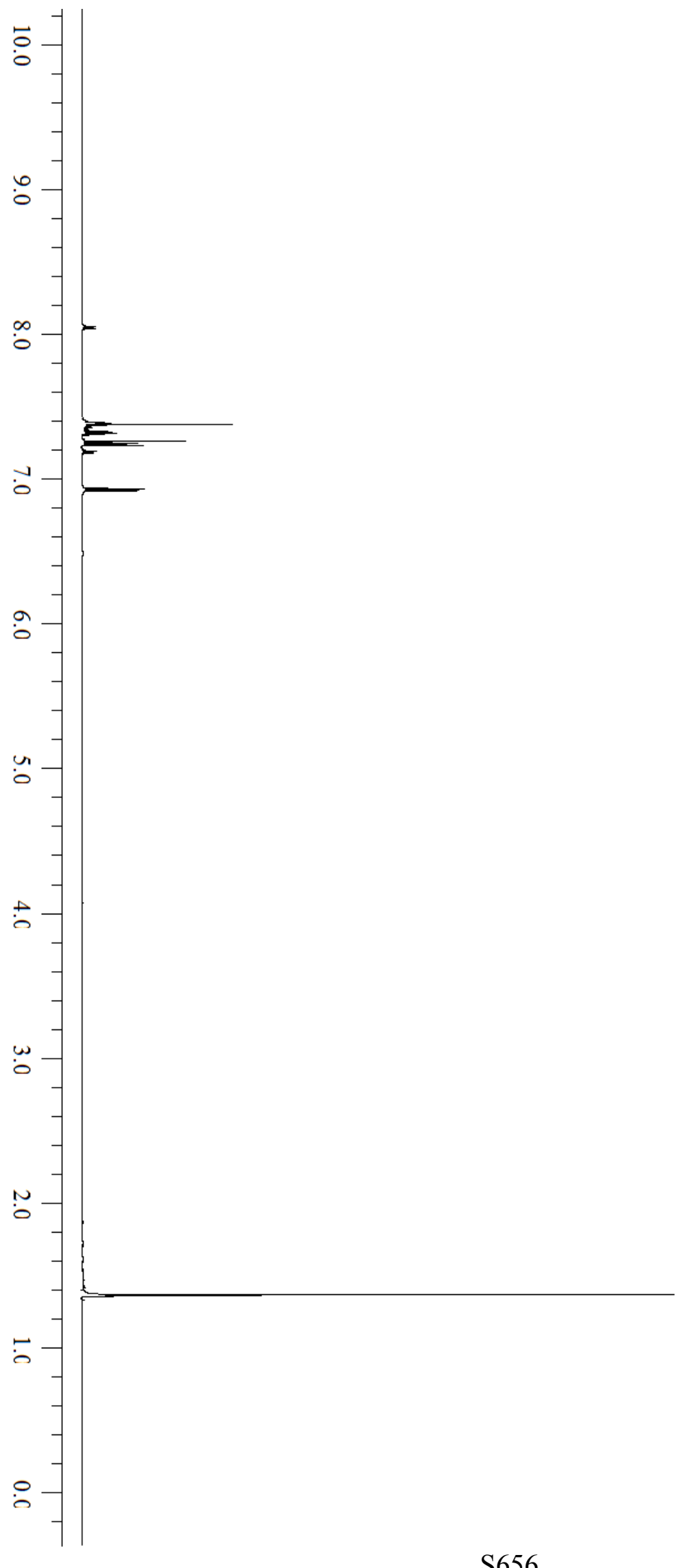

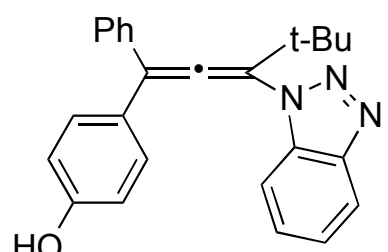

${ }^{1} \mathrm{H} 600 \mathrm{MHz}$ NMR $1.5 .4 \mathrm{k}$ 


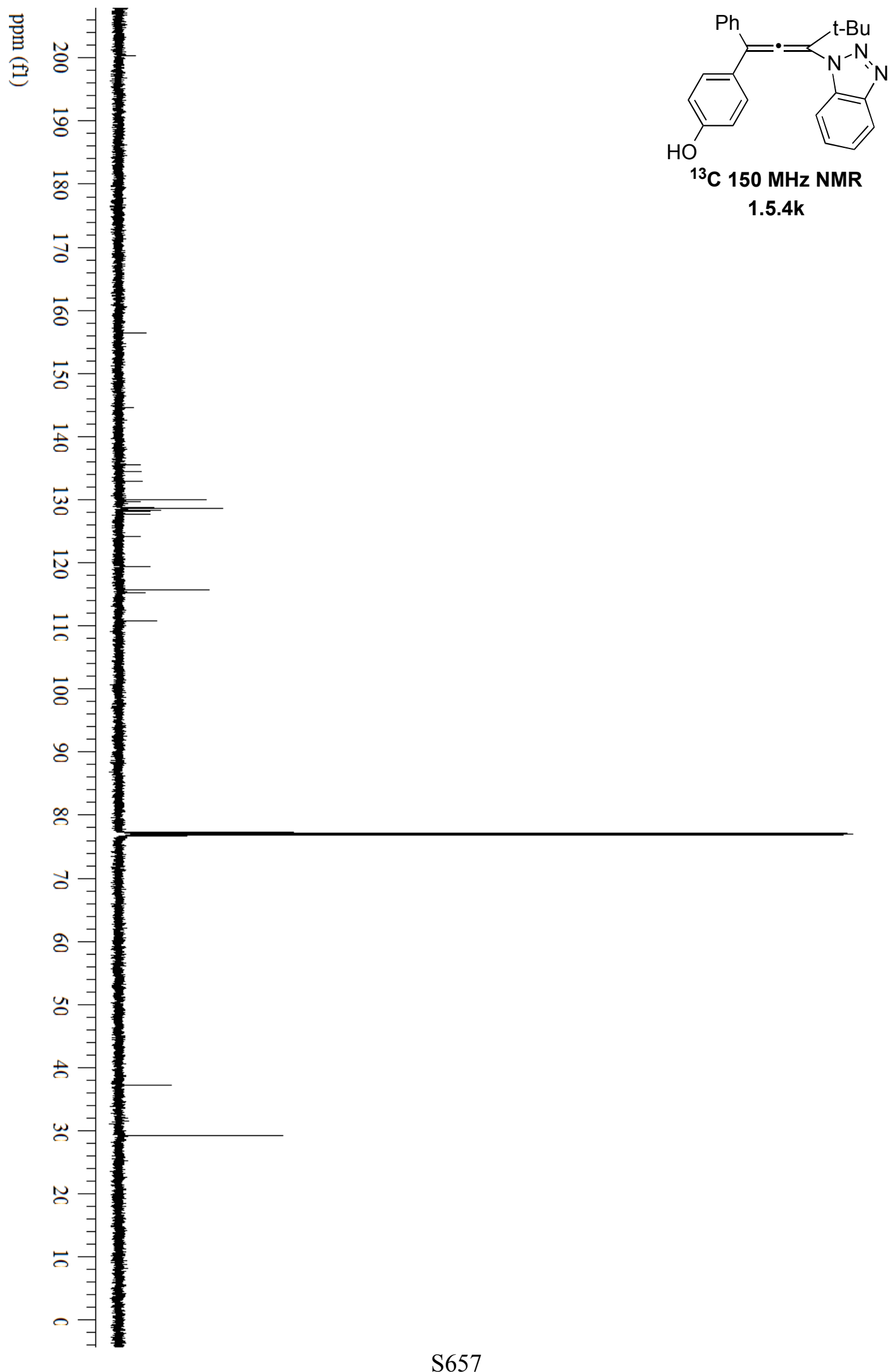




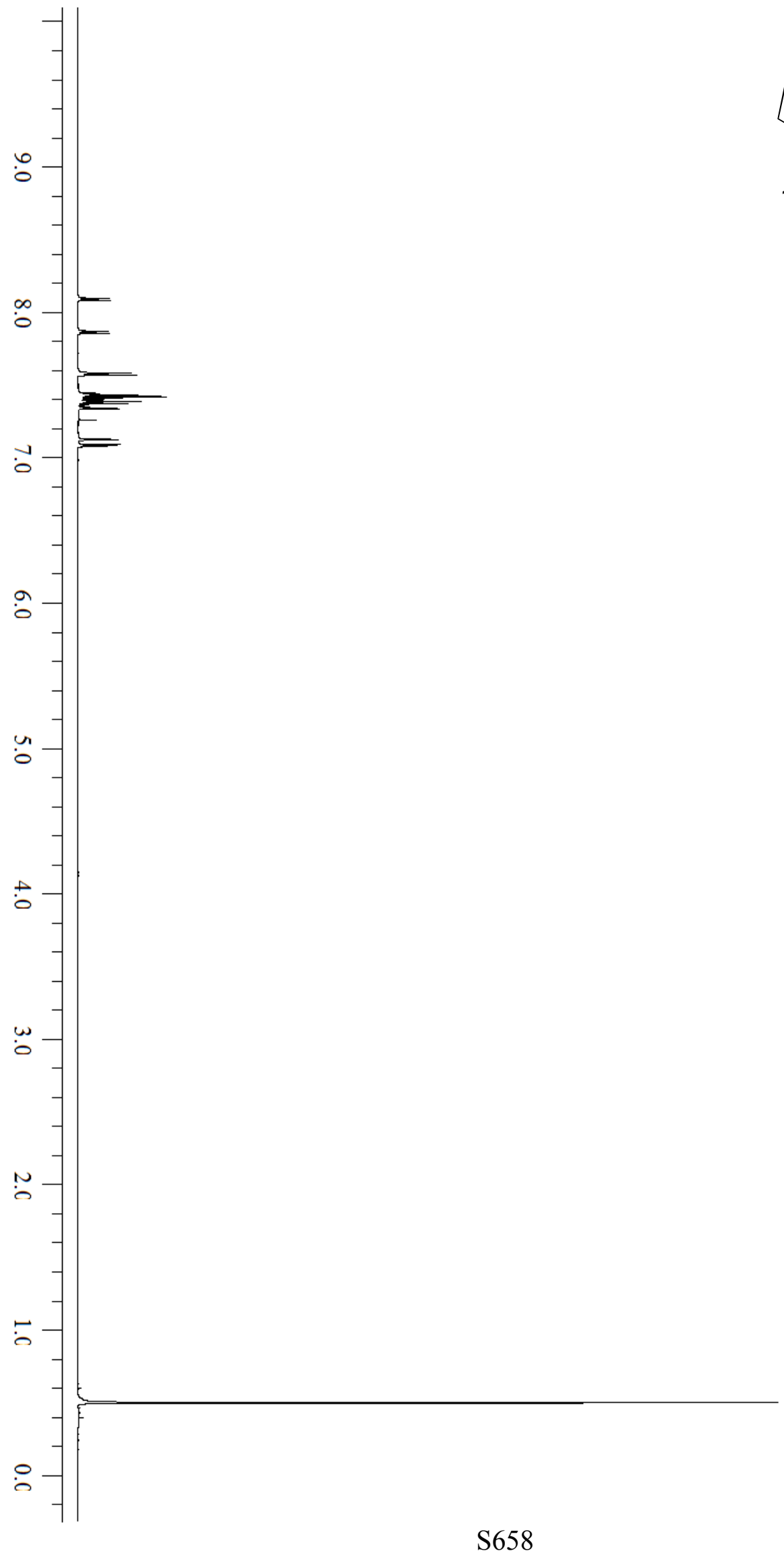

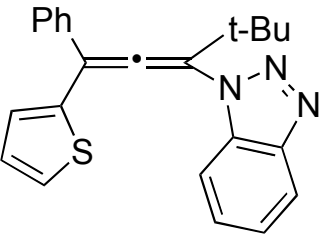

${ }^{1} \mathrm{H} 600 \mathrm{MHz}$ NMR

1.5.4I 


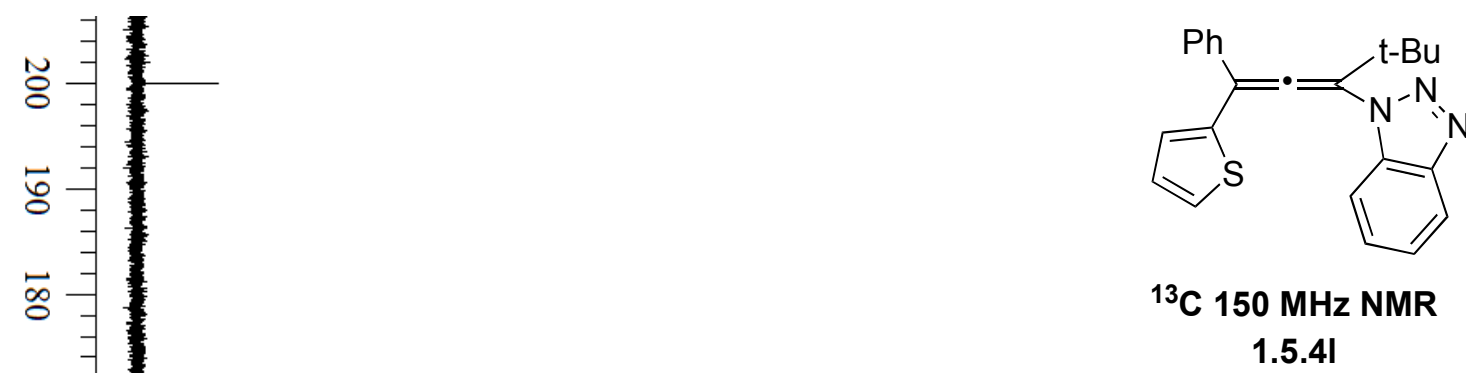



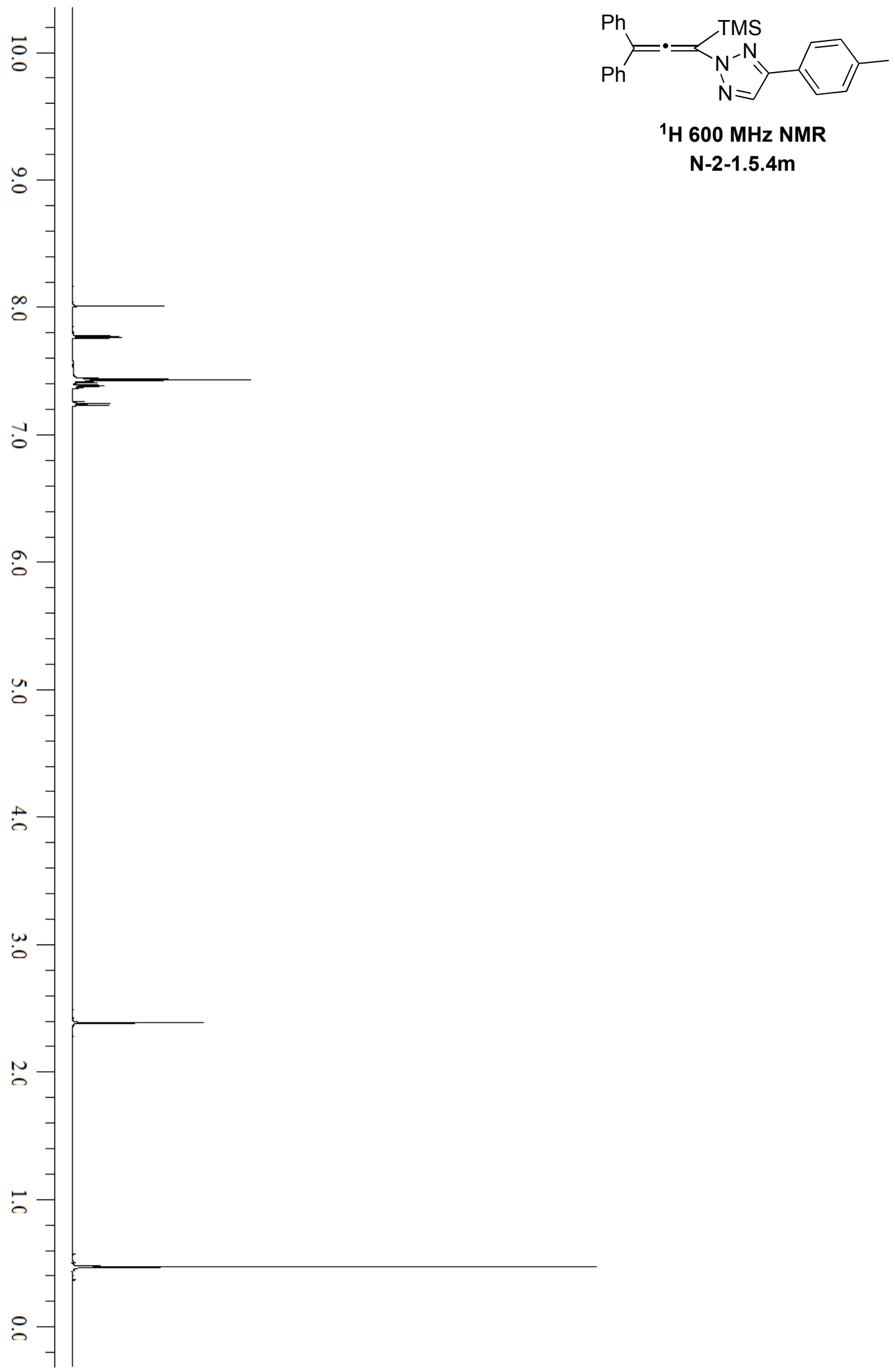

${ }^{1} \mathrm{H} 600 \mathrm{MHz}$ NMR

$\mathrm{N}-2-1.5 .4 \mathrm{~m}$ 


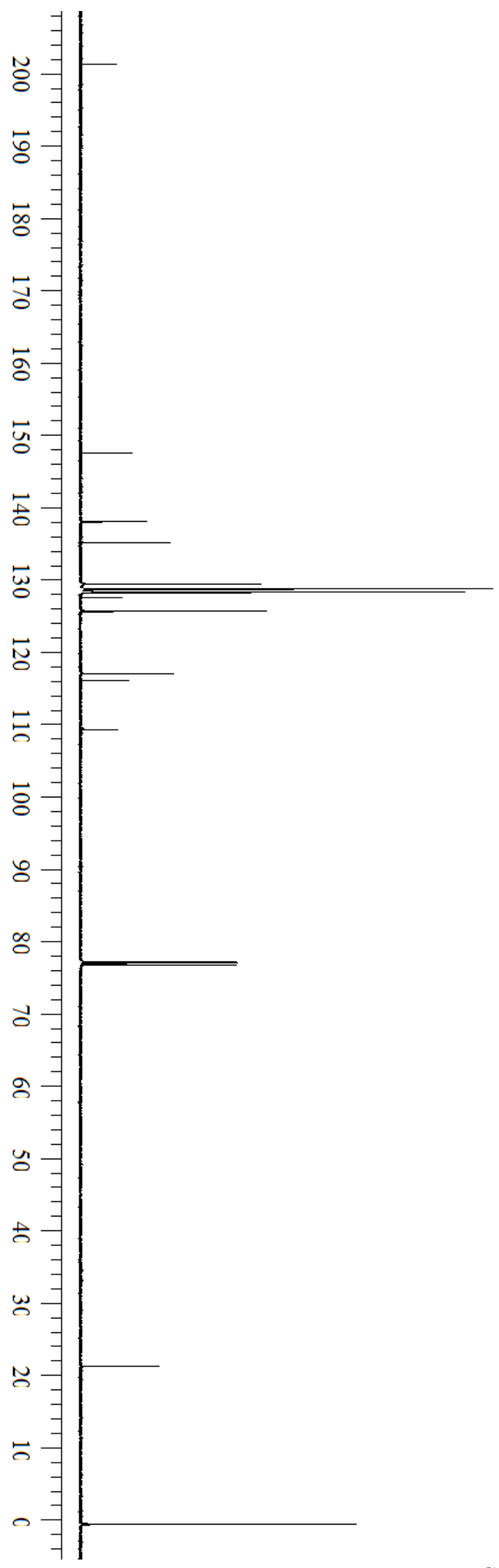

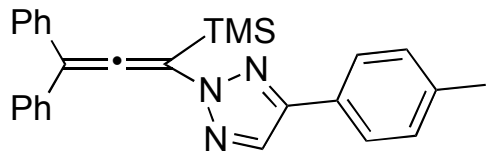

${ }^{13} \mathrm{C} 150 \mathrm{MHz}$ NMR $\mathrm{N}-2-1.5 .4 \mathrm{~m}$ 


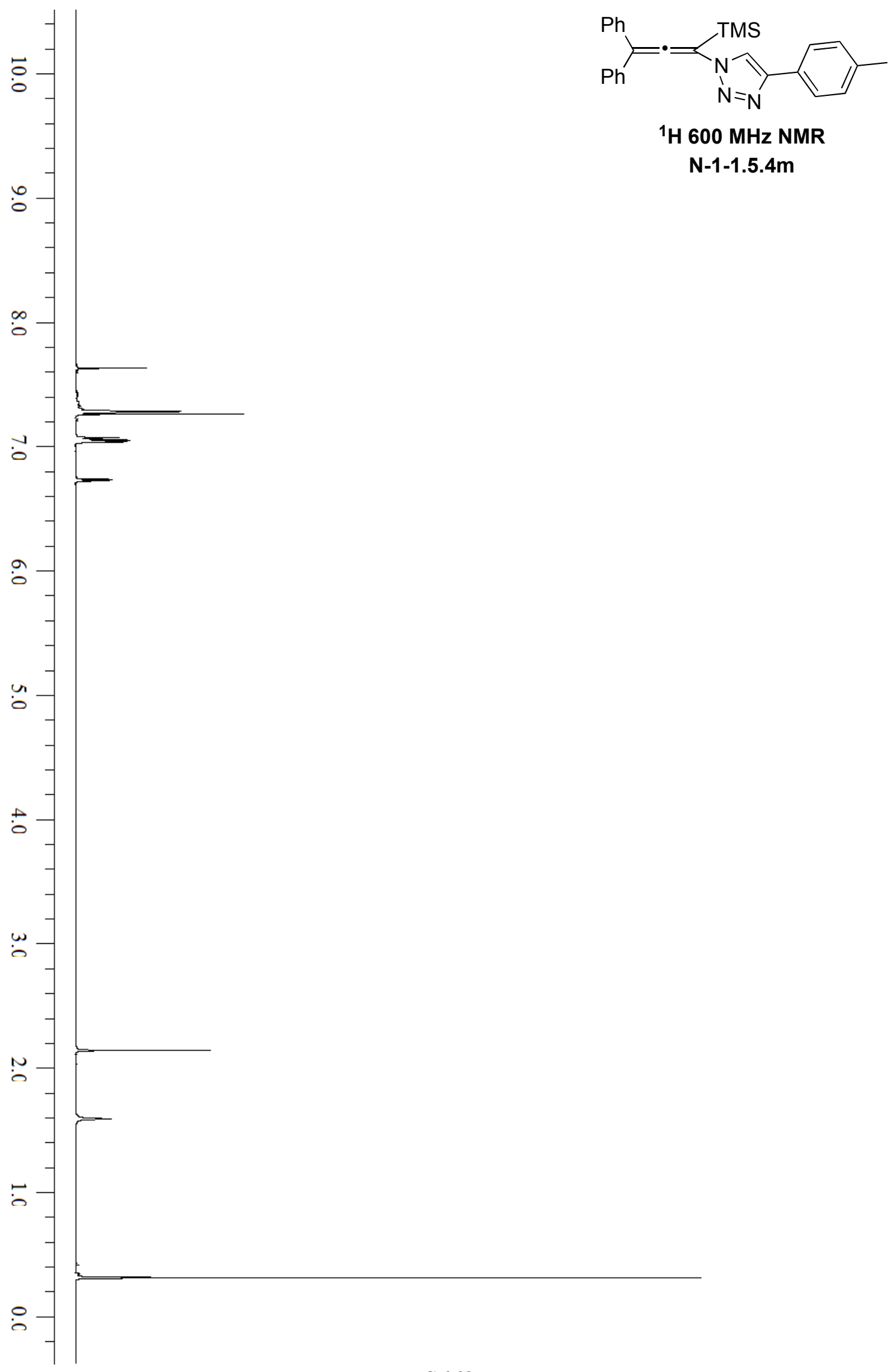




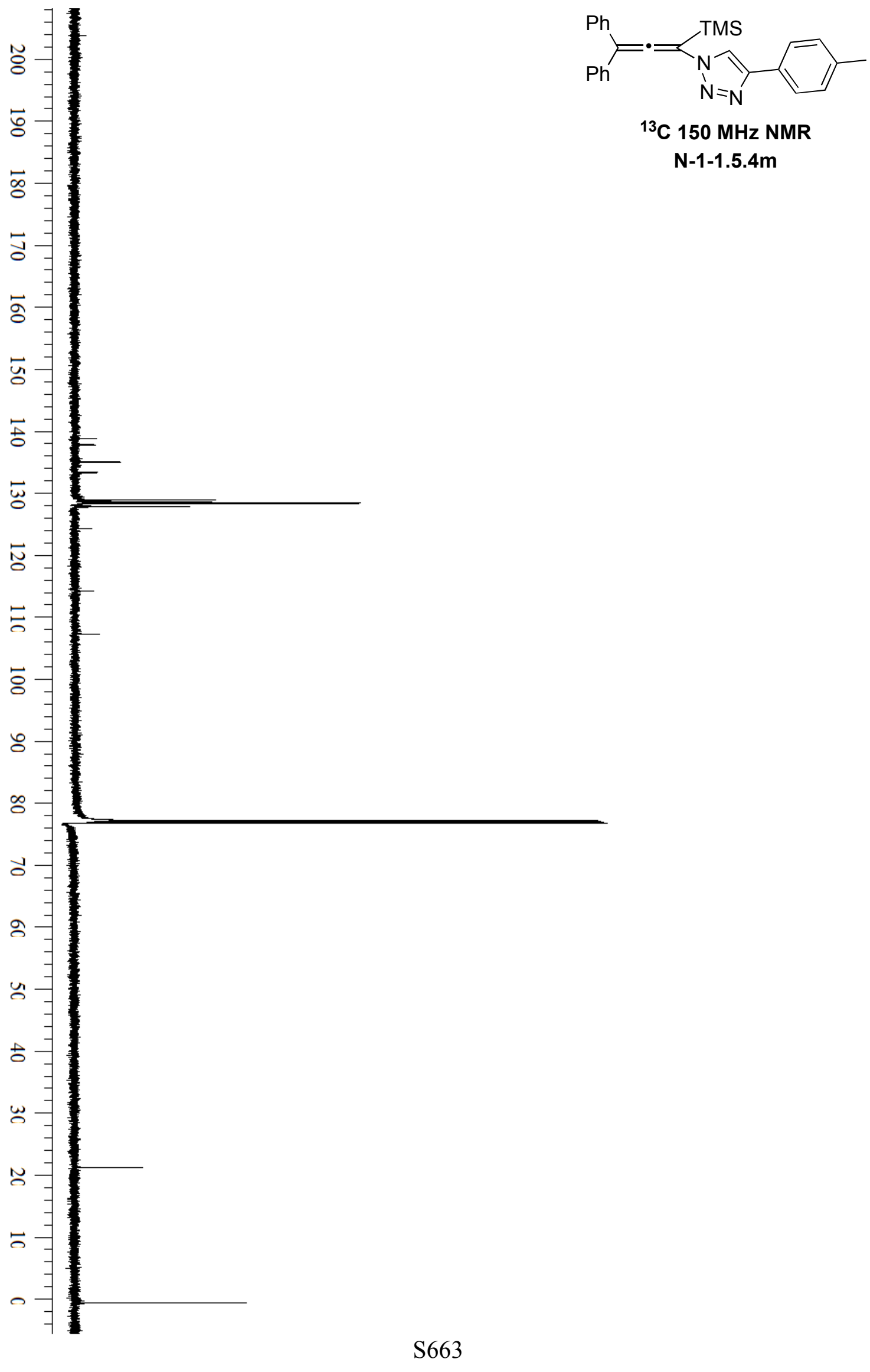




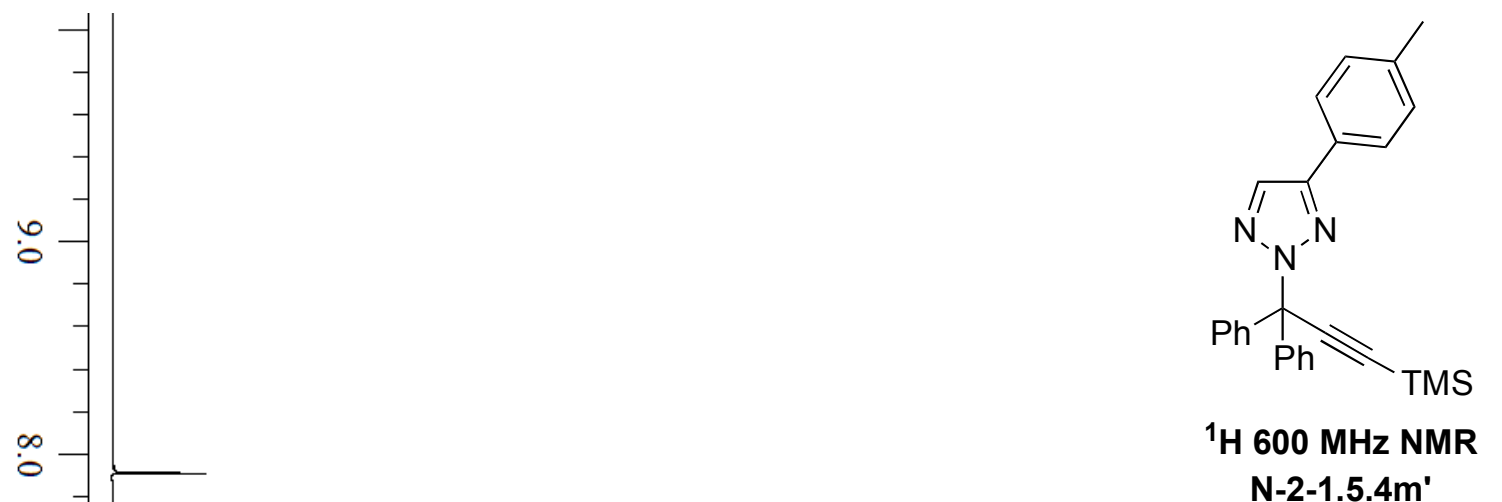

N-2-1.5.4m' 


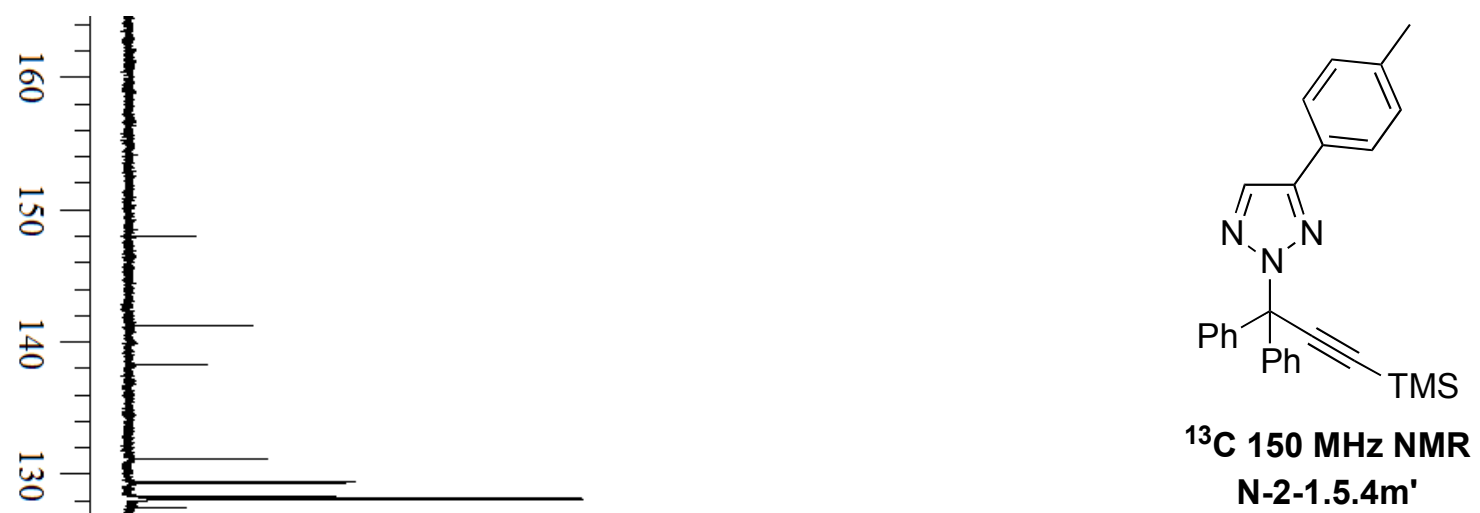

年

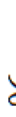

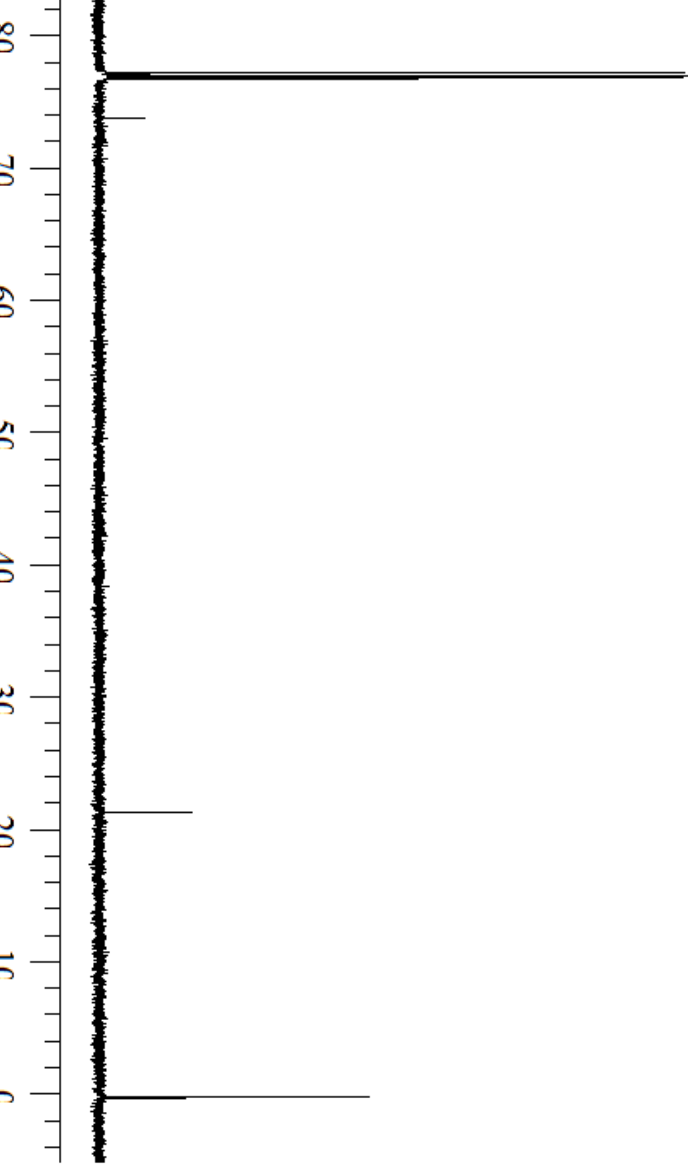




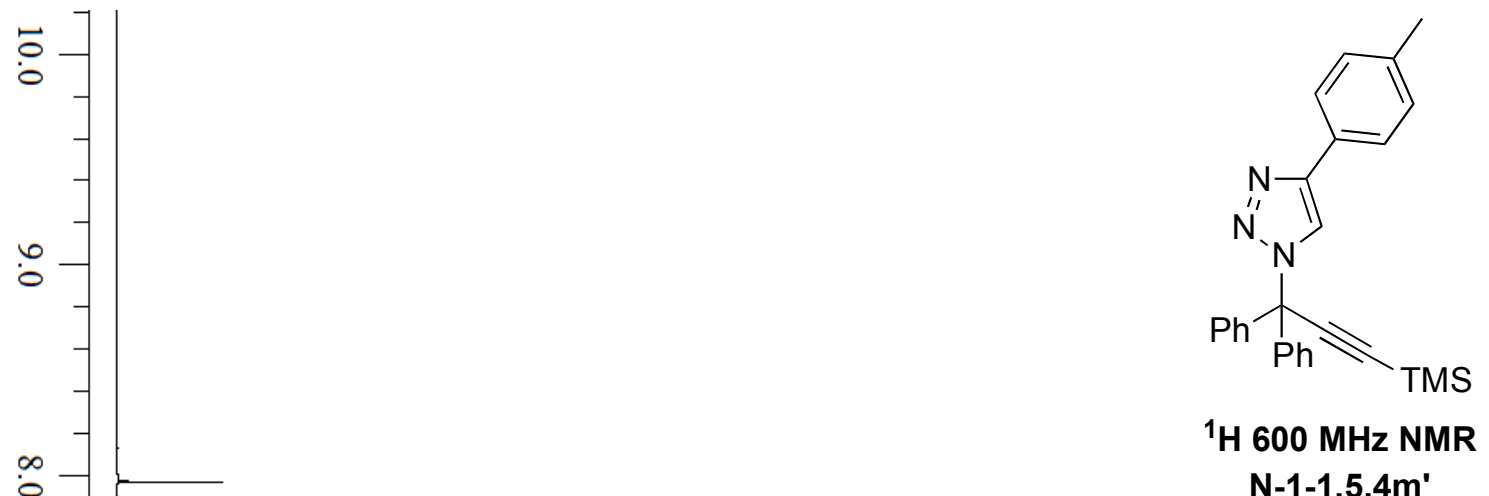

$\mathrm{N}-1-1.5 .4 \mathrm{~m}^{\prime}$ 

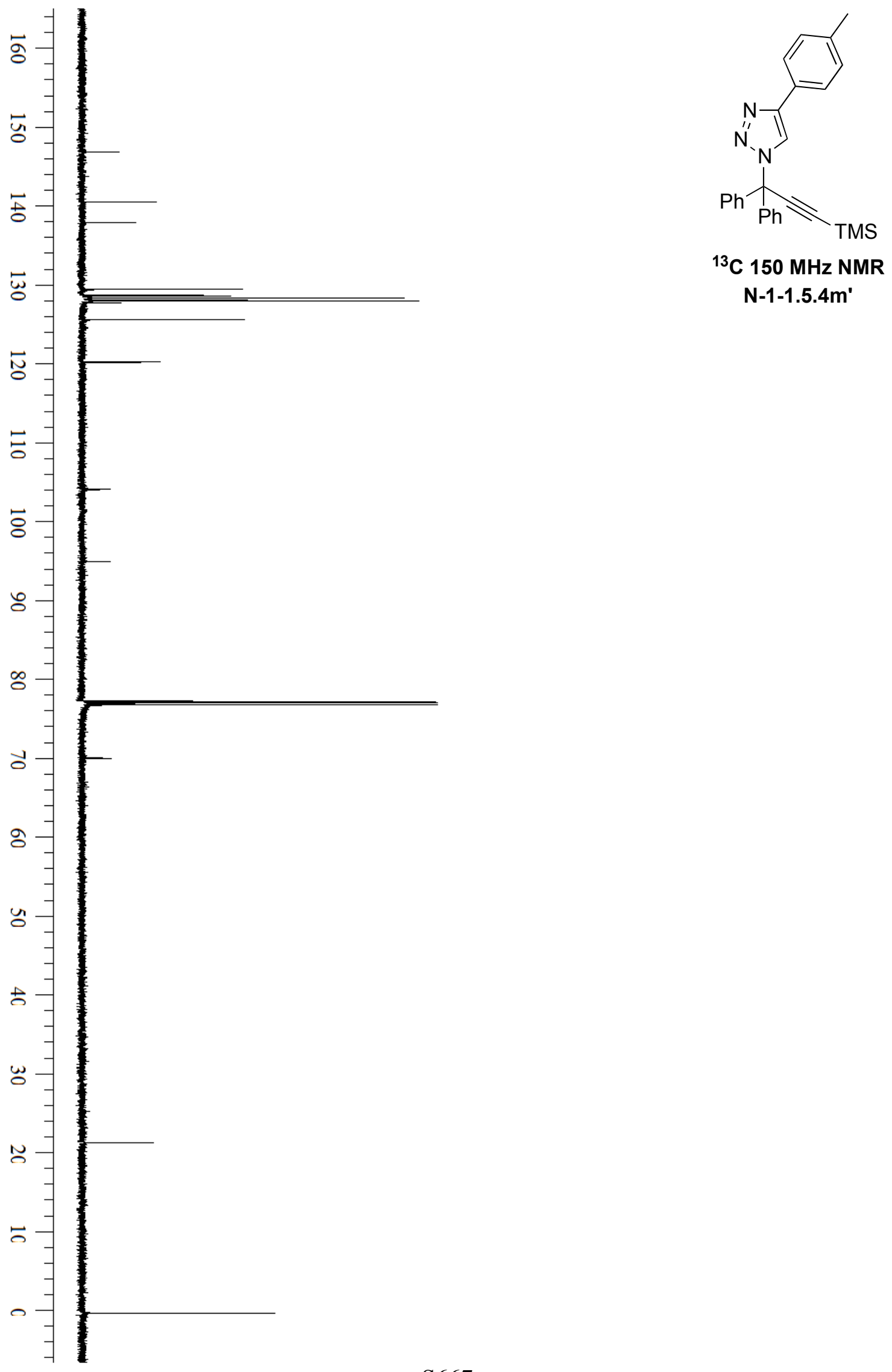

N-1-1.5.4m' 


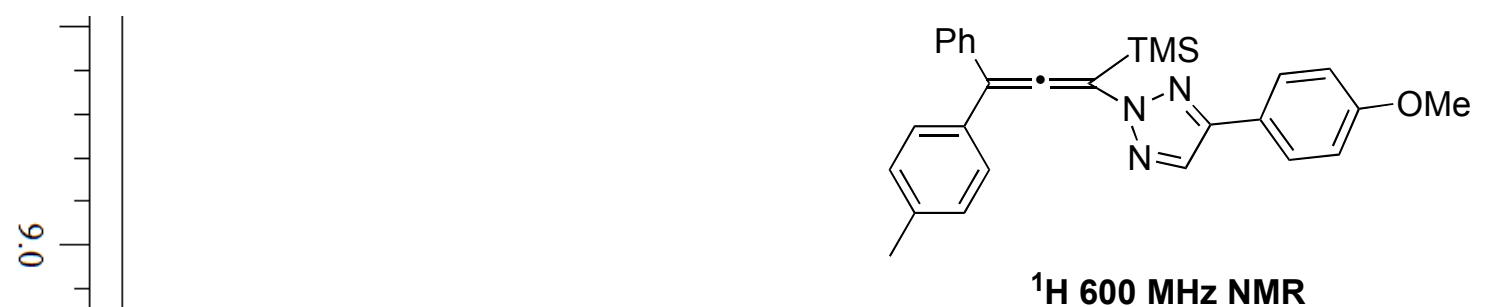

${ }^{1} \mathrm{H} 600 \mathrm{MHz}$ NMR

$\mathrm{N}-2-1.5 .4 \mathrm{n}$

$\infty$

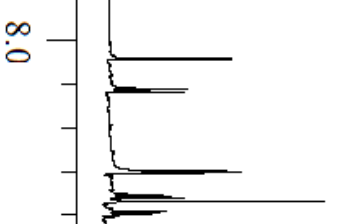



2

0

or

$\dot{0}$

co 

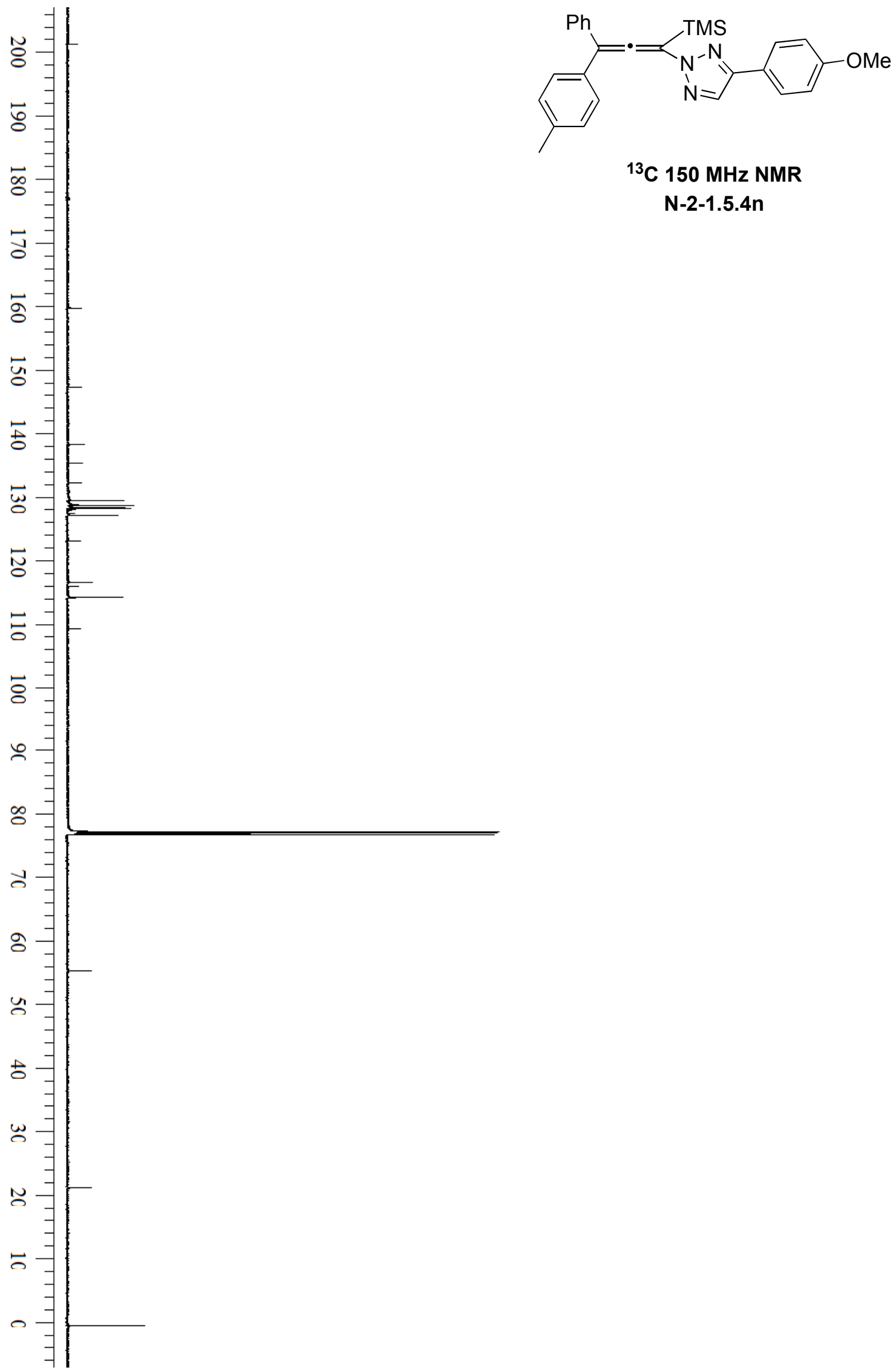

$\mathrm{N}-2-1.5 .4 \mathrm{n}$ 


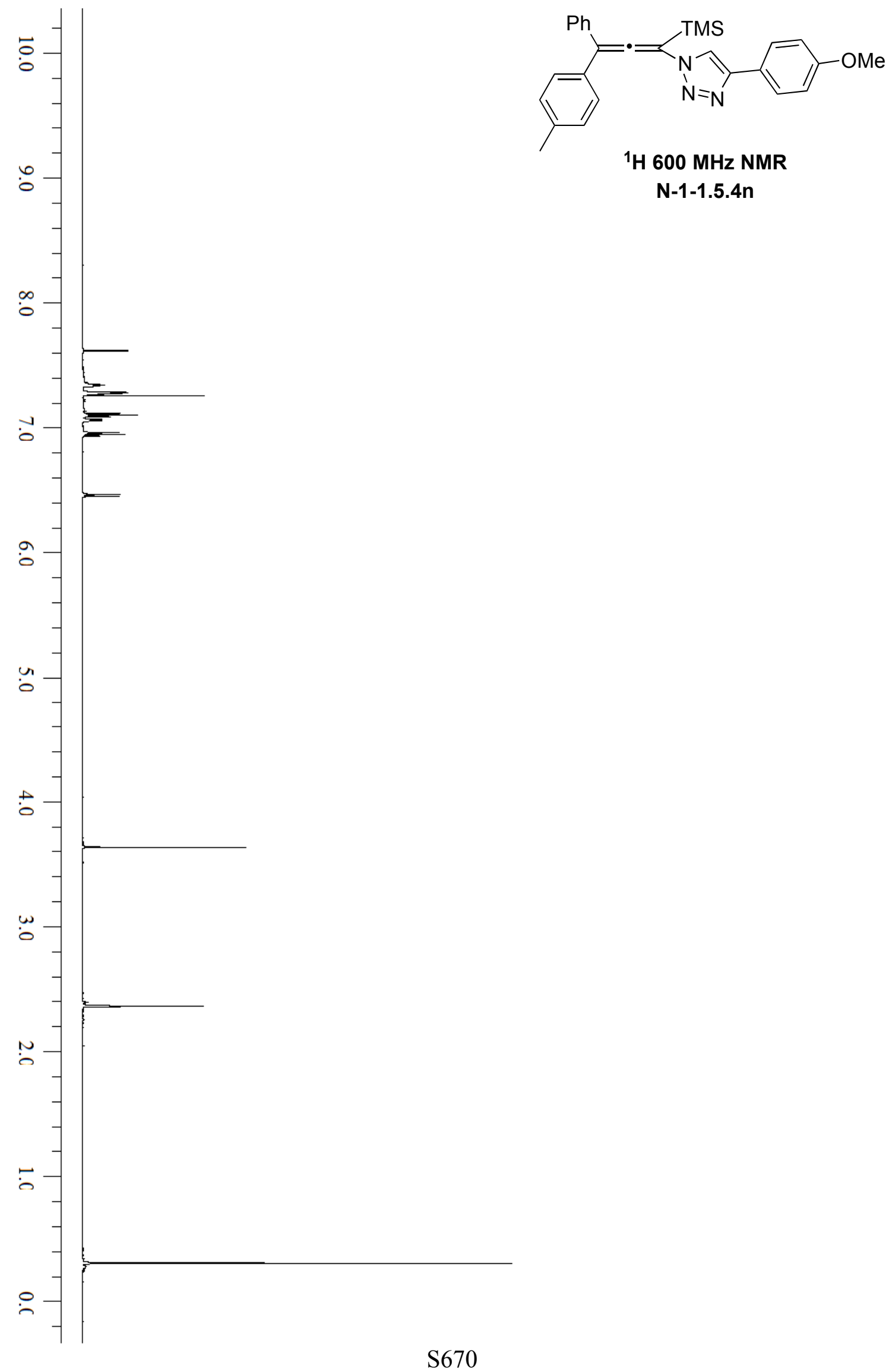




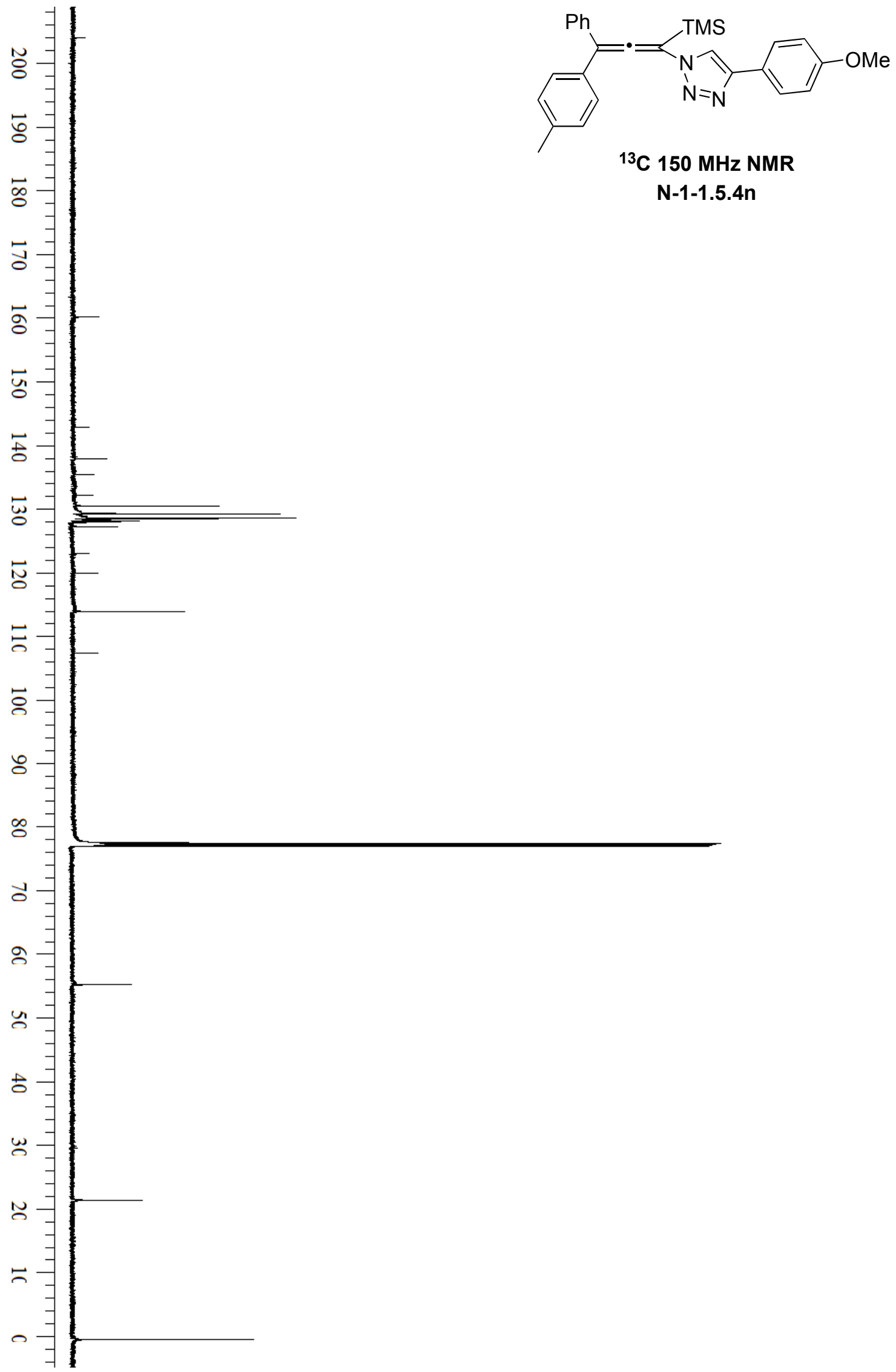



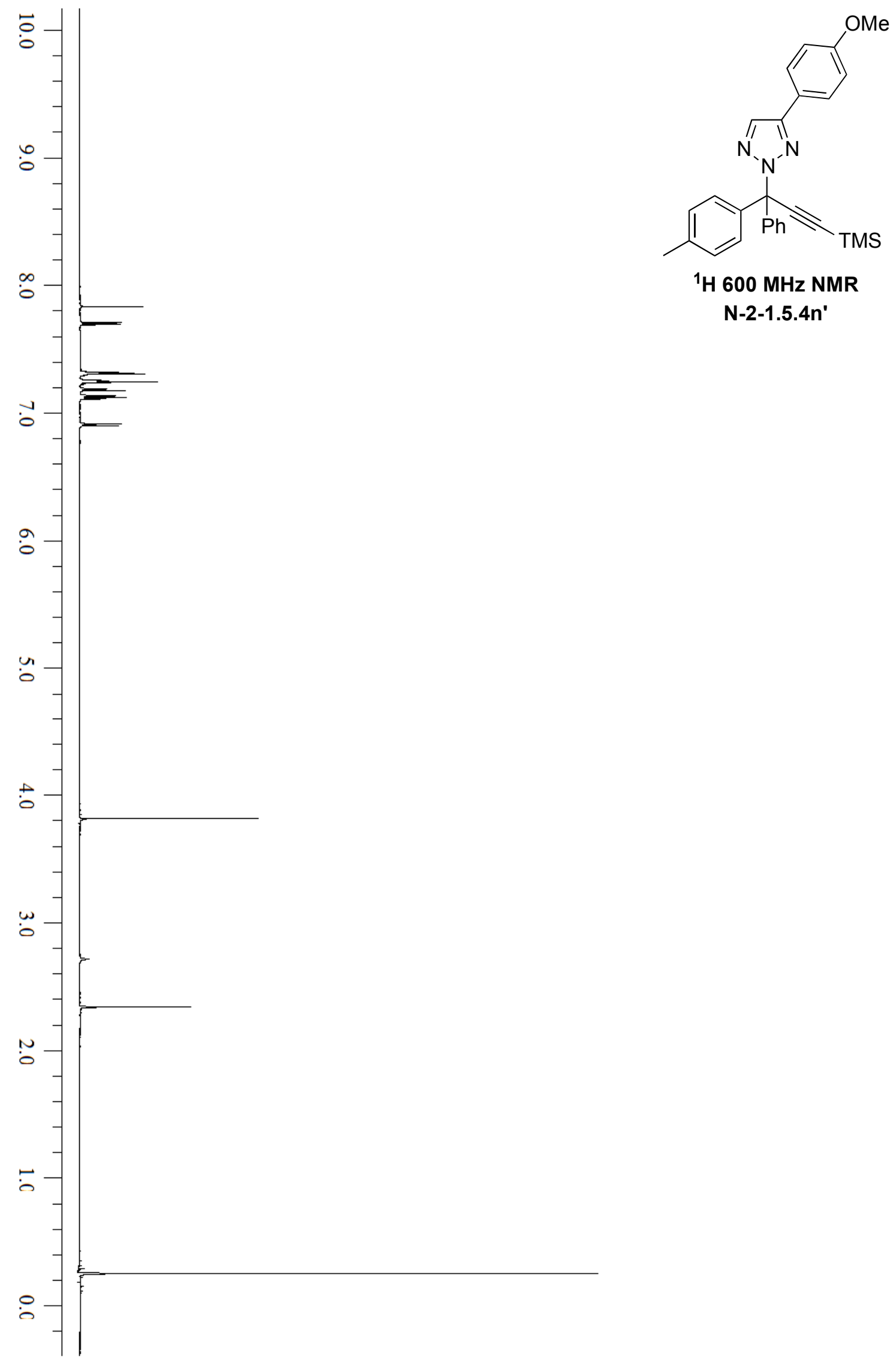

${ }^{1} \mathrm{H} 600 \mathrm{MHz}$ NMR

$\mathrm{N}-2-1.5 .4 n^{\prime}$ 

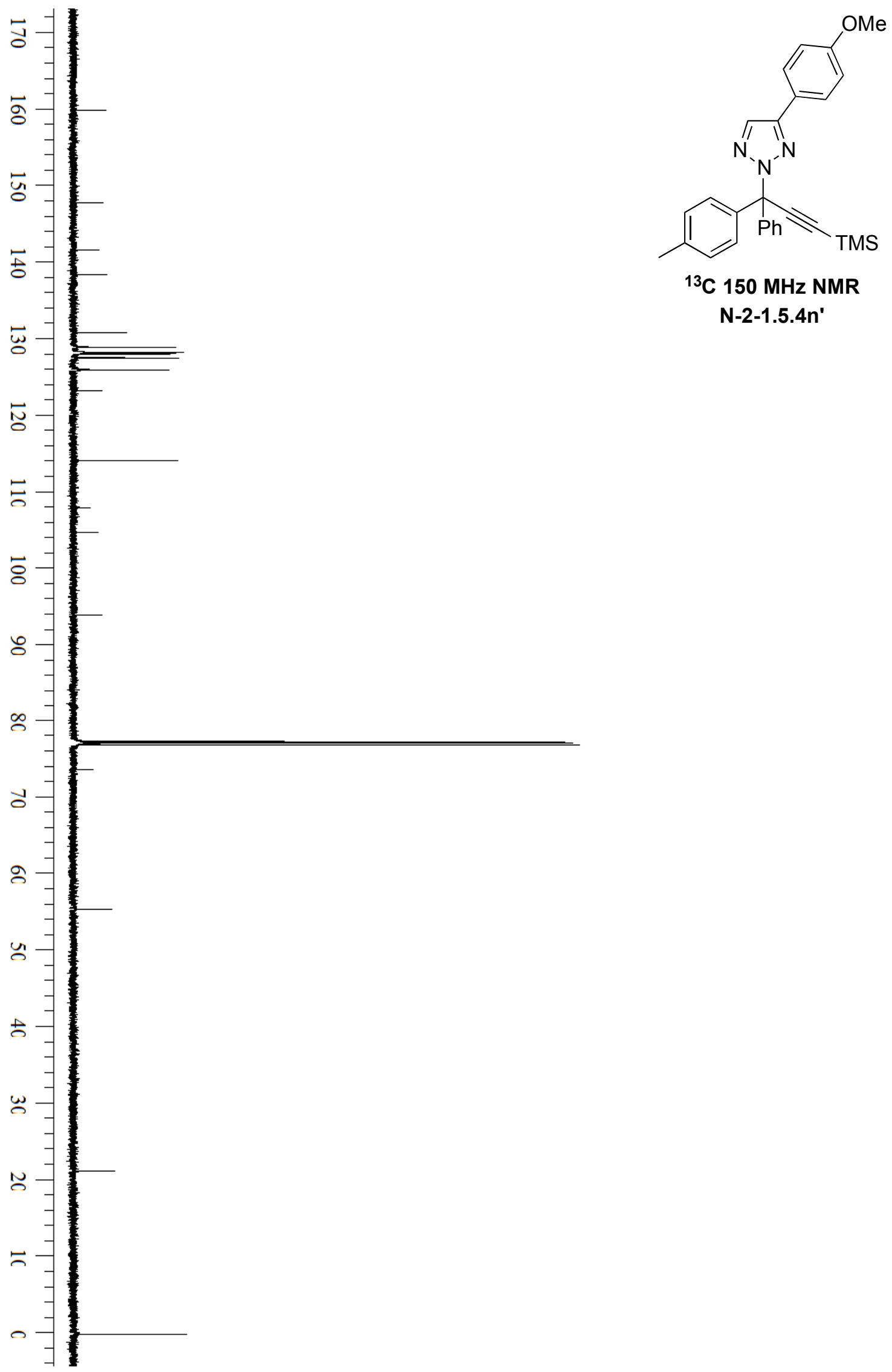

${ }^{3} \mathrm{C} 150 \mathrm{MHz}$ NMR

$\mathrm{N}-2-1.5 .4 n^{\prime}$ 


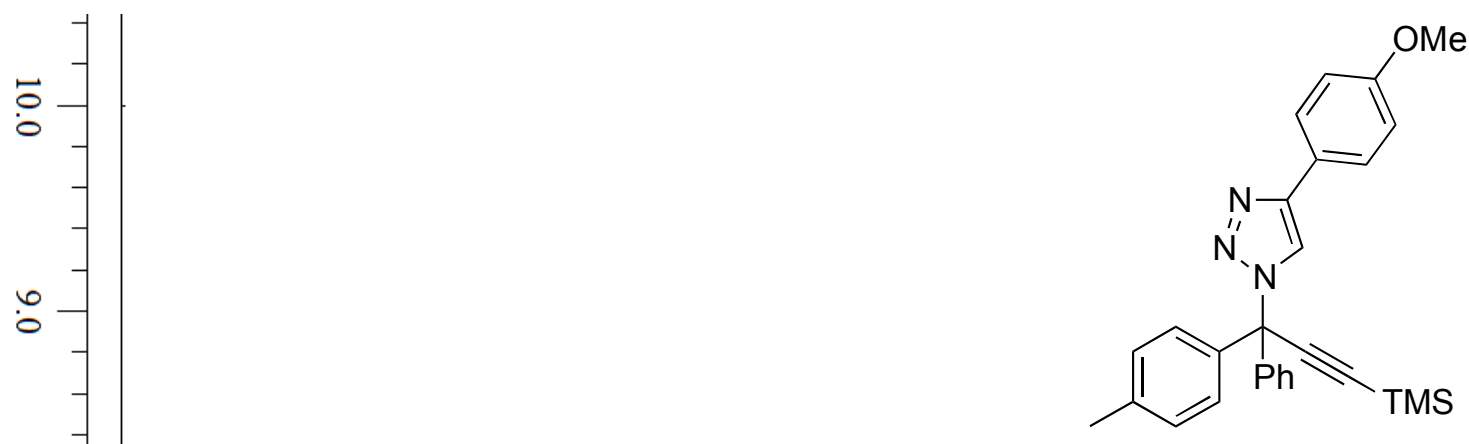

${ }^{1} \mathrm{H} 600 \mathrm{MHz}$ NMR

$\mathrm{N}-1-1.5 .4 n^{\prime}$

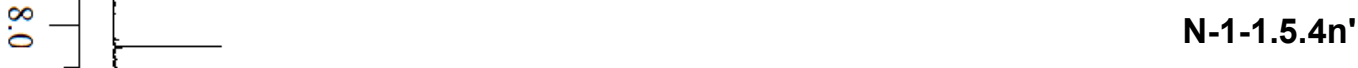

\%

$\ddot{0}$

$\circ-1$

w

-

o

un

-

ñ

:

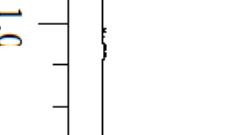

$\therefore-$ 

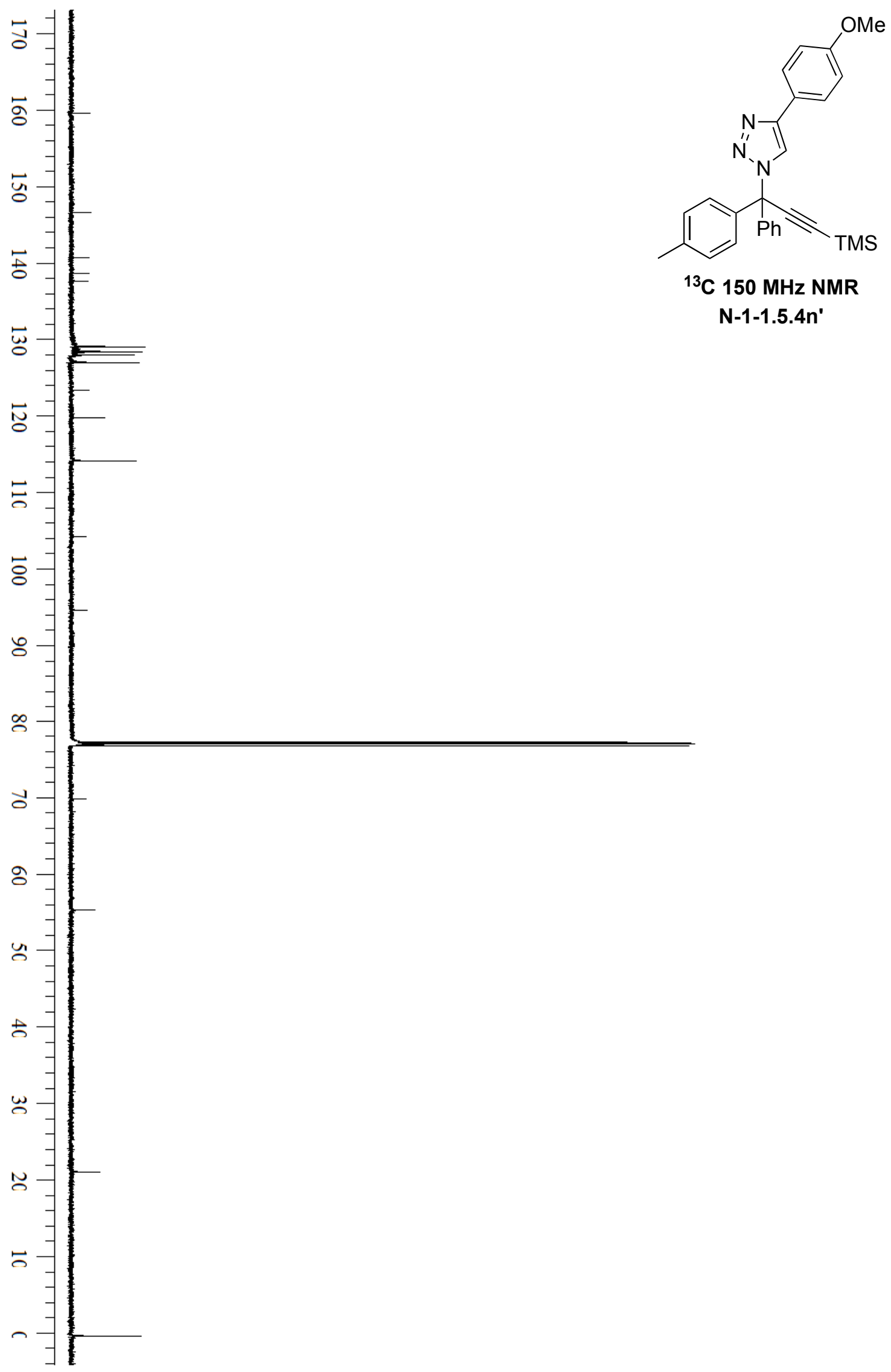

$\mathrm{N}-1-1.5 .4 n^{\prime}$ 


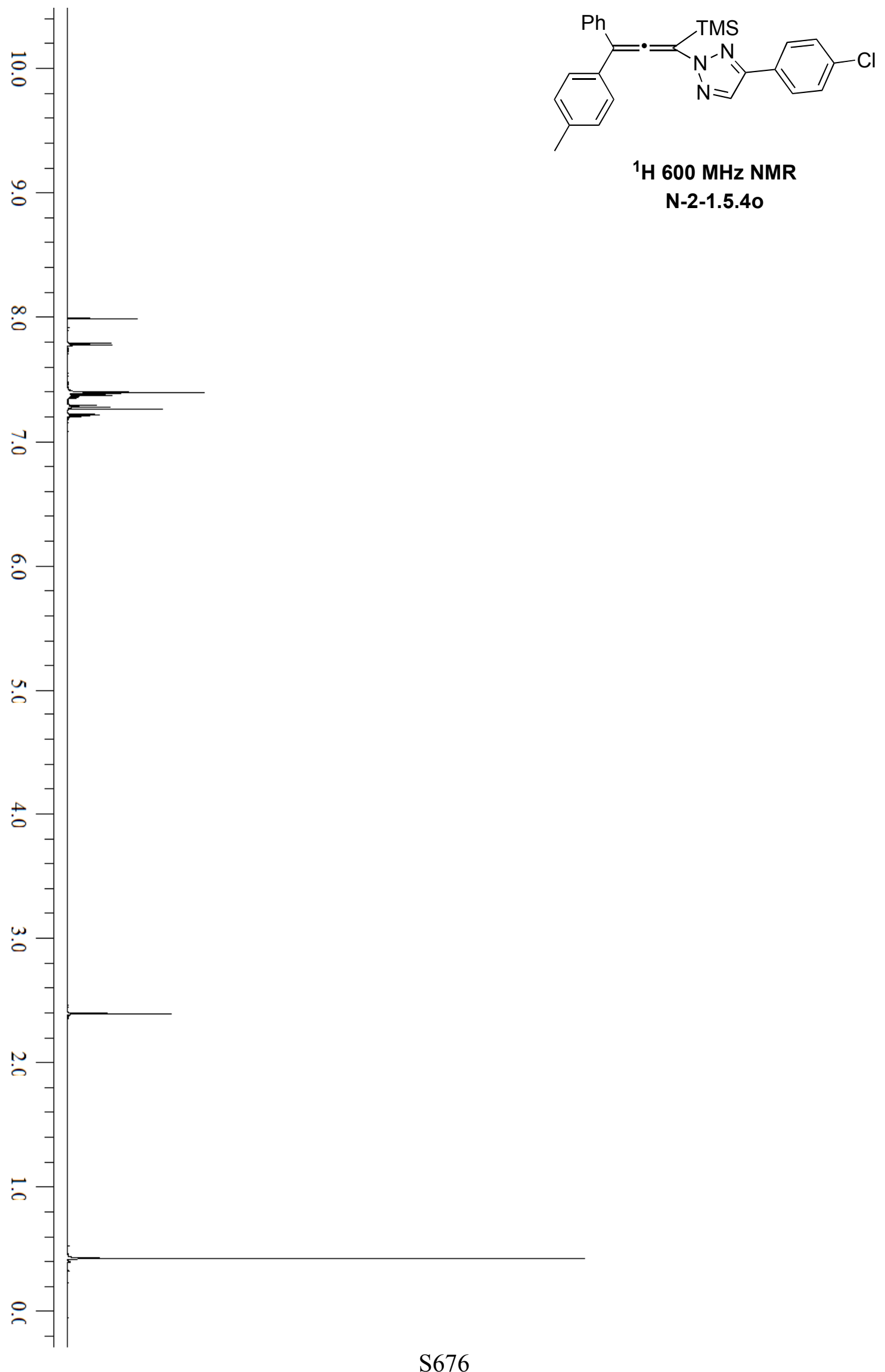



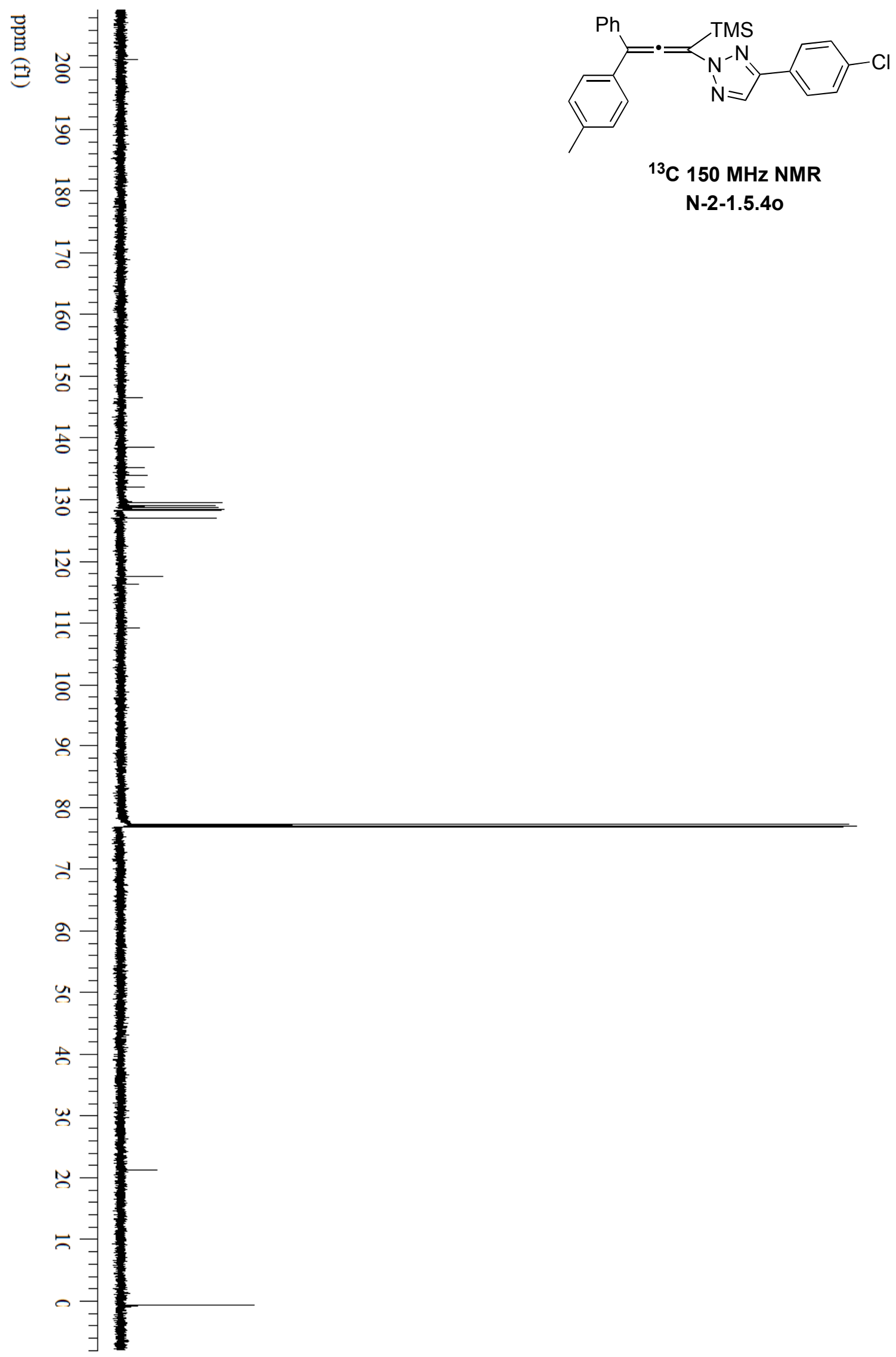

${ }^{13} \mathrm{C} 150 \mathrm{MHz}$ NMR

N-2-1.5.4o 


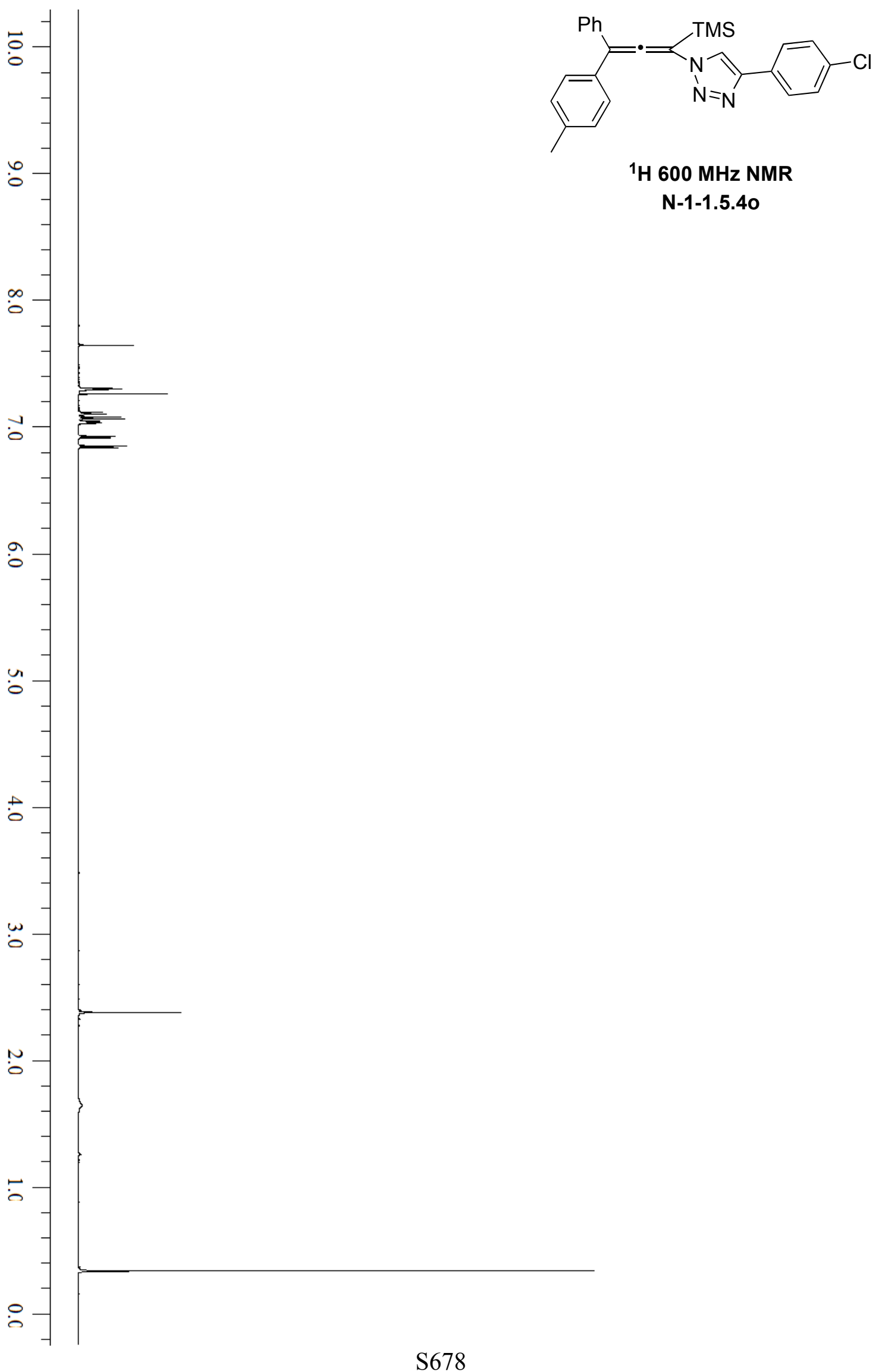




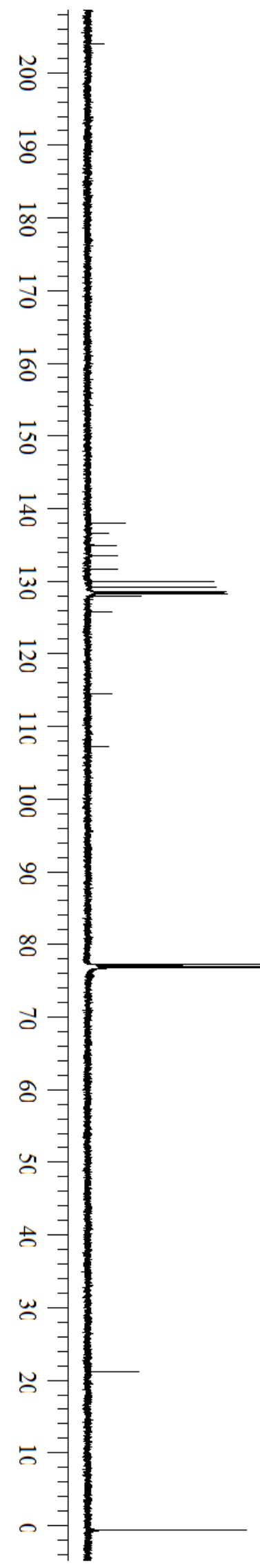

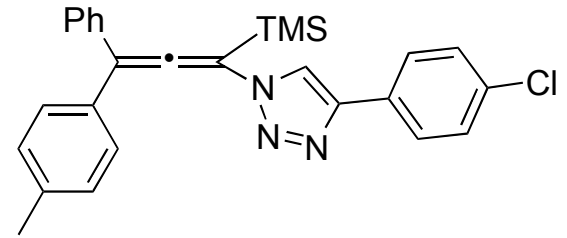

${ }^{13} \mathrm{C} 150 \mathrm{MHz}$ NMR

$\mathrm{N}-1-1.5 .40$ 

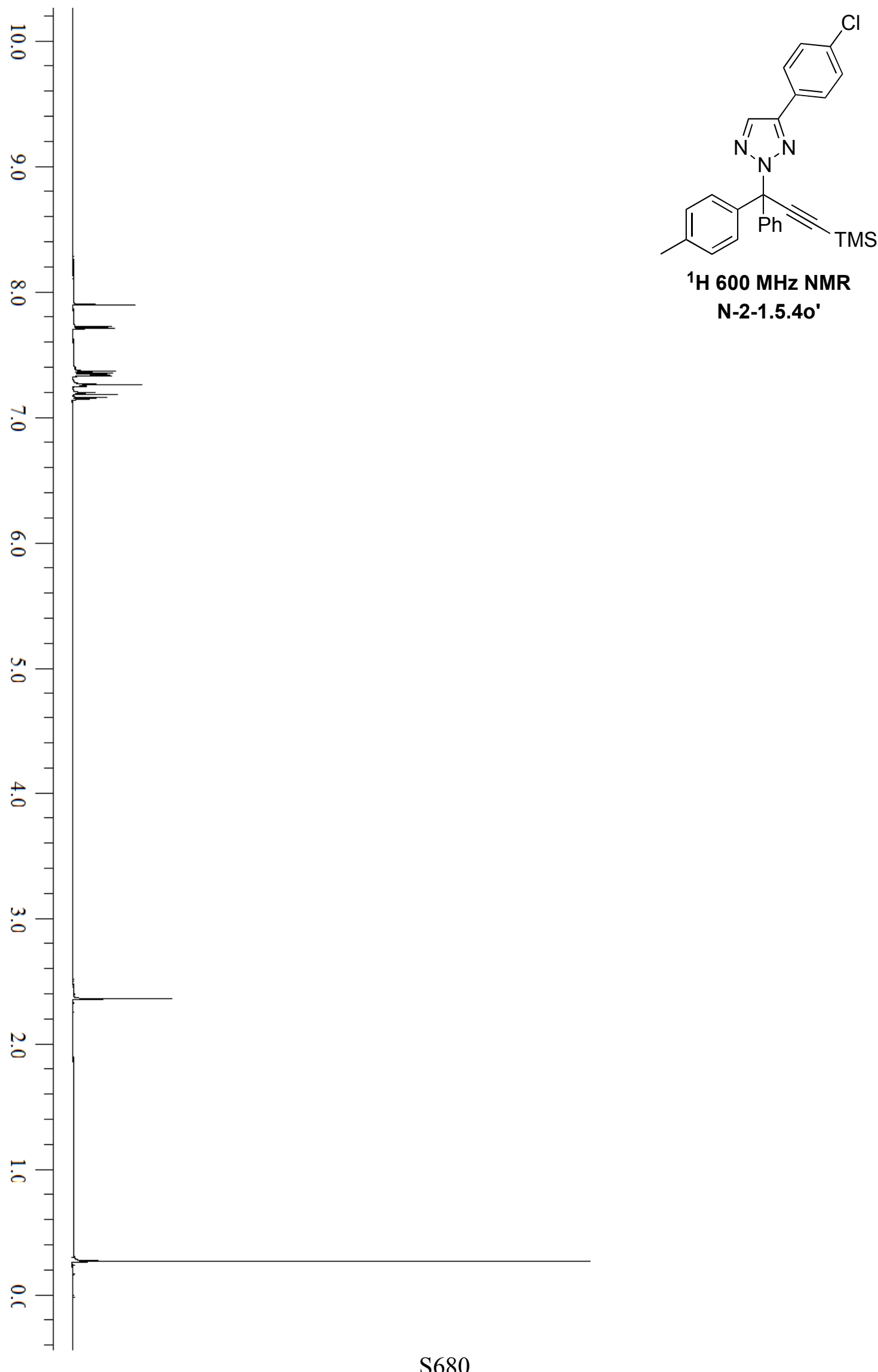

${ }^{1} \mathrm{H} 600 \mathrm{MHz}$ NMR

N-2-1.5.40' 

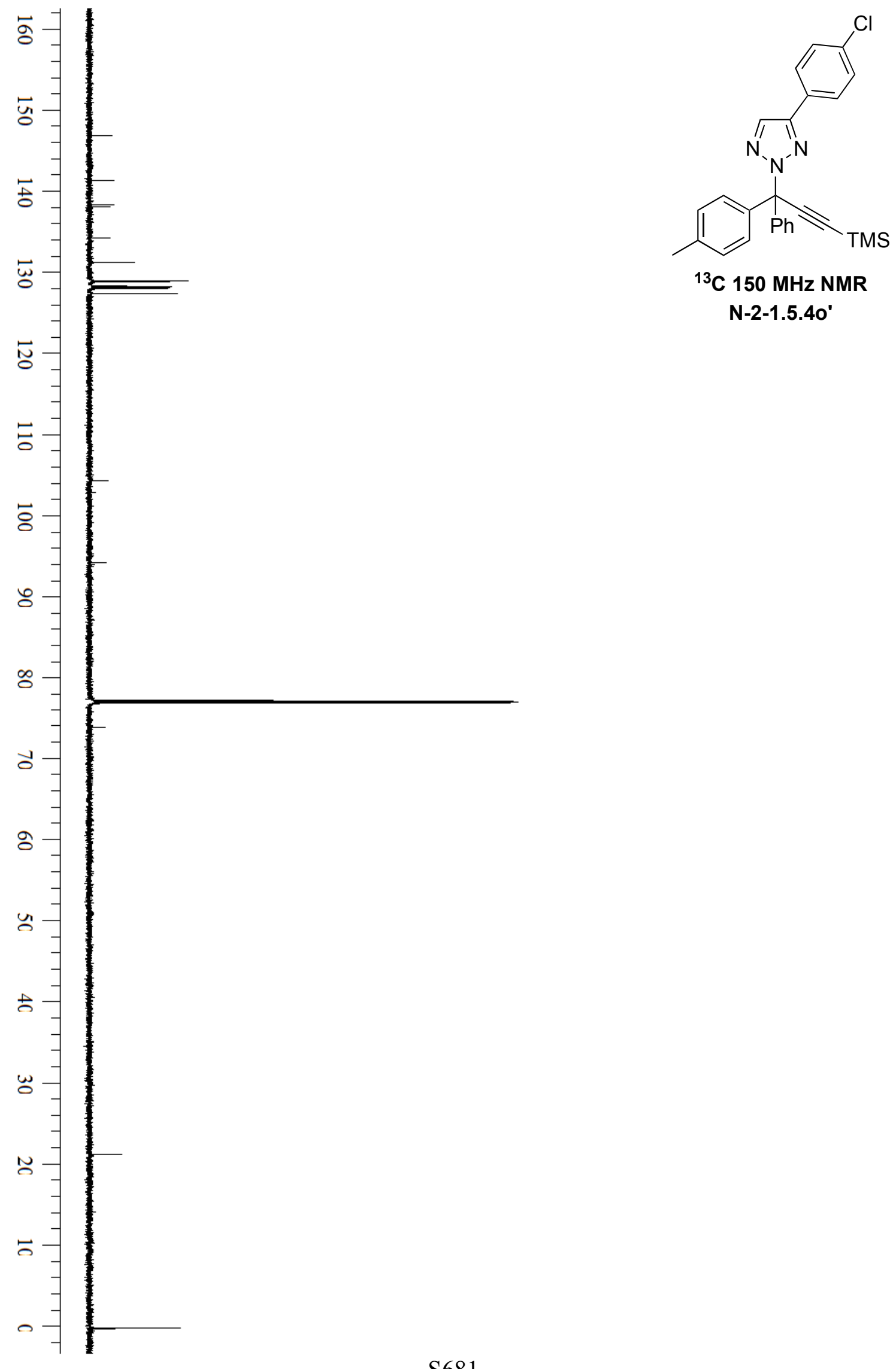

N-2-1.5.40' 


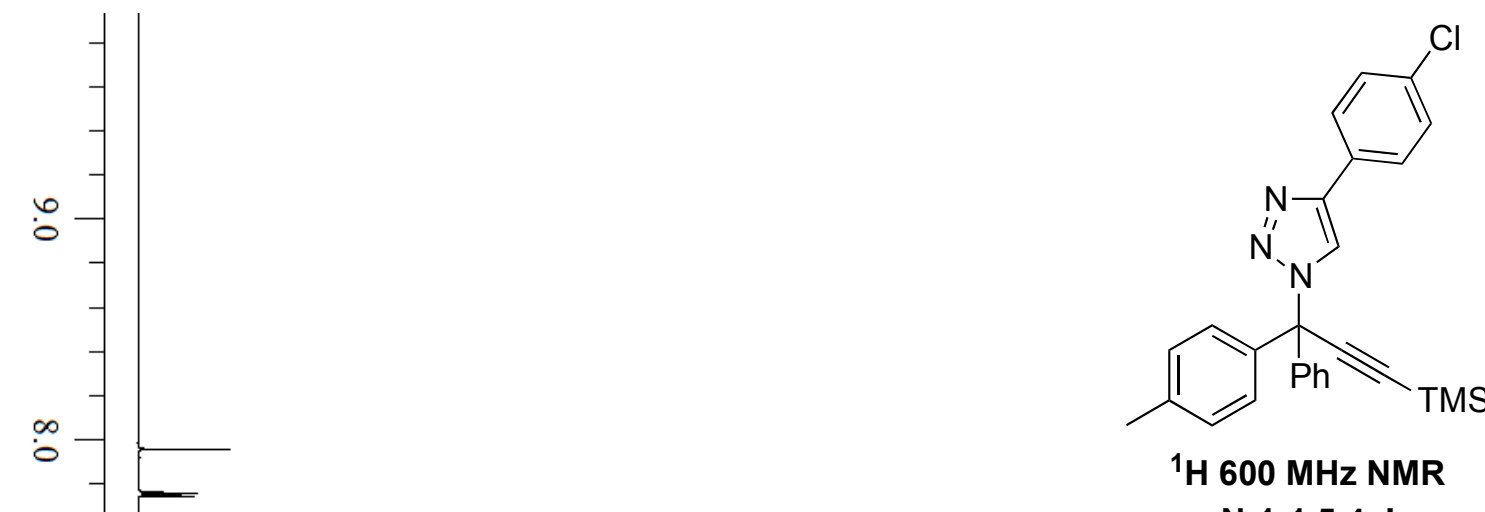

$\mathrm{N}-1-1.5 .40^{\circ}$

$E$

¿

0

$\dot{0}$

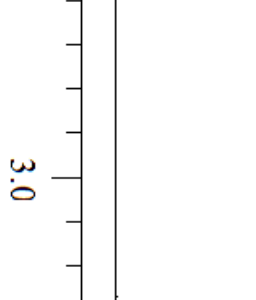

u

o

-

-

-
-1
-
-1
-1
-
-

0

$-1$ 


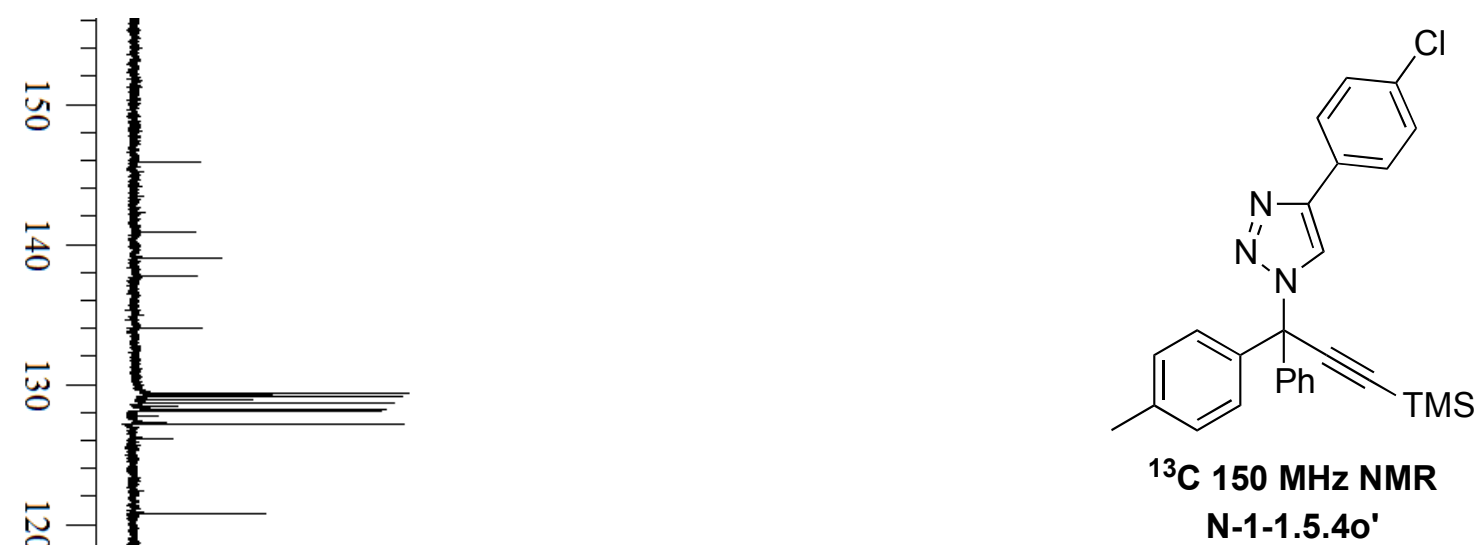

$\mathrm{N}-1-1.5 .40$ 


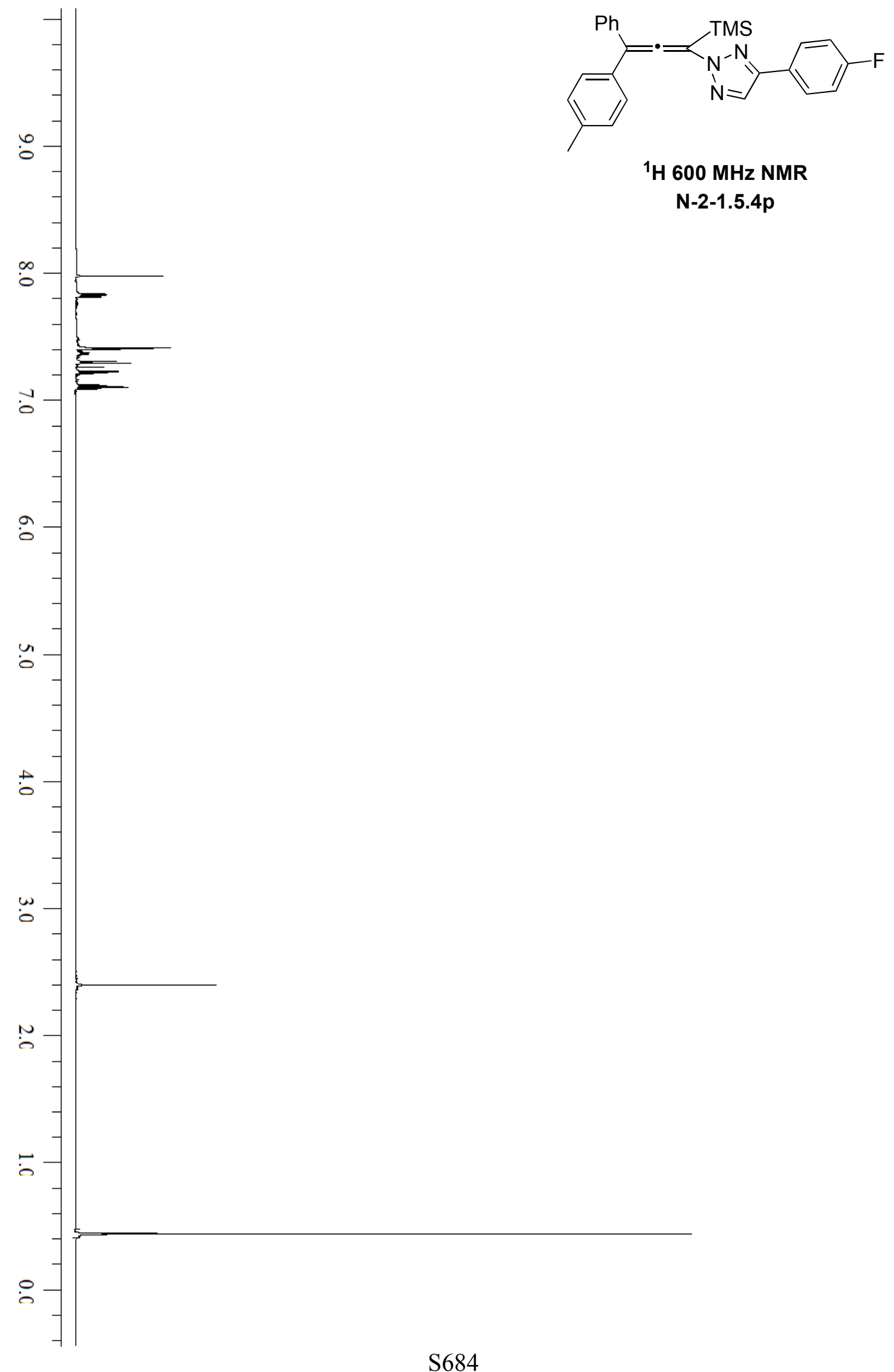



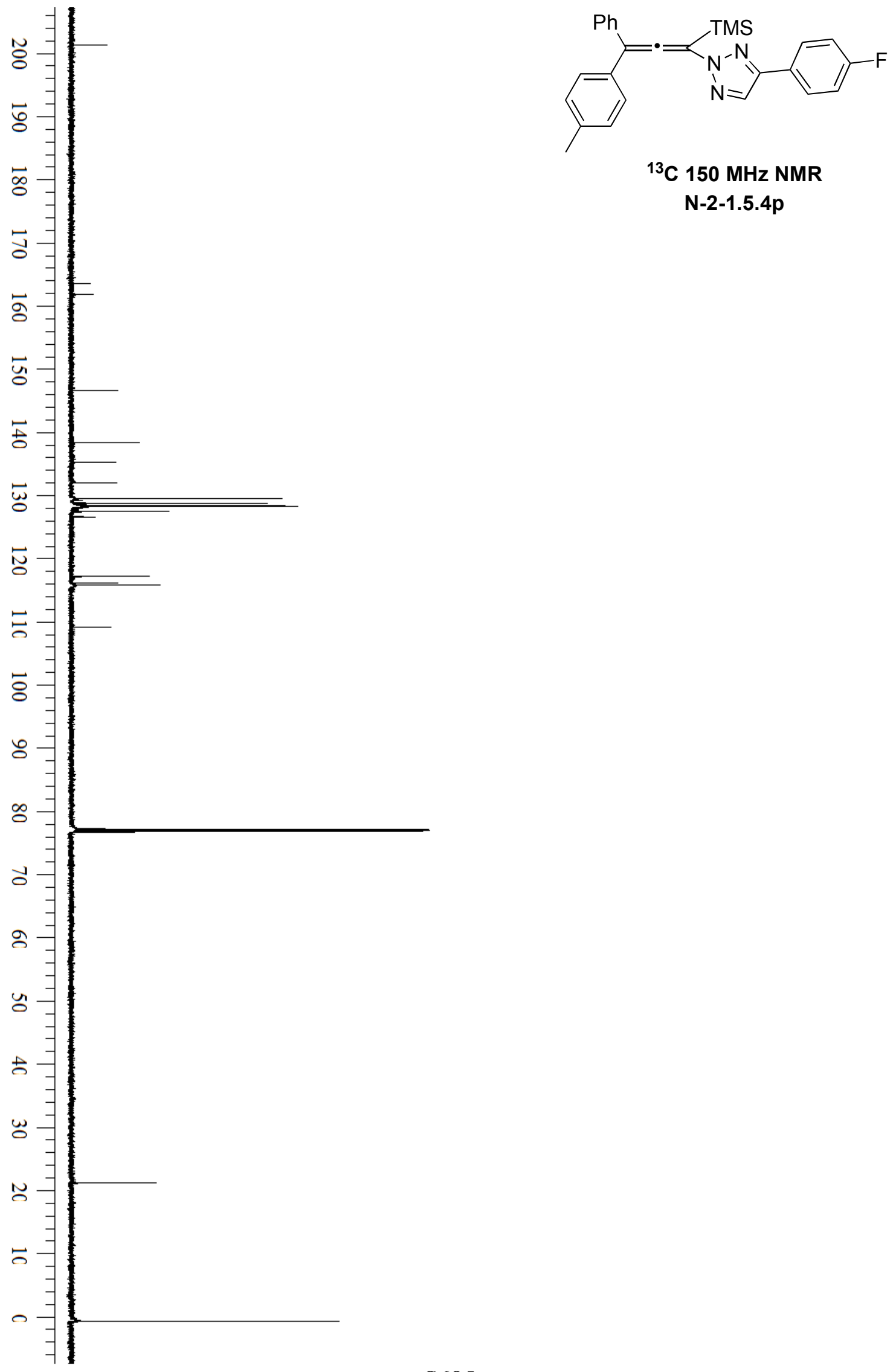


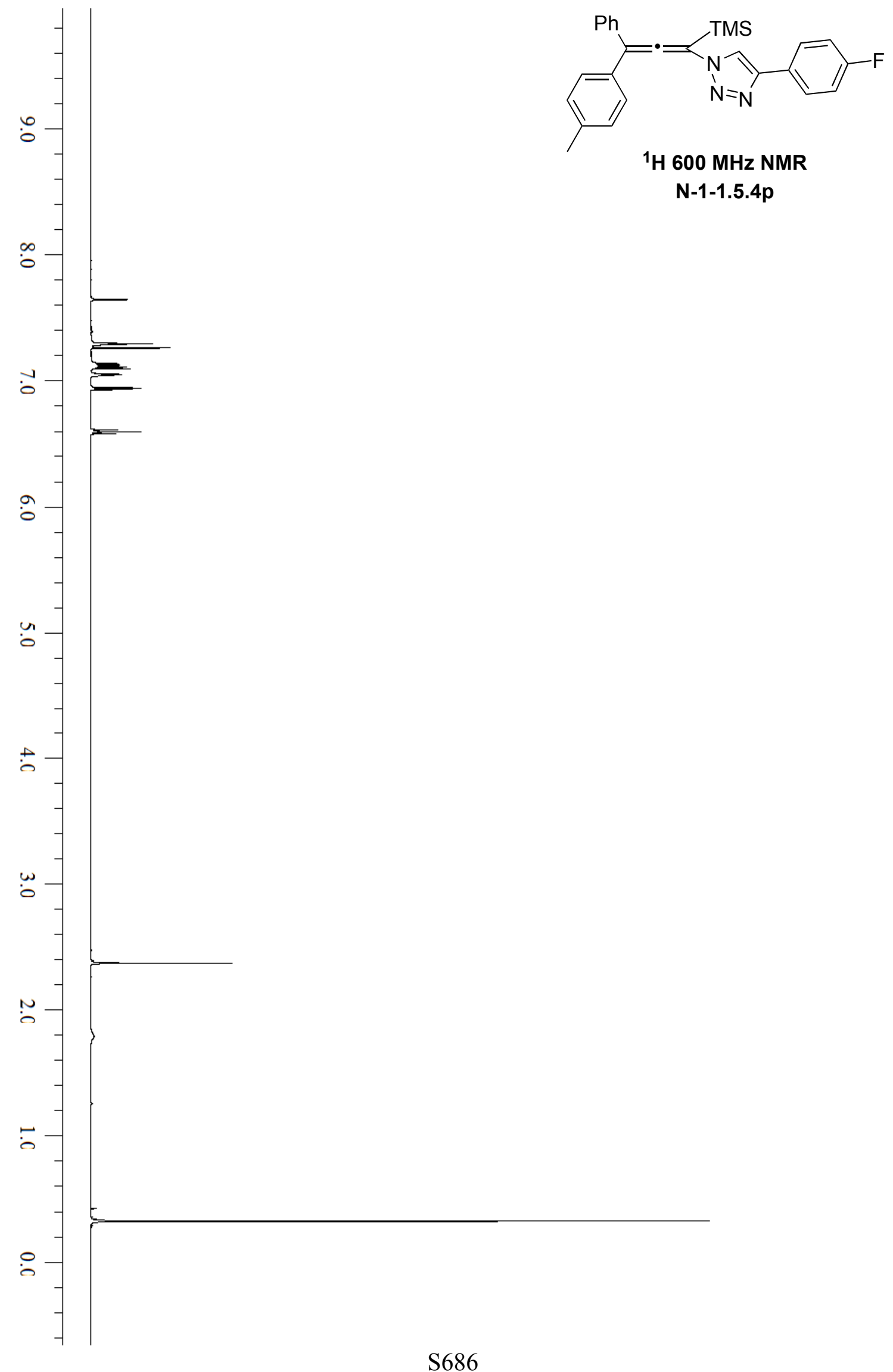



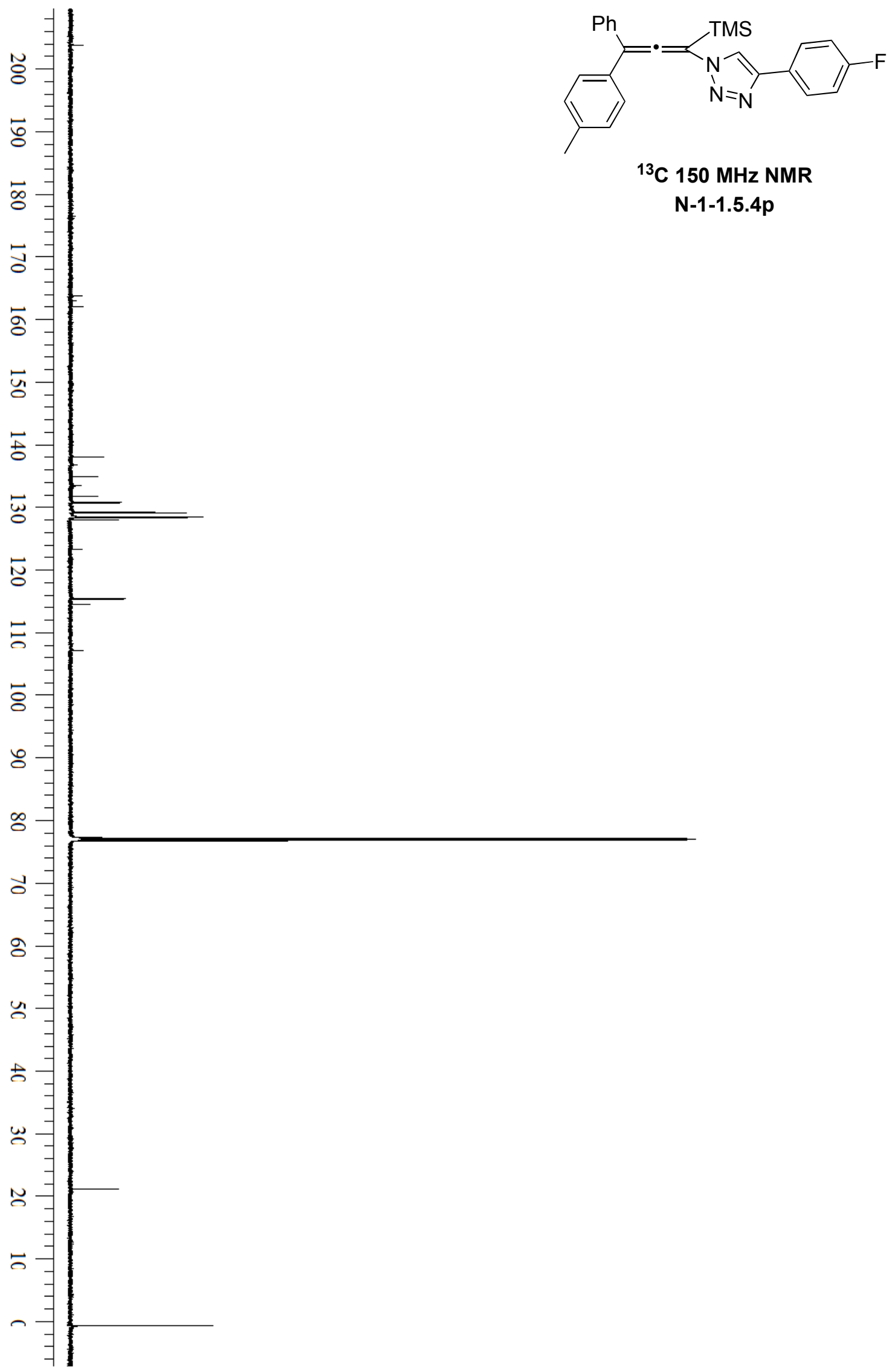

${ }^{13} \mathrm{C} 150$ MHz NMR

$\mathrm{N}-1-1.5 .4 p$ 

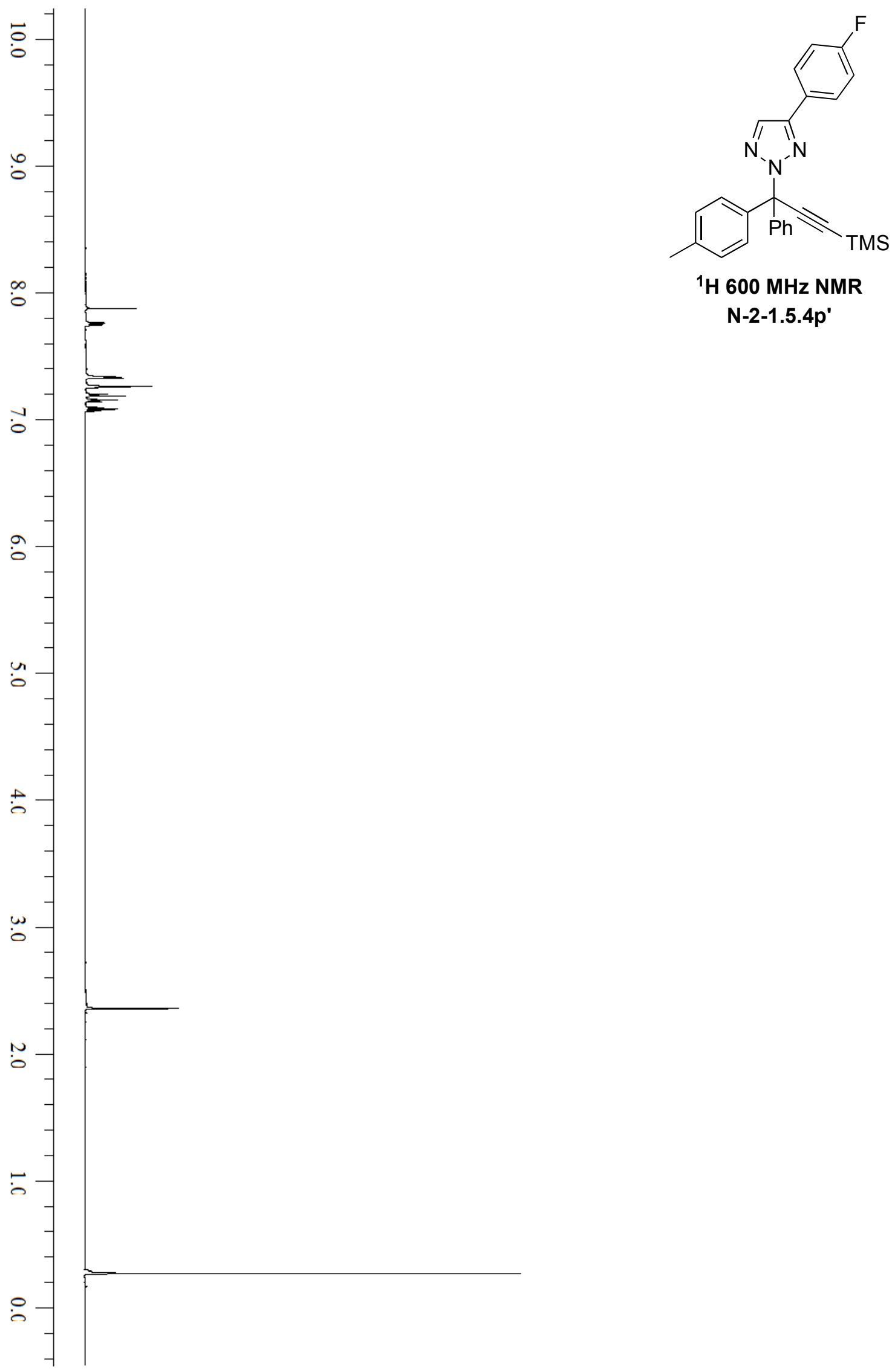

${ }^{1} \mathrm{H} 600 \mathrm{MHz}$ NMR

$\mathrm{N}-2-1.5 .4 p^{\prime}$ 

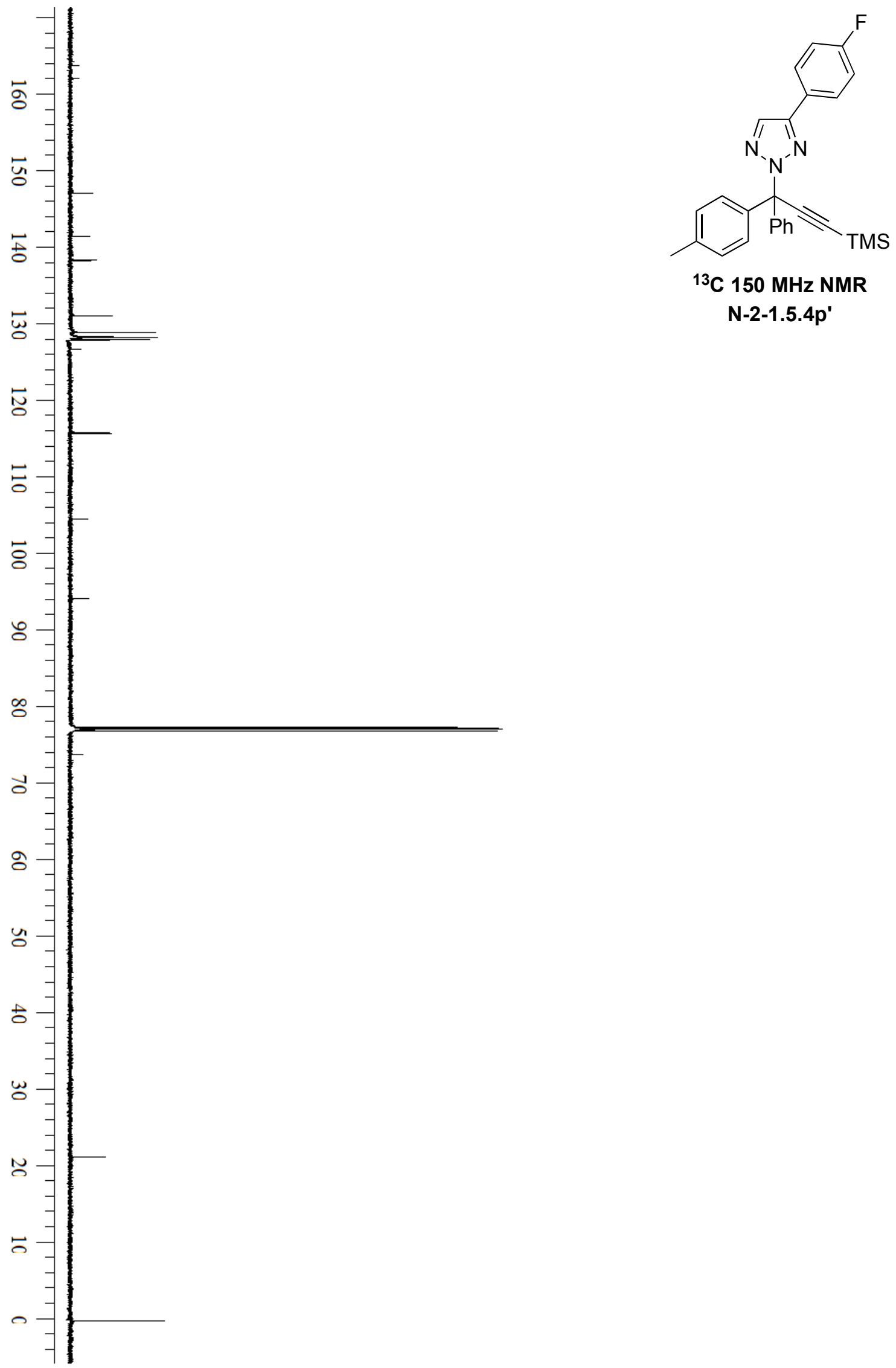

$\mathrm{N}-2-1.5 .4 p^{\prime}$ 


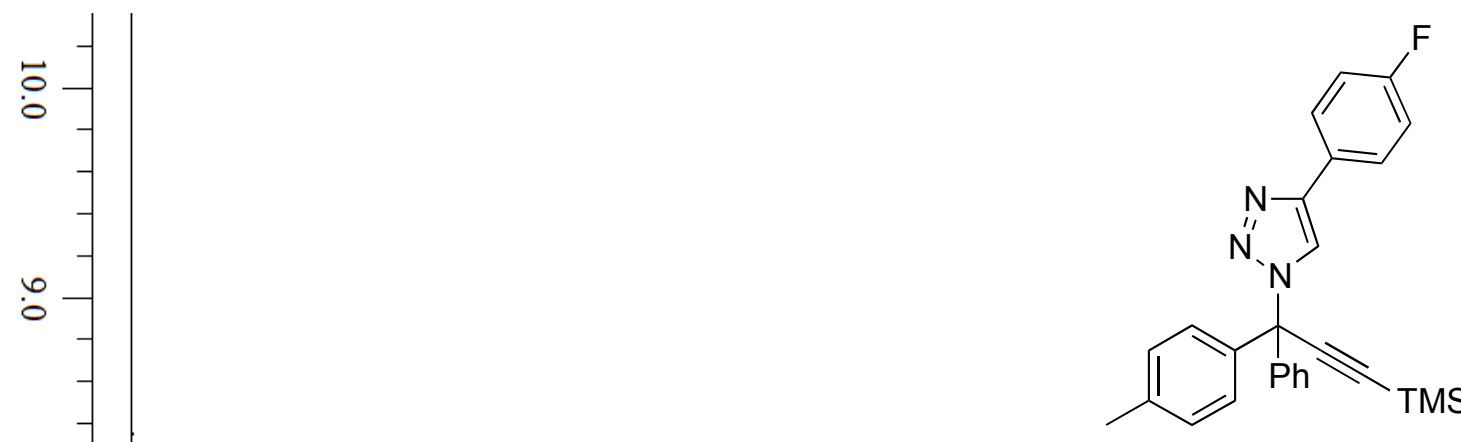

${ }^{1} \mathrm{H} 600 \mathrm{MHz}$ NMR

$\mathrm{N}-1-1.5 .4 p^{\prime}$

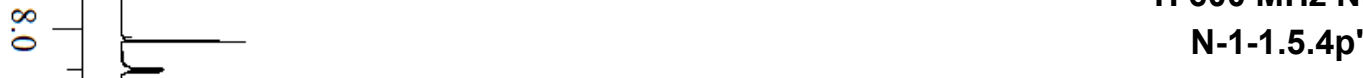

2

u

$\stackrel{0}{0}$

$=$

J
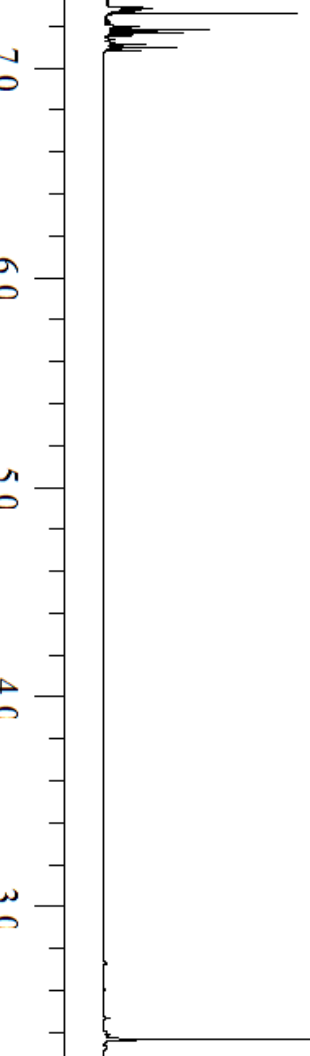

no

- 


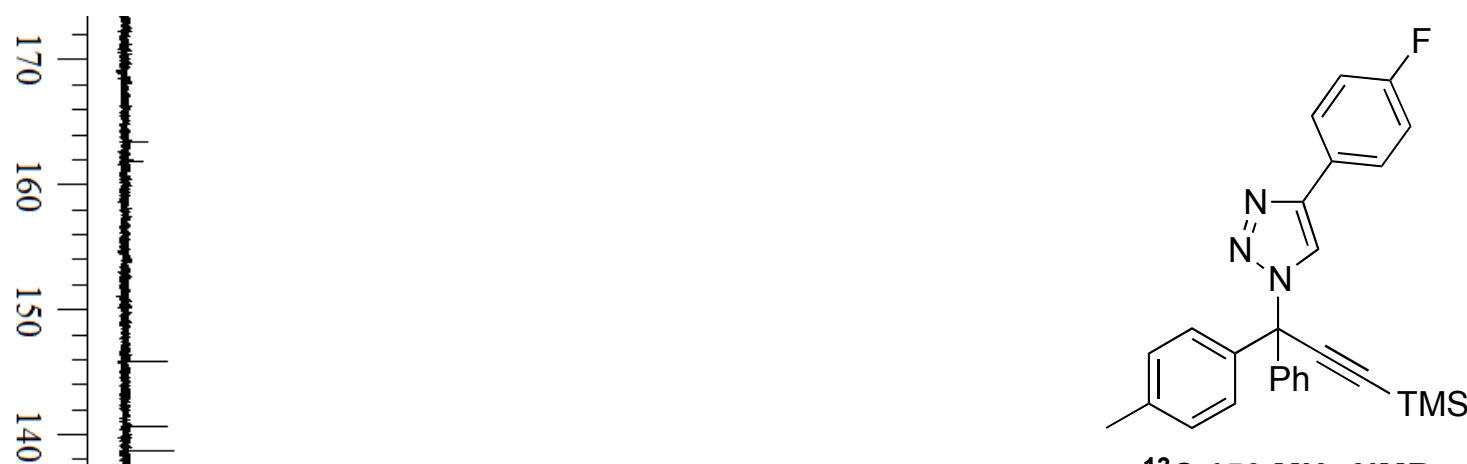

${ }^{13} \mathrm{C} 150 \mathrm{MHz}$ NMR $\mathrm{N}-1-1.5 .4 p^{\prime}$

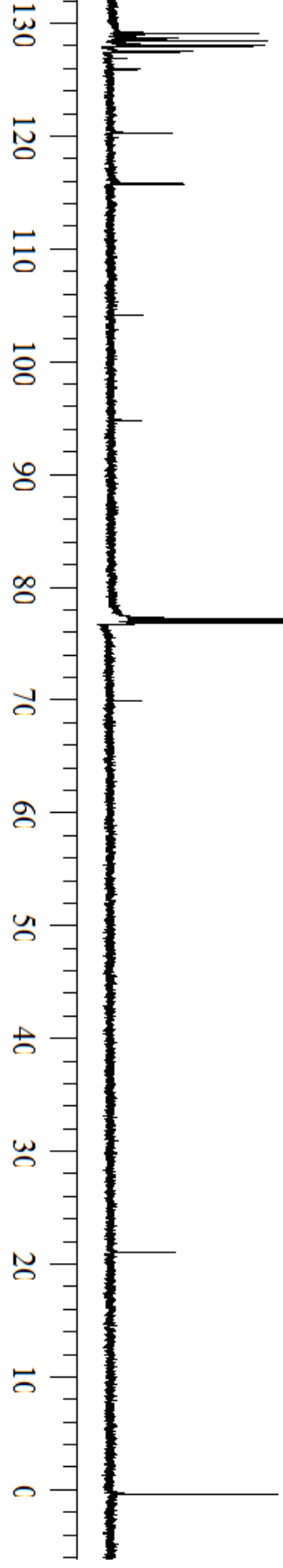




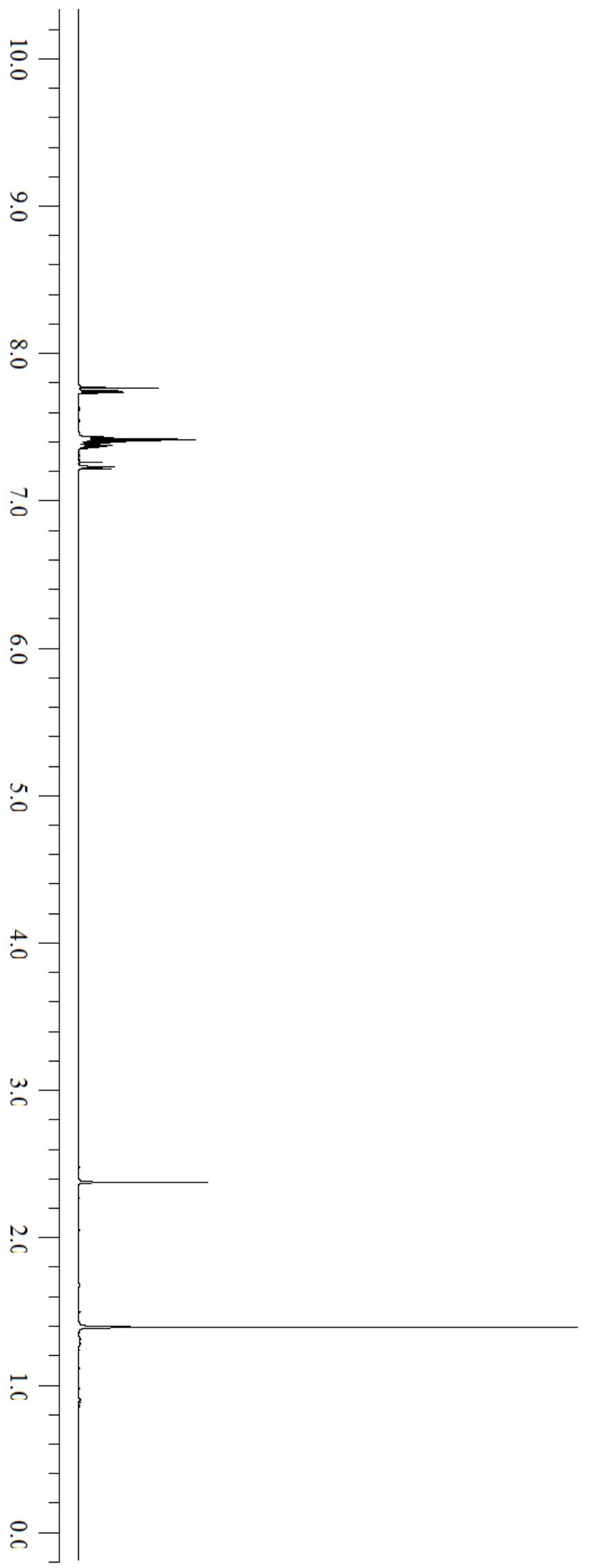

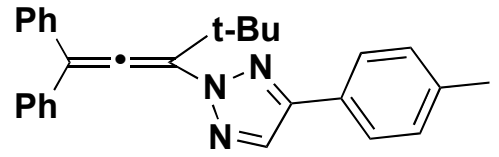

${ }^{1} \mathrm{H} 600 \mathrm{MHz}$ NMR

$\mathrm{N}-2-1.5 .4 \mathrm{q}$ 


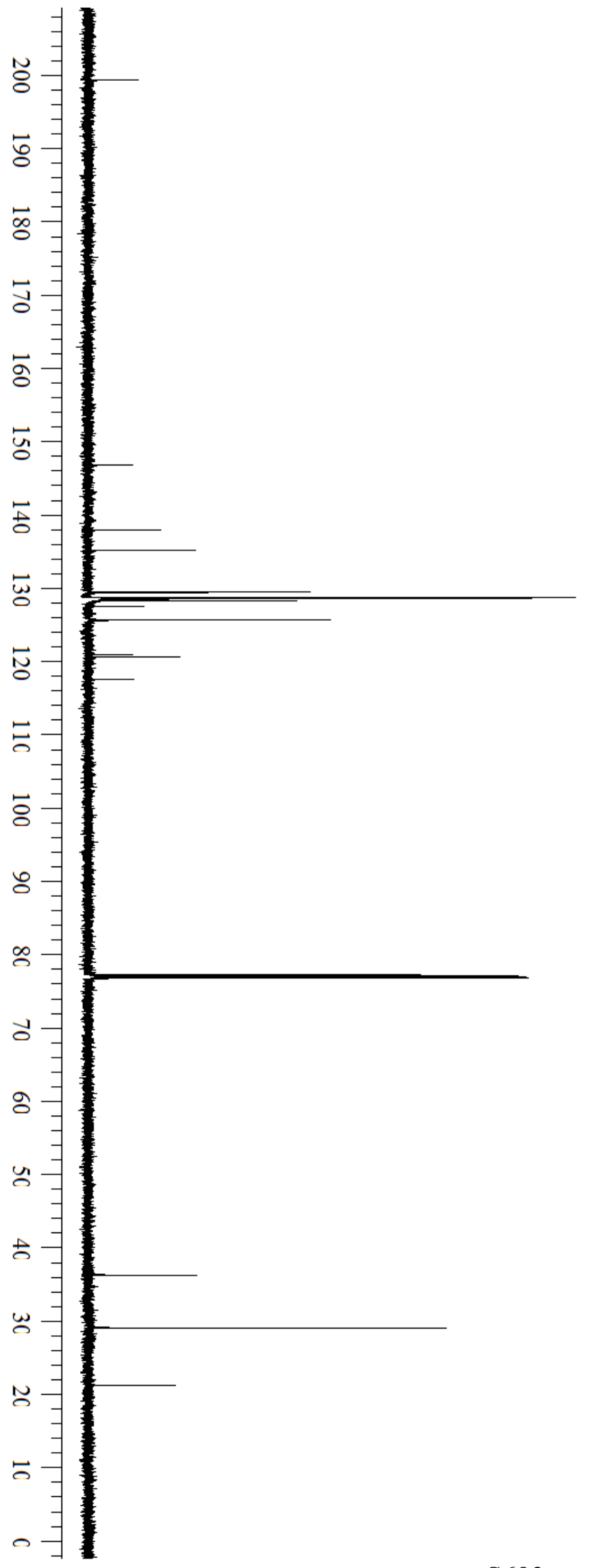

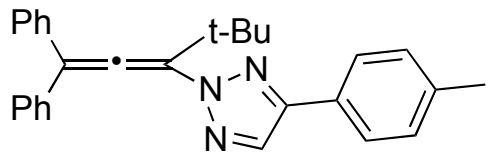

${ }^{13} \mathrm{C} 150 \mathrm{MHz}$ NMR

$\mathrm{N}-2-1.5 .4 \mathrm{q}$ 

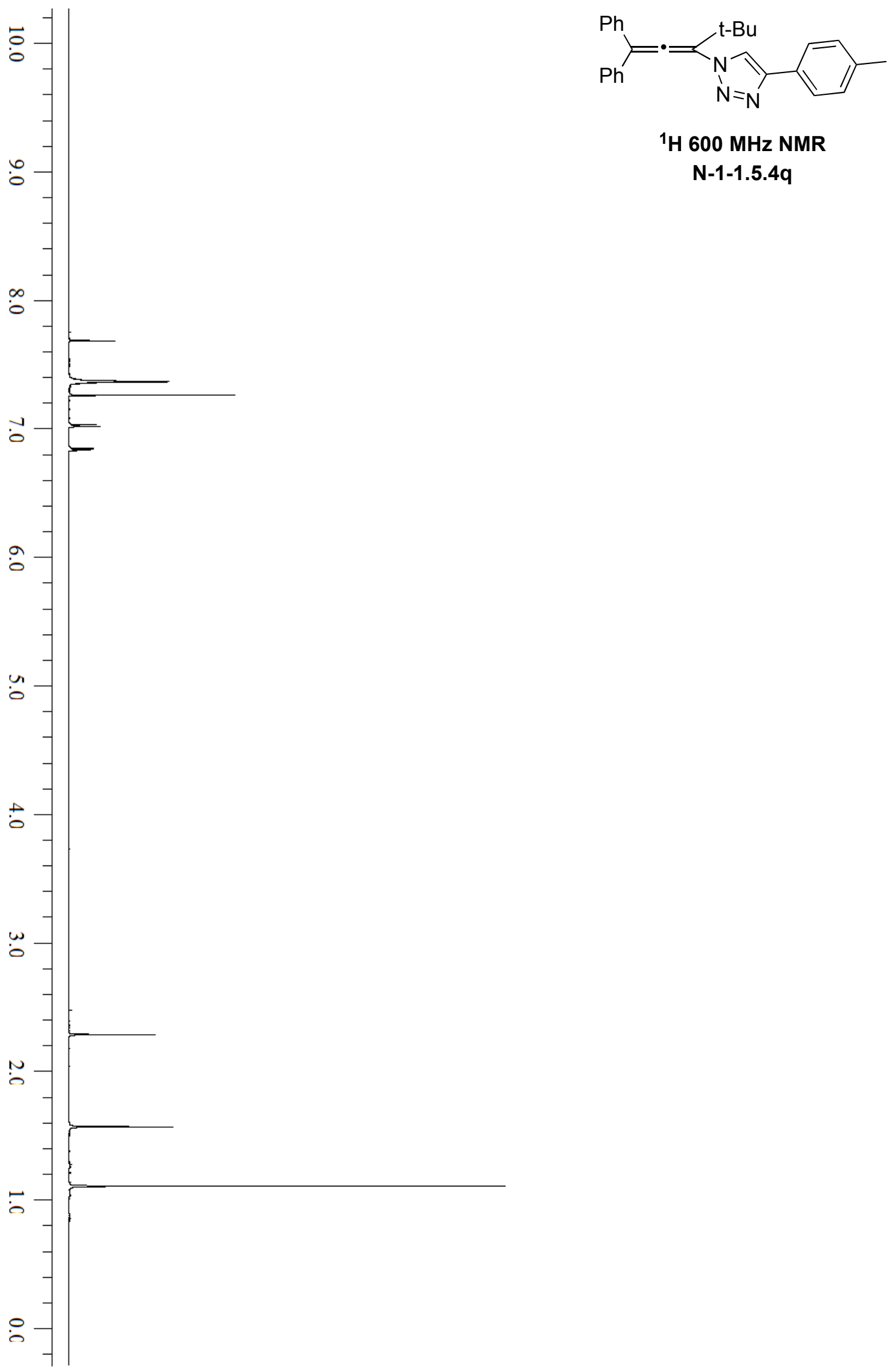

${ }^{1} \mathrm{H} 600 \mathrm{MHz}$ NMR

$\mathrm{N}-1-1.5 .4 q$ 


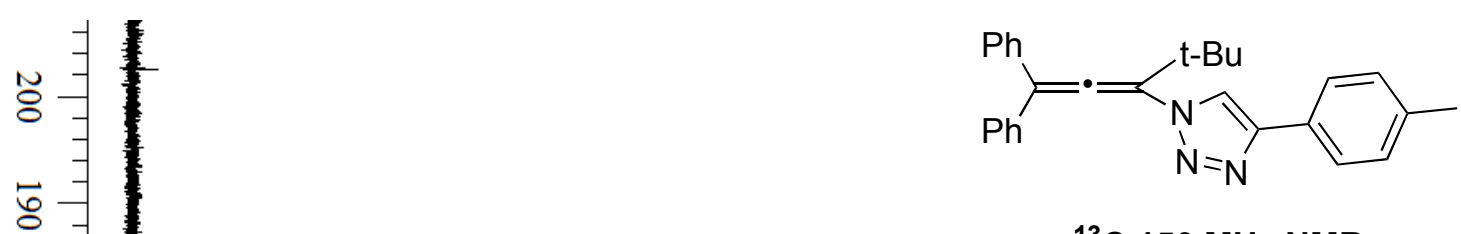

${ }^{13} \mathrm{C} 150 \mathrm{MHz}$ NMR

$\mathrm{N}-1-1.5 .4 q$

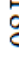

s

晏

동

咅

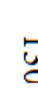

客

호

s
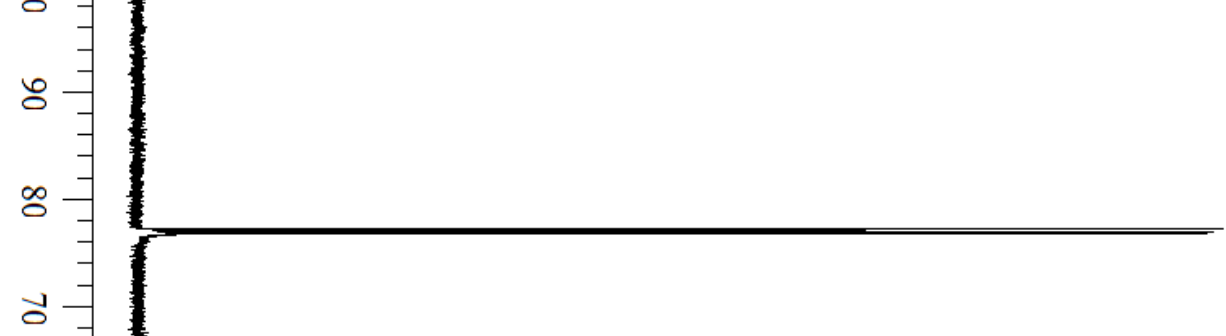

๖

g

-

닝-

a

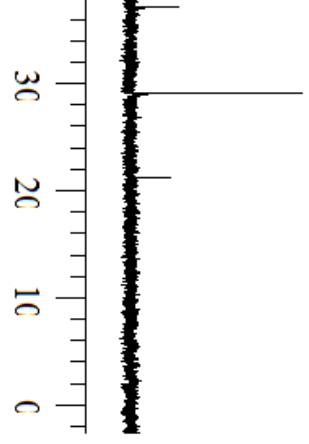




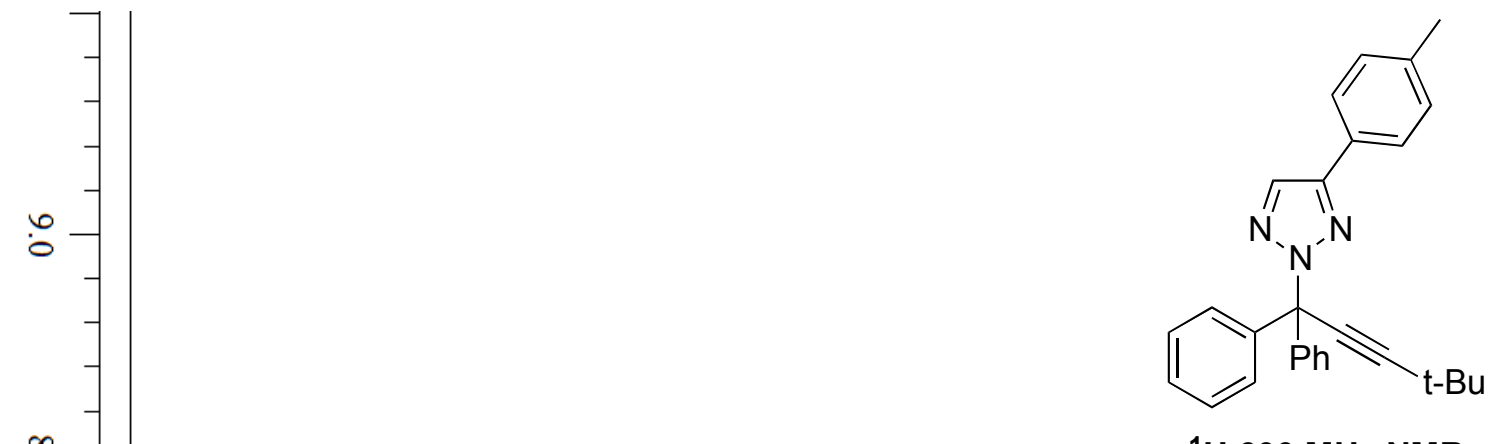

${ }^{1} \mathrm{H} 600 \mathrm{MHz}$ NMR

$\mathrm{N}-2-1.5 .4 q^{\prime}$ 

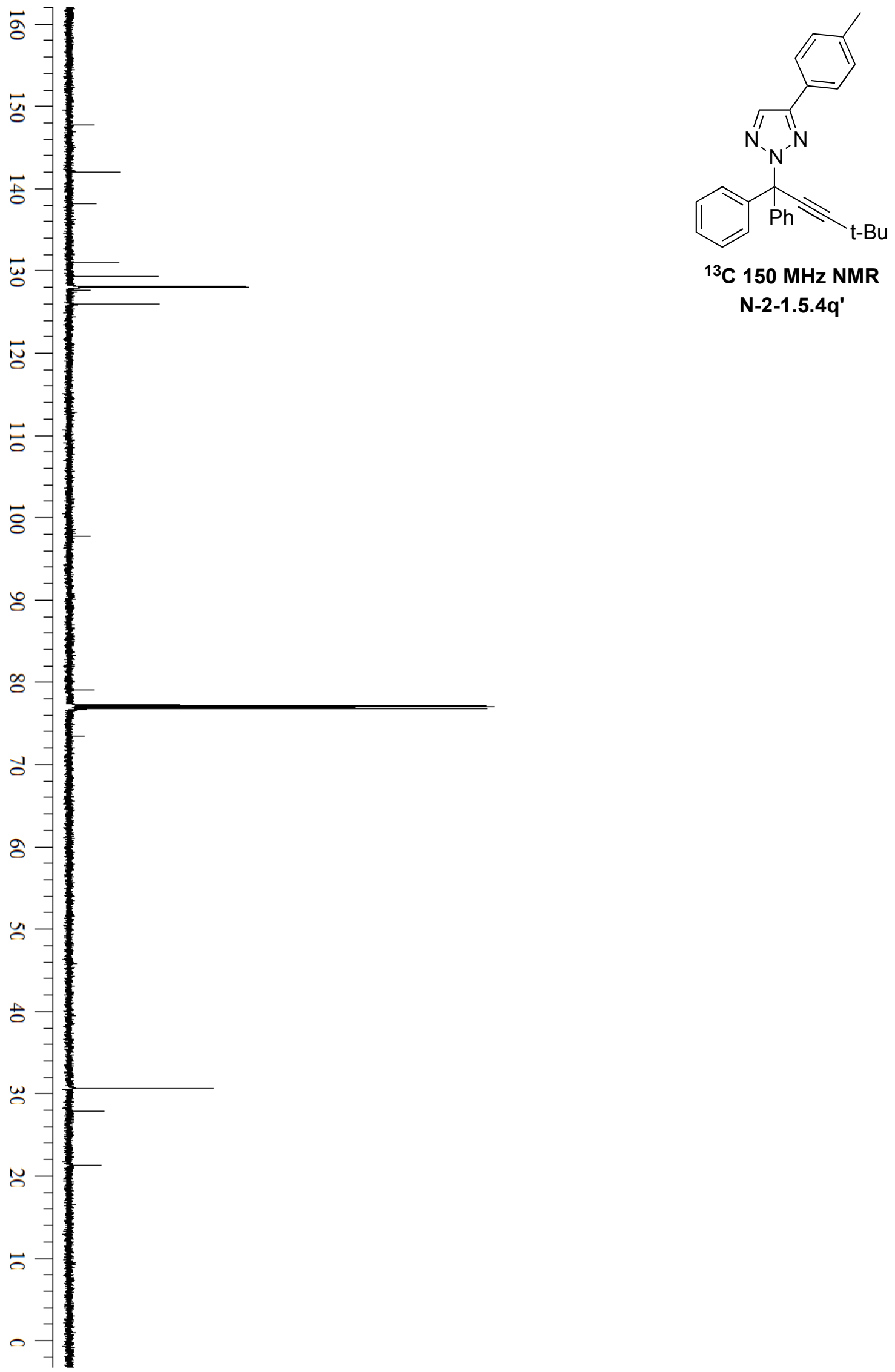

${ }^{13} \mathrm{C} 150 \mathrm{MHz}$ NMR

$\mathrm{N}-2-1.5 .4 q^{\prime}$ 


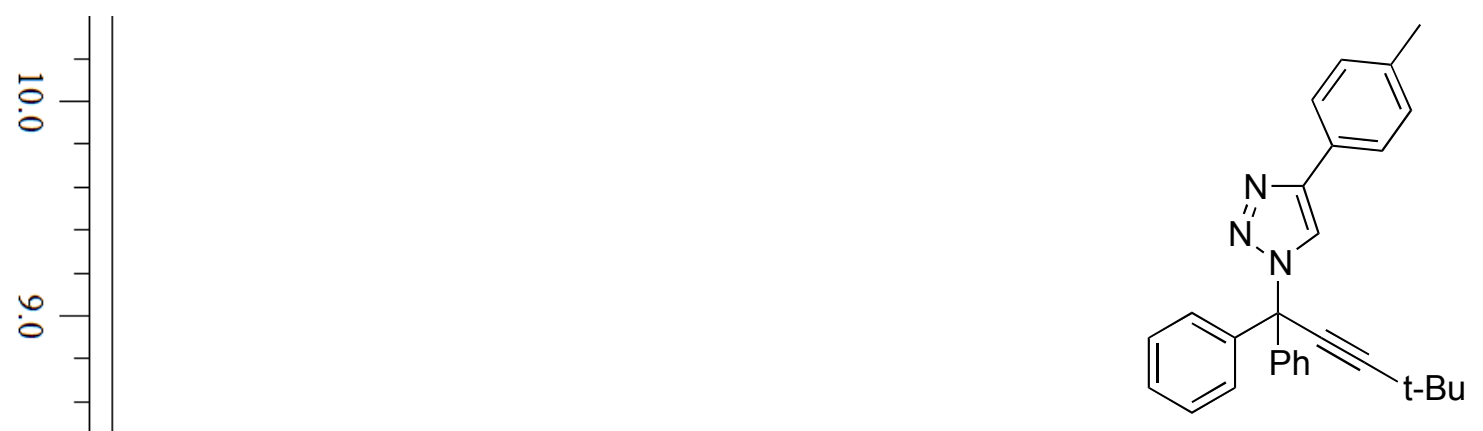

${ }^{1} \mathrm{H} 600 \mathrm{MHz}$ NMR

$\mathrm{N}-1-1.5 .4 q^{\prime}$

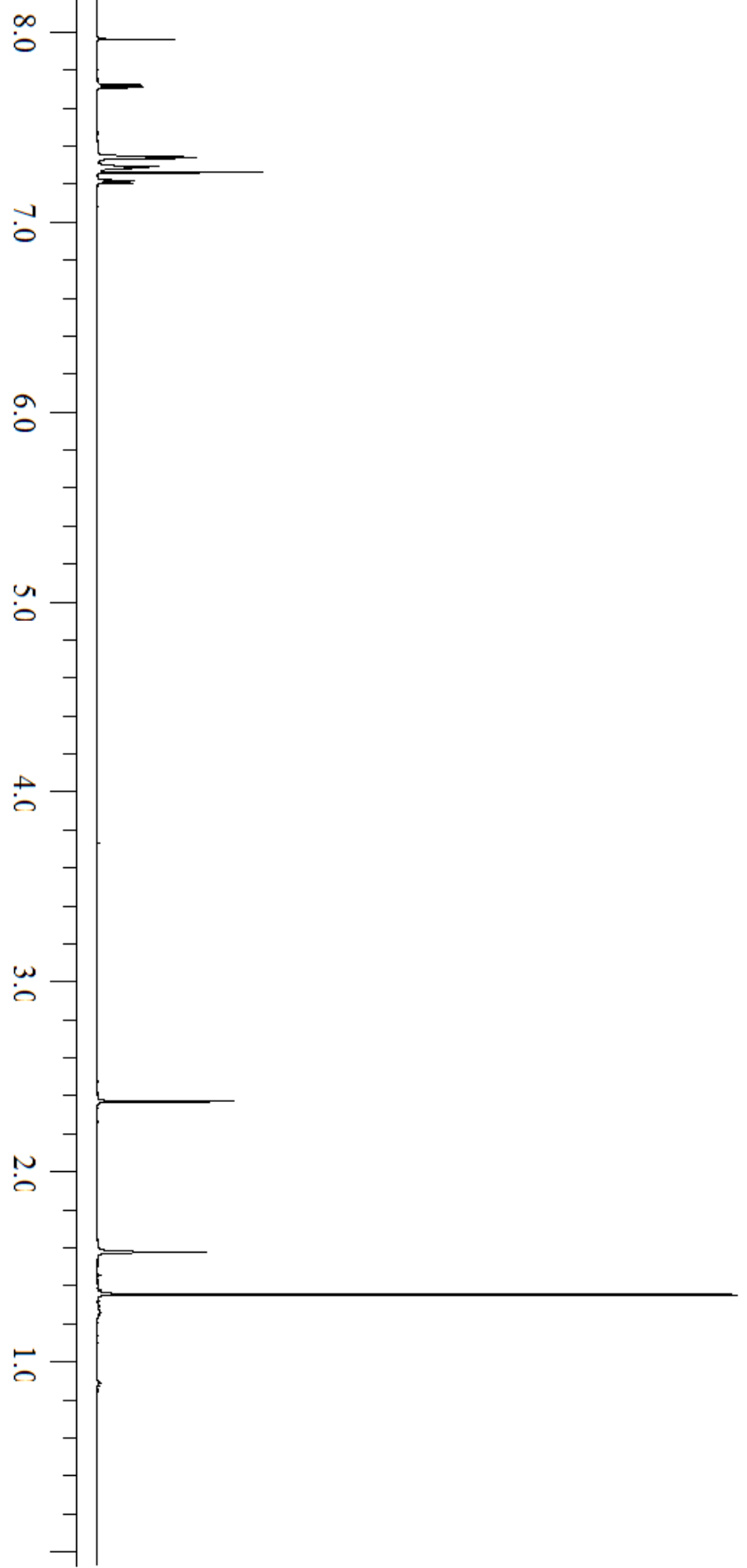




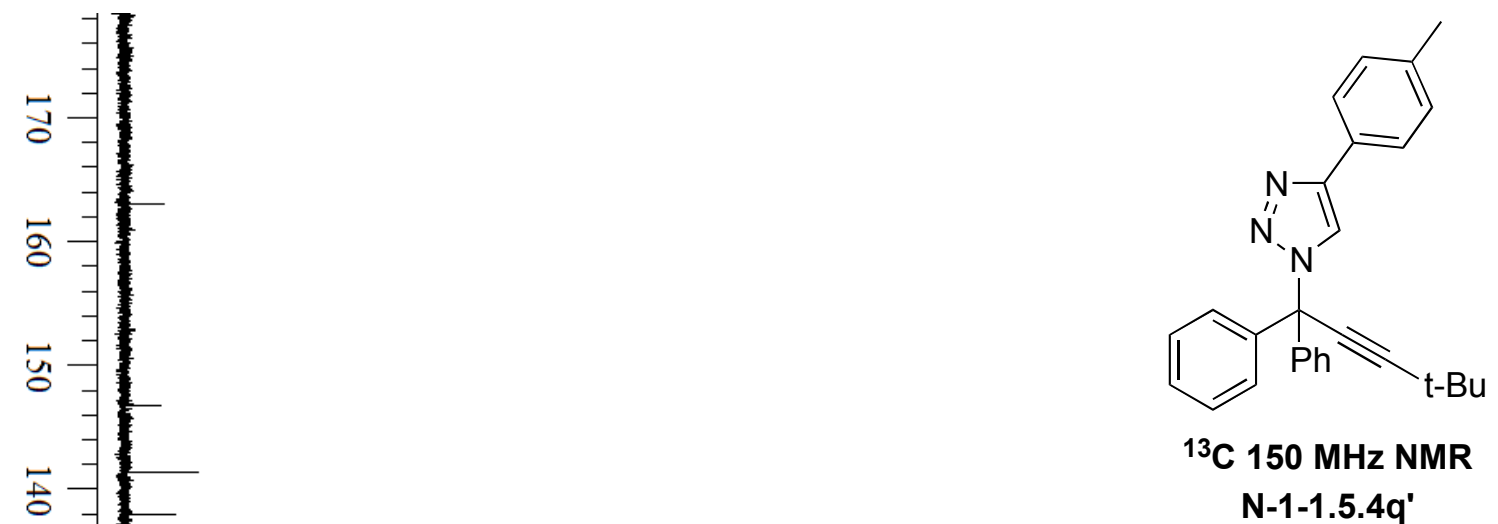

$\mathrm{N}-1-1.5 .4 q^{\prime}$ 


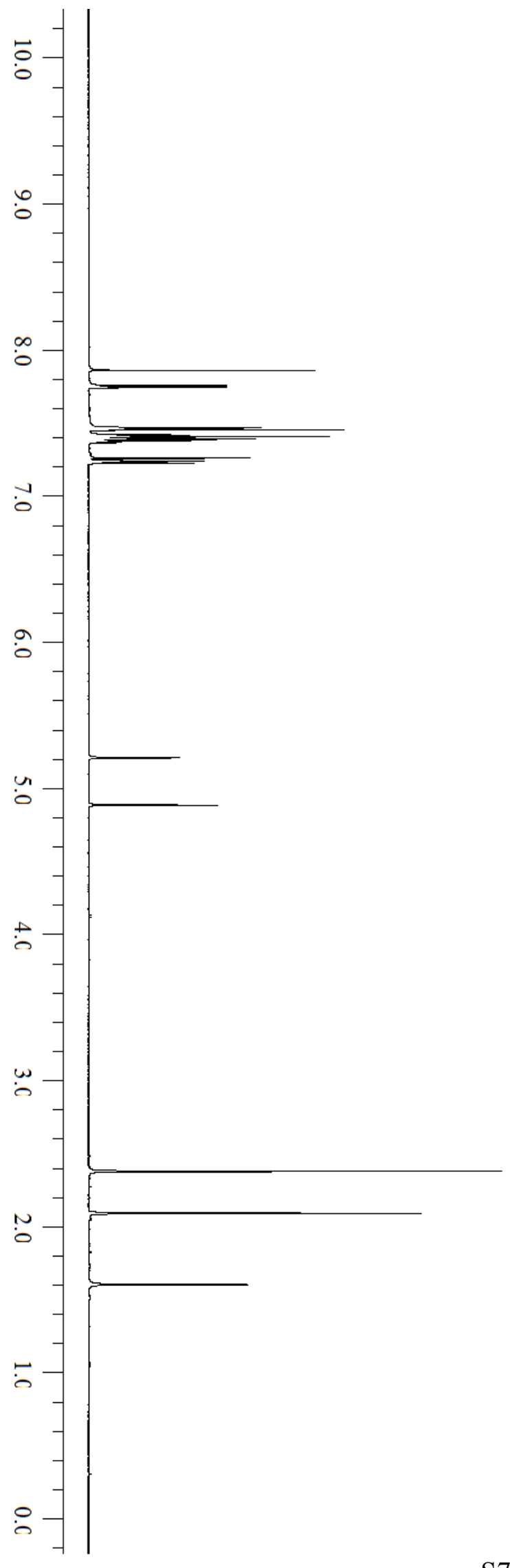

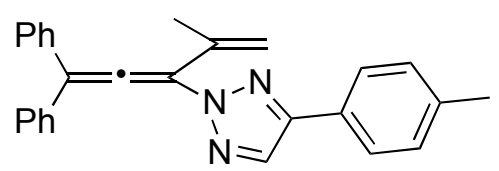

${ }^{1} \mathrm{H} 600 \mathrm{MHz}$ NMR

$\mathrm{N}-2-1.5 .4 \mathrm{r}$ 

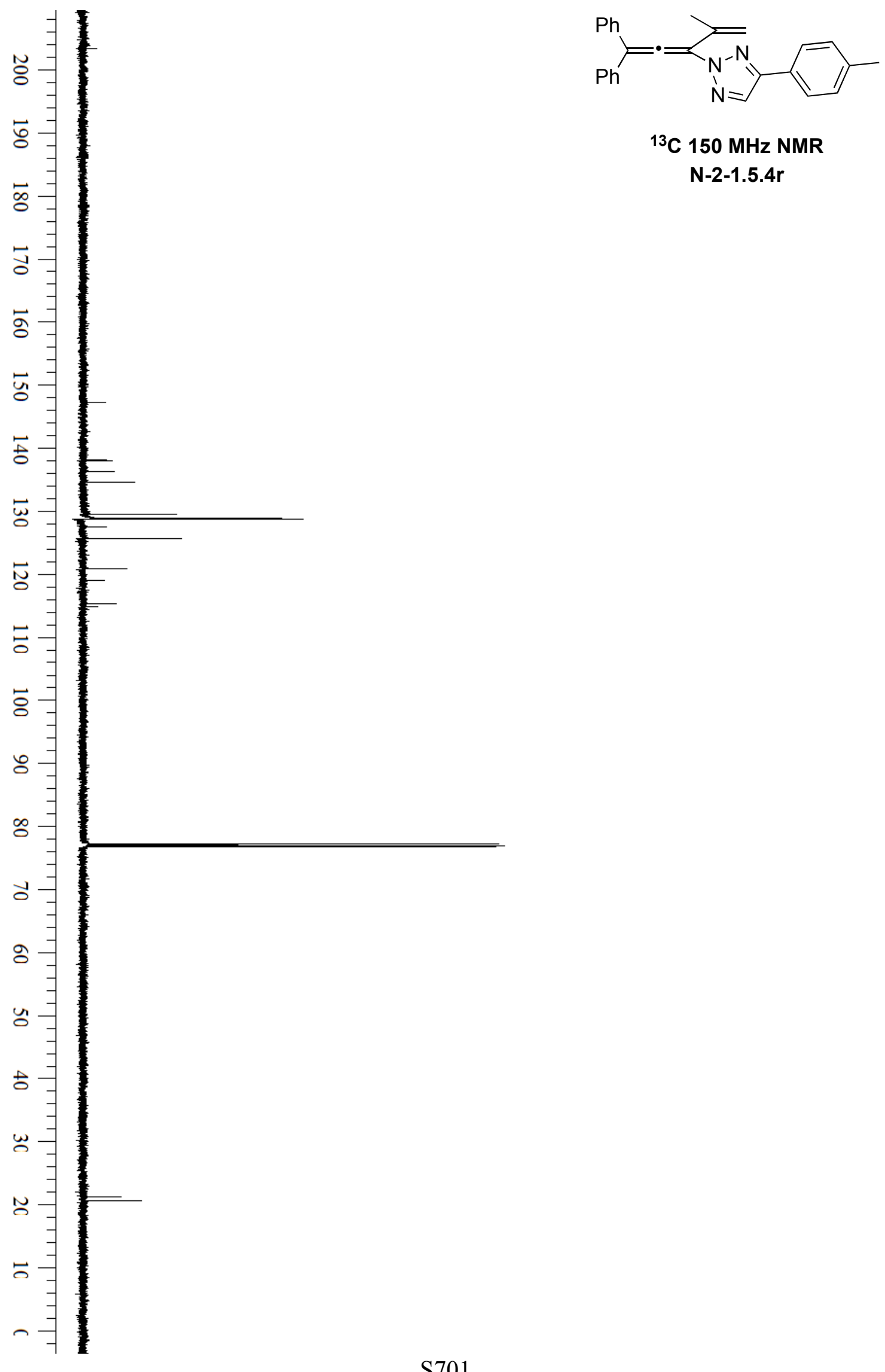

${ }^{13} \mathrm{C} 150 \mathrm{MHz}$ NMR

$\mathrm{N}-2-1.5 .4 \mathrm{r}$ 


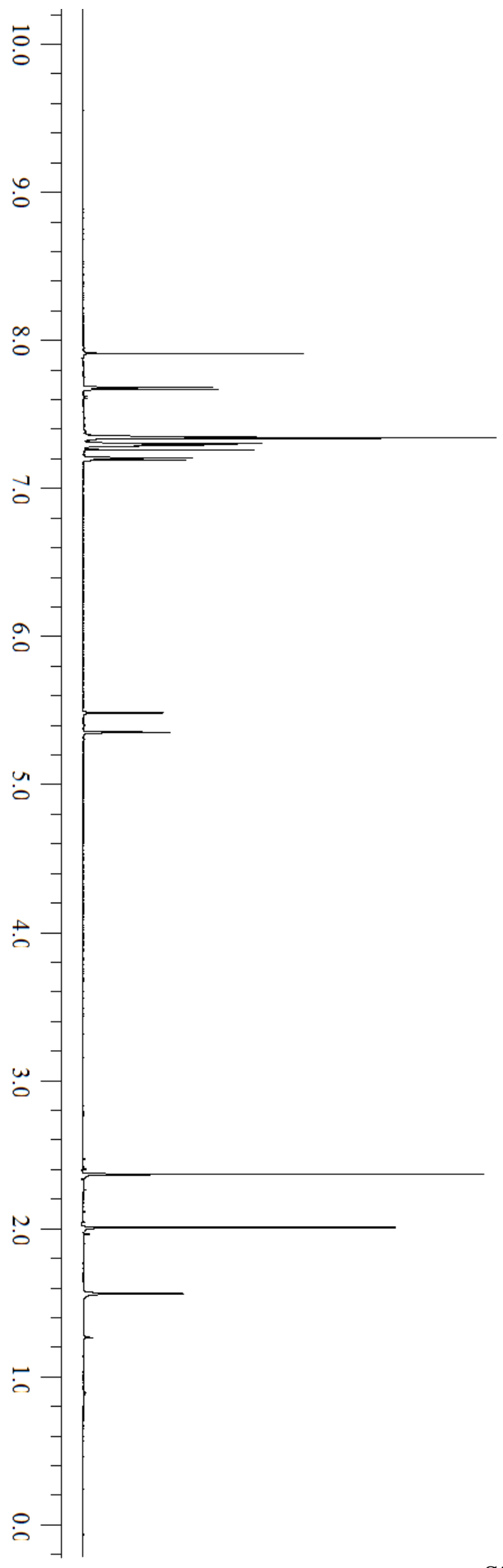

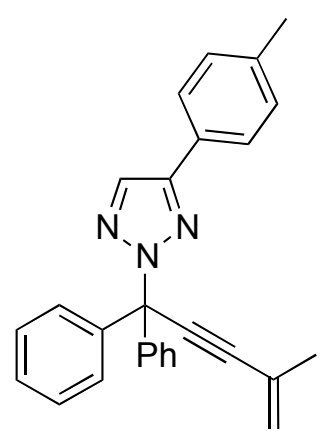

${ }^{1} \mathrm{H} 600 \mathrm{MHz}$ NMR

$\mathrm{N}-2-1.5 .4 \mathrm{r}^{\prime}$ 

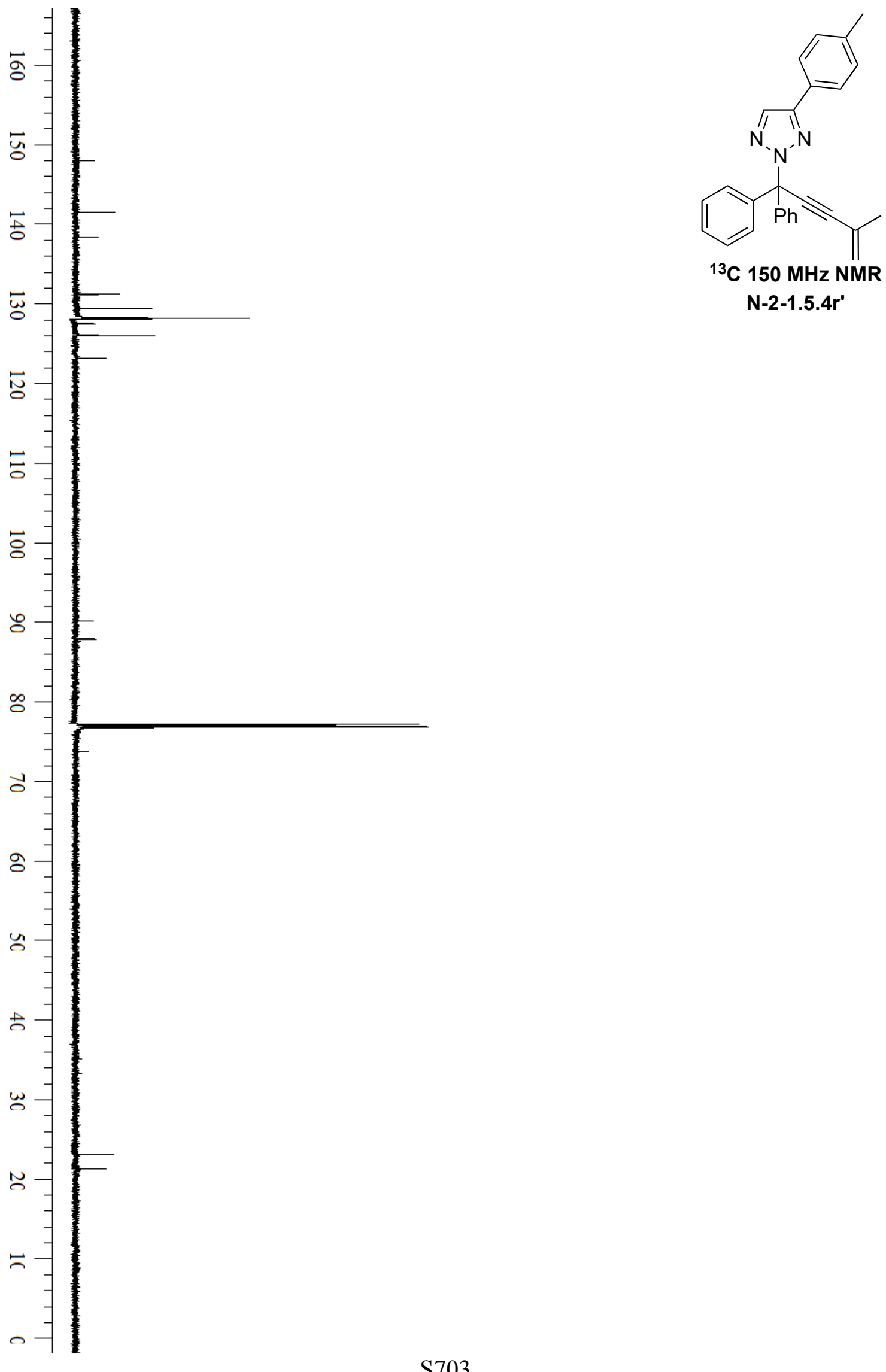

${ }^{13} \mathrm{C} 150 \mathrm{MHz}$ NMR

$\mathrm{N}-2-1.5 .4 r^{\prime}$ 


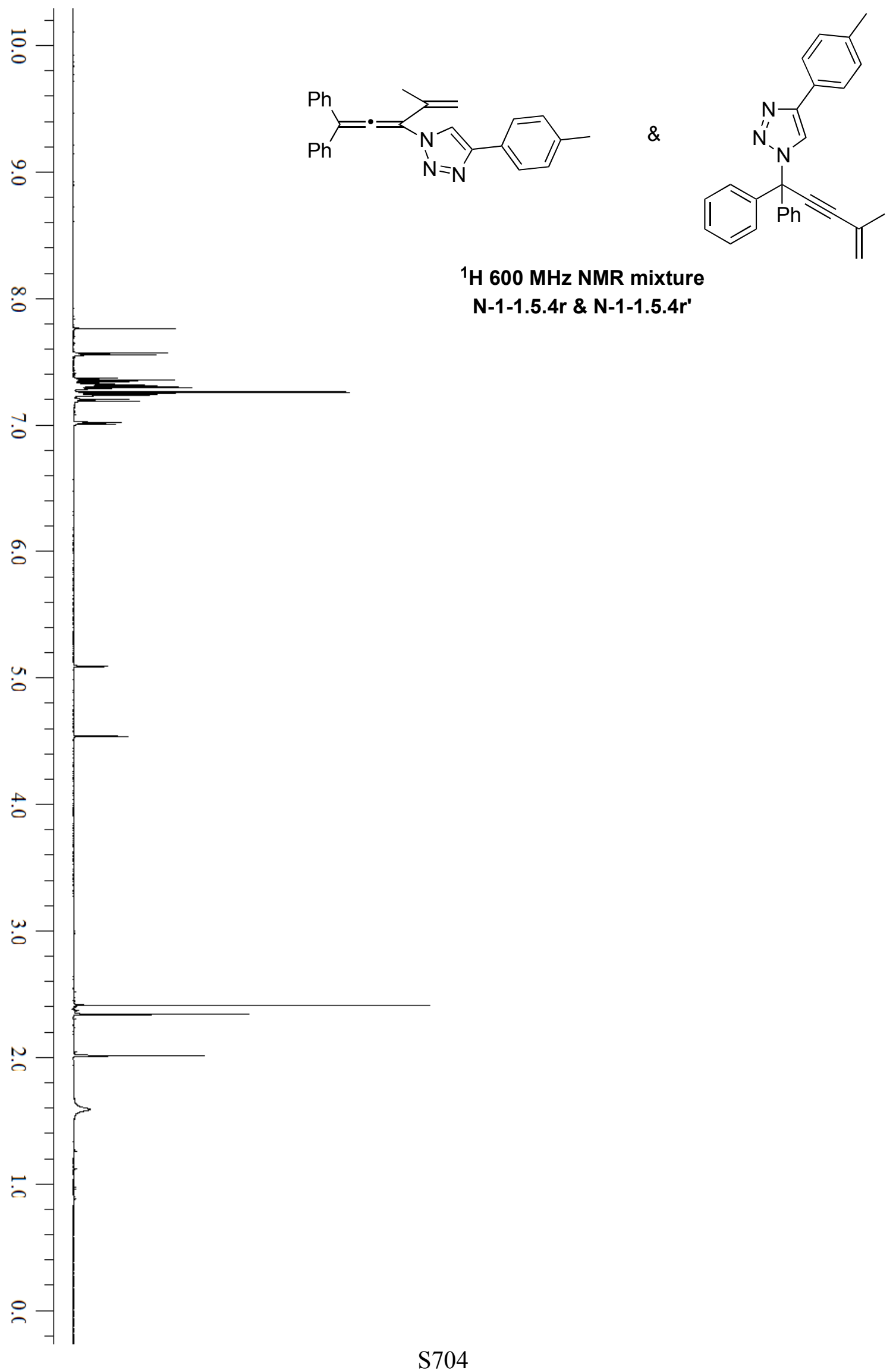




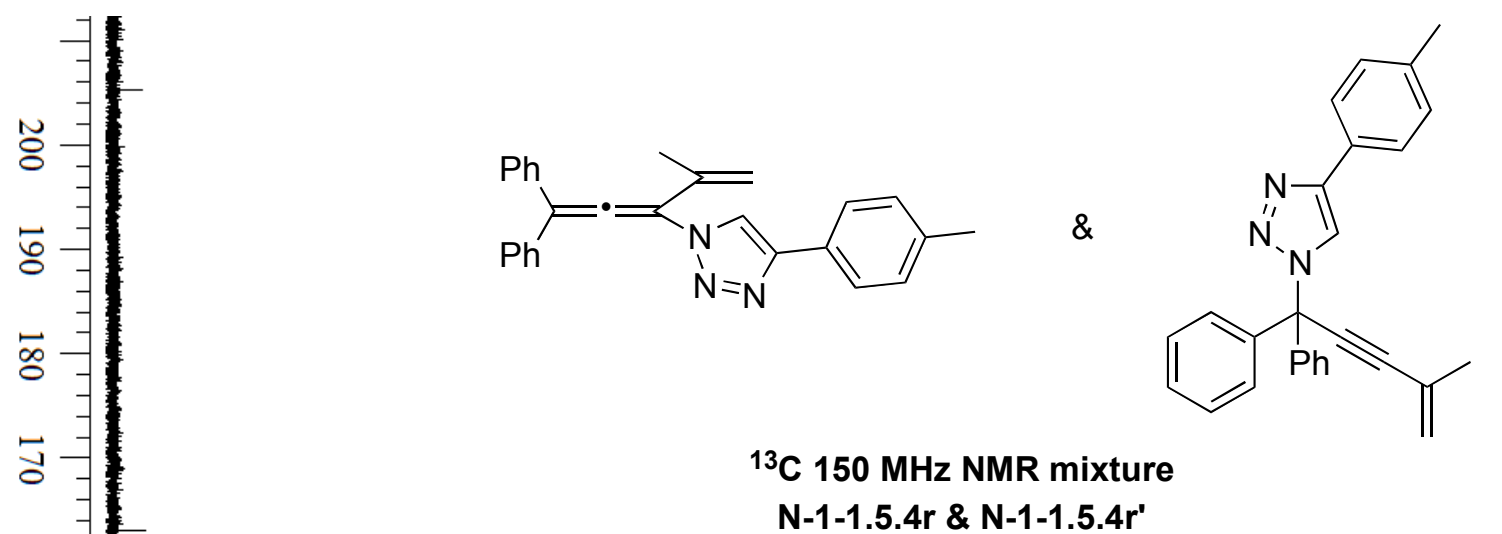

a

列

㝒

公

$$
\text { }
$$$$
\text { 훙 }
$$$$
\text { ๕ }
$$

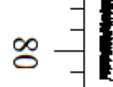$$
\text { ○) }
$$$$
\text { g) }
$$$$
\text { - }
$$$$
\text { पू }
$$$$
+-
$$$$
\text { क }
$$$$
\text { w }
$$$$
\text { Э }
$$$$
\text { N }
$$$$
\text { - }
$$$$
\rightleftharpoons-
$$$$
\text { o }
$$ 


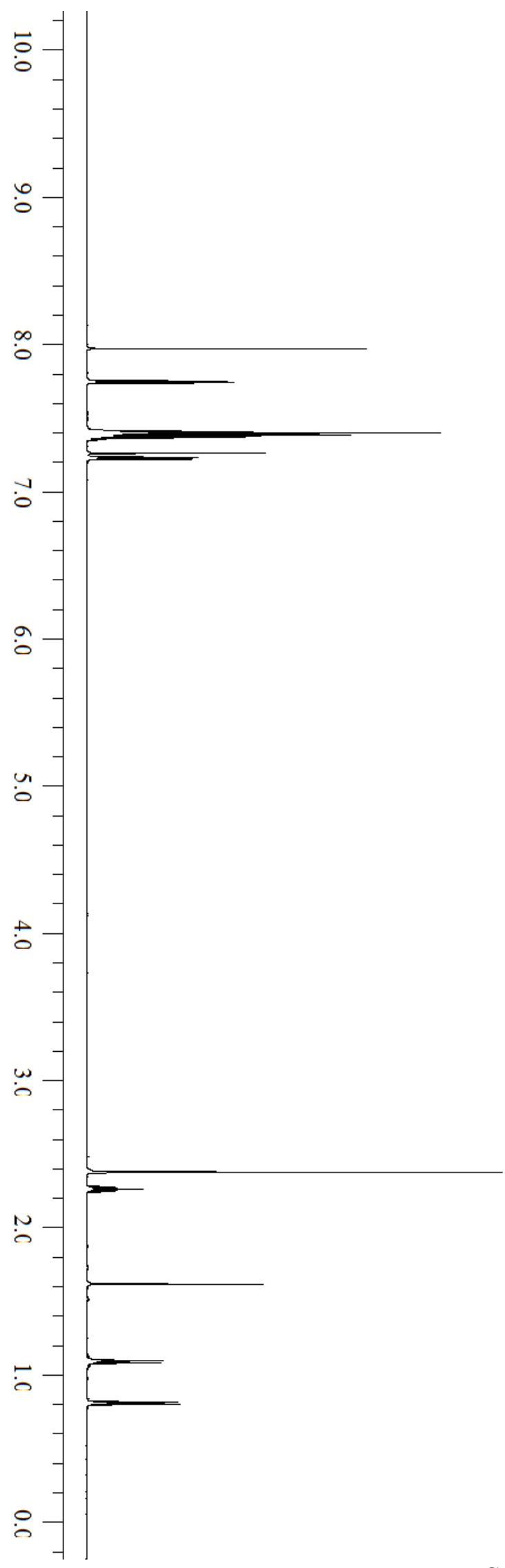

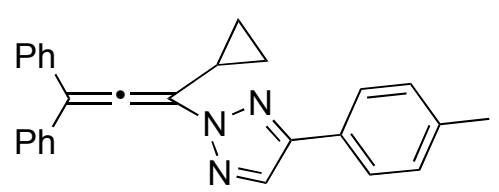

${ }^{1} \mathrm{H} 600 \mathrm{MHz}$ NMR

$\mathrm{N}-2-1.5 .4 \mathrm{~s}$ 


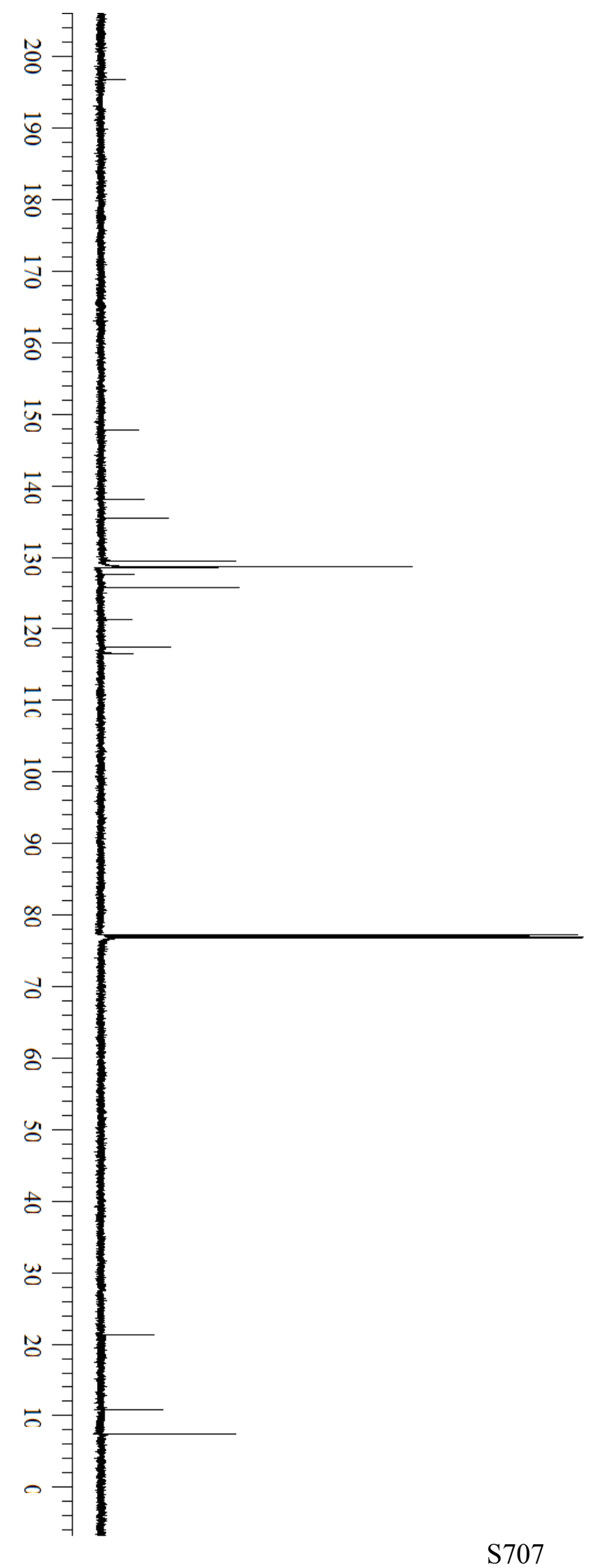

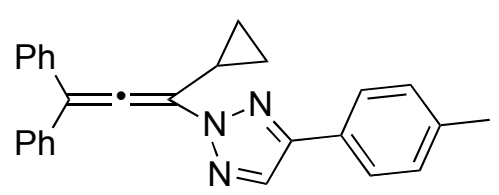

${ }^{13} \mathrm{C} 150 \mathrm{MHz}$ NMR

$\mathrm{N}-2-1.5 .4 \mathrm{~s}$ 


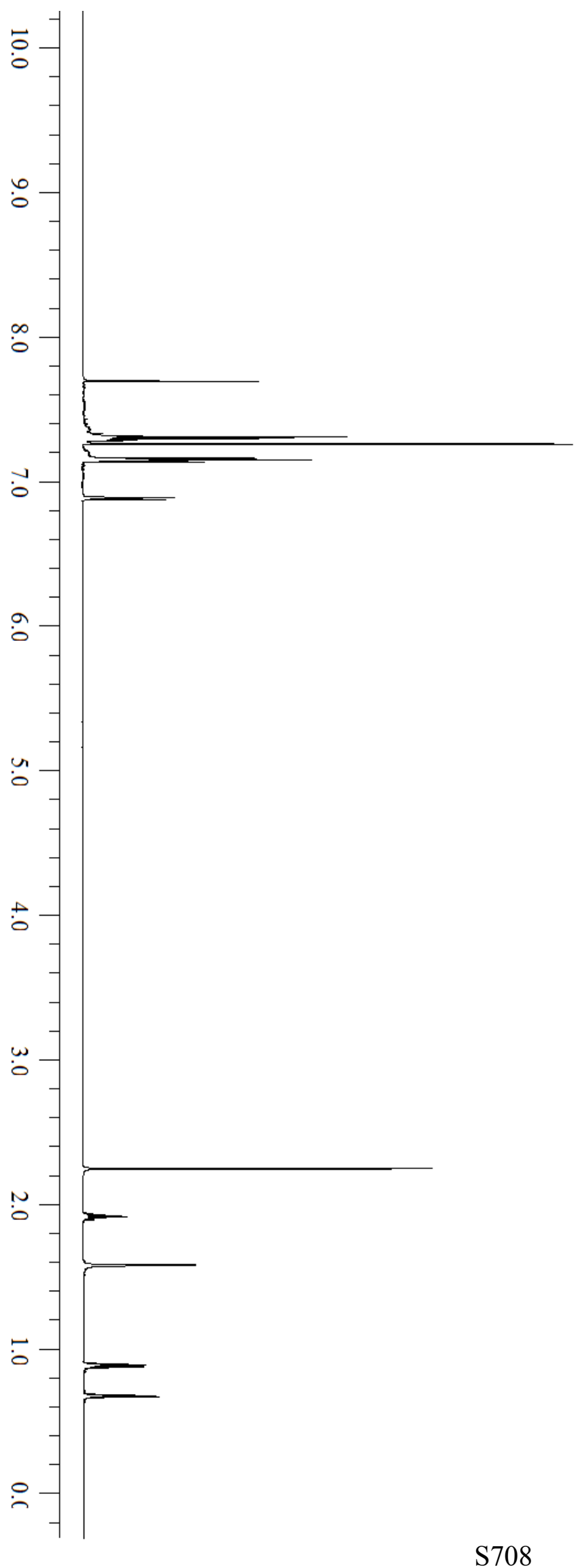

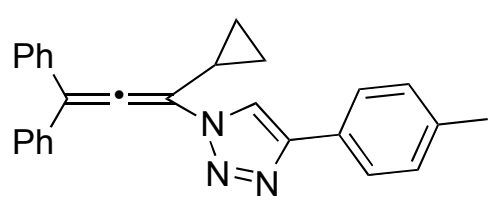

${ }^{1} \mathrm{H} 600 \mathrm{MHz}$ NMR

$\mathrm{N}-1-1.5 .4 \mathrm{~s}$ 


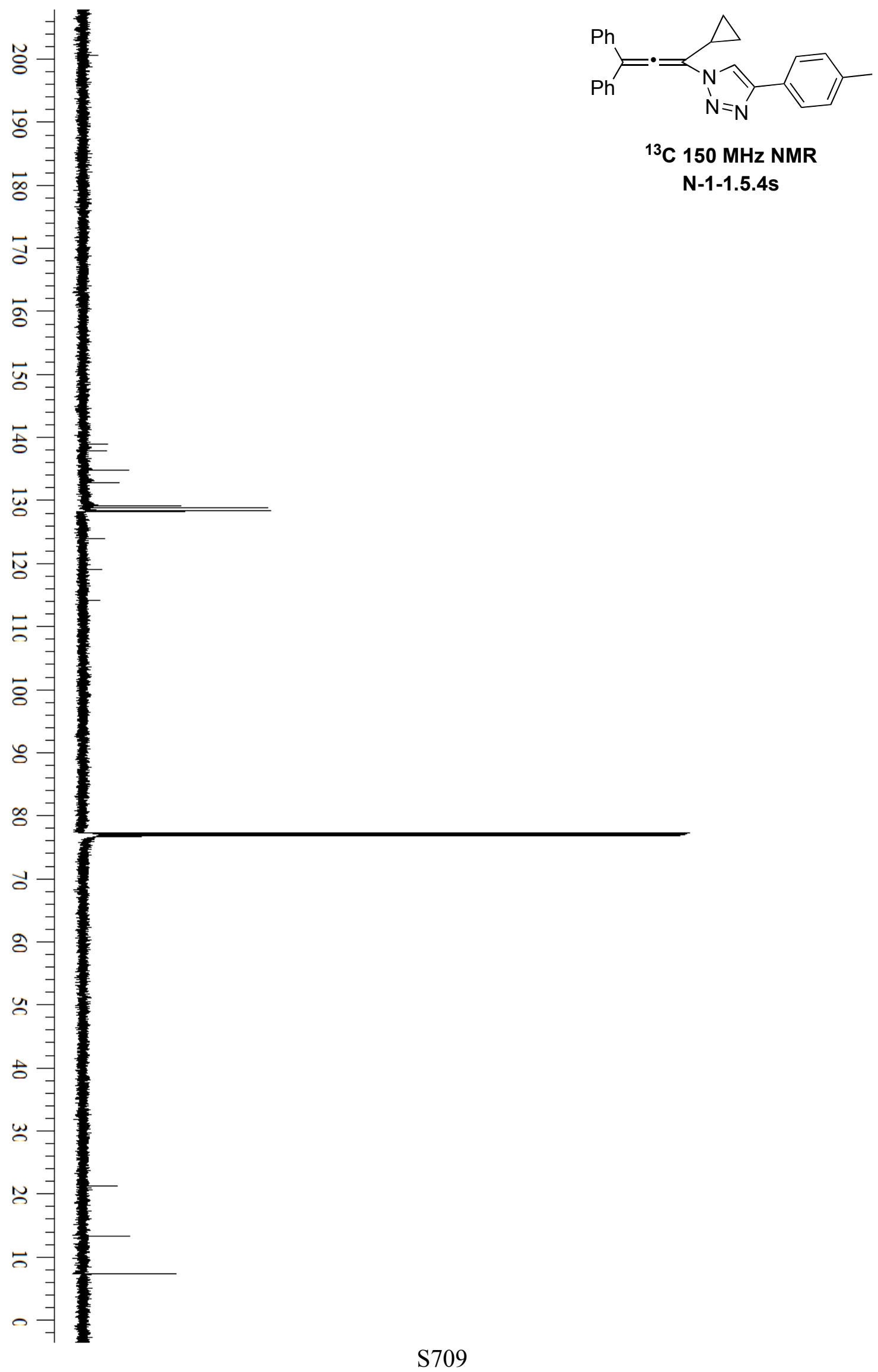



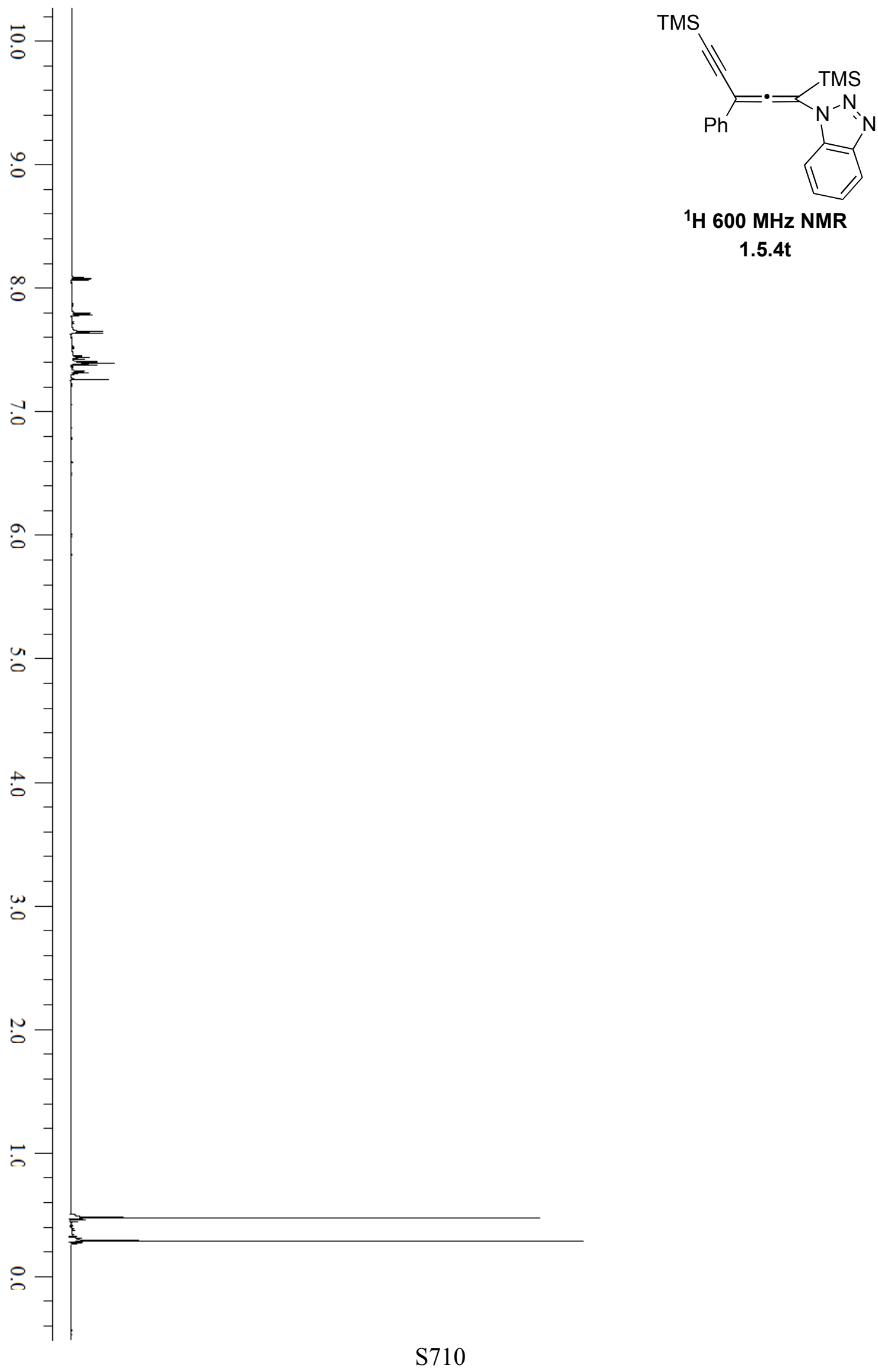

${ }^{1} \mathrm{H} 600 \mathrm{MHz}$ NMR

1.5.4t 


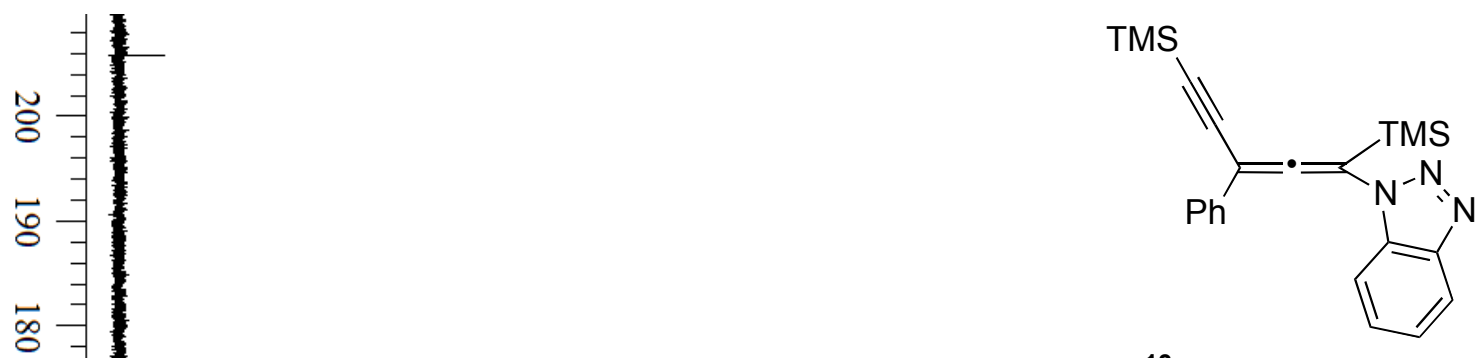

${ }^{13} \mathrm{C} 150 \mathrm{MHz}$ NMR

$1.5 .4 \mathrm{t}$ 


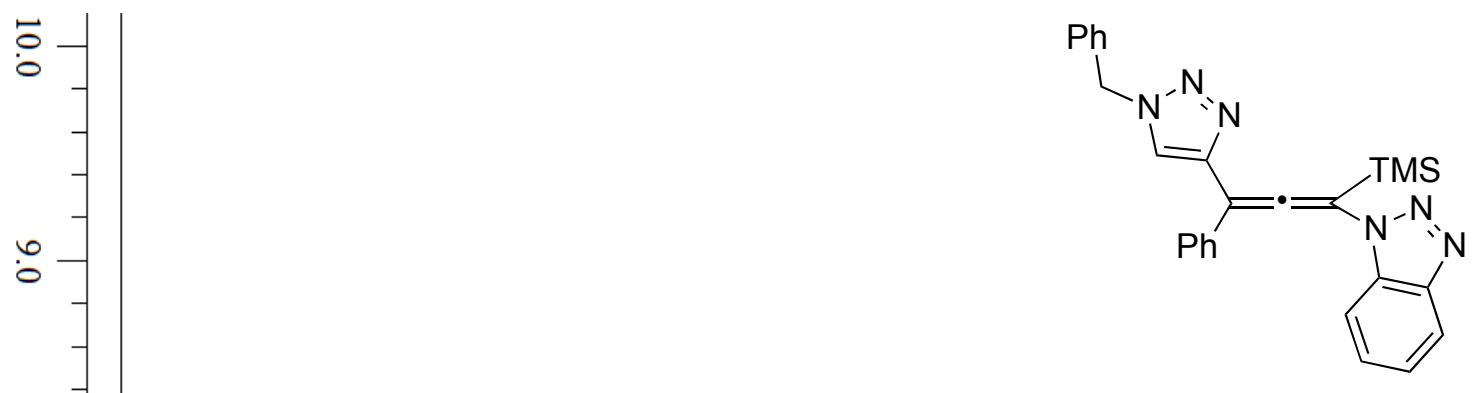

${ }^{1} \mathrm{H} 600 \mathrm{MHz}$ NMR

$1.5 .4 \mathrm{u}$

$\stackrel{\circ}{\circ}$

o

$\ddot{0}$

$\infty-1$

$=$

o

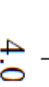

-

w

$\stackrel{n}{0}$

-

\%

$\therefore-1$ 

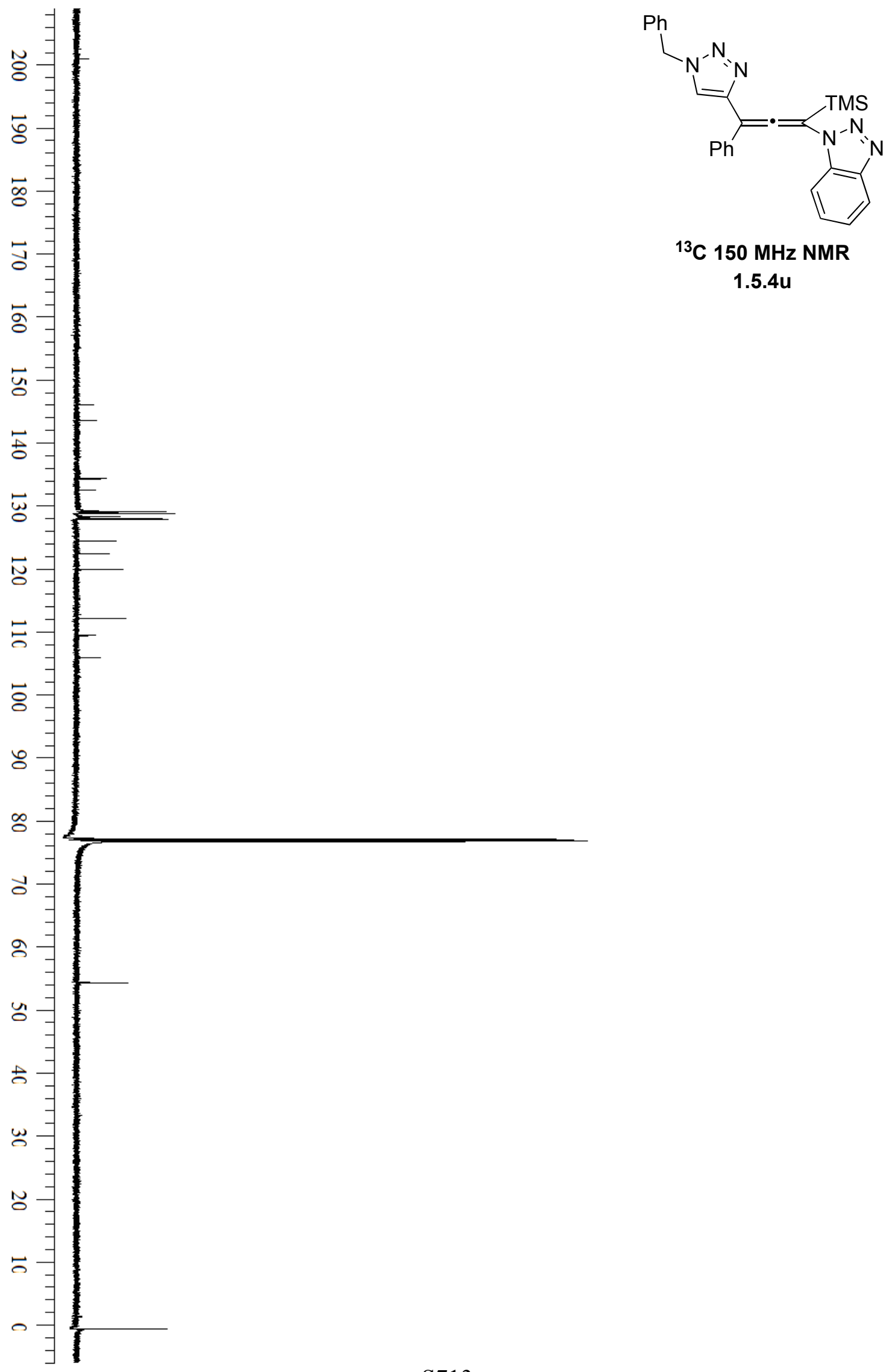

${ }^{13} \mathrm{C} 150 \mathrm{MHz}$ NMR

$1.5 .4 \mathrm{u}$ 


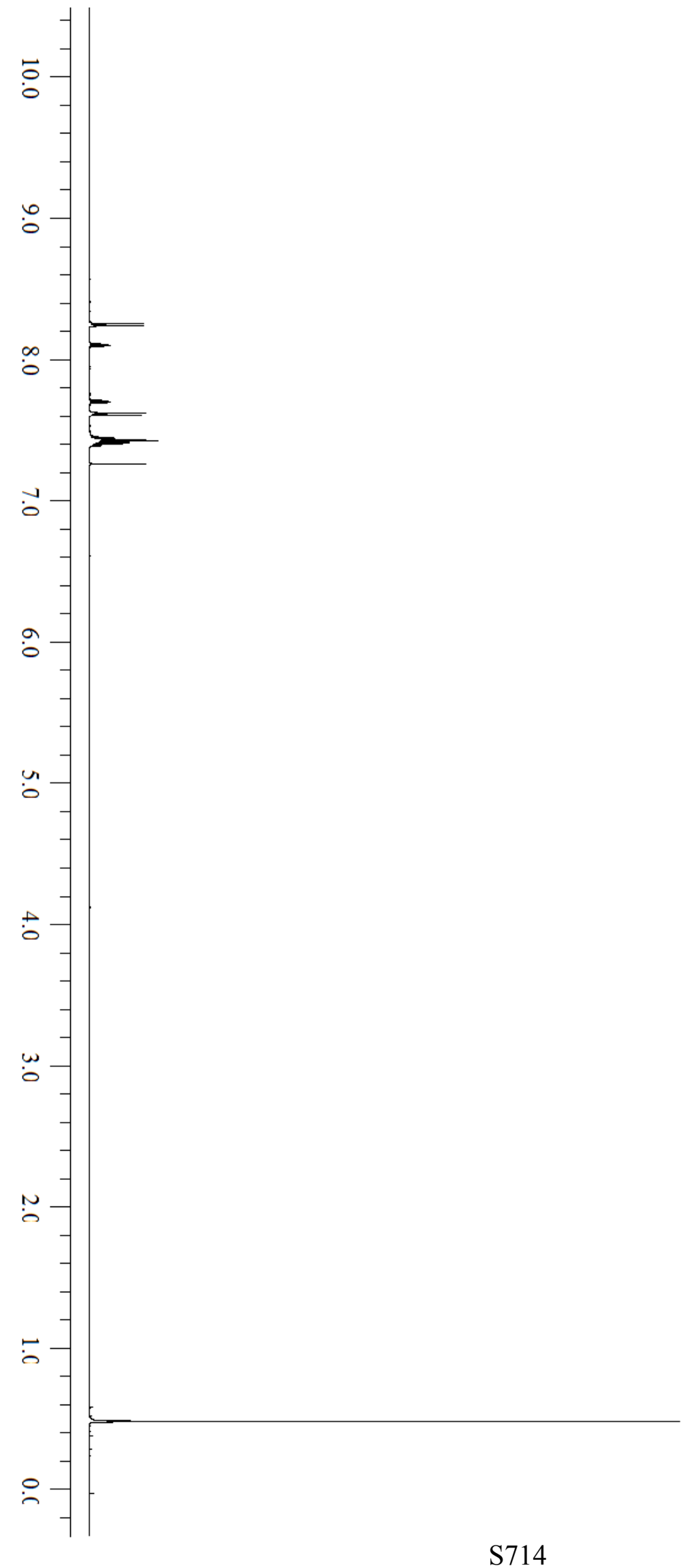

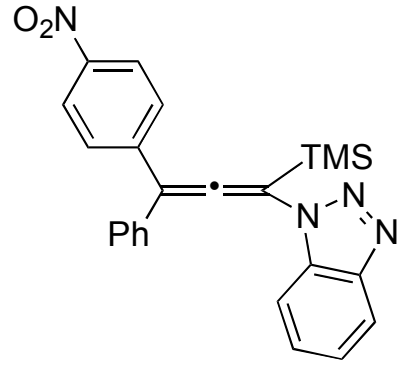

${ }^{1} \mathrm{H} 600 \mathrm{MHz}$ NMR

$1.5 .4 \mathrm{v}$ 

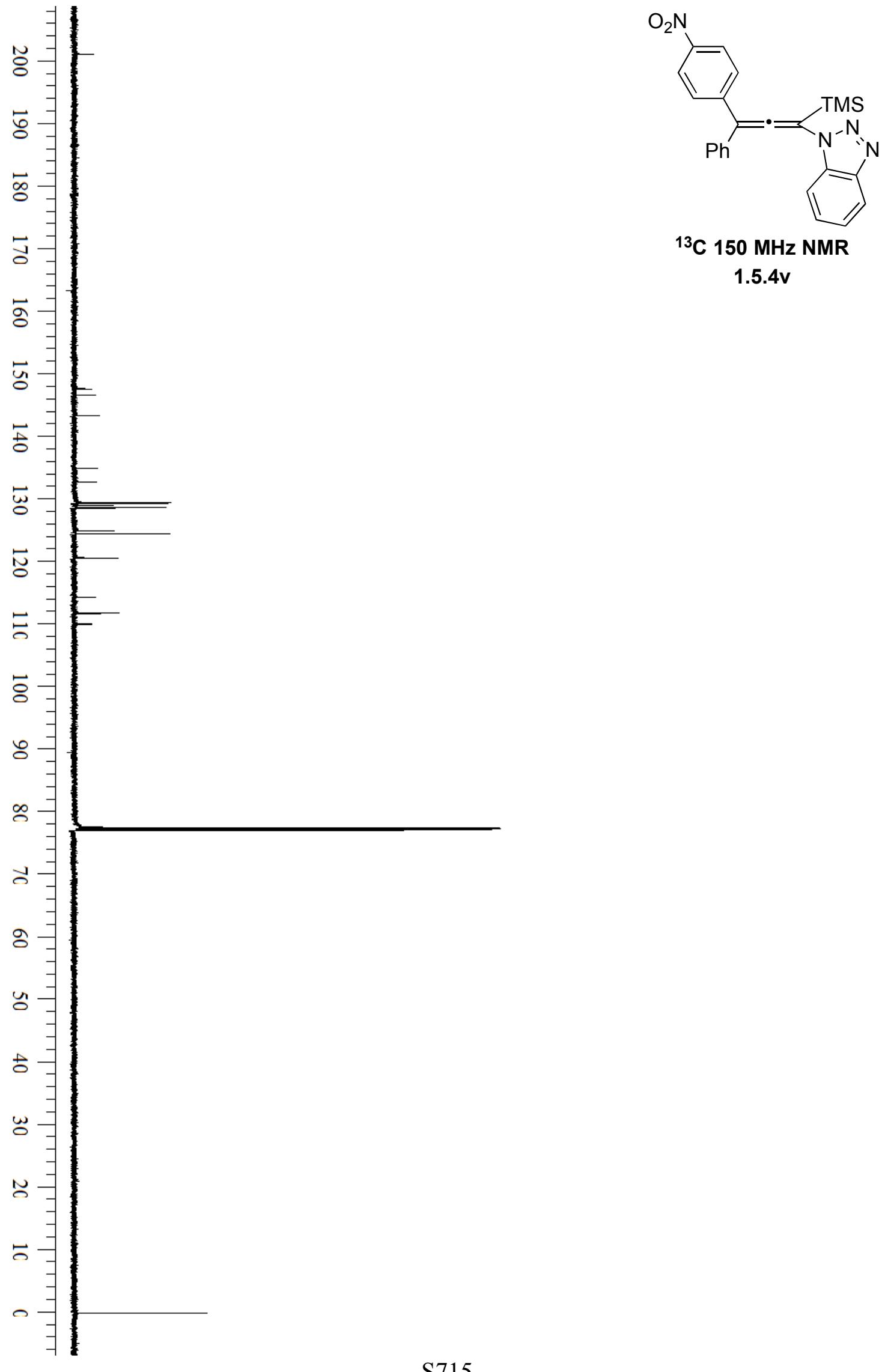

${ }^{13} \mathrm{C} 150 \mathrm{MHz}$ NMR

$1.5 .4 \mathrm{v}$ 


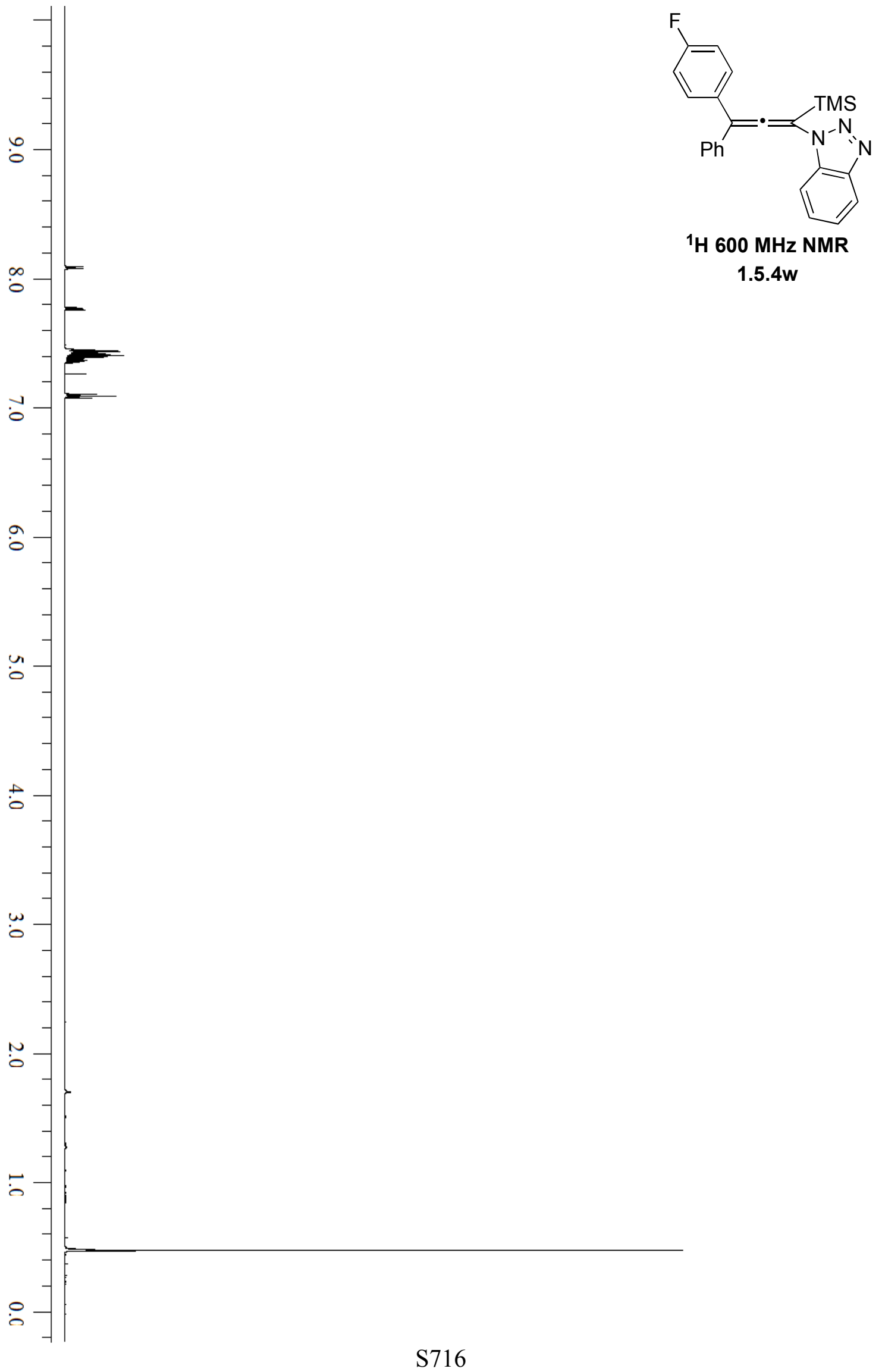



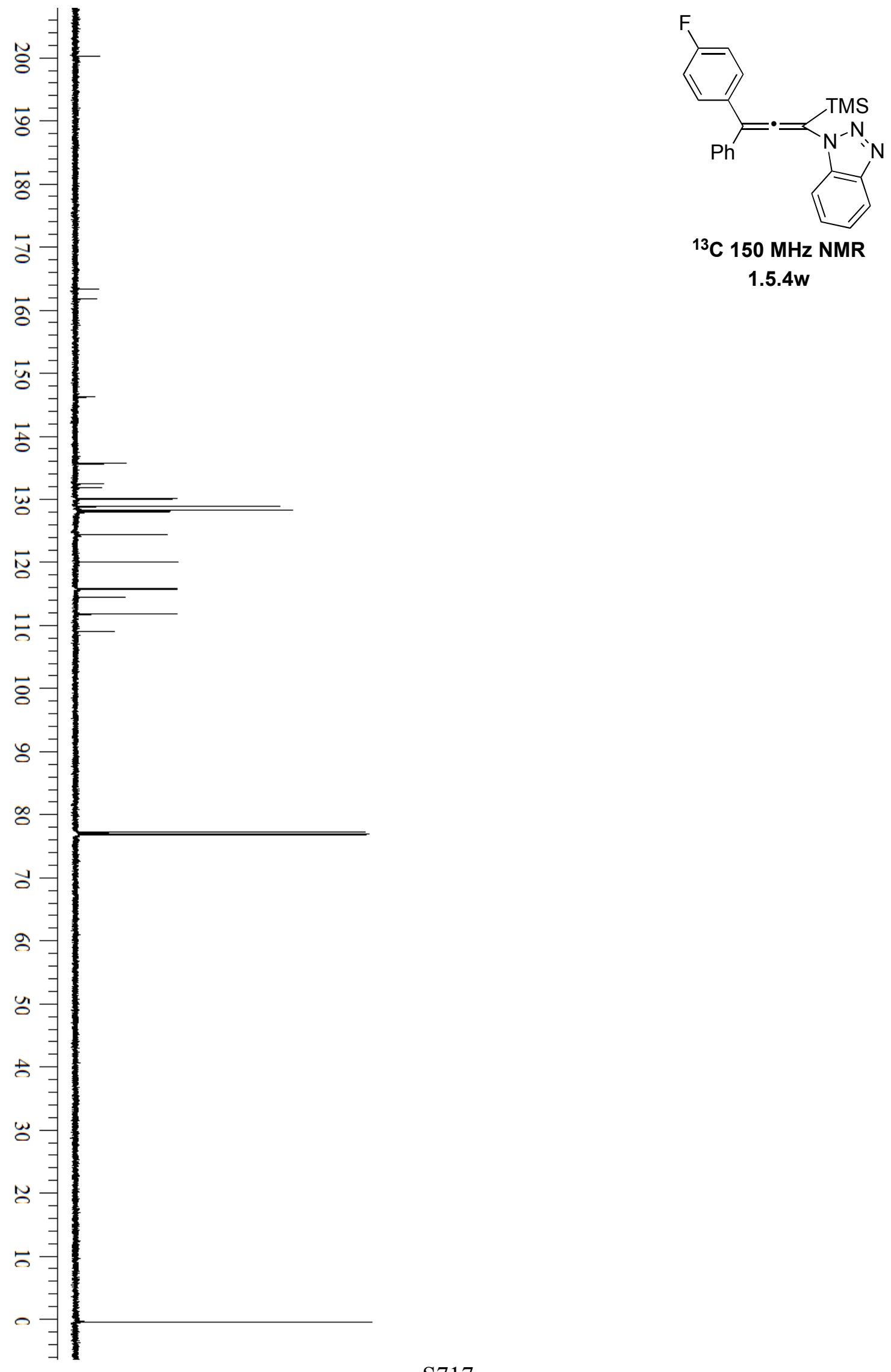

${ }^{13} \mathrm{C} 150 \mathrm{MHz}$ NMR

1.5.4w 


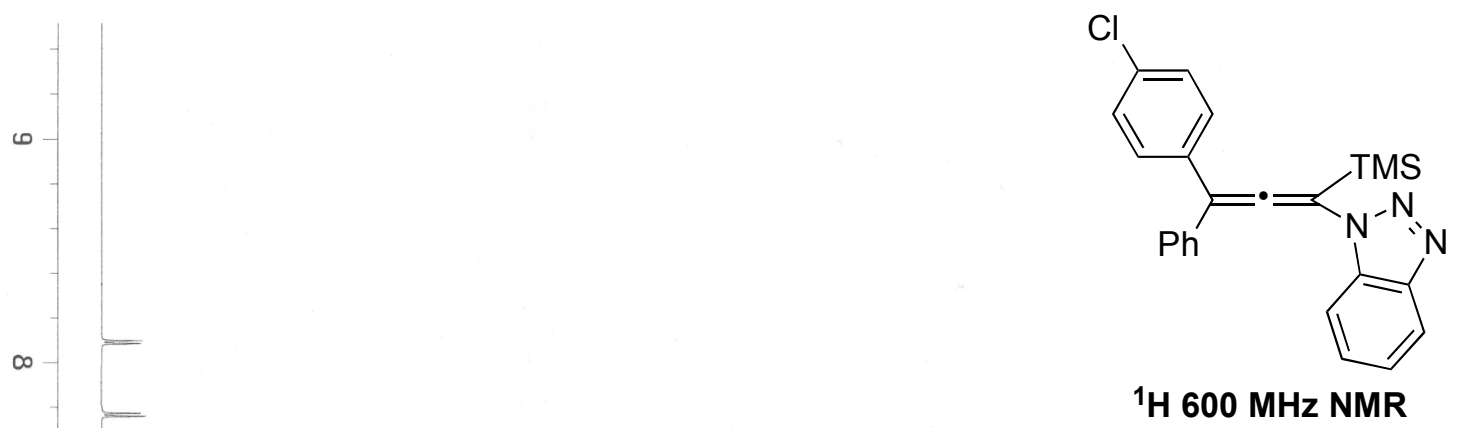

1.5.4x

v

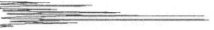




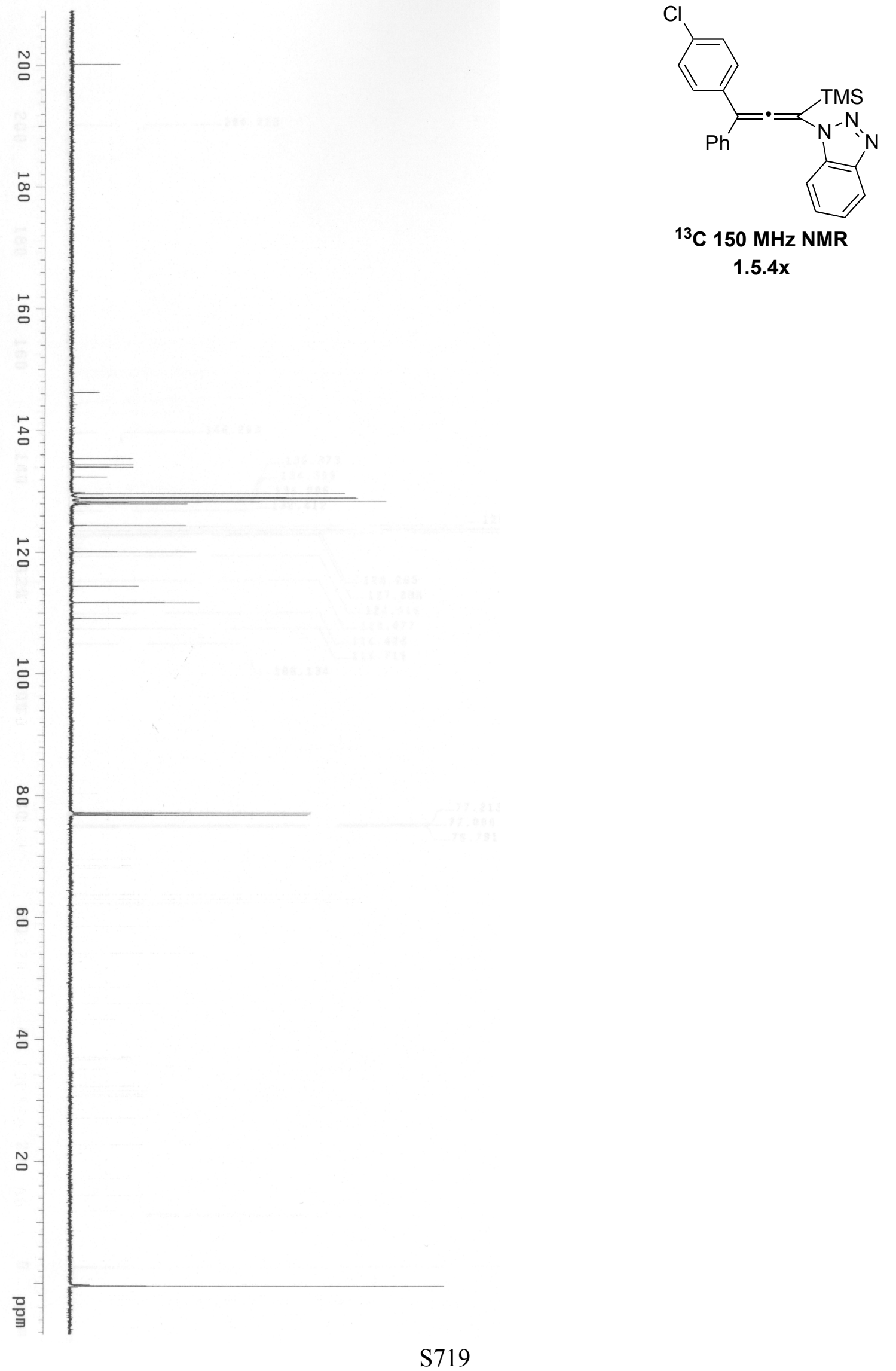



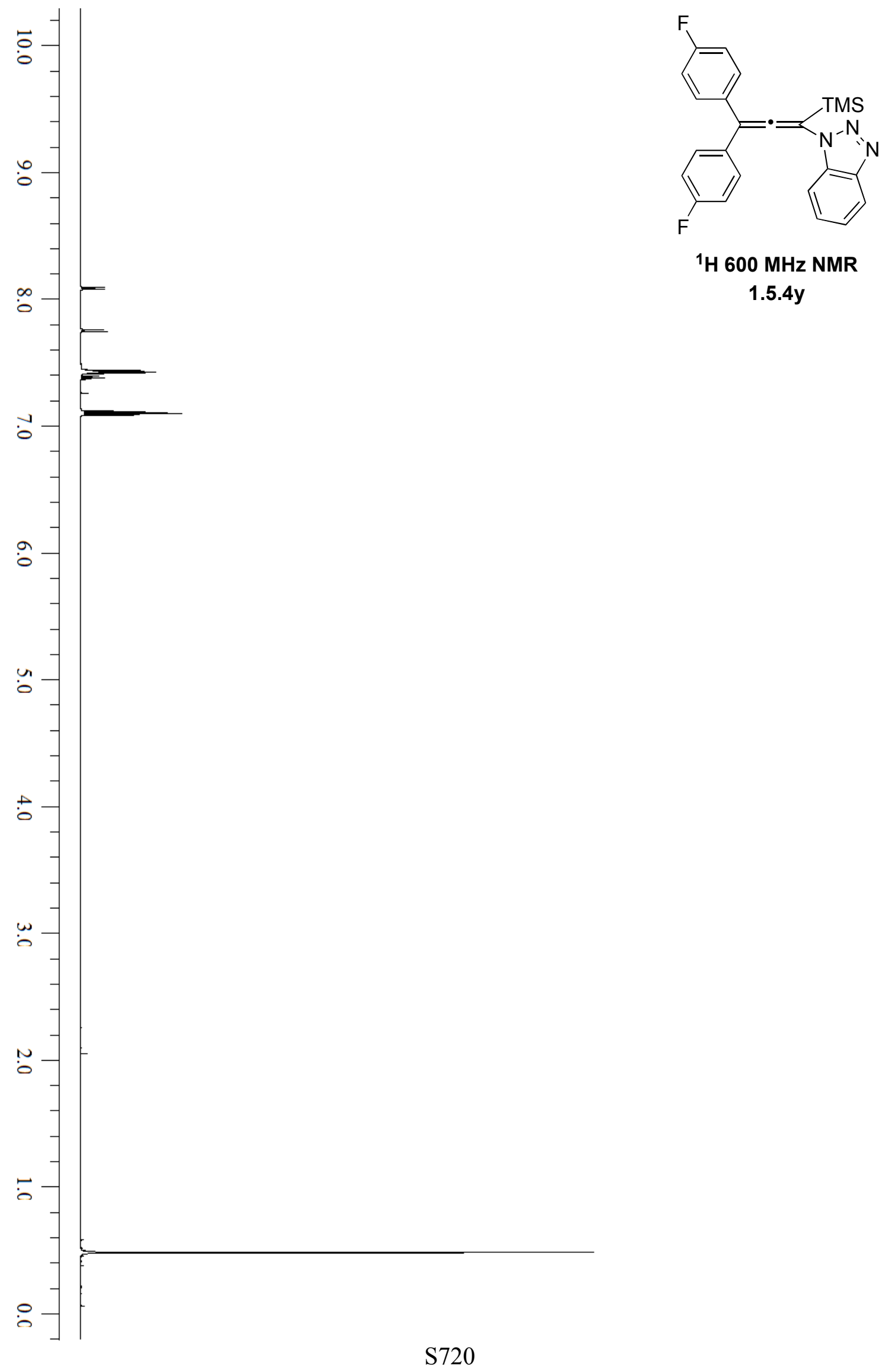

${ }^{1} \mathrm{H} 600 \mathrm{MHz}$ NMR

1.5.4y 


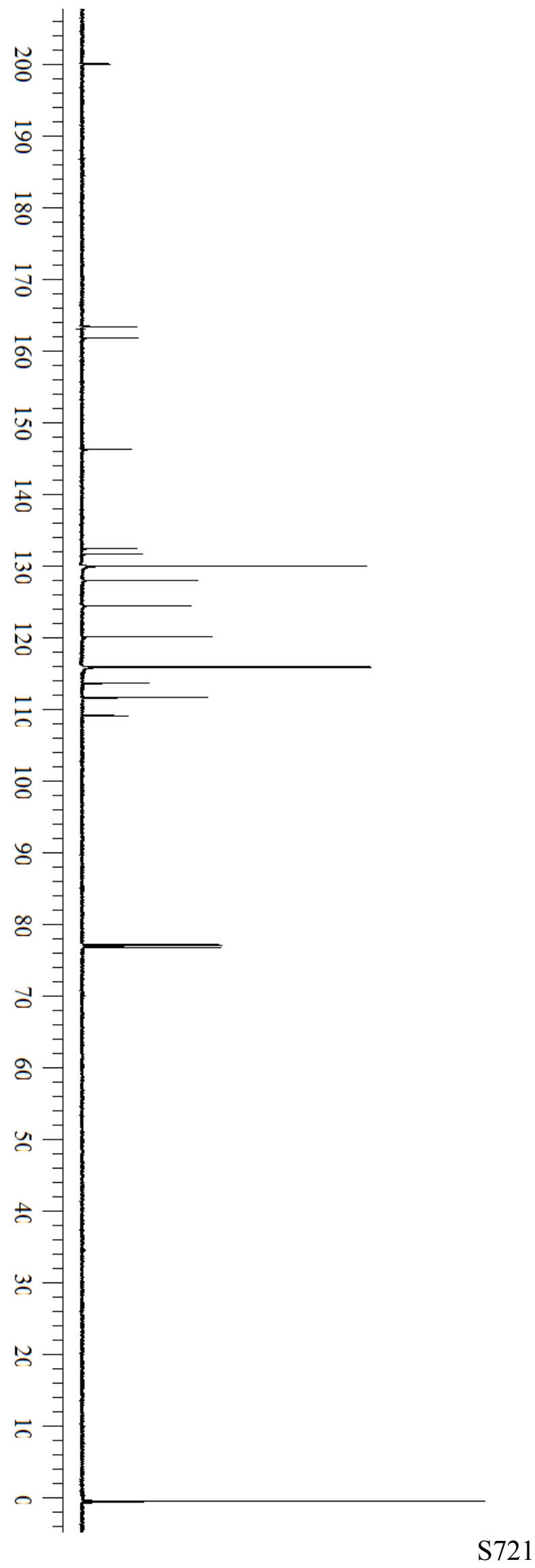

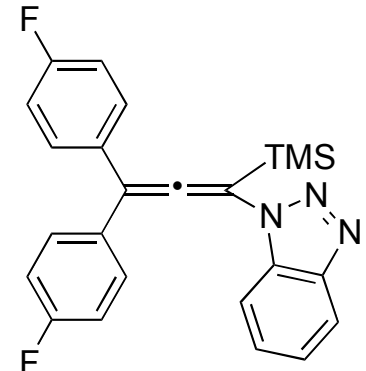

${ }^{13} \mathrm{C} 150 \mathrm{MHz}$ NMR

1.5.4y 


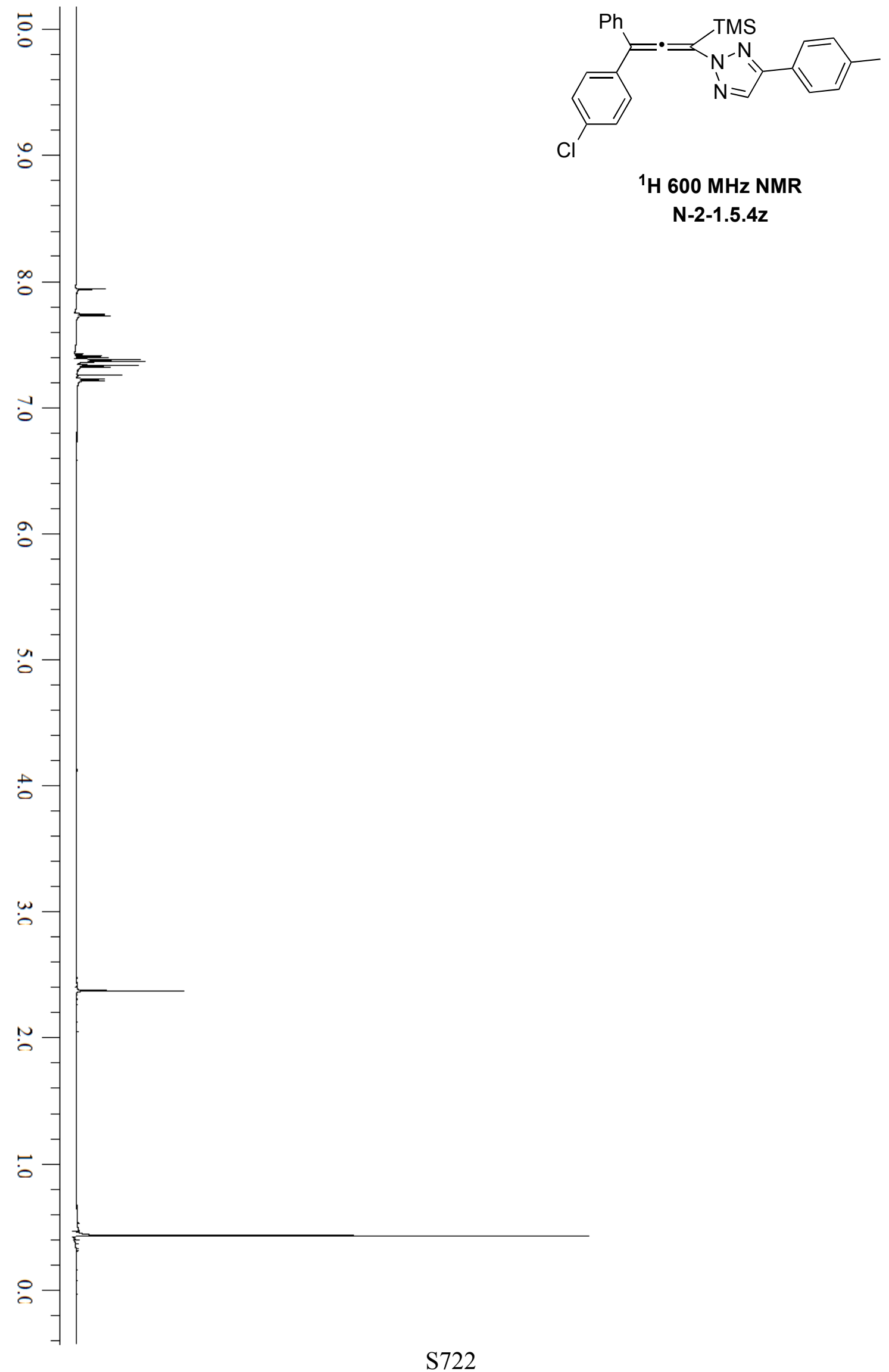




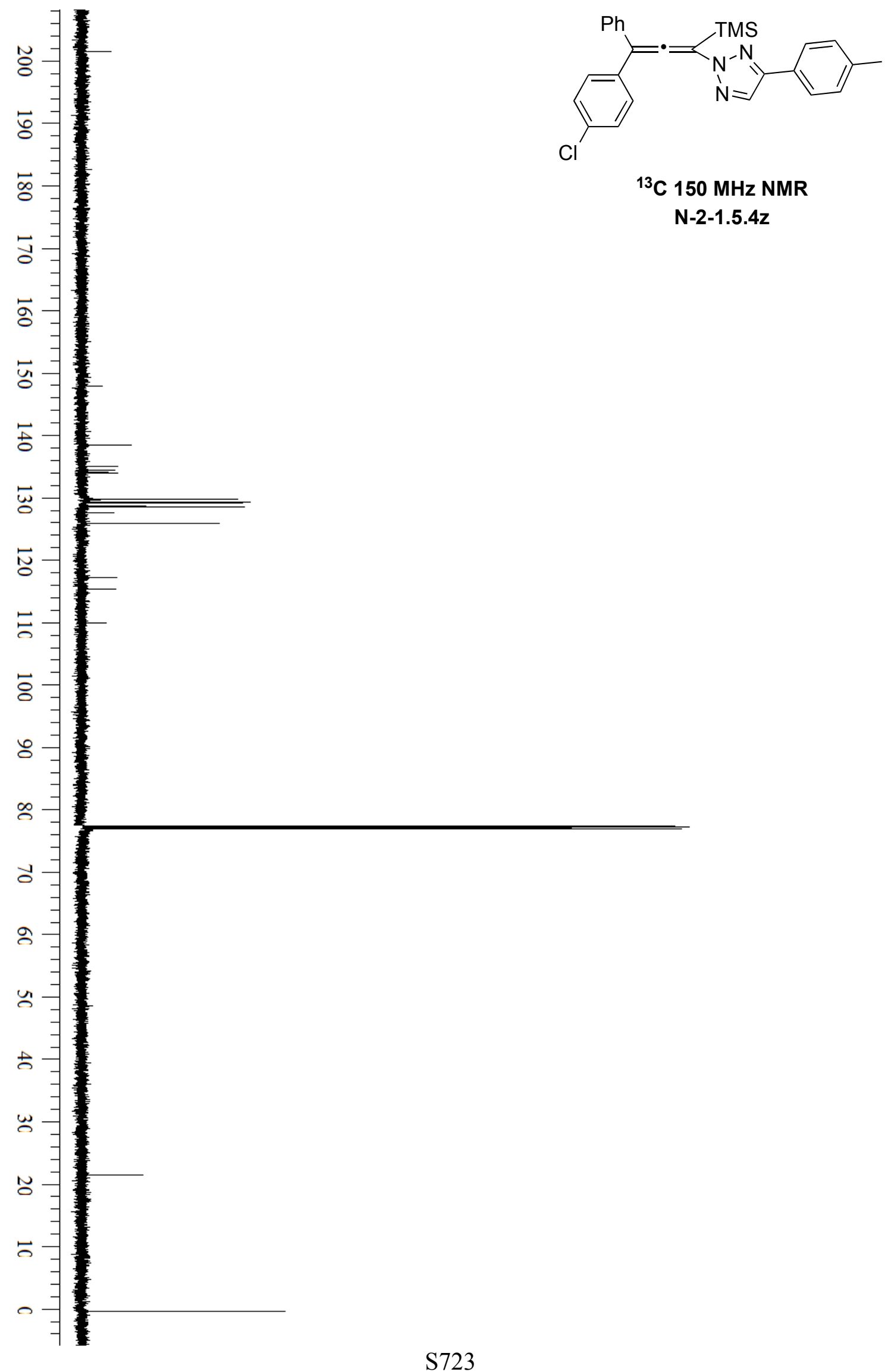




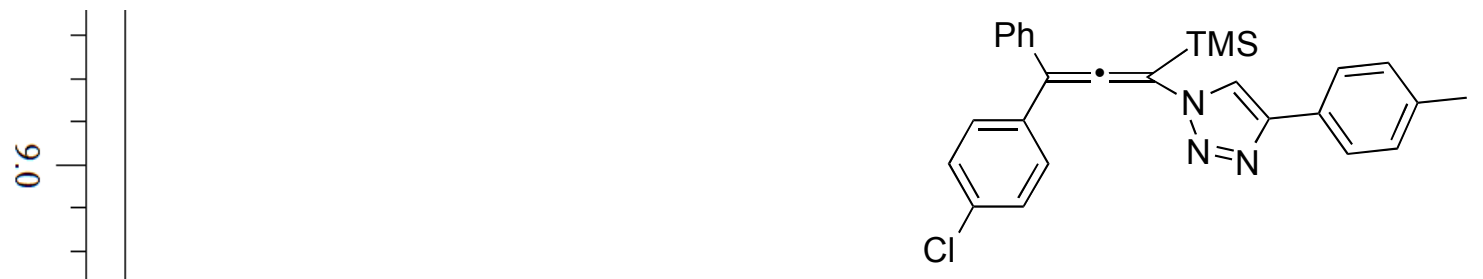

${ }^{1} \mathrm{H} 600 \mathrm{MHz}$ NMR

$\mathrm{N}-1-1.5 .4 z$

$\infty$

b-

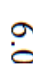

un

0

co

-

$\stackrel{n}{0}$

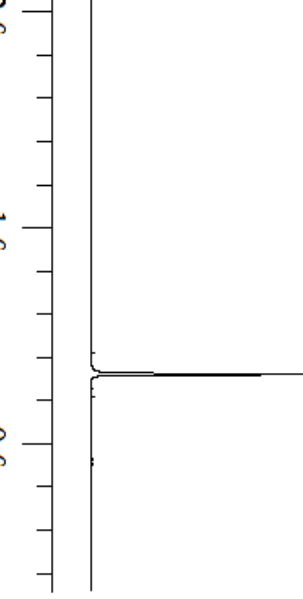



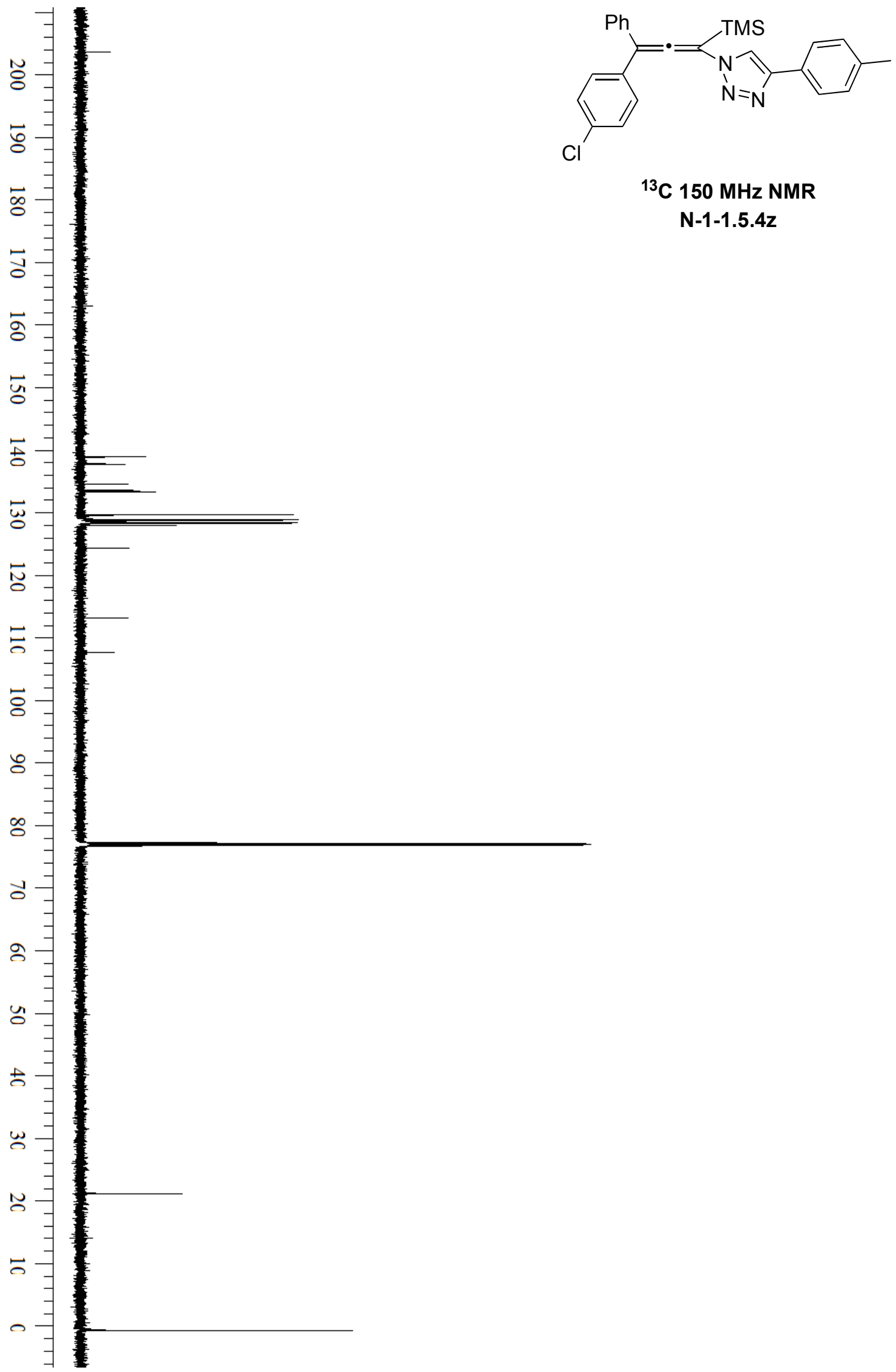

${ }^{13} \mathrm{C} 150 \mathrm{MHz}$ NMR

$\mathrm{N}-1-1.5 .4 \mathrm{z}$ 


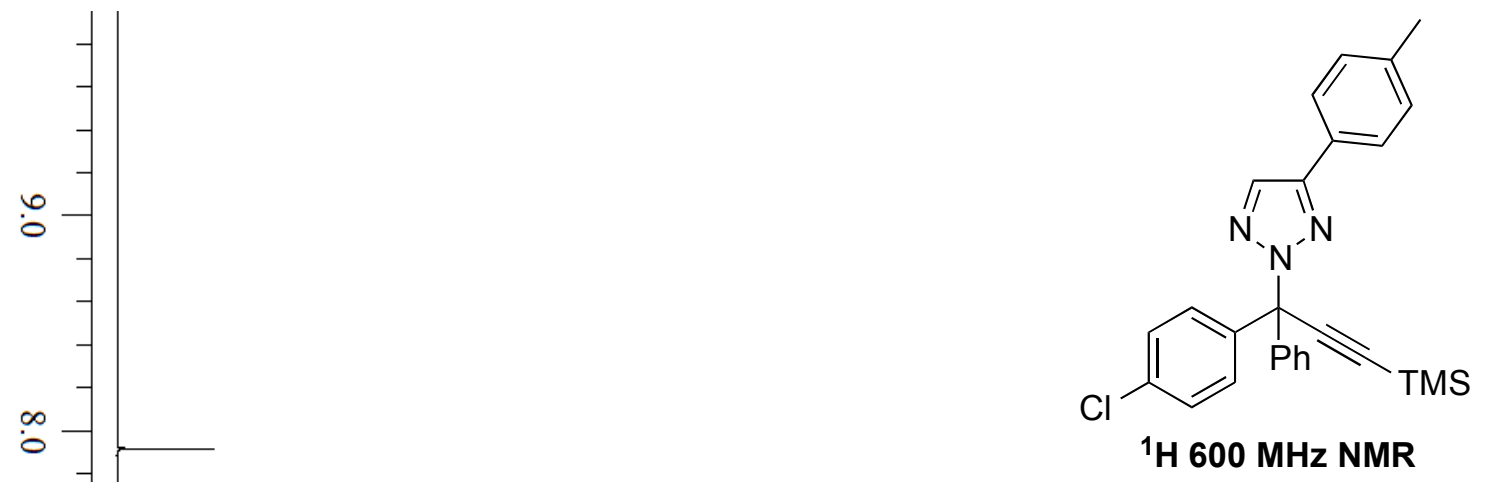

$$
\text { N-2-1.5.4z' }
$$

$\therefore-\sqrt{5}$

$\grave{0}$

in

$\dot{0}$

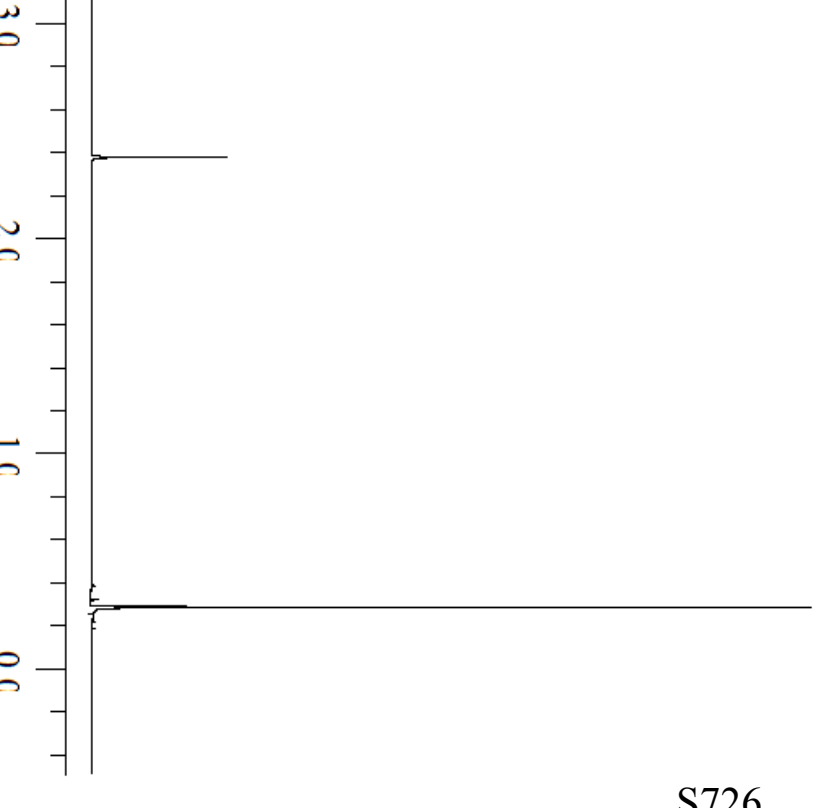




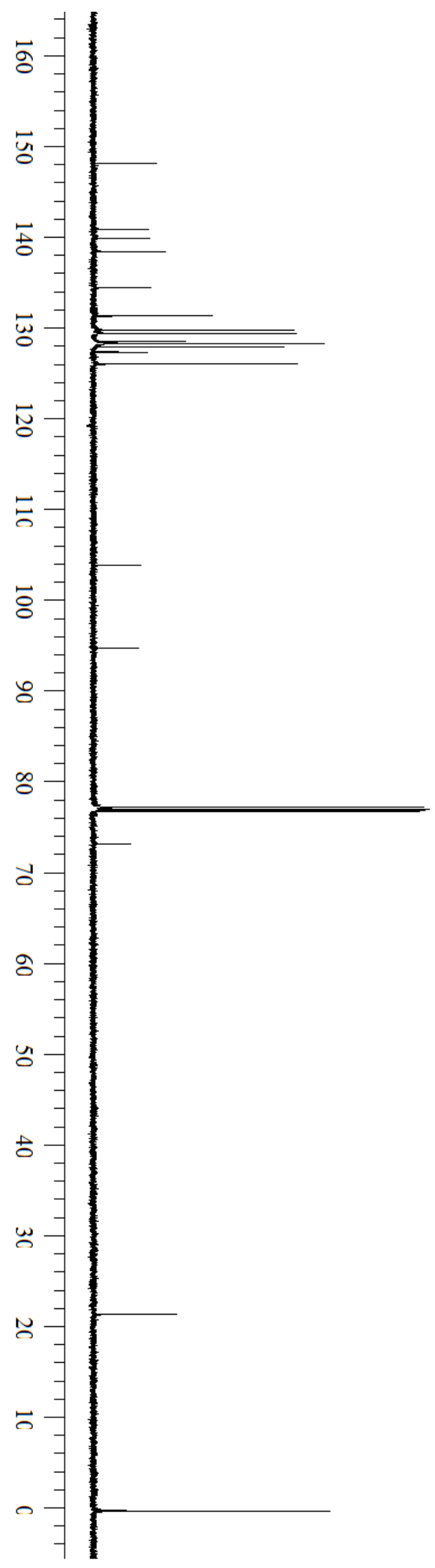

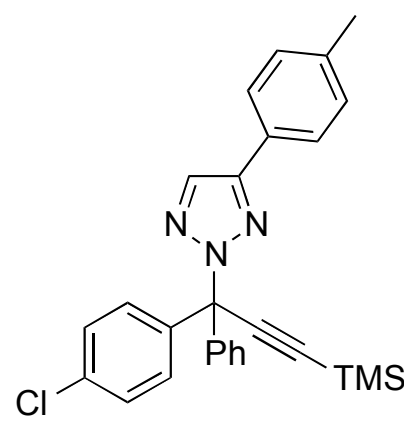

${ }^{13} \mathrm{C} 150 \mathrm{MHz}$ NMR N-2-1.5.4z' 


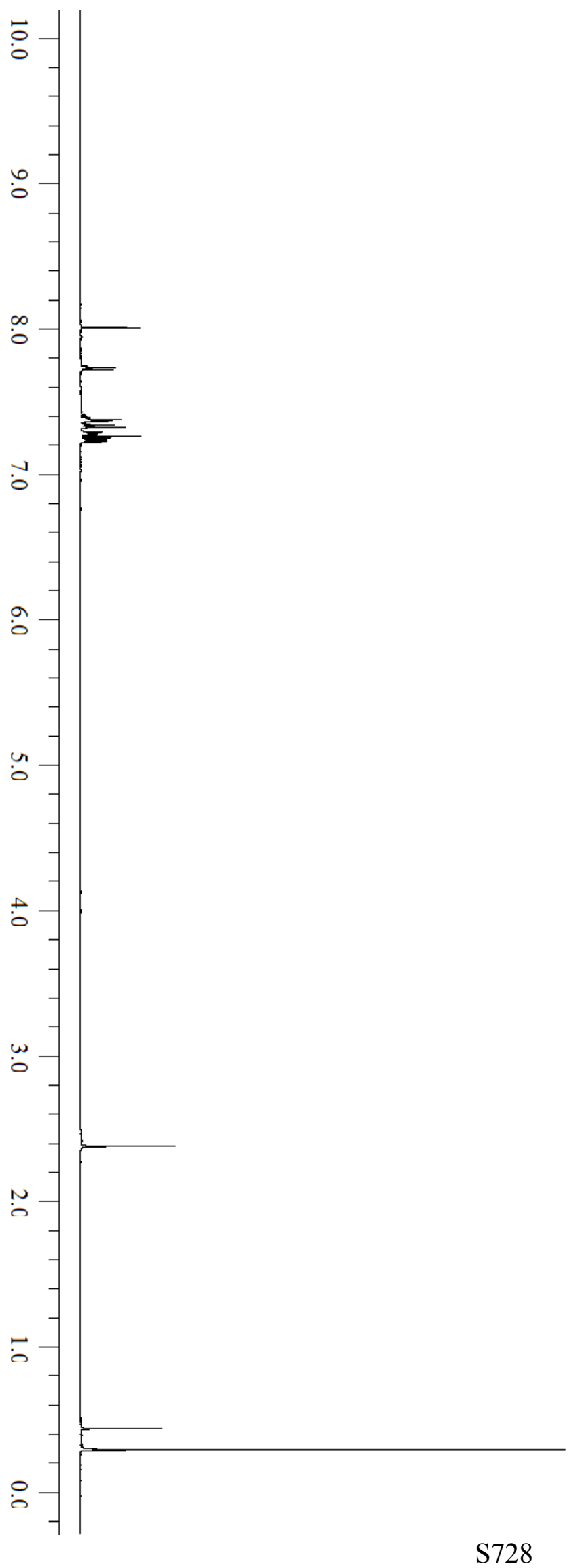

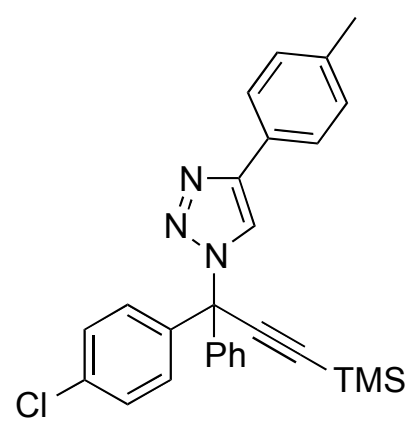

${ }^{1} \mathrm{H} 600 \mathrm{MHz}$ NMR

$\mathrm{N}-1-1.5 .4 z^{\prime}$ 


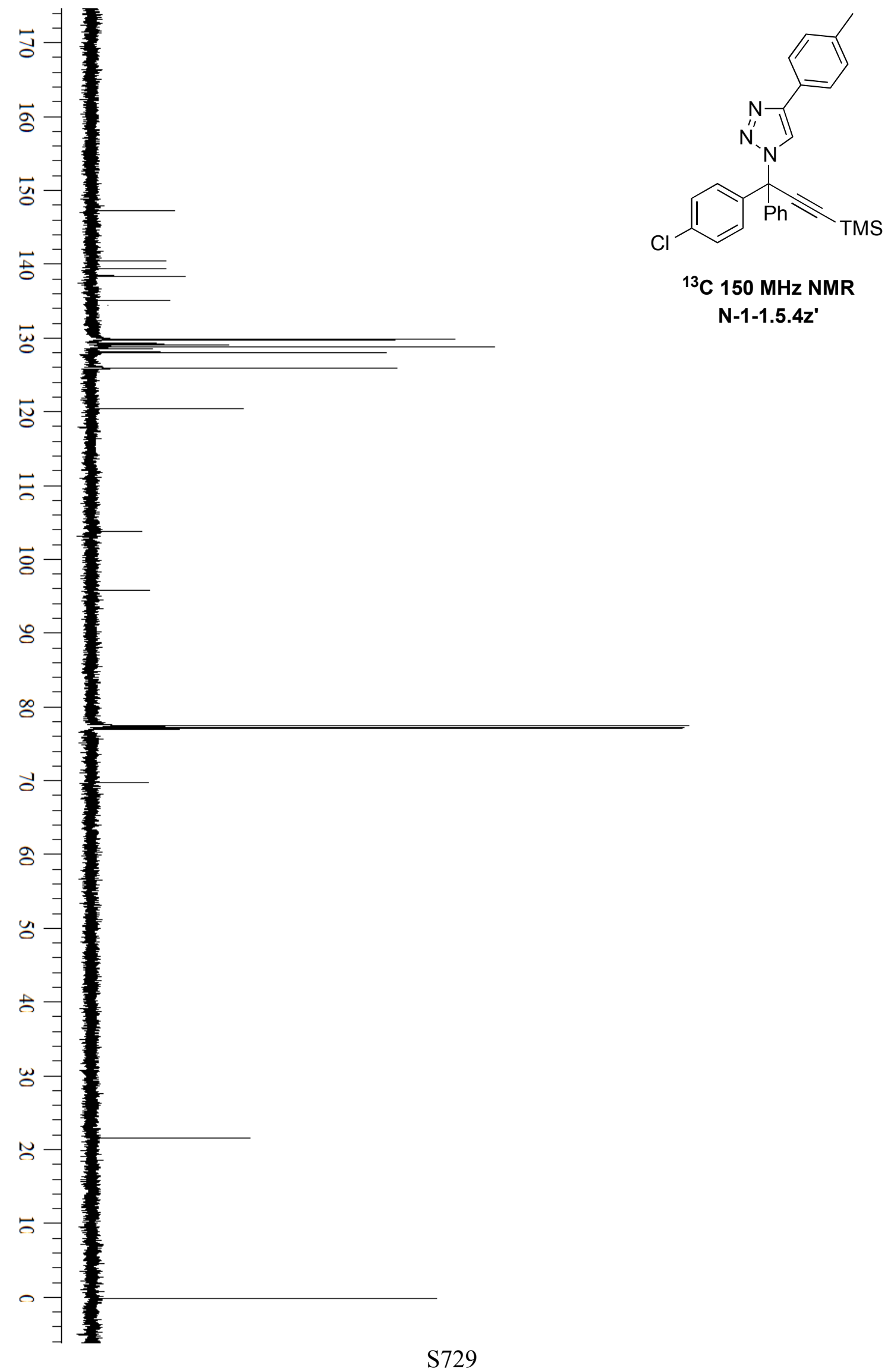




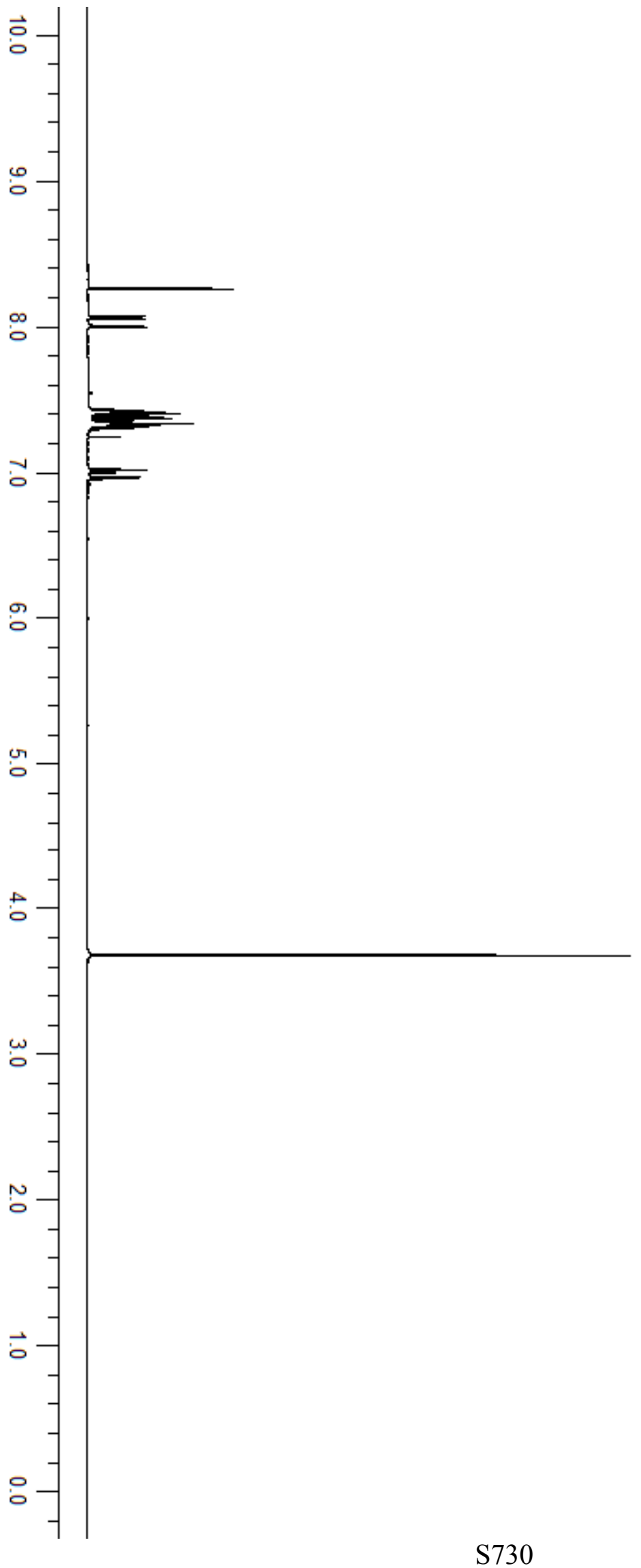

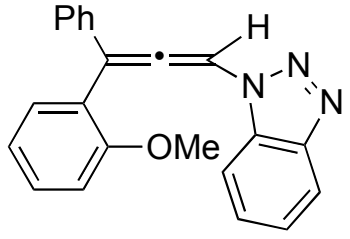

${ }^{1} \mathrm{H} 600 \mathrm{MHz}$ NMR

1.5.7a 


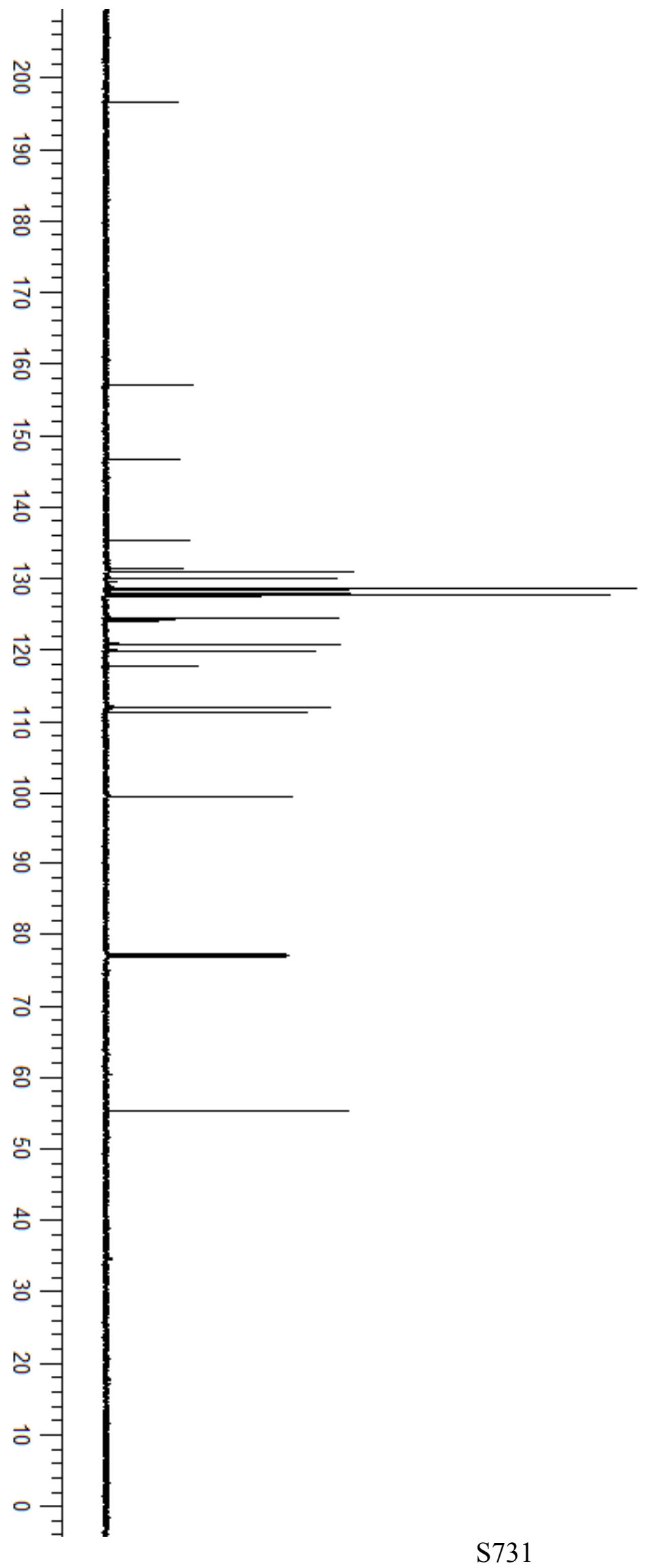

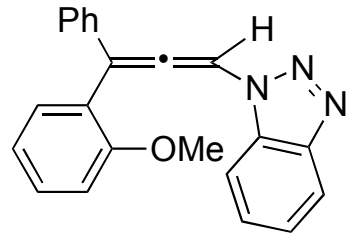

${ }^{13} \mathrm{C} 150 \mathrm{MHz}$ NMR

1.5.7a 


\subsection{HPLC Data and NMR Spectra Data}

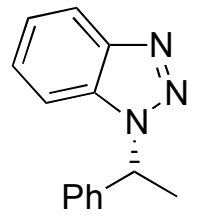

(R)-N-1-1.6.7a

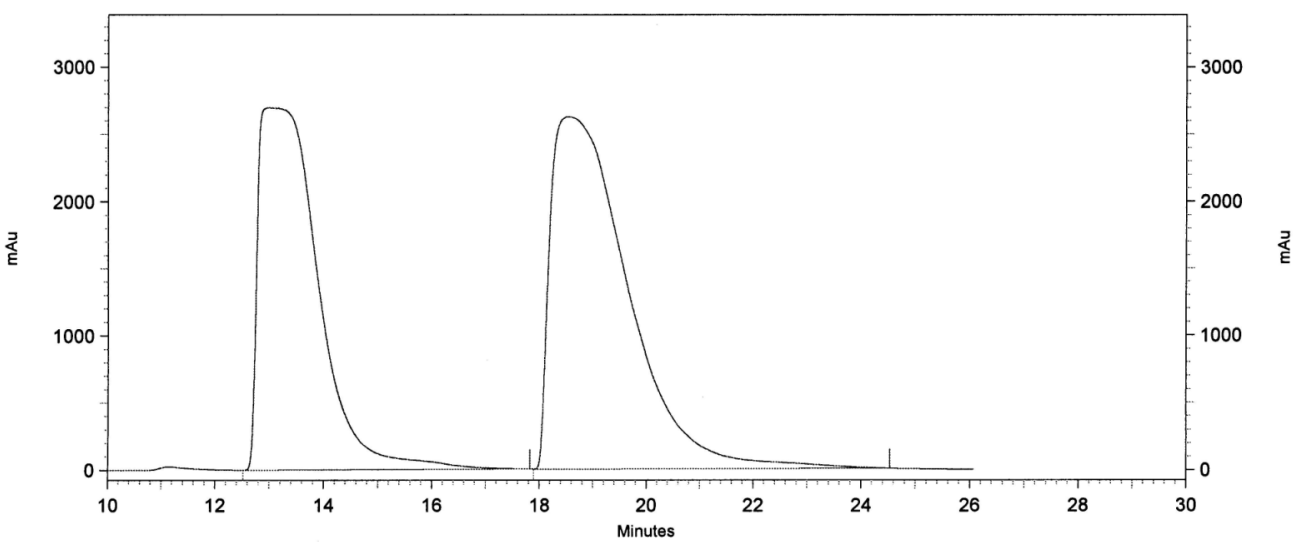

1: 254 nm, 4 nm Results

Retention Time 12.996 Area 263554817 Area \%

18.532

\begin{tabular}{|r|r|r|}
\hline Totals & 470935111 & 100.00 \\
\hline
\end{tabular}

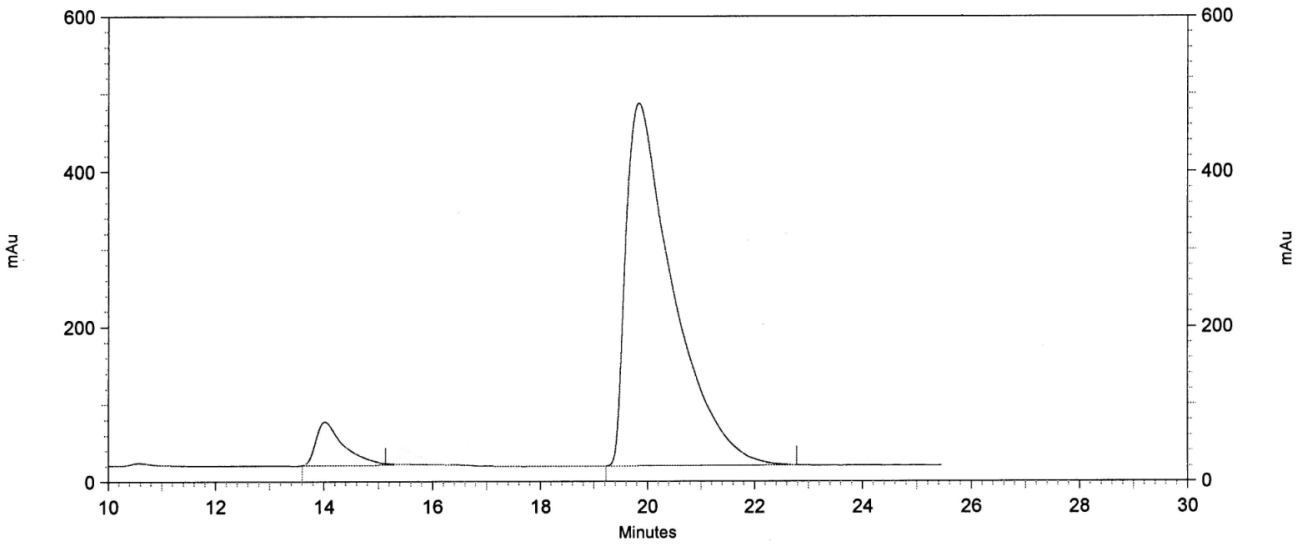

1: 254 nm, 4 nm Results

Retention Time

14.080 Area Area \%

19.856 1202352 54129512 2.17

Totals 55331864 


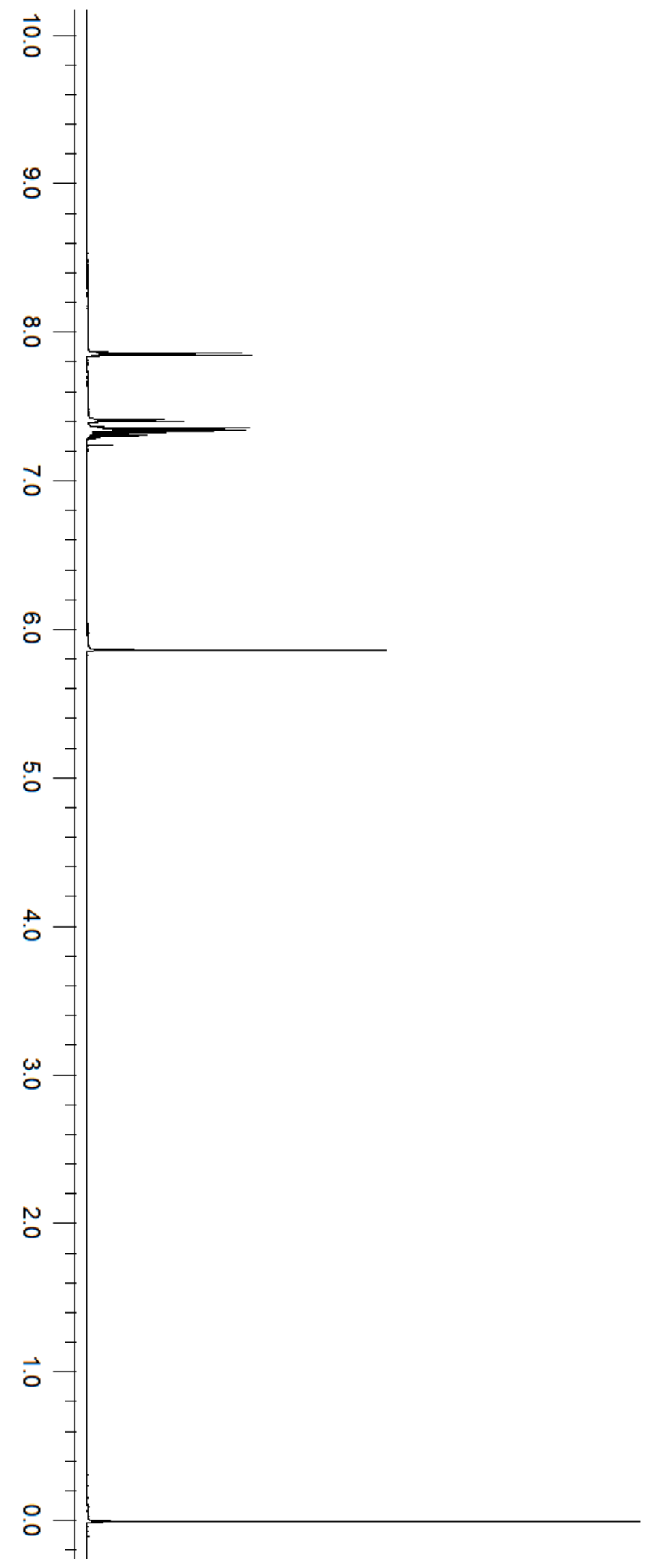

$\mathrm{C}_{N}^{\mathrm{N}}{ }^{\mathrm{Nh}}$

${ }^{1} \mathrm{H}$ 600MHz NMR

N-2-1.6.3a 

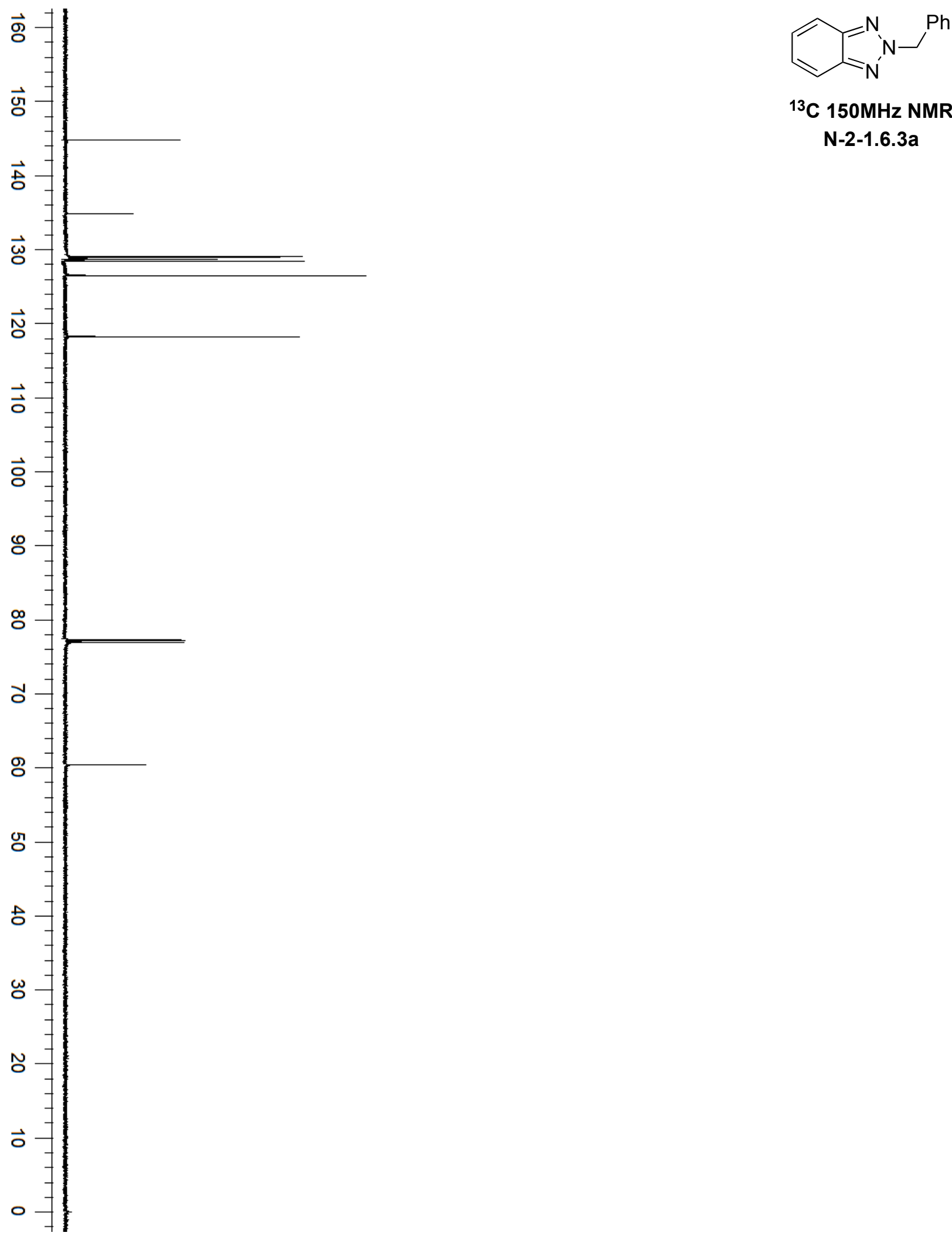

${ }^{13} \mathrm{C}$ 150MHz NMR N-2-1.6.3a

S734 

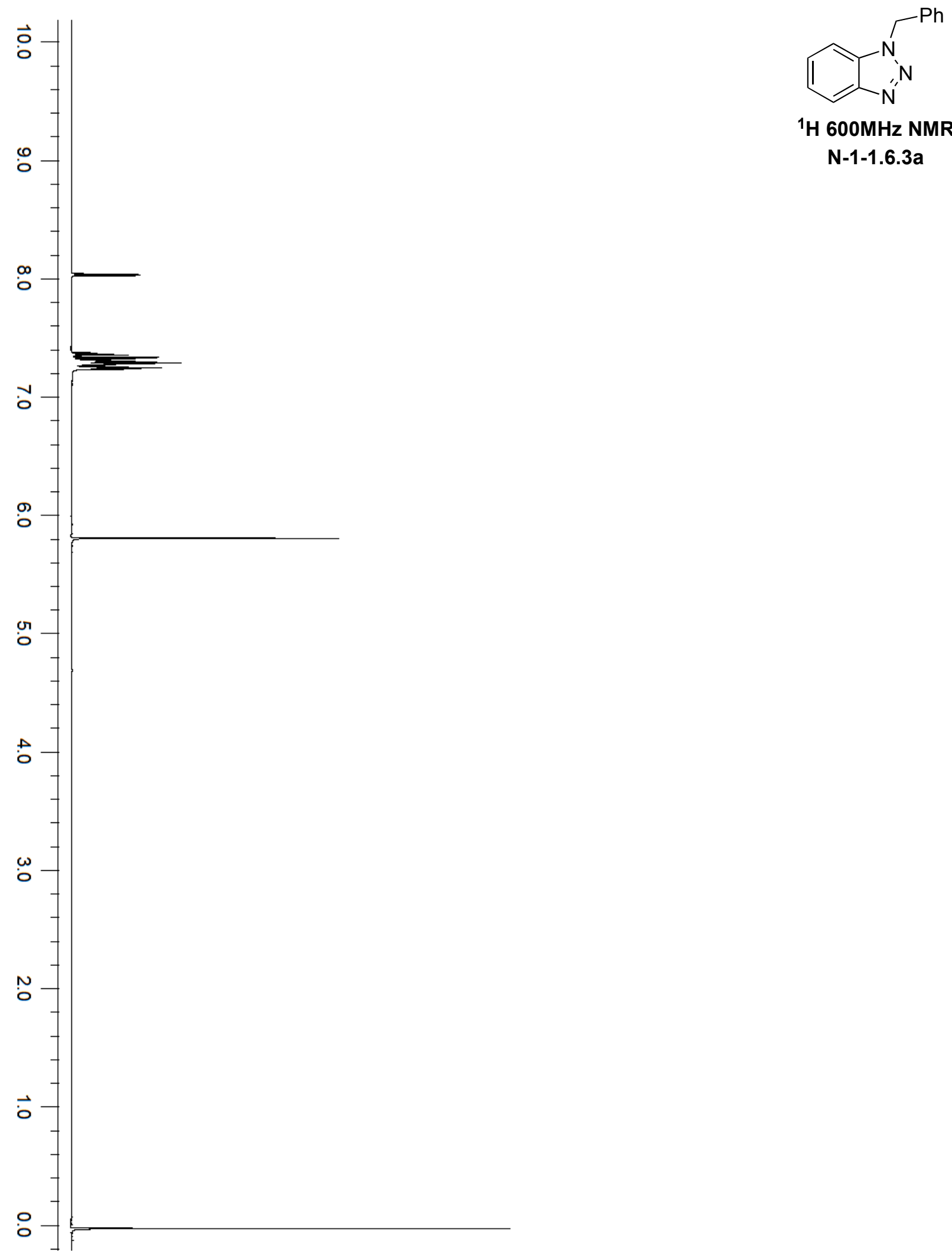

${ }^{1} \mathrm{H}$ 600MHz NMR

$\mathrm{N}-1-1.6 .3 a$ 


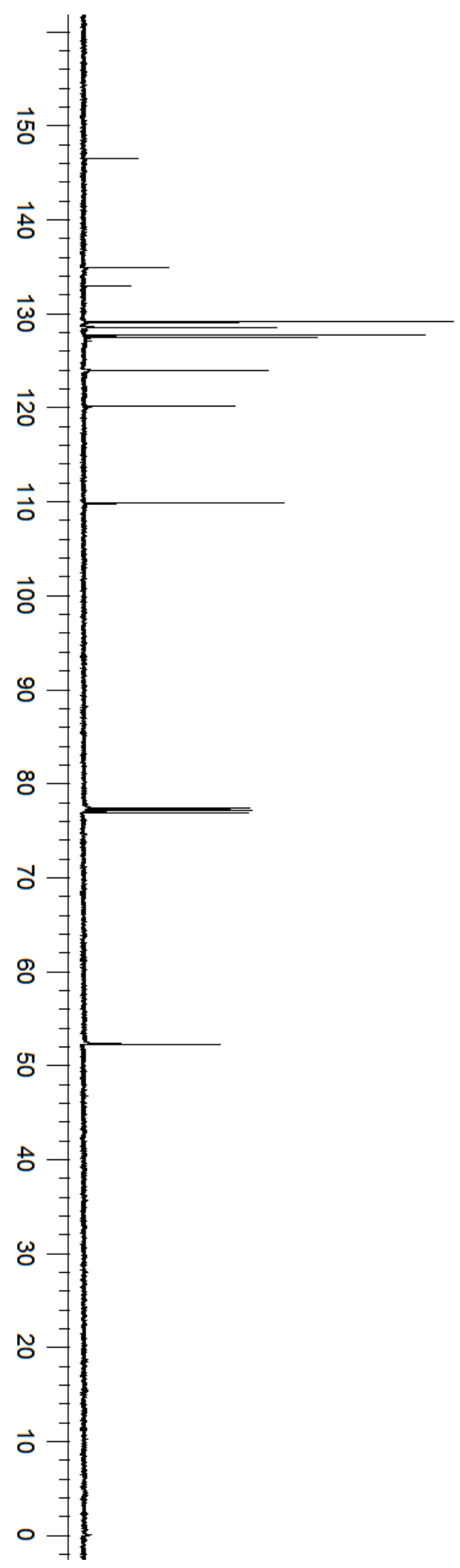

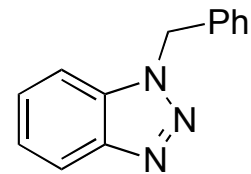

${ }^{13} \mathrm{C} 150 \mathrm{MHz}$ NMR $\mathrm{N}-1-1.6 .3 a$

S736 

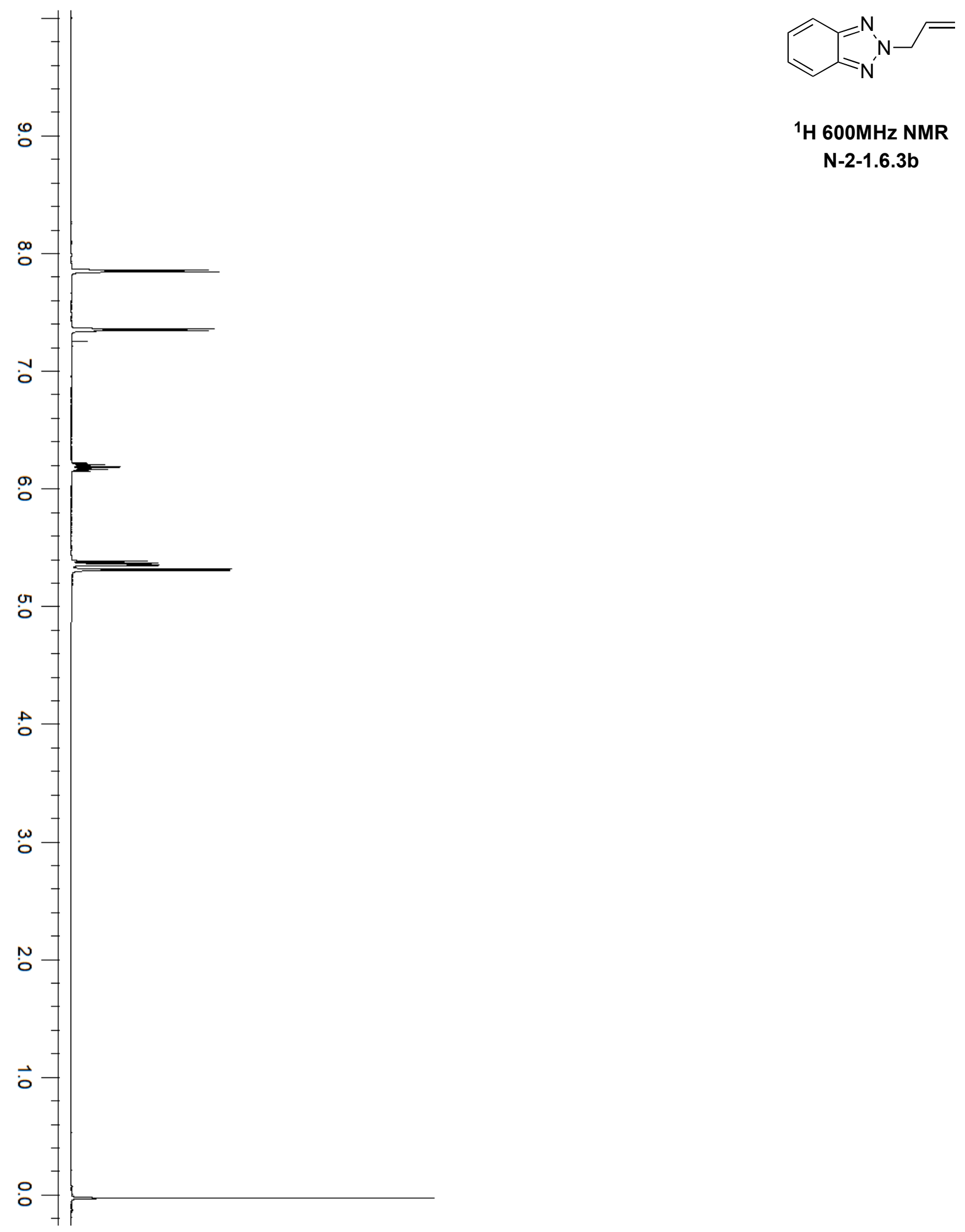

${ }^{1} \mathrm{H}$ 600MHz NMR $\mathrm{N}-2-1.6 .3 \mathrm{~b}$ 


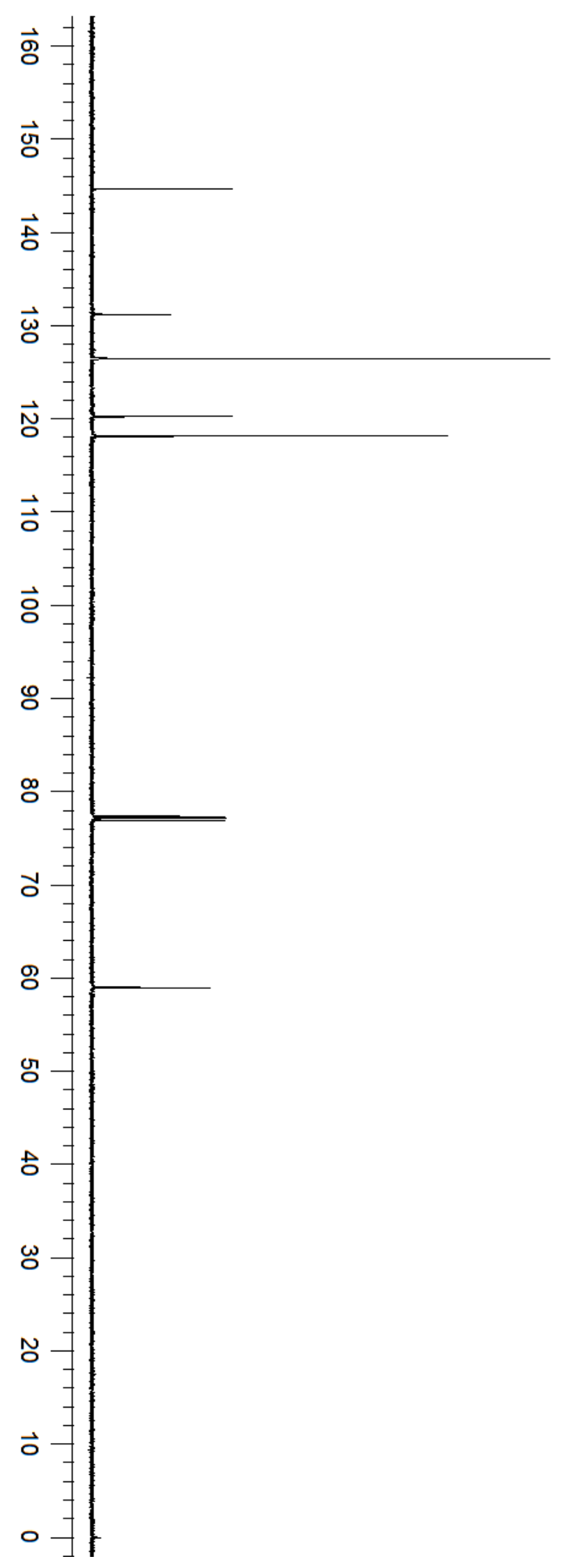

${ }^{13} \mathrm{C}$ 150MHz NMR

N-2-1.6.3b 

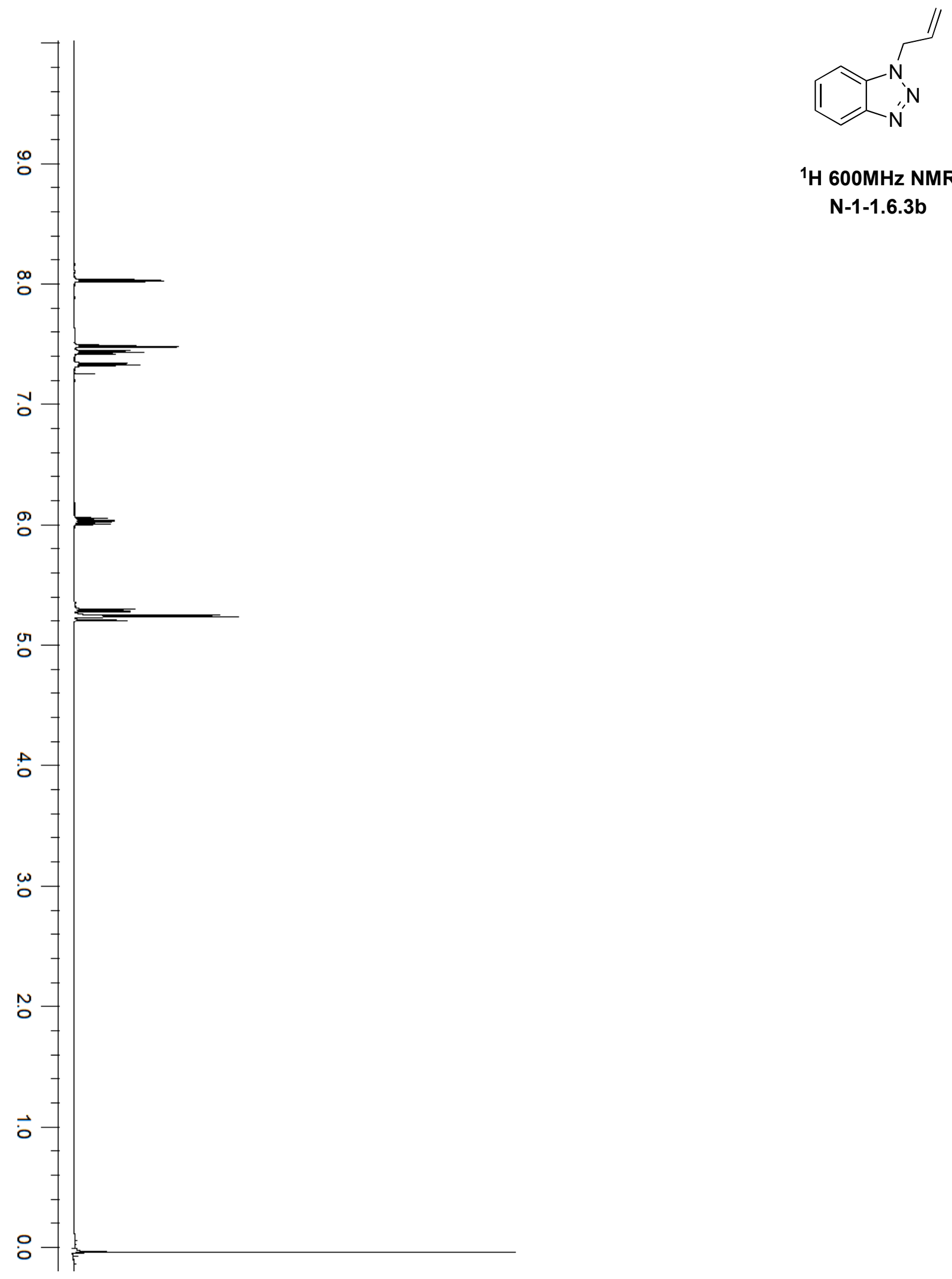

${ }^{1} \mathrm{H}$ 600MHz NMR

$\mathrm{N}-1-1.6 .3 \mathrm{~b}$ 

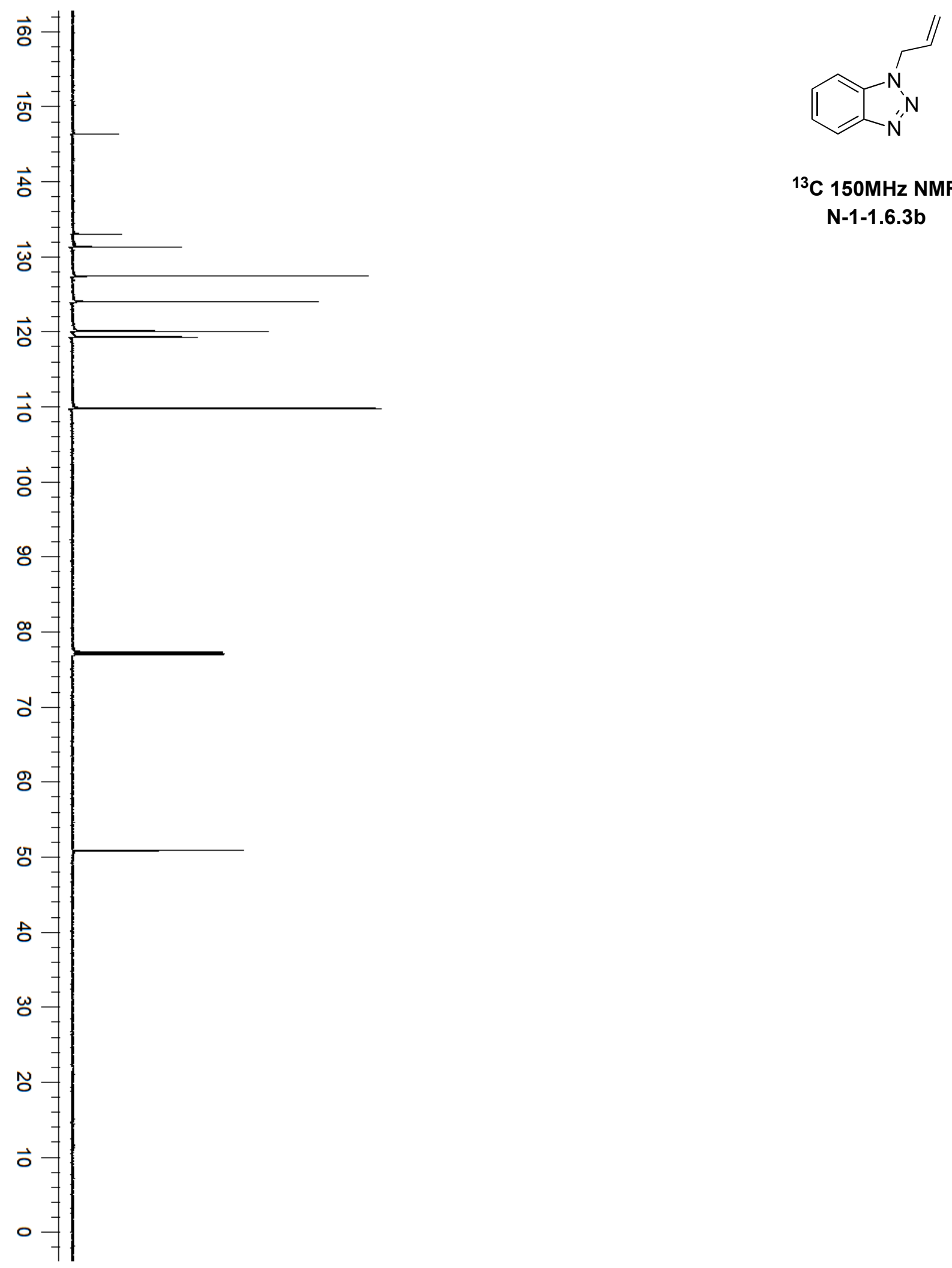

${ }^{13} \mathrm{C} 150 \mathrm{MHz}$ NMR $\mathrm{N}-1-1.6 .3 \mathrm{~b}$ 


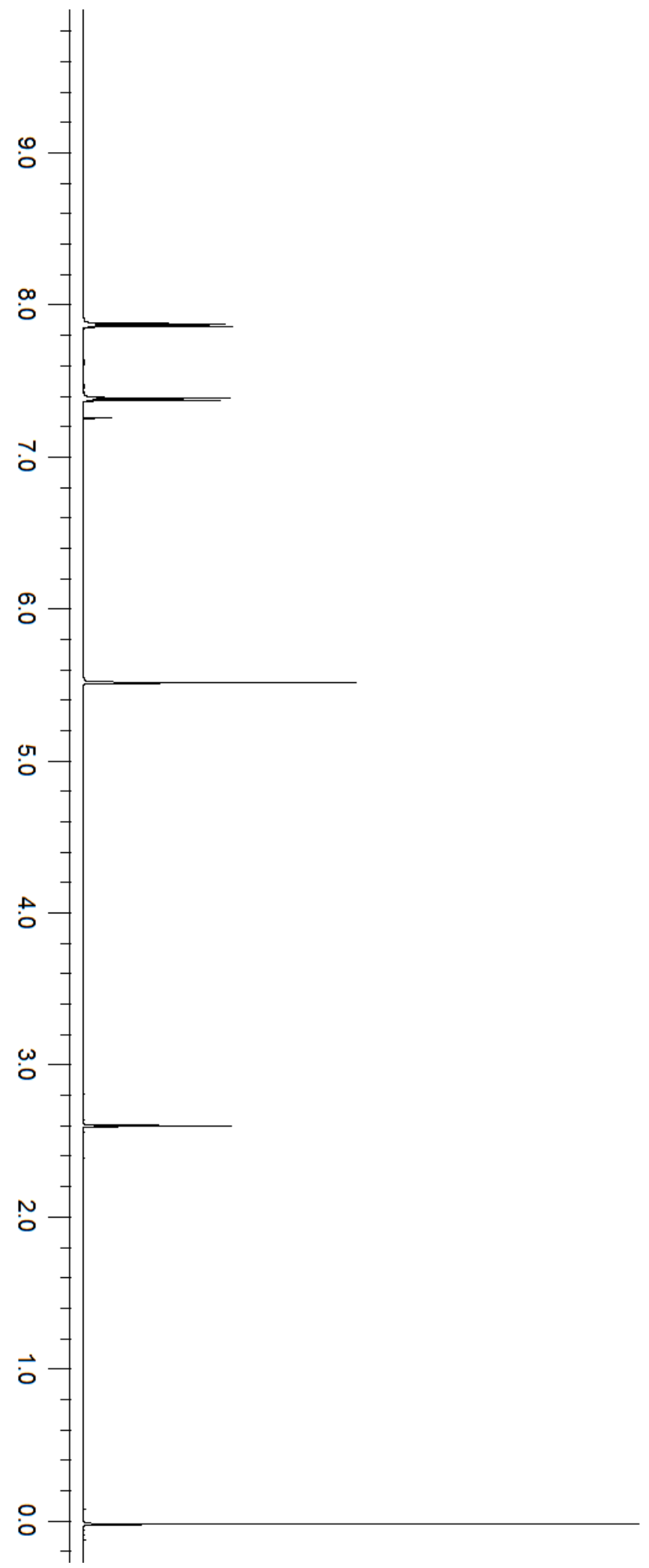

$C_{N^{N}}^{N}=$

${ }^{1} \mathrm{H}$ 600MHz NMR

$\mathrm{N}-2-1.6 .3 \mathrm{C}$ 

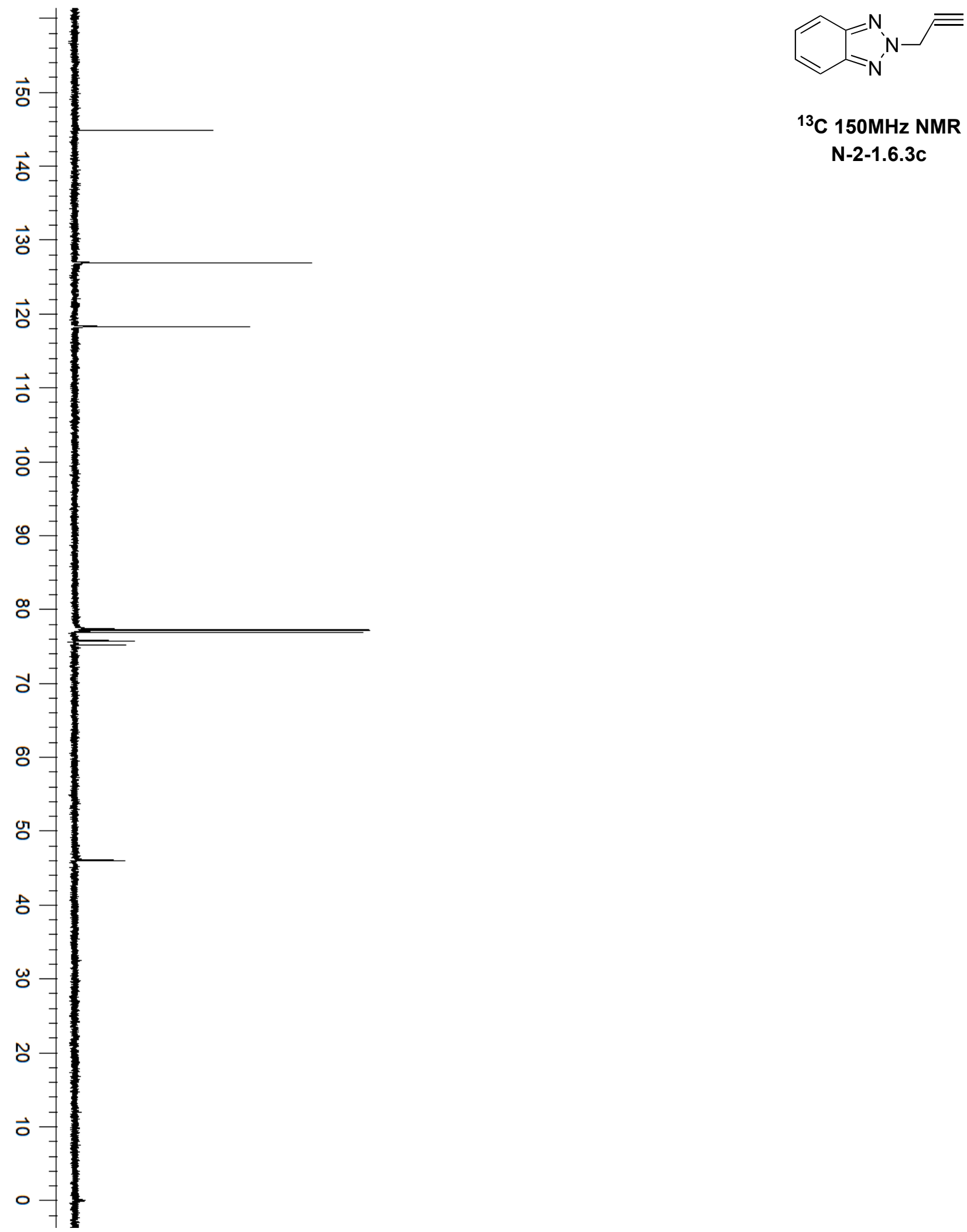

${ }^{13} \mathrm{C}$ 150MHz NMR

$\mathrm{N}-2-1.6 .3 \mathrm{c}$ 


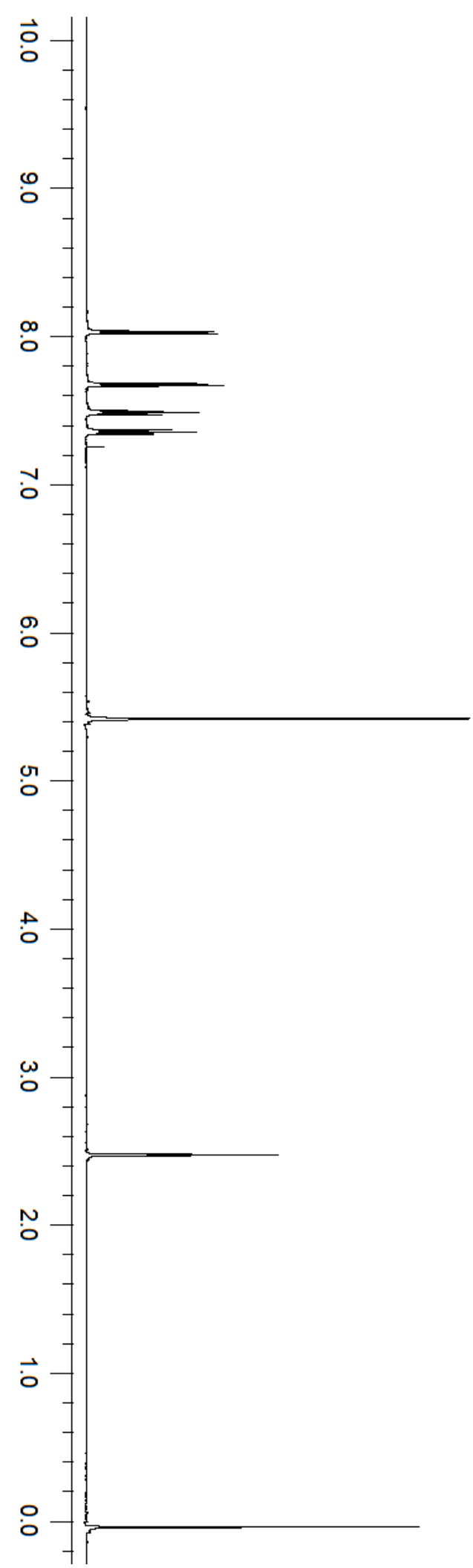

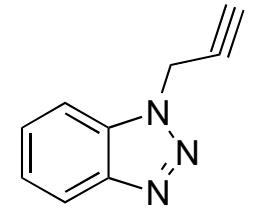

${ }^{1} \mathrm{H}$ 600MHz NMR

$\mathrm{N}-1-1.6 .3 \mathrm{C}$ 


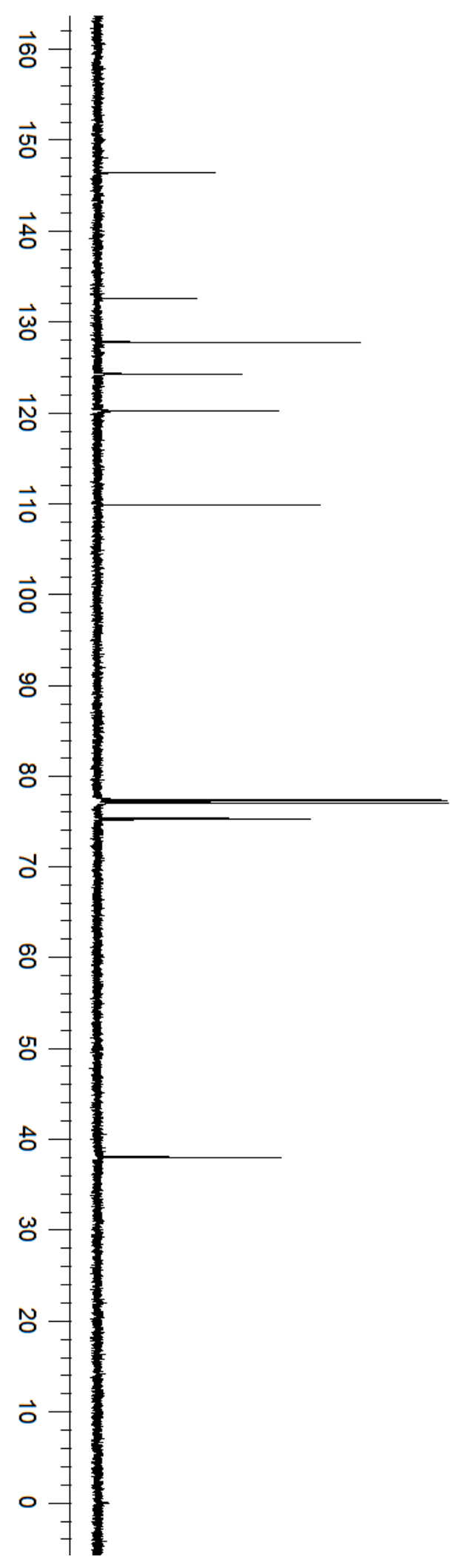

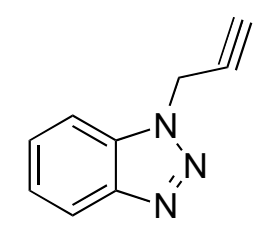

${ }^{13} \mathrm{C} 150 \mathrm{MHz}$ NMR $\mathrm{N}-1-1.6 .3 \mathrm{C}$ 


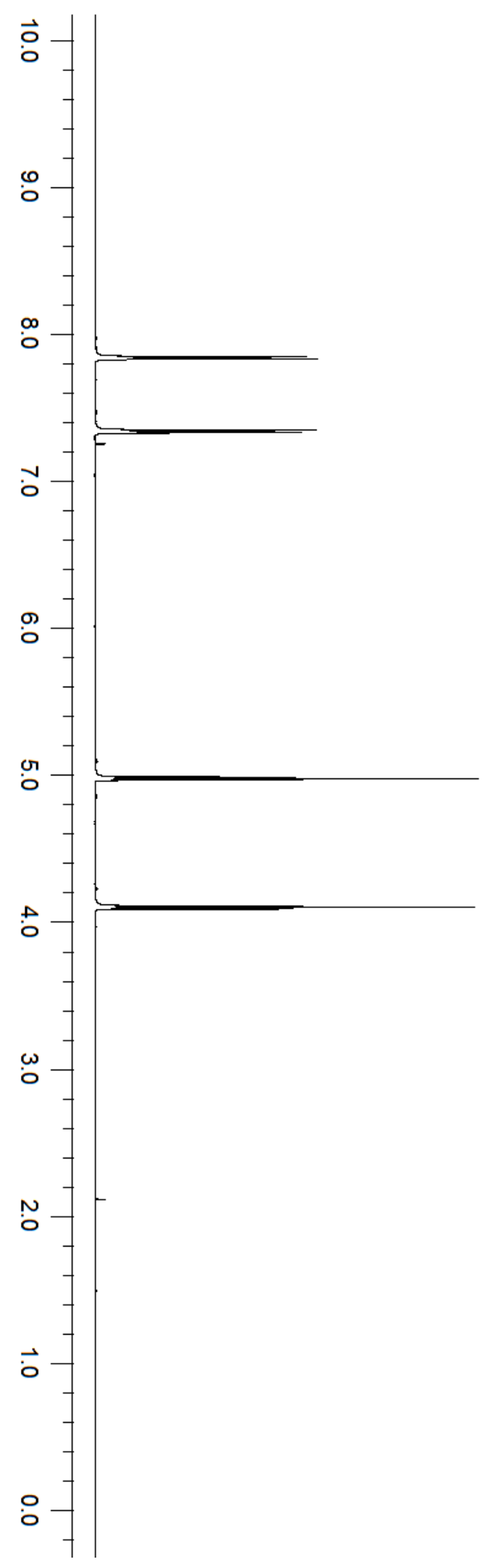

${ }^{1} \mathrm{H} 600 \mathrm{MHz}$ NMR

N-2-1.6.3d 


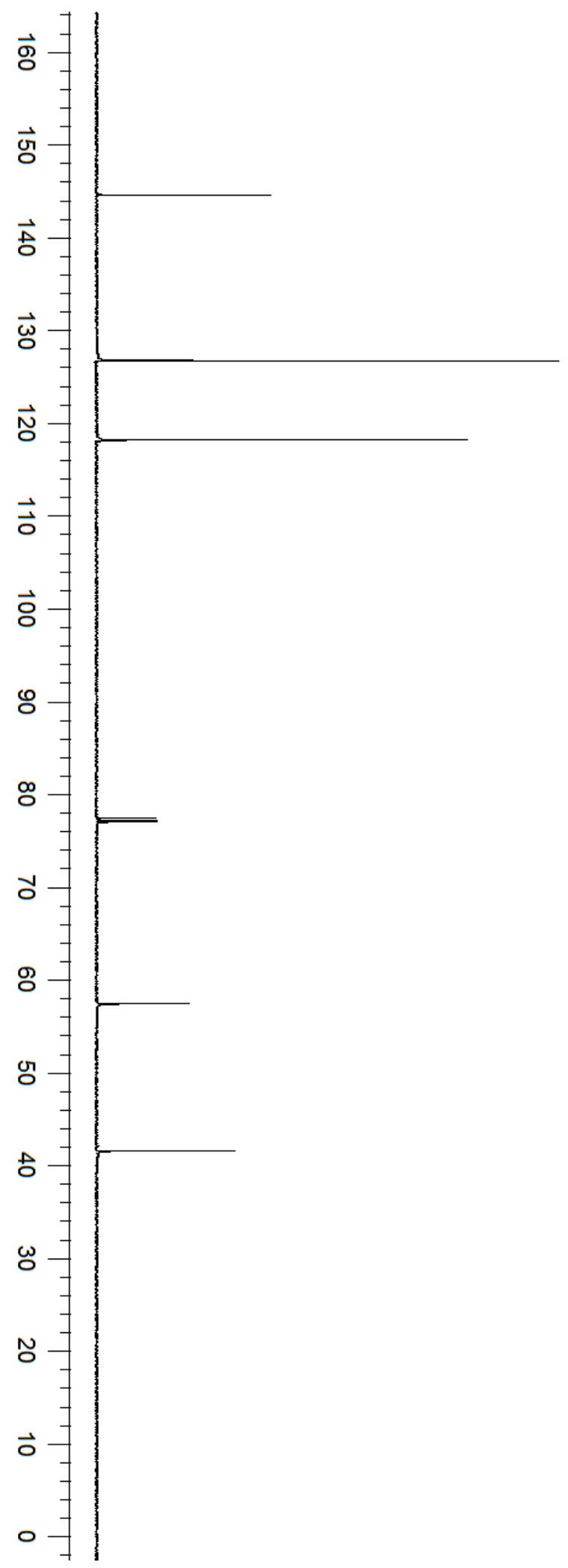

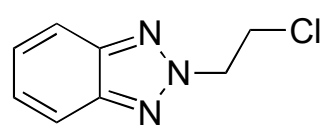

${ }^{13} \mathrm{C} 150 \mathrm{MHz}$ NMR

N-2-1.6.3d 


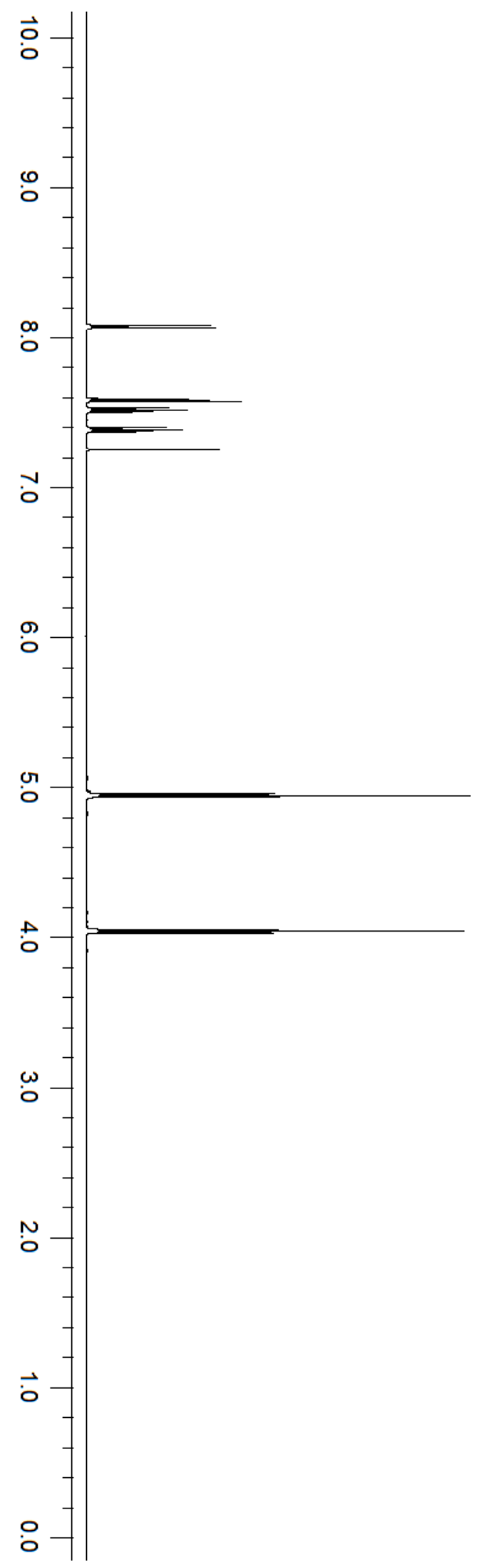

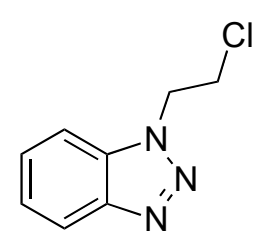

${ }^{1} \mathrm{H} 600 \mathrm{MHz}$ NMR $\mathrm{N}-1-1.6 .3 d$ 


$$
F
$$




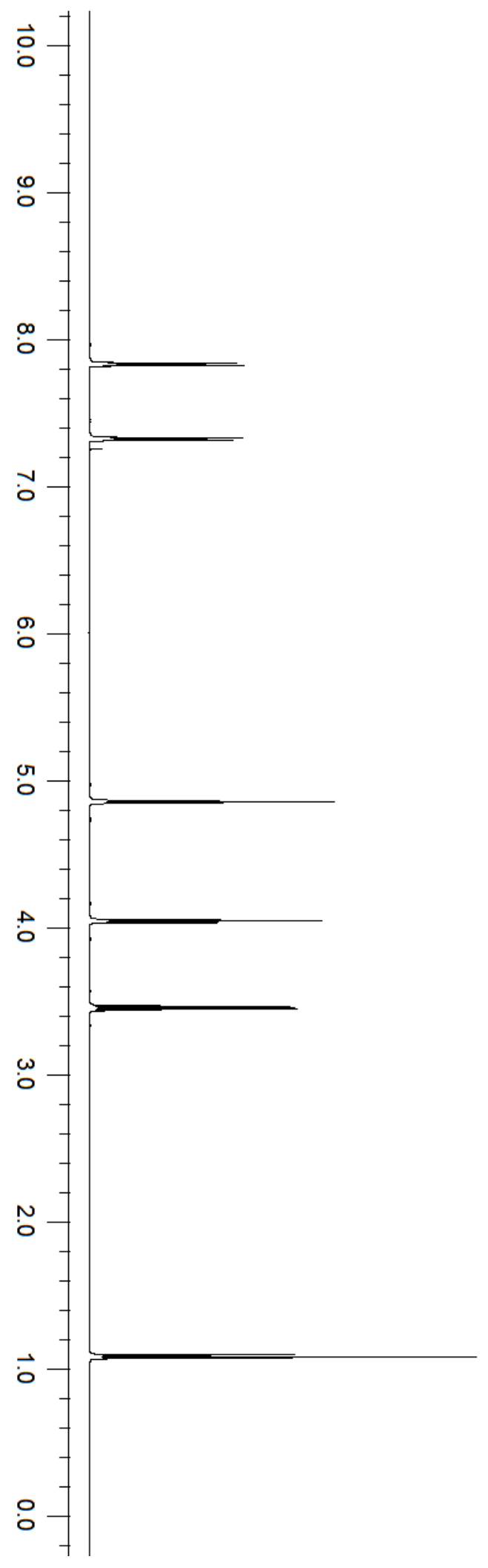

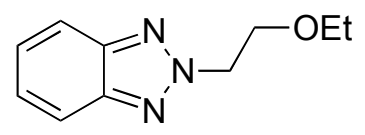

${ }^{1} \mathrm{H} 600 \mathrm{MHz}$ NMR N-2-1.6.3e 


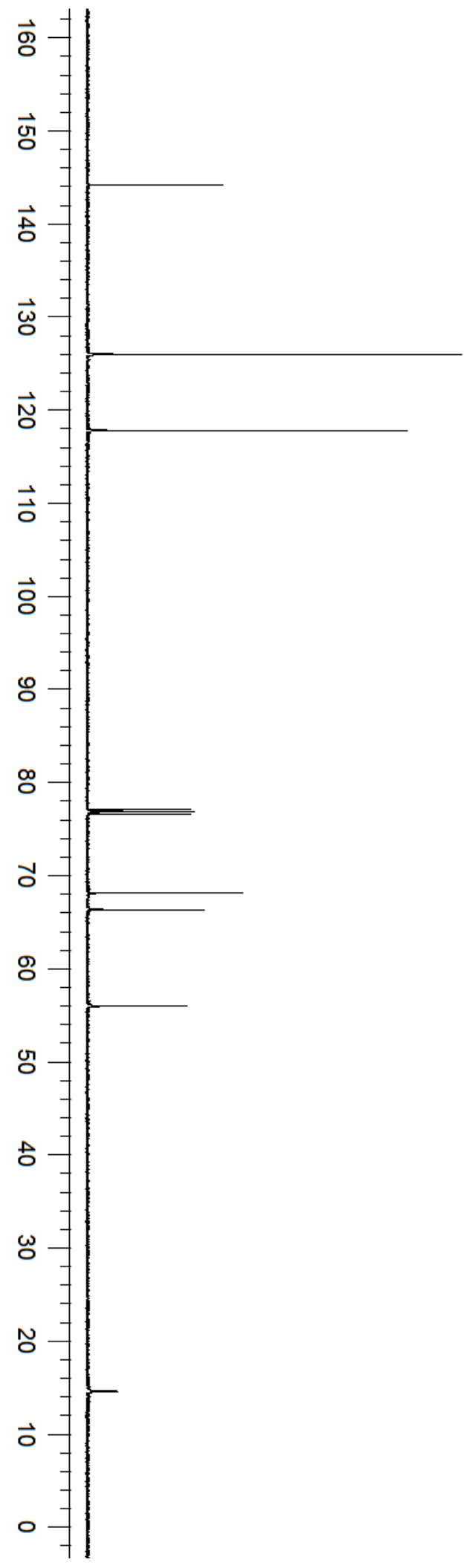

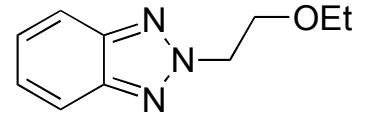

${ }^{13} \mathrm{C} 150 \mathrm{MHz}$ NMR $\mathrm{N}-2-1.6 .3 e$ 


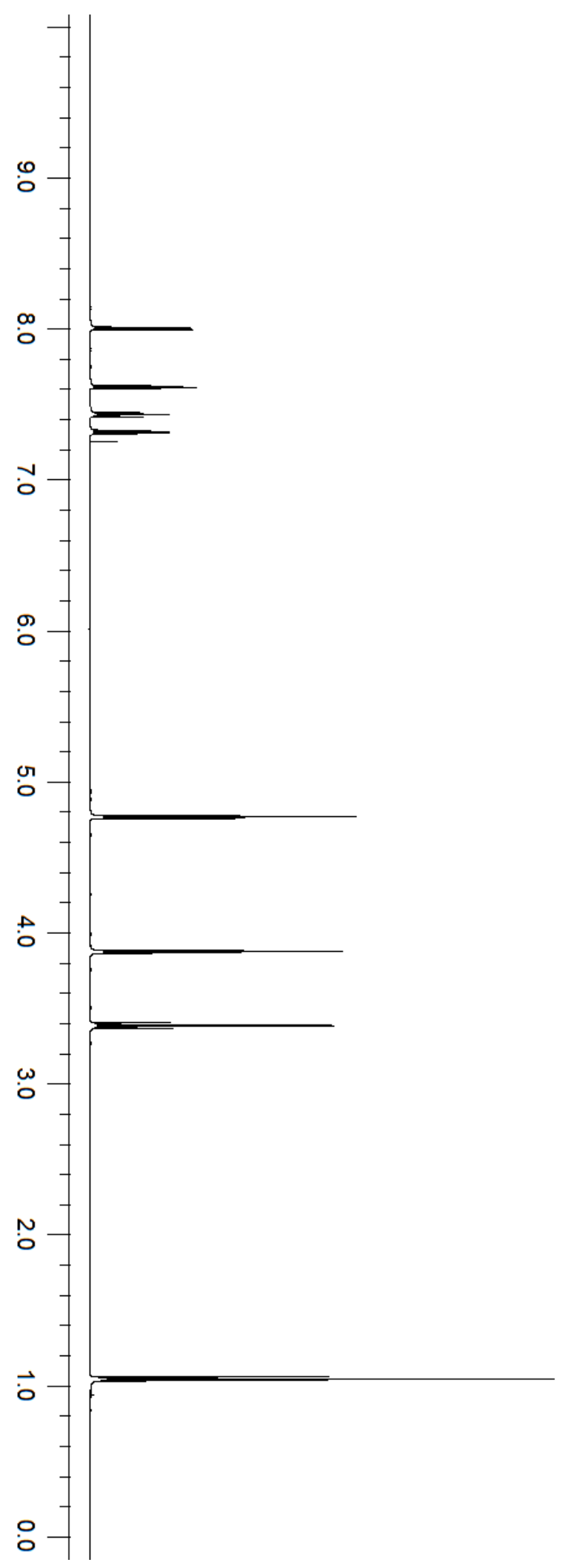

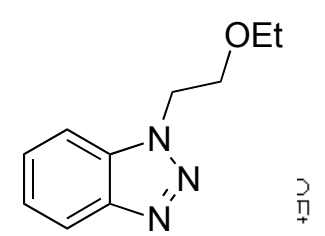

${ }^{1} \mathrm{H} 600 \mathrm{MHz}$ NMR

$\mathrm{N}-1-1.6 .3 e$ 

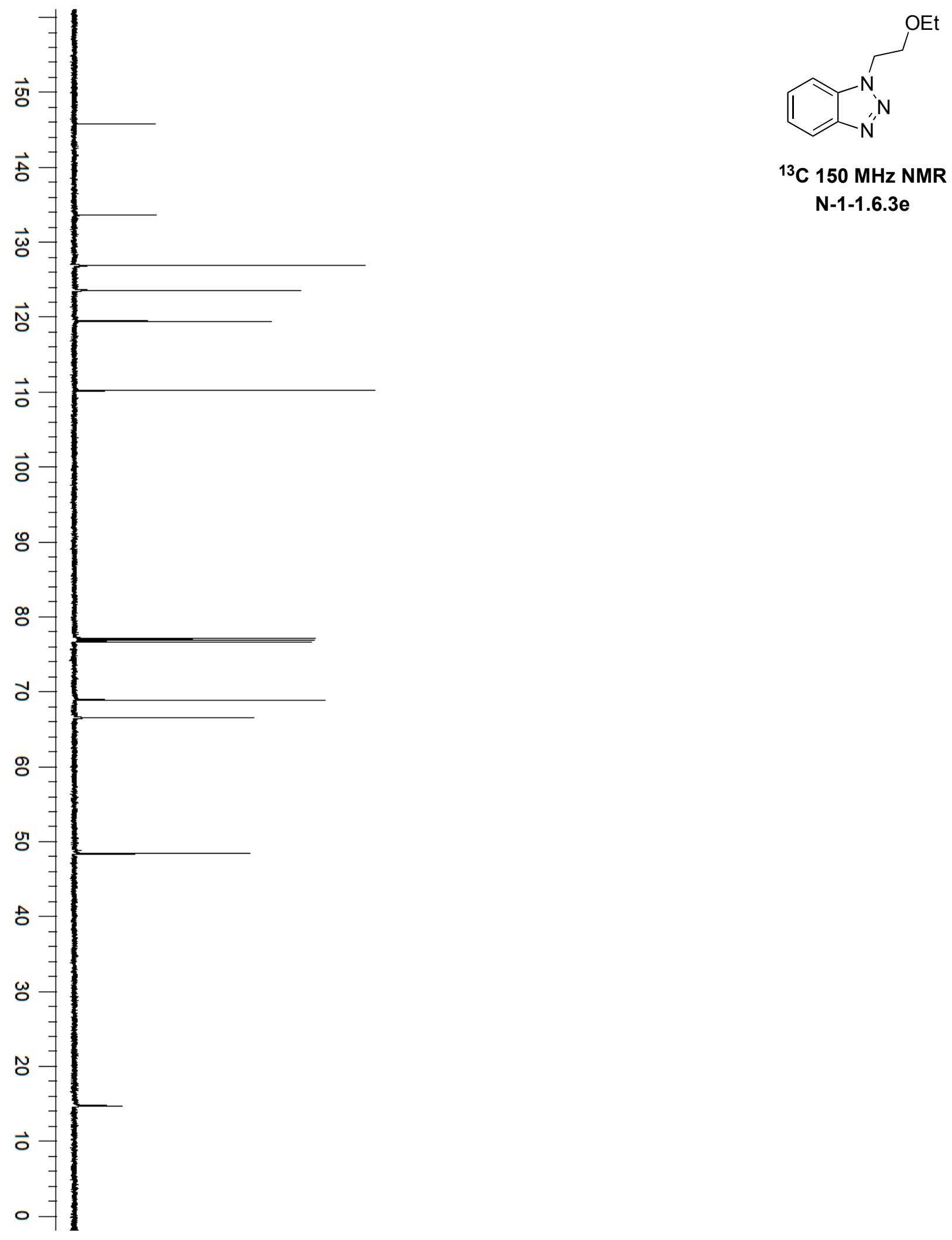

${ }^{13} \mathrm{C} 150 \mathrm{MHz}$ NMR $\mathrm{N}-1-1.6 .3 \mathrm{e}$ 


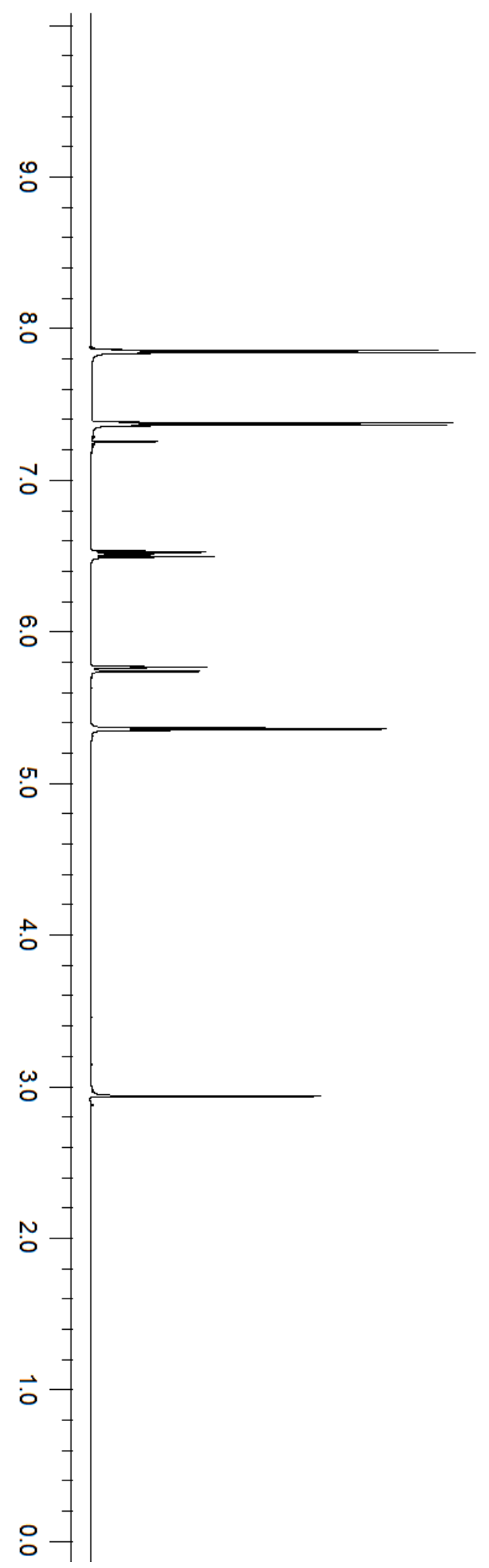

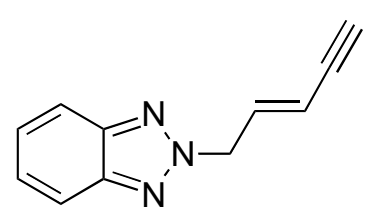

${ }^{1} \mathrm{H} 600 \mathrm{MHz}$ NMR

$\mathrm{N}-2-1.6 .3 f$ 

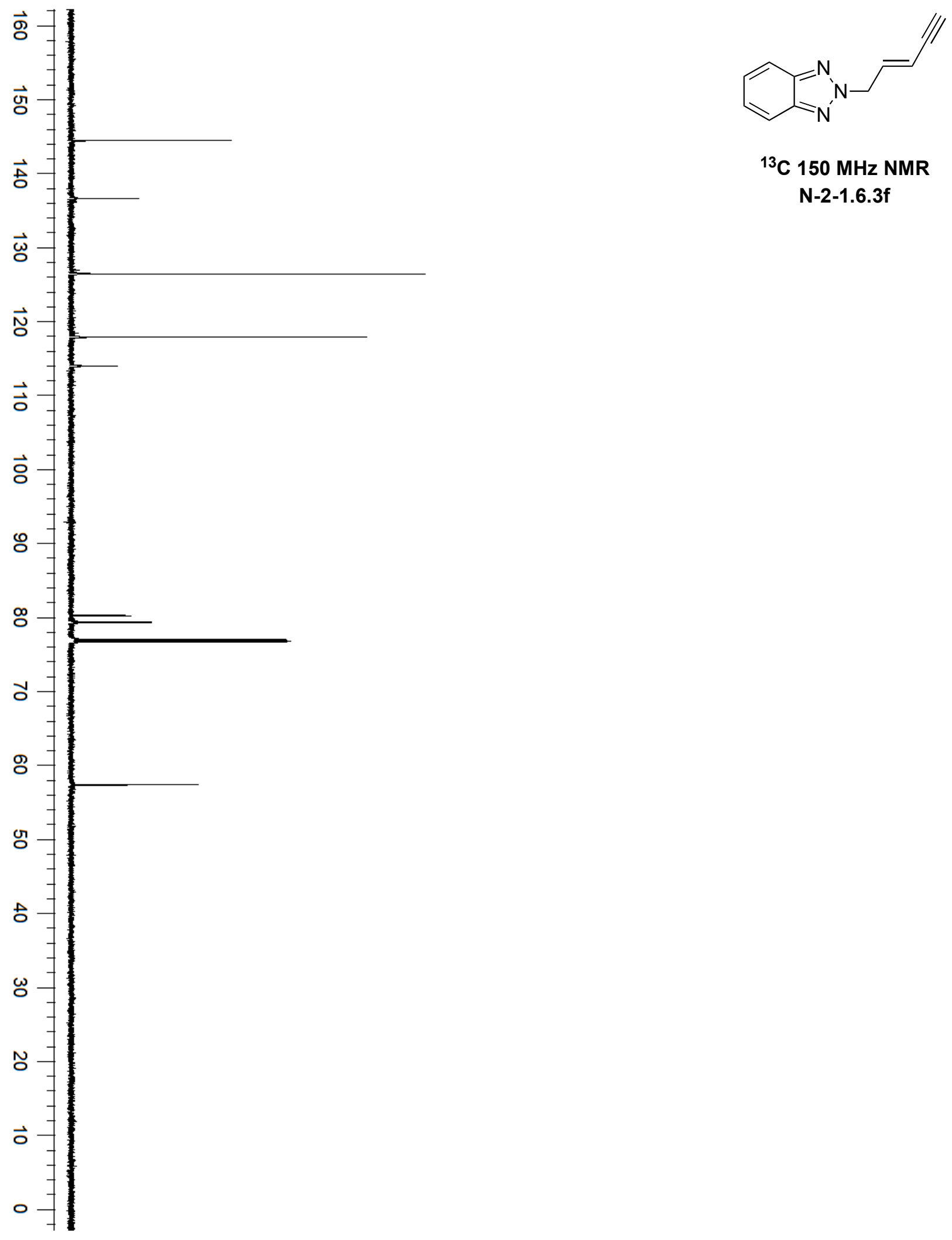

${ }^{13} \mathrm{C} 150 \mathrm{MHz}$ NMR

$\mathrm{N}-2-1.6 .3 \mathrm{f}$ 


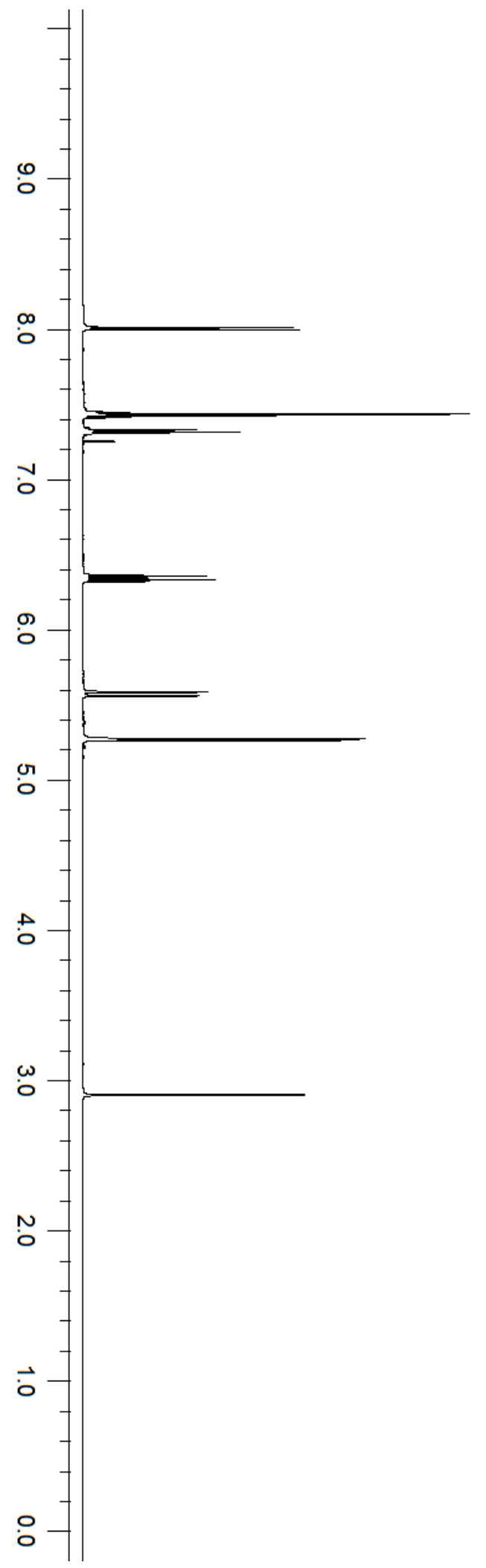

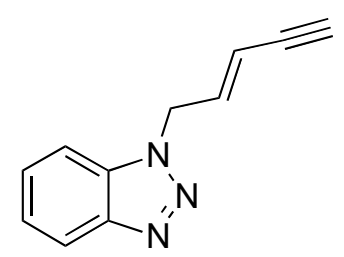

${ }^{1} \mathrm{H} 600 \mathrm{MHz}$ NMR N-1-1.6.3f 

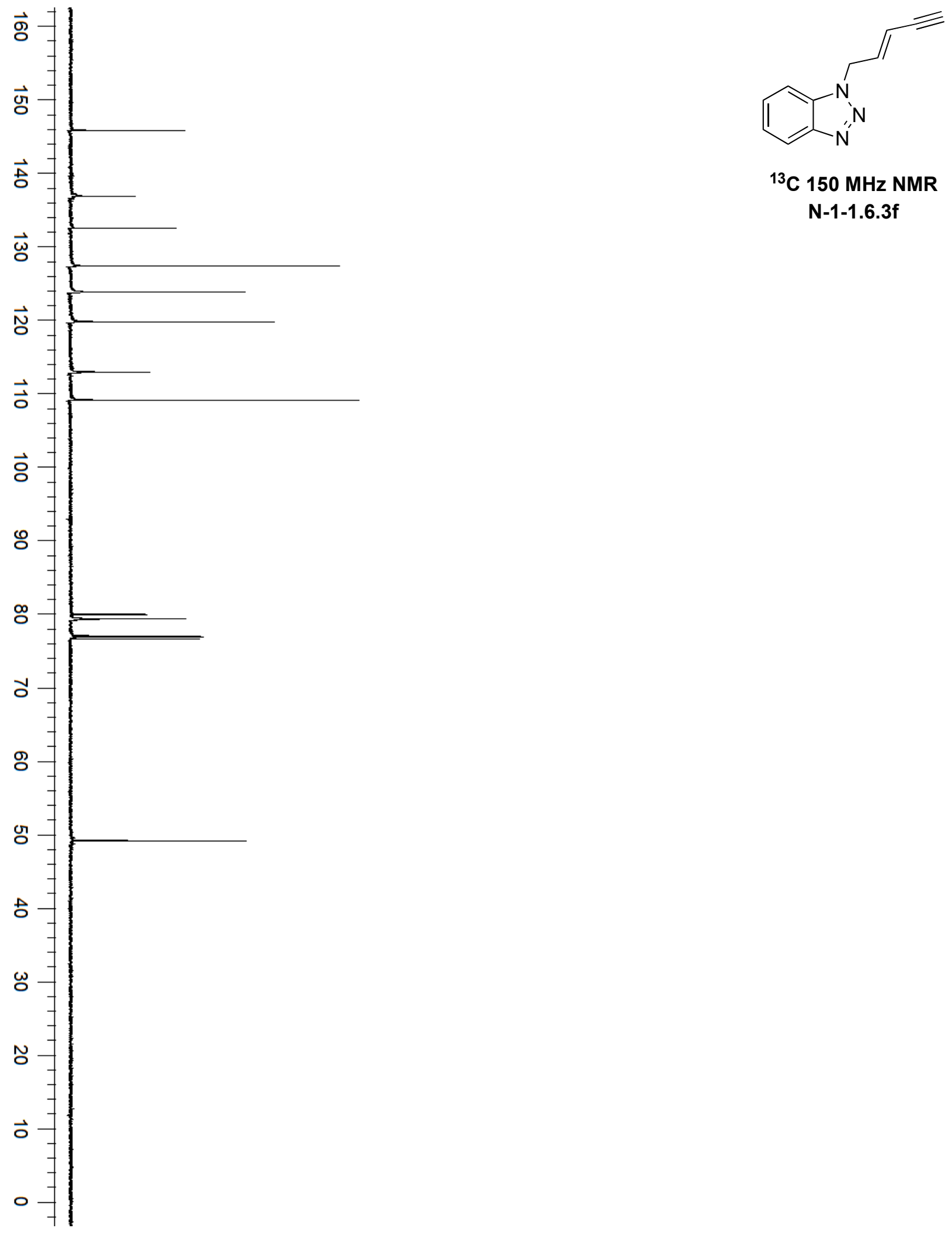

${ }^{13} \mathrm{C} 150 \mathrm{MHz}$ NMR N-1-1.6.3f 


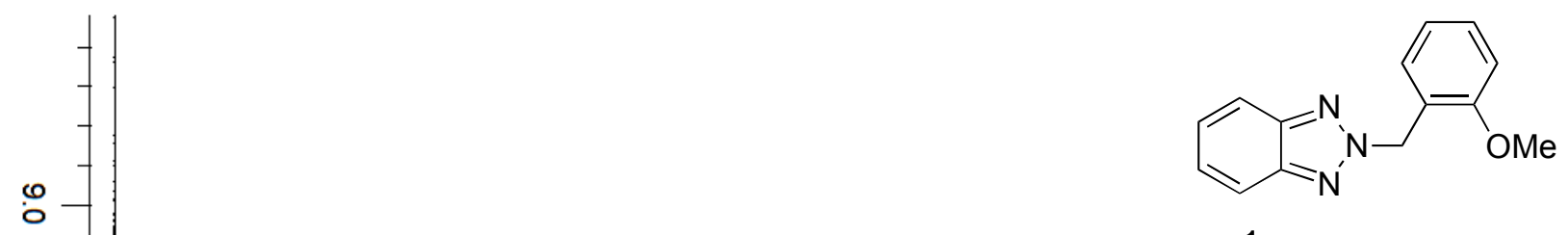

${ }^{1} \mathrm{H} 600 \mathrm{MHz}$ NMR

N-2-1.6.3g

$\infty$

0

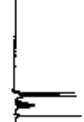

$\therefore-5$

9

cr

-1
-1
$0-1$

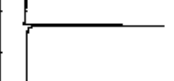

s.

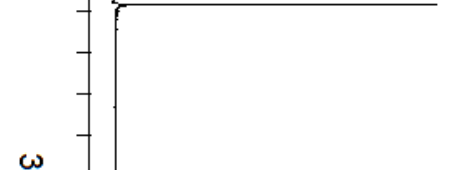

0

n

$\overrightarrow{0}$

$\circ-1$ 


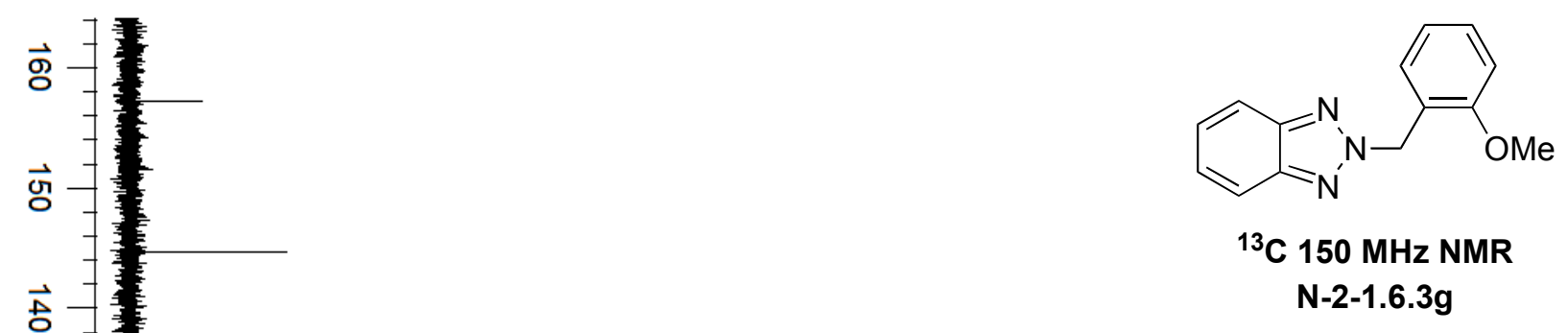




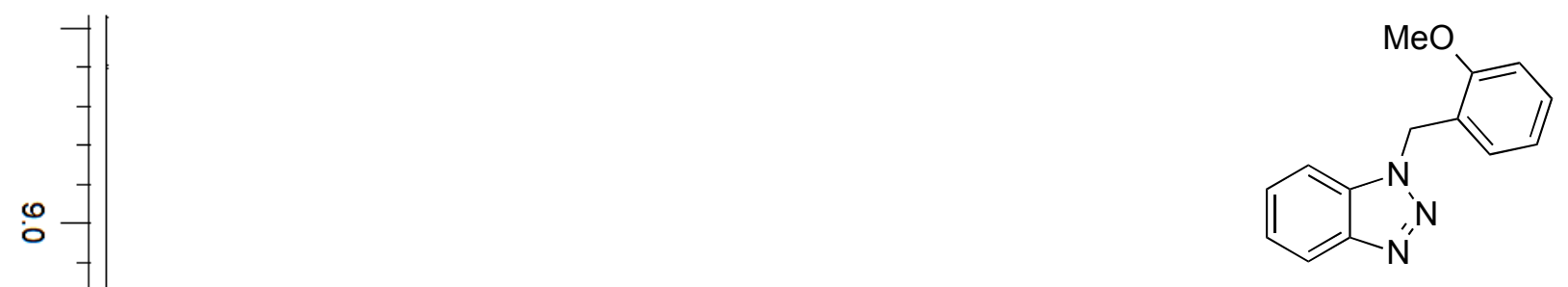

${ }^{1} \mathrm{H} 600 \mathrm{MHz}$ NMR

$\mathrm{N}-1-1.6 .3 \mathrm{~g}$

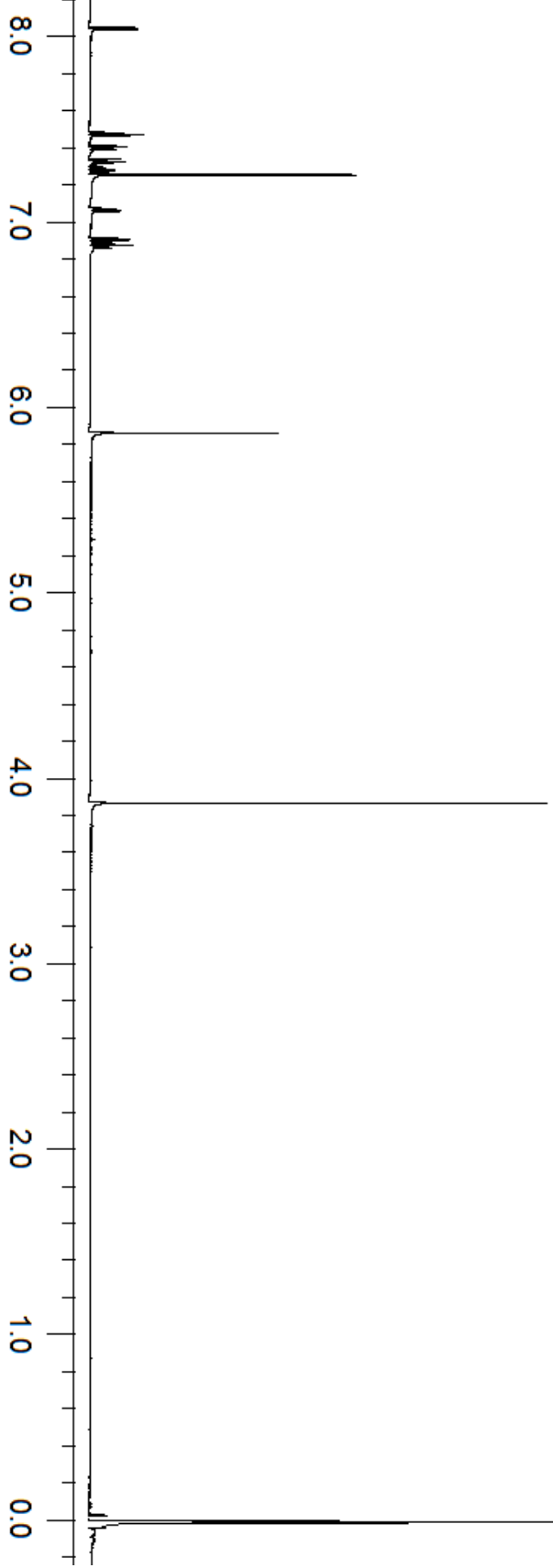




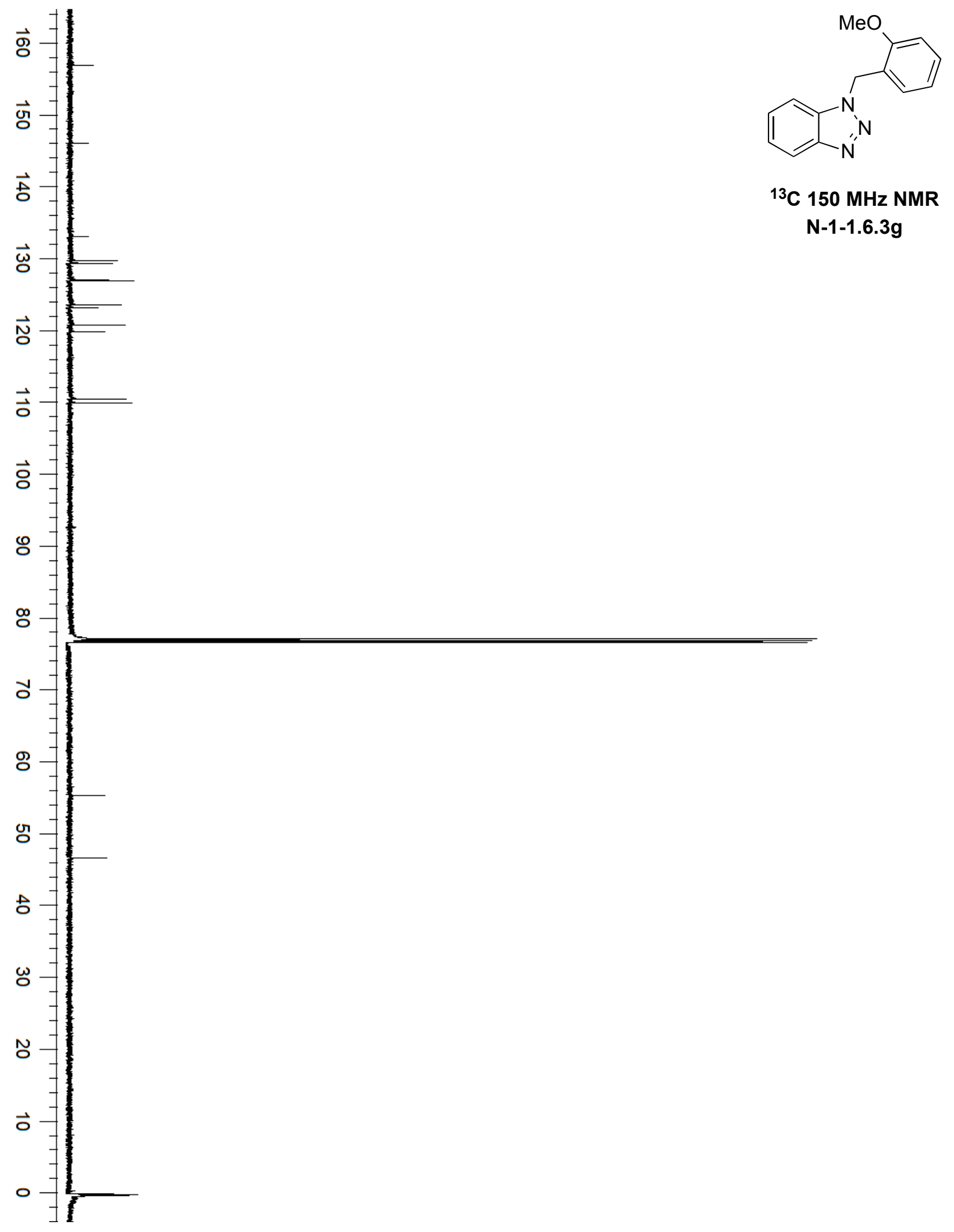




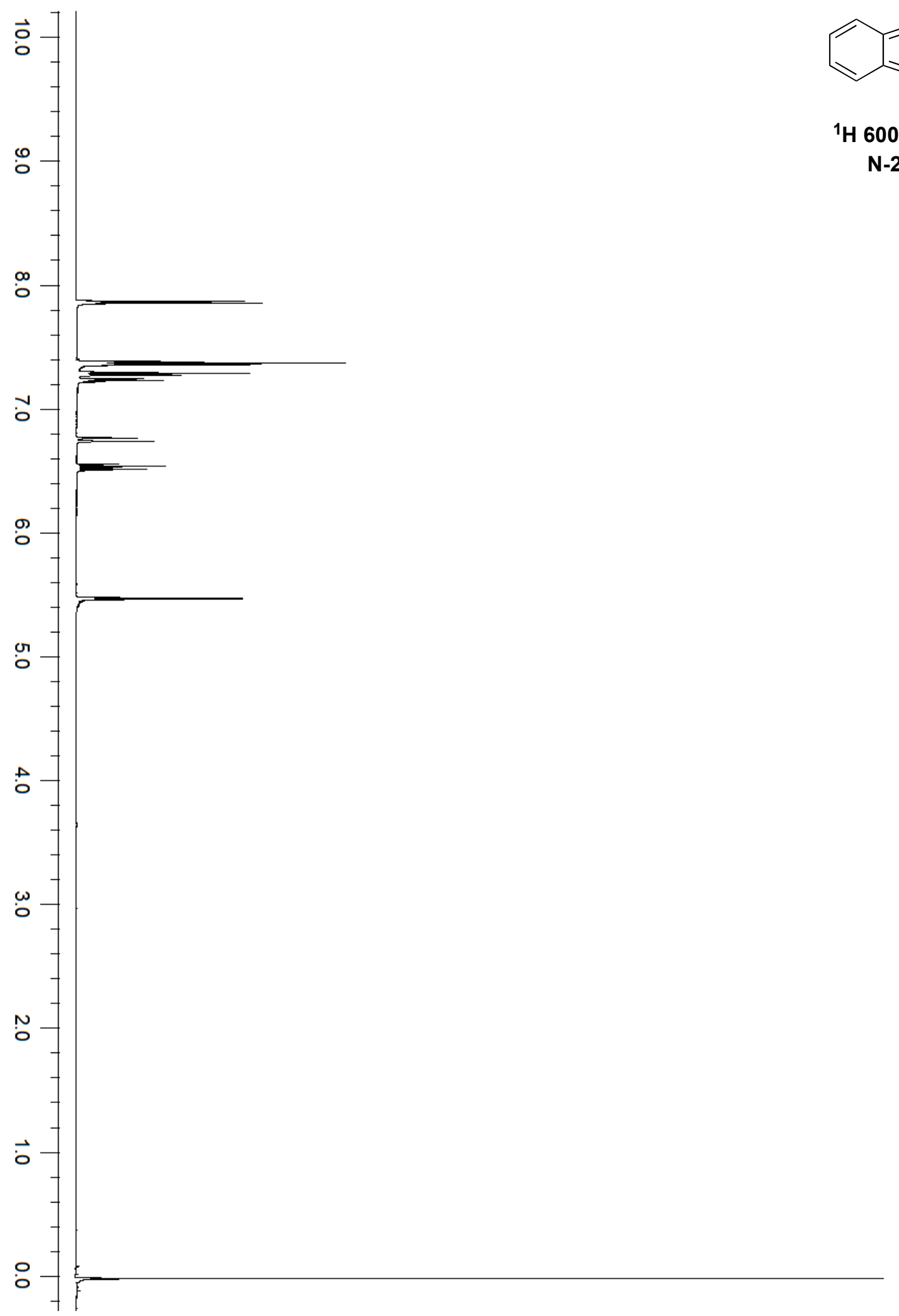



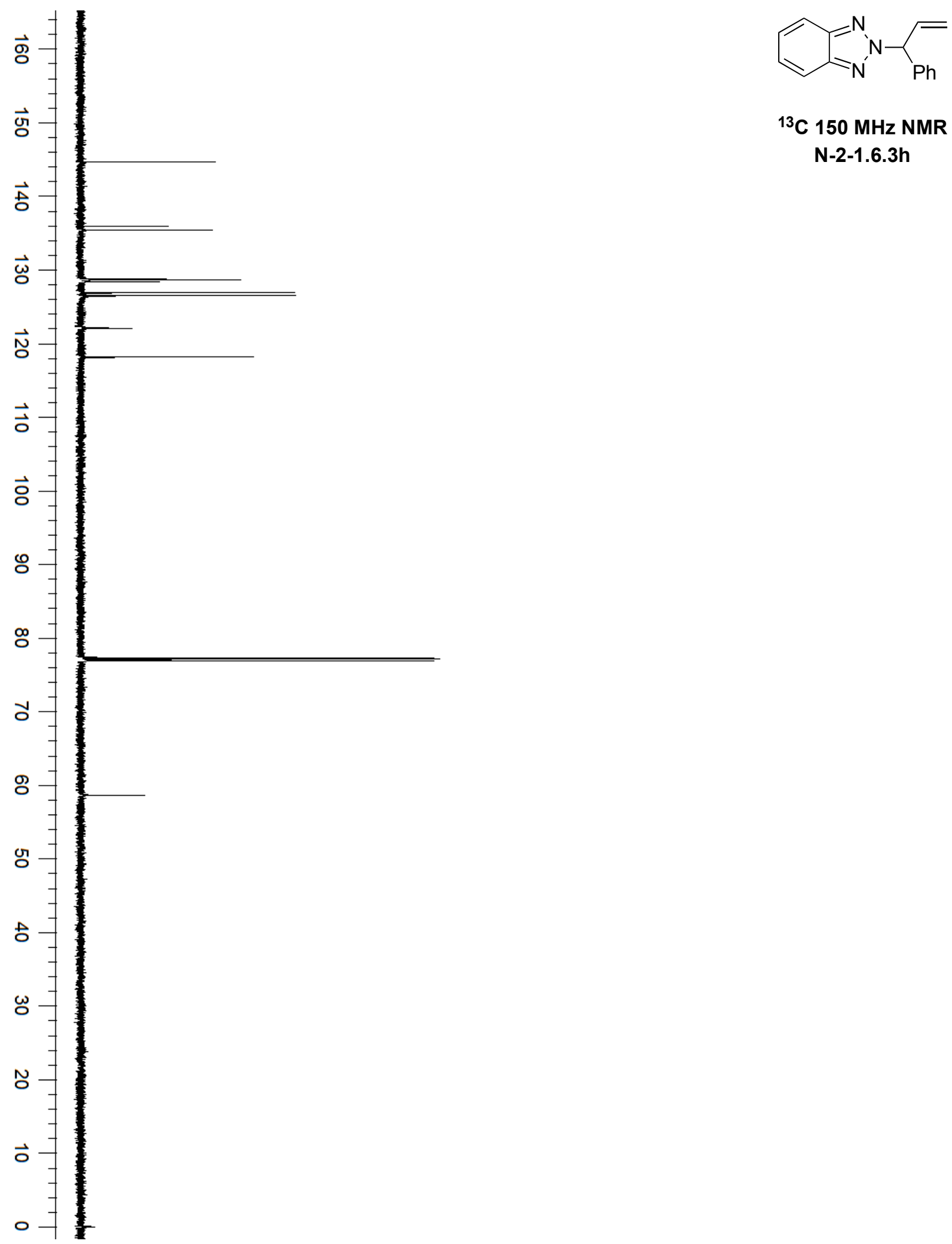

${ }^{13} \mathrm{C} 150 \mathrm{MHz}$ NMR $\mathrm{N}-2-1.6 .3 \mathrm{~h}$ 


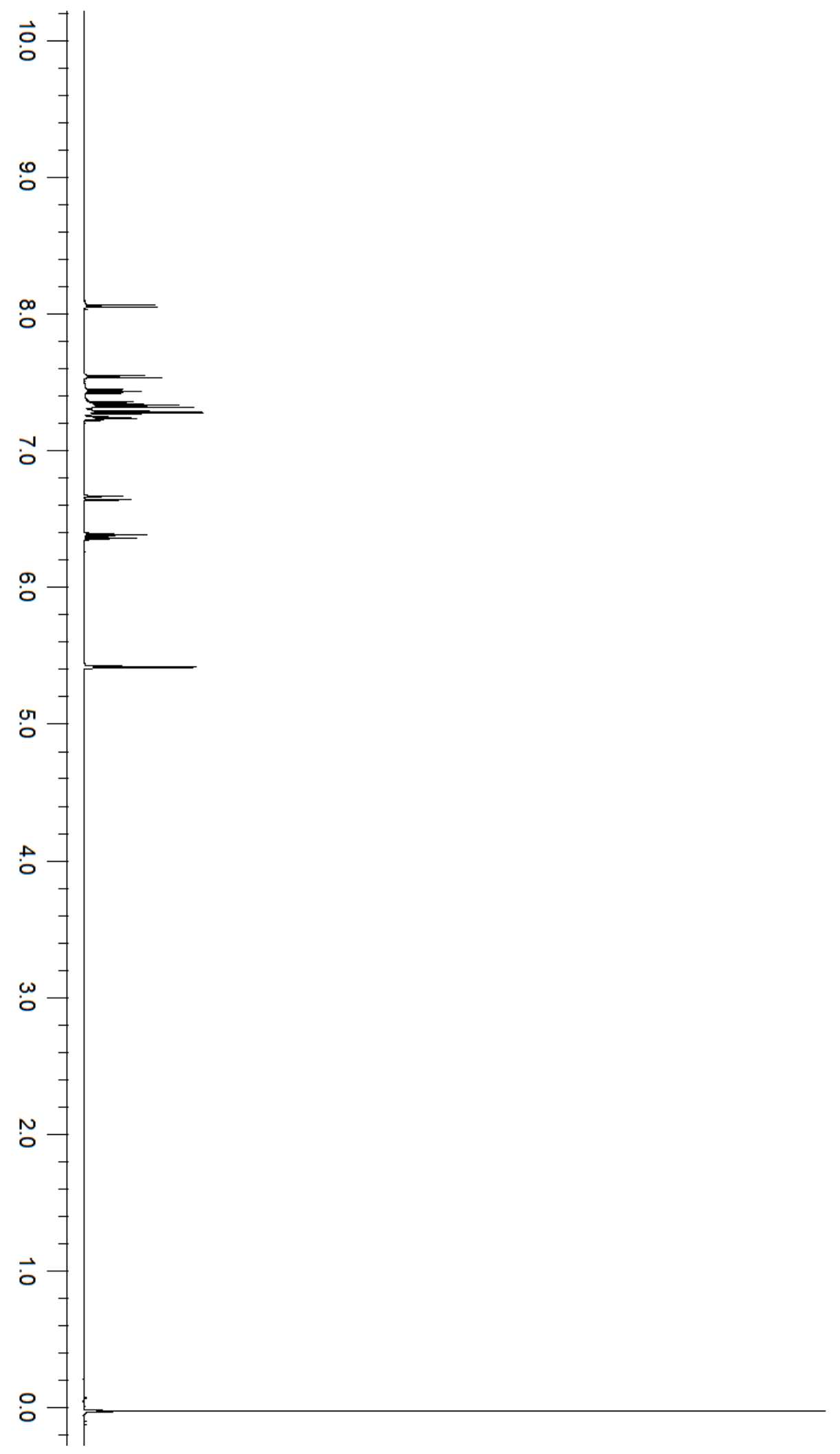

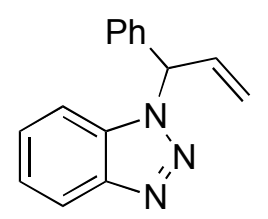

${ }^{1} \mathrm{H} 600 \mathrm{MHz}$ NMR $\mathrm{N}-1-1.6 .3 \mathrm{~h}$ 

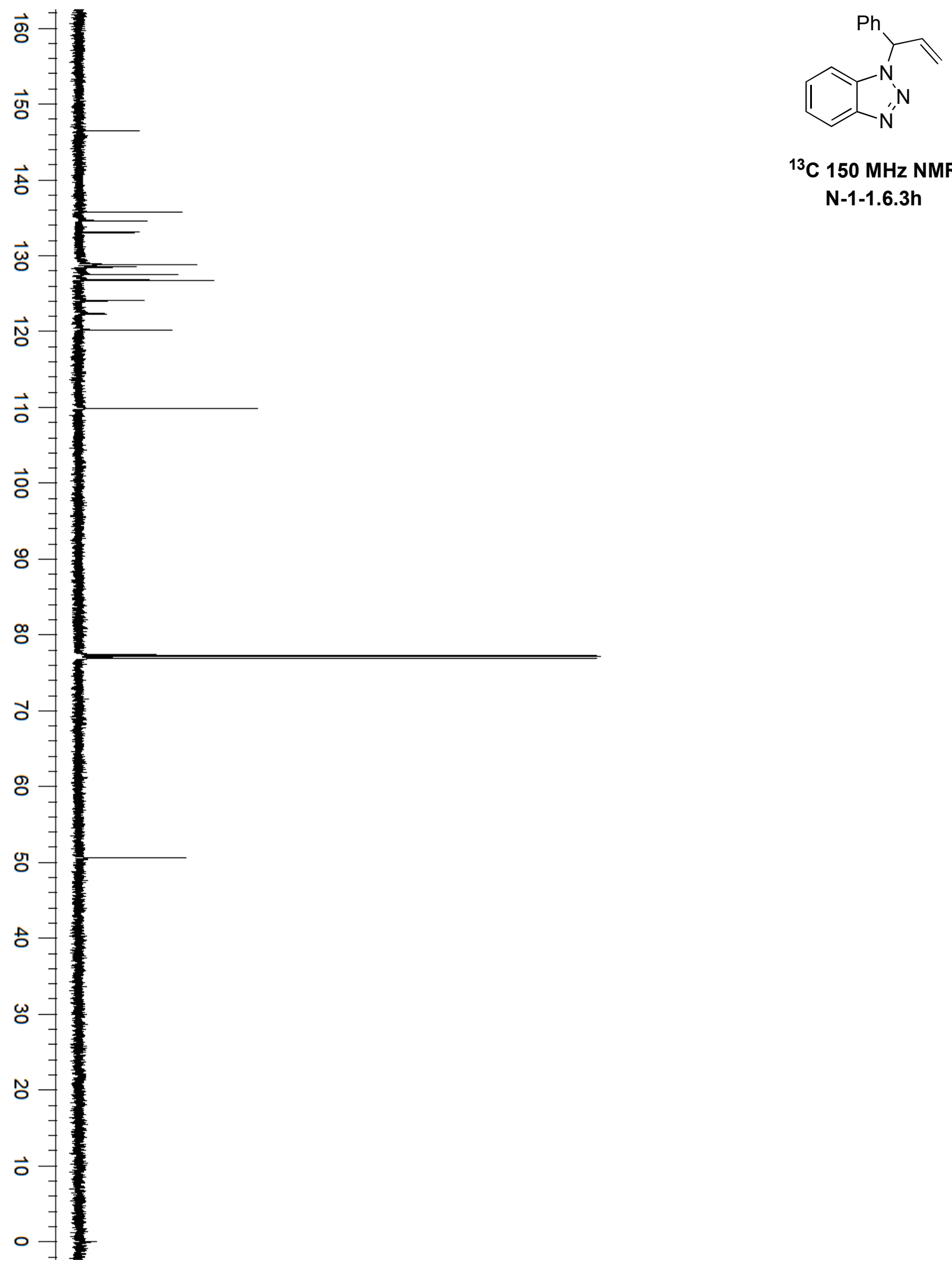

${ }^{13} \mathrm{C} 150 \mathrm{MHz}$ NMR $\mathrm{N}-1-1.6 .3 \mathrm{~h}$ 


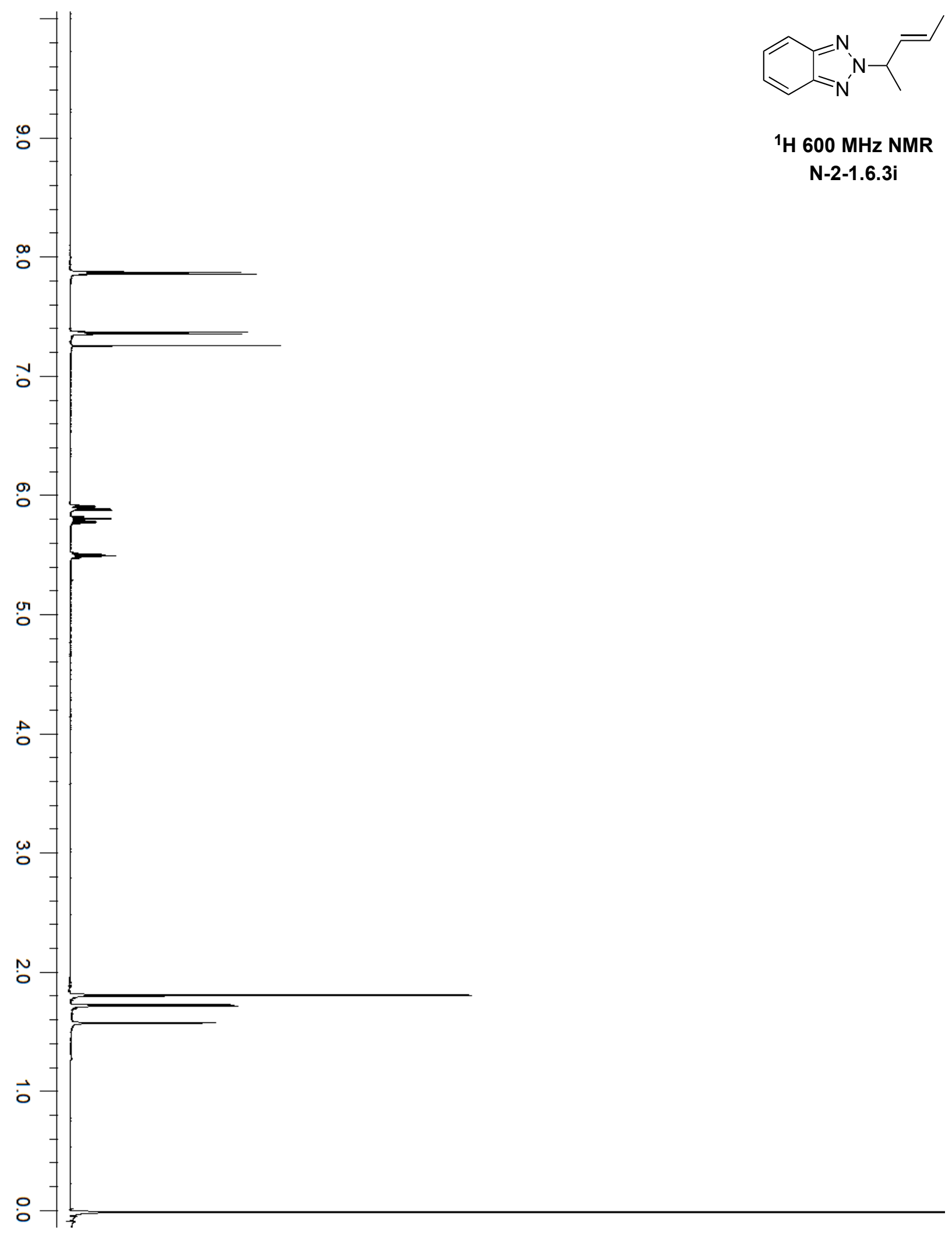




$$
L^{*}
$$




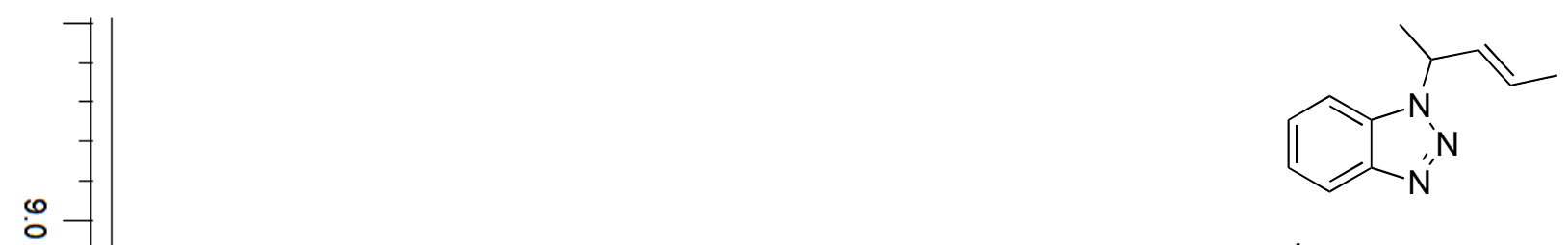

${ }^{1} \mathrm{H} 600 \mathrm{MHz}$ NMR N-1-1.6.3i

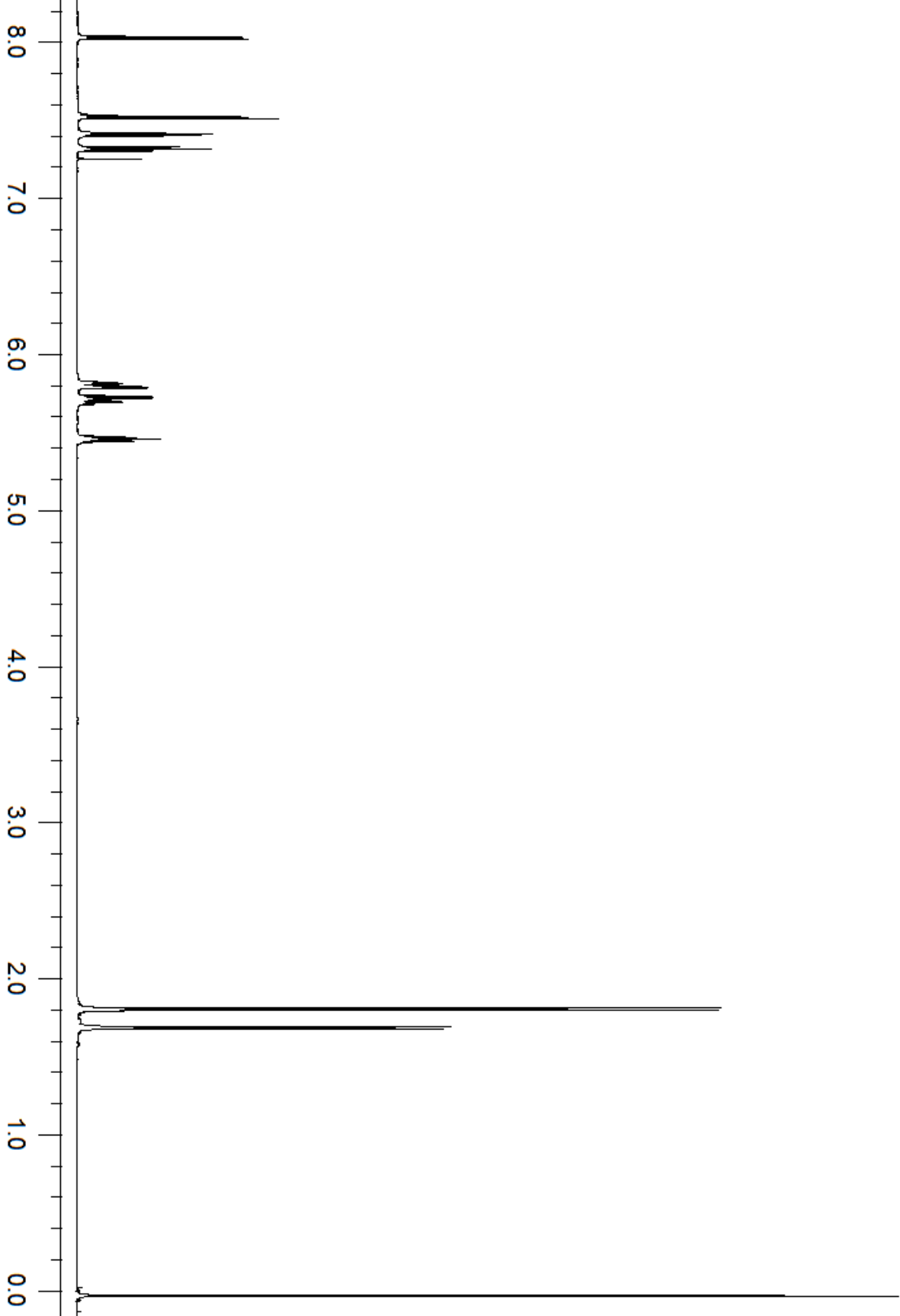




$$
F
$$




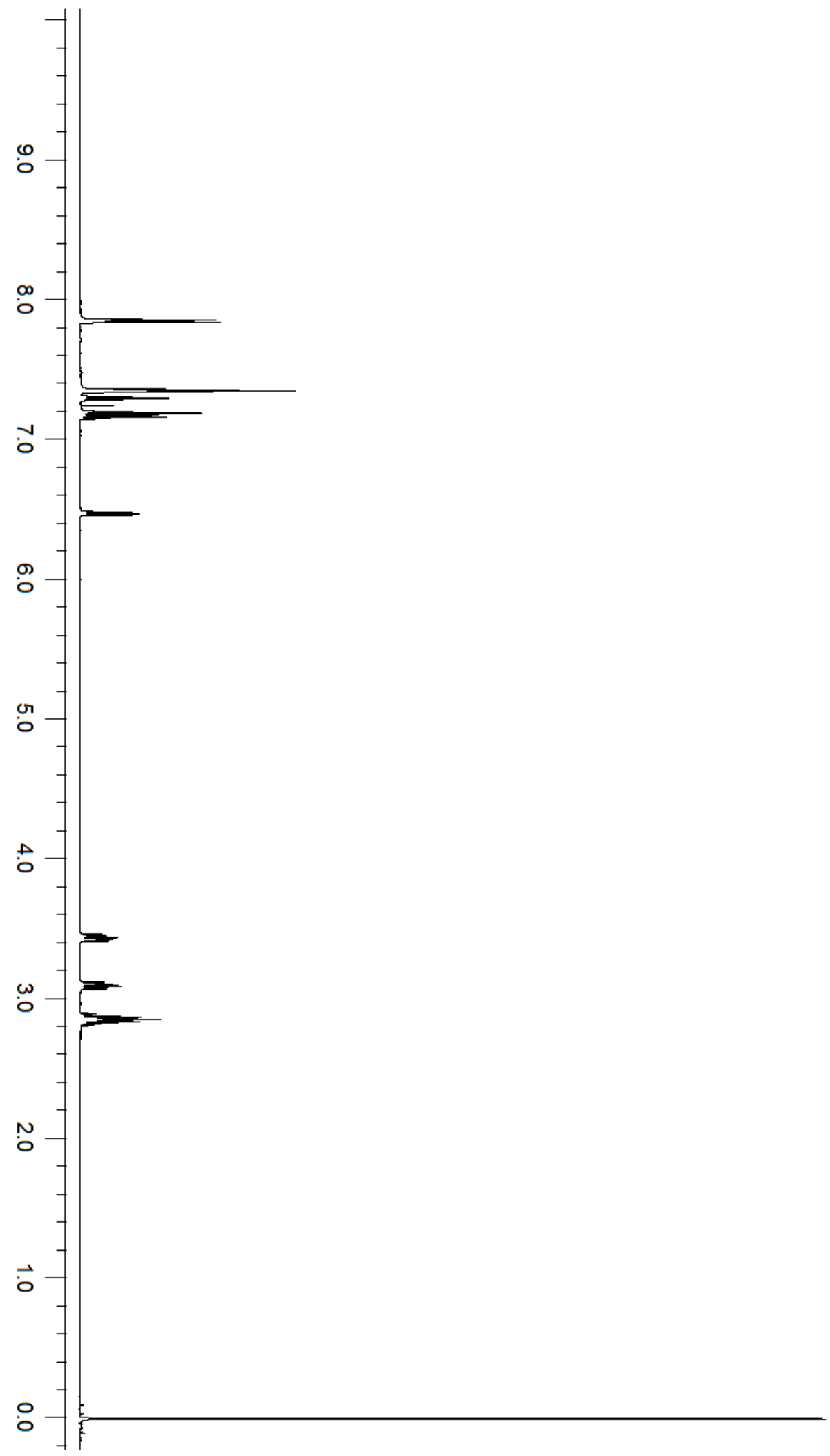

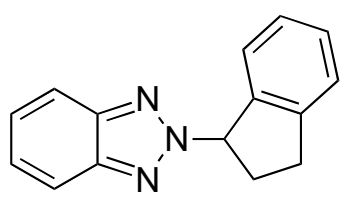

${ }^{1} \mathrm{H} 600 \mathrm{MHz}$ NMR

N-2-1.6.3j 


$$
=
$$




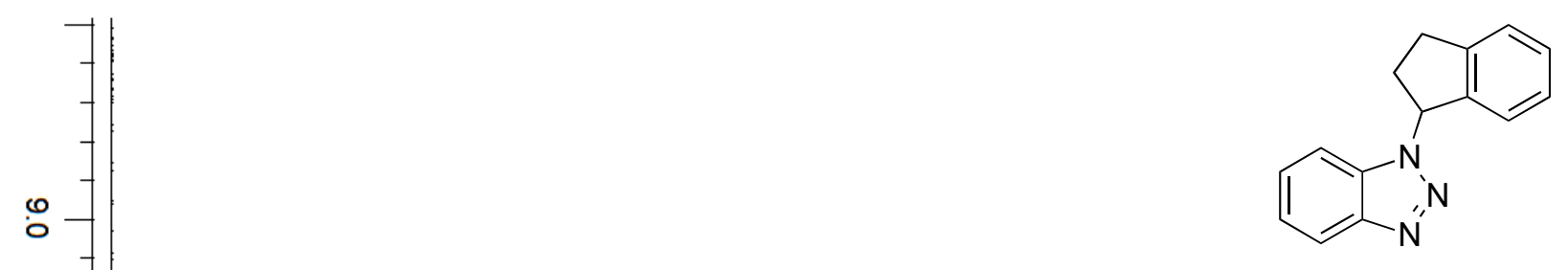

${ }^{1} \mathrm{H} 600 \mathrm{MHz}$ NMR

$\mathrm{N}-1-1.6 .3 \mathrm{j}$

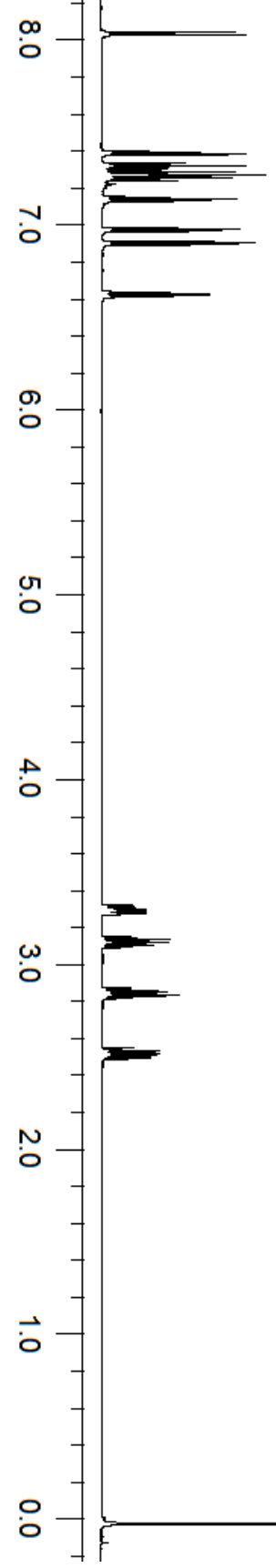



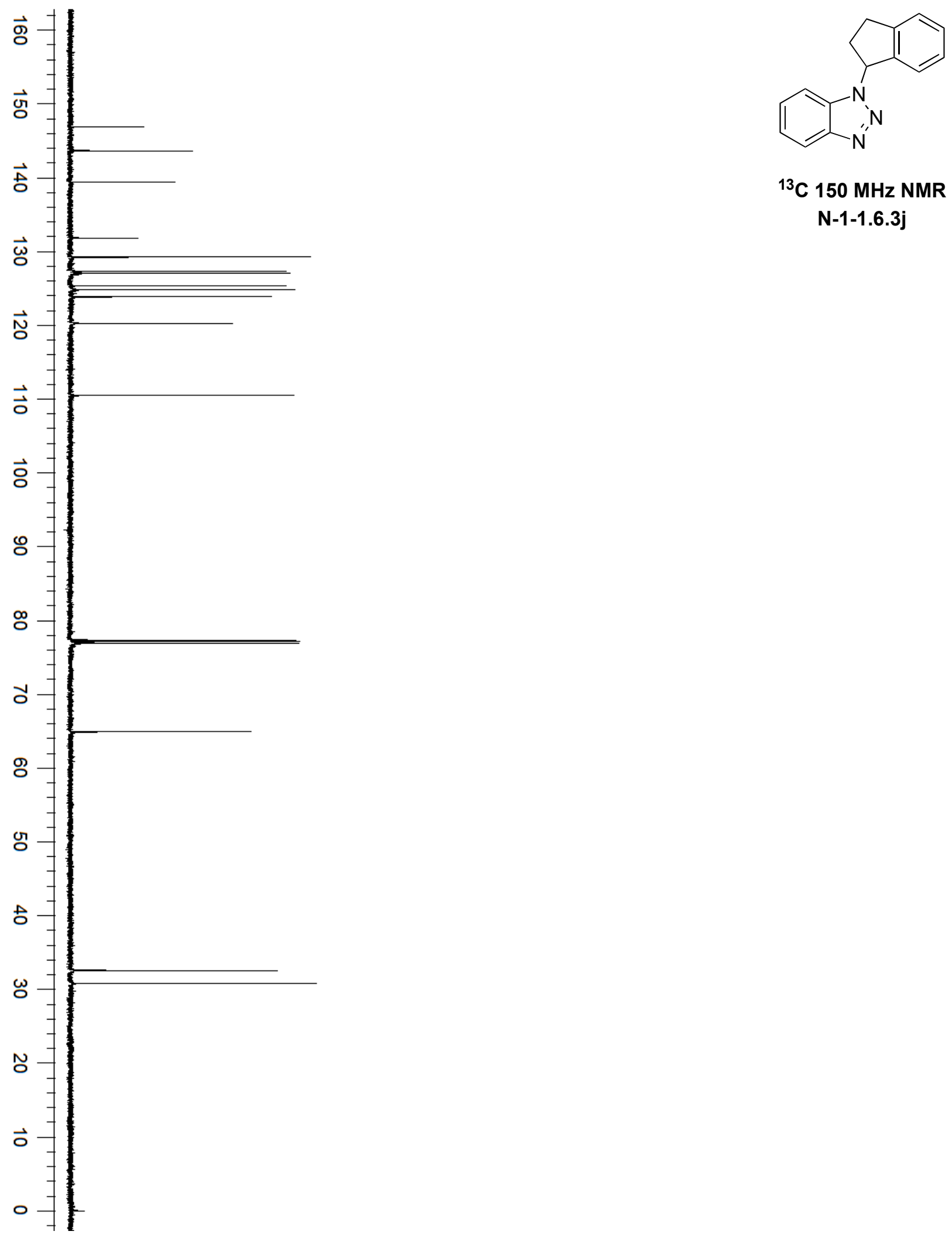

${ }^{13} \mathrm{C} 150 \mathrm{MHz}$ NMR

$\mathrm{N}-1-1.6 .3 \mathrm{j}$ 


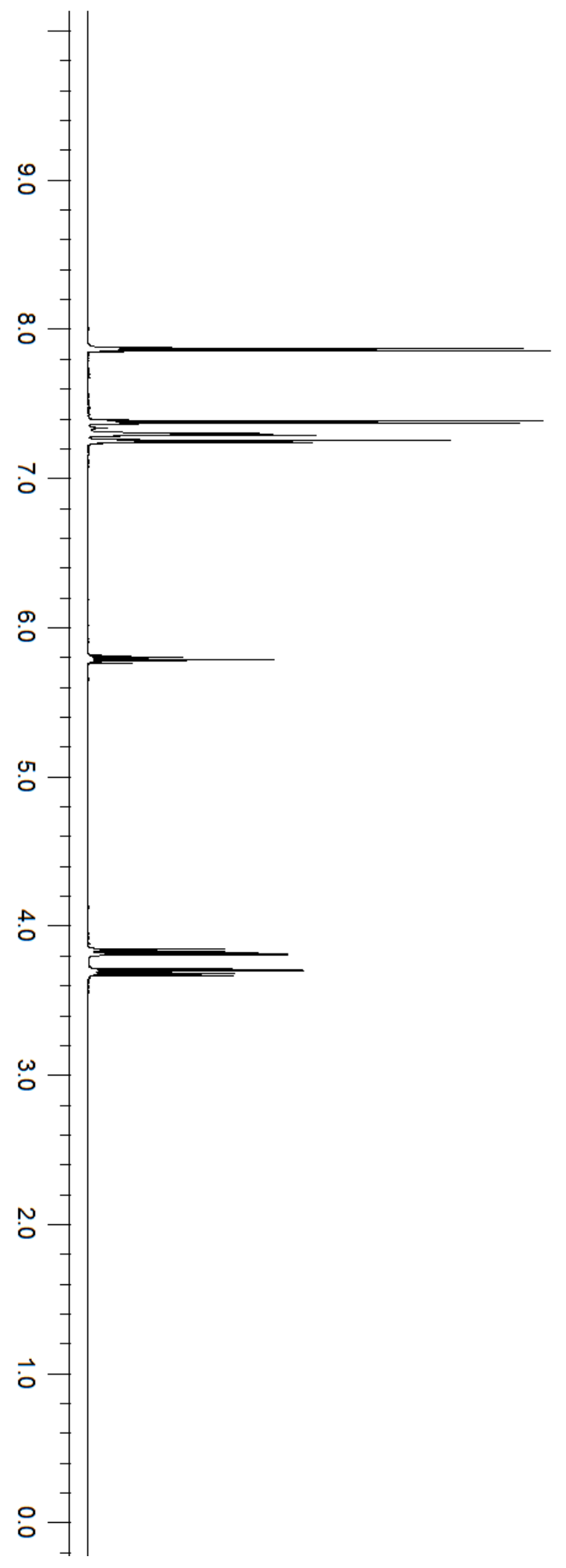

${ }^{1} \mathrm{H} 600 \mathrm{MHz}$ NMR

$\mathrm{N}-2-1.6 .3 \mathrm{k}$ 


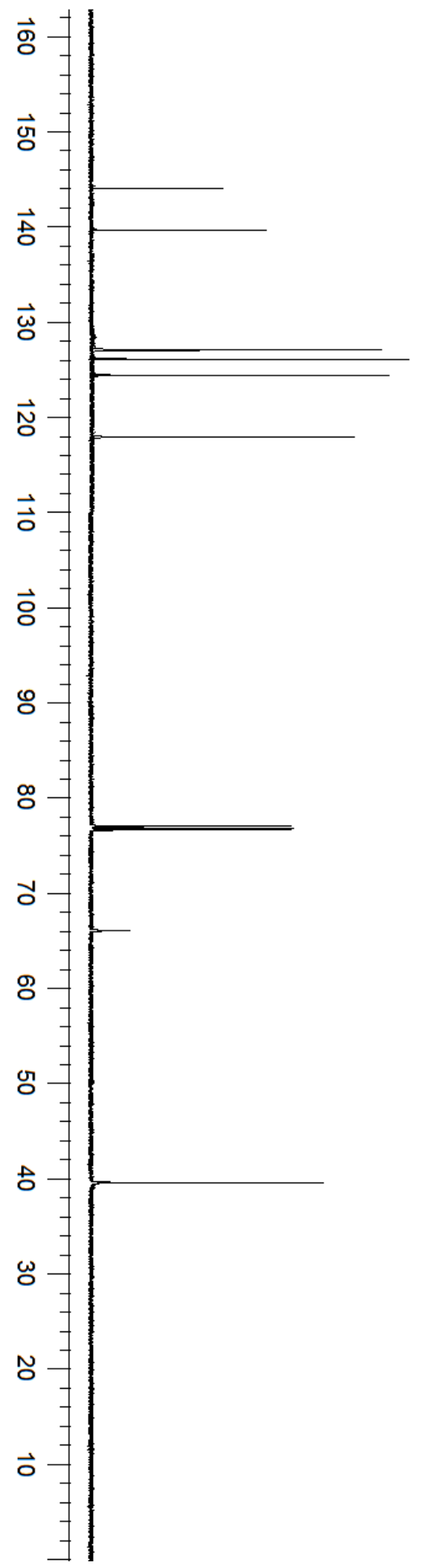

${ }^{13} \mathrm{C} 150 \mathrm{MHz}$ NMR N-2-1.6.3k 

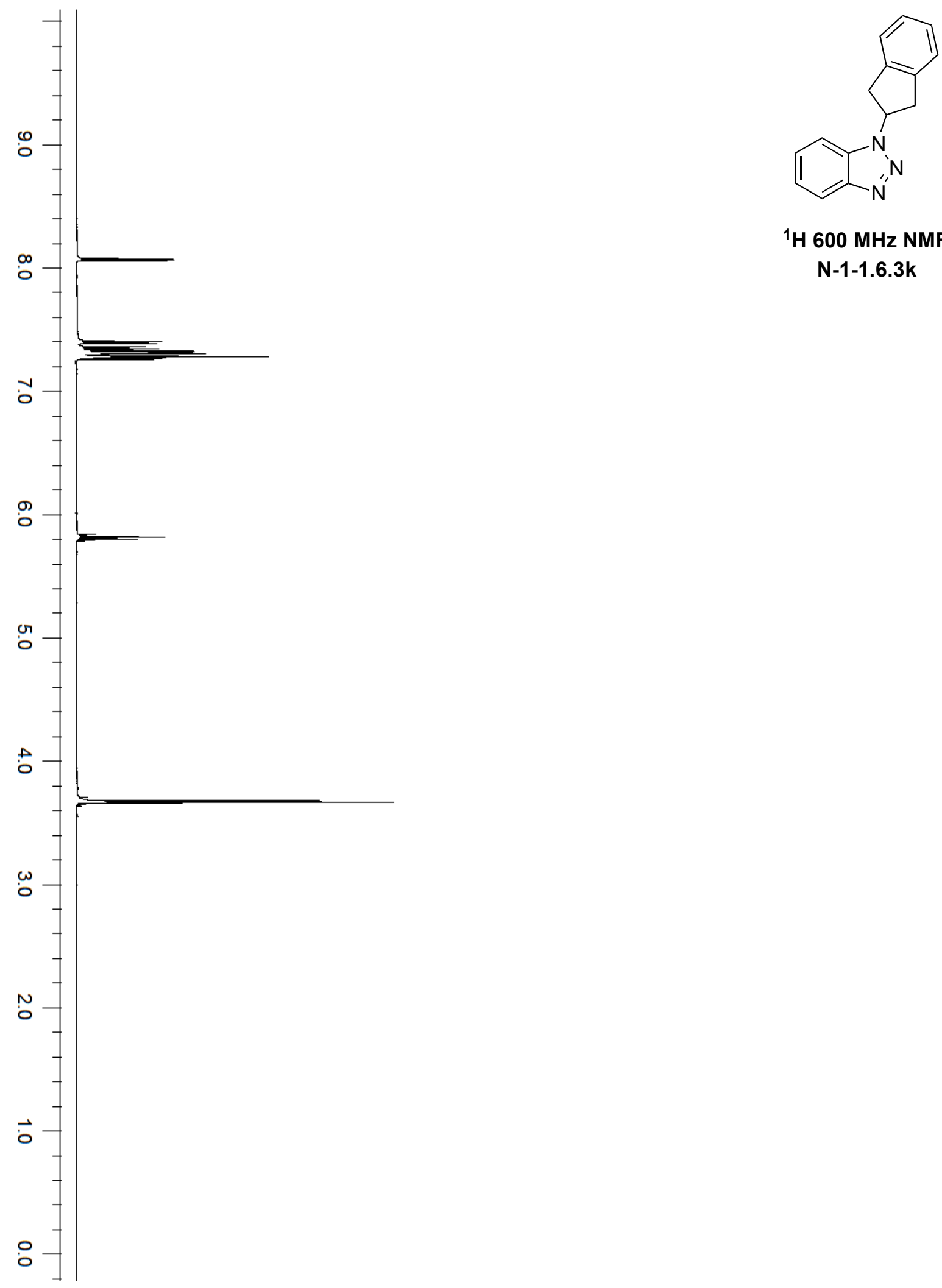

${ }^{1} \mathrm{H} 600 \mathrm{MHz}$ NMR $\mathrm{N}-1-1.6 .3 \mathrm{k}$ 


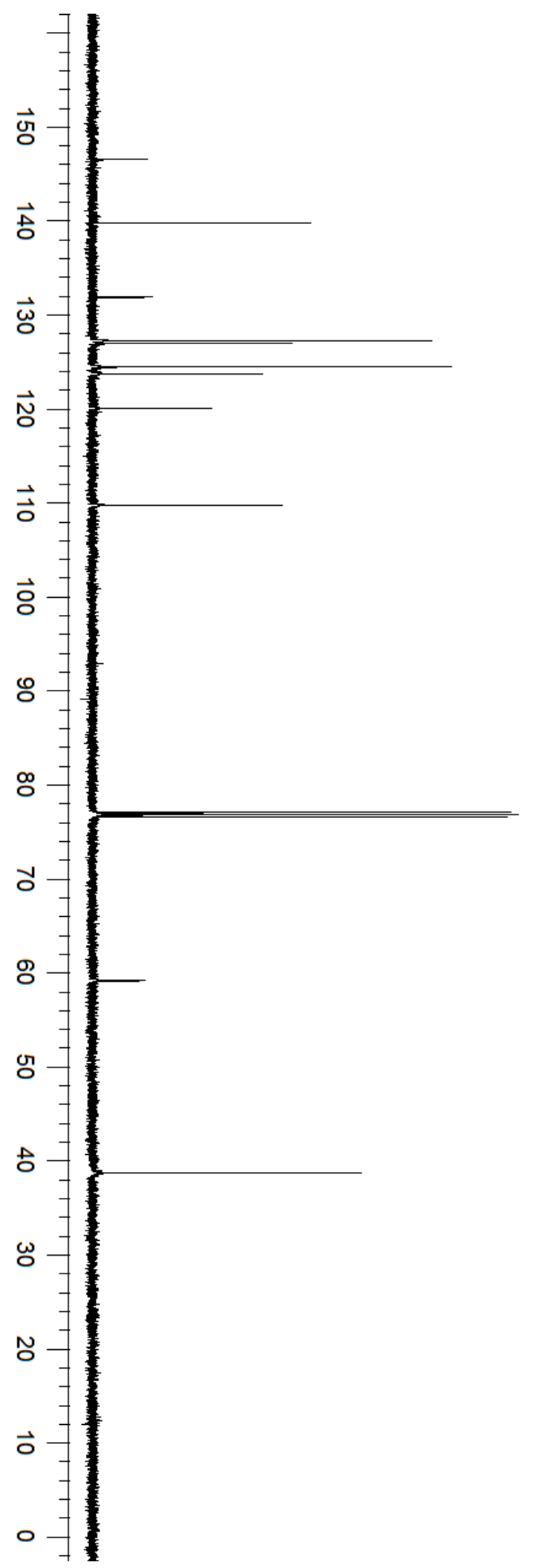

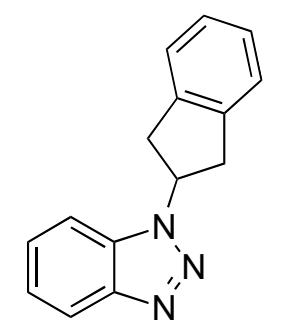

${ }^{13} \mathrm{C} 150 \mathrm{MHz}$ NMR $\mathrm{N}-1-1.6 .3 \mathrm{k}$ 

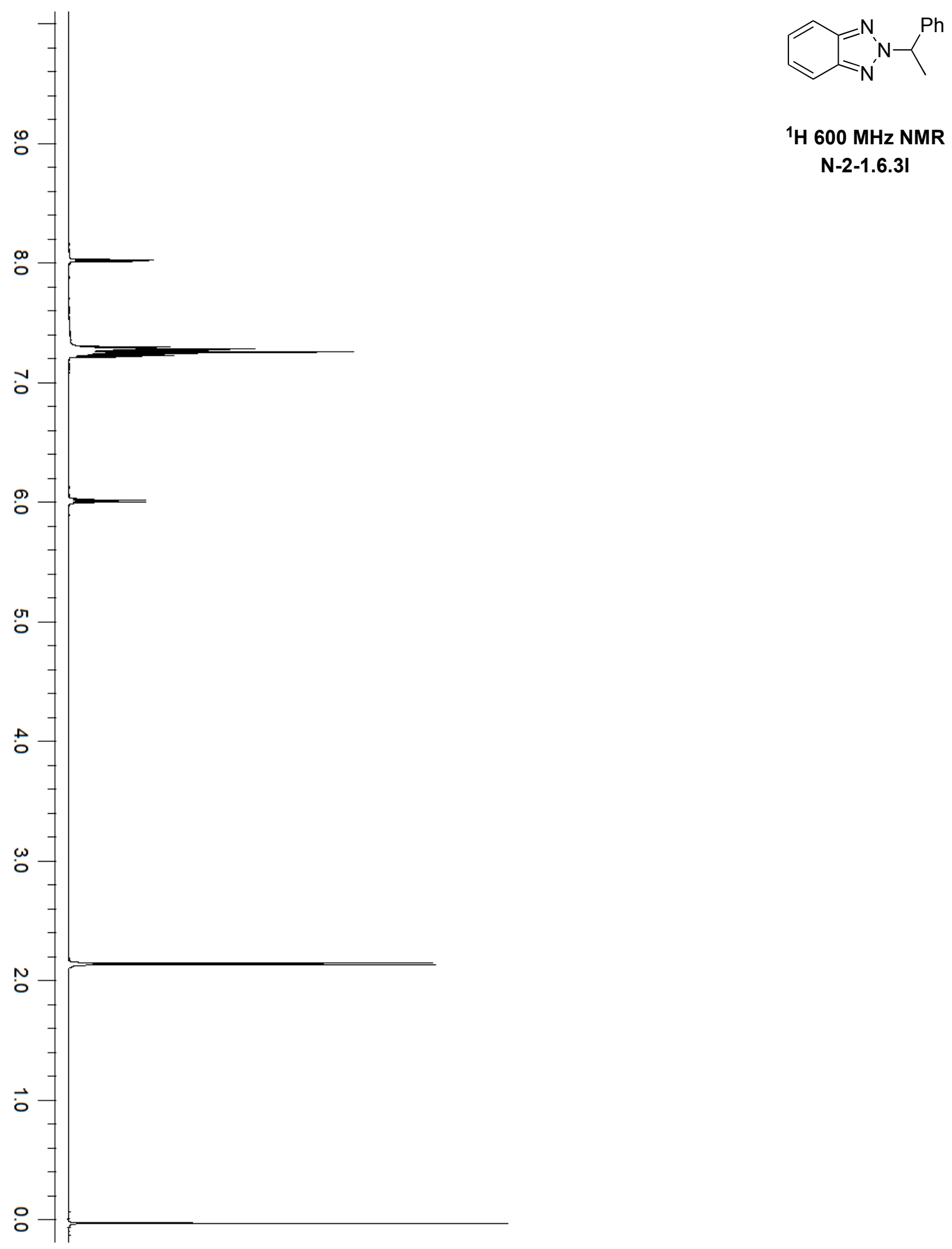

${ }^{1} \mathrm{H} 600 \mathrm{MHz}$ NMR

N-2-1.6.3। 


$$
F
$$



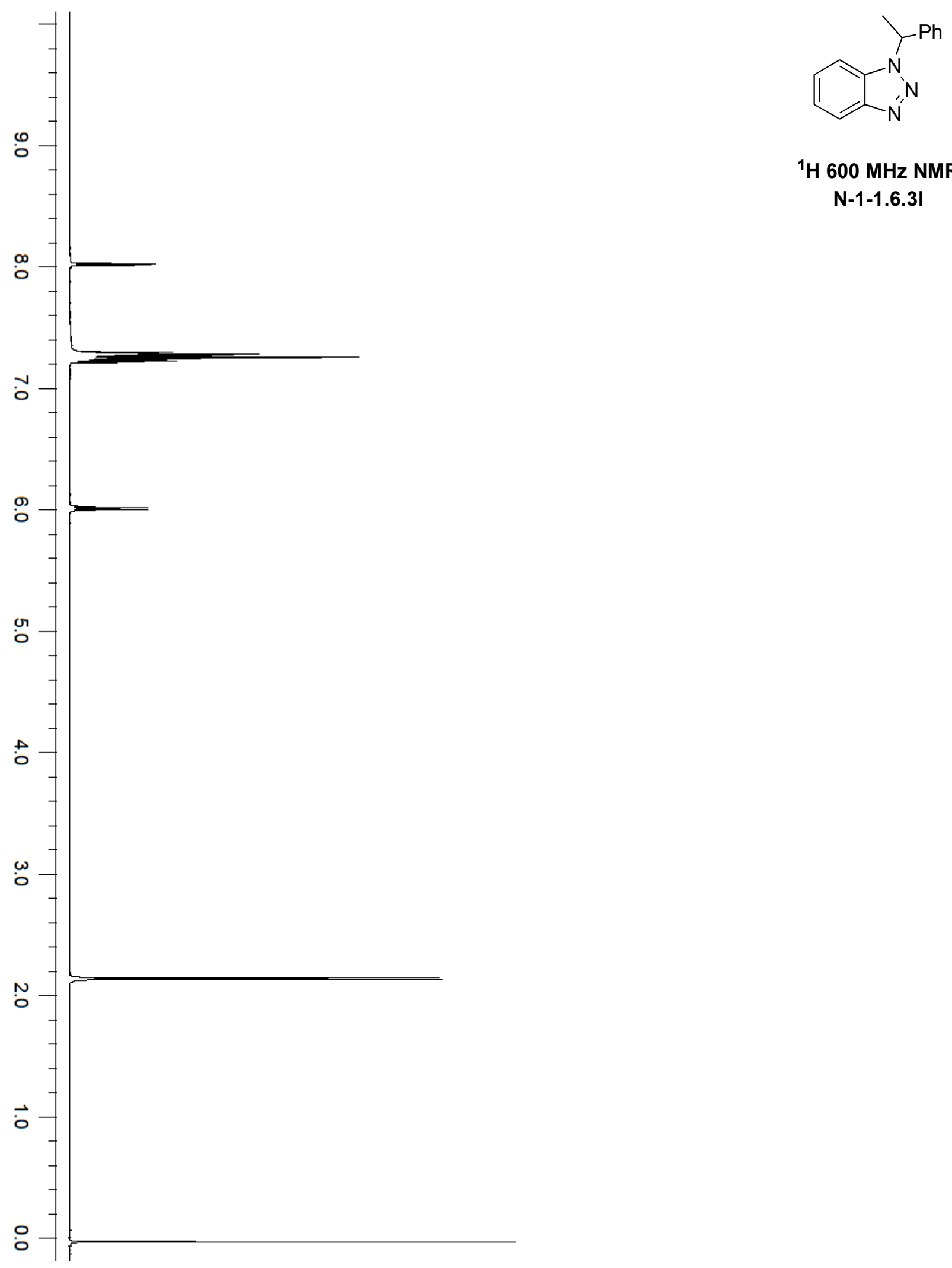

${ }^{1} \mathrm{H} 600 \mathrm{MHz}$ NMR

$\mathrm{N}-1-1.6 .3 \mid$ 


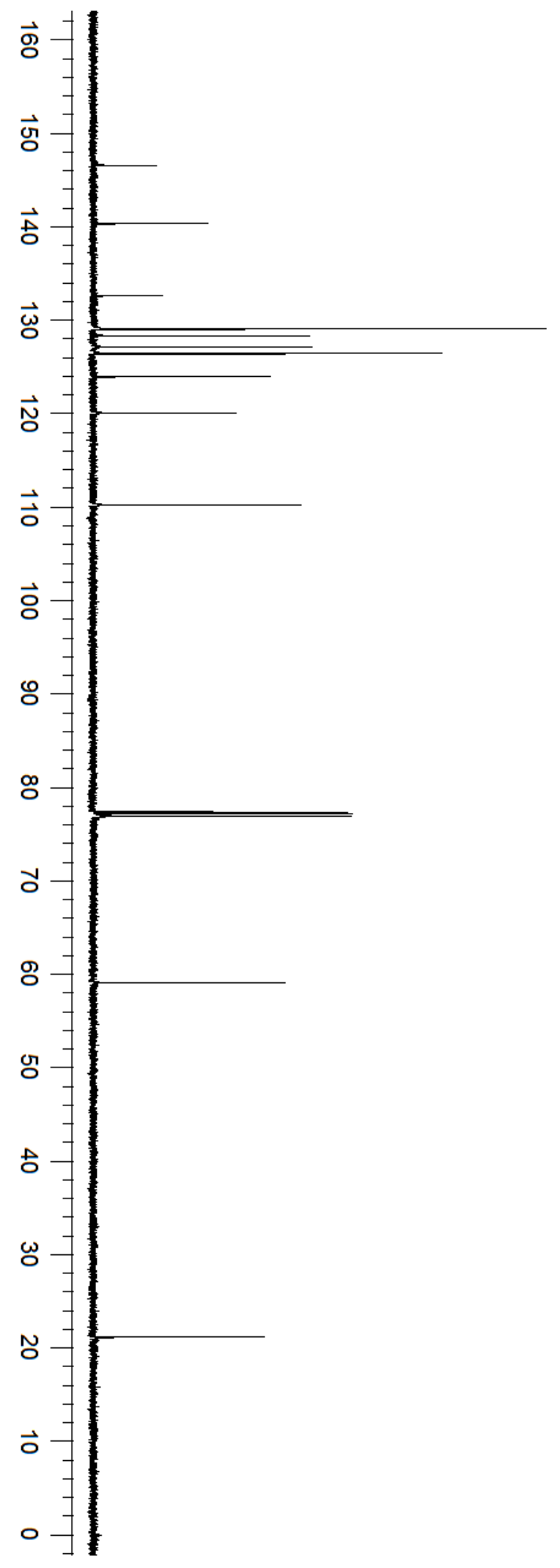

${ }^{13} \mathrm{C} 150 \mathrm{MHz}$ NMR

N-1-1.6.3। 


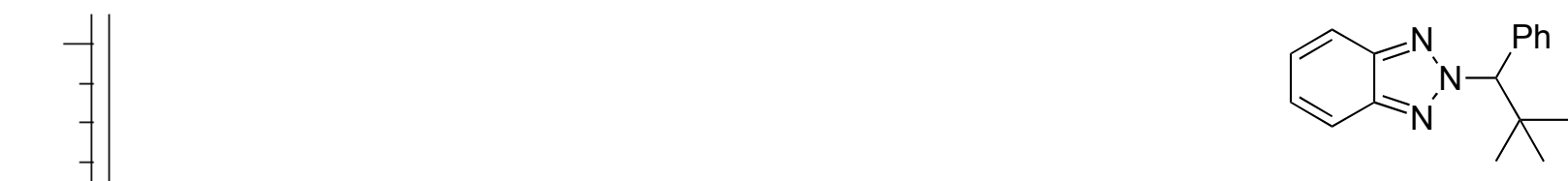

${ }^{1} \mathrm{H} 600 \mathrm{MHz}$ NMR

$\mathrm{N}-2-1.6 .3 \mathrm{~m}$

$\stackrel{\infty}{\circ}$

V

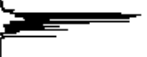

or

0

$-\longdiv { }$

$\ddot{0}$

w

$N$

음

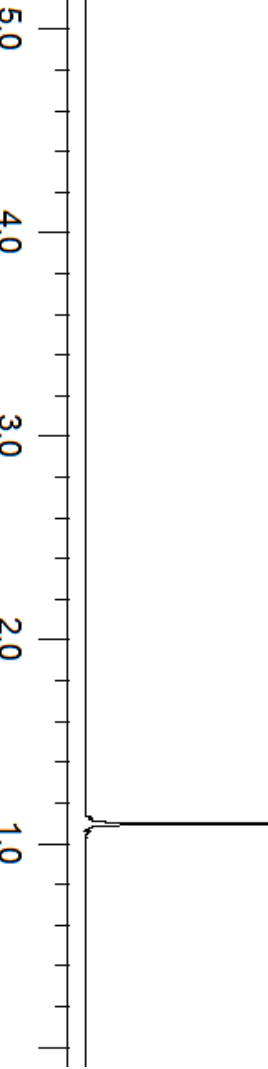




$$
1=
$$




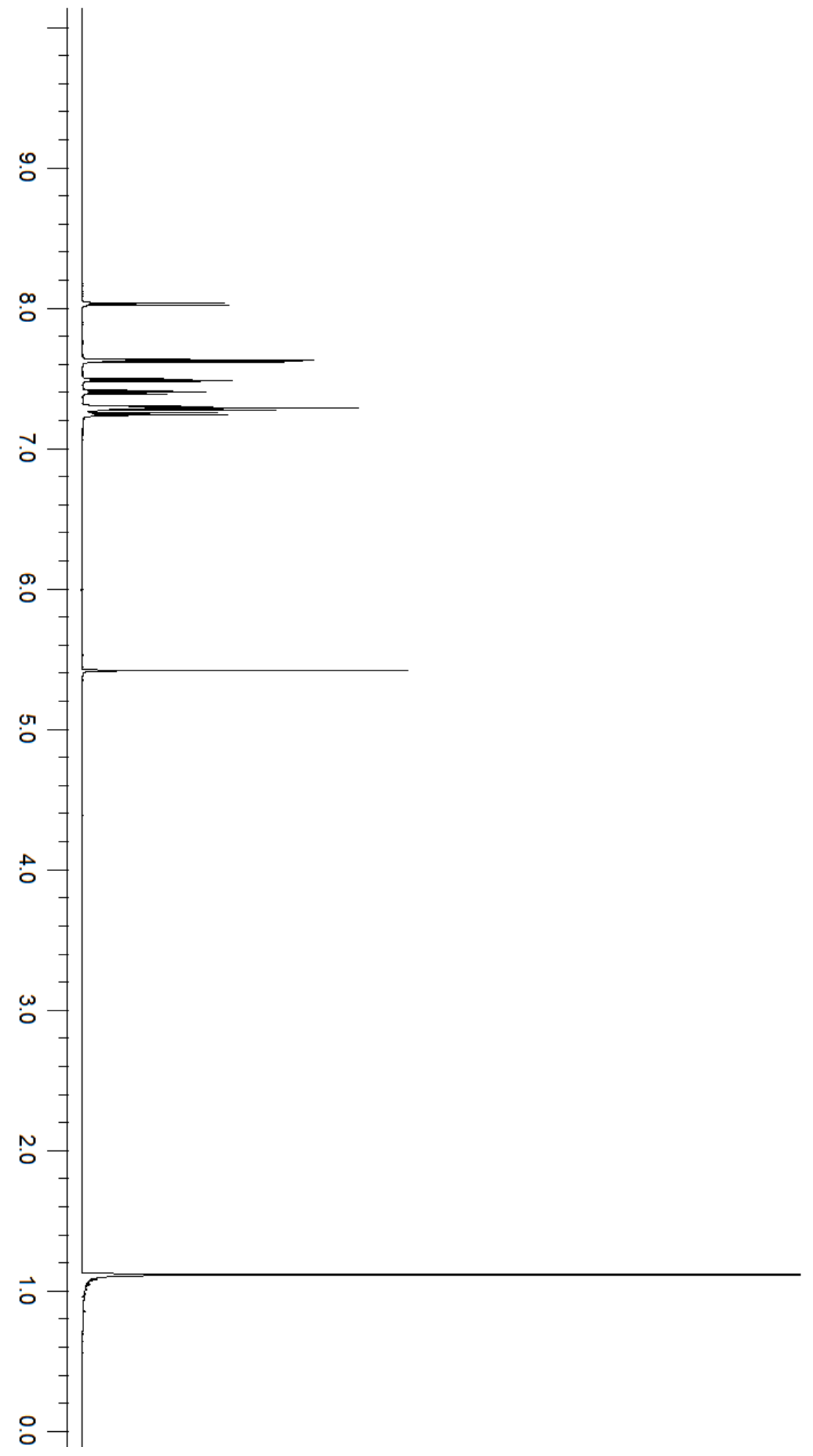

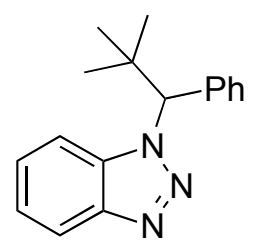

${ }^{1} \mathrm{H} 600 \mathrm{MHz}$ NMR

$\mathrm{N}-1-1.6 .3 \mathrm{~m}$ 

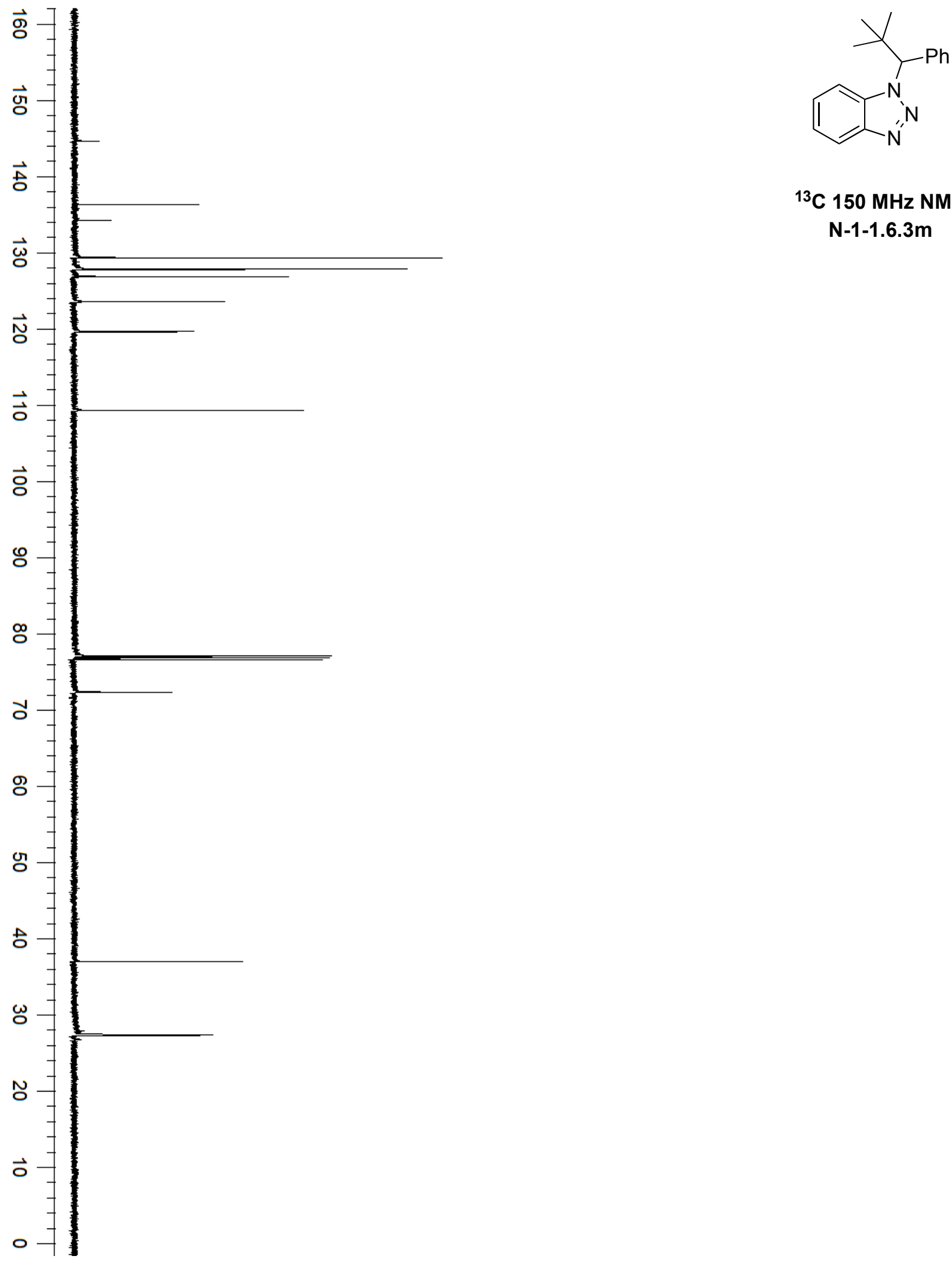

${ }^{13} \mathrm{C} 150 \mathrm{MHz}$ NMR

$\mathrm{N}-1-1.6 .3 \mathrm{~m}$

S784 


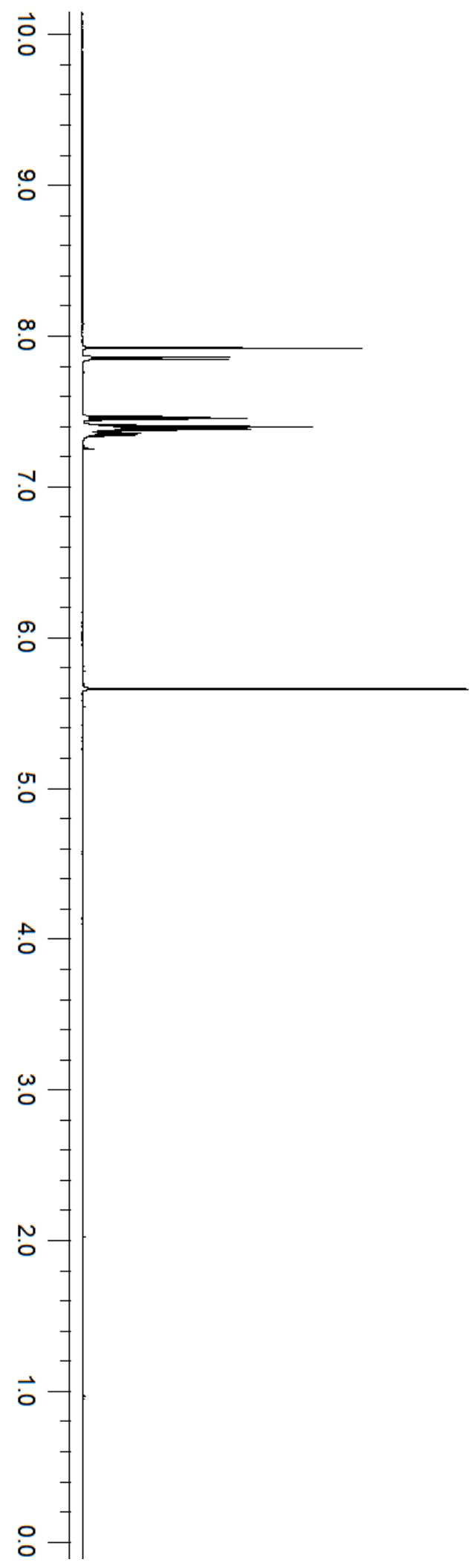

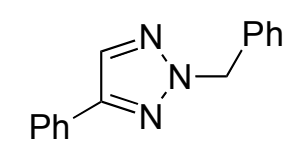

${ }^{1} \mathrm{H} 600 \mathrm{MHz}$ NMR

$\mathrm{N}-2-1.6 .4 \mathrm{a}$ 


$$
1
$$




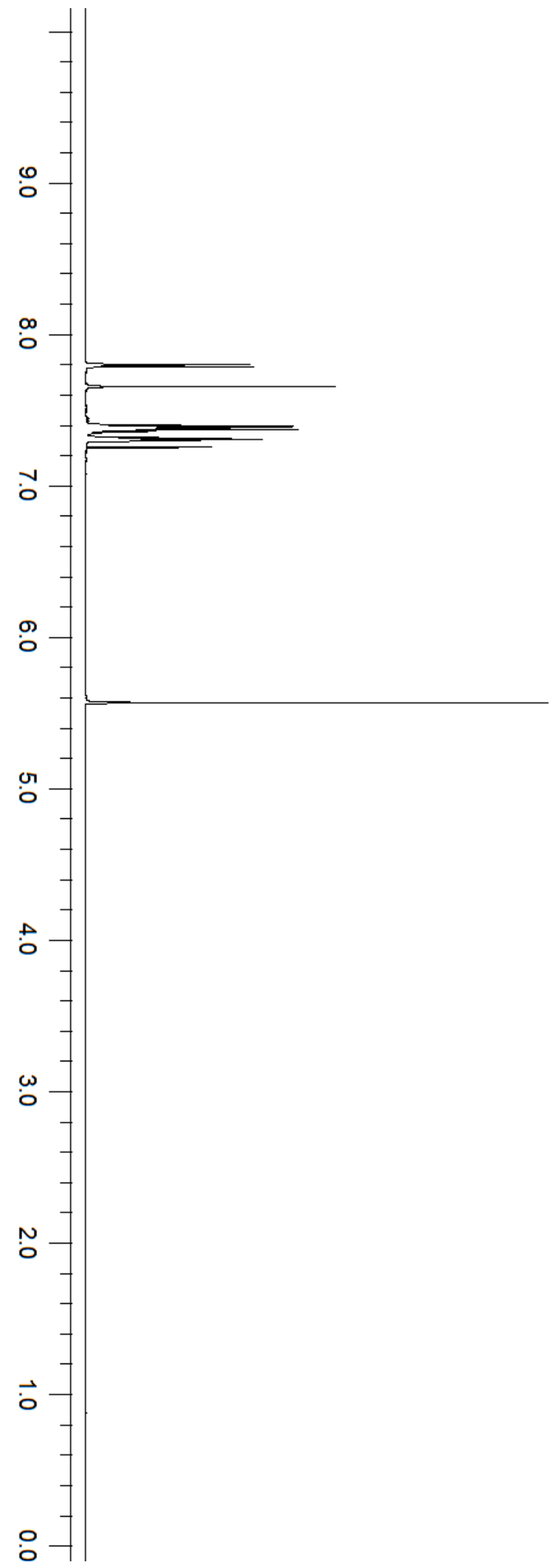

${ }^{1} \mathrm{H} 600 \mathrm{MHz}$ NMR $\mathrm{N}-1-1.6 .4 \mathrm{a}$ 


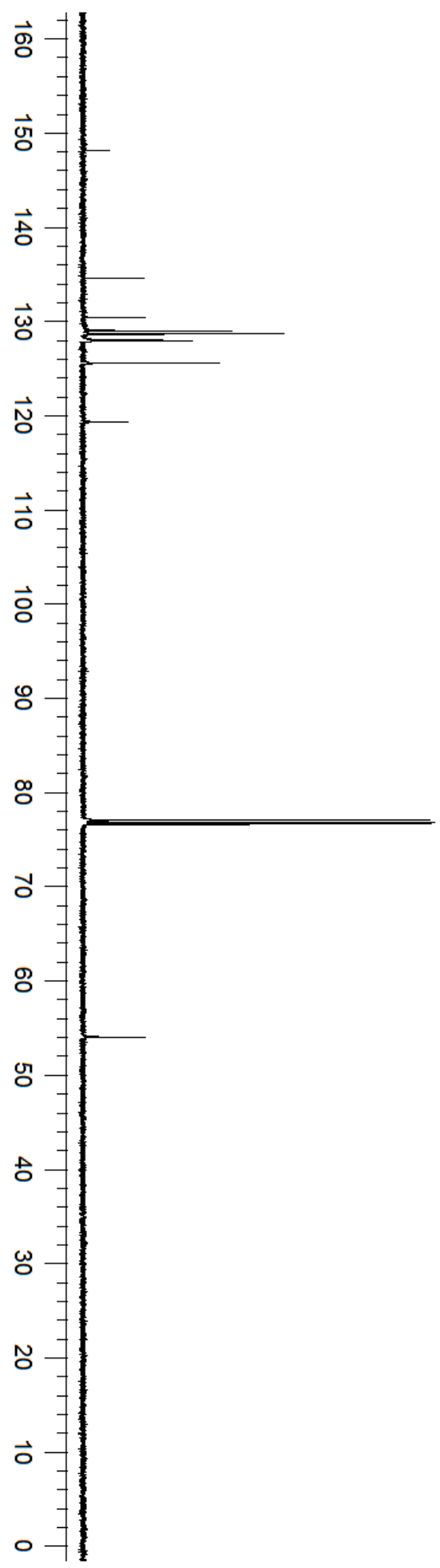<smiles>c1ccc(Cn2cc(-c3ccccc3)nn2)cc1</smiles>

${ }^{13} \mathrm{C} 150 \mathrm{MHz}$ NMR N-1-1.6.4a

S788 


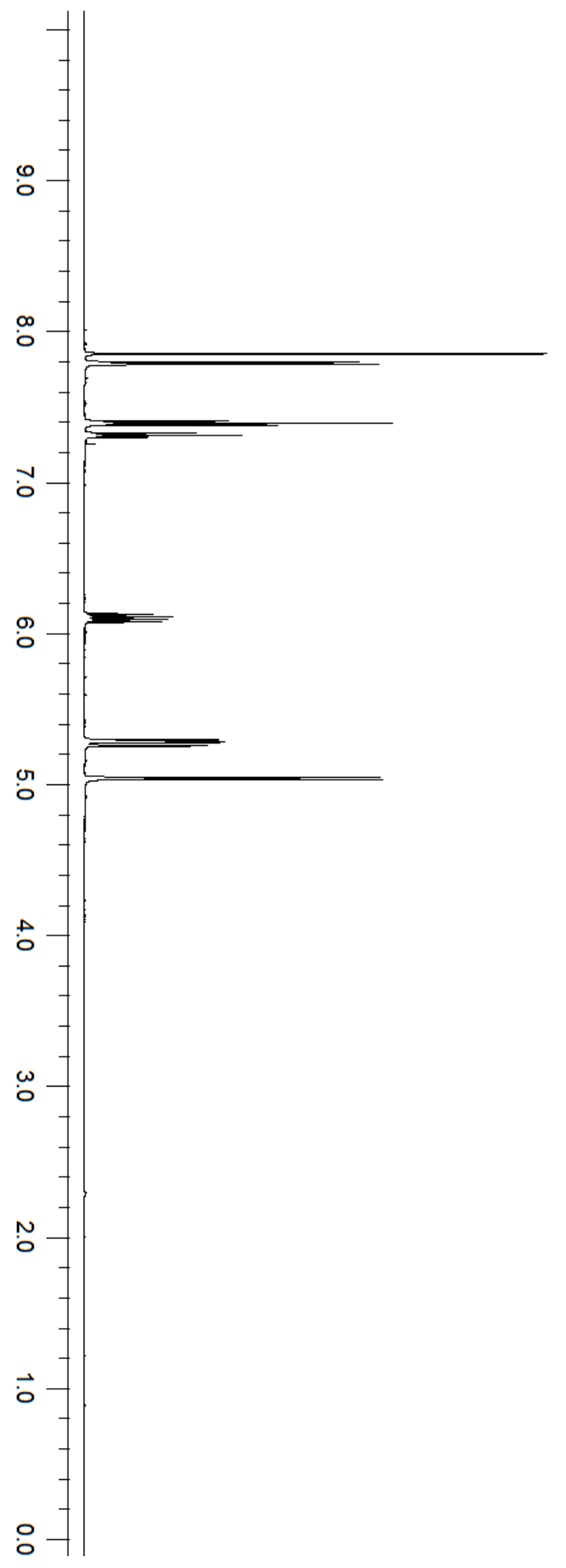

$\overbrace{\mathrm{Nh}}^{\mathrm{N}-}=$

${ }^{1} \mathrm{H} 600 \mathrm{MHz}$ NMR

$\mathrm{N}-2-1.6 .4 \mathrm{~b}$ 


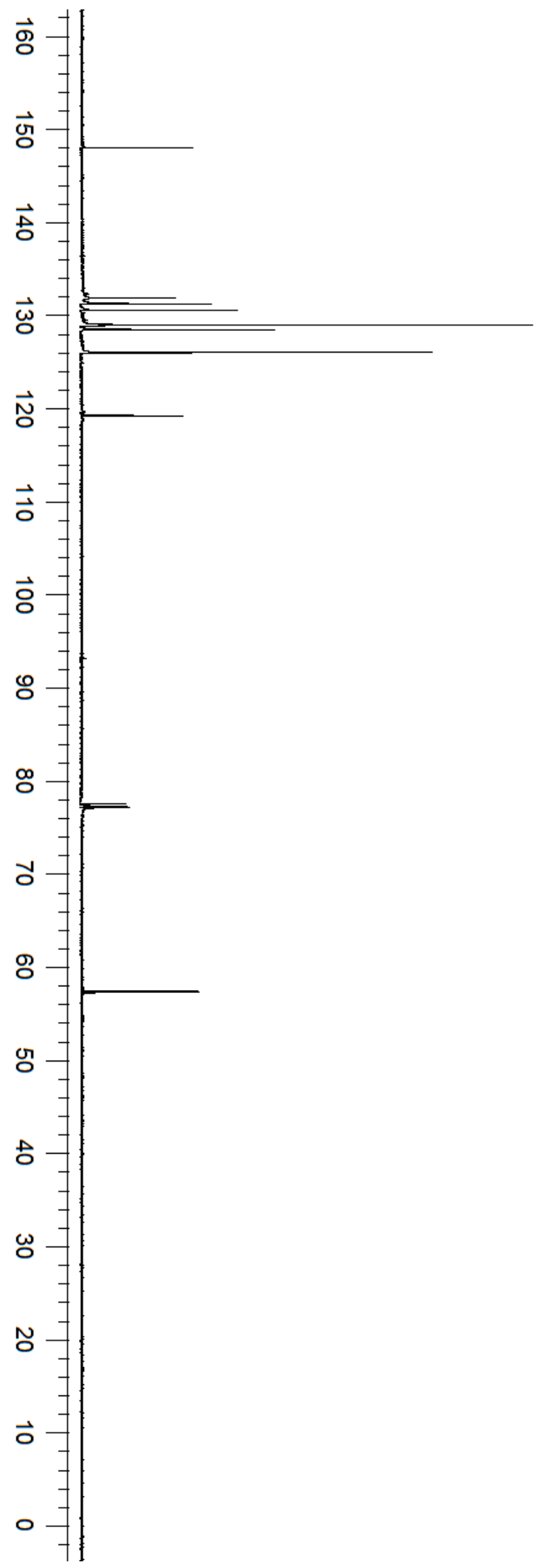

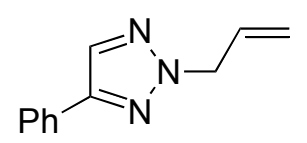

${ }^{13} \mathrm{C} 150 \mathrm{MHz}$ NMR $\mathrm{N}-2-1.6 .4 \mathrm{~b}$ 


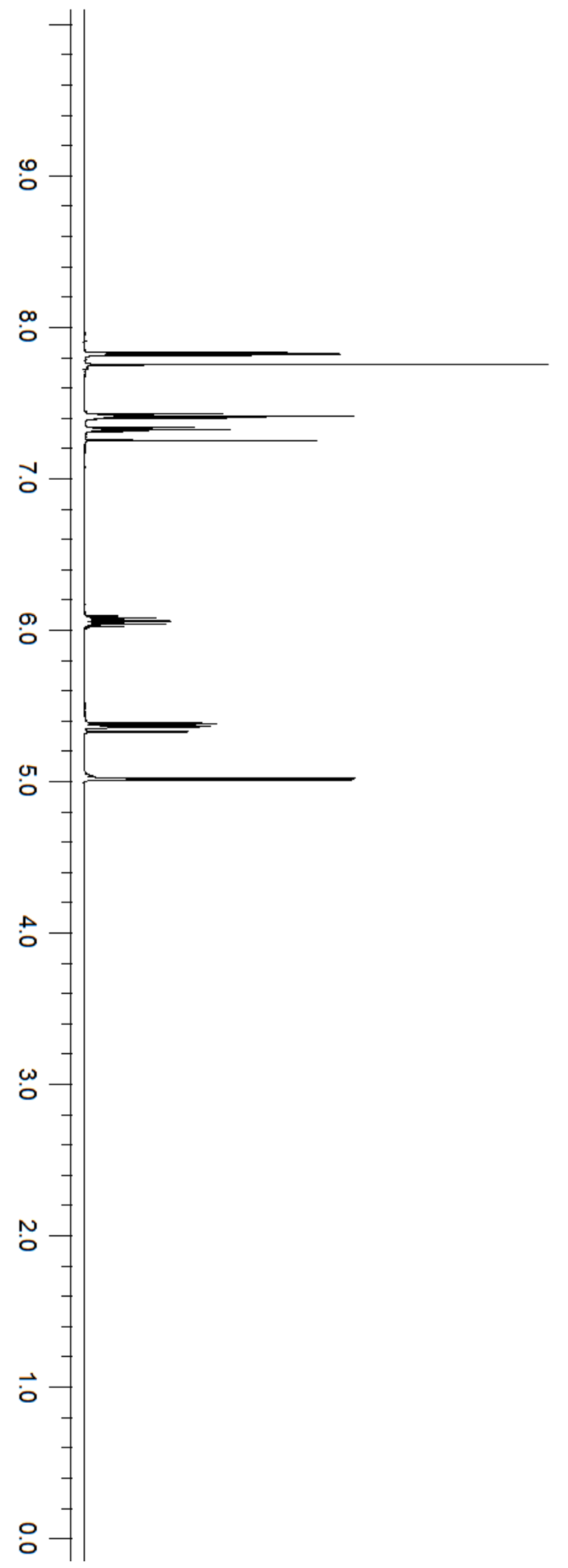

${ }^{1} \mathrm{H} 600 \mathrm{MHz}$ NMR

$\mathrm{N}-1-1.6 .4 \mathrm{~b}$ 


$$
F
$$




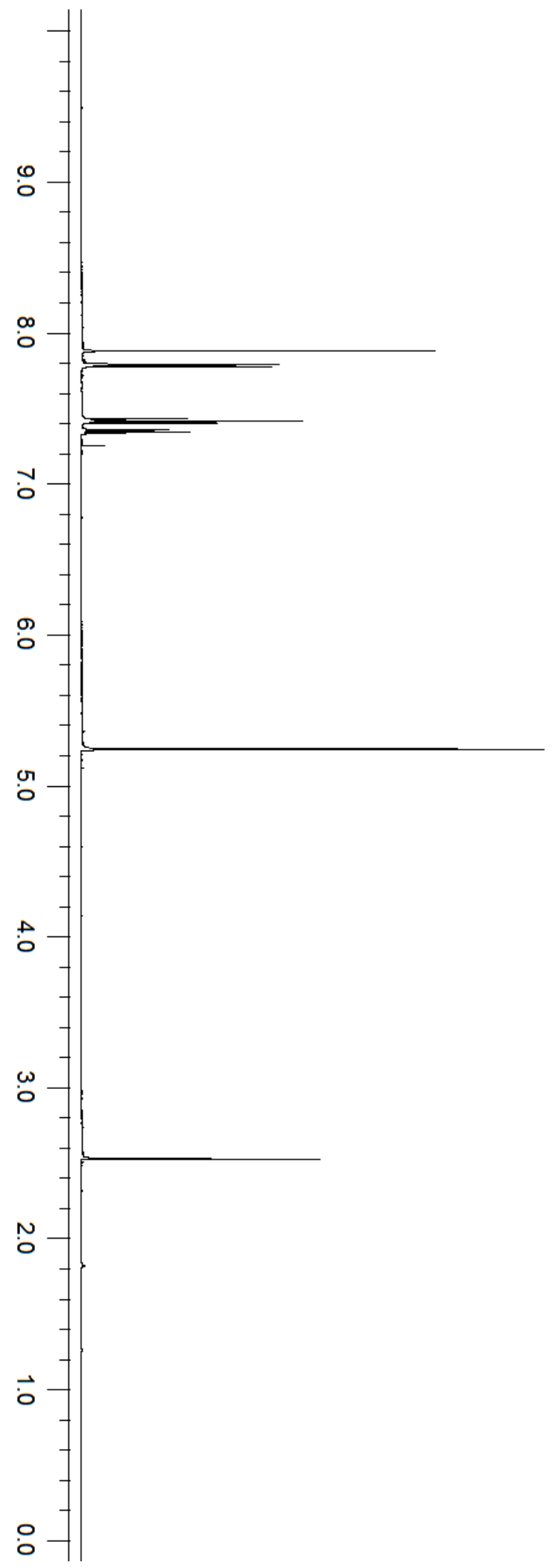

${ }^{1} \mathrm{H} 600 \mathrm{MHz}$ NMR

$\mathrm{N}-2-1.6 .4 \mathrm{c}$ 


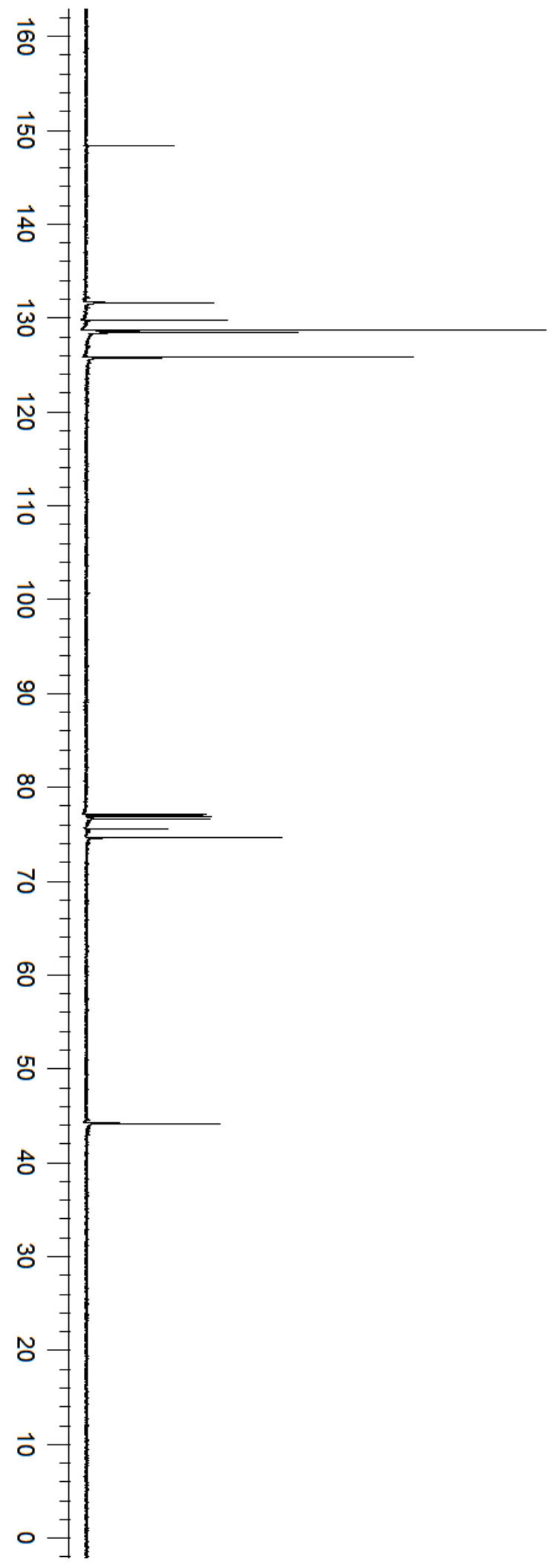

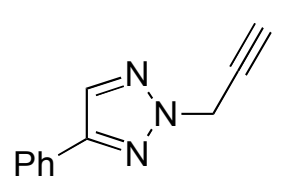

${ }^{13} \mathrm{C} 150 \mathrm{MHz}$ NMR

N-2-1.6.4C 


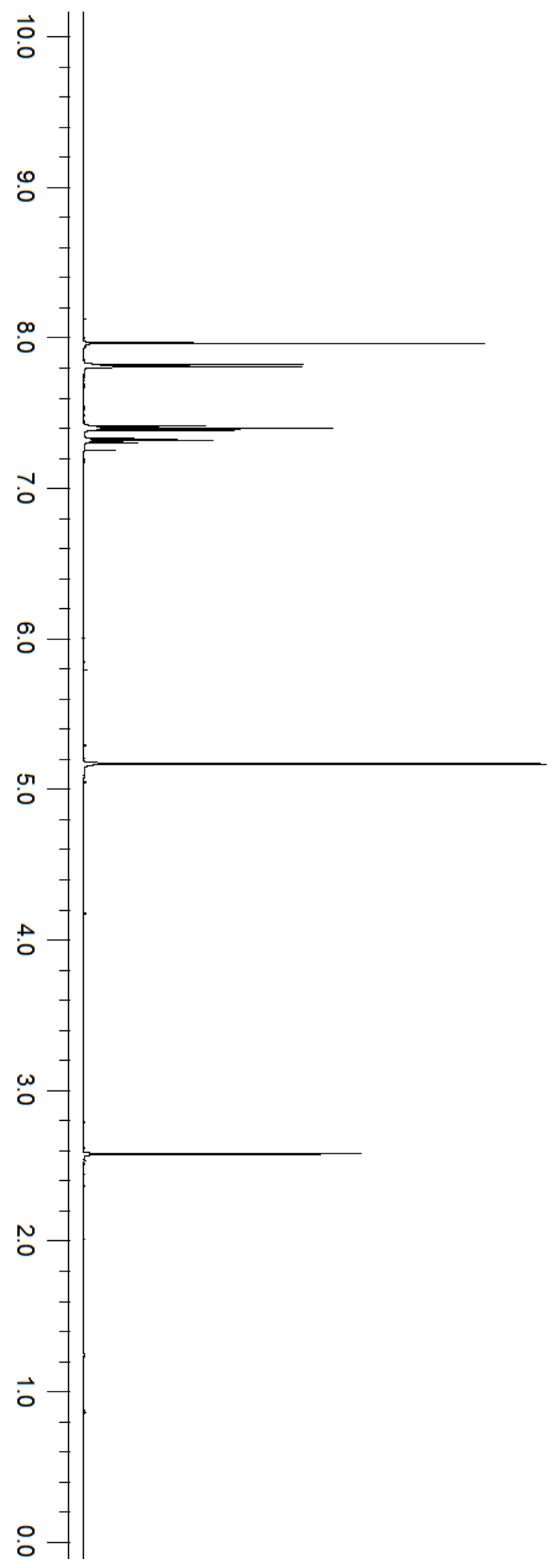

${ }^{1} \mathrm{H} 600 \mathrm{MHz}$ NMR $\mathrm{N}-1-1.6 .4 \mathrm{C}$ 


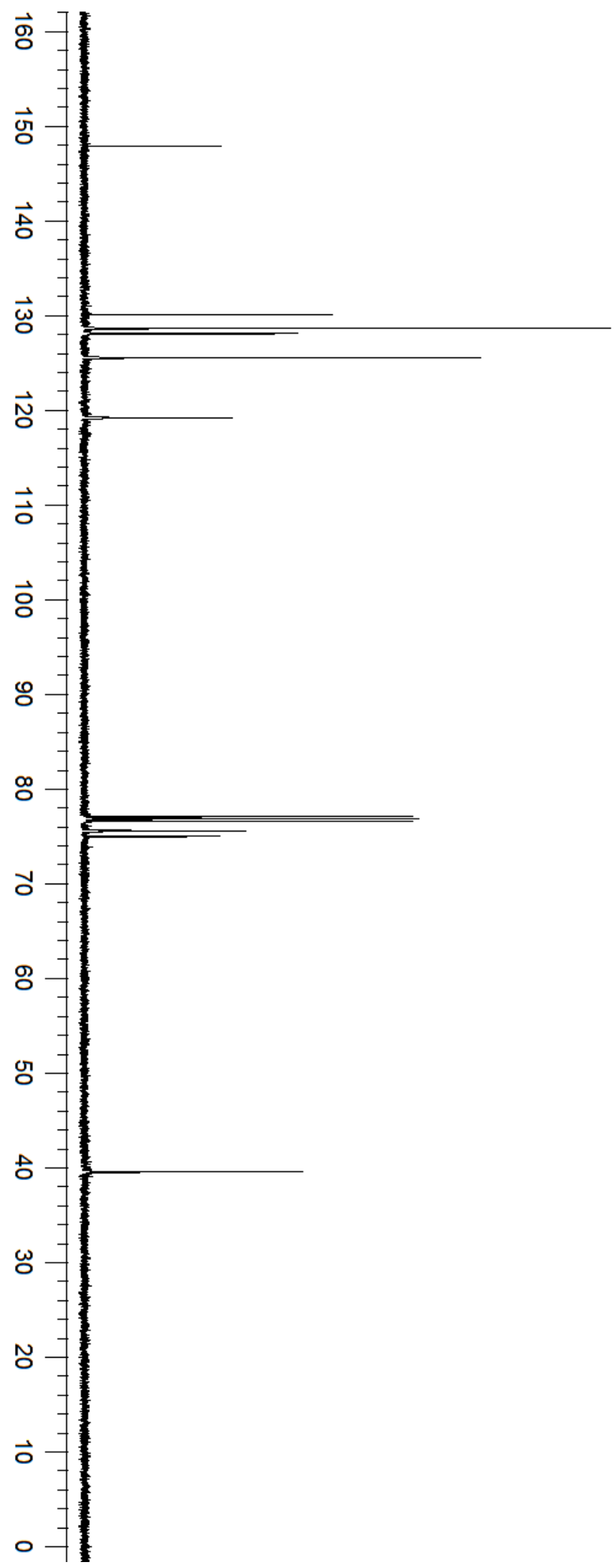<smiles>C#CCn1cc(-c2ccccc2)nn1</smiles>

${ }^{13} \mathrm{C} 150 \mathrm{MHz}$ NMR N-1-1.6.4C

S796 


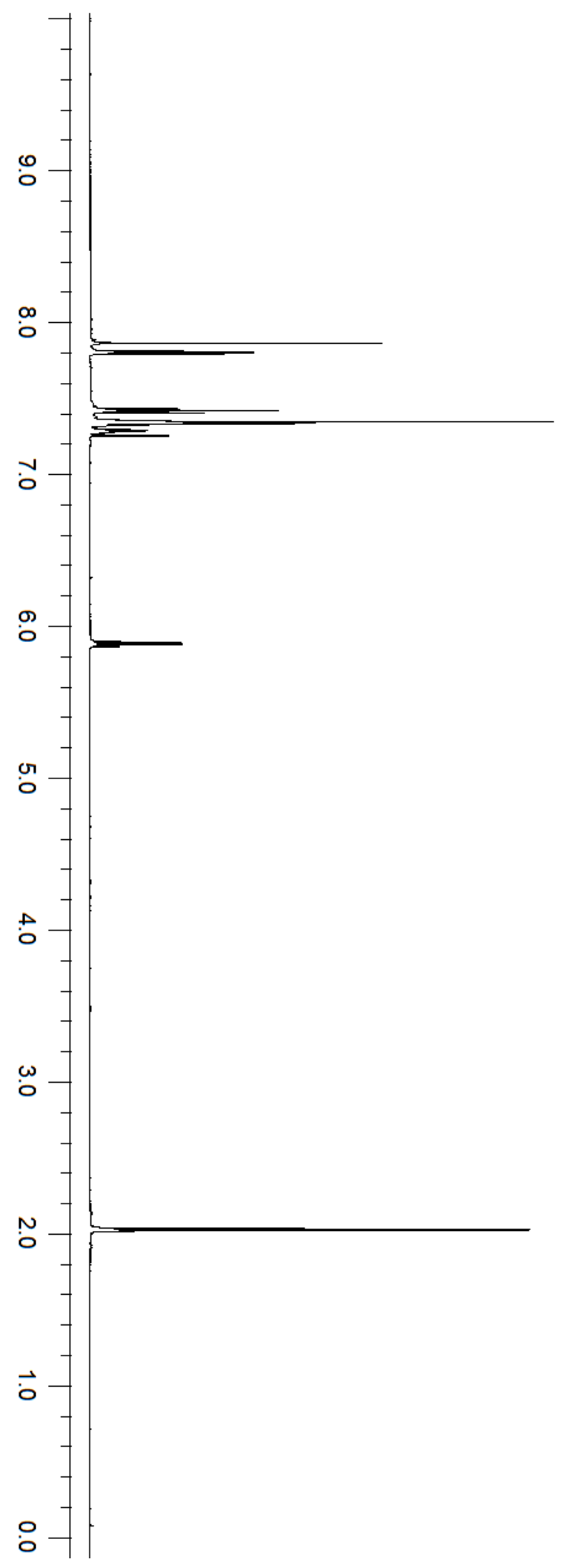

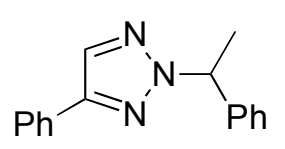

${ }^{1} \mathrm{H} 600 \mathrm{MHz}$ NMR N-2-1.6.4d 


$$
F
$$




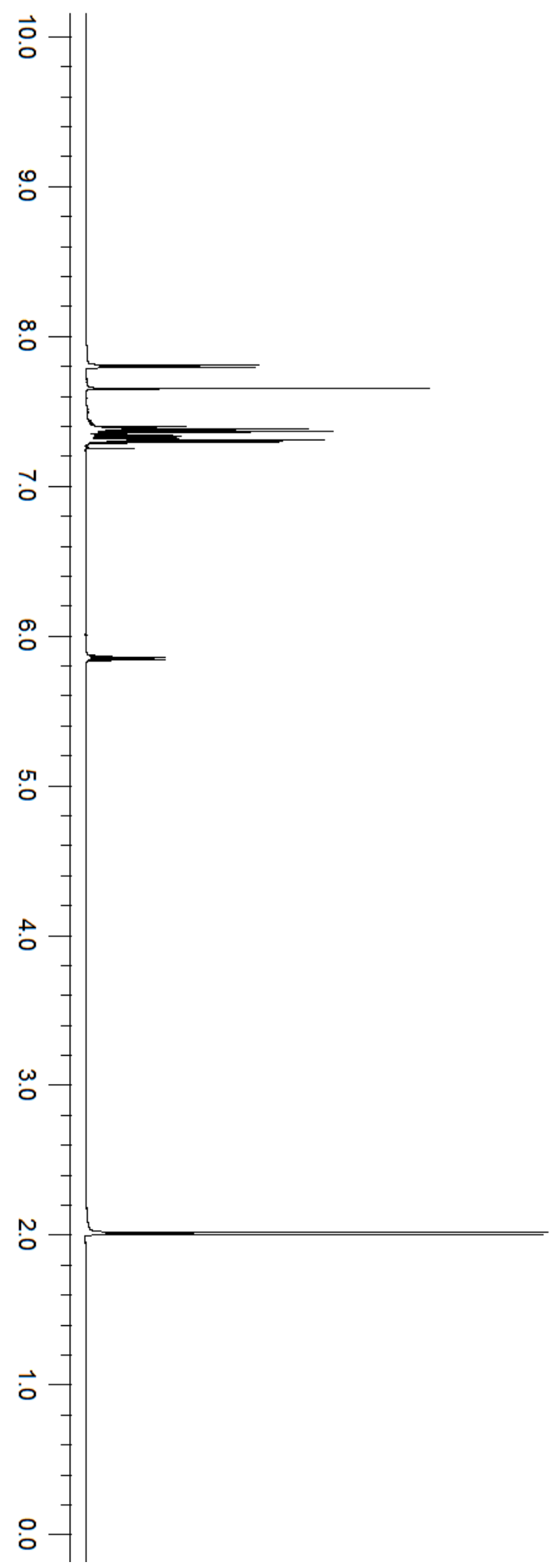

\section{${ }^{1} \mathrm{H} 600 \mathrm{MHz}$ NMR}

N-1-1.6.4d 


$$
F^{2}
$$



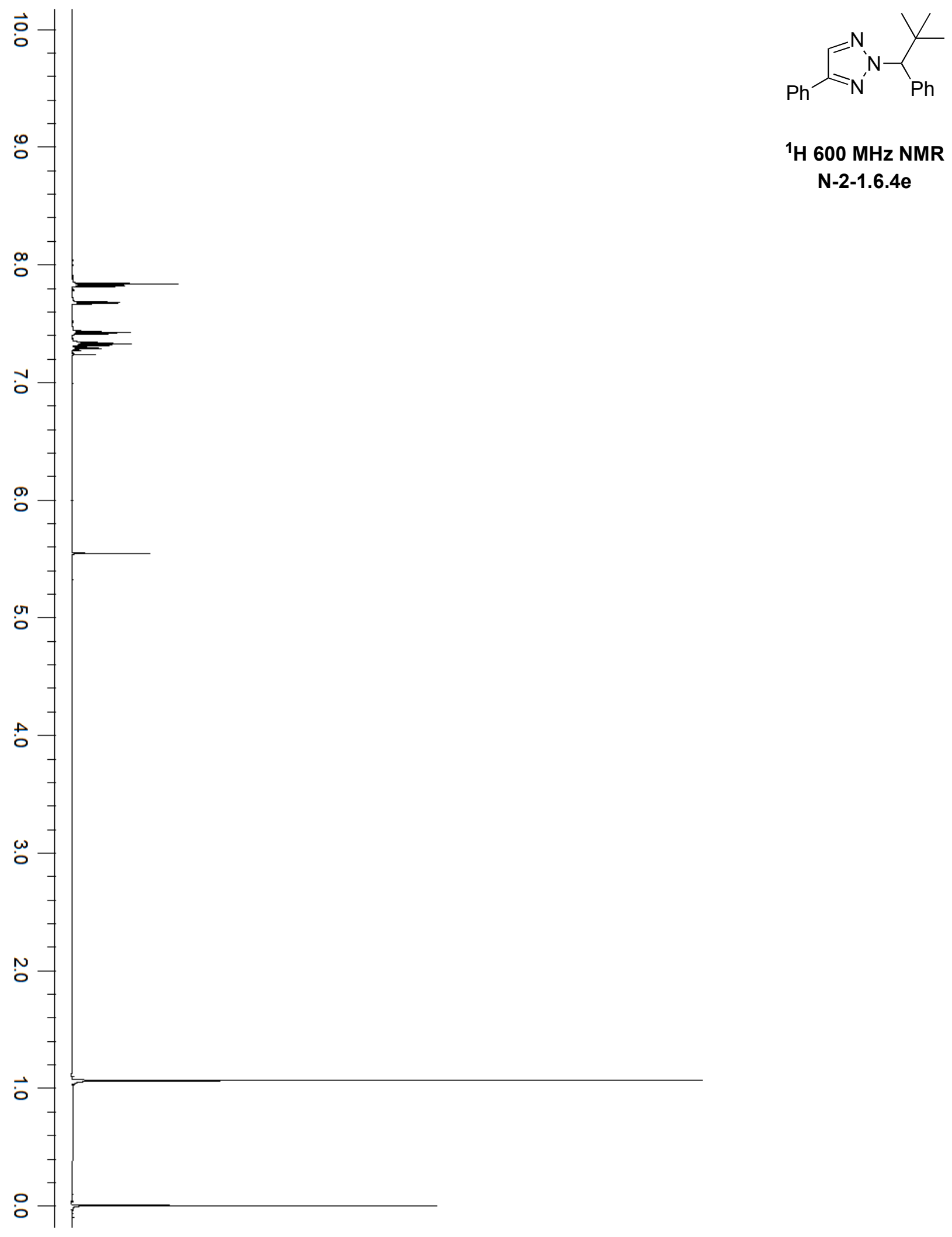

${ }^{1} \mathrm{H} 600 \mathrm{MHz}$ NMR

$\mathrm{N}-2-1.6 .4 \mathrm{e}$ 


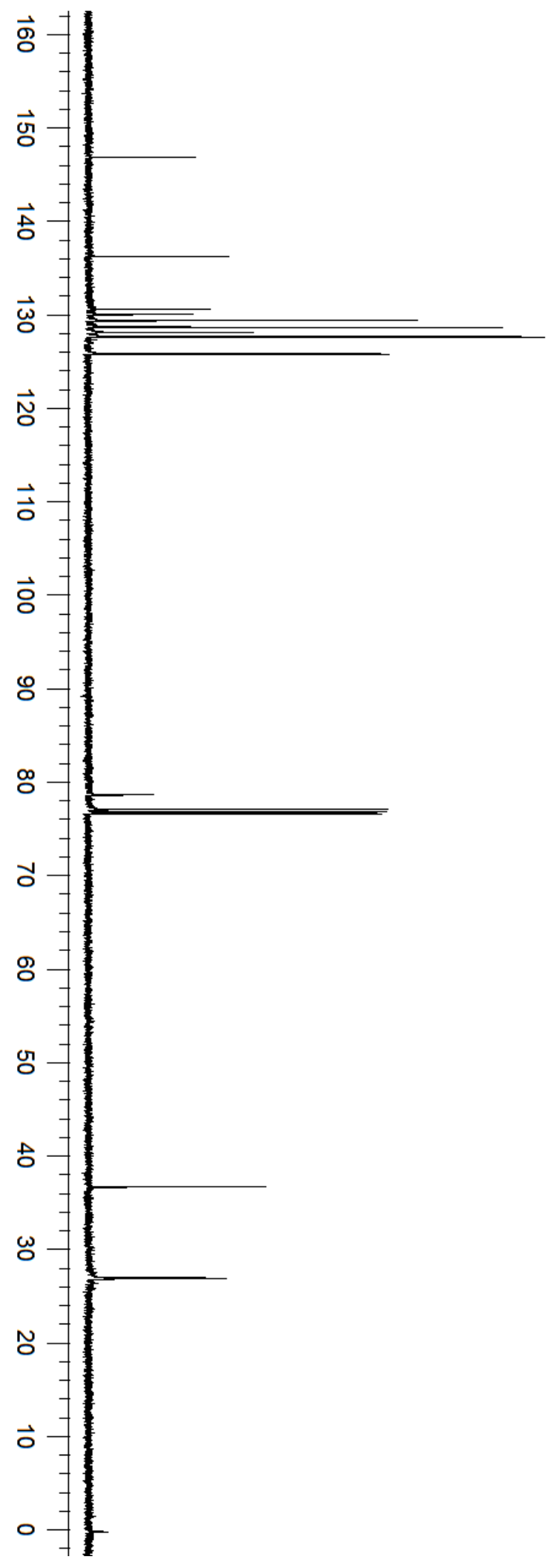

${ }^{13} \mathrm{C} 150 \mathrm{MHz}$ NMR

$\mathrm{N}-2-1.6 .4 \mathrm{e}$ 


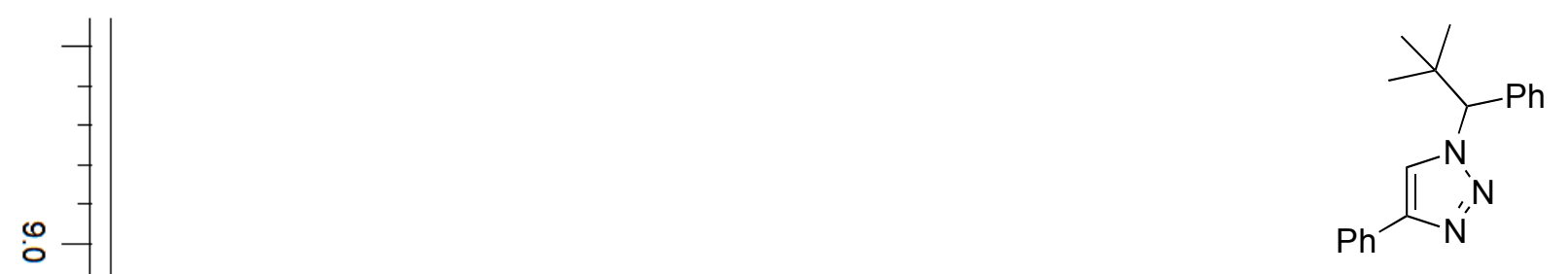

${ }^{1} \mathrm{H} 600 \mathrm{MHz}$ NMR $\mathrm{N}-1-1.6 .4 e$

$\stackrel{-1}{\circ}$

i

o

9

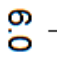

con

$\stackrel{\square}{\circ}$

$\omega$

$\stackrel{n}{\circ}$

$-1$

$\overrightarrow{0}$

$\circ$ 

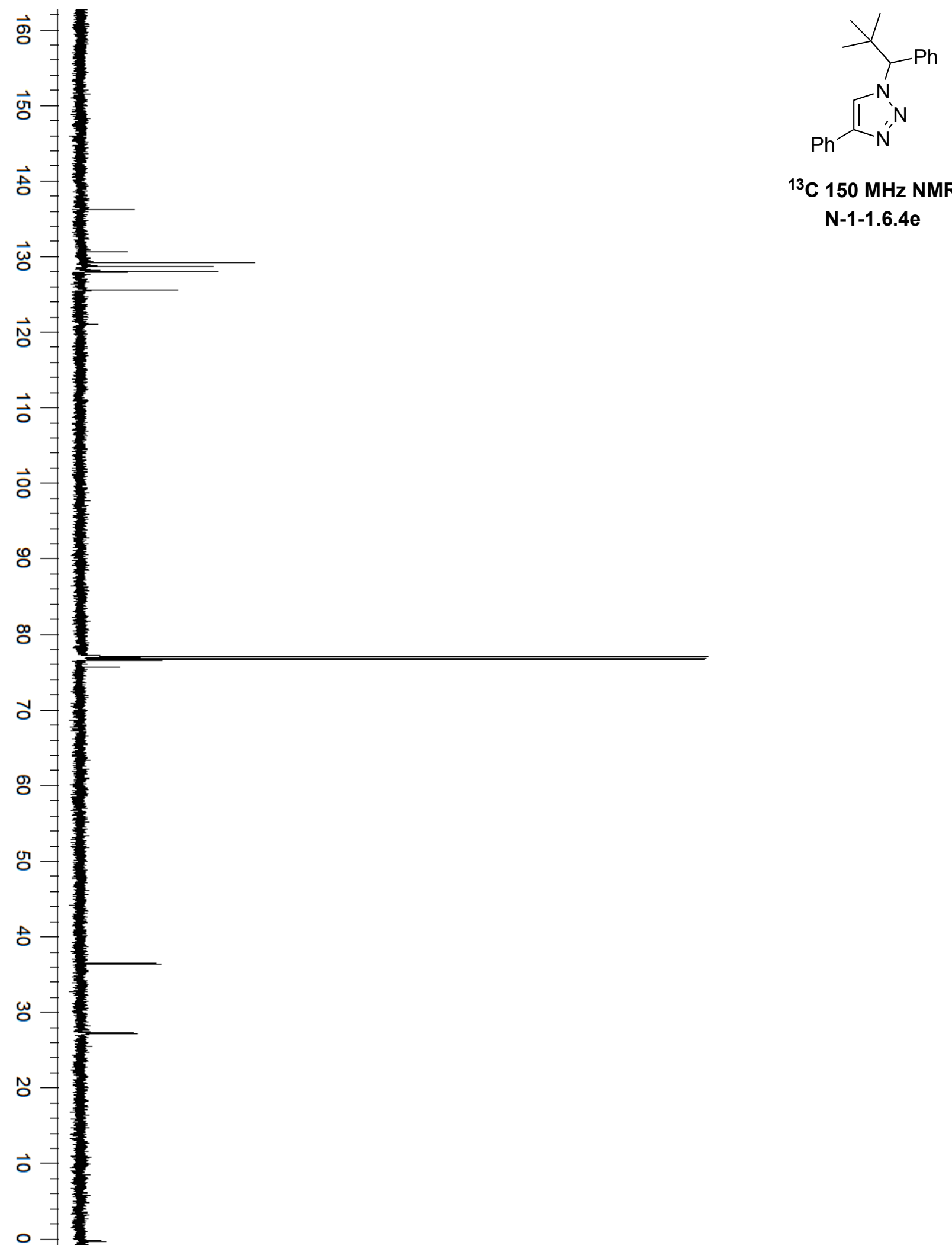

${ }^{13} \mathrm{C} 150 \mathrm{MHz}$ NMR $\mathrm{N}-1-1.6 .4 \mathrm{e}$ 


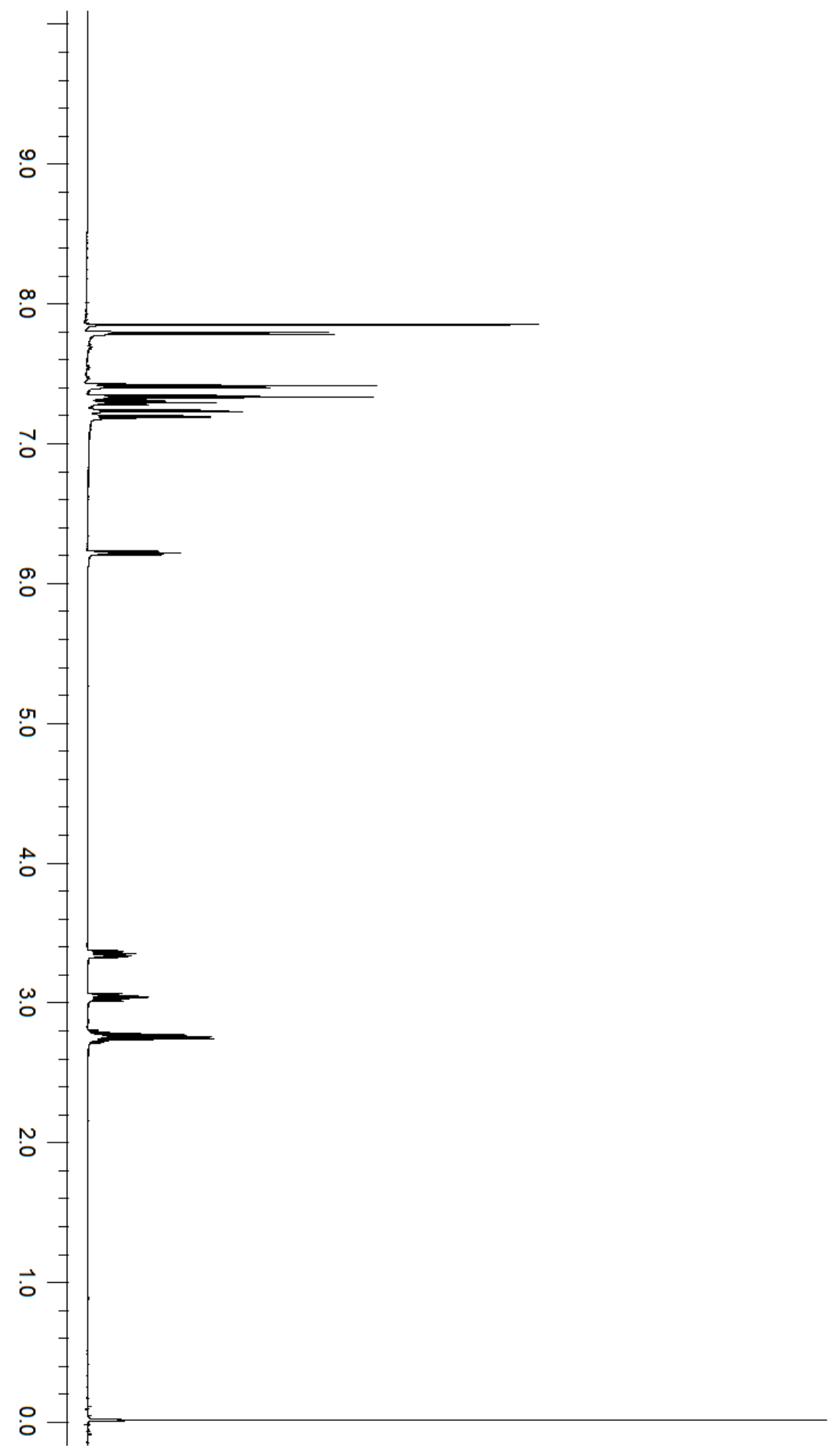

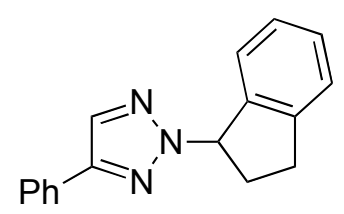

${ }^{1} \mathrm{H} 600 \mathrm{MHz}$ NMR

$\mathrm{N}-2-1.6 .4 \mathrm{f}$ 


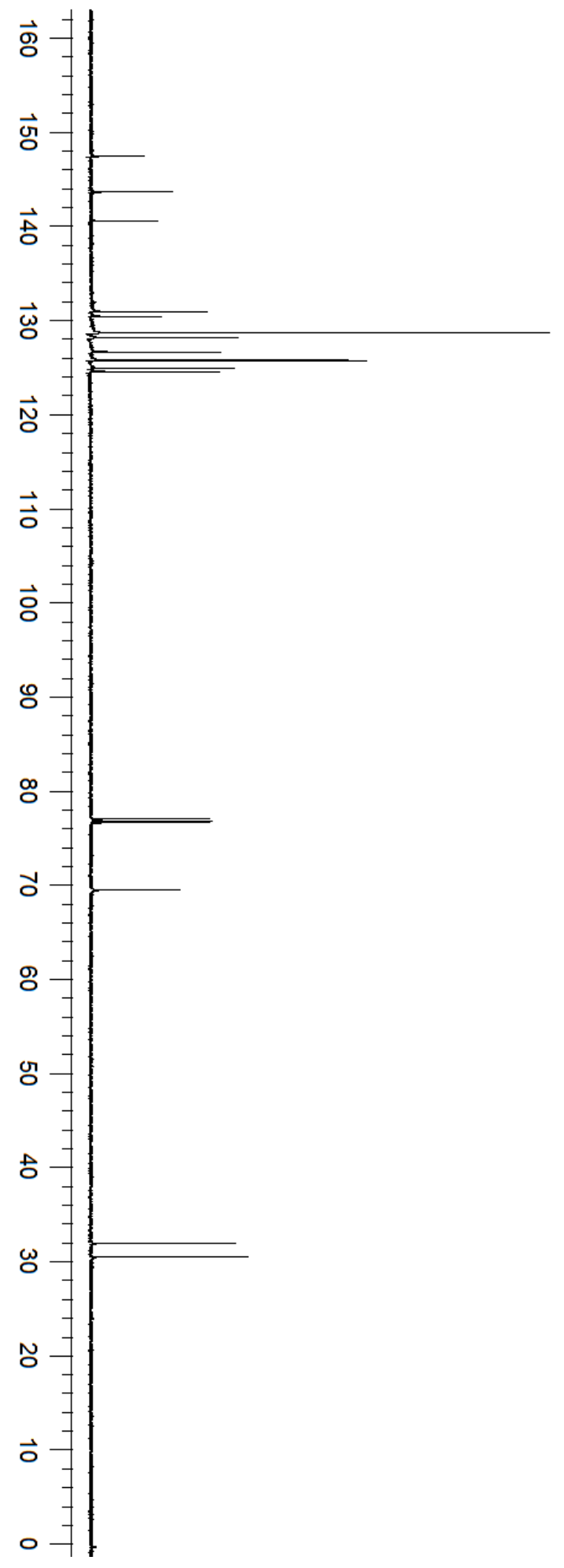

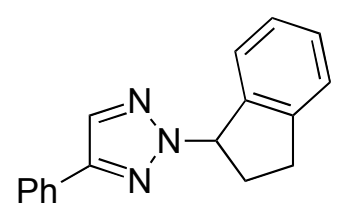

${ }^{13} \mathrm{C} 150 \mathrm{MHz}$ NMR N-2-1.6.4f 

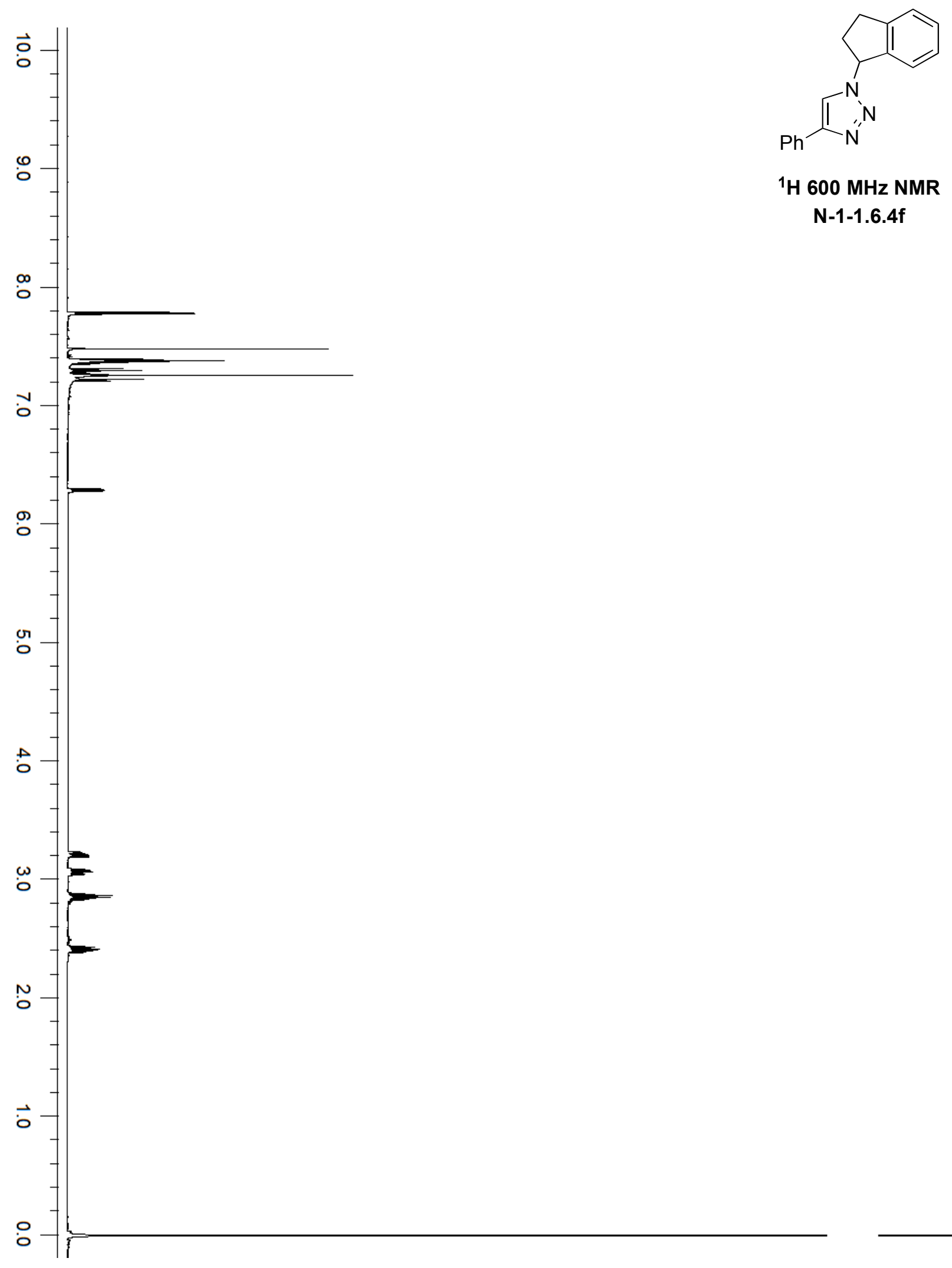

$600 \mathrm{MHz}$ NMR

N-1-1.6.4f 


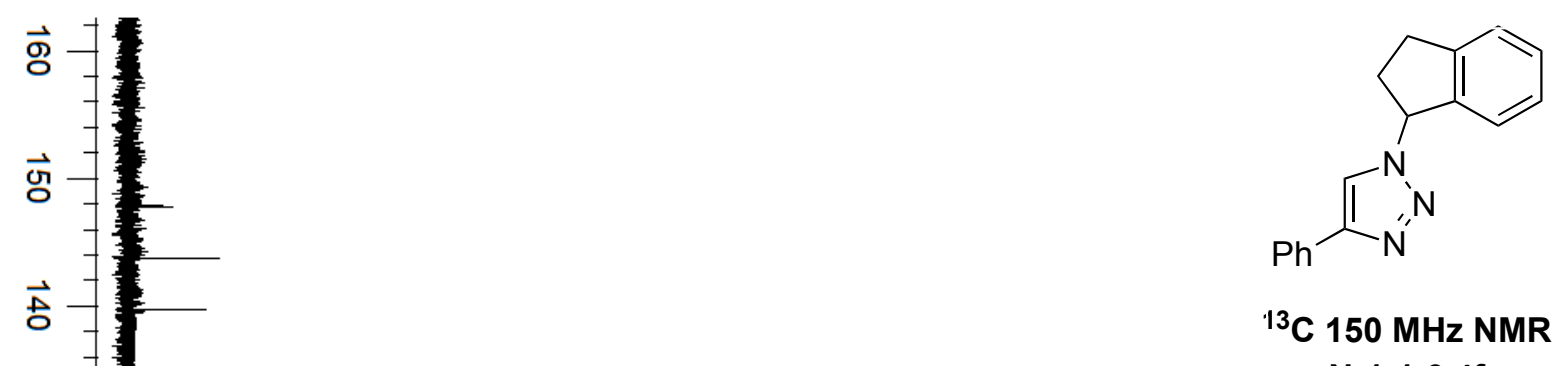
N-1-1.6.4f 

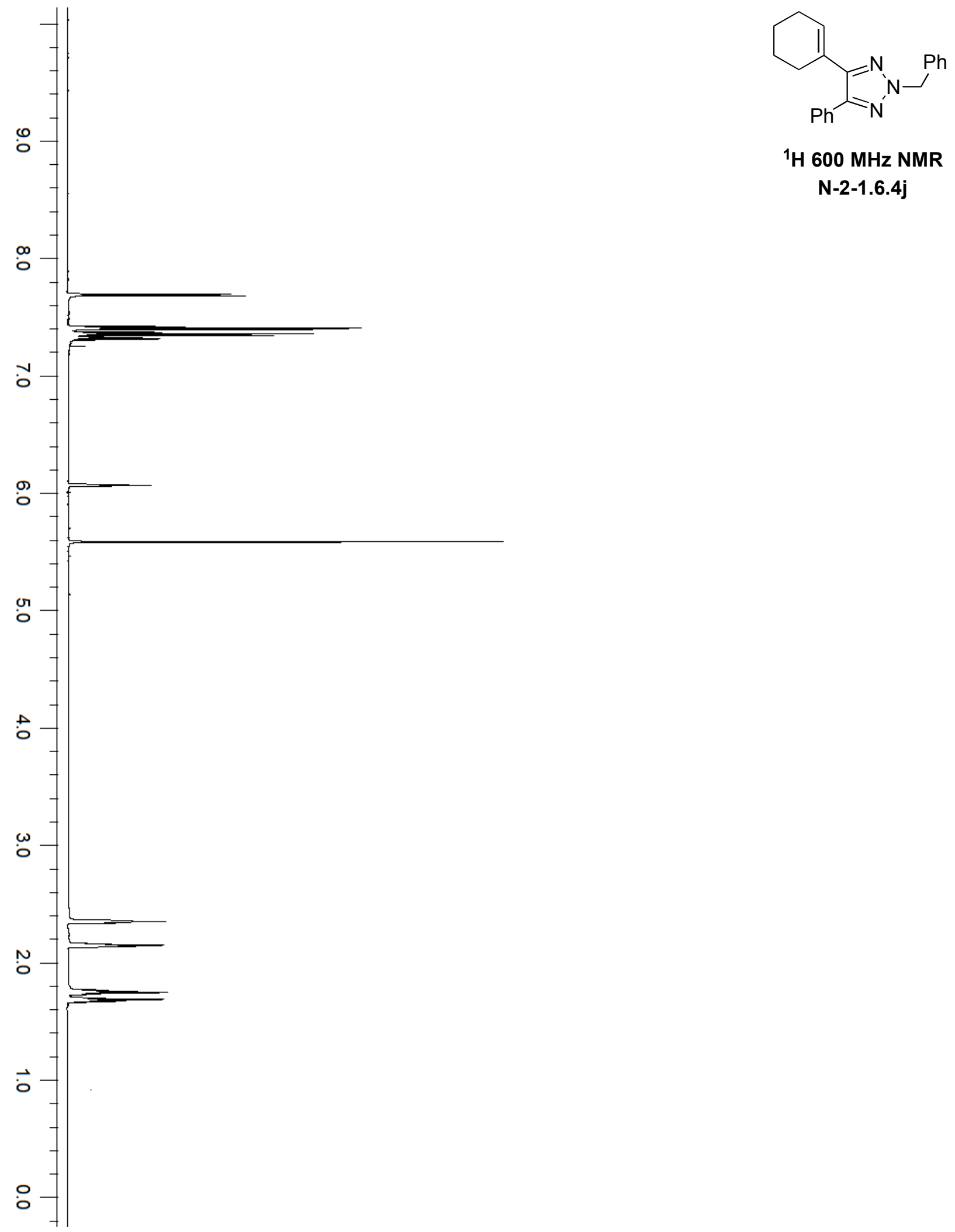

${ }^{1} \mathrm{H} 600 \mathrm{MHz}$ NMR

$\mathrm{N}-2-1.6 .4 \mathrm{j}$ 

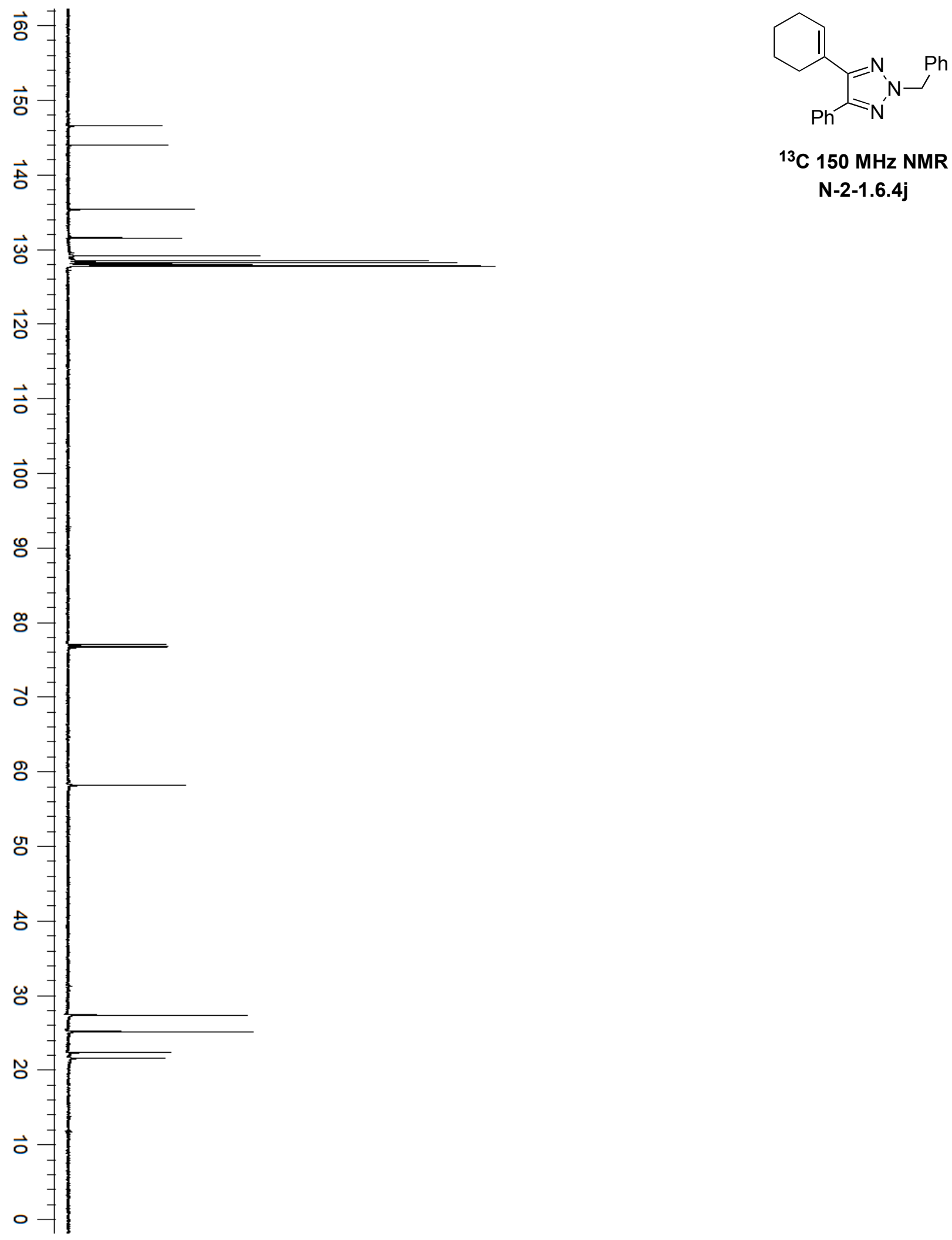

${ }^{13} \mathrm{C} 150 \mathrm{MHz}$ NMR N-2-1.6.4j

S810 

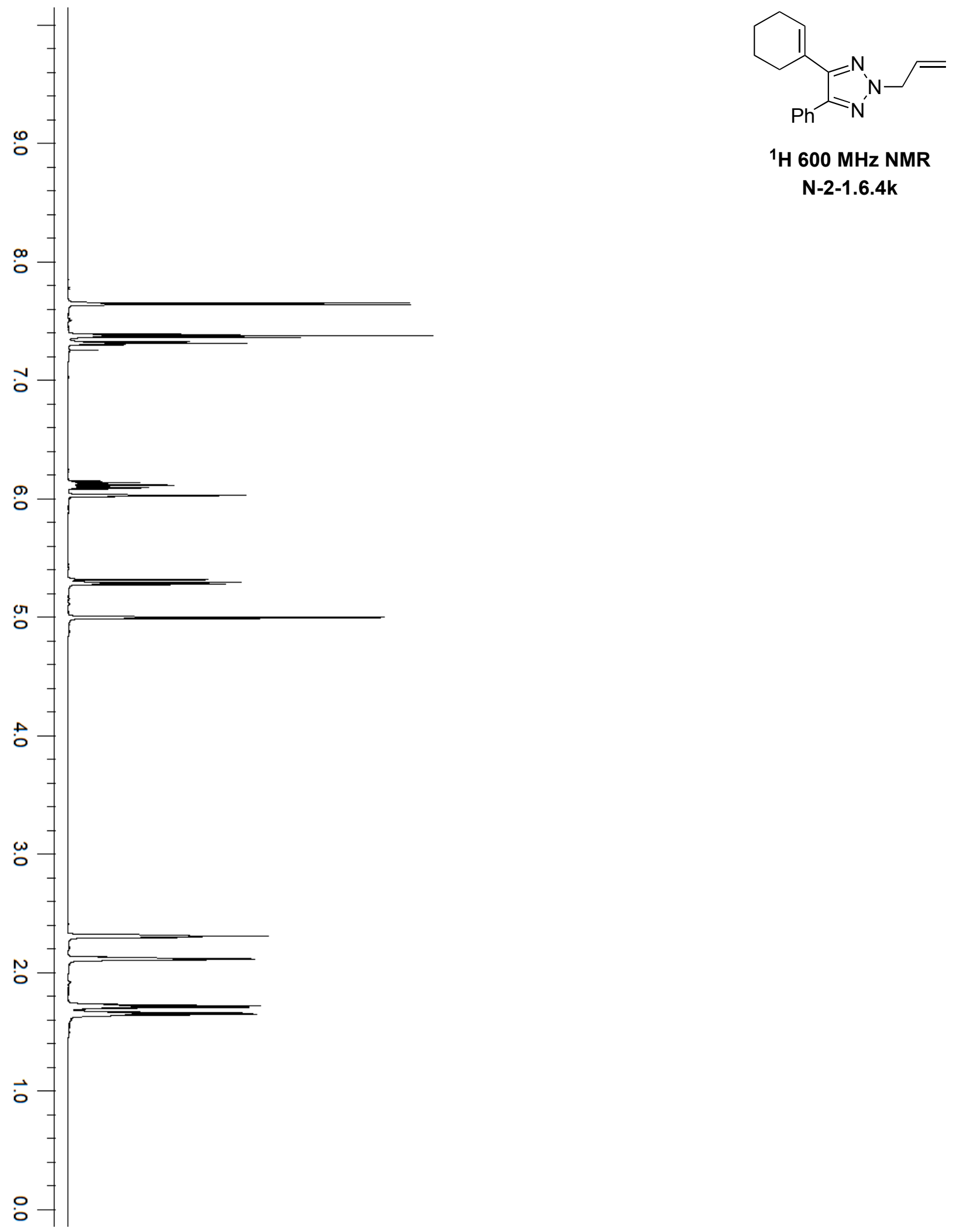

${ }^{1} \mathrm{H} 600 \mathrm{MHz}$ NMR

N-2-1.6.4k 

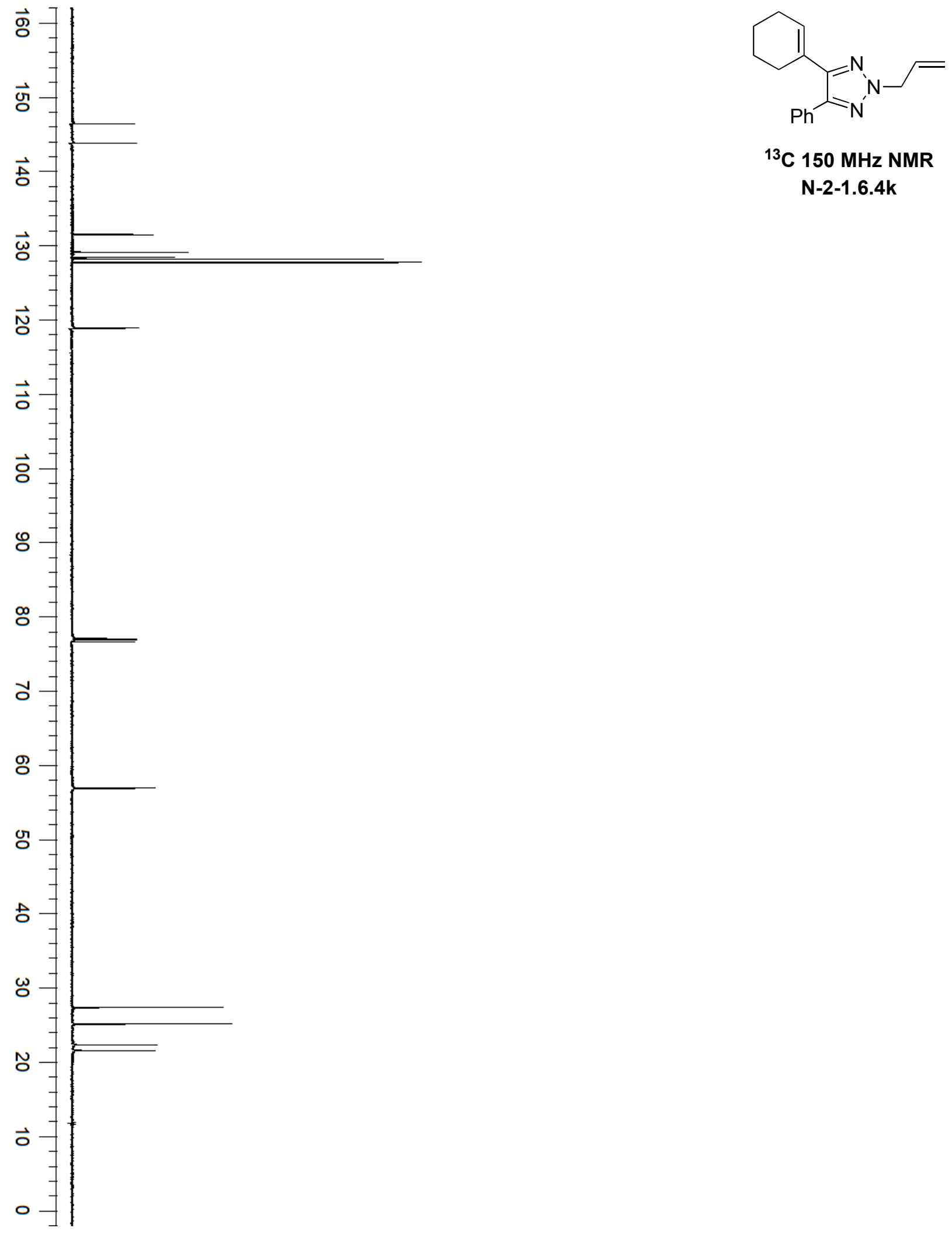

${ }^{13} \mathrm{C} 150 \mathrm{MHz}$ NMR N-2-1.6.4k 

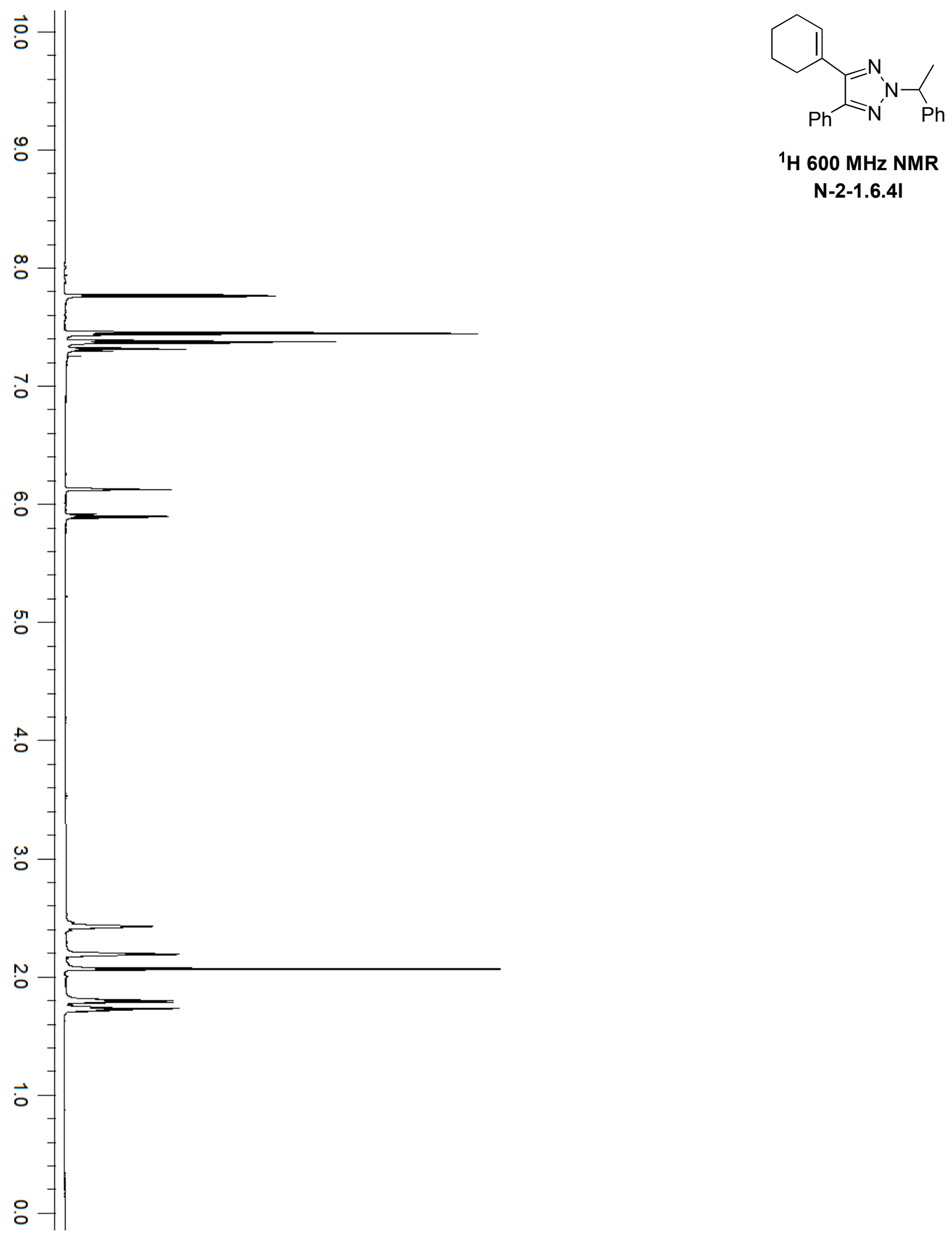

${ }^{1} \mathrm{H} 600 \mathrm{MHz}$ NMR

N-2-1.6.4 

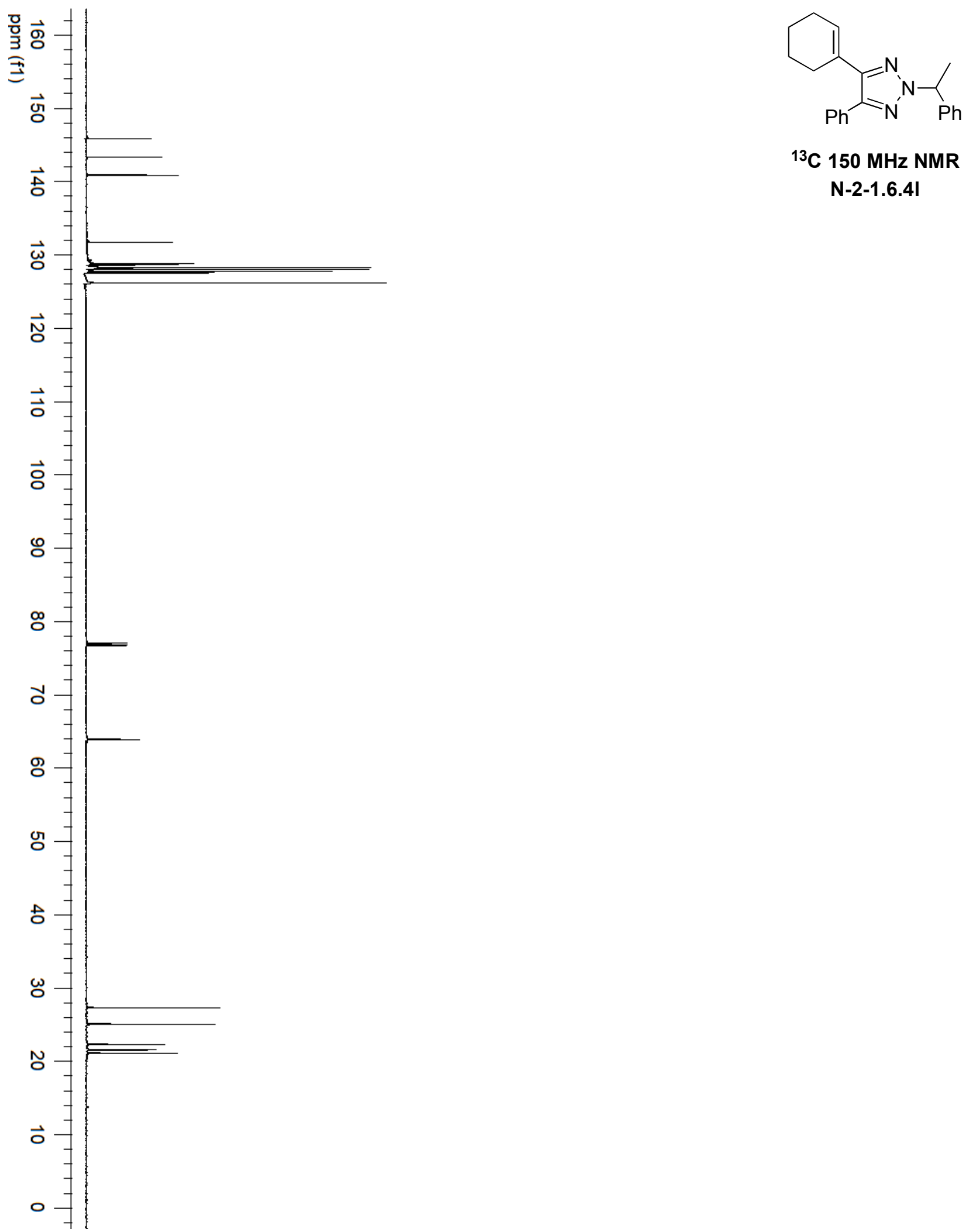

${ }^{13} \mathrm{C} 150 \mathrm{MHz}$ NMR

N-2-1.6.4I 


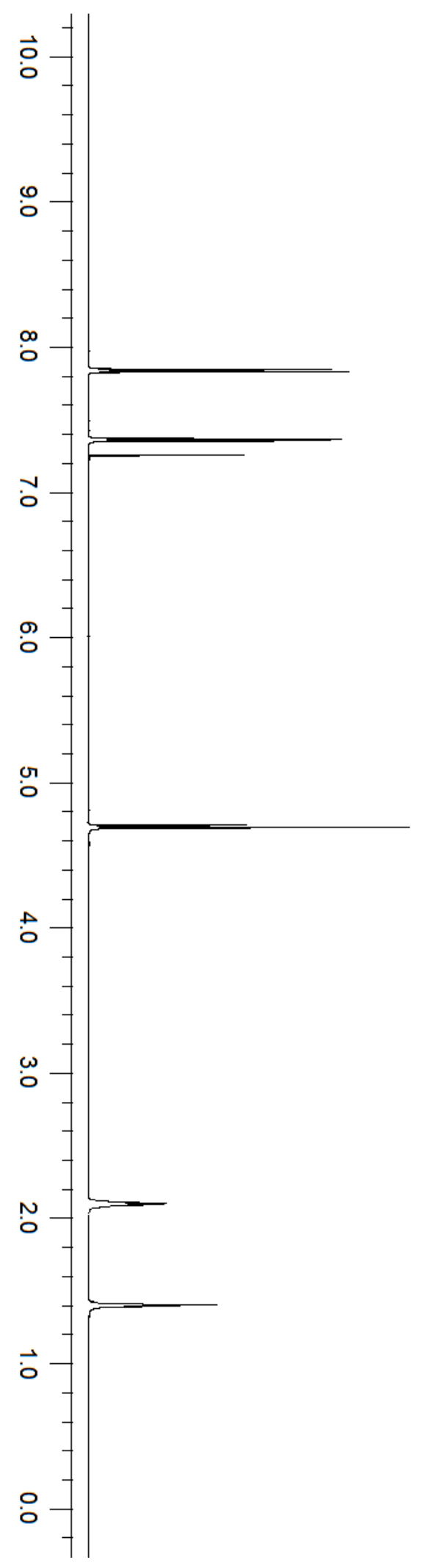

${ }^{1} \mathrm{H} 600 \mathrm{MHz}$ NMR

1.6.5a 


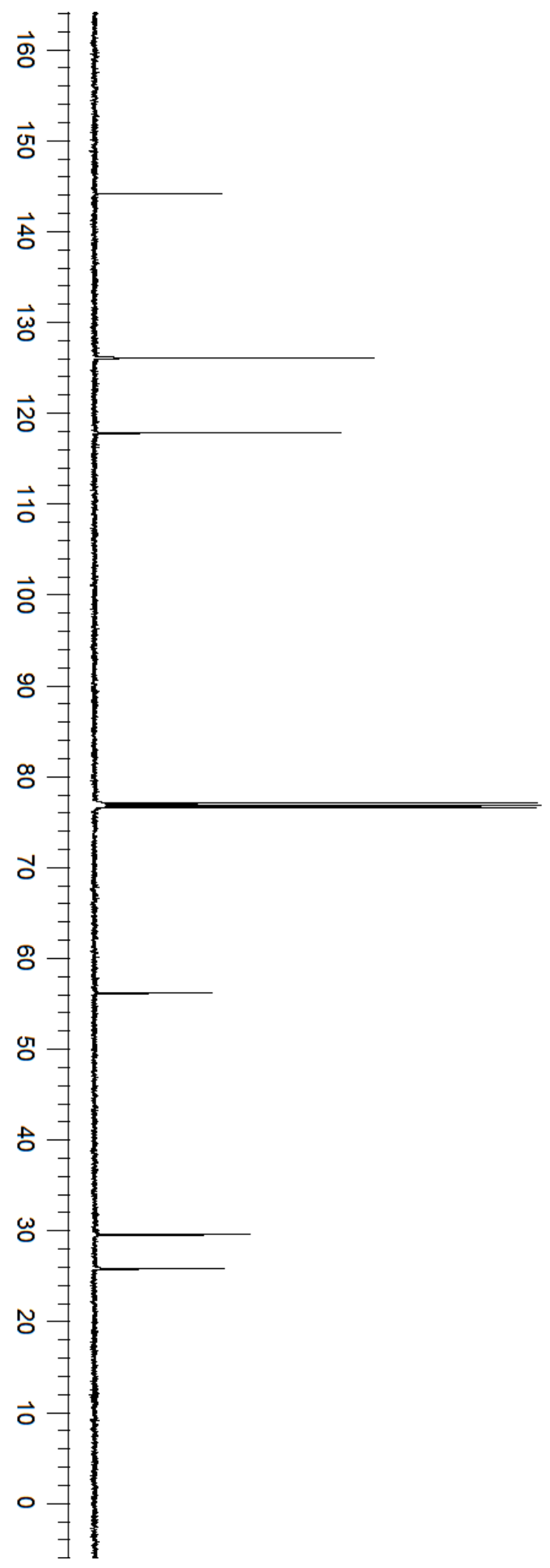

${ }^{13} \mathrm{C} 150 \mathrm{MHz}$ NMR

1.6.5a 


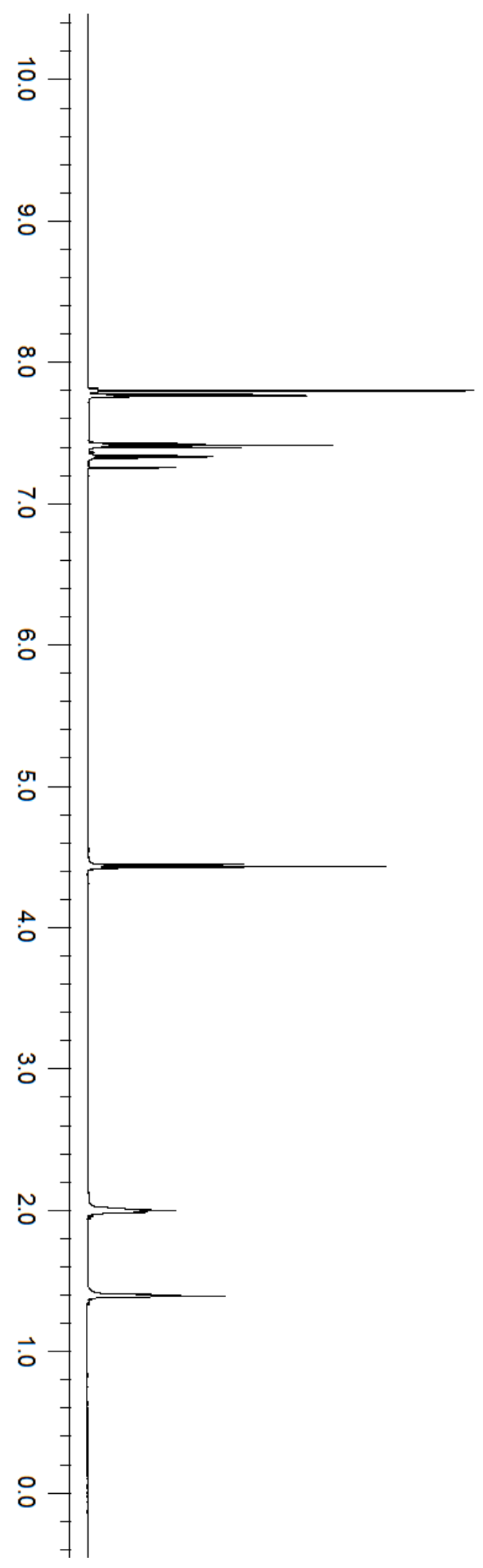

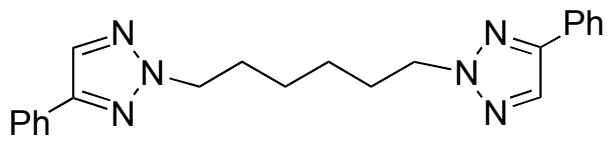

${ }^{1} \mathrm{H} 600 \mathrm{MHz}$ NMR

1.6.5b 


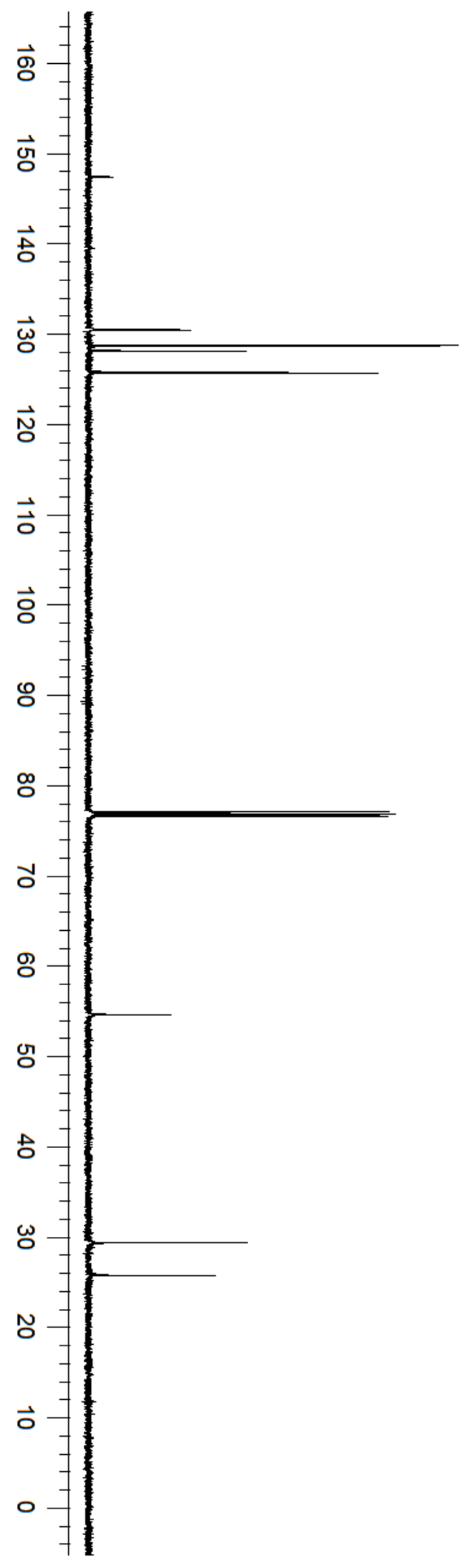

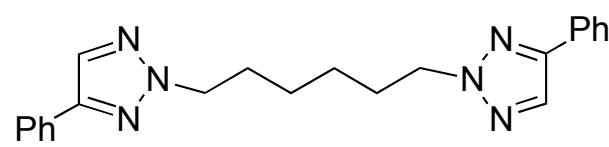

${ }^{13} \mathrm{C} 150 \mathrm{MHz}$ NMR

$1.6 .5 \mathrm{~b}$ 


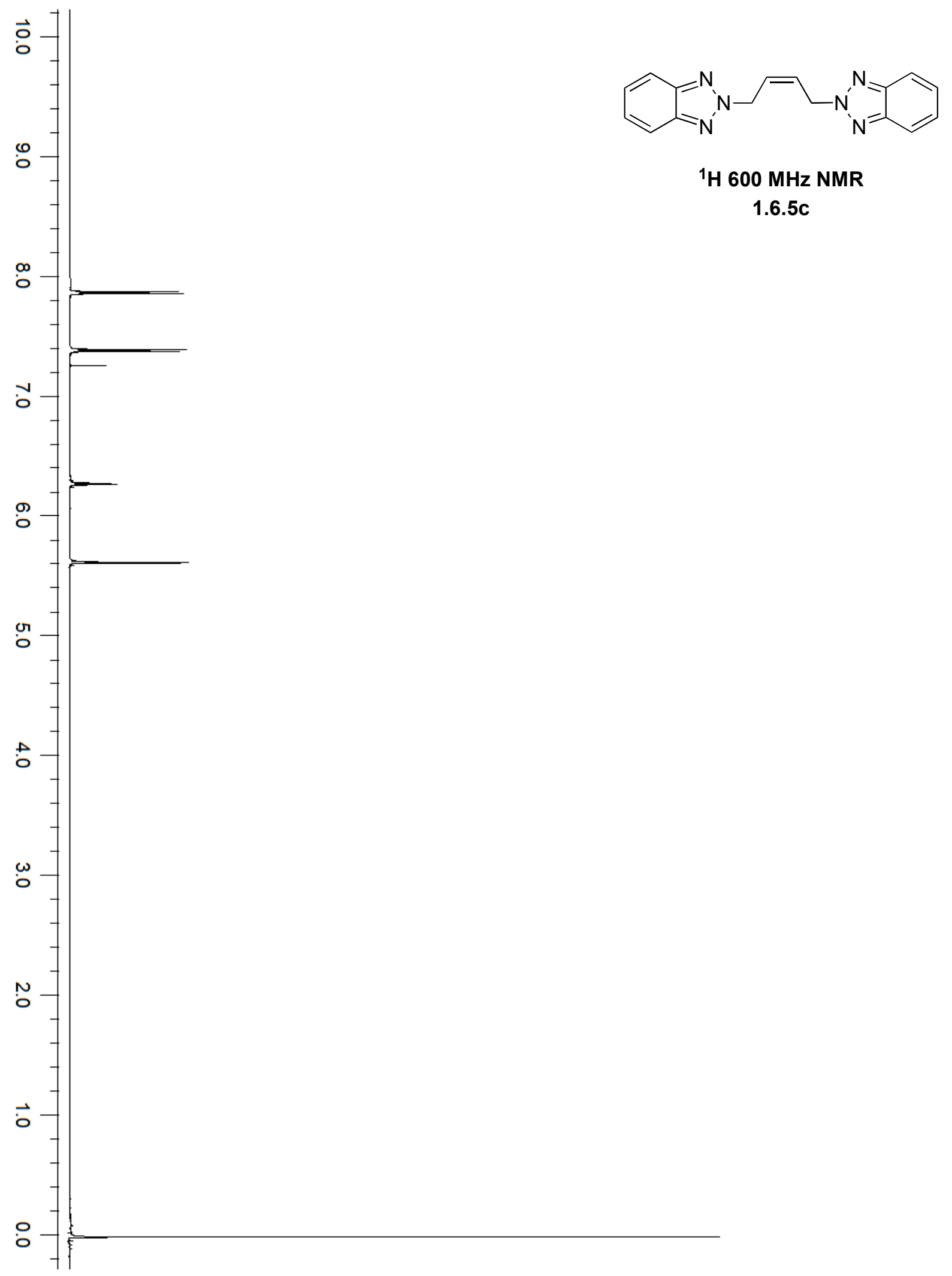




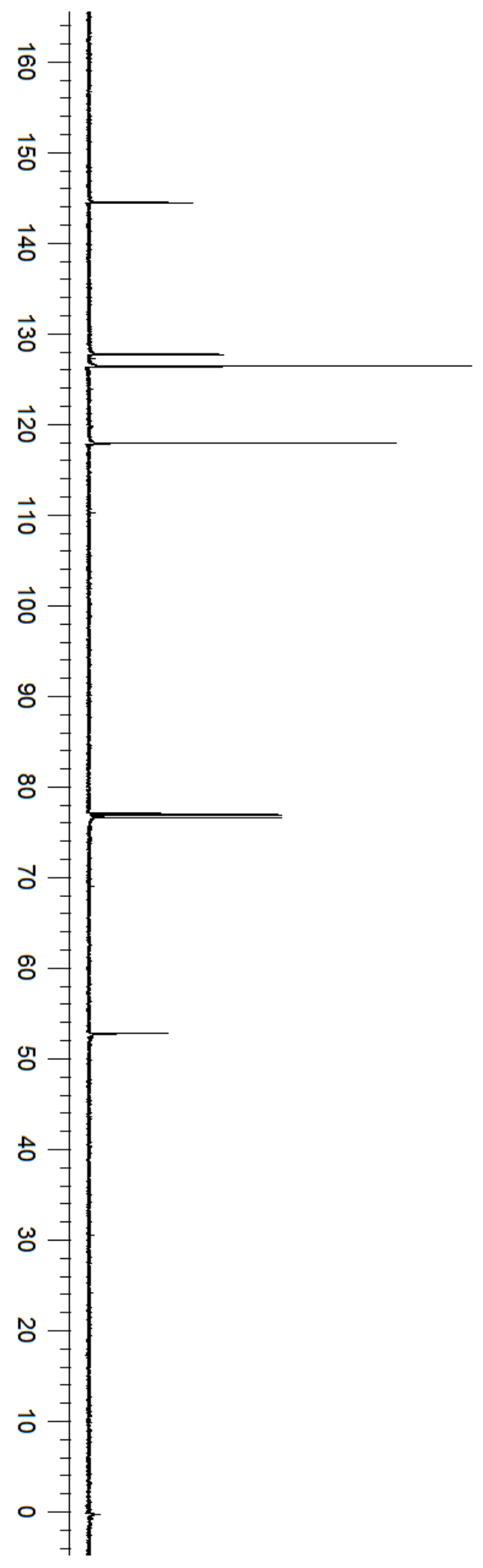

${ }^{13} \mathrm{C} 150 \mathrm{MHz}$ NMR

$1.6 .5 \mathrm{c}$ 

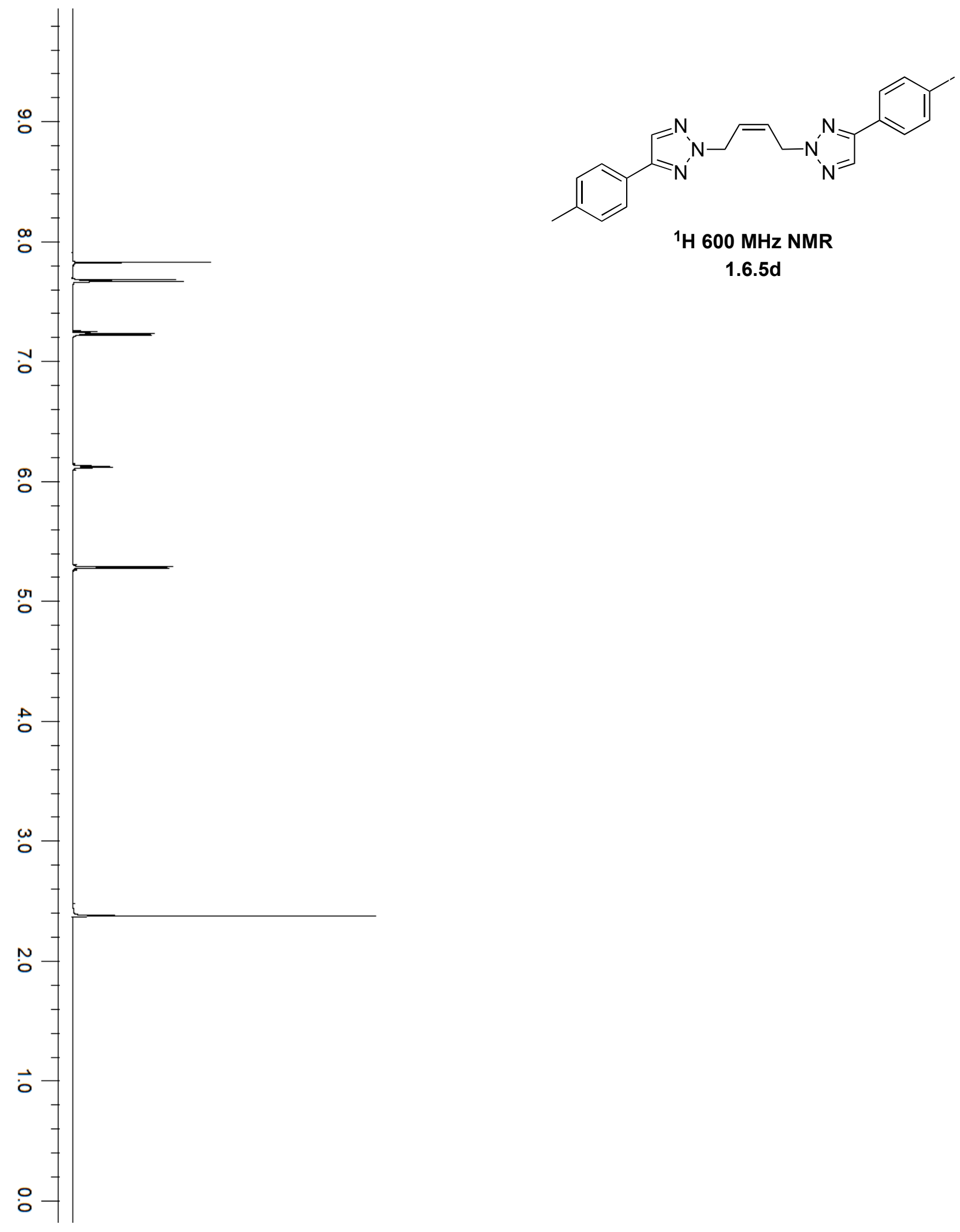

$1.6 .5 \mathrm{~d}$ 


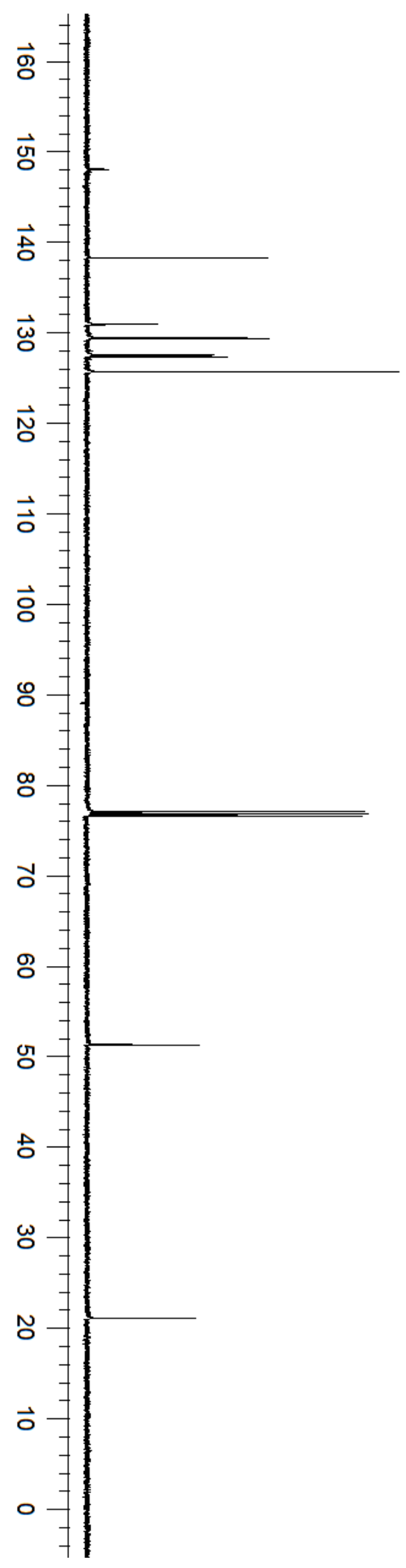




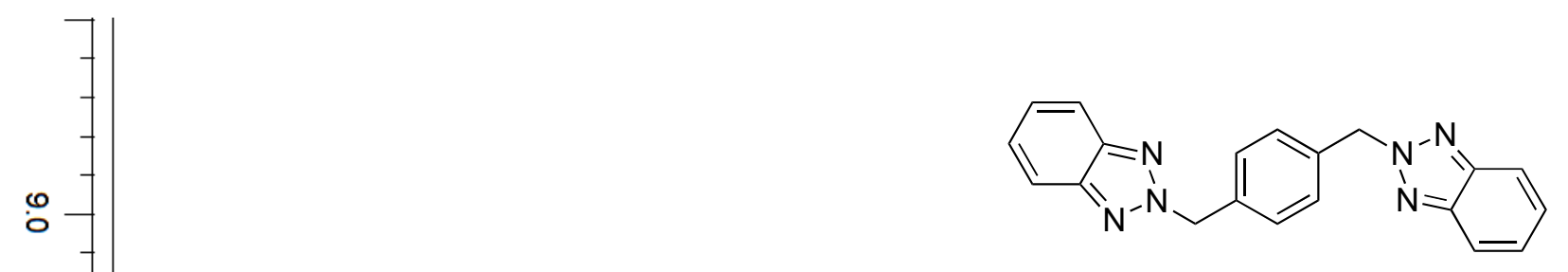

${ }^{1} \mathrm{H} 600 \mathrm{MHz}$ NMR

1.6.5e

$\infty$

c。

$\ddot{0}$

or

of

$\therefore$

$\omega$

nt

$\circ$

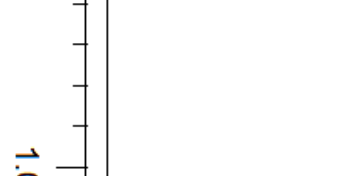

$\vec{\circ}$

$0-$ 

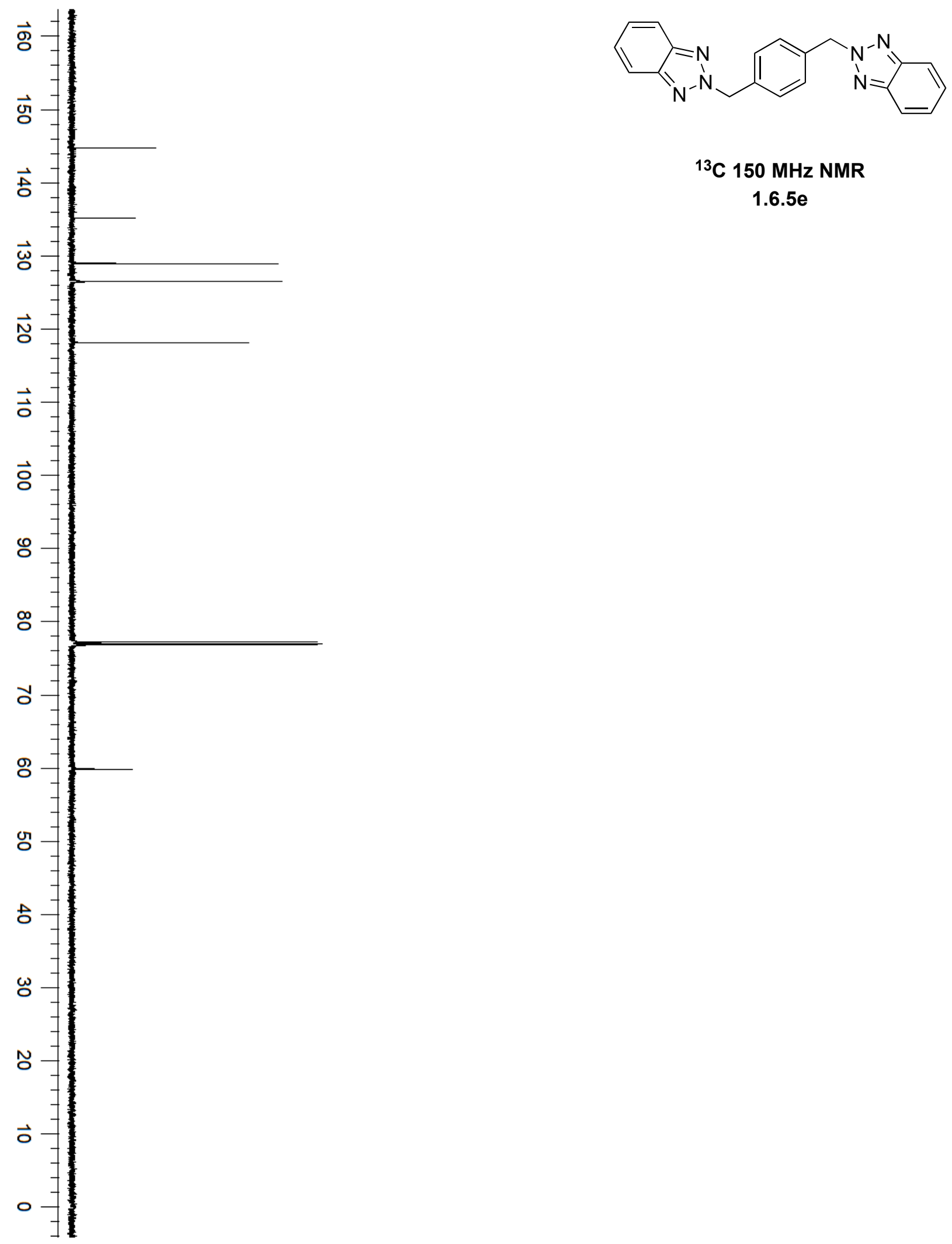

${ }^{13} \mathrm{C} 150 \mathrm{MHz}$ NMR

1.6.5e 


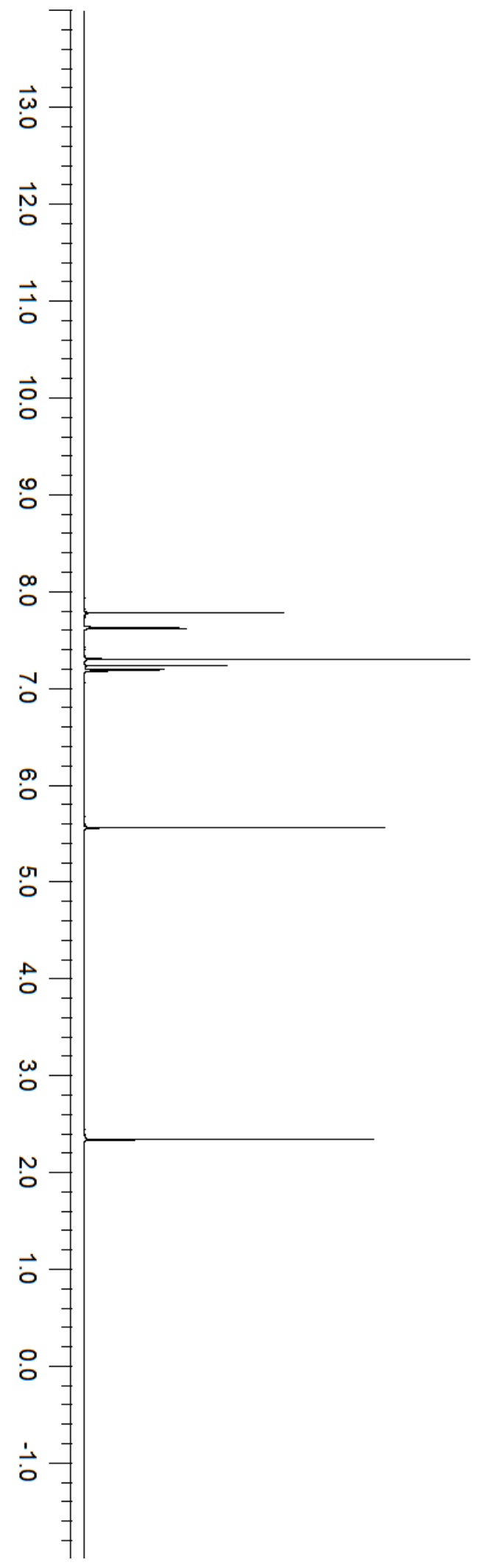

\section{${ }^{1} \mathrm{H} 600 \mathrm{MHz}$ NMR}

1.6.5f 


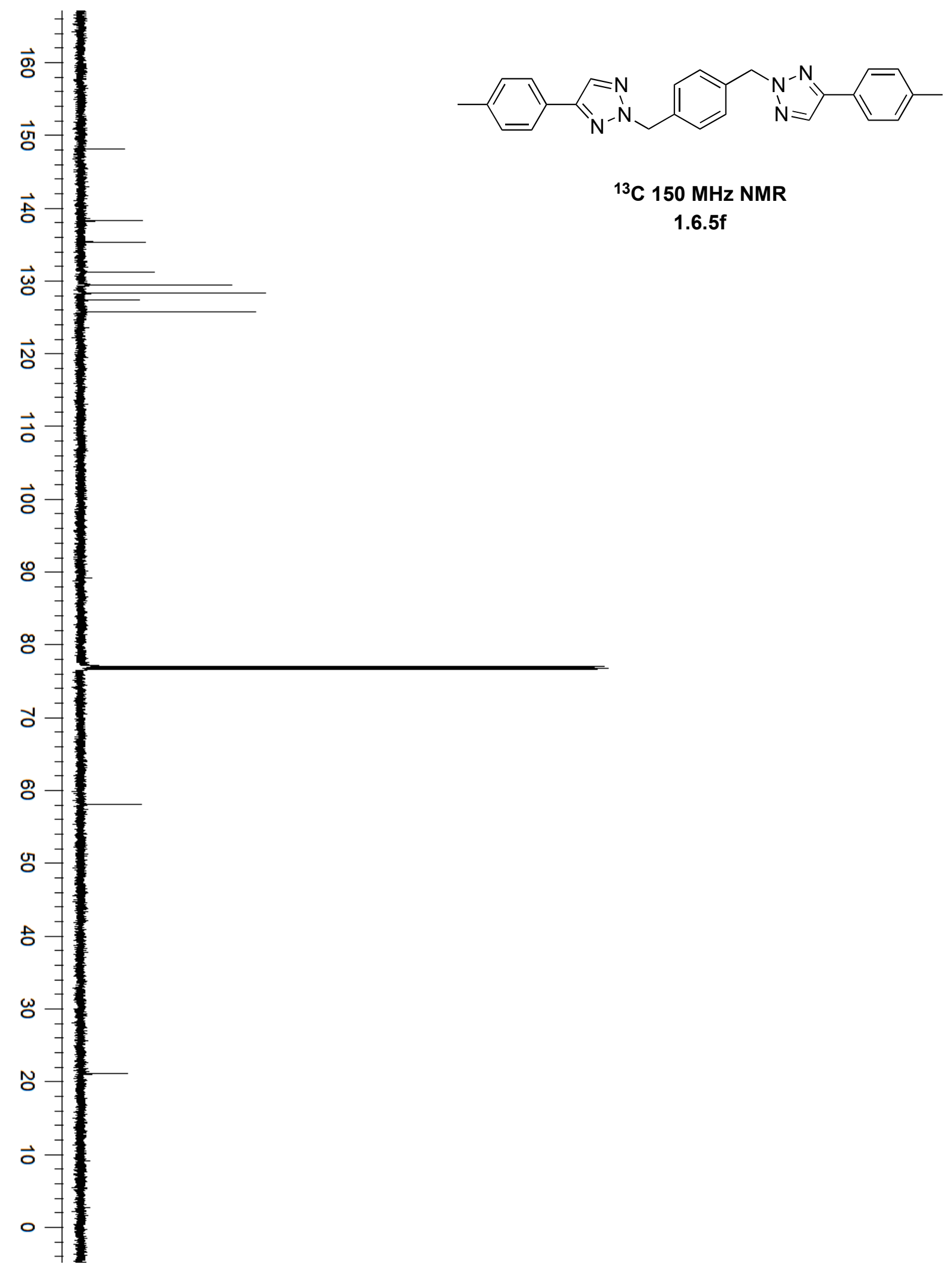




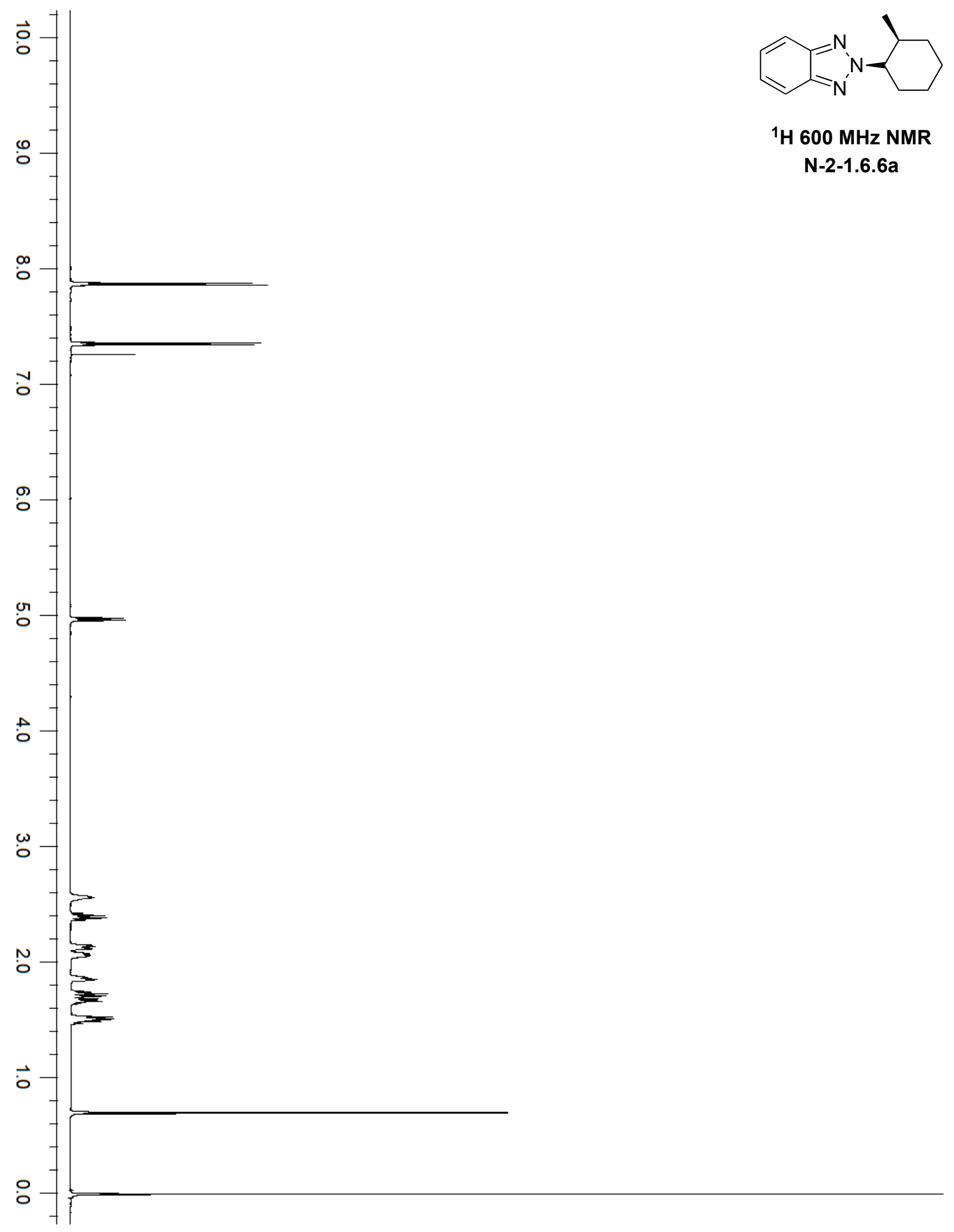



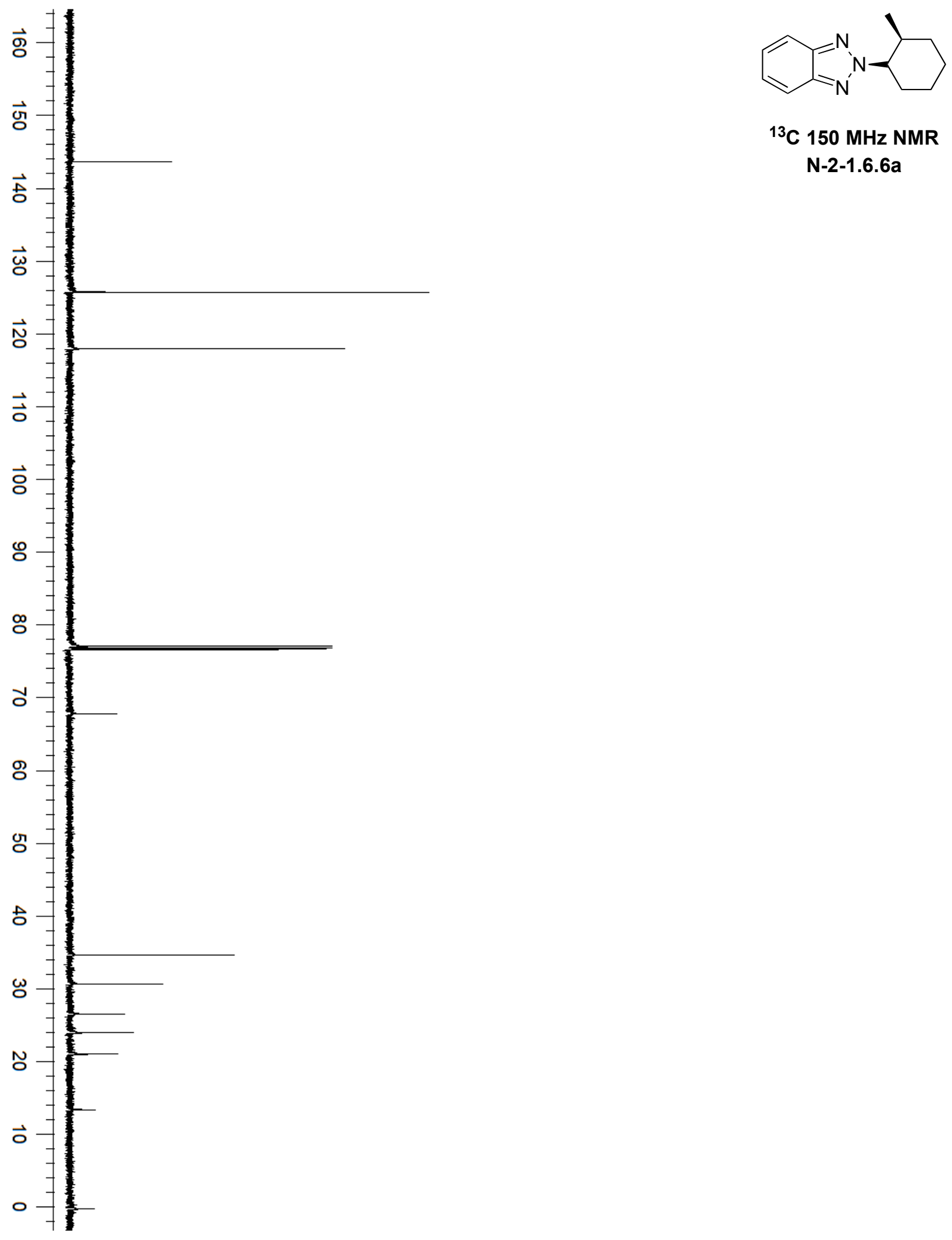

${ }^{13} \mathrm{C} 150 \mathrm{MHz}$ NMR N-2-1.6.6a 


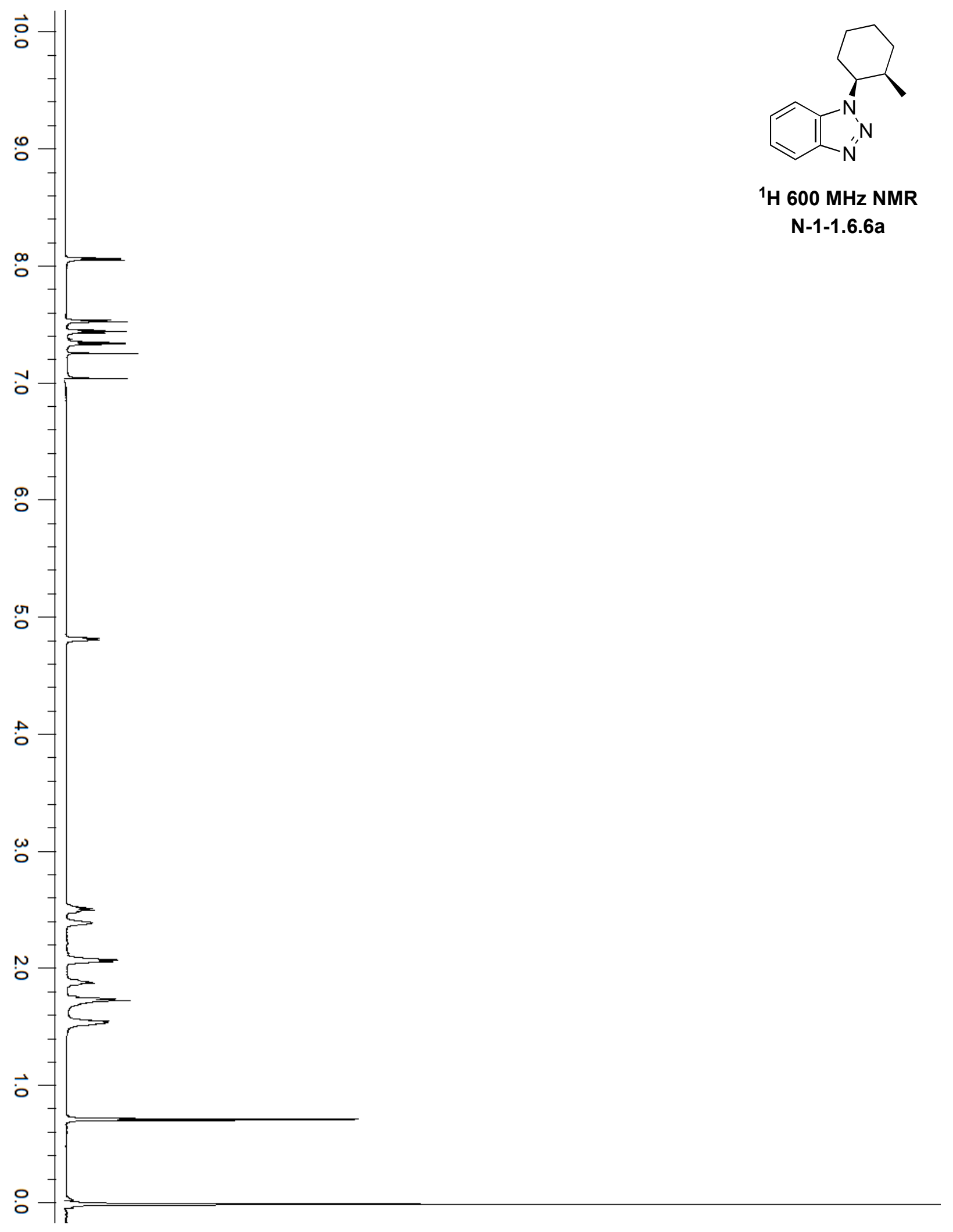




$$
L^{2}
$$



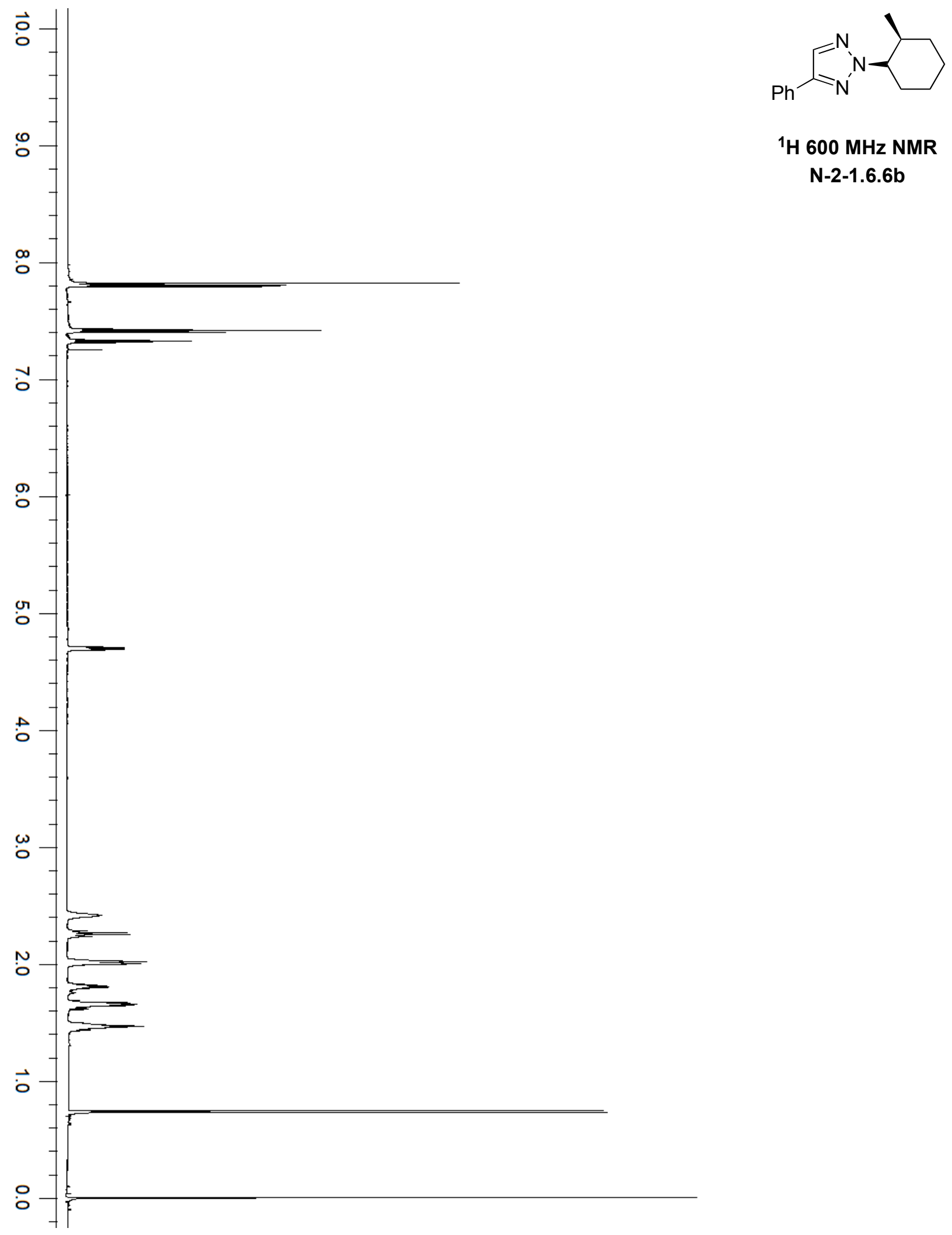

${ }^{1} \mathrm{H} 600 \mathrm{MHz}$ NMR N-2-1.6.6b 

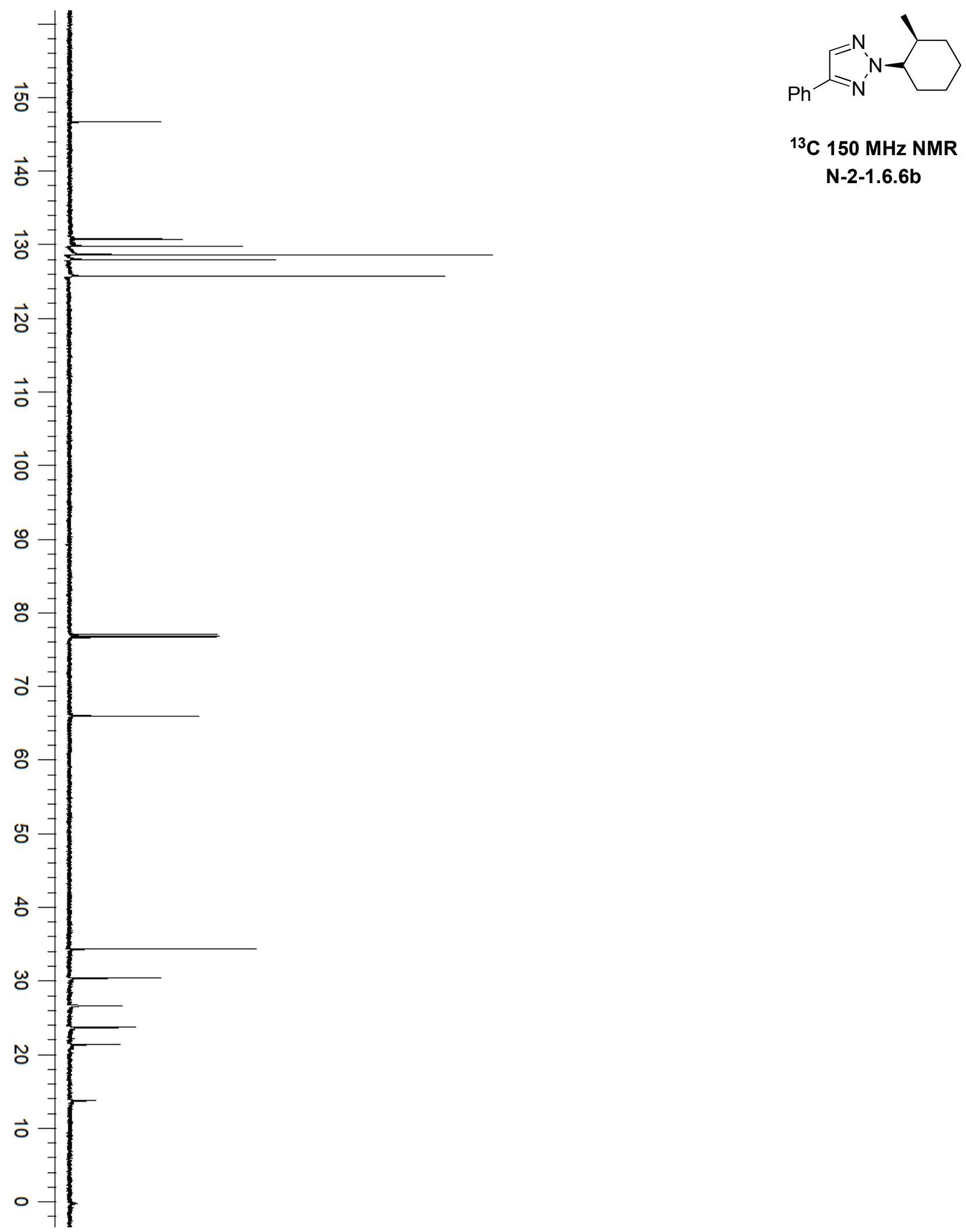

${ }^{13} \mathrm{C} 150 \mathrm{MHz}$ NMR

$\mathrm{N}-2-1.6 .6 \mathrm{~b}$ 


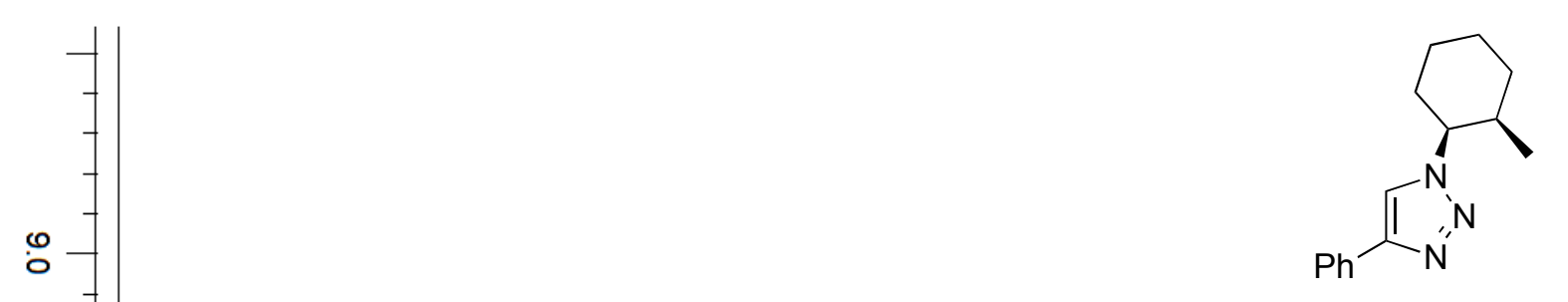

${ }^{1} \mathrm{H} 600 \mathrm{MHz}$ NMR

$\mathrm{N}-1-1.6 .6 \mathrm{~b}$

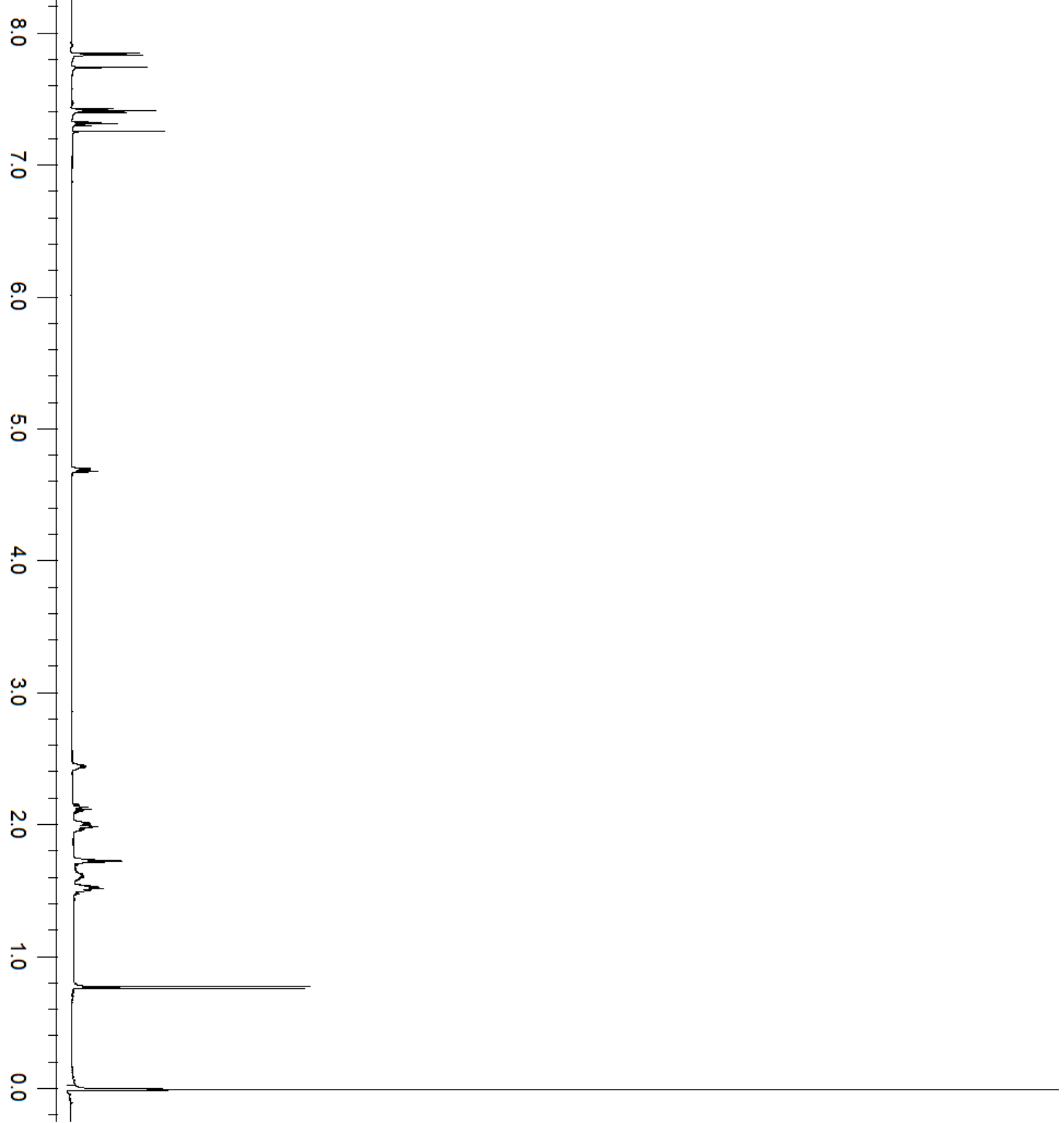



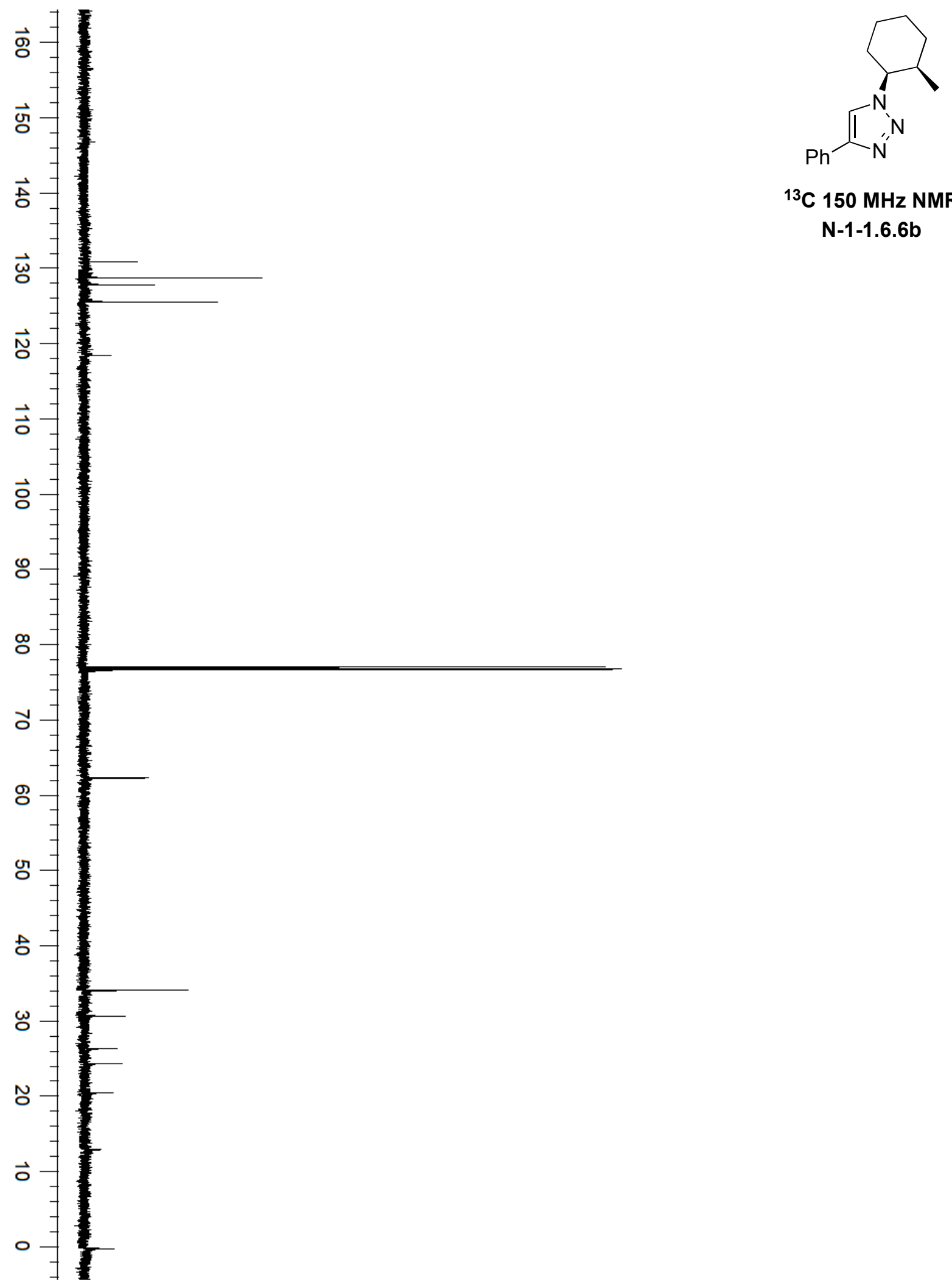

${ }^{13} \mathrm{C} 150 \mathrm{MHz}$ NMR $\mathrm{N}-1-1.6 .6 \mathrm{~b}$ 

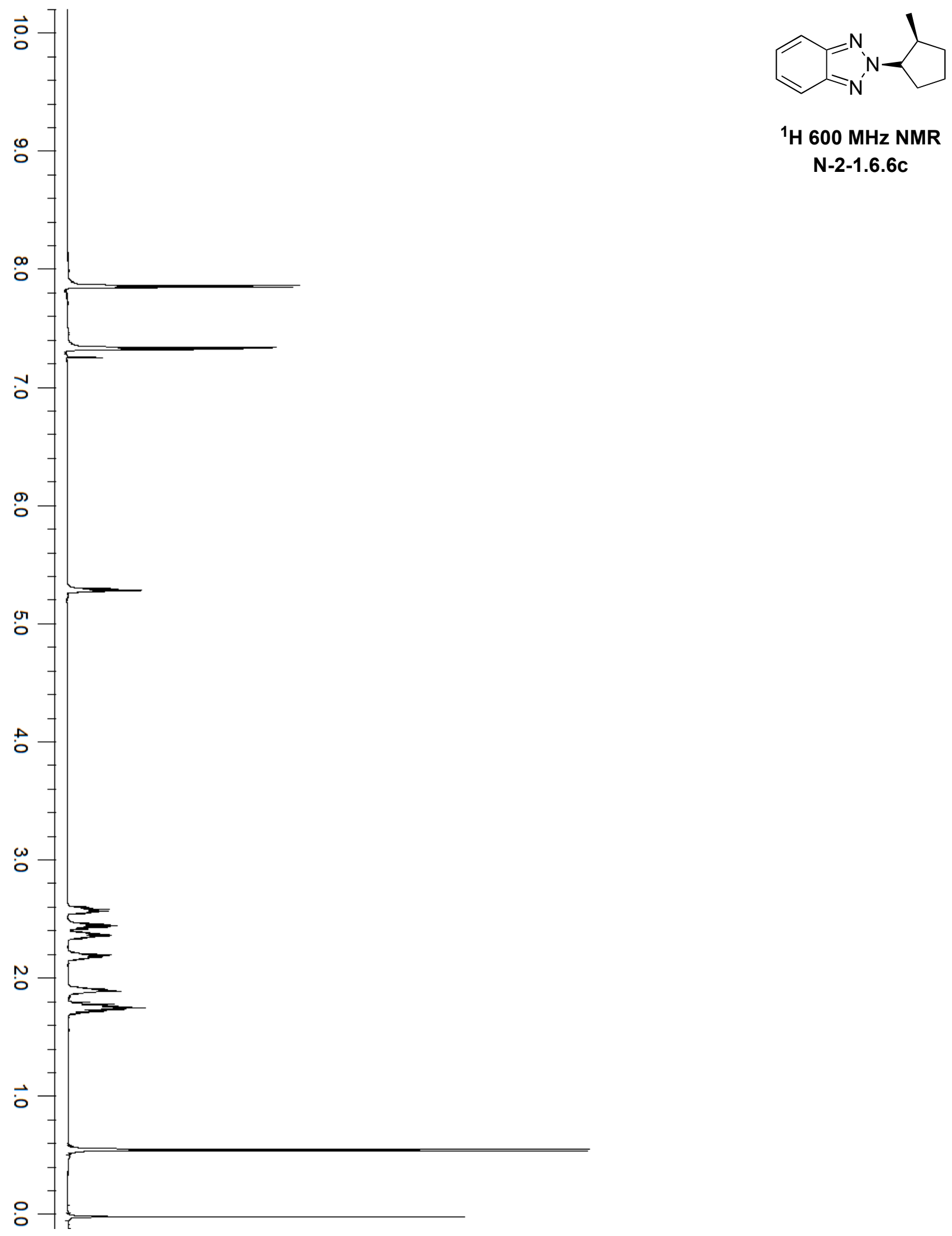

${ }^{1} \mathrm{H} 600 \mathrm{MHz}$ NMR

$\mathrm{N}-2-1.6 .6 \mathrm{c}$ 

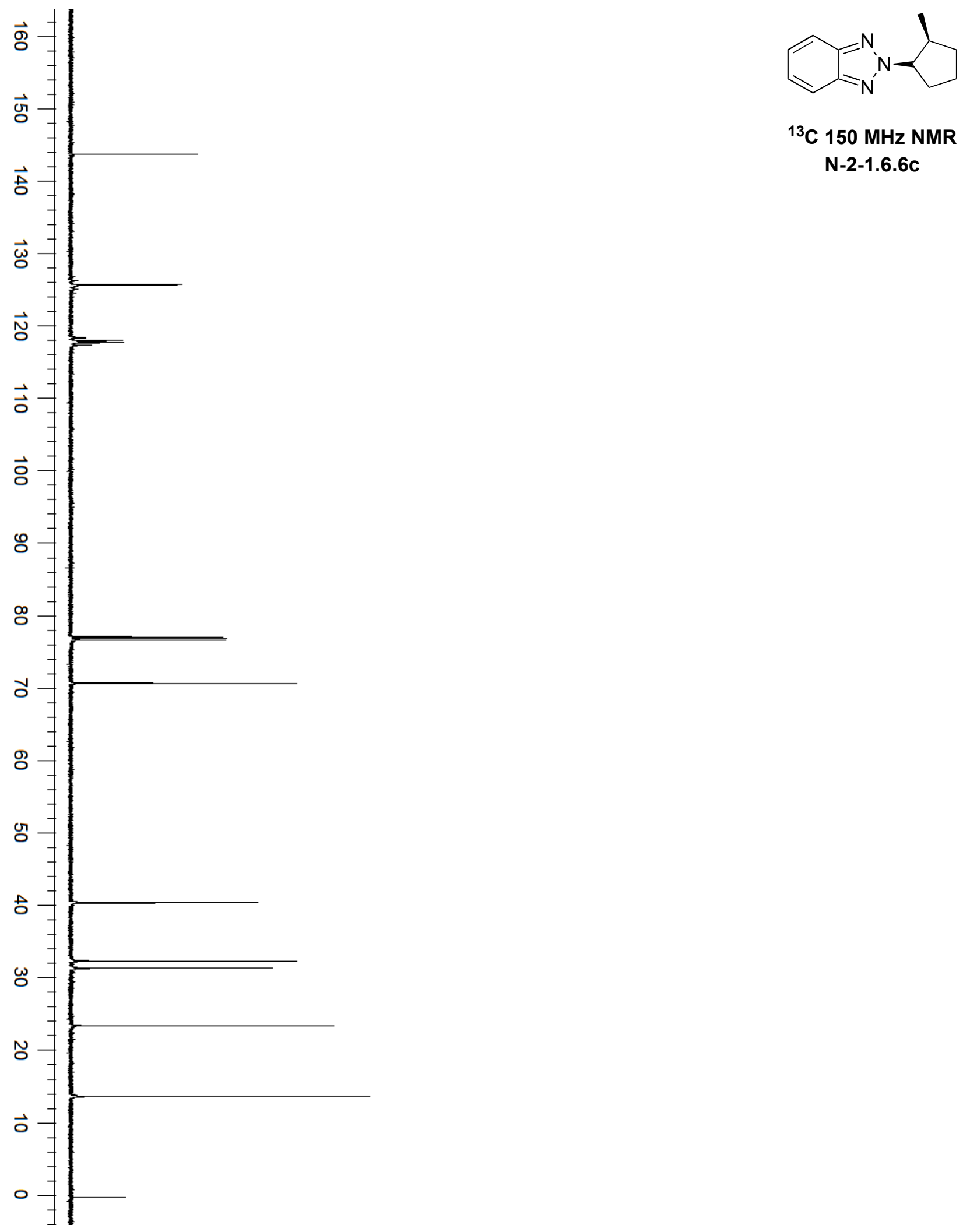

${ }^{13} \mathrm{C} 150 \mathrm{MHz}$ NMR $\mathrm{N}-2-1.6 .6 \mathrm{C}$ 

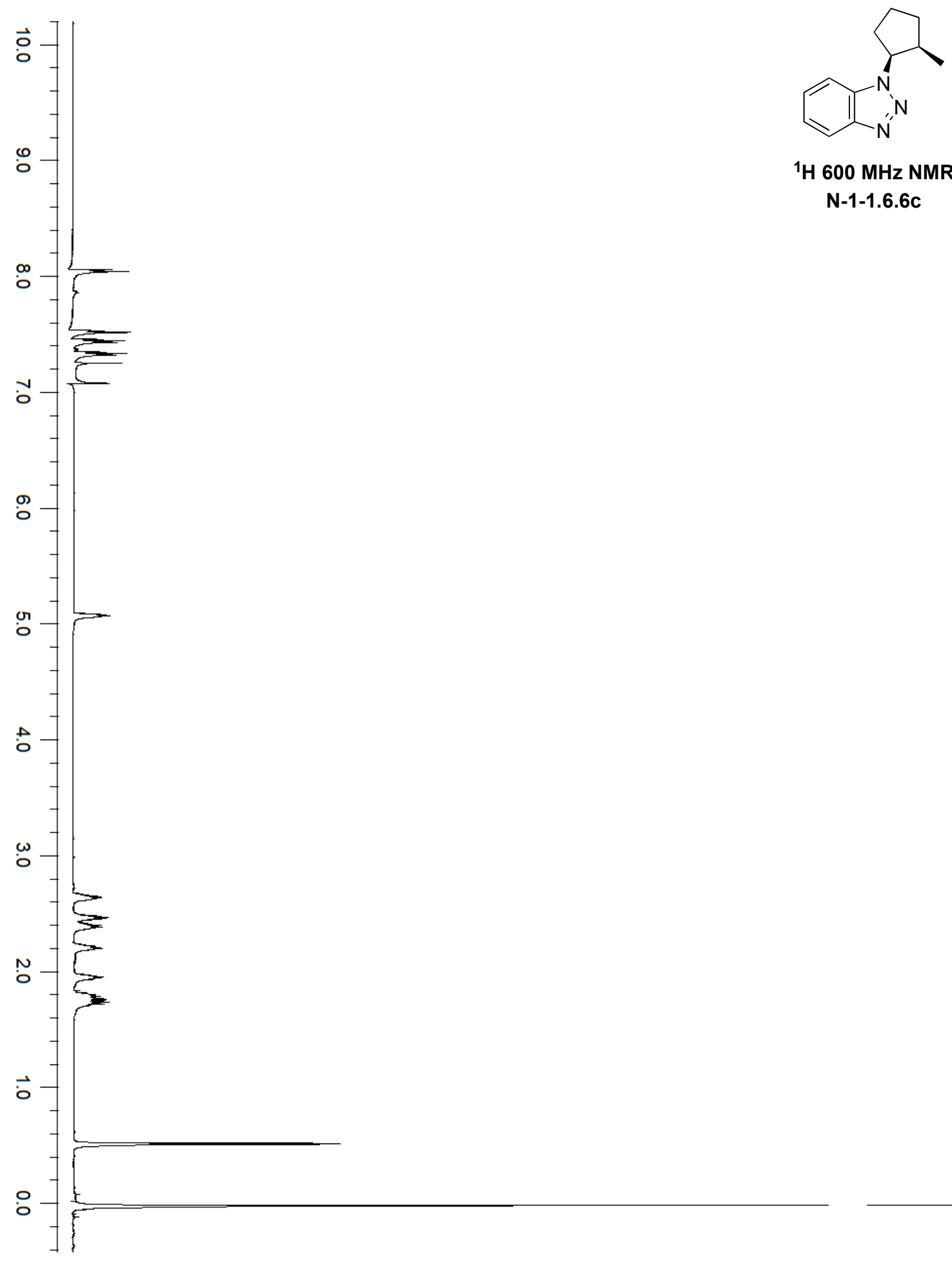

'H 600 MHz NMR

$\mathrm{N}-1-1.6 .6 \mathrm{c}$ 


$$
L^{2}
$$



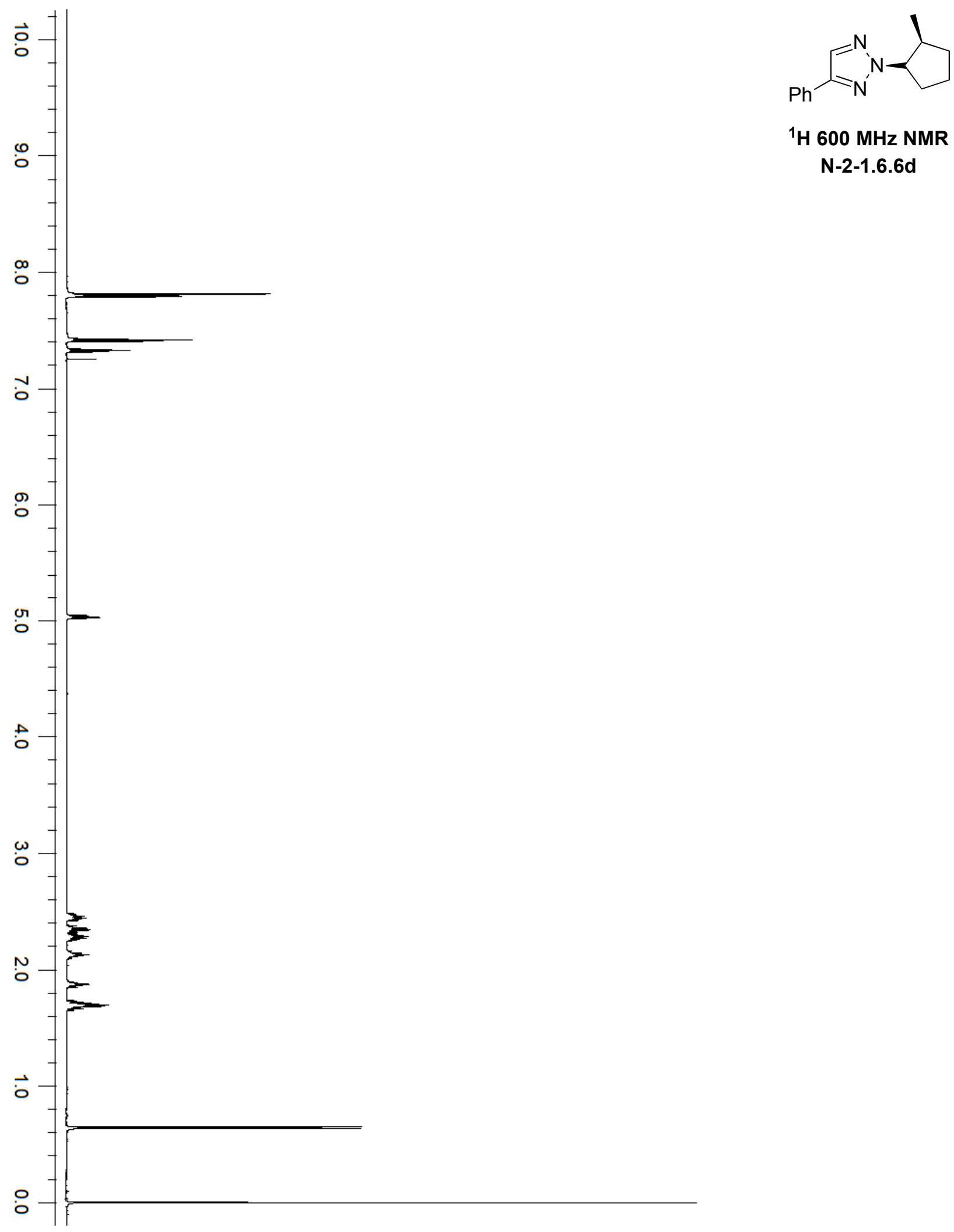

${ }^{1} \mathrm{H} 600 \mathrm{MHz}$ NMR

$\mathrm{N}-2-1.6 .6 \mathrm{~d}$ 


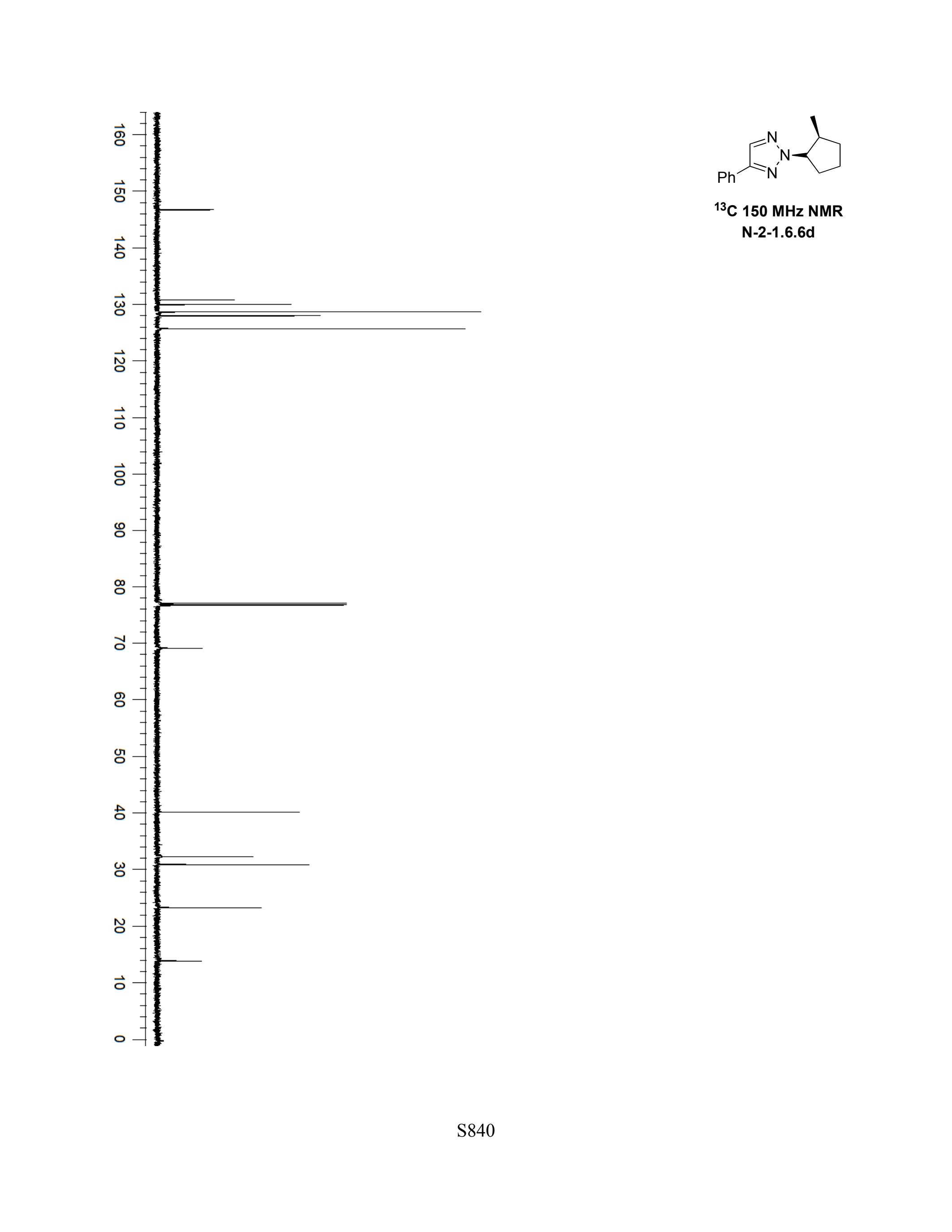




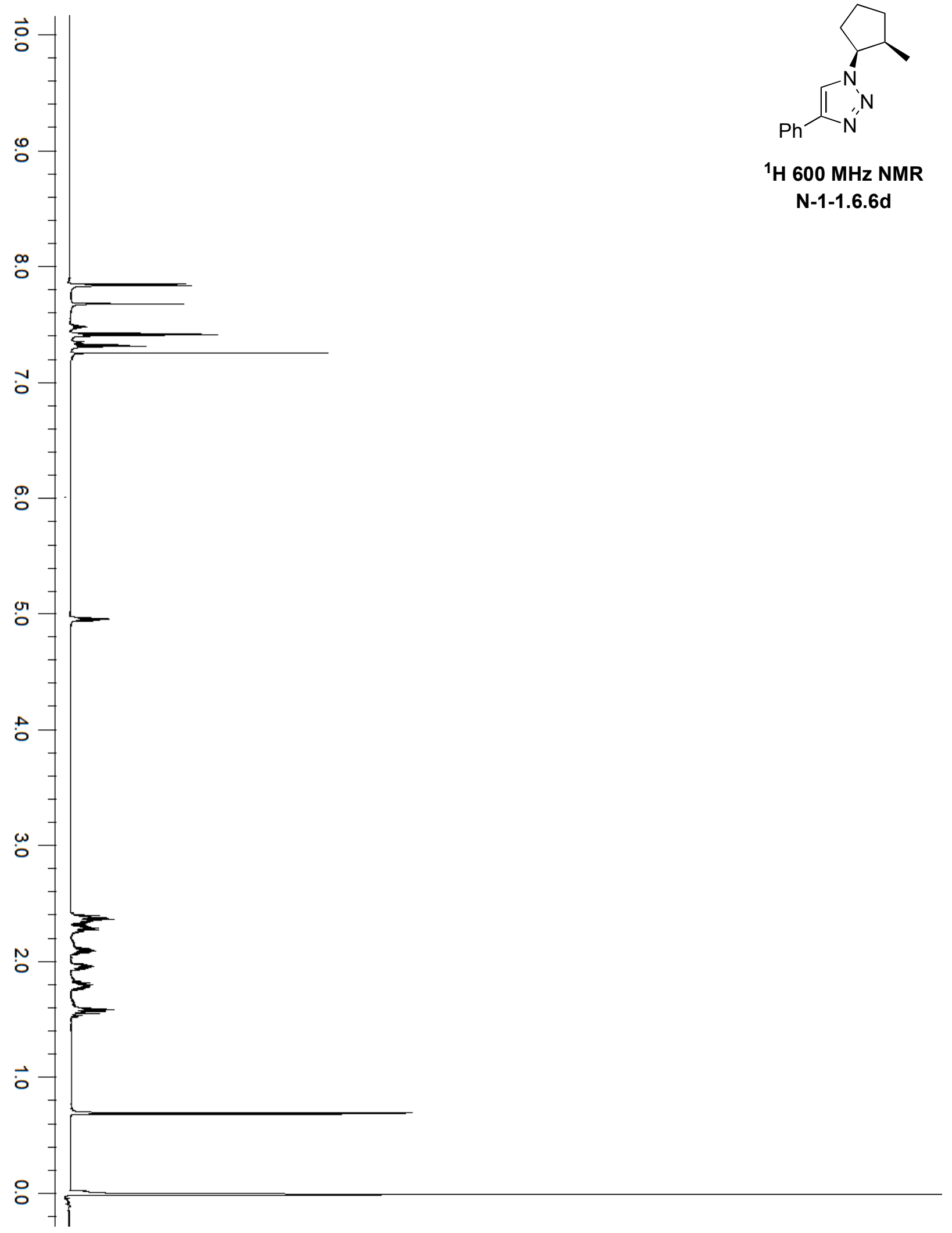




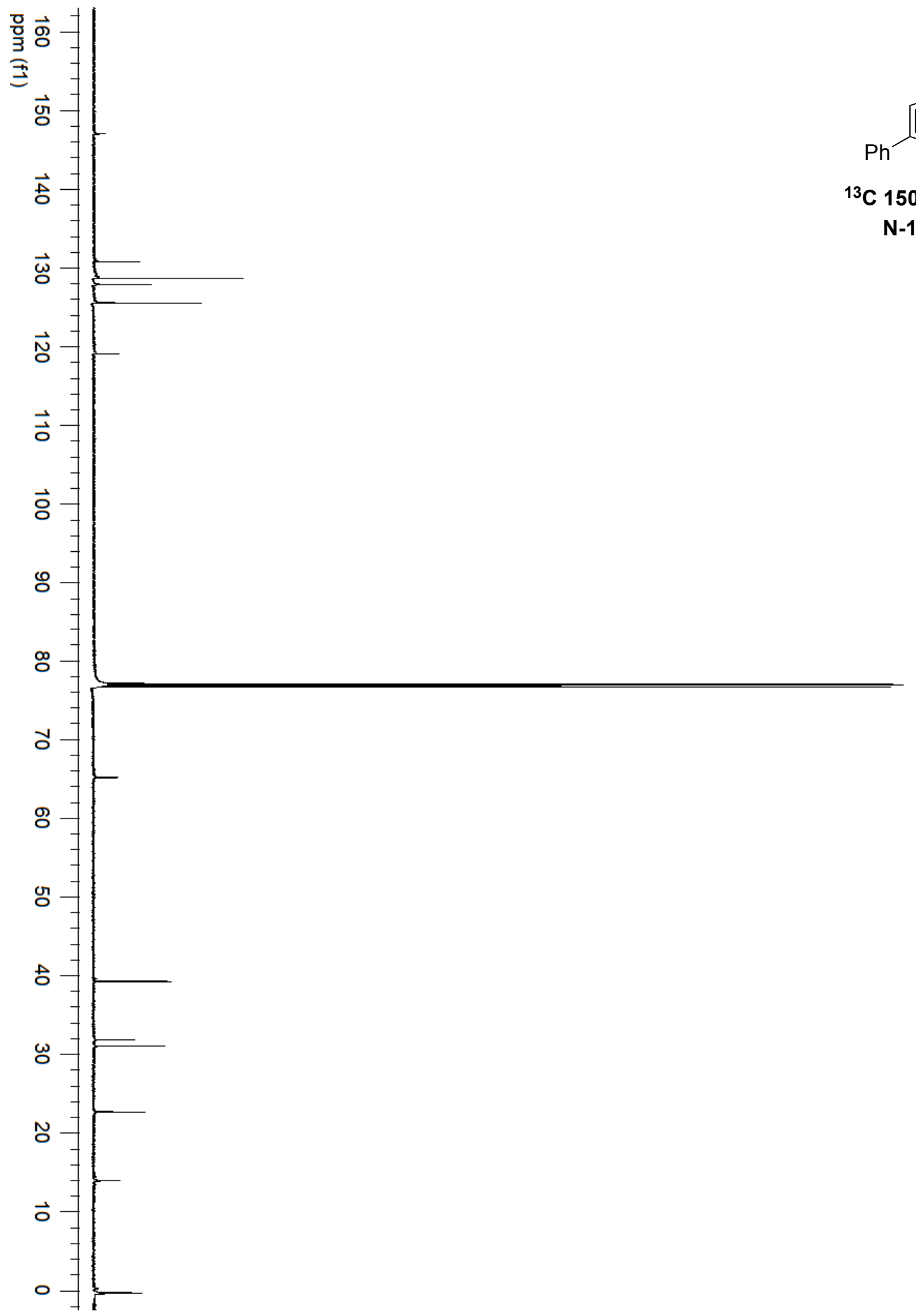




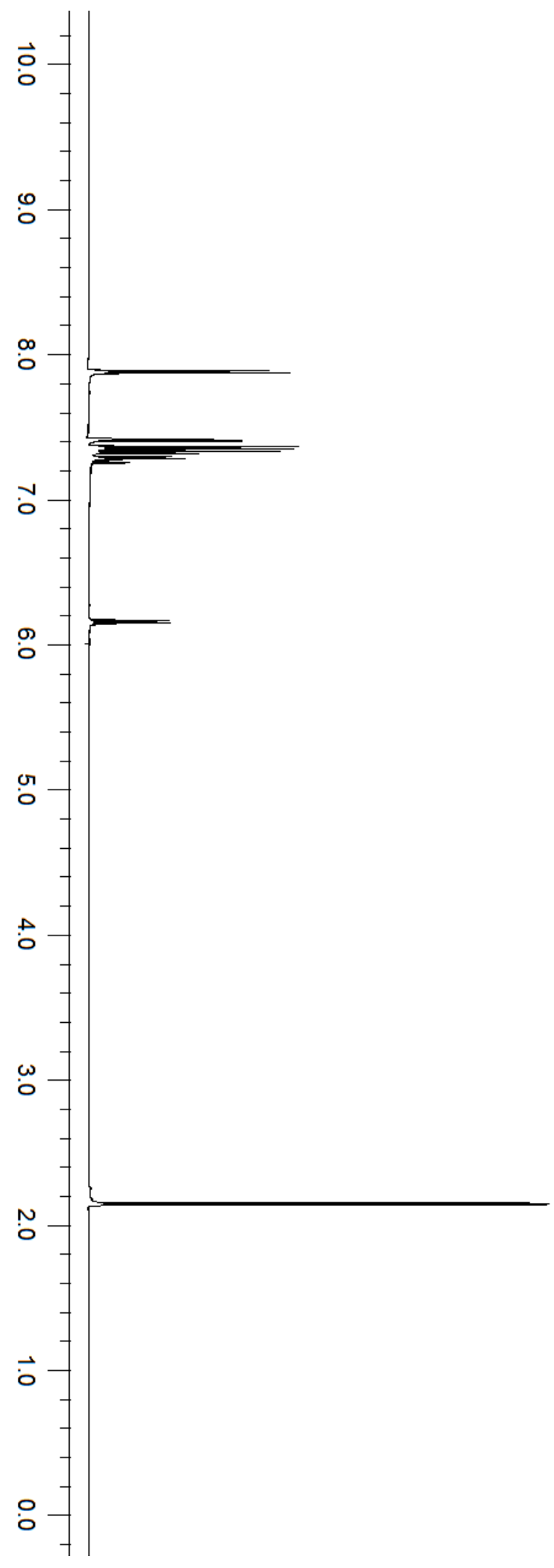

${ }^{1} \mathrm{H} 600 \mathrm{MHz}$ NMR

(R)-N-2-1.6.7a 

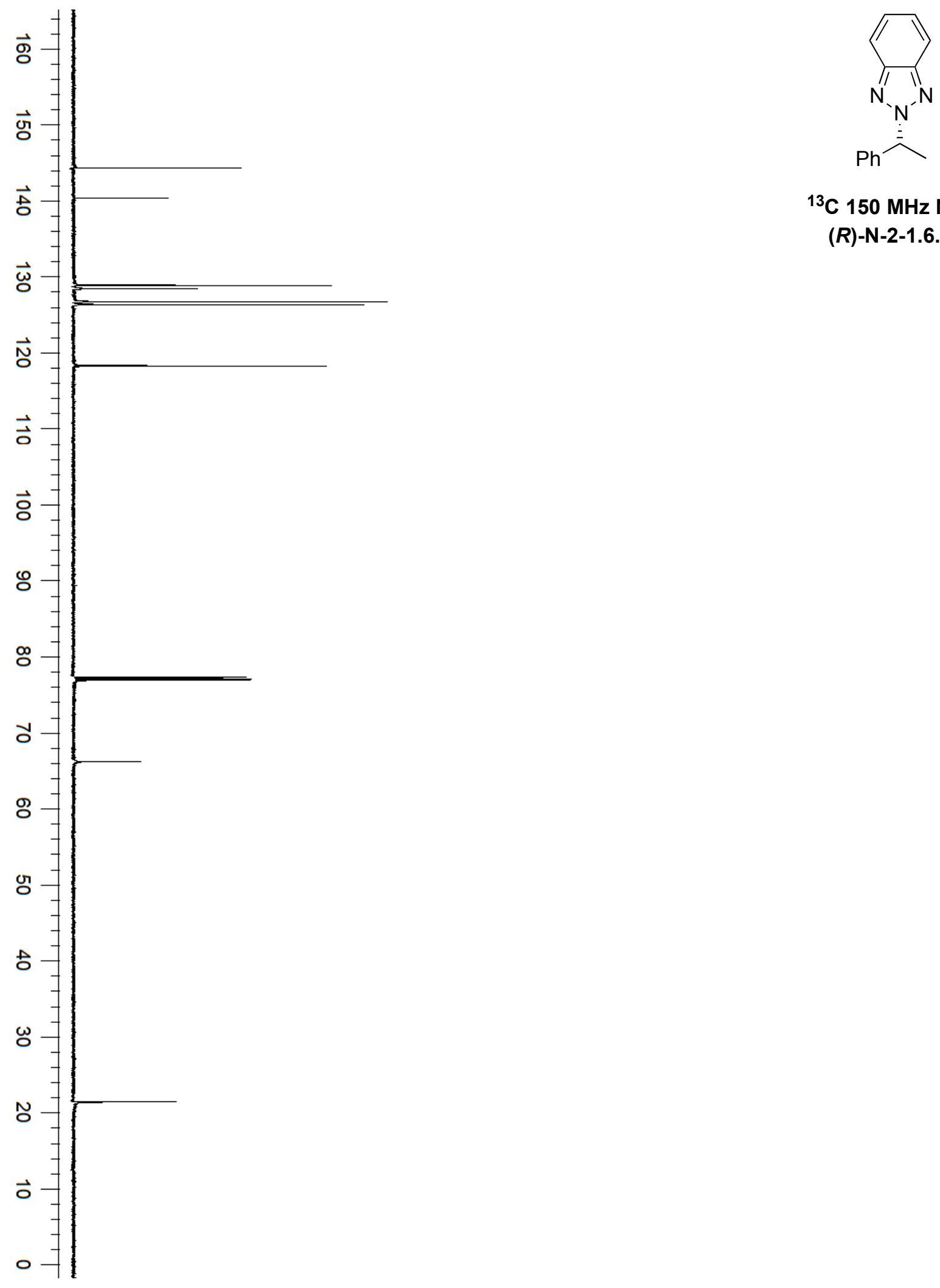

${ }^{13} \mathrm{C} 150 \mathrm{MHz}$ NMR (R)-N-2-1.6.7a 


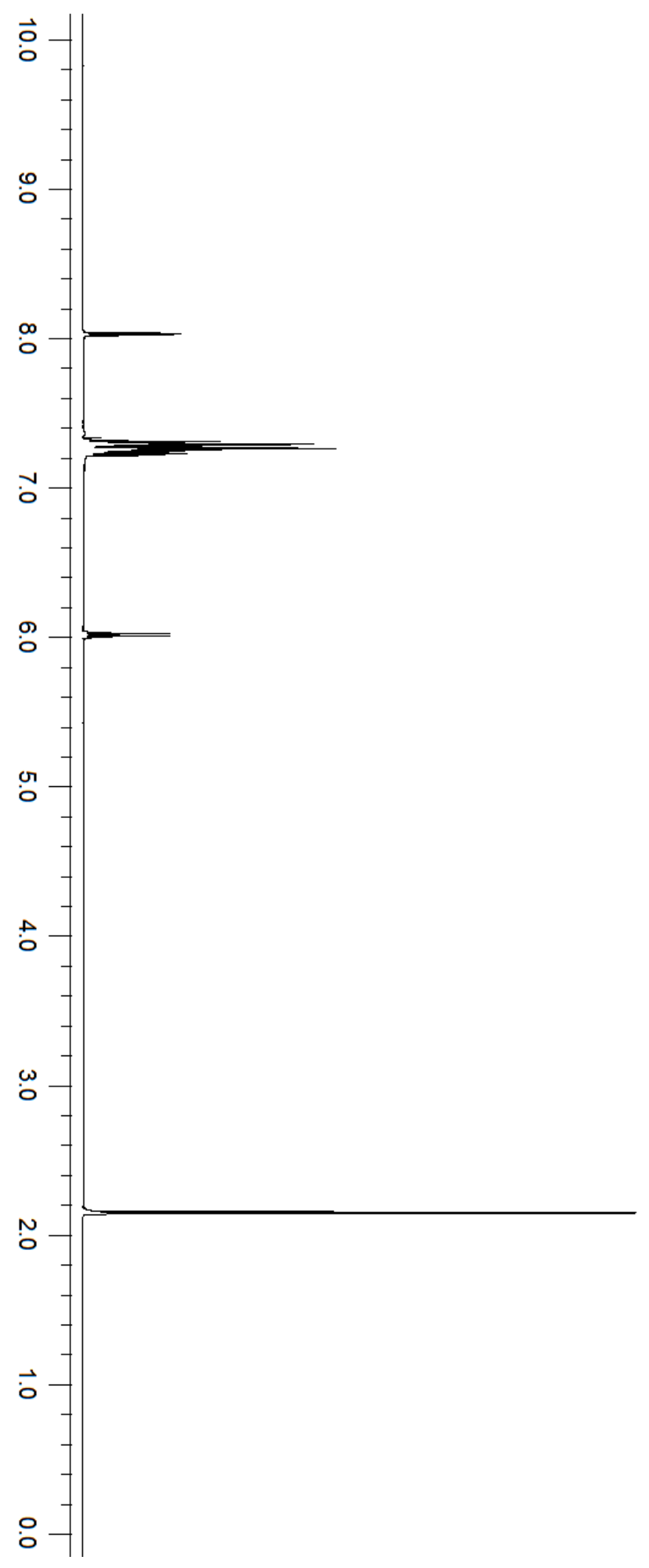

${ }^{1} \mathrm{H} 600 \mathrm{MHz}$ NMR

(R)-N-1-1.6.7a 


$$
E^{2}
$$




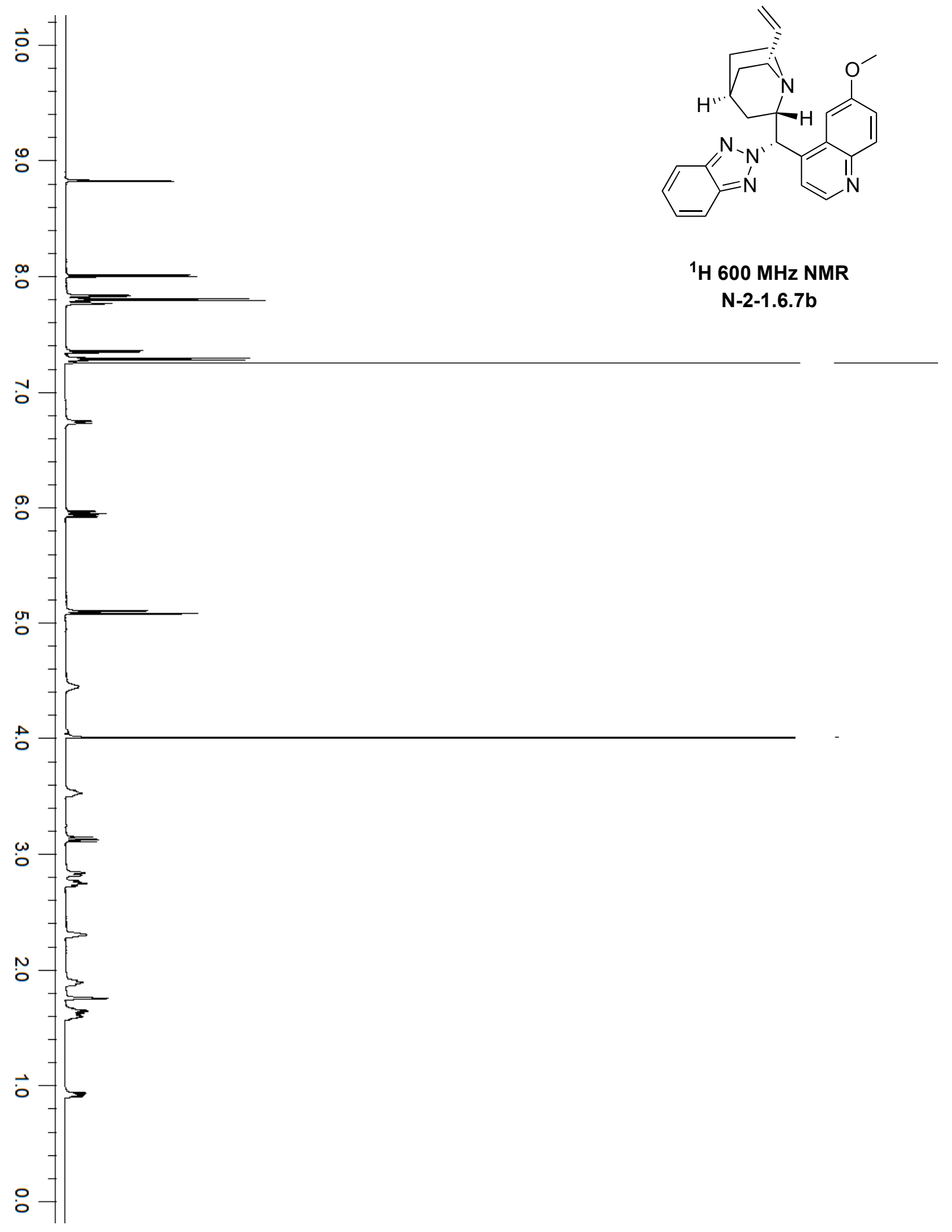




$$
F^{*}
$$




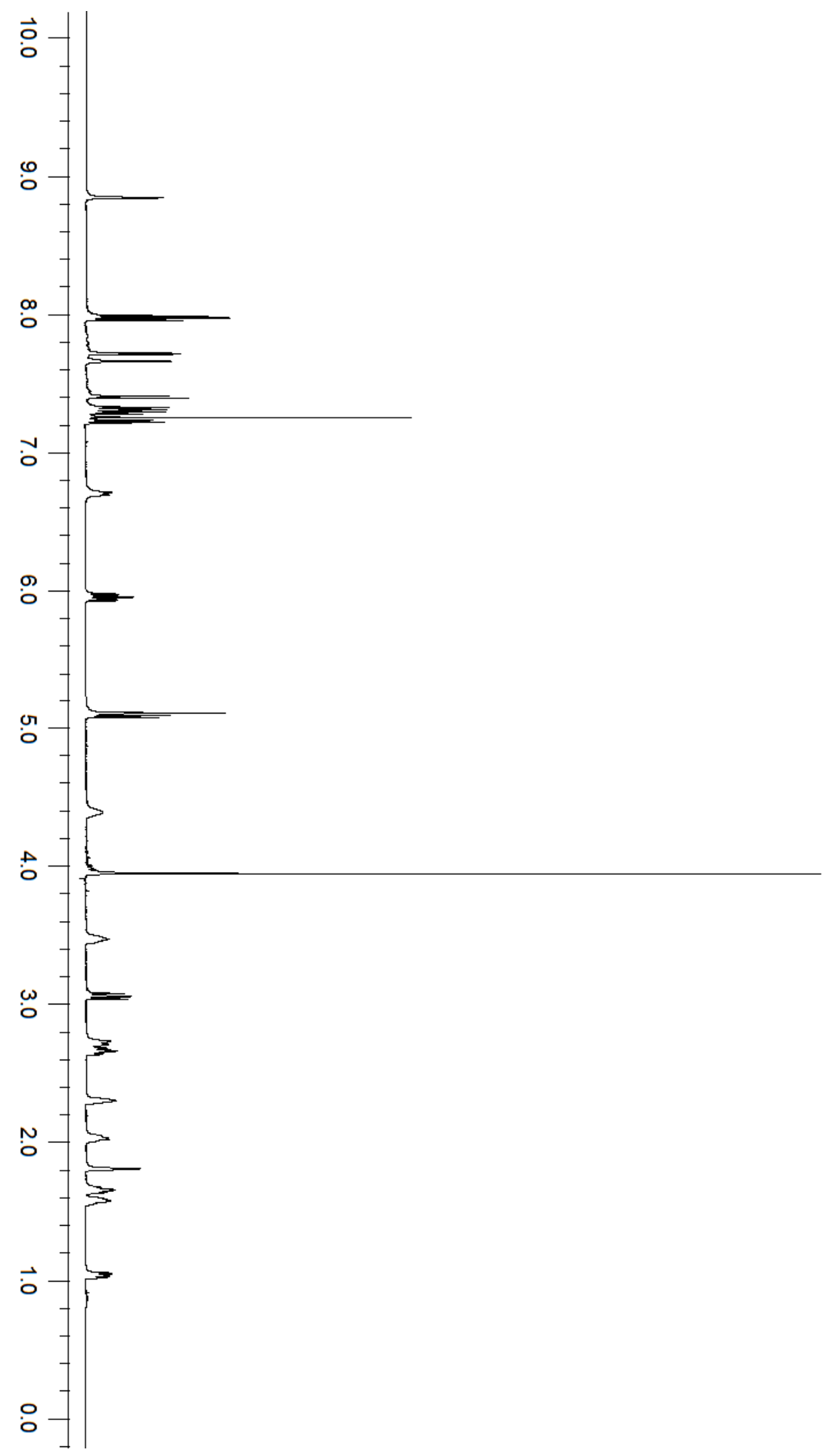

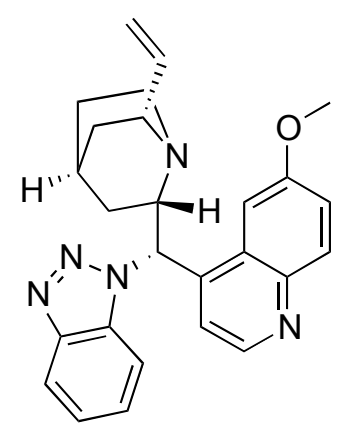

${ }^{1} \mathrm{H} 600 \mathrm{MHz}$ NMR $\mathrm{N}-1-1.6 .7 \mathrm{~b}$ 

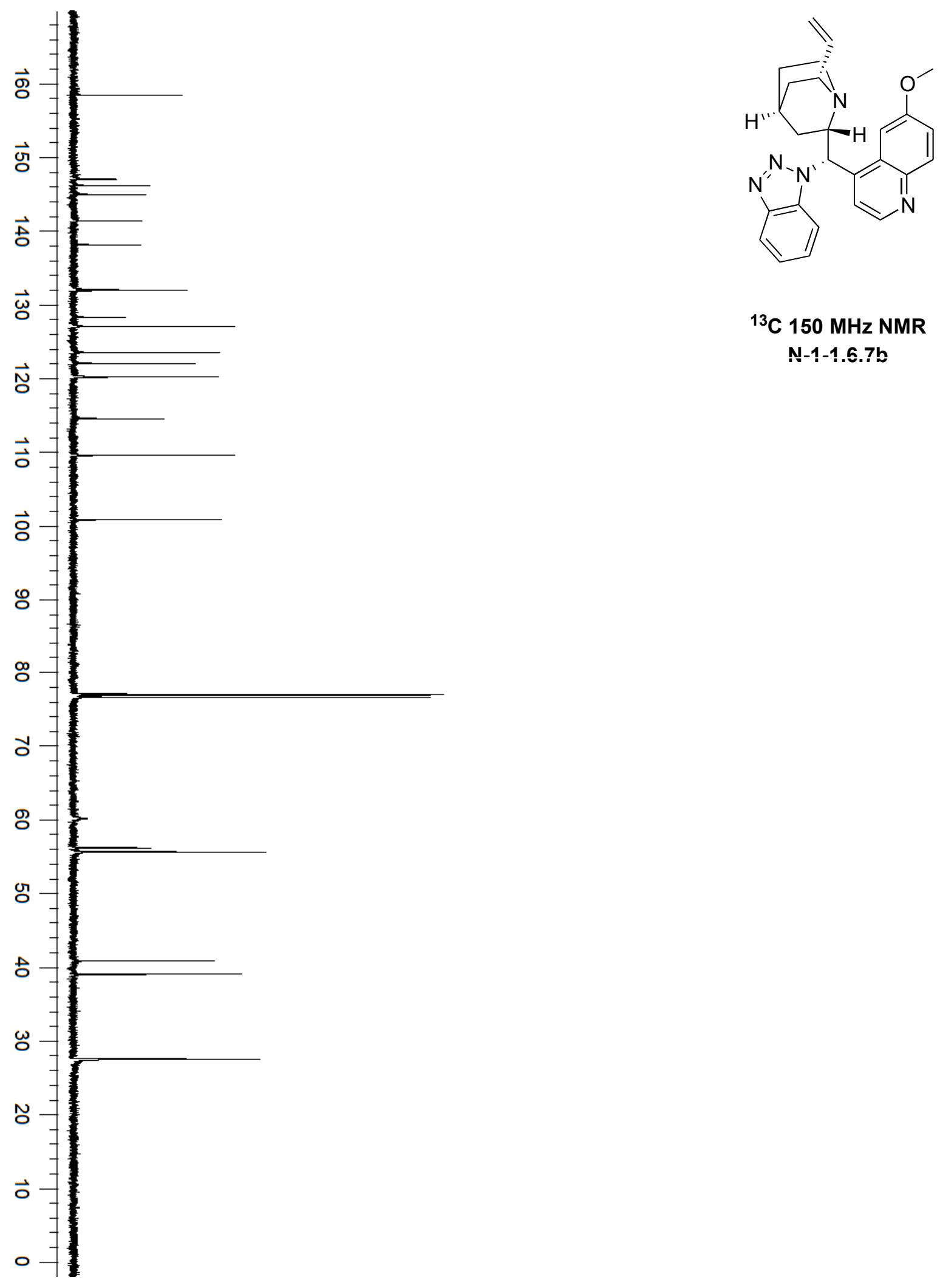

${ }^{13} \mathrm{C} 150 \mathrm{MHz}$ NMR

NN-1-1.6.7b 


\subsection{NMR Spectra Data}

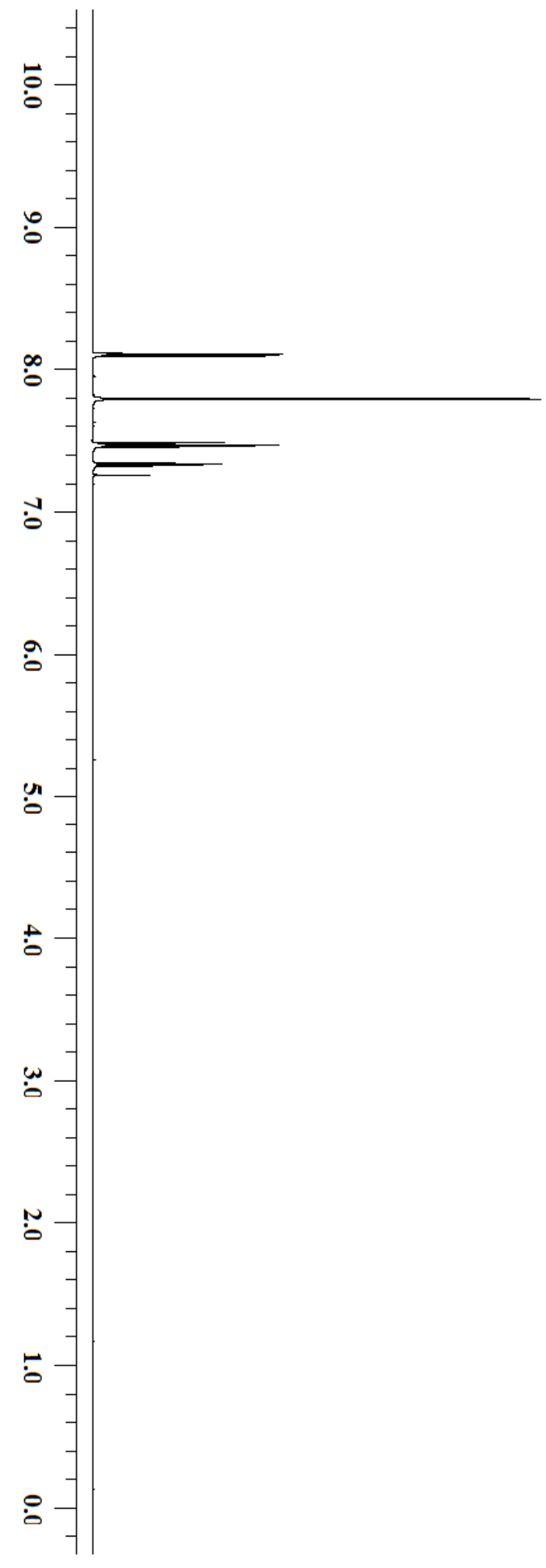

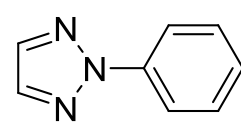

${ }^{1} \mathrm{H} 600 \mathrm{MHz}$ NMR

2.2.1 

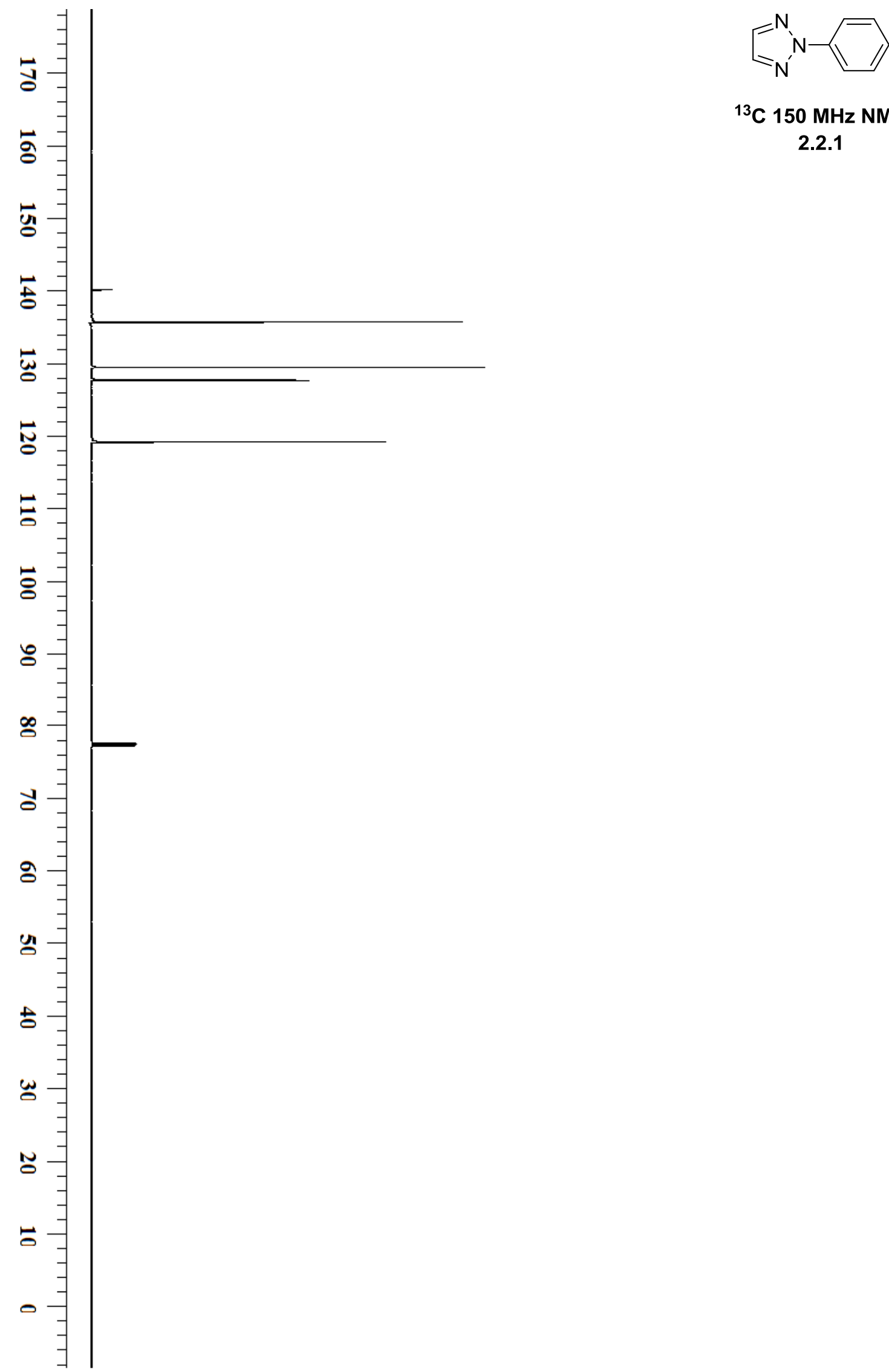

${ }^{13} \mathrm{C} 150 \mathrm{MHz}$ NMR

2.2.1 


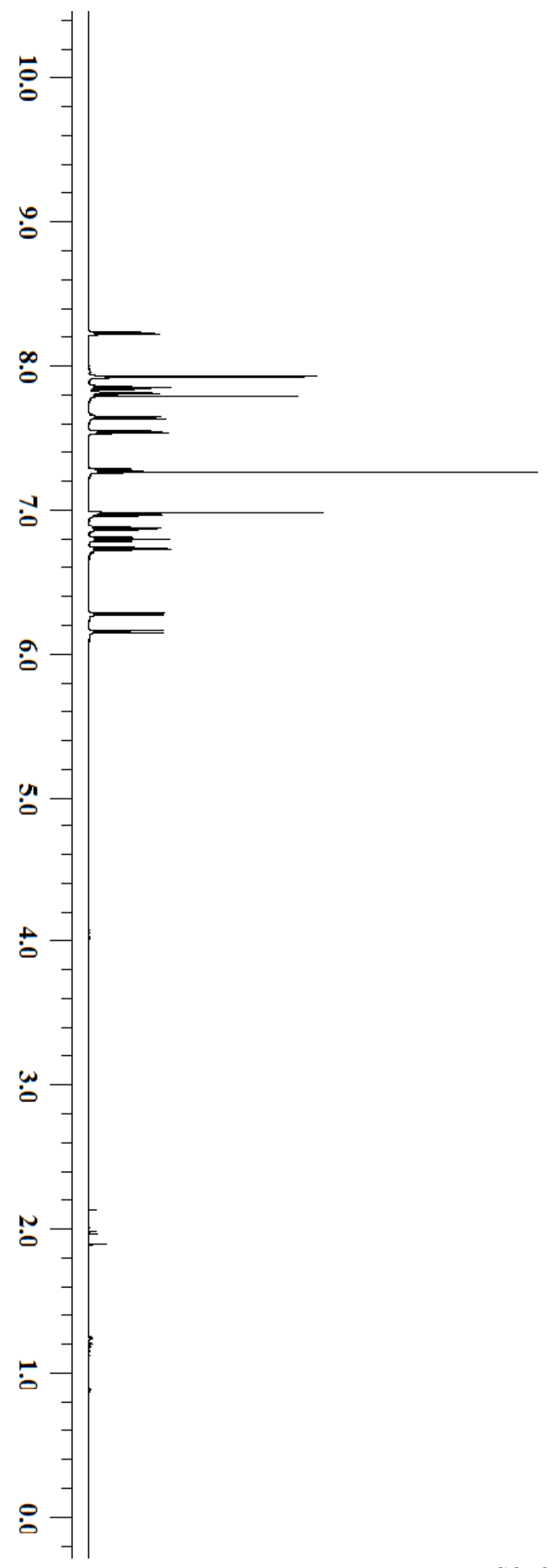

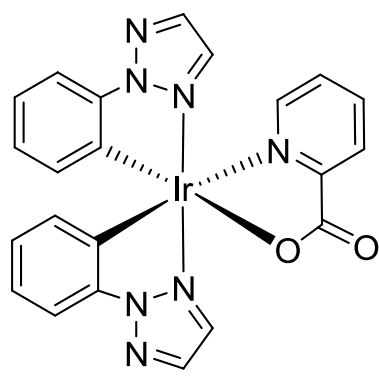

${ }^{1} \mathrm{H} 600 \mathrm{MHz}$ NMR

2.2.3e 

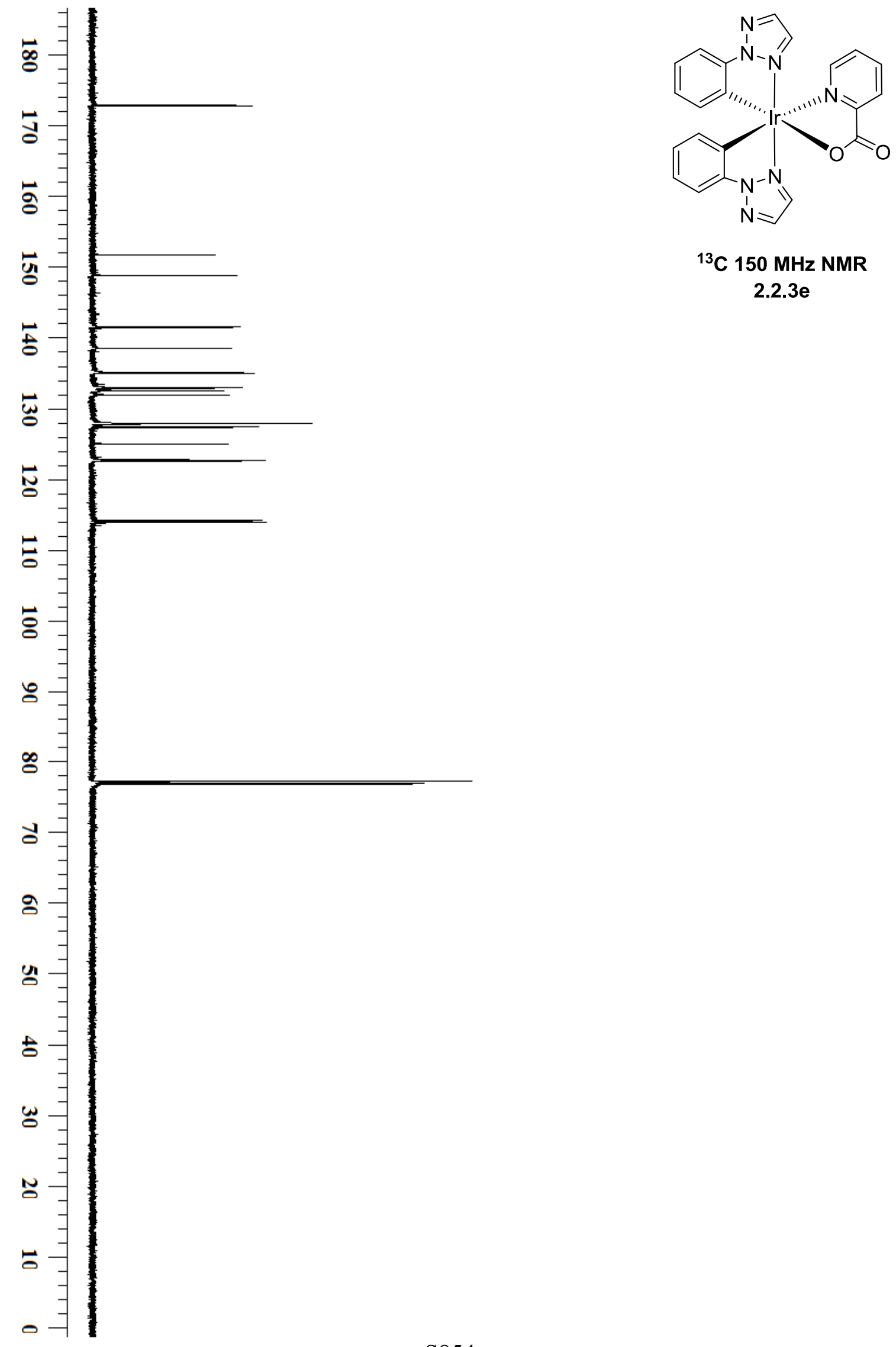

${ }^{13} \mathrm{C} 150 \mathrm{MHz}$ NMR 2.2.3e 


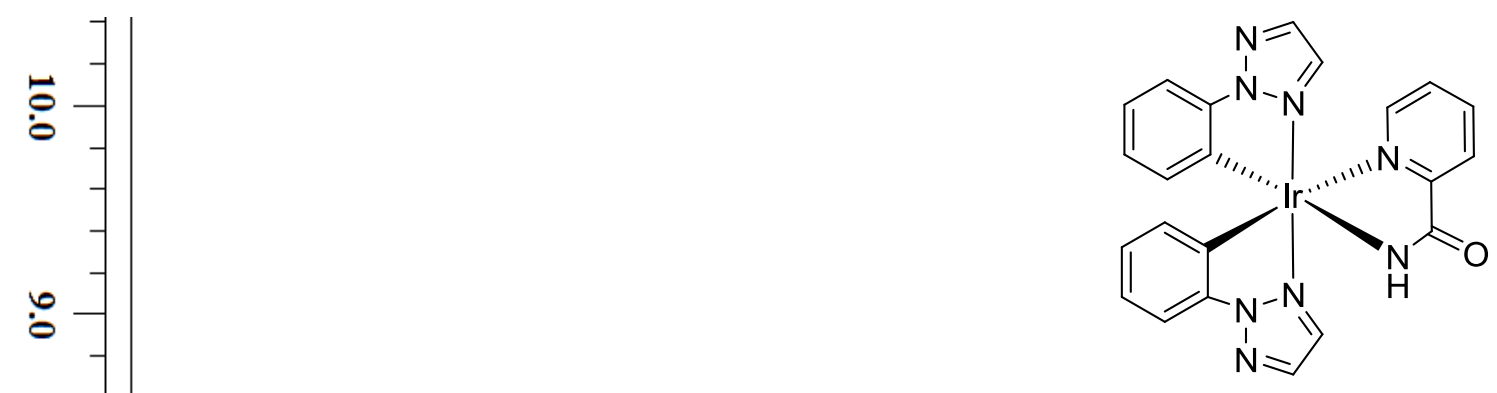

${ }^{1} \mathrm{H} 600 \mathrm{MHz}$ NMR 2.2.3f

!n

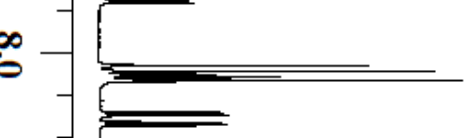



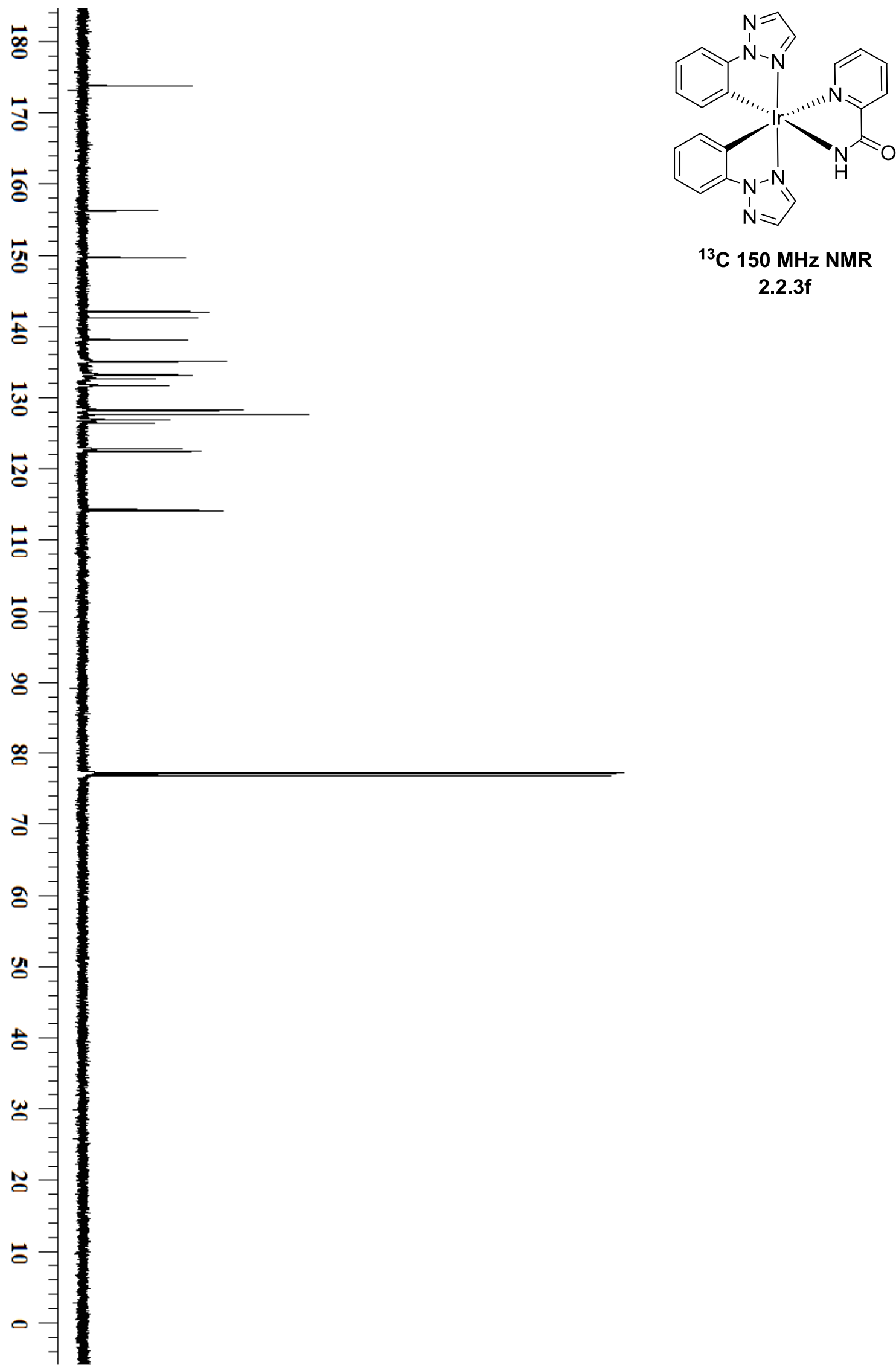

${ }^{13} \mathrm{C} 150 \mathrm{MHz}$ NMR 2.2.3f 


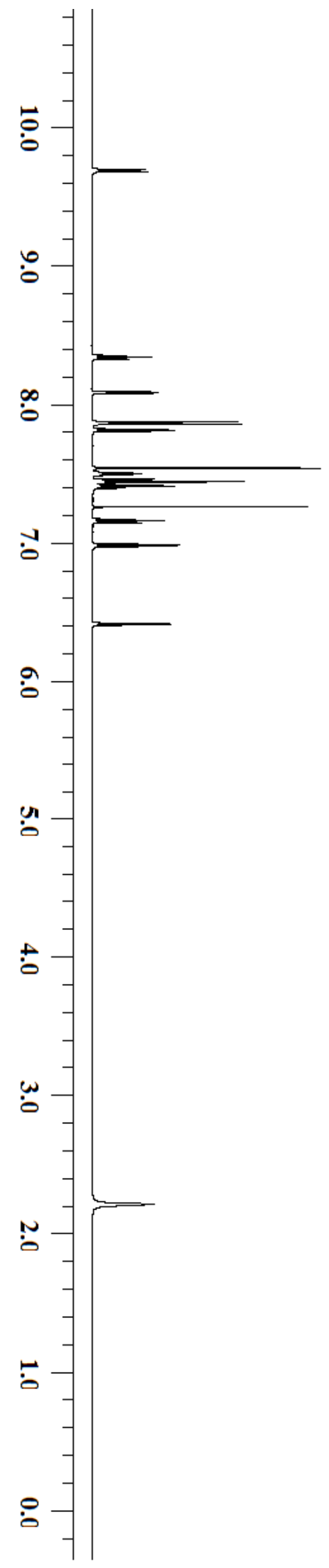

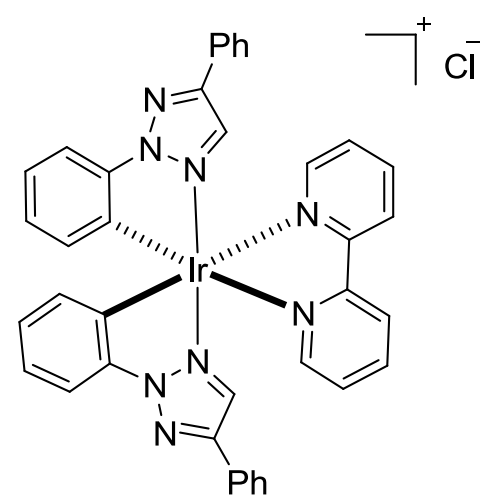

${ }^{1} \mathrm{H} 600 \mathrm{MHz}$ NMR

[Ir(bbpy)(ArTriaz) $\left.{ }_{2}\right]$ Cl 2.2.4 

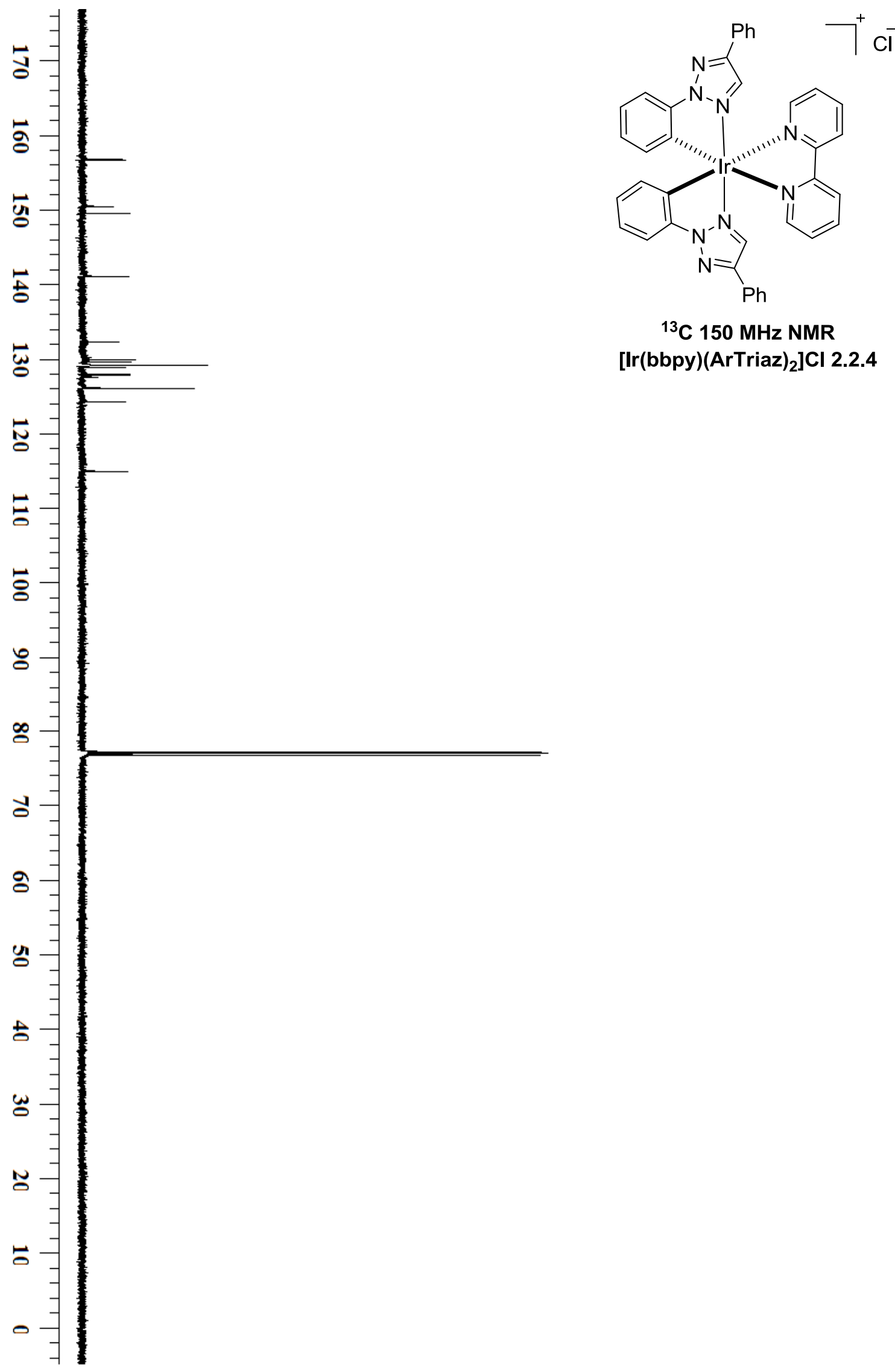

${ }^{13} \mathrm{C} 150 \mathrm{MHz}$ NMR

[Ir(bbpy)(ArTriaz) 2 ]Cl 2.2.4 


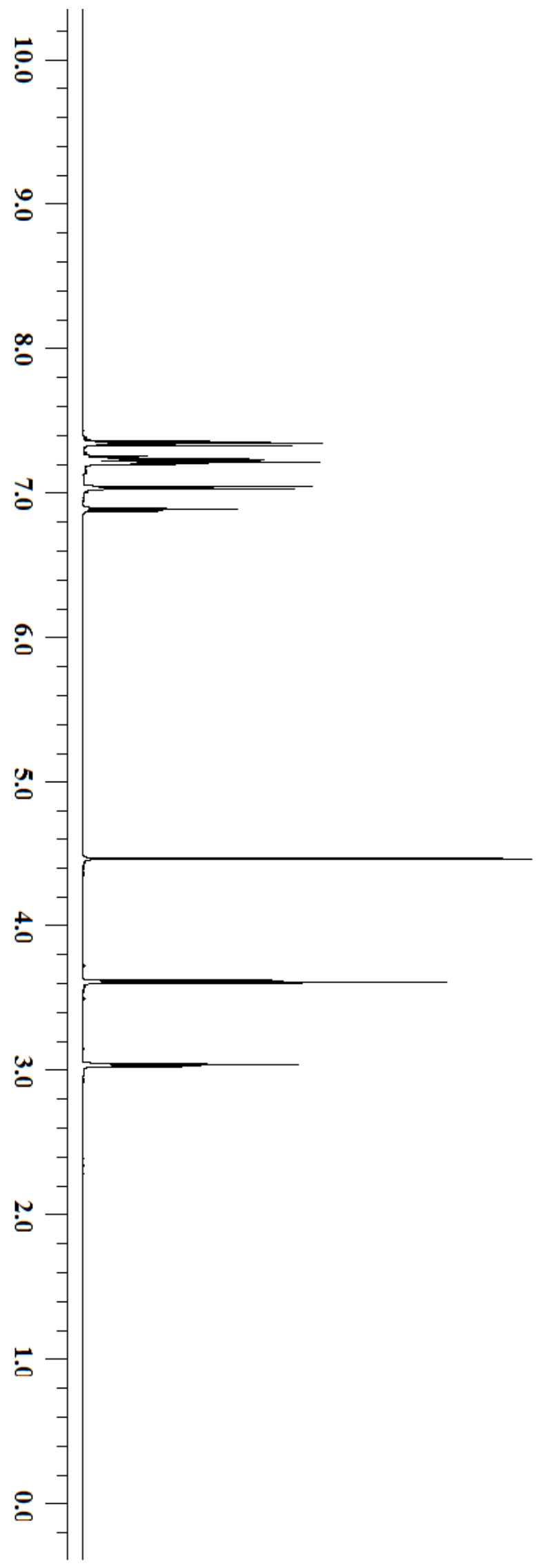

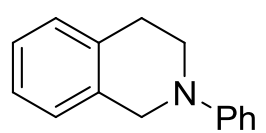

${ }^{1} \mathrm{H} 600 \mathrm{MHz}$ NMR

2.2.5a 

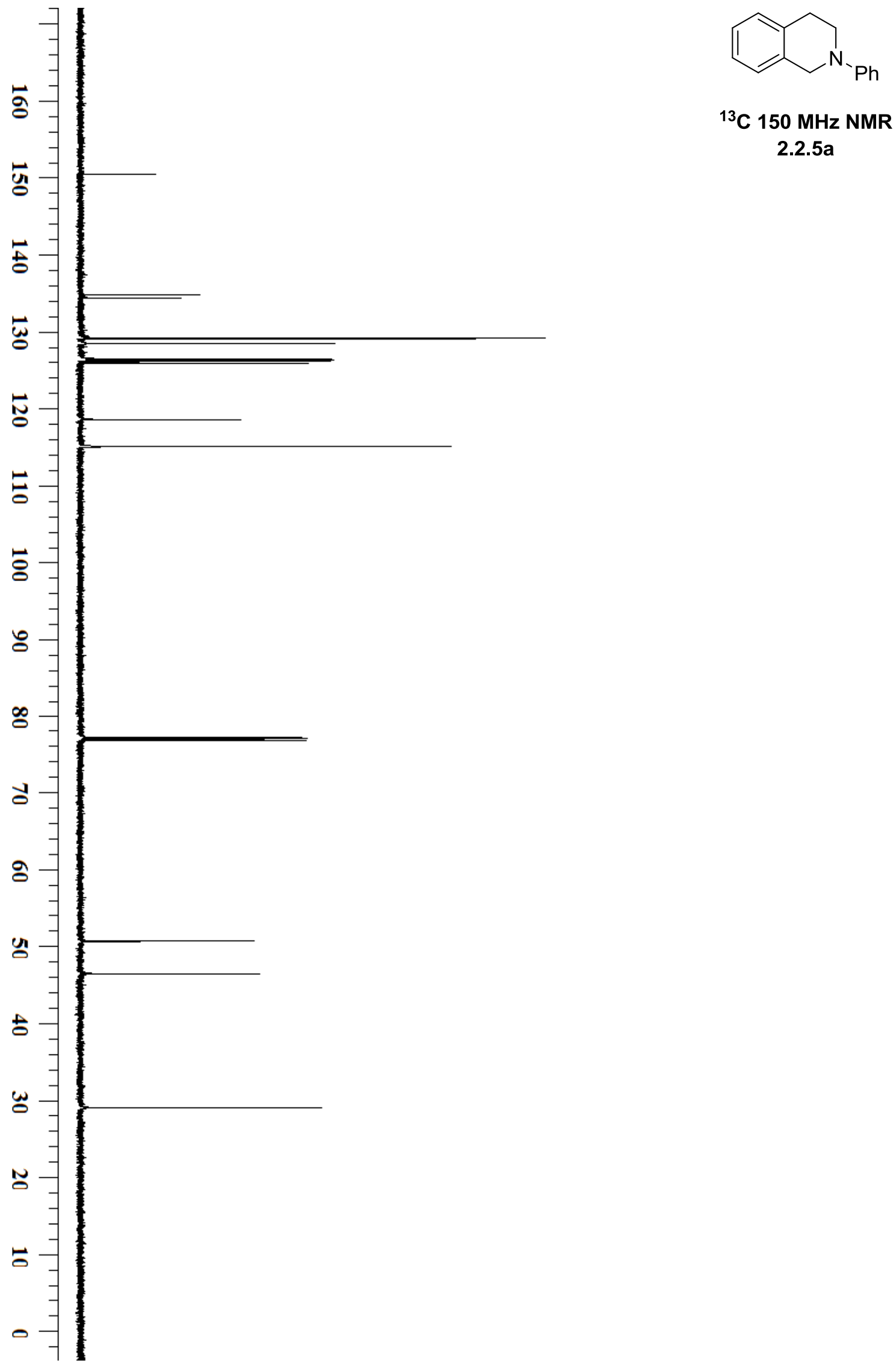

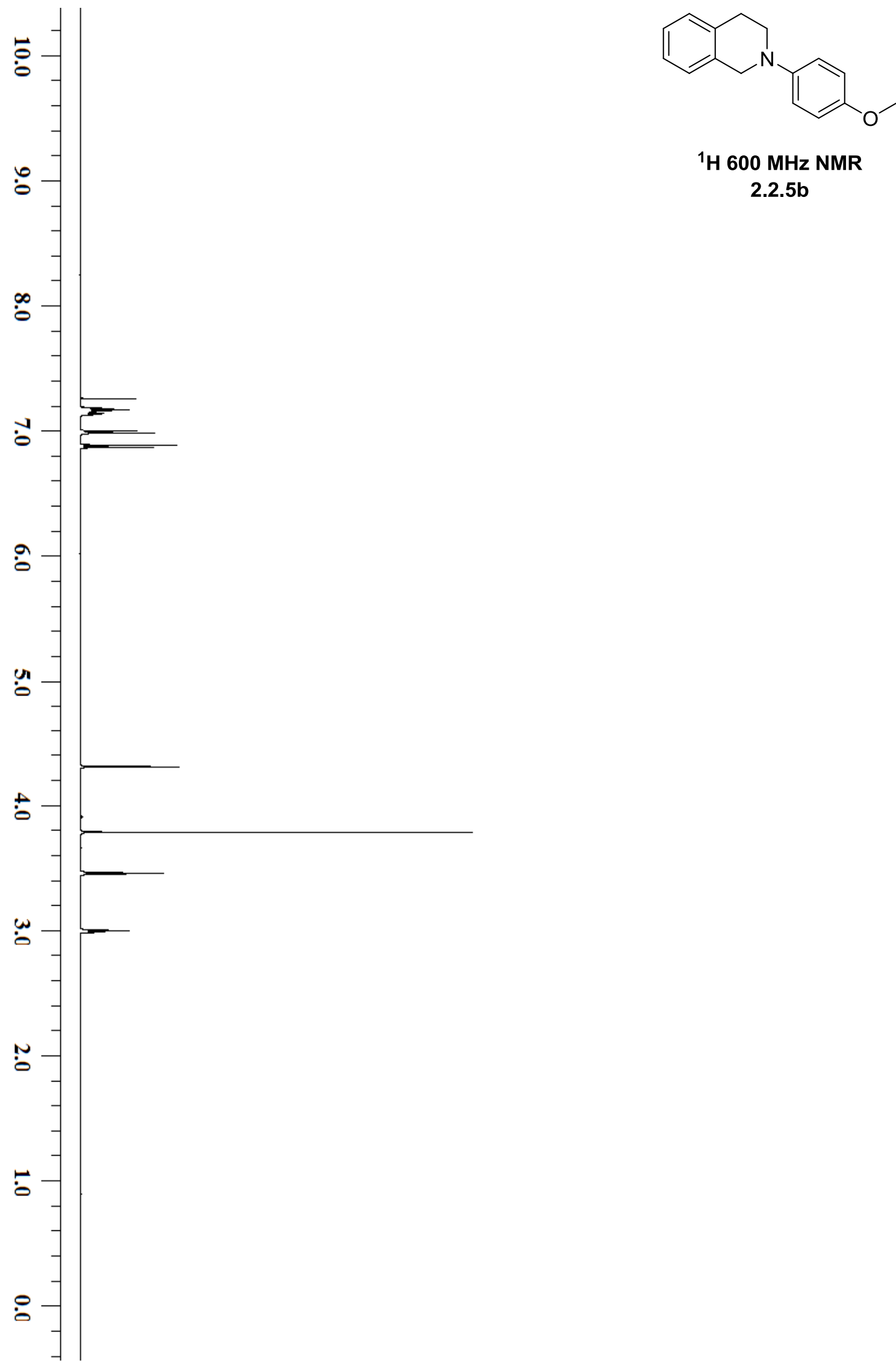

${ }^{1} \mathrm{H} 600 \mathrm{MHz}$ NMR

2.2.5b 

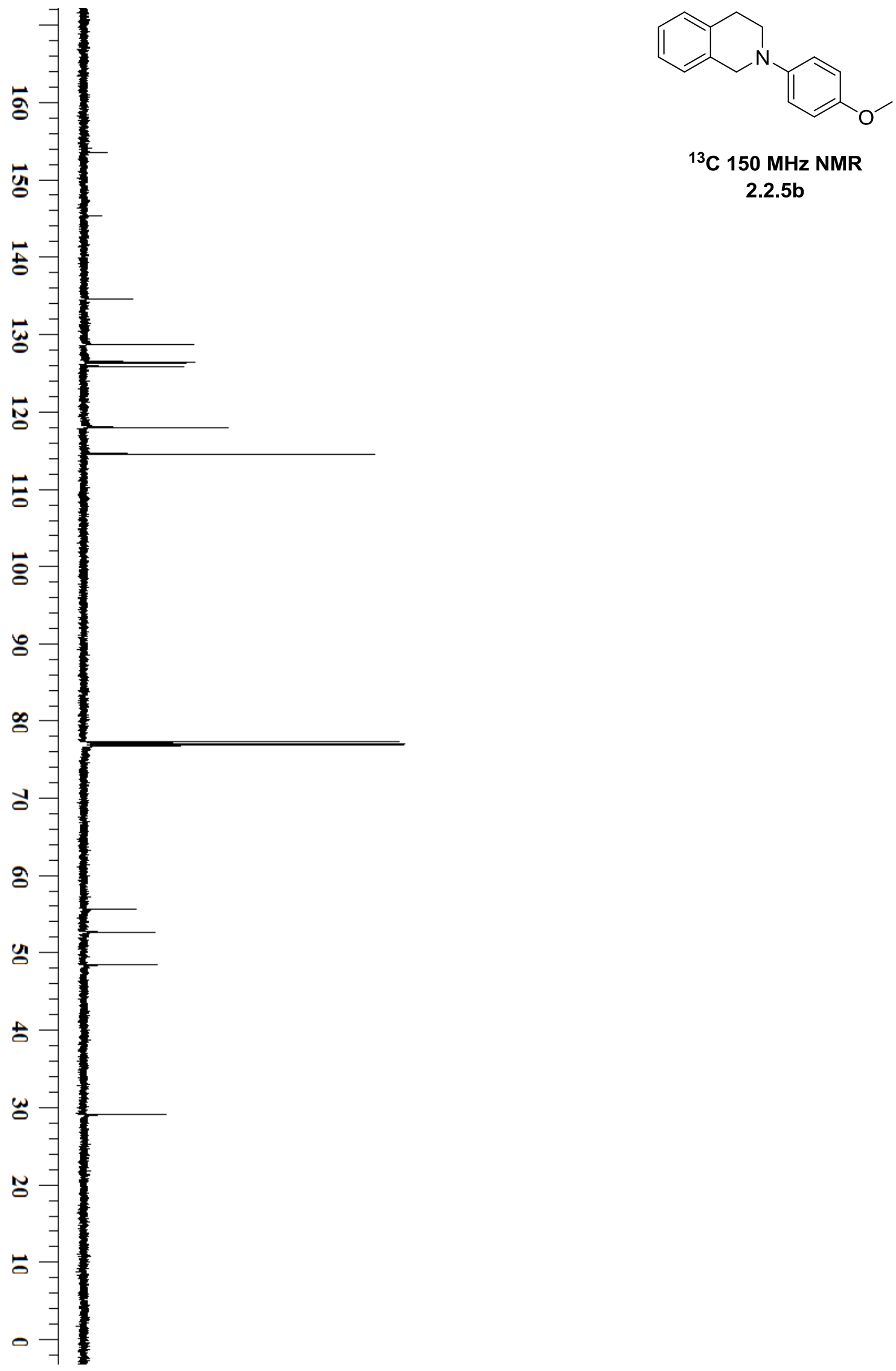

${ }^{13} \mathrm{C} 150 \mathrm{MHz}$ NMR

2.2.5b 


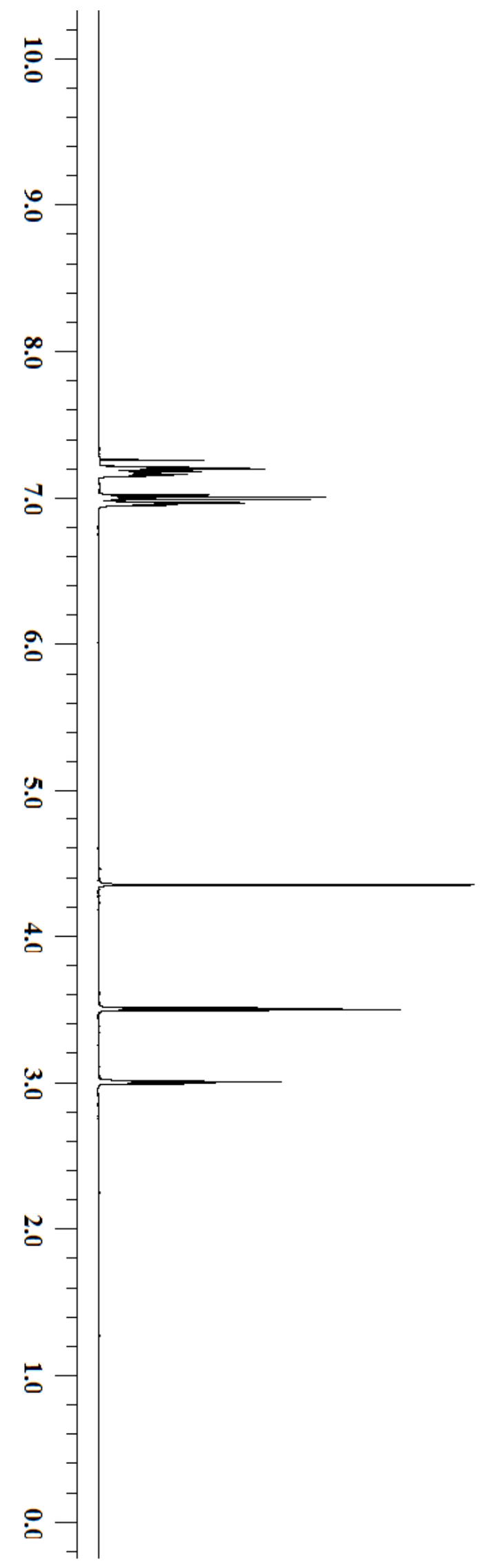

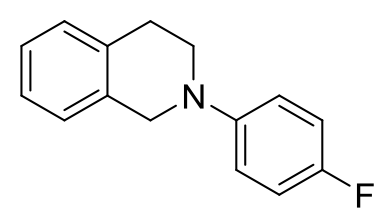

${ }^{1} \mathrm{H} 600 \mathrm{MHz}$ NMR

2.2.5c 


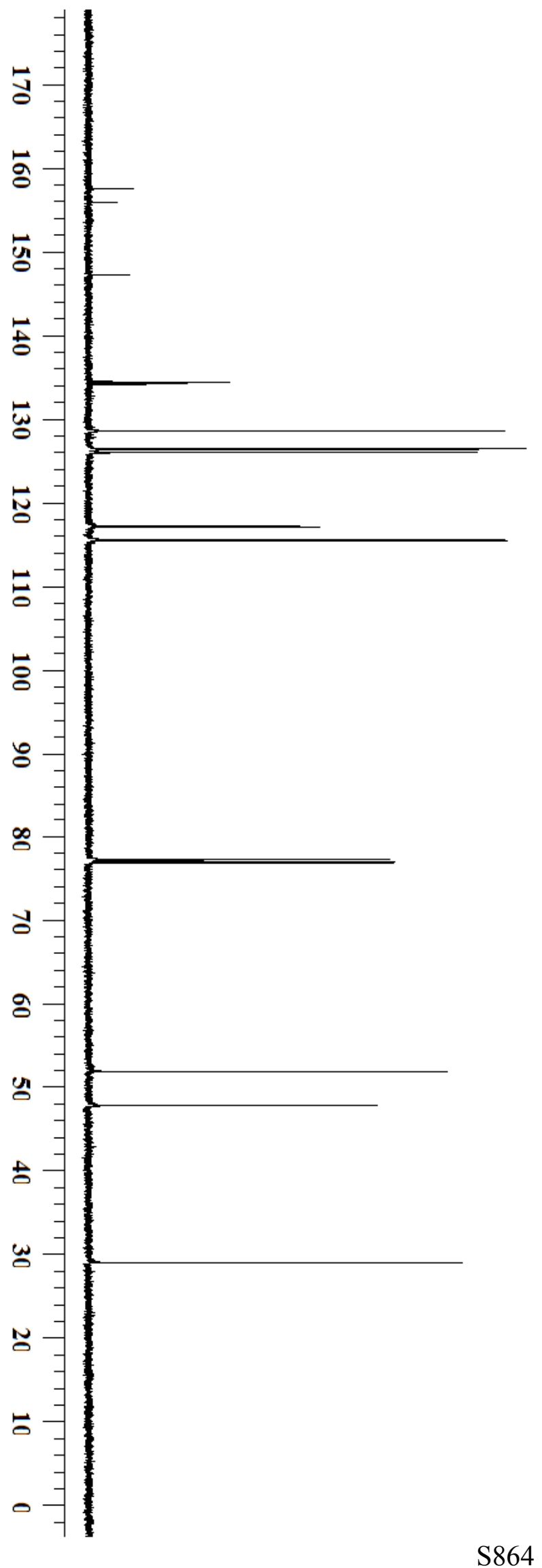

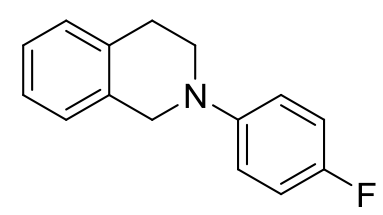

${ }^{13} \mathrm{C} 150 \mathrm{MHz}$ NMR

2.2.5c 

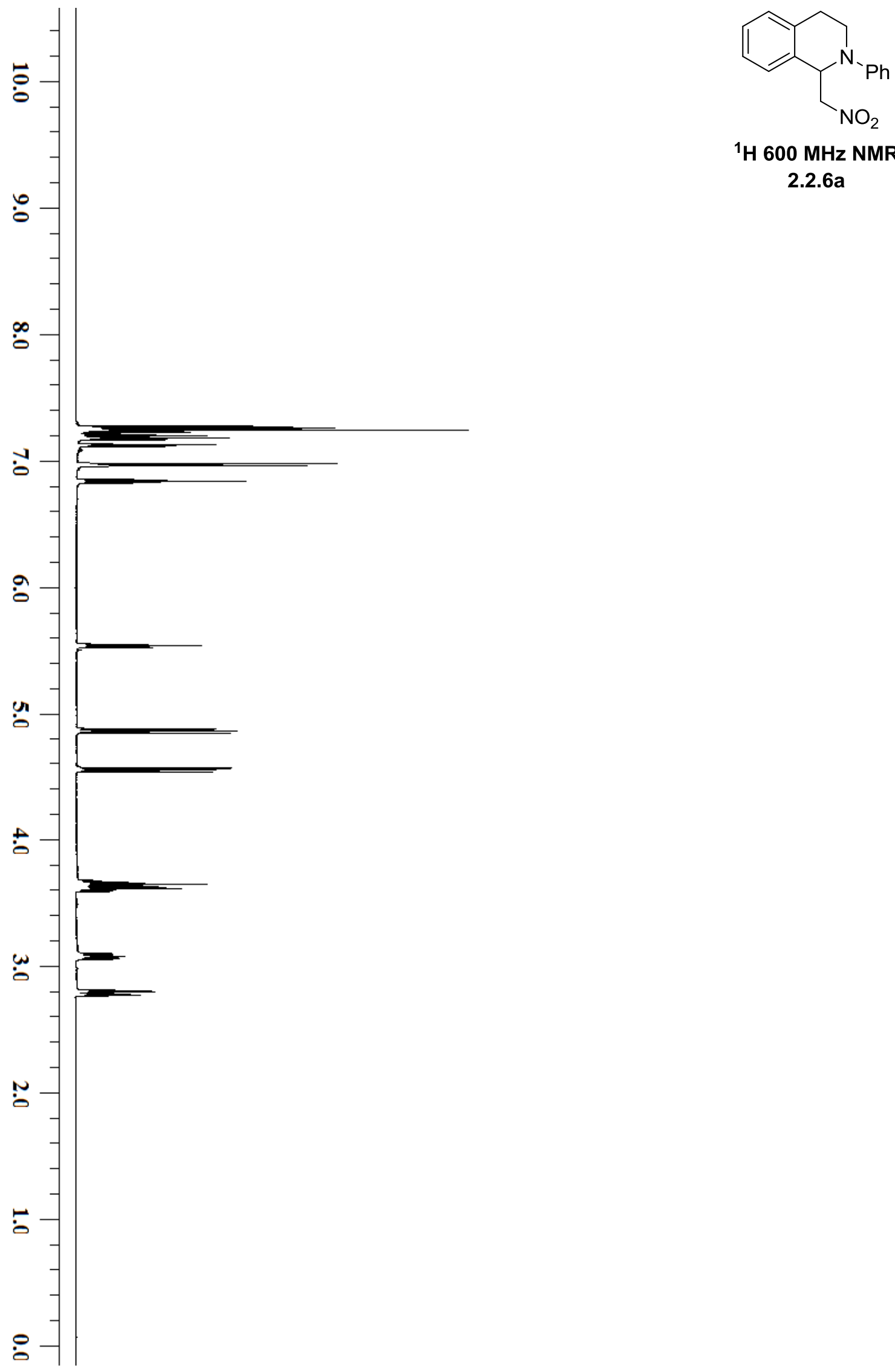

${ }^{1} \mathrm{H} 600 \mathrm{MHz}$ NMR

2.2.6a 


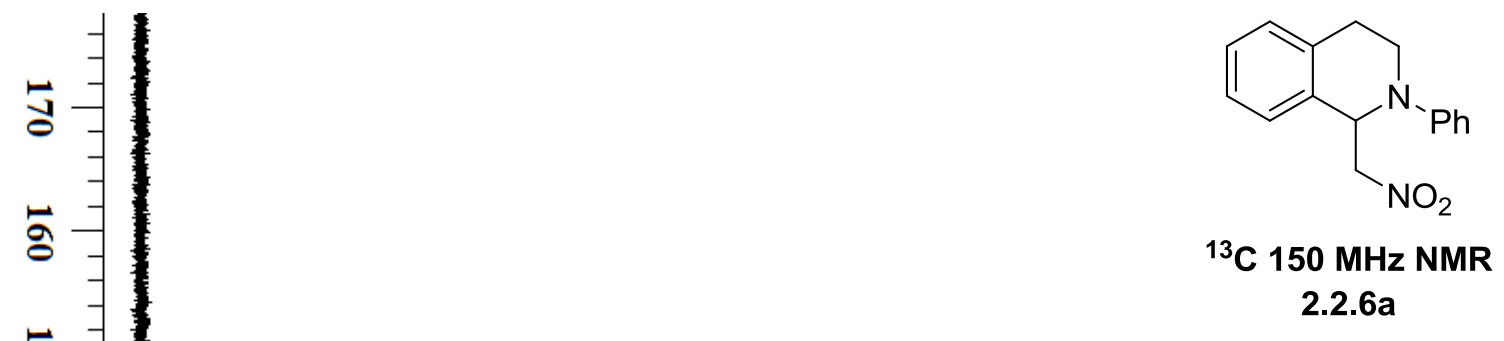

E
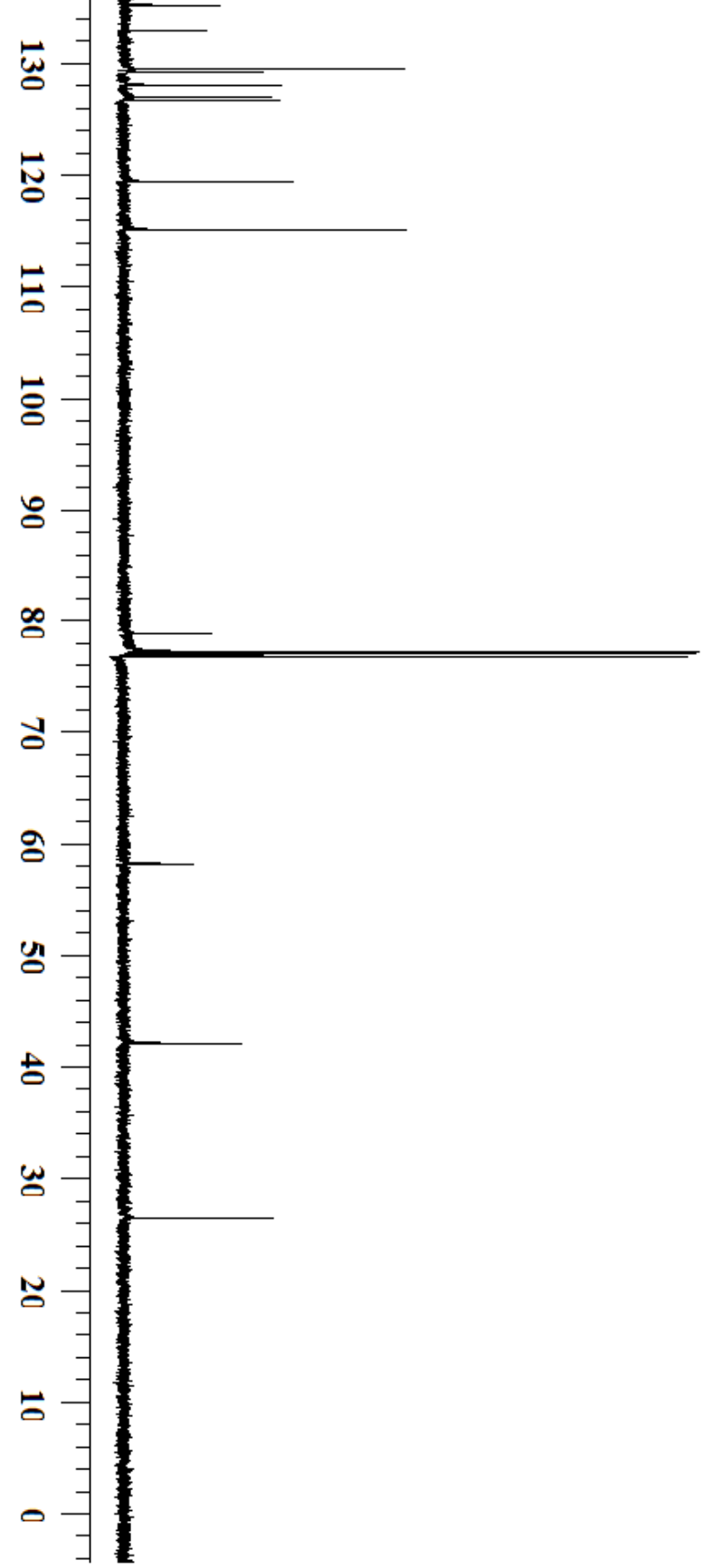


\subsection{NMR Spectra Data}
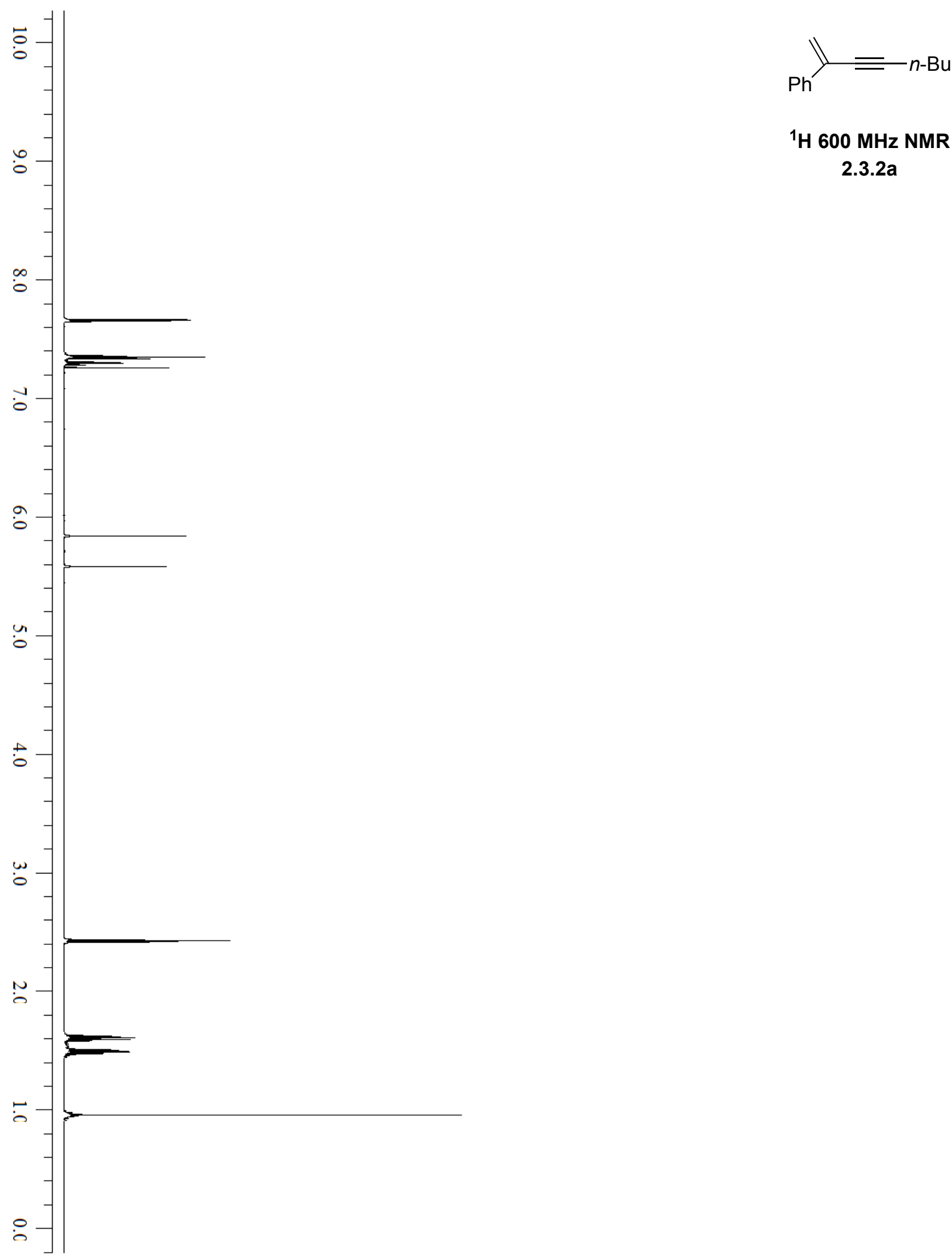

${ }^{1} \mathrm{H} 600 \mathrm{MHz}$ NMR

2.3.2a 

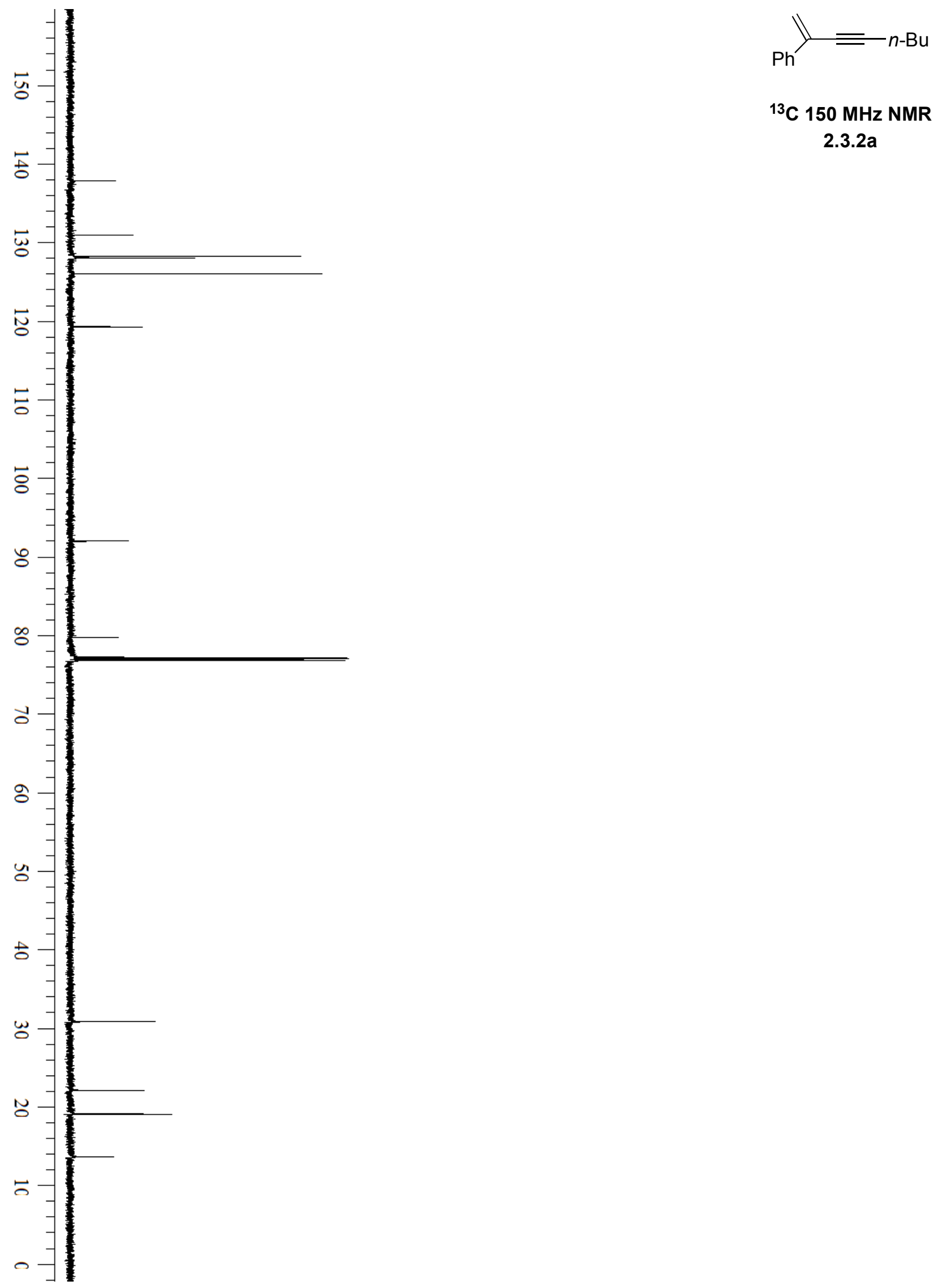

${ }^{13} \mathrm{C} 150 \mathrm{MHz}$ NMR

2.3.2a 

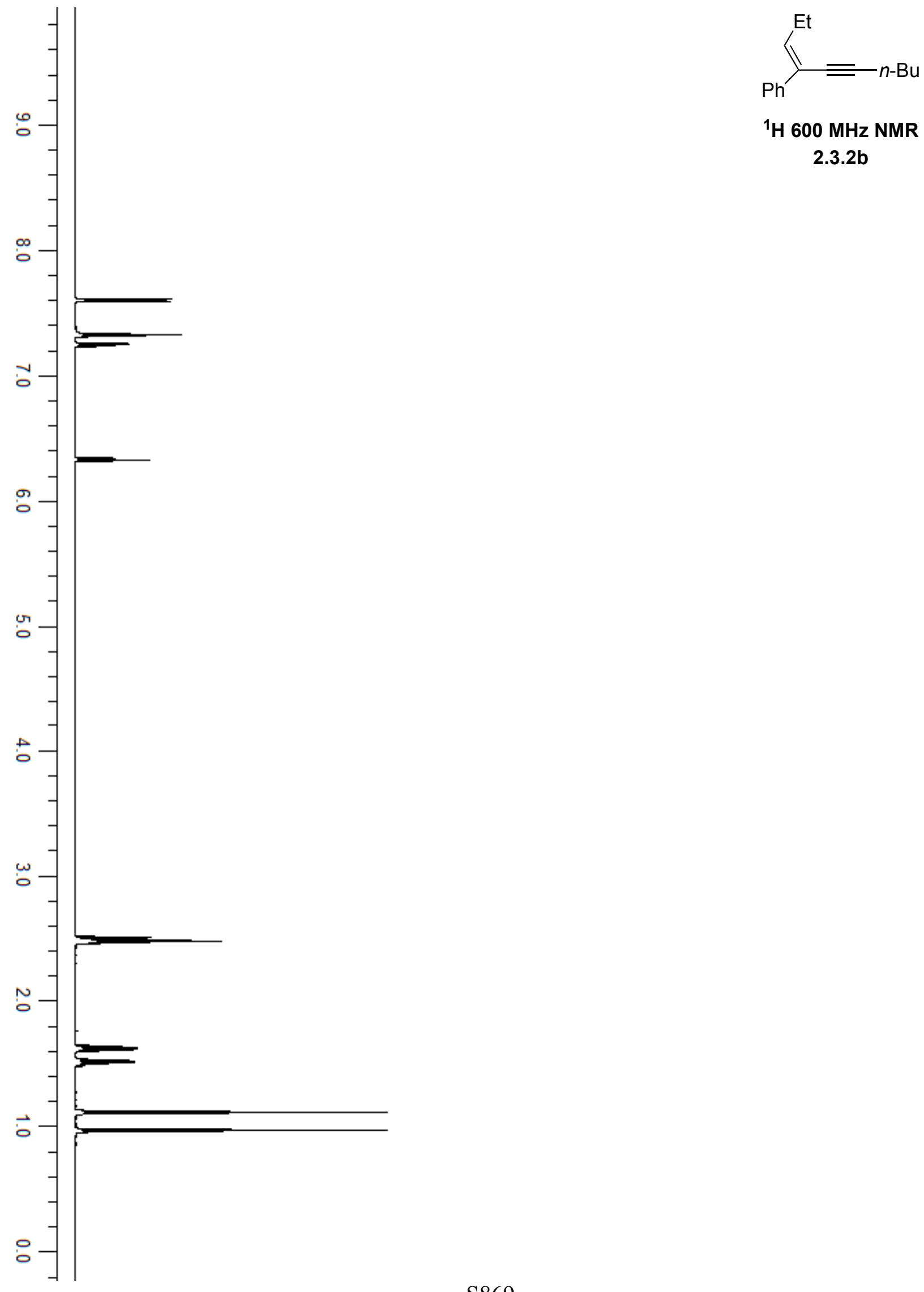

${ }^{1} \mathrm{H} 600 \mathrm{MHz}$ NMR

2.3.2b 

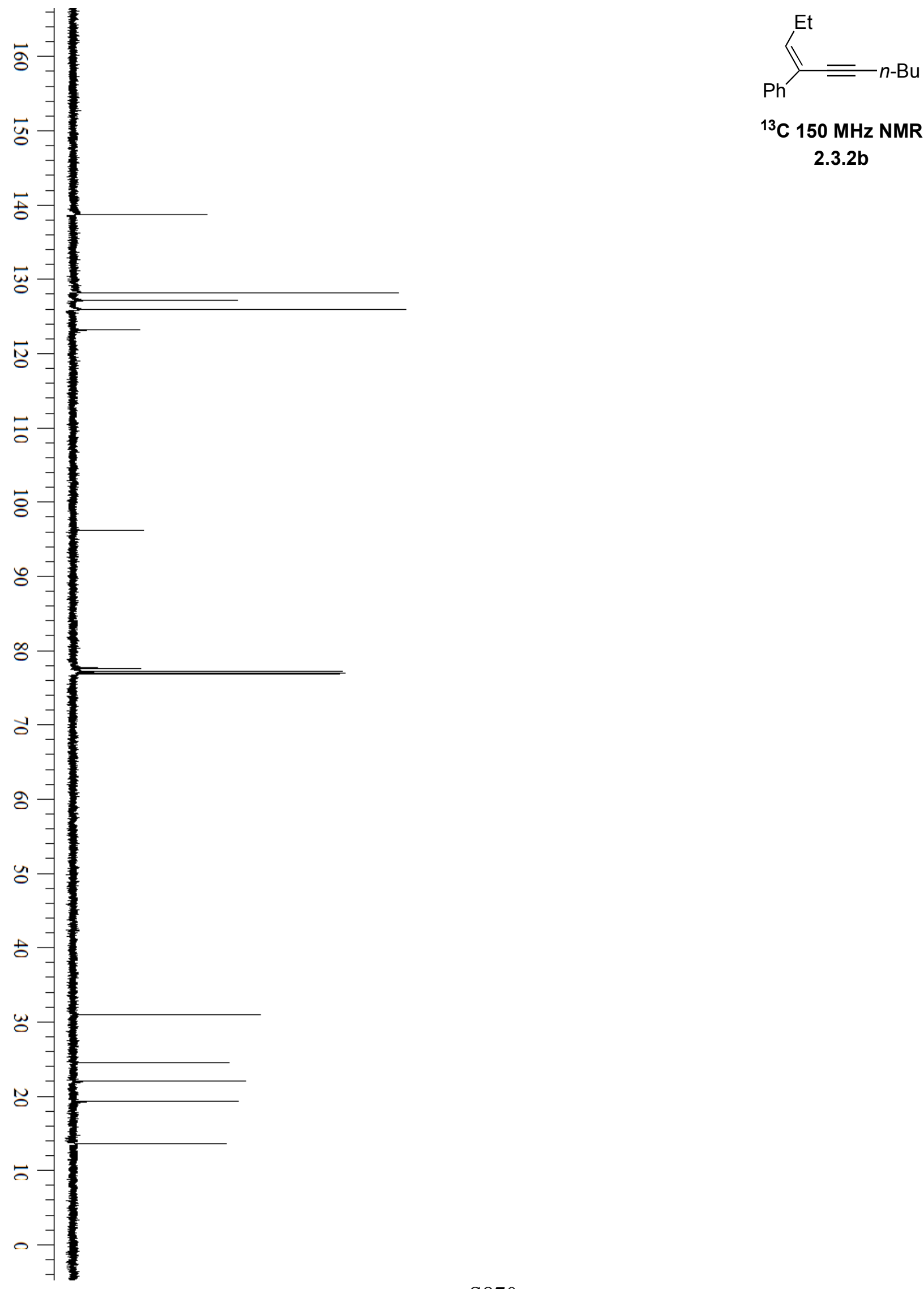

${ }^{13} \mathrm{C} 150 \mathrm{MHz}$ NMR

2.3.2b 

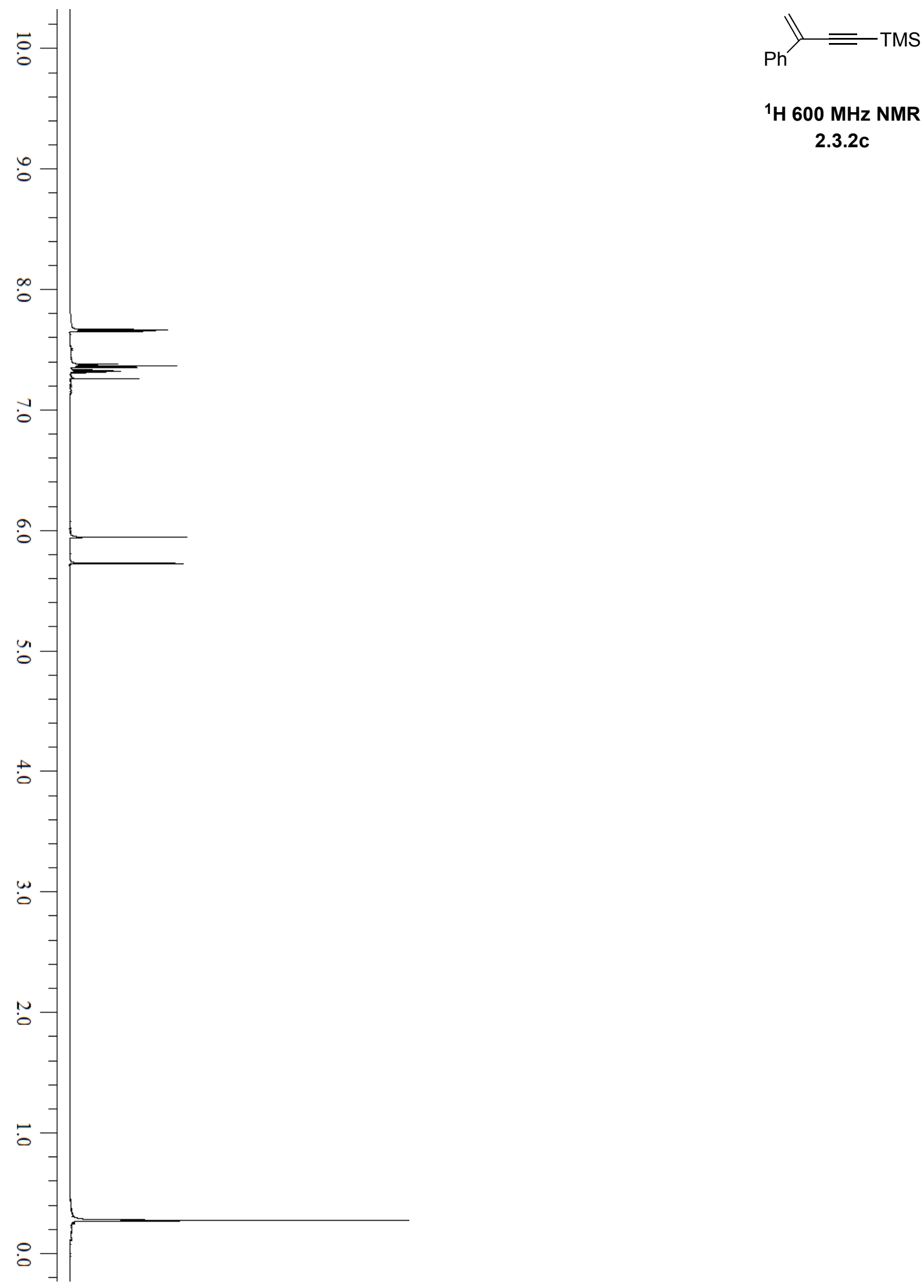

${ }^{1} \mathrm{H} 600 \mathrm{MHz}$ NMR

2.3.2c 

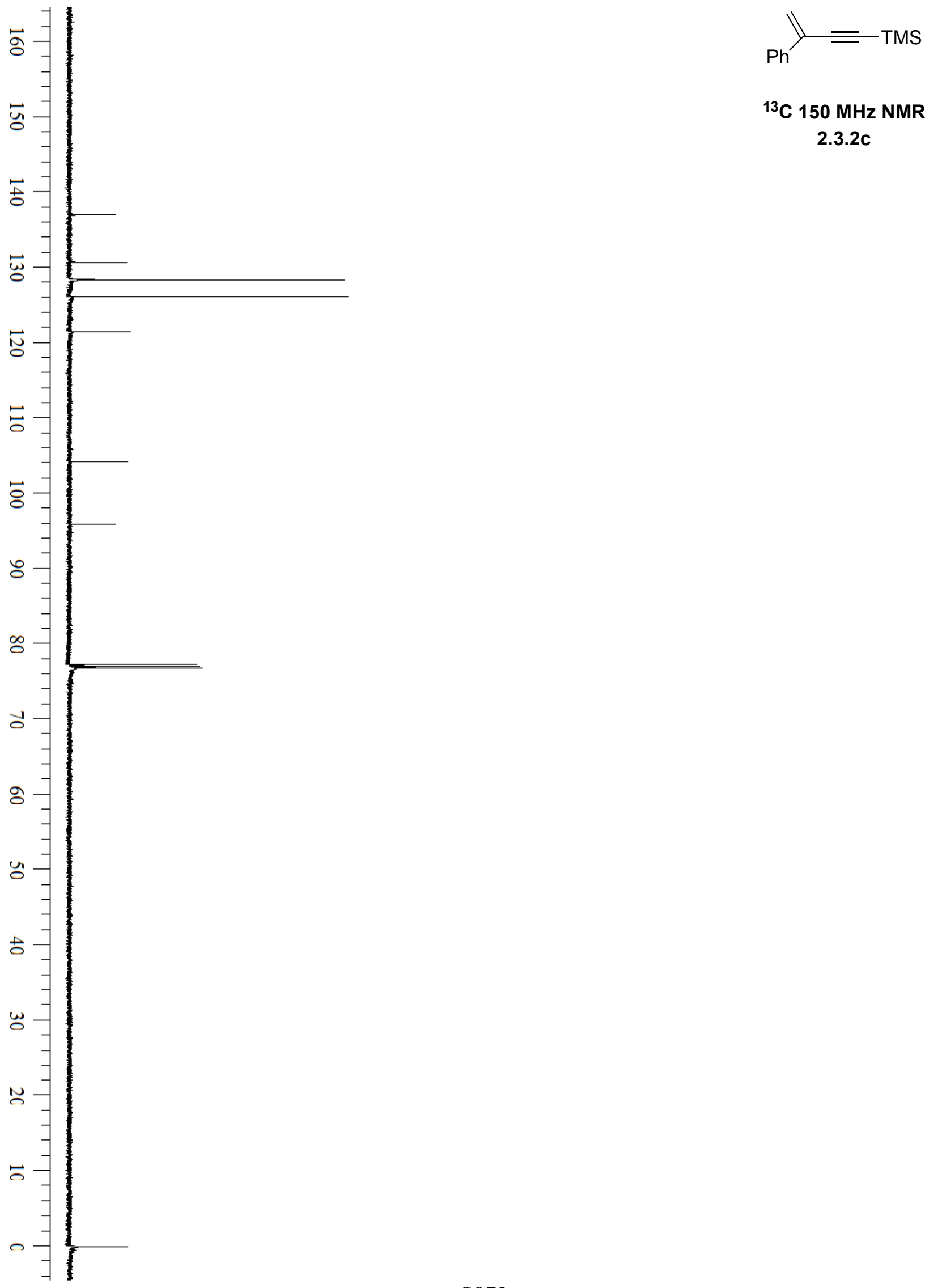

${ }^{13} \mathrm{C} 150 \mathrm{MHz}$ NMR

2.3.2c 


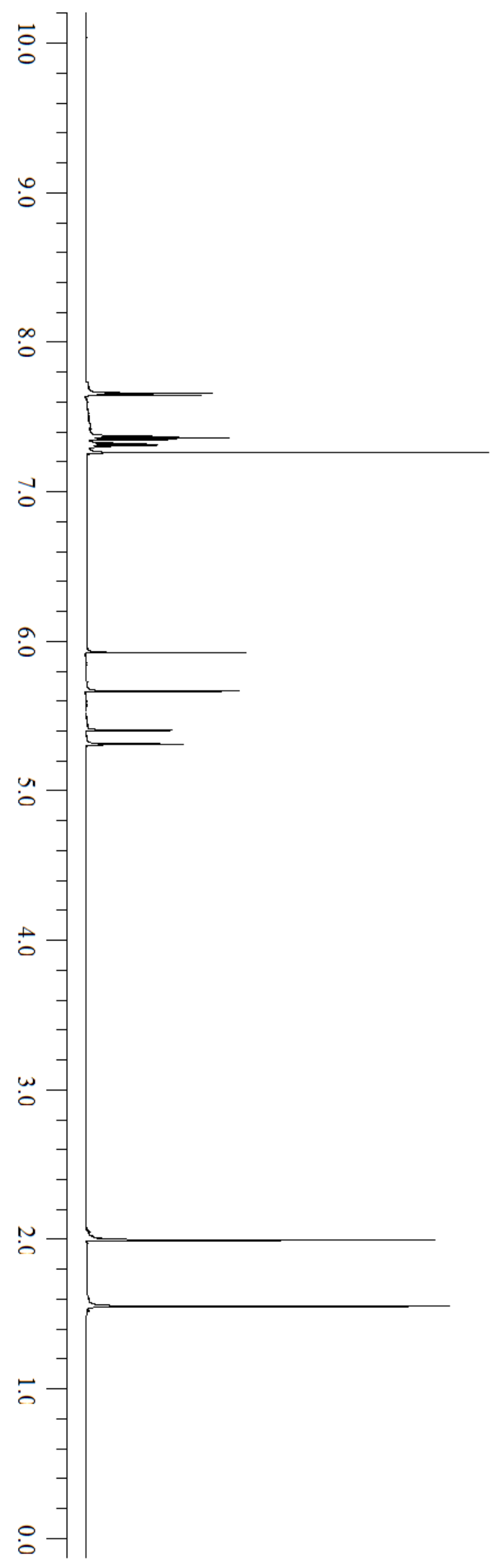

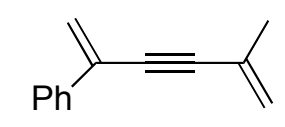

${ }^{1} \mathrm{H} 600 \mathrm{MHz}$ NMR

2.3.2d 

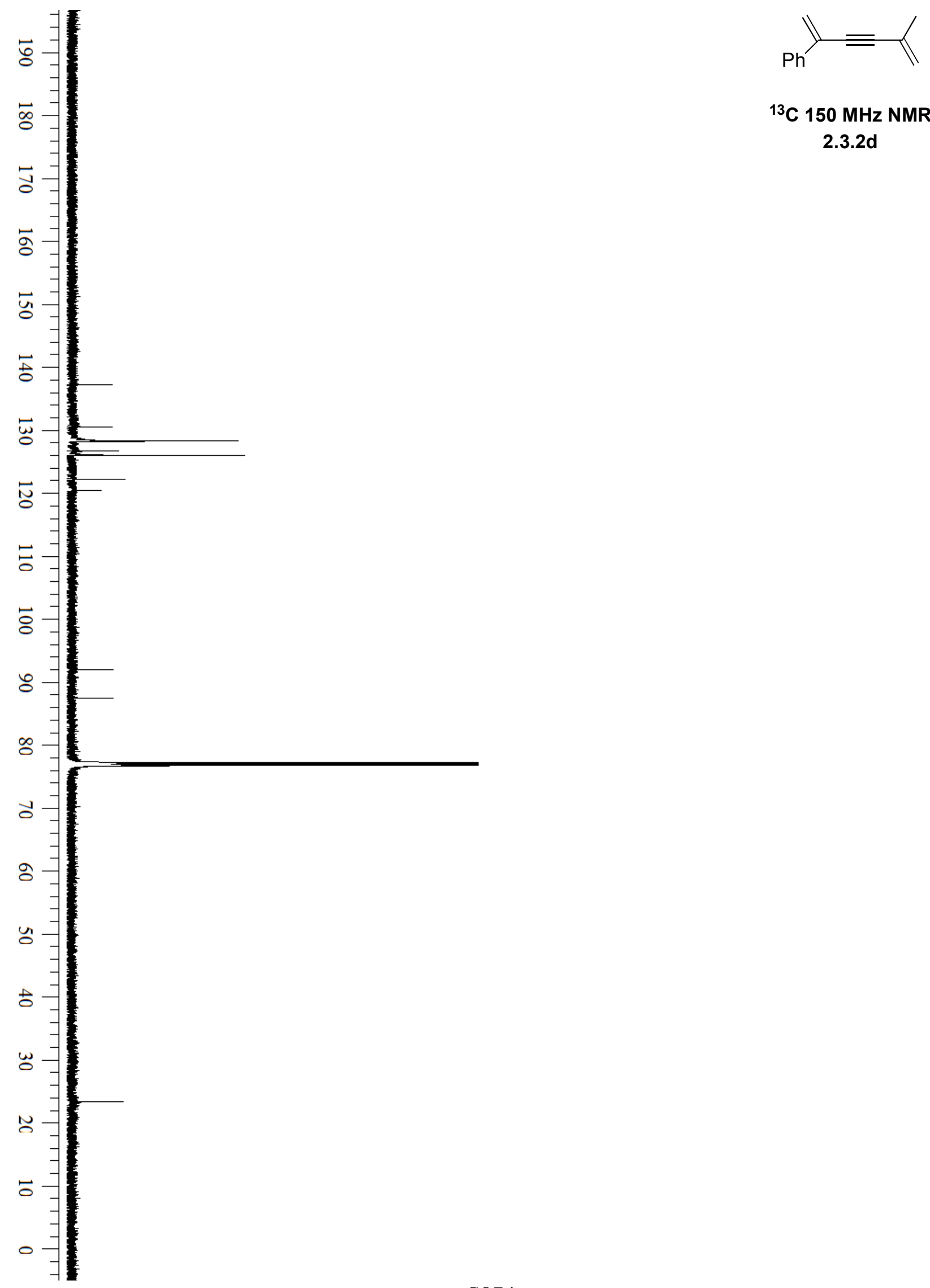

${ }^{13} \mathrm{C} 150 \mathrm{MHz}$ NMR

2.3.2d 

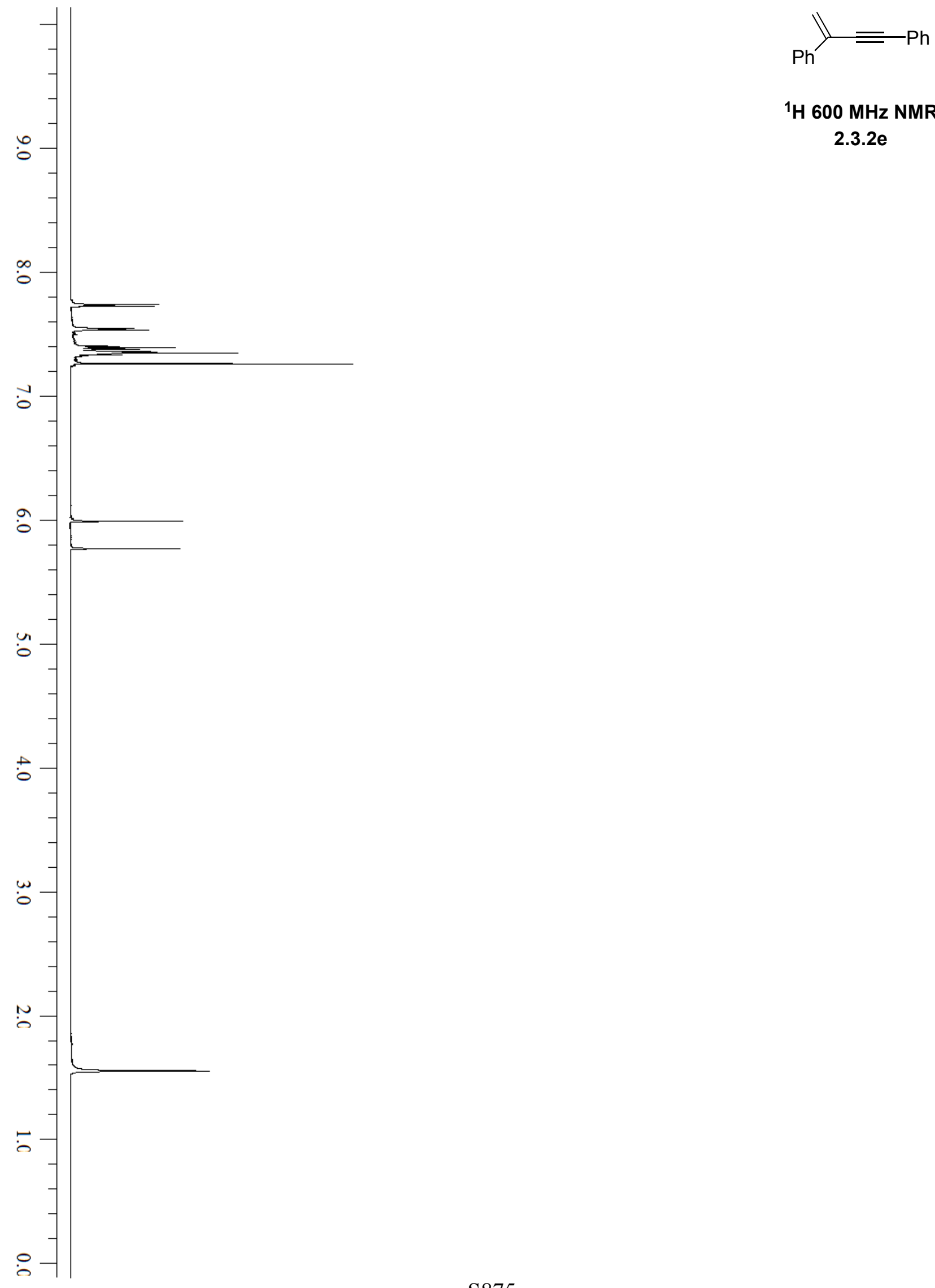

${ }^{1} \mathrm{H} 600 \mathrm{MHz}$ NMR

2.3.2e 

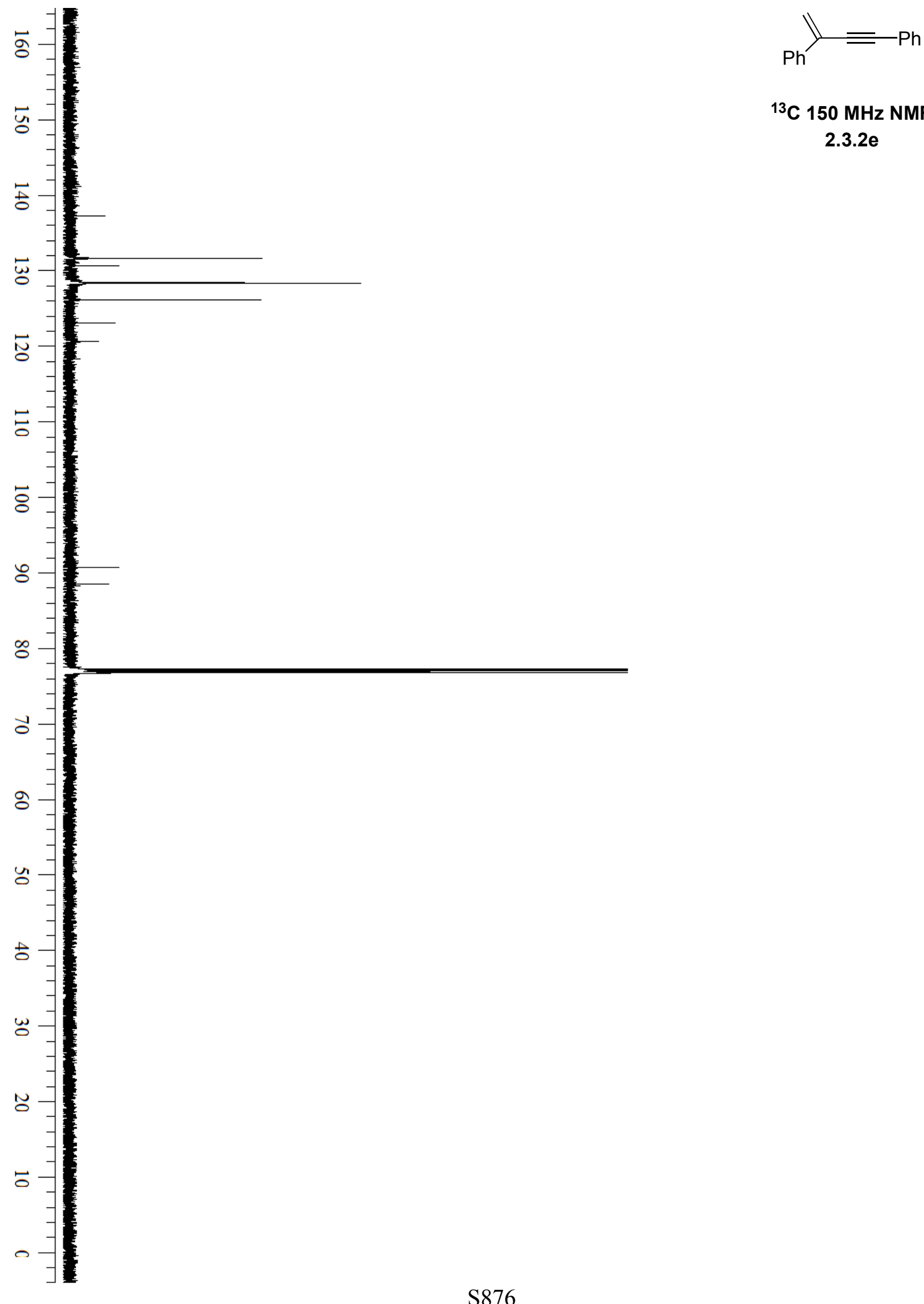

${ }^{13} \mathrm{C} 150 \mathrm{MHz}$ NMR

2.3.2e 

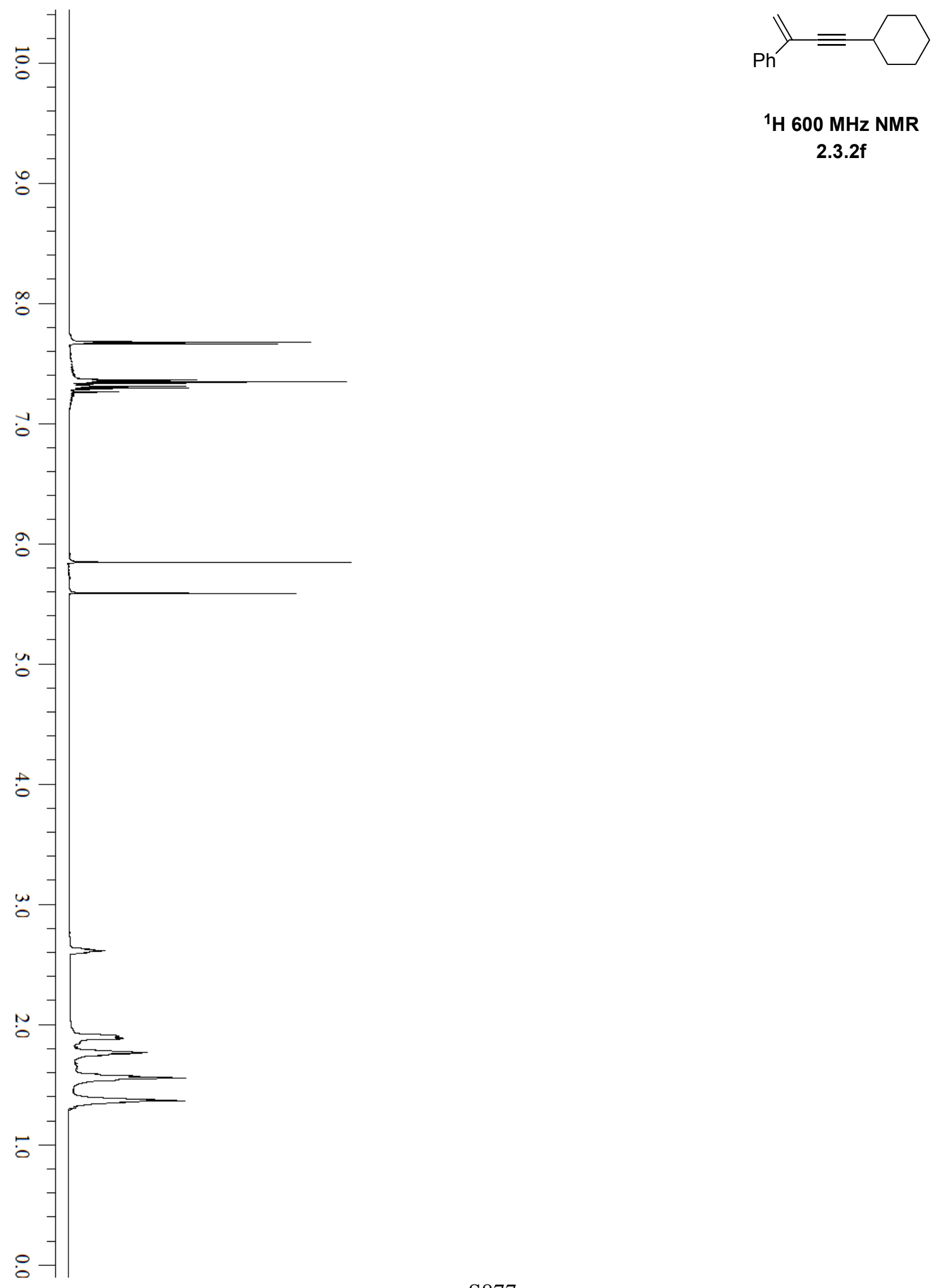

${ }^{1} \mathrm{H} 600 \mathrm{MHz}$ NMR

2.3.2f 

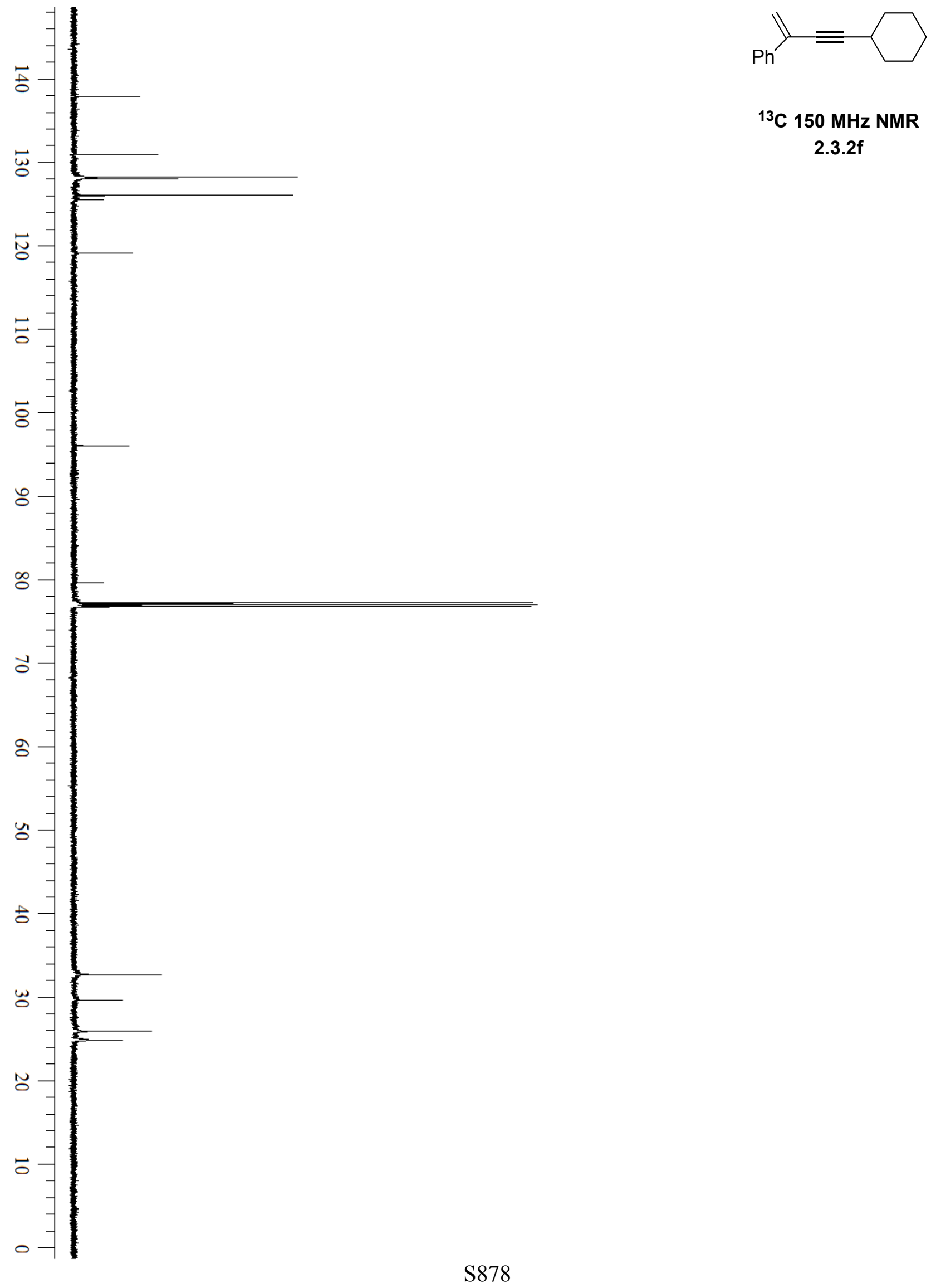

${ }^{13} \mathrm{C} 150 \mathrm{MHz}$ NMR

2.3.2f 


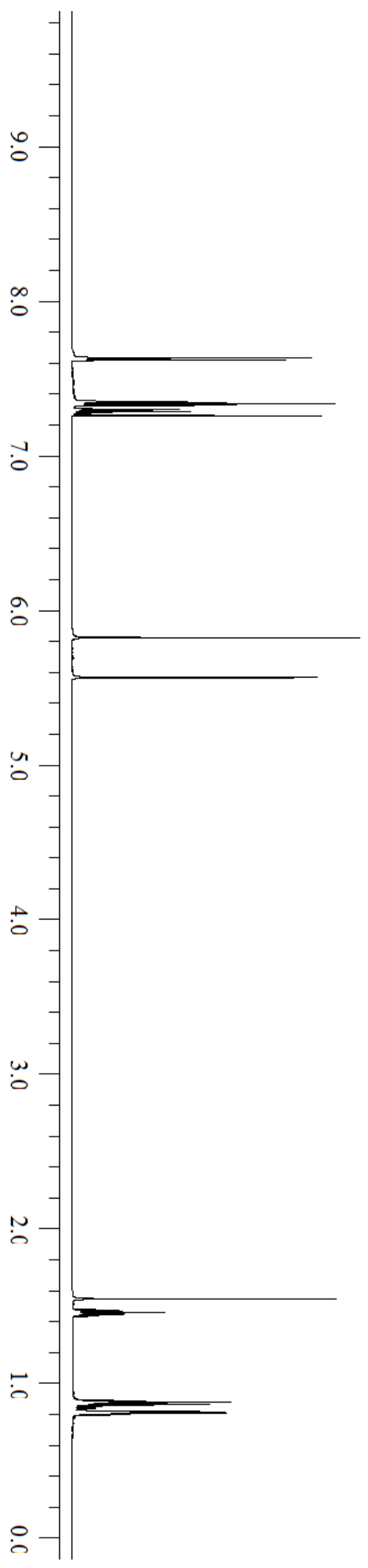

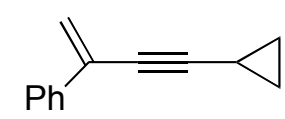

${ }^{1} \mathrm{H} 600 \mathrm{MHz}$ NMR

2.3.2g 

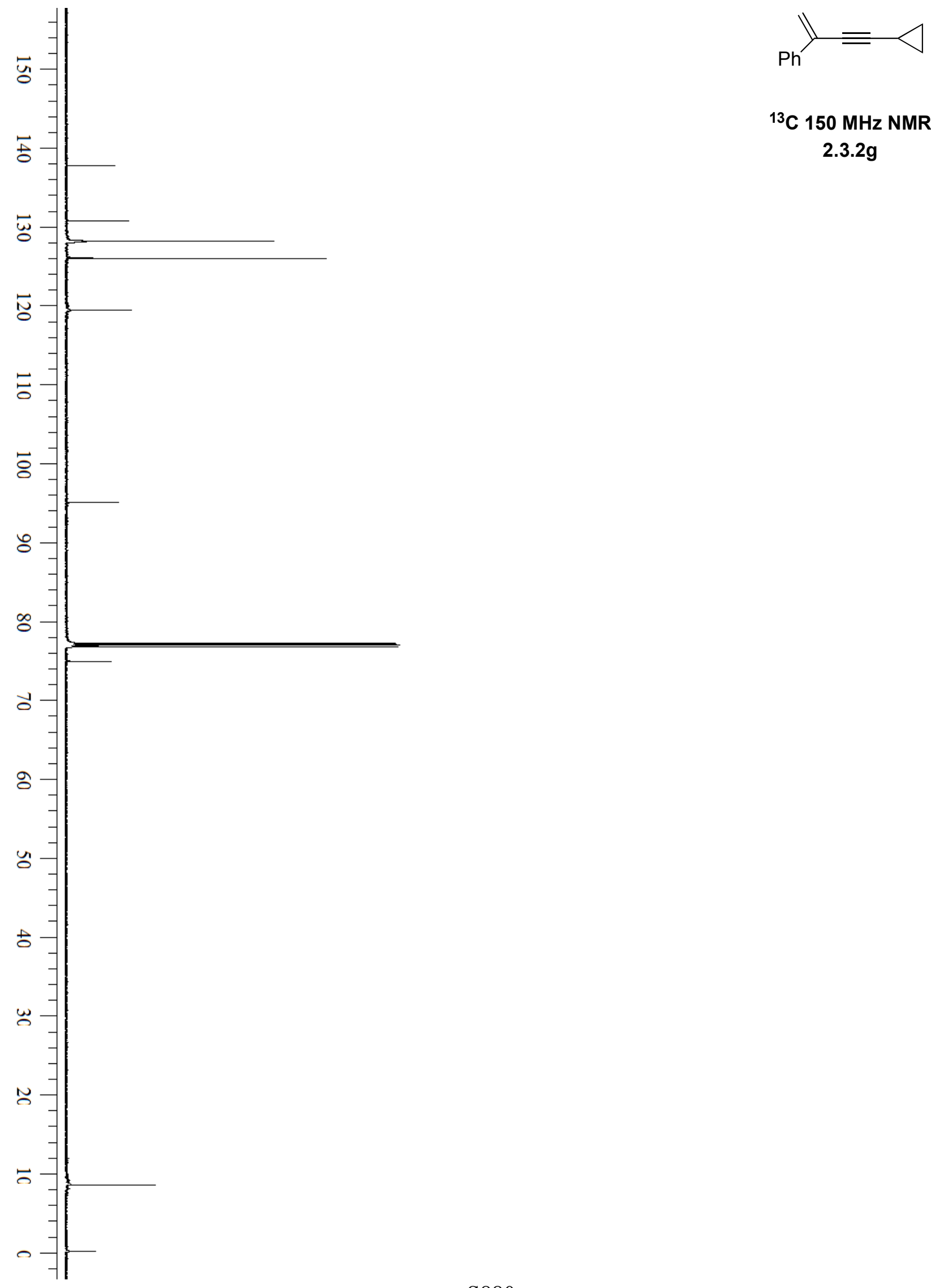

${ }^{13} \mathrm{C} 150 \mathrm{MHz}$ NMR

2.3.2g 

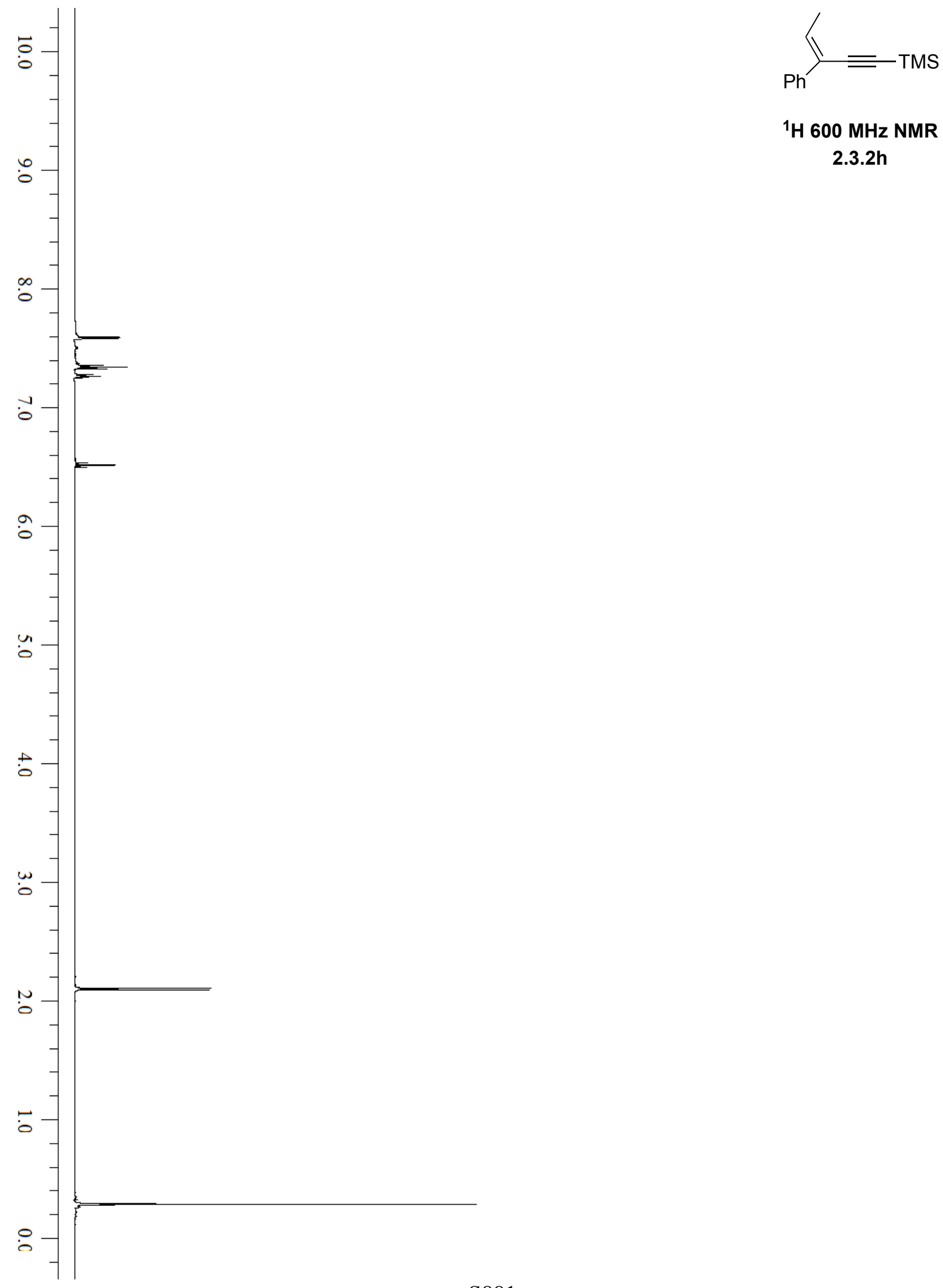

${ }^{1} \mathrm{H} 600 \mathrm{MHz}$ NMR

2.3.2h 

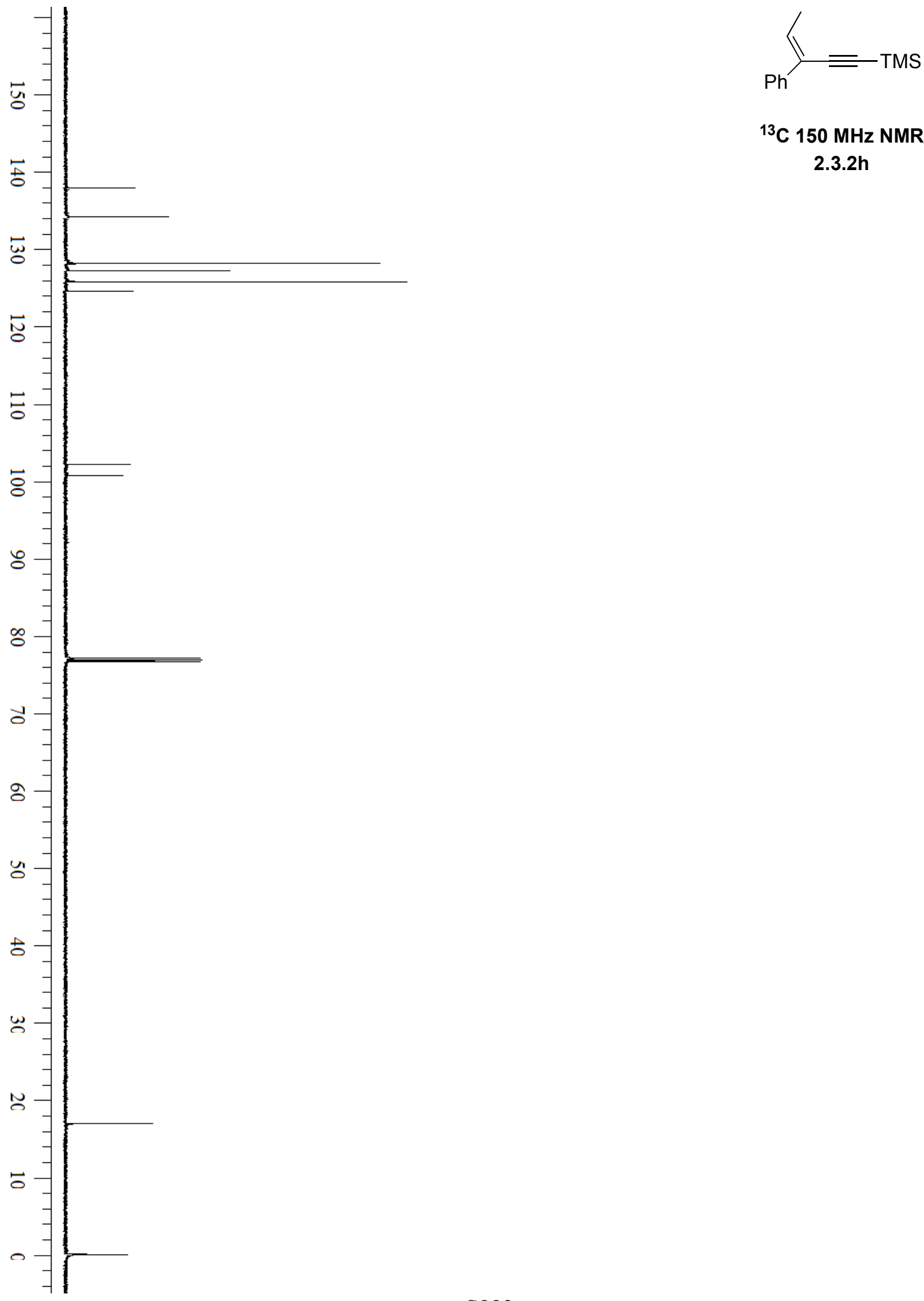

${ }^{13} \mathrm{C} 150 \mathrm{MHz}$ NMR

2.3.2h 

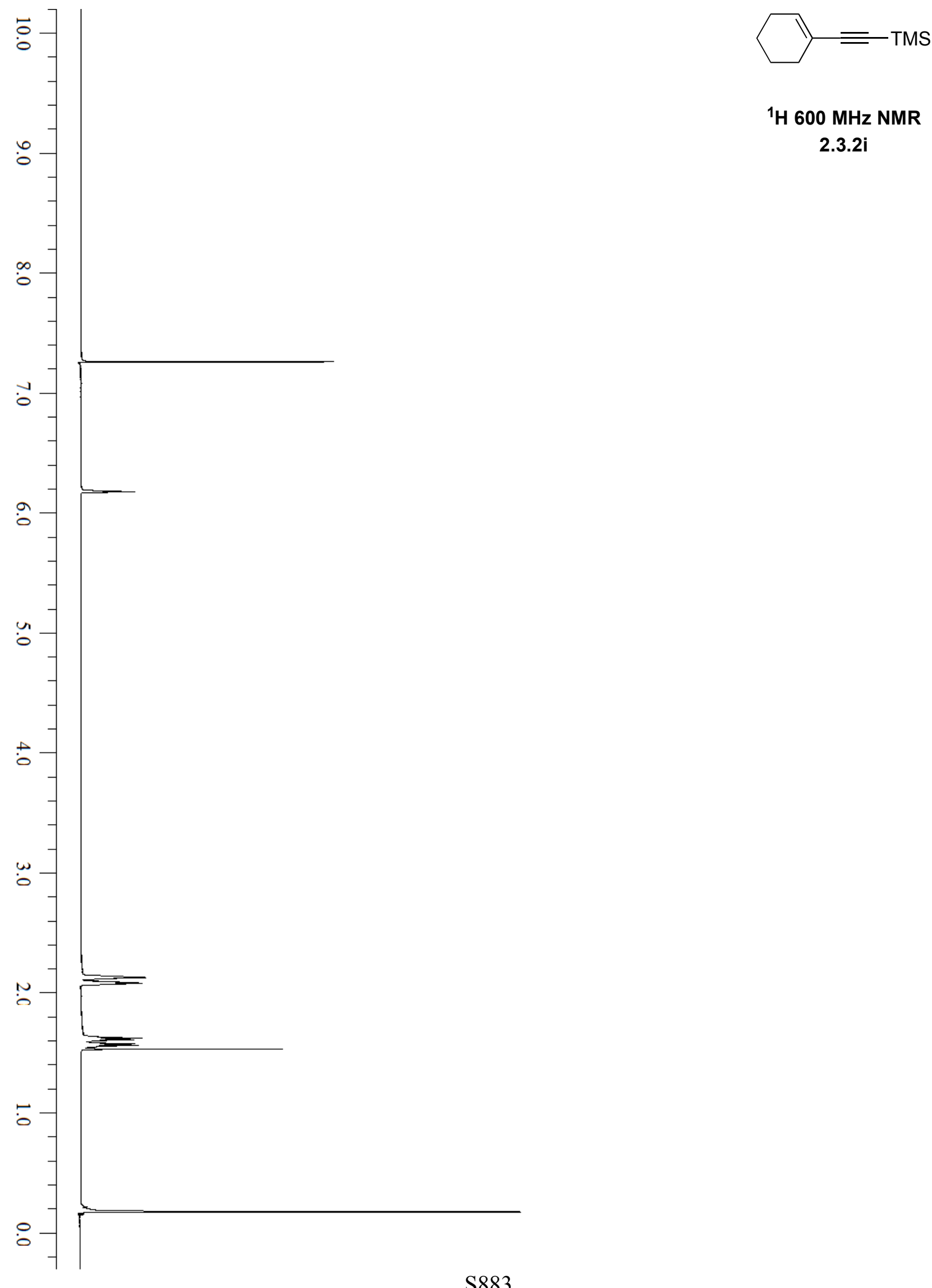

${ }^{1} \mathrm{H} 600 \mathrm{MHz}$ NMR

2.3.2i 


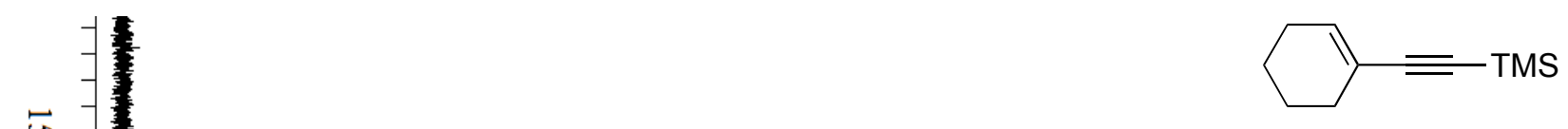

${ }^{13} \mathrm{C} 150 \mathrm{MHz}$ NMR

2.3.2i

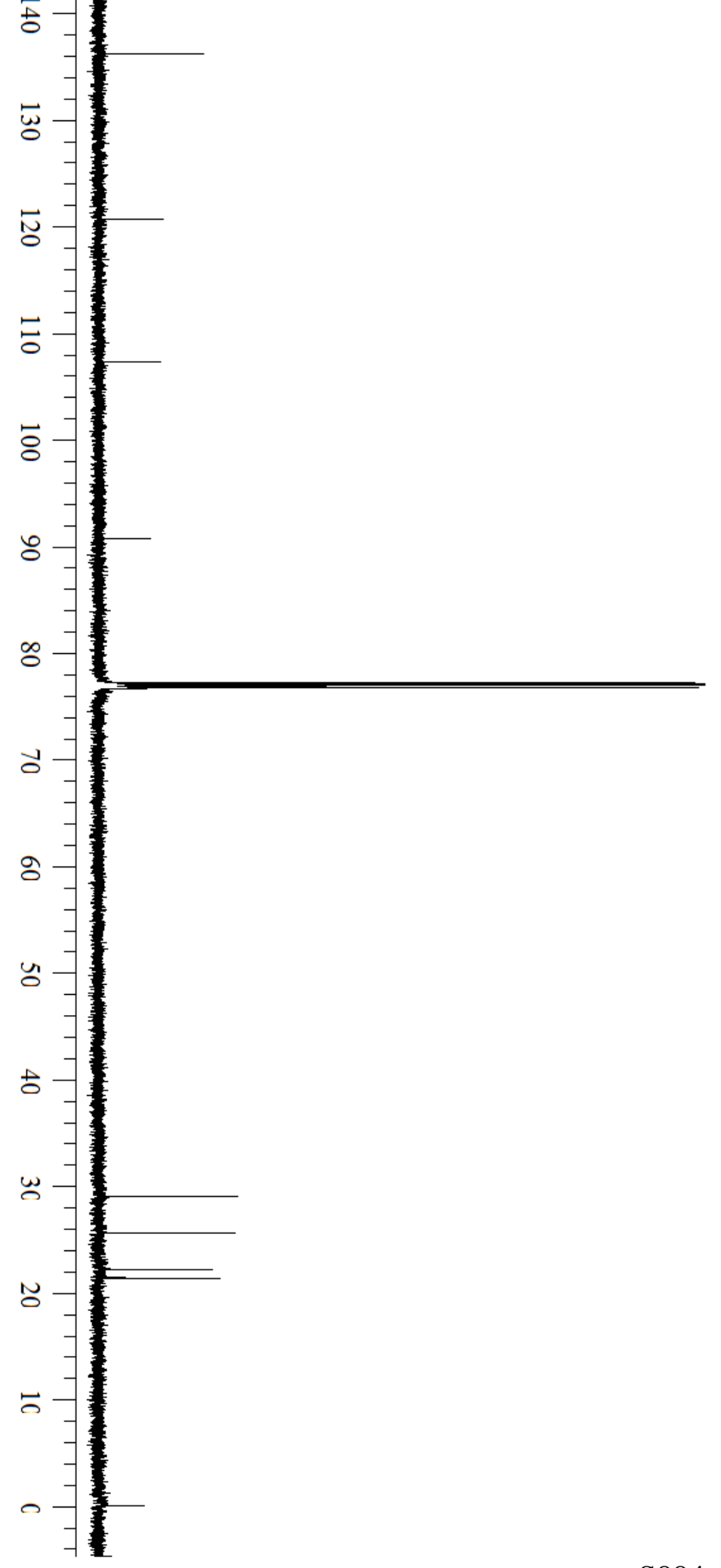



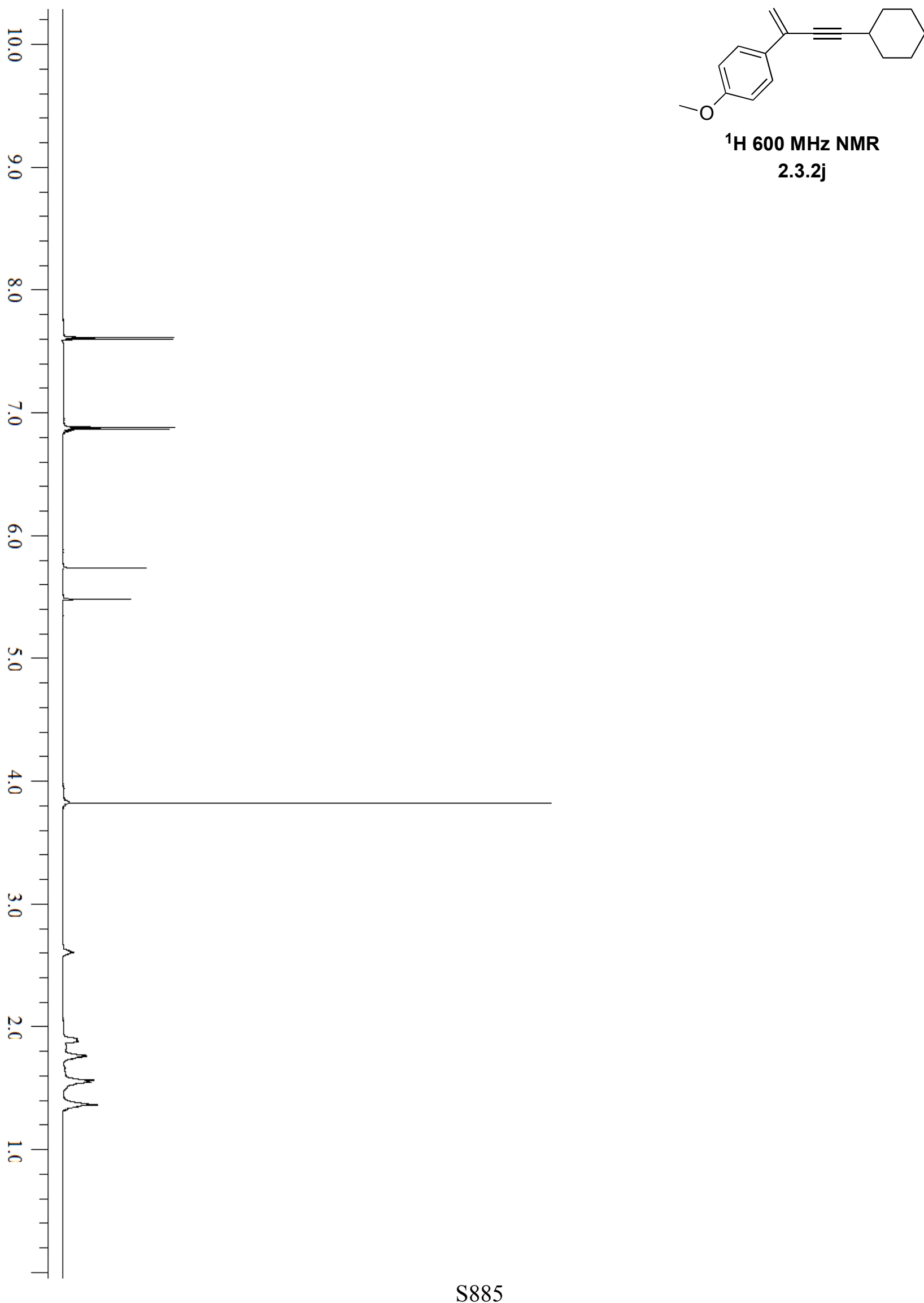

${ }^{1} \mathrm{H} 600 \mathrm{MHz}$ NMR

2.3.2j 

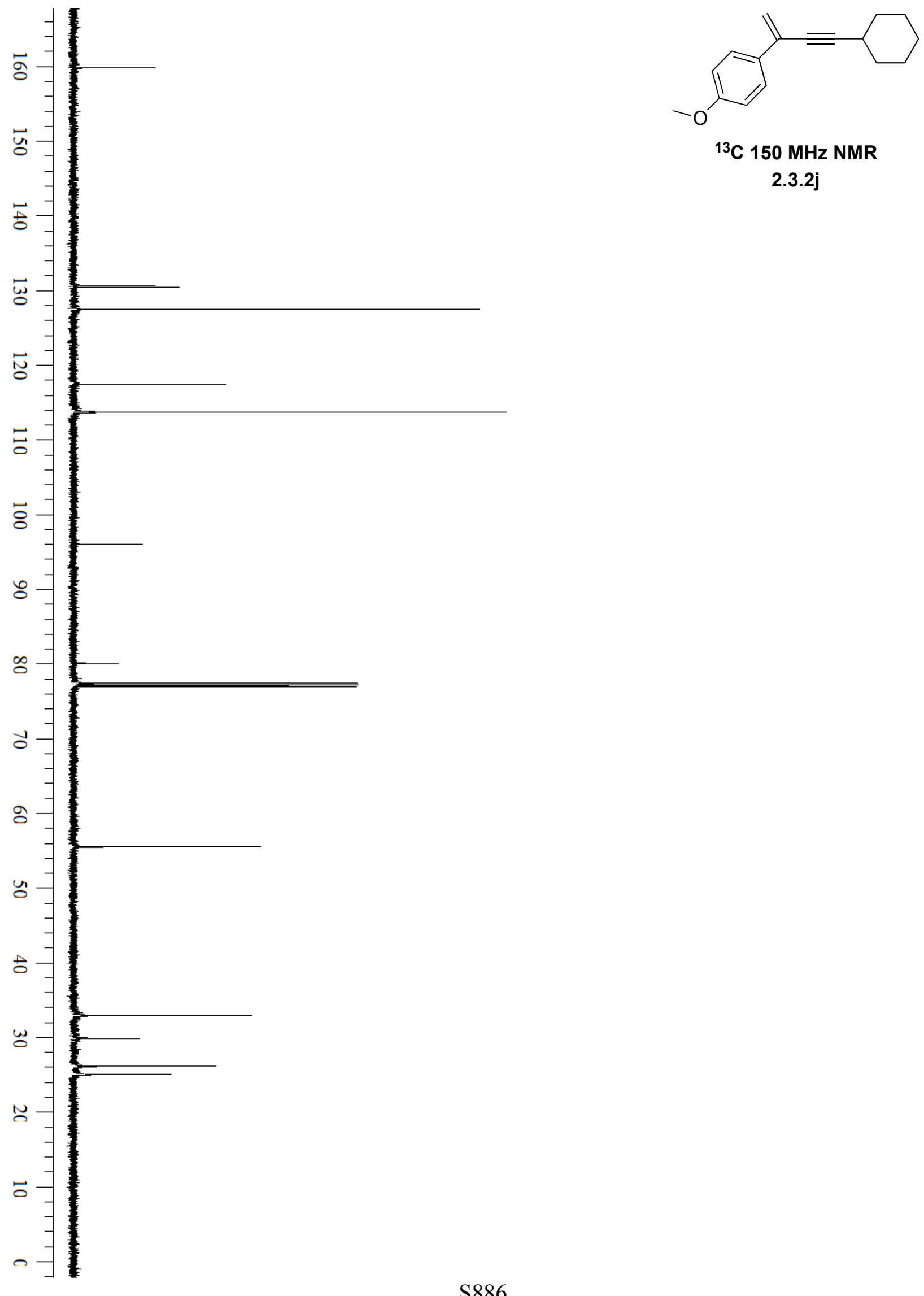

2.3.2j 

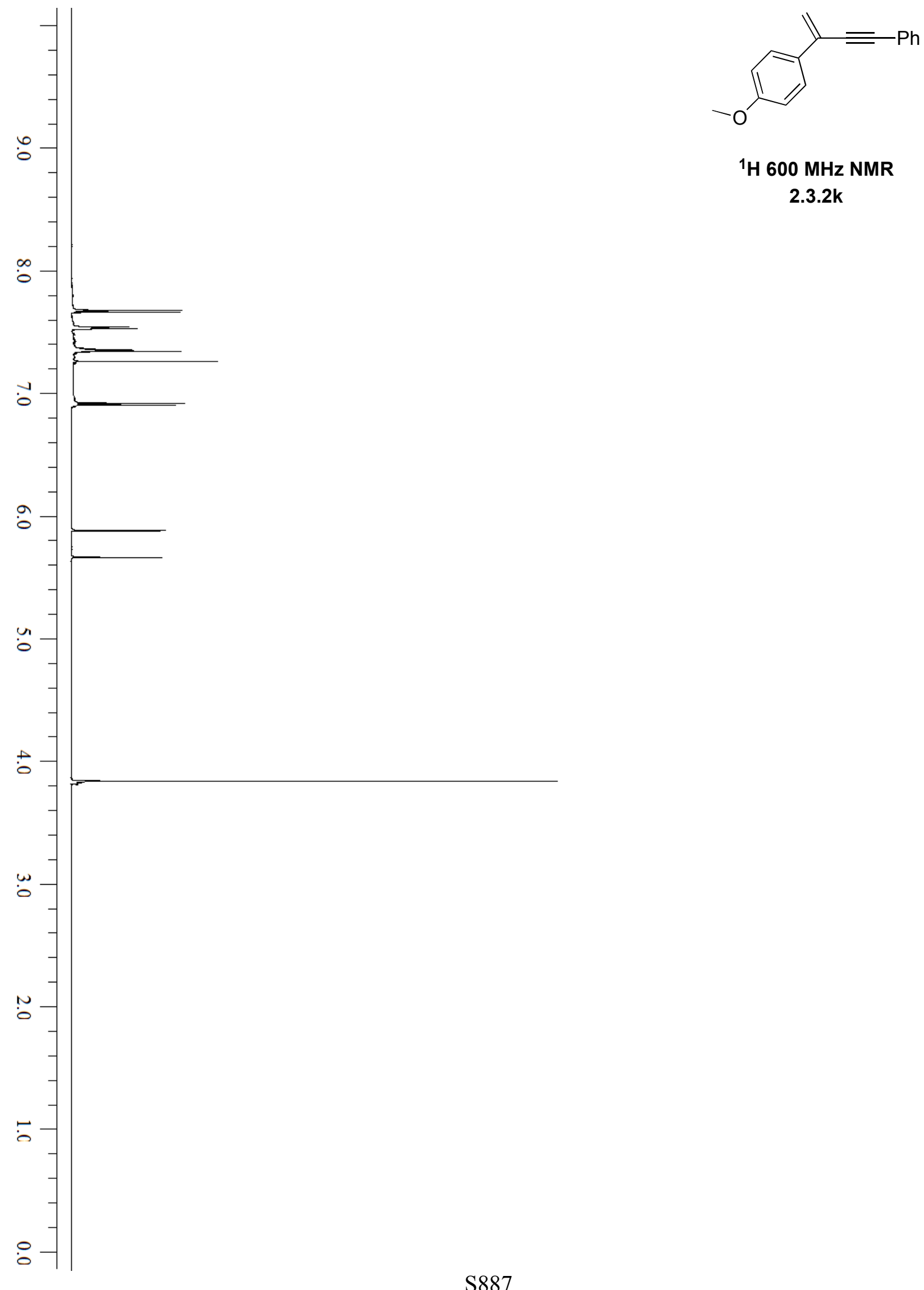

${ }^{1} \mathrm{H} 600 \mathrm{MHz}$ NMR

2.3.2k 


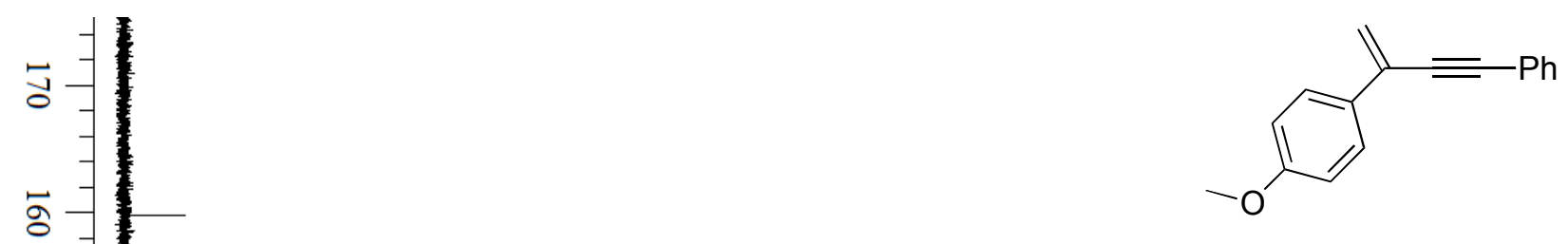

${ }^{13} \mathrm{C} 150 \mathrm{MHz}$ NMR

2.3.2k

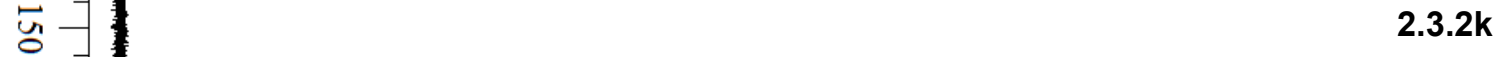

它

w

몽

官

용

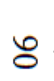

$\infty$

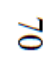

g

s

5

w

N

ह- 


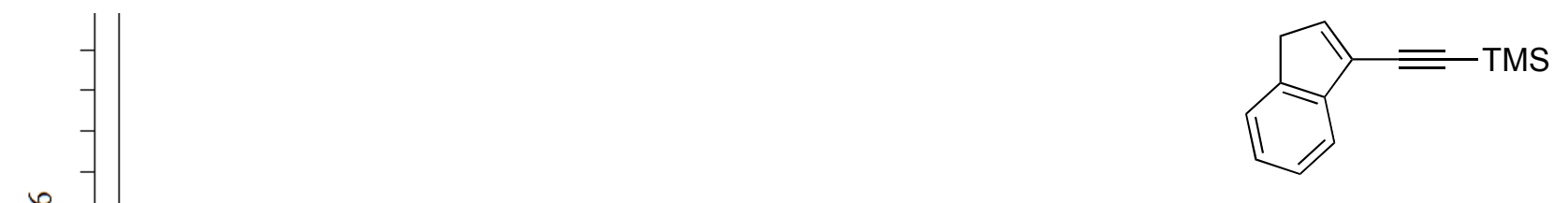

$\because$

${ }^{1} \mathrm{H} 600 \mathrm{MHz}$ NMR

2.3.2I

$\infty$

$\ddot{0}$

:

]

$\div$

w

-

$\stackrel{N}{0}$

$\therefore$

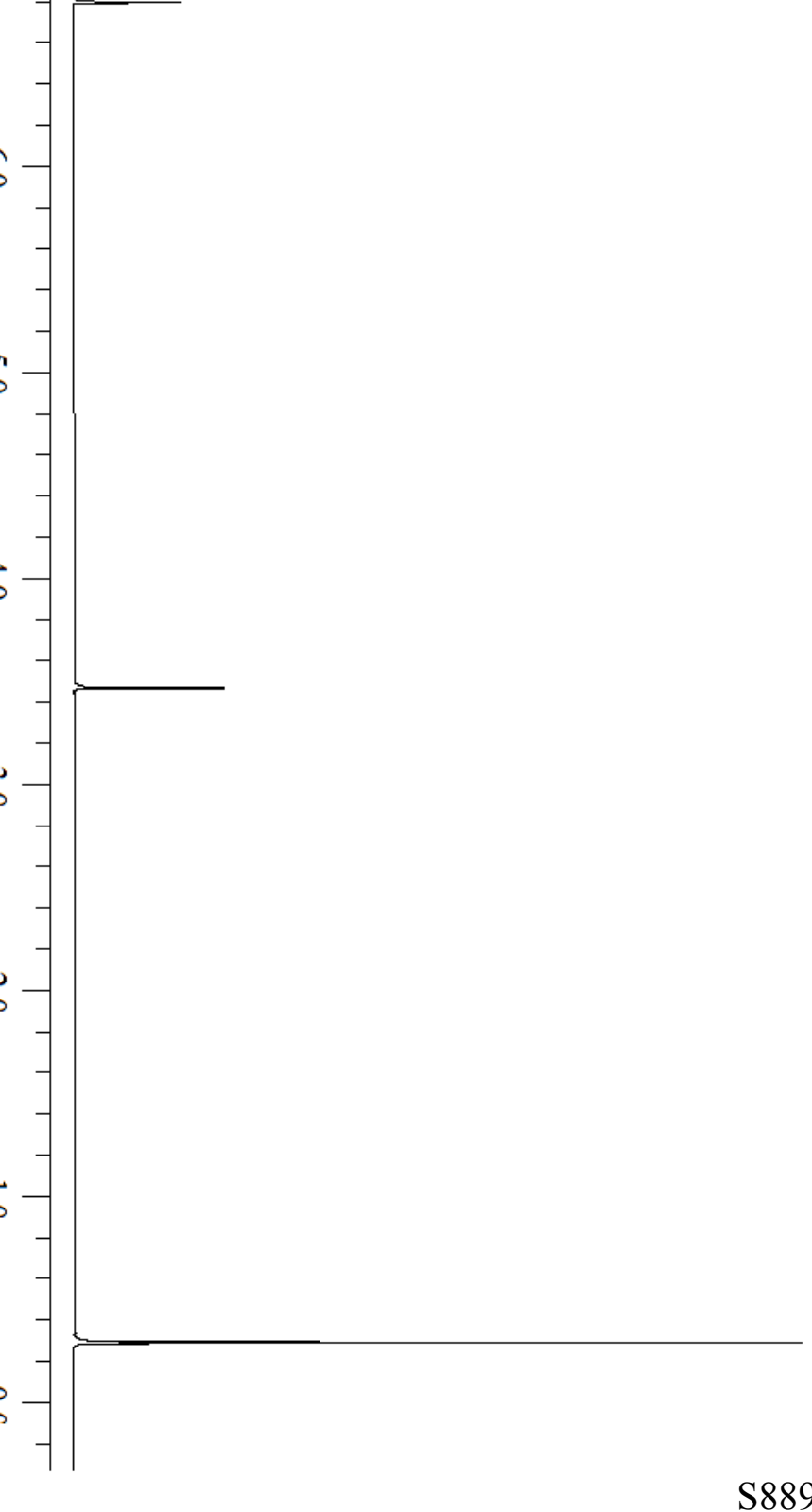



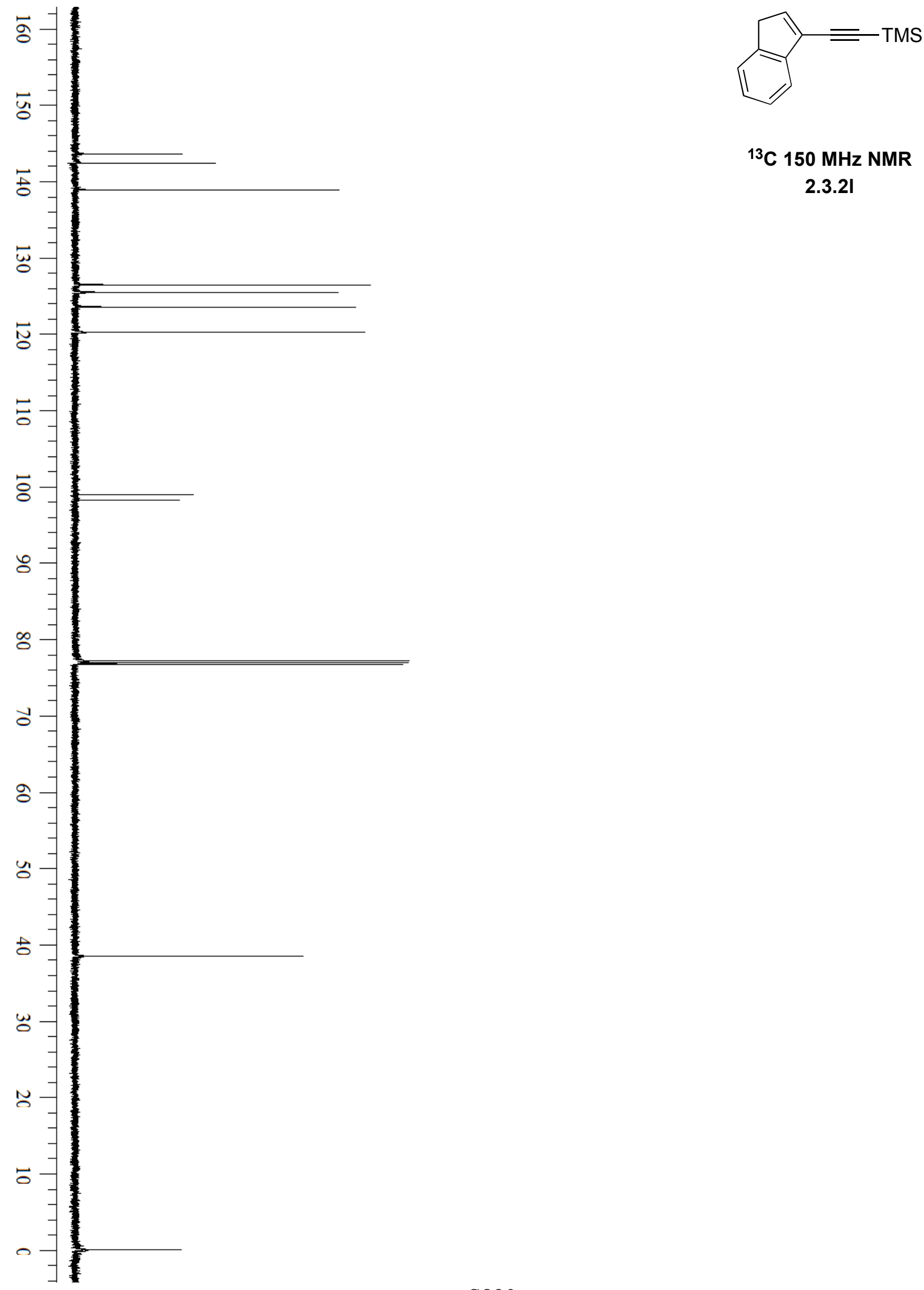

${ }^{13} \mathrm{C} 150 \mathrm{MHz}$ NMR

2.3.2I 


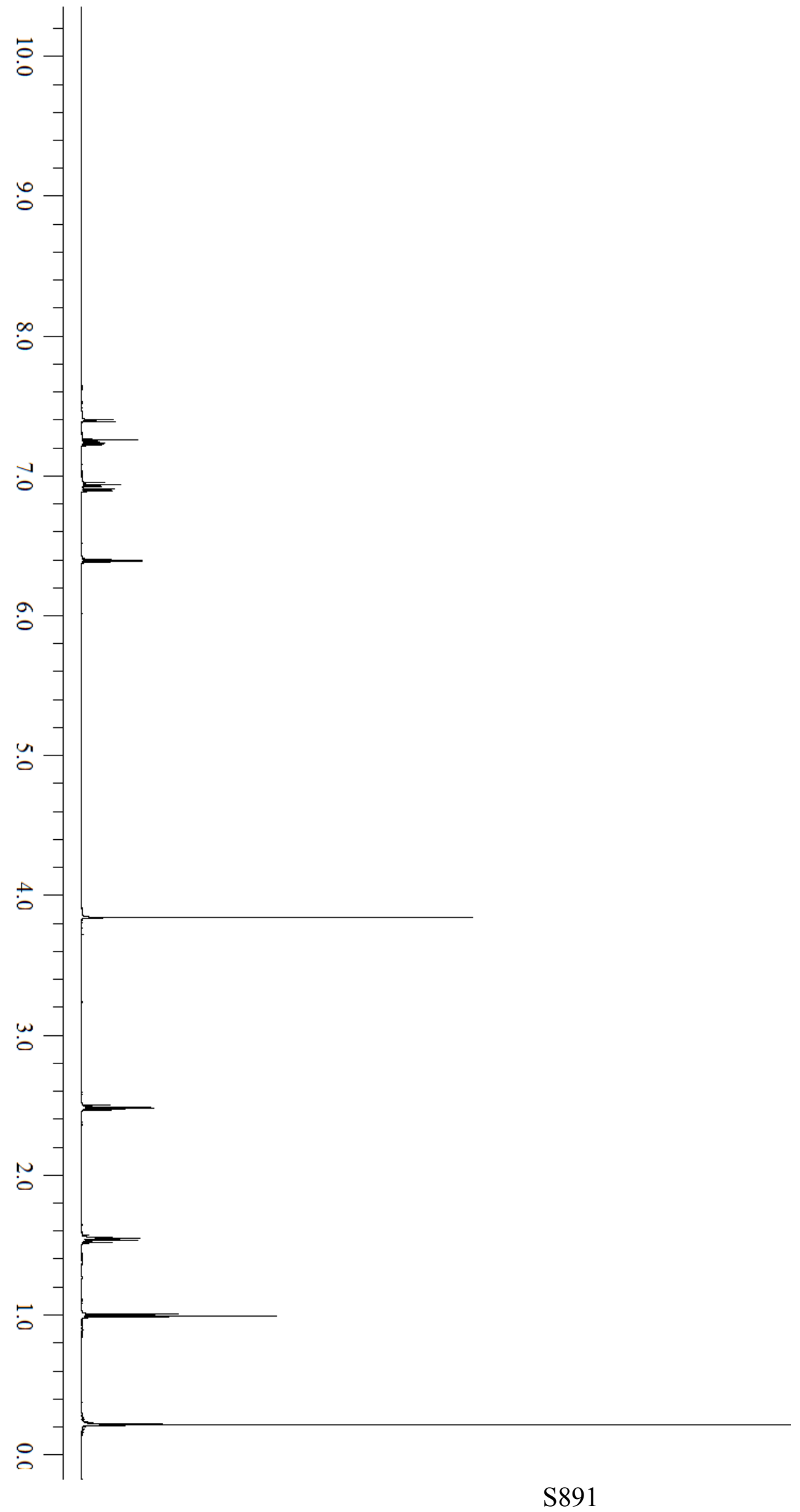

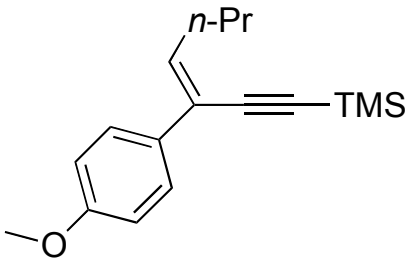

${ }^{1} \mathrm{H} 600 \mathrm{MHz}$ NMR

2.3.2m 

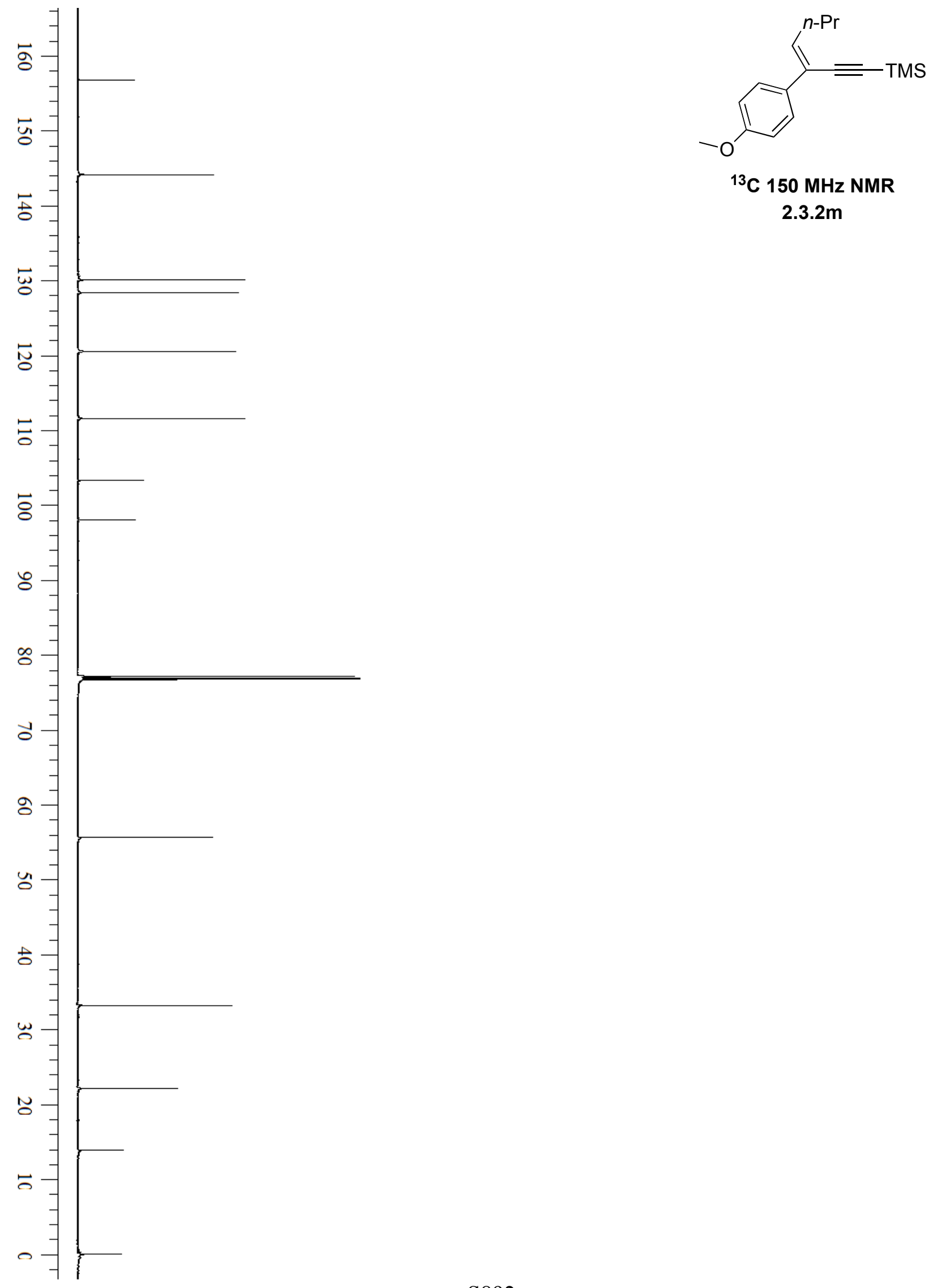

${ }^{13} \mathrm{C} 150 \mathrm{MHz}$ NMR

2.3.2m 

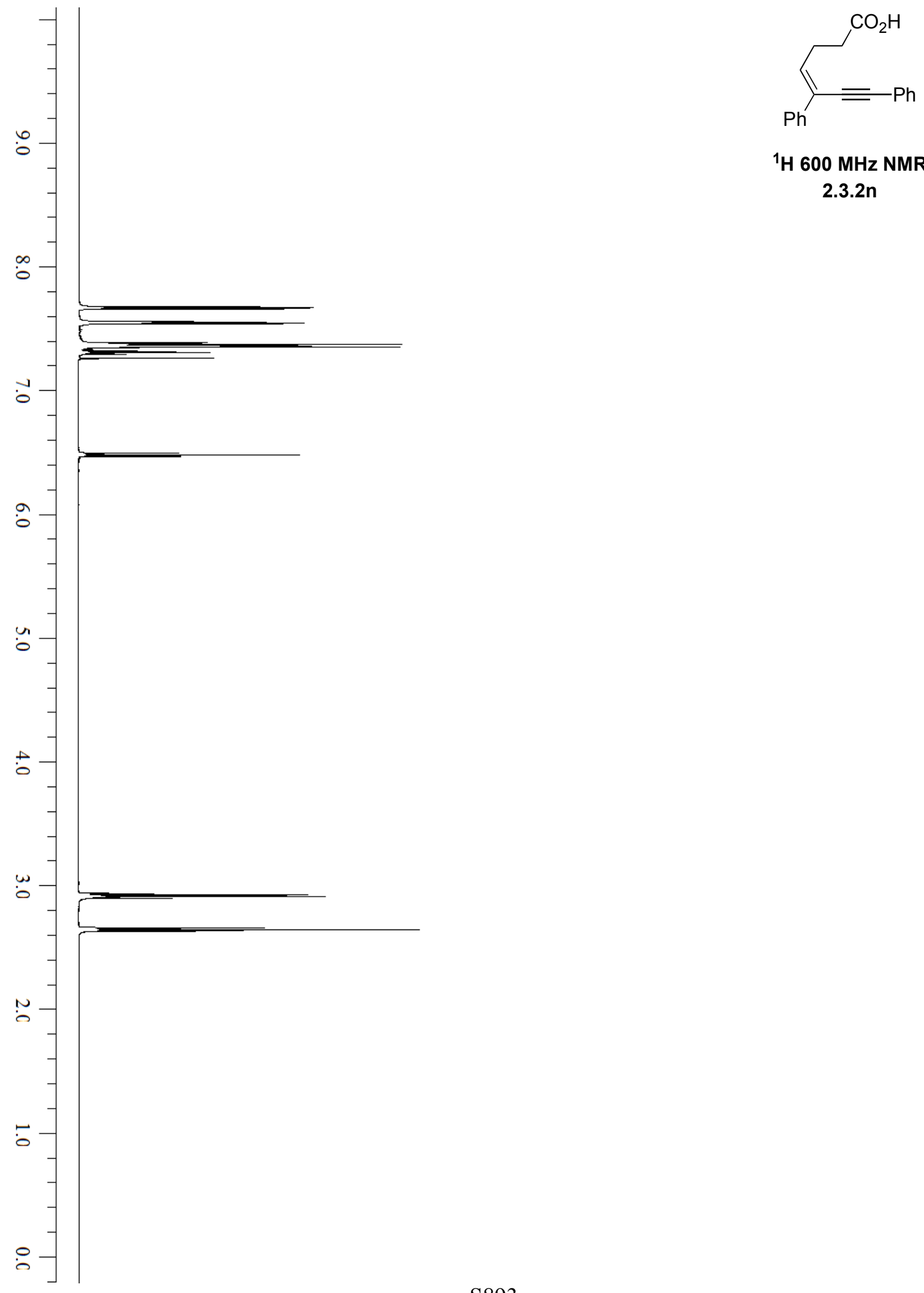

${ }^{1} \mathrm{H} 600 \mathrm{MHz}$ NMR

2.3.2n 

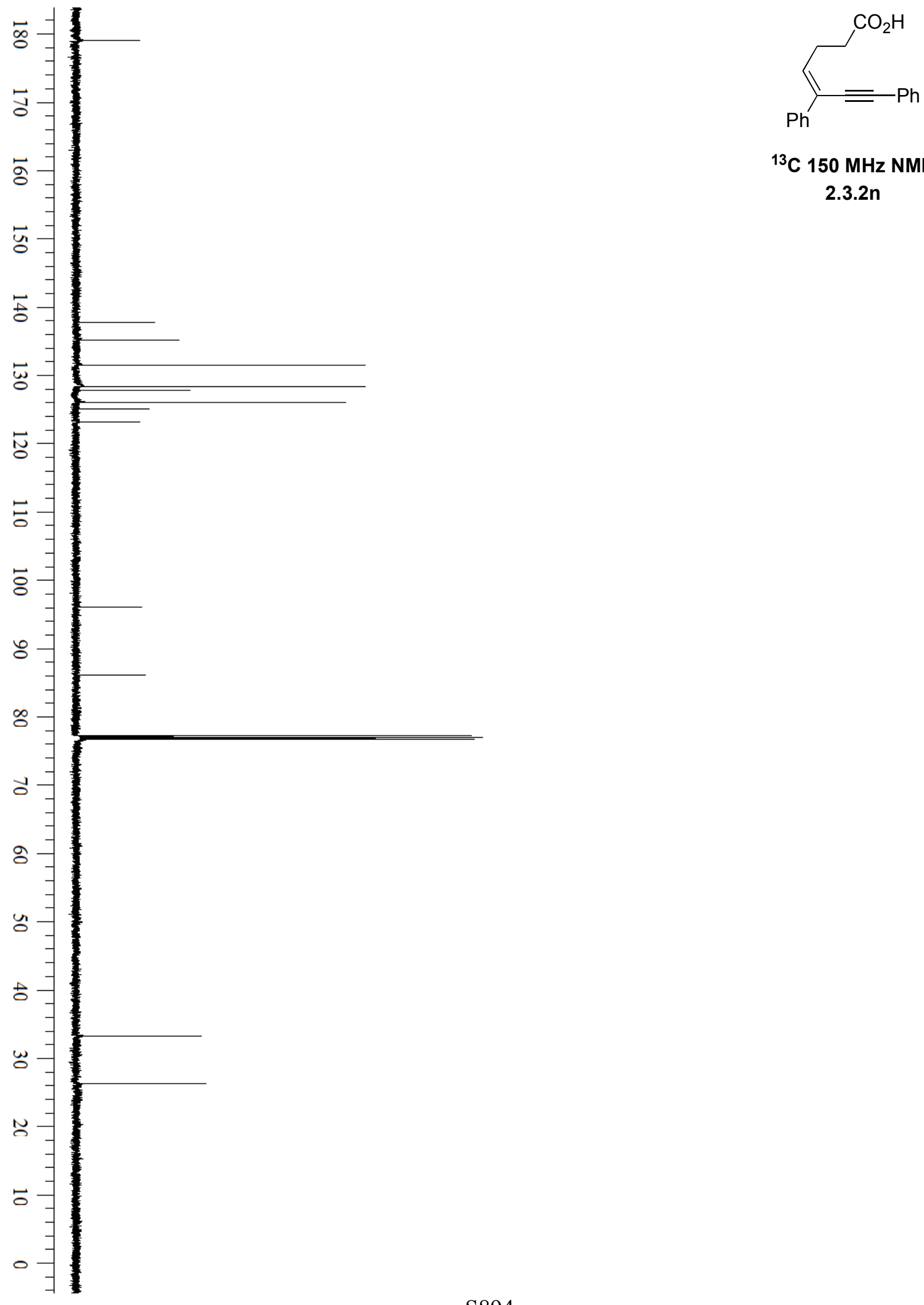

${ }^{13} \mathrm{C} 150 \mathrm{MHz}$ NMR

2.3.2n 


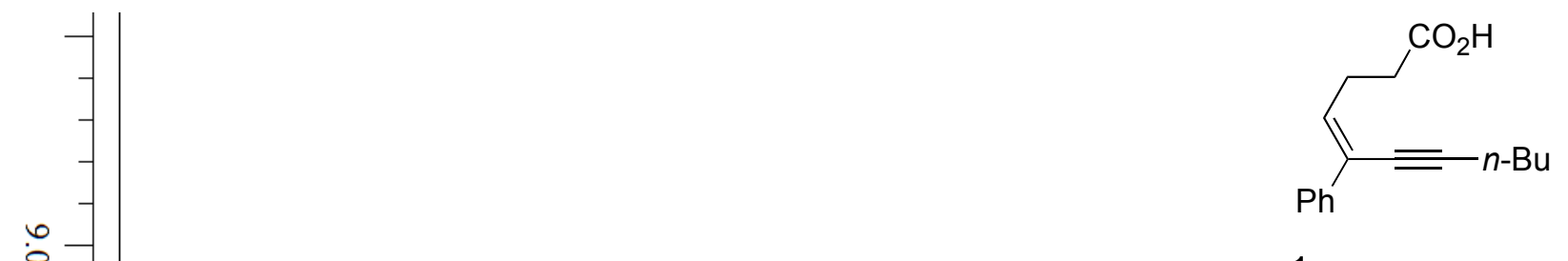

${ }^{1} \mathrm{H} 600 \mathrm{MHz}$ NMR

2.3.20

$\infty$

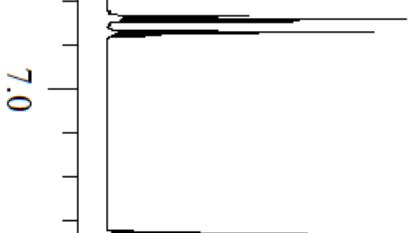

on

$\ddot{0}$

$w$

o

0

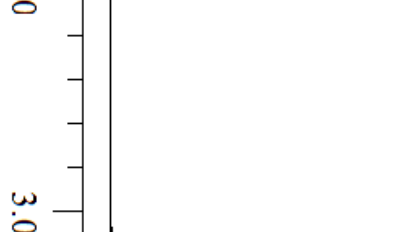

$-\sqrt{\square-}$

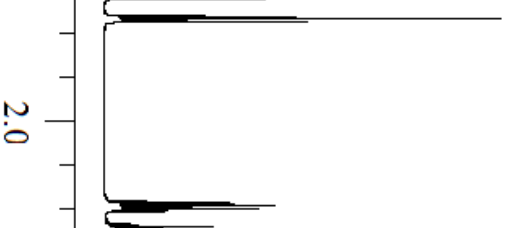

$\circ$

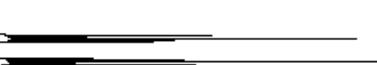

$\stackrel{0}{0}$ 

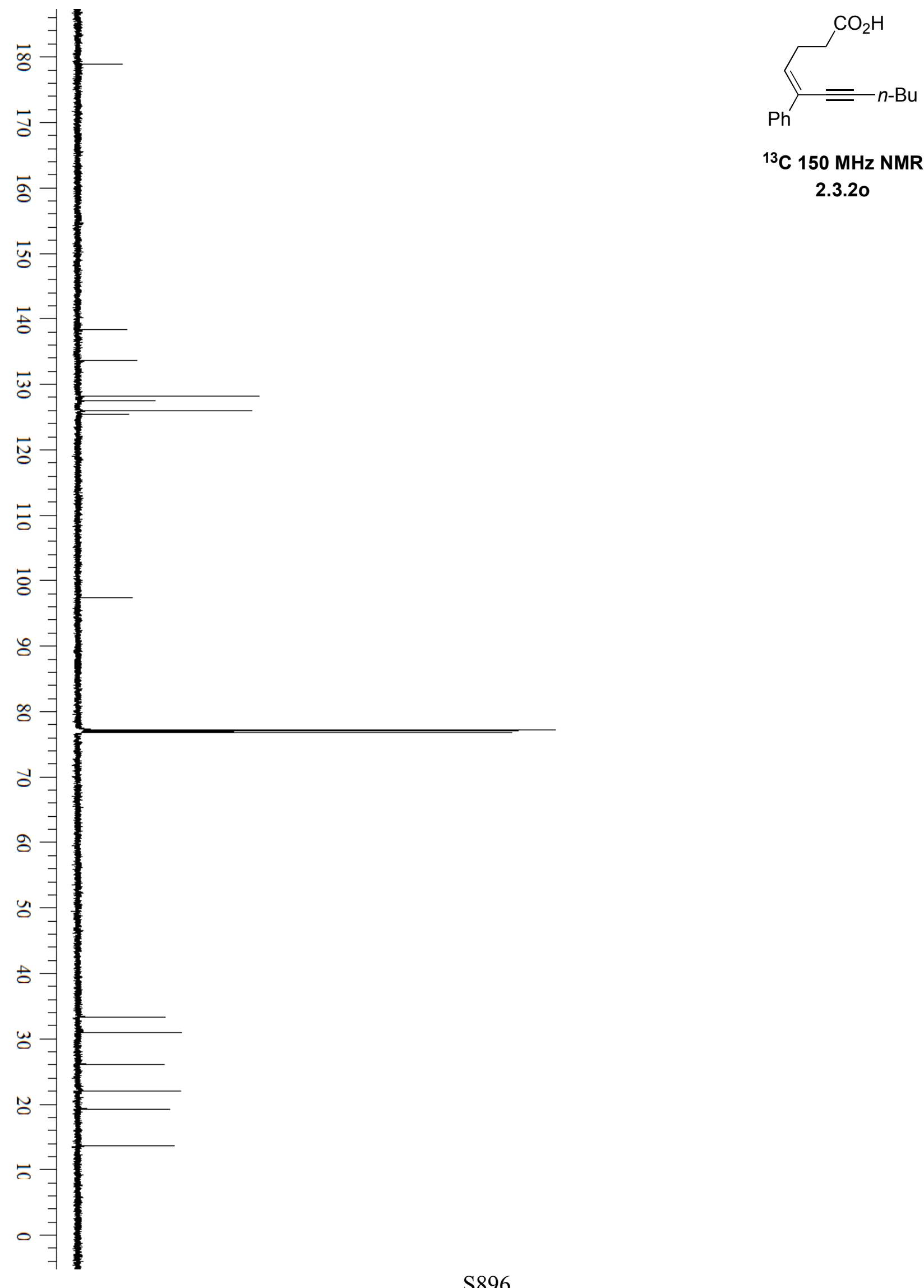

${ }^{13} \mathrm{C} 150 \mathrm{MHz}$ NMR

2.3.20 


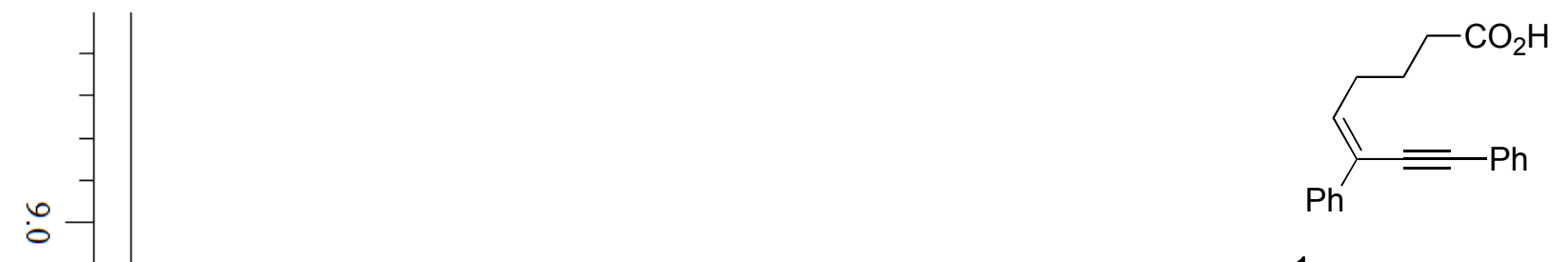

${ }^{1} \mathrm{H} 600 \mathrm{MHz}$ NMR

2.3.2p

$\infty$

○

0

0

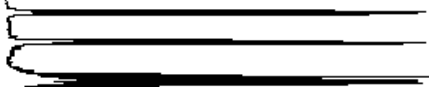

(1)
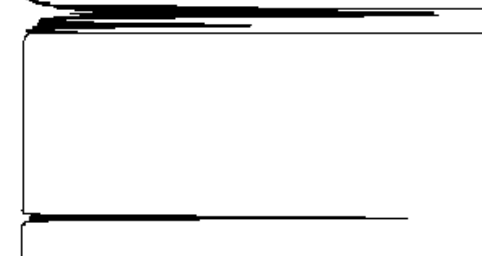

$\stackrel{\circ}{\circ}$

○

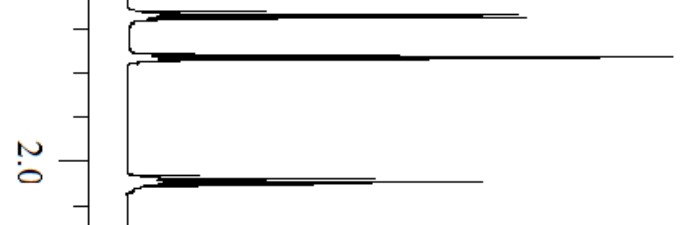

:

$\therefore$ 

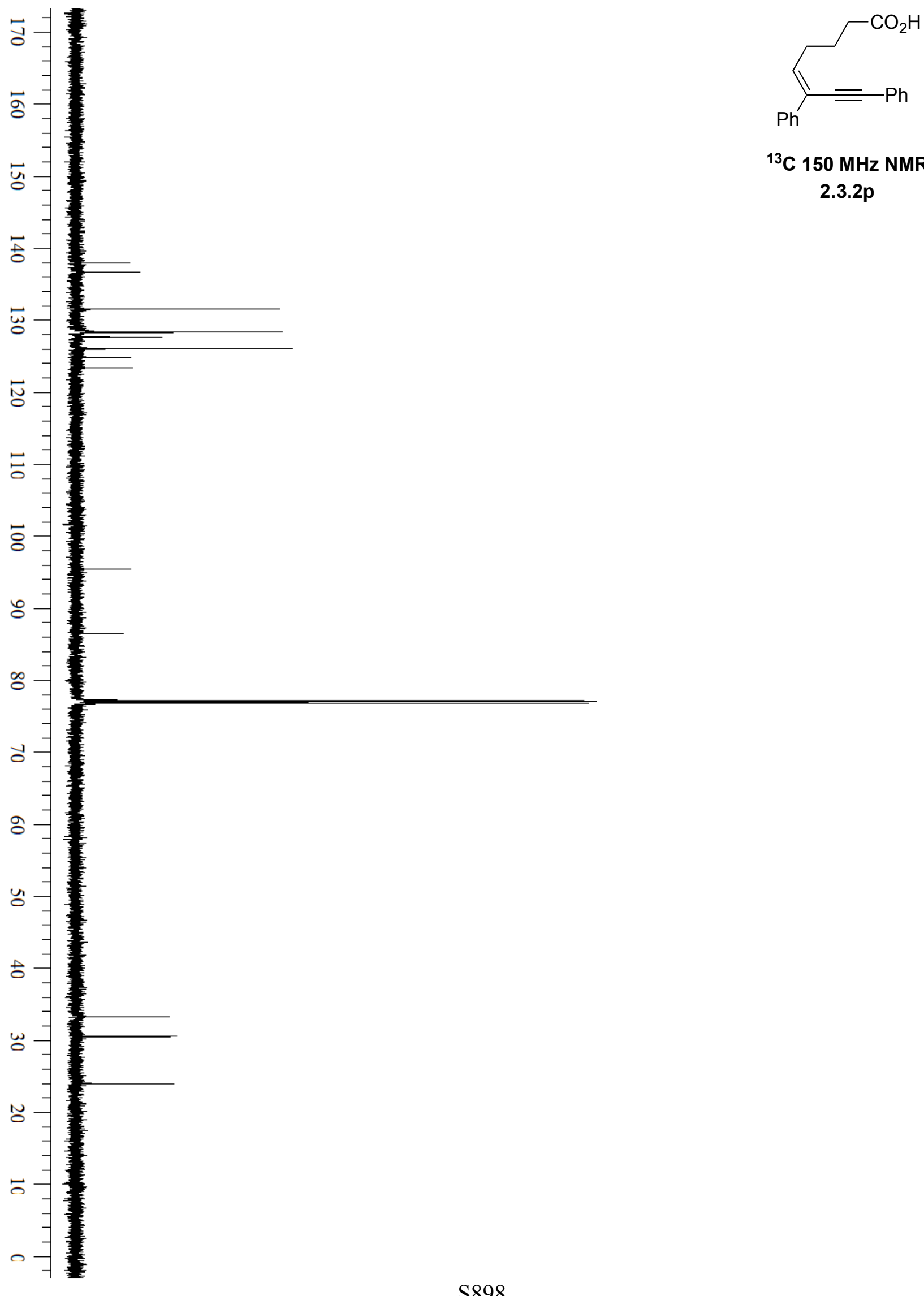

${ }^{13} \mathrm{C} 150 \mathrm{MHz}$ NMR

2.3.2p 

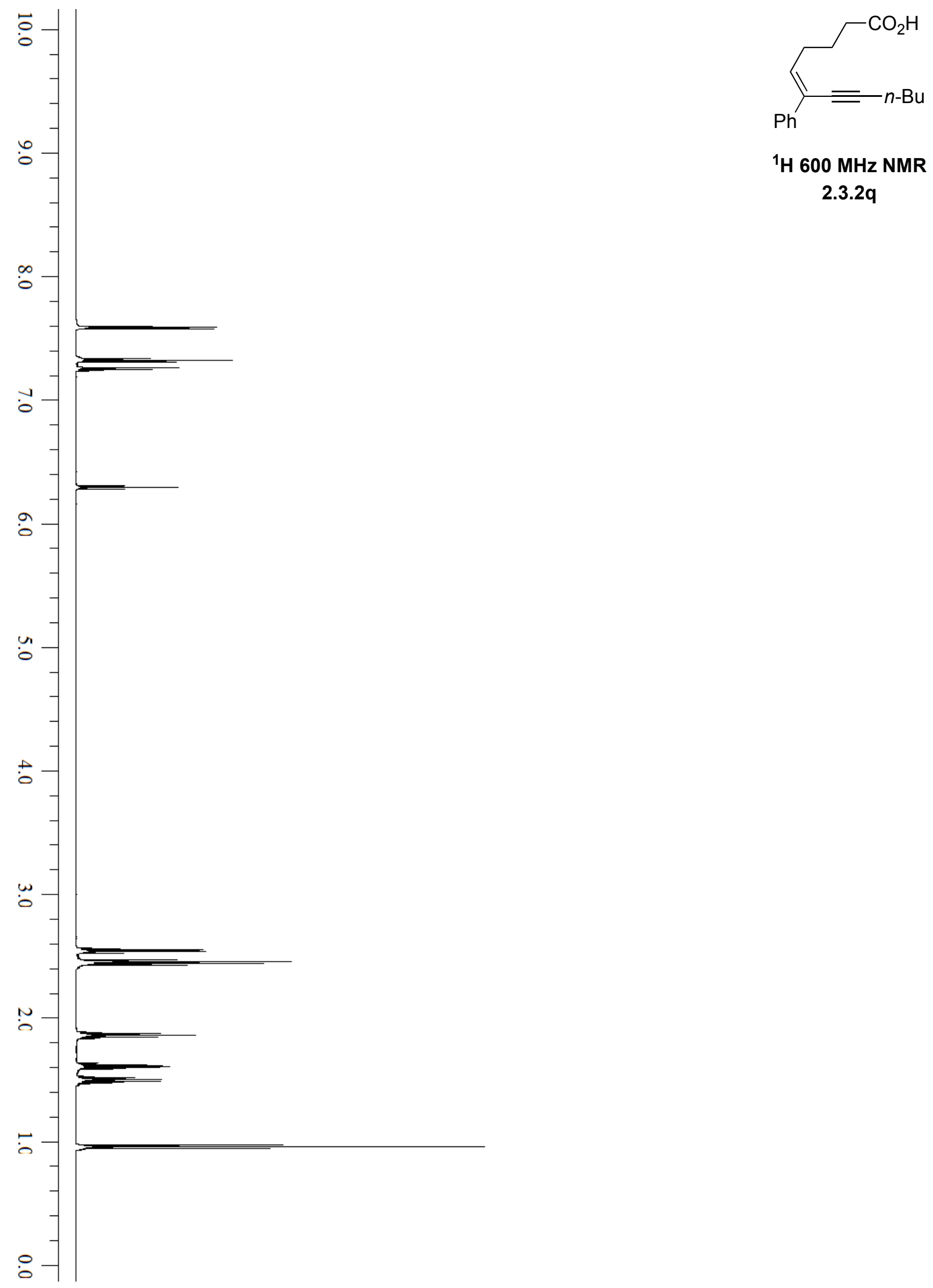

${ }^{1} \mathrm{H} 600 \mathrm{MHz}$ NMR

2.3.2q 

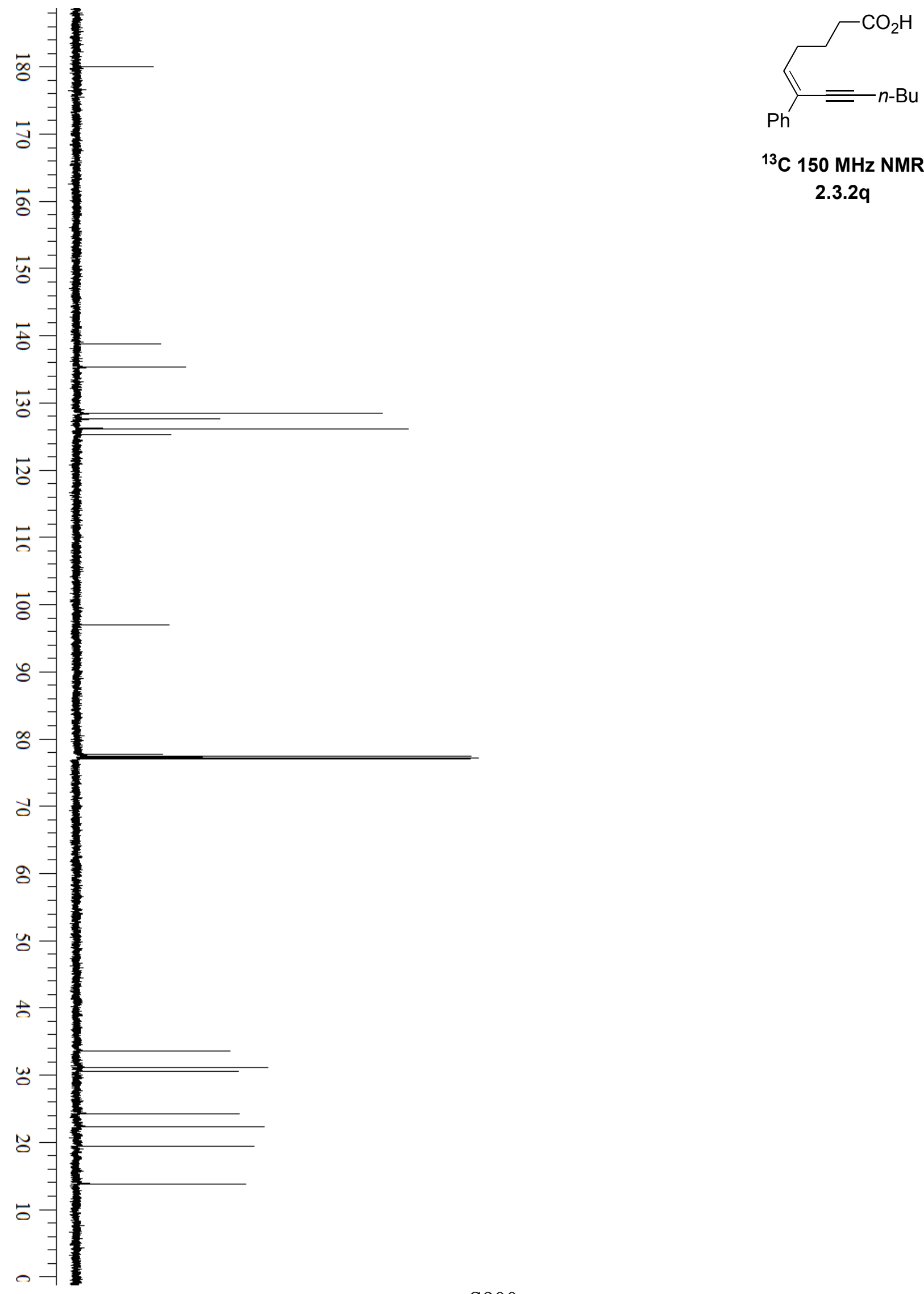

${ }^{13} \mathrm{C} 150 \mathrm{MHz}$ NMR

2.3.2q 

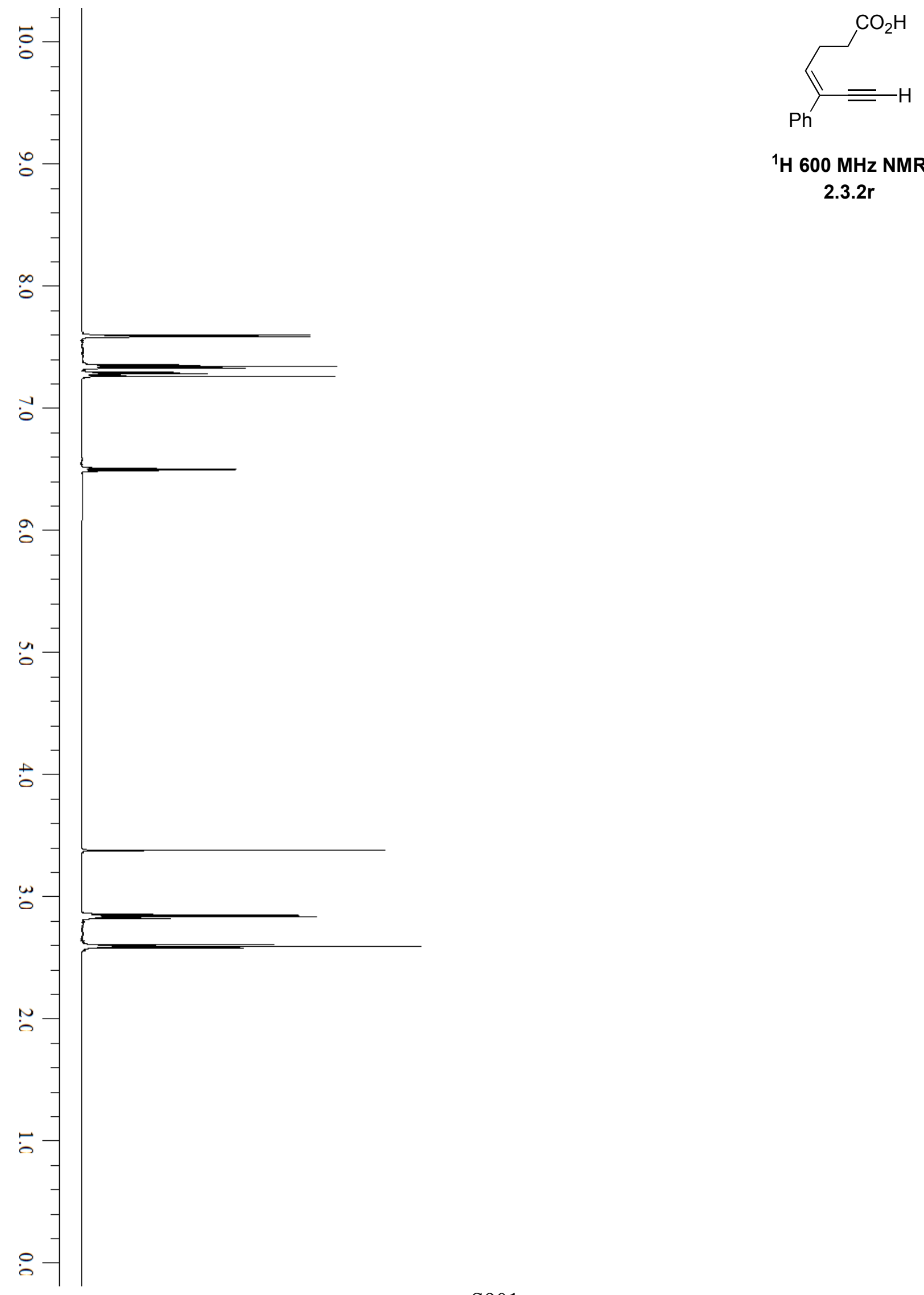

${ }^{1} \mathrm{H} 600 \mathrm{MHz}$ NMR

2.3.2r 

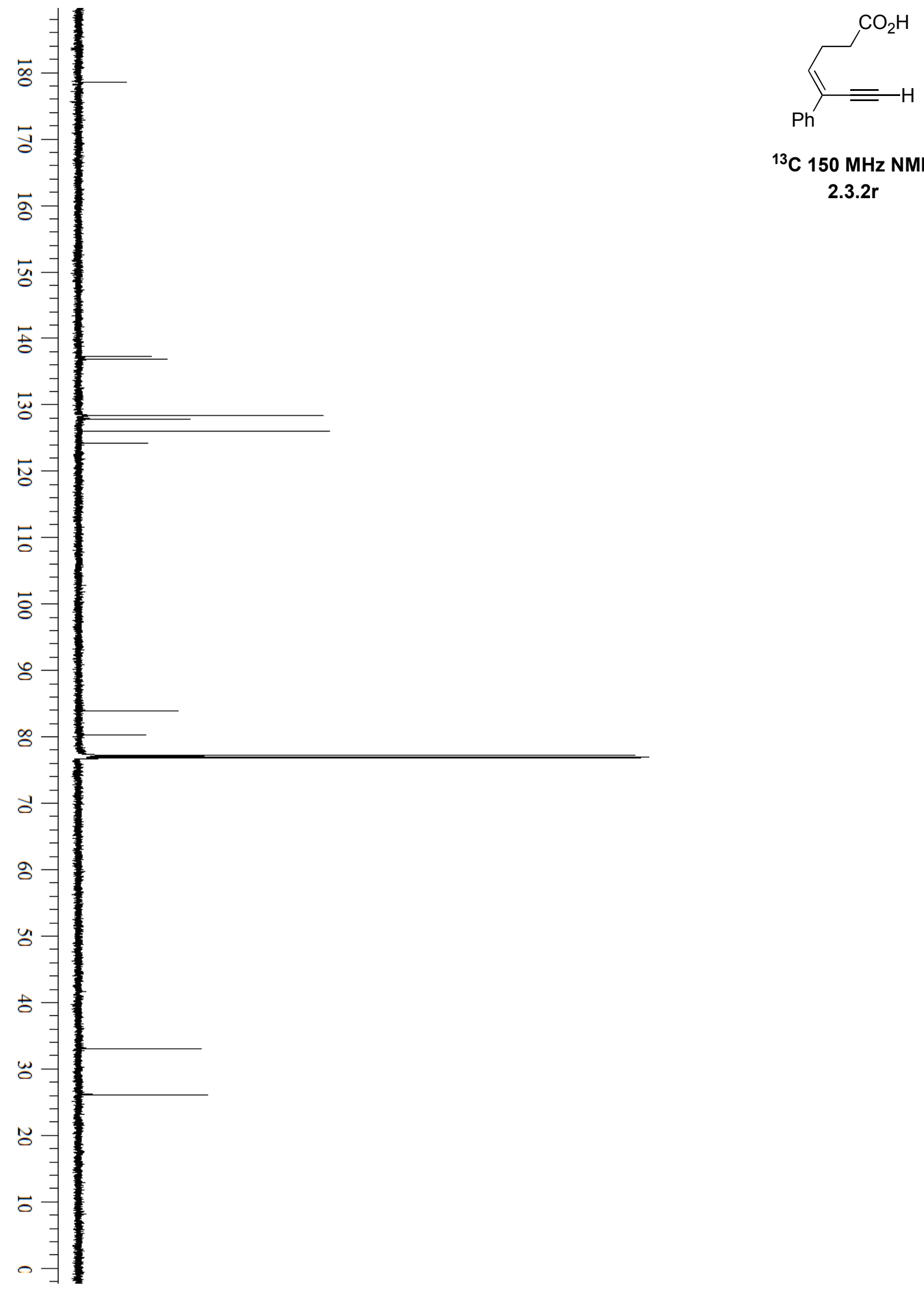

${ }^{13} \mathrm{C} 150 \mathrm{MHz}$ NMR

2.3.2r 

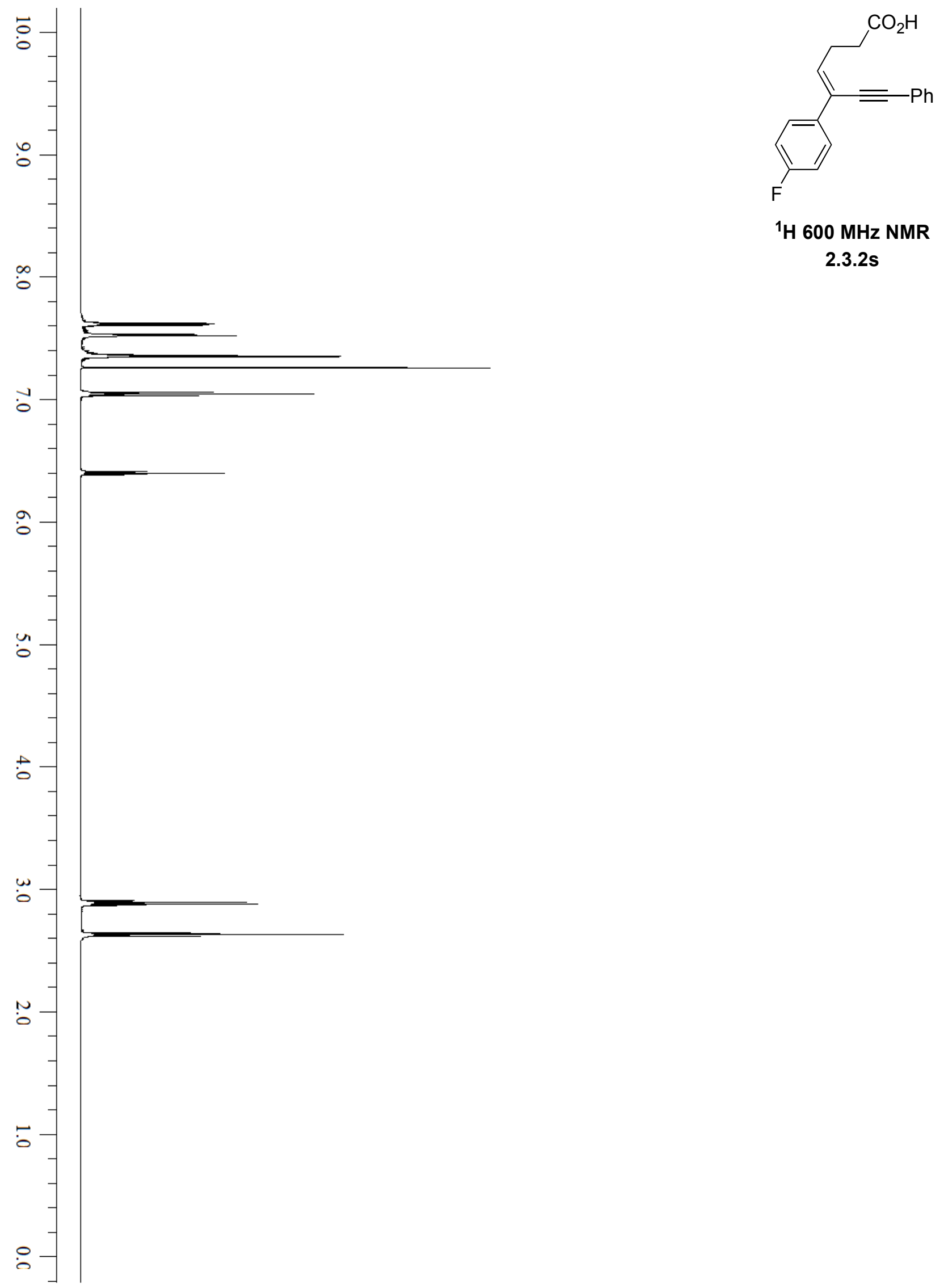

${ }^{1} \mathrm{H} 600 \mathrm{MHz}$ NMR

2.3.2s 


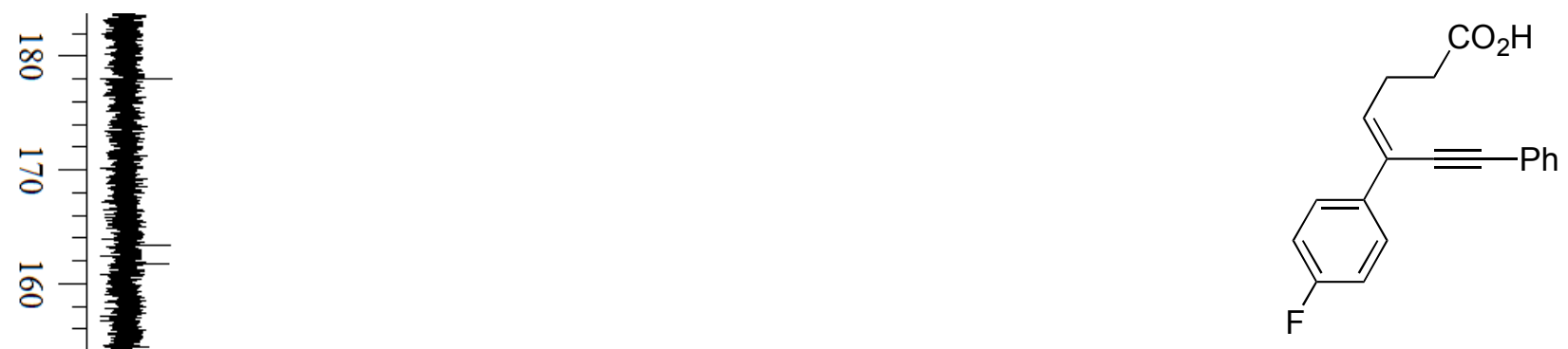

${ }^{13} \mathrm{C} 150 \mathrm{MHz}$ NMR

2.3.2s 


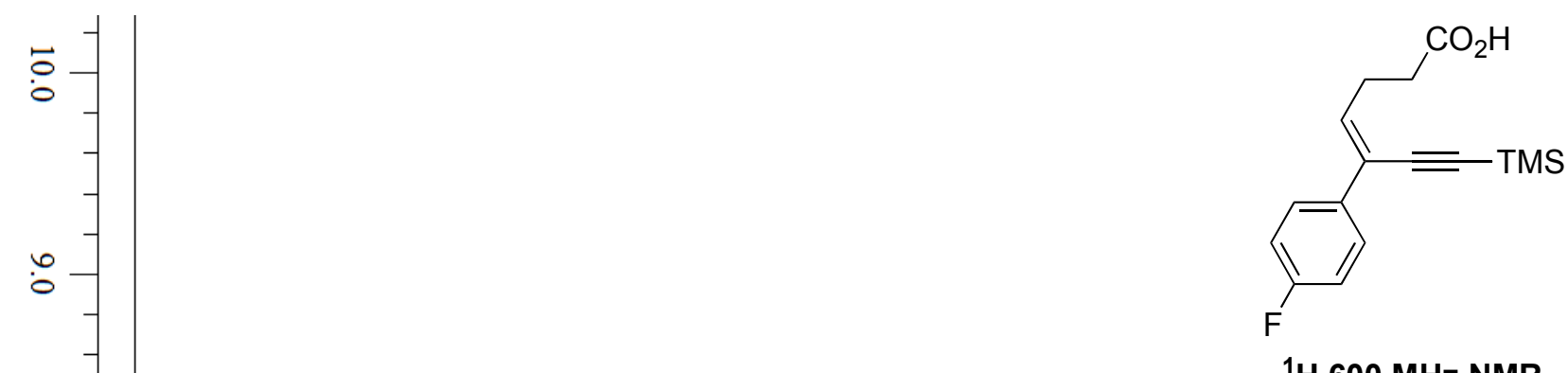

${ }^{1} \mathrm{H} 600 \mathrm{MHz}$ NMR

2.3.2t

$\infty$

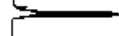

$\square$

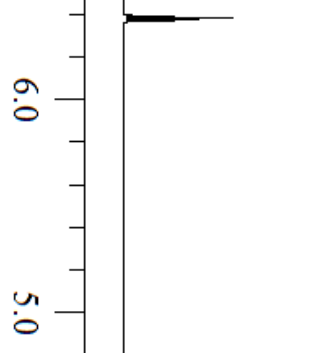

c

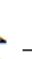

-

is

-

$\stackrel{n}{0}$

๑

$\therefore-1=$ 


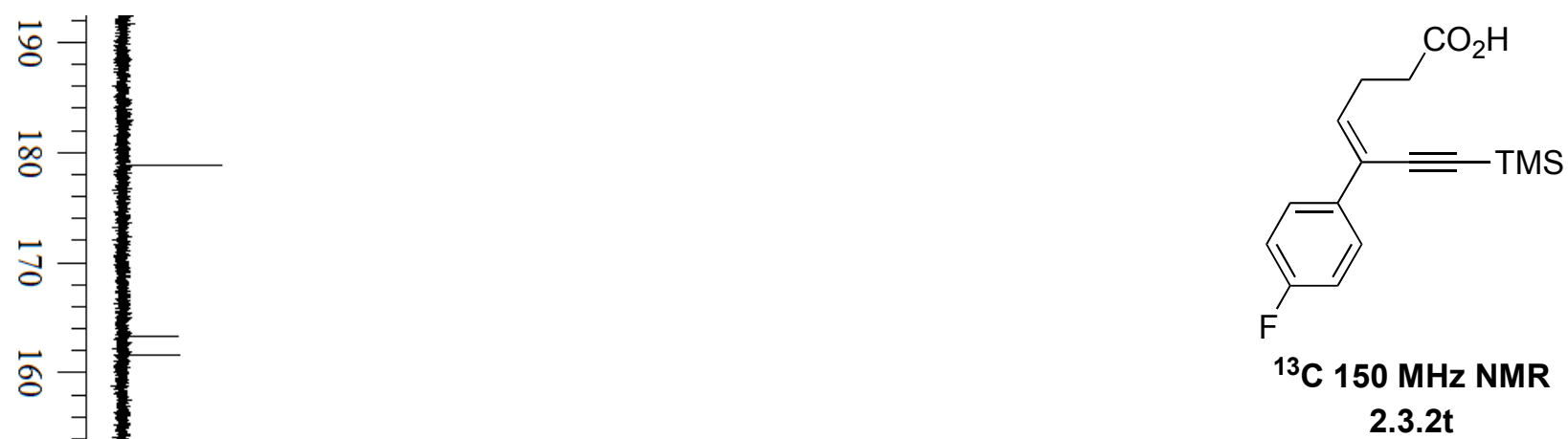

봉

点

岁寻

ㄴ.

E

응

๖

$\stackrel{\infty}{\circ}$

o

\&

4.

t

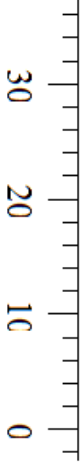

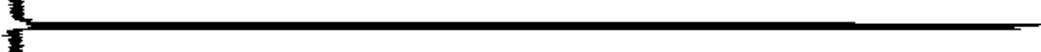

-

-

-

- 

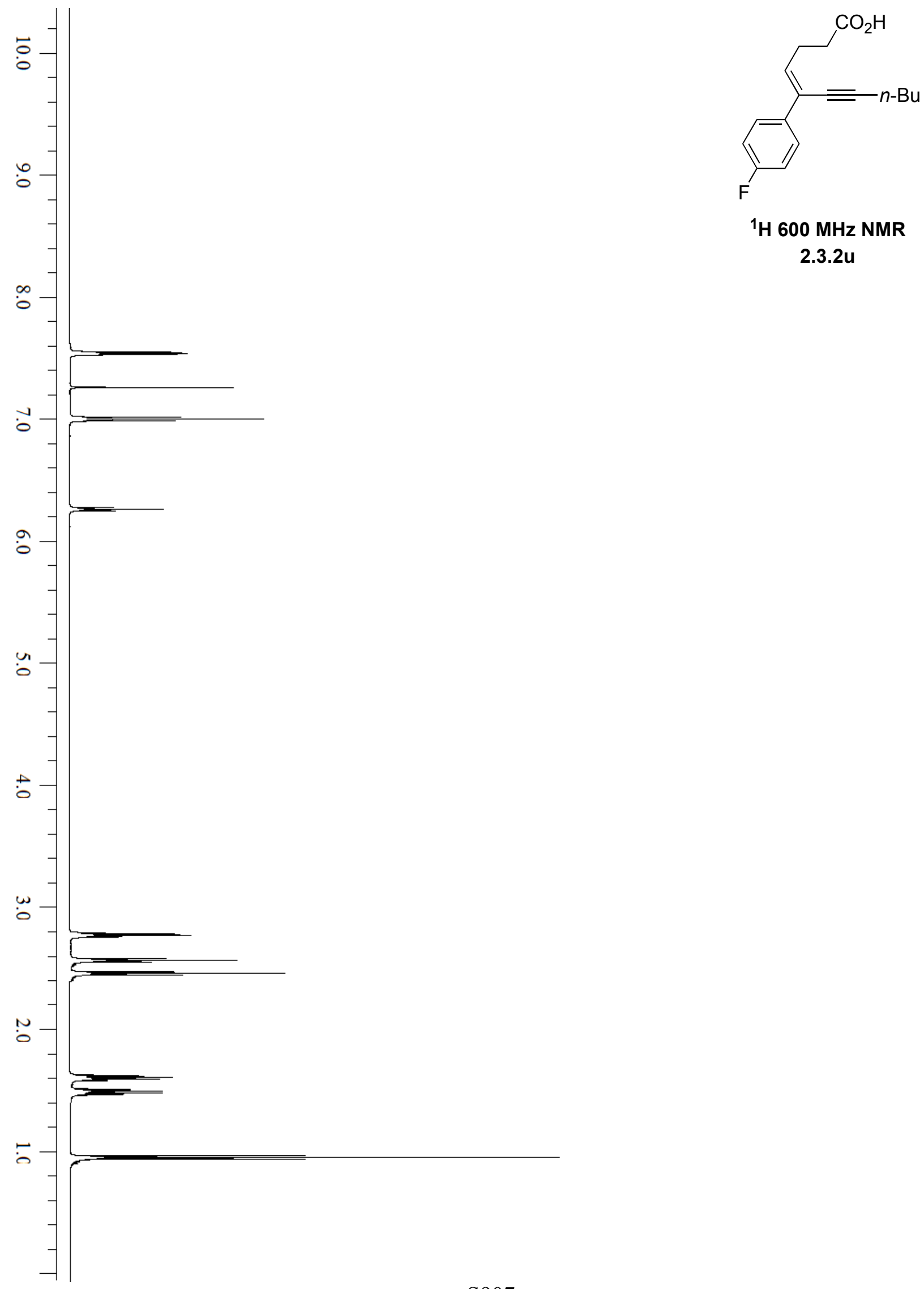

${ }^{1} \mathrm{H} 600 \mathrm{MHz}$ NMR

2.3.2u 

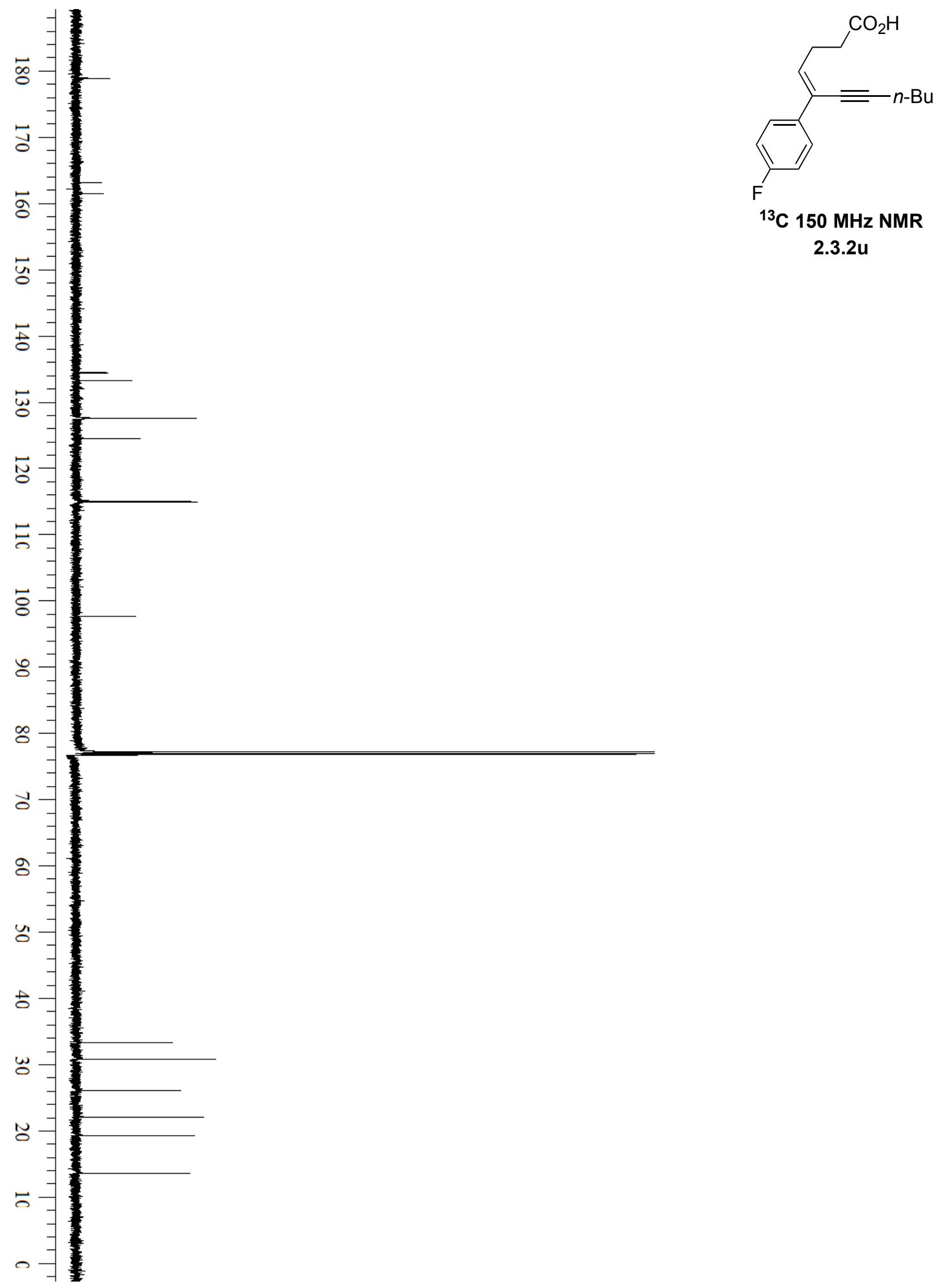

${ }^{13} \mathrm{C} 150 \mathrm{MHz}$ NMR

2.3.2u 


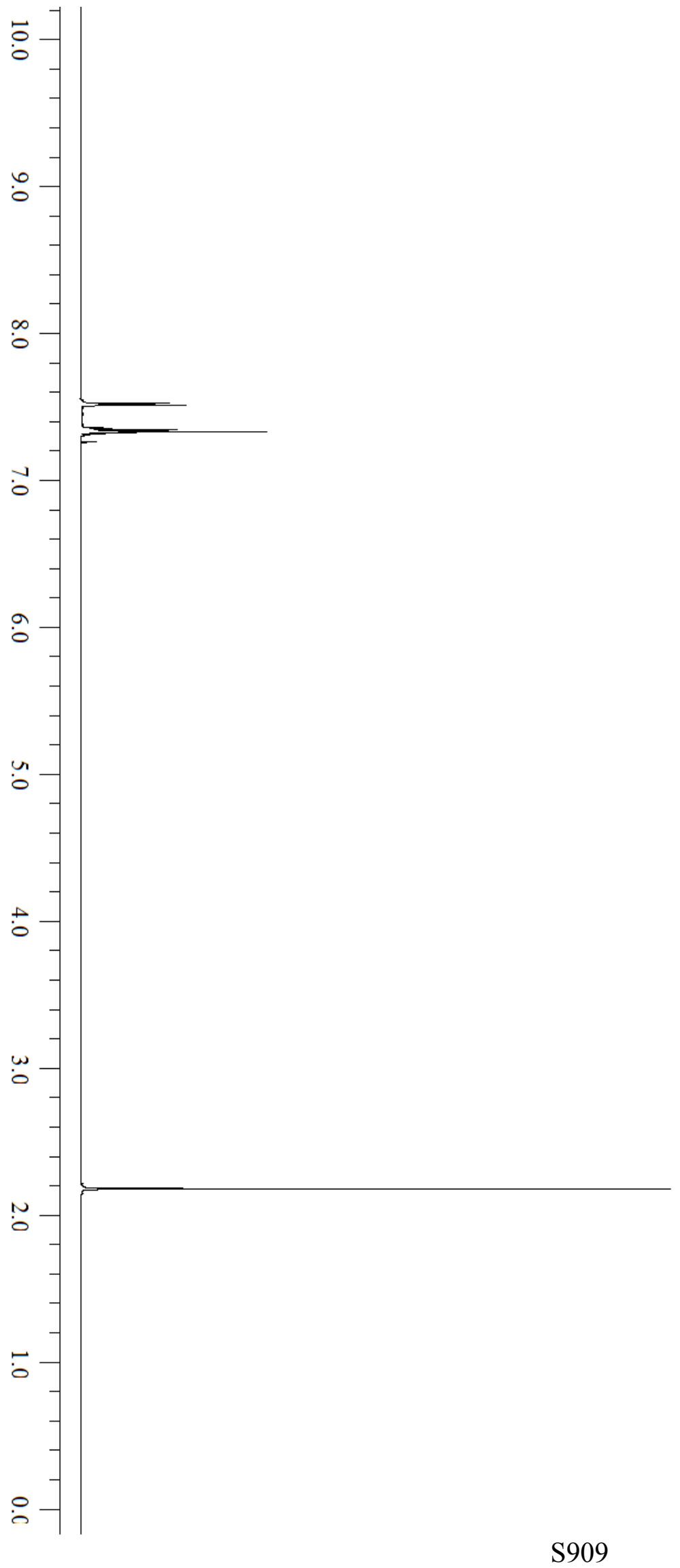

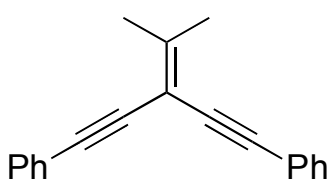

${ }^{1} \mathrm{H} 600 \mathrm{MHz}$ NMR

2.3.2v 

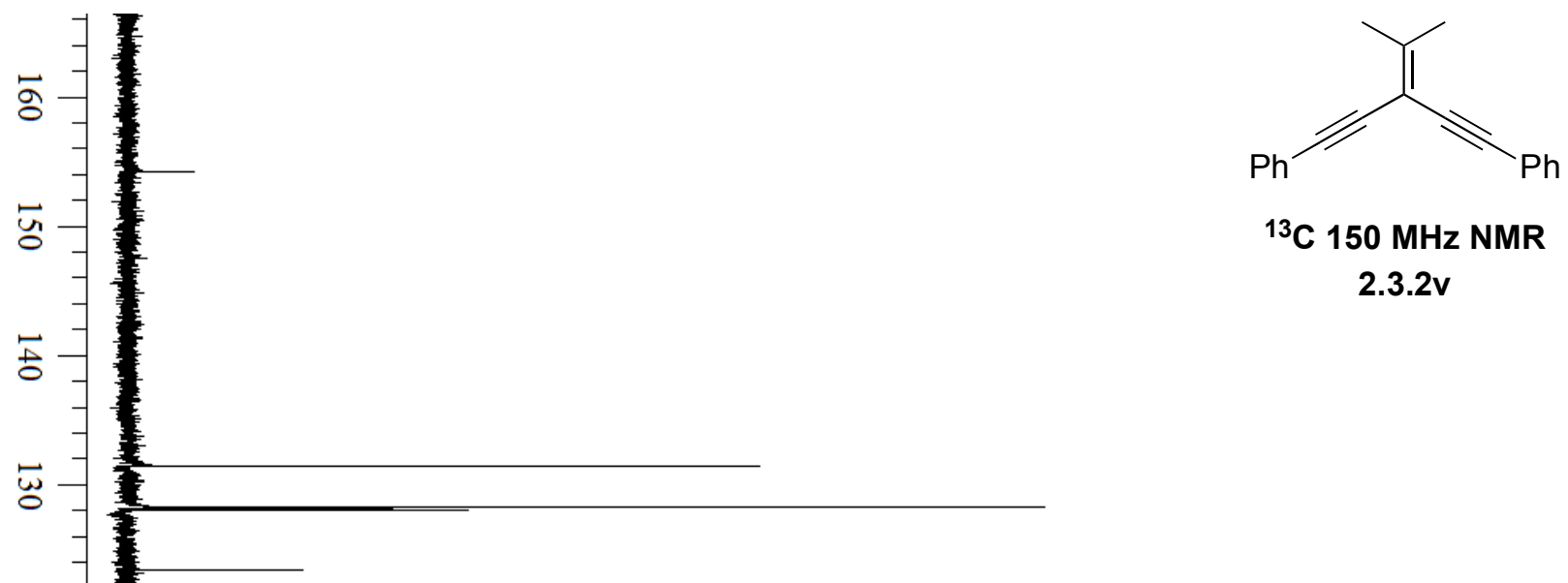

ㄱ.

。

옹

๕

$\stackrel{\circ}{\circ}$

๖

의

능-

b

w

n

N

○-

$\circ-1$

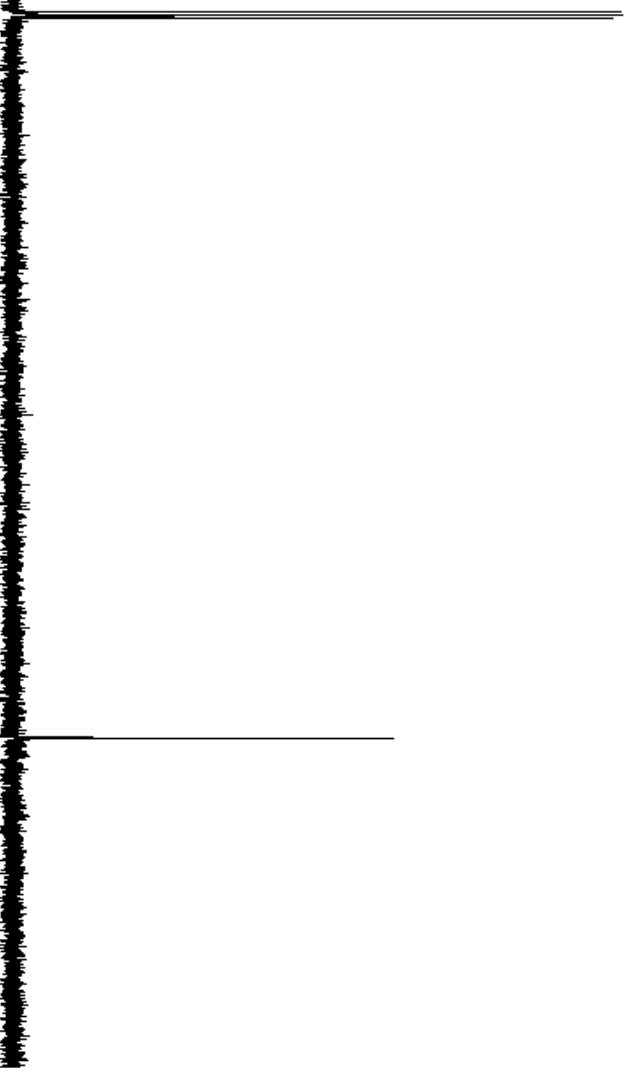



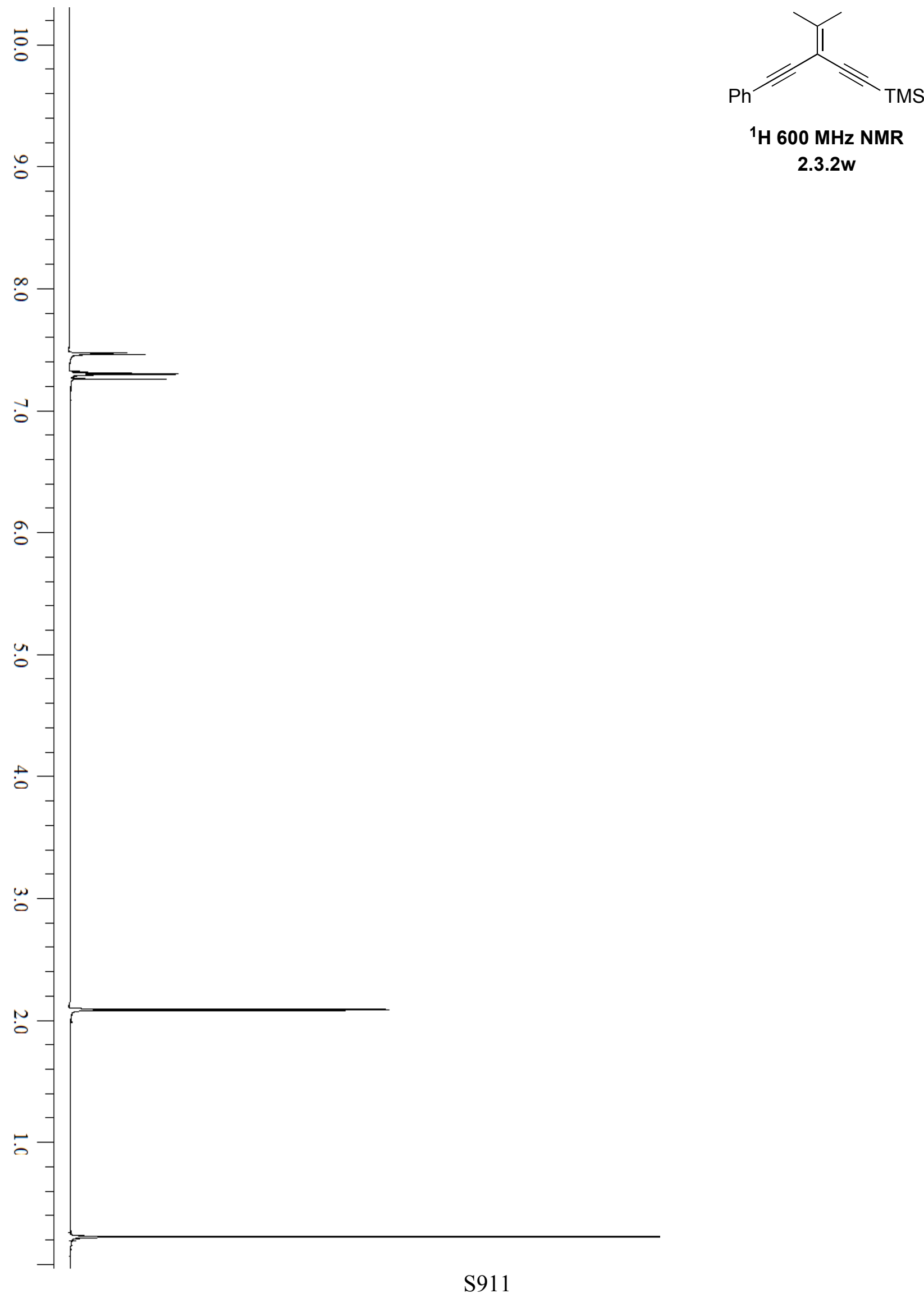

${ }^{1} \mathrm{H} 600 \mathrm{MHz}$ NMR

2.3.2w 


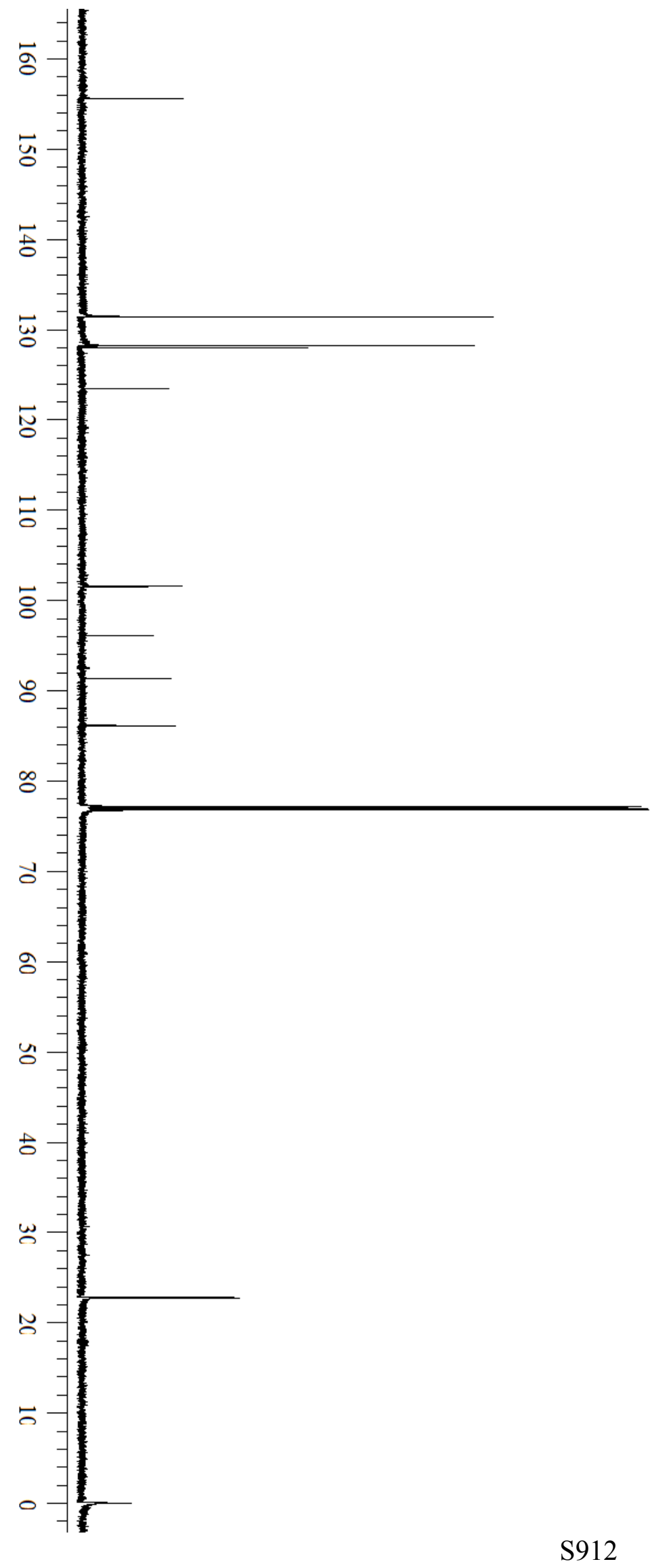

${ }^{13} \mathrm{C} 150 \mathrm{MHz}$ NMR

2.3.2w 

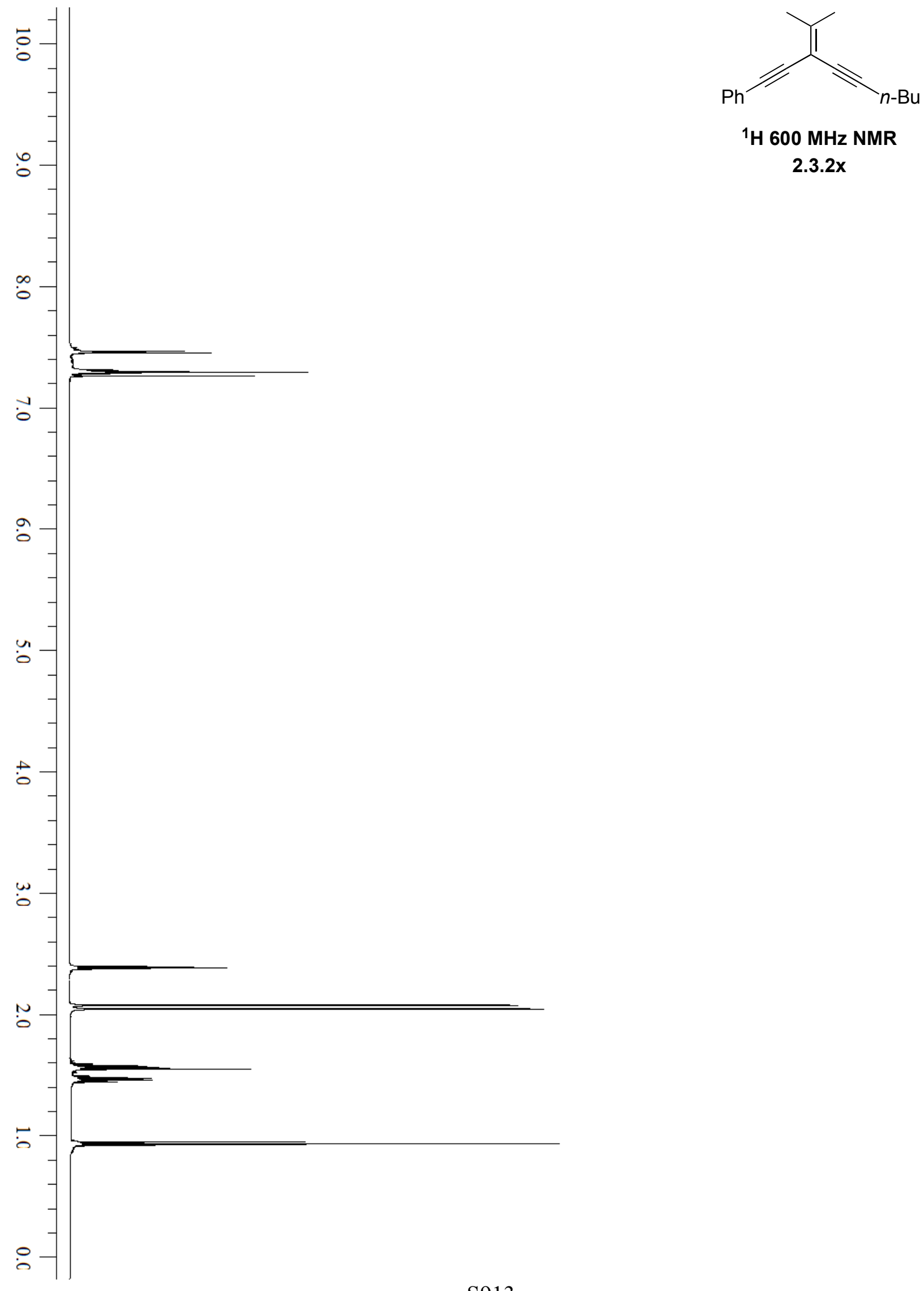

${ }^{1} \mathrm{H} 600 \mathrm{MHz}$ NMR

2.3.2x 


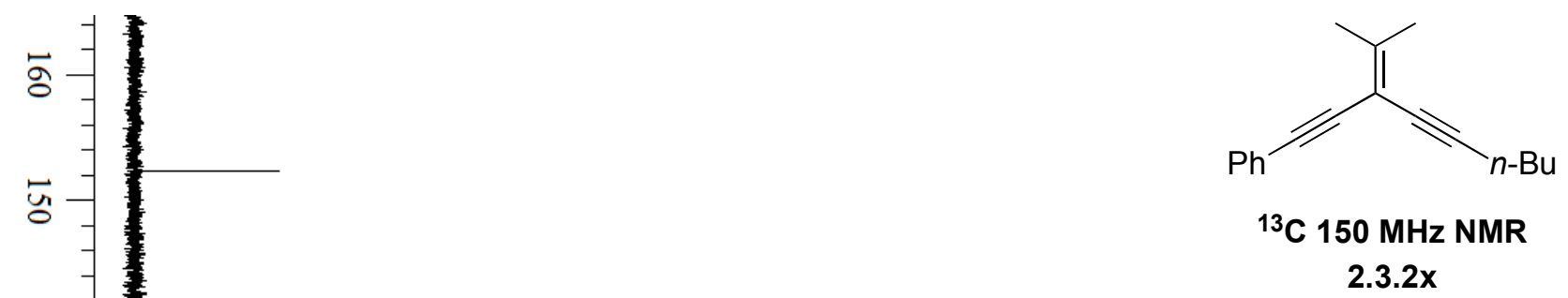

2.3.2x 


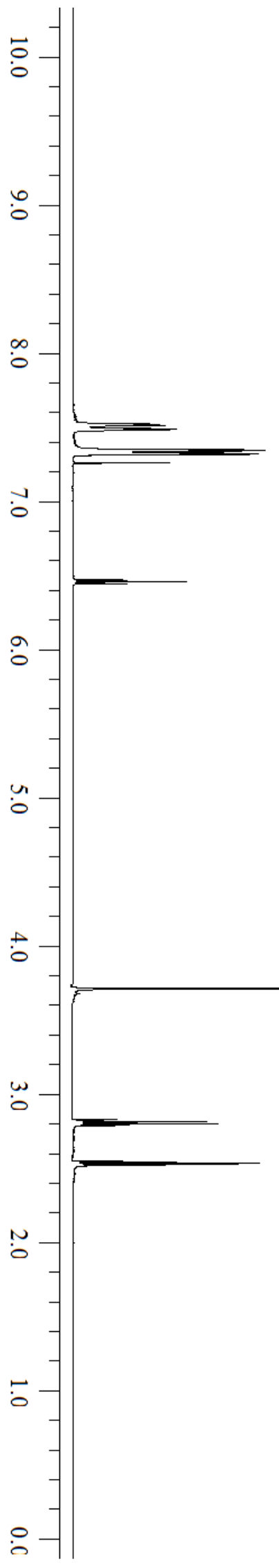

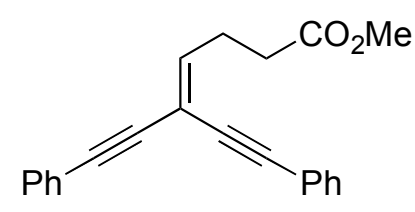

${ }^{1} \mathrm{H} 600 \mathrm{MHz}$ NMR

2.3.2y 

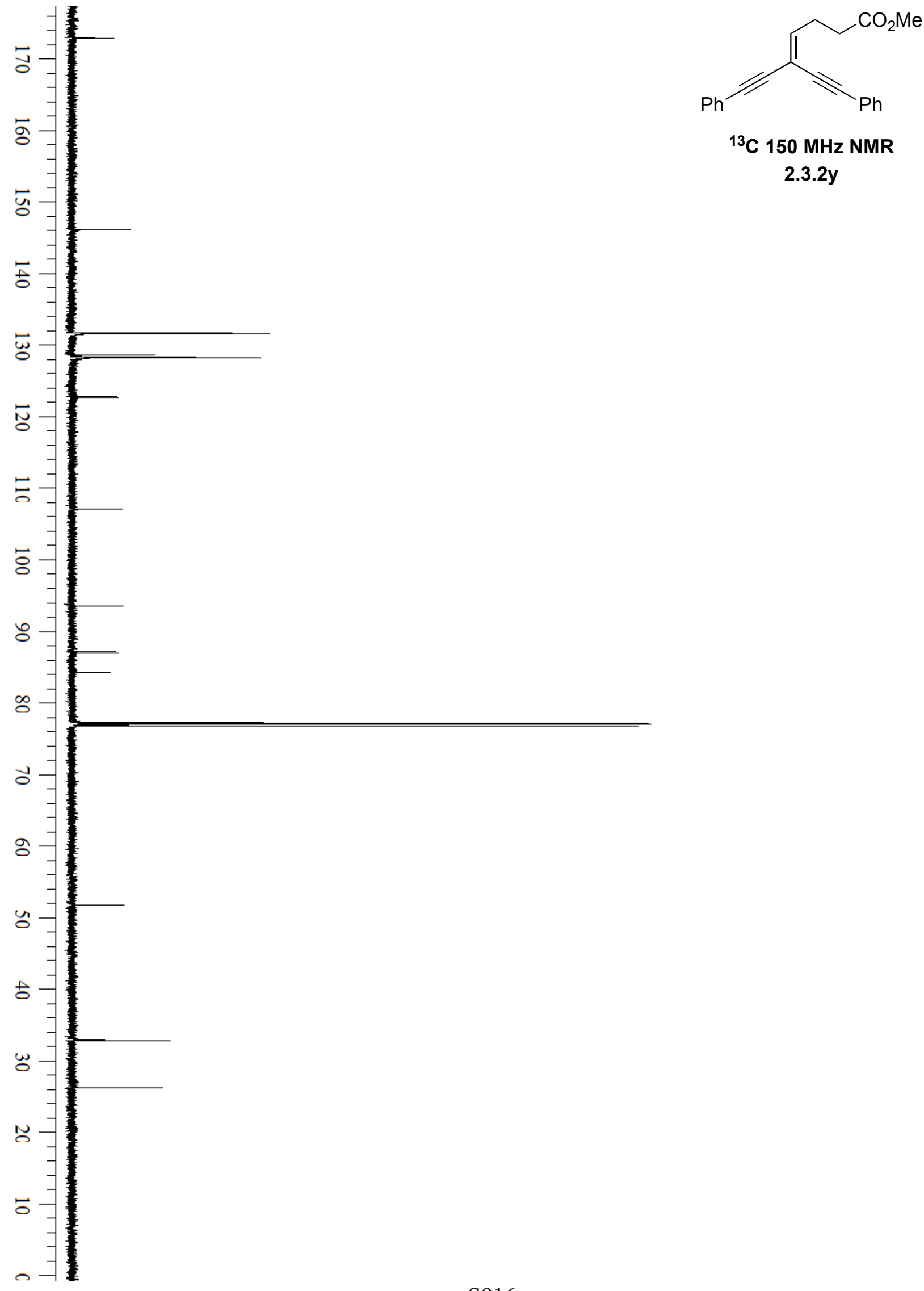


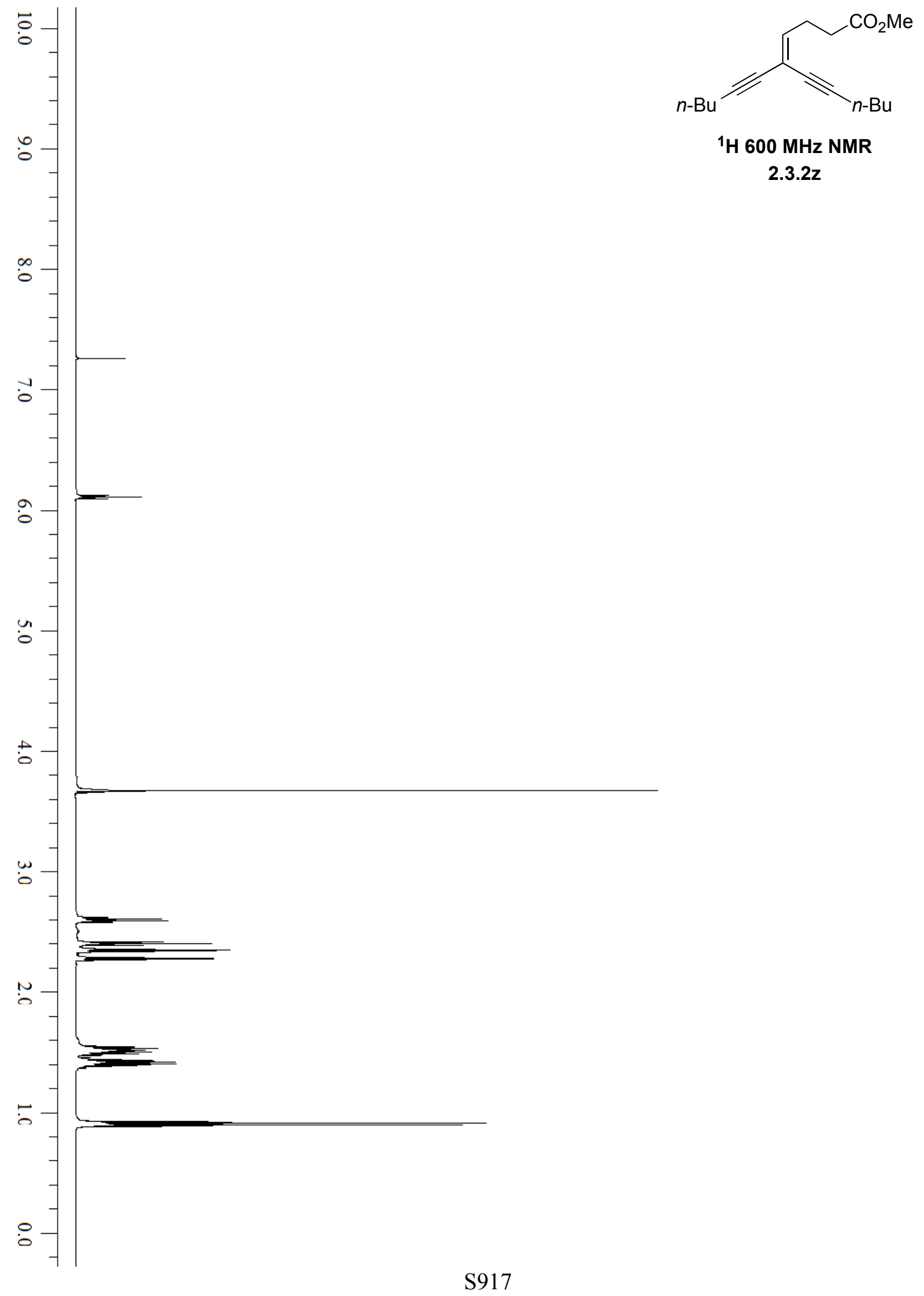



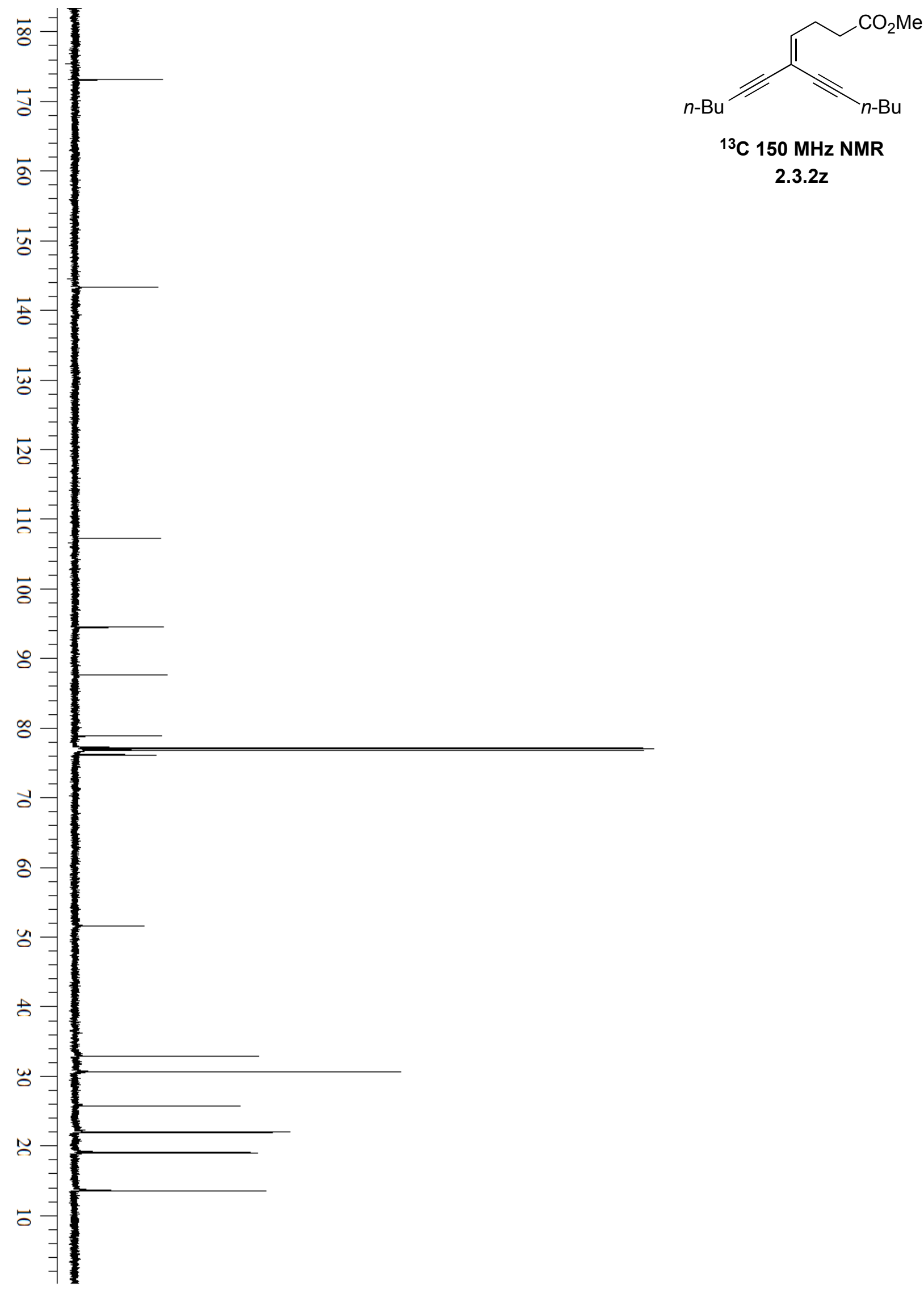

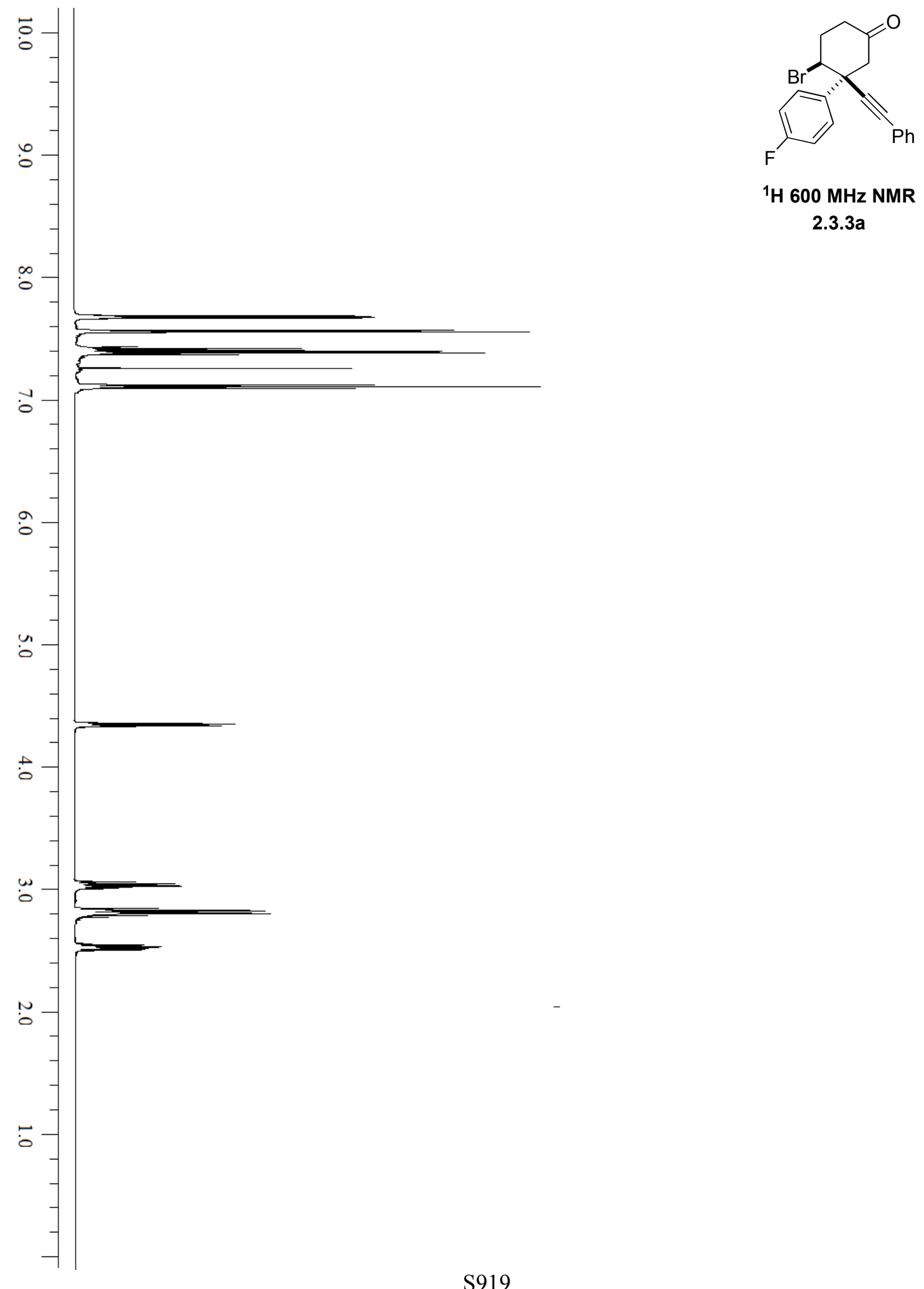

\section{${ }^{1} \mathrm{H} 600 \mathrm{MHz}$ NMR}

2.3.3a 

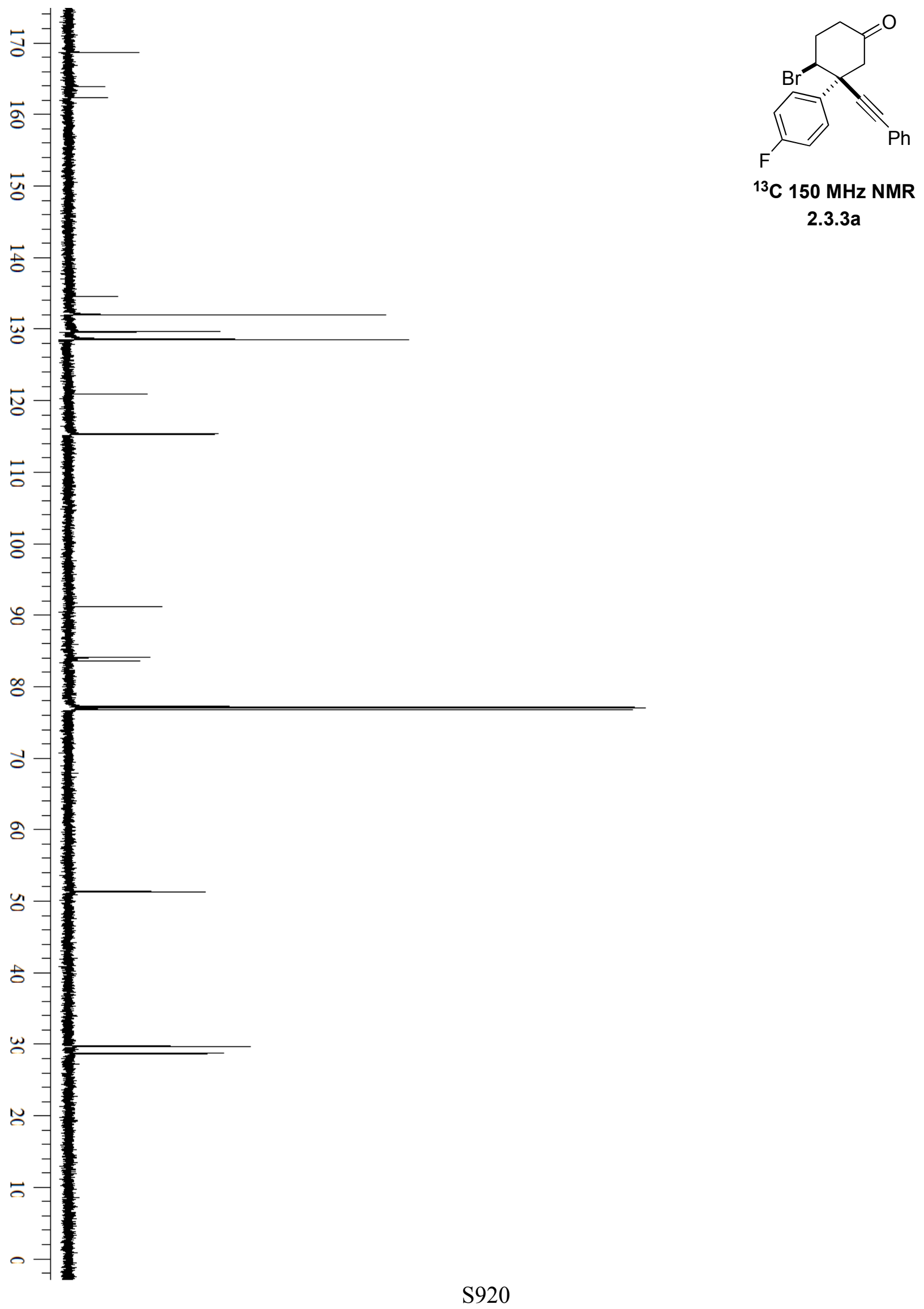

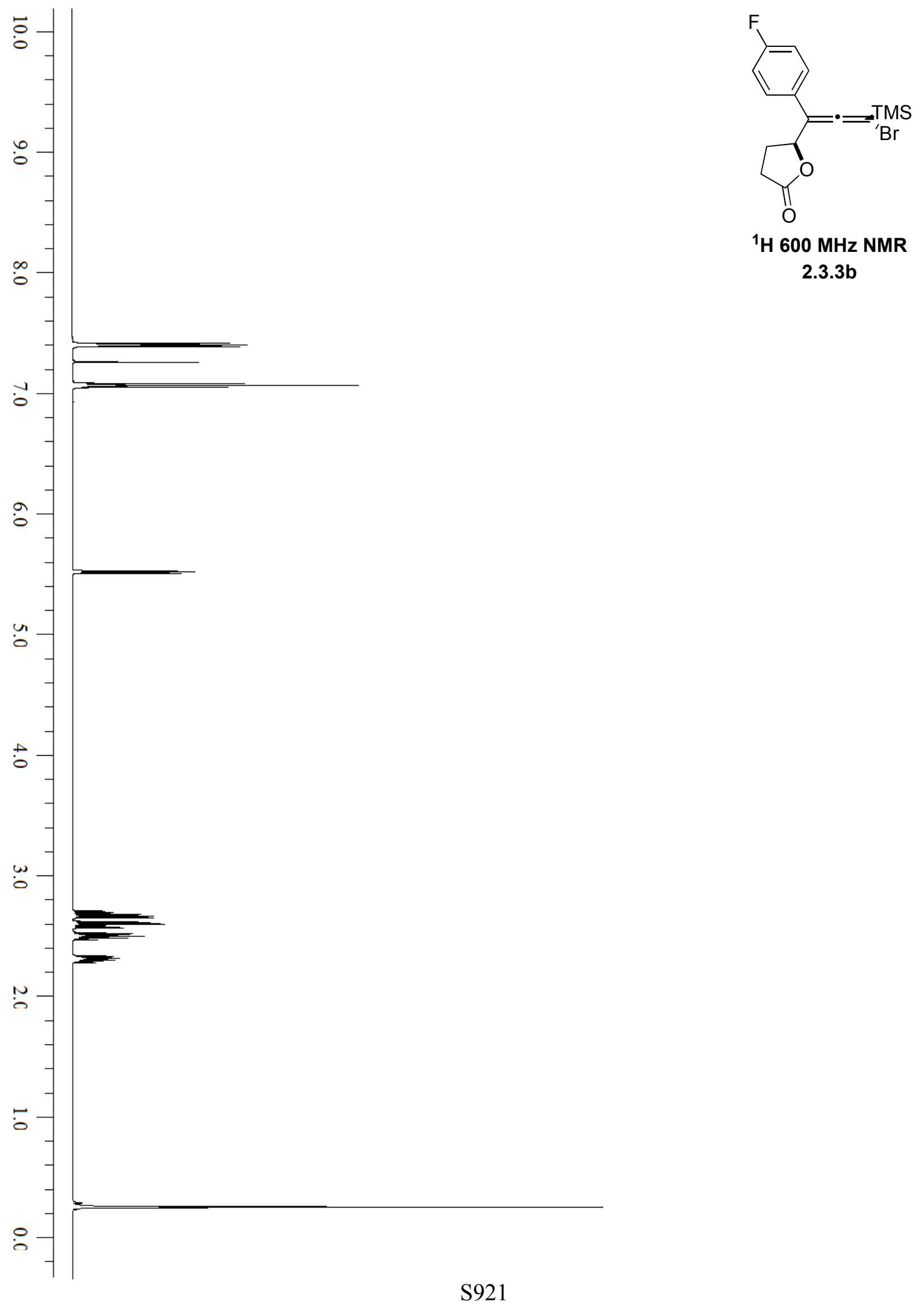

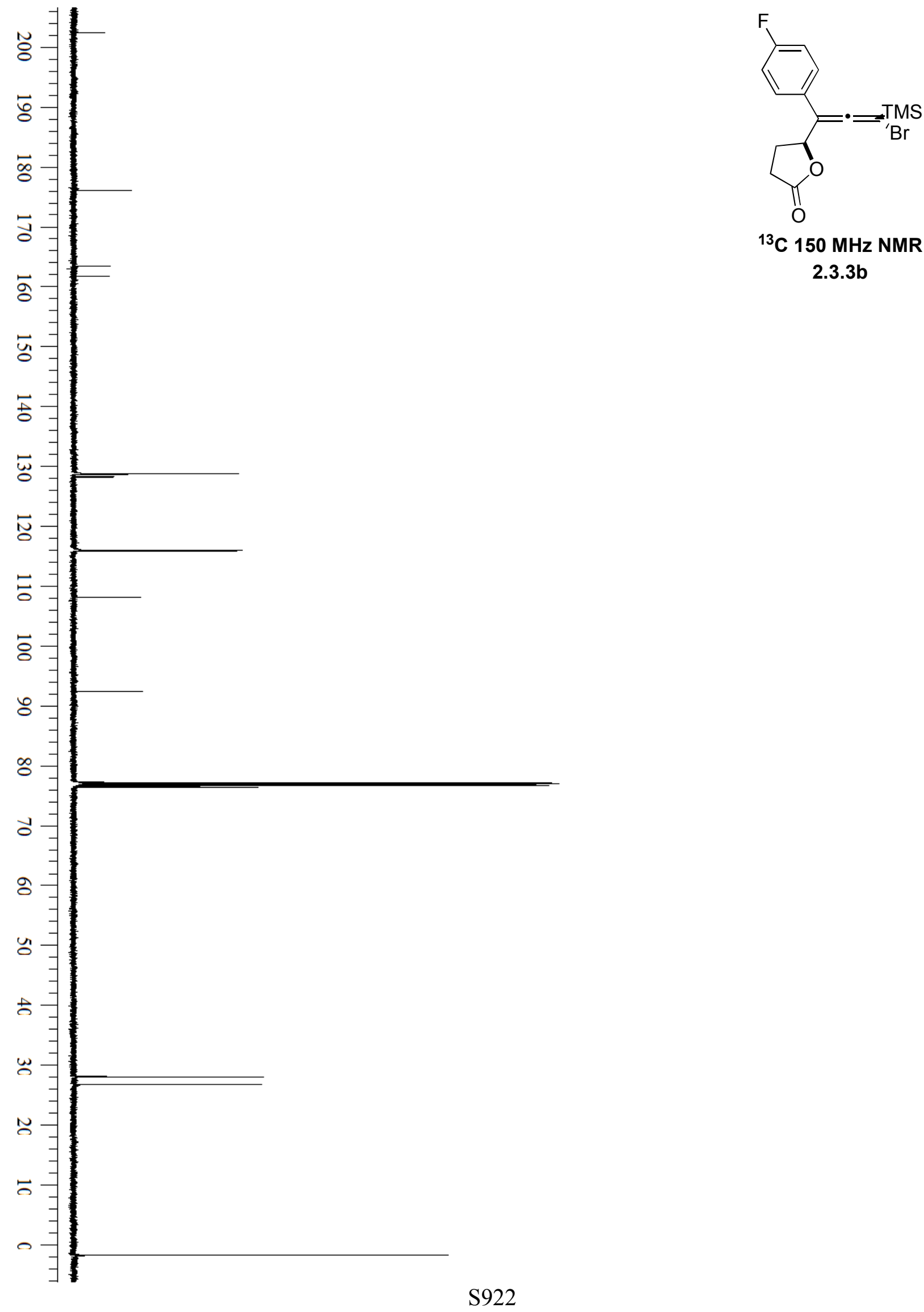

${ }^{13} \mathrm{C} 150 \mathrm{MHz}$ NMR

2.3.3b 


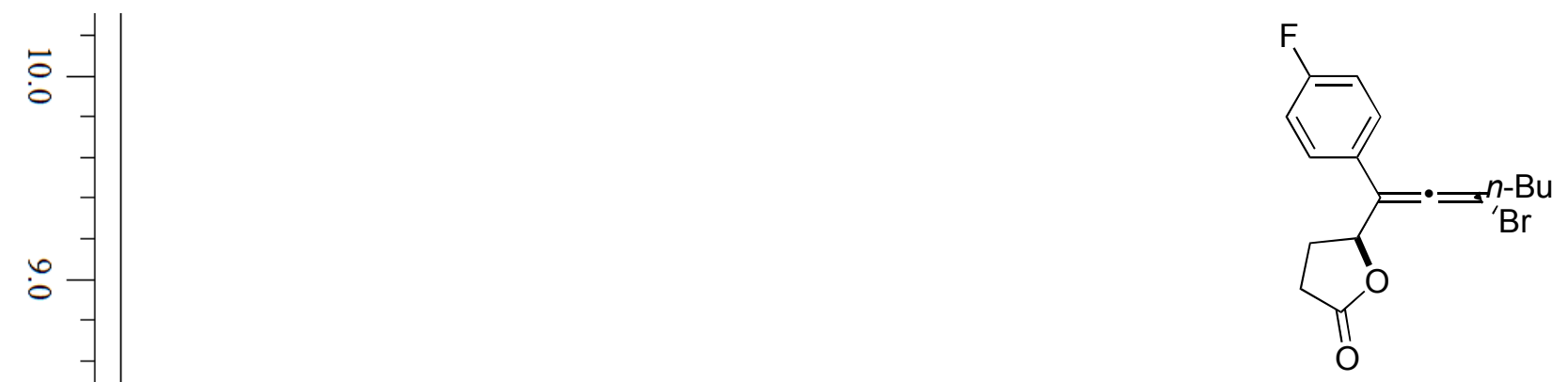

${ }^{1} \mathrm{H} 600 \mathrm{MHz}$ NMR

2.3.3c

$\infty$

0

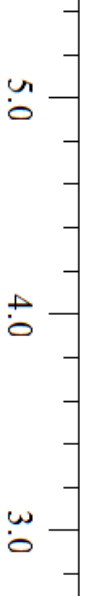

0
0
0

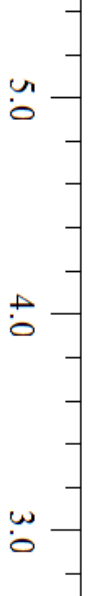

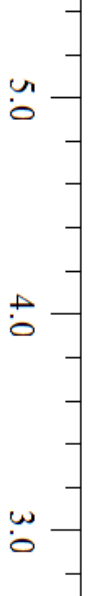

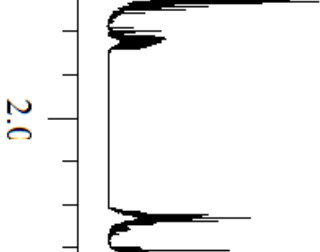

மே

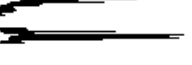

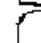
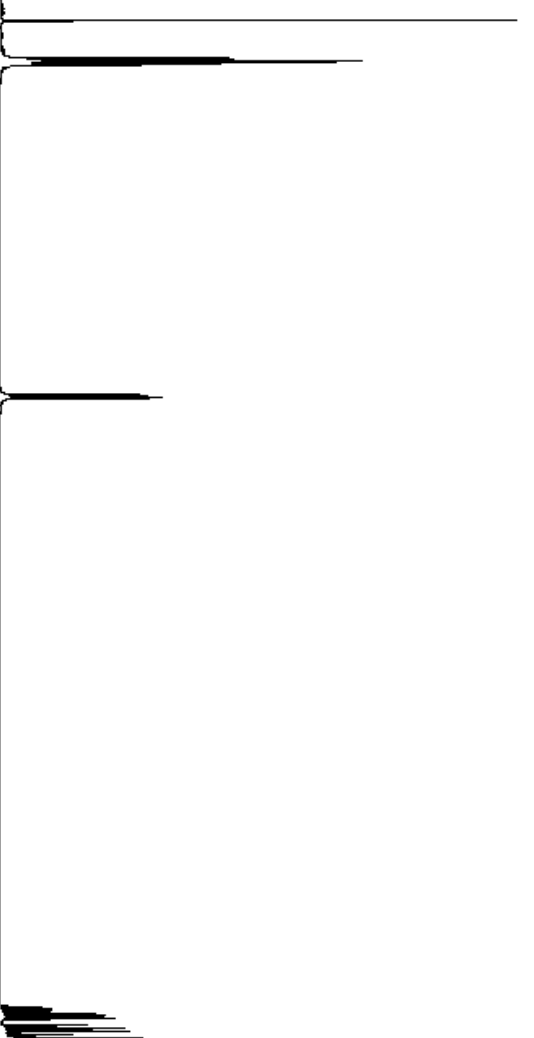


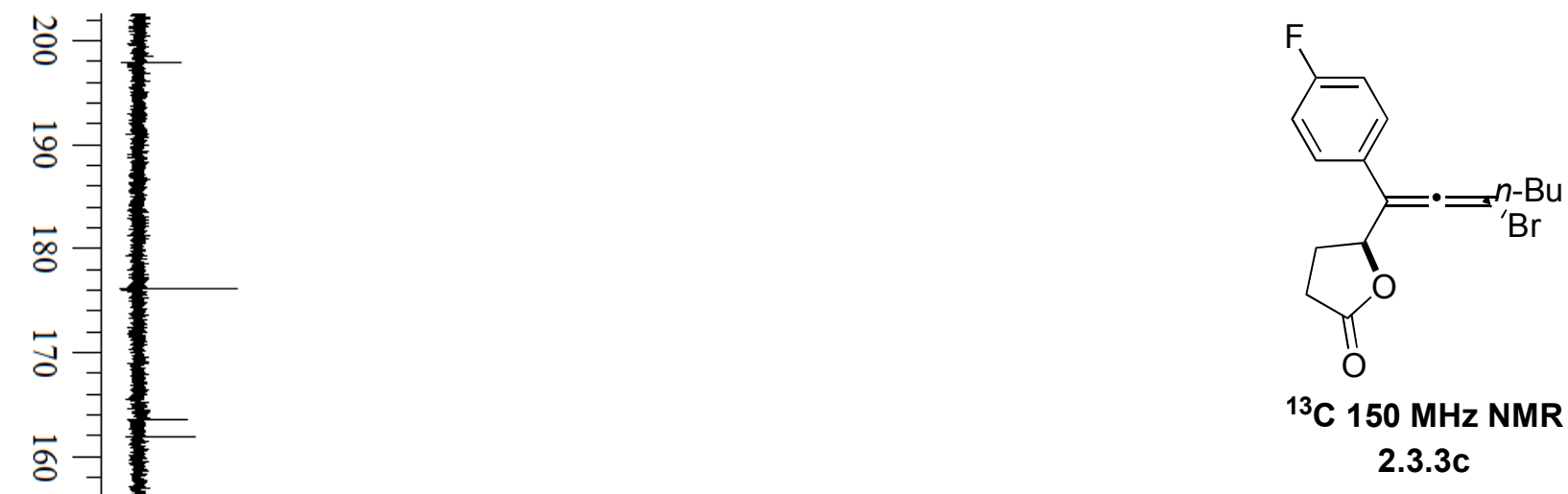




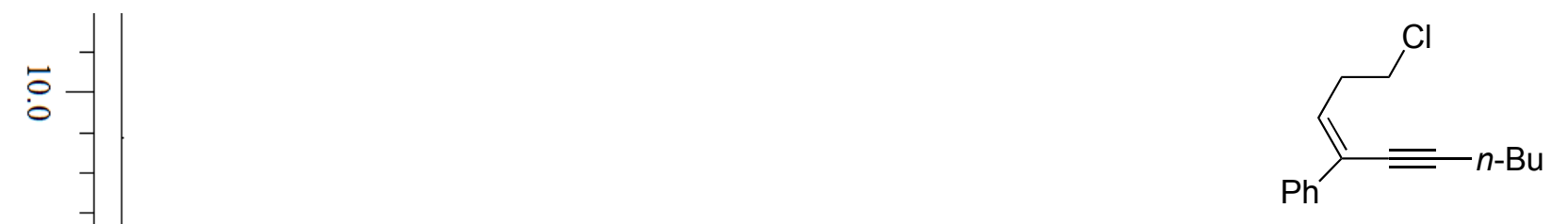

:0

${ }^{1} \mathrm{H} 600 \mathrm{MHz}$ NMR

2.3.5a

$\infty$

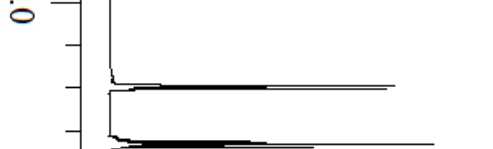

i

$\ddot{\circ}$

o

un

$\circ$

$\stackrel{\circ}{\circ}$

$\omega$

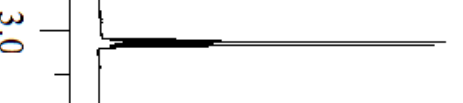

N

잉

0

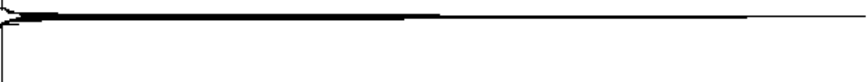




$$
E
$$



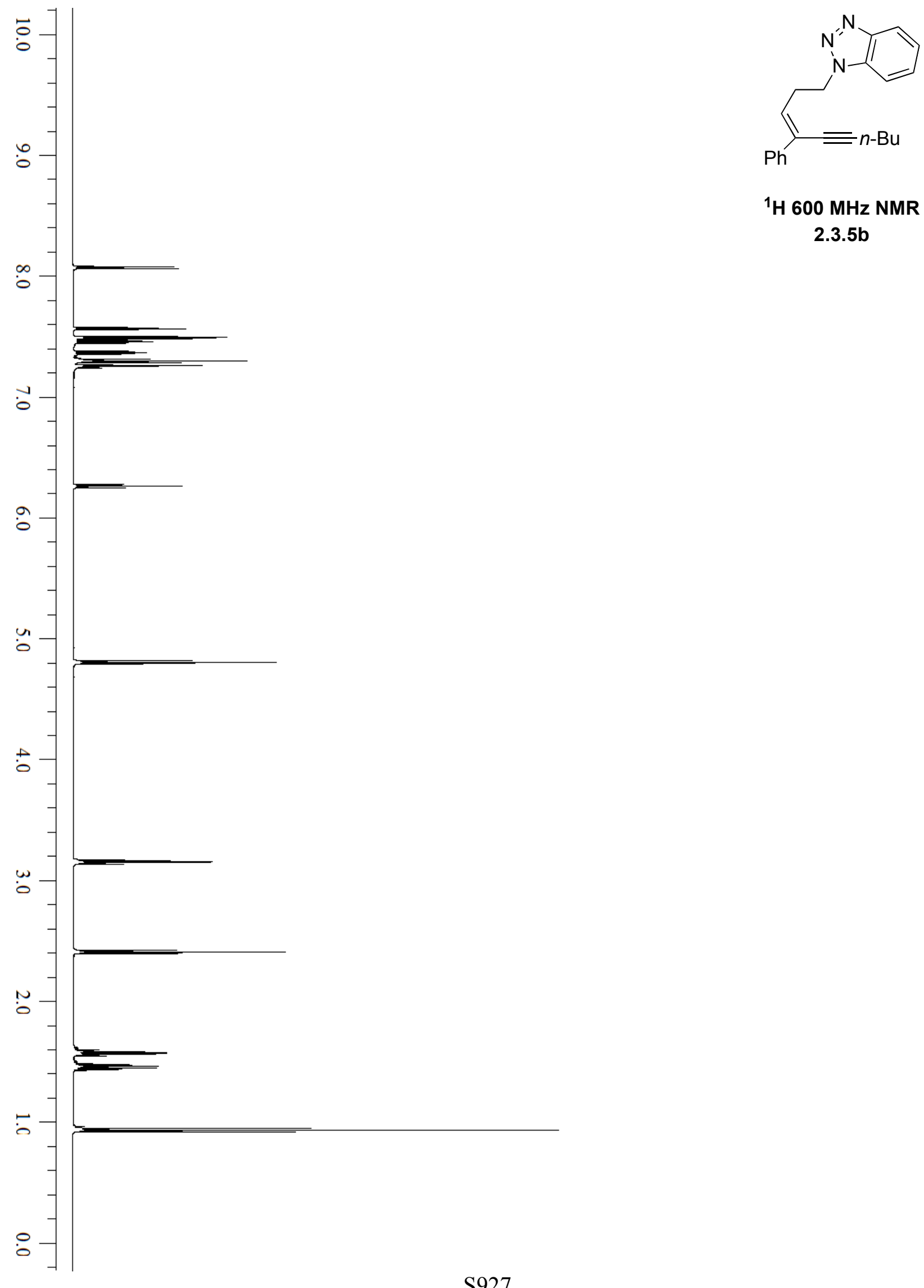

${ }^{1} \mathrm{H} 600 \mathrm{MHz}$ NMR

2.3.5b 

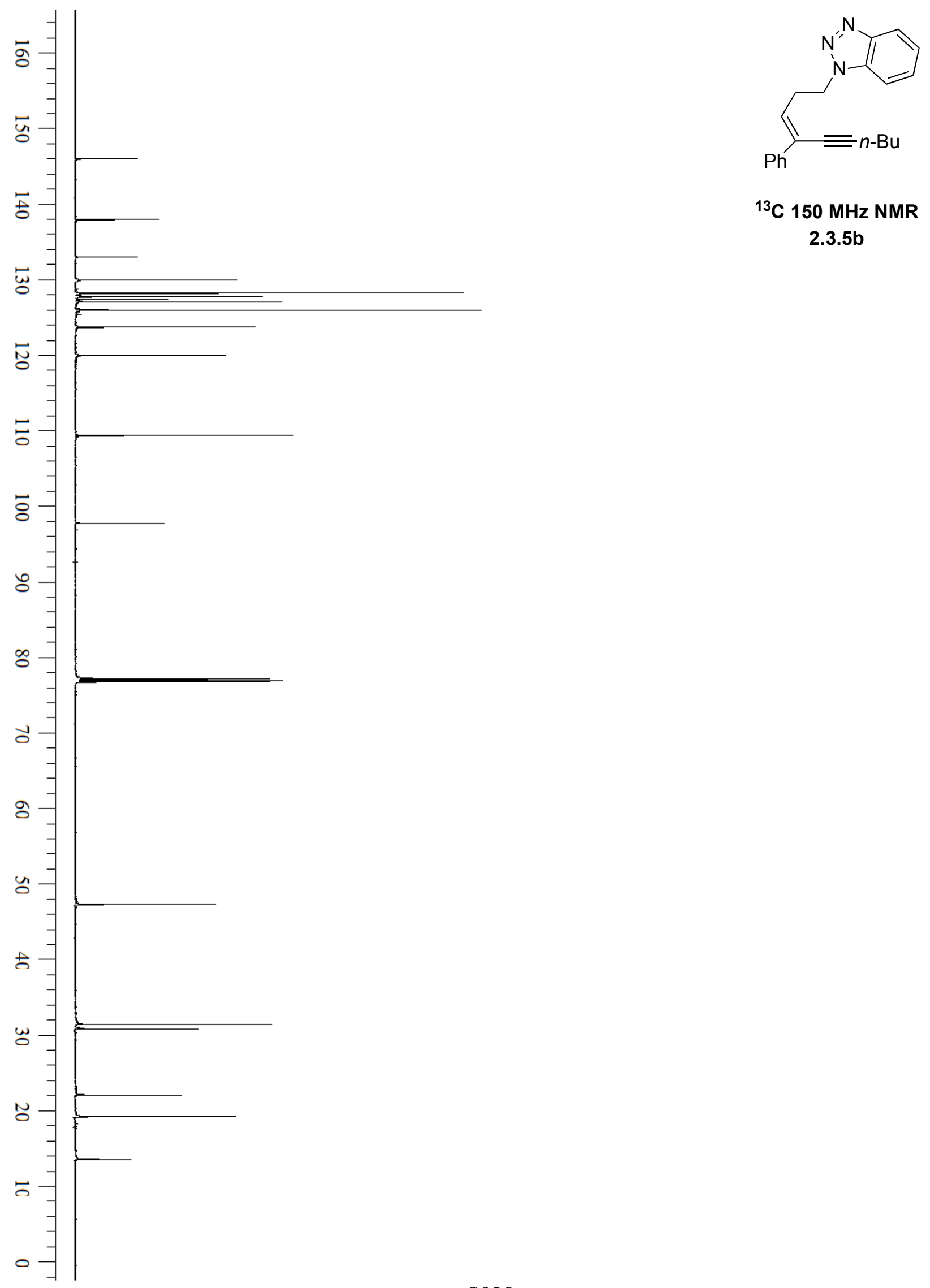

${ }^{13} \mathrm{C} 150 \mathrm{MHz}$ NMR

2.3.5b 


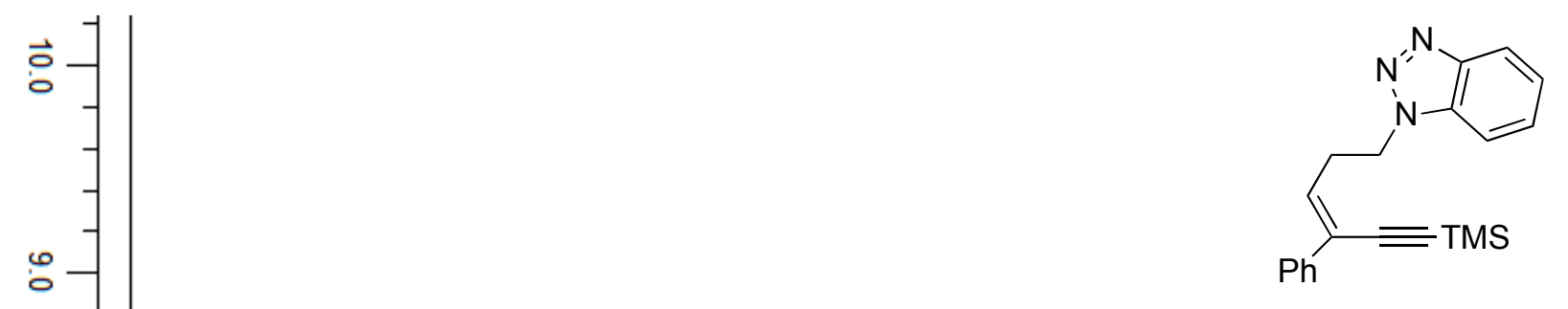

${ }^{1} \mathrm{H} 600 \mathrm{MHz}$ NMR

2.3.5c

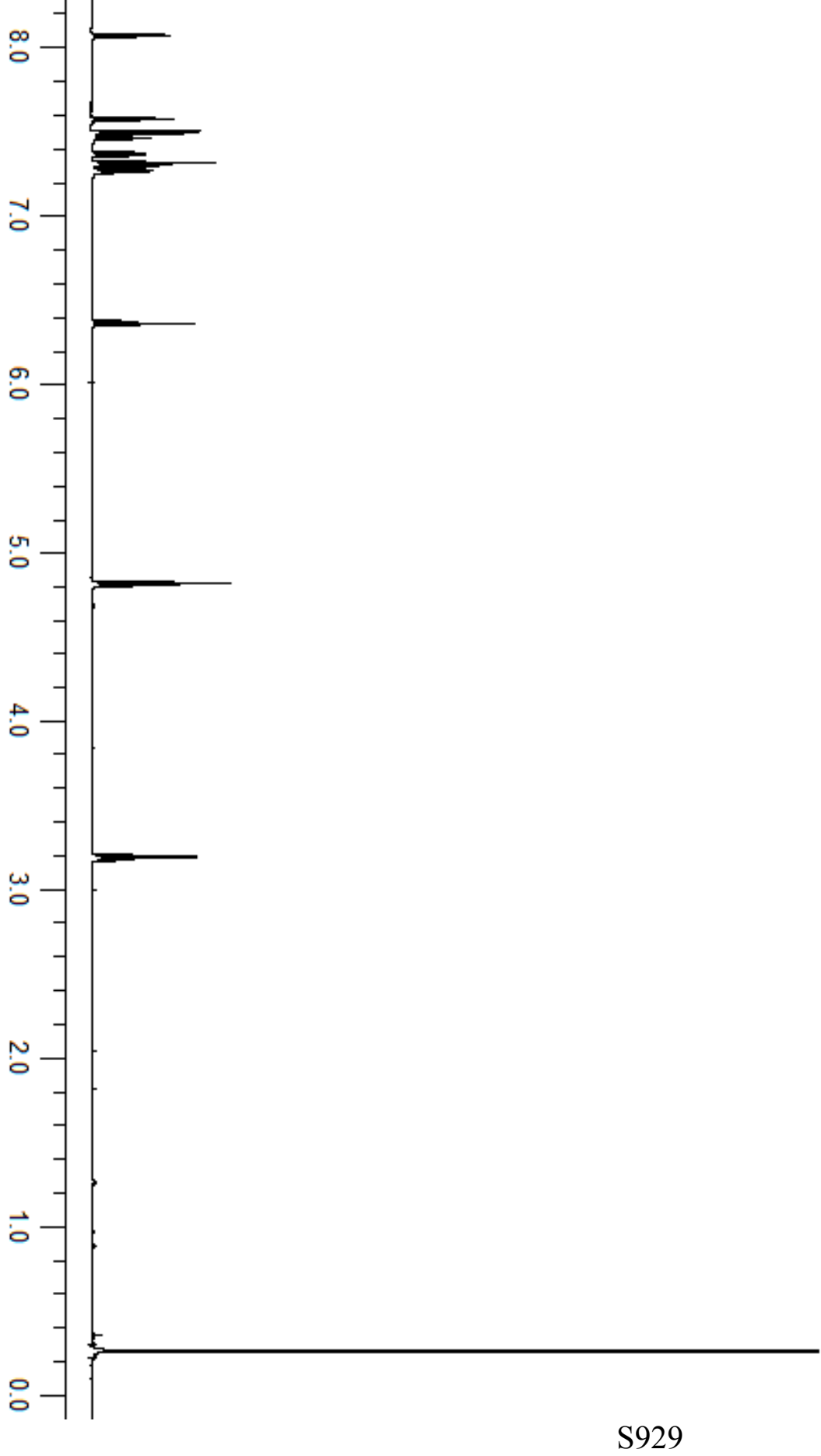



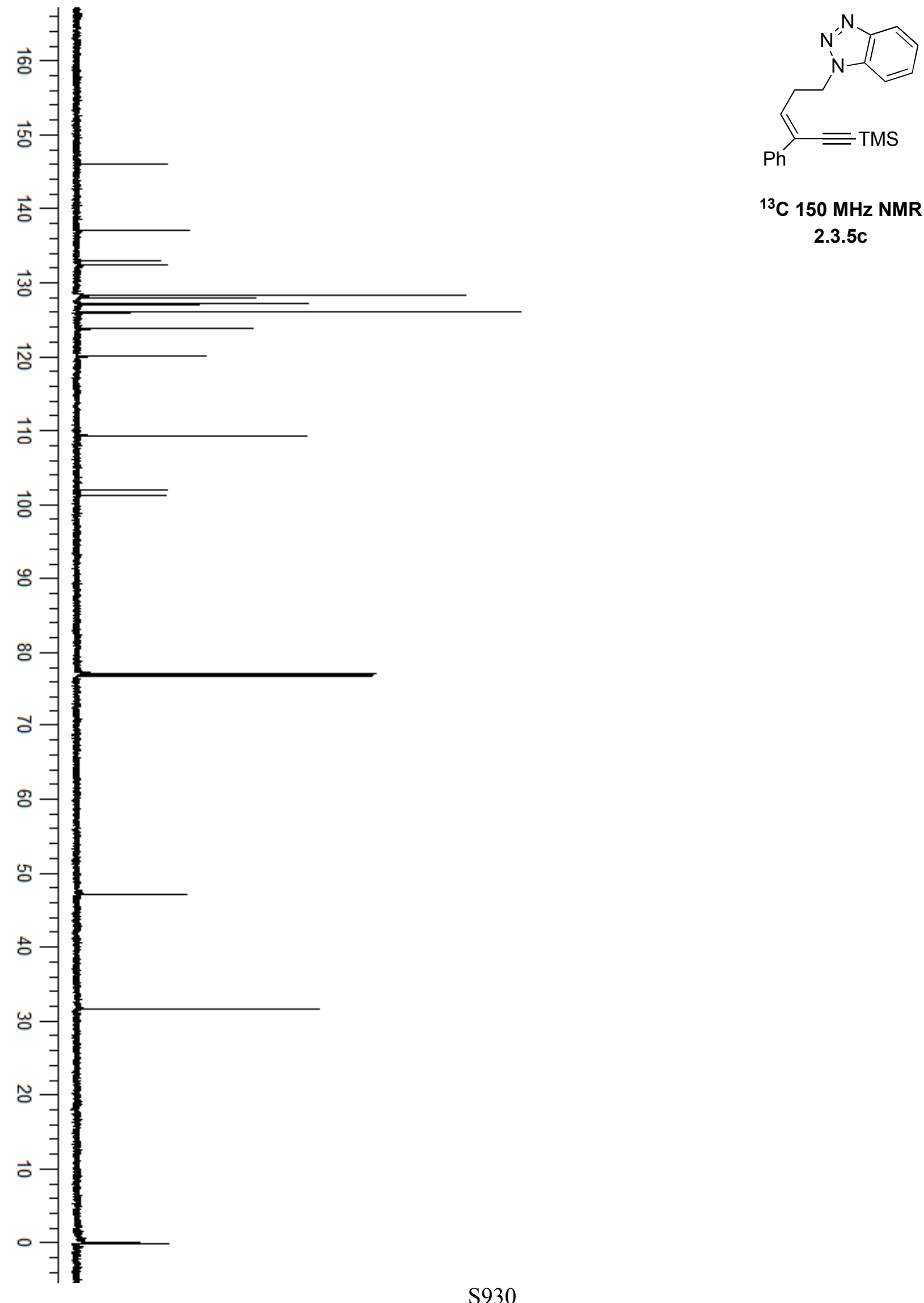

${ }^{13} \mathrm{C} 150 \mathrm{MHz}$ NMR

2.3.5c 


\section{NMR Spectra Data}

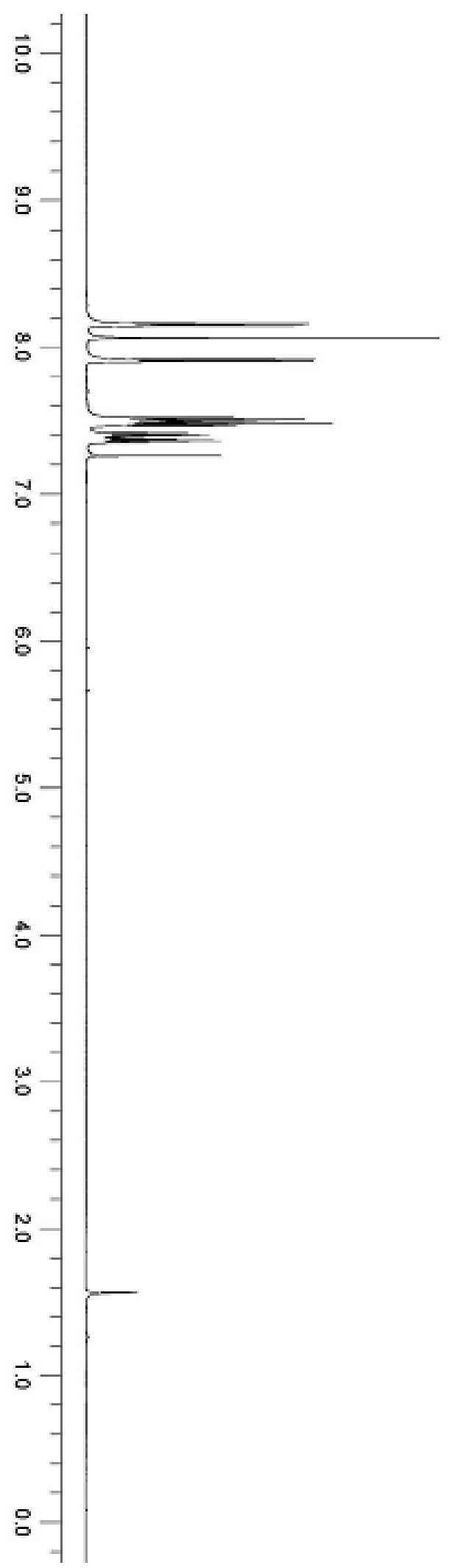

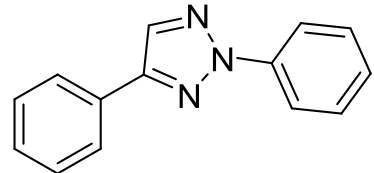

${ }^{1} \mathrm{H}$ 600MHz NMR

$3.1 \mathrm{e}$ 


$$
F=
$$




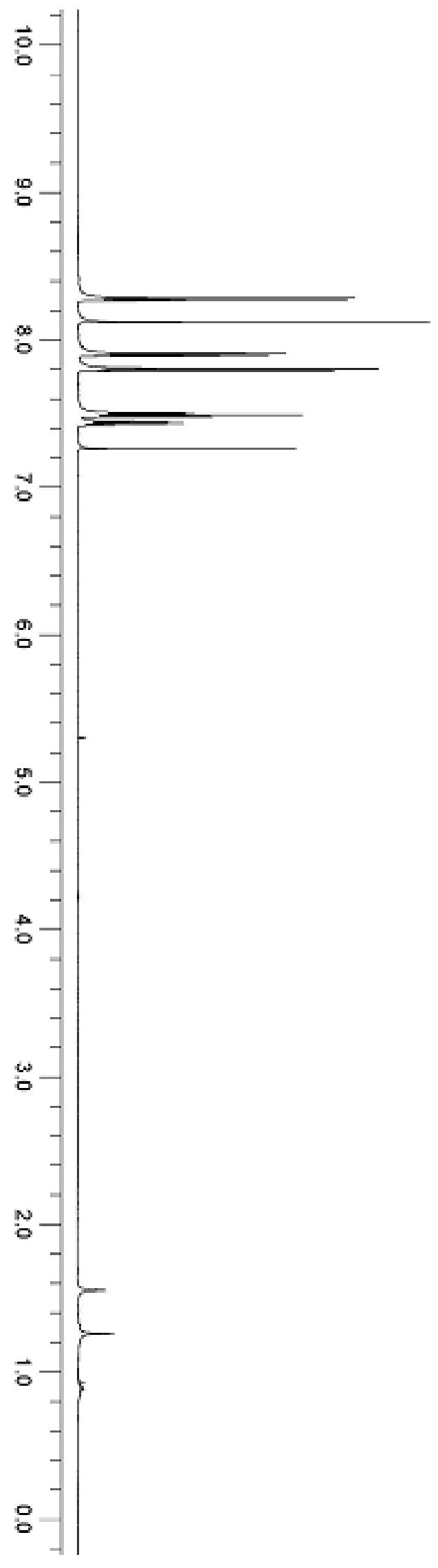

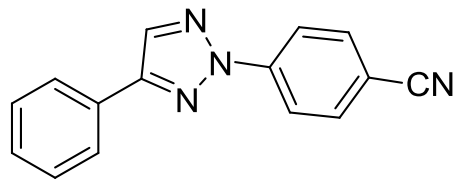

${ }^{1} \mathrm{H}$ 600MHz NMR

$3.2 \mathrm{a}$ 


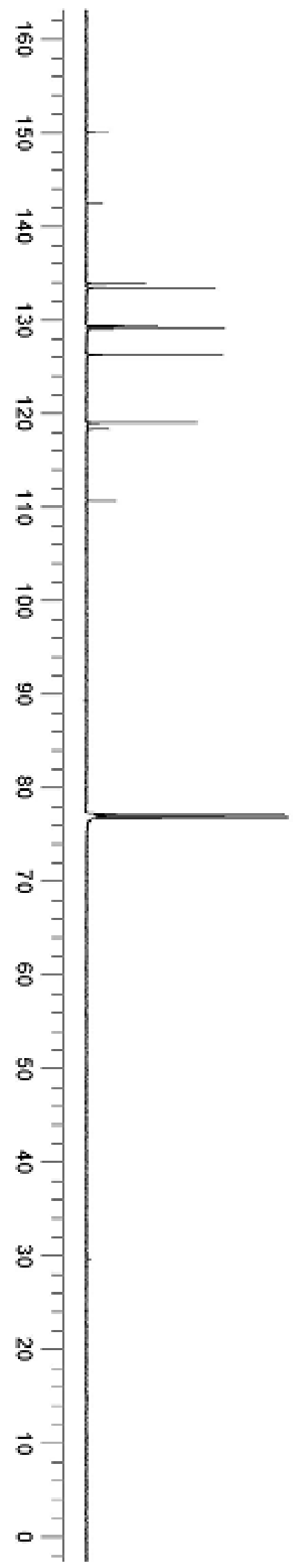

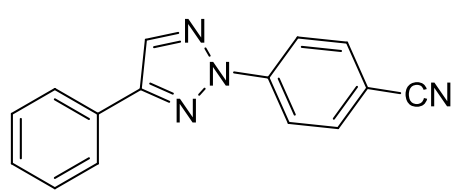

${ }^{13} \mathrm{C} 150 \mathrm{MHz}$ NMR

$3.2 \mathrm{a}$ 


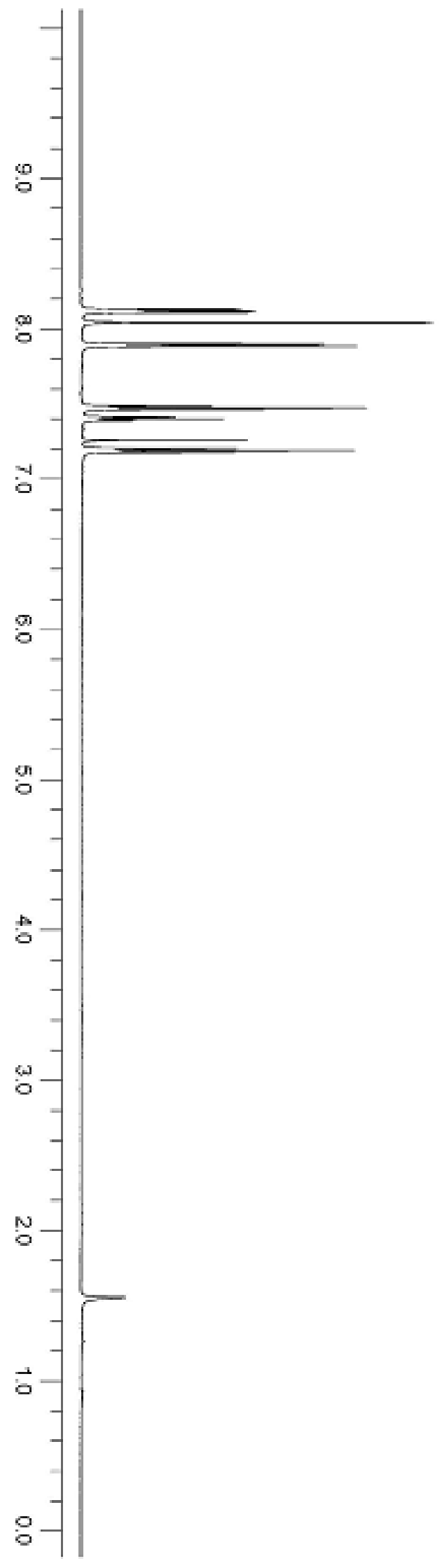

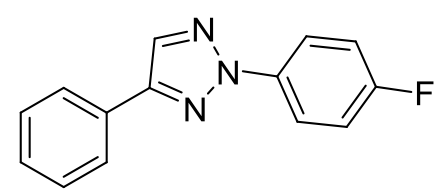

${ }^{1} \mathrm{H} 600 \mathrm{MHz}$ NMR

$3.2 \mathrm{~b}$ 

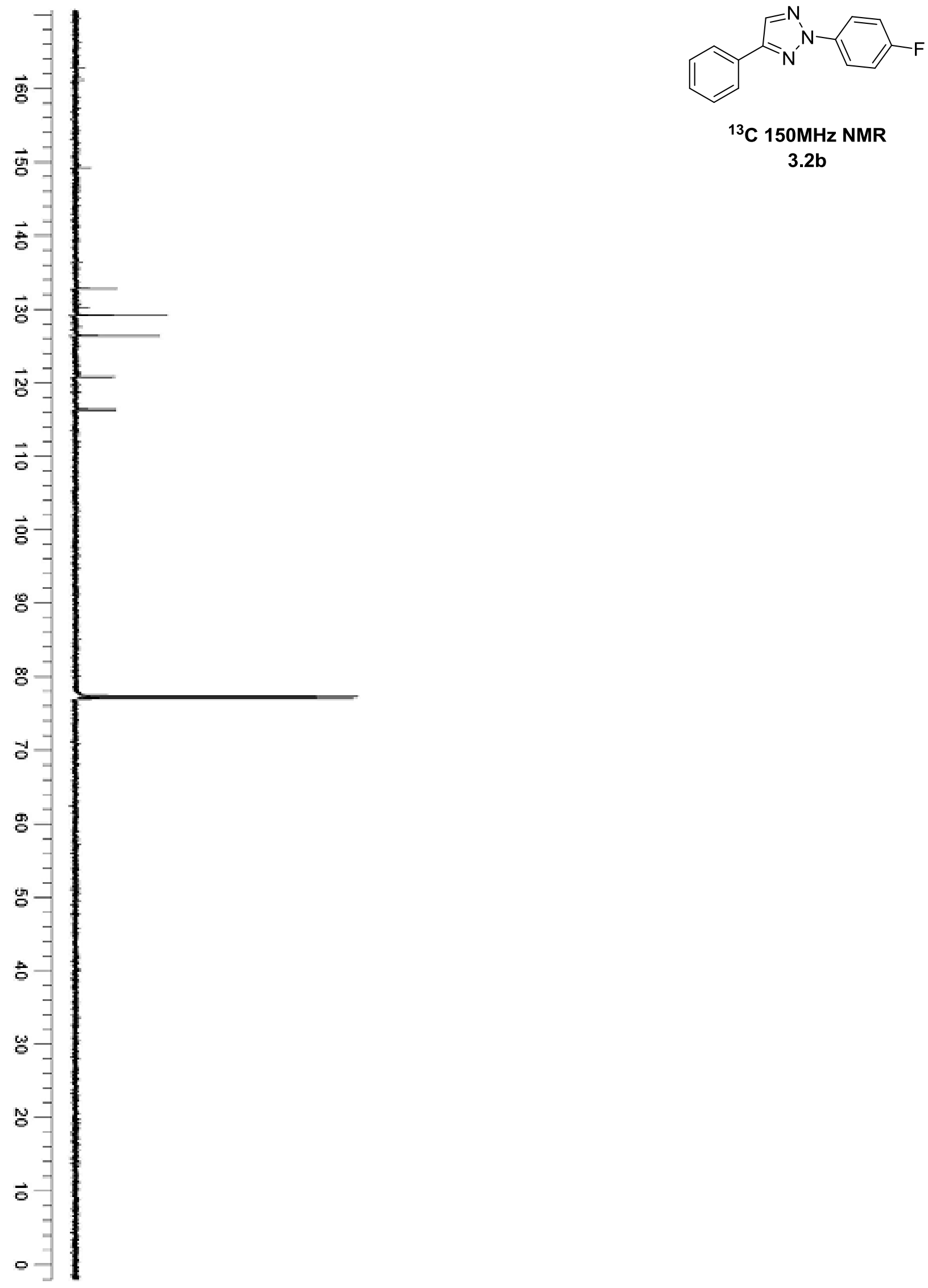

${ }^{13} \mathrm{C} 150 \mathrm{MHz}$ NMR 3.2b 


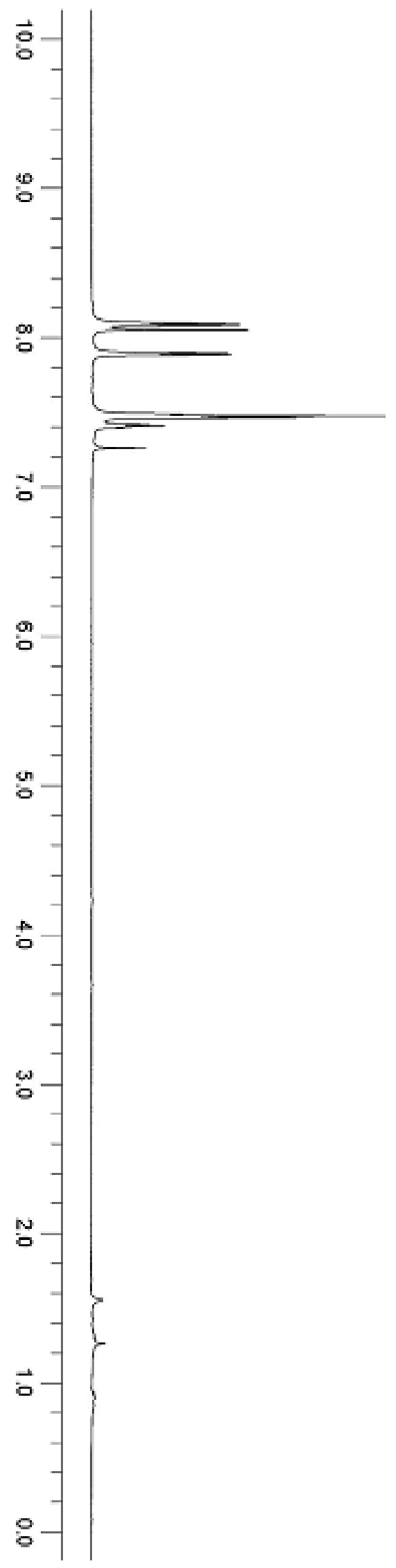

${ }^{1} \mathrm{H} 600 \mathrm{MHz}$ NMR $3.2 \mathrm{c}$ 


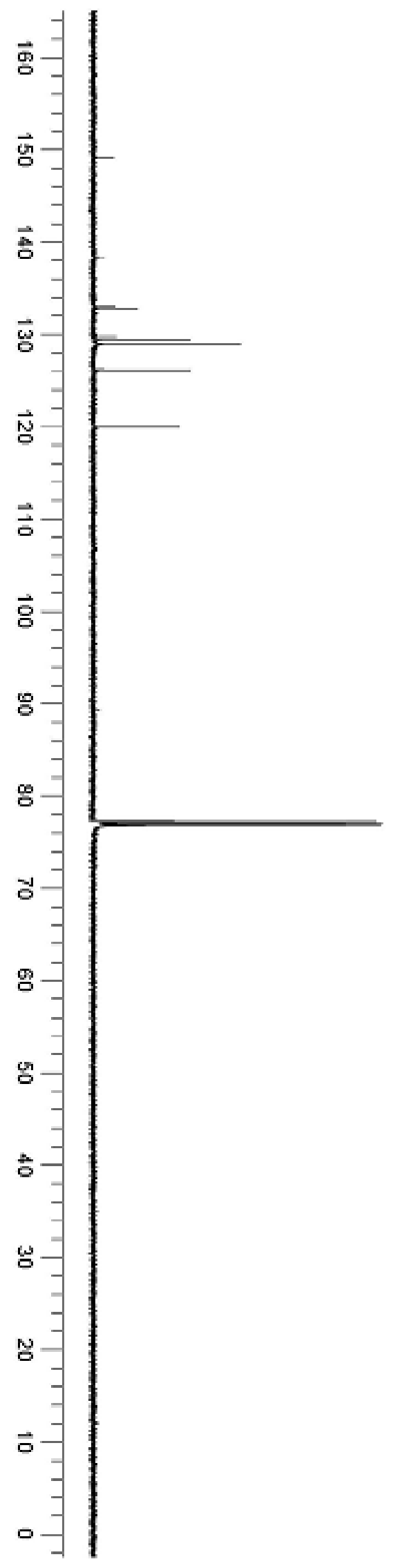

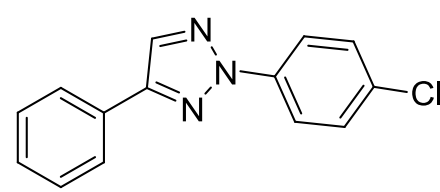

${ }^{13} \mathrm{C} 150 \mathrm{MHz}$ NMR 3.2c 


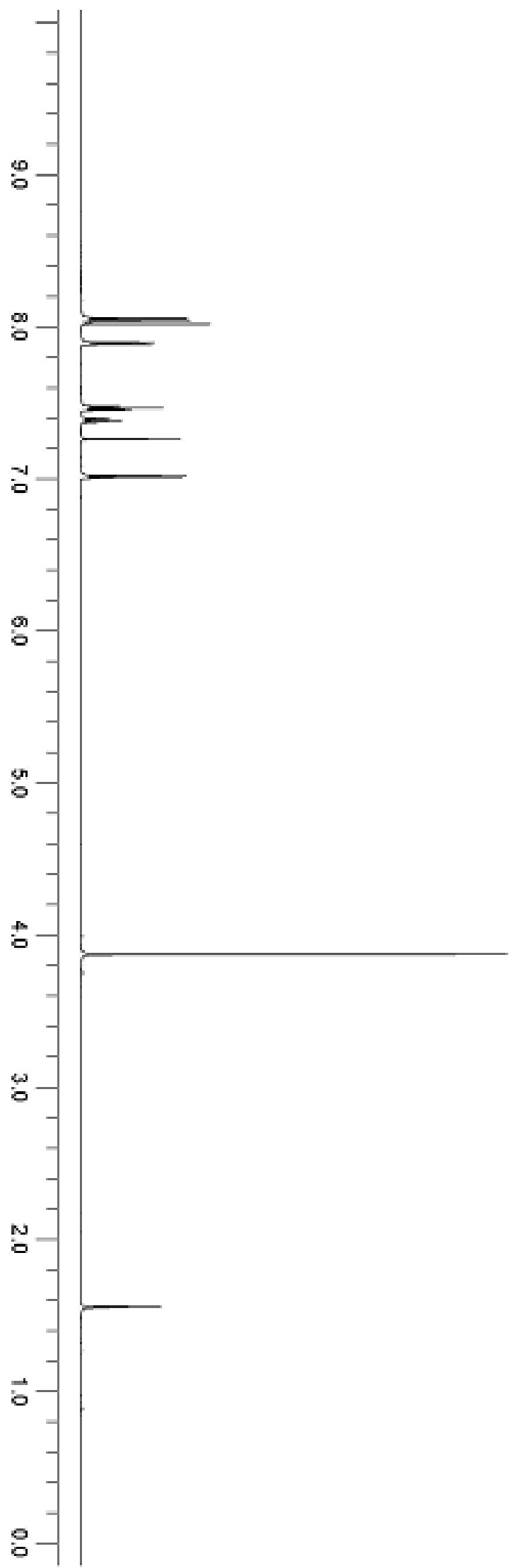

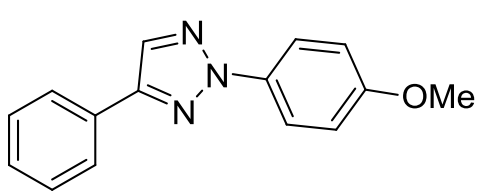

${ }^{1} \mathrm{H} 600 \mathrm{MHz}$ NMR

3.2d 

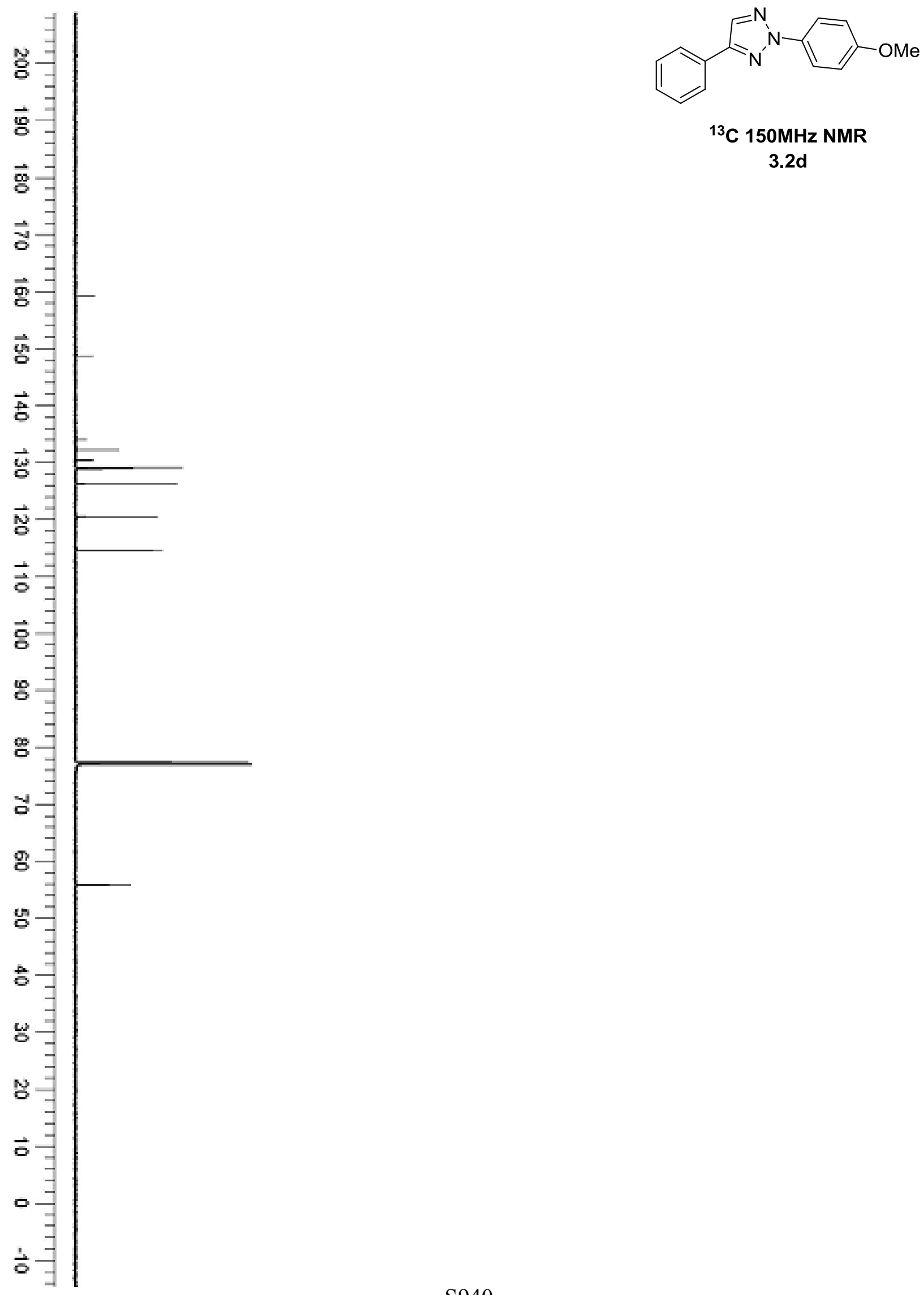

${ }^{13} \mathrm{C} 150 \mathrm{MHz}$ NMR

3.2d 


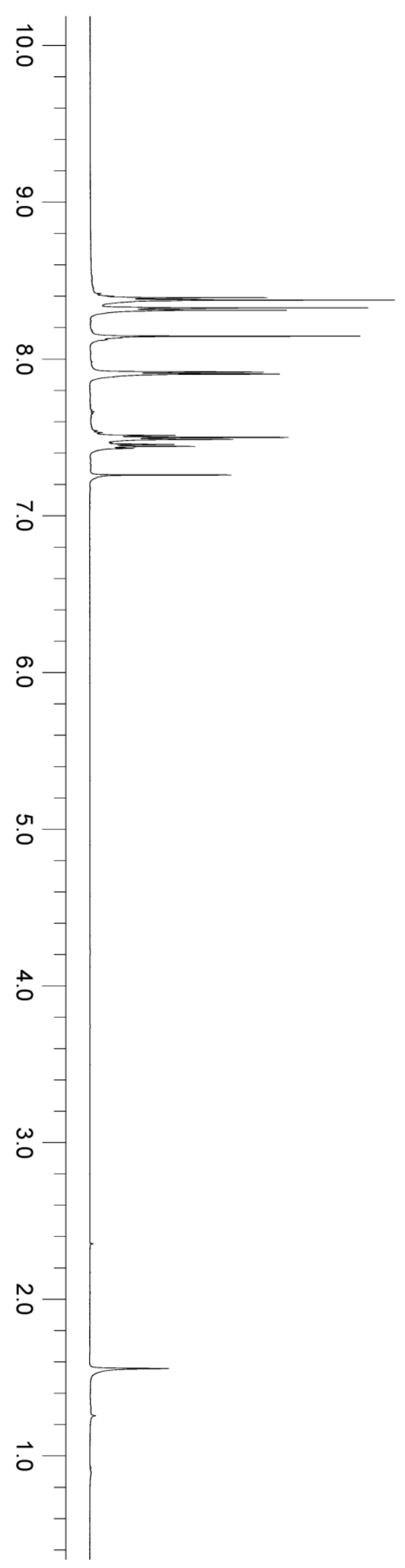

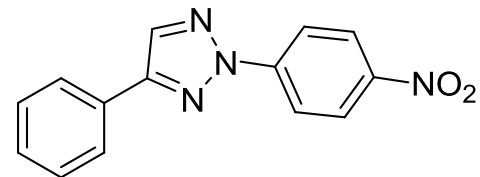

${ }^{1} \mathrm{H} 600 \mathrm{MHz}$ NMR

$3.2 \mathrm{e}$ 

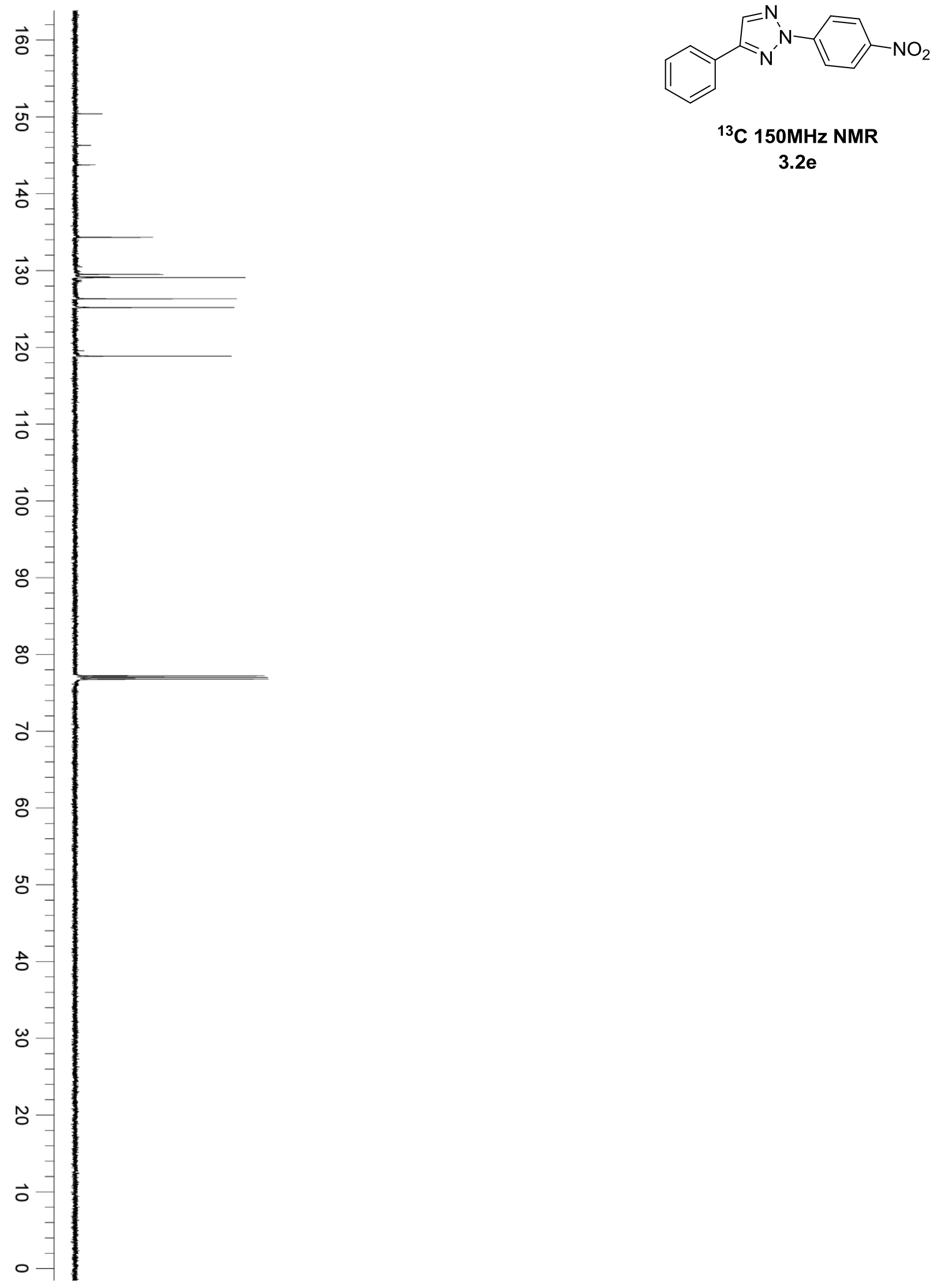

${ }^{13} \mathrm{C} 150 \mathrm{MHz}$ NMR $3.2 \mathrm{e}$ 


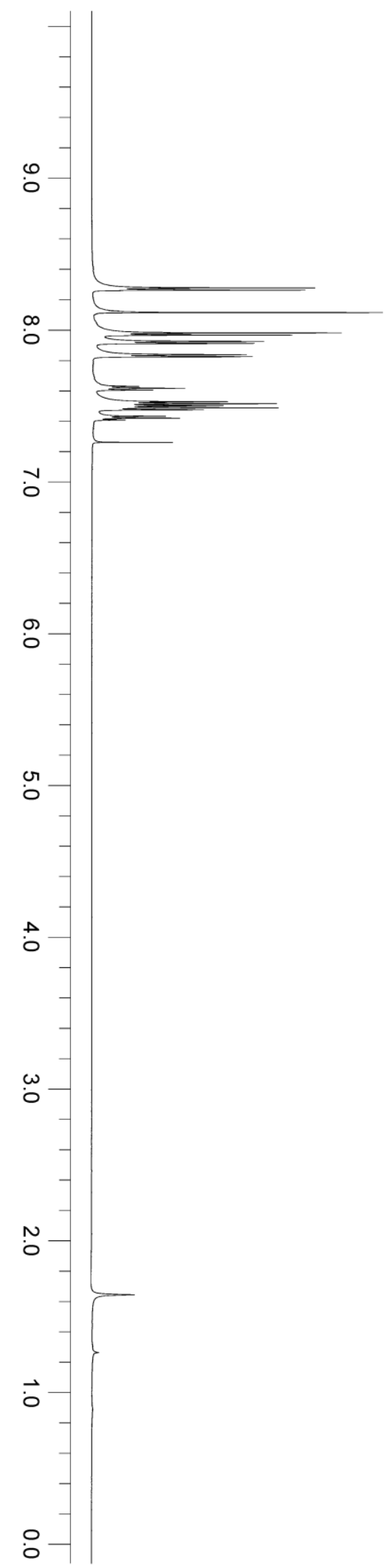

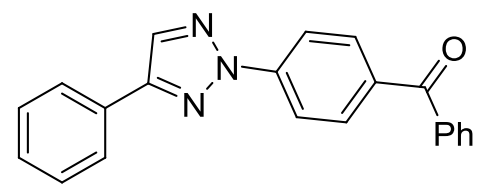

${ }^{1} \mathrm{H}$ 600MHz NMR $3.2 f$ 

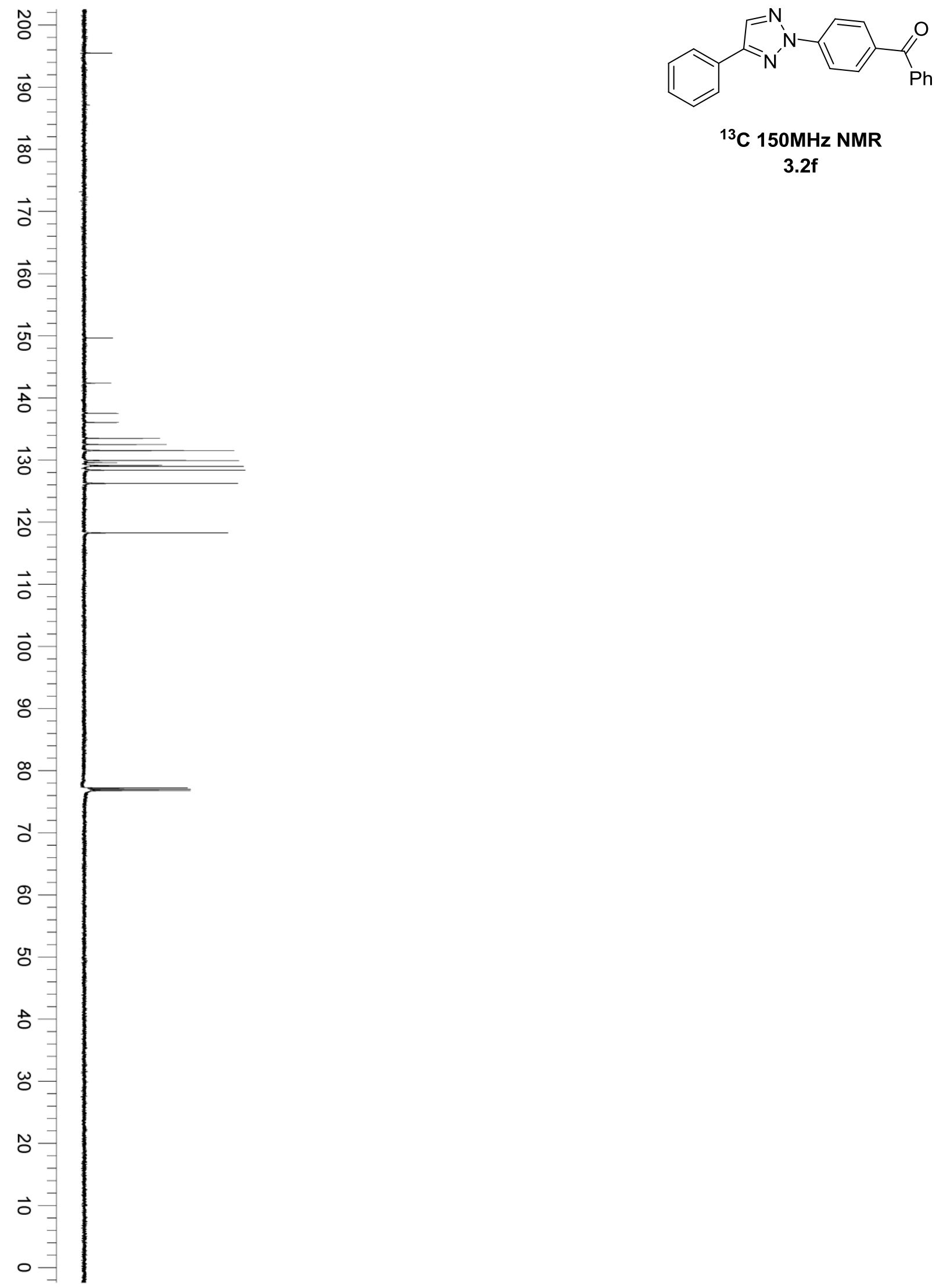


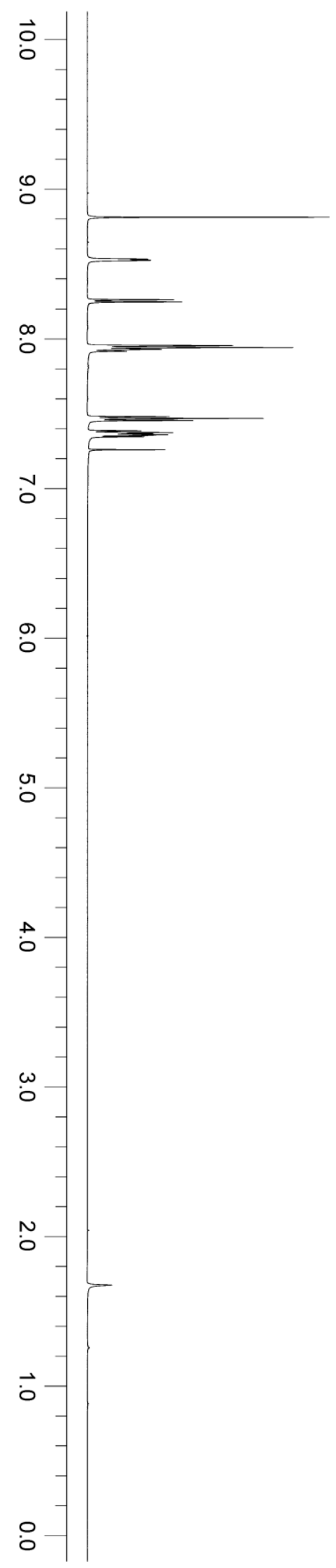

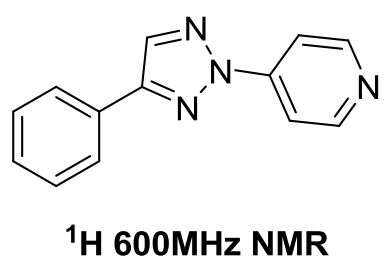

$3.2 \mathrm{~g}$ 

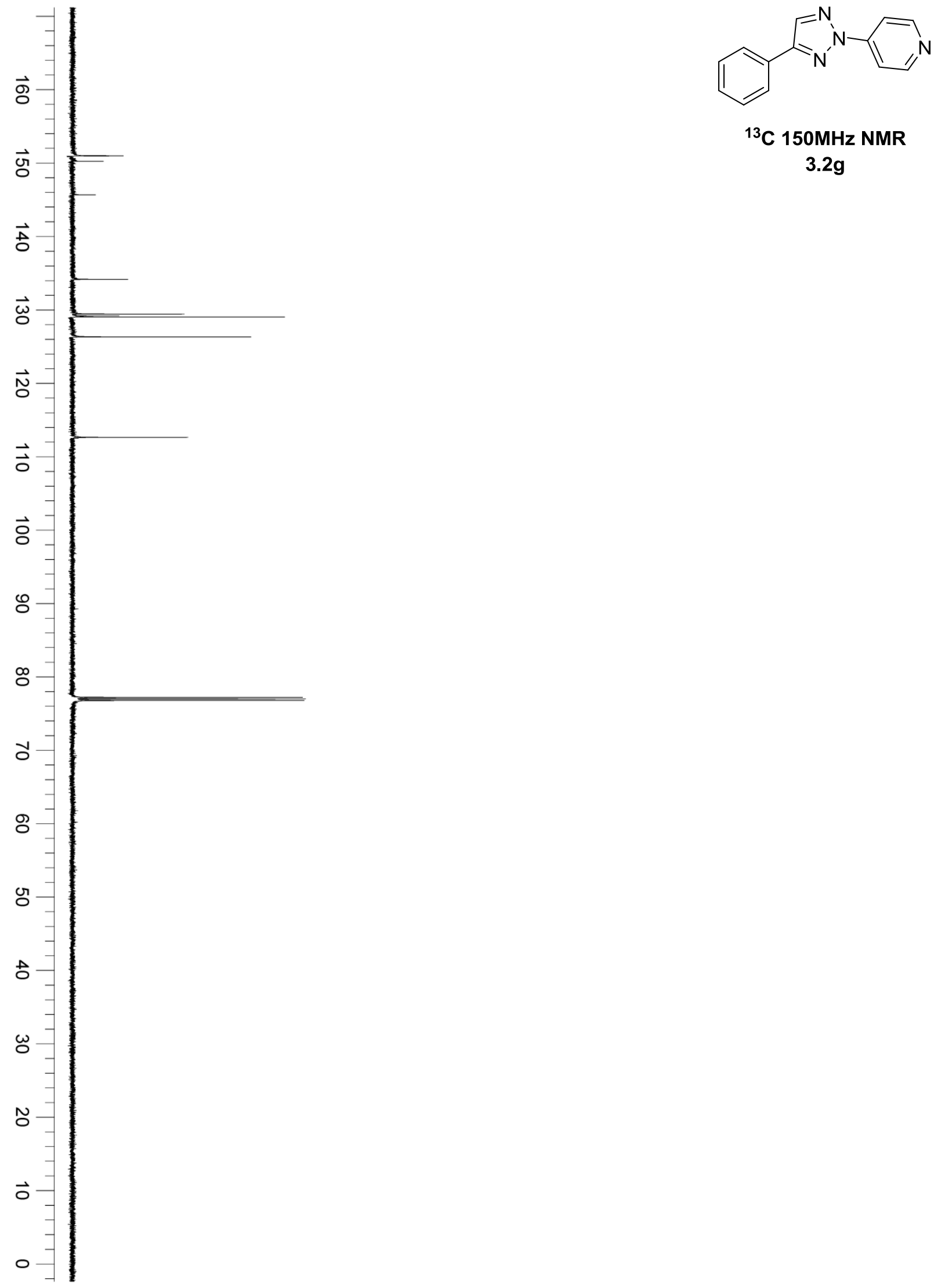

$3.2 \mathrm{~g}$ 


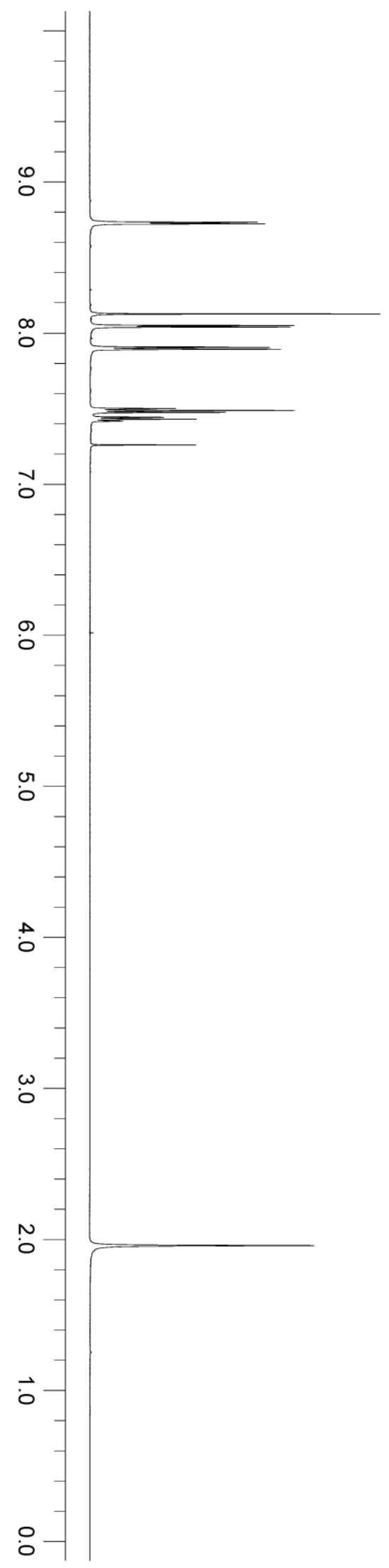

${ }^{1} \mathrm{H}$ 600MHz NMR

$3.2 \mathrm{~h}$ 


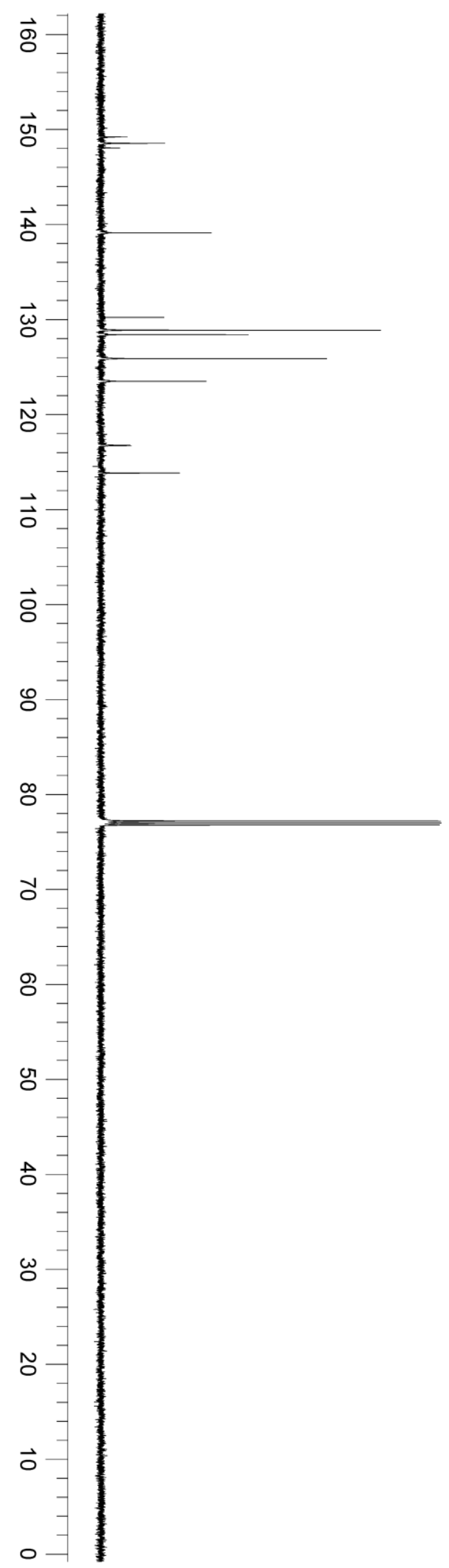




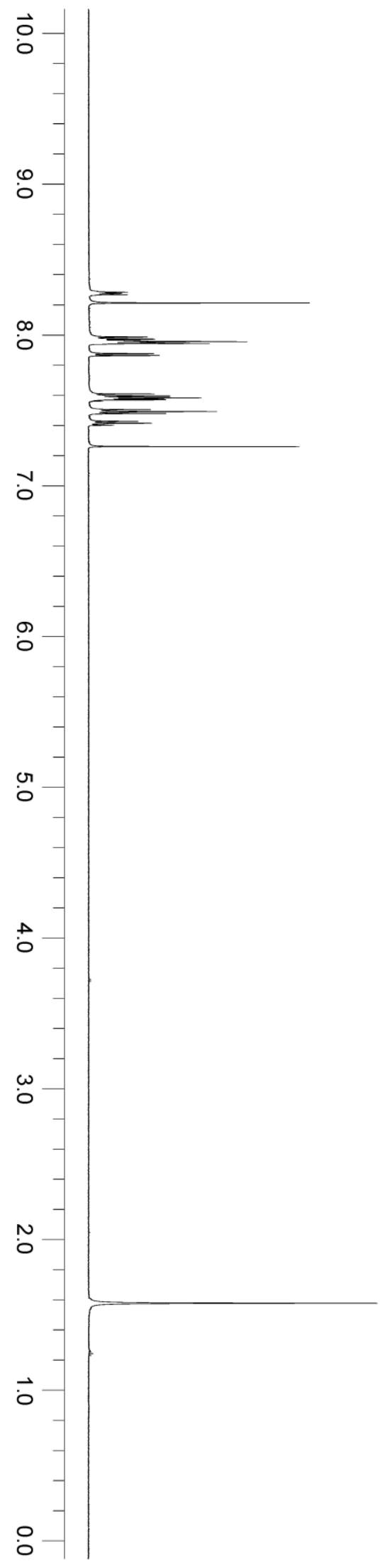

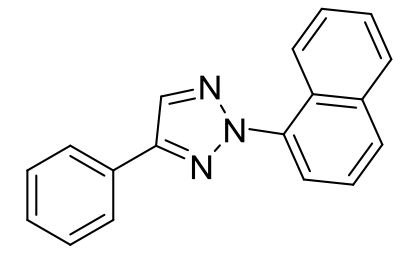

${ }^{1} \mathrm{H} 600 \mathrm{MHz}$ NMR

$3.2 \mathrm{i}$ 

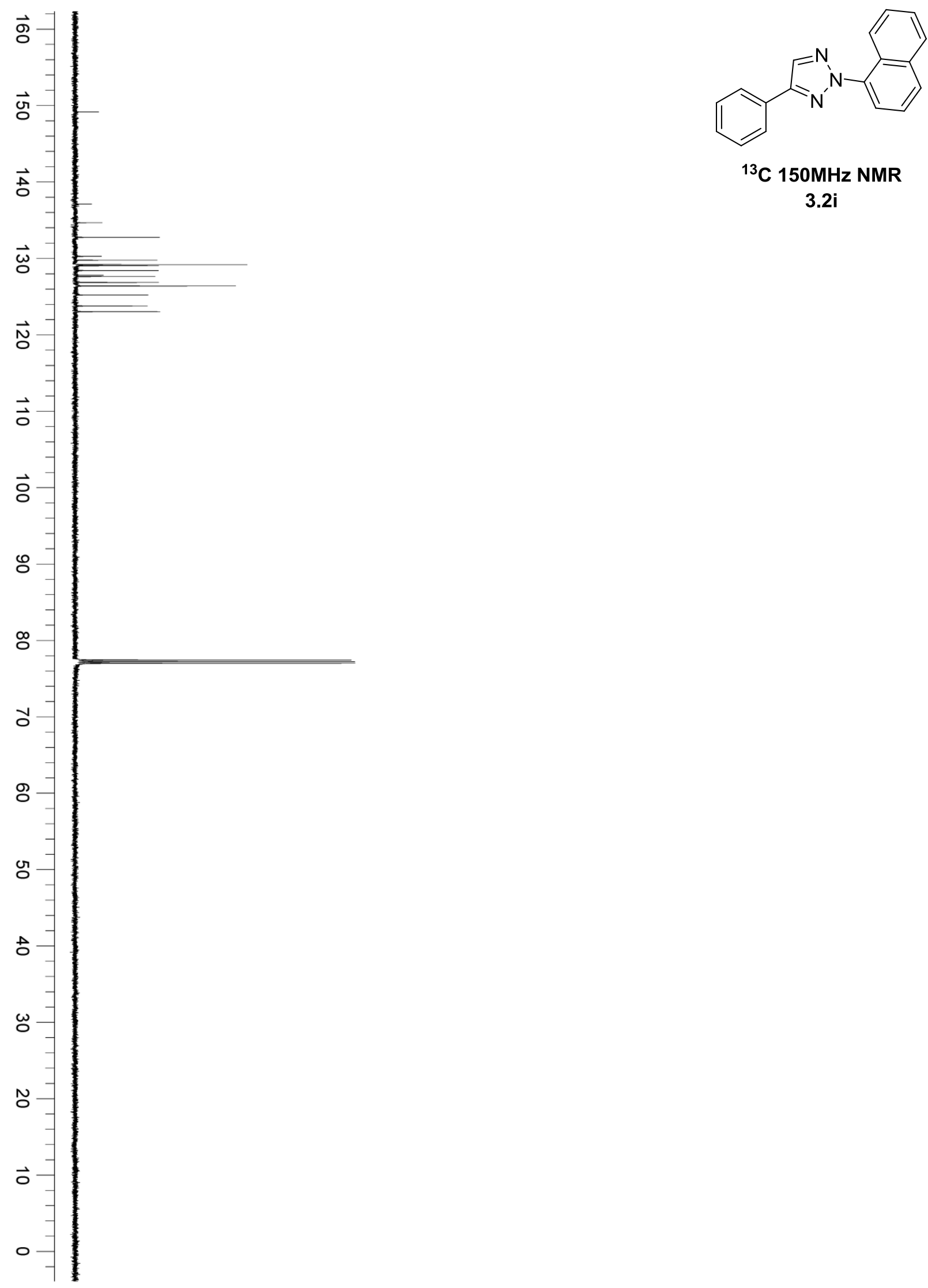

$3.2 \mathrm{i}$ 

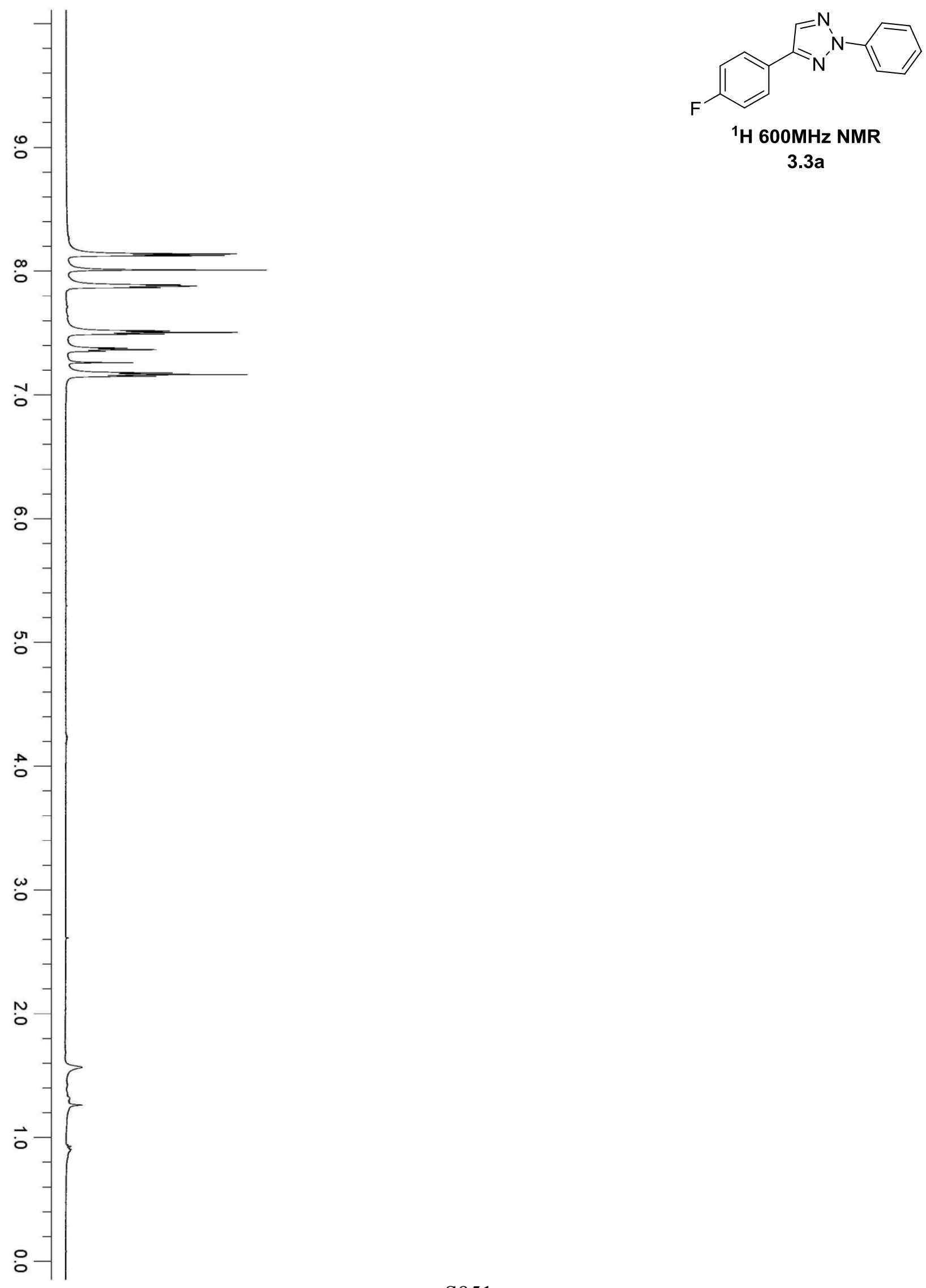


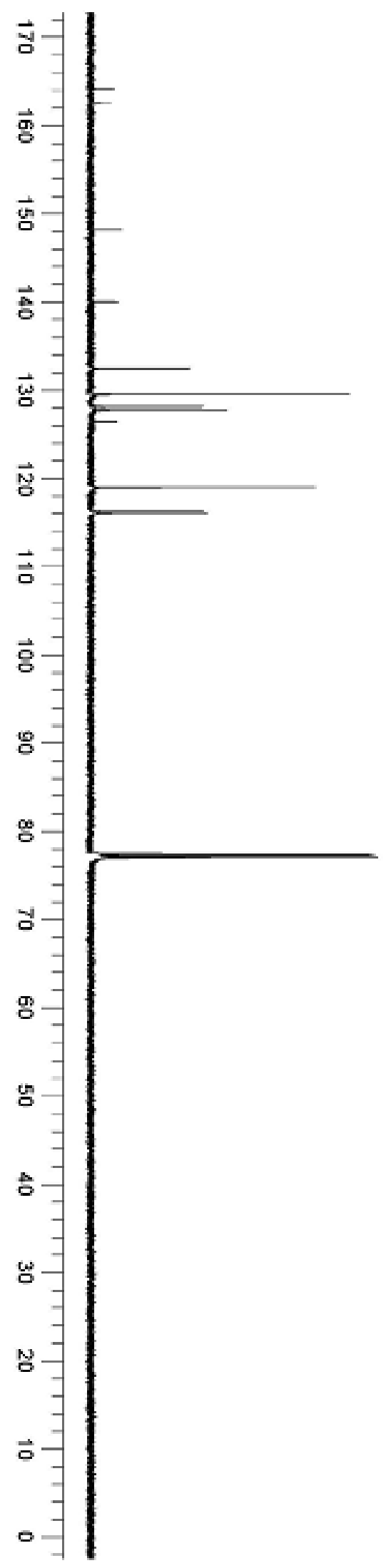

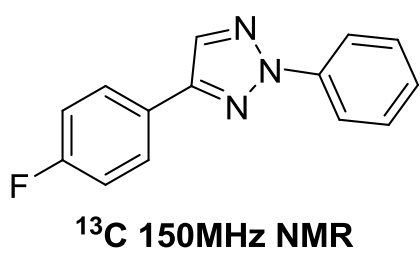

3.3a 


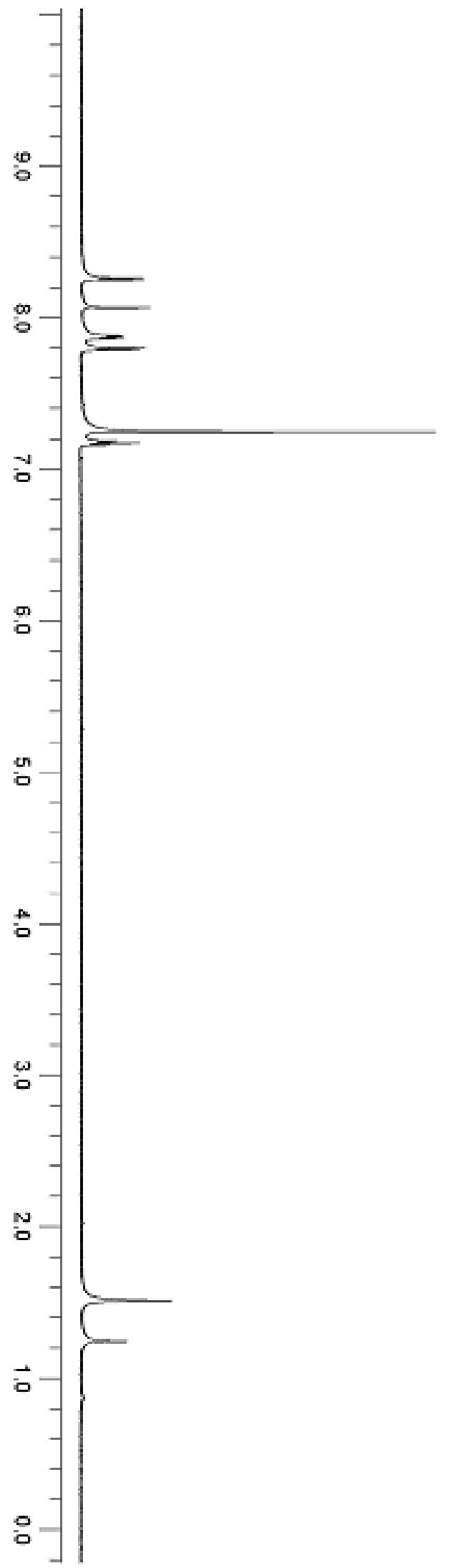

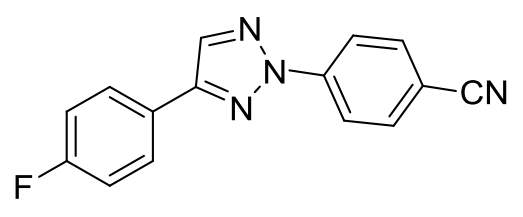

${ }^{1} \mathrm{H} 600 \mathrm{MHz}$ NMR

3.3b 


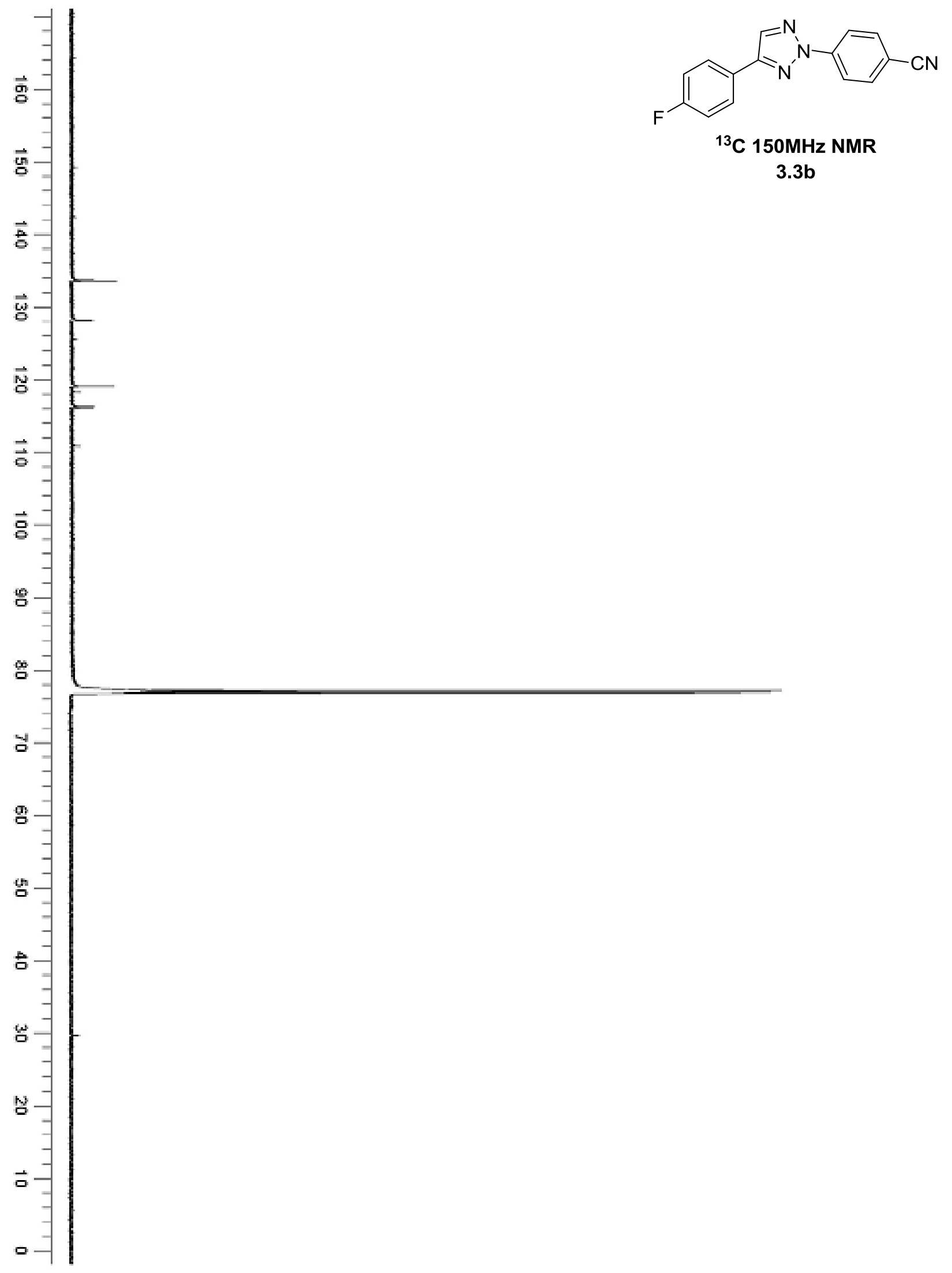




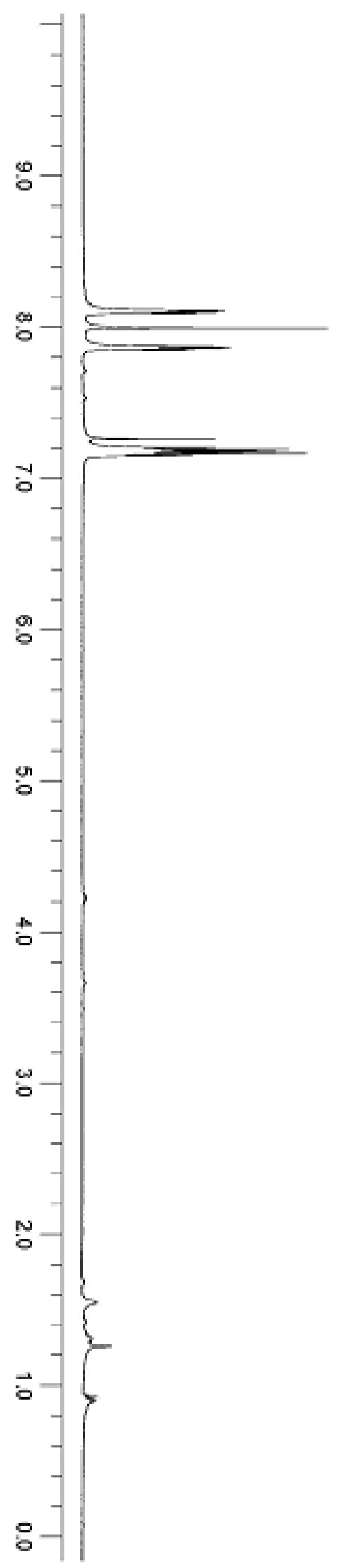

${ }^{1} \mathrm{H} 600 \mathrm{MHz}$ NMR

$$
3.3 \mathrm{c}
$$




$$
F^{*}
$$




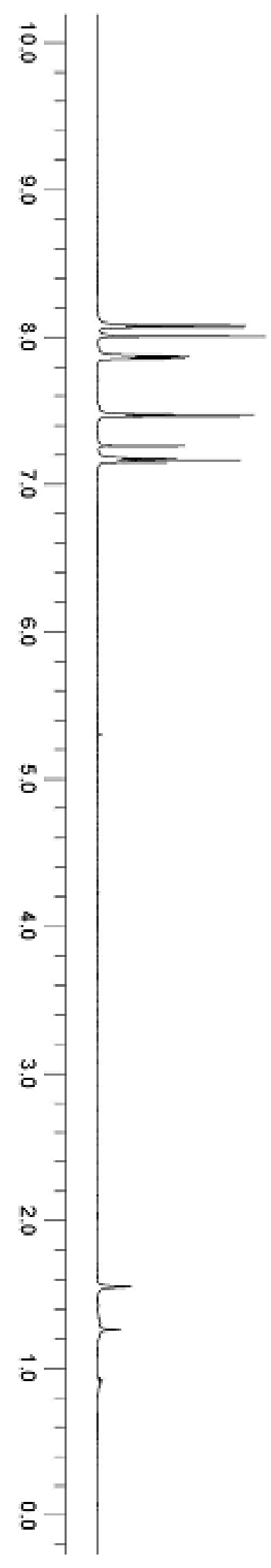

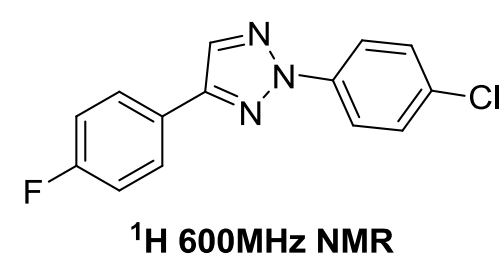

3.3d 

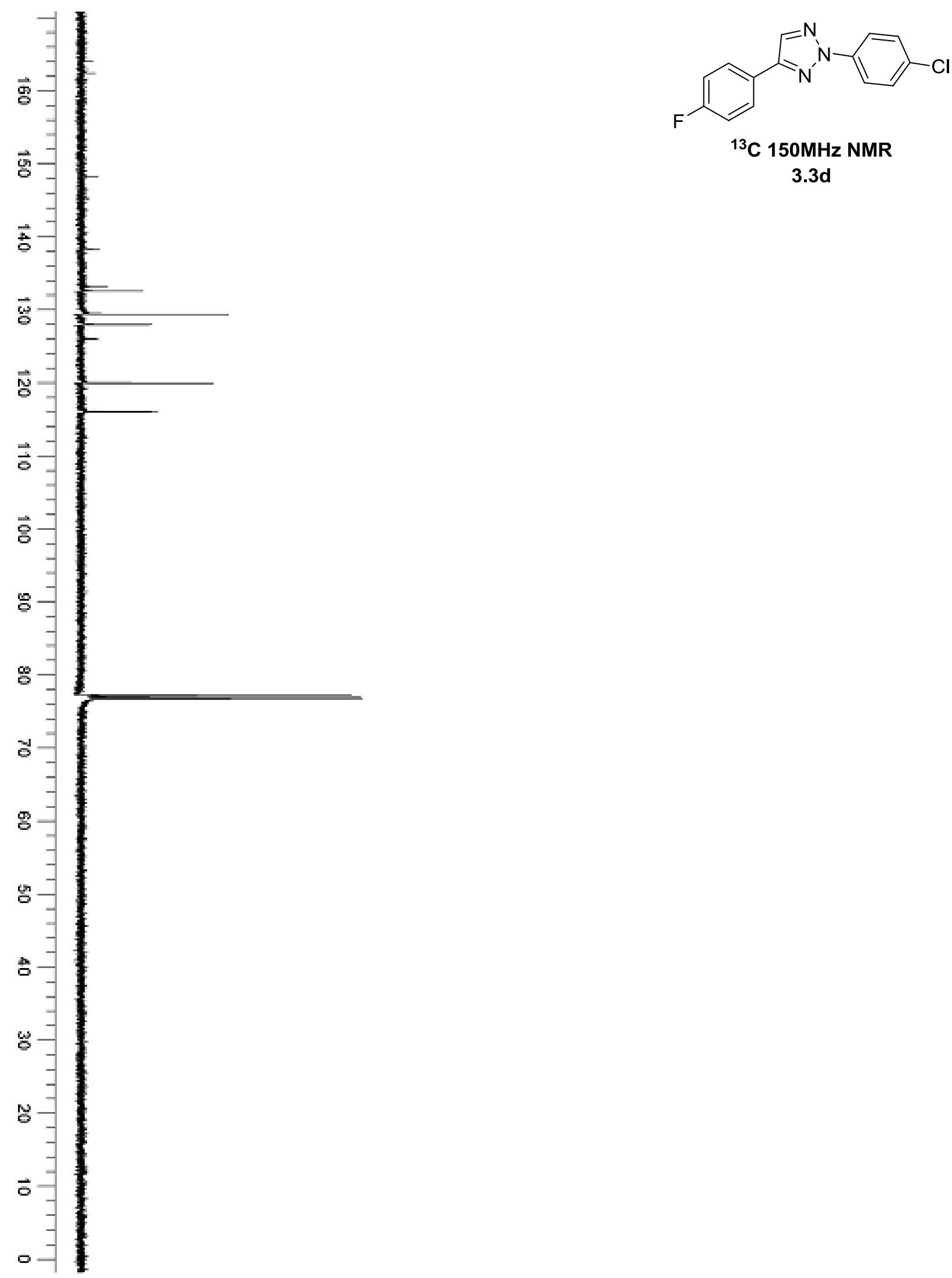


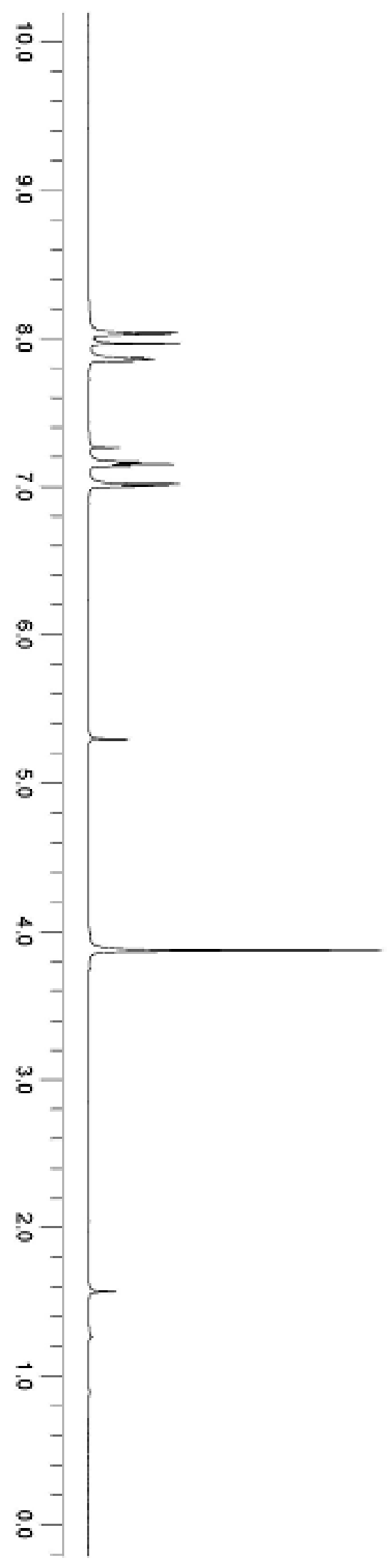

${ }^{1} \mathrm{H} 600 \mathrm{MHz}$ NMR

$3.3 \mathrm{e}$ 


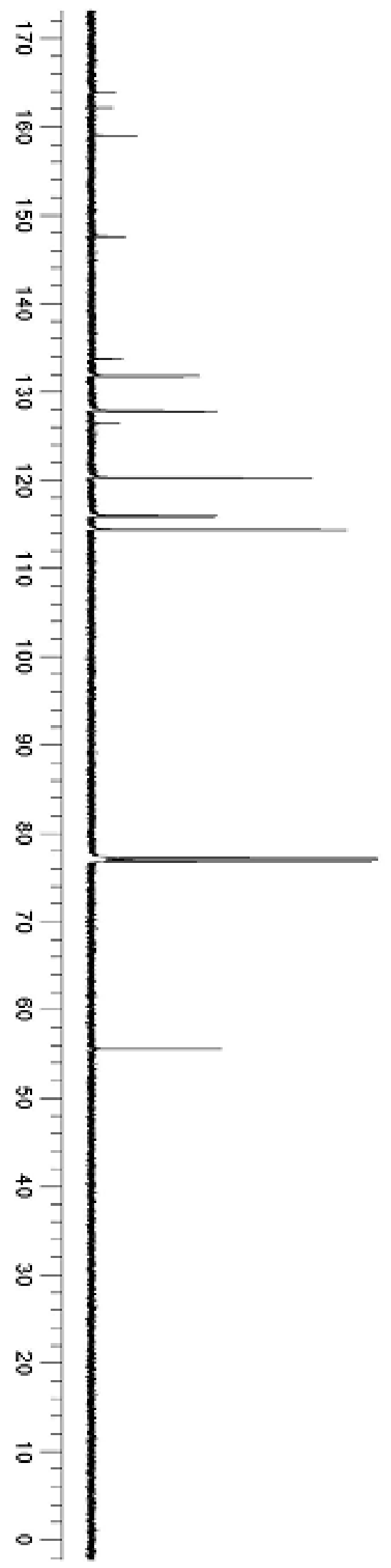

${ }^{13} \mathrm{C} 150 \mathrm{MHz}$ NMR

3.3e 


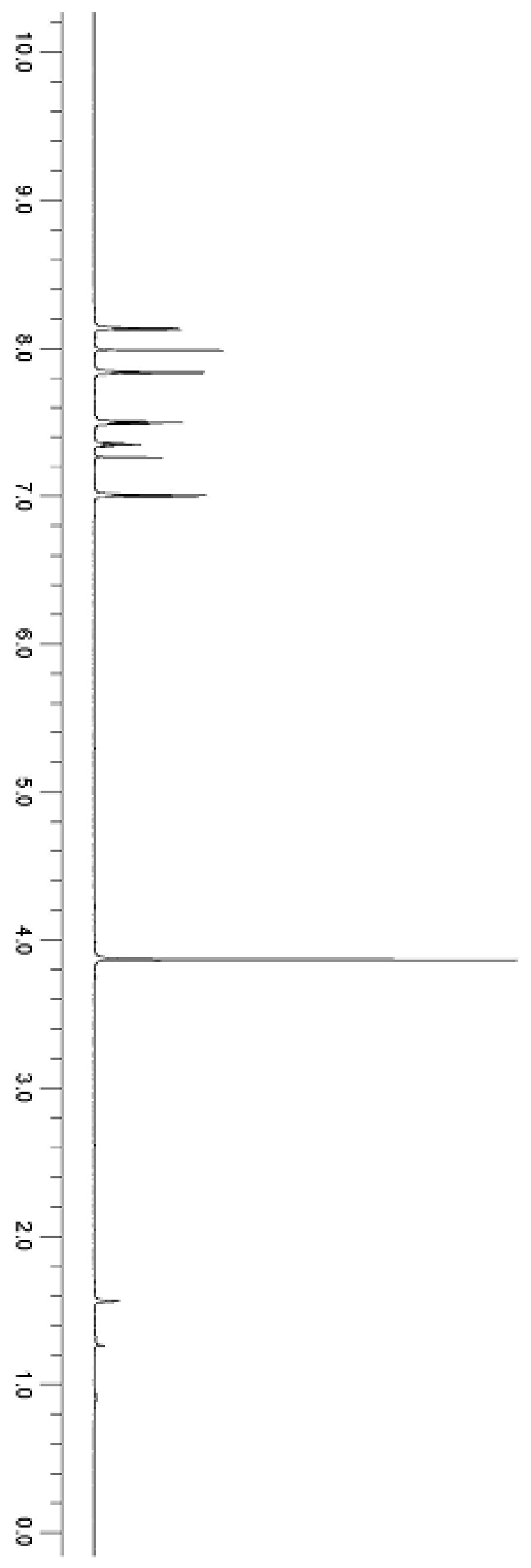

${ }^{1} \mathrm{H} 600 \mathrm{MHz}$ NMR

$3.3 f$ 


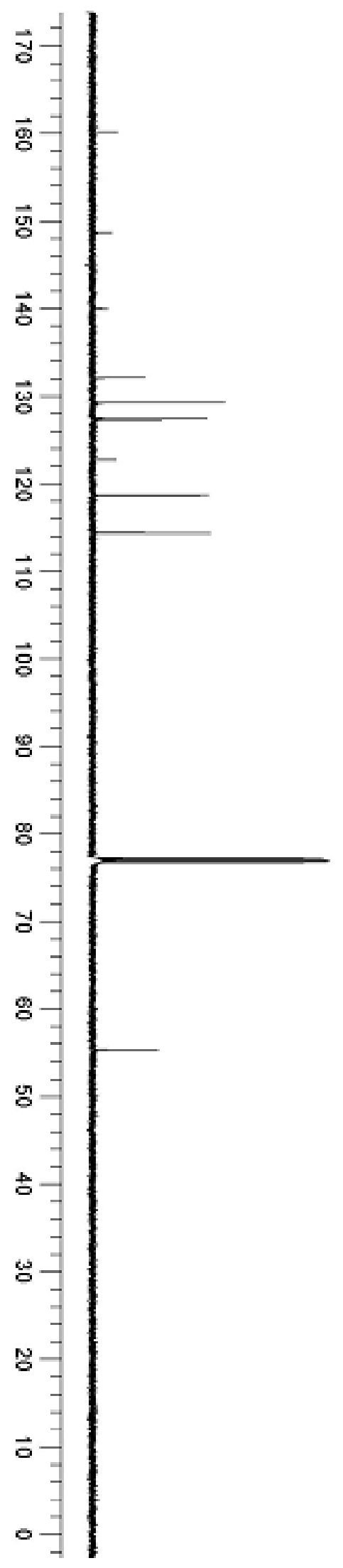

${ }^{13} \mathrm{C}$ 150MHz NMR

$3.3 f$ 


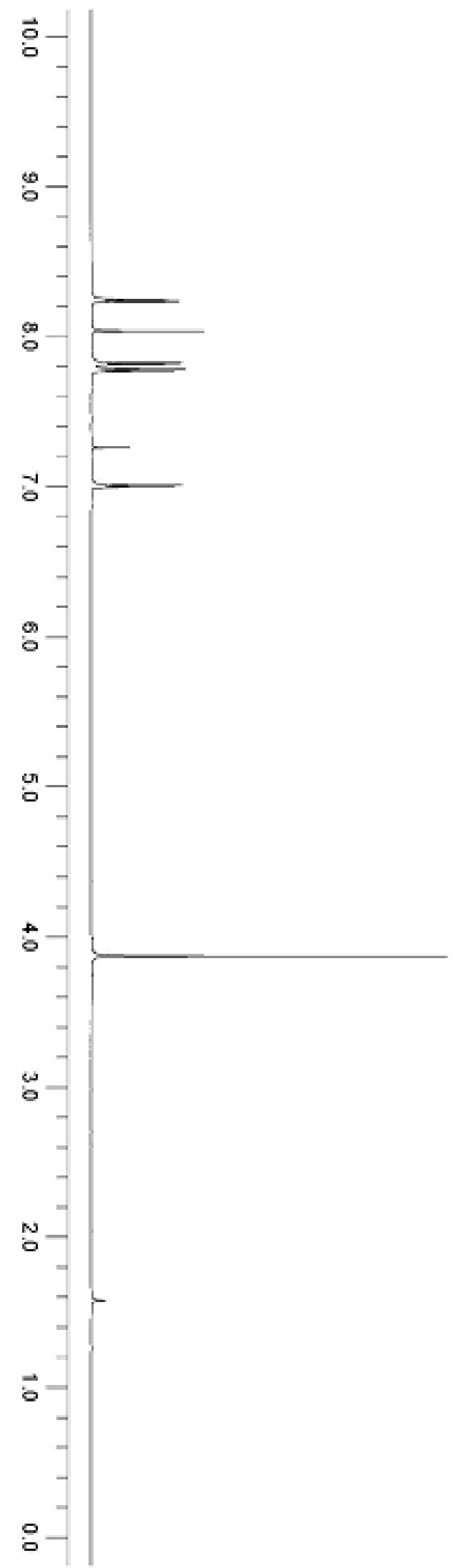

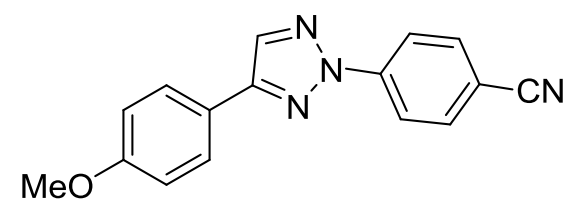

${ }^{1} \mathrm{H} 600 \mathrm{MHz}$ NMR

$3.3 \mathrm{~g}$ 


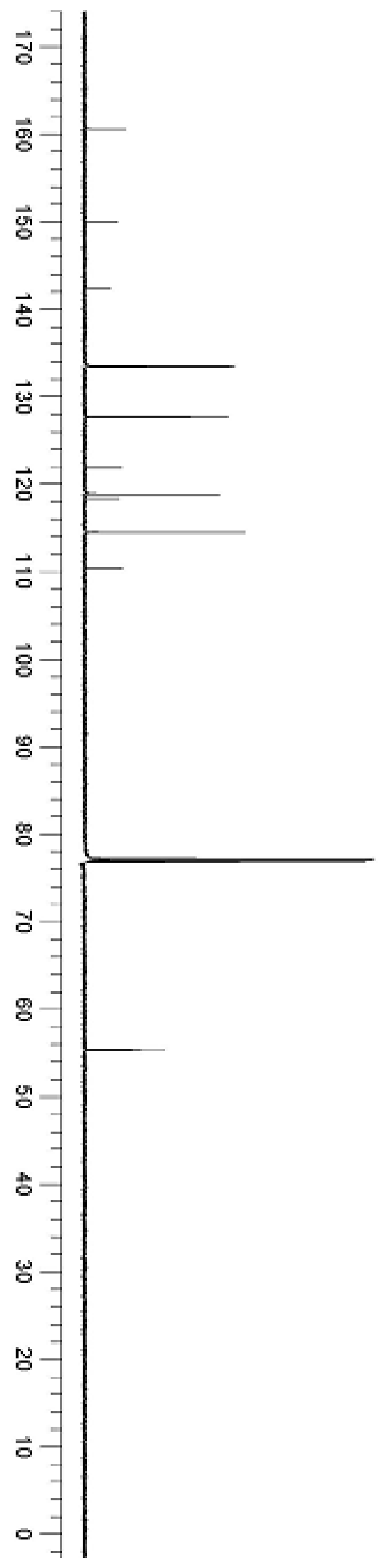

C 150MHz NMR

$$
3.3 \mathrm{~g}
$$




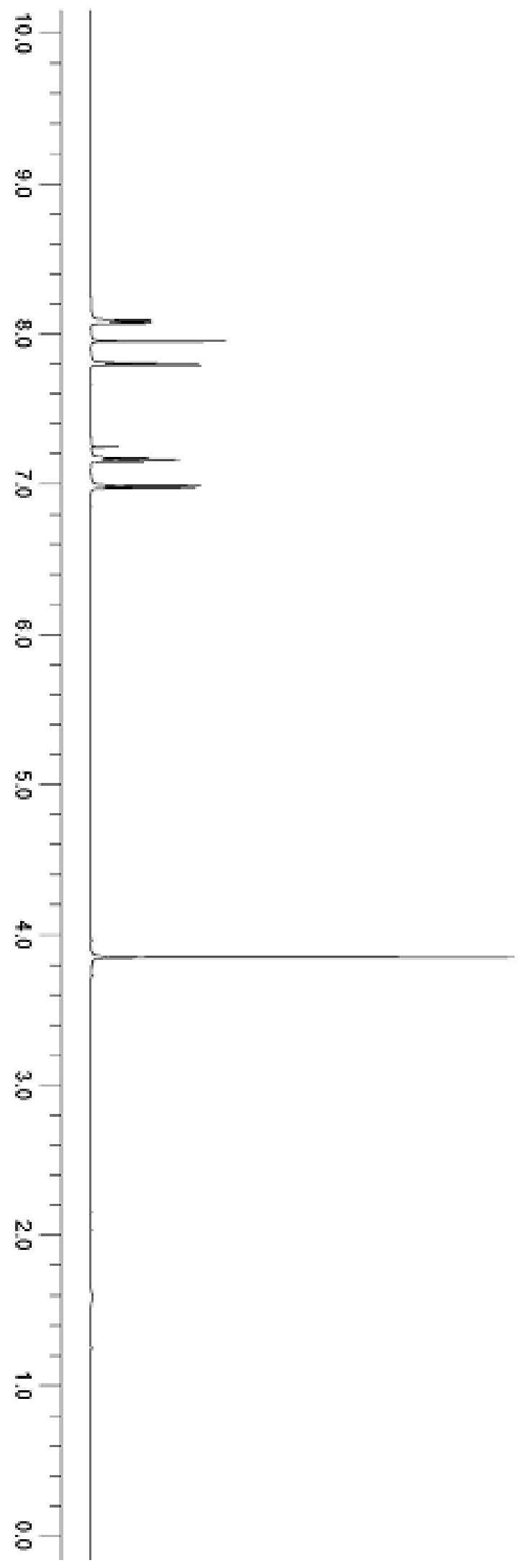

$3.3 \mathrm{~h}$ 


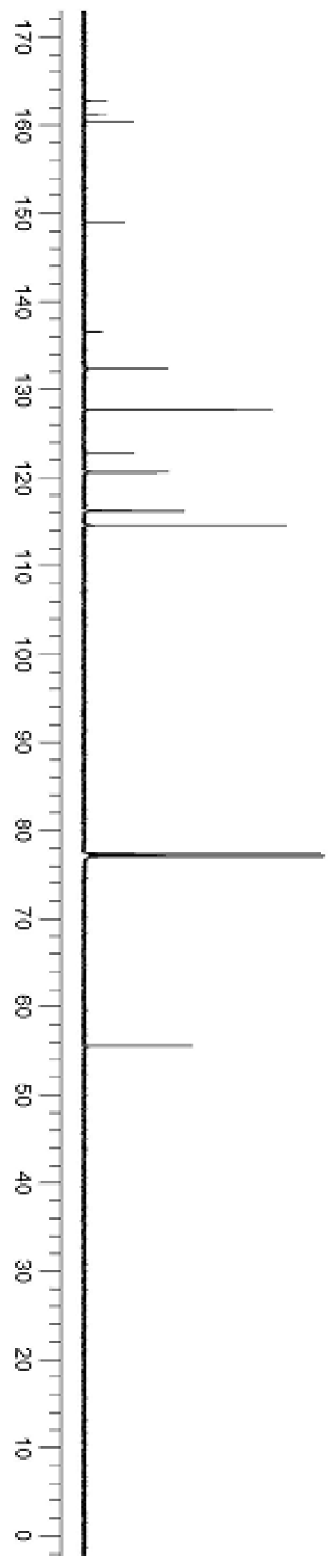




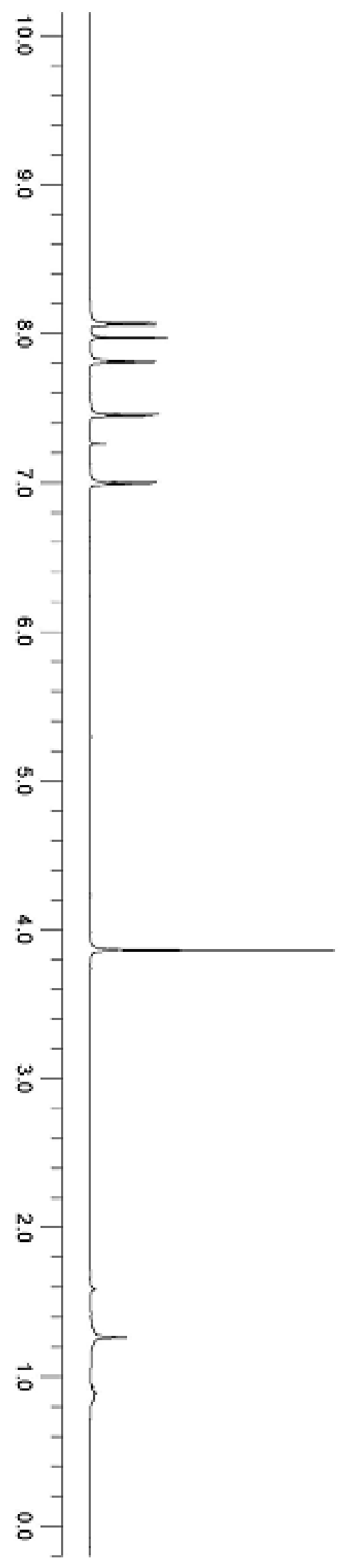

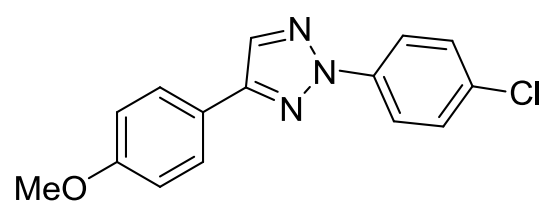

${ }^{1} \mathrm{H}$ 600MHz NMR 3.3i 

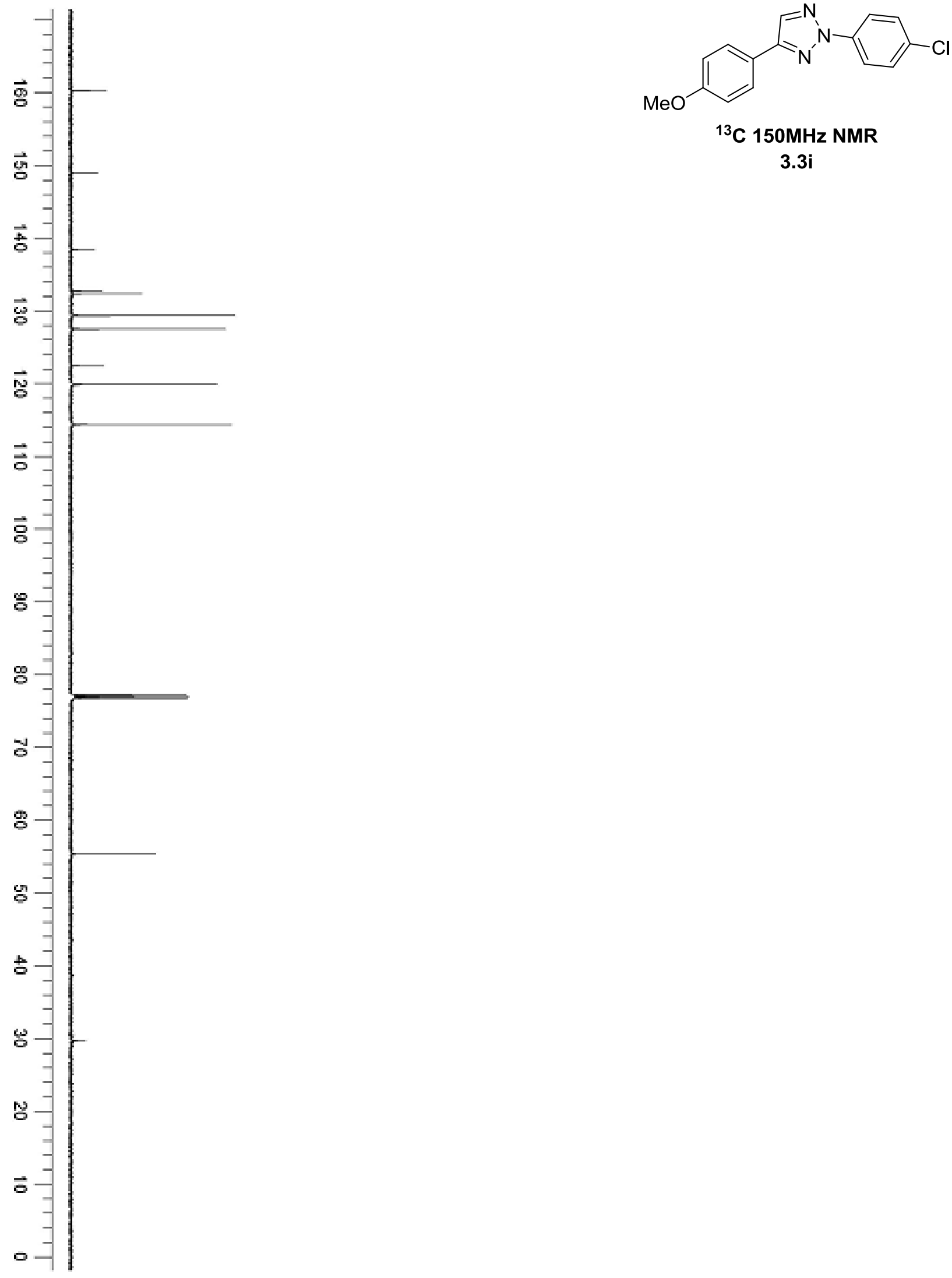

3.3i 


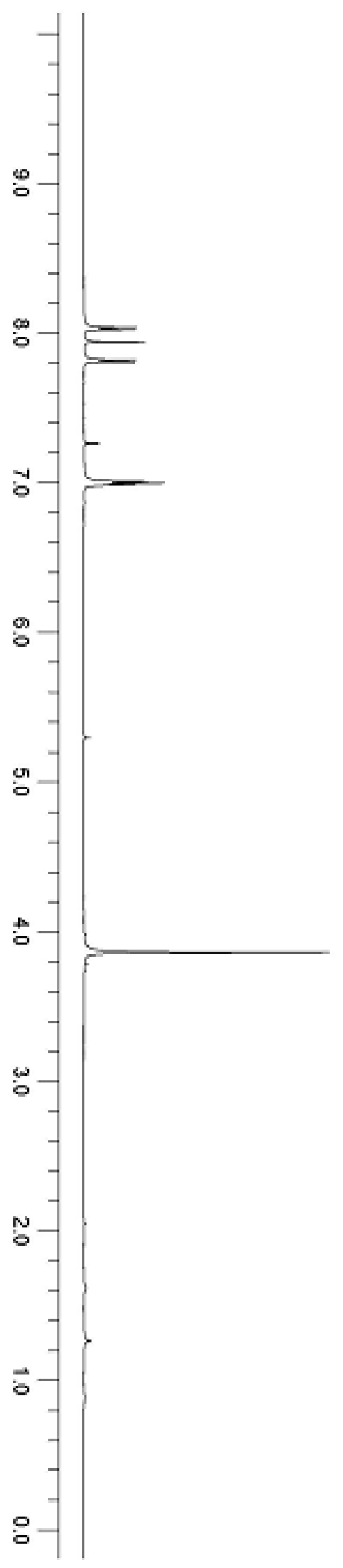

\section{3j}




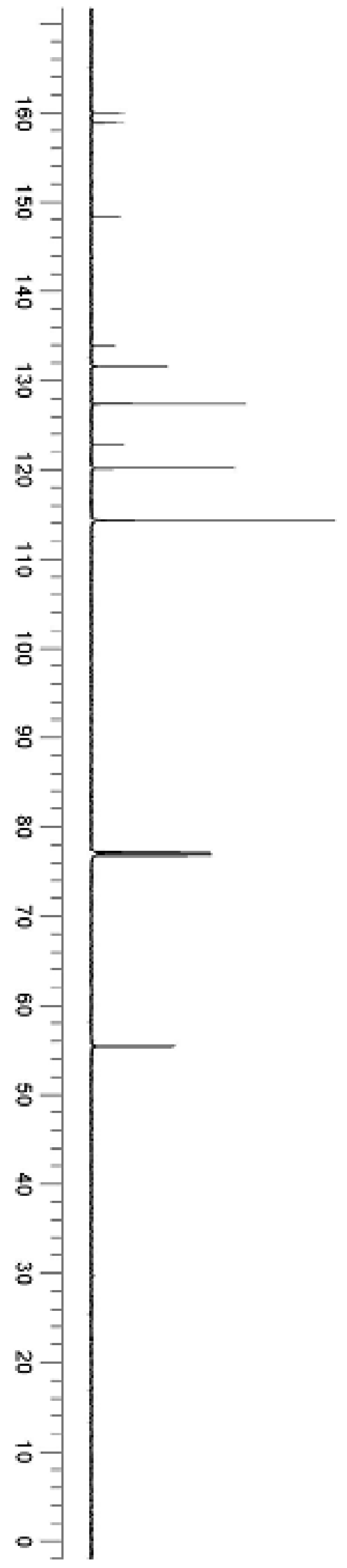

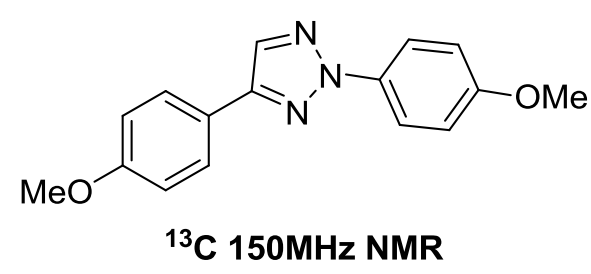

3.3j 


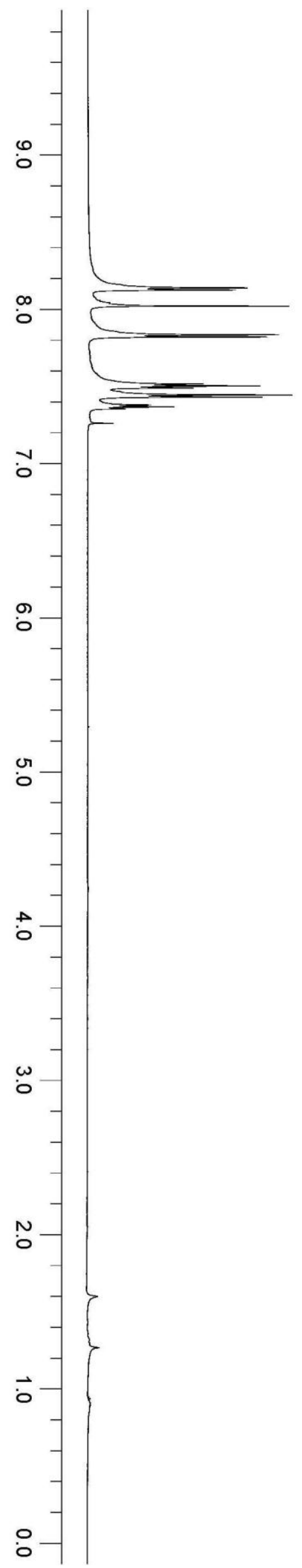

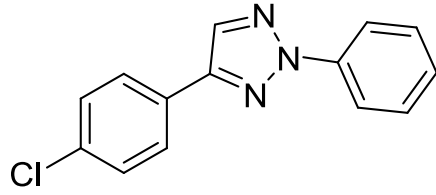

${ }^{1} \mathrm{H}$ 600MHz NMR $3.3 \mathrm{k}$ 

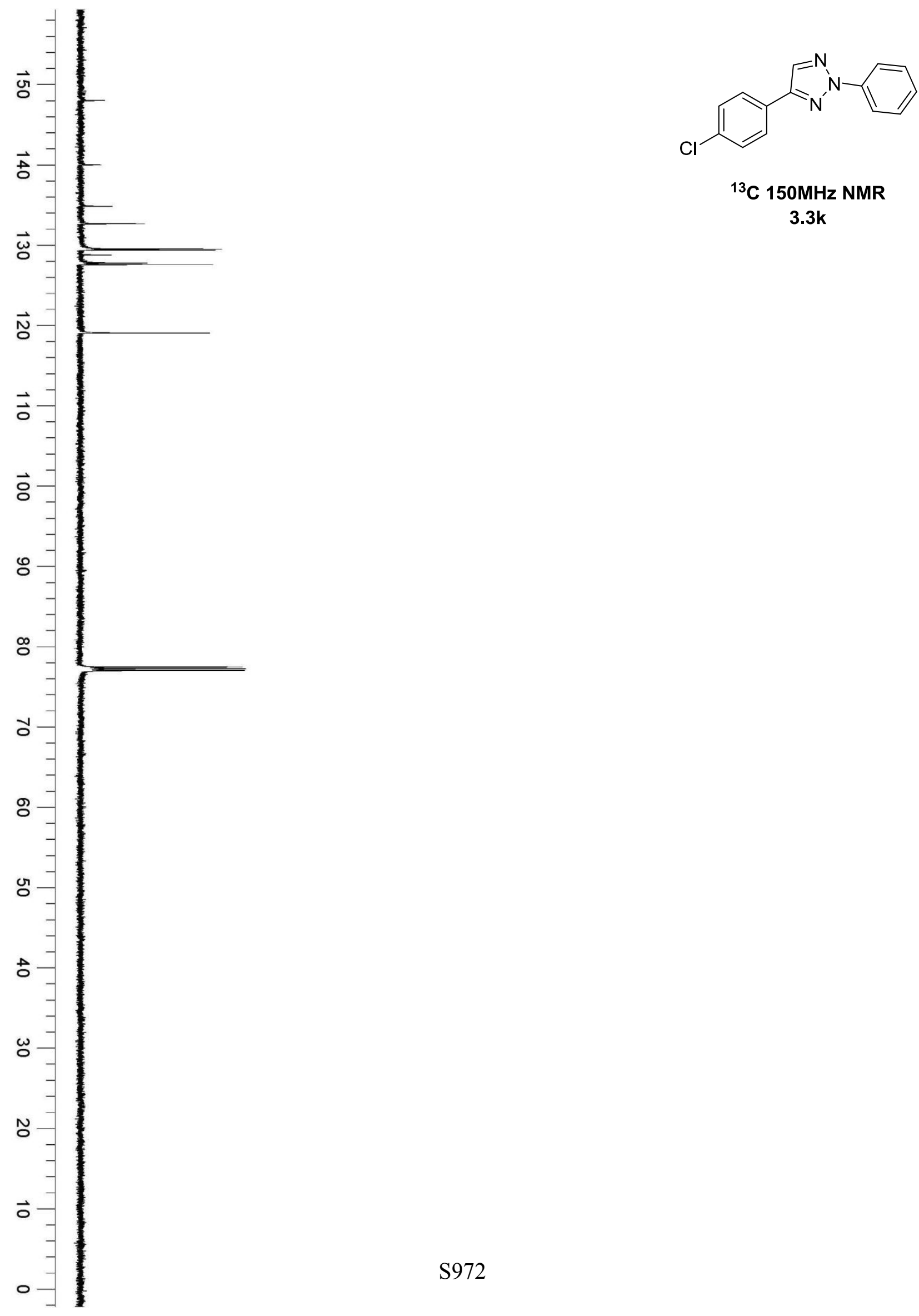

${ }^{13} \mathrm{C} 150 \mathrm{MHz}$ NMR

3.3k 


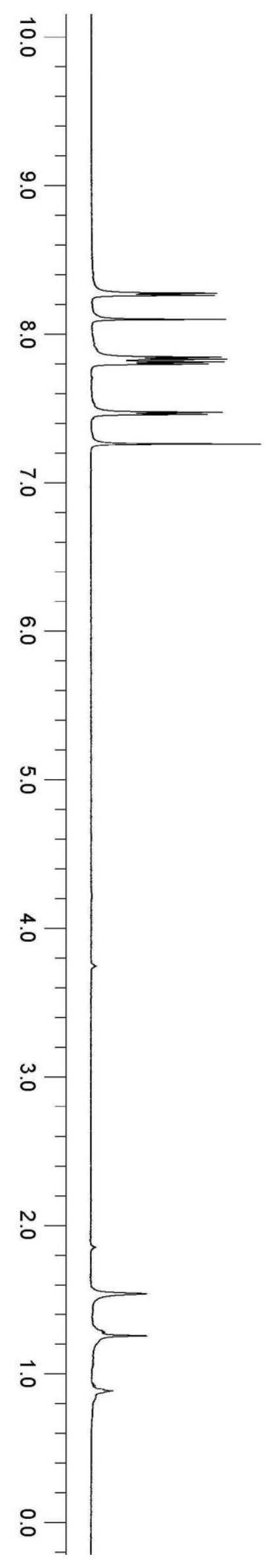

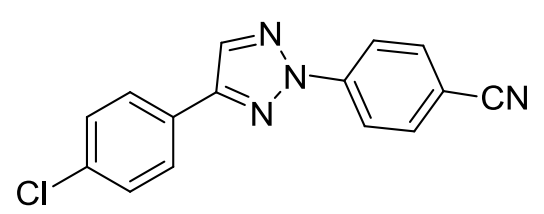

${ }^{1} \mathrm{H} 600 \mathrm{MHz}$ NMR

3.3I 

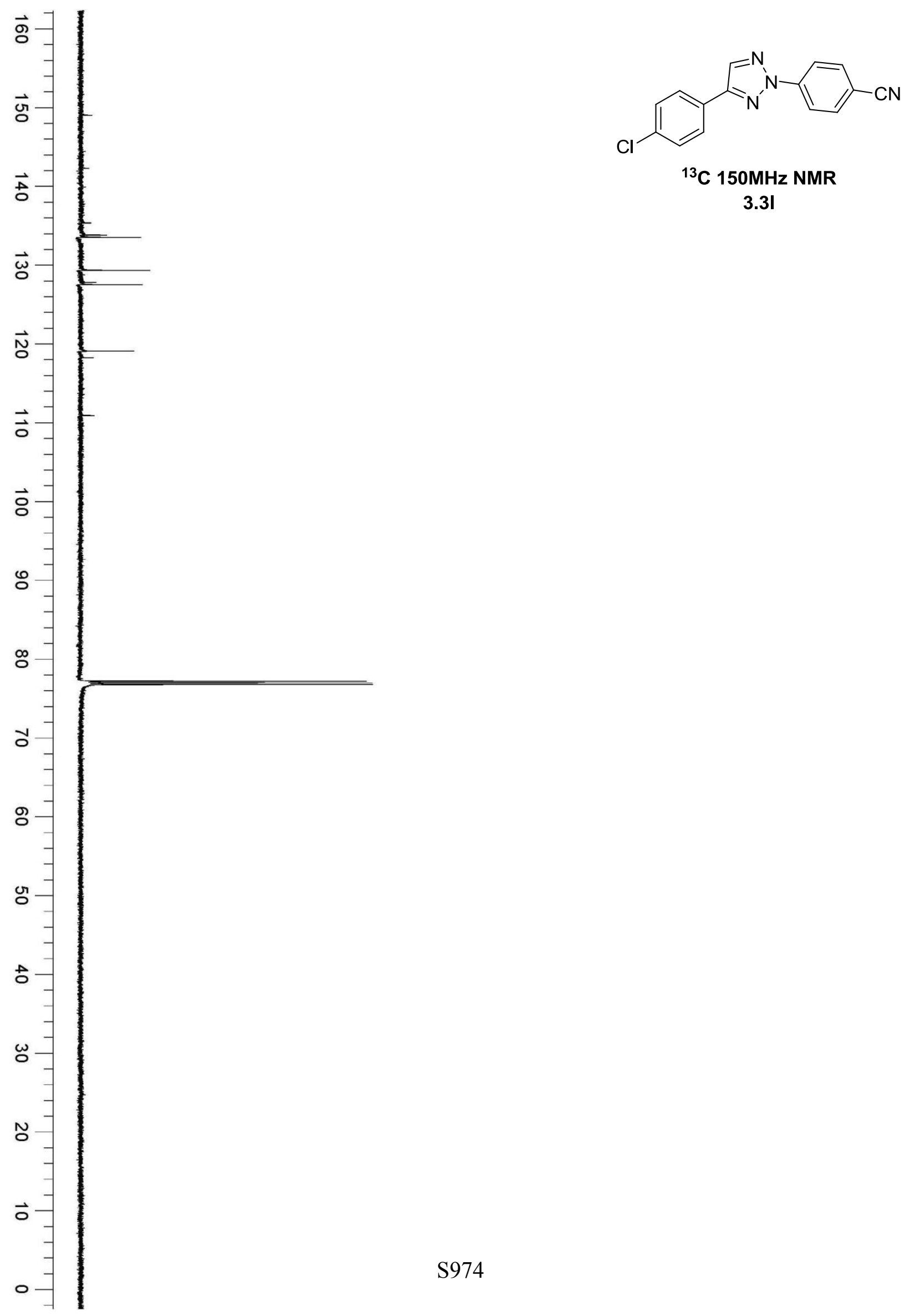

3.3I 


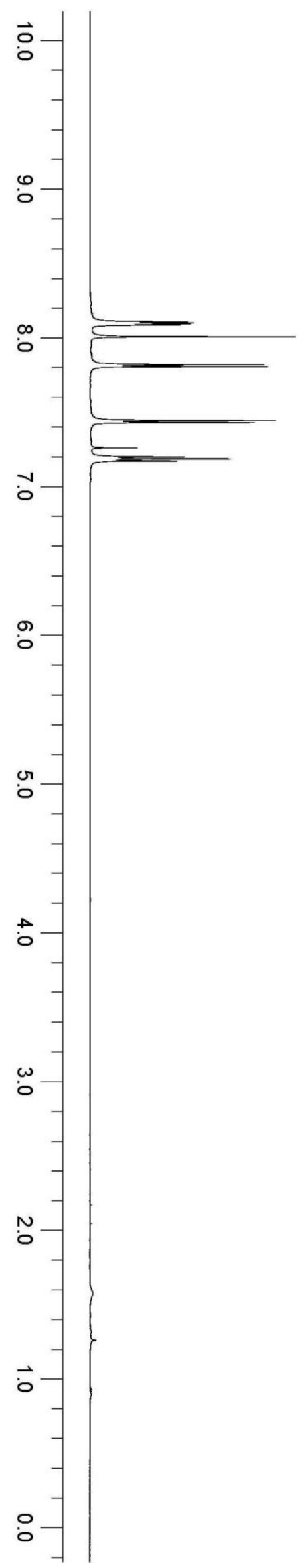

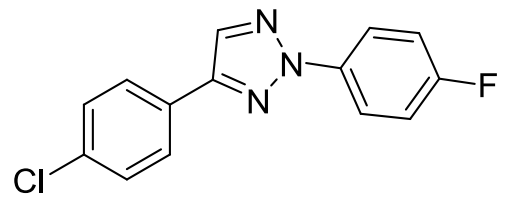

${ }^{1} \mathrm{H}$ 600MHz NMR

$3.3 \mathrm{~m}$ 

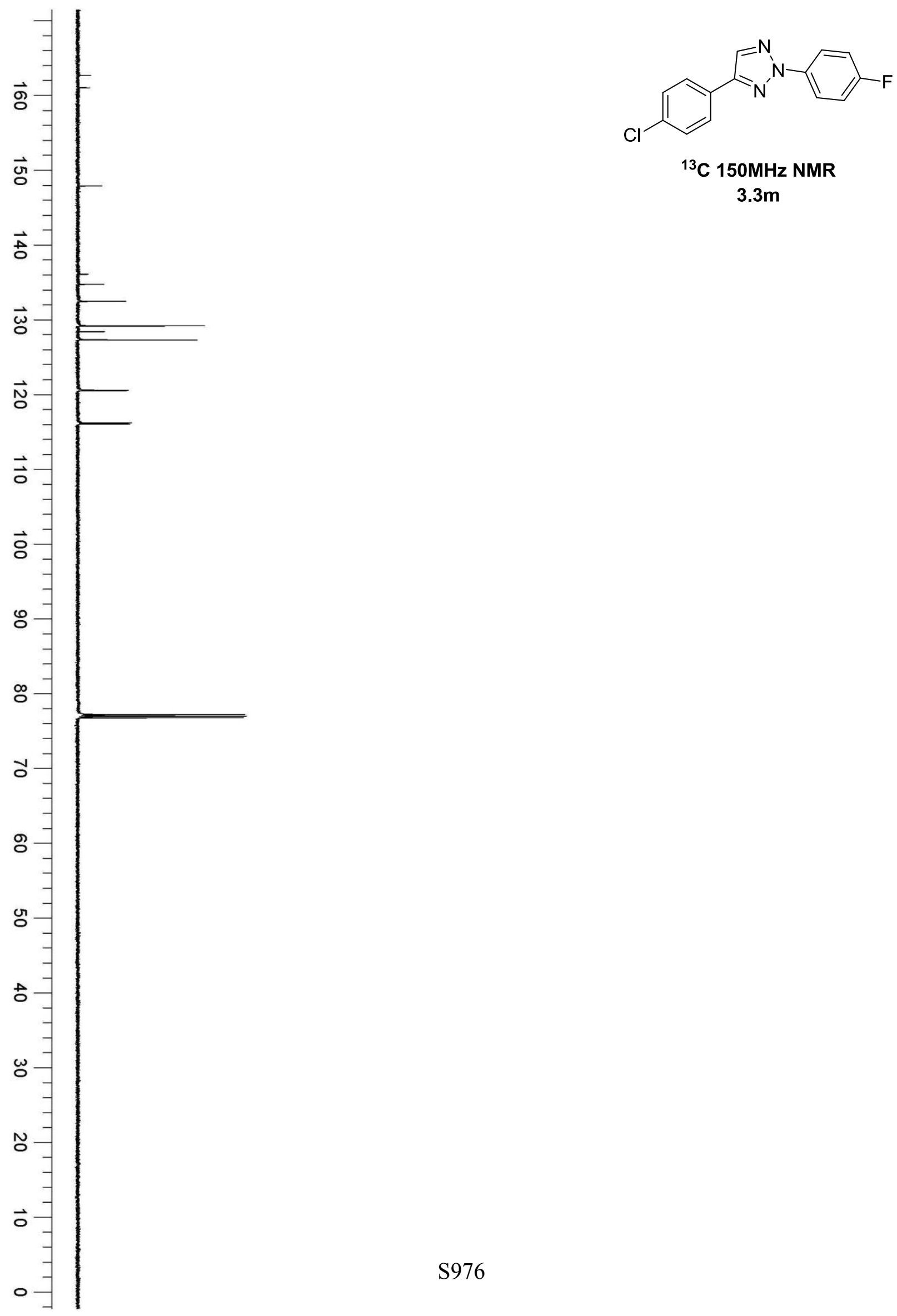

$3.3 \mathrm{~m}$ 


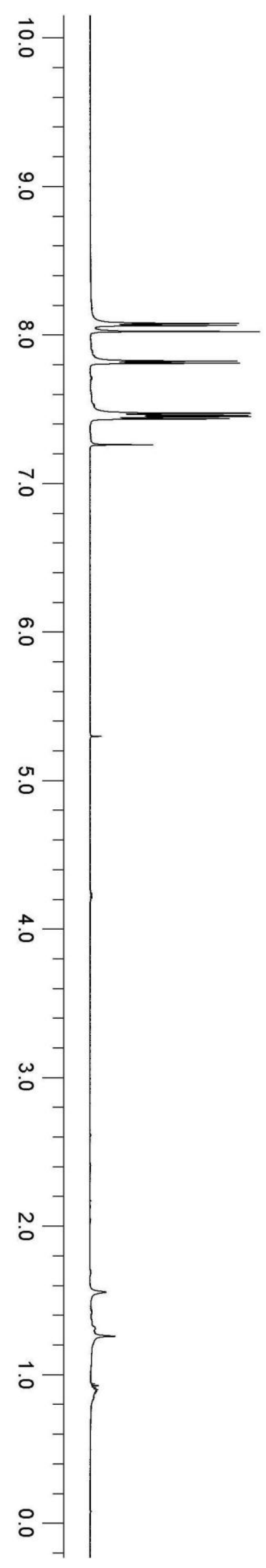

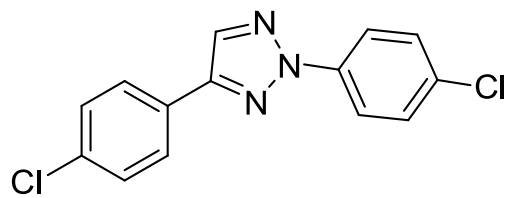

${ }^{1} \mathrm{H} 600 \mathrm{MHz}$ NMR

$3.3 n$ 

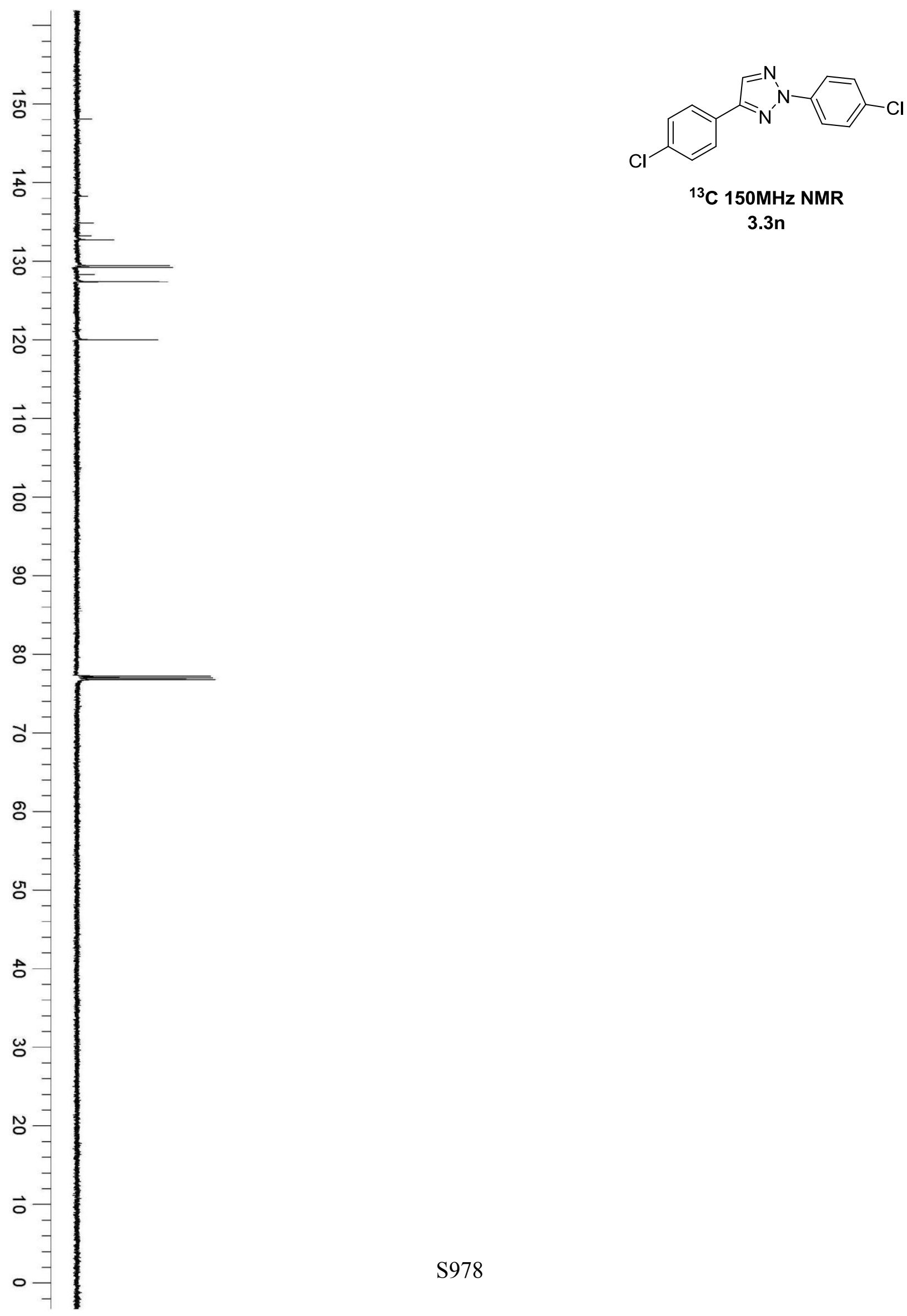


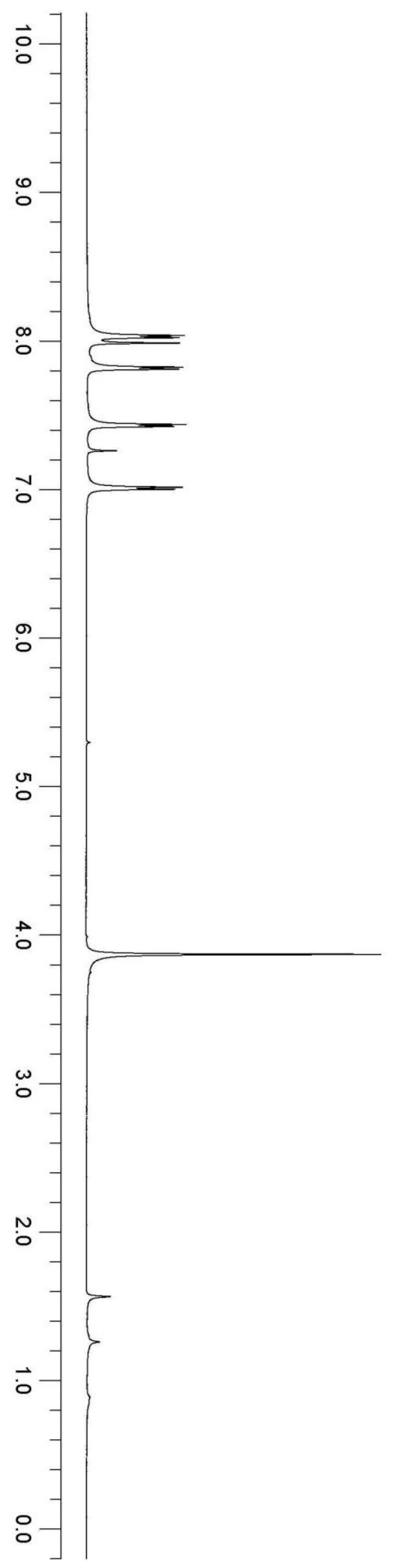

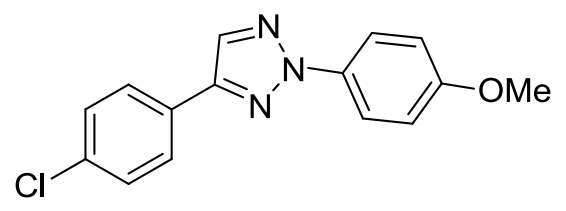

${ }^{1} \mathrm{H} 600 \mathrm{MHz}$ NMR

3.30 

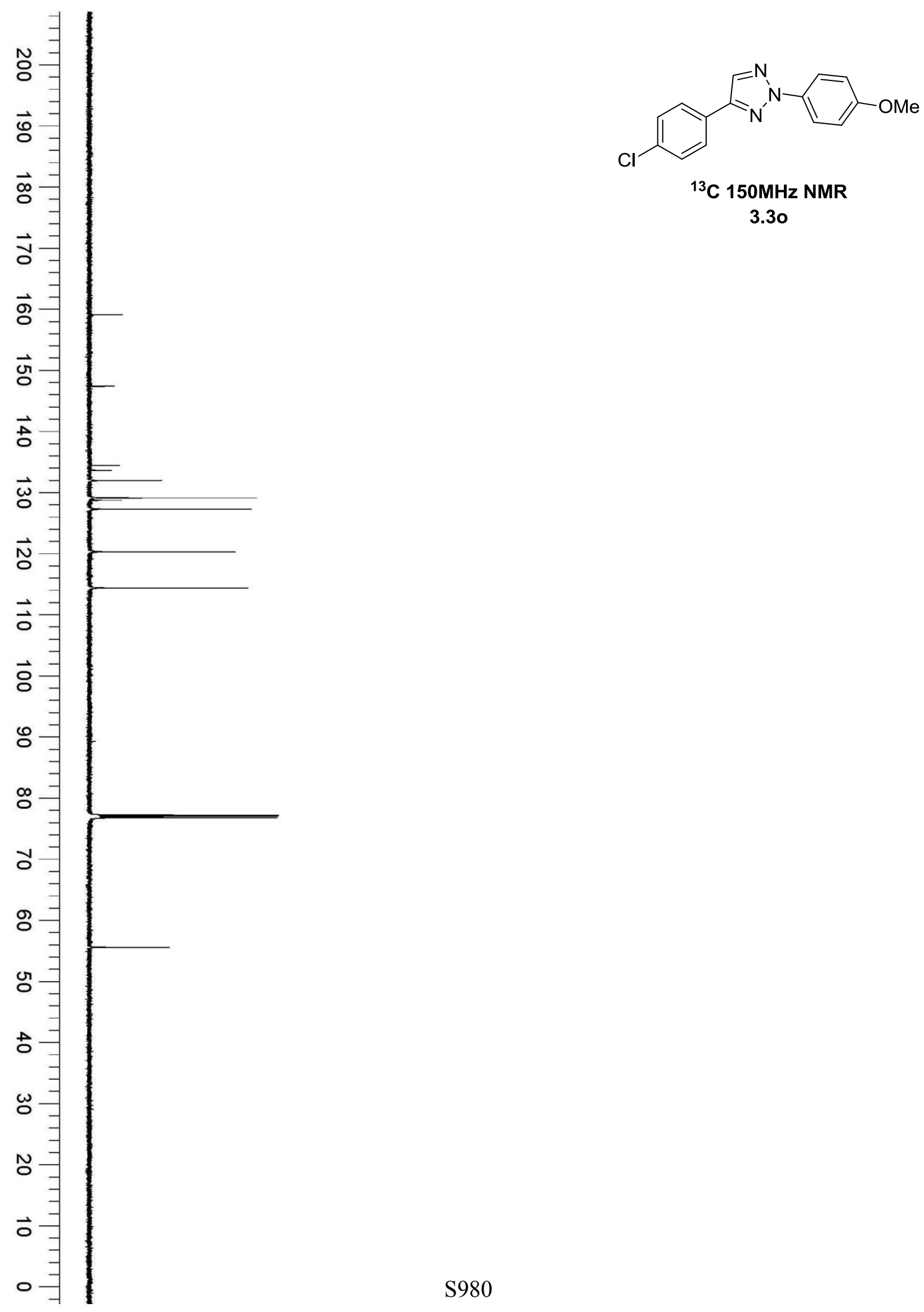

3.30 


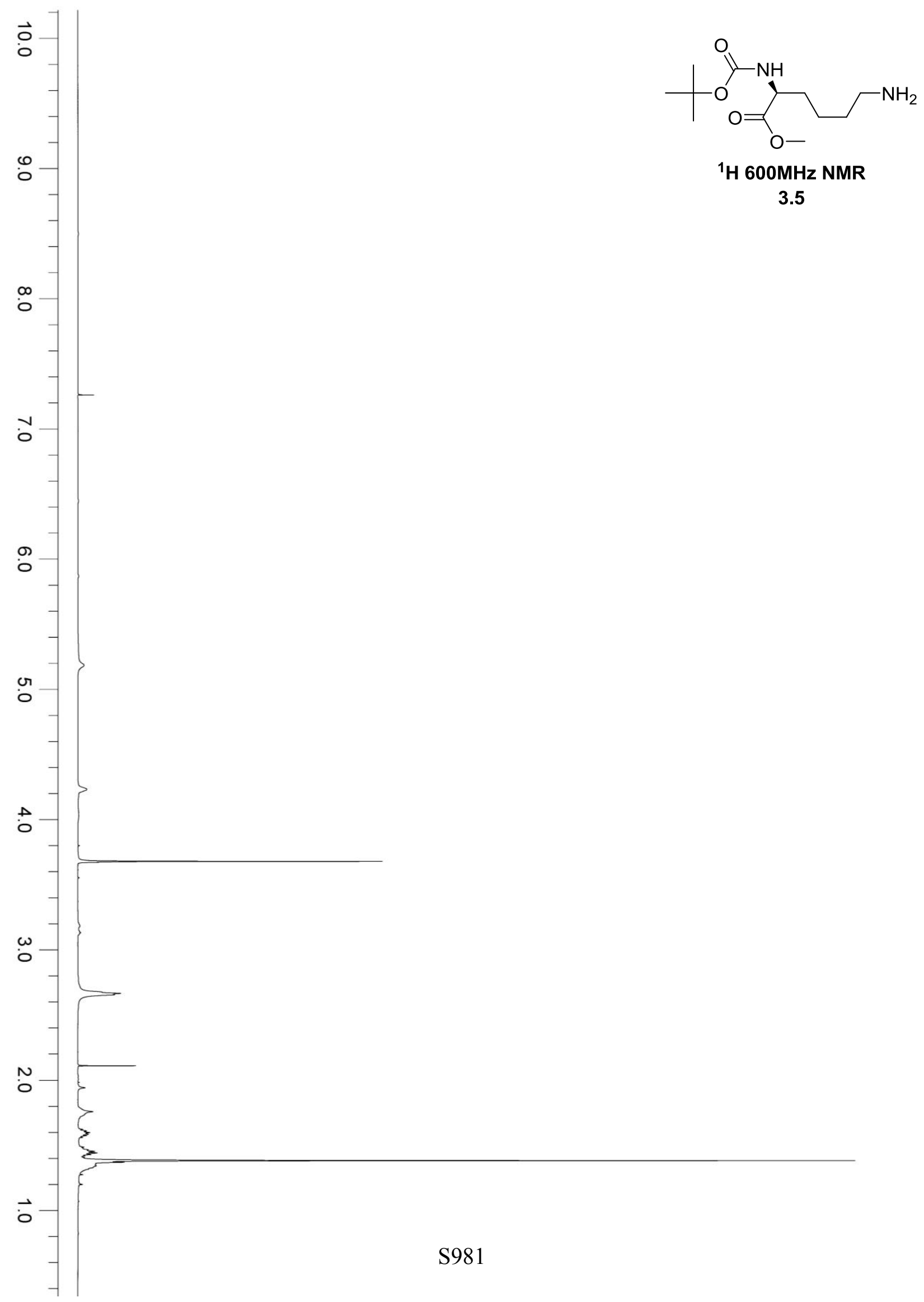



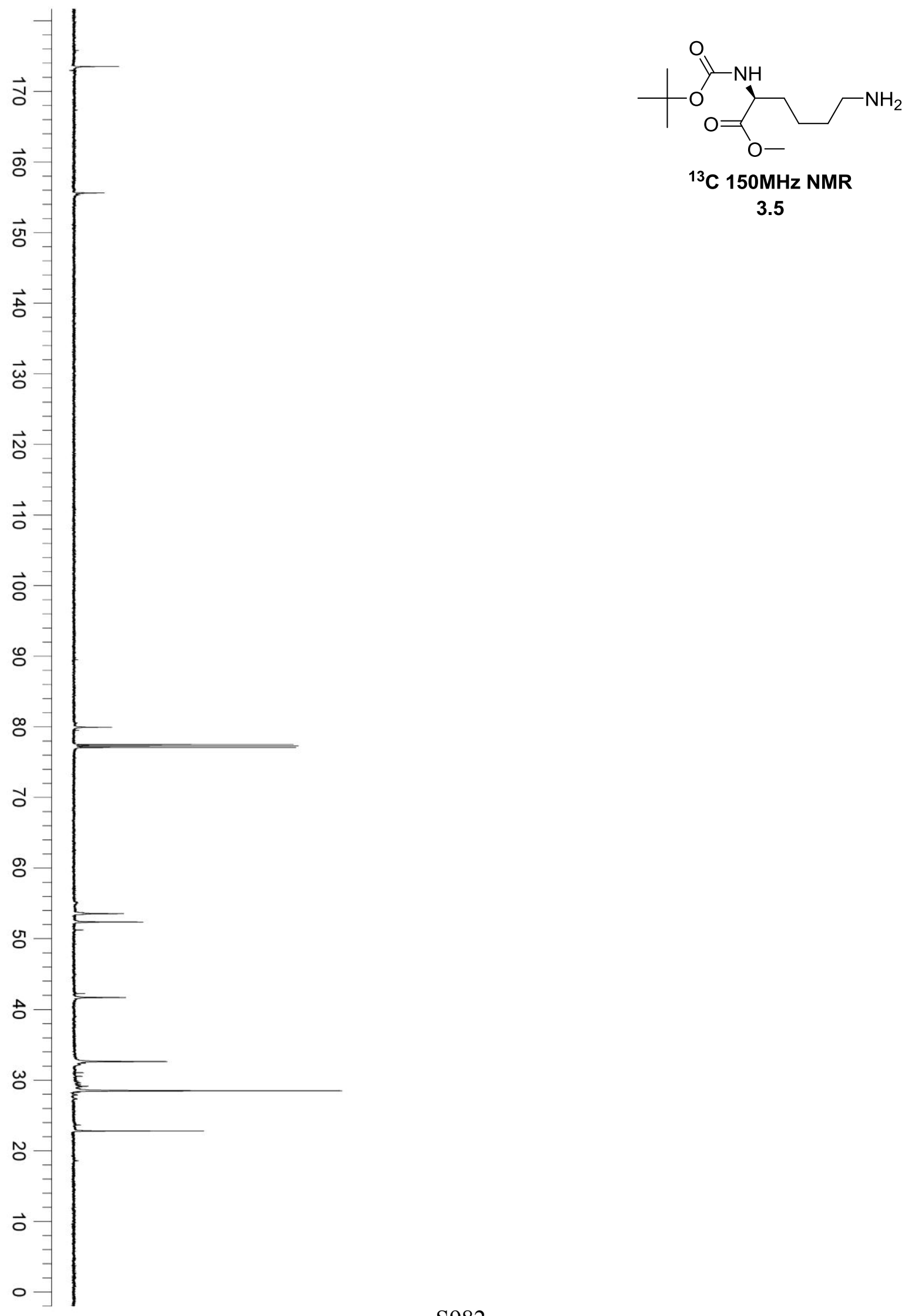

${ }^{13} \mathrm{C}$ 150MHz NMR

3.5 


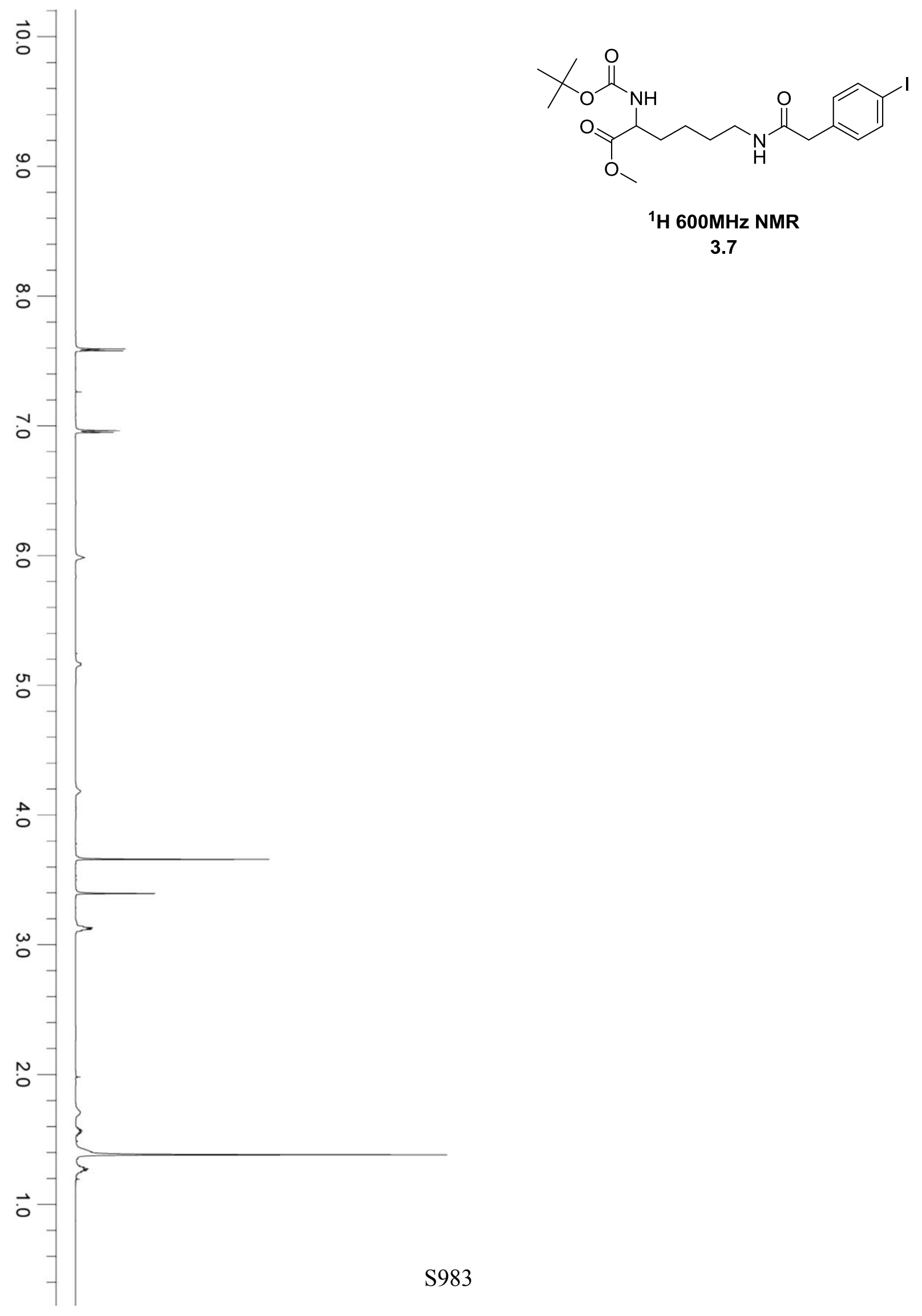




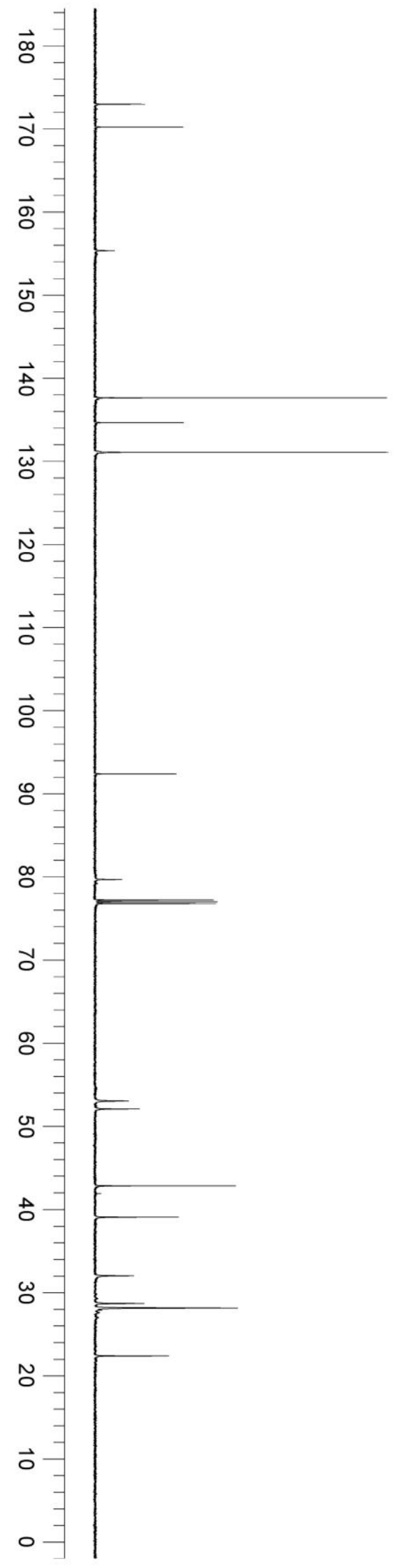

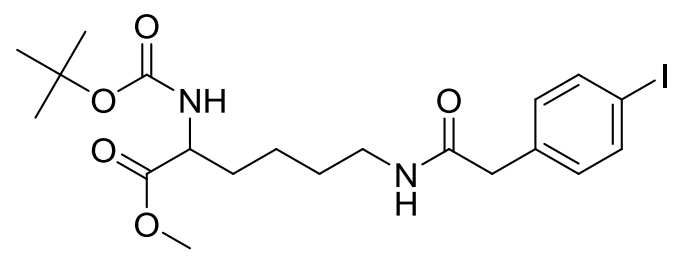

${ }^{13} \mathrm{C}$ 150MHz NMR

3.7 


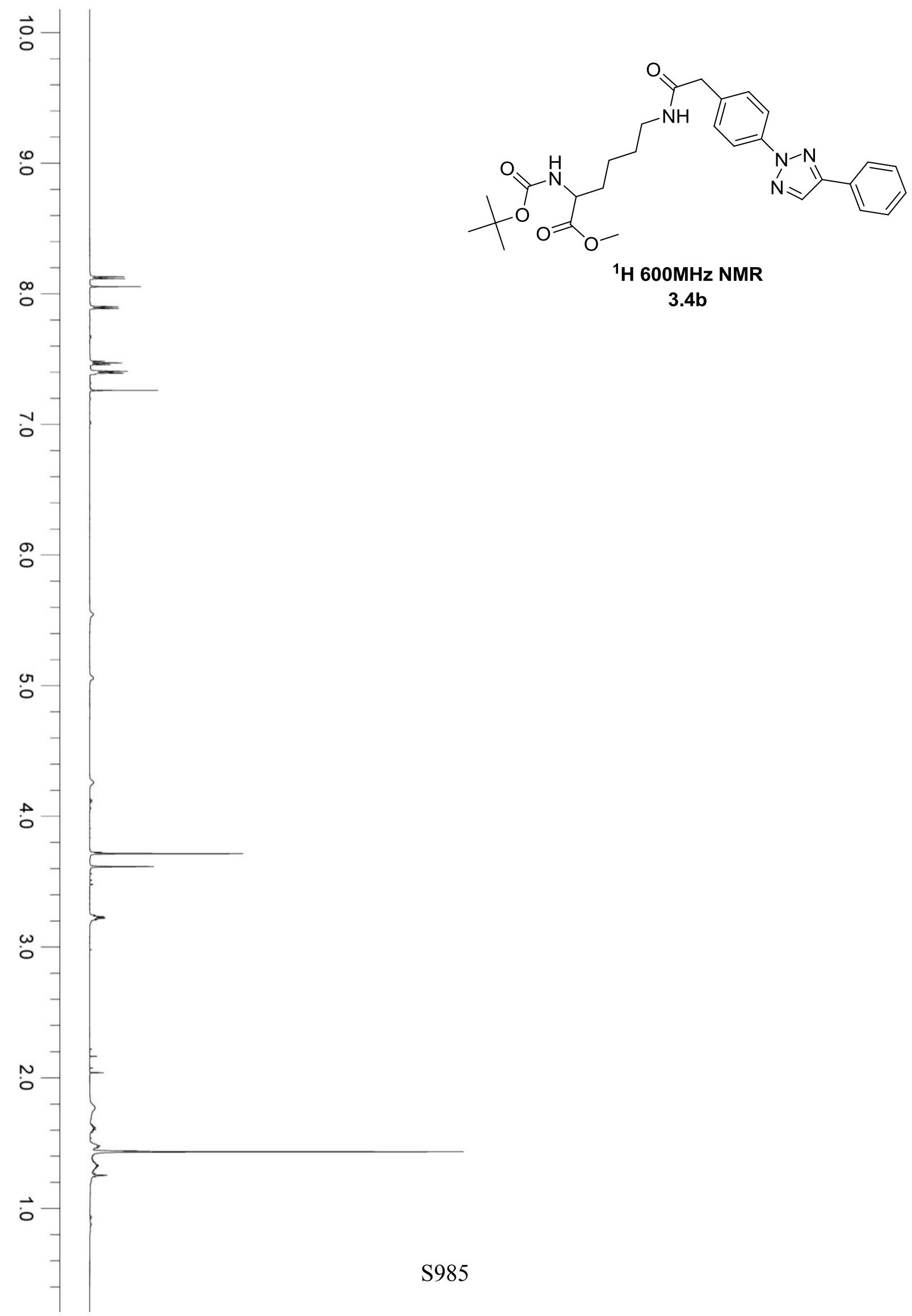




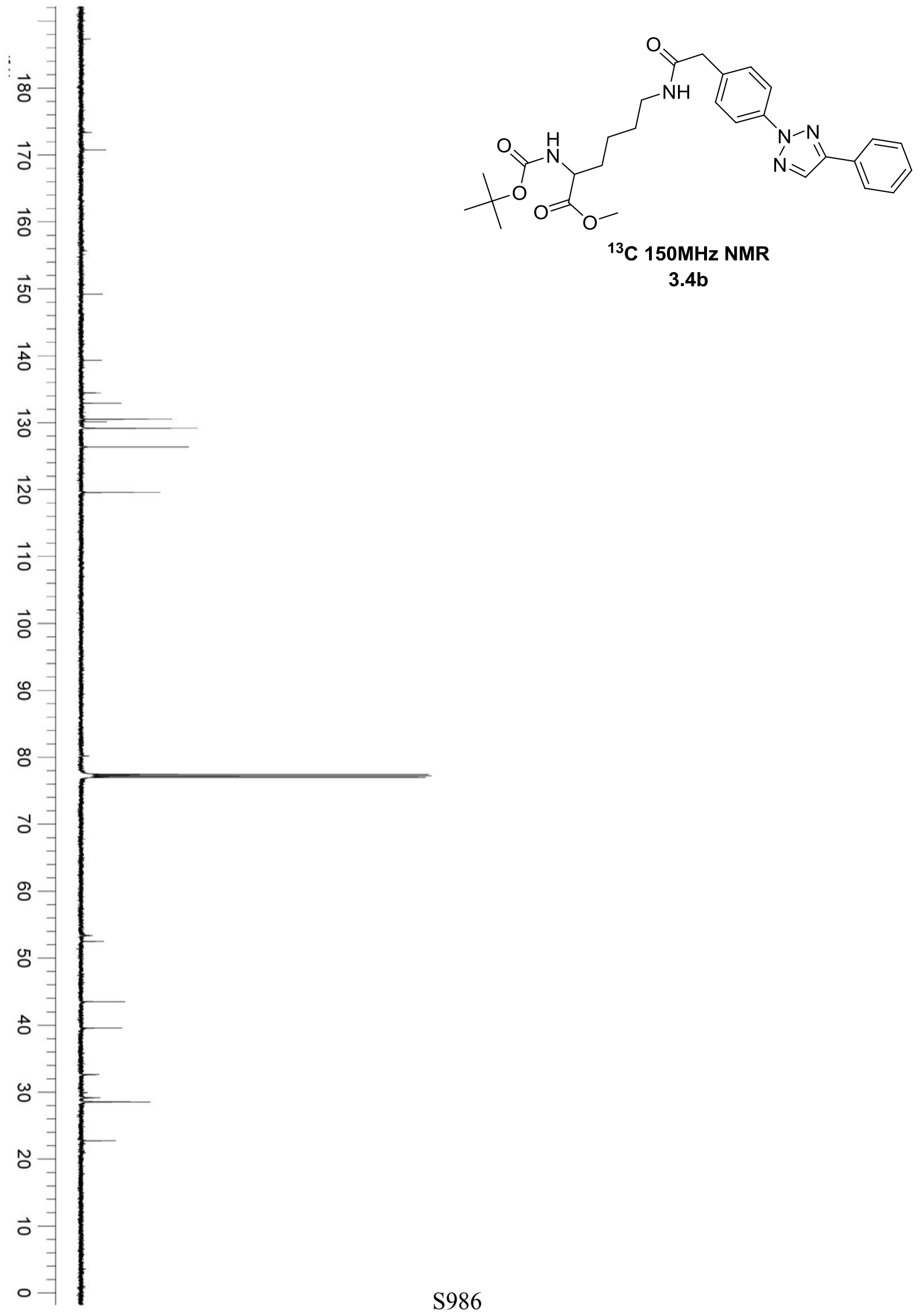

